Huayi CHEN Atsushi MORIWAKI

\title{
ARAKELOV GEOMETRY OVER ADELIC CURVES
}




\section{Huayi CHEN}

Institut de Mathématiques de Jussieu - Paris Rive Gauche, Université Paris Diderot.

E-mail : huayi.chen@imj-prg.fr

Atsushi MORIWAKI

University of Kyoto.

E-mail : moriwaki@math.kyoto-u.ac.jp 
ARAKELOV GEOMETRY

OVER ADELIC CURVES

Huayi CHEN, Atsushi MORIWAKI 



\section{CONTENTS}

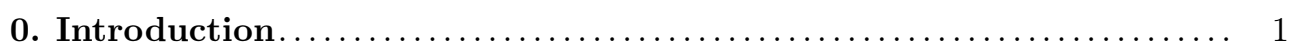

1. Metrized vector bundles: local theory $\ldots \ldots \ldots \ldots \ldots \ldots \ldots \ldots \ldots \ldots$

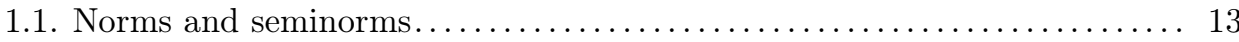

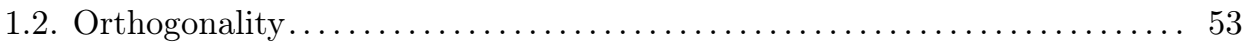

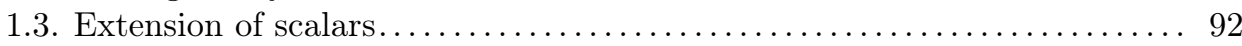

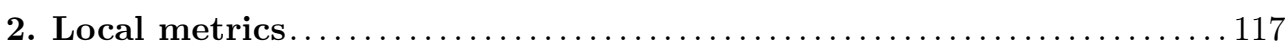

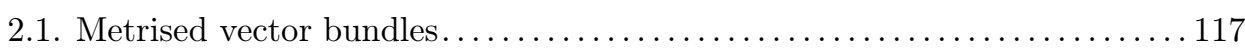

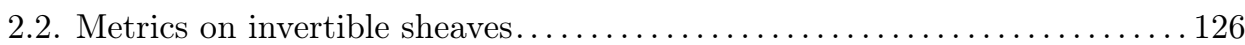

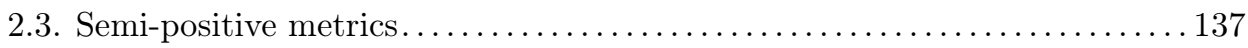

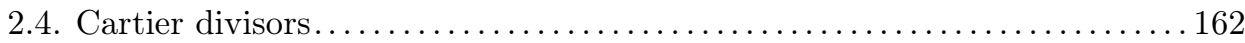

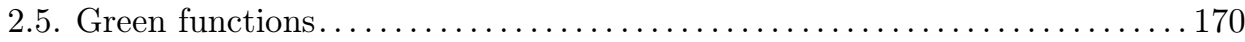

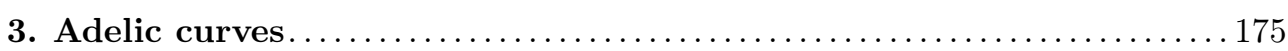

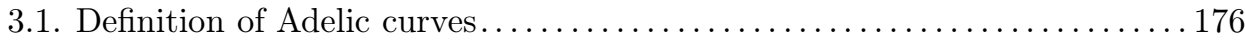

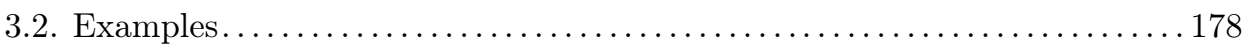

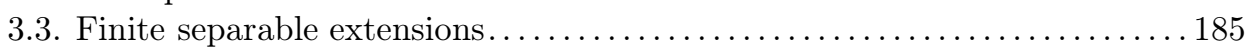

3.4. General algebraic extensions. . . . . . . . . . . . . . . . . . . . . . 195

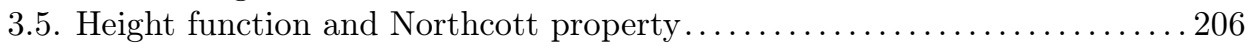

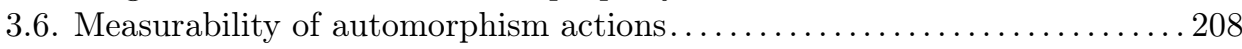

3.7. Morphisms of adelic curves....................................

4. Vector bundles on adelic curves: global theory $\ldots \ldots \ldots \ldots \ldots \ldots \ldots 213$

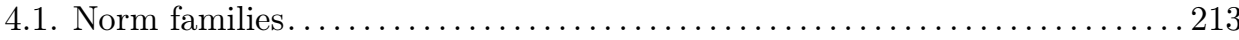

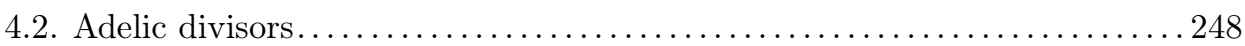

4.3. Arakelov degree and slopes............................... 249

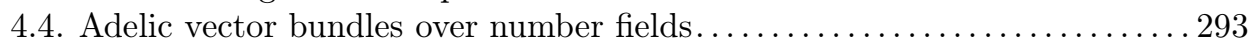

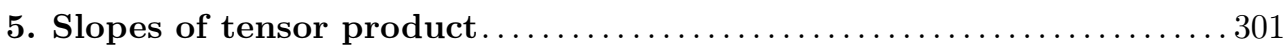

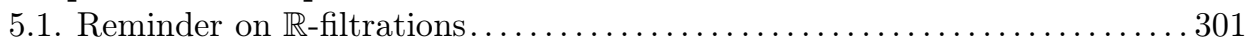


5.2. Reminder on geometric invariant theory ....................... 305

5.3. Estimate for the minimal slope under semi-stability assumption.........313

5.4. An interpretation of the geometric semistability .............. 315

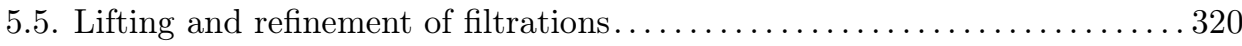

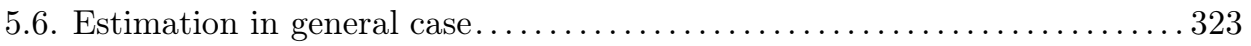

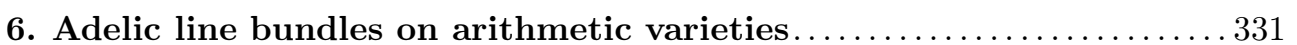

6.1. Metrised line bundles on an arithmetic variety . . . . . . . . . . . . 331

6.2. Adelic line bundle and Adelic divisors. . . . . . . . . . . . . . . . . . . . 349

6.3. Okounkov bodies and concave transform ......................... 359

6.4. Asymptotic invariants of graded linear series................... 384

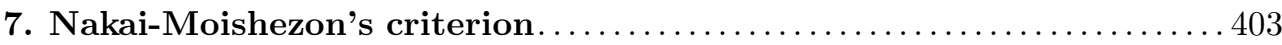

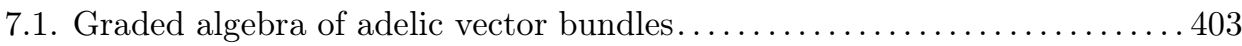

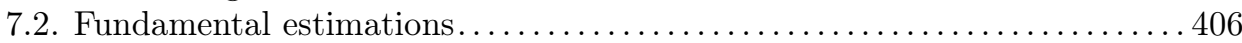

7.3. A consequence of the extension property of semipositive metrics....... 411

7.4. Nakai-Moishezon's criterion in a general settings . . . . . . . . . . . 413

7.5. Nakai-Moishezon's criterion over a number field .................416

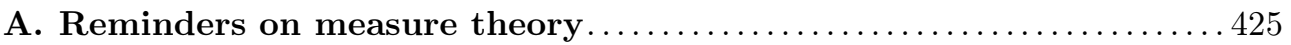

A.1. Monotone class theorems.................................. 425

A.2. Measurable selection theorem ............................. 427

A.3. Vague convergence and weak convergence of measures.............. 427

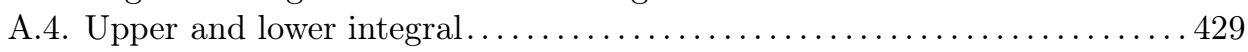

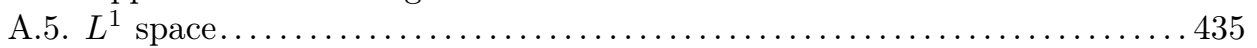

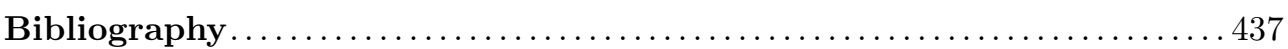




\section{CHAPTER 0}

\section{INTRODUCTION}

The purpose of this book is to build up the fundament of an Arakelov theory over adelic curves in order to provide a unified framework for the researches of arithmetic geometry in several directions.

Let us begin with a brief description of the main ideas of Arakelov geometry. In number theory, it is well known that number fields are similar to fields of rational functions over algebraic curves defined over a base field, which is often assumed to be finite. It is expected that the geometry of schemes of finite type over $\mathbb{Z}$ should be similar to the algebraic geometry of schemes of finite type over a regular projective curve. However, some nice properties, especially finiteness of cohomological groups (in the case where the base field is finite), fail to hold in the arithmetic setting, which prevents using geometrical methods to count the arithmetic objects. The core problem is that schemes over Spec $\mathbb{Z}$, even projective, are not "compact", and in general it is not possible to "compactify" them in the category of schemes. The seminal works of Arakelov [4, 3] propose to "compactify" a scheme of finite type over Spec $\mathbb{Z}$ by transcendental objects, such as the associated complex analytic variety, Hermitian metrics, Green functions, and differential forms etc. In the case of relative dimension zero, the idea of Arakelov corresponds to the classic approach in algebraic number theory to include the infinite places of a number field to obtain a product formula, and to introduce Hermitian norms on projective modules over an algebraic integer ring to study the geometry of numbers and counting problems. Most interestingly, the approach of Arakelov proposes an intersection theory for divisors on a projective arithmetic surface (relative dimension one case), which is similar to the intersection pairing of Cartier divisors on classic projective surfaces.

The works of Arakelov have opened a gate to a new geometric theory of arithmetic varieties (schemes of finite type over Spec $\mathbb{Z}$ ). Inspired by the classic algebraic geometry, many results have been obtained and enriched Arakelov's geometry. Among the wide literature, we can mention for example the arithmetic Hodge index theorem 
by Faltings [57 and Hriljac [84, arithmetic intersection theory of higher dimensional arithmetic varieties and arithmetic Riemann-Roch theorem by Gillet and Soulé [66, 67], see also 65. Arakelov geometry also provides an alternative approach (compared to the classic Weil height theory) to the height theory in arithmetic geometry, see $[4,3,133,58,22$, (see also the approach of Philippon [115, 116], and [129] for the comparison between Philippon height and Arakelov height). The Arakelov height is often more precise than the Weil height machine since the choice of a Hermitian metric on a line bundle permits to construct an explicit height function associated with that line bundle (in the Weil heigh machine, the height function is defined only up to a bounded function).

These advancements have led to fruitful applications in number theory, such as the proof of Mordell's conjecture by Faltings [55, [56] and the alternative proof by Vojta 139. (see also the proof of Bombieri [11] and the generalisation of Vojta's approach to the study of subvarieties in an Abelian variety [58]), equidistribution of algebraic points in an arithmetic variety and applications to Bogomolov's conjecture 132, 137, 150, algebraicity of formal leaves of algebraic foliation [17, etc.

Although the philosophy of Arakelov allows to inspire notions and results of algebraic geometry and has already led to a rich arithmetic theory, the realisation of Arakelov theory is rather different from that of the classic algebraic geometry and usually gets involved subtle tools in analysis. The transition of technics on the two sides is often obscure. For example, the $a b c$ conjecture, which can be easily established in the function field setting by algebraic geometry tools (see [99]), turns out to be very deep in the number field setting. Conversely, the Bogomolov's conjecture has been resolved in the number field setting, before the adaptation of its proof in the function field setting by using Berkovich analytic spaces (see [77, 142, 143). It is therefore an interesting problem to provide a uniform fundament for Arakelov geometry, both in the function field and number field settings, and the adelic approach is a natural choice for this goal. We would like however to mention that Durov [52] has proposed an approach of different nature to algebrify the Arakelov geometry over number fields.

The theory of adèles in the study of global fields was firstly introduced by Chevalley [46. Chapitre III] for function fields and by Weil [140] for number fields. This theory allows to consider all places of a global filed in a unified way. It also leads to a uniform approach in the geometry of numbers in global fields, either via the adelic version of Minkowski's theorems and Siegel's lemma developed by McFeat [101], BombieriVaaler [10, Thunder [135, Roy-Thunder [121, or via the study of adelic vector bundles developed by Gaudron [60, generalising the slope theory introduced by Bost 15, 17.

Several works have been realised in the adelification of Arakelov theory. Besides the result of Gaudron on adelic vector bundles over global fields mentioned above, 
we can for example refer to 149 for adelic metrics on arithmetic line bundles and applications to the Bogomolov problem for cycles. Moreover, Moriwaki [108] has studied the birational geometry of adelic line bundles over arithmetic varieties. The key point is to consider an arithmetic variety as a scheme of finite type over a global field, together with a family of analytic varieties (possibly equipped with metrised vector bundles) associated with the scheme, which is parametrised by the set of all places of the global field. Classic objects in Arakelov geometry can be naturally considered in this setting. For example, given a Hermitian line bundle over a classic arithmetic variety (scheme of finite type over $\operatorname{Spec} \mathbb{Z}$ ), the algebraic structure of the line bundle actually induces, for each finite place of $\mathbb{Q}$, a metric on the pull-back of the line bundle on the corresponding analytic space.

In this book, we introduce the notion of adelic curves and develop an Arakelov theory over them. By adelic curve we mean a field equipped with a family of absolute values parametrised by a measure space, such that the logarithmic absolute value of each non-zero element of the filed is an integrable function on the measure space, with 0 as its integral. This property is called product formula. Note that this notion has been studied by Gubler [76 in the setting of height theory and is also considered by Ben Yaakov and Hrushovski [7, 85 in a recent work on model theory of global fields. Clearly the notion of adelic curve generalises the classic one of global field, where the measure space is given by the set of all places of the global field equipped with the discrete measure of local degrees. However, this is certainly not the only motivation for the general notion of adelic curves. Our choice is rather inspired by several bunches of researches which are apparently transversal to each other, which we will resume as follows (we will explain further the reason for the choice of terminology "adelic curve").

1. Finitely generated extensions of a number field. From a point of view of birational geometry, we expect that the field of rational functions of an algebraic variety determines the geometric properties of the variety. In Arakelov geometry, we consider integral schemes of finite type over $\operatorname{Spec} \mathbb{Q}$, whose function field is a finitely generated extension of $\mathbb{Q}$. Moriwaki [102] has developed an Arakelov height theory for varieties over a finitely generated extension of a number field and applied it to the study of Bogomolov problem over such a field (see [103, see also [104] for a panoramic view). Burgos, Philippon and Sombra 33 have expressed the height of cycles in a projective variety over a finitely generated extension of $\mathbb{Q}$ as an integral of local heights over the set of places of the field.

2. Trivially valued field. In number theory, we usually consider non-trivial absolute values on fields. Note that on any field there exists a trivial absolute value which takes value 1 on each non-zero element of the field. Note that a trivial product formula is satisfied in this setting. Although the trivially valued fields are very simple, the corresponding geometry of numbers is rather rich, which has wide interactions with 
the classic geometry of lattices or Hermitian vector bundles. In fact, given a finitedimensional vector space over a trivially valued field, the ultrametric norms on it are canonically in bijection to the decreasing $\mathbb{R}$-filtrations on the vector space. The $\mathbb{R}$-filtration is a key method of the works $[\mathbf{3 8}, \mathbf{3 5}, \mathbf{3 7}$, where the main idea consists in associating to each Hermitian vector bundle an $\mathbb{R}$-filtration on the generic fibre, which captures the arithmetic information such as successive minima or successive slopes.

3. Harder-Narasimhan theory for vector bundles on higher dimensional varieties. Harder and Narasimhan theory [81] is an important tool in the study of vector bundles on a projective curve. In the geometry of Euclidean lattices, the counterpart of Harder-Narasimhan theory has been proposed by Stuhler 131 and Grayson [68. Later Bost [15] has generalised their work in the setting of Hermitian vector bundles on the spectrum of the ring of algebraic integers in a number field. Moreover, he has developed the slope inequalities in this framework and applied them to the study of algebraicity of formal schemes [17, 18, 19. Note that the slope function and the notion of semistability can be naturally defined for torsion-free coherent sheaves on a polarised projective variety [134. This allows Shatz [128] and Maruyama 98 to develop a Harder-Narasimhan theory for general torsion-free coherent sheaves. However, it seems that the analogue of their results in the arithmetic case is still missing.

4. Fields of algebraic numbers, Siegel fields. The geometry of numbers for algebraic (not necessarily finite) extensions plays an important role both in Diophantine problems and in Arakelov geometry. Recall that the Minkowski's theorem and Siegel's lemma in geometry of numbers admit an adelic version for number fields, see [10, 101]. They also have an absolute counterpart over $\overline{\mathbb{Q}}$, see [121, 122, 148]. In Arakelov geometry, a notion of Hermitian vector bundle over $\overline{\mathbb{Q}}$ has been proposed in the work [21] of Bost and Chen, on which the absolute Siegel's lemma applies and is useful in the study of tensorial semistability of classic Hermitian vector bundles. Similarly, in the approach of Gaudron and Rémond $\mathbf{6 2}$ to the tensorial semistability, the absolute Siegel's lemma is also a key argument. In [63], the notion of Siegel field has been proposed. A Siegel field is a subfield of $\overline{\mathbb{Q}}$ on which an analogue of Siegel's lemma is true. In order to formulate a geometry of numbers for a Siegel field, Gaudron and Rémond have introduced a topology on the space of all places of such a field and a Borel measure on it.

5. Algebraic extensions of function fields. In [48], Corvaja and Zannier have studied the arithmetic of algebraic extensions of function fields. They have characterised the infinite algebraic extensions of function fields of a curve which still satisfy a product formula. They have also discussed several examples of product formulas associated with algebraic surfaces in revealing the non-uniqueness of the extension of a product formula under finite field extensions. 
The above results are obtained in various settings of arithmetic geometry. It turns out that these settings can be naturally included in the framework of adelic curves (see $\$ 3.2$ for details) in order to treat the geometry of various fields analogously to that of vector bundles on projective curves. For example, on the field $\mathbb{Q}(T)$ of rational functions with coefficients in $\mathbb{Q}$, three types of absolute values are defined (see $\$ 3.2 .5$ for details): the valuation corresponding to closed points of $\mathbb{P}_{\mathbb{Q}}^{1}$, the natural extensions of $p$-adic absolute values, the Archimedean absolute value corresponding to divers embeddings of $\mathbb{Q}(T)$ in $\mathbb{C}$. Note that Jensen's formula for Mahler measure shows that these absolute values, once suitably parametrised by a measure space, satisfies a product formula. Thus we can consider it as an adelic curve. This is actually a particular case of polarised arithmetic projective varieties, where the polarisation provides a structure of adelic curve on the field of rational functions on the projective variety. Moreover, algebraic coverings of an adelic curve can be naturally constructed (see Section 3.4), which provides a framework for the study of the arithmetic of algebraic extensions.

Note that Gubler 76 has introduced a similar notion of $M$-field and extended the Arakelov height theory to this setting. An $M$-field is a field equipped with a measure space and a family of functions parametrised by the measure space which are absolute values almost everywhere, and the height of an arithmetic variety is defined as the integration along the measure space of local heights. However, our main concern is to build up a suitable geometry of numbers while the purpose of [76 is to extend the Arakelov height theory in a sufficiently general setting in order to include the theory of Nevanlinna. In Diophantine geometry the geometry of numbers is as important as a the height theory, particularly in the geometrisation of the method of "auxiliary polynomials". We propose the notion of adelic vector bundles on adelic curves, which consist of a finite-dimensional vector space over the underlying field, equipped with a measurable family of norms parametrised by the measure space. Our choice facilites the study of algebraic constructions of adelic vector bundles. The height of arithmetic varieties is described in a global way by the asymptotic behaviour of graded linear series equipped with structures of adelic vector bundles, rather than the integral of local heights.

In the framework of model theory, Ben Yaakov and Hrushovski [7, 85] also consider the formalisme of a field equipped with a family of absolute value parametrised by a measure space, which satisfied a product formula (called globally valued field in their terminologies). Their work permits to considered classic Diophantine geometry objects (in particular heights) in the model theory setting.

In order to set up a theory of adelic vector bundles over adelic curves, we present in the first chapter various constructions and properties of seminormed vector spaces over a complete valued field. Although the constructions and results are basic, the subtleties in the interaction and the compatibility of divers algebraic constructions, 
such as restriction, quotient, dual, tensor product, exterior powers etc, have not been clarified in the literature in a systematic way. In particular, several classic results in the functional analysis over $\mathbb{C}$ are no longer true in the non-Archimedean setting. We choose carefully our approach of presentation to unify the treatment of non-Archimedean and Archimedean cases whenever possible, and specify the differences and highlight the subtleties in detail. A particular attention is paid to the two constructions of tensor product seminorms: the $\pi$-tensor product and the $\varepsilon$-tensor product. These notions have been firstly introduced by Grothendieck [69, 75] in the setting of functional analysis over $\mathbb{C}$. It turns out that similar constructions can be defined more generally over an arbitrary complete valued field, and they are useful for example in the study of seminorms on exterior powers.

The orthogonality is another theme discussed in the first chapter. Classically the orthogonality is a natural notion in the study of inner product spaces. We consider an equivalent form of this notion, which can be defined in the setting of finitely generated seminormed vector spaces over an arbitrary complete valued field. This reformulation has been used in $\mathbf{3 2}$ to study the arithmetic positivity on toric varieties. Here it will serve as a fundamental tool to study ultrametrically normed spaces, inner product spaces and the construction of orthogonal tensor products. In particular, an analogue of the Gram-Schmidt process holds for finite-dimensional ultrametrically seminormed spaces, which plays a key role in the compatibility of the determinant norm with respect to short exact sequences.

We also discuss extension of seminorms under a valued extension of scalars. We distinguish three extensions of seminorms, corresponding to the three types of tensor product. The compatibility of extension of scalars with respect to divers algebraic construction is also explained. These constructions are used in the pull-back of an adelic vector bundle by an algebraic covering of the adelic curve.

Note that in the classic Arakelov theory, usually we consider a vector space over a global field equipped with a family of norms. However, from the point of view of birational geometry, it is natural to consider metrics which admits singularity, that is, degenerates on a closed subscheme (which is usually the base locus of a linear series) to a family of seminorms. Moreover, in the study of algebraicity of formal leaves of an arithmetic foliation, the canonical "metrics" on the tangent bundle are often seminorms. We refer the readers to [20 for more details. Motivated by these observations, we choose to present a panoramic view on the tools about general seminormed vector spaces which could be useful in Arakelov geometry later.

The second chapter is devoted to a presentation of metrised line bundles on a projective scheme over a complete valued field. It could be considered as a higher dimensional version of the results presented in Chapter 1. We use Berkovich topology to define continuous metrics on a vector bundle. Note that in the case where the base field is $\mathbb{C}$, our definition coincides with the classic definition of continuous metric on 
a vector bundle over a complex analytic space (associated with a complex projective scheme).

The Fubini-Study metric is another important ingredient of Chapter 2. It is closely related to the positivity of metrics on line bundles. More precisely, a continuous metric on a line bundle over a projective scheme defined over a complete valued field is said to be semipositive if it can be written as a uniform limit of FubiniStudy metrics. In the case where the absolute value is Archimedean, this definition is equivalent to the semipositivity of the curvature current of the metric. In the case where the absolute value is non-Archimedean and non-trivial, it is equivalent to the semipositivity condition proposed in [34, Section 6.8] and [79, Section 6]. However, in the trivial valuation case, it seems that our formulation is crucial to study the positivity of the metrics.

In classic Hermitian geometry, the positivity is closely related to the extension of sections of an ample line bundle with a control on the supremum norms. We establish a non-Archimedean analogue of the extension property, generalising the main result of 44 to the non-necessarily reduced case.

The third chapter is devoted to the fundament of adelic curves. We first give the formal definition of this notion and illustrate by various examples. The algebraic coverings of adelic curves occupy an important part of the chapter. As mentioned above, an adelic curve is a field equipped with a family of absolute values parametrised by a measure space, which satisfies a product formula. Given an algebraic extension of the underlying field, there is a canonical family of absolute values parametrised by a measure space fibered on the initial measure space and equipped with a disintegration kernel. This construction is important in the height theory for algebraic points and in the study of Siegel and Northcott properties. Contrary to the approach of [63], we do not assume the structural measurable space of an adelic curve to be a topological space and do not adopt the topological construction of algebraic coverings. Although it is possible to reduce the construction to the case of finite extensions by an argument of passage to projective limit, even for the simplest case of finite separable extension of the underlying field, the problem is highly non-trivial. The main subtleties come from the measurability of the fibre integral, which neither follows from the classic disintegration theory, nor from the property of extension of absolute values in algebraic number theory. The difficulty is resolved by using symmetric polynomials and Vandermonde matrix.

The analogue in the adelic curve setting of the geometry of numbers occupies the main part of the fourth chapter. Given an adelic curve, for any finite-dimensional vector space over the underlying field, we consider families of norms indexed by the structural measure space of the adelic curve. Natural measurability and dominancy conditions are defined for such norm families. An adelic vector bundle is a finitedimensional vector space over the underlying field of the adelic curve, equipped with 
a measurable and dominated norm family. In the case where the adelic curve arises from a global field, this notion corresponds essentially the notion of adelic vector bundle in the work $[\mathbf{6 0}]$. Note that in the classic global field case it is required that almost all norms in the structure of an adelic vector bundle come from a common integral model of the vector space. However, in our general setting of adelic curve, it is not adequate to discuss integral models since the integral ring in an adelic curves is not well defined. The condition of common integral model is replaced by the dominancy condition, which in the global field case can be considered as uniform limit of classic structure of adelic vector bundle.

The arithmetic invariants of adelic vector bundles are also discussed. For example, the Arakelov degree of an adelic vector bundle is defined as the integral of the logarithmic determinant norm of a non-zero maximal exterior power vector, similarly as in the classic case of Hermitian vector bundle over an arithmetic curve. Moreover, although the analogue of classic minima of lattices can not be reformulated in the adelic curve setting, due to the lack of integral models, the version of Roy and Thunder [121, which is based on the height function (or equivalently the Arakelov degree of the non-zero vectors), can be naturally generalised in our setting of adelic curves. However, it turns out that several fundamental results in geometry of numbers, such as Minkowski's theorems, are not true in the general setting, and the set of vectors in the adelic unit ball is not the good generalisation of lattice points of norm $\leqslant 1$. This phenomenon suggests that the slope method of Bost $1 \mathbf{1 5}$ might be more efficient in Diophantine geometry. In fact, inspired by the Harder-Narasimhan theory of vector bundles over curves, the notion of successive slopes has been proposed in [131, 68, for Euclidean lattices and generalised in [15, 17] with applications to the period and isogenies of abelian varieties, and algebraicity of formal schemes. In the setting of adelic vector bundles on adelic curves, we build up an analogue of Harder-Narasimhan theory and the slope method. In this sense, adelic vector bundles on adelic curves have very similar properties as those of vector bundles on a regular projective curve, or Hermitian vector bundles over an arithmetic curve. It is for this reason that we have chosen the terminology of adelic curve. However, although the successive minima and the successive slopes are close in the number field case (see [14, 42]), they can differ much in the general adelic curve setting, even for the simple case of a field equipped with several copies of the trivial absolute value. Note that the semistability of adelic vector bundles over such adelic curves plays an important role in Diophantine geometry of projective spaces, as for example in the work of Faltings and Wüstholz [59] (although not written explicitly in the language of the slope theory). Our general setting of adelic vector bundles helps to understand the roles of different arithmetic invariants should play in a Diophantine argument.

The adelic curve consisting of the trivial absolute value is also closely related to the geometric invariant theory. In the fifth chapter of the book, we explain this link 
and apply it to the estimation of the minimal slope of the tensor product of two adelic vector bundles. In fact, an ultrametrically normed vector space over a trivially valued field can be considered as a decreasing $\mathbb{R}$-filtration of the vector space. In the geometric invariant theory, an action of the multiplicative group on a finitedimensional vector space over a field corresponds to the decomposition of the vector space into the direct sum of eigensubspaces and thus determines an $\mathbb{R}$-filtration of the vector space by the eigenvalues. Therefore we can reformulate the Hilbert-Mumford criterion for general linear groups (or products of general linear groups) in terms of a slope inequality for adelic vector bundles on the adelic curve of one trivial absolute value.

Bogomolov (see [119]) has interpreted the semistability of a vector bundle over a projective curve as an inequality linking the $\mathbb{R}$-filtration and the Arakelov degree. This result can also be viewed as a link between the geometric invariant theory and the semistability in the theory of Harder-Narasimhan. Later Ramanan and Ramanathan 117. have given an algebraic proof of the semistability of the tensor product of two semistable vector bundles on a regular projective curve over a field of characteristic 0 . In the number field case, Bost [16 has conjectured that the arithmetic analogue of the tensorial semistability is also true. This conjecture is equivalent to the statement that the tensor product of two Hermitian vector bundles has a minimal slope which is bounded from below by the minimal slopes of the two Hermitian vector bundles.

In the setting of adelic vector bundles over adelic curves, we can consider the natural generalisation of Bost's conjecture stating that, if the underlying base field of the adelic curve is perfect, then the tensor product of two semistable Hermitian adelic vector bundles is also semistable. Besides the function field case proved by Ramanan and Ramanathan, the generalised conjecture is also true in the case where the adelic curve is given by a perfect field equipped with a finite number of copies of the trivial absolute value (see [136]). We prove here a weaker version of this conjecture, showing that the minimal slope of the tensor product of two (non-necessarily Hermitian) adelic vector bundles is bounded from below by the sum of the minimal slopes of the two adelic vector bundles, minus three half of the logarithm of the rank of the tensor product bundle times the measure of Archimedean places. In particular, the conjecture is true if the base field is perfect and all absolute values in the adelic curve structure are non-Archimedean. This result is similar to the works [36, 2, 62, 21] in the case where the adelic curve comes from a number field. However, the strategy of proof is different. In fact, the common point of the works cited above is a geometric version of Siegel's lemma proved by Zhang [148, which could be considered as an absolute version of Minkowski's second theorem, which is false for general adelic curves. Our method relies on the geometric invariant theory of grassmannian (with Plücker coordinates) and combines the technics of [36] and [21]. 
The sixth chapter is devoted to the study of metrised line bundles on arithmetic varieties over adelic curves. In the classic setting of adelic metrics such as [149, 108, it was required that an adelic metric should coincides with an integral model metric for all but finitely many places. Again the integral model metric is not adequate in our setting of adelic curves, the suitable notions of dominancy and measurability occupy thus an important part of the chapter. An adelic line bundle on a projective variety is then defined to be an invertible sheaf equipped with a dominated and measurable family of metrics parametrised by the adelic curve. In the setting of global fields, our definition is slightly more general than the classic one, which includes the limits of classic adelic line bundles. The analogue of some classic geometric invariants, such as height function, essential minimum, and arithmetic volume function is also discussed. In particular, in the definition of the arithmetic volume function, we use the positive degree instead of the logarithmic cardinal of the small sections since the latter is no longer adequate in the general setting. Note that the failure of Minkowski's first theorem brings several technical difficulties, notably the filtration by minima and the filtration by slopes do not lead to the same arithmetic invariants, on the contrary of the case of number fields as in 42 . Our strategy consists in introducing a refinement of the method of arithmetic Newton-Okounkov bodies [25, which allows to treat the case of graded linear series equipped with filtrations which are not necessarily additive.

In the seventh and the last chapter, we relate the asymptotic minimal slope to the absolute minimum of the height function of an adelic line bundle, which could be considered as a generalisation of Nakai-Moishezon's criterion in the setting of Arakelov geometry over an adelic curve. In the case where the analogue of a strong version of Minkowski's first theorem holds for the adelic curve, we deduce from the criterion an analogue of Siegel's lemma for adelic vector bundles on the adelic curve. Our work clarifies the arguments of geometric nature from several fundamental result in the classic geometry of numbers.

Limited by the volume of the monograph, many aspects are not included in the current text. First of all, an arithmetic intersection theory should be developed in the setting of Arakelov geometry over an adelic curve, which allows to interpret the height of arithmetic varieties as the arithmetic intersection number. Secondly, by using the adelic curve of several copies of the trivial absolute value, we expect to incorporate the conditions and results of geometric invariant theory into the arithmetic setting. Thirdly, the geometry of adelic vector bundles should lead to a Diophantine approximation theory of adelic curves. Finally, the fundamental works achieved in the monograph could be applied to the study of Nevanlinna theory of $M$-field proposed by Gubler. 
Acknowledgements. We are grateful to Jean-Benoît Bost, Carlo Gasbarri and Hugues Randriambololona for comments, and to Itaï Ben Yaacov and Ehud Hrushovski for having sent us their lecture notes and for letter communications. 



\section{CHAPTER 1}

\section{METRIZED VECTOR BUNDLES: LOCAL THEORY}

The purpose of this chapter is to explain the constructions and properties of normed vector spaces over a complete valued field. It will serve as the fundament for the global study of adelic vector bundles. Note that we need to consider both Archimedean and non-Archimedean cases. Hence we carefully choose the approach of presentation to unify the statements whenever possible, and to clarify the differences.

Throughout the chapter, let $k$ be a field equipped with an absolute value $|\cdot|$. We assume that $k$ is complete with respect to the topology induced by $|\cdot|$. We emphasise that $|\cdot|$ could be the trivial absolute value on $k$, namely $|a|=1$ for any $a \in k \backslash\{0\}$. If the absolute value $|\cdot|$ is Archimedean, then $k$ is either the field $\mathbb{R}$ of real numbers or the field $\mathbb{C}$ of complex numbers. For simplicity, we assume that $|\cdot|$ is the usual absolute value on $\mathbb{R}$ or $\mathbb{C}$ if it is Archimedean.

\subsection{Norms and seminorms}

Definition 1.1.1. - Let $V$ be a vector space over $k$. A map $\|\cdot\|: V \rightarrow \mathbb{R}_{\geqslant 0}$ is called a seminorm on $V$ if the following conditions (a) and (b) are satisfied:

(a) for any $a \in k$ and any $x \in V$, one has $\|a x\|=|a| \cdot\|x\|$;

(b) the triangle inequality: for any $(x, y) \in V \times V$, one has $\|x+y\| \leqslant\|x\|+\|y\|$.

The couple $(V,\|\cdot\|)$ is called a seminormed vector space over $k$. If in addition the following strong triangle inequality is satisfied

$$
\forall(x, y) \in V^{2}, \quad\|x+y\| \leqslant \max \{\|x\|,\|y\|\},
$$

we say that the seminorm $\|\cdot\|$ is ultrametric. Note that the existence of a nonidentically vanishing ultrametric seminorm on $V$ implies that the absolute value $|\cdot|$ on $k$ is non-Archimedean. Furthermore, if the following additional condition (c) is satisfied:

(c) for any $x \in V \backslash\{0\}$, one has $\|x\|>0$, 
the seminorm $\|\cdot\|$ is called a norm on $V$, and the couple $(V,\|\cdot\|)$ is called a normed vector space over $k$.

If $(V,\|\cdot\|)$ is a seminormed vector space over $k$, then

$$
N_{\|\cdot\|}:=\{x \in V:\|x\|=0\}
$$

is a vector subspace of $V$, called the null space of $\|\cdot\|$. Moreover, if we denote by $\pi: V \rightarrow V / N_{\|\cdot\|}$ the linear map of projection, then there is a unique norm $\|\cdot\|^{\sim}$ on $V / N_{\|\cdot\|}$ such that $\|\cdot\|=\|\cdot\|^{\sim} \circ \pi$. The norm $\|\cdot\|^{\sim}$ is called the norm associated with the seminorm $\|\cdot\|$.

Definition 1.1.2. - Let $f: W \rightarrow V$ be a linear map of vector spaces over $k$ and $\|\cdot\|$ be a seminorm on $V$. We define $\|\cdot\|_{f}: W \rightarrow \mathbb{R}_{\geqslant 0}$ to be

$$
\forall x \in W, \quad\|x\|_{f}:=\|f(x)\|,
$$

which is a seminorm on $W$, called the seminorm induced by $f$ and $\|\cdot\|$. Clearly, if $\|\cdot\|$ is ultrametric, then also is $\|\cdot\|_{f}$. In the case where $f$ is injective, $\|\cdot\|_{f}$ is often denoted by $\|\cdot\|_{W \hookrightarrow V}$ and is called the seminorm on $W$ induced by $\|\cdot\|$, or the restriction of $\|\cdot\|$ to $W$.

Notation 1.1.3. - Let $(V,\|\cdot\|)$ be a seminormed vector space over $k$. If $\epsilon$ is a non-negative real number, we denote by $(V,\|\cdot\|)_{\leqslant \epsilon}$ or simply by $V_{\leqslant \varepsilon}$ the closed ball $\{x \in V:\|x\| \leqslant \epsilon\}$ of radius $\epsilon$ centered at the origin. Similarly, we denote by $(V,\|\cdot\|)_{<\epsilon}$ or by $V_{<\epsilon}$ the open ball $\{x \in V:\|x\|<\epsilon\}$.

Proposition 1.1.4. - Assume that $|\cdot|$ is non-trivial. Let $\lambda \in] 0,1[$ such that

$$
\lambda<\sup \left\{|a|: a \in k^{\times},|a|<1\right\} .
$$

Let $(V,\|\cdot\|)$ be a seminormed vector space over $k$ and $x$ be a vector in $V$ such that $\|x\|>0$. There exists $b \in k^{\times}$such that $\lambda \leqslant\|b x\|<1$.

Proof. - Let $a$ be an element in $k^{\times}$such that $\lambda<|a|<1$. We take $b=a^{p}$ with

$$
p=\left\lfloor\frac{\ln (\lambda)-\ln \|x\|}{\ln |a|}\right\rfloor .
$$

By definition one has $p \leqslant(\ln (\lambda)-\ln \|x\|) / \ln |a|$. Hence $|b|=|a|^{p} \geqslant \lambda /\|x\|$, which leads to $\|b x\|=|b| \cdot\|x\| \geqslant \lambda$. Moreover, since $\lambda<|a|<1$ one has $\ln (\lambda)<\ln |a|<0$. Hence $\ln (\lambda) / \ln |a|>1$, which implies that $p>-\ln \|x\| / \ln |a|$. Hence $|b|=|a|^{p}<\|x\|^{-1}$, which leads to $\|b x\|<1$.

Proposition 1.1.5. - Let $(V,\|\cdot\|)$ be an ultrametrically seminormed vector space over $k$.

(1) If $x_{1}, \ldots, x_{n}$ are vectors of $V$ such that the numbers $\left\|x_{1}\right\|, \ldots,\left\|x_{n}\right\|$ are distinct, then one has $\left\|x_{1}+\cdots+x_{n}\right\|=\max _{i \in\{1, \ldots, n\}}\left\|x_{i}\right\|$. 
(2) The cardinal of the image of the composed map

$$
V \backslash N_{\|\cdot\|} \stackrel{\|\cdot\|}{\longrightarrow} \mathbb{R}_{>0} \longrightarrow \mathbb{R}_{>0} /\left|k^{\times}\right|
$$

is not greater than the rank of $V / N_{\|\cdot\|}$ over $k$, where $\mathbb{R}_{>0}$ denotes the multiplicative group of positive real numbers, and $\left|k^{\times}\right|$is the image of $k^{\times}$by $|\cdot|$.

Proof. - (1) The statement is trivial when $n=1$. Moreover, by induction it suffices to treat the case where $n=2$. Without loss of generality, we assume that $\left\|x_{1}\right\|<\left\|x_{2}\right\|$. Since $\|\cdot\|$ is ultrametric, one has $\left\|x_{1}+x_{2}\right\| \leqslant \max \left\{\left\|x_{1}\right\|,\left\|x_{2}\right\|\right\}=\left\|x_{2}\right\|$. Moreover,

$$
\left\|x_{2}\right\|=\left\|x_{1}+x_{2}+\left(-x_{1}\right)\right\| \leqslant \max \left\{\left\|x_{1}+x_{2}\right\|,\left\|x_{1}\right\|\right\} .
$$

Since $\left\|x_{2}\right\|>\left\|x_{1}\right\|$, one should have $\left\|x_{2}\right\| \leqslant\left\|x_{1}+x_{2}\right\|$. Therefore

$$
\left\|x_{1}+x_{2}\right\|=\left\|x_{2}\right\|=\max \left\{\left\|x_{1}\right\|,\left\|x_{2}\right\|\right\} .
$$

(2) By replacing $V$ by $V / N_{\|\cdot\|}$ and $\|\cdot\|$ by the associated norm, we may assume that $\|\cdot\|$ is actually a norm. Denote by $I$ the image of the composed map (1.1). For each element $\alpha$ in $I$, we pick a vector $x_{\alpha}$ in $V \backslash\{0\}$ such that the image of $x_{\alpha}$ by the composed map is $\alpha$. We will show that the family $\left\{x_{\alpha}\right\}_{\alpha \in I}$ is linearly independent over $k$ and hence the cardinal of $I$ is not greater than the rank of $V$ over $k$. Assume that $\alpha_{1}, \ldots, \alpha_{n}$ are distinct elements of the set $I$ and $\lambda_{1}, \ldots, \lambda_{n}$ are non-zero elements of $k$. Then the values $\left\|\lambda_{1} x_{\alpha_{1}}\right\|, \ldots,\left\|\lambda_{n} x_{\alpha_{n}}\right\|$ are distinct. As the norm $\|\cdot\|$ is ultrametric, by (1) one has

$$
\left\|\lambda_{1} x_{\alpha_{1}}+\cdots+\lambda_{n} x_{\alpha_{n}}\right\|=\max _{i \in\{1, \ldots, n\}}\left\|\lambda_{i} x_{i}\right\|>0 .
$$

Hence $\lambda_{1} x_{\alpha_{1}}+\cdots+\lambda_{n} x_{\alpha_{n}}$ is non-zero.

Corollary 1.1.6. - Let $(V,\|\cdot\|)$ be an ultrametrically seminormed vector space of finite rank over $k$. Then we have the following:

(1) If $|\cdot|$ is a discrete valuation (namely $\left|k^{\times}\right|$is a discrete subgroup of $\mathbb{R}_{>0}$ ), then the image of $V \backslash N_{\|\cdot\|}$ by $\|\cdot\|$ is a discrete subset of $\mathbb{R}_{>0}$.

(2) If $|\cdot|$ is the trivial absolute value, then the image of $V$ by $\|\cdot\|$ is a finite set, whose cardinal does not exceed $\operatorname{dim}_{k}\left(V / N_{\|\cdot\|}\right)+1$.

1.1.1. Topology. - Let $(V,\|\cdot\|)$ be a seminormed vector space over $k$. The seminorm $\|\cdot\|$ induces a pseudometric $\operatorname{dist}(\cdot, \cdot)$ on $V \operatorname{such}$ that $\operatorname{dist}(x, y):=\|x-y\|$ for any $(x, y) \in V^{2}$. We equip $V$ with the most coarse topology which makes continuous the functions $(y \in V) \mapsto\|x-y\|$ for any $x \in V$. In other words, a subset $U$ of $V$ is open if and only if, for any $x \in U$, there is a positive number $\epsilon$ such that $\{y \in V:\|y-x\|<\epsilon\} \subseteq U$. This topology is said to be induced by the seminorm $\|\cdot\|$. The set $V$ equipped with this topology forms a topological vector space. For any vector subspace $W$ of $V$, the closure of $W$ is also a vector subspace of $V$. In particular, if $W$ is a hyperplane in $V$ (namely the kernel of a linear form), then either $W$ is a 
closed vector subspace of $V$ or $W$ is dense in $V$. For any $x \in V$, the pseudodistance between $W$ and $x$ is defined as

$$
\operatorname{dist}(x, W):=\inf \{\|x-y\|: y \in W\} .
$$

Then $\operatorname{dist}(x, W)=0$ if and only if $x$ belongs to the closure of $W$. In particular, the null space of $(V,\|\cdot\|)$ is a closed subspace, which is the closure of the zero vector subspace $\{0\}$. Thus the topological vector space $V$ is separated if and only if $\|\cdot\|$ is a norm.

Proposition 1.1.7. - Let $\left(V_{1},\|\cdot\|_{1}\right)$ and $\left(V_{2},\|\cdot\|_{2}\right)$ be seminormed vector spaces over $k$, and $f: V_{1} \rightarrow V_{2}$ be a k-linear map. Then we have the following:

(1) If the map $f$ is continuous, then $f\left(N_{\|\cdot\|_{1}}\right) \subseteq N_{\|\cdot\|_{2}}$.

(2) If there is a non-negative constant $C$ such that $\|f(x)\|_{2} \leqslant C\|x\|_{1}$ for all $x \in V_{1}$, then the map $f$ is continuous. The converse is true if either (i) the absolute value $|\cdot|$ is non-trivial or (ii) $\operatorname{dim}_{k}\left(V_{2} / N_{\|\cdot\|_{2}}\right)<\infty$.

Proof. - (1) Since $N_{\|\cdot\|_{2}}$ is a closed subset of $V_{2}$, its inverse image by the continuous map $f$ is a closed subset of $V_{1}$, which clearly contains $0 \in V_{1}$. Hence $f^{-1}\left(N_{\|\cdot\|_{2}}\right)$ contains $N_{\|\cdot\|_{1}}$ since $N_{\|\cdot\|_{1}}$ is the closure of $\{0\}$ in $V_{1}$.

(2) Let $\left\{x_{n}\right\}_{n \in \mathbb{N}}$ be a sequence in $V_{1}$ which converges to a point $x \in V_{1}$. One has

$$
\left\|f\left(x_{n}\right)-f(x)\right\|_{2}=\left\|f\left(x_{n}-x\right)\right\|_{2} \leqslant C\left\|x_{n}-x\right\|_{1},
$$

so that the sequence $\left\{f\left(x_{n}\right)\right\}_{n \in \mathbb{N}}$ converges to $f(x)$. Hence the map $f$ is continuous.

Assume that $f$ is continuous. First we consider the case where the absolute value $|\cdot|$ is not trivial. The set $f^{-1}\left(\left(V_{2},\|\cdot\|_{2}\right)_{<1}\right)$ is an open subset of $V_{1}$ (see Notation 1.1.3). Hence there exists $\epsilon>0$ such that $f^{-1}\left(\left(V_{2},\|\cdot\|_{2}\right)_{<1}\right) \supseteq\left(V_{1},\|\cdot\|_{1}\right)_{<\epsilon}$. As the absolute value $|\cdot|$ is not trivial, there exists $a \in k$ such that $0<|a|<1$. Let us see that $\|f(x)\|_{2} \leqslant(\epsilon|a|)^{-1}\|x\|_{1}$ for all $x \in V_{1}$. If $x \in N_{\|\cdot\|_{1}}$, then the assertion is obvious by (1) so that we may assume that $x \notin N_{\|\cdot\|_{1}}$. Then there exists a unique integer $n$ such that

$$
\left\|a^{n} x\right\|_{1}<\epsilon \leqslant\left\|a^{n-1} x\right\|_{1}=|a|^{n-1} \cdot\|x\|_{1} .
$$

Thus $\left\|f\left(a^{n} x\right)\right\|_{2}<1$ and hence

$$
\|f(x)\|_{2}<|a|^{-n} \leqslant(\epsilon|a|)^{-1} \cdot\|x\|_{1},
$$

as desired.

Next we assume that the absolute value $|\cdot|$ is trivial and $\operatorname{dim}_{k}\left(V_{2} / N_{\|\cdot\|_{2}}\right)<\infty$. By (2) in Corollary 1.1.6 there exist positive numbers $r$ and $\delta$ such that $\|y\| \leqslant r$ for any $y \in V_{2}$ and that $\left(V_{2},\|\cdot\|_{2}\right)_{<\delta}=N_{\|\cdot\|_{2}}$. If $f$ is continuous, then there exists $\epsilon>0$ such that

$$
f^{-1}\left(N_{\|\cdot\|_{2}}\right)=f^{-1}\left(\left(V_{2},\|\cdot\|_{2}\right)_{<\delta}\right) \supseteq\left(V_{1},\|\cdot\|_{1}\right)_{<\epsilon} .
$$

Therefore one has $\|f(x)\|_{2} \leqslant(r / \epsilon)\|x\|_{1}$ for all $x \in V_{1}$. 
Remark 1.1.8. - The hypothesis of non-triviality of the absolute value or $\operatorname{dim}_{k}\left(V_{2} / N_{\|\cdot\|_{2}}\right)<\infty$ for the sufficiency part of the above proposition is essential. In fact, if $V$ is an infinite-dimensional vector space over a trivially valued field $k$, equipped with the norm $\|\cdot\|$ such that $\|x\|=1$ for any $x \in V \backslash\{0\}$, then the topology on $V$ induced by the norm $\|\cdot\|$ is discrete. In particular, any $k$-linear map from $V$ to a normed vector space over $k$ is continuous. However, one can take a basis $B$ of the vector space $V$ (which is an infinite set) and define a new norm $\|\cdot\|^{\prime}$ on $V$ such that

$$
\left\|\sum_{x \in B} n_{x} x\right\|^{\prime}=\max _{x \in B, n_{x} \neq 0} \varphi(x),
$$

where $\varphi: B \rightarrow] 0,+\infty[$ is a map which is not bounded. If $f$ is the identity map from $(V,\|\cdot\|)$ to $\left(V,\|\cdot\|^{\prime}\right)$, then one can not find a non-negative constant $C$ such that $\|x\|^{\prime} \leqslant C\|x\|$ for all $x \in V$.

1.1.2. Operator seminorm. - Let $\left(V_{1},\|\cdot\|_{1}\right)$ and $\left(V_{2},\|\cdot\|_{2}\right)$ be seminormed vector spaces over $k$. Let $f: V_{1} \rightarrow V_{2}$ be a $k$-linear map. We say that the linear map $f$ is bounded if there is a non-negative constant $C$ such that $\|f(x)\|_{2} \leqslant C\|x\|_{1}$ for all $x \in V_{1}$. Note that if $f$ is bounded, then $f$ is continuous and $f\left(N_{\|\cdot\|_{1}}\right) \subseteq N_{\|\cdot\|_{2}}$ by Proposition 1.1.7.

If $f\left(N_{\|\cdot\|_{1}}\right) \subseteq N_{\|\cdot\|_{2}}$, we denote by $\|f\|$ the element

$$
\sup _{x \in V_{1} \backslash N_{\|\cdot\|_{1}}} \frac{\|f(x)\|_{2}}{\|x\|_{1}} \in[0,+\infty] .
$$

If the relation $f\left(N_{\|\cdot\|_{1}}\right) \subseteq N_{\|\cdot\|_{2}}$ does not hold, then by convention $\|f\|$ is defined to be $+\infty$. With this notation, the linear map $f$ is bounded if and only if $\|f\|<+\infty$.

We denote by $\mathscr{L}\left(V_{1}, V_{2}\right)$ the set of all bounded $k$-linear maps from $V_{1}$ to $V_{2}$, which forms a vector space over $k$ since, for $(f, g) \in \mathscr{L}\left(V_{1}, V_{2}\right)^{2}$ and $x \in V_{1} \backslash N_{\|\cdot\|_{1}}$,

$$
\frac{\|(f+g)(x)\|_{2}}{\|x\|_{1}} \leqslant \begin{cases}\max \left\{\frac{\|f(x)\|_{2}}{\|x\|_{1}}, \frac{\|g(x)\|_{2}}{\|x\|_{1}}\right\} & \text { if }\|\cdot\|_{2} \text { is ultrametric, } \\ \frac{\|f(x)\|_{2}}{\|x\|_{1}}+\frac{\|g(x)\|_{2}}{\|x\|_{1}} & \text { otherwise. }\end{cases}
$$

The map $\|\cdot\|: \mathscr{L}\left(V_{1}, V_{2}\right) \longrightarrow[0,+\infty[$ defined above is a seminorm, called the operator seminorm. Moreover, from the above formula, we observe that, if $\|\cdot\|_{2}$ is ultrametric, then the operator seminorm is also ultrametric. If $\|\cdot\|_{2}$ is a norm, then the operator seminorm is actually a norm, called operator norm.

In the case where either the absolute value |.| is non-trivial or $\operatorname{dim}_{k}\left(V_{2} / N_{\|\cdot\|_{2}}\right)<\infty$, the space $\mathscr{L}\left(V_{1}, V_{2}\right)$ identifies with the vector space of all continuous $k$-linear maps from $V_{1}$ to $V_{2}$ (see Proposition 1.1.7). 
1.1.3. Quotient seminorm. - Let $g: V \rightarrow Q$ be a surjective linear map of vector spaces over $k$ and $\|\cdot\|$ be a seminorm on $V$. We define $\|\cdot\|_{V \rightarrow Q}$ to be

$$
\forall y \in Q, \quad\|y\|_{V \rightarrow Q}:=\inf \{\|x\|: x \in V, g(x)=y\} .
$$

Then we have the following proposition:

Proposition 1.1.9. — (1) $\|\cdot\|_{V \rightarrow Q}$ is a seminorm on $Q$. Moreover, if $\|\cdot\|$ is ultrametric, then also is $\|\cdot\|_{V \rightarrow Q}$.

(2) Let $N_{\|\cdot\|_{V \rightarrow Q}}$ be the null space of $\|\cdot\|_{V \rightarrow Q}$. Then $g^{-1}\left(N_{\|\cdot\|_{V \rightarrow Q}}\right)$ coincides with the closure of $\operatorname{Ker}(g)$ with respect to the topology induced by the seminorm $\|\cdot\|$. In particlar, if $\operatorname{Ker}(g)$ is closed, then $\|\cdot\|_{V \rightarrow Q}$ is a norm on $Q$.

Proof. - (1) In order to see the condition (a) in Definition 1.1.1 we may assume that $a \neq 0$ since otherwise the assertion is obvious. Then

$$
\begin{aligned}
\|a y\|_{V \rightarrow Q} & =\inf \left\{\left\|x^{\prime}\right\|: x^{\prime} \in V, g\left(x^{\prime}\right)=a y\right\}=\inf \{\|a x\|: x \in V, g(x)=y\} \\
& =|a| \inf \{\|x\|: x \in V, g(x)=y\}=|a| \cdot\|y\|_{V \rightarrow Q} .
\end{aligned}
$$

Fix $\left(y, y^{\prime}\right) \in Q^{2}$. For any $\epsilon>0$, we can find $\left(x, x^{\prime}\right) \in V^{2}$ such that $g(x)=y$, $g\left(x^{\prime}\right)=y^{\prime},\|x\| \leqslant\|y\|_{V \rightarrow Q}+\epsilon$ and $\left\|x^{\prime}\right\| \leqslant\left\|y^{\prime}\right\|_{V \rightarrow Q}+\epsilon$. Then $g\left(x+x^{\prime}\right)=y+y^{\prime}$ and

$$
\left\|y+y^{\prime}\right\|_{V \rightarrow Q} \leqslant\left\|x+x^{\prime}\right\| \leqslant\|x\|+\left\|x^{\prime}\right\| \leqslant\|y\|_{V \rightarrow Q}+\left\|y^{\prime}\right\|_{V \rightarrow Q}+2 \epsilon,
$$

and hence (b) holds. If $\|\cdot\|$ is ultrametric, in a similar way we can see that $\|\cdot\|_{V \rightarrow Q}$ is also ultrametric.

(2) Let $x \in V$ and $y=g(x)$. It is easy to see $\|y\|_{V \rightarrow Q}=\operatorname{dist}(x, \operatorname{Ker}(g))$. Therefore

$$
x \in \overline{\operatorname{Ker}(g)} \quad \Longleftrightarrow \quad \operatorname{dist}(x, \operatorname{Ker}(g))=0 \quad \Longleftrightarrow\|y\|_{V \rightarrow Q}=0,
$$

as required.

Given a vector subspace $W$ of a seminormed vector space $(V,\|\cdot\|)$, the seminorm $\|\cdot\|_{V \rightarrow V / W}$ on $V / W$ is called the quotient seminorm on $V / W$ of the seminorm $\|\cdot\|$ on $V$. For simplicity, the seminorm $\|\cdot\|_{V \rightarrow V / W}$ is often denoted by $\|\cdot\|_{V / W}$. If the vector subspace $W$ is closed, then the seminorm $\|\cdot\|_{V / W}$ is actually a norm, called the quotient norm of $\|\cdot\|$ by the quotient map $V \rightarrow V / W$. Note that the norm $\|\cdot\|^{\sim}$ identifies with the quotient norm of $\|\cdot\|$ by the quotient map $V \rightarrow V / N_{\|\cdot\|}$.

Proposition 1.1.10. - Let $(V,\|\cdot\|)$ be a seminormed vector space over $k$ and $W$ be a vector subspace of $V$. The topology on $V / W$ defined by the quotient seminorm coincides with the quotient topology. In particular, the quotient map $V \rightarrow V / W$ is continuous if we equip $V / W$ with the quotient seminorm.

Proof. - Recall that the quotient topology is the finest topology on $V / W$ which makes the quotient map $\pi: V \rightarrow V / W$ continuous. In other words, a subset $U$ of $V / W$ is open for the quotient topology if and only if $\pi^{-1}(U)$ is an open subset of $V$. If we equip $V / W$ with the topology induced by the quotient seminorm, then the 
quotient map is continuous since $\|\pi\| \leqslant 1$ (see Proposition 1.1.7). Moreover, if $U$ is a subset of $V / W$ such that $\pi^{-1}(U)$ is an open subset of $V$, then, for any $u \in U$ and any $x_{0} \in V$ such that $\pi\left(x_{0}\right)=u$, there exists $\epsilon>0$ such that

$$
\left\{x \in V:\left\|x-x_{0}\right\|<\epsilon\right\} \subseteq \pi^{-1}(U) .
$$

Hence for any $v \in V / W$ with $\|v-u\|<\epsilon$, there exists $x \in \pi^{-1}(U)$ such that $\pi(x)=v$. So $U$ is an open subset of $V / W$ for the topology defined by the quotient seminorm. The proposition is thus proved.

1.1.4. Topology of normed vector spaces of finite rank. - If $V$ is a finitedimensional $k$-vector space, then all norms on $V$ induce the same topology. More precisely, we have the following result (see [30] Chapter I, §2, no.3, Theorem 2 and the remark on the page I.15).

Proposition 1.1.11. - Assume that the vector space $V$ is of finite rank over $k$. If $\|\cdot\|$ and $\|\cdot\|^{\prime}$ are norms on $V$, then there are positive constants $C$ and $C^{\prime}$ such that $C\|\cdot\|^{\prime} \leqslant\|\cdot\| \leqslant C^{\prime}\|\cdot\|^{\prime}$ on $V$. In particular, $V$ is complete with respect to $\|\cdot\| .(1)$

Proof. - Let $\left\{e_{i}\right\}_{i=1}^{r}$ be a basis of $V$ and $f: k^{r} \rightarrow V$ be the isomorphism given by $f\left(a_{1}, \ldots, a_{r}\right)=a_{1} e_{1}+\cdots+a_{r} e_{r}$. Here we consider the product topology on $k^{r}$ and the topology induced by any norm $\|\cdot\|$ on $V$. By Proposition 1.1.7, it is sufficient to show that $f$ is a homeomorphism. Since

$$
\left\|a_{1} e_{1}+\cdots+a_{r} e_{r}\right\| \leqslant \max \left\{\left|a_{1}\right|, \ldots,\left|a_{r}\right|\right\} \sum_{i=1}^{r}\left\|e_{i}\right\|,
$$

$f$ is continuous. It remains to show that $f^{-1}$ is continuous.

We reason by induction on the rank $r$ of $V$. The case where $r=0$ is trivial. In the case where $r=1$, as $|a| /\left\|a e_{1}\right\|=1 /\left\|e_{1}\right\|$ for any $a \in k^{\times}, f^{-1}$ is continuous by Proposition 1.1.7.

Assume that the proposition has been proved for vector spaces of rank $<r$. Let $W$ be the vector subspace of $V$ generated by $e_{1}, \ldots, e_{r-1}$. By the induction hypothesis, the map $g: k^{r-1} \rightarrow W$ sending $\left(a_{1}, \ldots, a_{r-1}\right) \in k^{r-1}$ to $a_{1} e_{1}+\cdots+a_{r-1} e_{r-1}$ is a homeomorphism. In particular, the topological vector space $W$ is complete. As a consequence, $W$ is a closed vector subspace of $V$. By the rank 1 case of the proposition proved above, the map $\bar{f}$ from $k$ to $V / W$ sending $a \in k$ to $a\left[e_{r}\right]$ is a homeomorphism.

In the following, we show that, if $U$ is an open neighbourhood of $(0, \ldots, 0) \in k^{r}$, then there exists $\epsilon>0$ such that $f(U)$ contains all vectors $x \in V$ satisfying $\|x\|<\epsilon$. Without loss of generality, we may assume that $U$ is the open multidisc $B_{\delta}^{r}$, where

1. That is, for any sequence $\left\{x_{n}\right\}_{n \in \mathbb{N}}$ in $V$, if

$$
\lim _{N \rightarrow+\infty} \sup _{\substack{(n, m) \in \mathbb{N}^{2} \\ n \geqslant N, m \geqslant N}}\left\|x_{n}-x_{m}\right\|=0,
$$

then there exists $x \in V$ such that $\lim _{n \rightarrow \infty}\left\|x_{n}-x\right\|=0$. 
$B_{\delta}=\{a \in k:|a|<\delta\}$ and $\delta>0$. Since the map $g: k^{r-1} \rightarrow W$ is a homeomorphism, there exists $\epsilon_{1}>0$ such that

$$
g\left(B_{\delta}^{r-1}\right) \supseteq\left\{y \in W:\|y\|<\epsilon_{1}\right\} .
$$

Let $\delta^{\prime}=\min \left\{\epsilon_{1} /\left(2\left\|e_{r}\right\|\right), \delta\right\}$. Since the map $\bar{f}$ is a homeomorphism, there exists $\epsilon_{2}>0$ such that

$$
\bar{f}\left(B_{\delta^{\prime}}\right) \supseteq\left\{u \in V / W:\|u\|_{V / W}<\epsilon_{2}\right\},
$$

where we consider the quotient norm on $V / W$. We claim that

$$
f(U) \supseteq\{x \in V:\|x\|<\epsilon\}
$$

with $\epsilon=\frac{1}{2} \min \left\{\epsilon_{1}, \epsilon_{2}\right\}$. In fact, if $x$ is an element of $V$ such that $\|x\|<\epsilon$, then its class in $V / W$ has norm $<\epsilon_{2}$. Hence there exists $a_{r} \in B_{\delta^{\prime}}$ such that $[x]=a_{r}\left[e_{r}\right]$. Moreover, one has

$$
\left\|x-a_{r} e_{r}\right\| \leqslant\|x\|+\left|a_{r}\right| \cdot\left\|e_{r}\right\|<\frac{1}{2} \epsilon_{1}+\delta^{\prime}\left\|e_{r}\right\| \leqslant \epsilon_{1} .
$$

Hence there exists $\left(a_{1}, \ldots, a_{r-1}\right) \in B_{\delta}^{r-1}$ such that $g\left(a_{1}, \ldots, a_{r-1}\right)=x-a_{r} e_{r}$. Thus $\left(a_{1}, \ldots, a_{r}\right)$ is an element in $B_{\delta}^{r}$ such that $f\left(a_{1}, \ldots, a_{r}\right)=x$. The proposition is proved.

Corollary 1.1.12. - Let $f: V_{1} \rightarrow V_{2}$ be a linear map of vector spaces over $k$, and let $\|\cdot\|_{1}$ and $\|\cdot\|_{2}$ be seminorms on $V_{1}$ and $V_{2}$, respectively. We assume that $f\left(N_{\|\cdot\|_{1}}\right) \subseteq$ $N_{\|\cdot\|_{2}}$ and $\operatorname{dim}_{k}\left(V_{2} / N_{\|\cdot\|_{2}}\right)<\infty$. Then the following conditions are equivalent:

(a) the map $f$ is continuous;

(b) $f^{-1}\left(N_{\|\cdot\|_{2}}\right)$ is a closed vector subspace of $V$;

(c) $\|f\|$ is finite.

Proof. - ' (a) $\Longrightarrow(\mathrm{b})]$ ': Since $f^{-1}\left(N_{\|\cdot\|_{2}}\right)$ is the inverse image by $f$ of the closed subset $N_{\|\cdot\|_{2}}$ of $V_{2}$, if $f$ is continuous, then it is a closed subset of $V_{1}$.

'(b) $\Longrightarrow$ (c) : The assertion is trivial when $f\left(V_{1}\right) \subseteq N_{\|\cdot\|_{2}}$. In the following, we assume that $f\left(V_{1}\right) \nsubseteq N_{\|\cdot\|_{2}}$. We set

$$
Q:=f\left(V_{1}\right) /\left(f\left(V_{1}\right) \cap N_{\|\cdot\|_{2}}\right) \cong\left(f\left(V_{1}\right)+N_{\|\cdot\|_{2}}\right) / N_{\|\cdot\|_{2}} \neq\{0\} .
$$

Let $\|\cdot\|_{Q}$ be the quotient seminorm on $Q$ induced by $V_{1} \rightarrow f\left(V_{1}\right) \rightarrow Q$ and $\|\cdot\|_{1}$. By Proposition 1.1.9 and the condition (b), the seminorm $\|\cdot\|_{Q}$ is actually a norm. Moreover, we can consider $Q$ as a vector subspace of $V_{2} / N_{\|\cdot\|_{2}}$. Let $\|\cdot\|_{Q}^{\prime}$ be the restriction of $\|\cdot\|_{2}^{\sim}$ to $Q$, where $\|\cdot\|_{2}^{\sim}$ is the norm associated with the seminorm $\|\cdot\|_{2}$. By Proposition [1.1.11, there is a constant $C$ with $\|\cdot\|_{Q}^{\prime} \leqslant C\|\cdot\|_{Q}$. Thus, for any $x \in V_{1} \backslash N_{\|\cdot\|_{1}}$, one has

$$
\frac{\|f(x)\|_{2}}{\|x\|_{1}}=\frac{\|[f(x)]\|_{Q}^{\prime}}{\|x\|_{1}} \leqslant \frac{C\|[f(x)]\|_{Q}}{\|x\|_{1}} \leqslant C,
$$

which implies $\|f\| \leqslant C$.

$$
\text { ' }(\mathrm{c}) \Longrightarrow(\mathrm{a}) \text { ' follows from Proposition 1.1.7 }
$$


Corollary 1.1.13. — Let $(V,\|\cdot\|)$ be a finite-dimensional seminormed vector space over $k$. Then we have the following:

(1) Every vector subspace of $V$ containing $N_{\|\cdot\|}$ is closed.

(2) Let $\left(V^{\prime},\|\cdot\|^{\prime}\right)$ be a seminormed vector space over $k$ and $f: V \rightarrow V^{\prime}$ be a linear map of vector spaces over $k$ such that $f\left(N_{\|\cdot\|}\right) \subseteq N_{\|\cdot\|^{\prime}}$. Then $f$ is continuous and $\|f\|<+\infty$.

(3) A linear form on $V$ is bounded if and only if its kernel contains $N_{\|\cdot\|}$.

Proof. - (1) Let $\pi: V \rightarrow V / N_{\|\cdot\|}$ be the canonical projection map and $\|\cdot\|^{\sim}$ be the norm on $V / N_{\|\cdot\|}$ associated with $\|\cdot\|$. By Proposition 1.1.10, the linear map $\pi$ is continuous. If $W$ is a vector subspace of $V$ containing $N_{\|\cdot\|}$, then one has $W=$ $\pi^{-1}(\pi(W))$. By Proposition 1.1.11, $\pi(W)$ is complete with respect to the induced norm of $\|\cdot\|^{\sim}$ on $\pi(W)$, so that $\pi(W)$ is closed. Hence $W$ is also closed since it is the inverse image of a closed subset of $V / N_{\|\cdot\|}$ by a continuous linear map.

(2) By replacing $V^{\prime}$ by $f(V)$, we may assume that $\operatorname{dim}_{k}\left(V^{\prime}\right)<\infty$. Thus the assertion follows from (1) and Corollary 1.1.12.

(3) Let $f: V \rightarrow k$ be a linear form. If $f$ is bounded, by Corollary 1.1.12, the kernel of $f$ is a closed vector subspace of $V$, hence it contains the closure of $\{0\}$, which is $N_{\|\cdot\|}$. Conversely, if $\operatorname{Ker}(f) \supseteq N_{\|\cdot\|}$, then by (2), the linear form $f$ is bounded.

Proposition 1.1.14. - (1) Let $V \stackrel{\alpha}{\longrightarrow} W \stackrel{\beta}{\longrightarrow} Q$ be a sequence of surjective linear maps of finite-dimensional vector spaces over $k$. For any seminorm $\|\cdot\|$ on $V$, (2) Let one has $\|\cdot\|_{V \rightarrow W, W \rightarrow Q}=\|\cdot\|_{V \rightarrow Q}$.

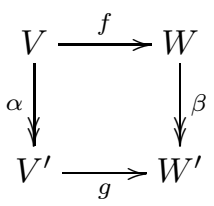

be a commutative diagram of linear maps of finite-dimensional vector spaces over $k$ such that $\alpha$ and $\beta$ are surjective. Then we have the following:

(2.a) Let $\|\cdot\|_{V}$ and $\|\cdot\|_{W}$ be seminorms on $V$ and $W$; let $\|\cdot\|_{V^{\prime}}$ and $\|\cdot\|_{W^{\prime}}$ be the quotient seminorms of $\|\cdot\|_{V}$ and $\|\cdot\|_{W}$ on $V^{\prime}$ and $W^{\prime}$, respectively. If $f\left(N_{\|\cdot\|_{V}}\right) \subseteq N_{\|\cdot\|_{W}}$, then $g\left(N_{\|\cdot\|_{V^{\prime}}}\right) \subseteq N_{\|\cdot\|_{W^{\prime}}}$ and $\|g\| \leqslant\|f\|$.

(2.b) We assume that $f$ and $g$ are injective. Let $\|\cdot\|_{W}$ be a seminorm on $W$. Then $\|\cdot\|_{W, V \hookrightarrow W, V \rightarrow V^{\prime}} \geqslant\|\cdot\|_{W, W \rightarrow W^{\prime}, V^{\prime} \hookrightarrow W^{\prime}}$. Moreover, if $\operatorname{Ker}(\beta) \subseteq$ $f(V)$, then the equality $\|\cdot\|_{W, V \hookrightarrow W, V \rightarrow V^{\prime}}=\|\cdot\|_{W, W \rightarrow W^{\prime}, V^{\prime} \hookrightarrow W^{\prime}}$ holds. 
Proof. - (1) For $q \in Q$, one has

$$
\begin{aligned}
\|q\|_{V \rightarrow W, W \rightarrow Q} & =\inf _{y \in W, \beta(y)=q}\|y\|_{V \rightarrow W}=\inf _{\substack{y \in W \\
\beta(y)=q \alpha(x)=y}} \inf _{\substack{x \in V \\
\alpha(x)}}\|x\|_{V} \\
& =\inf _{x \in V, \beta(\alpha(x))=q}\|x\|_{V}=\|q\|_{V \rightarrow Q},
\end{aligned}
$$

as desired.

(2.a) By Proposition 1.1.9, $\alpha^{-1}\left(N_{\|\cdot\|_{V^{\prime}}}\right)$ is the closure of $\operatorname{Ker}(\alpha)$ in $V$, hence is equal to $\operatorname{Ker}(\alpha)+N_{\|\cdot\|_{V}}$. Let $y^{\prime}$ be an element in $N_{\|\cdot\|_{V^{\prime}}}$. There then exists $y \in N_{\|\cdot\|_{V}}$ such that $\alpha(y)=y^{\prime}$. Therefore

$$
g\left(y^{\prime}\right)=g(\alpha(y))=\beta(f(y)) \in N_{\|\cdot\|_{W^{\prime}}}
$$

since $f(y) \in N_{\|\cdot\|_{W}}$.

It remains to prove that $\|g\| \leqslant\|f\|$. Let $x^{\prime}$ be an element of $V^{\prime}$. For any $x \in V$ with $\alpha(x)=x^{\prime}$, one has

$$
\left\|g\left(x^{\prime}\right)\right\|_{W^{\prime}}=\|g(\alpha(x))\|_{W^{\prime}}=\|\beta(f(x))\|_{W^{\prime}} \leqslant\|f(x)\|_{W} \leqslant\|f\| \cdot\|x\|_{V},
$$

which leads to

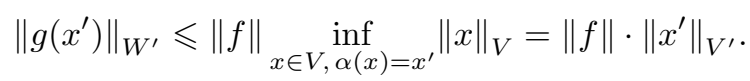

(2.b) Note that $f(\operatorname{Ker}(\alpha))=f(V) \cap \operatorname{Ker}(\beta)$. Therefore, for $v \in V$,

$$
\|\alpha(v)\|_{W, V \hookrightarrow W, V \rightarrow V^{\prime}}=\inf \left\{\|x\|_{W}: x \in f(v)+(f(V) \cap \operatorname{Ker}(\beta))\right\}
$$

and

$$
\|\alpha(v)\|_{W, W \rightarrow W^{\prime}, V^{\prime} \hookrightarrow W^{\prime}}=\inf \left\{\|x\|_{W}: x \in f(v)+\operatorname{Ker}(\beta)\right\},
$$

so that the first assertion follows. Moreover, if $\operatorname{Ker}(\beta) \subseteq f(V)$, then $f(V) \cap \operatorname{Ker}(\beta)=$ $\operatorname{Ker}(\beta)$. Thus the second assertion holds.

Proposition 1.1.15. - (1) Let $f: V \rightarrow W$ be a surjective linear map of vector spaces over $k,\|\cdot\|_{V}$ be a seminorm on $V$ and $\|\cdot\|_{W}$ be the quotient seminorm of $\|\cdot\|_{V}$ on $W$. If the seminorm $\|\cdot\|_{W}$ does not vanish, then $\|f\|=1$.

(2) Let

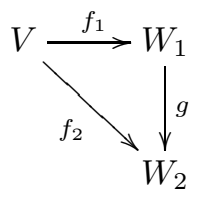

be a commutative digram of linear maps of finite-dimensional vector spaces over $k$ such that $g$ is an isomorphism and $\operatorname{dim}_{k}\left(W_{1}\right)=\operatorname{dim}_{k}\left(W_{2}\right)=1$. Let $\|\cdot\|_{V}$, $\|\cdot\|_{W_{1}}$ and $\|\cdot\|_{W_{2}}$ be seminorms of $V, W_{1}$ and $W_{2}$, respectively. Then $\left\|f_{2}\right\|=$ $\left\|f_{1}\right\| \cdot\|g\|$ provided that $f_{1}, f_{2}$ and $g$ are continuous. 
Proof. - (1) Since $\|v\|_{V} \geqslant\|f(v)\|_{W}$ for any $v \in V$, one has $\|f\| \leqslant 1$. Let $w$ be an element of $W$ such that $\|w\|_{W}>0$. Since

$$
\|w\|_{W}=\inf _{v \in V, f(v)=w}\|v\|_{V}
$$

one has

$$
1=\inf _{v \in V, f(v)=w} \frac{\|v\|_{V}}{\|f(v)\|_{W}} \geqslant\|f\|^{-1},
$$

which leads to $\|f\| \geqslant 1$.

(2) As $g$ is an isomorphism and $\operatorname{dim}_{k}\left(W_{1}\right)=\operatorname{dim}_{k}\left(W_{2}\right)=1$, for any $w_{1} \in W_{1}$, $\|g\| \cdot\left\|w_{1}\right\|_{W_{1}}=\left\|g\left(w_{1}\right)\right\|_{W_{2}}$, Therefore,

$$
\begin{aligned}
\left\|f_{2}\right\| & =\sup _{v \in V \backslash N_{\|\cdot\|_{V}}} \frac{\left\|f_{2}(v)\right\|_{W_{2}}}{\|v\| \|_{V}}=\sup _{v \in V \backslash N_{\|\cdot\|_{V}}} \frac{\left\|g\left(f_{1}(v)\right)\right\|_{W_{2}}}{\|v\|_{V}} \\
& =\sup _{v \in V \backslash N_{\|\cdot\|_{V}}}\|g\| \frac{\left\|f_{1}(v)\right\|_{W_{1}}}{\|v\|_{V}}=\|g\| \cdot\left\|f_{1}\right\|,
\end{aligned}
$$

as required.

Proposition 1.1.16. — Let $\left(V,\|\cdot\|_{V}\right)$ be a finite-dimensional seminormed vector space over $k, W$ be a vector subspace of $V$ and $Q$ be the quotient vector space $V / W$. We denote by $i: W \rightarrow V$ and $\pi: V \rightarrow Q$ the inclusion map and the projection map, respectively. Let $\|\cdot\|_{W}$ be the restriction of $\|\cdot\|_{V}$ to $W$ and $\|\cdot\|_{Q}$ be the quotient seminorm of $\|\cdot\|_{V}$ on $Q$. Then one has $i\left(N_{\|\cdot\|_{W}}\right) \subseteq N_{\|\cdot\|_{V}}$ and $\pi\left(N_{\|\cdot\|_{V}}\right) \subseteq N_{\|\cdot\|_{Q}}$. Moreover, the linear maps $i$ and $\pi$ induce short exact sequences

$$
0 \longrightarrow N_{\|\cdot\|_{W}} \longrightarrow N_{\|\cdot\|_{V}} \longrightarrow N_{\|\cdot\|_{Q}} \longrightarrow 0
$$

and

$$
0 \longrightarrow W / N_{\|\cdot\|_{W}} \longrightarrow V / N_{\|\cdot\|_{V}} \longrightarrow Q / N_{\|\cdot\|_{Q}} \longrightarrow 0
$$

and the induced norm (resp. quotient norm) of $\|\cdot\|_{V}^{\sim}$ on $W / N_{\|\cdot\|_{W}}\left(\operatorname{resp} . Q / N_{\|\cdot\|_{Q}}\right)$ identifies with $\|\cdot\|_{W}\left(\right.$ resp. $\left.\|\cdot\|_{Q}^{\sim}\right)$.

Proof. - The relations $i\left(N_{\|\cdot\|_{W}}\right) \subseteq N_{\|\cdot\|_{V}}$ and $\pi\left(N_{\|\cdot\|_{V}}\right) \subseteq N_{\|\cdot\|_{Q}}$ follow directly from the definition of induced and quotient seminorms. Moreover, by definition one has $N_{\|\cdot\|_{W}}=N_{\|\cdot\|_{V}} \cap W$.

For any element $x \in V, \pi(x)$ lies in $N_{\|\cdot\|_{Q}}$ if and only if $x \in W+N_{\|\cdot\|_{V}}$ since $W+N_{\|\cdot\|_{V}}$ is the closure of $W$ in $V$. Therefore one has

$$
N_{\|\cdot\|_{Q}} \cong\left(W+N_{\|\cdot\|_{V}}\right) / W \cong N_{\|\cdot\|_{V}} /\left(W \cap N_{\|\cdot\|_{V}}\right)=N_{\|\cdot\|_{V}} / N_{\|\cdot\|_{W}}
$$

which proves that (1.2) is an exact sequence. The exactness of (1.2) implies that of (1.3). Moreover, if $x$ is an element of $W$, then

$$
\|[x]\|_{W}^{\sim}=\|x\|_{W}=\|x\|_{V}=\|[x]\|_{V}^{\sim} .
$$


If $u$ is an element in $Q$, then

$$
\|[u]\|_{Q}^{\sim}=\|u\|_{Q}=\inf _{y \in V, \pi(y)=u}\|y\|_{V}=\inf _{v \in V / N_{\|\cdot\|_{V}}, \widetilde{\pi}(v)=[u]}\|v\|_{V}^{\sim},
$$

where $\tilde{\pi}: V / N_{\|\cdot\|_{V}} \rightarrow Q / N_{\|\cdot\|_{Q}}$ is the linear map induced by $\pi$. Hence $\|\cdot\|_{Q}^{\sim}$ identifies with the quotient norm of $\|\cdot\|_{V}^{\sim}$.

1.1.5. Dual norm. - Let $(V,\|\cdot\|)$ be a seminormed vector space over $k$. We denote by $V^{*}$ the vector space $\mathscr{L}(V, k)$ (where we consider $|\cdot|$ as a norm on $k$ ) of bounded $k$-linear forms on $V$ (which necessarily vanish on $N_{\|\cdot\|}$ ), called the dual normed vector space of $V$. The operator norm on $V^{*}$ is called the dual norm of $\|\cdot\|$, denoted by $\|\cdot\|_{*}$. Note that in general $V^{*}$ is different from the (algebraic) dual vector space $V^{\vee}:=\operatorname{Hom}_{k}(V, k)$. One has

$$
V^{*} \subseteq\left(V / N_{\|\cdot\|}\right)^{\vee}=\left\{\varphi \in V^{\vee}:\left.\varphi\right|_{N_{\|\cdot\|}}=0\right\} .
$$

Proposition 1.1.17. - Let $(V,\|\cdot\|)$ be a finite-dimensional seminormed vector space over $k$. Then the map $\left(V / N_{\|\cdot\|}\right)^{\vee} \rightarrow V^{\vee}$ sending $\varphi \in\left(V / N_{\|\cdot\|}\right)^{\vee}$ to its composition with the projection map $V \rightarrow V / N_{\|\cdot\|}$ defines an isomorphism between $\left(V / N_{\|\cdot\|}\right)^{\vee}$ and $V^{*}$. In particular, the equality $V^{*}=V^{\vee}$ holds when $\|\cdot\|$ is a norm.

Proof. - By Corollary 1.1.13, a linear form on $V$ is bounded if and only if its kernel contains $N_{\|\cdot\|}$. Therefore $V^{*}$ is canonically isomorphic to $\left(V / N_{\|\cdot\|}\right)^{\vee}$.

If $x$ is an element of $V$, for any $\alpha \in V^{*}$ one has

$$
|\alpha(x)| \leqslant\|\alpha\|_{*} \cdot\|x\| .
$$

Therefore the linear form on $V^{*}$ sending $\alpha \in V^{*}$ to $\alpha(x) \in k$ is bounded. Hence one obtains a $k$-linear map from $V$ to the double dual space $V^{* *}$ whose kernel contains $N_{\|\cdot\|}$. It is called the canonical linear map from $V$ to $V^{* *}$. The double dual norm $\|\cdot\|_{* *}$ on $V^{* *}$ induces by composition with the canonical $k$-linear map $V \rightarrow V^{* *}$ a seminorm on $V$ which we still denote by $\|\cdot\|_{* *}$ by abuse of notation. Moreover, by (1.4) we obtain

$$
\forall x \in V, \quad\|x\|_{* *} \leqslant\|x\| .
$$

We say that $(V,\|\cdot\|)$ is reflexive if the $k$-linear map $V \rightarrow V^{* *}$ described above induces an isometric $k$-linear isomorphism between the normed vector spaces $\left(V / N_{\|\cdot\|},\|\cdot\|^{\sim}\right)$ and $\left(V^{* *},\|\cdot\|_{* *}\right)$.

The following proposition shows that, in the Archimedean case, the seminorm $\|\cdot\|_{* *}$ on $V$ identifies with $\|\cdot\|$. In particular, a finite-dimensional seminormed vector space over an Archimedean complete field is always reflexive. We will see further in Corollary 1.2 .12 that any finite-dimensional ultrametrically seminormed vector space over $k$ is also reflexive. 
Proposition 1.1.18. - Assume that the absolute value $|\cdot|$ is Archimedean. Let $(V,\|\cdot\|)$ be a seminormed vector space over $k$. For any $x \in V$ one has $\|x\|=\|x\|_{* *}$.

Proof. - This is a direct consequence of Hahn-Banach theorem. In fact, if $x$ is a vector in $V \backslash N_{\|\cdot\|}$, then by Hahn-Banach theorem there exists a $k$-linear form $\tilde{f}: V / N_{\|\cdot\|} \rightarrow k$ such that $\tilde{f}(\pi(x))=\|\pi(x)\|^{\sim}$ and that $|\tilde{f}(\pi(y))| \leqslant\|\pi(y)\|^{\sim}$ for any $y \in V$, where $\pi: V \rightarrow V / N_{\|\cdot\|}$ is the canonical linear map. If we set $f=\tilde{f} \circ \pi$, then $f(x)=\|x\|$ and $|f(y)| \leqslant\|y\|$ for any $y \in V$. In particular, $\|f\|_{*}=1$. Hence

$$
\|x\|_{* *} \geqslant \frac{|f(x)|}{\|f\|_{*}}=\|x\| .
$$

Remark 1.1.19. - The above proposition is not true when the absolute value $|\cdot|$ is non-Archimedean. Let $(V,\|\cdot\|)$ be a normed vector space over $k$. If the absolute value $|\cdot|$ is non-Archimedean, then the dual norm $\|\cdot\|_{*}$ is necessarily ultrametric (cf. Subsection 1.1.2). For the same reason, the double dual norm $\|\cdot\|_{* *}$ is ultrametric, and hence cannot identify with $\|\cdot\|$ on $V$ once the norm $\|\cdot\|$ is not ultrametric. In the next section, we will establish the analogue of the above proposition in the case where $V$ is of finite rank over $k$ and $\|\cdot\|$ is ultrametric (see Corollary 1.2.12). We refer to [47] and [86] for more general results on non-Archimedean Hahn-Banach theorem.

Proposition 1.1.20. — Let $\left(V,\|\cdot\|_{V}\right)$ be a seminormed vector space over $k, W$ be a vector subspace of $V$ and $Q=V / W$ be the quotient space. Let $\|\cdot\|_{Q}$ be the quotient seminorm on $Q$ induced by $\|\cdot\|_{V}$. Then the map $Q^{*} \rightarrow V^{*}$ sending $\varphi \in Q^{*}$ to the composition of $\varphi$ with the projection map $V \rightarrow Q$ is an isometry from $Q^{*}$ to its image (equipped with the induced norm), where we consider the dual norms $\|\cdot\|_{Q, *}$ and $\|\cdot\|_{V, *}$ on $Q^{*}$ and $V^{*}$, respectively.

Proof. - Note that $\|v\|_{Q}^{-1}=\sup _{x \in V,[x]=v}\|x\|_{V}^{-1}$ for $v \in Q \backslash N_{\|\cdot\|_{Q}}$. Thus, for $\varphi \in Q^{*}$,

$$
\|\varphi\|_{Q, *}=\sup _{v \in Q \backslash N_{\|\cdot\|_{Q}}} \frac{|\varphi(v)|}{\|v\|_{Q}}=\sup _{x \in V \backslash N_{\|\cdot\|_{V}}} \frac{|\varphi([x])|}{\|x\|_{V}},
$$

as required.

Remark 1.1.21. - The dual statement of the above proposition for the dual of a restricted seminorm is much more subtle. Let $\left(V,\|\cdot\|_{V}\right)$ be a seminormed vector space over $k$ and $W$ be a vector subspace of $V$. We denote by $\|\cdot\|_{W}$ the restriction of the seminorm $\|\cdot\|_{V}$ to $W$. Then the restriction to $W$ of bounded linear forms on $V$ defines a $k$-linear map $\pi$ from $V^{*}$ to $W^{*}$. We are interested in the nature of the dual norm $\|\cdot\|_{W, *}$. In the case where $k$ is Archimedean, the $k$-linear map $\pi: V^{*} \rightarrow W^{*}$ is surjective and the norm $\|\cdot\|_{W, *}$ identifies with the quotient norm of $\|\cdot\|_{V, *}$. This is a direct consequence of Hahn-Banach theorem which asserts that any bounded linear form on $W$ extends to $V$ with the same operator norm (see Lemma 1.2 .48 for more 
details). However, the non-Archimedean analogue of this result is not true, even in the case where $V$ is finite-dimensional. In fact, assume that $(k,|\cdot|)$ is non-Archimedean and $V$ is a finite-dimensional vector space of rank $\geqslant 2$ over $k$, equipped with a norm $\|\cdot\|$ which is not ultrametric. Then the double dual norm $\|\cdot\|_{* *}$ on $V$ is bounded from above by $\|\cdot\|$ (see (1.5)), and there exists at least an element $x \in V$ such that $\|x\|_{* *}<\|x\|$ since $\|\cdot\|_{* *}$ is ultrametric but $\|\cdot\|$ is not. However, both norms $\|\cdot\|_{* *}$ and $\|\cdot\|$ induce the same dual norm on $V^{\vee}$ (see Proposition 1.2.14). Therefore the quotient norm of $\|\cdot\|_{*}$ on $(k x)^{\vee}$ can not identify with the dual norm of the restriction of $\|\cdot\|$ to $k x$. We will show in Proposition 1.2.35 that the non-Archimedean analogue of the above statement is true when the norm on $V$ is ultrametric.

1.1.6. Seminorm of the dual operator. - Let $\left(V_{1},\|\cdot\|_{1}\right)$ and $\left(V_{2},\|\cdot\|_{2}\right)$ be seminormed vector spaces over $k$, and $f: V_{1} \rightarrow V_{2}$ be a bounded linear map. Note that $f\left(N_{\|\cdot\|_{1}}\right) \subseteq N_{\|\cdot\|_{2}}$ by Proposition 1.1.7. For any $\alpha \in V_{2}^{*}$, we let $f^{*}(\alpha)$ be the linear form on $V_{1}$ which sends $x \in V_{1}$ to $\alpha(f(x))$. Note that for $x \in V_{1}$ one has

$$
\left|f^{*}(\alpha)(x)\right|=|\alpha(f(x))| \leqslant\|\alpha\|_{2, *} \cdot\|f(x)\|_{2} \leqslant\|\alpha\|_{2, *} \cdot\|f\| \cdot\|x\|_{1} .
$$

Therefore $f^{*}(\alpha)$ is a bounded linear form on $V_{1}$. Thus $f^{*}$ defines a linear map from $V_{2}^{*}$ to $V_{1}^{*}$.

Proposition 1.1.22. - Let $\left(V_{1},\|\cdot\|_{1}\right)$ and $\left(V_{2},\|\cdot\|_{2}\right)$ be seminormed vector spaces over $k$ and $f: V_{1} \rightarrow V_{2}$ be a bounded linear map. Then one has $\left\|f^{*}\right\| \leqslant\|f\|$. The equality holds when $\|\cdot\|_{2}=\|\cdot\|_{2, * *}$ on $V_{2}$.

Proof. - By (1.6) we obtain that, if $\alpha$ is an element of $V_{2}^{*}$, then one has

$$
\left\|f^{*}(\alpha)\right\|_{1, *} \leqslant\|f\| \cdot\|\alpha\|_{2, *} .
$$

Hence $\left\|f^{*}\right\| \leqslant\|f\|$. If we apply this inequality to $f^{*}$, we obtain $\left\|f^{* *}\right\| \leqslant\left\|f^{*}\right\| \leqslant\|f\|$. Let $\iota_{1}: V_{1} \rightarrow V_{1}^{* *}$ and $\iota_{2}: V_{2} \rightarrow V_{2}^{* *}$ be the canonical linear maps. For any vector $x$ in $V_{1}$, one has $f^{* *}\left(\iota_{1}(x)\right)=\iota_{2}(f(x))$. Moreover, if $\|\cdot\|_{2}=\|\cdot\|_{2, * *}$ on $V_{2}$, then

$$
\left\|f^{* *}\right\| \geqslant \sup _{x \in V_{1},\|x\|_{1, * *}>0} \frac{\left\|f^{* *}\left(\iota_{1}(x)\right)\right\|_{2, * *}}{\|x\|_{1, * *}} \geqslant \sup _{x \in V_{1},\|x\|_{1}>0} \frac{\|f(x)\|_{2}}{\|x\|_{1}}=\|f\|,
$$

as required.

1.1.7. Lattices and norms. - In this subsection, we assume that the absolute value $|\cdot|$ is non-Archimedean. Let $\mathfrak{o}_{k}:=\{a \in k:|a| \leqslant 1\}$ be the closed unit ball of $(k,|\cdot|)$. It is a valuation ring, namely for any $a \in k \backslash \mathfrak{o}_{k}$ one has $a^{-1} \in \mathfrak{o}_{k}$ (see [26] Chapter IV, $\S 1$, no.2). It is a discrete valuation ring (namely a Noetherian valuation ring) if and only if the absolute value $|\cdot|$ is discrete, namely the image of $k^{\times}$by $|\cdot|$ is a discrete subgroup of $\left(\mathbb{R}_{>0}, \times\right)$ (see [26] Chapter IV, $\S 3$, no.6). In this case, $\mathfrak{o}_{k}$ is a principal ideal domain. In particular, its maximal ideal $\{a \in k:|a|<1\}$ is generated by one element $\varpi$, called a uniformizing parameter of $k$. Note that, if the absolute 
value $|\cdot|$ is not discrete, then $\left|k^{\times}\right|$is a dense subgroup of $\left(\mathbb{R}_{>0}, \times\right)$. This results from the facts that a subgroup of $(\mathbb{R},+)$ is either discrete or dense (cf. [27] Chapter $\mathrm{V}, \S 1$, no.1 and $\S 4$, no.1) and that the exponential function defines an isomorphism between the topological groups $(\mathbb{R},+)$ and $\left(\mathbb{R}_{>0}, \times\right)$.

Definition 1.1.23. - Let $V$ be a finite-dimensional vector space over $k$. A sub$\mathfrak{o}_{k}$-module $\mathcal{V}$ of $V$ is called a lattice of $V$ if $\mathcal{V}$ generates $V$ as a vector space over $k$ (i.e. the natural linear map $\mathcal{V} \otimes_{\mathfrak{o}_{k}} k \rightarrow V$ is surjective) and $\mathcal{V}$ is bounded in $V$ for a certain norm on $V$ (or equivalently for any norm on $V$, see Proposition 1.1.11). In particular, if $\mathcal{V}$ is a sub-o $\mathfrak{o}_{k}$-module of finite type of $V$, which generates $V$ as a vector space over $k$, then it is a lattice in $V$. If $\mathcal{V}$ is a lattice of $V$, we define a function $\|\cdot\|_{\mathcal{V}}$ on $V$ as follows:(2)

$$
\forall x \in V \backslash\{0\}, \quad\|x\|_{\mathcal{V}}:=\inf \left\{|a|: a \in k^{\times}, a^{-1} x \in \mathcal{V}\right\}, \quad \text { and }\|0\|_{\mathcal{V}}:=0 .
$$

Clearly, if $\mathcal{V}$ and $\mathcal{V}^{\prime}$ are lattices of $V$ such that $\mathcal{V} \subseteq \mathcal{V}^{\prime}$, then one has $\|\cdot\|_{\mathcal{V}} \geqslant\|\cdot\|_{\mathcal{V}^{\prime}}$. Note that, if the absolute value $|\cdot|$ is trivial, then $\mathfrak{o}_{k}=k$ and the only lattice of $V$ is $V$ itself.

Proposition 1.1.24. - Let $V$ be a finite-dimensional vector space over $k, \mathcal{V}$ be a lattice of $V$ and $\|\cdot\|$ be a norm on $V$. Assume that $\mathcal{V}$ is contained in the unit ball of $(V,\|\cdot\|)$, then one has $\|\cdot\|_{\mathcal{V}} \geqslant\|\cdot\|$.

Proof. - Let $x \in V \backslash\{0\}$ and $a$ be an element of $k^{\times}$such that $a^{-1} x \in \mathcal{V}$. One has

$$
\left\|a^{-1} x\right\|=|a|^{-1} \cdot\|x\| \leqslant 1
$$

since $\mathcal{V}$ is contained in the unit ball of $(V,\|\cdot\|)$. Therefore $\|x\| \leqslant|a|$. Thus we deduce that

$$
\|x\| \leqslant \inf \left\{|a|: a \in k^{\times}, a^{-1} x \in \mathcal{V}\right\}=\|x\|_{\mathcal{V}}
$$

In the case where the absolute value $|\cdot|$ is non-trivial, the balls in an ultrametrically normed vector space are natural examples of lattices.

Proposition 1.1.25. - Assume that the absolute value $|\cdot|$ is non-trivial. Let $V$ be a finite-dimensional vector space over $k$, equipped with an ultrametric norm $\|\cdot\|$. For any $\epsilon>0$ the balls $V_{\leqslant \epsilon}=\{x \in V:\|x\| \leqslant \epsilon\}$ and $V_{<\epsilon}=\{x \in V:\|x\|<\epsilon\}$ are both lattices of $V$.

2. Note that, in the case where $|\cdot|$ is not the trivial absolute value, one has

$$
\inf \left\{|a|: a \in k^{\times}, a^{-1} 0 \in \mathcal{V}\right\}=0 .
$$

However, this equality does not hold when $|\cdot|$ is trivial. 
Proof. - Since the norm $\|\cdot\|$ is ultrametric, both balls $V_{\leqslant \epsilon}$ and $V_{<\epsilon}$ are stable by addition. Clearly they are also stable by the multiplication by an element in $\mathfrak{o}_{k}$. Therefore they are sub- $\mathfrak{o}_{k}$-modules of $V$. Moreover, by definition they are bounded subsets of $V$. It remains to verify that they generate $V$ as a vector space over $k$. It suffices to treat the open ball case. Let $\left\{e_{i}\right\}_{i=1}^{r}$ be a basis of $V$ over $k$. Since the absolute value $|\cdot|$ is non-trivial, there exists a non-zero element $a \in k$ such that $|a|<1$. For sufficiently large integer $n \in \mathbb{N}_{>0}$, one has $\left\|a^{n} e_{i}\right\|<\epsilon$ for any $i \in\{1, \ldots, r\}$. Hence $V_{<\epsilon}$ contains a basis of the vector space $V$.

The following proposition shows that each lattice defines a norm on the underlying vector space.

Proposition 1.1.26. - Let $V$ be a finite-dimensional vector space over $k$ and $\mathcal{V}$ be a lattice of $V$. The map $\|\cdot\|_{\mathcal{V}}$ is an ultrametric norm on $V$. Moreover, $\mathcal{V}$ is contained in the unit ball of $\left(V,\|\cdot\|_{\mathcal{V}}\right)$.

Proof. - In the case where the absolute value $|\cdot|$ is trivial, one has $\mathcal{V}=V$ and the function $\|\cdot\|_{\mathcal{V}}$ takes value 1 on $V \backslash\{0\}$ and vanishes on $\{0\}$. The result is clearly true in this case. In the following, we assume that $|\cdot|$ is non-trivial. For any $x \in V$, let $A_{x}$ be the set of all $a \in k^{\times}$such that $a^{-1} x \in \mathcal{V}$. We claim that $A_{x}$ is non-empty and hence $\|x\|_{\mathcal{V}}$ is finite. Let $\left\{e_{i}\right\}_{i=1}^{r}$ be a subset of $\mathcal{V}$ which forms a basis of $V$ over $k$. We write $x$ in the form $x=a_{1} e_{1}+\cdots+a_{r} e_{r}$ with $\left(a_{1}, \ldots, a_{r}\right) \in k^{r}$. Since $k$ is the fraction field of $\mathfrak{o}_{k}$, there exists $b \in k^{\times}$such that $b a_{1}, \ldots, b a_{r}$ are all in $\mathfrak{o}_{k}$. Thus $b x \in \mathcal{V}$ and hence $b^{-1} \in A_{x}$. Therefore $\|\cdot\|_{\mathcal{V}}$ is a map from $V$ to $\mathbb{R}_{\geqslant 0}$.

Let $x$ be an element of $V$ and $a \in k^{\times}$. The map $b \mapsto a b$ defines a bijection between $A_{x}$ and $A_{a x}$. Hence one has $\|a x\|_{\mathcal{V}}=|a| \cdot\|x\|_{\mathcal{V}}$.

Let $x$ and $y$ be elements of $V, a \in A_{x}$ and $b \in A_{y}$. One has $\left\{a^{-1} x, b^{-1} y\right\} \subseteq \mathcal{V}$. Note that

$$
\begin{aligned}
a^{-1}(x+y) & =a^{-1} x+a^{-1} y=a^{-1} x+\left(a^{-1} b\right)\left(b^{-1} y\right), \\
b^{-1}(x+y) & =b^{-1} x+b^{-1} y=\left(b^{-1} a\right)\left(a^{-1} x\right)+b^{-1} y .
\end{aligned}
$$

Since $\mathfrak{o}_{k}$ is a valuation ring, either $b^{-1} a \in \mathfrak{o}_{k}$, or $a^{-1} b \in \mathfrak{o}_{k}$. Hence, either $a \in A_{x+y}$ or $b \in A_{x+y}$. Therefore $\|x+y\|_{\mathcal{V}} \leqslant \max \left\{\|x\|_{\mathcal{V}},\|y\|_{\mathcal{V}}\right\}$.

It remains to verify that, if $\|x\|_{\mathcal{V}}=0$ then $x=0$. Assume that there exists a non-zero element $x \in V$ such that $\|x\|_{\mathcal{V}}=0$. Then there exists a sequence $\left\{a_{n}\right\}_{n \in \mathbb{N}}$ in $A_{x}$ such that $\lim _{n \rightarrow+\infty}\left|a_{n}\right|=0$. However, one has $a_{n}^{-1} x \in \mathcal{V}$ for any $n \in \mathbb{N}$. This contradicts the assumption that $\mathcal{V}$ is bounded.

If $x$ is an element in $\mathcal{V}$, then 1 belongs to $A_{x}$. Hence $\|x\|_{\mathcal{V}} \leqslant 1$.

Definition 1.1.2\%. - Let $V$ be a finite-dimensional vector space over $k$ and $\mathcal{V}$ be a lattice of $V$. We call $\|\cdot\|_{\mathcal{V}}$ the norm on $V$ induced by the lattice $\mathcal{V}$. 
Proposition 1.1.28. — Let $V$ be a finite-dimensional vector space over $k$ and $r$ be its rank over $k$. Let $\mathcal{V}$ be a lattice of $V$. Assume that $\mathcal{V}$ is an $\mathfrak{o}_{k}$-module of finite type. Then it is a free $\mathfrak{o}_{k}$-module of rank $r$.

Proof. - Since $\mathcal{V}$ is a sub-o ${ }_{k}$-module of $V$, it is torsion-free. By [26] Chapter VI, $\S 4$, no.6, Lemma 1, any torsion-free module of finite type over a valuation ring is free. Hence $\mathcal{V}$ is a free $\mathfrak{o}_{k}$-module. Finally, since $\mathcal{V}$ generates $V$ as a vector space over $k$, any basis of $\mathcal{V}$ over $\mathfrak{o}_{k}$ is also a basis of $V$ over $k$. Hence the rank of $\mathcal{V}$ over $\mathfrak{o}_{k}$ is $r$.

Definition 1.1.29. - Let $(V,\|\cdot\|)$ be a finite-dimensional seminormed vector space over $k$. We define the default of purity of $\|\cdot\|$ as

$$
\operatorname{dpur}(\|\cdot\|):=\sup _{x \in V \backslash N_{\|\cdot\|}} \operatorname{dist}\left(\ln \|x\|, \ln \left|k^{\times}\right|\right),
$$

with

$$
\operatorname{dist}\left(\ln \|x\|, \ln \left|k^{\times}\right|\right):=\inf \left\{|\ln \|x\|-\ln | a||: a \in k^{\times}\right\} .
$$

We say that the seminorm $\|\cdot\|$ is pure if $\operatorname{dpur}(\|\cdot\|)=0$, or equivalently, the image of $V \backslash N_{\|\cdot\|}$ by $\|\cdot\|$ is contained in the closure of $\left|k^{\times}\right|$in $\mathbb{R}_{>0}$. By definition, if the absolute value $|\cdot|$ is not discrete, then any seminorm on $V$ is pure; if $|\cdot|$ is discrete, then a seminorm $\|\cdot\|$ on $V$ is pure if and only if its image is contained in that of $|\cdot|$. In the case where $|\cdot|$ is discrete, Moreover, for any lattice $\mathcal{V}$ of $V$, the norm $\|\cdot\|_{\mathcal{V}}$ is pure.

In the following, we study the correspondance between ultrametric norms and lattices of a finite-dimensional vector space over $k$. Note that the behaviour depends much on the discreteness of the absolute value $|\cdot|$.

Proposition 1.1.30. - Assume that the absolute value $|\cdot|$ is discrete.

(1) For any lattice $\mathcal{V}$ of $V$, one has $\left(V,\|\cdot\|_{\mathcal{V}}\right)_{\leqslant 1}=\mathcal{V}$ (see Notation 1.1.3).

(2) Any lattice $\mathcal{V}$ of $V$ is a free $\mathfrak{o}_{k}$-module of rank $\operatorname{dim}_{k}(V)$.

(3) Assume in addition that the absolute value $|\cdot|$ is non-trivial. Let $\|\cdot\|$ be an ultrametric norm on $V$ and let $\mathcal{V}=(V,\|\cdot\|)_{\leqslant 1}$. Then one has $\|\cdot\| \leqslant\|\cdot\|_{\mathcal{V}} \leqslant$ $|\varpi|^{-1}\|\cdot\|$, where $\varpi$ is a uniformizing parameter of $k$. In particular, the default of purity of $\|\cdot\|$ is bounded from above by $-\ln |\varpi|$. Moreover, if the norm $\|\cdot\|$ is pure, then $\|\cdot\|_{\mathcal{V}}=\|\cdot\|$.

Proof. - (1) By Proposition 1.1.26, one has $\mathcal{V} \subseteq\left(V,\|\cdot\|_{\mathcal{V}}\right)_{\leqslant 1}$. Let $x$ be an element of $V$ such that $\|x\|_{\mathcal{V}} \leqslant 1$. In order to see that $x \in \mathcal{V}$, we may assume that $x \neq 0$. There is a sequence $\left\{\alpha_{n}\right\}_{n \in \mathbb{N}}$ in $k^{\times}$such that $\alpha_{n}^{-1} x \in \mathcal{V}$ and $\lim _{n \rightarrow \infty}\left|\alpha_{n}\right|=\|x\|_{\mathcal{V}}$. As $|\cdot|$ is discrete, there is $n \in \mathbb{N}$ such that $\left|\alpha_{n}\right|=\|x\|_{\mathcal{V}}$, so that $\alpha_{n} \in \mathfrak{o}_{k}$ because $\|x\|_{\mathcal{V}} \leqslant 1$. Therefore, $x \in \alpha_{n} \mathcal{V} \subseteq \mathcal{V}$, and hence $\left(V,\|\cdot\|_{\mathcal{V}}\right) \leqslant 1 \subseteq \mathcal{V}$.

(2) Let $\left\{e_{i}\right\}_{i=1}^{r}$ be a basis of $V$ over $k$. We equip $V$ with the norm $\|\cdot\|$ such that

$$
\left\|\lambda_{1} e_{1}+\cdots+\lambda_{r} e_{r}\right\|=\max \left\{\left|\lambda_{1}\right|, \ldots,\left|\lambda_{r}\right|\right\}
$$


for any $\left(\lambda_{1}, \ldots, \lambda_{r}\right) \in k^{r}$. For any $\epsilon>0$, the ball

$$
(V,\|\cdot\|)_{\leqslant \epsilon}=\{a \in k:|a| \leqslant \epsilon\}^{r}
$$

is a free $\mathfrak{o}_{k}$-module of rank $r$ since $\mathfrak{o}_{k}$ is a principal ideal domain. Let $\mathcal{V}$ be a lattice. Since it is bounded, it is contained in certain ball $(V,\|\cdot\|)_{\leqslant \epsilon}$. Thus $\mathcal{V}$ is an $\mathfrak{o}_{k}$-module of finite type, and hence a free $\mathfrak{o}_{k}$-module of rank $r$ by Proposition 1.1.28,

(3) By the definition of the uniformizing element, one has $\left|k^{\times}\right|=\left\{|\varpi|^{n}: n \in \mathbb{Z}\right\}$. If $x$ is a non-zero element in $V$ and if $A_{x}$ is the set of all $a \in k^{\times}$such that

$$
\left\|a^{-1} x\right\|=|a|^{-1} \cdot\|x\| \leqslant 1
$$

then one has

$$
\left\{|a|: a \in A_{x}\right\}=\left\{|\varpi|^{n}: n \in \mathbb{Z},|\varpi|^{n} \geqslant\|x\|\right\} .
$$

Since $\|x\|_{\mathcal{V}}=\inf \left\{|a|: a \in A_{x}\right\}$, one has $\|x\|_{\mathcal{V}} \geqslant\|x\|>|\varpi| \cdot\|x\|_{\mathcal{V}}$. Combined with the fact that the norm $\|\cdot\|_{\mathcal{V}}$ is pure, this implies the inequality $\operatorname{dpur}(\|\cdot\|) \leqslant-\ln |\varpi|$. If in addition the norm $\|\cdot\|$ is pure, $\|x\|_{\mathcal{V}}$ belongs to $\left\{|\varpi|^{n}: n \in \mathbb{Z}\right\}$. Hence $\|x\|_{\mathcal{V}}=$ $\|x\|$.

Remark 1.1.31. - Let $V$ be a finite-dimensional vector space over $k$. We denote by $\operatorname{Lat}(V)$ the set of all lattices of $V$, and by $\operatorname{Nor}(V)$ that of all ultrametric norms on $V$. The correspondance $(\mathcal{V} \in \operatorname{Lat}(V)) \mapsto\|\cdot\|_{\mathcal{V}}$ defines a map from $\operatorname{Lat}(V)$ to $\operatorname{Nor}(V)$. Proposition 1.1.30 shows that, if the absolute value $|\cdot|$ is discrete, then this map is injective, and its image is precisely the set of all pure ultrametric norms.

Proposition 1.1.32. - Assume that the absolute value $|\cdot|$ is not discrete.

(1) For any lattice $\mathcal{V}$ of $V$ one has $\left(V,\|\cdot\|_{\mathcal{V}}\right)_{<1} \subseteq \mathcal{V} \subseteq\left(V,\|\cdot\|_{\mathcal{V}}\right)_{\leqslant 1}$. If in addition there exists an ultrametric norm $\|\cdot\|$ on $V$ such that $\mathcal{V}=(V,\|\cdot\|) \leqslant 1$, then one has $\mathcal{V}=\left(V,\|\cdot\|_{\mathcal{V}}\right)_{\leqslant 1}$.

(2) Let $\|\cdot\|$ be an ultrametric norm on $V$ and $\mathcal{V}=(V,\|\cdot\|)_{\leqslant 1}$. Then $\|\cdot\|=\|\cdot\|_{\mathcal{V}}$.

Proof. - (1) If $x$ is an element of $\mathcal{V}$, by the relation $1 x=x \in \mathcal{V}$ we obtain that $\|x\|_{\mathcal{V}} \leqslant 1$. Hence $\mathcal{V} \subseteq\left(V,\|\cdot\|_{\mathcal{V}}\right)_{1}$. In the following, we prove the inclusion relation $\left(V,\|\cdot\|_{\mathcal{V}}\right)_{<1} \subseteq \mathcal{V}$. Let $x$ be an element in $V$ such that $\|x\|_{\mathcal{V}}<1$. By definition there exists $a \in k^{\times},|a|<1$, such that $a^{-1} x \in \mathcal{V}$. Since $|a|<1$ one has $a \in \mathfrak{o}_{k}$. Therefore $x=a\left(a^{-1} x\right) \in \mathcal{V}$.

The second assertion of (1) is a direct consequence of (2) In the following, we prove the statement $(2)$, Let $x$ be an element of $V$ and

$$
A_{x}=\left\{a \in k^{\times}: a^{-1} x \in \mathcal{V}\right\}=\left\{a \in k^{\times}:\|x\| \leqslant|a|\right\} .
$$

Since the image of $|\cdot|$ is dense in $\mathbb{R}_{+}$, one has $\|x\|_{\mathcal{V}}=\inf \left\{|a|: a \in A_{x}\right\}=\|x\|$. Hence $\|\cdot\|_{\mathcal{V}}=\|\cdot\|$.

Remark 1.1.33. - Let $V$ be a finite-dimensional vector space over $k$. Proposition 1.1 .32 shows that, if the absolute value $|\cdot|$ is not discrete, the map $\operatorname{Lat}(V) \rightarrow \operatorname{Nor}(V)$, 
sending any lattice $\mathcal{V}$ of $V$ to the norm $\|\cdot\|_{\mathcal{V}}$, is surjective (compare with Remark 1.1.31).

Proposition 1.1.34. - Let $V$ be a finite-dimensional vector space over $k$ and $\mathcal{V}$ be a lattice of $V$. Let $\mathcal{V}^{\vee}=\operatorname{Hom}_{\mathfrak{o}_{k}}\left(\mathcal{V}, \mathfrak{o}_{k}\right)$ be the dual $\mathfrak{o}_{k}$-module of $\mathcal{V}$. Then one has $\|\cdot\|_{\mathcal{V}, *}=\|\cdot\|_{\mathcal{V} \vee}$ on $V^{\vee}$.

Proof. - Let $f$ be a non-zero element of $V^{\vee}$. Assume that $a$ is an element of $k^{\times}$ such that $a^{-1} f \in \mathcal{V}^{\vee}$. Then for any $x \in V$ and any $b \in k^{\times}$such that $b^{-1} x \in \mathcal{V}$ one has $a^{-1} f\left(b^{-1} x\right)=(a b)^{-1} f(x) \in \mathfrak{o}_{k}$ and hence $|b| \geqslant|f(x)| /|a|$. Since $b$ is arbitrary one has $\|x\|_{\mathcal{V}} \geqslant|f(x)| /|a|$ for any $x \in V$ and hence $|a| \geqslant\|f\|_{\mathcal{V}, *}$. Since $a$ is arbitrary we obtain $\|f\|_{\mathcal{V} \vee} \geqslant\|f\|_{\mathcal{V}, *}$.

Conversely, suppose that the operator norm of a non-zero linear form $f: V \rightarrow k$ is bounded from above by 1 , where we consider the norm $\|\cdot\|_{\mathcal{V}}$ on $V$. Then for any $x \in \mathcal{V}$ one has $|f(x)| \leqslant\|x\|_{\mathcal{V}} \leqslant 1$ and hence $f(x) \in \mathfrak{o}_{k}$. This shows that $f \in \mathcal{V}^{\vee}$ and hence $\|f\|_{\mathcal{V} \vee} \leqslant 1$. Therefore the unit ball of $\|\cdot\|_{\mathcal{V} \vee}$ contains that of $\|\cdot\|_{\mathcal{V}, *}$. Moreover, since the norm $\|\cdot\|_{\mathcal{V}}$ is pure, also is its dual norm $\|\cdot\|_{\mathcal{V}, *}$. Therefore, the norm $\|\cdot\|_{\mathcal{V} . *}$ coincides with the norm induced by its unit ball (see Propositions 1.1.30 and 1.1.32). Therefore, $\|\cdot\|_{\mathcal{V} \vee} \leqslant\|\cdot\|_{\mathcal{V}, *}$. The proposition is thus proved.

1.1.8. Trivial valuation case. - In this subsection, we study ultrametrically normed vector spaces over a trivially valued field. We fix a field $k$ equipped with the trivial absolute value $|\cdot|$. If $V$ is a vector space over $k$, we denote by $\Theta(V)$ the set of all non-zero vector subspaces of $V$. The set $\Theta(V)$ is equipped with the partial order of inclusion. If $\|\cdot\|$ is an ultrametric norm on $V$, we denote by $\Psi(V,\|\cdot\|)$ the set of closed balls of $V$ (centered at the origin) which do not reduce to one point, namely (see Notation 1.1.3)

$$
\Psi(V,\|\cdot\|)=\left\{(V,\|\cdot\|)_{\leqslant r}: r>0,(V,\|\cdot\|)_{\leqslant r} \neq\{0\}\right\} .
$$

Proposition 1.1.35. - Let $V$ be a finite-dimensional vector space equipped with an ultrametric norm $\|\cdot\|$. The set $\Psi(V,\|\cdot\|)$ is a totally ordered subset of $\Theta(V)$, whose cardinal does not exceed the rank of $V$ over $k$.

Proof. - By definition the set $\Psi(V,\|\cdot\|)$ is totally ordered with respect to the partial order of inclusion. In the following, we show that any element $W \in \Psi(V,\|\cdot\|)$ is a vector subspace of $V$ and hence belongs to $\Theta(V)$. Assume that $W=(V,\|\cdot\|)_{\leqslant r}$ with $r>0$. Since the absolute value on $k$ is trivial, for any $x \in W$ and any $a \in k$ one has $\|a x\| \leqslant\|x\| \leqslant r$. Moreover, since the norm $\|\cdot\|$ is ultrametric, $W$ is stable by addition. Hence $\Psi(V,\|\cdot\|)$ is a totally ordered subset of $\Theta(V)$. In particular, the function $\operatorname{rk}_{k}(\cdot): \Psi(V,\|\cdot\|) \rightarrow \mathbb{N}_{\geqslant 1}$ is injective, which is bounded from above by $\operatorname{rk}_{k}(V)$. Therefore the cardinal of $\Psi(V,\|\cdot\|)$ does not exceed $\operatorname{rk}_{k}(V)$. 
The above proposition shows that the set $\Psi(V,\|\cdot\|)$ actually forms an increasing flag of non-zero vector subspaces of $V$. For any $W \in \Psi(V,\|\cdot\|)$, let

$$
\varphi_{\|\cdot\|}(W):=\sup \left\{t \in \mathbb{R}: W \subseteq(V,\|\cdot\|)_{\leqslant \mathrm{e}^{-t}}\right\} .
$$

Then $\varphi_{\|\cdot\|}$ is a strictly decreasing function on $\Psi(V,\|\cdot\|)$ in the sense that, if $W_{1}$ and $W_{2}$ are two elements of $\Psi(V,\|\cdot\|)$ such that $W_{1} \subsetneq W_{2}$, then one has $\varphi_{\|\cdot\|}\left(W_{1}\right)>\varphi_{\|\cdot\|}\left(W_{2}\right)$. The following proposition shows that the norm $\|\cdot\|$ is completely determined by the increasing flag $\Psi(V,\|\cdot\|)$ and the function $\varphi_{\|\cdot\|}$.

Proposition 1.1.36. - Let $\Psi$ be a totally ordered subset of $\Theta(V)$ and $\varphi:(\Psi, \supseteq) \rightarrow$ $(\mathbb{R}, \leqslant)$ be a function which preserves strictly the orders, that is, for any $\left(W_{1}, W_{2}\right) \in \Psi^{2}$ with $W_{1} \subsetneq W_{2}$, one has $\varphi\left(W_{1}\right)>\varphi\left(W_{2}\right)$. Then there exists a unique ultrametric norm $\|\cdot\|$ on $V$ such that $\Psi(V,\|\cdot\|)=\Psi$ and $\varphi_{\|\cdot\|}=\varphi$.

Proof. - We write $\Psi$ in the form of an increasing flag $V_{1} \subsetneq \ldots \subsetneq V_{n}$. For $i \in$ $\{1, \ldots, n\}$, let $a_{i}=\varphi\left(V_{i}\right)$. Since $\varphi$ preserves strictly the orders, one has $a_{1}>\ldots>$ $a_{n}$. Let $\boldsymbol{e}=\left\{e_{j}\right\}_{j=1}^{m}$ be a basis of $V$ which is compatible with the flag $\Psi$ (namely $\operatorname{card}\left(\mathbf{e} \cap V_{i}\right)=\operatorname{rk}_{k}\left(V_{i}\right)$ for any $\left.i \in\{1, \ldots, n\}\right)$. For any $j \in\{1, \ldots, m\}$, there exists a unique $i \in\{1, \ldots, n\}$ such that $e_{j} \in V_{i} \backslash V_{i-1}$ (where $V_{0}=\{0\}$ by convention) and we let $r_{j}=\mathrm{e}^{-a_{i}}$. Let $\|\cdot\|$ be the ultrametric norm on $V$ defined as

$$
\forall\left(\lambda_{1}, \ldots, \lambda_{m}\right) \in k^{m}, \quad\left\|\lambda_{1} e_{1}+\cdots+\lambda_{m} e_{m}\right\|=\max _{\substack{j \in\{1, \ldots, m\} \\ \lambda_{j} \neq 0}} r_{j} .
$$

Note that for $r \geqslant 0$ the ball $(V,\|\cdot\|)_{\leqslant r}$ identifies with the vector subspace generated by those $e_{j}$ with $r_{j} \leqslant r$. Hence one has $\Psi(V,\|\cdot\|)=\Psi$. Moreover, for any $i \in\{1, \ldots, n\}$ one has

$$
\varphi_{\|\cdot\|}\left(V_{i}\right)=\sup \left\{t \in \mathbb{R}: V_{i} \subseteq(V,\|\cdot\|)_{\leqslant \mathrm{e}^{-t}}\right\}=a_{i}=\varphi\left(V_{i}\right) .
$$

Let $\|\cdot\|^{\prime}$ be another ultrametric norm on $V$ verifying the relations $\Psi\left(V,\|\cdot\|^{\prime}\right)=\Psi$ and $\varphi_{\|\cdot\|^{\prime}}=\varphi$. For any $r \geqslant 0,\left(V,\|\cdot\|^{\prime}\right)_{\leqslant r}=V_{i}$ if and only if $r \in\left[\mathrm{e}^{-a_{i}}, \mathrm{e}^{-a_{i+1}}[\right.$, with the convention $a_{0}=+\infty$ and $a_{n+1}=-\infty$. Therefore one has $(V,\|\cdot\|) \leqslant r=\left(V,\|\cdot\|^{\prime}\right) \leqslant r$ for any $r \geqslant 0$, which leads to $\|\cdot\|=\|\cdot\|^{\prime}$.

Definition 1.1.3\% - Let $V$ be a finite-dimensional vector space over $k$. A family $\mathcal{F}=\left\{\mathcal{F}^{t}(V)\right\}_{t \in \mathbb{R}}$ of vector subspaces of $V$ parametrised by $\mathbb{R}$ is called an $\mathbb{R}$-filtration of $V$ if it is separated $\left(\mathcal{F}^{t}(V)=\{0\}\right.$ for sufficiently positive $\left.t\right)$, exhaustive $\left(\mathcal{F}^{t}(V)=V\right.$ for sufficiently negative $t$ ) and left-continuous (the function $(t \in \mathbb{R}) \rightarrow \operatorname{rk}_{k}\left(\mathcal{F}^{t}(V)\right)$ is left-continuous).

Definition 1.1.38. - Let $V$ be a finite-dimensional vector space over $k$ and $\mathcal{F}$ be an $\mathbb{R}$-filtration on $V$. Let $r$ be the rank of $V$ over $k$. We define a map $Z_{\mathcal{F}}:\{1, \ldots, r\} \rightarrow \mathbb{R}$ as follows:

$$
\forall i \in\{1, \ldots, r\}, \quad Z_{\mathcal{F}}(i):=\sup \left\{t \in \mathbb{R}: \operatorname{rk}_{k}\left(\mathcal{F}^{t}(V)\right) \geqslant i\right\}
$$


By definition, for any $t \in \mathbb{R}$ and any $i \in\{1, \ldots, r\}$ one has

$$
Z_{\mathcal{F}}(i) \geqslant t \Longleftrightarrow \operatorname{rk}_{k}\left(\mathcal{F}^{t}(V)\right) \geqslant i \text {. }
$$

Proposition 1.1.39. - Let $V$ be a finite-dimensional non-zero vector space over $k$ and $\mathcal{F}$ and $\mathcal{G}$ be $\mathbb{R}$-filtrations on $V$. Let $a \in \mathbb{R}$ such that, for any $t \in \mathbb{R}$ one has $\mathcal{F}^{t}(V) \subseteq \mathcal{G}^{t-a}(V)$. Then one has $Z_{\mathcal{F}}(i) \leqslant Z_{\mathcal{G}}(i)+$ a for any $i \in\left\{1, \ldots, \operatorname{rk}_{k}(V)\right\}$.

Proof. - By the relation (1.7), for any $i \in\left\{1, \ldots, \operatorname{rk}_{k}(V)\right\}$, if $Z_{\mathcal{F}}(i) \geqslant t$, then $\operatorname{rk}_{k}\left(\mathcal{F}^{t}(V)\right) \geqslant i$, which implies that $\operatorname{rk}_{k}\left(\mathcal{G}^{t-a}(V)\right) \geqslant i$ and hence (still by the relation (1.7)) $Z_{\mathcal{G}}(i) \geqslant t-a$. Therefore we obtain $Z_{\mathcal{F}}(i)-a \leqslant Z_{\mathcal{G}}(i)$.

Remark 1.1.40. — Let $V$ be a finite-dimensional vector space over $k$. There are canonical bijections between the following three sets:

(A) the set of all pairs

$$
\left(0=V_{0} \subsetneq V_{1} \subsetneq \ldots \subsetneq V_{n}=V, \mu_{1}>\ldots>\mu_{n}\right)
$$

such that $0=V_{0} \subsetneq V_{1} \subsetneq \ldots \subsetneq V_{n}=V$ is an increasing sequence of vector subspaces of $V$ and $\mu_{1}>\ldots>\mu_{n}$ is a decreasing sequence of real numbers.

(B) the set of all $\mathbb{R}$-filtrations $\mathcal{F}$ of $V$.

(C) the set of all ultrametric norms $\|\cdot\|$ of $V$ over $k$.

In the following, we explain the construction of these canonical maps.

- (A) $\rightarrow(\mathrm{B})$ The associated $\mathbb{R}$-filtration $\mathcal{F}$ on $V$ with the data $\left(V_{0} \subsetneq \ldots \subsetneq V_{n}, \mu_{1}>\right.$ $\left.\ldots>\mu_{n}\right)$ is defined by $\mathcal{F}^{t}(V):=V_{i}$ if $\left.\left.t \in\right] \mu_{i+1}, \mu_{i}\right] \cap \mathbb{R}$, where $\mu_{0}=+\infty$ and $\mu_{n+1}=-\infty$ by convention.

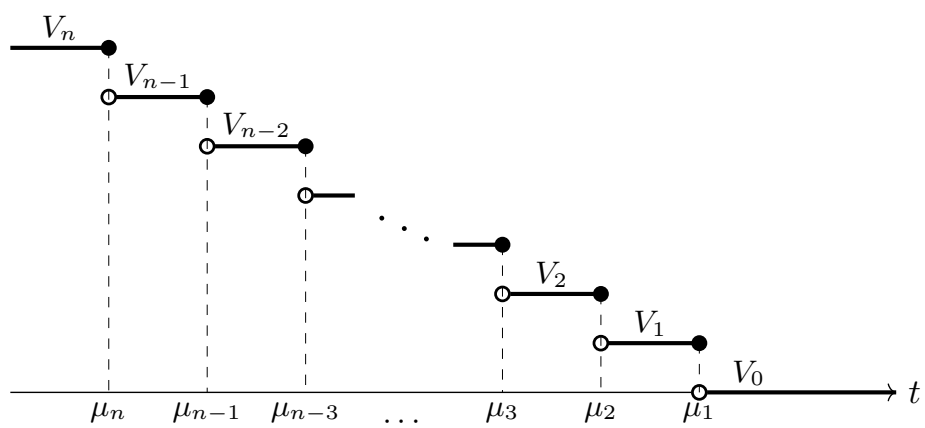

- (B) $(\mathrm{A})$ One has a sequence $0=V_{0} \subsetneq V_{1} \subsetneq \ldots \subsetneq V_{n}=V$ such that $\left\{\mathcal{F}^{t}(V): t \in \mathbb{R}\right\}=\left\{V_{0}, V_{1}, \ldots, V_{n}\right\}$. A sequence $\mu_{1}>\ldots>\mu_{n}$ in $\mathbb{R}$ is given by $\mu_{i}=\sup \left\{t: \mathcal{F}^{t}(V)=V_{i}\right\}$ for $i \in\{1, \ldots, n\}$. 
- $(\mathrm{A}) \rightarrow(\mathrm{C})$. The corresponding norm $\|\cdot\|$ to the data $\left(V_{0} \subsetneq \ldots \subsetneq V_{n}, \mu_{1}>\ldots>\mu_{n}\right)$ is given by

$$
\|x\|= \begin{cases}\mathrm{e}^{-\mu_{i}} & \text { if } x \in V_{i} \backslash V_{i-1} \\ 0 & \text { if } x=0\end{cases}
$$

- (C) (A) By Proposition 1.1.35, there is an increasing sequence

$$
0=V_{0} \subsetneq V_{1} \subsetneq \ldots \subsetneq V_{n}=V
$$

of subspaces of $V$ such that $\Psi(V,\|\cdot\|)=\left\{V_{1}, \ldots, V_{n}\right\}$. A decreasing sequence of real numbers is given by $\mu_{i}=\varphi_{\|\cdot\|}\left(V_{i}\right)$ for $i \in\{1, \ldots, n\}$.

- (B) $-(\mathrm{C})$ We define a function $\lambda_{\mathcal{F}}: V \rightarrow \mathbb{R} \cup\{+\infty\}$ such that

$$
\forall x \in V, \quad \lambda_{\mathcal{F}}(x):=\sup \left\{t \in \mathbb{R}: x \in \mathcal{F}^{t}(V)\right\} .
$$

Then the ultrametric norm $\|\cdot\|$ on $V$ corresponding to $\mathcal{F}$ is given by

$$
\forall x \in V, \quad\|x\|=\mathrm{e}^{-\lambda_{\mathcal{F}}(x)} .
$$

- (C) $\rightarrow(\mathrm{B})$ The corresponding filtration $\mathcal{F}$ to the norm $\|\cdot\|$ is given by $\mathcal{F}^{t}(V)=$ $(V,\|\cdot\|)_{\leqslant} \leqslant \mathrm{e}^{-t}$.

Let $\mathcal{F}$ be an $\mathbb{R}$-filtration on $V$, which corresponds to an increasing flag $0=V_{0} \subsetneq$ $V_{1} \subsetneq \ldots \subsetneq V_{n}=V$ together with a decreasing sequence $\mu_{1}>\ldots>\mu_{n}$ of real numbers. Note that the sets $\left\{\mu_{1}, \ldots, \mu_{n}\right\}$ and $\left\{Z_{\mathcal{F}}(1), \ldots, Z_{\mathcal{F}}(r)\right\}$ are actually equal, where $r$ denotes the rank of $V$ over $k$. Moreover, the value $\mu_{i}$ appears exactly $\operatorname{rk}_{k}\left(V_{i} / V_{i-1}\right)$ times in the sequence $Z_{\mathcal{F}}(1), \ldots, Z_{\mathcal{F}}(r)$.

1.1.9. Metric on the space of norms. - Let $V$ be a finite-dimensional vector space over $k$. We denote by $\mathcal{N}_{V}$ the set of all norms on $V$. If $\|\cdot\|_{1}$ and $\|\cdot\|_{2}$ are norms on $V$, by Proposition 1.1.11 we obtain that

$$
\sup _{s \in V \backslash\{0\}}\left|\ln \|s\|_{1}-\ln \|s\|_{2}\right|
$$

is finite. We denote by $d\left(\|\cdot\|_{1},\|\cdot\|_{2}\right)$ this number, called the distance between $\|\cdot\|_{1}$ and $\|\cdot\|_{2}$. It is easy to see that the function $d: \mathcal{N}_{V} \times \mathcal{N}_{V} \rightarrow \mathbb{R}_{\geqslant 0}$ satisfies the axioms of metric.

Remark 1.1.41. - Let $V$ be a finite-dimensional vector space over $k$ and $\|\cdot\|_{0}$ be a norm on $V$, which is the trivial norm if the absolute value $|\cdot|$ is trivial (namely $\|x\|_{0}=1$ for any $\left.x \in V \backslash\{0\}\right)$. Let $\lambda$ be a real number in ]0,1[. If the absolute value $|\cdot|$ is non-trivial, we require in addition that $\lambda<\sup \left\{|a|: a \in k^{\times},|a|<1\right\}$. We denote by $C$ the annulus $\left\{x \in V: \lambda \leqslant\|x\|_{0} \leqslant 1\right\}$. Note that one has $C=V \backslash\{0\}$ when $|\cdot|$ is trivial. For any norm $\|\cdot\|$ on $V$, the restriction of the function $\ln \|\cdot\|$ to $C$ is bounded, and the norm $\|\cdot\|$ is uniquely determined by its restriction to $C$ (this is a consequence of Proposition [1.1.4 when $|\cdot|$ is non-trivial). Thus we can identify $\mathcal{N}_{V}$ 
with a closed subset of $\mathcal{C}_{b}(C)$, the space of bounded and continuous functions on $C$ equipped with the sup norm. In particular, $\mathcal{N}_{V}$ is a complete metric space.

Proposition 1.1.42. - Let $V$ be a finite-dimensional vector space over $k$, and $\|\cdot\|_{1}$ and $\|\cdot\|_{2}$ be norms on $V$.

(1) Let $U$ be a vector subspace of $V,\|\cdot\|_{U, 1}$ and $\|\cdot\|_{U, 2}$ be the restrictions of $\|\cdot\|_{1}$ and $\|\cdot\|_{2}$ to $U$, respectively. Then one has $d\left(\|\cdot\|_{U, 1},\|\cdot\|_{U, 2}\right) \leqslant d\left(\|\cdot\|_{1},\|\cdot\|_{2}\right)$.

(2) Let $W$ be a quotient vector space of $V,\|\cdot\|_{W, 1}$ and $\|\cdot\|_{W, 2}$ be quotient norms of $\|\cdot\|_{1}$ and $\|\cdot\|_{2}$ on $W$, respectively. Then one has $d\left(\|\cdot\|_{W, 1},\|\cdot\|_{W, 2}\right) \leqslant d\left(\|\cdot\|_{1},\|\cdot\|_{2}\right)$.

Proof. - (1) follows directly from the definition of the distance function.

(2) It is sufficient to show that

$$
\left|\ln \|x\|_{W, 1}-\ln \|x\|_{W, 2}\right| \leqslant d\left(\|\cdot\|_{1},\|\cdot\|_{2}\right) .
$$

for $x \in W \backslash\{0\}$. Clearly we may assume that $\|x\|_{W, 1}>\|x\|_{W, 2}$. For $\epsilon>0$, one can choose $s \in V$ such that $[s]=x$ and $\|s\|_{2} \leqslant \mathrm{e}^{\epsilon}\|x\|_{2, W}$. Then

$$
\begin{aligned}
0 & <\ln \|x\|_{W, 1}-\ln \|x\|_{W, 2} \leqslant \ln \|s\|_{1}-\ln \left(\mathrm{e}^{-\epsilon}\|s\|_{2}\right) \\
& =\left(\ln \|s\|_{1}-\ln \|s\|_{2}\right)+\epsilon \leqslant d\left(\|\cdot\|_{1},\|\cdot\|_{2}\right)+\epsilon
\end{aligned}
$$

as desired.

Proposition 1.1.43. - Let $V$ and $W$ be finite-dimensional vector spaces over $k$, $\|\cdot\|_{V, 1}$ and $\|\cdot\|_{V, 2}$ be norms on $V$, and $\|\cdot\|$ be a norm on $W$. Let $\|\cdot\|_{1}$ and $\|\cdot\|_{2}$ be the operator norms on $\mathscr{L}(V, W)$, where we consider the norm $\|\cdot\|_{W}$ on $W$, and the norms $\|\cdot\|_{V, 1}$ and $\|\cdot\|_{V, 2}$ on $V$, respectively. Then one has

$$
d\left(\|\cdot\|_{1},\|\cdot\|_{2}\right) \leqslant d\left(\|\cdot\|_{V, 1},\|\cdot\|_{V, 2}\right) .
$$

In particular, one has

$$
d\left(\|\cdot\|_{V, 1, *},\|\cdot\|_{V, 2, *}\right) \leqslant d\left(\|\cdot\|_{V, 1} \cdot\|\cdot\|_{V, 2}\right),
$$

Moreover, the equality in (1.9) holds when both norms $\|\cdot\|_{V, 1}$ and $\|\cdot\|_{V, 2}$ are reflexive.

Proof. - For (1.8), it is sufficient to show

$$
\left|\ln \|f\|_{1}-\ln \|f\|_{2}\right| \leqslant d\left(\|\cdot\|_{V, 1},\|\cdot\|_{V, 2}\right)
$$

for $f \in \mathscr{L}(V, W) \backslash\{0\}$. Clearly we may assume that $\|f\|_{1}>\|f\|_{2}$. By definition one has

$$
\|f\|_{1}=\sup _{x \in V \backslash\{0\}} \frac{\|f(x)\|_{W}}{\|x\|_{V, 1}} \quad \text { and } \quad\|f\|_{2}=\sup _{x \in V \backslash\{0\}} \frac{\|f(x)\|_{W}}{\|x\|_{V, 2}}
$$


so that, for $\epsilon>0$, one can find $x \in V \backslash\{0\}$ such that $\mathrm{e}^{-\epsilon}\|f\|_{1} \leqslant\|f(x)\|_{W} /\|x\|_{V, 1}$. Therefore,

$$
\begin{aligned}
0 & <\ln \|f\|_{1}-\ln \|f\|_{2} \leqslant \ln \left(\mathrm{e}^{\epsilon} \frac{\|f(x)\|_{W}}{\|x\|_{V, 1}}\right)-\ln \left(\frac{\|f(x)\|_{W}}{\|x\|_{V, 2}}\right) \\
& =\left(\ln \|x\|_{V, 2}-\ln \|x\|_{V, 1}\right)+\epsilon \leqslant d\left(\|\cdot\|_{V, 1},\|\cdot\|_{V, 2}\right)+\epsilon
\end{aligned}
$$

as desired.

In order to obtain (1.9), it suffices to apply (1.8) to the case where $(W,\|\cdot\|)=(k,|\cdot|)$. If in addition both norms $\|\cdot\|_{V, 1}$ and $\|\cdot\|_{V, 2}$ are reflexive, then one has

$$
d\left(\|\cdot\|_{V, 1, *},\|\cdot\|_{V, 2, *}\right) \geqslant d\left(\|\cdot\|_{V, 1, * *},\|\cdot\|_{V, 2, * *}\right)=d\left(\|\cdot\|_{V, 1},\|\cdot\|_{V, 2}\right) .
$$

Hence the equality holds.

1.1.10. Direct sums. - Let $\mathscr{S}$ be the set of all convex and continuous functions $\psi:[0,1] \rightarrow[0,1]$ such that $\max \{t, 1-t\} \leqslant \psi(t)$ for any $t \in[0,1]$.

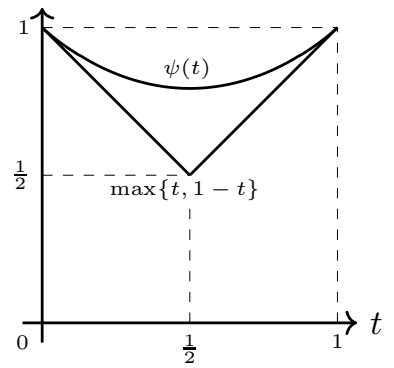

Let $\|\cdot\|$ be a norm on $\mathbb{R}^{2}$, where we consider the usual absolute value $|\cdot|_{\infty}$ on $\mathbb{R}$. We say that $\|\cdot\|$ is an absolute normalised norm if $\|(1,0)\|=\|(0,1)\|=1$ and if

$$
\forall(x, y) \in \mathbb{R}^{2}, \quad\|(x, y)\|=\left\|\left(|x|_{\infty},|y|_{\infty}\right)\right\| .
$$

By 13, §21, Lemma 3], the set of all absolute normalised norms on $\mathbb{R}^{2}$ can be parametrised by the functional space $\mathscr{S}$ (see [126 for the higher dimensional generalisation of this result). If $\|\cdot\|$ is the absolute normalised norm corresponding to $\psi \in \mathscr{S}$, one has

In particular, one always has

$$
\|(x, y)\|=(|x|+|y|) \psi\left(\frac{|x|}{|x|+|y|}\right) .
$$

$$
\|(x, y)\| \geqslant \max \left(|x|_{\infty},|y|_{\infty}\right)
$$

Conversely, given an absolute normalised norm $\|\cdot\|$ on $\mathbb{R}^{2}$, the corresponding function in $\mathscr{S}$ is

$$
(t \in[0,1]) \longmapsto\|(t, 1-t)\| .
$$

For example, the function $\psi(t)=\max \{t, 1-t\}, t \in[0,1]$ corresponds to the norm $(x, y) \mapsto \max \left\{|x|_{\infty},|y|_{\infty}\right\}$ on $\mathbb{R}^{2}$. If $p \geqslant 1$ is a real number, the function 
$\psi_{p}(t)=\left(t^{p}+(1-t)^{p}\right)^{1 / p}, t \in[0,1]$ belongs to $\mathscr{S}$; it corresponds to the $\ell^{p}$-norm $(x, y) \mapsto\left(|x|^{p}+|y|^{p}\right)^{1 / p}$.

Given a function $\psi$ in $\mathscr{S}$, or equivalently an absolute normalised norm on $\mathbb{R}^{2}$, for any couple of finite-dimensional seminormed vector spaces over $k$, one can naturally attach to the direct sum of the vector spaces a direct sum seminorm, which depends on the function $\psi$.

Lemma 1.1.44. - Let $a, b, a^{\prime}$ and $b^{\prime}$ be real numbers such that $0 \leqslant a \leqslant a^{\prime}$ and $0 \leqslant b \leqslant b^{\prime}$. We assume in addition that $a+b>0$. If $\psi$ is a function in $\mathscr{S}$, then

$$
(a+b) \psi\left(\frac{a}{a+b}\right) \leqslant\left(a^{\prime}+b^{\prime}\right) \psi\left(\frac{a^{\prime}}{a^{\prime}+b^{\prime}}\right) .
$$

Proof. - For any $t \in[0,1]$, the value $\psi(t)$ is bounded from below by $t$. Moreover, one has $\psi(1)=1$. The function $t \mapsto \psi(t) / t$ on $] 0,1]$ is non-increasing. In fact, for $0<s \leqslant t$, by the convexity of the function $\psi$ one has

$$
\begin{aligned}
\psi(t) & =\psi\left(\frac{t-s}{1-s}+\frac{1-t}{1-s} s\right) \leqslant \frac{t-s}{1-s} \psi(1)+\frac{1-t}{1-s} \psi(s) \\
& \leqslant \frac{t-s}{1-s} \cdot \frac{\psi(s)}{s}+\frac{1-t}{1-s} \psi(s)=\frac{t}{s} \psi(s) .
\end{aligned}
$$

In particular, one has

$$
(a+b) \psi\left(\frac{a}{a+b}\right) \leqslant\left(a+b^{\prime}\right) \psi\left(\frac{a}{a+b^{\prime}}\right) .
$$

Moreover, the function from $[0,1]$ to itself sending $t \in[0,1]$ to $\psi(1-t)$ also belongs to $\mathscr{S}$. By the above argument, we obtain that the function $t \mapsto \psi(1-t) / t$ is also non-increasing. Therefore

$$
\left(a+b^{\prime}\right) \psi\left(\frac{a}{a+b^{\prime}}\right)=\left(a+b^{\prime}\right) \psi\left(1-\frac{b^{\prime}}{a+b^{\prime}}\right) \leqslant\left(a^{\prime}+b^{\prime}\right) \psi\left(1-\frac{b^{\prime}}{a^{\prime}+b^{\prime}}\right) .
$$

The inequality (1.12) is thus proved.

Proposition 1.1.45. - Let $\left(V,\|\cdot\|_{V}\right)$ and $\left(W,\|\cdot\|_{W}\right)$ be finite-dimensional seminormed vector spaces over $k$. For any $\psi \in \mathscr{S}$, let $\|\cdot\|_{\psi}: V \oplus W \rightarrow \mathbb{R}_{\geqslant 0}$ be the map such that $\|(v, w)\|_{\psi}=0$ for $(v, w) \in N_{\|\cdot\|_{V}} \oplus N_{\|\cdot\|_{W}}$ and that, for any $(x, y) \in(V \oplus W) \backslash\left(N_{\|\cdot\|_{V}} \oplus N_{\|\cdot\|_{W}}\right)$,

$$
\|(x, y)\|_{\psi}:=(\|x\|+\|y\|) \psi\left(\frac{\|x\|}{\|x\|+\|y\|}\right) .
$$

Then $\|\cdot\|_{\psi}$ is a seminorm on $V \oplus W$ such that $N_{\|\cdot\|_{\psi}}=N_{\|\cdot\|_{V}} \oplus N_{\|\cdot\|_{W}}$. Moreover, for any $(x, y) \in V \times W$ one has

$$
\max \{\|x\|,\|y\|\} \leqslant\|(x, y)\|_{\psi} \leqslant\|x\|+\|y\| .
$$

Proof. - By definition, for any $(x, y) \in V \oplus W$ and any $a \in k$, one has

$$
\|(a x, a y)\|_{\psi}=|a| \cdot\|(x, y)\|_{\psi} .
$$


Moreover, for $(x, y) \notin N_{\|\cdot\|_{V}} \oplus N_{\|\cdot\|_{W}}$, one has $\|(x, y)\|_{\psi}>0$. Thus it remains to verify the triangle inequality.

Let $\left(x_{1}, y_{1}\right)$ and $\left(x_{2}, y_{2}\right)$ be two elements in $V \oplus W$ such that $\left(x_{1}+x_{2}, y_{1}+y_{2}\right)$ does not belong to $N_{\|\cdot\|_{V}} \oplus N_{\|\cdot\|_{W}}$. One has

$$
\left\|\left(x_{1}+x_{2}, y_{1}+y_{2}\right)\right\|_{\psi}=\left(\left\|x_{1}+x_{2}\right\|+\left\|y_{1}+y_{2}\right\|\right) \psi(u)
$$

where

$$
u=\frac{\left\|x_{1}+x_{2}\right\|}{\left\|x_{1}+x_{2}\right\|+\left\|y_{1}+y_{2}\right\|} .
$$

Since $\left\|x_{1}+x_{2}\right\| \leqslant\left\|x_{1}\right\|+\left\|x_{2}\right\|$ and $\left\|y_{1}+y_{2}\right\| \leqslant\left\|y_{1}\right\|+\left\|y_{2}\right\|$, by Lemma 1.1.44 one obtains that $\left\|\left(x_{1}+x_{2}, y_{1}+y_{2}\right)\right\|_{\psi}$ is bounded from above by

$$
\left(\left\|x_{1}\right\|+\left\|x_{2}\right\|+\left\|y_{1}\right\|+\left\|y_{2}\right\|\right) \psi(v),
$$

with

$$
v=\frac{\left\|x_{1}\right\|+\left\|x_{2}\right\|}{\left\|x_{1}\right\|+\left\|x_{2}\right\|+\left\|y_{1}\right\|+\left\|y_{2}\right\|}=\left(1+\frac{\left\|y_{1}\right\|+\left\|y_{2}\right\|}{\left\|x_{1}\right\|+\left\|x_{2}\right\|}\right)^{-1}
$$

if $\left\|x_{1}\right\|+\left\|x_{2}\right\|>0$, and $v=0$ otherwise. If $\left\|x_{1}\right\|>0$, let

$$
s=\frac{\left\|x_{1}\right\|}{\left\|x_{1}\right\|+\left\|y_{1}\right\|}=\left(1+\frac{\left\|y_{1}\right\|}{\left\|x_{1}\right\|}\right)^{-1}
$$

otherwise let $s=0$. Similarly, if $\left\|x_{2}\right\|>0$, let

$$
t=\frac{\left\|x_{2}\right\|}{\left\|x_{2}\right\|+\left\|y_{2}\right\|}=\left(1+\frac{\left\|y_{2}\right\|}{\left\|x_{2}\right\|}\right)^{-1}
$$

otherwise let $t=0$. In the case where $\left\|x_{1}\right\|$ and $\left\|x_{2}\right\|$ are both $>0$, one has

$$
\min \left\{\frac{\left\|y_{1}\right\|}{\left\|x_{1}\right\|}, \frac{\left\|y_{2}\right\|}{\left\|x_{2}\right\|}\right\} \leqslant \frac{\left\|y_{1}\right\|+\left\|y_{2}\right\|}{\left\|x_{1}\right\|+\left\|x_{2}\right\|} \leqslant \max \left\{\frac{\left\|y_{1}\right\|}{\left\|x_{1}\right\|}, \frac{\left\|y_{2}\right\|}{\left\|x_{2}\right\|}\right\},
$$

and therefore $\min \{s, t\} \leqslant v \leqslant \max \{s, t\}$. By the convexity of the function $\psi$ we obtain

Note that

$$
\psi(v) \leqslant \frac{v-t}{s-t} \psi(s)+\frac{s-v}{s-t} \psi(t)
$$

$$
\frac{v-t}{s-t}=\frac{\left\|x_{1}\right\|+\left\|y_{1}\right\|}{\left\|x_{1}\right\|+\left\|x_{2}\right\|+\left\|y_{1}\right\|+\left\|y_{2}\right\|}, \quad \frac{s-v}{s-t}=\frac{\left\|x_{2}\right\|+\left\|y_{2}\right\|}{\left\|x_{1}\right\|+\left\|x_{2}\right\|+\left\|y_{1}\right\|+\left\|y_{2}\right\|} .
$$

Thus we obtain the triangle inequality $\left\|\left(x_{1}+x_{2}, y_{1}+y_{2}\right)\right\|_{\psi} \leqslant\left\|\left(x_{1}, y_{1}\right)\right\|_{\psi}+\left\|\left(x_{2}, y_{2}\right)\right\|_{\psi}$.

We now proceed with the proof of the inequalities (1.13). The second inequality comes from the fact that $\psi$ takes values $\leqslant 1$. The first inequality is a consequence of Lemma 1.1.44. In fact, by (1.12), when $\|x\|>0$ one has

$$
\|x\|=(\|x\|+0) \psi\left(\frac{\|x\|}{\|x\|+0}\right) \leqslant\|(x, y)\|_{\psi} .
$$

Similarly, one has $\|y\| \leqslant\|(x, y)\|_{\psi}$. The proposition is thus proved.

Definition 1.1.46. - The seminorm $\|\cdot\|_{\psi}$ constructed in the above proposition is called the $\psi$-direct sum of the seminorms of $V$ and $W$. 
Proposition 1.1.47. - Let $\|\cdot\|$ be an absolute normalised norm on $\mathbb{R}^{2}$. Then the dual norm $\|\cdot\|_{*}$ is also an absolute normalised norm, where $\operatorname{Hom}_{\mathbb{R}}\left(\mathbb{R}^{2}, \mathbb{R}\right)$ is identified with $\mathbb{R}^{2}$ by using the isomorphism $\iota: \mathbb{R}^{2} \rightarrow \operatorname{Hom}_{\mathbb{R}}\left(\mathbb{R}^{2}, \mathbb{R}\right)$ given by $\iota(x, y)(a, b)=$ $a x+b y$.

Proof. - Let $(x, y)$ be an element of $\mathbb{R}^{2}$. One has (recall that $|\cdot|_{\infty}$ denotes the usual absolute value on $\mathbb{R}$ )

$$
\|(x, y)\|_{*}=\sup _{(0,0) \neq(a, b) \in \mathbb{R}^{2}} \frac{|a x+b y|_{\infty}}{\|(a, b)\|} .
$$

Since $\|\cdot\|$ is an absolute normalised norm on $\mathbb{R}^{2}$, from the above formula we deduce that $\|(x, y)\|_{*}=\left\|\left(|x|_{\infty},|y|_{\infty}\right)\right\|_{*}$ for any $(x, y) \in \mathbb{R}^{2}$. Moreover, by (1.10) one has

$$
\|(1,0)\|_{*}=\sup _{(0,0) \neq(a, b) \in \mathbb{R}} \frac{|a|_{\infty}}{\|(a, b)\|}=\sup _{0 \neq a \in \mathbb{R}} \frac{|a|_{\infty}}{\|(a, 0)\|}=1 .
$$

Similarly, $\|(0,1)\|_{*}=1$. Therefore, $\|\cdot\|_{*}$ is an absolute normalised norm on $\mathbb{R}^{2}$.

Definition 1.1.48. - Let $\psi$ be an element of $\mathscr{S}$, which corresponds to an absolute normalised norm $\|\cdot\|$ on $\mathbb{R}^{2}$. The above proposition shows that the dual norm $\|\cdot\|_{*}$ is also an absolute normalised norm. We denote by $\psi_{*}$ the element of $\mathscr{S}$ corresponding to this dual norm. Note that $\psi_{*}$ is actually given by

$$
\psi_{*}(t)=\sup _{\lambda \in] 0,1[}\left\{\frac{\lambda t+(1-\lambda)(1-t)}{\psi(\lambda)}\right\} .
$$

The following proposition studies the dual of a direct sum norm.

Proposition 1.1.49. - Let $\left(V,\|\cdot\|_{V}\right)$ and $\left(W,\|\cdot\|_{W}\right)$ be finite-dimensional seminormed vector spaces over $k, \psi$ be an element in $\mathscr{S}$, and $\|\cdot\|_{\psi}$ be the $\psi$-direct sum of $\|\cdot\|_{V}$ and $\|\cdot\|_{W}$. Let $\psi_{0} \in \mathscr{S}$ such that $\psi_{0}(t)=\max \{t, 1-t\}$ for any $t \in[0,1]$.

(1) Assume that the absolute value ||| is non-Archimedean. Then the dual norm $\|\cdot\|_{\psi, *}$ identifies with the $\psi_{0}$-direct sum of $\|\cdot\|_{V, *}$ and $\|\cdot\|_{W, *}$.

(2) Assume that the absolute value $|\cdot|$ is Archimedean. Then the dual norm $\|\cdot\|_{\psi, *}$ identifies with the $\psi_{*}$-direct sum of $\|\cdot\|_{V, *}$ and $\|\cdot\|_{W, *}$.

Proof. - Since the null space of the seminorm $\|\cdot\|$ is $N_{\|\cdot\|_{V}} \oplus N_{\|\cdot\|_{W}}$, we obtain that a linear form $(f, g) \in V^{\vee} \oplus W^{\vee}$ vanishes on $N_{\|\cdot\|_{\psi}}$ if and only if it belongs to $V^{*} \oplus W^{*}$. In other words, one has $(V \oplus W)^{*}=V^{*} \oplus W^{*}$.

(1) Let $(f, g)$ be an element in $V^{*} \oplus W^{*}$, one has

$$
\begin{aligned}
\|(f, g)\|_{\psi, *}= & \sup _{\substack{(s, t) \in V \oplus W \\
\max \left\{\|s\|_{V},\|t\|_{W}\right\}>0}} \frac{|f(s)+g(t)|}{\|(s, t)\|_{\psi}} \\
& \leqslant \sup _{\substack{(s, t) \in V \oplus W \\
\max \left\{\|s\|_{V},\|t\|_{W}\right\}>0}} \frac{\max \{|f(s)|,|g(t)|\}}{\max \left\{\|s\|_{V},\|t\|_{W}\right\}} \leqslant \max \left\{\|f\|_{V, *},\|g\|_{W, *}\right\},
\end{aligned}
$$


where the first inequality comes from (1.13) and the fact that the absolute value $|\cdot|$ is non-Archimedean. Moreover, one has

$$
\|(f, g)\|_{\psi, *} \geqslant \sup _{s \in V \backslash N_{\|\cdot\|_{V}}} \frac{|f(s)+g(0)|}{\|(s, 0)\|_{\psi}}=\|f\|_{V, *} .
$$

Similarly, one has

$$
\|(f, g)\|_{\psi, *} \geqslant \sup _{t \in W \backslash N_{\|\cdot\|_{W}}} \frac{|f(0)+g(t)|}{\|(0, t)\|_{\psi}}=\|g\|_{W, *} .
$$

Therefore $\|(f, g)\|_{\psi, *}=\max \left\{\|f\|_{V, *},\|g\|_{W, *}\right\}$.

(2) Let $\|\cdot\|$ be the absolute normalised norm on $\mathbb{R}^{2}$ corresponding to $\psi$ and let $\|\cdot\|_{*}$ be its dual norm. For any $(s, t) \in V \oplus W$, on has $\|(s, t)\|_{\psi}=\left\|\left(\|s\|_{V},\|t\|_{W}\right)\right\|$. Let $(f, g)$ be an element in $V^{*} \oplus W^{*}$. One has

$$
\begin{aligned}
& \|(f, g)\|_{\psi, *}=\sup _{\substack{(s, t) \in V \oplus W \\
\max \left\{\|s\|_{V},\|t\|_{W}\right\}>0}} \frac{|f(s)+g(t)|}{\|(s, t)\|_{\psi}} \\
\leqslant & \sup _{\substack{(s, t) \in V \oplus W \\
\max \left\{\|s\|_{V},\|t\|_{W}\right\}>0}} \frac{\|f\|_{V, *} \cdot\|s\|_{V}+\|g\|_{W, *} \cdot\|t\|_{W}}{\left\|\left(\|s\|_{V},\|t\|_{W}\right)\right\|}=\left\|\left(\|f\|_{V, *},\|g\|_{W, *}\right)\right\|_{*} .
\end{aligned}
$$

Moreover, since $k=\mathbb{R}$ or $\mathbb{C}$, by Hahn-Banach theorem, for any $a>0$, there exists $s \in V$ such that $\|s\|_{V}=a$ and that $f(s)=\|f\|_{V, *} \cdot\|s\|_{V}$. Similarly, for any $b>0$, there exists $t \in W$ such that $\|t\|_{W}=b$ and $g(t)=\|g\|_{W, *} \cdot\|t\|_{W}$. Therefore the inequality in the above formula is actually an equality.

Proposition 1.1.50. - Let $f: V \rightarrow V^{\prime}$ and $g: W \rightarrow W^{\prime}$ be surjective linear maps of finite-dimensional vector spaces over $k$. Let $\|\cdot\|_{V}$ and $\|\cdot\|_{W}$ be seminorms on $V$ and $W$, and let $\|\cdot\|_{V^{\prime}}$ and $\|\cdot\|_{W^{\prime}}$ be the quotient seminorms of $\|\cdot\|_{V}$ and $\|\cdot\|_{W}$ on $V^{\prime}$ and $W^{\prime}$, respectively. Then the quotient seminorm $\|\cdot\|_{V \oplus W, \psi, V \oplus W \rightarrow V^{\prime} \oplus W^{\prime}}$ of $\|\cdot\|_{V \oplus W, \psi}$ on $V^{\prime} \oplus W^{\prime}$ coincides with $\|\cdot\|_{V^{\prime} \oplus W^{\prime}, \psi}$.

Proof. - It is sufficient to see that

$$
\left\|\left(x^{\prime}, y^{\prime}\right)\right\|_{V^{\prime} \oplus W^{\prime}, \psi}=\left\|\left(x^{\prime}, y^{\prime}\right)\right\|_{V \oplus W, \psi, V \oplus W \rightarrow V^{\prime} \oplus W^{\prime}}
$$

for all $x^{\prime} \in V^{\prime}$ and $y^{\prime} \in W^{\prime}$ with $\left\|x^{\prime}\right\|_{V^{\prime}}+\left\|y^{\prime}\right\|_{W^{\prime}}>0$. Let $x \in V$ and $y \in W$ with $f(x)=x^{\prime}$ and $g(y)=y^{\prime}$. Then, as $\|x\|_{V} \geqslant\left\|x^{\prime}\right\|_{V^{\prime}}$ and $\|y\|_{W} \geqslant\left\|y^{\prime}\right\|_{W^{\prime}}$, by Lemma 1.1.44 one has $\left\|\left(x^{\prime}, y^{\prime}\right)\right\|_{V^{\prime} \oplus W^{\prime}, \psi} \leqslant\|(x, y)\|_{V \oplus W, \psi}$, so that

$$
\left\|\left(x^{\prime}, y^{\prime}\right)\right\|_{V^{\prime} \oplus W^{\prime}, \psi} \leqslant\left\|\left(x^{\prime}, y^{\prime}\right)\right\|_{V \oplus W, \psi, V \oplus W \rightarrow V^{\prime} \oplus W^{\prime}} .
$$

Let us consider the converse inequality. We choose sequences $\left\{x_{n}\right\}_{n \in \mathbb{N}}$ and $\left\{y_{n}\right\}_{n \in \mathbb{N}}$ in $V$ and $W$ such that $f\left(x_{n}\right)=x^{\prime}, g\left(y_{n}\right)=y^{\prime}, \lim _{n \rightarrow \infty}\left\|x_{n}\right\|_{V}=\left\|x^{\prime}\right\|_{V^{\prime}}$ and $\lim _{n \rightarrow \infty}\left\|y_{n}\right\|_{W}=\left\|y^{\prime}\right\|_{W^{\prime}}$. 
We assume that $\left\|x^{\prime}\right\|_{V^{\prime}}+\left\|y^{\prime}\right\|_{W^{\prime}}>0$. Then as $\left\|x_{n}\right\|_{V}+\left\|y_{n}\right\|_{W}>0$ for sufficiently large $n$ and $\psi$ is continuous, one has

$$
\begin{aligned}
\left\|\left(x^{\prime}, y^{\prime}\right)\right\|_{V^{\prime} \oplus W^{\prime}, \psi} & =\left(\left\|x^{\prime}\right\|_{V^{\prime}}+\left\|y^{\prime}\right\|_{W^{\prime}}\right) \psi\left(\frac{\left\|x^{\prime}\right\|_{V^{\prime}}}{\left\|x^{\prime}\right\|_{V^{\prime}}+\left\|y^{\prime}\right\|_{W^{\prime}}}\right) \\
& =\lim _{n \rightarrow \infty}\left(\left\|x_{n}\right\|_{V}+\left\|y_{n}\right\|_{W}\right) \psi\left(\frac{\left\|x_{n}\right\|_{V}}{\left\|x_{n}\right\|_{V}+\left\|y_{n}\right\|_{W}}\right) \\
& =\lim _{n \rightarrow \infty}\left\|\left(x_{n}, y_{n}\right)\right\|_{V \oplus W, \psi} \geqslant\left\|\left(x^{\prime}, y^{\prime}\right)\right\|_{V \oplus W, \psi, V \oplus W \rightarrow V^{\prime} \oplus W^{\prime}},
\end{aligned}
$$

as required. Otherwise, as

$$
0 \leqslant\left\|\left(x^{\prime}, y^{\prime}\right)\right\|_{V \oplus W, \psi, V \oplus W \rightarrow V^{\prime} \oplus W^{\prime}} \leqslant\left\|\left(x_{n}, y_{n}\right)\right\|_{V \oplus W, \psi} \leqslant\left\|x_{n}\right\|_{V}+\left\|y_{n}\right\|_{W}
$$

and $\lim _{n \rightarrow \infty}\left\|x_{n}\right\|_{V}+\left\|y_{n}\right\|_{W}=0$, one has

$$
\left\|\left(x^{\prime}, y^{\prime}\right)\right\|_{V \oplus W, \psi, V \oplus W \rightarrow V^{\prime} \oplus W^{\prime}}=0,
$$

as desired.

Remark 1.1.51. - Let $\psi$ be an element of $\mathscr{S}$. Let $\left\{\psi_{n}\right\}_{n=1}^{\infty}$ be a sequence of functions given in the following ways:

$$
\begin{cases}\forall a \in \mathbb{R}_{\geqslant 0}, & \psi_{1}(a)=a, \\ \forall(a, b) \in \mathbb{R}_{\geqslant 0}^{2}, & \psi_{2}(a, b)= \begin{cases}(a+b) \psi\left(\frac{a}{a+b}\right) & \text { if } a+b>0, \\ 0 & \text { if } a=b=0,\end{cases} \\ \forall\left(a_{1}, \ldots, a_{n}\right) \in \mathbb{R}_{\geqslant 0}^{n}, & \psi_{n}\left(a_{1}, \ldots, a_{n}\right)=\psi_{2}\left(\psi_{n-1}\left(a_{1}, \ldots, a_{n-1}\right), a_{n}\right) .\end{cases}
$$

Let $\left(V_{1},\|\cdot\|_{1}\right), \ldots,\left(V_{n},\|\cdot\|_{n}\right)$ be finite-dimensional normed vector spaces over $k$. If we define

$$
\left\|\left(x_{1}, \ldots, x_{n}\right)\right\|_{\psi}:=\psi_{n}\left(\left\|x_{1}\right\|_{1}, \ldots,\left\|x_{n}\right\|_{n}\right)
$$

for $\left(x_{1}, \ldots, x_{n}\right) \in V_{1} \oplus \cdots \oplus V_{n}$, then, by Proposition 1.1.45, it yields a norm on $V_{1} \oplus \cdots \oplus V_{n}$

We assume that

$$
\forall a_{1}, a_{2}, a_{3} \in \mathbb{R}_{\geqslant 0}, \quad \psi_{2}\left(a_{1}, \psi_{2}\left(a_{2}, a_{3}\right)\right)=\psi_{2}\left(\psi_{2}\left(a_{1}, a_{2}\right), a_{3}\right) .
$$

Then it is easy to see that $\psi_{n}\left(a_{1}, \ldots, a_{n}\right)=\psi_{2}\left(\psi_{i}\left(a_{1}, \ldots, a_{i}\right), \psi_{n-i}\left(a_{i+1}, \ldots, a_{n}\right)\right)$ for $i \in\{1, \ldots, n-1\}$, so that the construction of the norm $\|\cdot\|_{\psi}$ is associative. If we assume $\psi_{2}(a, b)=\psi_{2}(b, a)$ for all $(a, b) \in \mathbb{R}_{\geqslant 0}^{2}$ in addition to (1.14), then $\psi_{n}\left(a_{1}, \ldots, a_{n}\right)$ is symmetric, that is, for any permutation $\sigma, \psi_{n}\left(a_{\sigma(1)}, \ldots, a_{\sigma(n)}\right)=\psi_{n}\left(a_{1}, \ldots, a_{n}\right)$, which means that its construction is order independent. 
1.1.11. Tensor product seminorms. - Let $V$ and $W$ be seminormed vector spaces of finite rank over $k$. On the tensor product space $V \otimes_{k} W$ there are several natural ways to construct tensor product seminorms. We refer the readers to the original article 69] of Grothendieck for different constructions. In this subsection, we recall the $\pi$-tensor product and the $\varepsilon$-tensor product. We refer to the book [124] for a more detailed presentation in the Archimedean case.

Definition 1.1.52. - Let $\left(V_{1},\|\cdot\|_{1}\right), \ldots,\left(V_{n},\|\cdot\|_{n}\right)$ be seminormed vector spaces over $k$. We define a map $\|\cdot\|_{\pi}: V_{1} \otimes_{k} \cdots \otimes_{k} V_{n} \rightarrow[0,+\infty[$ such that, for any $\varphi \in V_{1} \otimes_{k} \cdots \otimes_{k} V_{n}$

$$
\|\varphi\|_{\pi}:=\inf \left\{\sum_{i=1}^{N}\left\|x_{1}^{(i)}\right\|_{1} \cdots\left\|x_{n}^{(i)}\right\|_{n}: \varphi=\sum_{i=1}^{N} x_{1}^{(i)} \otimes \cdots \otimes x_{n}^{(i)}\right\} .
$$

Note that $\|\cdot\|_{\pi}$ is a seminorm on $V_{1} \otimes_{k} \cdots \otimes_{k} V_{n}$. For example, the triangle inequality can be checked as follows: for $\varphi, \psi \in V_{1} \otimes_{k} \cdots \otimes_{k} V_{n}$ and a positive number $\epsilon$, we choose expressions

$$
\varphi=\sum_{i=1}^{N} x_{1}^{(i)} \otimes \cdots \otimes x_{n}^{(i)} \quad \text { and } \quad \psi=\sum_{j=1}^{M} y_{1}^{(j)} \otimes \cdots \otimes y_{n}^{(j)}
$$

such that

$$
\sum_{i=1}^{N}\left\|x_{1}^{(i)}\right\|_{1} \cdots\left\|x_{n}^{(i)}\right\|_{n} \leqslant\|\varphi\|_{\pi}+\epsilon \quad \text { and } \quad \sum_{j=1}^{M}\left\|y_{1}^{(j)}\right\|_{1} \cdots\left\|y_{n}^{(j)}\right\|_{n} \leqslant\|\psi\|_{\pi}+\epsilon .
$$

Then, as

$$
\varphi+\psi=\sum_{i=1}^{N} x_{1}^{(i)} \otimes \cdots \otimes x_{n}^{(i)}+\sum_{j=1}^{M} y_{1}^{(j)} \otimes \cdots \otimes y_{n}^{(i)}
$$

one has

$$
\|\varphi+\psi\|_{\pi} \leqslant \sum_{i=1}^{N}\left\|x_{1}^{(i)}\right\|_{1} \cdots\left\|x_{n}^{(i)}\right\|_{n}+\sum_{j=1}^{M}\left\|y_{1}^{(j)}\right\|_{1} \cdots\left\|y_{n}^{(j)}\right\|_{n} \leqslant\|\varphi\|_{\pi}+\|\psi\|_{\pi}+2 \epsilon
$$

as desired. We call $\|\cdot\|_{\pi}$ the $\pi$-tensor product of the seminorms $\|\cdot\|_{1}, \ldots,\|\cdot\|_{n}$.

Any element $\varphi$ in the tensor product space $V_{1} \otimes_{k} \cdots \otimes_{k} V_{n}$ can be considered as a multilinear form on $V_{1}^{*} \times \cdots \times V_{n}^{*}$. In particular, if $\varphi$ is of the form $x_{1} \otimes$ $\cdots \otimes x_{n}$, the corresponding multilinear form sends $\left(f_{1}, \ldots, f_{n}\right) \in V_{1}^{*} \times \cdots \times V_{n}^{*}$ to $f_{1}\left(x_{1}\right) \cdots f_{n}\left(x_{n}\right) \in k$. For any $\varphi \in V_{1} \otimes_{k} \cdots \otimes_{k} V_{n}$, viewed as a $k$-multilinear form on $V_{1}^{*} \times \cdots \times V_{n}^{*}$, let

$$
\|\varphi\|_{\varepsilon}:=\sup _{\substack{\left(f_{1}, \ldots, f_{n}\right) \in V_{1}^{*} \times \cdots \times V_{n}^{*} \\ \forall i \in\{1, \ldots, n\}, f_{i} \neq 0}} \frac{\left|\varphi\left(f_{1}, \ldots, f_{n}\right)\right|}{\left\|f_{1}\right\|_{1, *} \cdots\left\|f_{n}\right\|_{n, *}} .
$$

Then $\|\cdot\|_{\varepsilon}$ is a seminorm on the tensor product space $V_{1} \otimes_{k} \cdots \otimes_{k} V_{n}$, called the $\varepsilon$-tensor product of seminorms $\|\cdot\|_{1}, \ldots,\|\cdot\|_{n}$. It is a norm once the seminorms $\|\cdot\|_{1}, \ldots,\|\cdot\|_{n}$ are 
norms. Similarly to the dual norm case, if the absolute value $|\cdot|$ is non-Archimedean, then the $\varepsilon$-tensor product $\|\cdot\|_{\varepsilon}$ is ultrametric. By Proposition 1.2.14 in the next section, we obtain that, in the case where all $V_{i}$ are of finite type over $k$, the $\varepsilon$-tensor product of $\|\cdot\|_{1}, \ldots,\|\cdot\|_{n}$ identifies with that of $\|\cdot\|_{1, * *}, \ldots,\|\cdot\|_{n, * *}$.

Remark 1.1.53. - Let $\left(V_{1},\|\cdot\|_{1}\right)$ and $\left(V_{2},\|\cdot\|_{2}\right)$ be seminormed vector spaces over $k$. Let $\|\cdot\|$ be the operator seminorm on the vector space $\mathscr{L}\left(V_{1}^{*}, V_{2}\right)$, where we consider the dual norm $\|\cdot\|_{1, *}$ on $V_{1}^{*}$ and the double dual seminorm $\|\cdot\|_{2, * *}$ on $V_{2}$. One has a canonical $k$-linear map from $V_{1} \otimes_{k} V_{2}$ to $\mathscr{L}\left(V_{1}^{*}, V_{2}\right)$ sending $x \otimes y \in V_{1} \otimes_{k} V_{2}$ to the bounded linear map $\left(\alpha \in V_{1}^{*}\right) \mapsto \alpha(x) y$. We claim that the seminorm on $V_{1} \otimes_{k} V_{2}$ induced by $\|\cdot\|$ and the above canonical map identifies with the $\varepsilon$-tensor product $\|\cdot\|_{\varepsilon}$ of $\|\cdot\|_{1}$ and $\|\cdot\|_{2}$. In fact, for any $\varphi \in V_{1} \otimes_{k} V_{2}$ one has

$$
\|\varphi\|=\sup _{f_{1} \in V_{1}^{*} \backslash\{0\}} \frac{\left\|\varphi\left(f_{1}\right)\right\|_{2, * *}}{\left\|f_{1}\right\|_{1, *}}=\sup _{\substack{f_{1} \in V_{1}^{*} \backslash\{0\} \\ f_{2} \in V_{2}^{*} \backslash\{0\}}} \frac{\left|\varphi\left(f_{1}, f_{2}\right)\right|}{\left\|f_{1}\right\|_{1, *}\left\|f_{2}\right\|_{2, *}}=\|\varphi\|_{\varepsilon} .
$$

In particular, if $\|\cdot\|_{2}=\|\cdot\|_{2, * *}$ on $V_{2}$, then the $\varepsilon$-tensor product norm $\|\cdot\|_{\varepsilon}$ identifies with the operator seminorm if we consider tensors in $V_{1} \otimes_{k} V_{2}$ as $k$-linear operators from $\left(V_{1}^{*},\|\cdot\|_{1, *}\right)$ to $\left(V_{2},\|\cdot\|_{2}\right)$.

Proposition 1.1.54. - We keep the notation of Definition 1.1.52. If $\|\cdot\|$ is a seminorm on $V_{1} \otimes_{k} \cdots \otimes_{k} V_{n}$ such that $\left\|x_{1} \otimes \cdots \otimes x_{n}\right\| \leqslant\left\|x_{1}\right\|_{1} \cdots\left\|x_{n}\right\|_{n}$ for any $\left(x_{1}, \ldots, x_{n}\right) \in V_{1} \times \cdots \times V_{n}$, then one has $\|\cdot\| \leqslant\|\cdot\|_{\pi}$. In particular, the seminorm $\|\cdot\|_{\varepsilon}$ is bounded from above by $\|\cdot\|_{\pi}$. Moreover, if $\|\cdot\|_{1}, \ldots,\|\cdot\|_{n}$ are norms, then $\|\cdot\|_{\pi}$ is also a norm.

Proof. - Let $\varphi$ be an element of $V_{1} \otimes_{k} \cdots \otimes_{k} V_{n}$. If $\varphi$ is written in the form

$$
\varphi=\sum_{i=1}^{N} x_{1}^{(i)} \otimes \cdots \otimes x_{n}^{(i)},
$$

where $x_{j}^{(i)} \in V_{j}$ for any $j \in\{1, \ldots, n\}$, then one has

$$
\|\varphi\| \leqslant \sum_{i=1}^{N}\left\|x_{1}^{(i)} \otimes \cdots \otimes x_{n}^{(i)}\right\| \leqslant \sum_{i=1}^{N}\left\|x_{1}^{(1)}\right\|_{1} \cdots\left\|x_{n}^{(1)}\right\|_{n} .
$$

Therefore we obtain $\|\cdot\| \leqslant\|\cdot\|_{\pi}$. Note that, for any $\left(x_{1}, \ldots, x_{n}\right) \in V_{1} \times \cdots \times V_{n}$ one has

$$
\begin{aligned}
\left\|x_{1} \otimes \cdots \otimes x_{n}\right\|_{\varepsilon} & =\sup _{\substack{\left(f_{1}, \ldots, f_{n}\right) \in V_{1}^{*} \times \cdots \times V_{n}^{*} \\
\forall i \in\{1, \ldots, n\}, f_{i} \neq 0}} \frac{\left|f_{1}\left(x_{1}\right)\right| \cdots\left|f_{n}\left(x_{n}\right)\right|}{\left\|f_{1}\right\|_{1, *} \cdots\left\|f_{n}\right\|_{n, *}} \\
& =\left\|x_{1}\right\|_{1, * *} \cdots\left\|x_{n}\right\|_{n, * *} \leqslant\left\|x_{1}\right\|_{1} \cdots\left\|x_{n}\right\|_{n} .
\end{aligned}
$$

Therefore, one has $\|\cdot\|_{\varepsilon} \leqslant\|\cdot\|_{\pi}$. If the seminorms $\|\cdot\|_{i}(i \in\{1, \ldots, n\})$ are norms, then $\|\cdot\|_{\varepsilon}$ is a norm and hence $\|\cdot\|_{\pi}$ is also a norm. 
Remark 1.1.55. - From the definition we observe that the $\varepsilon$-tensor product and $\pi$ tensor product are commutative. Namely, if $V_{1}$ and $V_{2}$ are finite-dimensional normed vector spaces over $k$, then the canonical isomorphism $V_{1} \otimes_{k} V_{2} \rightarrow V_{2} \otimes_{k} V_{1}$ is an isometry if we consider $\varepsilon$-tensor products or $\pi$-tensor product norms on both sides. The $\varepsilon$-tensor product and the $\pi$-tensor product are also associative. Namely, if $V_{1}$, $V_{2}$ and $V_{3}$ are finite-dimensional normed vector spaces over $k$, then the canonical isomorphisms $\left(V_{1} \otimes_{k} V_{2}\right) \otimes_{k} V_{3} \rightarrow V_{1} \otimes_{k} V_{2} \otimes_{k} V_{3}$ and $V_{1} \otimes_{k}\left(V_{2} \otimes_{k} V_{3}\right) \rightarrow V_{1} \otimes_{k} V_{2} \otimes_{k} V_{3}$ are both isometries.

Remark 1.1.56. - Let $\left(V_{1},\|\cdot\|_{1}\right), \ldots,\left(V_{n},\|\cdot\|_{n}\right)$ be finite-dimensional seminormed vector spaces over $k$. From the definition, we observe that, if $\left(u_{1}, \ldots, u_{n}\right)$ is an element of $V_{1} \times \cdots \times V_{n}$, then one has

$$
\left\|u_{1} \otimes \cdots \otimes u_{n}\right\|_{\varepsilon}=\left\|u_{1}\right\|_{1, * *} \cdots\left\|u_{n}\right\|_{n, * *} .
$$

If the seminormed vector spaces $\left(V_{1},\|\cdot\|_{1}\right), \ldots,\left(V_{n},\|\cdot\|_{n}\right)$ are reflexive, by (1.16) and Proposition 1.1.54, we obtain that, for any $\left(u_{1}, \ldots, u_{n}\right) \in V_{1} \times \cdots \times V_{n}$, one has

$$
\prod_{i=1}^{n}\left\|u_{i}\right\|_{i}=\left\|u_{1} \otimes \cdots \otimes u_{n}\right\|_{\varepsilon} \leqslant\left\|u_{1} \otimes \cdots \otimes u_{n}\right\|_{\pi} .
$$

Moreover, by definition one has $\left\|u_{1} \otimes \cdots \otimes u_{n}\right\|_{\pi} \leqslant\left\|u_{1}\right\|_{1} \cdots\left\|u_{n}\right\|_{n}$. Therefore

$$
\left\|u_{1} \otimes \cdots \otimes u_{n}\right\|_{\varepsilon}=\left\|u_{1} \otimes \cdots \otimes u_{n}\right\|_{\pi}=\left\|u_{1}\right\|_{1} \cdots\left\|u_{n}\right\|_{n} .
$$

In particular, if $V_{1}, \ldots, V_{n}$ are seminormed vector spaces of rank 1 over $k$ (in this case they are necessarily reflexive), then their $\varepsilon$-tensor product and $\pi$-tensor product norms are the same. We simply call it the tensor product of the seminorms $\|\cdot\|_{1}, \ldots,\|\cdot\|_{n}$.

Proposition 1.1.5\%. - Let $\left(V_{1},\|\cdot\|_{1}\right), \ldots,\left(V_{n},\|\cdot\|_{n}\right)$ be finite-dimensional seminormed vector spaces over $k$. Let $\|\cdot\|_{*, \pi}$ and $\|\cdot\|_{*, \varepsilon}$ be respectively the $\pi$-tensor product and the $\varepsilon$-tensor product of the dual norms $\|\cdot\|_{1, *}, \ldots,\|\cdot\|_{n, *}$. The $\varepsilon$-tensor product of $\|\cdot\|_{1}, \ldots,\|\cdot\|_{n}$ identifies with the seminorm induced by the dual norm $\|\cdot\|_{*, \pi, *}$ on $\left(V_{1}^{*} \otimes_{k} \cdots \otimes_{k} V_{n}^{*}\right)^{*}$ by the natural linear map $V_{1} \otimes_{k} \cdots \otimes_{k} V_{n} \rightarrow\left(V_{1}^{*} \otimes_{k} \cdots \otimes_{k} V_{n}^{*}\right)^{*}$. If the absolute value $|\cdot|$ is Archimedean, then the $\pi$-tensor product of $\|\cdot\|_{1}, \ldots,\|\cdot\|_{n}$ identifies with seminorm induced by the dual norm $\|\cdot\|_{*, \varepsilon, *}$ on $\left(V_{1}^{*} \otimes_{k} \cdots \otimes_{k} V_{n}^{*}\right)^{*}$ by the natural linear map $V_{1} \otimes_{k} \cdots \otimes_{k} V_{n} \rightarrow\left(V_{1}^{*} \otimes_{k} \cdots \otimes_{k} V_{n}^{*}\right)^{*}$.

Proof. - Let $\varphi$ be an element in $V_{1} \otimes_{k} \cdots \otimes_{k} V_{n}$, which can also be viewed as a $k$-multilinear form on $V_{1}^{*} \times \cdots \times V_{n}^{*}$ or a linear form on $V_{1}^{*} \otimes_{k} \cdots \otimes_{k} V_{n}^{*}$. Let $\alpha$ be an element in $V_{1}^{*} \otimes_{k} \cdots \otimes_{k} V_{n}^{*}$. If $\alpha$ is written in the form

$$
\alpha=\sum_{i=1}^{N} f_{1}^{(i)} \otimes \cdots \otimes f_{n}^{(i)},
$$


where $f_{j}^{(i)} \in V_{j}^{*}$, then one has $\varphi(\alpha)=\sum_{i=1}^{N} \varphi\left(f_{1}^{(i)}, \ldots, f_{n}^{(i)}\right)$ and hence

$$
|\varphi(\alpha)| \leqslant \sum_{i=1}^{N}\left|\varphi\left(f_{1}^{(i)}, \ldots, f_{n}^{(i)}\right)\right|
$$

Thus we obtain

$$
\frac{|\varphi(\alpha)|}{\sum_{i=1}^{N}\left\|f_{1}^{(i)}\right\|_{1, *} \cdots\left\|f_{n}^{(i)}\right\|_{n, *}} \leqslant \frac{\sum_{i=1}^{N}\left|\varphi\left(f_{1}^{(i)}, \ldots, f_{n}^{(i)}\right)\right|}{\sum_{i=1}^{N}\left\|f_{1}^{(i)}\right\|_{1, *} \cdots\left\|f_{n}^{(i)}\right\|_{n, *}} \leqslant\|\varphi\|_{\varepsilon} .
$$

Therefore $\varphi$ is a bounded linear form on $\left(V_{1}^{*} \otimes_{k} \cdots \otimes_{k} V_{n}^{*},\|\cdot\|_{*, \pi}\right)$ and $\|\varphi\|_{*, \pi, *} \leqslant\|\varphi\|_{\varepsilon}$.

For any $\left(f_{1}, \ldots, f_{n}\right) \in\left(V_{1}^{*} \backslash\{0\}\right) \times \cdots \times\left(V_{n}^{*} \backslash\{0\}\right)$ one has

$$
\frac{\left|\varphi\left(f_{1} \otimes \cdots \otimes f_{n}\right)\right|}{\left\|f_{1} \otimes \cdots \otimes f_{n}\right\|_{*, \pi}}=\frac{\left|\varphi\left(f_{1}, \ldots, f_{n}\right)\right|}{\left\|f_{1} \otimes \cdots \otimes f_{n}\right\|_{*, \pi}} \geqslant \frac{\left|\varphi\left(f_{1}, \ldots, f_{n}\right)\right|}{\left\|f_{1}\right\|_{1, *} \cdots\left\|f_{n}\right\|_{n, *}} .
$$

Therefore one has $\|\varphi\|_{\varepsilon} \leqslant\|\varphi\|_{*, \pi, *}$. The first assertion is thus proved.

If $|\cdot|$ is Archimedean, any finite-dimensional normed vector space is reflexive. By the first assertion, the dual norm of the $\pi$-tensor product of $\|\cdot\|_{1}, \ldots,\|\cdot\|_{n}$ is the $\varepsilon$ tensor product of $\|\cdot\|_{1, *}, \ldots,\|\cdot\|_{n, *}$. By taking the double dual seminorm we obtain that the $\pi$-tensor product of $\|\cdot\|_{1}, \ldots,\|\cdot\|_{n}$ identifies with the seminorm induced by $\|\cdot\|_{*, \varepsilon, *} \cdot$

Proposition 1.1.58. - Let $V$ and $W$ be seminormed vector spaces over $k$, and $Q$ be a quotient space of $V$, equipped with the quotient seminorm. Let $V_{0}$ be the kernel of the projection map $V \rightarrow Q$. Then the canonical isomorphism $\left(V \otimes_{k} W\right) /\left(V_{0} \otimes_{k} W\right) \rightarrow$ $Q \otimes_{k} W$ is an isometry, where we consider the $\pi$-tensor product seminorms on $V \otimes_{k} W$ and $Q \otimes_{k} W$, and the quotient seminorm on $\left(V \otimes_{k} W\right) /\left(V_{0} \otimes_{k} W\right)$.

Proof. - Let $\psi$ be an element of $Q \otimes_{k} W$. One has

$$
\begin{aligned}
\|\psi\|_{\pi} & =\inf \left\{\sum_{i=1}^{N}\left\|\alpha_{i}\right\| \cdot\left\|y_{i}\right\|: N \in \mathbb{N}, \psi=\sum_{i=1}^{N} \alpha_{i} \otimes y_{i}\right\} \\
= & \inf _{N \in \mathbb{N}} \inf _{\substack{\left(\alpha_{i}\right)_{i=1}^{N} \in Q^{N} \\
\left(y_{i}\right)_{i=1}^{N} \in W^{N} \\
\psi=\sum_{i=1}^{N} \alpha_{i} \otimes y_{i}}} \inf _{\substack{\left(x_{i}\right)_{i=1}^{N} \in V^{N} \\
\left[x_{i}\right]=\alpha_{i}}} \sum_{i=1}^{N}\left\|x_{i}\right\| \cdot\left\|y_{i}\right\|=\inf _{\substack{\varphi \in V \otimes W \\
[\varphi]=\psi}}\|\varphi\|_{\pi} .
\end{aligned}
$$

Remark 1.1.59. - We consider the $\varepsilon$-tensor product analogue of the above proposition. Let $f$ be an element of $V \otimes_{k} W$, viewed as a $k$-bilinear form on $V^{*} \times W^{*}$. Then its image $g$ in $Q \otimes_{k} W$ corresponds to the restriction of $f$ to $Q^{*} \times W^{*}$. By Proposition 1.1.20, the dual norm on $Q^{*}$ of the quotient seminorm identifies with the restriction to $Q^{*}$ of the dual norm on $V^{*}$. Therefore, one has $\|g\|_{\varepsilon} \leqslant\|f\|_{\varepsilon}$. However, in the case 
where the absolute value $|\cdot|$ is Archimedean, in general the inequality

$$
\|g\|_{\varepsilon} \leqslant \inf _{\substack{\left.f \in V \otimes_{k} W \\ f\right|_{Q^{*} \times W^{*}=g}}}\|f\|_{\varepsilon}
$$

is strict. In fact, this problem is closely related to the extension property of the normed vector space $V^{*}$, which consists of extending a linear operator defined on a vector subspace of $V^{*}$ and valued in another seminormed vector space while keeping the operator seminorm. In the case where the linear operator is a linear form (namely valued in $k$ ), it is just a consequence of Hahn-Banach theorem. However, in general the extension property does not hold, except in the cases where $\operatorname{rk}_{k}(V) \leqslant 2$ or the norm on $V$ comes from a symmetric semipositive bilinear form (see \$1.2.1 for the notation). We refer the readers to [89, 125] for more details.

In the case where the absolute value $|\cdot|$ is non-Archimedean, any dual norm is ultrametric, we will give a proof for the $\varepsilon$-tensor product analogue of Proposition 1.1.58, by using the ultrametric Gram-Schmidt process (see Proposition 1.2.36).

Proposition 1.1.60. — Let $\left(V,\|\cdot\|_{V}\right)$ and $\left(W,\|\cdot\|_{W}\right)$ be seminormed vector spaces over $k, V_{0}$ be a vector subspace of $V$ and $\|\cdot\|_{V_{0}}$ be the restriction of $\|\cdot\|_{V}$ to $V_{0}$.

(1) Let $\|\cdot\|_{\pi}$ be the $\pi$-tensor product of $\|\cdot\|_{V}$ and $\|\cdot\|_{W},\|\cdot\|_{\pi, 0}$ be the $\pi$-tensor product of $\|\cdot\|_{V_{0}}$ and $\|\cdot\|_{W}$. Then the seminorm $\|\cdot\|_{\pi, 0}$ is bounded from below by the restriction of $\|\cdot\|_{\pi}$ to $V_{0} \otimes_{k} W$.

(2) Let $\|\cdot\|_{\varepsilon}$ be the $\varepsilon$-tensor product of $\|\cdot\|_{V}$ and $\|\cdot\|_{W}$, and $\|\cdot\|_{\varepsilon, 0}$ be the $\varepsilon$-tensor product of $\|\cdot\|_{V_{0}}$ and $\|\cdot\|_{W}$. Then the seminorm $\|\cdot\|_{\varepsilon, 0}$ is bounded from below by the restriction of $\|\cdot\|_{\varepsilon}$ to $V_{0} \otimes_{k} W$.

Proof. - (1) Let $\varphi$ be an element of $V_{0} \otimes_{k} W$. By definition, for any writing of $\varphi$ as $\sum_{i=1}^{N} x_{i} \otimes y_{i}$ with $\left\{x_{1}, \ldots, x_{N}\right\} \subseteq V_{0}$ and $\left\{y_{1}, \ldots, y_{N}\right\} \subseteq W$, one has

$$
\|\varphi\|_{\pi} \leqslant \sum_{i=1}^{N}\left\|x_{i}\right\|_{V_{0}} \cdot\left\|y_{i}\right\|_{W}
$$

Therefore $\|\varphi\|_{\pi} \leqslant\|\varphi\|_{\pi, 0}$.

(2) We consider the canonical linear map $V^{*} \rightarrow V_{0}^{*}$ sending a bounded linear form on $V$ to its restriction to $V_{0}$. Note that for any $f \in V^{*}$ one has $\left\|f_{0}\right\|_{V_{0}, *} \leqslant\|f\|_{V, *}$, where $f_{0}$ is the restriction of $f$ to $V_{0}$. Therefore, for any element $\varphi$ of $V_{0} \otimes_{k} W$, viewed as a bilinear form on $V_{0}^{*} \times W^{*}$ or as a bilinear form on $V^{*} \times W^{*}$ via the inclusion $V_{0} \otimes_{k} W \subseteq V \otimes_{k} W$, one has

$$
\|\varphi\|_{\varepsilon, 0}=\sup _{\substack{\left(f_{0}, g\right) \in V_{0}^{*} \times W^{*} \\ f_{0} \neq 0, g \neq 0}} \frac{\left|\varphi\left(f_{0}, g\right)\right|}{\|f\|_{V_{0}, *}\|g\|_{W, *}} \geqslant \sup _{\substack{f, g) \in V^{*} \times W^{*} \\ f \neq 0, g \neq 0}} \frac{|\varphi(f, g)|}{\|f\|_{V, *}\|g\|_{W, *}}=\|\varphi\|_{\varepsilon} .
$$


Proposition 1.1.61. - Let $n$ be a positive integer and $\left\{\left(V_{j},\|\cdot\|_{V_{j}}\right)\right\}_{j=1}^{n}$ and $\left\{\left(W_{j},\|\cdot\|_{W_{j}}\right)\right\}_{j=1}^{n}$ be finite-dimensional seminormed vector spaces over $k$. For any $j \in\{1, \ldots, n\}$, let $f_{j}: V_{j} \rightarrow W_{j}$ be a bounded $k$-linear map. Let $f: V_{1} \otimes_{k} \cdots \otimes V_{n} \rightarrow$ $W_{1} \otimes_{k} \cdots \otimes_{k} W_{n}$ be the $k$-linear map sending $x_{1} \otimes \cdots \otimes x_{n}$ to $f_{1}\left(x_{1}\right) \otimes \cdots \otimes f_{n}\left(x_{n}\right)$.

(1) We equip the vector spaces $V_{1} \otimes_{k} \cdots \otimes_{k} V_{n}$ and $W_{1} \otimes_{k} \cdots \otimes_{k} W_{n}$ with the $\pi$ tensor product seminorms of $\left\{\|\cdot\|_{V_{j}}\right\}_{j=1}^{n}$ and of $\left\{\|\cdot\|_{W_{j}}\right\}_{j=1}^{n}$, respectively. Then the operator seminorm of $f$ is bounded from above by $\left\|f_{1}\right\| \cdots\left\|f_{n}\right\|$.

(2) We equip the vector spaces $V_{1} \otimes_{k} \cdots \otimes_{k} V_{n}$ and $W_{1} \otimes_{k} \cdots \otimes_{k} W_{n}$ with the $\varepsilon$ tensor product seminorms of $\left\{\|\cdot\|_{V_{j}}\right\}_{j=1}^{n}$ and of $\left\{\|\cdot\|_{W_{j}}\right\}_{j=1}^{n}$, respectively. Then the operator seminorm of $f$ is bounded from above by $\left\|f_{1}^{*}\right\| \cdots\left\|f_{n}^{*}\right\|$.

Proof. - (1) Let $\varphi$ be an element in $V_{1} \otimes_{k} \cdots \otimes_{k} V_{n}$, which is written as

$$
\varphi=\sum_{i=1}^{N} x_{1}^{(i)} \otimes \cdots \otimes x_{n}^{(i)}
$$

where $x_{j}^{(i)} \in V_{j}$ for any $j \in\{1, \ldots, n\}$. By definition, one has

$$
f(\varphi)=\sum_{i=1}^{N} f_{1}\left(x_{1}^{(i)}\right) \otimes \cdots \otimes f_{n}\left(x_{n}^{(i)}\right) .
$$

Therefore

$$
\|f(\varphi)\|_{\pi} \leqslant \sum_{i=1}^{N}\left\|f_{1}\left(x_{1}^{(i)}\right)\right\|_{W_{1}} \cdots\left\|f_{n}\left(x_{n}^{(i)}\right)\right\|_{W_{n}} \leqslant\left(\prod_{i=1}^{n}\left\|f_{i}\right\|\right) \sum_{i=1}^{N}\left\|x_{1}^{(i)}\right\|_{V_{1}} \cdots\left\|x_{n}^{(i)}\right\|_{V_{n}} .
$$

Thus $\|f(\varphi)\|_{\pi} \leqslant\left\|f_{1}\right\| \cdots\left\|f_{n}\right\| \cdot\|\varphi\|_{\pi}$.

(2) Let $\varphi$ be an element in $V_{1} \otimes_{k} \cdots \otimes_{n} V_{n}$, which can be viewed as a multilinear form on $V_{1}^{*} \times \cdots \times V_{n}^{*}$. Then the element $f(\varphi) \in W_{1} \otimes_{k} \cdots \otimes_{k} W_{n}$, viewed as a multilinear form on $W_{1}^{*} \times \cdots \times W_{n}^{*}$, sends $\left(\beta_{1}, \ldots, \beta_{n}\right) \in W_{1}^{*} \times \cdots \times W_{n}^{*}$ to $\varphi\left(f_{1}^{*}\left(\beta_{1}\right), \ldots, f_{n}^{*}\left(\beta_{n}\right)\right)$. Thus for $\left(\beta_{1}, \ldots, \beta_{n}\right) \in\left(W_{1}^{*} \backslash\{0\}\right) \times \cdots \times\left(W_{n}^{*} \backslash\{0\}\right)$, one has

$$
\frac{\left|f(\varphi)\left(\beta_{1}, \ldots, \beta_{n}\right)\right|}{\left\|\beta_{1}\right\|_{W_{1}, *} \cdots\left\|\beta_{n}\right\|_{W_{n}, *}} \leqslant \frac{\|\varphi\|_{\varepsilon}\left\|f_{1}^{*}\left(\beta_{1}\right)\right\|_{V_{1}, *} \cdots\left\|f_{n}^{*}\left(\beta_{n}\right)\right\|_{V_{n}, *}}{\left\|\beta_{1}\right\|_{W_{1}, *} \cdots\left\|\beta_{n}\right\|_{W_{n}, *}} \leqslant\|\varphi\|_{\varepsilon}\left\|f_{1}^{*}\right\| \cdots\left\|f_{n}^{*}\right\|,
$$

so that $\|f(\varphi)\|_{\varepsilon} \leqslant\|\varphi\|_{\varepsilon}\left\|f_{1}^{*}\right\| \cdots\left\|f_{n}^{*}\right\|$, as required.

1.1.12. Exterior power seminorm. - Let $V$ be a vector space over $k$ and $r$ be the rank of $V$ over $k$. For any $i \in \mathbb{N}$, we let $\Lambda^{i} V$ be the $i^{\text {th }}$ exterior power of the vector space $V$. It is a quotient vector space of $V^{\otimes i}$.

Definition 1.1.62. — Let $\|\cdot\|$ be a seminorm on the vector space $V$ and $\|\cdot\|_{\pi}$ be the $\pi$-tensor power of $\|\cdot\|$ on $V^{\otimes i}$. The $i^{\text {th }} \pi$-exterior power seminorm of $\|\cdot\|$ on $\Lambda^{i} V$ is by definition the quotient seminorm on $\Lambda^{i} V$ of $\|\cdot\|_{\pi}$ induced by the canonical projection map $V^{\otimes i} \rightarrow \Lambda^{i} V$ sending $x_{1} \otimes \cdots \otimes x_{i}$ to $x_{1} \wedge \cdots \wedge x_{i}$, denoted by $\|\cdot\|_{\Lambda_{\pi}^{i}}$, or simply 
by $\|\cdot\|_{\Lambda^{i}}$. Similarly, the $\varepsilon$-tensor product seminorm $\|\cdot\|_{\varepsilon}$ on $V^{\otimes i}$ induces by quotient a seminorm on $\Lambda^{i} V$, called the $i^{\text {th }} \varepsilon$-exterior power of $\|\cdot\|$, denoted by $\|\cdot\|_{\Lambda_{\varepsilon}^{i}}$.

Proposition 1.1.63. - Let $(V,\|\cdot\|)$ be a seminormed vector space over $k$ and $i$ be a natural number. For any $\left(x_{1}, \ldots, x_{i}\right) \in V^{i}$ one has

$$
\left\|x_{1} \wedge \cdots \wedge x_{i}\right\|_{\Lambda_{\varepsilon}^{i}} \leqslant\left\|x_{1} \wedge \cdots \wedge x_{i}\right\|_{\Lambda_{\pi}^{i}} \leqslant\left\|x_{1}\right\| \cdots\left\|x_{i}\right\| .
$$

Proof. - The first inequality follows from Proposition 1.1.54

Note that $x_{1} \wedge \cdots \wedge x_{i}$ is the image of $x_{1} \otimes \cdots \otimes x_{i}$ by the canonical projection map $V^{\otimes i} \rightarrow \Lambda^{i} V$. Therefore one has

$$
\left\|x_{1} \wedge \cdots \wedge x_{i}\right\|_{\Lambda_{\pi}^{i}} \leqslant\left\|x_{1} \otimes \cdots \otimes x_{i}\right\|_{\pi} \leqslant\left\|x_{1}\right\| \cdots\left\|x_{i}\right\|,
$$

where $\|\cdot\|_{\pi}$ denotes the $\pi$-tensor power of $\|\cdot\|$.

Proposition 1.1.64. - Let $V$ and $W$ be seminormed vector spaces over $k$ and $f: V \rightarrow W$ be a bounded $k$-linear map. Let $i$ be a positive integer. The $k$-linear map $f$ induces by passing to the $i^{\text {th }}$ exterior power a k-linear map $\Lambda^{i} f: \Lambda^{i} V \rightarrow \Lambda^{i} W$.

(1) If we equip $\Lambda^{i} V$ and $\Lambda^{i} W$ with the $i^{\text {th }} \pi$-exterior power seminorms, then the operator seminorm of $\Lambda^{i} f$ is bounded from above by $\|f\|^{i}$.

(2) If we equip $\Lambda^{i} V$ and $\Lambda^{i} W$ with the $i^{\text {th }}$-exterior power seminorms, then the operator seminorm of $\Lambda^{i} f$ is bounded from above by $\left\|f^{*}\right\|^{i}$.

Proof. - Let us consider a commutative diagram:

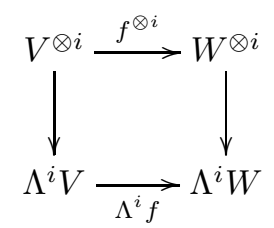

By (2.a) in Proposition 1.1.14, we obtain $\left\|\Lambda^{i} f\right\| \leqslant\left\|f^{\otimes i}\right\|$. Thus the assertions follow from Proposition 1.1.61.

1.1.13. Determinant seminorm. - Let $V$ be a finite-dimensional vector space over $k$. Recall that the determinant of $V$ is defined as the maximal exterior power $\Lambda^{r} V$ of the vector space $V$, where $r$ is the rank of $V$ over $k$. It is a quotient space of rank 1 of $V^{\otimes r}$. We denote by $\operatorname{det}(V)$ the determinant of $V$.

Definition 1.1.65. - Assume that the vector space $V$ is equipped with a seminorm $\|\cdot\|$. We call the determinant seminorm of $\|\cdot\|$ on $\operatorname{det}(V)$ and we denote by $\|\cdot\|_{\operatorname{det}}$ the $\pi$-exterior power seminorm of $\|\cdot\|$, that is, quotient seminorm induced by the $\pi$-tensor power seminorm on $V^{\otimes r}$. 
Proposition 1.1.66 (Hadamard's inequality). - Let $(V,\|\cdot\|)$ be a finitedimensional seminormed vector space of rank $r>0$ over $k$. For any $\eta \in \operatorname{det}(V)$,

$$
\|\eta\|_{\text {det }}=\inf \left\{\left\|x_{1}\right\| \cdots\left\|x_{r}\right\|: \eta=x_{1} \wedge \cdots \wedge x_{r}\right\} .
$$

In particular, the determinant seminorm is a norm if and only if $\|\cdot\|$ is a norm.

Proof. - If $\eta$ is written in the form $\eta=x_{1} \wedge \cdots \wedge x_{r}$, where $x_{1}, \ldots, x_{r}$ are elements in $V$, then it is the image of $x_{1} \otimes \cdots \otimes x_{r}$ by the canonical projection $V^{\otimes r} \rightarrow \operatorname{det}(V)$. Therefore one has $\|\eta\|_{\text {det }} \leqslant\left\|x_{1}\right\| \cdots\left\|x_{r}\right\|$. Thus we obtain

$$
\|\eta\|_{\text {det }} \leqslant \inf \left\{\left\|x_{1}\right\| \cdots\left\|x_{r}\right\|: \eta=x_{1} \wedge \cdots \wedge x_{r}\right\} .
$$

In the following, we prove the converse inequality. It suffices to treat the case where $\eta \neq 0$. By definition one has

$$
\|\eta\|_{\text {det }}=\inf \left\{\sum_{i=1}^{N}\left\|x_{1}^{(i)}\right\| \cdots\left\|x_{r}^{(i)}\right\|: \eta=\sum_{i=1}^{N} x_{1}^{(i)} \wedge \cdots \wedge x_{r}^{(i)}\right\} .
$$

Let $\left\{x_{j}^{(i)}\right\}_{i \in\{1, \ldots, N\}, j \in\{1, \ldots, r\}}$ be elements in $V$ such that $\eta=\sum_{i=1}^{N} x_{1}^{(i)} \wedge \cdots \wedge x_{r}^{(i)}$. Let $\left\{e_{j}\right\}_{j=1}^{r}$ be a basis of $V$ and $\eta_{0}=e_{1} \wedge \cdots \wedge e_{r}$. For any $i \in\{1, \ldots, N\}$, there exists $a_{i} \in k$ such that $x_{1}^{(i)} \wedge \cdots \wedge x_{r}^{(i)}=a_{i} \eta_{0}$. Without loss of generality, we may assume that all $a_{i}$ are non-zero and that

$$
\frac{\left\|x_{1}^{(1)}\right\| \cdots\left\|x_{r}^{(1)}\right\|}{\left|a_{1}\right|}=\min _{i \in\{1, \ldots, N\}} \frac{\left\|x_{1}^{(i)}\right\| \cdots\left\|x_{r}^{(i)}\right\|}{\left|a_{i}\right|} .
$$

Note that one has

$$
\eta=\left(a_{1}+\cdots+a_{N}\right) \eta_{0}=\left(1+\frac{a_{2}}{a_{1}}+\cdots+\frac{a_{N}}{a_{1}}\right) x_{1}^{(1)} \wedge \cdots \wedge x_{r}^{(1)},
$$

and

$$
\begin{aligned}
& \left|1+\frac{a_{2}}{a_{1}}+\cdots+\frac{a_{N}}{a_{1}}\right| \cdot\left\|x_{1}^{(1)}\right\| \cdots\left\|x_{r}^{(1)}\right\| \\
\leqslant & \left(1+\left|\frac{a_{2}}{a_{1}}\right|+\cdots+\left|\frac{a_{N}}{a_{1}}\right|\right)\left\|x_{1}^{(1)}\right\| \cdots\left\|x_{r}^{(1)}\right\| \leqslant \sum_{i=1}^{N}\left\|x_{1}^{(i)}\right\| \cdots\left\|x_{r}^{(i)}\right\| .
\end{aligned}
$$

The proposition is thus proved.

Remark 1.1.67. - Let $(V,\|\cdot\|)$ be a non-zero finite-dimensional normed vector space over $k$. Let $r$ be the rank of $V$ over $k$. Proposition 1.1.66 shows that

$$
\inf \left\{\frac{\left\|x_{1}\right\| \cdots\left\|x_{r}\right\|}{\left\|x_{1} \wedge \cdots \wedge x_{r}\right\|_{\text {det }}}:\left(x_{1}, \ldots, x_{r}\right) \in V^{r}, x_{1} \wedge \cdots \wedge x_{r} \neq 0\right\}=1 .
$$

If the infimum is attained by some $\left(e_{1}, \ldots, e_{r}\right) \in V^{r}$, then $\left\{e_{i}\right\}_{i=1}^{r}$ is called an Hadamard basis of $(V,\|\cdot\|)$. By convention, the empty subset of the zero normed vector space is considered as an Hadamard basis. 
Corollary 1.1.68. - Let $V$ be a finite-dimensional seminormed vector space over $k$ and $W$ be a vector subspace of $V$. Twhe canonical isomorphism (see [28] Chapter III, $\S 7$, no. 7 )

$$
\operatorname{det}(W) \otimes \operatorname{det}(V / W) \longrightarrow \operatorname{det}(V)
$$

has seminorm $\leqslant 1$, where we consider the determinant seminorm of the induced seminorm on $\operatorname{det}(W)$ and that of the quotient seminorm on $\operatorname{det}(V / W)$, and the tensor product seminorm on $\operatorname{det}(W) \otimes \operatorname{det}(V / W)$ (see Remark 1.1.56).

Proof. - Let $\left\{x_{1}, \ldots, x_{n}\right\}$ be a basis of $W$ and $\left\{y_{1}, \ldots, y_{m}\right\}$ be elements in $V \backslash W$ whose image in $V / W$ forms a basis of $V / W$. By Proposition 1.1.66 one has

$$
\left\|x_{1} \wedge \cdots \wedge x_{n} \wedge y_{1} \wedge \cdots \wedge y_{m}\right\|_{\text {det }} \leqslant\left\|x_{1}\right\| \cdots\left\|x_{n}\right\| \cdot\left\|y_{1}\right\| \cdots\left\|y_{m}\right\| .
$$

Note that if we replace each $y_{i}$ by an element $y_{i}^{\prime}$ in the same equivalent class, one has

$$
x_{1} \wedge \cdots \wedge x_{n} \wedge y_{1} \wedge \cdots \wedge y_{m}=x_{1} \wedge \cdots \wedge x_{n} \wedge y_{1}^{\prime} \wedge \cdots \wedge y_{m}^{\prime} .
$$

Hence we obtain

$$
\left\|x_{1} \wedge \cdots \wedge x_{n} \wedge y_{1} \wedge \cdots \wedge y_{m}\right\| \leqslant\left\|x_{1}\right\| \cdots\left\|x_{n}\right\| \cdot\left\|\left[y_{1}\right]\right\| \cdots\left\|\left[y_{m}\right]\right\| .
$$

Therefore, for any $\eta \in \operatorname{det}(W)$ and $\eta^{\prime} \in \operatorname{det}(V / W)$ one has

$$
\left\|\eta \wedge \eta^{\prime}\right\|_{\text {det }} \leqslant\left(\inf _{\substack{\left(x_{1}, \ldots, x_{n}\right) \in W^{n} \\ x_{1} \wedge \cdots \wedge x_{n}=\eta}}\left\|x_{1}\right\| \cdots\left\|x_{n}\right\|\right)\left(\inf _{\substack{\left(y_{1}, \ldots, y_{m}\right) \in(V \backslash W)^{m} \\\left[y_{1}\right] \wedge \cdots \wedge\left[y_{m}\right]=\eta^{\prime}}}\left\|\left[y_{1}\right]\right\| \cdots\left\|\left[y_{m}\right]\right\|\right),
$$

which leads to, by Proposition 1.1.66, the inequality $\left\|\eta \wedge \eta^{\prime}\right\|_{\text {det }} \leqslant\|\eta\|_{\text {det }} \cdot\left\|\eta^{\prime}\right\|_{\text {det }}$.

Proposition 1.1.69. — Let $\left(V,\|\cdot\|_{V}\right)$ and $\left(W,\|\cdot\|_{W}\right)$ be finite-dimensional seminormed vector spaces over $k$, and $n$ and $m$ be respectively the ranks of $V$ and $W$ over $k$. We equip $V \otimes_{k} W$ with the $\pi$-tensor product seminorm $\|\cdot\|_{\pi}$. Then the natural $k$-linear isomorphism $\operatorname{det}\left(V \otimes_{k} W\right) \cong \operatorname{det}(V)^{\otimes m} \otimes_{k} \operatorname{det}(W)^{\otimes n}$ is an isometry, where we consider the determinant seminorm of $\|\cdot\|_{\pi}$ on $\operatorname{det}\left(V \otimes_{k} W\right)$ and the tensor product of determinant seminorms on $\operatorname{det}(V)^{\otimes m} \otimes_{k} \operatorname{det}(W)^{\otimes n}$.

Proof. - Let $\|\cdot\|^{\prime}$ be the seminorm on $\operatorname{det}(V)^{\otimes m} \otimes \operatorname{det}(W)^{\otimes n}$ given by tensor product of determinant seminorms. By Proposition 1.1.58, the seminorm $\|\cdot\|^{\prime}$ identifies with the quotient of the $\pi$-tensor power on $\left(V \otimes_{k} W\right)^{\otimes n m}$ of the seminorm $\|\cdot\|_{\pi}$ on $V \otimes_{k} W$. In other words, $\|\cdot\|^{\prime}$ identifies with $\|\cdot\|_{\pi, \text { det }}$.

Proposition 1.1.70. — Let $(V,\|\cdot\|)$ be a finite-dimensional seminormed vector space over $k$ and $r$ be the rank of $V$ over $k$. Let $i$ be a positive integer. Then the canonical $k$-linear isomorphism $\operatorname{det}\left(\Lambda^{i} V\right) \rightarrow \operatorname{det}(V)^{\otimes\left(\begin{array}{c}r-1 \\ i-1\end{array}\right)}$ is an isometry, where we consider the $i^{\text {th }} \pi$-exterior power seminorm on $\Lambda^{i} V$. 
Proof. - Consider the following commutative diagram

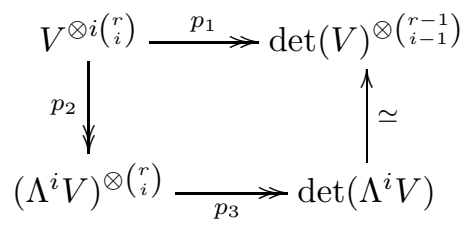

By definition, if we equip $V^{\otimes i}\left(\begin{array}{l}r \\ i\end{array}\right)$ with the $\pi$-tensor product seminorm, then its

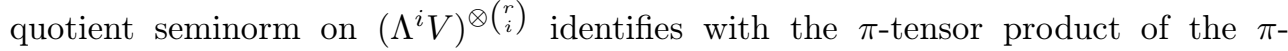
exterior power seminorm. Moreover, by Proposition 1.1.58, the quotient seminorm on $\operatorname{det}\left(\Lambda^{i} V\right)$ (induced by $p_{3}$ ) of the tensor product of the $\pi$-exterior power seminorm identifies with the determinant seminorm of the latter. Still by the same proposition, the quotient seminorm on $\operatorname{det}(V)^{\otimes\left(\begin{array}{c}r-1 \\ i-1\end{array}\right)}$ induced by $p_{1}$ identifies with the tensor power of the determinant seminorm. Therefore the natural isomorphism $\operatorname{det}\left(\Lambda^{i} V\right) \rightarrow \operatorname{det}(V)^{\otimes\left(\begin{array}{c}r-1 \\ i-1\end{array}\right)}$ preserves actually the seminorms by using [1) in Proposition 1.1.14

1.1.14. Seminormed graded algebra. - Let $R_{\bullet}=\bigoplus_{n \in \mathbb{N}} R_{n}$ be a graded $k$ algebra such that, for any $n \in \mathbb{N}, R_{n}$ is of finite rank over $k$. For any $n \in \mathbb{N}$, let $\|\cdot\|_{n}$ be a seminorm on $R_{n}$. We say that $\bar{R} \cdot=\left\{\left(R_{n},\|\cdot\|_{n}\right)\right\}_{n \in \mathbb{N}}$ is a seminormed graded algebra over $k$ if the following submultiplicativity condition is satisfied: for any $(n, m) \in \mathbb{N}^{2}$ and any $(a, b) \in R_{n} \times R_{m}$, one has

$$
\|a \cdot b\|_{n+m} \leqslant\|a\|_{n} \cdot\|b\|_{m} .
$$

Furthermore, we say that $\bar{R}$. is of finite type if the underlying graded $k$-algebra $R_{\bullet}$ is of finite type over $k$.

Let $M_{\bullet}=\bigoplus_{m \in \mathbb{Z}} M_{m}$ be a $\mathbb{Z}$-graded $k$-linear space and $h$ be a positive integer. We say that $M_{\bullet}$ is an $h$-graded $R_{\bullet}$-module if $M_{\bullet}$ is equipped with a structure of $R_{\bullet}$-module such that

$$
\forall(n, m) \in \mathbb{N} \times \mathbb{Z}, \quad \forall(a, x) \in R_{n} \times M_{m}, \quad a x \in M_{n h+m} .
$$

Let $M_{\bullet}$ be an $h$-graded $R_{\bullet}$-module. Assume that each homogeneous component $M_{m}$ is of finite rank over $k$ and is equipped with a seminorm $\|\cdot\|_{M_{m}}$. We say that $\bar{M}$. = $\left\{\left(M_{m},\|\cdot\|_{M_{m}}\right)\right\}_{m \in \mathbb{Z}}$ is a seminormed $h$-graded $\bar{R}_{\bullet}$-module if the following condition is satisfied: for any $(n, m) \in \mathbb{N} \times \mathbb{Z}$ and any $(a, x) \in R_{n} \times M_{m}$, one has

$$
\|a \cdot x\|_{M_{n h+m}} \leqslant\|a\|_{n} \cdot\|x\|_{M_{m}} .
$$

We say that an $h$-graded $\bar{R}_{\bullet}$-module $\bar{M}_{\bullet}$ is of finite type if the underlying $h$-graded $R_{\bullet}$-module $M_{\bullet}$ is of finite type.

Proposition 1.1.71. - Let $\bar{R} \cdot=\left\{\left(R_{n},\|\cdot\|_{n}\right)\right\}_{n \in \mathbb{N}}$ be a seminormed graded algebra over $k$. Let $I_{\bullet}$ be a homogenous ideal of $R_{\bullet}$ and $R_{\bullet}^{\prime}:=R_{\bullet} / I_{\bullet}$. 
(1) Let $\|\cdot\|_{n}^{\prime}$ be the quotient seminorm on $R_{n}^{\prime}$ induced by $\|\cdot\|_{n}$ and $R_{n} \rightarrow R_{n}^{\prime}$. Then $\bar{R}_{\bullet}^{\prime}=\left\{\left(R_{n}^{\prime},\|\cdot\|_{n}^{\prime}\right)\right\}_{n \in \mathbb{N}}$ forms a seminormed graded algebra over $k$.

(2) Let $\bar{M}_{\bullet}=\left\{\left(M_{m},\|\cdot\|_{M_{m}}\right)\right\}_{m \in \mathbb{N}}$ be a normed $h$-graded $\bar{R}_{\bullet}$-module and $f_{\bullet}: M_{\bullet} \rightarrow$ $N_{\bullet}$ be a homomorphism of h-graded modules over $R_{\bullet}(3)$ We assume that $I_{\bullet} \cdot N_{\bullet}=0$ and $f_{m}: M_{m} \rightarrow N_{m}$ is surjective for all $m \in \mathbb{Z}$. Let $\|\cdot\|_{N_{m}}$ be the quotient seminorm on $N_{m}$ induced by $\|\cdot\|_{M_{m}}$ and $f_{m}$. Then $\bar{N}_{\bullet}=$ $\left\{\left(N_{m},\|\cdot\|_{N_{m}}\right)\right\}_{m \in \mathbb{N}}$ forms a seminormed $h$-graded $\bar{R}_{\bullet}^{\prime}$-module.

Proof. — First let us see the following:

$$
\forall(n, m) \in \mathbb{N} \times \mathbb{Z},\left(a^{\prime}, y\right) \in R_{n}^{\prime} \times N_{m}, \quad\left\|a^{\prime} \cdot y\right\|_{N_{n h+m}} \leqslant\left\|a^{\prime}\right\|_{n}^{\prime} \cdot\|y\|_{N_{m}} .
$$

Indeed, for a fixed positive number $\epsilon$, one can find $a \in R_{n}$ and $x \in M_{m}$ such that

$$
\left\{\begin{array}{l}
{[a]=a^{\prime}, \quad\|a\|_{n} \leqslant \mathrm{e}^{\epsilon}\left\|a^{\prime}\right\|_{n}^{\prime},} \\
f_{m}(x)=y, \quad\|x\|_{M_{m}} \leqslant \mathrm{e}^{\epsilon}\|y\|_{N_{m}} .
\end{array}\right.
$$

Then, as $f_{m}(a \cdot x)=a^{\prime} \cdot y$,

$$
\left\|a^{\prime} \cdot y\right\|_{N_{n h+m}} \leqslant\|a \cdot x\|_{M_{n h+m}} \leqslant\|a\|_{n} \cdot\|x\|_{M_{m}} \leqslant \mathrm{e}^{2 \epsilon}\left\|a^{\prime}\right\|_{n}^{\prime} \cdot\|y\|_{N_{m}},
$$

which implies (1.20) because $\epsilon$ is an arbitrary positive number. Applying (1.20) to the case where $\bar{M}_{\bullet}=\bar{R}_{\bullet}$ and $\bar{N}_{\bullet}=\bar{R}_{\bullet}^{\prime}$, one has

$$
\forall\left(n, n^{\prime}\right) \in \mathbb{N}^{2}, \quad \forall\left(a^{\prime}, b^{\prime}\right) \in R_{n}^{\prime} \times R_{n^{\prime}}^{\prime}, \quad\left\|a^{\prime} \cdot b^{\prime}\right\|_{n+n^{\prime}}^{\prime} \leqslant\left\|a^{\prime}\right\|_{n}^{\prime} \cdot\left\|b^{\prime}\right\|_{n^{\prime}}^{\prime} .
$$

Thus (1) is proved, so that (2) is also proved by (1.20).

1.1.15. Norm of polynomial. - Let $k[X]$ be the polynomial ring of one variable over $k$. For $f=a_{n} X^{n}+\cdots+a_{1} X+a_{0} \in k[X]$, We define $\|f\|$ to be

$$
\|f\|:=\max \left\{\left|a_{n}\right|, \ldots,\left|a_{1}\right|,\left|a_{0}\right|\right\} \text {. }
$$

It is easy to see that $\|\cdot\|$ yields a norm of $k[X]$ over $k$.

Proposition 1.1.72. - For $f, g \in k[X]$, one has the following:

(1) If the absolute value of $k$ is Archimedean, then

$$
\|f g\| \leqslant \min \{\operatorname{deg}(f)+1, \operatorname{deg}(g)+1\}\|f\| \cdot\|g\|,
$$

where the degree of the zero polynomial is defined to be -1 by convention.

(2) If the absolute value of $k$ is non-Archimedean, then $\|f g\|=\|f\| \cdot\|g\|$.

Proof. - Clearly we may assume that $f \neq 0, g \neq 0$ and $\operatorname{deg}(f) \leqslant \operatorname{deg}(g)$. We set

$$
\left\{\begin{array}{l}
f=a_{n} X^{n}+\cdots+a_{1} X+a_{0}, \\
g=b_{m} X^{m}+\cdots+b_{1} X+b_{0}, \\
f g=c_{n+m} X^{n+m}+\cdots+c_{1} X+c_{0},
\end{array}\right.
$$

3. That is, for each $m \in \mathbb{Z}, f_{m}: M_{m} \rightarrow N_{m}$ is a $k$-linear map such that $f_{n h+m}(a \cdot x)=[a] \cdot f_{m}(x)$ for all $a \in R_{n}$ and $x \in M_{m}$. 
where $n=\operatorname{deg}(f)$ and $m=\operatorname{deg}(g)$. Then

$$
c_{l}=\sum_{(i, j) \in \Delta(l)} a_{i} b_{j},
$$

where

$$
\Delta(l)=\{(i, j): i+j=l, i \in\{0, \ldots, n\}, j \in\{0, \ldots, m\}\},
$$

so that, as $\operatorname{card}(\Delta(l)) \leqslant n+1$, one has

$$
\left|c_{l}\right| \leqslant \begin{cases}\sum_{(i, j) \in \Delta(l)}\left|a_{i}\right| \cdot\left|b_{j}\right| \leqslant(n+1)\|f\| \cdot\|g\| & \text { (Archimedean case), } \\ \max _{(i, j) \in \Delta(l)}\left\{\left|a_{i}\right| \cdot\left|b_{j}\right|\right\} \leqslant\|f\| \cdot\|g\| \quad \text { (non-Archimedean case). }\end{cases}
$$

Thus (1) and the inequality $\|f g\| \leqslant\|f\| \cdot\|g\|$ in the non-Archimedean case are obtained.

Finally let us consider the converse inequality in the non-Archimedean case. We set

$$
\alpha=\min \left\{i:\left|a_{i}\right|=\|f\|\right\} \quad \text { and } \quad \beta=\min \left\{j:\left|b_{j}\right|=\|g\|\right\} .
$$

Note that if $i+j=\alpha+\beta$ and $(i, j) \neq(\alpha, \beta)$, then $\left|a_{i}\right| \cdot\left|b_{j}\right|<\|f\| \cdot\|g\|$ because either $i<\alpha$ or $j<\beta$. Therefore, $\left|c_{\alpha+\beta}\right|=\|f\| \cdot\|g\|$ by Proposition 1.1.5 and hence $\|f g\| \geqslant\|f\| \cdot\|g\|$.

\subsection{Orthogonality}

The orthogonality of bases plays an important role in the study of finite-dimensional normed vector spaces. In the classic functional analysis over $\mathbb{R}$ or $\mathbb{C}$, the orthogonality often refers to a property related to an inner product. This property actually has an equivalent form, which has an analogue in the non-Archimedean case. However, in a finite-dimensional normed vector space over a non-Archimedean valued field, there may not exist an orthogonal basis. One can remedy this problem by introducing an approximative variant of the orthogonality. This technic is useful in the study of determinant norms.

1.2.1. Inner product. - In this subsection, we assume that the absolute value $|\cdot|$ is Archimedean. In this case the field $k$ is either $\mathbb{R}$ or $\mathbb{C}$ and we assume that $|\cdot|$ is the usual absolute value.

Let $V$ be a vector space over $k$. A map $\langle\rangle:, V \times V \rightarrow k$ is called a semidefinite inner product on $V$ if the following conditions are satisfied:

(i) $\langle x, a y+b z\rangle=a\langle x, y\rangle+b\langle x, z\rangle$ for all $(x, y, z) \in V^{3}$ and $(a, b) \in k^{2}$.

(ii) $\langle x, y\rangle=\overline{\langle y, x\rangle}$ for any $(x, y) \in V^{2}$, where $\overline{\langle y, x\rangle}$ is the complex conjugation of $\langle y, x\rangle$.

(iii) $\langle x, x\rangle \in \mathbb{R}_{\geqslant 0}$ for any $x \in V$. 
If $\langle x, x\rangle>0$ for any $x \in V \backslash\{0\}$, we just say that $\langle$,$\rangle is an inner product. Namely,$ an inner product means either a scalar product or a Hermitian product according to $k=\mathbb{R}$ or $\mathbb{C}$. Note that the semidefinite inner product $\langle$,$\rangle induces a seminorm \|\cdot\|$ on $V$ such that $\|x\|=\langle x, x\rangle^{1 / 2}$ for any $x \in V$.

Proposition 1.2.1. - Let $V$ be a vector space over $k,\langle$,$\rangle be a semidefinite inner$ product on $V$ and $\|\cdot\|$ be the seminorm induced by $\langle$,$\rangle .$

(1) For any $x \in N_{\|\cdot\|}$ and any $y \in V$ one has $\langle x, y\rangle=\langle y, x\rangle=0$.

(2) The semidefinite inner product $\langle$,$\rangle induces by passing to quotient an inner prod-$ uct $\langle,\rangle \sim$ on $V / N_{\|\cdot\|}$ such that

$$
\forall(x, y) \in V^{2}, \quad\langle[x],[y]\rangle^{\sim}=\langle x, y\rangle,
$$

where $[x]$ and $[y]$ are the classes of $x$ and $y$ in $V / N_{\|\cdot\|}$, respectively. Moreover, one has $\left(\|\alpha\|^{\sim}\right)^{2}=\langle\alpha, \alpha\rangle^{\sim}$ for any $\alpha \in V / N_{\|\cdot\|}$.

(3) Assume that $V$ is of finite rank over $k$. For any bounded linear form $f$ on $V$ there exists an element $y$ in $V$ such that $f(x)=\langle y, x\rangle$ for any $x \in V$. Moreover, the element $y$ is unique up to addition by an element in $N_{\|\cdot\| \cdot}$.

Proof. - (1) By Cauchy-Schwarz inequality, one has $|\langle x, y\rangle|^{2} \leqslant\|x\|^{2} \cdot\|y\|^{2}=0$. Hence $\langle x, y\rangle=0$. Similarly, $\langle y, x\rangle=0$.

(2) By (1) and the properties (i) and (ii) of semidefinite inner product, we obtain that, if $x, x^{\prime}, y$ and $y^{\prime}$ are vectors in $V$ such that $x-x^{\prime} \in N_{\|\cdot\|}$ and $y-y^{\prime} \in N_{\|\cdot\|}$, then $\langle x, y\rangle=\left\langle x^{\prime}, y^{\prime}\right\rangle$. Therefore the semidefinite inner product $\langle$,$\rangle induces by passing to$ quotient a function

$$
\langle,\rangle^{\sim}:\left(V / N_{\|\cdot\|}\right) \times\left(V / N_{\|\cdot\|}\right) \rightarrow k .
$$

From the definition it is straightforward to check that $\langle,\rangle^{\sim}$ is a semidefinite inner product and $\langle\alpha, \alpha\rangle^{\sim}=\left(\|\alpha\|^{\sim}\right)^{2}$ for any $\alpha \in V / N_{\|\cdot\|}$. It remains to verify that $\langle,\rangle^{\sim}$ is definite. Let $x$ be an element in $V$ such that $\langle[x],[x]\rangle^{\sim}=0$. Then one has $\langle x, x\rangle=$ $\|x\|^{2}=0$. Hence $\|x\|=0$, namely $x \in N_{\|\cdot\| \cdot}$.

(3) Since $f$ is a bounded linear form, it vanishes on $N_{\|\cdot\|}$. Hence there exists a unique linear form $\tilde{f}: V / N_{\|\cdot\|} \rightarrow k$ such that $\tilde{f} \circ \pi=f$, where $\pi: V \rightarrow V / N_{\|\cdot\|}$ is the projection map. Moreover, by Riesz's representation theorem for usual finitedimensional inner product space, there exists a unique $\beta \in V / N_{\|\cdot\|}$ such that $\tilde{f}(\alpha)=$ $\langle\beta, \alpha\rangle \sim$ for any $\alpha \in V / N_{\|\cdot\|}$. Hence we obtain that the equivalence class $\beta$ equals the set of $y \in V$ such that $f(x)=\langle y, x\rangle$ for any $x \in V$.

Let $V$ be a finite-dimensional vector space over $k$ equipped with a seminorm $\|\cdot\|$. We say that the seminorm $\|\cdot\|$ is Euclidean (resp. Hermitian) if $k=\mathbb{R}($ resp. $k=\mathbb{C}$ ) and if the seminorm $\|\cdot\|$ is induced by a semidefinite inner product. Note that if a seminorm $\|\cdot\|$ on $V$ is Euclidean (resp. Hermitian), then also is its dual norm on $V^{*}$. In fact, if $\langle$,$\rangle is a semidefinite inner product on V$ and $\|\cdot\|$ is the corresponding 
seminorm, then it induces (by Riesz's representation theorem) an $\mathbb{R}$-linear isometry $\iota:\left(V / N_{\|\cdot\|},\|\cdot\|^{\sim}\right) \rightarrow\left(V^{*},\|\cdot\|_{*}\right)$ such that

$$
\forall(x, y) \in V^{2}, \quad \iota([x])(y)=\langle x, y\rangle .
$$

Moreover, for $a \in k$ and $x \in V$ one has $\iota(a x)=\bar{a} \iota(x)$. Then the dual norm on $V^{*}$ is induced by the following inner product $\langle,\rangle_{*}$ :

$$
\forall(\alpha, \beta) \in\left(V^{*}\right)^{2}, \quad\langle\alpha, \beta\rangle_{*}=\overline{\left\langle\iota^{-1}(\alpha), \iota^{-1}(\beta)\right\rangle^{\sim}} .
$$

Remark 1.2.2. - Let $\psi:[0,1] \rightarrow[0,1]$ be the function $t \mapsto\left(t^{2}+(1-t)^{2}\right)^{1 / 2}$. If $V$ and $W$ are finite-dimensional vector spaces over $k$ equipped with semidefinite inner products, then the direct sum seminorm $\|\cdot\|_{\psi}$ on $V \oplus W$ as constructed in $\$ 1.1 .10$ is induced by the semidefinite inner product on $V \oplus W$ defined as $\left\langle(x, y),\left(x^{\prime}, y^{\prime}\right)\right\rangle:=$ $\left\langle x, x^{\prime}\right\rangle+\left\langle y, y^{\prime}\right\rangle$. The seminorm $\|\cdot\|_{\psi}$ is called the orthogonal direct sum of the seminorms on $V$ and $W$ corresponding to their semidefinite inner products.

1.2.2. Orthogonal basis of an inner product. - In this subsection, we assume that the absolute value $|\cdot|$ is Archimedean. Let $V$ be a finite-dimensional vector space over $k$ equipped with a semidefinite inner product $\langle$,$\rangle . Let \|\cdot\|$ be the seminorm induced by $\langle$,$\rangle . We say that a basis \left\{e_{1}, \ldots, e_{r}\right\}$ of $V$ is orthogonal if $\left\langle e_{i}, e_{j}\right\rangle=0$ for distinct indices $i$ and $j$ in $\{1, \ldots, r\}$. If in addition $\left\langle e_{i}, e_{i}\right\rangle=1$ for any $i \in\{1, \ldots, r\}$ such that $e_{i} \in V \backslash N_{\|\cdot\|}$, we say that $\left\{e_{1}, \ldots, e_{r}\right\}$ is an orthonormal basis. Note that, if $\left\{e_{1}, \ldots, e_{r}\right\}$ is an orthogonal basis, then

$$
\forall\left(\lambda_{1}, \ldots, \lambda_{r}\right) \in k^{r}, \quad\left\|\lambda_{1} e_{1}+\cdots+\lambda_{r} e_{r}\right\|^{2}=\sum_{i=1}^{r}\left|\lambda_{i}\right|^{2} \cdot\left\|e_{i}\right\|^{2} .
$$

Moreover, by the Gram-Schmidt process, there always exists an orthonormal basis of $V$ (cf. the proof of Proposition 1.2.30).

The following proposition provides an alternative form for the orthogonality condition of a basis in a finite-dimensional vector space equipped with a semidefinite inner product.

Proposition 1.2.3. - Let $V$ be a finite-dimensional vector space over $k$, equipped with a semidefinite inner product $\langle$,$\rangle . Let \left\{e_{i}\right\}_{i=1}^{r}$ be a basis of $V$. Then it is an orthogonal basis if and only if the following condition is satisfied:

$$
\forall\left(\lambda_{1}, \ldots, \lambda_{r}\right) \in k^{r}, \quad\left\|\lambda_{1} e_{1}+\cdots+\lambda_{r} e_{r}\right\| \geqslant \max _{i \in\{1, \ldots, r\}}\left\|\lambda_{i} e_{i}\right\| .
$$

Proof. - If $\left\{e_{i}\right\}_{i=1}^{r}$ is an orthogonal basis of $V$, then by (1.22) we obtain that the inequality (1.23) holds. Conversely, assume given a basis $\left\{e_{i}\right\}_{i=1}^{r}$ of $V$ which verifies the condition (1.23). Then for any $\left(\lambda_{1}, \ldots, \lambda_{r-1}\right) \in k^{r-1}$, one has

$$
\left\|\lambda_{1} e_{1}+\cdots+\lambda_{r-1} e_{r-1}+e_{r}\right\| \geqslant\left\|e_{r}\right\|,
$$


which implies that $e_{r}$ is orthogonal to the vector subspace generated by $e_{1}, \ldots, e_{r-1}$. Indeed, $\left\|( \pm \epsilon) e_{i}+e_{r}\right\| \geqslant\left\|e_{r}\right\|$ for $\epsilon>0$, which implies that $\epsilon\left\|e_{i}\right\|^{2} \pm 2\left\langle e_{i}, e_{r}\right\rangle \geqslant 0$, and hence $\pm\left\langle e_{i}, e_{r}\right\rangle \geqslant 0$ by taking the limit when $\epsilon \rightarrow 0$, as required. Therefore by induction we obtain that the basis $\left\{e_{i}\right\}_{i=1}^{r}$ is an orthogonal basis.

1.2.3. Orthogonality in general cases. - In this subsection, we consider a general valued field $(k,|\cdot|)$, which is not necessarily Archimedean. Let $V$ be a finitedimensional vector space over $k$ and $\|\cdot\|$ be a seminorm on $V$. We say that a basis $\left\{e_{i}\right\}_{i=1}^{r}$ of $V$ is orthogonal if for any $\left(a_{1}, \ldots, a_{r}\right) \in k^{r}$ one has

$$
\left\|a_{1} e_{1}+\cdots+a_{r} e_{r}\right\| \geqslant \max _{i \in\{1, \ldots, r\}}\left\|a_{i} e_{i}\right\| .
$$

If in addition $\left\|e_{i}\right\|=1$ for any $i \in\{1, \ldots, r\}$ such that $e_{i} \in V \backslash N_{\|\cdot\|}$, we say that the basis $\left\{e_{i}\right\}_{i=1}^{r}$ is orthonormal. We have seen in Proposition 1.2 .3 that this definition is equivalent to the definition in $\$ 1.2 .2$ when the absolute value $|\cdot|$ is Archimedean and the seminorm $\|\cdot\|$ is induced by a semidefinite inner product.

The existence of an orthogonal basis in the non-Archimedean case is not always true. We refer the readers to [113, Example 2.3.26] for a counter-example. Thus we need a refinement of the notion of orthogonality.

Definition 1.2.4. - Let $(V,\|\cdot\|)$ be a finite-dimensional seminormed vector space over $k$, and $\alpha \in] 0,1]$. We say that a basis $\left\{e_{1}, \ldots, e_{r}\right\}$ of $V$ is $\alpha$-orthogonal if for any $\left(\lambda_{1}, \ldots, \lambda_{r}\right) \in k^{r}$ one has

$$
\left\|\lambda_{1} e_{1}+\cdots+\lambda_{r} e_{r}\right\| \geqslant \alpha \max \left(\left|\lambda_{1}\right| \cdot\left\|e_{1}\right\|, \ldots,\left|\lambda_{r}\right| \cdot\left\|e_{r}\right\|\right)
$$

Note that the 1-orthogonality is just the orthogonality defined in the beginning of the subsection. We refer the readers to [113, §2.3] for more details about this notion.

Proposition 1.2.5. - Let $(V,\|\cdot\|)$ be a finite-dimensional seminormed vector space over $k, \alpha$ be an element in $] 0,1]$, and $\boldsymbol{e}=\left\{e_{i}\right\}_{i=1}^{r}$ be an $\alpha$-orthogonal basis of $(V,\|\cdot\|)$. Then the intersection of $\boldsymbol{e}$ with $N_{\|\cdot\|}$ forms a basis of $N_{\|\cdot\| \cdot}$.

Proof. - Without loss of generality, we assume that $\boldsymbol{e} \cap N_{\|\cdot\|}=\left\{e_{1}, \ldots, e_{n}\right\}$, where $n \in \mathbb{N}, n \leqslant r$. Suppose that $N_{\|\cdot\|}$ is not generated by $\boldsymbol{e} \cap N_{\|\cdot\|}$, then there exists an element $x=\lambda_{1} e_{1}+\cdots+\lambda_{r} e_{r}$ in $N_{\|\cdot\|}$ which does not belong to the vector subspace of $V$ generated by $\boldsymbol{e} \cap N_{\|\cdot\|}$. Therefore there exists $i \in\{n+1, \ldots, r\}$ such that $\lambda_{i} \neq 0$. Since the basis $\boldsymbol{e}$ is $\alpha$-orthogonal, one has

$$
0=\|x\| \geqslant \alpha\left|\lambda_{i}\right| \cdot\left\|e_{i}\right\|>0,
$$

which leads to a contradiction.

Proposition 1.2.6. - Let $(V,\|\cdot\|)$ be a finite-dimensional seminormed vector space over $k, \alpha \in] 0,1]$ and $\boldsymbol{e}$ be an $\alpha$-orthogonal basis of $V$. Let $\boldsymbol{e}^{\prime}$ be a subset of $\boldsymbol{e}$ and $W$ be the vector subspace of $V$ generated by all vectors in $\boldsymbol{e}^{\prime}$. 
(1) The set $\boldsymbol{e}^{\prime}$ is an $\alpha$-orthogonal basis of $W$ with respect to the restriction of $\|\cdot\|$ to $W$.

(2) The image of $\boldsymbol{e} \cap(V \backslash W)$ in $V / W$ forms an $\alpha$-orthogonal basis of $V / W$ with respect to the quotient seminorm of $\|\cdot\|$. Moreover, for any $x \in \boldsymbol{e} \cap(V \backslash W)$, the quotient seminorm of the class of $x$ is bounded from below by $\alpha\|x\|$. In particular, if $\alpha=1$, namely $e$ is an orthogonal basis, then for any element $x \in \boldsymbol{e} \cap(V \backslash W)$, the quotient seminorm of the class of $x$ in $V / W$ is equal to $\|x\|$.

Proof. - (1) Assume that $\boldsymbol{e}^{\prime}=\left\{e_{1}, \ldots, e_{n}\right\}$. Since $W$ is generated by the vectors in $\boldsymbol{e}^{\prime},\left\{e_{1}, \ldots, e_{n}\right\}$ is a basis of $W$. Since $\boldsymbol{e}$ is an $\alpha$-orthogonal basis of $V$, for any $\left(\lambda_{1}, \ldots, \lambda_{n}\right) \in k^{n}$ one has

$$
\left\|\lambda_{1} e_{1}+\cdots+\lambda_{n} e_{n}\right\| \geqslant \alpha \max \left\{\left|\lambda_{1}\right| \cdot\left\|e_{1}\right\|, \ldots,\left|\lambda_{n}\right| \cdot\left\|e_{n}\right\|\right\} .
$$

Therefore $\left\{e_{1}, \ldots, e_{n}\right\}$ is an $\alpha$-orthogonal basis of $W$.

(2) Assume that $\boldsymbol{e}^{\prime}=\left\{e_{1}, \ldots, e_{n}\right\}$ and $\boldsymbol{e} \cap(V \backslash W)=\left\{e_{n+1}, \ldots, e_{r}\right\}$. It is clear that the canonical image of $\boldsymbol{e} \cap(V \backslash W)$ in $V / W$ forms a basis of $V / W$. It remains to show that it is an $\alpha$-orthogonal basis. Let $\|\cdot\|^{\prime}$ be the quotient seminorm of $\|\cdot\|$ on $V / W$. For any $i \in\{n+1, \ldots, r\}$, let $y_{i}$ be the canonical image of $e_{i}$ in $V / W$. Let $\left(\lambda_{1}, \ldots, \lambda_{r}\right)$ be an element in $k^{r}$. Since $\boldsymbol{e}$ is an $\alpha$-orthogonal basis of $(V,\|\cdot\|)$, for any $\left(\lambda_{1}, \ldots, \lambda_{r}\right) \in k^{r}$ one has

$$
\left\|\lambda_{1} e_{1}+\cdots+\lambda_{r} e_{r}\right\| \geqslant \alpha \max _{i \in\{1, \ldots, r\}}\left|\lambda_{i}\right| \cdot\left\|e_{i}\right\| \geqslant \alpha \max _{i \in\{n+1, \ldots, r\}}\left|\lambda_{i}\right| \cdot\left\|e_{i}\right\| .
$$

Therefore, for any $\left(\lambda_{n+1}, \ldots, \lambda_{r}\right) \in k^{r-n}$ one has (note that $y_{j}=\left[e_{j}\right]$ )

$$
\left\|\lambda_{n+1} y_{n+1}+\cdots+\lambda_{r} y_{r}\right\|^{\prime} \geqslant \alpha \max _{i \in\{n+1, \ldots, r\}}\left|\lambda_{i}\right| \cdot\left\|e_{i}\right\| \geqslant \alpha \max _{i \in\{n+1, \ldots, r\}}\left|\lambda_{i}\right| \cdot\left\|y_{i}\right\|^{\prime} .
$$

Hence $\left\{y_{i}\right\}_{i=n+1}^{r}$ is an $\alpha$-orthogonal basis of $V / W$. The first inequality also implies that $\left\|y_{i}\right\|^{\prime} \geqslant \alpha\left\|e_{i}\right\|$ for any $i \in\{n+1, \ldots, r\}$. If $\alpha=1$, for any $i \in\{n+1, \ldots, r\}$ one has

$$
\left\|y_{i}\right\|^{\prime} \geqslant\left\|e_{i}\right\| \geqslant\left\|y_{i}\right\|^{\prime}
$$

which leads to the equality $\left\|y_{i}\right\|^{\prime}=\left\|e_{i}\right\|$.

The following proposition shows that, in the Archimedean case, any finitedimensional normed vector space admits an orthogonal basis. In general case, for any $\alpha \in$ ]0,1[, any finite-dimensional normed vector space admits an $\alpha$-orthogonal basis.

Proposition 1.2.7. — Let $(V,\|\cdot\|)$ be a finite-dimensional normed vector space over $k$. Then we have the following:

(1) For any $\alpha \in] 0,1[$, there exists an $\alpha$-orthogonal basis of $V$.

(2) Any Hadamard basis of $V$ is orthogonal (see Remark 1.1.67).

(3) If the field $k$ is locally compact and the absolute value $|\cdot|$ is not trivial, then $V$ admits an Hadamard basis, which is also an orthogonal basis. 
Proof. - (1) (2) By Proposition 1.1.66, we can choose a basis $\boldsymbol{e}=\left\{e_{i}\right\}_{i=1}^{r}$ such that

$$
\frac{\left\|e_{1}\right\| \cdots\left\|e_{r}\right\|}{\left\|e_{1} \wedge \cdots \wedge e_{r}\right\|} \leqslant \alpha^{-1}
$$

We claim that $\boldsymbol{e}=\left\{e_{i}\right\}_{i=1}^{r}$ is $\alpha$-orthogonal. Let $\left(\lambda_{1}, \ldots, \lambda_{r}\right)$ be an element in $k^{r}$ and $x=\lambda_{1} e_{1}+\cdots+\lambda_{r} e_{r}$. For any $i \in\{1, \ldots, r\}$, by Proposition 1.1.66 one has

$$
\frac{\left\|e_{1}\right\| \cdots\left\|e_{r}\right\|}{\left\|e_{1} \wedge \cdots \wedge e_{r}\right\|} \leqslant \alpha^{-1} \frac{\left\|e_{1}\right\| \cdots\left\|e_{i-1}\right\| \cdot\|x\| \cdot\left\|e_{i+1}\right\| \cdots\left\|e_{r}\right\|}{\left\|e_{1} \wedge \cdots e_{i-1} \wedge x \wedge e_{i+1} \wedge \cdots \wedge e_{r}\right\|}
$$

Therefore one has $\|x\| \geqslant \alpha\left|\lambda_{i}\right| \cdot\left\|e_{i}\right\|$. Since $i \in\{1, \ldots, r\}$ is arbitrary, we obtain that $\left\{e_{i}\right\}_{i=1}^{r}$ is an $\alpha$-orthogonal basis. A similar argument also shows that an Hadamard basis is necessarily orthogonal.

(3) We assume that $k$ is locally compact and $|\cdot|$ is not trivial. Then, as $V$ is locally compact, there is $a_{0} \in k^{\times}$such that $(V,\|\cdot\|)_{\leqslant\left|a_{0}\right|}$ is compact. By Proposition 1.1.4, if we choose $\lambda$ with $\lambda<\sup \left\{|a|: a \in k^{\times},|a|<1\right\}$, then, for any $x \in V \backslash\{0\}$, there is $b \in k^{\times}$such that $\lambda \leqslant\|b x\|<1$. Here we set

$$
C=\left\{x \in V: \lambda\left|a_{0}\right| \leqslant\|x\| \leqslant\left|a_{0}\right|\right\},
$$

which is a compact set in $V$. For $\left(x_{1}, \ldots, x_{r}\right) \in(V \backslash\{0\})^{r}$, there are $b_{1}, \ldots, b_{r} \in k^{\times}$ such that $\lambda \leqslant\left\|b_{i} x_{i}\right\|<1$ for all $i$, so that $\left(a_{0} b_{1} x_{1}, \ldots, a_{0} b_{r} x_{r}\right) \in C^{r}$ and

$$
\frac{\left\|\left(a_{0} b_{1} x_{1}\right) \wedge \cdots \wedge\left(a_{0} b_{r} x_{r}\right)\right\|}{\left\|a_{0} b_{1} x_{1}\right\| \cdots\left\|a_{0} b_{r} x_{r}\right\|}=\frac{\left\|x_{1} \wedge \cdots \wedge x_{r}\right\|}{\left\|x_{1}\right\| \cdots\left\|x_{r}\right\|} .
$$

Hence the function

$$
\left(x_{1}, \ldots, x_{r}\right) \longmapsto \frac{\left\|x_{1} \wedge \cdots \wedge x_{r}\right\|}{\left\|x_{1}\right\| \cdots\left\|x_{r}\right\|}
$$

attains its maximal value on $(V \backslash\{0\})^{r}$, which is equal to 1 . The proposition is thus proved.

Remark 1.2.8. - (1) In the case where $|\cdot|$ is trivial and $\|\cdot\|$ is ultrametric, there is an orthogonal basis $e$ for $\|\cdot\|$ by Proposition 1.2.30. Thus, by Proposition 1.2.23, $e$ is an Hadamard basis of $(V,\|\cdot\|)$.

(2) We assume that $k$ is an infinite field and the absolute value $|\cdot|$ is trivial. Fix a map $\lambda: \mathbb{P}^{1}(k) \rightarrow\left[\frac{1}{2}, 1\right]$. Let $\pi: k^{2} \backslash\{(0,0)\} \rightarrow \mathbb{P}^{1}(k)$ be the natural map. We set

$$
\forall x \in k^{2}, \quad\|x\|_{\lambda}:= \begin{cases}\lambda(\pi(x)) & \text { if } x \neq(0,0), \\ 0 & \text { if } x=(0,0) .\end{cases}
$$

It is easy to see that $\|\cdot\|_{\lambda}$ satisfies the axioms of norm: (1) $\|a x\|_{\lambda}=|a| \cdot\|x\|_{\lambda} ;$ (2) $\|x+y\|_{\lambda} \leqslant\|x\|_{\lambda}+\|y\|_{\lambda} ;(3)\|x\|_{\lambda}=0 \Longleftrightarrow x=0$. Choosing an infinite subset $S=\left\{\zeta_{1}, \zeta_{2}, \ldots, \zeta_{n}, \ldots\right\}$ of $\mathbb{P}^{1}(k)$, we consider $\lambda$ given by

$$
\lambda(\zeta):= \begin{cases}\frac{1}{2}+\left(\frac{1}{2}\right)^{n} & \text { if } \zeta \in S \text { and } \zeta=\zeta_{n}, \\ 1 & \text { otherwise. }\end{cases}
$$


Then $\left.\left.\lambda\left(\mathbb{P}^{1}(k)\right) \subseteq\right] \frac{1}{2}, 1\right]$, and for any $\epsilon>1 / 2$ there is $\zeta \in \mathbb{P}^{1}(k)$ with $\lambda(\zeta)<\epsilon$. Obviously $\left\{\|x\|_{\lambda}: x \in k^{2}\right\}$ is an infinite set, which means that (2) in Corollary 1.1.6 does not holds without the assumption that the norm is ultrametric. Moreover, let us see that there is no orthogonal basis for $\|\cdot\|_{\lambda}$. Indeed, we assume that $\left\{e_{1}, e_{2}\right\}$ is an orthogonal basis for $\|\cdot\|_{\lambda}$. By the property of $\lambda$, there is $x \in k^{2} \backslash\{(0,0)\}$ such that $\|x\|_{\lambda}<\min \left\{\left\|e_{1}\right\|_{\lambda},\left\|e_{2}\right\|_{\lambda}\right\}$. If we set $x=a e_{1}+b e_{2}$ with $(a, b) \neq(0,0)$, then

$$
\|x\|_{\lambda} \geqslant \max \left\{|a| \cdot\left\|e_{1}\right\|_{\lambda},|b| \cdot\left\|e_{2}\right\|_{\lambda}\right\} \geqslant \min \left\{\left\|e_{1}\right\|_{\lambda},\left\|e_{2}\right\|_{\lambda}\right\},
$$

which is a contradiction.

Corollary 1.2.9. - Let $(V,\|\cdot\|)$ be a finite-dimensional seminormed vector space over $k$. For any $\alpha \in] 0,1[$, there exists an $\alpha$-orthogonal basis of $(V,\|\cdot\|)$. If the absolute value $|\cdot|$ is non-trivial and $(k,|\cdot|)$ is locally compact, then $(V,\|\cdot\|)$ admits an orthogonal basis.

Proof. - If the absolute value $|\cdot|$ is non-trivial and $(k,|\cdot|)$ is locally compact, let $\alpha$ be an element in ]0,1], otherwise let $\alpha$ be an element in ]0,1[. Let $W$ be the quotient vector space $V / N_{\|\cdot\|}$, equipped with the quotient norm $\|\cdot\|^{\sim}$. By Proposition 1.2.7. the normed vector space $\left(W,\|\cdot\|^{\sim}\right)$ admits an $\alpha$-orthogonal basis $\left\{x_{i}\right\}_{i=1}^{n}$. For any $i \in\{1, \ldots, n\}$, let $e_{i}$ be an element in the class $x_{i}$. We also choose a basis $\left\{e_{j}\right\}_{j=n+1}^{r}$ of $N_{\|\cdot\|}$. Hence $\left\{e_{i}\right\}_{i=1}^{r}$ becomes a basis of $V$. For any $\left(\lambda_{1}, \ldots, \lambda_{r}\right) \in k^{r}$ one has

$$
\begin{aligned}
\left\|\lambda_{1} e_{1}+\cdots+\lambda_{r} e_{r}\right\| & =\left\|\lambda_{1} x_{1}+\cdots+\lambda_{n} x_{n}\right\|^{\sim} \geqslant \alpha \max _{i \in\{1, \ldots, n\}}\left|\lambda_{i}\right| \cdot\left\|x_{i}\right\|^{\sim} \\
& =\alpha \max _{i \in\{1, \ldots, n\}}\left|\lambda_{i}\right| \cdot\left\|e_{i}\right\|=\alpha \max _{i \in\{1, \ldots, r\}}\left|\lambda_{i}\right| \cdot\left\|e_{i}\right\| .
\end{aligned}
$$

Lemma 1.2.10. - Let $(V,\|\cdot\|)$ be a finite-dimensional seminormed vector space over $k$, and $\alpha \in] 0,1]$. If $\boldsymbol{e}=\left\{e_{i}\right\}_{i=1}^{r}$ is an $\alpha$-orthogonal basis of $V$ and if $\left\{e_{i}^{\vee}\right\}_{i=1}^{r}$ is its dual basis, then, for any $i \in\{1, \ldots, r\}, e_{i} \notin N_{\|\cdot\|}$ if and only if $e_{i}^{\vee} \in V^{*}$, and in this case one has

$$
1 \leqslant\left\|e_{i}^{\vee}\right\|_{*} \cdot\left\|e_{i}\right\| \leqslant \alpha^{-1}
$$

Proof. - The hypothesis that $e_{i} \notin N_{\|\cdot\|}$ actually implies that $e_{i}^{\vee}$ vanishes on $N_{\|\cdot\|}$ since $N_{\|\cdot\|}$ is generated by $\boldsymbol{e} \cap N_{\|\cdot\|}$ (see Proposition 1.2.5). By Corollary 1.1.13, $e_{i}^{\vee}$ belongs to $V^{*}$. Conversely, if $e_{i}$ belongs to $N_{\|\cdot\|}$ then $e_{i}^{\vee}$ is not a bounded linear form on $V$ since it takes non-zero value on $e_{i} \in N_{\|\cdot\|}$.

The first inequality of (1.24) comes from the formula (1.4) in $\$ 1.1 .5$. In the following, we prove the second inequality. For any $\left(\lambda_{1}, \ldots, \lambda_{r}\right) \in k^{r}$ one has

$$
e_{i}^{\vee}\left(\lambda_{1} e_{1}+\cdots+\lambda_{r} e_{r}\right)=\lambda_{i}
$$


Hence

$$
\left\|e_{i}^{\vee}\right\|_{*}=\sup _{\substack{\left(\lambda_{1}, \ldots, \lambda_{r}\right) \in k^{r} \\ \lambda_{i} \neq 0}} \frac{\left|\lambda_{i}\right|}{\left\|\lambda_{1} e_{1}+\cdots+\lambda_{r} e_{r}\right\|} \leqslant \alpha^{-1}\left\|e_{i}\right\|^{-1},
$$

where the inequality comes from the hypothesis that the basis $\left\{e_{i}\right\}_{i=1}^{r}$ is $\alpha$-orthogonal (so that $\left\|\lambda_{1} e_{1}+\cdots+\lambda_{r} e_{r}\right\| \geqslant \alpha\left|\lambda_{i}\right| \cdot\left\|e_{i}\right\|$ ).

Proposition 1.2.11. - Let $V$ be a finite-dimensional seminormed vector space over $k$ and $\alpha \in] 0,1]$. If $\left\{e_{i}\right\}_{i=1}^{r}$ is an $\alpha$-orthogonal basis of $V$ and if $\left\{e_{i}^{\vee}\right\}_{i=1}^{r}$ is the dual basis of $\left\{e_{i}\right\}_{i=1}^{r}$, then $\left\{e_{i}^{\vee}\right\}_{i=1}^{r} \cap V^{*}$ is an $\alpha$-orthogonal basis of $V^{*}$. Moreover, $\left\{e_{i}\right\}_{i=1}^{r}$ is an $\alpha$-orthogonal basis of $\left(V,\|\cdot\|_{* *}\right)$ and one has

$$
\alpha\left\|e_{i}\right\| \leqslant\left\|e_{i}\right\|_{* *} \leqslant\left\|e_{i}\right\| .
$$

Proof. - By Proposition 1.2.5 and Lemma 1.2.10, the cardinal of $\left\{e_{i}^{\vee}\right\}_{i=1}^{r} \cap V^{*}$, which is equal to that of $\left\{e_{i}\right\}_{i=1}^{r} \cap\left(V \backslash N_{\|\cdot\|}\right)$, is $\operatorname{dim}_{k}\left(V^{*}\right)=\operatorname{dim}_{k}\left(V / N_{\|\cdot\|}\right)$. Therefore $\left\{e_{i}^{\vee}\right\}_{i=1}^{r} \cap V^{*}$ is a basis of $V^{*}$.

Consider $\xi=a_{1} e_{1}^{\vee}+\cdots+a_{r} e_{r}^{\vee}$ in $V^{*}$. As $\xi\left(e_{i}\right)=a_{i}$ we get that

$$
\|\xi\|_{*} \geqslant \frac{\left|a_{i}\right|}{\left\|e_{i}\right\|} \geqslant \alpha\left|a_{i}\right| \cdot\left\|e_{i}^{\vee}\right\|_{*}
$$

for any $i \in\{1, \ldots, r\}$ such that $\left\|e_{i}\right\| \neq 0$, where the second inequality comes from Lemma 1.2.10. This implies that $\left\{e_{i}^{\vee}\right\}_{i=1}^{r} \cap V^{*}$ is an $\alpha$-orthogonal basis of $V^{*}$.

Let $x=\lambda_{1} e_{1}+\cdots+\lambda_{r} e_{r}$ be an element in $V$. Without loss of generality, we assume that $\boldsymbol{e} \cap N_{\|\cdot\|}=\left\{e_{n+1}, \ldots, e_{r}\right\}$. By definition, for $i \in\{1, \ldots, n\}$ one has

$$
\|x\|_{* *} \geqslant \frac{\left|e_{i}^{\vee}(x)\right|}{\left\|e_{i}^{\vee}\right\|_{*}}=\frac{\left|\lambda_{i}\right|}{\left\|e_{i}^{\vee}\right\|_{*}} .
$$

By Lemma 1.2.10, one has $1 \leqslant\left\|e_{i}^{\vee}\right\|_{*} \cdot\left\|e_{i}\right\| \leqslant \alpha^{-1}$ and hence

$$
\|x\|_{* *} \geqslant \alpha\left|\lambda_{i}\right| \cdot\left\|e_{i}\right\| \geqslant \alpha\left|\lambda_{i}\right| \cdot\left\|e_{i}\right\|_{* *},
$$

where the last inequality comes from (1.5). For $i \in\{n+1, \ldots, r\}$ one has $\left\|e_{i}\right\|_{* *}=$ $\left\|e_{i}\right\|=0$. Hence

$$
\|x\|_{* *} \geqslant \alpha \max _{i \in\{1, \ldots, r\}}\left|\lambda_{i}\right| \cdot\left\|e_{i}\right\|_{* *},
$$

which shows that $\left\{e_{i}\right\}_{i=1}^{r}$ is an $\alpha$-orthogonal basis of $\left(V,\|\cdot\|_{* *}\right)$. Moreover, (1.27) also implies that $\left\|e_{i}\right\|_{* *} \geqslant \alpha\left\|e_{i}\right\|$ for $i \in\{1, \ldots, n\}$, which, joint with the relation

$$
\forall i \in\{n+1, \ldots, r\}, \quad\left\|e_{i}\right\|=\left\|e_{i}\right\|_{* *}=0,
$$

leads to the first inequality of (1.25). The second inequality of (1.25) comes from (1.5).

Corollary 1.2.12. - We suppose that the absolute value $|\cdot|$ is non-Archimedean. Let $(V,\|\cdot\|)$ be a finite-dimensional seminormed vector space over $k$. Then the double dual seminorm $\|\cdot\|_{* *}$ on $V$ is the largest ultrametric seminorm on $V$ which is bounded 
from above by $\|\cdot\|$, and one has $\|\cdot\| \leqslant \operatorname{rk}(V)\|\cdot\|_{* *}$. If the seminorm $\|\cdot\|$ is ultrametric, then one has $\|\cdot\|_{* *}=\|\cdot\|$.

Proof. - We have seen in Remark 1.1.19 that the double dual seminorm $\|\cdot\|_{* *}$ is ultrametric, and in the formula (1.5) of 1.1 .5 that it is bounded from above by $\|\cdot\|$. Let $\|\cdot\|^{\prime}$ be an ultrametric seminorm on $V$ such that $\|\cdot\|^{\prime} \leqslant\|\cdot\|$. We will show that $\|\cdot\|^{\prime} \leqslant\|\cdot\|_{* *}$ and $\|\cdot\| \leqslant r\|\cdot\|_{* *}$, where $r$ is the rank of $V$ over $k$.

Let $\alpha \in] 0,1\left[\right.$. By Proposition 1.2.7 there exists an $\alpha$-orthogonal basis $\left\{e_{i}\right\}_{i=1}^{r}$ of $(V,\|\cdot\|)$. For any vector $x=\lambda_{1} e_{1}+\cdots+\lambda_{r} e_{r}$ in $V$ one has

$$
\alpha^{2}\|x\|^{\prime} \leqslant \alpha^{2} \max _{i \in\{1, \ldots, r\}}\left|\lambda_{i}\right| \cdot\left\|e_{i}\right\| \leqslant \alpha \max _{i \in\{1, \ldots, r\}}\left|\lambda_{i}\right| \cdot\left\|e_{i}\right\|_{* *} \leqslant\|x\|_{* *},
$$

where the second inequality comes from (1.25) and the third inequality follows from the fact that $\left\{e_{i}\right\}_{i=1}^{r}$ is an $\alpha$-orthogonal basis for $\|\cdot\|_{* *}$ (see Proposition 1.2.11). Moreover, by the triangle inequality one has

$$
\alpha^{2}\|x\| \leqslant \alpha^{2} \sum_{i=1}^{r}\left|\lambda_{i}\right| \cdot\left\|e_{i}\right\| \leqslant r \alpha^{2} \max _{i \in\{1, \ldots, r\}}\left|\lambda_{i}\right| \cdot\left\|e_{i}\right\| \leqslant r\|x\|_{* *}
$$

Since $\alpha \in] 0,1\left[\right.$ is arbitrary, we obtain $\|\cdot\|^{\prime} \leqslant\|\cdot\|_{* *}$ and $\|\cdot\| \leqslant r\|\cdot\|_{* *}$. The first assertion of the proposition is thus proved.

If $\|\cdot\|$ is ultrametric, it is certainly the largest ultrametric norm bounded from above by $\|\cdot\|$. Hence one has $\|\cdot\|=\|\cdot\|_{* *}$.

Remark 1.2.13. - In Corollary 1.2.12, the constant $\operatorname{rk}(V)$ in the inequality $\|\cdot\| \leqslant$ $\operatorname{rk}(V)\|\cdot\|_{* *}$ is optimal. We can consider for example the vector space $V=k^{r}$ equipped with the $\ell^{1}$-norm

$$
\forall\left(a_{1}, \ldots, a_{r}\right) \in k^{r}, \quad\left\|\left(a_{1}, \ldots, a_{r}\right)\right\|_{\ell^{1}}=\left|a_{1}\right|+\cdots+\left|a_{r}\right| .
$$

Then its double dual norm is given by

$$
\forall\left(a_{1}, \ldots, a_{r}\right) \in k^{r}, \quad\left\|\left(a_{1}, \ldots, a_{r}\right)\right\|_{\ell^{1}, * *}=\max \left\{\left|a_{1}\right|, \ldots\left|a_{r}\right|\right\} .
$$

In particular, one has $\|(1, \ldots, 1)\|_{\ell^{1}}=r\|(1, \ldots, 1)\|_{\ell^{1}, * *}$.

Proposition 1.2.14. — Let $(V,\|\cdot\|)$ be a finite-dimensional seminormed vector space over the field $k$.

(1) The seminorm $\|\cdot\|$ and its double dual seminorm $\|\cdot\|_{* *}$ induce the same dual norm on the vector space $V^{*}$ of bounded linear forms.

(2) If $W$ is a quotient space of rank 1 of $V$, then the seminorms $\|\cdot\|$ and $\|\cdot\|_{* *}$ induce the same quotient seminorm on $W$.

Proof. - (1) The Archimedean case follows from Proposition 1.1.18, It suffices to treat the case where the absolute value $|\cdot|$ is non-Archimedean. By (1.5) of \$1.1.5, one has $\|\cdot\|_{* *} \leqslant\|\cdot\|$. Moreover, by Corollary [1.2.9, for any $\left.\alpha \in\right] 0,1[$ there exists an $\alpha$-orthogonal basis $\boldsymbol{e}=\left\{e_{i}\right\}_{i=1}^{r}$ of $(V,\|\cdot\|)$. By Proposition 1.2.11, this basis is 
also an $\alpha$-orthogonal basis of $\left(V,\|\cdot\|_{* *}\right)$ and one has $\alpha\left\|e_{i}\right\| \leqslant\left\|e_{i}\right\|_{* *}$ for any $i \in$ $\{1, \ldots, r\}$. By Proposition 1.2.5, this leads to $N_{\|\cdot\|}=N_{\|\cdot\|_{* *}}$ and hence the space of bounded linear forms on $\left(V,\|\cdot\|_{* *}\right)$ identifies with $(V,\|\cdot\|)^{*}$, and the relation $\|\cdot\|_{* *} \leqslant\|\cdot\|$ leads to $\|\cdot\|_{*} \leqslant\|\cdot\|_{* *, *}$. Let $\left\{e_{i}^{\vee}\right\}_{i=1}^{r}$ be the dual basis of $\left\{e_{i}\right\}_{i=1}^{r}$ and assume that $\left\{e_{i}^{\vee}\right\}_{i=1}^{r} \cap V^{*}=\left\{e_{i}^{\vee}\right\}_{i=1}^{n}$. Then by Proposition 1.2.11 the familiy $\left\{e_{i}^{\vee}\right\}_{i=1}^{n}$ is an $\alpha$ orthogonal basis of $V^{*}$ both for $\|\cdot\|_{*}$ and $\|\cdot\|_{* *, *}$. Moreover, by Lemma 1.2.10, for any $i \in\{1, \ldots, n\}$ one has

$$
\left\|e_{i}^{\vee}\right\|_{*} \geqslant\left\|e_{i}\right\|^{-1} \geqslant \alpha\left\|e_{i}\right\|_{* *}^{-1} \geqslant \alpha^{2}\left\|e_{i}^{\vee}\right\|_{* *, *},
$$

where the middle inequality comes from Proposition 1.2.11. Since the absolute value $|\cdot|$ is non-Archimedean, the norm $\|\cdot\|_{* *, *}$ is ultrametric. Hence for any $\varphi=\lambda_{1} e_{1}^{\vee}+$ $\cdots+\lambda_{n} e_{n}^{\vee} \in V^{*}$ one has

$$
\|\varphi\|_{* *, *} \leqslant \max _{i \in\{1, \ldots, n\}}\left|\lambda_{i}\right| \cdot\left\|e_{i}^{\vee}\right\|_{* *, *} \leqslant \alpha^{-2} \max _{i \in\{1, \ldots, n\}}\left|\lambda_{i}\right| \cdot\left\|e_{i}^{\vee}\right\|_{*} \leqslant \alpha^{-1}\|\varphi\|_{*},
$$

where the last inequality comes from the fact that $\left\{e_{i}^{\vee}\right\}_{i=1}^{n}$ is an $\alpha$-orthogonal basis of $V^{*}$. Since $\left.\alpha \in\right] 0,1\left[\right.$ is arbitrary, we obtain $\|\cdot\|_{* *, *}=\|\cdot\|_{*}$.

(2) If the kernel of the quotient map $V \rightarrow W$ does not contain $N_{\|\cdot\|}$, then the quotient seminorm of $\|\cdot\|$ on $W$ vanishes because $\operatorname{dim}_{K} W=1$. The quotient seminorm of $\|\cdot\|_{* *}$ on $W$ also vanishes since we have observed in the proof of (1) that $N_{\|\cdot\|}=N_{\|\cdot\|_{* *}}$. In the following we treat the case where the kernel of the projection map $V \rightarrow W$ contains $N_{\|\cdot\|}$, or equivalent, the quotient seminorms of $\|\cdot\|$ and $\|\cdot\|_{* *}$ on $W$ are actually norms. Since $W$ is of rank 1 , any norm on $W$ is uniquely determined by its dual norm on $W^{\vee}$. Let $\|\cdot\|_{W}$ be the quotient norm on $W$ induced by $\|\cdot\|$. By Proposition 1.1.20, the dual norm $\|\cdot\|_{W, *}$ identifies with the restriction of $\|\cdot\|_{*}$ to $W^{\vee}$ (viewed as a vector subspace of $V^{*}$ ). By (1), the norm $\|\cdot\|_{*}$ identifies with the dual norm of $\|\cdot\|_{* *}$. As a consequence, $\|\cdot\|_{W}$ coincides with the quotient norm of $\|\cdot\|_{* *}$.

Proposition 1.2.15. - We assume that the absolute value $|\cdot|$ is non-Archimedean. Let $(V,\|\cdot\|)$ be a finite-dimensional seminormed vector space over $k$ and let $r$ be the rank of $V$. Then the quotient norm of the $\varepsilon$-tensor product seminorm $\|\cdot\|_{\varepsilon}$ on $V^{\otimes r}$ by the canonical quotient map $V^{\otimes r} \rightarrow \operatorname{det}(V)$ identifies with the determinant seminorm on $\operatorname{det}(V)$ induced by $\|\cdot\|$. In particular, $\|\cdot\|$ and $\|\cdot\|_{* *}$ induce the same determinant seminorm on $\operatorname{det}(V)$.

Proof. - Denote by $\|\cdot\|_{\operatorname{det}_{\varepsilon}}$ the quotient seminorm on $\operatorname{det}(V)$ of the $\varepsilon$-tensor product seminorm on $V^{\otimes r}$. We have seen in Proposition 1.1.54 that the $\varepsilon$-tensor product seminorm is always bounded from above by the $\pi$-tensor product seminorm. Therefore, one has $\|\cdot\|_{\operatorname{det}_{\varepsilon}} \leqslant\|\cdot\|_{\text {det }}$. Moreover, if $\|\cdot\|$ is not a norm, then the seminorm $\|\cdot\|_{\text {det }}$ vanishes. Hence the seminorm $\|\cdot\|_{\operatorname{det}_{\varepsilon}}$ also vanishes. To prove the first assertion of the proposition, it remains to verify the inequality $\|\cdot\|_{\text {det }} \leqslant\|\cdot\|_{\operatorname{det}_{\varepsilon}}$ in the case where $\|\cdot\|$ is a norm 
Consider a tensor vector $\varphi$ in $V^{\otimes r}$, which is also viewed as a $k$-multilinear form on $\left(V^{\vee}\right)^{r}$. By definition, one has

$$
\|\varphi\|_{\varepsilon}=\sup _{\substack{\left(f_{1}, \ldots, f_{r}\right) \in\left(V^{\vee}\right)^{r} \\ \forall i \in\{1, \ldots, r\}, f_{i} \neq 0}} \frac{\left|\varphi\left(f_{1}, \ldots, f_{r}\right)\right|}{\left\|f_{1}\right\|_{*} \cdots\left\|f_{r}\right\|_{*}} .
$$

Let $\alpha \in] 0,1\left[,\left\{x_{i}\right\}_{i=1}^{r}\right.$ be an $\alpha$-orthogonal basis of $V$, and $\left\{x_{i}^{\vee}\right\}_{i=1}^{r}$ be its dual basis of $V^{\vee}$. Assume that $\varphi$ is written in the form

$$
\varphi=\sum_{I=\left(i_{1}, \ldots, i_{r}\right) \in\{1, \ldots, r\}^{r}} a_{I}\left(x_{i_{1}} \otimes \cdots \otimes x_{i_{r}}\right),
$$

where $a_{I} \in k$. Then one has

$$
\forall\left(i_{1}, \ldots, i_{r}\right) \in\{1, \ldots, r\}^{r}, \quad \varphi\left(x_{i_{1}}^{\vee}, \ldots, x_{i_{r}}^{\vee}\right)=a_{\left(i_{1}, \ldots, i_{r}\right)} .
$$

In particular,

$$
\forall\left(i_{1}, \ldots, i_{r}\right) \in\{1, \ldots, r\}^{r}, \quad\|\varphi\|_{\varepsilon} \geqslant \frac{\left|a_{\left(i_{1}, \ldots, i_{r}\right)}\right|}{\left\|x_{i_{1}}^{\vee}\right\|_{*} \cdots\left\|x_{i_{r}}^{\vee}\right\|_{*}} .
$$

Note that the canonical image $\eta$ of $\varphi$ in $\operatorname{det}(V)$ is

$$
\left(\sum_{\sigma \in \mathfrak{S}_{r}} \operatorname{sgn}(\sigma) a_{(\sigma(1), \ldots, \sigma(r))}\right) x_{1} \wedge \cdots \wedge x_{r},
$$

where $\mathfrak{S}_{r}$ is the symmetric group of order $r$, namely the group of all bijections from the set $\{1, \ldots, r\}$ to itself, and $\operatorname{sgn}(\cdot): \mathfrak{S}_{r} \rightarrow\{ \pm 1\}$ denotes the character of signature. Hence

$$
\begin{aligned}
\|\eta\|_{\text {det }} & =\left|\sum_{\sigma \in \mathfrak{S}_{r}} \operatorname{sgn}(\sigma) a_{(\sigma(1), \ldots, \sigma(r))}\right| \cdot\left\|x_{1} \wedge \cdots \wedge x_{r}\right\|_{\text {det }}, \\
& \leqslant\left|\sum_{\sigma \in \mathfrak{S}_{r}} \operatorname{sgn}(\sigma) a_{(\sigma(1), \ldots, \sigma(r))}\right| \cdot\left\|x_{1}\right\| \cdots\left\|x_{r}\right\|,
\end{aligned}
$$

where the inequality follows from (1.18). Since the absolute value $|\cdot|$ is nonArchimedean, one has

$$
\|\eta\|_{\text {det }} \leqslant\|\varphi\|_{\varepsilon} \cdot\left\|x_{1}\right\| \cdots\left\|x_{r}\right\| \cdot\left\|x_{1}^{\vee}\right\|_{*} \cdots\left\|x_{r}^{\vee}\right\|_{*} \leqslant\|\varphi\|_{\varepsilon} \alpha^{-r},
$$

where the second inequality comes from Lemma 1.2.10. Since $\alpha \in] 0,1[$ is arbitrary, the first assertion is proved.

We proceed with the proof of the second assertion. By Proposition 1.2.14, the seminorms $\|\cdot\|$ and $\|\cdot\|_{* *}$ induce the same dual norm on $V^{*}$, and hence induce the same $\varepsilon$-tensor product seminorm on $V^{\otimes r}$. Therefore, by the first assertion of the proposition, we obtain that they induce the same determinant seminorm on $\operatorname{det}(V)$.

Remark 1.2.16. - In the above proposition, the non-Archimedean assumption on the absolute value is essential. In the Archimedean case, the inequality (1.28) only 
leads to a weaker estimate $\|\cdot\|_{\text {det }} \leqslant r !\|\cdot\|_{\operatorname{det}_{\varepsilon}}$, where $\|\cdot\|_{\operatorname{det}_{\varepsilon}}$ is the quotient seminorm on $\operatorname{det}(V)$ induced by the $\varepsilon$-tensor product seminorm.

Definition 1.2.17. - Let $(V,\|\cdot\|)$ be a finite-dimensional seminormed vector space over $k$ and $r$ be the dimension of $V$ over $k$. We denote by $\|\cdot\|_{\operatorname{det}_{\varepsilon}}$ the quotient seminorm of the $\varepsilon$-tensor power of $\|\cdot\|$ on $V^{\otimes r}$ by the canonical projection map $V^{\otimes r} \rightarrow \operatorname{det}(V)$, called the $\varepsilon$-determinant seminorm of $\|\cdot\|$.

Proposition 1.2.18. - We assume that the absolute value $|\cdot|$ is Archimedean. Let $(V,\|\cdot\|)$ be a finite-dimensional seminormed vector space over $k$ and let $r$ be the rank of $V / N_{\|\cdot\|}$ over $k$. Then the $\varepsilon$-determinant norm of the dual norm $\|\cdot\|_{*}$ on $\operatorname{det}\left(V^{*}\right)$ is bounded from below by $(r !)^{-1}\|\cdot\|_{\mathrm{det}, *}^{\sim}$, where $\|\cdot\|_{\mathrm{det}, *}^{\sim}$ is the dual norm of the determinant of the norm $\|\cdot\|^{\sim}$.

Proof. - By Corollary 1.1.13, one has $V^{*}=\left(V / N_{\|\cdot\|}\right)^{\vee}$. Moreover one has $\|\cdot\|_{*}=$ $\|\cdot\|_{*}^{\sim}$. Hence, by replacing $(V,\|\cdot\|)$ by $\left(V / N_{\|\cdot\|},\|\cdot\|^{\sim}\right)$, we may assume without loss of generality that $\|\cdot\|$ is a norm.

Let $\varphi$ be an element in $V^{\vee \otimes r}$. Viewed as a $k$-multilinear form on $V^{r}$, one has

$$
\|\varphi\|_{*, \varepsilon}=\sup _{\left(x_{1}, \ldots, x_{r}\right) \in(V \backslash\{0\})^{r}} \frac{\left|\varphi\left(x_{1}, \ldots, x_{r}\right)\right|}{\left\|x_{1}\right\| \cdots\left\|x_{r}\right\|} .
$$

Let $\left\{e_{i}\right\}_{i=1}^{r}$ be a basis of $V$ and $\left\{e_{i}^{\vee}\right\}_{i=1}^{r}$ be its dual basis of $V^{\vee}$. Assume that $\varphi$ is written in the form

$$
\varphi=\sum_{I=\left(i_{1}, \ldots, i_{r}\right) \in\{1, \ldots, r\}^{r}} a_{I}\left(e_{i_{1}}^{\vee} \otimes \cdots \otimes e_{i_{r}}^{\vee}\right),
$$

where $a_{I} \in k$. Then for any $\left(i_{1}, \ldots, i_{r}\right)$ one has

$$
\varphi\left(e_{i_{1}}, \ldots, e_{i_{r}}\right)=a_{\left(i_{1}, \ldots, i_{r}\right)} .
$$

In particular,

$$
\|\varphi\|_{*, \varepsilon} \geqslant \frac{\left|a_{\left(i_{1}, \ldots, i_{r}\right)}\right|}{\left\|e_{i_{1}}\right\| \cdots\left\|e_{i_{r}}\right\|} .
$$

Note that the canonical image $\eta$ of $\varphi$ in $\operatorname{det}(V)$ is

$$
\left(\sum_{\sigma \in \mathfrak{S}_{r}} \operatorname{sgn}(\sigma) a_{(\sigma(1), \ldots, \sigma(r))}\right) e_{1}^{\vee} \wedge \cdots \wedge e_{r}^{\vee} .
$$

Therefore,

$$
\begin{aligned}
\|\eta\|_{\text {det }, *} & =\left|\sum_{\sigma \in \mathfrak{S}_{r}} \operatorname{sgn}(\sigma) a_{(\sigma(1), \ldots, \sigma(r))}\right| \cdot\left\|e_{1}^{\vee} \wedge \wedge \wedge e_{r}^{\vee}\right\|_{\text {det }, *} \\
& =\left|\sum_{\sigma \in \mathfrak{S}_{r}} \operatorname{sgn}(\sigma) a_{(\sigma(1), \ldots, \sigma(r))}\right| \cdot \frac{1}{\left\|e_{1} \wedge \cdots \wedge e_{r}\right\|_{\text {det }}} \leqslant r !\|\varphi\|_{*, \varepsilon} \frac{\left\|e_{1}\right\| \cdots\left\|e_{r}\right\|}{\left\|e_{1} \wedge \cdots \wedge e_{r}\right\|_{\text {det }}} .
\end{aligned}
$$

By (1.18), we obtain $\|\eta\|_{\operatorname{det}, *} \leqslant r !\|\varphi\|_{*, \varepsilon}$. The proposition is thus proved. 
Proposition 1.2.19. - Let $\left\{\left(V_{j},\|\cdot\|_{j}\right)\right\}_{j=1}^{d}$ be a finite family of finite-dimensional seminormed $k$-vector spaces and let $\alpha$ be a real number in $] 0,1]$. For any $j \in$ $\{1, \ldots, d\}$, let $\left\{e_{i}^{(j)}\right\}_{i=1}^{n_{j}}$ be an $\alpha$-orthogonal basis of $V_{j}$. Then

$$
e_{i_{1}}^{(1)} \otimes \cdots \otimes e_{i_{d}}^{(d)}, \quad\left(i_{1}, \ldots, i_{d}\right) \in \prod_{j=1}^{d}\left\{1, \ldots, n_{j}\right\}
$$

form an $\alpha^{d}$-orthogonal basis of $V_{1} \otimes_{k} \cdots \otimes_{k} V_{d}$ for the $\varepsilon$-tensor product norm $\|\cdot\|_{\varepsilon}$ of $\left\{\|\cdot\|_{j}\right\}_{j=1}^{d} \cdot$ Moreover, if $\|\cdot\|$ is an ultrametric norm on $V_{1} \otimes_{k} \cdots \otimes_{k} V_{d}$ such that

$$
\left\|x_{1} \otimes \cdots \otimes x_{d}\right\| \leqslant\left\|x_{1}\right\|_{1, * *} \cdots\left\|x_{d}\right\|_{d, * *}
$$

for any $\left(x_{1}, \ldots, x_{d}\right) \in V_{1} \times \cdots \times V_{d}$, then $\|\cdot\| \leqslant\|\cdot\|_{\varepsilon}$.

Proof. - Let

$$
T=\sum_{\left(i_{1}, \ldots, i_{d}\right) \in \prod_{j=1}^{d}\left\{1, \ldots, n_{j}\right\}} a_{i_{1}, \ldots, i_{d}} e_{i_{1}}^{(1)} \otimes \cdots e_{i_{d}}^{(d)}
$$

be a tensor in $V_{1} \otimes_{k} \cdots \otimes_{k} V_{d}$, where $a_{i_{1}, \ldots, i_{d}} \in k$. We consider it as a $k$-multilinear form on $V_{1}^{\vee} \times \cdots \times V_{d}^{\vee}$. For any $j \in\{1, \ldots, d\}$, let $\left\{\varphi_{i}^{(j)}\right\}_{i=1}^{n_{j}}$ be the dual basis of $\left\{e_{i}^{(j)}\right\}_{i=1}^{n_{j}}$ and assume that

$$
\left\{\varphi_{i}^{(j)}\right\}_{i=1}^{n_{j}} \cap V^{*}=\left\{\varphi_{i}^{(j)}\right\}_{i=1}^{n_{j}^{\prime}} .
$$

By Proposition 1.2.11 $\left\{\varphi_{i}^{(j)}\right\}_{i=1}^{n_{j}^{\prime}}$ is an $\alpha$-orthogonal basis of $V^{(j), *}$. For any $\left(i_{1}, \ldots, i_{d}\right) \in \prod_{j=1}^{d}\left\{1, \ldots, n_{j}^{\prime}\right\}$ we have $T\left(\varphi_{i_{1}}^{(1)}, \ldots, \varphi_{i_{d}}^{(d)}\right)=a_{i_{1}, \ldots, i_{d}}$, which leads to

$$
\begin{aligned}
\|T\|_{\varepsilon} \geqslant \frac{\left|a_{i_{1}, \ldots, i_{d}}\right|}{\left\|\varphi_{i_{1}}^{(1)}\right\|_{1, *} \cdots\left\|\varphi_{i_{d}}^{(d)}\right\|_{d, *}} & \geqslant \alpha^{d}\left|a_{i_{1}, \ldots, i_{d}}\right| \cdot\left\|e_{i_{1}}^{(1)}\right\|_{1, * *} \cdots\left\|e_{i_{d}}^{(d)}\right\|_{d, * *} \\
& =\alpha^{d}\left|a_{i_{1}, \ldots, i_{d}}\right| \cdot\left\|e_{i_{1}}^{(1)} \otimes \cdots \otimes e_{i_{d}}^{(d)}\right\|_{\varepsilon},
\end{aligned}
$$

where the second inequality follows from (1.24) and the equality comes from Remark 1.1.56. This completes the proof of the proposition because

$$
\left\|e_{i_{1}}^{(1)} \otimes \cdots \otimes e_{i_{d}}^{(d)}\right\|_{\varepsilon}=\left\|e_{i_{1}}^{(1)}\right\|_{1, * *} \cdots\left\|e_{i_{d}}^{(d)}\right\|_{d, * *}
$$

vanishes once $\left(i_{1}, \ldots, i_{d}\right) \notin \prod_{j=1}^{d}\left\{1, \ldots, n_{j}^{\prime}\right\}$ (see Lemma 1.2.10). Moreover, if $\|\cdot\|$ is an ultrametric norm on $V_{1} \otimes_{k} \cdots \otimes_{k} V_{d}$ such that

$$
\left\|x_{1} \otimes \cdots \otimes x_{d}\right\| \leqslant\left\|x_{1}\right\|_{1, * *} \cdots\left\|x_{d}\right\|_{d, * *}
$$

for any $\left(x_{1}, \ldots, x_{d}\right) \in V_{1} \times \cdots \times V_{d}$, then

$$
\begin{aligned}
& \|T\| \leqslant \max _{\left(i_{1}, \ldots, i_{d}\right) \in \prod_{j=1}^{d}\left\{1, \ldots, n_{j}\right\}}\left|a_{i_{1}, \ldots, i_{d}}\right| \cdot\left\|e_{i_{1}}^{(1)} \otimes \cdots \otimes e_{i_{d}}^{(d)}\right\| \\
& \quad \leqslant \max _{\left(i_{1}, \ldots, i_{d}\right) \in \prod_{j=1}^{d}\left\{1, \ldots, n_{j}\right\}}\left|a_{i_{1}, \ldots, i_{d}}\right| \cdot\left\|e_{i_{1}}^{(1)}\right\|_{1, * *} \cdot\left\|e_{i_{d}}\right\|_{d, * *} \leqslant \alpha^{-d}\|T\|_{\varepsilon}
\end{aligned}
$$

By Proposition 1.2.7, for any $\alpha \in] 0,1\left[\right.$, there exist $\alpha$-orthogonal bases of $V_{1}, \ldots, V_{d}$ respectively. Hence $\|\cdot\| \leqslant\|\cdot\|_{\varepsilon}$. 
Corollary 1.2.20. - Assume that the absolute value $|\cdot|$ is non-Archimedean. Let $\left\{\left(V_{j},\|\cdot\|_{j}\right)\right\}_{j=1}^{d}$ be a finite family of finite-dimensional seminormed vector spaces over $k$, and $\|\cdot\|_{\varepsilon}$ be the $\varepsilon$-tensor product of the seminorms $\|\cdot\|_{1}, \ldots,\|\cdot\|_{d}$. Then the dual norm $\|\cdot\|_{\varepsilon, *}$ coincides with the $\varepsilon$-tensor product of the dual norms $\|\cdot\|_{1, *}, \ldots,\|\cdot\|_{d, *}$.

Proof. - Let $\alpha$ be an element of $] 0,1\left[\right.$. For $j \in\{1, \ldots, d\}$, let $\left\{e_{i}^{(j)}\right\}_{i=1}^{n_{j}}$ be an $\alpha$ orthogonal basis of $V_{j}$ over $k$ (see Proposition 1.2.7) and $\left\{\varphi_{i}^{(j)}\right\}_{i=1}^{n_{j}}$ be the dual basis of $\left\{e_{i}^{(j)}\right\}_{i=1}^{n_{j}}$, and assume that $\left\{\varphi_{i}^{(j)}\right\}_{i=1}^{n_{j}} \cap V_{j}^{*}=\left\{\varphi_{i}^{(j)}\right\}_{i=1}^{n_{j}^{\prime}}$. Note that for any $\left(i_{1}, \ldots, i_{d}\right) \in$ $\prod_{j=1}^{d}\left\{1, \ldots, n_{j}\right\}$

$$
\left\|e_{i_{1}}^{(1)} \otimes \cdots \otimes e_{i_{d}}^{(d)}\right\|_{\varepsilon}=\left\|e_{i_{1}}\right\|_{1, * *} \cdot\left\|e_{i_{d}}\right\|_{d, * *} \neq 0
$$

if and only if $\left(i_{1}, \ldots, i_{d}\right) \prod_{j=1}^{d} \in\left\{1, \ldots, n_{i}^{\prime}\right\}$. Therefore,

$$
\left\{\varphi_{i_{1}}^{(1)} \otimes \cdots \otimes \varphi_{i_{d}}^{(d)}\right\}_{\left(i_{1}, \ldots, i_{d}\right) \in \prod_{j=1}^{d}\left\{1, \ldots, n_{j}^{\prime}\right\}}
$$

forms a basis of the vector space of bounded linear forms on $\left(V_{1} \otimes_{k} \cdots \otimes_{k} V_{d},\|\cdot\|_{\varepsilon}\right)$, which shows that $\left(V_{1} \otimes_{k} \cdots \otimes_{k} V_{d}\right)^{*} \cong V_{1}^{*} \otimes_{k} \cdots \otimes_{k} V_{d}^{*}$.

Let $\|\cdot\|^{\prime}$ be the $\varepsilon$-tensor product of $\|\cdot\|_{1, *}, \ldots,\|\cdot\|_{d, *}$. By definition, for any $T$ in $V_{1}^{*} \otimes_{k} \cdots \otimes_{k} V_{d}^{*}$, one has

$$
\begin{aligned}
\|T\|^{\prime}= & \sup _{\substack{\left(s_{1}, \ldots, s_{d}\right) \in V_{1} \times \cdots \times V_{d} \\
\min \left\{\left\|s_{1}\right\|_{1}, \ldots,\left\|s_{d}\right\|_{d}\right\}>0}} \frac{\left|T\left(s_{1}, \ldots, s_{d}\right)\right|}{\left\|s_{1}\right\|_{1, * *} \cdots\left\|s_{d}\right\|_{d, * *}} \\
= & \sup _{\substack{\left(s_{1}, \ldots, s_{d}\right) \in V_{1} \times \cdots \times V_{d} \\
\min \left\{\left\|s_{1}\right\|_{1}, \ldots,\left\|s_{d}\right\|_{d}\right\}>0}} \frac{\left|T\left(s_{1}, \ldots, s_{d}\right)\right|}{\left\|s_{1} \otimes \cdots \otimes s_{d}\right\|_{\varepsilon}} \leqslant\|T\|_{\varepsilon, *},
\end{aligned}
$$

where the second equality comes from (1.16). Conversely, if $T$ is of the form $\psi_{1} \otimes$ $\cdots \otimes \psi_{d}$, where $\left(\psi_{1}, \ldots, \psi_{d}\right) \in V_{1}^{*} \times \cdots \times V_{d}^{*}$, then

$$
\|T\|_{\varepsilon, *}=\sup _{\substack{f \in V_{1} \otimes_{k} \cdots \otimes_{k} V_{d} \\\|f\|_{\varepsilon} \neq 0}} \frac{\left|f\left(\psi_{1}, \ldots, \psi_{d}\right)\right|}{\|f\|_{\varepsilon}} \leqslant\left\|\psi_{1}\right\|_{1, *} \cdots\left\|\psi_{d}\right\|_{d, *} .
$$

By Proposition 1.2.19, we obtain $\|\cdot\|_{\varepsilon, *} \leqslant\|\cdot\|^{\prime}$.

1.2.4. Orthogonality and lattice norms. - In this subsection, we assume that the absolute value $|\cdot|$ is non-Archimedean and we denote by $\mathfrak{o}_{k}$ the valuation ring of $(k,|\cdot|)$. Let $V$ be a finite-dimensional vector space over $k$ and let $r$ be the rank of $V$.

Proposition 1.2.21. - Let $\mathcal{V}$ be a lattice of $V$ which is an $\mathfrak{o}_{k}$-module of finite type (and hence a free $\mathfrak{o}_{k}$-module of rank $r$ ). Then any basis of $\mathcal{V}$ over $\mathfrak{o}_{k}$ is an orthonormal basis of $\left(V,\|\cdot\|_{\mathcal{V}}\right)$.

Proof. - Let $\left\{e_{i}\right\}_{i=1}^{r}$ be a basis of $\mathcal{V}$ over $\mathfrak{o}_{k}$. Note that an element $a_{1} e_{1}+\cdots+a_{r} e_{r}$ of $V$ (with $\left(a_{1}, \ldots, a_{r}\right) \in k^{r}$ ) belongs to $\mathcal{V}$ if and only if all $a_{i}$ are in $\mathfrak{o}_{k}$. Let $x=$ $\lambda_{1} e_{1}+\cdots+\lambda_{r} e_{r}$ be an element of $V$. If $a$ is an element in $k^{\times}$such that $a^{-1} x$ belongs 
to $\mathcal{V}$, then one has $a^{-1} \lambda_{i} \in \mathfrak{o}_{k}$ and hence $\left|\lambda_{i}\right| \leqslant|a|$ for any $i \in\{1, \ldots, r\}$. Therefore $\max \left\{\left|\lambda_{1}\right|, \ldots,\left|\lambda_{r}\right|\right\} \leqslant\|x\|_{\mathcal{V}}$. Conversely, if $j \in\{1, \ldots, r\}$ is such that

$$
\left|\lambda_{j}\right|=\max \left\{\left|\lambda_{1}\right|, \ldots,\left|\lambda_{r}\right|\right\}>0,
$$

then one has $\lambda_{i} \lambda_{j}^{-1} \in \mathfrak{o}_{k}$ for any $i \in\{1, \ldots, r\}$. Hence $\lambda_{j}^{-1} x \in \mathcal{V}$ and

$$
\|x\|_{\mathcal{V}} \leqslant\left|\lambda_{j}\right|=\max \left\{\left|\lambda_{1}\right|, \ldots,\left|\lambda_{r}\right|\right\}
$$

Proposition 1.2.22. - Assume that the absolute value $|\cdot|$ is non-trivial. Let $\lambda \in$ ]0,1[ be a real number such that

$$
\lambda<\sup \left\{|a|: a \in k^{\times},|a|<1\right\} .
$$

Then, for any ultrametric norm $\|\cdot\|$ on $V$ there exists a lattice of finite type $\mathcal{V}$ of $V$, such that $\|\cdot\| \leqslant\|\cdot\|_{\mathcal{V}} \leqslant \lambda^{-1}\|\cdot\|$.

Proof. - Let $\alpha \in] 0,1\left[\operatorname{such}\right.$ that $\lambda / \alpha<\sup \left\{|a|: a \in k^{\times},|a|<1\right\}$. Let $\left\{e_{i}\right\}_{i=1}^{r}$ be an $\alpha$-orthogonal basis of $V$ (the existence of which has been proved in Proposition 1.2.7). By Proposition 1.1.4, by dilating the vectors $e_{i}, i \in\{1, \ldots, r\}$, we may assume that $\lambda / \alpha \leqslant\left\|e_{i}\right\|<1$ for any $i$. Let $\mathcal{V}$ be the free $\mathfrak{o}_{k}$-module generated by $\left\{e_{i}\right\}_{i=1}^{r}$. It is a lattice of $V$. Moreover, by Proposition 1.2.21, $\left\{e_{i}\right\}_{i=1}^{r}$ is an orthonormal basis of $\left(V,\|\cdot\|_{\mathcal{V}}\right)$. In particular, for any vector $x=a_{1} e_{1}+\cdots+a_{r} e_{r}$ in $V$, one has

$$
\|x\|_{\mathcal{V}}=\max _{i \in\{1, \ldots, r\}}\left|a_{i}\right| \geqslant \max _{i \in\{1, \ldots, r\}}\left|a_{i}\right| \cdot\left\|e_{i}\right\| \geqslant\|x\| .
$$

Moreover, by the $\alpha$-orthogonality of $\left\{e_{i}\right\}_{i=1}^{r}$ one has

$$
\|x\| \geqslant \alpha \max _{i \in\{1, \ldots, r\}}\left|a_{i}\right| \cdot\left\|e_{i}\right\| \geqslant \lambda\|x\|_{\mathcal{V}} .
$$

The proposition is thus proved.

1.2.5. Orthogonality and Hadamard property. - We have seen in Proposition 1.2.7 that an Hadamard basis of a finite-dimensional normed vector space is necessarily orthogonal. The converse of this assertion is also true when the absolute value $|\cdot|$ is non-Archimedean.

Proposition 1.2.23. - We assume that the absolute value $|\cdot|$ is non-Archimedean. Let $(V,\|\cdot\|)$ be a finite-dimensional seminormed vector space over $k$, and let $r$ be the rank of $V$ over $k$. If $\alpha$ is an element in $] 0,1]$ and if $\left\{x_{i}\right\}_{i=1}^{r}$ is an $\alpha$-orthogonal basis of $V$, then one has

$$
\left\|x_{1} \wedge \cdots \wedge x_{r}\right\| \geqslant \alpha^{r}\left\|x_{1}\right\| \cdots\left\|x_{r}\right\| .
$$

In particular, if $\|\cdot\|$ is a norm, any orthogonal basis of $V$ is an Hadamard basis. 
Proof. - If $N_{\|\cdot\|}$ is non-zero, then the interserction of $\left\{x_{i}\right\}_{i=1}^{r}$ with $N_{\|\cdot\|}$ is not empty (see Proposition 1.2.5) and hence the inequality (1.30) holds. In the following, we assume that $\|\cdot\|$ is a norm. Note that the case where $V=\{0\}$ is trivial. Hence we may assume that $r>0$. Let $\left\{x_{i}\right\}_{i=1}^{r}$ be an $\alpha$-orthogonal basis of $V$. Let $\left\{y_{i}\right\}_{i=1}^{r}$ be an arbitrary basis of $V$ and $A=\left(a_{i j}\right)_{i \in\{1, \ldots, r\}, j \in\{1, \ldots, r\}} \in k^{r \times r}$ be the transition matrix from $\left\{x_{i}\right\}_{i=1}^{r}$ to $\left\{y_{i}\right\}_{i=1}^{r}$, namely $y_{i}=\sum_{j=1}^{r} a_{i j} x_{j}$ for any $i \in\{1, \ldots, r\}$. By the $\alpha$-orthogonality of the basis $\left\{x_{i}\right\}_{i=1}^{r}$ one has

$$
\left|a_{i j}\right| \leqslant \alpha^{-1} \frac{\left\|y_{i}\right\|}{\left\|x_{j}\right\|}
$$

Thus, by the assumption that the absolute value $|\cdot|$ is non-Archimedean, one has

$$
\left\|y_{1} \wedge \cdots \wedge y_{r}\right\|=|\operatorname{det}(A)| \cdot\left\|x_{1} \wedge \cdots \wedge x_{r}\right\| \leqslant \alpha^{-r} \frac{\left\|y_{1}\right\| \cdots\left\|y_{r}\right\|}{\left\|x_{1}\right\| \cdots\left\|x_{r}\right\|} \cdot\left\|x_{1} \wedge \cdots \wedge x_{r}\right\|
$$

and hence

$$
\frac{\left\|x_{1} \wedge \cdots \wedge x_{r}\right\|}{\left\|x_{1}\right\| \cdots\left\|x_{r}\right\|} \geqslant \alpha^{r} \frac{\left\|y_{1} \wedge \cdots \wedge y_{r}\right\|}{\left\|y_{1}\right\| \cdots\left\|y_{r}\right\|} .
$$

Since the basis $\left\{y_{i}\right\}_{i=1}^{r}$ is arbitrary, by Proposition 1.1.66 (see also Remark 1.1.67) we obtain that

$$
\frac{\left\|x_{1} \wedge \cdots \wedge x_{r}\right\|}{\left\|x_{1}\right\| \cdots\left\|x_{r}\right\|} \geqslant \alpha^{r}
$$

The proposition is thus proved.

Remark 1.2.24. - The Archimedean analogue of Proposition 1.2.23 is not true in general. One can consider for example the case where $V=\mathbb{R}^{2}$ equipped with the norm $\|\cdot\|$ such that $\|(a, b)\|=\max \{|a|,|b|\}$, where $|\cdot|$ is the usual absolute value on $\mathbb{R}$. Let $e_{1}=(1,0)$ and $e_{2}=(0,1)$. The basis $\left\{e_{1}, e_{2}\right\}$ is orthonormal. However,

$$
\left\|e_{1} \wedge e_{2}\right\|=\left\|\frac{1}{\sqrt{2}}\left(e_{1}+e_{2}\right) \wedge \frac{1}{\sqrt{2}}\left(e_{1}-e_{2}\right)\right\|=\frac{1}{2} .
$$

Therefore $\left\{e_{1}, e_{2}\right\}$ is not an Hadamard basis.

The following proposition shows that the Archimedean analogue of Proposition 1.2 .23 is true provided that the norm is induced by an inner product.

Proposition 1.2.25. - Let $(V,\|\cdot\|)$ be a finite-dimensional normed vector space over $k$. Assume that the absolute value $|\cdot|$ is Archimedean and that the norm $\|\cdot\|$ is induced by an inner product. Then any orthogonal basis of $V$ is an Hadamard basis.

Proof. - The field $k$ is locally compact, therefore $V$ admits an Hadamard basis $\boldsymbol{e}=\left\{e_{i}\right\}_{i=1}^{r}$, which is necessarily an orthogonal basis (see Proposition 1.2.7). Without loss of generality, we may assume that $\boldsymbol{e}$ is an orthonormal basis. Let $\boldsymbol{e}^{\prime}=\left\{e_{i}^{\prime}\right\}_{i=1}^{r}$ be another orthonormal basis. There exists a unitary matrix $A$ such that $\boldsymbol{e}^{\prime}=A \boldsymbol{e}$. One has $|\operatorname{det}(A)|=1$ and hence

$$
\left\|e_{1}^{\prime} \wedge \cdots \wedge e_{r}^{\prime}\right\|_{\text {det }}=\left\|e_{1} \wedge \cdots \wedge e_{r}\right\|_{\text {det }}=1=\left\|e_{1}^{\prime}\right\| \cdots\left\|e_{r}^{\prime}\right\| .
$$


Therefore the basis $\left\{e_{i}^{\prime}\right\}_{i=1}^{r}$ is also an Hadamard basis. Thus we have proved that any orthonormal basis is an Hadamard basis. If $\left\{x_{i}\right\}_{i=1}^{r}$ is an orthogonal basis, and if $e_{i}=\left\|x_{i}\right\|^{-1} x_{i}$ for any $i \in\{1, \ldots, r\}$, then $\left\{e_{i}\right\}_{i=1}^{r}$ is an orthonormal basis of $V$, which is an Hadamard basis. We then deduce that $\left\{x_{i}\right\}_{i=1}^{r}$ is also an Hadamard basis.

Proposition 1.2.26. - We assume that the absolute value $|\cdot|$ is trivial. Let $(V,\|\cdot\|)$ be an $r$-dimensional $\left(r \in \mathbb{N}_{>0}\right)$, ultrametrically normed vector space over $k$, which corresponds to an increasing sequence

$$
0=V_{0} \subsetneq V_{1} \subsetneq \ldots \subsetneq V_{n}=V
$$

of vector subspaces of $V$ and a decreasing sequence $\mu_{1}>\ldots>\mu_{n}$ of real numbers as described in Remark 1.1.40.

(1) A basis $\left\{x_{j}\right\}_{j=1}^{r}$ of $V$ is orthogonal if and only if $\operatorname{card}\left(\left\{x_{j}\right\}_{j=1}^{r} \cap V_{i}\right)=\operatorname{dim}_{k}\left(V_{i}\right)$ for any $i \in\{1, \ldots, n\}$.

(2) Let $\alpha$ be an element of $] 0,1]$ such that

$$
\forall i \in\{1, \ldots, n\}, \quad \alpha>\mathrm{e}^{-\left(\mu_{i}-\mu_{i+1}\right) / r},
$$

where $\mu_{n+1}=-\infty$ by convention. Then any $\alpha$-orthogonal basis of $(V,\|\cdot\|)$ is orthogonal.

Proof. - (1) Note that the restriction of $\|\cdot\|$ on each $V_{i} \backslash V_{i-1}$ is constant and takes $\mathrm{e}^{-\mu_{i}}$ as its value for $i \in\{1, \ldots, n\}$. Let

$$
\lambda_{1} \leqslant \ldots \leqslant \lambda_{r}
$$

be the increasing sequence of positive real numbers such that $\mathrm{e}^{-\mu_{i}}$ appears exactly $\operatorname{dim}_{k}\left(V_{i}\right)-\operatorname{dim}_{k}\left(V_{i-1}\right)$ times. Let $\left\{x_{j}\right\}_{j=1}^{r}$ be a basis of $V$ such that $\left\|x_{1}\right\| \leqslant \ldots \leqslant\left\|x_{r}\right\|$. For each $i \in\{1, \ldots, n\}$, the cardinal of $\left\{x_{j}\right\}_{j=1}^{r} \cap V_{i}$ does not exceed $\operatorname{dim}_{k}\left(V_{i}\right)$, so that $\left\|x_{j}\right\| \geqslant \lambda_{j}$ for any $j \in\{1, \ldots, r\}$ and hence

$$
\prod_{j=1}^{r}\left\|x_{j}\right\| \geqslant \prod_{j=1}^{r} \lambda_{j} .
$$

Moreover, if the equality $\operatorname{card}\left(\left\{x_{j}\right\}_{j=1}^{r} \cap V_{i}\right)=\operatorname{dim}_{k}\left(V_{i}\right)$ holds for any $i \in\{1, \ldots, n\}$, then the basis $\left\{x_{j}\right\}_{j=1}^{r}$ is an Hadamard basis, and hence is an orthogonal basis (by Proposition 1.2.7 (2) . If there exists an index $i \in\{1, \ldots, n\}$ such that $\operatorname{card}\left(\left\{x_{j}\right\}_{j=1}^{r} \cap\right.$ $\left.V_{i}\right)<\operatorname{dim}_{k}\left(V_{i}\right)$, then there exists an element

$$
x=\lambda_{1} x_{1}+\cdots+\lambda_{r} x_{r}
$$

of $V_{i}$ and a $j \in\{1, \ldots, r\}$ such that $\lambda_{j} \neq 0$ and $x_{j} \in V \backslash V_{i}$. As $\|x\| \leqslant \mu_{i}<\left\|x_{j}\right\|$, the basis $\left\{x_{j}\right\}_{j=1}^{r}$ is not orthogonal.

(2) Let $\left\{e_{j}\right\}_{j=1}^{r}$ be an $\alpha$-orthogonal basis of $(V,\|\cdot\|)$. Without loss of generality, we assume that $\left\|e_{1}\right\| \leqslant \ldots \leqslant\left\|e_{r}\right\|$. One has

$$
\left\{\left\|e_{1}\right\|, \ldots,\left\|e_{r}\right\|\right\} \subseteq\left\{\lambda_{1}, \ldots, \lambda_{r}\right\}=\left\{\mathrm{e}^{-\mu_{1}}, \ldots, \mathrm{e}^{-\mu_{n}}\right\} .
$$


Moreover, since

$$
\operatorname{card}\left(\left\{e_{j}\right\}_{j=1}^{r} \cap V_{i}\right) \leqslant \operatorname{dim}_{k}\left(V_{i}\right)
$$

for any $i \in\{1, \ldots, n\}$, one has $\left\|e_{j}\right\| \geqslant \lambda_{j}$ for any $j \in\{1, \ldots, r\}$. Therefore, if the strict inequality $\left\|e_{j}\right\|>\lambda_{j}$ holds for some $j \in\{1, \ldots, r\}$, then, by (1.31), one can find $m$ such that $m>j,\left\|e_{j}\right\| \geqslant \lambda_{m}$ and $\lambda_{m} \lambda_{j}^{-1}=\mathrm{e}^{\mu_{i}-\mu_{i+1}}$ for some $i$, so that, by our assumption,

$$
\left\|e_{j}\right\| \geqslant \lambda_{j}\left(\lambda_{m} \lambda_{j}^{-1}\right)=\lambda_{j} \mathrm{e}^{\mu_{i}-\mu_{i+1}}>\lambda_{j} \alpha^{-r} .
$$

Therefore,

$$
\prod_{\ell=1}^{r}\left\|e_{\ell}\right\|>\alpha^{-r} \prod_{\ell=1}^{r} \lambda_{\ell}=\alpha^{-r}\left\|e_{1} \wedge \cdots \wedge e_{r}\right\|_{\operatorname{det}} .
$$

This contradicts Proposition 1.2.23. Therefore one has $\left\|e_{j}\right\|=\lambda_{j}$ for any $j \in$ $\{1, \ldots, r\}$ and hence, for each $i \in\{1, \ldots, n\}$, the cardinal of $\left\{e_{j}\right\}_{j=1}^{r} \cap V_{i}$ is equal to $\operatorname{dim}_{k}\left(V_{i}\right)$, which implies that $\left\{e_{j}\right\}_{j=1}^{r}$ is an orthogonal basis.

1.2.6. Ultrametric Gram-Schimdt process. - In this subsection, we consider a refinement of Proposition 1.2.7. First we recall the spherically completeness of a metric space.

Definition 1.2.27. - We say that a metric space $(X, d)$ is spherically complete if, for any decreasing sequence

$$
B_{1} \supseteq B_{2} \supseteq \cdots \supseteq B_{n} \supseteq B_{n+1} \supseteq \cdots
$$

of non-empty closed balls in $X$, one has $\bigcap_{n=1}^{\infty} B_{n} \neq \varnothing$. A normed vector space $(V,\|\cdot\|)$ over $k$ is said to be spherically complete if $(V,\|\cdot\|)$ is spherically complete as a metric space. If $(k,|\cdot|)$, viewed as a normed vector space over $k$, is spherically complete, we say that the valued field $(k,|\cdot|)$ is spherically complete.

Remark 1.2.28. - If $(k,|\cdot|)$ is a discrete valuation field, then $(k,|\cdot|)$ is spherically complete by [127, Proposition 20.2]. In particular, any locally compact nonArchimedean valued field is spherically complete.

Lemma 1.2.29. - Let $(V,\|\cdot\|)$ be an ultrametrically normed vector space of finite rank over $k$. Then we have the following:

(1) Let $W$ be a vector subspace of $V$ over $k$. If $W$ equipped with the restriction $\|\cdot\|_{W}$ of $\|\cdot\|$ to $W$ is spherically complete, then, for $x \in V$, there is $w \in W$ such that $\operatorname{dist}(x, W)=\|x-w\|$.

(2) If $(V,\|\cdot\|)$ has an orthogonal basis $\left\{e_{i}\right\}_{i=1}^{r}$ and $(k,|\cdot|)$ is spherically complete, then $(V,\|\cdot\|)$ is also spherically complete.

Proof. - For $a \in V$ and $\delta \in \mathbb{R}_{\geqslant 0}$, we set

$$
B(a ; \delta):=\{x \in V:\|x-a\| \leqslant \delta\} .
$$


As $\|\cdot\|$ is ultrametric, we can easily see that

$$
B(a ; \delta)=B\left(a^{\prime} ; \delta\right)
$$

for all $\delta \in \mathbb{R}_{\geqslant 0}$ and $a, a^{\prime} \in V$ with $\left\|a-a^{\prime}\right\| \leqslant \delta$.

(1) We can choose a decreasing sequence $\left\{\delta_{n}\right\}_{n=1}^{\infty}$ of positive numbers and a sequence $\left\{w_{n}\right\}_{n=1}^{\infty}$ in $W$ such that $\left\|x-w_{n}\right\| \leqslant \delta_{n}$ and $\lim _{n \rightarrow \infty} \delta_{n}=\operatorname{dist}(x, W)$. As $B\left(x ; \delta_{n}\right) \cap W=B\left(w_{n} ; \delta_{n}\right) \cap W$ by (1.32), $\left\{B\left(x ; \delta_{n}\right) \cap W\right\}_{n=1}^{\infty}$ yields a decreasing sequence of non-empty closed balls in $W$. Thus, by our assumption, there is $w \in \bigcap_{n=1}^{\infty} B\left(x ; \delta_{n}\right) \cap W$, so that $\|x-w\| \leqslant \delta_{n}$ for all $n$, that is, $\|x-w\| \leqslant \operatorname{dist}(x, W)$, as required.

(2) Note that $\left\|a_{1} e_{1}+\cdots+a_{r} e_{r}\right\|=\max \left\{\left|a_{1}\right| \cdot\left\|e_{1}\right\|, \ldots,\left|a_{r}\right| \cdot\left\|e_{r}\right\|\right\}$, so that

$$
B(a ; \delta)=B_{k}\left(a_{1} ; \delta /\left\|e_{1}\right\|\right) e_{1}+\cdots+B_{k}\left(a_{r} ; \delta /\left\|e_{r}\right\|\right) e_{r}
$$

for $a=a_{1} e_{1}+\cdots+a_{r} e_{r} \in V$ and $\delta \in \mathbb{R}_{\geqslant 0}$, where

$$
B_{k}\left(\lambda ; \delta^{\prime}\right)=\left\{t \in k:|t-\lambda| \leqslant \delta^{\prime}\right\}
$$

for $\lambda \in k$ and $\delta^{\prime} \in \mathbb{R}_{\geqslant 0}$. Therefore the assertion follows.

In the case of an ultrametrically normed finite-dimensional vector space, Proposition 1.2.7 has the following refined form. This could be considered as an ultrametric analogue of Gram-Schmidt orthogonalisation process.

Proposition 1.2.30. - Let $(V,\|\cdot\|)$ be an ultrametrically seminormed $k$-vector space of $r a n k r \geqslant 1$. Let

$$
0=V_{0} \subsetneq V_{1} \subsetneq V_{2} \subsetneq \ldots \subsetneq V_{r}=V
$$

be a complete flag of subspaces of $V$. Fix a real number $\alpha$ such that

$$
\alpha \in \begin{cases}] 0,1], & \text { if }(k,|\cdot|) \text { is spherically complete, } \\ ] 0,1[, & \text { otherwise. }\end{cases}
$$

Then there exists an $\alpha$-orthogonal basis e of $V$ such that, for any $i \in\{1, \ldots, r\}$, $\operatorname{card}\left(V_{i} \cap \boldsymbol{e}\right)=i$.

Proof. - If a basis $\boldsymbol{e}$ of $V$ is such that, for any $i \in\{1, \ldots, r\}, \operatorname{card}\left(V_{i} \cap \boldsymbol{e}\right)=i$, we say that the basis $\boldsymbol{e}$ is compatible with the flag (1.33).

We begin with the proof of the particular case where $\|\cdot\|$ is a norm by induction on $r$, the dimension of $V$ over $k$. The case where $r=1$ is trivial. Assume that the proposition holds for all vector spaces of dimension $<r$, where $r \geqslant 2$. Applying the induction hypothesis to $V_{r-1}$ and the flag $0=V_{0} \subsetneq V_{1} \subsetneq \ldots \subsetneq V_{r-1}$ we get a basis $\left\{e_{1}, \ldots, e_{r-1}\right\}$ of $V_{r-1}$ compatible with the flag such that, for any $\left(\lambda_{1}, \ldots, \lambda_{r-1}\right) \in$ $k^{r-1}$

$$
\left\|\lambda_{1} e_{1}+\cdots+\lambda_{r-1} e_{r-1}\right\| \geqslant \alpha^{1 / 2} \max _{i \in\{1, \ldots, r-1\}}\left|\lambda_{i}\right| \cdot\left\|e_{i}\right\| .
$$


Let $x$ be an element of $V \backslash V_{r-1}$. The distance between $x$ and $V_{r-1}$ is strictly positive since $V_{r-1}$ is closed in $V$ (see Proposition 1.1.11). Hence there exists $y \in V_{r-1}$ such that

$$
\|x-y\| \leqslant \alpha^{-1 / 2} \operatorname{dist}\left(x, V_{r-1}\right) .
$$

In the case where $\alpha=1$ and $(k,|\cdot|)$ is spherically complete, the existence of $y$ follows from Lemma 1.2.29. We choose $e_{r}=x-y$. The basis $\left\{e_{1}, \ldots, e_{r}\right\}$ is compatible with the flag $0=V_{0} \subsetneq V_{1} \subsetneq \ldots \subsetneq V_{r}=V$.

Let $\left(\lambda_{1}, \ldots, \lambda_{r}\right)$ be an element of $k^{r}$. We wish to find a lower bound for the norm of $z=\lambda_{1} e_{1}+\cdots+\lambda_{r} e_{r}$. By (1.35) we have that

$$
\|z\| \geqslant\left|\lambda_{r}\right| \cdot \operatorname{dist}\left(x, V_{r-1}\right) \geqslant \alpha^{1 / 2}\left|\lambda_{r}\right| \cdot\left\|e_{r}\right\| .
$$

This provides our lower bound when $\left\|\lambda_{r} e_{r}\right\| \geqslant\left\|\lambda_{1} e_{1}+\cdots+\lambda_{r-1} e_{r-1}\right\|$. If $\left\|\lambda_{r} e_{r}\right\|<$ $\left\|\lambda_{1} e_{1}+\cdots+\lambda_{r-1} e_{r-1}\right\|$ then we have

$$
\|z\|=\left\|\lambda_{1} e_{1}+\cdots+\lambda_{r-1} e_{r-1}\right\|
$$

because the norm is ultrametric (see Proposition 1.1.5). By the induction hypothesis (1.34) we have that $\|z\| \geqslant \alpha\left|\lambda_{i}\right| \cdot\left\|e_{i}\right\|$ for any $i \in\{1, \ldots, r-1\}$. This completes the proof of the proposition in the case where $\|\cdot\|$ is a norm.

We now consider the general seminorm case. Let $W$ be the quotient vector space $V / N_{\|\cdot\|}$. For each $i \in\{0, \ldots, r\}$, let $W_{i}$ be $\left(V_{i}+N_{\|\cdot\|}\right) / N_{\|\cdot\|}$. Applying the particular case of the proposition to $\left(W,\|\cdot\|^{\sim}\right)$, we obtain the existence of an $\alpha$-orthogonal basis $\widetilde{\boldsymbol{e}}$ of $W$ such that $\operatorname{card}\left(\widetilde{\boldsymbol{e}} \cap W_{i}\right)=\operatorname{rk}_{k}\left(W_{i}\right)$. We set

$$
I=\left\{i \in\{1, \ldots, r\}: W_{i-1} \subsetneq W_{i}\right\} \quad \text { and } \quad J=\left\{j \in\{1, \ldots, r\}: W_{j-1}=W_{j}\right\} .
$$

If $i \in I$, then there is a unique element $u_{i} \in \widetilde{\boldsymbol{e}} \cap\left(W_{i} \backslash W_{i-1}\right)$, so that we can choose $e_{i} \in V_{i}$ such that the class of $e_{i}$ in $V / N_{\|\cdot\|}$ is $u_{i}$. If $j \in J$, then we can pick up $e_{j} \in\left(N_{\|\cdot\|} \cap V_{j}\right) \backslash V_{j-1}$. Indeed, as $V_{j} \backslash V_{j-1} \neq \varnothing$ and $V_{j} \subseteq V_{j-1}+N_{\|\cdot\|}$, we can find $x \in V_{j} \backslash V_{j-1}, y \in V_{j-1}$ and $e_{j} \in N_{\|\cdot\|}$ with $x=y+e_{j}$, and hence $e_{j} \in\left(N_{\|\cdot\|} \cap V_{j}\right) \backslash V_{j-1}$. By construction, $\boldsymbol{e}:=\left\{e_{i}\right\}_{i=1}^{r}$ satisfies $\operatorname{card}\left(V_{i} \cap \boldsymbol{e}\right)=i$ for $i \in\{0, \ldots, r\}$. In particular, $e$ forms a basis of $V$.

Let us see that $\boldsymbol{e}$ is $\alpha$-orthogonal. For any $\left(\lambda_{1}, \ldots, \lambda_{r}\right) \in k^{r}$, if we let $x=\lambda_{1} e_{1}+$ $\cdots+\lambda_{r} e_{r}$ and $u=\sum_{i \in I} \lambda_{i} u_{i}$, then one has

$$
\|x\|=\|u\|^{\sim} \geqslant \alpha \max _{i \in I}\left|\lambda_{i}\right| \cdot\left\|u_{i}\right\|^{\sim}=\alpha \max _{i \in\{1, \ldots, r\}}\left|\lambda_{i}\right| \cdot\left\|e_{i}\right\|,
$$

where the inequality comes from the $\alpha$-orthogonality of $\widetilde{\boldsymbol{e}}$, as required.

Corollary 1.2.31. - Let $(V,\|\cdot\|)$ be an ultrametrically seminormed vector space of finite rank over $k$. If $(k,|\cdot|)$ is spherically complete, then $(V,\|\cdot\|)$ has an orthogonal basis. In particular, $(V,\|\cdot\|)$ is spherically complete. 
Remark 1.2.32. - Assume that the absolute value $|\cdot|$ is Archimedean. Let $V$ be a finite-dimensional vector space over $k$ and $\|\cdot\|$ be a seminorm on $V$ which is induced by an inner product. Given a complete flag

$$
0=V_{0} \subsetneq V_{1} \subsetneq V_{2} \subsetneq \ldots \subsetneq V_{r}=V
$$

of $V$, the Gram-Schmidt process permits to construct an orthogonal basis $e$ of $V$ such that $\operatorname{card}\left(\boldsymbol{e} \cap V_{i}\right)=i$ for any $i \in\{1, \ldots, r\}$, along the same line as in the proof of Proposition 1.2.30, The main point is that the field $k$ is locally compact, and hence the distance in (1.35) is actually attained by some point in $V_{r-1}$. For a general seminorm, even though an orthogonal basis always exists, it is not always possible to find an orthogonal basis which is compatible with a given flag.

Proposition 1.2.30 and the usual Gram-Schmidt process lead to the following projection result.

Corollary 1.2.33. - Let $V$ be a finite-dimensional vector space over $k$ equipped with a seminorm $\|\cdot\|$ which is either ultrametric or induced by a semidefinite inner product. Let $V_{0}$ be a vector subspace of $V$. For any $\left.\alpha \in\right] 0,1[$ there exists a $k$-linear projection $\pi: V \rightarrow V_{0}$ (namely $\pi$ is a k-linear map and its restriction to $V_{0}$ is the identity map) such that $\|\pi\| \leqslant \alpha^{-1}$. If $(k,|\cdot|)$ is non-Archimedean and spherically complete, or if $\|\cdot\|$ is induced by a semidefinite inner product, we can choose the $k$-linear projection $\pi$ such that $\|\pi\| \leqslant 1$.

Proof. - We first consider the ultrametric case. By Proposition 1.2.30 there exists an $\alpha$-orthogonal basis $\boldsymbol{e}=\left\{e_{i}\right\}_{i=1}^{n}$ of $V$ such that $\operatorname{card}\left(\boldsymbol{e} \cap V_{0}\right)=\operatorname{rk}_{k}\left(V_{0}\right)$. Without loss of generality, we may assume that $\boldsymbol{e} \cap V_{0}=\left\{e_{i}\right\}_{i=1}^{m}$, where $m=\operatorname{rk}_{k}\left(V_{0}\right)$. Let $\pi$ : $V \rightarrow V_{0}$ be the $k$-linear map sending $\lambda_{1} e_{1}+\cdots+\lambda_{n} e_{n} \in V$ to $\lambda_{1} e_{1}+\cdots+\lambda_{m} e_{m} \in V_{0}$. Since the basis $\boldsymbol{e}$ is $\alpha$-orthogonal, one has

$$
\left\|\lambda_{1} e_{1}+\cdots+\lambda_{n} e_{n}\right\| \geqslant \alpha \max _{i \in\{1, \ldots, n\}}\left\|\lambda_{i} e_{i}\right\| \geqslant \alpha\left\|\lambda_{1} e_{1}+\cdots+\lambda_{m} e_{m}\right\|,
$$

which implies that $\|\pi\| \leqslant \alpha^{-1}$.

If $(k,|\cdot|)$ is non-Archimedean and spherically complete, or if $\|\cdot\|$ is induced by an inner product, we use the existence of an orthogonal basis $\boldsymbol{e} \operatorname{such}$ that $\operatorname{card}\left(\boldsymbol{e} \cap V_{0}\right)=$ $\mathrm{rk}_{k}\left(V_{0}\right)$. By the same agrument as above, we obtain the existence of a linear projection $\pi: V \rightarrow V_{0}$ such that $\|\pi\| \leqslant 1$.

Corollary 1.2.34. - Let $V$ be a finite-dimensional vector space over $k$ and $\|\cdot\|_{1}$ and $\|\cdot\|_{2}$ be two seminorms on $V$. We assume that $\|\cdot\|_{1} \leqslant\|\cdot\|_{2}$ and that the seminorm $\|\cdot\|_{2}$ is either ultrametric or induced by a semidefinite inner product. If there exists a vector $x \in V$ such that $\|x\|_{1}<\|x\|_{2}$, then one has $\|\cdot\|_{1, \operatorname{det}}^{\prime}<\|\cdot\|_{2, \operatorname{det}}^{\sim}$ on $\operatorname{det}\left(V / N_{\|\cdot\|_{2}}\right) \backslash\{0\}$, where $\|\cdot\|_{1}^{\prime}$ denotes the quotient seminorm of $\|\cdot\|_{1}$ on $V / N_{\|\cdot\|_{2}}$. 
Proof. - The condition $\|\cdot\|_{1} \leqslant\|\cdot\|_{2}$ implies that $N_{\|\cdot\|_{2}} \subseteq N_{\|\cdot\|_{1}}$. In particular, for any $x \in V$, if we denote by $[x]$ the class of $x$ in $V / N_{\|\cdot\|_{2}}$, then one has

$$
\|[x]\|_{1}^{\prime}=\|x\|_{1}, \quad\|[x]\|_{2}^{\sim}=\|x\|_{2} .
$$

Therefore, by replacing $V$ by $V / N_{\|\cdot\|_{2}},\|\cdot\|_{1}$ by $\|\cdot\|_{1}^{\prime}$, and $\|\cdot\|_{2}$ by $\|\cdot\|_{2}^{\sim}$, we may assume without loss of generality that $\|\cdot\|_{2}$ is a norm. Moreover, the case where $\|\cdot\|_{1}$ is not a norm is trivial since the seminorm $\|\cdot\|_{1, \text { det }}$ vanishes. Hence it suffices to treat the case where both seminorms $\|\cdot\|_{1}$ and $\|\cdot\|_{2}$ are norms.

Let $\lambda=\|x\|_{2} /\|x\|_{1}>1$. We first consider the ultrametric case. By Proposition 1.2.30, for any $\alpha \in] 0,1\left[\right.$ there exists an $\alpha$-orthogonal basis $\left\{e_{i}\right\}_{i=1}^{r}$ of $\left(V,\|\cdot\|_{2}\right)$ such that $e_{1}=x$. Hence See Proposition 1.2 .23 for the first inequality

$$
\begin{aligned}
\left\|e_{1} \wedge \cdots \wedge e_{r}\right\|_{2, \operatorname{det}} \geqslant \alpha^{r}\left\|e_{1}\right\|_{2} \cdots\left\|e_{r}\right\|_{2} & \\
& \geqslant \lambda \alpha^{r}\left\|e_{1}\right\|_{1} \cdots\left\|e_{r}\right\|_{1} \geqslant \lambda \alpha^{r}\left\|e_{1} \wedge \cdots \wedge e_{r}\right\|_{1, \operatorname{det} .}
\end{aligned}
$$

Since $\alpha \in] 0,1\left[\right.$ is arbitrary, one has $\|\cdot\|_{2, \operatorname{det}} /\|\cdot\|_{1, \operatorname{det}} \geqslant \lambda>1$.

The Archimedean case is very similar. There exists an orthogonal basis $\left\{e_{i}\right\}_{i=1}^{r}$ of $(V,\|\cdot\|)$ such that $e_{1}=x$. We then proceed as above in replacing $\alpha$ by 1 .

Proposition 1.2.35. - Let $V$ be a finite-dimensional vector space over $k$, equipped with a seminorm $\|\cdot\|_{V}$, which is ultrametric or induced by a semidefinite inner product. Let $\left(W,\|\cdot\|_{W}\right)$ be a seminormed vector space over $k$. For any $k$-vector subspace $V_{0}$ of $V$, the $k$-linear map $\mathscr{L}(V, W) \rightarrow \mathscr{L}\left(V_{0}, W\right)$, sending $f \in \mathscr{L}(V, W)$ to its restriction to $V_{0}$, is surjective, and the operator seminorm on $\mathscr{L}\left(V_{0}, W\right)$ coincides with the quotient seminorm induced by the operator seminorm on $\mathscr{L}(V, W)$. In particular, the dual norm on $V_{0}^{*}$ identifies with the quotient of the dual norm on $V^{*}$ by the canonical quotient map $V^{*} \rightarrow V_{0}^{*}$.

Proof. - For any $f \in \mathscr{L}(V, W)$, the operator seminorm of $\left.f\right|_{V_{0}}$ does not exceed that of $f$. In the following, we show that, for any linear map $g \in \mathscr{L}\left(V_{0}, W\right)$ and any $\alpha \in$ ]0, $1[$, there exists a $k$-linear map $f: V \rightarrow W$ extending $g$ such that $\alpha\|f\| \leqslant\|g\|$. By Corollary 1.2.33, there exists a $k$-linear projection $\pi: V \rightarrow V_{0}$ such that $\|\pi\| \leqslant \alpha^{-1}$. Let $f=g \circ \pi$. Then $\|f\| \leqslant \alpha^{-1}\|g\|$. The proposition is thus proved.

Proposition 1.2.36. - Let $V$ and $W$ be finite-dimensional seminormed vector spaces over $k, V_{0}$ be a k-vector subspace of $V$, and $Q$ be the quotient vector space $V / V_{0}$. We assume that, either the absolute value $|\cdot|$ is non-Archimedean, or the seminorm on $V$ is induced by a semidefinite inner product. Then the canonique isomorphism $\left(V \otimes_{k} W\right) /\left(V_{0} \otimes_{k} W\right) \rightarrow Q \otimes_{k} W$ is an isometry, where we consider the $\varepsilon$-tensor product seminorms on $V \otimes_{k} W$ and $Q \otimes_{k} W$, and the quotient seminorm on $\left(V \otimes_{k} W\right) /\left(V_{0} \otimes_{k} W\right)$.

Proof. - We have seen in Remark 1.1.59 that, for any $f \in V \otimes_{k} W$ viewed as a $k$ bilinear form on $V^{*} \times W^{*}$, its restriction to $Q^{*} \times W^{*}$ has an $\varepsilon$-tensor product norm not 
greater than that of $f$. In the following, we consider an element $g \in Q \otimes_{k} W$, viewed as a $k$-bilinear form on $Q^{*} \otimes_{k} W^{*}$. We will show that, for any $\left.\alpha \in\right] 0,1[$, there exists a $k$-bilinear form $f$ on $V^{*} \times W^{*}$ extending $g$ such that $\|f\|_{\varepsilon} \leqslant \alpha^{-1}\|g\|_{\varepsilon}$. By Proposition 1.1.20, the dual norm on $Q^{*}$ identifies with the restriction of the dual norm on $V^{*}$. By Corollary 1.2.33, there exists a $k$-linear projection $\pi: V^{*} \rightarrow Q^{*}$ such that $\|\pi\| \leqslant \alpha^{-1}$ (in the non-Archimedean case, we use the fact that any dual norm is ultrametric). We let $f$ be the $k$-bilinear form on $V^{*} \times W^{*}$ such that $f(\xi, \eta)=g(\pi(\xi), \eta)$. Then for $(\xi, \eta) \in\left(V^{*} \backslash\{0\}\right) \times\left(W^{*} \backslash\{0\}\right)$ one has

$$
\frac{|f(\xi, \eta)|}{\|\xi\|_{*}\|\eta\|_{*}}=\frac{|g(\pi(\xi), \eta)|}{\|\xi\|_{*}\|\eta\|_{*}} \leqslant \alpha^{-1} \frac{|g(\pi(\xi), \eta)|}{\|\pi(\xi)\|_{*}\|\eta\|_{*}} \leqslant \alpha^{-1}\|g\|_{\varepsilon} .
$$

The proposition is thus proved.

Corollary 1.2.3\%. - We assume that the absolute value $|\cdot|$ is non-Archimedean. Let $(V,\|\cdot\|)$ be a finite-dimensional seminormed vector space over $k$ and $r$ be the rank of $V$ over $k$. Let $i$ be a positive integer. Then the canonical k-linear isomorphism $\operatorname{det}\left(\Lambda^{i} V\right) \rightarrow \operatorname{det}(V)^{\otimes\left(\begin{array}{c}r-1 \\ i-1\end{array}\right)}$ is an isometry, where we consider the $i^{\text {th }} \varepsilon$-exterior power seminorm on $\Lambda^{i} V$.

Proof. - Consider the following commutative diagram

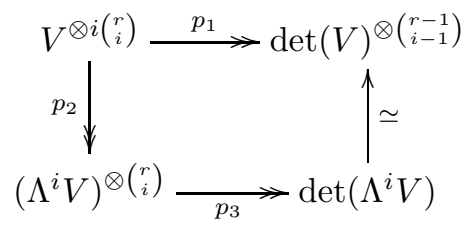

By Proposition 1.2.36, if we equip $V^{\otimes i\left(\begin{array}{l}r \\ i\end{array}\right)}$ with the $\varepsilon$-tensor product seminorm, then its quotient seminorm on $\left(\Lambda^{i} V\right)^{\otimes}\left(\begin{array}{c}r \\ i\end{array}\right)$ identifies with the $\varepsilon$-tensor product of the $\varepsilon$ exterior power seminorm. Moreover, by Proposition 1.2.15, the quotient seminorm on $\operatorname{det}\left(\Lambda^{i} V\right)$ (induced by $p_{3}$ ) of the tensor product of the $\varepsilon$-exterior power seminorm identifies with the determinant seminorm of the latter. Still by the same proposition, the quotient seminorm on $\operatorname{det}(V)^{\otimes\left(\begin{array}{c}r-1 \\ i-1\end{array}\right)}$ induced by $p_{1}$ identifies with the tensor power of the determinant seminorm. Therefore the natural isomorphism $\operatorname{det}\left(\Lambda^{i} V\right) \rightarrow$ $\operatorname{det}(V)^{\otimes\left(\begin{array}{c}r-1 \\ i-1\end{array}\right)}$ is actually an isometry by using (1) in Proposition 1.1.14.

Corollary 1.2.38. - We assume that the absolute value $|\cdot|$ is non-Archimedean. Let $(V,\|\cdot\|)$ be a finite-dimensional normed vector space over $k$ and $i$ be a positive integer. Then the $i^{\text {th }} \varepsilon$-exterior power norm on $\Lambda^{i} V$ is the double dual norm of the $i^{\text {th }} \pi$-exterior power norm.

Proof. - By definition, the $i^{\text {th }} \varepsilon$-exterior power norm $\|\cdot\|_{\Lambda_{\varepsilon}^{i}}$ on $\Lambda^{i} V$ is ultrametric and is bounded from above by the $i^{\text {th }} \pi$-exterior power norm. By Corollary 1.2.12, 
$\|\cdot\|_{\Lambda_{\pi}^{i}, * *}$ is the largest ultrametric norm bounded from above by the $i^{\text {th }} \pi$-exterior power norm $\|\cdot\|_{\Lambda_{\pi}^{i}}$. In particular, one has $\|\cdot\|_{\Lambda_{\pi}^{i}, * *} \geqslant\|\cdot\|_{\Lambda_{\varepsilon}^{i}}$ and hence

$$
\|\cdot\|_{\Lambda_{\varepsilon}^{i}, \operatorname{det}} \leqslant\|\cdot\|_{\Lambda_{\pi}^{i}, * *, \operatorname{det}} \leqslant\|\cdot\|_{\Lambda_{\pi}^{i}, \operatorname{det}} .
$$

By Corollary 1.2.37, one has $\|\cdot\|_{\Lambda_{\varepsilon}^{i}, \text { det }}=\|\cdot\|_{\Lambda_{\pi}^{i} \text {,det }}$ and hence the above inequalities are actually equalities. By Corollary 1.2.34, we obtain $\|\cdot\|_{\Lambda_{\varepsilon}^{i}}=\|\cdot\|_{\Lambda_{\pi}^{i}, * *}$.

Proposition 1.2.39. - Assume that $|\cdot|$ is non-Archimedean. Let $V$ and $W$ be finite-dimensional seminormed vector spaces over $k$ and $n$ and $m$ be respectively the ranks of $V$ and $W$ over $k$. We equip $V \otimes_{k} W$ with the $\varepsilon$-tensor product seminorm $\|\cdot\|_{\varepsilon}$. Then the natural $k$-linear isomorphism $\operatorname{det}\left(V \otimes_{k} W\right) \cong \operatorname{det}(V)^{\otimes m} \otimes_{k} \operatorname{det}(W)^{\otimes n}$ is an isometry, where we consider the determinant seminorm of $\|\cdot\|_{\varepsilon}$ on $\operatorname{det}\left(V \otimes_{k} W\right)$ and the tensor product of determinant seminorms on $\operatorname{det}(V)^{\otimes m} \otimes_{k} \operatorname{det}(W)^{\otimes n}$.

Proof. — Let $\|\cdot\|^{\prime}$ be the seminorm on $\operatorname{det}(V)^{\otimes m} \otimes \operatorname{det}(W)^{\otimes n}$ induced by tensor product of determinant seminorms. By Propositions 1.2.36 and 1.2.15, the seminorm $\|\cdot\|^{\prime}$ identifies with the quotient of the $\varepsilon$-tensor power seminorm on $\left(V \otimes_{k} W\right)^{\otimes n m}$ of $\|\cdot\|_{\varepsilon}$. Therefore, by Proposition 1.2 .15 the seminorm $\|\cdot\|^{\prime}$ identifies with $\|\cdot\|_{\varepsilon, \text { det }}$.

Proposition 1.2.40. - Let $V$ be a finite-dimensional vector space over $k$ and $\|\cdot\|$ be a seminorm on $V$. We assume that the seminorm $\|\cdot\|$ is either ultrametric or induced by a semidefinite inner product. For any vector subspace $W$ of $V$, the canonical isomorphism

$$
\operatorname{det}(W) \otimes_{k} \operatorname{det}(V / W) \longrightarrow \operatorname{det}(V)
$$

is an isometry, where we consider the determinant seminorm of the induced seminorm on $\operatorname{det}(W)$ and that of the quotient seminorm on $\operatorname{det}(V / W)$, and the tensor product seminorm on the tensor product space $\operatorname{det}(W) \otimes_{k} \operatorname{det}(V / W)$ (see Remark 1.1.56).

Proof. - By Proposition 1.1.16, if the seminorm $\|\cdot\|$ is not a norm, then either its restriction to $W$ is not a norm, or its quotient seminorm on $V / W$ is not a norm. In both cases, the seminorms on $\operatorname{det}(W) \otimes_{k} \operatorname{det}(V / W)$ and on $\operatorname{det}(V)$ vanish. Therefore we may assume without loss of generality that $\|\cdot\|$ is a norm.

Let $f: \operatorname{det}(W) \otimes_{k} \operatorname{det}(V / W) \rightarrow \operatorname{det}(V)$ be the canonical isomorphism. We have seen in Corollary 1.1.68 that the operator norm of $f$ is $\leqslant 1$. Since $f$ is an isomorphism between vector spaces of dimension 1 over $k$, to prove that $f$ is an isometry, it suffices to verify that $\|f\| \geqslant 1$.

We first treat the case where the norm $\|\cdot\|$ is ultrametric. By Proposition 1.2.30, for any $\alpha \in] 0,1\left[\right.$, there exists an $\alpha$-orthogonal basis $\boldsymbol{e}=\left\{e_{i}\right\}_{i=1}^{r}$ of $V$ such that $\operatorname{card}(\boldsymbol{e} \cap W)=\operatorname{rk}(W)$. Without loss of generality, we assume that $\left\{e_{1}, \ldots, e_{n}\right\}$ forms a basis of $W$, and $e_{n+1}, \ldots, e_{r}$ are vectors in $V \backslash W$. For any $i \in\{n+1, \ldots, r\}$, let $\bar{e}_{i}$ 
be the image of $e_{i}$ in $V / W$. By Proposition 1.2.23, one has

$$
\begin{aligned}
\left\|e_{1} \wedge \cdots \wedge e_{r}\right\|_{\text {det }} & \geqslant \alpha^{r} \cdot\left\|e_{1}\right\| \cdots\left\|e_{r}\right\| \geqslant \alpha^{r}\left\|e_{1}\right\| \cdots\left\|e_{n}\right\| \cdot\left\|\bar{e}_{n+1}\right\| \cdots\left\|\bar{e}_{r}\right\| \\
& \geqslant \alpha^{r}\left\|e_{1} \wedge \cdots \wedge e_{n}\right\|_{\text {det }} \cdot\left\|\bar{e}_{n+1} \wedge \cdots \wedge \bar{e}_{r}\right\|_{\text {det }},
\end{aligned}
$$

where the last equality comes from Corollary 1.1.68. Therefore the operator norm of $f$ is bounded from below by $\alpha^{r}$. Since $\left.\alpha \in\right] 0,1[$ is arbitrary, one has $\|f\| \geqslant 1$.

For the Archimedean case where the norm $\|\cdot\|$ is induced by an inner product, by the classic Gram-Schmidt process we can construct an orthonormal basis $e$ of $V$ such that $\operatorname{card}(\boldsymbol{e} \cap W)=\operatorname{rk}(W)$. By Proposition 1.2.23, $\boldsymbol{e}$ is an Hadamard basis. We then proceed as above in replacing $\alpha$ by 1 to conclude.

In Proposition 1.2.40, the assumption on the seminorm is crucial. In order to study the behaviour of the determinant seminorms of an exact sequence of general seminormed vector spaces, we introduce the following invariant.

Definition 1.2.41. — Let $(V,\|\cdot\|)$ be a finite-dimensional seminormed vector space over $k$. Let $\mathcal{H}(V,\|\cdot\|)$ be the set of all normes $\|\cdot\|_{h}$ on $V / N_{\|\cdot\|}$ which is either ultrametric or induced by an inner product, and such that $\|\cdot\|_{h} \geqslant\|\cdot\|^{\sim}$. We define $\Delta(V,\|\cdot\|)$ to be the number (if $\|\cdot\|$ vanishe, by convention $\Delta(V,\|\cdot\|$ ) is defined to be 1 )

$$
\inf \left\{\frac{\|\cdot\|_{h, \text { det }}}{\|\cdot\|_{\text {det }}^{\sim}}:\|\cdot\|_{h} \in \mathcal{H}(V,\|\cdot\|)\right\} \in[1,+\infty[,
$$

where $\|\cdot\|_{h, \text { det }}$ and $\|\cdot\|_{\text {det }}^{\sim}$ are respectively determinant norms on $\operatorname{det}\left(V / N_{\|\cdot\|}\right)$ induced by the norms $\|\cdot\|_{h}$ and $\|\cdot\|^{\sim}$. By definition, one has $\Delta(V,\|\cdot\|)=\Delta\left(V / N_{\|\cdot\|},\|\cdot\|^{\sim}\right)$. Moreover, if $\|\cdot\|$ is ultrametric or induced by a semidefinite inner product, then $\Delta(V,\|\cdot\|)=1$.

Proposition 1.2.42. - Assume that $|\cdot|$ is non-Archimedean. Let $(V,\|\cdot\|)$ be a finitedimensional seminormed vector space over $k$. One has

$$
\ln \Delta(V,\|\cdot\|) \leqslant \operatorname{rk}\left(V / N_{\|\cdot\|}\right) \sup _{x \in V \backslash N_{\|\cdot\|}}\left(\ln \|x\|-\ln \|x\|_{* *}\right) .
$$

In particular, $\ln \Delta(V,\|\cdot\|) \leqslant \operatorname{rk}\left(V / N_{\|\cdot\|}\right) \ln \left(\operatorname{rk}\left(V / N_{\|\cdot\|}\right)\right)$.

Proof. - By replacing $(V,\|\cdot\|)$ by $\left(V / N_{\|\cdot\|},\|\cdot\|^{\sim}\right)$, we may assume without loss of generality that $\|\cdot\|$ is a norm. Let

$$
\lambda=\sup _{x \in V \backslash\{0\}}\left(\ln \|x\|-\ln \|x\|_{* *}\right) .
$$

By definition one has $\|\cdot\| \leqslant \mathrm{e}^{\lambda}\|\cdot\|_{* *}$. Note that the norm $\mathrm{e}^{\lambda}\|\cdot\|_{* *}$ is ultrametric. Therefore

$$
\Delta(V,\|\cdot\|) \leqslant \frac{\mathrm{e}^{r \lambda}\|\cdot\|_{* *, \operatorname{det}}}{\|\cdot\|_{\text {det }}}=\mathrm{e}^{r \lambda},
$$


where $r$ is the rank of $V$ over $k$, and the equality comes from Proposition 1.2.15. The inequality (1.36) is thus proved. The last inequality results from (1.36) and Corollary 1.2 .12 .

Proposition 1.2.43. - Let $(V,\|\cdot\|)$ be a finite-dimensional normed vector space over $k$. For any vector subspace $W$ of $V$, the norm of the canonical isomorphism

$$
f: \operatorname{det}(W) \otimes \operatorname{det}(V / W) \longrightarrow \operatorname{det}(V)
$$

is bounded from below by

$$
\frac{\Delta\left(W,\|\cdot\|_{W}\right) \Delta\left(V / W,\|\cdot\|_{V / W}\right)}{\Delta(V,\|\cdot\|)} \geqslant \Delta(V,\|\cdot\|)^{-1},
$$

where $\|\cdot\|_{W}$ is the restriction of the norm $\|\cdot\|$ to the vector subspace $W$ and $\|\cdot\|_{V / W}$ is the quotient norm of $\|\cdot\|$ on the quotient space $V / W$.

Proof. - Let $\|\cdot\|_{h}$ be a norm in $\mathcal{H}(V,\|\cdot\|)$. Let $\|\cdot\|_{h, W}$ and $\|\cdot\|_{h, V / W}$ be respectively the restriction of $\|\cdot\|_{h}$ to $W$ and the quotient norm of $\|\cdot\|_{h}$ on $V / W$. By Proposition 1.2.40, the canonical isomorphism

$$
\operatorname{det}\left(W,\|\cdot\|_{h, W}\right) \otimes \operatorname{det}\left(V / W,\|\cdot\|_{h, V / W}\right) \longrightarrow \operatorname{det}\left(V,\|\cdot\|_{V, h}\right)
$$

is an isometry. Hence

$$
\frac{\|\cdot\|_{h, \mathrm{det}}}{\|\cdot\|_{\operatorname{det}}}=\frac{\|\cdot\|_{h, W, \mathrm{det}}\|\cdot\|_{h, V / W, \operatorname{det}}}{\|f\| \cdot\|\cdot\|_{W, \operatorname{det}}\|\cdot\|_{V / W, \operatorname{det}}} \geqslant \frac{1}{\|f\|} \cdot \Delta\left(W,\|\cdot\|_{W}\right) \Delta\left(V / W,\|\cdot\|_{V / W}\right) .
$$

Since $\|\cdot\|_{h} \in \mathcal{H}(V,\|\cdot\|)$ is arbitrary, we obtain the lower bound announced in the proposition.

Corollary 1.2.44. - Let $\left(V,\|\cdot\|_{V}\right)$ be a finite-dimensional seminormed vector space over $k, W$ be a vector subspace of $V,\|\cdot\|_{W}$ be the restriction of $\|\cdot\|_{V}$ to $W$, and $\|\cdot\|_{V / W}$ be the quotient of $\|\cdot\|_{V}$ on $V / W$. One has

$$
\Delta\left(W,\|\cdot\|_{W}\right) \Delta\left(V / W,\|\cdot\|_{V / W}\right) \leqslant \Delta(V,\|\cdot\|) .
$$

In particular, $\Delta\left(W,\|\cdot\|_{W}\right) \leqslant \Delta(V,\|\cdot\|)$ and $\Delta\left(V / W,\|\cdot\|_{V / W}\right) \leqslant \Delta(V,\|\cdot\|)$.

Proof. - By Proposition 1.1.16, we can assume without loss of generality that $\|\cdot\|_{V}$ is a norm. By Corollary 1.1.68 if we denote by $f: \operatorname{det}(W) \otimes \operatorname{det}(V / W) \rightarrow \operatorname{det}(V)$ the canonical isomorphism, then $\|f\| \leqslant 1$. The inequality (1.37) thus follows from Proposition 1.2.43. Finally, by definition one has $\Delta\left(W,\|\cdot\|_{W}\right) \geqslant 1$ and $\Delta\left(V / W,\|\cdot\|_{V / W}\right) \geqslant$ 1 , thus we deduce from (1.37) the last two inequalities stated in the corollary.

Remark 1.2.45. - Let $(V,\|\cdot\|)$ be a finite-dimensional normed vector space over $k$. We assume that the rank $r$ of $V / N_{\|\cdot\|}$ is positive. In the case where the absolute value $|\cdot|$ is non-Archimedean, Corollary 1.2 .12 provides the upper bound $\Delta(V,\|\cdot\|) \leqslant r^{r}$. This result is also true in the Archimedean case (which follows from the existence of an orthogonal basis, see the beginning of $\$ 1.2 .8$ for details). However, as we will see 
in the next subsection (cf. Theorem[1.2.54), in the Archimedean case one has a better upper bound $\Delta(V,\|\cdot\|) \leqslant r^{r / 2}$.

1.2.7. Dual determinant norm. - Let $(V,\|\cdot\|)$ be a finite-dimensional seminormed vector space over $k$. We denote by $\|\cdot\|_{\text {det }}^{\sim}$ the determinant norm on $\operatorname{det}\left(V / N_{\|\cdot\|}\right)$ induced by $\|\cdot\|^{\sim}$, and denote by $\|\cdot\|_{\text {det,* }}^{\sim}$ the dual norm of $\|\cdot\|_{\text {det. }}^{\sim}$ Let $\|\cdot\|_{*, \text { det }}$ be the determinant norm on $\operatorname{det}\left(V^{*}\right) \cong \operatorname{det}\left(V / N_{\|\cdot\|}\right)^{*}$ of the dual norm $\|\cdot\|_{*}$ on $V^{*}$. The purpose of this subsection is to compare these two norms. We denote by $\delta(V,\|\cdot\|)$ the ratio

$$
\delta(V,\|\cdot\|):=\frac{\|\cdot\|_{\mathrm{det}, *}^{\sim}}{\|\cdot\|_{*, \mathrm{det}}} .
$$

In the case where there is no ambiguity on the seminorm $\|\cdot\|$ on $V$, we also use the abbreviate notation $\delta(V)$ to denote $\delta(V,\|\cdot\|)$. By definition, if $\eta$ is a non-zero element in $\operatorname{det}\left(V / N_{\|\cdot\|}\right)$ and if $\eta^{\vee}$ is its dual element in $\operatorname{det}\left(V^{*}\right)$, then one has

$$
\delta(V,\|\cdot\|)^{-1}=\|\eta\|_{\operatorname{det}} \cdot\left\|\eta^{\vee}\right\|_{*, \operatorname{det}} .
$$

In particular, one has (see Proposition 1.2.15)

$$
\delta(V,\|\cdot\|)=\delta\left(V / N_{\|\cdot\|},\|\cdot\|^{\sim}\right)=\delta\left(V^{*},\|\cdot\|_{*}\right) .
$$

Proposition 1.2.46. - Let $(V,\|\cdot\|)$ be a finite-dimensional seminormed vector space over $k$. One has $\delta(V,\|\cdot\|) \geqslant 1$.

Proof. - By (1.39) we may assume without loss of generality that $\|\cdot\|$ is a norm.

Let $\left\{e_{i}\right\}_{i=1}^{r}$ be a basis of $V$, and $\left\{e_{i}^{\vee}\right\}_{i=1}^{r}$ be its dual basis. One has

$$
\left\|e_{1}^{\vee} \wedge \cdots \wedge e_{r}^{\vee}\right\|_{\text {det }, *}=\left\|e_{1} \wedge \cdots \wedge e_{r}\right\|_{\text {det }}^{-1} .
$$

Therefore

$$
\begin{aligned}
\delta(V,\|\cdot\|)^{-1} & =\left\|e_{1} \wedge \cdots \wedge e_{r}\right\|_{\operatorname{det}} \cdot\left\|e_{1}^{\vee} \wedge \cdots \wedge e_{r}^{\vee}\right\|_{*, \operatorname{det}} \\
& \leqslant\left\|e_{1}\right\| \cdots\left\|e_{r}\right\| \cdot\left\|e_{1}^{\vee}\right\|_{*} \cdots\left\|e_{r}^{\vee}\right\|_{*},
\end{aligned}
$$

where the inequality comes from Proposition 1.1.66. If the basis $\left\{e_{i}\right\}_{i=1}^{r}$ is $\alpha$ orthogonal, where $\alpha \in] 0,1\left[\right.$, by Lemma 1.2 .10 one has $\delta(V,\|\cdot\|)^{-1} \leqslant \alpha^{-r}$. Since for any $\alpha \in] 0,1[$ there exists an $\alpha$-orthogonal basis (see Proposition 1.2.7), one has $\delta(V,\|\cdot\|) \geqslant 1$.

Proposition 1.2.4\% - Let $(V,\|\cdot\|)$ be a finite-dimensional seminormed vector space over $k$. Assume that the absolute value $|\cdot|$ is non-Archimedean, or the seminorm $\|\cdot\|$ is induced by a semidefinite inner product. Then one has $\delta(V,\|\cdot\|)=1$.

Proof. - By (1.39) we may assume without loss of generality that $\|\cdot\|$ is a norm.

We first treat the case where the absolute value $|\cdot|$ is non-Archimedean. Let $\alpha \in$ ]0,1 [ and $\left\{e_{i}\right\}_{i=1}^{r}$ be an $\alpha$-orthogonal basis of $(V,\|\cdot\|)$ (see Proposition 1.2 .7 for the existence of an $\alpha$-orthogonal basis). Then the dual basis $\left\{e_{i}^{\vee}\right\}_{i=1}^{r}$ is $\alpha$-orthogonal with 
respect to the dual norm $\|\cdot\|_{*}$ (see Proposition 1.2.11). In particular, by Proposition 1.2 .23 one has

$$
\frac{\left\|e_{1} \wedge \cdots \wedge e_{r}\right\|_{\mathrm{det}}}{\left\|e_{1}\right\| \cdots\left\|e_{r}\right\|} \geqslant \alpha^{r}, \quad \frac{\left\|e_{1}^{\vee} \wedge \cdots \wedge e_{r}^{\vee}\right\|_{*, \mathrm{det}}}{\left\|e_{1}^{\vee}\right\|_{*} \cdots\left\|e_{r}^{\vee}\right\|_{*}} \geqslant \alpha^{r}
$$

Therefore

$$
\delta(V,\|\cdot\|)=\frac{\left\|e_{1} \wedge \cdots \wedge e_{r}\right\|_{\operatorname{det}}^{-1}}{\left\|e_{1}^{\vee} \wedge \cdots \wedge e_{r}^{\vee}\right\|_{*, \operatorname{det}}} \leqslant \alpha^{-2 r} \frac{1}{\left\|e_{1}\right\| \cdots\left\|e_{r}\right\| \cdot\left\|e_{1}^{\vee}\right\|_{*} \cdots\left\|e_{r}^{\vee}\right\|_{*}} \leqslant \alpha^{-2 r},
$$

where the last inequality comes from Lemma 1.2.10, Since $\alpha$ is arbitrary, one has $\delta(V,\|\cdot\|) \leqslant 1$.

The proof of the Archimedean case is quite similar, where we use the existence of an orthogonal basis, which is also an Hadamard basis (see Proposition 1.2.25). We omit the details.

The following Lemma is the Archimedean counterpart of Proposition 1.2.35 (see also the comparison in Remark 1.1.21).

Lemma 1.2.48. - Assume that the absolute value $|\cdot|$ is Archimedean. Let $\left(V,\|\cdot\|_{V}\right)$ be a finite-dimensional seminormed vector space over $k, W$ be a vector subspace of $V$, and $\|\cdot\|_{W}$ be the restriction of the seminorm $\|\cdot\|_{V}$ to $W$. Then the map $F: V^{*} \rightarrow W^{*}$, which sends $\varphi \in V^{*}$ to its restriction to $W$, is surjective. Moreover, the quotient norm on $W^{*}$ induced by the dual norm $\|\cdot\|_{V, *}$ coincides with the norm $\|\cdot\|_{W, *}$.

Proof. - Let $\psi$ be an element in $W^{*}$. If $\varphi$ is an element in $V^{*}$ which extends $\psi$, then clearly one has $\|\varphi\|_{V, *} \geqslant\|\psi\|_{W, *}$. Moreover, by Hahn-Banach theorem, there exists $\varphi_{0} \in V^{*}$ which extends $\psi$ and such that $\left\|\varphi_{0}\right\|_{V, *}=\|\psi\|_{W, *}$. Therefore, the map $F$ is surjective and the quotient norm on $W^{\vee}$ induced by $\|\cdot\|_{V, *}$ coincides with $\|\cdot\|_{W, *}$.

Proposition 1.2.49. - Let $\left(V,\|\cdot\|_{V}\right)$ and $\left(W,\|\cdot\|_{W}\right)$ be finite-dimensional seminormed vector spaces over $k, V_{0}$ be a $k$-vector subspace of $V$ and $\|\cdot\|_{V_{0}}$ be the restriction of $\|\cdot\|_{V}$ on $V_{0}$. Denote by $\|\cdot\|_{\varepsilon}$ and $\|\cdot\|_{\pi}$ the $\varepsilon$-tensor product and the $\pi$-tensor product of the seminorms $\|\cdot\|_{V}$ and $\|\cdot\|_{W}$, respectively.

(1) Assume that, either the absolute value $|\cdot|$ is Archimedean, or the seminorm $\|\cdot\|_{V}$ is ultrametric. Then the $\varepsilon$-tensor product $\|\cdot\|_{\varepsilon, 0}$ of $\|\cdot\|_{V_{0}}$ and $\|\cdot\|_{W}$ identifies with the restriction of $\|\cdot\|_{\varepsilon}$ to $V_{0} \otimes_{k} W$.

(2) Assume that the seminorm $\|\cdot\|_{V}$ is either ultrametric or induced by a semidefinite inner product. Then the $\pi$-tensor product $\|\cdot\|_{\pi, 0}$ of $\|\cdot\|_{V_{0}}$ and $\|\cdot\|_{W}$ coincides with the restriction of $\|\cdot\|_{\pi}$ to $V_{0} \otimes_{k} W$.

Proof. - (1) Let $\varphi$ be a tensor in $V_{0} \otimes_{k} W$, viewed as a bilinear form on $V_{0}^{*} \times W^{*}$. By definition, one has

$$
\|\varphi\|_{\varepsilon, 0}=\sup _{\substack{\left(f_{0}, g\right) \in V_{0}^{*} \times W^{*} \\ f_{0} \neq 0, g \neq 0}} \frac{\left|\varphi\left(f_{0}, g\right)\right|}{\left\|f_{0}\right\|_{V_{0}, *} \cdot\|g\|_{W, *}} .
$$


Since the absolute value $|\cdot|$ is Archimedean or the norm $\|\cdot\|_{V}$ is ultrametric, by Proposition 1.2.35 (for the ultrametric case) and Lemma 1.2.48 (for the Archimedean case), the norm $\|\cdot\|_{V_{0}, *}$ identifies with the quotient of $\|\cdot\|_{V, *}$ by the canonical surjective map $V^{*} \rightarrow V_{0}^{*}$. Therefore, one has

$$
\sup _{\substack{\left(f_{0}, g\right) \in V_{0}^{*} \times W^{*} \\ f_{0} \neq 0, g \neq 0}} \frac{\left|\varphi\left(f_{0}, g\right)\right|}{\left\|f_{0}\right\|_{V_{0}, *} \cdot\|g\|_{W, *}}=\sup _{\substack{f, g) \in V^{*} \times W^{*} \\ f \neq 0, g \neq 0}} \frac{|\varphi(f, g)|}{\|f\|_{V, *} \cdot\|g\|_{W, *}},
$$

which shows $\|\varphi\|_{\varepsilon, 0}=\|\varphi\|_{\varepsilon}$.

(2) We have already seen in Proposition 1.1.60 (1) that $\|\cdot\|_{\pi, 0}$ is bounded from below by the restriction of $\|\cdot\|_{\pi}$ to $V_{0} \otimes_{k} W$. Let $T$ be an element of $V_{0} \otimes_{k} W$, which is written, as an element of $V \otimes_{k} W$, in the form $T=\sum_{i=1}^{N} x_{i} \otimes y_{i}$, where $\left\{x_{1}, \ldots, x_{N}\right\} \subseteq V$ and $\left\{y_{1}, \ldots, y_{N}\right\} \subseteq W$. By Corollary 1.2.33, for any $\left.\alpha \in\right] 0,1[$, there exists a linear projection $\pi_{\alpha}: V \rightarrow V_{0}$ such that $\left\|\pi_{\alpha}\right\| \leqslant \alpha^{-1}$. Since $T$ belongs to $V_{0} \otimes_{k} W$ one has $T=\sum_{i=1}^{N} \pi_{\alpha}\left(x_{i}\right) \otimes y_{i}$. Moreover,

$$
\|T\|_{0, \pi} \leqslant \sum_{i=1}^{N}\left\|\pi_{\alpha}\left(x_{i}\right)\right\|_{V_{0}} \cdot\left\|y_{i}\right\|_{W} \leqslant \alpha^{-1} \sum_{i=1}^{N}\left\|x_{i}\right\|_{V} \cdot\left\|y_{i}\right\|_{W} .
$$

Since $\alpha$ and the writing $T=\sum_{i=1}^{N} x_{i} \otimes y_{i}$ are arbitrary, we obtain $\|T\|_{0, \pi} \leqslant\|T\|_{\pi}$.

Proposition 1.2.50. — Let $\left(V,\|\cdot\|_{V}\right)$ and $\left(W,\|\cdot\|_{W}\right)$ be seminormed vector spaces over $k$, and $\|\cdot\|_{\pi}$ be the $\pi$-tensor product norm of $\|\cdot\|_{V} \cdot$ We assume that $\|\cdot\|_{W}$ is ultrametric. For any $(x, y) \in V \times W$, one has $\|x \otimes y\|_{\pi}=\|x\|_{V} \cdot\|y\|_{W}$.

Proof. - By definition on has $\|x \otimes y\|_{\pi} \leqslant\|x\|_{V} \cdot\|y\|_{W}$. It then suffices to show that, for any writing of $x \otimes y$ as

$$
\sum_{i=1}^{N} x_{i} \otimes y_{i}
$$

with $\left(x_{1}, \ldots, x_{n}\right) \in V^{n}$ and $\left(y_{1}, \ldots, y_{n}\right) \in W^{n}$, one has

$$
\|x\|_{V} \cdot\|y\|_{W} \leqslant \sum_{i=1}^{N}\left\|x_{i}\right\|_{V} \cdot\left\|y_{i}\right\|_{W} .
$$

Therefore we may assume without loss of generality that $V$ and $W$ are finitedimensional vector spaces over $k$. Consider the $k$-linear map $\ell$ from $W^{*}$ to $V$ sending $\varphi \in W^{*}$ to

$$
\varphi(y) x=\sum_{i=1}^{N} \varphi\left(y_{i}\right) x_{i}
$$

We equip $W^{*}$ with the dual norm $\|\cdot\|_{W, *}$ and consider the operator norm of $\ell$. On one hand, one has

$$
\|\ell\|=\sup _{\varphi \in W^{*} \backslash\{0\}} \frac{\|\varphi(y) x\|_{V}}{\|\varphi\|_{W, *}}=\sup _{\varphi \in W^{*} \backslash\{0\}} \frac{|\varphi(y)| \cdot\|x\|_{V}}{\|\varphi\|_{W, *}}=\|y\|_{W, * *} \cdot\|x\|_{V}=\|y\|_{W} \cdot\|x\|_{V},
$$


where the last equality comes from Corollary 1.2 .12 and the hypothesis that $\|\cdot\|_{W}$ is ultrametric. On the other hand, one has

$$
\begin{aligned}
\|\ell\| & =\sup _{\varphi \in W^{*} \backslash\{0\}} \frac{\left\|\varphi\left(y_{1}\right) x_{1}+\cdots+\varphi\left(y_{N}\right) x_{N}\right\|_{V}}{\|\varphi\|_{W, *}} \\
& \leqslant \sup _{\varphi \in W^{*} \backslash\{0\}} \sum_{i=1}^{N} \frac{\left|\varphi\left(y_{i}\right)\right| \cdot\left\|x_{i}\right\|_{V}}{\|\varphi\|_{W, *}} \leqslant \sum_{i=1}^{N}\left\|x_{i}\right\|_{V} \cdot\left\|y_{i}\right\|_{W, * *}=\sum_{i=1}^{N}\left\|x_{i}\right\|_{V} \cdot\left\|y_{i}\right\|_{W},
\end{aligned}
$$

where the last equality follows from Corollary 1.2 .12 and the hypothesis that $\|\cdot\|_{W}$ is ultrametric again. The proposition is thus proved.

The following result provides a variant of Proposition 1.2.43. Note that it generalises (by using Proposition 1.2.47) Proposition 1.2.40.

Proposition 1.2.51. — Let $(V,\|\cdot\|)$ be a finite-dimensional normed vector space over $k$. Assume that the absolute value $|\cdot|$ is Archimedean or the norm $\|\cdot\|$ is ultrametric. For any vector subspace $W$ of $V$, the norm of the canonical isomorphism

$$
f: \operatorname{det}(W) \otimes \operatorname{det}(V / W) \longrightarrow \operatorname{det}(V)
$$

is bounded from below by

$$
\frac{\delta\left(W,\|\cdot\|_{W}\right) \delta\left(V / W,\|\cdot\|_{V / W}\right)}{\delta(V,\|\cdot\|)} \geqslant \delta(V,\|\cdot\|)^{-1},
$$

where we consider the restriction $\|\cdot\|_{W}$ of the norm $\|\cdot\|$ to the vector subspace $W$ and the quotient norm $\|\cdot\|_{V / W}$ of $\|\cdot\|$ on the quotient space $V / W$. In particular, one has

$$
\max \left\{\delta\left(W,\|\cdot\|_{W}\right), \delta\left(V / W,\|\cdot\|_{V / W}\right)\right\} \leqslant \delta(V,\|\cdot\|) .
$$

Proof. — Let $\|\cdot\|_{V / W}$ be the quotient norm on $V / W$ induced by $\|\cdot\|_{V}$. By Proposition 1.1.20, the dual norm $\|\cdot\|_{V / W, *}$ coincides with the restriction of the norm $\|\cdot\|_{*}$ to $(V / W)^{\vee}$. Moreover, by Lemma 1.2.48 (for the Archimedean case) and Proposition 1.2.35 (for the non-Archimedean case), the quotient norm on $W^{\vee}$ induced by $\|\cdot\|_{*}$ identifies with the dual norm $\|\cdot\|_{W, *}$. Let $\alpha$ and $\beta$ be respectively non-zero elements in $\operatorname{det}(W)$ and $\operatorname{det}(V / W)$. Let $\alpha^{\vee} \in \operatorname{det}\left(W^{\vee}\right)$ and $\beta^{\vee} \in \operatorname{det}\left((V / W)^{\vee}\right)$ be their dual elements, $\eta$ be the image of $\alpha \otimes \beta$ by the canonical isomorphism $\operatorname{det}(W) \otimes$ $\operatorname{det}(V / W) \rightarrow \operatorname{det}(V)$, and $\eta^{\vee}$ be the image of $\alpha^{\vee} \otimes \beta^{\vee}$ by the canonical isomorphism $\operatorname{det}\left(W^{\vee}\right) \otimes \operatorname{det}\left((V / W)^{\vee}\right) \rightarrow \operatorname{det}\left(V^{\vee}\right)$. Then $\eta^{\vee}$ is the dual element of $\eta$.

By Proposition 1.1.68, one has

$$
\left\|\eta^{\vee}\right\|_{*, \operatorname{det}} \leqslant\left\|\alpha^{\vee}\right\|_{W, *, \operatorname{det}} \cdot\left\|\beta^{\vee}\right\|_{V / W, *, \operatorname{det}} \cdot
$$


Hence by (1.38) one has

$$
\begin{aligned}
\frac{\delta\left(W,\|\cdot\|_{W}\right) \delta\left(V / W,\|\cdot\|_{V / W}\right)}{\delta(V,\|\cdot\|)} & =\frac{\left\|\eta^{\vee}\right\|_{*, \operatorname{det}} \cdot\|\eta\|_{\text {det }}}{\left\|\alpha^{\vee}\right\|_{W, *, \operatorname{det}}\|\alpha\|_{W, \operatorname{det}} \cdot\|\beta\|_{V / W, \operatorname{det}}\left\|\beta^{\vee}\right\|_{V / W, *, \operatorname{det}}} \\
& \leqslant \frac{\|\eta\|_{\text {det }}}{\|\alpha\|_{W, \operatorname{det}} \cdot\|\beta\|_{V / W, \operatorname{det}}}=\|f\| .
\end{aligned}
$$

Finally, by Corollary 1.1.68, we obtain

$$
\delta\left(W,\|\cdot\|_{W}\right) \delta\left(V / W,\|\cdot\|_{V / W}\right) \leqslant \delta(V,\|\cdot\|) .
$$

Since $\delta\left(W,\|\cdot\|_{W}\right)$ and $\delta\left(V / W,\|\cdot\|_{V / W}\right)$ are $\geqslant 1$ (see Proposition 1.2.46), we obtain the last inequality.

Corollary 1.2.52. - Let $V$ be a finite-dimensional vector space over $k$ and $\|\cdot\|$ be a norm on $V$. We assume that, either the norm $\|\cdot\|$ is ultrametric or the absolute value $|\cdot|$ is Archimedean. If $W_{1}$ and $W_{2}$ are two k-vector subspaces of $V$, then the canonical isomorphism

$$
\operatorname{det}\left(W_{1}\right) \otimes \operatorname{det}\left(W_{2}\right) \longrightarrow \operatorname{det}\left(W_{1} \cap W_{2}\right) \otimes \operatorname{det}\left(W_{1}+W_{2}\right)
$$

induced by the short exact sequence

$$
0 \longrightarrow W_{1} \cap W_{2} \longrightarrow W_{1} \oplus W_{2} \longrightarrow W_{1}+W_{2} \longrightarrow 0
$$

has operator norm $\leqslant \min \left\{\delta\left(W_{1}\right), \delta\left(W_{2}\right)\right\} / \delta\left(W_{1} \cap W_{2}\right)$, where in the above formulae we consider the restricted norms on the vector subspaces of $V$. In particular, if $\|\cdot\|$ is an ultrametric norm, then the linear map (1.40) has norm $\leqslant 1$.

Proof. - Consider the short exact sequence

$$
0 \longrightarrow W_{1} \cap W_{2} \longrightarrow W_{1} \longrightarrow W_{1} /\left(W_{1} \cap W_{2}\right) \longrightarrow 0 \text {. }
$$

By Proposition 1.2.51, the canonical element $\eta$ in

$$
\operatorname{det}\left(W_{1}\right)^{\vee} \otimes \operatorname{det}\left(W_{1} \cap W_{2}\right) \otimes \operatorname{det}(G)
$$

has norm $\leqslant \delta\left(W_{1}\right) / \delta\left(W_{1} \cap W_{2}\right) \delta(G)$, where $G$ denotes the vector space $W_{1} /\left(W_{1} \cap W_{2}\right)$ equipped with the quotient norm $\|\cdot\|_{G}$.

Similarly, consider the short exact sequence

$$
0 \longrightarrow W_{2} \longrightarrow W_{1}+W_{2} \longrightarrow\left(W_{1}+W_{2}\right) / W_{2} \longrightarrow 0 \text {. }
$$

By Corollary 1.1.68, the canonical element $\eta^{\prime}$ in

$$
\operatorname{det}\left(W_{2}\right)^{\vee} \otimes \operatorname{det}\left(G^{\prime}\right)^{\vee} \otimes \operatorname{det}\left(W_{1}+W_{2}\right)
$$

has norm $\leqslant 1$, where $G^{\prime}$ denotes the vector space $\left(W_{1}+W_{2}\right) / W_{2}$ equipped with the quotient norm $\|\cdot\|_{G^{\prime}}$. Therefore we obtain

$$
\left\|\eta \otimes \eta^{\prime}\right\| \leqslant \delta\left(W_{1}\right) / \delta\left(W_{1} \cap W_{2}\right) .
$$


Let $f: G \rightarrow G^{\prime}$ be the canonical isomorphism. One has $\|f(x)\|_{G^{\prime}} \leqslant\|x\|_{G}$ for any $x \in G$. In particular, the canonical element of $\operatorname{det}(G) \otimes \operatorname{det}\left(G^{\prime}\right)^{\vee}$ has norme $\geqslant 1$. We deduce that the canonical element of

$$
\operatorname{det}\left(W_{1}\right)^{\vee} \otimes \operatorname{det}\left(W_{2}\right)^{\vee} \otimes \operatorname{det}\left(W_{1} \cap W_{2}\right) \otimes \operatorname{det}\left(W_{1}+W_{2}\right)
$$

has norm $\leqslant \delta\left(W_{1}\right) / \delta\left(W_{1} \cap W_{2}\right)$. By the symmetry between $W_{1}$ and $W_{2}$, we obtain the announced inequality.

Remark 1.2.53. - We assume that the absolute value $|\cdot|$ is non-Archimedean. The result of Corollary 1.2 .52 is not true in general if the norm $\|\cdot\|$ is not ultrametic. However, we can combine the proof of Corollary 1.2.52 and Proposition 1.2.43 to show that the canonical isomorphism (1.40) in Corollary 1.2.52 has an operator norm bounded from above by

$$
\frac{\min \left\{\Delta\left(W_{1}\right), \Delta\left(W_{2}\right)\right\}}{\Delta\left(W_{1} \cap W_{2}\right)} .
$$

The same argument also works in the Archimedean case.

1.2.8. Ellipsoid of John and Löwner. - We assume that the absolute value $|\cdot|$ is Archimedean. Let $V$ be a finite-dimensional vector space over $k$, equipped with a norm $\|\cdot\|$. In this subsection, we discuss the approximation of the norm $\|\cdot\|$ by Euclidean or Hermitian norms. Note that Proposition 1.2.7 provides a result in this direction. Let $\left\{e_{i}\right\}_{i=1}^{r}$ be an orthonormal basis of $V$. Let $\langle$,$\rangle be an inner product$ on $V$ such that $\left\{e_{i}\right\}_{i=1}^{r}$ is orthogonal with respect to the inner product, and that $\left\langle e_{i}, e_{i}\right\rangle=r$ for any $i \in\{1, \ldots, r\}$. If $\|\cdot\|_{h}$ denotes the norm on $V$ induced by the inner product $\langle$,$\rangle , then for any x=\lambda_{1} e_{1}+\cdots+\lambda_{r} e_{r} \in V$ one has

$$
\frac{1}{r}\|x\|_{h}=\left(\frac{\left|\lambda_{1}\right|^{2}+\cdots+\left|\lambda_{r}\right|^{2}}{r}\right)^{1 / 2} \leqslant \max \left\{\left|\lambda_{1}\right|, \ldots,\left|\lambda_{r}\right|\right\} \leqslant\|x\|
$$

and $\|x\| \leqslant\left|\lambda_{1}\right|+\cdots+\left|\lambda_{r}\right| \leqslant r^{1 / 2}\left(\left|\lambda_{1}\right|^{2}+\cdots+\left|\lambda_{r}\right|^{2}\right)^{1 / 2}=\|x\|_{h}$.

The works of John $\mathbf{8 8}$ and Löwner provide a stronger result on the comparison of inner product norms and general norms. We refer to the expository article of Henk 83 for the history of this theory. For the convenience of the readers, we include the statement and the proof of this result.

Theorem 1.2.54 (John-Löwner). — Let $V$ be a non-zero finite-dimensional vector space over $k$, equipped with a norm $\|\cdot\|$. There exists a unique Euclidean or Hermitian norm $\|\cdot\|_{J}$ bounded from above by $\|\cdot\|$ such that, for any Euclidean or Hermitian norm $\|\cdot\|_{h}$ satisfying $\|\cdot\|_{h} \leqslant\|\cdot\|$, one has $\|\cdot\|_{h, \text { det }} \leqslant\|\cdot\|_{J, \text { det }}$. Moreover, for any $x \in V$, one has $\|x\|_{h} \leqslant\|x\| \leqslant r^{1 / 2}\|x\|_{h}$, where $r$ is the rank of $V$ over $k$.

Proof. - We fix an arbitrary inner product $\langle,\rangle^{\prime}$ on $V$ and denote by $\Theta$ the vector space (over $\mathbb{R}$ ) of all endomorphisms of $V$ which are self-adjoint with respect to the 
inner product $\langle,\rangle^{\prime}$. Recall that a $k$-linear map $u: V \rightarrow V$ is said to be self-adjoint with respect to $\langle,\rangle^{\prime}$ if and only if

$$
\forall x, y \in V, \quad\langle u(x), y\rangle^{\prime}=\langle x, u(y)\rangle^{\prime} .
$$

Let $\Theta^{+}$be the set of all positive definite self-adjoint operators. Since any pair of selfadjoint operator can be simultaneously diagonalised by a basis of $V$, we obtain that $\Theta^{+}$is a convex open subset of $\Theta$ and that the function $\log \operatorname{det}(\cdot)$ is strictly concave on $\Theta^{+}$.

Let $B=\{x \in V:\|x\| \leqslant 1\}$ be the unit ball of the norm $\|\cdot\|$. For any $u \in \Theta^{+}$, let $B_{u}=\left\{x \in V:\langle x, u(x)\rangle^{\prime} \leqslant 1\right\}$, which is the unit ball of the Euclidean or Hermitian norm $\|\cdot\|_{u}$ on $V$ defined as

$$
\forall x \in V, \quad\|x\|_{u}^{2}=\langle x, u(x)\rangle^{\prime} .
$$

Let $\Theta_{0}$ be the set of all $u \in \Theta^{+}$such that $B_{u} \supseteq B$. Then for any $u_{0} \in \Theta_{0}$, the set

$$
\Theta\left(u_{0}\right):=\left\{u \in \Theta_{0}: \operatorname{det}(u) \geqslant \operatorname{det}\left(u_{0}\right)\right\}
$$

is a convex and compact subset of $\Theta$. In fact, from the concavity and the continuity of the function $\log \operatorname{det}(\cdot)$ we obtain that the set $\Theta\left(u_{0}\right)$ is convex and closed. Moreover, the condition $B_{u} \supseteq B$ for $u \in \Theta_{0}$ implies that the set $\Theta\left(u_{0}\right)$ is bounded in $\Theta$. Therefore the restriction of the function $\operatorname{det}(\cdot)$ to $\Theta_{0}$ attains its maximal value on a unique point $u_{1} \in \Theta_{0}$.

Let $\langle$,$\rangle be the inner product on V$ such that

$$
\forall(x, y) \in V \times V, \quad\langle x, y\rangle=\left\langle x, u_{1}(y)\right\rangle^{\prime} .
$$

We call it the John inner product associated with the norm $\|\cdot\|$. The corresponding Euclidean or Hermitian norm $\|\cdot\|_{J}$ is called the John norm associated with $\|\cdot\|$.

In the following, we prove the relation

$$
\forall x \in V, \quad\|x\|_{J} \leqslant\|x\| \leqslant r^{1 / 2}\|x\|_{J}
$$

under the supplementary assumption that the unit ball $B$ is the convex hull of finitely many orbits of the action of $\{a \in k:|a|=1\}$ on $V$.

Without loss of generality, we assume that $\langle,\rangle^{\prime}=\langle$,$\rangle . For any x \in V$ such that $\|x\| \leqslant 1$, let $\varphi_{x}: \Theta \rightarrow \mathbb{R}$ be the linear functional which sends $u \in \Theta$ to $\langle x, u(x)\rangle$. If $u: V \rightarrow V$ is a self-adjoint linear operator such that $\varphi_{x}(u) \leqslant 0$ for any $x \in B$ such that $\langle x, x\rangle=1$, then one has $\operatorname{Tr}(u) \leqslant 0$. In fact, the condition

$$
\forall x \in B, \quad\langle x, x\rangle=1 \Longrightarrow \varphi_{x}(u) \leqslant 0
$$

implies that Id $+\varepsilon u \in \Theta_{0}$ for sufficiently small $\varepsilon>0$ (here we use the supplementary assumption that the convex body $B$ is spanned by a finite number of orbits). Therefore one has $\operatorname{det}(\operatorname{Id}+\varepsilon u) \leqslant \operatorname{det}(\mathrm{Id})=1$, which leads to $\operatorname{Tr}(u) \leqslant 0$. Therefore, the linear 
form $\operatorname{Tr}(\cdot)$ lies in the closure of the positive cone of $\Theta^{\vee}$ generated by $\varphi_{x}(\cdot)(x \in B$, $\langle x, x\rangle=1)$, namely there exist a sequence of elements $\left\{x_{n}\right\}_{n \in \mathbb{N}}$ in

$$
B \cap\{x \in V:\langle x, x\rangle=1\}
$$

and a sequence $\left\{\lambda_{n}\right\}_{n \geqslant 0}$ of real numbers such that

$$
\operatorname{Tr}(u)=\sum_{n \in \mathbb{N}} \lambda_{n}\left\langle x_{n}, u\left(x_{n}\right)\right\rangle
$$

for any $u \in \Theta$. If we apply the identity to $u=\mathrm{Id}$, we obtain

$$
r=\sum_{n \in \mathbb{N}} \lambda_{n}\left\langle x_{n}, x_{n}\right\rangle=\sum_{n \in \mathbb{N}} \lambda_{n}
$$

Let $y$ be an element in $V$ such that $\langle y, y\rangle=1$. We apply the identity (1.41) to the linear map $u(x)=\langle y, x\rangle y$, and obtain

$$
1=\sum_{n \in \mathbb{N}} \lambda_{n}\left|\left\langle x_{n}, y\right\rangle\right|_{\infty}^{2}
$$

Thus there should exist $n \in \mathbb{N}$ such that $\left|\left\langle x_{n}, y\right\rangle\right|_{\infty} \geqslant r^{-1 / 2}$ since otherwise we have

$$
1<\sum_{n \in \mathbb{N}} \frac{1}{r} \lambda_{n}=\frac{1}{r} \cdot r=1,
$$

where the first equality comes from (1.42), which leads to a contradiction. Since the unit ball $B=\{x \in V:\|x\|=1\}$ is invariant by the multiplication by any $\lambda \in k$ with $|\lambda|=1$, we obtain that, for any $y \in V$ such that $\langle y, y\rangle=1$, there exists $x \in B$ such that $\operatorname{Re}\langle y, x\rangle \geqslant r^{-1 / 2}$.

We claim that the unit ball $B=\{x \in V:\|x\| \leqslant 1\}$ contains the set of all $x \in V$ such that $\langle x, x\rangle \leqslant 1 / r$. In fact, if $x_{0} \in V$ is a point such that $\left\langle x_{0}, x_{0}\right\rangle \leqslant 1 / r$ and that $\|x\|>1$, we can choose an $\mathbb{R}$-affine function $f: V \rightarrow \mathbb{R}$ such that $f\left(x_{0}\right)=0$ and that $f(x)<0$ for any $x \in B$. Note that $\operatorname{Re}\langle$,$\rangle defines an inner product on V$, where $V$ is viewed as a vector space over $\mathbb{R}$ if $k=\mathbb{C}$. By Riesz's theorem there exists $y \in V$ such that

$$
\forall x \in V, \quad f(x)=\operatorname{Re}\langle y, x\rangle+f(0) .
$$

Without loss of generality, we may assume that $\langle y, y\rangle=1$. One has

$$
0=f\left(x_{0}\right)=\operatorname{Re}\left\langle y, x_{0}\right\rangle+f(0) \leqslant\langle y, y\rangle^{1 / 2}\left\langle x_{0}, x_{0}\right\rangle^{1 / 2}+f(0)=\frac{1}{\sqrt{r}}+f(0) .
$$

Hence $f(0) \geqslant-r^{-1 / 2}$. However, the above argument shows that there exists $x \in B$ such that $\operatorname{Re}\langle y, x\rangle \geqslant r^{-1 / 2}$. Hence one has

$$
0>f(x)=\operatorname{Re}\langle y, x\rangle+f(0) \geqslant 0,
$$

which leads a contradiction.

Since $B \subseteq\{x \in V:\langle x, x\rangle \leqslant 1\}$, one has $\|x\|_{J} \leqslant\|x\|$ for any $x \in V$. Moreover, the relation

$$
\{x \in V:\langle x, x\rangle \leqslant 1 / r\} \subseteq B=\{x \in V:\|x\| \leqslant 1\}
$$


implies that $\|x\| \leqslant r^{1 / 2}\|x\|_{J}$. The theorem is thus proved under the supplementary hypothesis.

For the general case, we can construct a decreasing sequence of norms $\left\{\|\cdot\|_{n}\right\}_{n \in \mathbb{N}}$ such that each unit ball $\left\{x \in V:\|x\|_{n} \leqslant 1\right\}$ verifies the supplementary hypothesis mentioned above and that the sequence

$$
\sup _{0 \neq x \in V} \frac{\|x\|_{n}}{\|x\|}
$$

converges to 1 when $n \rightarrow+\infty$. For each $n \in \mathbb{N}$, let $\|\cdot\|_{n, J}$ be the John norm associated to the norm $\|\cdot\|_{n}$. If we identify the set of Euclidean or Hermitian norms on $V$ with $\Theta^{+}$, we obtain that these John norms actually lies in a bounded subset of $\Theta$. Therefore there exists a subsequence of $\left\{\|\cdot\|_{n, J}\right\}_{n \in \mathbb{N}}$ which converges in $\Theta$, whose limite should be the John norm associated with $\|\cdot\|$ by the uniqueness of the John norm. Without loss of generality we may assume that $\left\{\|\cdot\|_{n, J}\right\}_{n \in \mathbb{N}}$ converges in $\Theta$. By what we have established above, for any $n \in \mathbb{N}$ one has

$$
\forall x \in V, \quad\|x\|_{n, J} \leqslant\|x\|_{n} \leqslant r^{1 / 2}\|x\|_{n, J} .
$$

By taking the limit when $n \rightarrow+\infty$, we obtain the result announced in the theorem.

Remark 1.2.55. - Let $(V,\|\cdot\|)$ be a finite-dimensional normed vector space over $\mathbb{R}$ or $\mathbb{C}$ (equipped with the usual absolute value). Since $(V,\|\cdot\|)$ is reflexive (see Proposition 1.1.18), we deduce that the dual norm $\|\cdot\|_{J, *}$ is the unique norm on $V^{\vee}$ which is bounded from below by $\|\cdot\|_{*}$ and such that the corresponding determinant norm $\|\cdot\|_{J, *, \text { det }}$ is minimal. In particular, one has

$$
\Delta\left(V^{\vee},\|\cdot\|_{*}\right)=\frac{\|\cdot\|_{J, *, \operatorname{det}}}{\|\cdot\|_{*, \operatorname{det}}} .
$$

Similarly, one has

$$
\Delta(V,\|\cdot\|)=\frac{\|\cdot\|_{L, \operatorname{det}}}{\|\cdot\|_{\operatorname{det}}},
$$

where $\|\cdot\|_{L}$ is the unique Euclidean or Hermitian norm on $V$ which is bounded from below by $\|\cdot\|$ and such that $\|\cdot\|_{L, \operatorname{det}}$ is minimal (called the Löwner norm of $\|\cdot\|$ ), which is also equal to $\|\cdot\|_{*, J, *}$. Theorem 1.2 .54 then leads to

$$
\max \left\{\Delta(V,\|\cdot\|), \Delta\left(V^{\vee},\|\cdot\|_{*}\right)\right\} \leqslant \operatorname{rk}(V)^{\operatorname{rk}(V) / 2}
$$

We denote by $\lambda(V,\|\cdot\|)$ the constant $\|\eta\|_{L, \text { det }} \cdot\left\|\eta^{\vee}\right\|_{J, * \text {,det }}$, where $\eta$ is an arbitrary nonzero element in $\operatorname{det}(V)$, and $\eta^{\vee}$ is its dual element in $\operatorname{det}\left(V^{\vee}\right)$. With this notation, by (1.38) in $\$ 1.2 .7$ one has

$$
\Delta\left(V^{\vee},\|\cdot\|_{*}\right) \Delta(V,\|\cdot\|)=\lambda(V,\|\cdot\|) \delta(V,\|\cdot\|) .
$$


Note that one has $\|\cdot\|_{J} \leqslant\|\cdot\|$ by definition. Hence we obtain

$$
\lambda(V,\|\cdot\|)=\frac{\|\cdot\|_{L, \operatorname{det}}}{\|\cdot\|_{J, \operatorname{det}}} \geqslant \frac{\|\cdot\|_{L, \operatorname{det}}}{\|\cdot\|_{\operatorname{det}}}=\Delta(V,\|\cdot\|),
$$

where the first equality comes from Proposition 1.2.47. Therefore the relation (1.45) leads to $\Delta\left(V^{\vee},\|\cdot\|_{*}\right) \geqslant \delta(V,\|\cdot\|)$. Since $\delta(V,\|\cdot\|)$ and $\lambda(V,\|\cdot\|)$ are both invariant by duality, one obtains

$$
\begin{aligned}
\delta(V,\|\cdot\|) & \leqslant \min \left\{\Delta(V,\|\cdot\|), \Delta\left(V^{\vee},\|\cdot\|_{*}\right)\right\} \\
& \leqslant \max \left\{\Delta(V,\|\cdot\|), \Delta\left(V^{\vee},\|\cdot\|_{*}\right)\right\} \leqslant \lambda(V,\|\cdot\|) .
\end{aligned}
$$

Remark 1.2.56. - We can deduce from Theorem 1.2.54 a similar result for seminorms. Let $(V,\|\cdot\|)$ be a finite-dimensional seminormed vector space over $k$. Let $\|\cdot\|_{J}$ be the John norm associated with $\|\cdot\|^{\sim}$. It is induced by an inner product on $V / N_{\|\cdot\|}$. Let $\|\cdot\|_{J}$ be the seminorm on $V$ given by the composition of $\|\cdot\|_{J}^{\sim}$ with the canonical projection $V \rightarrow V /\|\cdot\|$. It is a seminorm induced by a semidefinite inner product. Moreover, the following inequalities hold

$$
\|\cdot\|_{J} \leqslant\|\cdot\| \leqslant \operatorname{rk}_{k}\left(V / N_{\|\cdot\|}\right)^{1 / 2}\|\cdot\|_{J} .
$$

1.2.9. Hilbert-Schmidt tensor norm. - In this subsection, we assume that the absolute value $|\cdot|$ is Archimedean.

Let $V$ and $W$ be finite-dimensional vector spaces over $k$, equipped with semidefinite inner products. For $f \in \operatorname{Hom}_{k}\left(V^{*}, W\right)$, the adjoint operator $f^{*}: W \rightarrow V^{*}$ of $f$ is defined by $\langle f(\alpha), y\rangle=\left\langle\alpha, f^{*}(y)\right\rangle_{*}$ for all $\alpha \in V^{*}$ and $y \in W$. Note that the adjoint operator $f^{*}$ exists for any $f$ because the product $\langle,\rangle_{*}$ on $V^{*}$ is positive definite. We can equip $\operatorname{Hom}_{k}\left(V^{*}, W\right)$ with the following semidefinite inner product $\langle,\rangle_{\mathrm{HS}}$ :

$$
\forall f, g \in \operatorname{Hom}_{k}\left(V^{*}, W\right), \quad\langle f, g\rangle_{\mathrm{HS}}:=\operatorname{Tr}\left(f^{*} \circ g\right) .
$$

This semidefinite inner product defines a seminorm on $\operatorname{Hom}_{k}\left(V^{*}, W\right)$, which induces by the canonical linear map $V \otimes_{k} W \rightarrow \operatorname{Hom}_{k}\left(V^{*}, W\right)$ a seminorm $\|\cdot\|_{\mathrm{HS}}$ on $V \otimes_{K} W$, called the orthogonal tensor product of the seminorms of $V$ and $W$, or Hilbert-Schmidt seminorm. Note that if $\left\{x_{i}\right\}_{i=1}^{n}$ and $\left\{y_{j}\right\}_{j=1}^{m}$ are respectively orthogonal basis of $V$ and $W$, then $\left\{x_{i} \otimes y_{j}\right\}_{i \in\{1, \ldots, n\}, j \in\{1, \ldots, m\}}$ is an orthogonal basis of $V \otimes_{k} W$ with respect to $\langle,\rangle_{\mathrm{HS}}$. Moreover, for $x \in V$ and $y \in W$ one has

$$
\|x \otimes y\|_{\mathrm{HS}}=\|x\| \cdot\|y\| .
$$

In particular, if $V$ and $W$ are both of rank 1 over $k$, then the orthogonal tensor product seminorm on $V \otimes_{k} W$ coincides with the $\varepsilon$-tensor product and the $\pi$-tensor product seminorms. In this case we just call it the tensor product seminorm.

The dual norm on $V^{*} \otimes_{k} W^{*}$ of the Hilbert-Schmidt seminorm on $V \otimes_{k} W$ coincides with the orthogonal tensor product of the dual norms on $V^{*}$ and $W^{*}$. Moreover, the orthogonal tensor product is commutative, namely the isomorphism from $V \otimes_{k} W$ to $W \otimes_{k} V$ given by the transposition is actually an isometry under orthogonal tensor 
product seminorms. Similarly, the orthogonal tensor product is associative. More precisely, given three finite-dimensional vector spaces $U, V$ and $W$ over $k$, equipped with semidefinite inner products, the natural isomorphism from $\left(U \otimes_{k} V\right) \otimes_{k} W$ to $U \otimes_{k}\left(V \otimes_{k} W\right)$ is an isometry for orthogonal tensor product seminorms.

The following assertion, which is similar to Proposition 1.2.36, studies the quotient norm of the orthogonal tensor product.

Proposition 1.2.5\%. - Let $V$ and $W$ be finite-dimensional seminormed vector spaces over $k, V_{0}$ be a k-vector subspace of $V$, and $Q$ be the quotient vector space $V / V_{0}$ equipped with the quotient seminorm. We assume that the seminorms of $V$ and $W$ are induced by semidefinite inner products. Then the canonical isomorphism $\left(V \otimes_{k} W\right) /\left(V_{0} \otimes_{k} W\right) \rightarrow Q \otimes_{k} W$ is an isometry, where we consider the orthogonal tensor product seminorms on $V \otimes_{k} W$ and $Q \otimes_{k} W$, and the quotient seminorm on $\left(V \otimes_{k} W\right) /\left(V_{0} \otimes_{k} W\right)$.

Proof. - By the Gram-Schmidt process we can identify the quotient space $Q$ with the orthogonal supplementary of $V_{0}$ in $V$. Let $\boldsymbol{e}=\left\{e_{i}\right\}_{i=1}^{n}$ be an orthogonal basis of $V$ such that card $\left(\boldsymbol{e} \cap V_{0}\right)=\operatorname{rk}_{k}\left(V_{0}\right)$. Then the projection $V \rightarrow Q$ defines an isometry between $Q$ and the vector subspace $V_{1}$ of $V$ generated by $\boldsymbol{e} \backslash V_{0}$. Let $\boldsymbol{f}=\left\{f_{j}\right\}_{j=1}^{m}$ be an orthogonal basis of $W$. Then the basis $\boldsymbol{e} \otimes \boldsymbol{f}=\left\{e_{i} \otimes f_{j}\right\}_{(i, j) \in\{1, \ldots, n\} \times\{1, \ldots, m\}}$ of $V \otimes_{k} W$ is orthogonal. Moreover, one has

$$
\operatorname{card}\left((\boldsymbol{e} \otimes \boldsymbol{f}) \cap\left(V_{0} \otimes_{k} W\right)\right)=\operatorname{rk}_{k}\left(V_{0} \otimes_{k} W\right) .
$$

Thus $\left(\boldsymbol{e} \backslash V_{0}\right) \otimes \boldsymbol{f}$ forms an orthogonal basis of $Q \otimes_{k} W$ equipped with the quotient seminorm (where we identify $Q$ with $V_{0}^{\perp}$ ). Hence the quotient seminorm on $Q \otimes_{k} W$ identifies with the orthogonal tensor product seminorm.

Proposition 1.2.58. - Let $\left(V,\|\cdot\|_{V}\right)$ and $\left(W,\|\cdot\|_{W}\right)$ be finite-dimensional seminormed vector space over $k, V_{0}$ be a k-vector subspace of $V$ and $\|\cdot\|_{V_{0}}$ be the restriction of $\|\cdot\|_{V}$ on $V_{0}$. We assume that the absolute value $|\cdot|$ is Archimedean and that the seminorms $\|\cdot\|_{V}$ and $\|\cdot\|_{W}$ are induced by semidefinite inner products. Let $\|\cdot\|$ be the orthogonal tensor product of $\|\cdot\|_{V}$ and $\|\cdot\|_{W}$, and $\|\cdot\|_{0}$ be the orthogonal tensor product of $\|\cdot\|_{V_{0}}$ and $\|\cdot\|_{W}$. Then $\|\cdot\|_{0}$ identifies with the restriction of $\|\cdot\|$ to $V_{0} \otimes_{k} W$.

Proof. - Note that $\|\cdot\|_{*}$ identifies with the orthogonal tensor product of $\|\cdot\|_{V, *}$ and $\|\cdot\|_{W, *}$, and $\|\cdot\|_{0, *}$ identifies with the orthogonal tensor product of $\|\cdot\|_{V_{0}, *}$ and $\|\cdot\|_{W, *}$. Moreover, by Lemma 1.2.48, $\|\cdot\|_{V_{0}, *}$ identifies with the quotient norm of $\|\cdot\|_{*}$ by the canonical surjective map $V^{*} \rightarrow V_{0}^{*}$. By Proposition 1.2.57, we obtain that $\|\cdot\|_{0, *}$ identifies with the quotient norm of $\|\cdot\|_{*}$ by the canonical surjective map $V^{*} \otimes_{k}$ $W^{*} \rightarrow V_{0}^{*} \otimes_{k} W^{*}$. Therefore, by Proposition 1.1.20, $\|\cdot\|_{0}$ is the restriction of $\|\cdot\|$ to $V_{0} \otimes_{k} W$.

The following proposition compares $\varepsilon$-tensor product to orthogonal tensor product. 
Proposition 1.2.59. - Let $V$ and $W$ be finite-dimensional vector spaces over $k$, equipped with semidefinite inner products. Let $\|\cdot\|_{\varepsilon}$ and $\|\cdot\|_{\mathrm{HS}}$ be respectively the $\varepsilon$ tensor product seminorm and the orthogonal tensor product seminorm on $V \otimes_{k} W$. Then $\|\cdot\|_{\varepsilon} \leqslant\|\cdot\|_{\mathrm{HS}} \leqslant \min \left\{\operatorname{rk}_{k}\left(V^{*}\right), \operatorname{rk}_{k}\left(W^{*}\right)\right\}^{1 / 2}\|\cdot\|_{\varepsilon}$.

Proof. - Without loss of generality, we may assume that $\operatorname{rk}_{k}\left(V^{*}\right) \leqslant \operatorname{rk}_{k}\left(W^{*}\right)$. Let $\varphi$ be an element of $V \otimes_{k} W$, viewed as a $k$-linear map from $V^{*}$ to $W$. Let $\lambda_{1} \geqslant \ldots \geqslant \lambda_{r}$ be the eigenvalues of the positive semidefinite operator $\varphi^{*} \circ \varphi$. By definition, the Hilbert-Schmidt seminorm of $\varphi$ is $\|\varphi\|_{\mathrm{HS}}=\left(\lambda_{1}+\ldots+\lambda_{r}\right)^{1 / 2}$. Moreover, the operator seminorm of $\varphi$ is $\lambda_{1}^{1 / 2}$. In fact, if $\alpha_{1}, \ldots, \alpha_{r}$ are eigenvectors of $\varphi^{*} \circ \varphi$ of eigenvalues $\lambda_{1}, \ldots, \lambda_{r}$, respectively, then for any $\left(a_{1}, \ldots, a_{r}\right) \in k^{r}$ one has

$$
\begin{aligned}
& \left\langle\varphi\left(a_{1} \alpha_{1}+\ldots+a_{r} \alpha_{r}\right), \varphi\left(a_{1} \alpha_{1}+\ldots+a_{r} \alpha_{r}\right)\right\rangle \\
= & \left\langle\varphi^{*}\left(\varphi\left(a_{1} \alpha_{1}+\ldots+a_{r} \alpha_{r}\right)\right), a_{1} \alpha_{1}+\ldots+a_{r} \alpha_{r}\right\rangle=\sum_{i=1}^{r}\left|a_{i}\right|^{2} \lambda_{i} .
\end{aligned}
$$

Therefore one has $\|\varphi\|_{\varepsilon} \leqslant\|\varphi\|_{\mathrm{HS}} \leqslant \sqrt{r}\|\varphi\|_{\varepsilon}$.

By using the duality between the $\varepsilon$-tensor product and $\pi$-tensor product (see Proposition 1.1.57), we deduce from the previous proposition the following corollary.

Corollary 1.2.60. — Let $V$ and $W$ be finite-dimensional vector spaces over $k$, equipped with semidefinite inner products. Let $\|\cdot\|_{\pi}$ and $\|\cdot\|_{\mathrm{HS}}$ be respectively the $\pi$ tensor product and the orthogonal tensor product norms on $V \otimes_{k} W$. Then one has

$$
\|\cdot\|_{\pi} \geqslant\|\cdot\|_{\mathrm{HS}} \geqslant \min \left\{\operatorname{rk}_{k}\left(V^{*}\right), \operatorname{rk}_{k}\left(W^{*}\right)\right\}^{-1 / 2}\|\cdot\|_{\pi} .
$$

The following proposition expresses the Hilbert-Schmidt norm of endomorphisms in terms of the operator norm.

Proposition 1.2.61. - Let $V$ be a vector space of finite rank $r$ over $k$, equipped with an inner product $\langle$,$\rangle . Let f: V \rightarrow V$ be an endomorphism of $V$. Then one has

$$
\langle f, f\rangle_{\mathrm{HS}}=\sum_{i=1}^{r} \inf _{\substack{g \in \operatorname{End}_{k}(V) \\ \operatorname{rk}(g) \leqslant i-1}}\|f-g\|^{2},
$$

where $\|\cdot\|$ denotes the operator norm on $\operatorname{End}_{k}(V)$.

Proof. - Let $\left\{e_{i}\right\}_{i=1}^{r}$ be an orthonormal basis of $V$ consisting of the eigenvectors of the self-adjoint operator $f^{*} \circ f$. For any $i \in\{1, \ldots, r\}$, let $\lambda_{i}$ be the eigenvalue of $f^{*} \circ f$ corresponding to the eigenvector $e_{i}$. Without loss of generality, we may assume that $\lambda_{1} \geqslant \ldots \geqslant \lambda_{r}$. Since the self-adjoint operator $f^{*} \circ f$ is positive semidefinite, one has $\lambda_{r} \geqslant 0$. By definition, one has $\langle f, f\rangle_{\mathrm{HS}}=\sum_{i=1}^{r} \lambda_{i}$. In the following, we prove that, for any $i \in\{1, \ldots, r\}$, one has

$$
\inf _{\substack{g \in \operatorname{End}_{k}(V) \\ \operatorname{rk}(g) \leqslant i-1}}\|f-g\|^{2}=\lambda_{i} .
$$


Let $\pi$ be the orthogonal projection of $V$ to the vector subspace generated by $\left\{e_{1}, \ldots, e_{i-1}\right\}$. Then the endomorphism $f \circ \pi$ has rank $\leqslant i-1$. Moreover, since any orthogonal projection is self-adjoint, one has

$$
(f-f \pi)^{*}(f-f \pi)=\left(f^{*}-\pi f^{*}\right)(f-f \pi)=f^{*} f+\pi f^{*} f \pi-\pi f^{*} f-f^{*} f \pi .
$$

In particular, the linear endomorphism $(f-f \pi)^{*}(f-f \pi)$ sends an element $a_{1} e_{1}+$ $\cdots+a_{r} e_{r}$ in $V$ to $a_{i} \lambda_{i} e_{i}+\cdots+a_{r} \lambda_{r} e_{r}$. Hence the operator norm of $(f-f \pi)^{*}(f-f \pi)$, which is equal to the square of the operator norm of $f-f \pi$, is $\lambda_{i}$.

It remains to prove that, for any $k$-linear endomorphism $g \in \operatorname{End}_{k}(V)$ of rank $\leqslant i-1$, one has $\|f-g\|^{2} \geqslant \lambda_{i}$. Let $W$ be the vector subspace of $V$ generated by $\left\{e_{1}, \ldots, e_{i}\right\}$. Since $g$ has rank $\leqslant i-1$, one has $\operatorname{Ker}(g) \cap W \neq\{0\}$. Let $x$ be a non-zero vector in $\operatorname{Ker}(g) \cap W$. One has

$$
\|(f-g)(x)\|^{2}=\|f(x)\|^{2}=\langle f(x), f(x)\rangle=\left\langle f^{*}(f(x)), x\right\rangle .
$$

Since $x \in W$, one obtains $\|(f-g)(x)\|^{2} \geqslant \lambda_{i}\|x\|^{2}$. Therefore $\|f-g\|^{2} \geqslant \lambda_{i}$. The proposition is thus proved.

Proposition 1.2.62. - Let $V$ be a finite-dimensional vector space over $k$, equipped with a semidefinite inner product $\langle\rangle,$,$r be the rank of V$, and $\|\cdot\|_{\operatorname{det}^{\prime}}$ be the quotient seminorm of the orthogonal tensor product seminorm on $V^{\otimes r}$ by the canonical quotient map $V^{\otimes r} \rightarrow \operatorname{det}(V)$. Then one has $\|\cdot\|_{\text {det }}=(r !)^{1 / 2}\|\cdot\|_{\operatorname{det}^{\prime}}$.

Proof. - If the seminorm associated with the semidefinite inner product on $V$ is not a norm, then both seminorms $\|\cdot\|_{\text {det }}$ and $\|\cdot\|_{\operatorname{det}^{\prime}}$ vanish. It then suffices to treat the case where $\langle$,$\rangle is an inner product.$

Let $\varphi$ be an element in $V^{\otimes r}$. Let $\left\{e_{i}\right\}_{i=1}^{r}$ be an orthonormal basis of $V$. We write $\varphi$ into the form

$$
\varphi=\sum_{I=\left(i_{1}, \ldots, i_{r}\right) \in\{1, \ldots, r\}^{r}} a_{I}\left(e_{i_{1}} \otimes \cdots e_{i_{r}}\right) .
$$

Then the canonical image $\eta$ of $\varphi$ in $\operatorname{det}(V)$ is

$$
\left(\sum_{\sigma \in \mathfrak{S}_{r}} \operatorname{sgn}(\sigma) a_{(\sigma(1), \ldots, \sigma(r))}\right) e_{1} \wedge \cdots \wedge e_{r}
$$

where $\mathfrak{S}_{r}$ is the symmetric group of order $r$. Hence the Cauchy-Schwarz inequality leads to

$$
\|\eta\|_{\text {det }}=\left|\sum_{\sigma \in \mathfrak{S}_{r}} \operatorname{sgn}(\sigma) a_{(\sigma(1), \ldots, \sigma(r))}\right| \leqslant(r !)^{1 / 2}\|\varphi\|_{\mathrm{HS}},
$$

where $\|\cdot\|_{\mathrm{HS}}$ denotes the orthogonal tensor product norm on $V^{\otimes r}$. The equality is attained when $\varphi$ is of the form $\sum_{\sigma \in \mathfrak{S}_{r}} \operatorname{sgn}(\sigma) e_{\sigma(1)} \otimes \cdots \otimes e_{\sigma(r)}$. The proposition is thus proved.

Proposition 1.2.63. - Let $V$ and $W$ be finite-dimensional seminormed vector spaces. We assume that the seminorms of $V$ and $W$ are induced by semidefinite inner products. Let $n$ and $m$ be respectively the ranks of $V$ and $W$ over $k$. We equip 
the tensor product $V \otimes_{k} W$ with the orthogonal tensor product seminorm $\|\cdot\|_{\mathrm{HS}}$. Then the canonical isomorphism $\operatorname{det}\left(V \otimes_{k} W\right) \rightarrow \operatorname{det}(V)^{\otimes m} \otimes \operatorname{det}(W)^{\otimes n}$ is an isometry, where we consider the determinant of the Hilbert-Schmidt seminorm on $\operatorname{det}\left(V \otimes_{k} W\right)$, and the tensor product $\|\cdot\|^{\prime}$ of determinant seminorms on $\operatorname{det}(V)^{\otimes m} \otimes \operatorname{det}(W)^{\otimes n}$.

Proof. - The assertion is trivial when at least one of the seminorms of $V$ and $W$ is not a norm since in this case both seminorms $\|\cdot\|_{\text {HS, det }}$ and $\|\cdot\|^{\prime}$ vanish.

In the following, we assume that $V$ and $W$ are equipped with inner products. Let $\left\{e_{i}\right\}_{i=1}^{n}$ and $\left\{f_{j}\right\}_{j=1}^{m}$ be respectively orthonormal bases of $V$ and $W$, which are also Hadamard bases (by Proposition 1.2.25). Then $\left\{e_{i} \otimes f_{j}\right\}_{(i, j) \in\{1, \ldots, n\} \times\{1, \ldots, m\}}$ is an orthonormal basis of $V \otimes_{k} W$. By Proposition 1.2.25, it is also an Hadamard basis. Hence one has

$$
\left\|\bigwedge_{i=1}^{n} \bigwedge_{j=1}^{m}\left(e_{i} \otimes f_{j}\right)\right\|_{\mathrm{HS}, \mathrm{det}}=1=\left\|\left(e_{1} \wedge \cdots \wedge e_{n}\right)^{\otimes m} \otimes\left(f_{1} \wedge \cdots \wedge f_{m}\right)^{\otimes n}\right\|^{\prime} .
$$

The proposition is thus proved.

\subsection{Extension of scalars}

In this section, we suppose given a field extension $K$ of $k$ equipped with a complete absolute value which extends $|\cdot|$ on $k$. By abuse of notation, we still use the notation $|\cdot|$ to denote the extended absolute value on $K$. We can thus consider $K$ as a normed vector space over $k$, which is ultrametric if and only if the absolute value $|\cdot|$ on $k$ is non-Archimedean.

Let $(V,\|\cdot\|)$ be a finite-dimensional seminormed vector space over $k$. We consider the natural $K$-linear map from $V_{K}=V \otimes_{k} K$ to $\mathscr{L}\left(V^{*}, K\right)$ which sends $x \otimes a \in V \otimes_{k} K$ (with $x \in V$ and $a \in K)$ to the $k$-linear map $\left(f \in V^{*}\right) \mapsto a f(x)$. We equip $V^{*}$ with the dual norm and $\mathscr{L}\left(V^{*}, K\right)$ with the operator norm, which induces by this natural $K$-linear map a seminorm on $V \otimes_{k} K$ denoted by $\|\cdot\|_{K, \varepsilon}$ and called the seminorm induced by $\|\cdot\|$ by $\varepsilon$-extension of scalars. Note that the seminorm $\|\cdot\|_{K, \varepsilon}$ is necessarily ultrametric if $k$ is non-Archimedean. Moreover, if $(K,|\cdot|)$ is reflexive as normed vector space over $k$ (this condition is satisfied notably when $K / k$ is a finite extension), then the seminorm $\|\cdot\|_{K, \varepsilon}$ is the $\varepsilon$-tensor product of $\|\cdot\|$ and the absolute value on $K$ (viewed as a norm on the $k$-vector space $K$ ), see Remark 1.1.53.

We denote by $\|\cdot\|_{K, \pi}$ the $\pi$-tensor product seminorm on $V \otimes_{k} K$ of the seminorm $\|\cdot\|$ on $V$ and the absolute value $|\cdot|$ on $K$, called the seminorm induced by $\|\cdot\|$ by $\pi$-extension of scalars. If $|\cdot|$ is Archimedean and if the seminorm $\|\cdot\|$ is induced by a semidefinite inner product, we denote by $\|\cdot\|_{K, \mathrm{HS}}$ the orthogonal tensor product of the seminorm $\|\cdot\|$ on $V$ and the absolute value $|\cdot|$ on $K$ (in the Archimedean case the extension $K / k$ is always finite), called the seminorm induced by $\|\cdot\|$ by orthogonal extension of scalars. 
In what follows, an element $x \in V$ is often considered as an element of $V_{K}=V \otimes_{k} K$ by the inclusion map $V \rightarrow V \otimes_{k} K$ sending $x$ to $x \otimes 1$.

1.3.1. Basic properties. - In this subsection, we discuss some basic behaviour of norms induced by extension of scalars.

Proposition 1.3.1. — Let $(V,\|\cdot\|)$ be a finite-dimensional seminormed vector space over $k$.

(1) For any $x \in V$ one has $\|x\|_{K, \varepsilon}=\|x\|_{* *}$, where $\|\cdot\|_{* *}$ denotes the double dual seminorm of $\|\cdot\|$. In particular, if either $(k,|\cdot|)$ is Archimedean or $(V,\|\cdot\|)$ is ultrametric, then one has $\|x\|_{K, \varepsilon}=\|x\|$ for any $x \in V$.

(2) For any $x \in V$ one has $\|x\|_{K, \pi}=\|x\|$. If $|\cdot|$ is Archimedean and $\|\cdot\|$ is induced by a semidefinite inner product, for any $x \in V$ one has $\|x\|_{K, \mathrm{HS}}=\|x\|$.

(3) For any $y \in V_{K}$ one has $\|y\|_{K, \varepsilon} \leqslant\|y\|_{K, \pi}$. If $(k,|\cdot|)$ is $\mathbb{R}$ equipped with the usual absolute value, $K=\mathbb{C}$, and $\|\cdot\|$ is induced by a semidefinite inner product, then for any $y \in V_{\mathbb{C}}$ one has

$$
\begin{gathered}
\|y\|_{\mathbb{C}, \varepsilon} \leqslant\|y\|_{\mathbb{C}, \mathrm{HS}} \leqslant\|y\|_{\mathbb{C}, \pi}, \\
\min \left\{\operatorname{rk}_{\mathbb{R}}\left(V^{*}\right), 2\right\}^{-1 / 2}\|y\|_{\mathbb{C}, \pi} \leqslant\|y\|_{\mathbb{C}, \mathrm{HS}} \leqslant \min \left\{\operatorname{rk}_{\mathbb{R}}\left(V^{*}\right), 2\right\}^{1 / 2}\|y\|_{\mathbb{C}, \varepsilon} .
\end{gathered}
$$

Proof. - (1) Let $\ell_{x}: V^{*} \rightarrow k$ be the $k$-linear map sending any bounded linear form $f \in V^{*}$ to $f(x)$. Let $\widetilde{\ell}_{x}: V^{*} \rightarrow K$ be the composition $\ell_{x}$ with the inclusion map $k \rightarrow K$. The operator norms of $\ell_{x}$ and $\tilde{\ell}_{x}$ are the same. Therefore one has $\|x\|_{K}=\|x\|_{* *}$. The last assertion comes from Proposition 1.1.18 and Corollary 1.2.12

The first assertion of (2) follow from Remark 1.1.56 in the Archimedean case and from Proposition 1.2.50 in the non-Archimedean case (note that the absolute value on $K$, viewed as a norm when we consider $K$ as a vector space over $k$, is ultrametric once $|\cdot|$ is non-Archimedean). The second assertion follows from (1.48) in $\$ 1.2 .9$,

(3) The first assertion follows from (1) and Proposition 1.1.54.

In the case where $(k,|\cdot|)$ is $\mathbb{R}$ equipped with the usual absolute value, $K=\mathbb{C}$, and $\|\cdot\|$ is induced by an inner product, the inequalities follow from Proposition 1.2.59 and Corollary 1.2 .60 .

\section{Remark 1.3.2. -}

(1) Note that $\|\cdot\|$ and its double dual seminorm $\|\cdot\|_{* *}$ induce the same dual norm on $V^{*}$ (see Proposition 1.2.14). Hence they induce the same seminorm on $V_{K}$ by $\varepsilon$-extension of scalars. Moreover, if $K=k$, then $\|\cdot\|_{K, \varepsilon}$ identifies with the double dual seminorm of $\|\cdot\|$ on $V$.

(2) Assume that $k=\mathbb{R}, K=\mathbb{C}$ and $|\cdot|$ is the usual absolute value on $\mathbb{R}$. Suppose that the norm $\|\cdot\|$ is induced by a semidefinite inner product $\langle$,$\rangle . Note that \langle$, induces a semidefinite inner product $\langle,\rangle_{\mathbb{C}}$, given by

$$
\forall x, y, x^{\prime}, y^{\prime} \in V, \quad\left\langle x+i y, x^{\prime}+i y^{\prime}\right\rangle_{\mathbb{C}}=\left\langle x, x^{\prime}\right\rangle+\left\langle y, y^{\prime}\right\rangle+i\left(\left\langle x, y^{\prime}\right\rangle-\left\langle y, x^{\prime}\right\rangle\right) .
$$


Note that the seminorm corresponding to $\langle,\rangle_{\mathbb{C}}$ identifies with the orthogonal tensor product $\|\cdot\|_{\mathbb{C}, H S}$ of $\|\cdot\|$ and $|\cdot|$. Moreover, an orthogonal basis of $(V,\langle\rangle$, remains to be an orthogonal basis of $\left(V_{\mathbb{C}},\langle,\rangle\right)_{\mathbb{C}}$, which implies that $\|\cdot\|_{\mathbb{C}, \mathrm{HS}}$ is the unique seminorm on $V_{\mathbb{C}}$ extending $\|\cdot\|$ which is induced by a semidefinite inner product. In particular, one has $\langle,\rangle_{*, \mathbb{C}}=\langle,\rangle_{\mathbb{C}, *}$, where $\langle,\rangle_{*}$ denotes the dual inner product of $\langle$,$\rangle (see \$ 1.2 .1$ ), and hence $\|\cdot\|_{\mathbb{C}, H S, *}=\|\cdot\|_{*, \mathbb{C}, \mathrm{HS}}$.

(3) Let $V$ be a seminormed vector space of rank 1 on $k$. Then the norms $\|\cdot\|_{K, \varepsilon}$ and $\|\cdot\|_{K, \pi}$ are the same since they take the same value on a non-zero vector of $V$ (by Proposition 1.3.1). Similarly, if $|\cdot|$ is Archimedean then one has $\|\cdot\|_{K, \varepsilon}=$ $\|\cdot\|_{K, \mathrm{HS}}=\|\cdot\|_{K, \pi}$. We just call this seminorm the seminorm induced by $\|\cdot\|$ by extension of scalars and denote it by $\|\cdot\|_{K}$.

Proposition 1.3.3. - Let $(V,\|\cdot\|)$ be a finite-dimensional seminormed vector space over $k$ and $N=N_{\|\cdot\|}$ be the null space of $\|\cdot\|$.

(1) The null spaces of the seminorms $\|\cdot\|_{K, \varepsilon}$ and $\|\cdot\|_{K, \pi}$ are both equal to $N_{K}$.

(2) A linear form on the $K$-vector space $V_{K}$ is bounded with respect to the seminorm $\|\cdot\|_{K, \varepsilon}$ if and only if it is bounded with respect to $\|\cdot\|_{K, \pi}$. Moreover, the underlying vector spaces of $\left(V_{K},\|\cdot\|_{K, \varepsilon}\right)^{*}$ and $\left(V_{K},\|\cdot\|_{K, \pi}\right)^{*}$ are both canonically isomorphic to $\left(V_{K} / N_{K}\right)^{\vee}$.

(3) The quotient norm on $V_{K} / N_{K}$ induced by $\|\cdot\|_{K, \varepsilon}$ (resp. $\|\cdot\|_{K, \pi}$ ) identifies with the $\varepsilon$-extension of scalars $\|\cdot\|_{K, \varepsilon}^{\sim}$ (resp. the $\pi$-extension of scalars $\|\cdot\|_{K, \pi}^{\sim}$ ) of the norm $\|\cdot\|^{\sim}$.

Proof. - (1) Note that the relation $\|\cdot\|_{K, \varepsilon} \leqslant\|\cdot\|_{K, \pi}$ holds (see Proposition 1.3.1 (3)], so that it is sufficient to see that (i) $\|x\|_{K, \pi}=0$ for $x \in N_{K}$ and (ii) $\|x\|_{K, \varepsilon}>0$ for $x \in V_{K} \backslash N_{K}$. Let $\left\{e_{i}\right\}_{i=1}^{n}$ be a basis of $V$ such that $\left\{e_{i}\right\}_{i=1}^{r}$ forms a basis of $N$.

(i) We write $x$ in the form $x=\lambda_{1} e_{1}+\cdots+\lambda_{r} e_{r}$ with $\left(\lambda_{1}, \ldots, \lambda_{r}\right) \in K^{r}$. One has

$$
0 \leqslant\|x\|_{K, \pi} \leqslant \sum_{i=1}^{r}\left|\lambda_{i}\right| \cdot\left\|e_{i}\right\|_{K, \pi}=\sum_{i=1}^{r}\left|\lambda_{i}\right| \cdot\left\|e_{i}\right\|=0,
$$

where the first equality comes from Proposition $1.3 .1(2)$

(ii) We set $x=\lambda_{1} e_{1}+\cdots+\lambda_{n} e_{n}\left(\lambda_{1}, \ldots, \lambda_{n} \in K\right)$. If $x$ does not belong to $N_{K}$, then there exists $j \in\{r+1, \ldots, n\}$ such that $\lambda_{j} \neq 0$. Note that $e_{j}^{\vee}$ belongs to $V^{*}$. Hence

$$
\|x\|_{K, \varepsilon} \geqslant \frac{\left|\lambda_{j}\right|}{\left\|e_{j}^{\vee}\right\|_{*}}>0 .
$$

(2) By Corollary 1.1.13 (3), a linear form on a finite-dimensional seminormed vector space is bounded if and only if it vanishes on the null space of the seminorm. By (1) we obtain that both seminorms $\|\cdot\|_{K, \varepsilon}$ and $\|\cdot\|_{K, \pi}$ admit $N_{K}$ as the null space. Hence we obtain the required result.

(3) We identify $V^{*}$ with $(V / N)^{\vee}$ and then the norm $\|\cdot\|_{*}$ identifies with the dual norm of $\|\cdot\|^{\sim}$. Therefore by definition for any $x \in V_{K}$ one has $\|x\|_{K, \varepsilon}=\|[x]\|_{K, \varepsilon}^{\sim}$, 
where $[x]$ denotes the class of $x$ in $V_{K} / N_{K}$. The case of $\pi$-extension of scalars comes from Proposition 1.1.58

The following proposition proves a universal property of the $\pi$-extension of scalars.

Proposition 1.3.4. - Let $(V,\|\cdot\|)$ be a finite-dimensional seminormed vector space over $k$. If $\|\cdot\|^{\prime}$ is a seminorm on $V_{K}$ whose restriction to $V$ is bounded from above by $\|\cdot\|$, then the seminorm $\|\cdot\|^{\prime}$ is bounded from above by $\|\cdot\|_{K, \pi}$. In particular, $\|\cdot\|_{K, \pi}$ is the largest seminorm on $V_{K}=V \otimes_{k} K$ extending $\|\cdot\|$.

Proof. - For any $x \in V$ and $a \in K$ one has

$$
\|x \otimes a\|^{\prime}=|a| \cdot\|x \otimes 1\|^{\prime} \leqslant|a| \cdot\|x\| .
$$

By Proposition 1.1.54, we obtain $\|\cdot\|^{\prime} \leqslant\|\cdot\|_{K, \pi}$.

Proposition 1.3.5. - Let $\left(V_{1},\|\cdot\|_{1}\right)$ and $\left(V_{2},\|\cdot\|_{2}\right)$ be finite-dimensional seminormed vector spaces over $k$, and $\|\cdot\|$ be the $\pi$-tensor product seminorm of $\|\cdot\|_{1}$ and $\|\cdot\|_{2}$. Then the norm $\|\cdot\|_{K, \pi}$ identifies with the $\pi$-tensor product of $\|\cdot\|_{1, K, \pi}$ and $\|\cdot\|_{2, K, \pi} \cdot$

Proof. - Let $\|\cdot\|^{\prime}$ be the $\pi$-tensor product of $\|\cdot\|_{1, K, \pi}$ and $\|\cdot\|_{2, K, \pi}$. If $s$ is an element of $V_{1} \otimes_{k} V_{2}$, which is written as $s=x_{1} \otimes y_{1}+\cdots+x_{n} \otimes y_{n}$, with $\left(x_{1}, \ldots, x_{n}\right) \in V_{1}^{n}$ and $\left(y_{1}, \ldots, y_{2}\right) \in V_{2}^{n}$. Then one has

$$
\|s\|^{\prime} \leqslant \sum_{i=1}^{n}\left\|x_{i}\right\|_{1, K, \pi} \cdot\left\|y_{i}\right\|_{2, K, \pi}=\sum_{i=1}^{n}\left\|x_{i}\right\|_{1} \cdot\left\|y_{i}\right\|_{2} .
$$

Therefore one has $\|s\|^{\prime} \leqslant\|s\|$. By Proposition 1.3.4, the norm $\|\cdot\|^{\prime}$ is bounded from above by $\|\cdot\|_{K, \pi}$.

To prove the converse inequality, by Proposition 1.1.54 it suffices to show that, for any split tensor $u \otimes v$ in $V_{1, K} \otimes_{K} V_{2, K}$ one has $\|u \otimes v\|_{K, \pi} \leqslant\|u\|_{1, K, \pi} \cdot\|v\|_{2, K, \pi}$. Assume that $u$ and $v$ are written as $u=\lambda_{1} x_{1}+\cdots+\lambda_{n} x_{n}$ and $v=\mu_{1} y_{1}+\cdots+\mu_{m} y_{m}$ with $\left(\lambda_{1}, \ldots, \lambda_{n}\right) \in K^{n},\left(x_{1}, \ldots, x_{n}\right) \in V_{1}^{n},\left(\mu_{1}, \ldots, \mu_{m}\right) \in K^{m}$ and $\left(y_{1}, \ldots, y_{m}\right) \in V_{2}^{m}$. Then one has

$$
\begin{aligned}
\|u \otimes v\|_{K, \pi} & \leqslant \sum_{i=1}^{n} \sum_{j=1}^{m}\left|\lambda_{i} \mu_{j}\right| \cdot\left\|x_{i} \otimes y_{j}\right\|_{K, \pi}=\sum_{i=1}^{n} \sum_{j=1}^{m}\left|\lambda_{i} \mu_{j}\right| \cdot\left\|x_{i} \otimes y_{j}\right\| \\
& =\sum_{i=1}^{n} \sum_{j=1}^{m}\left|\lambda_{i} \mu_{j}\right| \cdot\left\|x_{i}\right\|_{1} \cdot\left\|y_{j}\right\|_{2}=\left(\sum_{i=1}^{n}\left|\lambda_{i}\right| \cdot\left\|x_{i}\right\|_{1}\right)\left(\sum_{j=1}^{m}\left|\mu_{j}\right| \cdot\left\|y_{j}\right\|_{2}\right) .
\end{aligned}
$$

Since the decompositions $u=\lambda_{1} x_{1}+\cdots+\lambda_{n} x_{n}$ and $v=\mu_{1} y_{1}+\cdots+\mu_{m} y_{m}$ are arbitrary, we obtain

$$
\|u \otimes v\|_{K, \pi} \leqslant\|u\|_{1, K, \pi} \cdot\|v\|_{2, K, \pi} .
$$


Proposition 1.3.6. - Assume that $(k,|\cdot|)$ is the field $\mathbb{R}$ equipped with the usual absolute value and $K=\mathbb{C}$. Let $\left(V_{1},\langle,\rangle_{1}\right)$ and $\left(V_{2},\langle,\rangle_{2}\right)$ be finite-dimensional vector spaces over $\mathbb{R}$ equipped with semidefinite inner products, $\|\cdot\|_{1}$ and $\|\cdot\|_{2}$ be seminorms corresponding to $\langle,\rangle_{1}$ and $\langle,\rangle_{2}$, respectively, and $\|\cdot\|$ be the orthogonal tensor product of $\|\cdot\|_{1}$ and $\|\cdot\|_{2}$. Then the seminorm $\|\cdot\|_{\mathbb{C}, \text { HS }}$ identifies with the orthogonal tensor product of $\|\cdot\|_{1, \mathbb{C}, \mathrm{HS}}$ and $\|\cdot\|_{2, \mathbb{C}, \mathrm{HS}}$.

Proof. - Let $\left\{x_{i}\right\}_{i=1}^{n}$ and $\left\{y_{j}\right\}_{j=1}^{m}$ be orthogonal bases of $\left(V_{1},\|\cdot\|_{1}\right)$ and $\left(V_{2},\|\cdot\|_{2}\right)$, respectively. Then $\left\{x_{i} \otimes y_{j}\right\}_{(i, j) \in\{1, \ldots, n\} \times\{1, \ldots, m\}}$ is an orthogonal basis of $\left(V_{1} \otimes_{\mathbb{R}} V_{2},\|\cdot\|\right)$ and hence is an orthogonal basis of $\left(V_{1, \mathbb{C}} \otimes_{\mathbb{C}} V_{2, \mathbb{C}},\|\cdot\|_{\mathbb{C}, \mathrm{HS}}\right)$. Moreover, $\left\{x_{i}\right\}_{i=1}^{n}$ and $\left\{y_{j}\right\}_{j=1}^{m}$ are also orthogonal bases of $\left(V_{1, \mathbb{C}},\|\cdot\|_{1, \mathbb{C}, \mathrm{HS}}\right)$ and $\left(V_{2, \mathbb{C}},\|\cdot\|_{2, \mathbb{C}, \mathrm{HS}}\right)$, respectively. Hence $\left\{x_{i} \otimes y_{j}\right\}_{(i, j) \in\{1, \ldots, n\} \times\{1, \ldots, m\}}$ is an orthogonal basis of $V_{1, \mathbb{C}} \otimes \mathbb{C} V_{2, \mathbb{C}}$ with respect to the orthogonal tensor product of $\|\cdot\|_{1, \mathbb{C}, \mathrm{HS}}$ and $\|\cdot\|_{2, \mathbb{C}, \mathrm{HS}}$. The proposition is thus proved.

Proposition 1.3.7. - Let $V$ be a finite-dimensional vector space over $k$, and $\|\cdot\|_{1}$ and $\|\cdot\|_{2}$ be two norms on $V$.

(1) One has

$$
d\left(\|\cdot\|_{1, K, \varepsilon},\|\cdot\|_{2, K, \varepsilon}\right)=d\left(\|\cdot\|_{1, * *},\|\cdot\|_{2, * *}\right) \leqslant d\left(\|\cdot\|_{1},\|\cdot\|_{2}\right) .
$$

In particular, if both norms $\|\cdot\|_{1}$ and $\|\cdot\|_{2}$ are reflexive, then

$$
d\left(\|\cdot\|_{1, K, \varepsilon},\|\cdot\|_{2, K, \varepsilon}\right)=d\left(\|\cdot\|_{1},\|\cdot\|_{2}\right) .
$$

(2) One has $d\left(\|\cdot\|_{1, K, \pi},\|\cdot\|_{2, K, \pi}\right)=d\left(\|\cdot\|_{1},\|\cdot\|_{2}\right)$.

(3) Assume that $|\cdot|$ is Archimedean and that $\|\cdot\|_{1}$ and $\|\cdot\|_{2}$ are induced by inner products. Then $d\left(\|\cdot\|_{1, K, \mathrm{HS}},\|\cdot\|_{2, K, \mathrm{HS}}\right)=d\left(\|\cdot\|_{1},\|\cdot\|_{2}\right)$.

Proof. - (1) By Proposition 1.1.43, one has $d\left(\|\cdot\|_{1, *},\|\cdot\|_{2, *}\right) \leqslant d\left(\|\cdot\|_{1},\|\cdot\|_{2}\right)$. By the same argument as that of the proof of Proposition 1.1.43, we can show that $d\left(\|\cdot\|_{1, K, \varepsilon},\|\cdot\|_{2, K, \varepsilon}\right) \leqslant d\left(\|\cdot\|_{1, *},\|\cdot\|_{2, *}\right)$. Hence we obtain the inequality $d\left(\|\cdot\|_{1, K, \varepsilon},\|\cdot\|_{2, K, \varepsilon}\right) \leqslant d\left(\|\cdot\|_{1},\|\cdot\|_{2}\right)$. By Proposition 1.2.14, for $i \in\{1,2\},\|\cdot\|_{i}$ and $\|\cdot\|_{i, * *}$ induce the same dual norm on $V^{*}$, and hence $\|\cdot\|_{i, K, \varepsilon}=\|\cdot\|_{i, * *, K, \varepsilon}$. Therefore the above argument actually leads to $d\left(\|\cdot\|_{1, K, \varepsilon},\|\cdot\|_{2, K, \varepsilon}\right) \leqslant d\left(\|\cdot\|_{1, * *},\|\cdot\|_{2, * *}\right)$. Conversely, by Proposition 1.3.1] we obtain that $\|\cdot\|_{1, K, \varepsilon}$ and $\|\cdot\|_{2, K, \varepsilon}$ extend $\|\cdot\|_{1, * *}$ and $\|\cdot\|_{2, * *}$, respectively. Hence one has $d\left(\|\cdot\|_{1, K, \varepsilon},\|\cdot\|_{2, K, \varepsilon}\right) \geqslant d\left(\|\cdot\|_{1, * *},\|\cdot\|_{2, * *}\right)$.

The inequality $d\left(\|\cdot\|_{1, * *},\|\cdot\|_{2, * *}\right) \leqslant d\left(\|\cdot\|_{1},\|\cdot\|_{2}\right)$ comes from Proposition 1.1.43 The equality holds when both norms $\|\cdot\|_{1}$ and $\|\cdot\|_{2}$ are reflexive.

(2) By Proposition 1.3.1 (2), $\|\cdot\|_{1, K, \pi}$ and $\|\cdot\|_{2, K, \pi}$ extend $\|\cdot\|_{1}$ and $\|\cdot\|_{2}$, respectively, and hence $d\left(\|\cdot\|_{1, K, \pi},\|\cdot\|_{2, K, \pi}\right) \geqslant d\left(\|\cdot\|_{1},\|\cdot\|_{2}\right)$. In the following, we prove the converse inequality. If we set $\delta=d\left(\|\cdot\|_{1},\|\cdot\|_{2}\right)$, then $\mathrm{e}^{-\delta} \leqslant\|s\|_{1} /\|s\|_{2} \leqslant \mathrm{e}^{\delta}$ for $s \in V \backslash\{0\}$, that is, $\|\cdot\|_{1} \leqslant \mathrm{e}^{\delta}\|\cdot\|_{2}$ and $\|\cdot\|_{2} \leqslant \mathrm{e}^{\delta}\|\cdot\|_{1}$. By Proposition 1.3.4, one has $\|\cdot\|_{1, K, \pi} \leqslant \mathrm{e}^{\delta}\|\cdot\|_{2, K, \pi}$. By the same reason, $\|\cdot\|_{2, K, \pi} \leqslant \mathrm{e}^{\delta}\|\cdot\|_{1, K, \pi}$. Hence the inequality $d\left(\|\cdot\|_{1, K, \pi},\|\cdot\|_{2, K, \pi}\right) \leqslant \delta=d\left(\|\cdot\|_{1},\|\cdot\|_{2}\right)$ holds. 
(3) It suffices to treat the case where $k=\mathbb{R}$ and $K=\mathbb{C}$. By Proposition 1.3.1 (2) $\|\cdot\|_{1, K, \mathrm{HS}}$ and $\|\cdot\|_{2, K, \mathrm{HS}}$ extend $\|\cdot\|_{1}$ and $\|\cdot\|_{2}$, respectively, and hence $d\left(\|\cdot\|_{1, K, \mathrm{HS}},\|\cdot\|_{2, K, \mathrm{HS}}\right) \geqslant d\left(\|\cdot\|_{1},\|\cdot\|_{2}\right)$. As in (2), if we set $\delta=d\left(\|\cdot\|_{1},\|\cdot\|_{2}\right)$, then $\|\cdot\|_{1} \leqslant \mathrm{e}^{\delta}\|\cdot\|_{2}$ and $\|\cdot\|_{2} \leqslant \mathrm{e}^{\delta}\|\cdot\|_{1}$. Let $z$ be an element of $V_{\mathbb{C}}$, which is written as $z=x+i y$, where $x$ and $y$ are vectors in $V$. Then one has

$$
\|z\|_{1, \mathbb{C}, \mathrm{HS}}^{2}=\|x\|_{1}^{2}+\|y\|_{1}^{2} \leqslant \mathrm{e}^{2 \delta}\left(\|x\|_{2}^{2}+\|y\|_{2}^{2}\right)=\mathrm{e}^{2 \delta}\|z\|_{2, \mathbb{C}, \mathrm{HS}}^{2} .
$$

Therefore $\|\cdot\|_{1, \mathrm{HS}, \mathbb{C}} \leqslant \mathrm{e}^{\delta}\|\cdot\|_{2, \mathrm{HS}, \mathbb{C} \cdot}$. Similarly, $\|\cdot\|_{2, \mathbb{C}, \mathrm{HS}} \leqslant \mathrm{e}^{\delta}\|\cdot\|_{1, \mathbb{C}, \mathrm{HS}}$, so that the inequality $d\left(\|\cdot\|_{1, \mathbb{C}, \mathrm{HS}},\|\cdot\|_{2, \mathbb{C}, \mathrm{HS}}\right) \leqslant \delta=d\left(\|\cdot\|_{1},\|\cdot\|_{2}\right)$ holds.

Proposition 1.3.8. - Let $\left(V_{1},\|\cdot\|_{1}\right)$ and $\left(V_{2},\|\cdot\|_{2}\right)$ be finite-dimensional seminormed vector spaces over $k$, and $f: V_{1} \rightarrow V_{2}$ be a bounded $k$-linear map. Let $f_{K}: V_{1, K} \rightarrow V_{2, K}$ be the $K$-linear map induced by $f$.

(1) If we consider the seminorms $\|\cdot\|_{1, K, \varepsilon}$ and $\|\cdot\|_{2, K, \varepsilon}$ on $V_{1, K}$ and $V_{2, K}$, respectively, then the operator seminorm of $f_{K}$ is bounded from above by that of $f^{*}$ (which is bounded from above by $\|f\|$, see Proposition 1.1.22). The equality $\left\|f_{K}\right\|=\|f\|$ holds when $\left(V_{2},\|\cdot\|_{2}\right)$ is reflexive.

(2) If we consider the seminorms $\|\cdot\|_{1, K, \pi}$ and $\|\cdot\|_{2, K, \pi}$ on $V_{1, K}$ and $V_{2, K}$, respectively, then the operator seminorms of $f_{K}$ and $f$ are the same.

(3) Assume that $(k,|\cdot|)$ is $\mathbb{R}$ equipped with the usual absolute value, $K=\mathbb{C}$ and that $\|\cdot\|_{1}$ and $\|\cdot\|_{2}$ are induced by semidefinite inner products. If we consider the norms $\|\cdot\|_{1, K, \mathrm{HS}}$ and $\|\cdot\|_{2, K, \mathrm{HS}}$ on $V_{1, K}$ and $V_{2, K}$, respectively, then the operator seminorms of $f_{K}$ and $f$ are the same.

Proof. - (1) Let $\varphi$ be an element of $V_{1, K}$, viewed as a $k$-linear map from $V_{1}^{*}$ to $K$. Then the element $f_{K}(\varphi) \in V_{2, K}$, viewed as a $k$-linear form from $V_{2}^{*}$ to $K$, sends $\beta \in V_{2}^{*}$ to $\varphi\left(f^{*}(\beta)\right) \in K$. One has

$$
\left|\varphi\left(f^{*}(\beta)\right)\right| \leqslant\|\varphi\|_{1, K, \varepsilon} \cdot\left\|f^{*}\right\| \cdot\|\beta\|_{2, *} .
$$

Therefore $\left\|f_{K}(\varphi)\right\|_{2, K, \varepsilon} \leqslant\left\|f^{*}\right\| \cdot\|\varphi\|_{1, K, \varepsilon}$. Since $\varphi$ is arbitrary, one has $\left\|f_{K}\right\| \leqslant\left\|f^{*}\right\|$. The first assertion is thus proved.

Assume that $\left(V_{2},\|\cdot\|_{2}\right)$ is reflexive. For any element $x \in V_{1}$ one has

$$
\left\{\begin{array}{l}
\|x\|_{1, K}=\|x\|_{1, * *} \leqslant\|x\|_{1}, \\
\left\|f_{K}(x)\right\|_{2, K, \varepsilon}=\|f(x)\|_{2, K, \varepsilon}=\|f(x)\|_{2, * *}=\|f(x)\|_{2}
\end{array}\right.
$$

since $\left(V_{2},\|\cdot\|_{2}\right)$ is reflexive. Therefore one has

$$
\left\|f_{K}\right\| \geqslant \sup _{x \in V_{1} \backslash N_{\|\cdot \cdot\|_{1}}} \frac{\left\|f_{K}(x)\right\|_{2, K}}{\|x\|_{1, K}} \geqslant \sup _{x \in V_{1} \backslash N_{\|\cdot\|_{1}}} \frac{\|f(x)\|_{2}}{\|x\|_{1}}=\|f\| .
$$

(2) Since the norms $\|\cdot\|_{1, K, \pi}$ and $\|\cdot\|_{2, K, \pi}$ extend $\|\cdot\|_{1}$ and $\|\cdot\|_{2}$, respectively (see Proposition 1.3.1), the operator seminorm $\|f\|$ is bounded from above by $\left\|f_{K}\right\|$. It suffices to prove the converse inequality. Let $y$ be an element in $V_{1, K}$, which is written 
as $y=x_{1} \otimes a_{1}+\cdots+x_{n} \otimes a_{n}$, where $\left(x_{1}, \ldots, x_{n}\right) \in V_{1}^{n}$ and $\left(a_{1}, \ldots, a_{n}\right) \in K^{n}$. Then one has $f_{K}(y)=f\left(x_{1}\right) \otimes a_{1}+\cdots+f\left(x_{n}\right) \otimes a_{n}$. Hence

$$
\left\|f_{K}(y)\right\|_{2, K, \pi} \leqslant \sum_{i=1}^{n}\left|a_{i}\right| \cdot\left\|f\left(x_{i}\right)\right\|_{2} \leqslant\|f\| \sum_{i=1}^{n}\left|a_{i}\right| \cdot\left\|x_{i}\right\|_{1} .
$$

As the decomposition $y=x_{1} \otimes a_{1}+\cdots+x_{n} \otimes a_{n}$ is arbitrary, we obtain

$$
\|f(y)\|_{2, K, \pi} \leqslant\|f\| \cdot\|y\|_{1, K, \pi} .
$$

(3) Since the seminorms $\|\cdot\|_{1, \mathbb{C}, \mathrm{HS}}$ and $\|\cdot\|_{2, \mathbb{C}, \mathrm{HS}}$ extend $\|\cdot\|_{1}$ and $\|\cdot\|_{2}$, respectively (see Proposition 1.3.1), the operator seminorm $\|f\|$ is bounded from above by $\left\|f_{\mathbb{C}}\right\|$. Let $z$ be an element of $V_{1, \mathbb{C}}$, written as $u+i v$, where $u$ and $v$ are vectors in $V_{1}$. Then one has $f_{\mathbb{C}}(z)=f(u)+i f(v)$. Therefore

$$
\left\|f_{\mathbb{C}}(z)\right\|^{2}=\|f(u)\|_{2}^{2}+\|f(v)\|_{2}^{2} \leqslant\|f\|^{2}\left(\|u\|_{1}^{2}+\|u\|_{2}^{2}\right)=\|f\|^{2} \cdot\|z\|_{1, \mathbb{C}, \mathrm{FS}}^{2} .
$$

Hence $\left\|f_{\mathbb{C}}\right\|^{2}=\|f\|^{2}$.

1.3.2. Direct sums. - In this subsection, we discuss the behaviour of direct sums under scalar extension. We fix a continuous and convex function $\psi:[0,1] \rightarrow[0,1]$ such that $\max \{t, 1-t\} \leqslant \psi(t)$ for any $t \in[0,1]$ (cf. \$1.1.10).

Proposition 1.3.9. - Let $\left(V,\|\cdot\|_{V}\right)$ and $\left(W,\|\cdot\|_{W}\right)$ be finite-dimensional seminormed vector spaces over $k$. Let $\|\cdot\|_{\psi}$ be the $\psi$-direct sum of $\|\cdot\|_{V}$ and $\|\cdot\|_{W}$. Then for $(f, g) \in V_{K} \oplus W_{K}$ one has

$$
\max \left\{\|f\|_{V, K, \varepsilon},\|g\|_{W, K, \varepsilon}\right\} \leqslant\|(f, g)\|_{\psi, K, \varepsilon} .
$$

The equality holds if either $(k,|\cdot|)$ is non-Archimedean or $\psi(t)=\max \{t, 1-t\}$ for any $t \in[0,1]$. Moreover, for any $(f, g) \in V_{K} \oplus W_{K}$ one has

$$
\|(f, 0)\|_{\psi, K, \varepsilon}=\|f\|_{V, K, \varepsilon}, \quad\|(0, g)\|_{\psi, K, \varepsilon}=\|g\|_{W, K, \varepsilon} .
$$

Proof. - By Proposition 1.1.49, the dual norm $\|\cdot\|_{\psi, *}$ is a certain direct sum of $\|\cdot\|_{V, *}$ and $\|\cdot\|_{W, *}$. Hence one has

$$
\|\alpha\|_{V, *}+\|\beta\|_{W, *} \geqslant\|(\alpha, \beta)\|_{\psi, *} \geqslant \max \left\{\|\alpha\|_{V, *},\|\beta\|_{V, *}\right\} .
$$

Therefore, for any $(f, g) \in V_{K} \oplus W_{K}$ one has

$$
\|(f, g)\|_{\psi, K, \varepsilon} \geqslant \sup _{\substack{(\alpha, \beta) \in V^{*} \oplus W^{*} \\(\alpha, \beta) \neq(0,0)}} \frac{|f(\alpha)+g(\beta)|}{\|\alpha\|_{V, *}+\|\beta\|_{W, *}}=\max \left\{\|f\|_{V, K, \varepsilon},\|g\|_{V, K, \varepsilon}\right\}
$$

which proves (1.51). Moreover, for any $f \in V_{K}$ one has

$$
\|(f, 0)\|_{\psi, K, \varepsilon} \leqslant \sup _{\substack{(\alpha, \beta) \in V^{*} \oplus W^{*} \\(\alpha, \beta) \neq(0,0)}} \frac{|f(\alpha)|}{\max \left\{\|\alpha\|_{V, *},\|\beta\|_{W, *}\right\}}=\|f\|_{V, K, \varepsilon} .
$$

Therefore, by (1.51) we obtain the equality $\|(f, 0)\|_{\psi, K, \varepsilon}=\|f\|_{V, K}$. Similarly, for any $g \in W_{K}$ one has $\|(0, g)\|_{\psi, K, \varepsilon}=\|g\|_{W, K, \varepsilon}$. 
Finally, we proceed with the proof of the equality part of (1.51). If $(k,|\cdot|)$ is non-Archimedean, then the seminorm $\|\cdot\|_{\psi, K, \varepsilon}$ is ultrametric and hence by (1.52) one has

$$
\forall(f, g) \in V_{K} \oplus W_{K}, \quad\|(f, g)\|_{\psi, K, \varepsilon} \leqslant \max \left\{\|f\|_{V, K, \varepsilon},\|g\|_{W, K, \varepsilon}\right\},
$$

which leads to (by (1.51) ) the equality

$$
\forall(f, g) \in V_{K} \oplus W_{K}, \quad\|(f, g)\|_{\psi, K, \varepsilon}=\max \left\{\|f\|_{V, K, \varepsilon},\|g\|_{W, K, \varepsilon}\right\} .
$$

In the case where $k$ is Archimedean and $\psi(t)=\max \{t, 1-t\}$ for any $t \in[0,1]$, one has $\|(\alpha, \beta)\|_{\psi, *}=\|\alpha\|_{V, *}+\|\beta\|_{W, *}$ for any $(\alpha, \beta) \in V^{\vee} \oplus W^{\vee}$. Therefore

$$
\|(f, g)\|_{\psi, K, \varepsilon}=\sup _{\substack{(\alpha, \beta) \in V^{*} \oplus W^{*} \\(\alpha, \beta) \neq(0,0)}} \frac{|f(\alpha)+g(\beta)|}{\|\alpha\|_{V, *}+\|\beta\|_{W, *}}=\max \left\{\|f\|_{V, K, \varepsilon},\|g\|_{V, K, \varepsilon}\right\} .
$$

Remark 1.3.10. - Let $\psi$ be an element in $\mathscr{S}$ (see \$1.1.10), which corresponds to an absolute normalised norm $\|\cdot\|$ on $\mathbb{R}^{2}$. Let $\psi_{*}$ be the element in $\mathscr{S}$ corresponding to the dual norm $\|\cdot\|_{*}$ (see Definition 1.1.48). Suppose given finite-dimensional seminormed vector spaces $\left(V,\|\cdot\|_{V}\right)$ and $\left(W,\|\cdot\|_{W}\right)$ over $\mathbb{R}$ (equipped with the usual absolute value). By Proposition 1.1.49 (2), the dual norm of $\|\cdot\|_{\psi}$ (the $\psi$-direct sum of $\|\cdot\|_{V}$ and $\left.\|\cdot\|_{W}\right)$ identifies with the $\psi_{*}$-direct sum of $\|\cdot\|_{V, *}$ and $\|\cdot\|_{W, *}$. Therefore, for any $(f, g) \in V_{\mathbb{C}} \oplus W_{\mathbb{C}}$, one has

$$
\begin{aligned}
\|(f, g)\|_{\psi, \mathbb{C}, \varepsilon} & =\sup _{\substack{(\alpha, \beta) \in V^{*} \oplus W^{*} \\
(\alpha, \beta) \neq(0,0)}} \frac{|f(\alpha)+g(\beta)|}{\left\|\left(\|\alpha\|_{V, *},\|\beta\|_{W, *}\right)\right\|_{*}} \\
& \leqslant \sup _{\substack{(\alpha, \beta) \in V^{*} \oplus W^{*} \\
(\alpha, \beta) \neq(0,0)}} \frac{\|f\|_{V, \mathbb{C}, \varepsilon} \cdot\|\alpha\|_{V, *}+\|g\|_{W, \mathbb{C}, \varepsilon} \cdot\|\beta\|_{W, *}}{\left\|\left(\|\alpha\|_{V, *},\|\beta\|_{W, *}\right)\right\|_{*}} \\
& =\left\|\left(\|f\|_{V, \mathbb{C}, \varepsilon},\|g\|_{W, \mathbb{C}, \varepsilon}\right)\right\| .
\end{aligned}
$$

In other words, the seminorm $\|\cdot\|_{\psi, \mathbb{C}, \varepsilon}$ is bounded from above by the $\psi$-direct sum of $\|\cdot\|_{V, \mathbb{C}, \varepsilon}$ and $\|\cdot\|_{W, \mathbb{C}, \varepsilon}$.

Proposition 1.3.11. - Let $\left(V,\|\cdot\|_{V}\right)$ and $\left(W,\|\cdot\|_{W}\right)$ be finite-dimensional seminormed vector spaces over $k$. Let $\|\cdot\|_{\psi}$ be the $\psi$-direct sum of $\|\cdot\|_{V}$ and $\|\cdot\|_{W}$, and $\|\cdot\|_{K, \pi, \psi}$ be the $\psi$-direct sum of $\|\cdot\|_{V, K, \pi}$ and $\|\cdot\|_{W, K, \pi}$. Then $\|\cdot\|_{K, \pi, \psi} \leqslant\|\cdot\|_{\psi, K, \pi}$.

Proof. - Let $(x, y)$ be an element in $V \oplus W$. One has

$$
\begin{aligned}
\|(x, y)\|_{K, \pi, \psi} & =\left(\|x\|_{V, K, \pi}+\|y\|_{W, K, \pi}\right) \psi\left(\frac{\|x\|_{V, K, \pi}}{\|x\|_{V, K, \pi}+\|y\|_{W, K, \pi}}\right) \\
& =\left(\|x\|_{V}+\|y\|_{W}\right) \psi\left(\frac{\|x\|_{V}}{\|x\|_{V}+\|y\|_{W}}\right)=\|(x, y)\|_{\psi},
\end{aligned}
$$

where the second equality comes from Proposition 1.3.1 (2) Therefore the seminorm $\|\cdot\|_{K, \pi, \psi}$ extends $\|\cdot\|_{\psi}$. By Proposition 1.3.4, it is bounded from above by $\|\cdot\|_{\psi, K, \pi}$. 
Proposition 1.3.12. - Assume that $(k,|\cdot|)$ is the real field $\mathbb{R}$ equipped with the usual absolute value. Let $\left(V,\langle,\rangle_{V}\right)$ and $\left(W,\langle,\rangle_{W}\right)$ be finite-dimensional vector spaces over $\mathbb{R}$, equipped with semidefinite inner products, $\|\cdot\|_{V}$ and $\|\cdot\|_{W}$ be seminorms associated with $\langle,\rangle_{V}$ and $\langle,\rangle_{W}$, respectively, and $\|\cdot\|$ be the orthogonal direct sum of $\|\cdot\|_{V}$ and $\|\cdot\|_{W}$. Then $\|\cdot\|_{\mathbb{C}, \mathrm{HS}}$ is the orthogonal direct sum of $\|\cdot\|_{V, \mathbb{C}, \mathrm{HS}}$ and $\|\cdot\|_{W, \mathbb{C}, \mathrm{HS}}$.

Proof. - Let $\|\cdot\|^{\prime}$ be the orthogonal direct sum of $\|\cdot\|_{V, \mathbb{C}, \mathrm{HS}}$ and $\|\cdot\|_{W, \mathbb{C}, \mathrm{HS}}$. It is a seminorm on $V_{\mathbb{C}} \oplus W_{\mathbb{C}}$ which is induced by a semidefinite inner product. Moreover, for any $(x, y) \in V \oplus W$ one has

$$
\|(x, y)\|^{\prime}=\left(\|x\|_{V, \mathbb{C}, \mathrm{HS}}^{2}+\|y\|_{V, \mathbb{C}, \mathrm{HS}}^{2}\right)^{1 / 2}=\left(\|x\|_{V}^{2}+\|y\|_{V}^{2}\right)^{1 / 2}=\|(x, y)\|,
$$

where the second equality comes from Proposition $1.3 .1,(2)$ Therefore, $\|\cdot\|^{\prime}$ is a seminorm extending $\|\cdot\|$ which is induced by a semidefinite inner product and hence one has $\|\cdot\|^{\prime}=\|\cdot\|_{\mathbb{C}, \mathrm{HS}}($ see Remark 1.3.2) .

1.3.3. Orthogonality. - In this subsection, we discuss the preservation of the orthogonality under extension of scalars, and its consequences. We have seen in Remark 1.3.2 (2) that the orthonormality is preserved by the orthogonal extension of scalars.

Proposition 1.3.13. — Let $(V,\|\cdot\|)$ be a finite-dimensional seminormed vector space over $k$, and $\alpha$ be a real number in $] 0,1]$. If $\boldsymbol{e}=\left\{e_{i}\right\}_{i=1}^{r}$ is an $\alpha$-orthogonal basis of $V$ with respect to the norm $\|\cdot\|$, then it is also an $\alpha$-orthogonal basis of $V_{K}$ with respect to the norms $\|\cdot\|_{K, \varepsilon}$ and $\|\cdot\|_{K, \pi}$.

Proof. - Let $\boldsymbol{e}^{\vee}=\left\{e_{i}^{\vee}\right\}_{i=1}^{r}$ be the dual basis of $\boldsymbol{e}$. By Proposition 1.2.11 the intersection $e^{\vee} \cap V^{*}$ is an $\alpha$-orthogonal bases of $V^{*}$, and one has $\left\|e_{i}^{\vee}\right\|_{*} \leqslant \alpha^{-1}\left\|e_{i}\right\|^{-1}$ for any $e_{i}^{\vee} \in e^{\vee} \cap V^{*}$ (see Lemma 1.2.10). If $x=a_{1} e_{1}+\cdots+a_{r} e_{r}$ is an element in $V_{K}$, where $\left(a_{1}, \ldots, a_{r}\right) \in K^{r}$, and if $\ell_{x}: V^{*} \rightarrow K$ is the $k$-linear map sending $\varphi \in V^{*}$ to $a_{1} \varphi\left(e_{1}\right)+\cdots+a_{r} \varphi\left(e_{r}\right)$, then for any $i \in\{1, \ldots, r\}$ such that $e_{i}^{\vee} \in V^{*}$ (or equivalently, $\left.e_{i} \notin N_{\|\cdot\|}\right)$ one has

$$
\left\|\ell_{x}\right\|_{K, \varepsilon} \geqslant \frac{\left|\ell_{x}\left(e_{i}^{\vee}\right)\right|}{\left\|e_{i}^{\vee}\right\|_{*}}=\frac{\left|a_{i}\right|}{\left\|e_{i}^{\vee}\right\|_{*}} \geqslant \alpha\left|a_{i}\right| \cdot\left\|e_{i}\right\| \geqslant \alpha\left|a_{i}\right| \cdot\left\|e_{i}\right\|_{K, \varepsilon},
$$

where the last inequality comes from Proposition 1.3.1 and the relation (1.5). Therefore $\boldsymbol{e}$ is also an $\alpha$-orthogonal basis for $\|\cdot\|_{K, \varepsilon}$.

By Proposition 1.3.1 $(3)$, one has $\|\cdot\|_{K, \varepsilon} \leqslant\|\cdot\|_{K, \pi}$. Therefore

$$
\|x\|_{K, \pi} \geqslant\|x\|_{K, \varepsilon} \geqslant \alpha \max _{i \in\{1, \ldots, r\}}\left|a_{i}\right| \cdot\left\|e_{i}\right\|=\alpha \max _{i \in\{1, \ldots, r\}}\left|a_{i}\right| \cdot\left\|e_{i}\right\|_{K, \pi},
$$

where the last equality comes from Proposition 1.3 .1$]$

By using the preservation of orthogonality of bases, we prove an universal property of $\varepsilon$-extension of scalars, which is an ultrametric analogue of Proposition 1.3.4. 
Proposition 1.3.14. - Assume that the absolute value $|\cdot|$ is non-Archimedean. Let $V$ be a finite-dimensional vector space over $k$, equipped with a seminorm $\|\cdot\|$. Let $\|\cdot\|_{K}^{\prime}$ be an ultrametric seminorm on $V_{K}$ whose restriction to $V$ is bounded from above by $\|\cdot\|_{* *}$. Then one has $\|\cdot\|_{K}^{\prime} \leqslant\|\cdot\|_{K, \varepsilon}$. In particular, $\|\cdot\|_{K, \varepsilon}$ is the largest ultrametric seminorm on $V_{K}$ which extends $\|\cdot\|_{* *}$.

Proof. - By Proposition [1.3.13, if $\alpha$ is an element of ]0, $1\left[\right.$ and if $\left\{e_{i}\right\}_{i=1}^{r}$ is an $\alpha$ orthogonal basis of $(V,\|\cdot\|)$, then $\left\{e_{i}\right\}_{i=1}^{r}$ is also an $\alpha$-orthogonal basis of $\left(V_{K},\|\cdot\|_{K, \varepsilon}\right)$. In particular, for any $\left(\lambda_{1}, \ldots, \lambda_{r}\right) \in K^{r}$ one has

$$
\left\|\lambda_{1} e_{1}+\cdots+\lambda_{r} e_{r}\right\|_{K}^{\prime} \leqslant \max _{i \in\{1, \ldots, r\}}\left|\lambda_{i}\right| \cdot\left\|e_{i}\right\|_{* *} \leqslant \alpha^{-1}\left\|\lambda_{1} e_{1}+\cdots+\lambda_{r} e_{r}\right\|_{K, \varepsilon} .
$$

Since $(V,\|\cdot\|)$ admits an $\alpha$-orthogonal basis for any $\alpha \in] 0,1\left[\right.$, we obtain $\|\cdot\|_{K}^{\prime} \leqslant\|\cdot\|_{K, \varepsilon}$ for any ultrametric seminorm $\|\cdot\|_{K}^{\prime}$ with $\|\cdot\|_{K}^{\prime} \leqslant\|\cdot\|_{* *}$ on $V$.

Corollary 1.3.15. - Let $K^{\prime}$ be an extension of $K$ equipped with a complete absolute value extending that on $K$. Let $(V,\|\cdot\|)$ be a finite-dimensional seminormed vector space over $k$. One has $\|\cdot\|_{K, \downarrow, K^{\prime}, \natural}=\|\cdot\|_{K^{\prime}, \natural}$ on $V_{K^{\prime}}$, where $\natural=\varepsilon$ or $\pi$.

Proof. - The assertion is trivial when the absolute value $|\cdot|$ is Archimedean since in this case $k=\mathbb{R}$ or $\mathbb{C}$ and hence either $k=K$ or $K=K^{\prime}$. In the following, we assume that the absolue value $|\cdot|$ is non-Archimedean.

By Proposition 1.3.14 $\|\cdot\|_{K^{\prime}, \varepsilon}$ is the largest ultrametric seminorm on $V_{K^{\prime}}$ extending the seminorm $\|\cdot\|_{* *}$ on $V$. Moreover, by Proposition 1.3.1, $\|\cdot\|_{K, \varepsilon}$ is an ultrametric seminorm on $V_{K}$ extending $\|\cdot\|_{* *}$, and the seminorm $\|\cdot\|_{K, \varepsilon, K^{\prime}, \varepsilon}$ extends $\|\cdot\|_{K, \varepsilon}$. Therefore one has $\|\cdot\|_{K, \varepsilon, K^{\prime}, \varepsilon} \leqslant\|\cdot\|_{K^{\prime}, \varepsilon}$. By the same reason, as the norm $\|\cdot\|_{K^{\prime}, \varepsilon}$ extends $\|\cdot\|_{* *}$, its restriction to $V_{K}$ is bounded from above by $\|\cdot\|_{K, \varepsilon}$ and hence the restriction of $\|\cdot\|_{K^{\prime}, \varepsilon}$ to $V_{K}$ coincides with $\|\cdot\|_{K, \varepsilon}$ (since we have already shown that $\left.\|\cdot\|_{K, \varepsilon, K^{\prime}, \varepsilon} \leqslant\|\cdot\|_{K^{\prime}, \varepsilon}\right)$. Therefore one has $\|\cdot\|_{K, \varepsilon, K^{\prime}, \varepsilon} \geqslant\|\cdot\|_{K^{\prime}, \varepsilon}$, still by the maximality property (for $\|\cdot\|_{K, \varepsilon, K^{\prime}, \varepsilon}$ ) proved in Proposition 1.3.14.

The case of $\pi$-extension of scalars is quite similar. By Proposition 1.3.1 (2), the seminorm $\|\cdot\|_{K, \pi, K^{\prime}, \pi}$ extends $\|\cdot\|_{K, \pi}$ on $V_{K}$ and hence extends $\|\cdot\|$ on $V$. By the maximality property proved in Proposition 1.3.4, we obtain that $\|\cdot\|_{K, \pi, K^{\prime}, \pi} \leqslant\|\cdot\|_{K^{\prime}, \pi}$. In particular, the restriction of $\|\cdot\|_{K^{\prime}, \pi}$ to $V_{K}$ is bounded from below by $\|\cdot\|_{K, \pi}$. Moreover, this restricted seminorm extends $\|\cdot\|$. Still by the maximality property proved in Proposition 1.3.4, we obtain that the restriction of $\|\cdot\|_{K^{\prime}, \pi}$ to $V_{K}$ is bounded from above by $\|\cdot\|_{K, \pi}$. Therefore the restriction of $\|\cdot\|_{K^{\prime}, \pi}$ to $V_{K}$ coincides with $\|\cdot\|_{K, \pi}$. By Proposition 1.3.4, the norm $\|\cdot\|_{K^{\prime}, \pi}$ is bounded from above by $\|\cdot\|_{K, \pi, K^{\prime}, \pi}$. The proposition is thus proved.

Proposition 1.3.16. — Let $\left(V,\|\cdot\|_{V}\right)$ be a finite-dimensional seminormed vector space over $k, Q$ be a quotient vector space of $V$, and $\|\cdot\|_{Q}$ be the quotient seminorm of $\|\cdot\|_{V}$ on $Q$. 
(1) The seminorm $\|\cdot\|_{Q, K, \pi}$ identifies with the quotient of $\|\cdot\|_{V, K, \pi}$ on $Q_{K}$.

(2) The seminorm $\|\cdot\|_{Q, K, \varepsilon}$ is bounded from above by the quotient seminorm of $\|\cdot\|_{V, K, \varepsilon}$ on $Q_{K}$. The equality holds if one of the following conditions is satisfied: (i) | | | is non-Archimedean; (ii) $|\cdot|$ is Archimedean and $\|\cdot\|_{V}$ is induced by a semidefinite inner product; (iii) $Q$ is of dimension 1 over $k$.

(3) Assume that $|\cdot|$ is Archimedean and $\|\cdot\|_{V}$ is induced by a semidefinite inner product. Then $\|\cdot\|_{Q, K, \mathrm{HS}}$ identifies with the quotient of $\|\cdot\|_{V, K, \mathrm{HS}}$ on $Q_{K}$.

Proof. - (1) follows directly from Proposition 1.1.58

(2) Let $\|\cdot\|_{Q, K}^{\prime}$ be the quotient of the seminorm $\|\cdot\|_{V, K, \varepsilon}$ on $Q_{K}$. Let $p: V \rightarrow Q$ be the canonical linear map. Note that $Q^{*} \subseteq V^{*}$ via $\psi \mapsto \psi \circ p$. Moreover, by Proposition 1.1.20. $\|\psi \circ p\|_{V, *}=\|\psi\|_{Q, *}$ for $\psi \in Q^{*}$. Thus, for $s \in Q_{K}$,

$$
\begin{aligned}
\|s\|_{Q, K}^{\prime} & =\inf _{\substack{x \in V_{K} \\
p_{K}(x)=s}} \sup _{\varphi \in V^{*} \backslash\{0\}} \frac{\left|\varphi_{K}(x)\right|}{\|\varphi\|_{V, *}} \geqslant \inf _{\substack{x \in V_{K} \\
p_{K}(x)=s}} \sup _{\psi \in Q^{*} \backslash\{0\}} \frac{\left|\psi_{K} \circ p_{K}(x)\right|}{\|\psi \circ p\|_{V, *}} \\
& =\sup _{\psi \in Q^{*} \backslash\{0\}} \frac{\left|\psi_{K}(s)\right|}{\|\psi\|_{Q, *}}=\|s\|_{Q, K, \varepsilon},
\end{aligned}
$$

and hence the first assertion holds.

In the following, we prove the equality $\|\cdot\|_{Q, K}^{\prime}=\|\cdot\|_{Q, K, \varepsilon}$ under each of the three conditions (i), (ii) and (iii). We first assume that the condition (i) or (ii) is satisfied. By Proposition 1.1.20, the dual norm $\|\cdot\|_{Q, *}$ identifies with the restriction of $\|\cdot\|_{V, *}$ to $Q^{*}$. By Proposition 1.2.35, we obtain that the seminorm $\|\cdot\|_{Q, K, \varepsilon}$ identifies with the quotient seminorm of $\|\cdot\|_{V, K, \varepsilon}$ on $Q_{K}$.

Assume that the condition (iii) is satisfied and that the absolute value $|\cdot|$ is Archimedean (the non-Archimedean case has already been proved above). Let $f$ be a continuous $k$-linear operator from $Q^{*}$ to $K$. Since $Q$ is assumed to be of dimension 1 over $k$, the image of $f$ is contained in a $k$-linear subspace of dimension 1 in $K$. Therefore by Hahn-Banach theorem we obtain that there exists a continuous $k$-linear map $g: V^{*} \rightarrow K$ extending $f$ such that $f$ and $g$ have the same operator seminorm. Hence the seminorm $\|\cdot\|_{Q, K, \varepsilon}$ identifies with the quotient seminorm of $\|\cdot\|_{V, K, \varepsilon}$ on $Q_{K}$.

(3) follows directly from Proposition 1.2 .57

Proposition 1.3.1\%. - Let $\left(V,\|\cdot\|_{V}\right)$ be a finite-dimensional seminormed vector space over $k$ and $W$ be a vector subspaces of $V$. Let $\|\cdot\|_{W}$ be the restriction of $\|\cdot\|_{V}$ to $W$.

(1) The restriction of $\|\cdot\|_{V, K, \varepsilon}$ to $W_{K}$ is bounded from above by $\|\cdot\|_{W, K, \varepsilon}$. If $|\cdot|$ is Archimedean or $\|\cdot\|_{V}$ is ultrametric, then the restriction of $\|\cdot\|_{V, K, \varepsilon}$ to $W_{K}$ coincides with $\|\cdot\|_{W, K, \varepsilon}$. 
(2) The restriction of $\|\cdot\|_{V, K, \pi}$ to $W_{K}$ is bounded from above by $\|\cdot\|_{W, K, \pi}$. It identifies with $\|\cdot\|_{W, K, \pi}$ if $\|\cdot\|_{V}$ is ultrametric or induced by a semidefinite inner product.

(3) Assume that $|\cdot|$ is Archimedean and that $\|\cdot\|_{V}$ is induced by a semidefinite inner product. Then the restriction of $\|\cdot\|_{V, K, \mathrm{HS}}$ to $W_{K}$ identifies with $\|\cdot\|_{W, K, \mathrm{HS}}$.

Proof. - (1) Assume that $|\cdot|$ is non-Archimedean. By Proposition 1.3.1 (1), the seminorm $\|\cdot\|_{V, K, \varepsilon}$ extends $\|\cdot\|_{V, * *}$. The restriction of $\|\cdot\|_{V, K, \varepsilon}$ to $V$ is then bounded from above by $\|\cdot\|_{V}$, which implies that the restriction of $\|\cdot\|_{V, K, \varepsilon}$ to $W$ is bounded from above by $\|\cdot\|_{W}$. Since $\|\cdot\|_{W, * *}$ is the largest ultrametric seminorm on $W$ which is bounded from above by $\|\cdot\|_{W}$ (see Corollary 1.2.12), we deduce from Proposition 1.3 .14 that the restriction of $\|\cdot\|_{V, K, \varepsilon}$ to $W$ is bounded from above by $\|\cdot\|_{W, * *}$. By Proposition 1.3.14, we obtain that the restriction of $\|\cdot\|_{V, K, \varepsilon}$ to $W_{K}$ is bounded from above by $\|\cdot\|_{W, K, \varepsilon}$.

If $\|\cdot\|_{V}$ is ultrametric or $|\cdot|$ is Archimedean, the dual norm $\|\cdot\|_{W, *}$ coincides with the quotient norm of $\|\cdot\|_{V, *}$ induced by the canonical quotient map $V^{*} \rightarrow W^{*}$ (see Proposition 1.2.35 for the ultrametric case, and Remark 1.1.21 for the Archimedean case). Therefore, any $f \in W_{K}$, viewed as a $k$-linear operator from $W^{*}$ to $K$ or as a $k$-linear operator from $V^{*}$ to $K$, has the same operator norm. In other words, the restriction of $\|\cdot\|_{V, K}$ to $W_{K}$ coincides with $\|\cdot\|_{W, K}$.

(2) follows directly from Proposition 1.2.49 (2)

(3) follows directly from Proposition 1.2 .58

Proposition 1.3.18. - Let $(V,\|\cdot\|)$ be a finite-dimensional normed vector space over $k$. We assume that either $|\cdot|$ is non-Archimedean or the norm $\|\cdot\|$ is induced by an inner product. If $\left\{e_{i}\right\}_{i=1}^{r}$ is an Hadamard basis of $V$, then it is also an Hadamard basis of $V_{K}$ with respect to the norm $\|\cdot\|_{K, \varepsilon}$.

Proof. - By Proposition 1.2.7, $\left\{e_{i}\right\}_{i=1}^{r}$ is an orthogonal basis with respect to $\|\cdot\|$. By Proposition 1.3.13, it is also an orthogonal basis with respect to $\|\cdot\|_{K, \varepsilon}$. Hence it is an Hadamard basis with respect to $\|\cdot\|_{K, \varepsilon}$ (see Propositions 1.2.23 and 1.2.25).

Proposition 1.3.19. - Let $(V,\|\cdot\|)$ be a finite-dimensional seminormed vector space over $k$. Let $\|\cdot\|_{\mathrm{det}, K}$ be the seminorm induced by the determinant seminorm $\|\cdot\|_{\mathrm{det}}$ of $\|\cdot\|$ by extension of scalars.

(1) If either $|\cdot|$ is non-Archimedean or the seminorm $\|\cdot\|$ is induced by a semidefinite inner product, then the determinant seminorm $\|\cdot\|_{K, \varepsilon, \operatorname{det}}$ of $\|\cdot\|_{K, \varepsilon}$ on $\operatorname{det}\left(V_{K}\right)$ coincides with $\|\cdot\|_{\mathrm{det}, K}$.

(2) The determinant seminorm $\|\cdot\|_{K, \pi, \operatorname{det}}$ of $\|\cdot\|_{K, \pi}$ coincides with $\|\cdot\|_{\operatorname{det}, K}$.

(3) Assume that $(k,|\cdot|)$ is $\mathbb{R}$ equipped with the usual absolute value and $\|\cdot\|$ is a seminorm associated with a semidefinite inner product $\langle$,$\rangle . Then the determinant$ seminorm $\|\cdot\|_{\mathbb{C}, \mathrm{HS}, \mathrm{det}}$ of $\|\cdot\|_{\mathbb{C}, \mathrm{HS}}$ coincides with $\|\cdot\|_{\mathrm{det}, \mathbb{C}}$. 
Proof. - (1) If $\|\cdot\|$ is not a norm, then $\|\cdot\|_{K, \varepsilon}$ is not a norm either. In this case both seminorms $\|\cdot\|_{K, \varepsilon, \operatorname{det}}$ and $\|\cdot\|_{\operatorname{det}, K}$ vanish. Hence we may assume without loss of generality that $\|\cdot\|$ is a norm.

We first assume that $(V,\|\cdot\|)$ admits an Hadamard basis $\left\{e_{i}\right\}_{i=1}^{r}$. By Proposition 1.3.18, it is also an Hadamard basis of $\left(V_{K},\|\cdot\|_{K, \varepsilon}\right)$. Moreover, by Propositions 1.2.11 and 1.3.1. for any $i \in\{1, \ldots, r\}$, one has $\left\|e_{i}\right\|=\left\|e_{i}\right\|_{K, \varepsilon}$. In particular, the vector $e_{1} \wedge \cdots \wedge e_{r}$ has the same length under the determinant norms induced by $\|\cdot\|$ and $\|\cdot\|_{K, \varepsilon}$. This establishes the proposition in the particular case where $(V,\|\cdot\|)$ admits an Hadamard basis (and hence in the case where $\|\cdot\|$ is induced by an inner product, see Proposition 1.2.7).

In the following, we assume that the absolute value $|\cdot|$ is non-Archimedean. Let $\alpha$ be an element in $] 0,1\left[\right.$ and $\left\{e_{i}\right\}_{i=1}^{r}$ be an $\alpha$-orthogonal basis of $(V,\|\cdot\|)$. By Proposition 1.3.13, it is also an $\alpha$-orthogonal basis of $\left(V,\|\cdot\|_{K, \varepsilon}\right)$. By Proposition 1.2.23, one has

$$
\begin{aligned}
\left\|e_{1} \wedge \cdots \wedge e_{r}\right\|_{K, \varepsilon, \mathrm{det}} & \geqslant \alpha^{r}\left\|e_{1}\right\|_{K, \varepsilon} \cdots\left\|e_{r}\right\|_{K, \varepsilon} \\
& \geqslant \alpha^{2 r}\left\|e_{1}\right\| \cdots\left\|e_{r}\right\| \geqslant \alpha^{2 r}\left\|e_{1} \wedge \cdots \wedge e_{r}\right\|_{\text {det }},
\end{aligned}
$$

where the second inequality comes from Propositions 1.3 .1 and 1.2.11 Conversely, one has

$$
\left\|e_{1} \wedge \cdots e_{r}\right\|_{K, \varepsilon \operatorname{det}} \leqslant\left\|e_{1}\right\|_{K, \varepsilon} \cdots\left\|e_{r}\right\|_{K, \varepsilon} \leqslant\left\|e_{1}\right\| \cdots\left\|e_{r}\right\| \leqslant \alpha^{-r}\left\|e_{1} \wedge \cdots \wedge e_{r}\right\|_{\text {det }},
$$

where the second inequality comes from Proposition 1.3 .1 and the formula (1.5) in 1.1.5. and the last inequality results from Proposition 1.2.23. Thus one has

$$
\alpha^{-r}\|\cdot\|_{\text {det }} \geqslant\|\cdot\|_{K, \operatorname{det}} \geqslant \alpha^{2 r}\|\cdot\|_{\operatorname{det}} .
$$

Since $\alpha \in] 0,1\left[\right.$ is arbitrary, we obtain $\|\cdot\|_{\operatorname{det}, K}=\|\cdot\|_{K, \varepsilon, \mathrm{det}}$.

(2) Let $r$ be the rank of $V$ over $k$. Note that the $r$-th $\pi$-tensor power of the norm $\|\cdot\|_{K, \pi}$ on $V_{K}^{\otimes K^{r}} \cong\left(V^{\otimes_{k} r}\right) \otimes_{k} K$ coincides with the $\pi$-tensor product of $r$ copies of $\|\cdot\|$ and the absolute value $|\cdot|$ on $K$ (see Proposition 1.3.5). Hence by Proposition 1.1.58 its quotient norm on $\operatorname{det}\left(V_{K}\right)$ coincides with $\|\cdot\|_{\operatorname{det}, K}$.

(3) Let $r$ be the rank of $V$ over $k$. Note that the $r$-th orthogonal tensor power of the norm $\|\cdot\|_{\mathbb{C}, \pi}$ on $V_{\mathbb{C}}^{\otimes_{\mathbb{C}} r} \cong\left(V^{\otimes_{\mathbb{R}} r}\right) \otimes_{\mathbb{R}} \mathbb{C}$ coincides with the orthogonal tensor product of $r$ copies of $\|\cdot\|$ and the usual absolute value $|\cdot|$ on $\mathbb{C}$ (see Proposition 1.3.6). Hence by Proposition 1.2 .62 its quotient seminorm on $\operatorname{det}\left(V_{\mathbb{C}}\right)$ coincides with $\|\cdot\|_{\operatorname{det}, \mathbb{C}}$.

Proposition 1.3.20. — Let $(V,\|\cdot\|)$ be a finite-dimensional seminormed vector space over $k$.

(1) Let $\|\cdot\|_{K, \varepsilon, *}$ be the dual norm of $\|\cdot\|_{K, \varepsilon}$ and $\|\cdot\|_{*, K, \varepsilon}$ be the norm induced by $\|\cdot\|_{*}$ by the $\varepsilon$-extension of scalars. Then we have $\|\cdot\|_{K, \varepsilon, *} \geqslant\|\cdot\|_{*, K, \varepsilon}$, and the restrictions to $V^{*}$ of these two norms are both equal to the dual norm $\|\cdot\|_{*}$. Moreover, the equality $\|\cdot\|_{K, \varepsilon, *}=\|\cdot\|_{*, K, \varepsilon}$ holds if $|\cdot|$ is non-Archimedean or if $V$ is of rank 1 over $k$. 
(2) The dual norm $\|\cdot\|_{K, \pi, *}$ of $\|\cdot\|_{K, \pi}$ is equal to $\|\cdot\|_{*, K, \varepsilon}$ on $V_{K}$.

Proof. - (1) Let $\varphi$ be an element in $V_{K}^{*}$. By definition one has

$$
\|\varphi\|_{K, \varepsilon, *}=\sup _{x \in V_{K} \backslash N_{\|\cdot\|_{K, \varepsilon}}} \frac{|\varphi(x)|}{\|x\|_{K}} \geqslant \sup _{x \in V \backslash N_{\|\cdot\|_{* *}}} \frac{|\varphi(x)|}{\|x\|_{* *}}=\|\varphi\|_{*, K, \varepsilon} .
$$

Note that for any $x \in V_{K}$ one has

$$
\|x\|_{K, \varepsilon}=\sup _{\alpha \in V^{*} \backslash\{0\}} \frac{|\alpha(x)|}{\|\alpha\|_{*}} .
$$

Therefore, if $\varphi \in V^{*} \backslash\{0\}$ then one has $\|x\|_{K, \varepsilon} \geqslant|\varphi(x)| /\|\varphi\|_{*}$, which leads to

$$
\|\varphi\|_{K, \varepsilon, *} \leqslant\|\varphi\|_{*}=\|\varphi\|_{*, K, \varepsilon}
$$

where the equality comes from Proposition 1.3.1 (in the non-Archimedean case we use the fact that the norm $\|\cdot\|_{*}$ is ultrametric).

In the following we prove the equality $\|\cdot\|_{K, \varepsilon, *}=\|\cdot\|_{*, K, \varepsilon}$ under the assumption that $|\cdot|$ is non-Archimedean or $\operatorname{rk}_{k}(V)=1$. We treat firstly the case where $\operatorname{rk}_{k}(V)=1$. In this case, either the seminorm $\|\cdot\|$ vanishes and $V_{K}^{*}$ is the trivial vector space, which has only one norm, or the seminorm $\|\cdot\|$ is a norm and for any non-zero element $\eta$ in $V$ one has

$$
\left\|\eta^{\vee}\right\|_{K, \varepsilon, *}=\|\eta\|_{K, \varepsilon}^{-1}=\|\eta\|^{-1}=\left\|\eta^{\vee}\right\|_{*}=\left\|\eta^{\vee}\right\|_{*, K, \varepsilon},
$$

where $\eta^{\vee}$ denotes the dual element of $\eta$ in $V^{*}=V^{\vee}$. Hence the equality $\|\cdot\|_{K, \varepsilon, *}=$ $\|\cdot\|_{*, K, \varepsilon}$ always holds.

We now treat the case where the absolute value $|\cdot|$ is non-Archimedean. Note that $\|\cdot\|_{K, \varepsilon, *}$ is an ultrametric norm on $V^{*} \otimes_{k} K$ extending $\|\cdot\|_{*}$. Hence by Proposition 1.3 .14 one has $\|\cdot\|_{K, \varepsilon, *} \leqslant\|\cdot\|_{*, K, \varepsilon}$. Therefore the equality $\|\cdot\|_{*, K, \varepsilon}=\|\cdot\|_{K, \varepsilon, *}$ holds.

$(2)$ If $(k,|\cdot|)$ is $\mathbb{R}$ equipped with the usual absolute value and if $K=\mathbb{C}$, then by Proposition 1.1.57, the norm $\|\cdot\|_{*, \mathbb{C}, \varepsilon, *}$ identifies with the $\pi$-tensor product of $\|\cdot\|_{*}$ and $|\cdot|$ (here we consider the absolute value $|\cdot|$ on $\mathbb{C}$ as a norm on a vector space over $\mathbb{R}$ ). Hence it is equal to the norm $\|\cdot\|_{\mathbb{C}, \pi, * *}$ on $V_{\mathbb{C}}^{* *}$, which implies the equality $\|\cdot\|_{*, \mathbb{C}, \varepsilon}=\|\cdot\|_{\mathbb{C}, \pi, *}$ since any finite-dimensional normed vector space over $\mathbb{R}$ is reflexive.

Assume that $|\cdot|$ is non-Archimedean. By $(1)$ and the fact that $\|\cdot\|_{K, \varepsilon} \leqslant\|\cdot\|_{K, \pi}$ (which results from Proposition 1.3.4 and Proposition 1.3.1](1) , one has

$$
\|\cdot\|_{*, K, \varepsilon}=\|\cdot\|_{K, \varepsilon, *} \geqslant\|\cdot\|_{K, \pi, *},
$$

which leads to

$$
\|\cdot\|_{K, \pi, * *} \geqslant\|\cdot\|_{K, \varepsilon, * *}=\|\cdot\|_{K, \varepsilon},
$$

where the equality comes from the fact that the norm $\|\cdot\|_{K, \varepsilon}$ is ultrametric. Note the the restriction of $\|\cdot\|_{K, \pi, * *}$ to $V$ is bounded from above by $\|\cdot\|$ since $\|\cdot\|$ identifies with the restriction of $\|\cdot\|_{K, \pi}$ to $V$ (see Proposition 1.3.1 $\left.(2)\right]$. As $\|\cdot\|_{K, \pi, * *}$ is ultrametric, by Proposition 1.3 .14 we obtain $\|\cdot\|_{K, \pi, * *} \leqslant\|\cdot\|_{K, \varepsilon}$, which leads to the equality 
$\|\cdot\|_{K, \pi, * *}=\|\cdot\|_{K, \varepsilon}$. By passing to the dual norms, using Proposition 1.2.14 (1) we obtain $\|\cdot\|_{K, \pi, *}=\|\cdot\|_{K, \varepsilon, *}=\|\cdot\|_{*, K, \varepsilon}$.

The following proposition is an $\varepsilon$-tensor analogue of Propositions 1.3.5 and 1.3.6.

Proposition 1.3.21. - We assume that the absolute value $|\cdot|$ on $k$ is nonArchimedean. Let $\left(V_{1},\|\cdot\|_{1}\right)$ and $\left(V_{2},\|\cdot\|_{2}\right)$ be finite-dimensional ultrametrically seminormed vector space over $k$, and $\|\cdot\|$ be the $\varepsilon$-tensor product norm of $\|\cdot\|_{1}$ and $\|\cdot\|_{2}$. Then $\|\cdot\|_{K, \varepsilon}$ identifies with the $\varepsilon$-tensor product of $\|\cdot\|_{1, K, \varepsilon}$ and $\|\cdot\|_{2, K, \varepsilon}$.

Proof. - Let $\|\cdot\|_{\varepsilon}^{\prime}$ be the $\varepsilon$-tensor product of the norms $\|\cdot\|_{1, K, \varepsilon}$ and $\|\cdot\|_{2, K, \varepsilon}$. By Remark 1.1.53, it identifies with the seminorm induced by the operator seminorm on the $K$-vector space $\operatorname{Hom}_{K}\left(V_{1, K}^{*}, V_{2, K}\right)$ by the canonical $K$-linear map

$$
V_{1, K} \otimes_{K} V_{2, K} \longrightarrow \operatorname{Hom}_{K}\left(V_{1, K}^{*}, V_{2, K}\right),
$$

where we consider the dual norm of $\|\cdot\|_{1, K, \varepsilon}$ on $V_{1, K}^{*}$, which identifies with the norm $\|\cdot\|_{1, *, K, \varepsilon}$ induced by $\|\cdot\|_{1, *}$ by $\varepsilon$-extension of scalars (see (1) in Proposition 1.3.20). By Proposition 1.3.8, for any $f \in \operatorname{Hom}_{k}\left(V_{1}^{*}, V_{2}\right)$, the seminorm of $f_{K}$ identifies with that of $f$. Therefore $\|\cdot\|_{\varepsilon}^{\prime}$ is an ultrametric norm on $V_{1, K} \otimes_{K} V_{2, K}$ which extends the $\varepsilon$-tensor product $\|\cdot\|_{\varepsilon}$ of $\|\cdot\|_{1}$ and $\|\cdot\|_{2}$. By Proposition 1.3.14, one has $\|\cdot\|_{\varepsilon}^{\prime} \leqslant\|\cdot\|_{K, \varepsilon}$.

In the following, we prove the converse inequality $\|\cdot\|_{K, \varepsilon} \leqslant\|\cdot\|_{\varepsilon}^{\prime}$. Let $\alpha \in$ ]0,1[ and $\left\{e_{i}\right\}_{i=1}^{n}$ and $\left\{f_{j}\right\}_{j=1}^{m}$ be respectively $\alpha$-orthogonal bases of $\left(V_{1},\|\cdot\|_{1}\right)$ and $\left(V_{2},\|\cdot\|_{2}\right)$. By Proposition 1.2.19 they are also $\alpha$-orthogonal bases of $\left(V_{1, K},\|\cdot\|_{1, K, \varepsilon}\right)$ and $\left(V_{2, K},\|\cdot\|_{2, K, \varepsilon}\right)$, respectively. By Propsition [1.2.19, the basis $\left\{e_{i} \otimes f_{j}\right\}_{i \in\{1, \ldots, n\}, j \in\{1, \ldots, m\}}$ of $V_{1, K} \otimes_{K} V_{2, K}$ is $\alpha^{2}$-orthogonal with respect to the seminorm $\|\cdot\|_{\varepsilon}^{\prime}$. Hence for $\left(a_{i j}\right)_{i \in\{1, \ldots, n\}, j \in\{1, \ldots, m\}} \in K^{n \times m}$ and $T=\sum_{i, j} a_{i j} e_{i} \otimes f_{j} \in V_{1, K} \otimes_{K} V_{2, K}$, one has

$$
\|T\|_{\varepsilon}^{\prime} \geqslant \alpha^{2} \max _{\substack{i \in\{1, \ldots, n\} \\ j \in\{1, \ldots, m\}}}\left|a_{i j}\right| \cdot\left\|e_{i} \otimes f_{j}\right\|_{\varepsilon} \geqslant \alpha^{2}\|T\|_{K, \varepsilon} .
$$

Since $\alpha \in] 0,1\left[\right.$ is arbitrary, we obtain the inequality $\|\cdot\|_{\varepsilon}^{\prime} \geqslant\|\cdot\|_{K, \varepsilon}$.

Proposition 1.3.22. - We assume that the absolute value $|\cdot|$ of $k$ is trivial. Let $(V,\|\cdot\|)$ be an ultrametrically seminormed vector space of finite rank over $k$. Let $\left(K,|\cdot|_{K}\right)$ be an extension of $(k,|\cdot|)$ such that $|\cdot|_{K}$ is non-trivial and complete. Let $\mathfrak{o}_{K}$ be the valuation ring of $\left(K,|\cdot|_{K}\right)$ and $\mathfrak{m}_{K}$ be the maximal ideal of $\mathfrak{o}_{K}$. Suppose the following assumptions (1) and (2).

(1) the natural map $k \rightarrow \mathfrak{o}_{K}$ induces an isomorphism $k \stackrel{\sim}{\longrightarrow} \mathfrak{o}_{K} / \mathfrak{m}_{K}$,

(2) $\left\{\left\|v^{\prime}\right\| /\|v\|: v, v^{\prime} \in V \backslash N_{\|\cdot\|}\right\} \cap\left|K^{\times}\right|_{K} \subseteq\{1\}$.

Let $\|\cdot\|_{K, \varepsilon}$ be the seminorm of $V_{K}$ induced by $\|\cdot\|$ by $\varepsilon$-extension of scalars. Then $\|\cdot\|_{K, \varepsilon}$ is the only ultrametric seminorm on $V_{K}$ extending $\|\cdot\|$. 
Proof. - We prove the assertion by induction on the rank $n$ of $V$ over $k$. The case where $n=1$ is trivial. In the following, we suppose that the assertion has been proved for seminormed vector spaces of rank $<n$ over $k$. Since $\|\cdot\|$ is ultrametric, one has $\|\cdot\|=\|\cdot\|_{* *}$ (see Corollary 1.2.12). Let $\|\cdot\|^{\prime}$ be another ultrametric seminorm on $V_{K}$ extending $\|\cdot\|$. By Proposition 1.3.14 one has $\|\cdot\|^{\prime} \leqslant\|\cdot\|_{K, \varepsilon}$.

Let $r$ be the rank of $V / N_{\|\cdot\|}$ and $\left\{e_{i}\right\}_{i=1}^{n}$ be an orthogonal basis of $V$ such that $\left\{e_{i}\right\}_{i=r+1}^{n}$ forms a basis of $N_{\|\cdot\|}$ (see Proposition 1.2.5). If the equality $\|\cdot\|^{\prime}=\|\cdot\|_{K, \varepsilon}$ does not hold, then there exists a vector $x \in V_{K}$ such that $\|x\|^{\prime}<\|x\|_{K, \varepsilon}$. We write $x$ in the form $x=a_{1} e_{1}+\cdots+a_{n} e_{n}$ with $\left(a_{1}, \ldots, a_{n}\right) \in K^{n}$. Note that

$$
\left\|a_{r+1} e_{r+1}+\cdots+a_{n} e_{n}\right\|^{\prime} \leqslant \max _{i \in\{r+1, \ldots, n\}}\left|a_{i}\right| \cdot\left\|e_{i}\right\|=0 .
$$

For the same reason, $\left\|a_{r+1} e_{r+1}+\cdots+a_{n} e_{n}\right\|_{K, \varepsilon}=0$. Therefore one has

$$
\left\|a_{1} e_{1}+\cdots+a_{r} e_{r}\right\|^{\prime}=\|x\|^{\prime}<\|x\|_{K, \varepsilon}=\left\|a_{1} e_{1}+\cdots+a_{r} e_{r}\right\|_{K, \varepsilon} .
$$

By replacing $x$ by $a_{1} e_{1}+\cdots+a_{r} e_{r}$ we many assume without loss of generality that $a_{r+1}=\cdots=a_{n}=0$.

We will prove that $\left|a_{i}\right|_{K} \cdot\left\|e_{i}\right\|$ are the same for $i \in\{1, \ldots, r\}$ by contradiction. Without loss of generality, we assume on the contrary that

$$
\left|a_{1}\right|_{K} \cdot\left\|e_{1}\right\| \leqslant \cdots \leqslant\left|a_{j}\right|_{K} \cdot\left\|e_{j}\right\|<\left|a_{j+1}\right|_{K} \cdot\left\|e_{j+1}\right\|=\cdots=\left|a_{r}\right|_{K} \cdot\left\|e_{r}\right\|
$$

with $j \in\{1, \ldots, r-1\}$. Note that

$$
\|x\|^{\prime}<\|x\|_{K, \varepsilon}=\max _{i \in\{1, \ldots, r\}}\left|a_{i}\right|_{K} \cdot\left\|e_{i}\right\|=\left|a_{r}\right|_{K} \cdot\left\|e_{r}\right\| .
$$

Moreover, by the induction hypothesis, the norms $\|\cdot\|^{\prime}$ and $\|\cdot\|_{K, \varepsilon}$ coincide on $K e_{j+1}+$ $\cdots+K e_{r}$. In particular, one has $\left\|a_{j+1} e_{j+1}+\cdots+a_{r} e_{r}\right\|^{\prime}=\left|a_{r}\right|_{K} \cdot\left\|e_{r}\right\|$. Therefore, if we let $y=a_{1} e_{1}+\cdots+a_{j} e_{j}$, then one has

$\|y\|^{\prime}=\left\|x-\left(a_{j+1} e_{j+1}+\cdots+a_{r} e_{r}\right)\right\|^{\prime}=\left|a_{r}\right|_{K} \cdot\left\|e_{r}\right\|>\max _{i \in\{1, \ldots, j\}}\left|a_{i}\right|_{K} \cdot\left\|e_{i}\right\|=\|y\|_{K, \varepsilon}$,

which leads to a contradiction since $\|\cdot\|^{\prime} \leqslant\|\cdot\|_{K, \varepsilon}$. Hence we should have

$$
\left|a_{1}\right|_{K} \cdot\left\|e_{1}\right\|=\cdots=\left|a_{r}\right|_{K} \cdot\left\|e_{r}\right\| .
$$

By the condition $(2)$, we have $\left\|e_{1}\right\|=\cdots=\left\|e_{r}\right\|$ (namely the function $\|\cdot\|$ is constant on $\left.V \backslash N_{\|\cdot\| \|}\right)$ and hence $\left|a_{1}\right|_{K}=\cdots=\left|a_{r}\right|_{K}>0$. As $\left|a_{i} / a_{r}\right|_{K}=1$ for any $i \in\{1, \ldots, r\}$, by the assumption (1) there exists a $b_{i} \in k^{\times}$such that $\left|a_{i} / a_{r}-b_{i}\right|_{K}<1$, that is, $\left|a_{i}-b_{i} a_{r}\right|_{K}<\left|a_{r}\right|_{K}$. Thus, by Proposition 1.1.5,

$$
\|x\|^{\prime}=\left\|a_{r} \sum_{i=1}^{r} b_{i} e_{i}+\sum_{i=1}^{r}\left(a_{i}-b_{i} a_{r}\right) e_{i}\right\|^{\prime}=\left|a_{r}\right|_{K} \cdot\left\|e_{r}\right\|=\|x\|_{K, \varepsilon}
$$

because

$$
\left\|a_{r} \sum_{i=1}^{r} b_{i} e_{i}\right\|^{\prime}=\left|a_{r}\right|_{K}\left\|\sum_{i=1}^{r} b_{i} e_{i}\right\|^{\prime}=\left|a_{r}\right|_{K}\left\|e_{r}\right\| \text { and }\left\|\sum_{i=1}^{r}\left(a_{i}-b_{i} a_{r}\right) e_{i}\right\|^{\prime}<\left|a_{r}\right|_{K}\left\|e_{r}\right\| .
$$


This leads to a contradiction. The proposition is thus proved.

Proposition 1.3.23. - We assume that $k=\mathbb{R}$ and that $|\cdot|$ is the usual absolute value. Let $\left\{\left(V_{i},\|\cdot\|_{i}\right)\right\}_{i=1}^{n}$ be finite-dimensional seminormed vector spaces over $k$. We assume that the seminorms $\|\cdot\|_{i}$ are induced by semidefinite inner products $\langle,\rangle_{i}$ and we let $\|\cdot\|_{\mathrm{HS}}$ be their orthogonal tensor product. For $i \in\{1, \ldots, n\}$, let $\pi_{i}: V_{i, \mathbb{C}} \rightarrow W_{i}$ be a quotient spaces of rank 1 of $V_{i, \mathbb{C}}$, and $\|\cdot\|_{W_{i}}$ be the quotient seminorm on $W_{i}$ induced by $\|\cdot\|_{i, \mathbb{C}}$. Let $\|\cdot\|_{W}$ be the quotient seminorm on $W=\bigotimes_{i=1}^{n} W_{i}$ induced by $\|\cdot\|_{\mathrm{HS}, \mathbb{C}}$ and let $\|\cdot\|$ be the tensor product of $\|\cdot\|_{W_{i}}$. Then one has

$$
\frac{1}{\sqrt{2}}\|\cdot\| \leqslant\|\cdot\|_{W} \leqslant(\sqrt{2})^{n}\|\cdot\| \cdot
$$

Proof. - For any $i \in\{1, \ldots, n\}$, let $\|\cdot\|_{i}^{\prime}$ be the seminorm on $V_{i, \mathbb{C}}$ induced by the semidefinite inner product $\langle,\rangle_{i, \mathbb{C}}$. One has $\|\cdot\|_{i, \mathbb{C}} \leqslant\|\cdot\|_{i}^{\prime} \leqslant \sqrt{2}\|\cdot\|_{i, \mathbb{C}}$ (see Remark 1.3 .2 $(2)]$. Let $\langle,\rangle_{\mathrm{HS}}$ be the semidefinite inner product corresponding to the orthogonal tensor product seminorm $\|\cdot\|_{\mathrm{HS}}$ and $\|\cdot\|^{\prime}$ be the seminorm on $\bigotimes_{i=1}^{n} V_{i, \mathbb{C}}$ induced by $\langle,\rangle_{\mathrm{HS}, \mathbb{C}}$. Still by Remark $1.3 .2,(2)$ one has $\|\cdot\|_{\mathrm{HS}, \mathbb{C}} \leqslant\|\cdot\|^{\prime} \leqslant \sqrt{2}\|\cdot\|_{\mathrm{HS}, \mathbb{C}}$. Moreover, $\|\cdot\|^{\prime}$ coincides with the orthogonal tensor product of the seminorms $\|\cdot\|_{i}^{\prime}$.

For $i \in\{1, \ldots, n\}$, let $\|\cdot\|_{W_{i}}^{\prime}$ be the quotient seminorms on $W_{i}$ induced by $\|\cdot\|_{i}^{\prime}$. Let $\|\cdot\|_{W}^{\prime}$ be the quotient seminorm on $W$ induced by $\|\cdot\|^{\prime}$. By Proposition 1.2.57 $\|\cdot\|_{W}^{\prime}$ coincides with the tensor product of the seminorms $\|\cdot\|_{W_{i}}^{\prime}$. Moreover, by the relations $\|\cdot\|_{i, \mathbb{C}} \leqslant\|\cdot\|_{i}^{\prime} \leqslant \sqrt{2}\|\cdot\|_{i, \mathbb{C}}$ we obtain $\|\cdot\|_{W_{i}} \leqslant\|\cdot\|_{W_{i}}^{\prime} \leqslant \sqrt{2}\|\cdot\|_{W_{i}}$, which implies

$$
\|\cdot\| \leqslant\|\cdot\|_{W}^{\prime} \leqslant(\sqrt{2})^{n}\|\cdot\|
$$

by the relation $\|\cdot\|_{\mathrm{HS}, \mathbb{C}} \leqslant\|\cdot\|^{\prime} \leqslant \sqrt{2}\|\cdot\|_{\mathrm{HS}, \mathbb{C}}$ we obtain

$$
\|\cdot\|_{W} \leqslant\|\cdot\|_{W}^{\prime} \leqslant \sqrt{2}\|\cdot\|_{W} .
$$

Combining (1.54) and (1.55), we obtain $\frac{1}{\sqrt{2}}\|\cdot\| \leqslant\|\cdot\|_{W} \leqslant(\sqrt{2})^{n}\|\cdot\|$. The proposition is thus proved.

Proposition 1.3.24. - Let $(V,\|\cdot\|)$ be a finite-dimensional seminormed vector space over $k$ and $W$ be a quotient vector space of rank 1 of $V$. Let $\|\cdot\|_{W}$ be the quotient seminorm on $W$ induced by $\|\cdot\|$.

(1) The seminorm $\|\cdot\|_{W, K}$ coincides with the quotient seminorm on $W_{K}$ induced by $\|\cdot\|_{K, \natural}$, where $\downarrow=\varepsilon$ or $\pi$.

(2) Assume that $(k,|\cdot|)$ is $\mathbb{R}$ equipped with the usual absolute value, $K=\mathbb{C}$, and $\|\cdot\|$ is induced by a semidefinite inner product. Then the seminorm $\|\cdot\|_{W, \mathbb{C}}$ coincides with the quotient seminorm on $W_{\mathbb{C}}$ induced by $\|\cdot\|_{\mathbb{C}, H S}$.

Proof. - (1) The case where $\downarrow=\pi$ follows directly from Proposition 1.1.58, In the following, we consider the case where $\measuredangle=\varepsilon$.

Let $\|\cdot\|_{W_{K}}$ be the quotient seminorm on $W_{K}$ induced by the seminorm $\|\cdot\|_{K, \varepsilon}$. If the kernel of the quotient map $V \rightarrow W$ does not contain $N_{\|\cdot\|}$, then the quotient 
seminorm $\|\cdot\|_{W}$ vanishes since $W$ is of dimension 1 over $k$. In this case the quotient seminorm $\|\cdot\|_{W_{K}}$ also vanishes since the kernel of the quotient map $V_{K} \rightarrow W_{K}$ does not contain $N_{\|\cdot\|_{K, \varepsilon}}=N_{\|\cdot\|} \otimes_{k} K$ (see Proposition 1.3.3).

In the following, we assume that that seminorm $\|\cdot\|_{W}$ is a norm. In this case $\|\cdot\|_{W_{K}}$ is also a norm since the kernel of the quotient map $V_{K} \rightarrow W_{K}$ contains $N_{\|\cdot\|_{K, \varepsilon}}=$ $N_{\|\cdot\|} \otimes_{k} K$. We will show that the dual norms $\|\cdot\|_{W_{K}, *}$ and $\|\cdot\|_{W, K, \varepsilon, *}$ on $W_{K}^{\vee}$ are equal. Since $W$ is a vector space of rank 1 , it suffices to show that the restrictions of these norms to $W^{\vee}$ are the same. We identify $W_{K}^{\vee}$ with a vector subspace of rank 1 of $V_{K}^{\vee}$. By Proposition 1.1.20 the norm $\|\cdot\|_{W_{K}, *}$ coincides with the restriction of $\|\cdot\|_{K, \varepsilon, *}$ to $W_{K}^{\vee}$, where $\|\cdot\|_{K, \varepsilon, *}$ denotes the dual seminorm of $\|\cdot\|_{K, \varepsilon}$. By (1) in Proposition 1.3.20, the restriction of $\|\cdot\|_{K, \varepsilon, *}$ to $V^{\vee}$ coincides with $\|\cdot\|_{*}$. Therefore, the restriction of $\|\cdot\|_{K, \varepsilon, *}$ to $W^{\vee}$ coincides with the restriction of $\|\cdot\|_{*}$ to $W^{\vee}$, which identifies with the dual norm of $\|\cdot\|_{W}$ (by Proposition 1.1.20). By (1) in Proposition 1.3.20, one has $\|\cdot\|_{W, K, *}=\|\cdot\|_{W, *, K}$. Finally, since $W$ is of rank 1 , if $k$ is non-Archimedean, then any norm on $W^{\vee}$ is ultrametric. Hence by Proposition 1.3.1 (for both Archimedean and non-Archimedean cases), the restriction of $\|\cdot\|_{W, K, *}=\|\cdot\|_{W, *, K}$ to $W^{\vee}$ identifies with $\|\cdot\|_{W, *}$. The assertion is thus proved.

(2) follows directly from Proposition 1.2.57

Proposition 1.3.25. — Let $(V,\|\cdot\|)$ be a finite dimension seminormed vector space over $k$ and $W$ be a quotient space of rank 1 of $V_{K}=V \otimes_{k} K$. We equip $V_{K}$ with the seminorm $\|\cdot\|_{K, \pi}$ induced by $\|\cdot\|$ by $\pi$-extension of scalars and $W$ with the quotient seminorm $\|\cdot\|_{W}$ of $\|\cdot\|_{K, \pi}$. Then for any $\ell \in W$ one has

$$
\|\ell\|_{W}=\inf _{\substack{s \in V, \lambda \in K^{\times} \\[s]=\lambda \ell}}|\lambda|^{-1}\|s\| .
$$

Proof. — By definition one has

$$
\|\ell\|_{W}=\inf _{\substack{s \in V_{K}, \lambda \in K^{\times} \\[s]=\lambda \ell}}|\lambda|^{-1} \cdot\|s\|_{K, \pi} \leqslant \inf _{\substack{s \in V, \lambda \in K^{\times} \\[s]=\lambda \ell}}|\lambda|^{-1} \cdot\|s\|_{K, \pi}=\inf _{\substack{s \in V, \lambda \in K^{\times} \\[s]=\lambda \ell}}|\lambda|^{-1} \cdot\|s\|,
$$

where the last equality comes from Proposition 1.3.1.

Without loss of generality, we may assume that $\ell \neq 0$. Let $s$ be an element in $V_{K}$, which is written as $s=a_{1} x_{1}+\cdots+a_{n} x_{n}$, where $\left(a_{1}, \ldots, a_{n}\right) \in K^{n}$ and $\left(x_{1}, \ldots, x_{n}\right) \in V$. For any $i \in\{1, \ldots, n\}$, let $\lambda_{i}$ be the element of $K$ such that $\left[x_{i}\right]=\lambda_{i} \ell$. Then $[s]=\lambda \ell$ with $\lambda=a_{1} \lambda_{1}+\cdots+a_{r} \lambda_{r}$. Let

$$
h=\inf _{\substack{t \in V, \lambda \in K^{\times} \\[t]=\lambda \ell}}|\lambda|^{-1} \cdot\|t\| .
$$

For any $i \in\{1, \ldots, n\}$ one has $\left\|x_{i}\right\| \geqslant\left|\lambda_{i}\right| h$. Hence

$$
|\lambda|^{-1} \sum_{i=1}^{n}\left|a_{i}\right| \cdot \| x_{i}|| \geqslant|\lambda|^{-1} \sum_{i=1}^{n}\left|a_{i}\right| \cdot\left|\lambda_{i}\right| h \geqslant h .
$$


The proposition is thus proved.

Proposition 1.3.26. - Let $(V,\|\cdot\|)$ be a finite-dimensional seminormed vector space over $k$. We assume one of the following conditions:

(i) $(k,|\cdot|)$ is non-Archimedean;

(ii) $k=\mathbb{C}$ equipped with the usual absolute value.

Let $W$ be a quotient space of rank 1 of $V \otimes_{k} K$. Let $\|\cdot\|_{W}$ be the quotient seminorm on $W$ induced by $\|\cdot\|_{K, \varepsilon}$ (the seminorm on $V \otimes_{k} K$ induced by $\|\cdot\|$ by $\varepsilon$-extension of scalars). Then, for any $\ell \in W$ one has

$$
\|\ell\|_{W}=\inf _{\substack{s \in V, \lambda \in K^{\times} \\[s]=\lambda \ell}}|\lambda|^{-1}\|s\| .
$$

Proof. - The case where $k=\mathbb{C}$ equipped with the usual absolute value is trivial since $K=k$. In the following, we assume that $(k,|\cdot|)$ is non-Archimedean.

By definition one has

$$
\begin{aligned}
\|\ell\|_{W} & =\inf _{\substack{s \in V_{K}, \lambda \in K^{\times} \\
[s]=\lambda \ell}}|\lambda|^{-1} \cdot\|s\|_{K, \varepsilon} \leqslant \inf _{\substack{s \in V, \lambda \in K^{\times} \\
[s]=\lambda \ell}}|\lambda|^{-1} \cdot\|s\|_{K, \varepsilon} \\
& =\inf _{\substack{s \in V, \lambda \in K^{\times} \\
[s]=\lambda \ell}}|\lambda|^{-1} \cdot\|s\| \leqslant \inf _{\substack{s \in V, \lambda \in K^{\times} \\
[s]=\lambda \ell}}|\lambda|^{-1} \cdot\|s\|,
\end{aligned}
$$

where the last equality comes from Proposition 1.3.1

We then prove the converse inequality. Let $\alpha$ be a real number in $] 0,1[$. By Proposition 1.2.7, there exists an $\alpha$-orthogonal basis $\left\{s_{i}\right\}_{i=1}^{r}$ of $(V,\|\cdot\|)$. By Proposition 1.3.13, $\left\{s_{i}\right\}_{i=1}^{r}$ is also an $\alpha$-orthogonal basis of $\left(V_{K},\|\cdot\|_{K}\right)$. For each $i \in\{1, \ldots, r\}$, let $\lambda_{i} \in K$ such that $\left[s_{i}\right]=\lambda_{i} \ell$. Let $s=a_{1} s_{1}+\cdots+a_{r} s_{r}$ be an element in $V \otimes_{k} K$, where $\left(a_{1}, \ldots, a_{r}\right) \in K^{r}$. Assume that $[s]$ is of the form $\lambda \ell$, where $\lambda \in K^{\times}$. Then one has $\lambda=a_{1} \lambda_{1}+\cdots+a_{r} \lambda_{r}$, which leads to $|\lambda| \leqslant \max _{i \in\{1, \ldots, r\}}\left|a_{i}\right| \cdot\left|\lambda_{i}\right|$ since the absolute value is non-Archimedean. By the $\alpha$-orthogonality of the basis $\left\{s_{i}\right\}_{i=1}^{r}$, we obtain

$$
\begin{aligned}
& |\lambda|^{-1} \cdot\|s\|_{K, \varepsilon} \geqslant \frac{\alpha}{|\lambda|} \max _{i \in\{1, \ldots, r\}}\left|a_{i}\right| \cdot\left\|s_{i}\right\|_{* *} \\
& \quad \geqslant \alpha \min _{i \in\{1, \ldots, r\}}\left|\lambda_{i}\right|^{-1}\left\|s_{i}\right\|_{* *} \geqslant \alpha^{2} \min _{i \in\{1, \ldots, r\}}\left|\lambda_{i}\right|^{-1}\left\|s_{i}\right\|,
\end{aligned}
$$

where the last inequality comes from Proposition 1.2.11. The proposition is thus proved.

Corollary 1.3.27. - We keep the notation and hypotheses of Proposition 1.3.26. Let $V^{\prime}$ be a quotient $k$-vector space of $V$, equipped with the quotient seminorm $\|\cdot\|^{\prime}$ induced by $\|\cdot\|$. We assume that the projection map $\pi: V_{K} \rightarrow W$ factorises through $V_{K}^{\prime}$. Then the quotient seminorm on $W$ induced by $\|\cdot\|_{K, \varepsilon}^{\prime}$ coincides with $\|\cdot\|_{W}$. 
Proof. - Let $\|\cdot\|_{W}^{\prime}$ be the quotient seminorm on $W$ induced by $\|\cdot\|_{K, \varepsilon}^{\prime}$. We apply Proposition 1.3.26 to $\left(V^{\prime},\|\cdot\|^{\prime}\right)$ and $W$ to obtain that, for any $\ell \in W$, one has

$$
\|\ell\|_{W}^{\prime}=\inf _{\substack{t \in V^{\prime}, \lambda \in K^{\times} \\[t]=\lambda \ell}}|\lambda|^{-1}\|t\|^{\prime}=\inf _{\substack{s \in V, \lambda \in K^{\times} \\[s]=\lambda \ell}}|\lambda|^{-1}\|s\| .
$$

Still by Proposition 1.3.26, we obtain $\|\ell\|_{W}^{\prime}=\|\ell\|_{W}$.

1.3.4. Extension of scalars in the real case. - In this subsection, we assume that $(k,|\cdot|)$ is the field $\mathbb{R}$ of real numbers equipped with the usual absolute value.

Definition 1.3.28. - Let $V$ be a vector space over $\mathbb{R}$. We say that a seminorm $\|\cdot\|$ on $V_{\mathbb{C}}:=V \otimes_{\mathbb{R}} \mathbb{C}$ is invariant under the complex conjugation if the equality $\|x+i y\|=\|x-i y\|$ holds for any $(x, y) \in V^{2}$.

Proposition 1.3.29. — Let $(V,\|\cdot\|)$ be a finite-dimensional seminormed vector space over $\mathbb{R}$. The seminorms $\|\cdot\|_{\mathbb{C}, \varepsilon}$ and $\|\cdot\|_{\mathbb{C}, \pi}$ are invariant under the complex conjugation. If $\|\cdot\|$ is induced by a semidefinite inner product, then $\|\cdot\|_{\mathbb{C}, \mathrm{HS}}$ is invariant under the complex conjugation.

Proof. - These statements follow directly from the definition of different tensor product seminorms and the fact that the absolute value on $\mathbb{C}$ is invariant under the complex conjugation (namely $|a+i b|=|a-i b|$ for any $(a, b) \in \mathbb{R}^{2}$ ).

Proposition 1.3.30. — Let $(V,\|\cdot\|)$ be a finite-dimensional vector space over $\mathbb{R}$ (equipped with the usual absolute value) and $\|\cdot\|^{\prime}$ be a seminorm on $V_{\mathbb{C}}$ extending $\|\cdot\|$. Assume that $\|\cdot\|^{\prime}$ is invariant under the complex conjugation. Then for any $(x, y) \in V^{2}$ one has $\max \{\|x\|,\|y\|\} \leqslant\|x+i y\|^{\prime} \leqslant\|x\|+\|y\|$.

Proof. - One has

$$
\begin{aligned}
& 2\|x\|=\|2 x\|=\|2 x\|^{\prime} \leqslant\|x+i y\|^{\prime}+\|x-i y\|^{\prime}=2\|x+i y\|^{\prime}, \\
& 2\|y\|=\|2 y\|=\|2 i y\|^{\prime} \leqslant\|x+i y\|^{\prime}+\|i y-x\|^{\prime}=2\|x+i y\|^{\prime} .
\end{aligned}
$$

Therefore $\|x+i y\|^{\prime} \geqslant \max \{\|x\|,\|y\|\}$. The relation $\|x+i y\|^{\prime} \leqslant\|x\|+\|y\|$ comes from the triangle inequality.

Proposition 1.3.31. — Let $(V,\|\cdot\|)$ be a seminormed vector space over $\mathbb{R}$. For any $(x, y) \in V^{2}$ one has

$$
\max \{\|x\|,\|y\|\} \leqslant\|x+i y\|_{\mathbb{C}, \varepsilon} \leqslant\left(\|x\|^{2}+\|y\|^{2}\right)^{1 / 2} .
$$

Moreover, for any seminorm $\|\cdot\|^{\prime}$ on $V_{\mathbb{C}}$ extending $\|\cdot\|$ which is invariant under the complex conjugation, one has

$$
\begin{gathered}
\frac{1}{2}\|\cdot\|^{\prime} \leqslant\|\cdot\|_{\mathbb{C}, \varepsilon} \leqslant \sqrt{2}\|\cdot\|^{\prime}, \\
\|\cdot\|^{\prime} \leqslant\|\cdot\|_{\mathbb{C}, \pi} \leqslant 2\|\cdot\|^{\prime} .
\end{gathered}
$$


Proof. - The first inequality of (1.56) comes from Propositions 1.3 .29 and 1.3 .30 , Moreover, one has

$$
\begin{aligned}
\|x+i y\|_{\mathbb{C}, \varepsilon} & =\sup _{\varphi \in V^{*} \backslash\{0\}} \frac{\sqrt{\varphi(x)^{2}+\varphi(y)^{2}}}{\|\varphi\|_{*}} \\
& \leqslant \sup _{\left(\varphi_{1}, \varphi_{2}\right) \in\left(V^{*} \backslash\{0\}\right)^{2}}\left(\frac{\varphi_{1}(x)^{2}}{\left\|\varphi_{1}\right\|_{*}^{2}}+\frac{\varphi_{2}(y)^{2}}{\left\|\varphi_{2}\right\|_{*}^{2}}\right)^{1 / 2}=\left(\|x\|^{2}+\|y\|^{2}\right)^{1 / 2},
\end{aligned}
$$

which proves the second inequality of (1.56).

By Proposition 1.3.30, for any $(x, y) \in V^{2}$, one has

$$
\frac{1}{2}\|x+i y\|^{\prime} \leqslant \frac{1}{2}(\|x\|+\|y\|) \leqslant \max \{\|x\|,\|y\|\} \leqslant\|x+i y\|_{\mathbb{C}, \varepsilon},
$$

where the last inequality comes from (1.56). Moreover, still by (1.56) one has

$$
\|x+i y\|_{\mathbb{C}, \varepsilon} \leqslant\left(\|x\|^{2}+\|y\|^{2}\right)^{1 / 2} \leqslant \sqrt{2} \max \{\|x\|,\|y\|\} \leqslant\|x+i y\|^{\prime},
$$

where the last inequality comes from Proposition 1.3.30 Hence (1.57) is proved.

Since the seminorm $\|\cdot\|^{\prime}$ extends $\|\cdot\|$, by Proposition 1.3 .4 one has $\|\cdot\|^{\prime} \leqslant\|\cdot\|_{\mathbb{C}, \pi}$. Moreover, for any $(x, y) \in V^{2}$ one has

$$
\|x+i y\|_{\mathbb{C}, \pi} \leqslant\|x\|+\|y\| \leqslant 2 \max \{\|x\|,\|y\|\} \leqslant 2\|x+i y\|^{\prime},
$$

where the last inequality comes from Proposition 1.3.30 Hence (1.58) is proved.

Proposition 1.3.32. - Let $\left(V,\|\cdot\|_{V}\right)$ be a finite-dimensional seminormed vector space over $\mathbb{R}, Q$ be a quotient vector space of $V$ and $\|\cdot\|_{Q}$ be the quotient seminorm of $\|\cdot\|_{V}$ on $Q$. Let $\|\cdot\|$ be a seminorm on $V_{\mathbb{C}}$ extending $\|\cdot\|_{V}$, which is invariant under the complex conjugation. Then the quotient seminorm of $\|\cdot\|$ on $Q_{\mathbb{C}}$ extends $\|\cdot\|_{Q}$. It is moreover invariant under the complex conjugation.

Proof. - Denote by $\|\cdot\|^{\prime}$ the quotient seminorm of $\|\cdot\|$ on $Q_{\mathbb{C}}$. For $q \in Q$ one has

$$
\|q\|^{\prime}=\inf _{\substack{(x, y) \in V^{2},[x]=q,[y]=0}}\|x+i y\| \leqslant \inf _{x \in V,[x]=q}\|x\| .
$$

Since $\|\cdot\|$ is invariant under the complex conjugation, for any $(x, y) \in V^{2}$ one has $\|x+i y\| \geqslant\|x\|$. Hence $\|q\|^{\prime} \geqslant \inf _{x \in V,[x]=q}\|x\|$, so that $\|q\|^{\prime}=\inf _{x \in V,[x]=q}\|x\|$. Therefore,

$$
\|q\|^{\prime}=\inf _{x \in V,[x]=q}\|x\|=\inf _{x \in V,[x]=q}\|x\|_{V}=\|q\|_{Q} .
$$

Finally, for any $(p, q) \in Q^{2}$ one has

$$
\|p+i q\|^{\prime}=\inf _{\substack{(x, y) \in V^{2} \\([x],[y])=(p, q)}}\|x+i y\|=\inf _{\substack{(x, y) \in V^{2} \\([x],[y])=(p, q)}}\|x-i y\|=\|p-i q\|^{\prime} .
$$


Remark 1.3.33. - Let $(V,\|\cdot\|)$ be finite-dimensional seminormed vector space over $\mathbb{R}, W$ be a quotient vector space of rank one of $V_{\mathbb{C}}:=V \otimes_{\mathbb{R}} \mathbb{C}$. Let $\|\cdot\|_{W}$ be the quotient seminorm on $W$ induced by $\|\cdot\|_{\mathbb{C}, \varepsilon}$. If $\ell$ is a vector of $W$, then clearly one has

$$
\|\ell\|_{W} \leqslant \inf _{\substack{s \in V, \lambda \in \mathbb{C}^{\times} \\[s]=\lambda \ell}}|\lambda|^{-1}\|s\| .
$$

The equality is in general not satisfied (see the counter-example in Remark 1.3.37). However, we can show that

$$
2\|\ell\|_{W} \geqslant \inf _{\substack{s \in V, \lambda \in \mathbb{C}^{\times} \\[s]=\lambda \ell}}|\lambda|^{-1}\|s\|
$$

In fact, by definition one has

$$
\|\ell\|_{W}=\inf _{\substack{s \in V_{\mathbb{C}}, \lambda \in \mathbb{C}^{\times} \\[s]=\lambda \ell}}|\lambda|^{-1}\|s\|_{\mathbb{C}, \varepsilon} .
$$

Let $s$ be an element in $V_{\mathbb{C}}$, which is written as $s=s_{1}+i s_{2}$, where $s_{1}$ and $s_{2}$ are vectors in $V$. Assume that $\lambda_{1}$ and $\lambda_{2}$ are complex numbers such that $\left[s_{1}\right]=\lambda_{1} \ell$ and $\left[s_{2}\right]=\lambda_{2} \ell$. Then one has $[s]=\left(\lambda_{1}+i \lambda_{2}\right) \ell$. By Proposition 1.3.31

$$
\|s\|_{\mathbb{C}, \varepsilon} \geqslant \max \left\{\left\|s_{1}\right\|,\left\|s_{2}\right\|\right\} \geqslant \frac{1}{2}\left(\left\|s_{1}\right\|+\left\|s_{2}\right\|\right),
$$

and $\left|\lambda_{1}+i \lambda_{2}\right| \leqslant\left|\lambda_{1}\right|+\left|\lambda_{2}\right|$. Hence

$$
\frac{\|s\|_{\mathbb{C}, \varepsilon}}{\left|\lambda_{1}+i \lambda_{2}\right|} \geqslant \frac{1}{2} \cdot \frac{\left\|s_{1}\right\|+\left\|s_{2}\right\|}{\left|\lambda_{1}\right|+\left|\lambda_{2}\right|} \geqslant \frac{1}{2} \inf _{\substack{s \in V, \lambda \in \mathbb{C}^{\times} \\[s]=\lambda \ell}}|\lambda|^{-1}\|s\| .
$$

Thus we obtain (1.59).

In particular, if $V^{\prime}$ is a quotient vector space of $V$ such that the projection map $V_{\mathbb{C}} \rightarrow W$ factorises through $V_{\mathbb{C}}^{\prime},\|\cdot\|^{\prime}$ is the quotient seminorm on $V^{\prime}$ induced by $\|\cdot\|$, and $\|\cdot\|_{W}^{\prime}$ is the quotient seminorm on $W$ induced by $\|\cdot\|_{\mathbb{C}, \varepsilon}^{\prime}$, then one has

$$
\|\cdot\|_{W}^{\prime} \leqslant\|\cdot\|_{W} \leqslant 2\|\cdot\|_{W}^{\prime} .
$$

In fact, by the above argument, for any non-zero element $\ell \in W$ one has

$$
\|\ell\|_{W}^{\prime} \leqslant \inf _{\substack{t \in V^{\prime}, \lambda \in \mathbb{C}^{\times} \\[t]=\lambda \ell}}|\lambda|^{-1}\|t\|=\inf _{\substack{s \in V, \lambda \in \mathbb{C}^{\times} \\[s]=\lambda \ell}}|\lambda|^{-1}\|s\| \leqslant 2\|\ell\|_{W}^{\prime} .
$$

The following proposition should be compared with $(2)$ and $(3)$ in Propositions 1.3 .19 .

Proposition 1.3.34. - Let $(V,\|\cdot\|)$ be a finite-dimensional seminormed vector space over $\mathbb{R}$. Denote by $\|\cdot\|_{\text {det }}$ and $\|\cdot\|_{\mathbb{C}, \varepsilon, \text { det }}$ the determinant seminorms induced by $\|\cdot\|$ and $\|\cdot\|_{\mathbb{C}, \varepsilon}$, respectively. Then one has

$$
\|\cdot\|_{\mathbb{C}, \varepsilon, \operatorname{det}} \leqslant\|\cdot\|_{\operatorname{det}, \mathbb{C}} \leqslant \frac{\delta\left(V_{\mathbb{C}},\|\cdot\|_{\mathbb{C}, \varepsilon}\right)}{\delta(V,\|\cdot\|)}\|\cdot\|_{\mathbb{C}, \varepsilon, \operatorname{det}},
$$


where $r$ is the rank of $V$ and $\|\cdot\|_{\operatorname{det}, \mathbb{C}}$ is the seminorm on $\operatorname{det}(V) \otimes_{\mathbb{R}} \mathbb{C}$ induced by $\|\cdot\|_{\operatorname{det}}$ by extension of scalars.

Proof. - In the case where $\|\cdot\|$ is not a norm, both seminorms $\|\cdot\|_{\mathbb{C}, \varepsilon, \operatorname{det}}$ and $\|\cdot\|_{\operatorname{det}, \mathbb{C}}$ vanish. In the following, we treat the case where $\|\cdot\|$ is a norm.

Let $\left\{e_{i}\right\}_{i=1}^{r}$ be an Hadamard basis of $(V,\|\cdot\|)$. One has

$$
\left\|e_{1} \wedge \cdots \wedge e_{r}\right\|_{\mathbb{C}, \varepsilon, \operatorname{det}} \leqslant\left\|e_{1}\right\|_{\mathbb{C}, \varepsilon} \cdots\left\|e_{r}\right\|_{\mathbb{C}, \varepsilon}=\left\|e_{1}\right\| \cdots\left\|e_{r}\right\|=\left\|e_{1} \wedge \cdots \wedge e_{r}\right\|_{\text {det }},
$$

where the first equality comes from Propositions 1.3.1 and 1.1.18. Hence we obtain

$$
\|\cdot\|_{\mathbb{C}, \varepsilon, \operatorname{det}} \leqslant\|\cdot\|_{\operatorname{det}, \mathbb{C}} .
$$

Similarly, if $\left\{\alpha_{i}\right\}_{i=1}^{r}$ is an Hadamard basis of $\left(V^{\vee},\|\cdot\|_{*}\right)$, one has

$$
\left\|\alpha_{1} \wedge \cdots \wedge \alpha_{r}\right\|_{*, \operatorname{det}, \mathbb{C}}=\left\|\alpha_{1} \wedge \cdots \wedge \alpha_{r}\right\|_{*, \operatorname{det}}=\left\|\alpha_{1}\right\|_{*} \cdots\left\|\alpha_{r}\right\|_{*},
$$

where $\|\cdot\|_{*, \text { det }}$ denotes the determinant norm of $\|\cdot\|_{*}$. Since $\alpha_{1}, \ldots, \alpha_{r}$ are elements in $V^{\vee}$, by (1) in Proposition 1.3.20 one has $\left\|\alpha_{i}\right\|_{*}=\left\|\alpha_{i}\right\|_{\mathbb{C}, \varepsilon, *}$ for any $i \in\{1, \ldots, r\}$, where $\|\cdot\|_{\mathbb{C}, \varepsilon, *}$ is the dual norm of $\|\cdot\|_{\mathbb{C}, \varepsilon}$. Hence we obtain

$$
\left\|\alpha_{1} \wedge \cdots \wedge \alpha_{r}\right\|_{*, \operatorname{det}, \mathbb{C}}=\left\|\alpha_{1}\right\|_{\mathbb{C}, *} \cdots\left\|\alpha_{r}\right\|_{\mathbb{C}, \varepsilon, *} \geqslant\left\|\alpha_{1} \wedge \cdots \wedge \alpha_{r}\right\|_{\mathbb{C}, \varepsilon, *, \operatorname{det}},
$$

where $\|\cdot\|_{\mathbb{C}, \varepsilon, *, \text { det }}$ denotes the determinant norm of $\|\cdot\|_{\mathbb{C}, \varepsilon, *}$.

Let $\eta$ be a non-zero element of $\operatorname{det}(V)$, and $\eta^{\vee}$ be its $\operatorname{dual}$ element in $\operatorname{det}\left(V^{\vee}\right)$. By definition (see $\$ 1.2 .7$ ) one has

$$
\|\eta\|_{\text {det }}=\delta(V,\|\cdot\|)^{-1}\left\|\eta^{\vee}\right\|_{*, \text { det }}^{-1},
$$

where $\|\cdot\|_{*, \text { det }}$ is the determinant norm of the dual norm $\|\cdot\|_{*}$ on $V^{\vee}$. Since $\eta^{\vee}$ belongs to $V^{\vee}$, by (1.61) we obtain $\left\|\eta^{\vee}\right\|_{*, \text { det }}=\left\|\eta^{\vee}\right\|_{*, \text { det, } \mathbb{C}} \geqslant\left\|\eta^{\vee}\right\|_{\mathbb{C}, \varepsilon, *, \text { det }}$. Hence we obtain

$$
\|\eta\|_{\text {det }} \leqslant \delta(V,\|\cdot\|)^{-1}\left\|\eta^{\vee}\right\|_{\mathbb{C}, \varepsilon, *, \operatorname{det}}^{-1}=\frac{\delta\left(V_{\mathbb{C}},\|\cdot\|_{\mathbb{C}}\right)}{\delta(V,\|\cdot\|)}\|\eta\|_{\mathbb{C}, \varepsilon, \operatorname{det}} .
$$

The proposition is thus proved.

The following proposition shows that, in the Archimedean case, the norm obtained by extension of scalars is "almost the largest" norm extending the initial one.

Proposition 1.3.35. - Let $(V,\|\cdot\|)$ be a finite-dimensional seminormed vector space over $\mathbb{R}$. Let $\|\cdot\|^{\prime}$ be a seminorm on $V_{\mathbb{C}}$ which extends $\|\cdot\|$. Then one has $\|\cdot\|^{\prime} \leqslant 2\|\cdot\|_{\mathbb{C}, \varepsilon}$.

Proof. - Let $s+i t$ be an element of $V_{\mathbb{C}}$, where $(s, t) \in V^{2}$. One has $\|s+i t\|^{\prime} \leqslant$ $\|s\|+\|t\|$. By Proposition 1.3.31, $\max \{\|s\|,\|t\|\} \leqslant\|s+i t\|_{\mathbb{C}, \varepsilon}$. Hence we obtain $\|s+i t\|^{\prime} \leqslant 2\|s+i t\|_{\mathbb{C}, \varepsilon}$.

Proposition 1.3.36. — Let $(V,\|\cdot\|)$ be a finite-dimensional seminormed vector space over $\mathbb{R}$. Let $\|\cdot\|_{\mathbb{C}, \varepsilon, *}$ be the dual norm of $\|\cdot\|_{\mathbb{C}, \varepsilon}$ and $\|\cdot\|_{*, \mathbb{C}, \varepsilon}$ be the norm on $E_{\mathbb{C}}^{*}$ induced by $\|\cdot\|_{*}$ by $\varepsilon$-extension of scalars. One has $\|\cdot\|_{\mathbb{C}, \varepsilon, *} \leqslant 2\|\cdot\|_{*, \mathbb{C}, \varepsilon}$. 
Proof. - By (1) in Proposition 1.3.20, the restriction of $\|\cdot\|_{\mathbb{C}, \varepsilon, *}$ to $V^{*}$ coincides with $\|\cdot\|_{*}$. Hence Proposition 1.3 .35 leads to the inequality $\|\cdot\|_{\mathbb{C}, \varepsilon, *} \leqslant 2\|\cdot\|_{*, \mathbb{C}, \varepsilon}$.

Remark 1.3.37. - The results of Proposition 1.3.19 is not necessarily true for a general seminormed vector space over an Archimedean valued field. Consider the vector space $V=\mathbb{R}^{2}$ equipped with the norm $\|\cdot\|$ such that

$$
\forall(a, b) \in \mathbb{R}^{2}, \quad\|(a, b)\|=\left(\max \{a, b, 0\}^{2}+\min \{a, b, 0\}^{2}\right)^{1 / 2} .
$$

In other words, if $a$ and $b$ have the same sign, one has $\|(a, b)\|=\max \{|a|,|b|\}$; otherwise $\|(a, b)\|=\left(a^{2}+b^{2}\right)^{1 / 2}$. The unit disc of this norm is represented by Figure 1 Let $\left\{e_{1}, e_{2}\right\}$ be the canonical basis of $\mathbb{R}^{2}$, where $e_{1}=(1,0)$ and $e_{1}=(0,1)$. One has

Figure 1. Unit ball of the norm $\|\cdot\|$

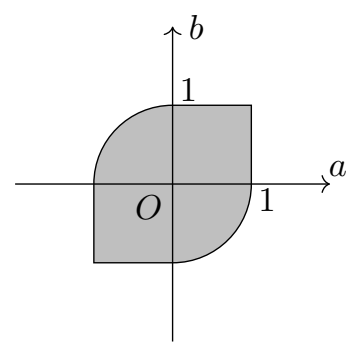

$$
\left\|e_{1} \wedge e_{2}\right\|_{\text {det }}=\inf _{a d-b c \neq 0} \frac{\|(a, b)\| \cdot\|(c, d)\|}{|a d-b c|}
$$

where $\|\cdot\|_{\text {det }}$ is the determinant norm induced by $\|\cdot\|$. Note that if $a, b, c, d$ are four real numbers such that $\max \{|a|,|b|,|c|,|d|\} \leqslant 1$ and that abcd $\geqslant 0$, then one has $|a d-b c| \leqslant \max \{|a d|,|b c|\} \leqslant 1$ since $a d$ and $b c$ have the same sign. Hence

$$
\left\|e_{1} \wedge e_{2}\right\|_{\text {det }}=\inf _{\substack{a d-b c \neq 0 \\ a b c d<0}} \frac{\|(a, b)\| \cdot\|(c, d)\|}{|a d-b c|}=\frac{1}{\sqrt{2}} .
$$

Moreover, $\left(e_{1}+e_{2}, e_{1}-e_{2}\right)$ forms an Hadamard basis of $(V,\|\cdot\|)$.

The dual norm of $\|\cdot\|$ is given by the following formula

$$
\forall(\lambda, \mu) \in \mathbb{R}^{2}, \quad\left\|\lambda e_{1}^{\vee}+\mu e_{2}^{\vee}\right\|_{*}= \begin{cases}|\lambda|+|\mu|, & \lambda \mu<0, \\ \left(\lambda^{2}+\mu^{2}\right)^{1 / 2}, & \lambda \mu \geqslant 0 .\end{cases}
$$

The unit disc of the dual norm is represented by Figure 2 ,

Consider now a vector $x+i y \in V \otimes_{\mathbb{R}} \mathbb{C}$, where $x$ and $y$ are vectors in $V$, and $i$ is the imaginary unit. One has

$$
\|x+i y\|_{\mathbb{C}, \varepsilon}=\sup _{\varphi \in V^{\vee} \backslash\{0\}} \frac{|\varphi(x)+i \varphi(y)|}{\|\varphi\|_{*}}=\sup _{\varphi \in V^{\vee} \backslash\{0\}} \frac{\left(\varphi(x)^{2}+\varphi(y)^{2}\right)^{1 / 2}}{\|\varphi\|_{*}} .
$$


Figure 2. Unit ball of the norm $\|\cdot\|_{*}$

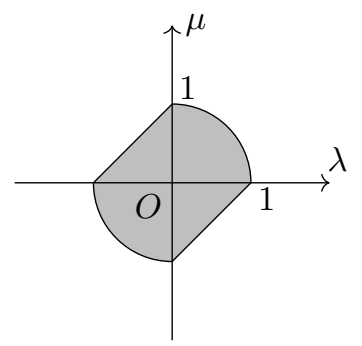

In particular, one has

$$
\left\|e_{1}+i e_{2}\right\|_{\mathbb{C}, \varepsilon}=\sup _{(\lambda, \mu) \neq(0,0)} \frac{\left(\lambda^{2}+\mu^{2}\right)^{1 / 2}}{f(\lambda, \mu)}
$$

where

$$
f(\lambda, \mu)= \begin{cases}|\lambda|+|\mu|, & \lambda \mu<0 \\ \left(\lambda^{2}+\mu^{2}\right)^{1 / 2}, & \lambda \mu \geqslant 0 .\end{cases}
$$

Hence one has $\left\|e_{1}+i e_{2}\right\|_{\mathbb{C}, \varepsilon}=1$. Similarly, one has $\left\|i e_{1}+e_{2}\right\|_{\mathbb{C}, \varepsilon}=1$. Therefore

$$
\left\|e_{1} \wedge e_{2}\right\|_{\mathbb{C}, \varepsilon, \operatorname{det}}=\frac{1}{2}\left\|\left(e_{1}+i e_{2}\right) \wedge\left(i e_{1}+e_{2}\right)\right\|_{\mathbb{C}, \varepsilon, \operatorname{det}} \leqslant \frac{1}{2},
$$

where $\|\cdot\|_{\mathbb{C}, \varepsilon, \text { det }}$ is the determinant norm associated with $\|\cdot\|_{\mathbb{C}, \varepsilon}$. In particular, $\left(e_{1}+\right.$ $\left.e_{2}, e_{1}-e_{2}\right)$ is no longer an Hadamard basis of $\left(V \otimes_{\mathbb{R}} \mathbb{C},\|\cdot\|_{\mathbb{C}, \varepsilon}\right)$.

The above construction also provides a counter-example to the statement of Proposition 1.3.26 in the case where $(k,|\cdot|)$ is $\mathbb{R}$ equipped with the usual absolute value and $K=\mathbb{C}$. Consider the surjective $\mathbb{C}$-linear map $\pi$ from $\mathbb{C}^{2}$ to $\mathbb{C}$ which sends $\left(z_{1}, z_{2}\right) \in \mathbb{C}^{2}$ to $z_{1}-i z_{2}$. Let $\|\cdot\|^{\prime}$ be the quotient norm on $\mathbb{C}$ induced by $\|\cdot\|_{\mathbb{C}, \varepsilon}$. Since $\pi(1, i)=2$ we obtain that

$$
\|1\|^{\prime} \leqslant \frac{1}{2}\|(1, i)\|_{\mathbb{C}, \varepsilon}=\frac{1}{2}
$$

However, for any non-zero element $(\lambda, \mu) \in \mathbb{R}^{2}$ one has

$$
\frac{\|(\lambda, \mu)\|}{|\pi(\lambda, \mu)|}=\frac{\|(\lambda, \mu)\|}{\sqrt{\lambda^{2}+\mu^{2}}}= \begin{cases}\max (|\lambda|,|\mu|) / \sqrt{\lambda^{2}+\mu^{2}}, & \lambda \mu \geqslant 0 \\ 1, & \lambda \mu<0\end{cases}
$$

which is bounded from below by $1 / \sqrt{2}$. 


\section{CHAPTER 2}

\section{LOCAL METRICS}

Throughout the chapter, let $k$ be a field equipped with an absolute value $|\cdot|$. We assume that $k$ is complete with respect to this absolute value. If $|\cdot|$ is Archimedean, we assume that it is the usual absolute value on $\mathbb{R}$ or $\mathbb{C}$.

\subsection{Metrised vector bundles}

Let $\pi: X \rightarrow$ Spec $k$ be a $k$-scheme. Let $F_{X}$ be the functor from the category $\mathbf{A}_{k}$ of all $k$-algebras to that of sets, which sends any $k$-algebra $A$ to the set of all $k$-points of $X$ valued in $A$. Recall that a $k$-point of $X$ valued in $A$ is by definition a $k$-morphism from $\operatorname{Spec} A$ to $X$. If we denote by $\mathbf{E}_{k}$ the full subcategory of $\mathbf{A}_{k}$ of all field extensions of $k$, then the scheme $X$ identifies (as a set) with the colimit (1) of the functor $F_{X}$ restricted to $\mathbf{E}_{k}$ (see [50] page 18, théorème de comparaison).

2.1.1. Berkovich space associated to a scheme. - The Berkovich space associated with a $k$-scheme can also be constructed as a colimit. By valued extension of $k$, we refer to a field extension $k^{\prime}$ of $k$ equipped with an absolute value which extends $|\cdot|$ on $k$. If $\left(k_{1},|\cdot|_{1}\right)$ and $\left(k_{2},|\cdot|_{2}\right)$ are two valued extensions of $k$, we call morphism from $\left(k_{1},|\cdot|_{1}\right)$ to $\left(k_{2},|\cdot|_{2}\right)$ any field homomorphism $k_{1} \rightarrow k_{2}$ which preserves the absolute values. The valued extensions of $k$ and morphisms between them form a category which we denote by $\mathbf{V E}_{k}$. One has a forgetful functor $w$ from $\mathbf{V E}_{k}$ to $\mathbf{A}_{k}$ which consists of forgetting the absolute values.

Definition 2.1.1. - Let $X$ be a $k$-scheme. The Berkovich space (as a set) associated with $X$ is defined as the colimit of the composed functor $F_{X} \circ w$ from $\mathbf{V E}_{k}$

1. One can fix two Grothendieck universes $\mathcal{U}$ and $\mathcal{V}$ such that $k \in \mathcal{U} \in \mathcal{V}$, and take $\mathbf{A}_{k}$ as the category of $k$-algebras whose underlying sets lie in $\mathcal{U}$. It is then a small category with respect to the univers $\mathcal{V}$. Thus we can consider the colimite of a functor from $\mathbf{A}_{k}$ to that of all sets in $\mathcal{U}$. 
to the category of sets, denoted by $X^{\text {an }}$. The universal property of colimits defines a map $j$ from $X^{\text {an }}$ to $X$, called the specification map.

Let $x$ be a point of $X^{\text {an }}$. We denote by $\kappa(x)$ the residue field of the point $j(x)$ of the scheme $X$, called the residue field of $x$. We consider the point $j(x)$ as a $k$-morphism from Spec $\kappa(x)$ to $X$. By definition, if $y: \operatorname{Spec} k^{\prime} \rightarrow X$ is a $k$-point of $X$ taking values in some valued extension $\left(k^{\prime},|\cdot|_{y}\right)$ of $k$, which represents the point $x \in X^{\text {an }}$, then, as a $k$-morphism of schemes, it factorises through the point $j(x)$. Therefore the residue field $\kappa(x)$ is a subfield of $k^{\prime}$. Note that the restriction of $|\cdot|_{y}$ to $\kappa(x)$ does not depend on the choice of the representative $y$. Hence we obtain an absolute value on $\kappa(x)$ extending $|\cdot|$ on $k$, denoted by $|\cdot|_{x}$, and called the absolute value induced by $x$. Note that two different points of $X^{\text {an }}$ may have the same residue field. However in this case they induce different absolute values on the residue field.

On the Berkovich space $X^{\text {an }}$, one can naturally define the Zariski topology, which is the most coarse topology making the specification map $j: X^{\text {an }} \rightarrow X$ continuous. Moreover, according to Berkovich [8], the construction of $X^{\text {an }}$ allows to define a finer topology, which we describe as follows. Let $U$ be an open subscheme of $X$. The ring $\mathcal{O}_{X}(U)$ of all regular functions on $U$ can be identified with the set of all $k$-morphisms from $U$ to the affine line $\mathbb{A}_{k}^{1}$. Let $f$ be a regular function on $U$. If $k^{\prime}$ is a valued extension of $k$ and $y: \operatorname{Spec} k^{\prime} \rightarrow U$ is a $k$-point of $U$ valued in $k^{\prime}$, then the evaluation of $f$ at $y$ determines an element in $k^{\prime}$ which we denote by $f(y)$. Since $k^{\prime}$ is equipped with an absolute value extending $|\cdot|$, we can evaluate the absolute value of the element $f(y)$, which we denote by $|f|(y)$. Note that the value of $|f|(y)$ only depends on the equivalence class of $y$ in $X^{\text {an }}$. Thus we obtain a non-negative function $|f|$ defined on $U^{\text {an }}=j^{-1}(U)$.

Definition 2.1.2. - Let $X$ be a scheme over Spec $k$. The Berkovich topology on $X^{\text {an }}$ is defined as the most coarse topology on $X^{\text {an }}$ which makes the specification map $j: X^{\text {an }} \rightarrow X$ and all functions of the form $|f|$ continuous, where $f$ runs over the set of all regular functions on Zariski open subsets of the scheme $X$. We refer the readers to $[8, \S 3.4]$ for more details.

The construction of Berkovich topological spaces associated with $k$-schemes is functorial. Let $X$ and $Y$ be $k$-schemes and $\varphi: X \rightarrow Y$ be a $k$-morphism. It induces a morphism of functors from $F_{X}$ to $F_{Y}$, which determines, by passing to colimit, a map $\varphi^{\text {an }}: X^{\text {an }} \rightarrow Y^{\text {an }}$, called the map associated with the morphism of $k$-schemes $X \rightarrow Y$.

Proposition 2.1.3. - Let $\varphi: X \rightarrow Y$ be a morphism of k-schemes. Then the map $\varphi^{\text {an }}$ between Berkovich spaces is continuous with respect to the Berkovich topologies.

Proof. - Clearly the map $\varphi^{\text {an }}$ is continuous with respect to the Zariski topologies. It suffices to prove that, for any regular function $f$ on a Zariski open subset $U$ of $Y$, the function $|f| \circ \varphi^{\text {an }}$ is continuous. Let $g$ be the image of $f$ by the morphism of sheaves $\mathcal{O}_{Y} \rightarrow \varphi_{*}\left(\mathcal{O}_{X}\right)$ in the structure of the morphism of schemes $\varphi$. It is a regular function 
on $\varphi^{-1}(U)$. For any $x \in \varphi^{-1}(U)^{\text {an }}$, the residue field $\kappa(x)$ is a valued extension of $\kappa(y)$ with $y=\varphi^{\text {an }}(x)$. Moreover, $g(x)$ is the canonical image of $f(y)$ in $\kappa(x)$. Therefore, one has $|f| \circ \varphi^{\text {an }}=|g|$, which is a continuous function.

Remark 2.1.4. - Assume that $k$ is an Archimedean valued field, that is, $k=\mathbb{R}$ or $\mathbb{C}$. If $X$ is a $k$-scheme, then the Berkovich space $X^{\text {an }}$ identifies (as a set) with the set $X(\mathbb{C})$ of all complex points of $X$ modulo the action of the Galois group $\operatorname{Gal}(\mathbb{C} / k)$. In particular, if $X$ is the affine line $\mathbb{A}_{k}^{1}$, then the Berkovich space associated with $X$ is $\mathbb{C}$ when $k=\mathbb{C}$, and is $\mathbb{C} / \tau$ when $k=\mathbb{R}$, where $\tau$ denotes the complex conjugation. In this case the Berkovich topology on $X^{\text {an }}$ is generated by functions of the form $|P(z)|$, where $P$ is a polynomial in $k[z]$. Therefore, it coincides with the usual topology on $\mathbb{C}$ or on $\mathbb{C} / \tau$. In fact, in the case where $k=\mathbb{C}$, the usual topology on $\mathbb{C}$ is generated by the functions $(z \in \mathbb{C}) \mapsto|z-a|$ (where $a \in \mathbb{C}$ ). In the case where $k=\mathbb{R}$, the usual topology on $\mathbb{C} / \tau$ is generated by the functions

$$
z \longmapsto|z-a| \cdot|z-\bar{a}|=\left|z^{2}-2 \operatorname{Re}(a) z+a \bar{a}\right|, \text { where } a \in \mathbb{C} .
$$

For a general $k$-scheme $X$, any regular function $f$ on $X$ determines a function $f$ an on $X^{\text {an }}$ valued in $\mathbb{C}$ (in the case where $k=\mathbb{R}$, we identify $\left(\mathbb{A}_{k}^{1}\right)$ an with the upper half-plane in $\mathbb{C}$ ). By Proposition 2.1.3, the map $f^{\text {an }}$ is a continuous complex function on $X^{\text {an }}$.

Proposition 2.1.5. — Let $X$ be a $k$-scheme, $X_{\text {red }}$ be the reduced scheme associated with $X$, and $i: X_{\mathrm{red}} \rightarrow X$ be the canonical morphism. Then the associated continuous map of Berkovich spaces $i^{\text {an }}: X_{\text {red }}^{\text {an }} \rightarrow X^{\text {an }}$ is a homeomorphism.

Proof. - Note that the restrictions of the functors $F_{X}$ and $F_{X_{\text {red }}}$ to $\mathbf{E}_{k}$ are the same. Therefore $i^{\text {an }}$ is a bijection of sets. Moreover, it is an homeomorphism if we equip $X_{\text {red }}^{\text {an }}$ and $X^{\text {an }}$ with the Zariski topologies. Let $U$ be a Zariski open subset of $X$. By definition $\mathcal{O}_{X_{\text {red }}}(U)$ is the reduced ring associated with $\mathcal{O}_{X}(U)$. For any nilpotent element $s$ in $\mathcal{O}_{X}(U)$ one has $s(x)=0$ for any $x \in X$. As a consequence, if $f$ is a regular function of $X$ on $U$ and if $\bar{f}$ is its canonical image in $\mathcal{O}_{X_{\text {red }}}(U)$, then one has $|\bar{f}|=|f|$ on $U^{\text {an }}$. Thus the Berkovich topologies on $X^{\text {an }}$ and $X_{\text {red }}^{\text {an }}$ are the same.

Remark 2.1.6. - We assume that the absolute value $|\cdot|$ is non-Archimedean and non-trivial. Let $\mathfrak{o}_{k}$ be the valuation ring of $(k,|\cdot|)$. Let $\mathscr{A}$ be a finitely generated $\mathfrak{o}_{k^{-}}$ algebra, which contains $\mathfrak{o}_{k}$ as a subring. Let $A=\mathscr{A} \otimes_{\mathfrak{o}_{k}} k$, which identifies with the localisation of $\mathscr{A}$ with respect to $\mathfrak{o}_{k} \backslash\{0\}$. Note that the Berkovich space $(\operatorname{Spec} A)^{\text {an }}$ identifies with the set of all multiplicative seminorms on $A$ extending the absolute value $|\cdot|$ on $k$. If $x$ is a point of $(\operatorname{Spec} A)^{\text {an }}$, we denote by $\widehat{\kappa}(x)$ the completion of the residue field $\kappa(x)$ with respect to the absolute value $|\cdot|_{x}$, and we let $p_{x}$ be the $k$-morphism from $\operatorname{Spec} \widehat{\kappa}(x)$ to $\operatorname{Spec} A$ corresponding to the point $j(x) \in \operatorname{Spec} A$ (see Definition 2.1.1 for the specification map $j$ ). Then $p_{x}$ extends to an $\mathfrak{o}_{k}$-morphism $\mathscr{P}_{x}$ from Spec $\mathfrak{o}_{x}$ to $\operatorname{Spec} \mathscr{A}$ if and only if $|a|_{x} \leqslant 1$ for any $a \in \mathscr{A}$. In this case the image 
of the maximal ideal $\mathfrak{m}_{x}$ of $\mathfrak{o}_{x}$ by the morphism $\mathscr{P}_{x}:$ Spec $\mathfrak{o}_{x} \rightarrow$ Spec $\mathscr{A}$ identifies with the prime ideal

$$
\left(\mathscr{A},|\cdot|_{x}\right)_{<1}:=\left\{a \in \mathscr{A}:|a|_{x}<1\right\} \text { of } \mathscr{A}
$$

which lies in the fibre $(\operatorname{Spec} \mathscr{A})$ 。 of Spec $\mathscr{A}$ over the maximal ideal of $\mathfrak{o}_{k}$. We denote by $(\operatorname{Spec} A)_{\mathscr{A}}^{\text {an }}$ the subset of $(\operatorname{Spec} A)^{\text {an }}$ of points $x$ such that

$$
\sup _{a \in \mathscr{A}}|a|_{x} \leqslant 1
$$

and by $r_{\mathscr{A}}:(\operatorname{Spec} A)_{\mathscr{A}}^{\mathrm{an}} \rightarrow(\operatorname{Spec} \mathscr{A})$ 。 the map sending $x \in(\operatorname{Spec} A)_{\mathscr{A}}^{\text {an }}$ to $\left(\mathscr{A},|\cdot|_{x}\right)_{<1}$, called the reduction map. Note that the reduction map is always surjective (cf. 8 Proposition 2.4.4] or [78, 4.13 and Proposition 4.14]).

Proposition 2.1.7. - We assume that the absolute value $|\cdot|$ is non-trivial and nonArchimedean and let $\mathfrak{o}_{k}$ be the valuation ring of $(k,|\cdot|)$. Let $\mathscr{A}$ be a finitely generated $\mathfrak{o}_{k}$-algebra and $A$ be the localisation of $\mathscr{A}$ with respect to $\mathfrak{o}_{k} \backslash\{0\}$. Then the integral closure of $\mathscr{A}$ in $A$ identifies with

$$
\bigcap_{x \in(\operatorname{Spec} A)_{\mathscr{A}}^{\mathrm{an}}}\left(A,|\cdot|_{x}\right)_{\leqslant 1}
$$

where

$$
\left(A,|\cdot|_{x}\right)_{\leqslant 1}=\left\{a \in A:|a|_{x} \leqslant 1\right\} .
$$

In particular, if $(k,|\cdot|)$ is discrete, $\mathscr{A}$ is flat over $\mathfrak{o}_{k}$ and $\mathscr{A} / \varpi \mathscr{A}$ is reduced, then

$$
\mathscr{A}=\bigcap_{x \in(\operatorname{Spec} A)^{\mathrm{an}}}\left(A,|\cdot|_{x}\right)_{\mathbb{S}},
$$

where $\varpi$ is a uniformizing parameter of $(k,|\cdot|)$.

Proof. - Let $\mathscr{B}$ be the integral closure of $\mathscr{A}$ in $A$. We first show that $\mathscr{B}$ is contained in $\left(A,|\cdot|_{x}\right)_{\leqslant 1}$ for any $x \in(\operatorname{Spec} A)_{\mathscr{A}}^{\text {an }}$. If $a \in \mathscr{B}$, then there are $a_{1}, \ldots, a_{n} \in \mathscr{A}$ such that $a^{n}+a_{1} a^{n-1}+\cdots+a_{n}=0$. Therefore

$$
|a|_{x}^{n}=\left|a^{n}\right|_{x}=\left|a_{1} a^{n-1}+\cdots+a_{n}\right|_{x} \leqslant \max _{i \in\{1, \ldots, n\}}\left|a_{i}\right|_{x} \cdot|a|_{x}^{n-i} \leqslant \max _{i \in\{1, \ldots, n\}}|a|_{x}^{n-i},
$$

which implies that $|a|_{x} \leqslant 1$.

Let $a \in A$ such that $a$ is not integral over $\mathscr{A}$. Since $A$ is a $k$-algebra of finite type, it is a Noetherian ring which is non-zero (since $a \in A$ ). In particular, it admits only finitely many minimal prime ideals $S^{-1} \mathfrak{p}_{1}, \ldots, S^{-1} \mathfrak{p}_{n}$, where $\mathfrak{p}_{1}, \ldots, \mathfrak{p}_{n}$ are prime ideals of $\mathscr{A}$ which do not intersect $S:=\mathfrak{o}_{k} \backslash\{0\}$. We show that there exists $j \in$ $\{1, \ldots, n\}$ such that the canonical image of $a$ in $A / S^{-1} \mathfrak{p}_{j}$ is not integral over $\mathscr{A} / \mathfrak{p}_{j}$. Assume that, for any $i \in\{1, \ldots, n\}, f_{i}$ is a monic polynomial in $\left(\mathscr{A} / \mathfrak{p}_{i}\right)[T]$ such that $f_{i}\left(\lambda_{i}\right)=0$, where $\lambda_{i}$ is the class of $a$ in $A / S^{-1} \mathfrak{p}_{i}$. Let $F_{i}$ be a monic polynomial in $\mathscr{A}[T]$ whose reduction modulo $\mathfrak{p}_{i}[T]$ coincides with $f_{i}$. One has $F_{i}(a) \in S^{-1} \mathfrak{p}_{i}$ for any $i \in\{1, \ldots, n\}$. Let $F$ be the product of the polynomials $F_{1}, \ldots, F_{n}$. Then $F(a)$ belongs to the intersection $\bigcap_{i=1}^{n} S^{-1} \mathfrak{p}_{i}$, hence is nilpotent, which implies that $a$ is 
integral over $\mathscr{A}$. To show that there exists $x \in(\operatorname{Spec} A)_{\mathscr{A}}^{\text {an }}$ such that $|a|_{x}>1$ we may replace $\mathscr{A}$ (resp. $A$ ) by $\mathscr{A} / \mathfrak{p}_{j}$ (resp. $\left.A / S^{-1} \mathfrak{p}_{j}\right)$ and hence assume that $\mathscr{A}$ is an integral domain without loss of generality.

Let $b=a^{-1}$ in the fraction field of $A$. We assert that

$$
b \mathscr{A}[b] \cap \mathfrak{o}_{k} \neq\{0\} \quad \text { and } \quad 1 \notin b \mathscr{A}[b] .
$$

We set $a=a^{\prime} / s$ for some $a^{\prime} \in \mathscr{A}$ and $s \in S$. Then $s=b a^{\prime} \in b \mathscr{A}[b] \cap \mathfrak{o}_{k}$, so that $b \mathscr{A}[b] \cap \mathfrak{o}_{k} \neq\{0\}$. Next we assume that $1 \in b \mathscr{A}[b]$. Then there exist $m \in \mathbb{N}_{\geqslant 1}$ and $\left(a_{1}^{\prime}, \ldots, a_{m}^{\prime}\right) \in \mathscr{A}^{m}$ such that

$$
1=a_{1}^{\prime} b+a_{2}^{\prime} b^{2}+\cdots+a_{m}^{\prime} b^{m}
$$

or equivalently $a^{m}=a_{1}^{\prime} a^{m-1}+\cdots+a_{m}^{\prime}$, which is a contradiction.

Let $\mathfrak{p}$ be a maximal ideal of $\mathscr{A}[b]$ such that $b \mathscr{A}[b] \subseteq \mathfrak{p}$. As $\mathfrak{p} \cap \mathfrak{o}_{k} \neq\{0\}$ and $\mathfrak{p} \cap \mathfrak{o}_{k} \subseteq \mathfrak{m}_{k}$ (where $\mathfrak{m}_{k}$ is the maximal ideal of $\mathfrak{o}_{k}$ ), we have $\mathfrak{p} \cap \mathfrak{o}_{k}=\mathfrak{m}_{k}$ (since the Krull dimension of $\mathfrak{o}_{k}$ is $1(2)$ ), and hence $\mathfrak{p}$ lies in the fibre (Spec $\mathscr{A}[b]$ )。 of Spec $\mathscr{A}[b]$ over $\mathfrak{m}_{k}$. Note that $\mathscr{A}[b]$ is finitely generated over $\mathfrak{o}_{k}$ and $\mathscr{A}[b] \otimes_{\mathfrak{o}_{k}} k=A[b]$. Thus, since the reduction map

$$
r_{\mathscr{A}[b]}:(\operatorname{Spec} A[b])_{\mathscr{A}[b]}^{\mathrm{an}} \longrightarrow(\operatorname{Spec} \mathscr{A}[b]) \text { 。 }
$$

is surjective, there is $x \in(\operatorname{Spec} A[b])_{\mathscr{A}[b]}^{\text {an }}$ such that $r_{\mathscr{A}[b]}(x)=\mathfrak{p}$. Clearly $x \in$ $(\operatorname{Spec} A)_{\mathscr{A}}^{\text {an }}$. As $b \in \mathfrak{p}$, we have $|b|_{x}<1$, so that $|a|_{x}>1$ because $a b=1$. Therefore,

$$
a \notin \bigcap_{x \in(\operatorname{Spec} A)_{\mathscr{A}}^{\operatorname{an}}}\left(A,|\cdot|_{x}\right)_{\leqslant 1},
$$

as required.

Finally we consider the last assertion. We assume that there is

$$
a \in \bigcap_{x \in(\operatorname{Spec} A)_{\mathscr{A}}^{\mathrm{an}}}\left(A,|\cdot|_{x}\right)_{\leqslant 1} \backslash \mathscr{A} .
$$

By the previous result, there are $a_{1}, \ldots, a_{n} \in \mathscr{A}$ such that

$$
a^{n}+a_{1} a^{n-1}+\cdots+a_{n-1} a+a_{n}=0 .
$$

One can choose a positive integer $e$ such that $\varpi^{e} a \in \mathscr{A}$ and $\varpi^{e-1} a \notin \mathscr{A}$. As

$$
\left(\varpi^{e} a\right)^{n}+\varpi^{e} a_{1}\left(\varpi^{e} a\right)^{n-1}+\cdots+\varpi^{e(n-1)} a_{n-1}\left(\varpi^{e} a\right)+\varpi^{e n} a_{n}=0,
$$

$\left(\varpi^{e} a\right)^{n}=0$ in $\mathscr{A} / \varpi \mathscr{A}$, so that $\varpi^{e} a=0$ in $\mathscr{A} / \varpi \mathscr{A}$ because $\mathscr{A} / \varpi \mathscr{A}$ is reduced. Therefore there is $a^{\prime} \in \mathscr{A}$ such that $\varpi^{e} a=\varpi a^{\prime}$, and hence $\varpi^{e-1} a=a^{\prime} \in \mathscr{A}$ because $\mathscr{A}$ is flat over $\mathfrak{o}_{k}$. This is a contradiction.

2. It suffices to see $\mathfrak{m}_{k} \subseteq \mathfrak{p}$ for a non-zero prime ideal $\mathfrak{p}$ of $\mathfrak{o}_{k}$. Fix $e \in \mathfrak{p} \backslash\{0\}$. If $x \in \mathfrak{m}_{k}$, then $x^{n} e^{-1} \in \mathfrak{o}_{k}$ for some positive integer $n$ because $|x|<1$, so that $x^{n} \in \mathfrak{o}_{k} e \subseteq \mathfrak{p}$, and hence $x \in \mathfrak{p}$. 
2.1.2. Metric on a vector bundle. - Let $X$ be a scheme over Spec $k$. We denote by $\mathcal{F}_{X}$ an the sheaf of all real-valued functions on the Berkovich topological space $X^{\text {an }}$. Let $\mathcal{C}_{X}^{0}$ an be the subsheaf of $\mathcal{F}_{X}$ an of continuous functions.

Definition 2.1.8. - Let $E$ be a locally free $\mathcal{O}_{X}$-module of finite rank. We call metric on $E$ any family $\varphi=\left\{|\cdot|_{\varphi}(x)\right\}_{x \in X^{\text {an }}}$, where each $|\cdot|_{\varphi}(x)$ is a norm on $E(x):=$ $E \otimes \widehat{\kappa}(x), \widehat{\kappa}(x)$ being the completion of $\kappa(x)$ with respect to the absolute value $|\cdot|_{x}$. We use the symbol $|\cdot|_{\varphi}$ instead of the usual double bar symbol in order to distinguish a local norm from a global seminorm (cf. Definition 2.1.15).

Note that the family $\varphi$ actually defines a morphism of sheaves (of sets) from $E$ to $j_{*}\left(\mathcal{F}_{X^{\text {an }}}\right)$, which sends each section $s$ of $E$ over a Zariski open subset $U$ of $X$ to the function $|s|_{\varphi}: U^{\text {an }} \rightarrow \mathbb{R}_{\geqslant 0}$ sending $x \in U^{\text {an }}$ to

$$
|s|_{\varphi}(x):=|s(x)|_{\varphi}(x),
$$

where $s(x)$ denotes the reduction of $s$ in $E(x)$. If this morphism of sheaves takes values in $j_{*}\left(\mathcal{C}_{X^{\text {an }}}^{0}\right)$ (namely, for any section $s$ of $E$ on a Zariski open subset of $X$, the function $|s|_{\varphi}$ is continuous with respect to the Berkovich topology), we say that the metric $\varphi$ is continuous.

Remark 2.1.9. - Let $E$ be a locally free $\mathcal{O}_{X}$-module of finite rank, equipped with a continuous metric $\varphi$. Let $F$ be a locally free sub- $\mathcal{O}_{X}$-module of $E$. For any $x \in X^{\text {an }}$, the restriction of the norm $|\cdot|_{\varphi}(x)$ to $F(x)$ defines a norm on $F(x)$. These norms actually define a continuous metric on $F$. However, we don't know if, for any quotient vector bundle of $E$, the quotient norms of $\left.\left.\right|_{\varphi}\right|_{\varphi}(x)\left(x \in X^{\text {an }}\right)$ define a continuous metric on the quotient bundle.

The following lemma is used in the proof of Proposition 2.1.12.

Lemma 2.1.10. — Let $M$ be a topological space and $f$ be a non-negative function on $M$. Suppose that, for any $\alpha \in] 0,1\left[\right.$, there exists a continuous function $f_{\alpha}$ on $M$ such that $\alpha f_{\alpha} \leqslant f \leqslant f_{\alpha}$. Then the function $f$ is continuous.

Proof. - Let $x_{0}$ be a point of $M$. From the inequalities $\alpha f_{\alpha} \leqslant f \leqslant f_{\alpha}$, we deduce

$$
\liminf _{x \rightarrow x_{0}} \alpha f_{\alpha}(x) \leqslant \liminf _{x \rightarrow x_{0}} f(x) \leqslant \limsup _{x \rightarrow x_{0}} f(x) \leqslant \limsup _{x \rightarrow x_{0}} f_{\alpha}(x) .
$$

Since the function $f_{\alpha}$ is continuous, we obtain

$$
\alpha f_{\alpha}\left(x_{0}\right) \leqslant \liminf _{x \rightarrow x_{0}} f(x) \leqslant \limsup _{x \rightarrow x_{0}} f(x) \leqslant f_{\alpha}\left(x_{0}\right) .
$$

Moreover, one has $\alpha f_{\alpha}\left(x_{0}\right) \leqslant f\left(x_{0}\right) \leqslant f_{\alpha}\left(x_{0}\right)$. Hence

$$
\liminf _{x \rightarrow x_{0}} f(x) \leqslant \limsup _{x \rightarrow x_{0}} f(x) \leqslant \alpha^{-1} f\left(x_{0}\right) \leqslant \alpha^{-1} f_{\alpha}\left(x_{0}\right) \leqslant \alpha^{-2} \liminf _{x \rightarrow x_{0}} f(x) .
$$

Since $\alpha \in] 0,1\left[\right.$ is arbitrary and $\liminf _{x \rightarrow x_{0}} f(x)$ is finite, we obtain

$$
\liminf _{x \rightarrow x_{0}} f(x)=\limsup _{x \rightarrow x_{0}} f(x)=f\left(x_{0}\right) .
$$


The proposition is thus proved.

Definition 2.1.11. - Let $\pi: X \rightarrow \operatorname{Spec}(k)$ be a $k$-scheme and $\bar{V}=(V,\|\cdot\|)$ be a finite-dimensional normed vector space over $k$. For any $x \in X^{\text {an }}$, let $|\cdot|_{\bar{V}, \varepsilon}(x)$ be the norm on $V \otimes_{k} \widehat{\kappa}(x)$ induced by $\|\cdot\|$ by $\varepsilon$-extension of scalars, and $|\cdot|_{\bar{V}, \pi}$ be the norm on $V \otimes_{k} \widehat{\kappa}(x)$ induced by $\|\cdot\|$ by $\pi$-extension of scalars (see $\left.\$ 1.3\right)$. If $|\cdot|$ is Archimedean and if the norm $\|\cdot\|$ is induced by an inner product, for any $x \in X^{\text {an }}$, we denote by $|\cdot|_{\bar{V}, \mathrm{HS}}(x)$ the norm on $V \otimes_{k} \widehat{\kappa}(x)$ induced by $\|\cdot\|$ by orthogonal extension of scalars. For simplicity, the norms $|\cdot|_{\bar{V}, \varepsilon}(x),|\cdot|_{\bar{V}, \pi}(x)$ and $|\cdot|_{\bar{V}, \mathrm{HS}}(x)$ are often denoted by $|\cdot|_{\varepsilon}(x)$, $|\cdot|_{\pi}(x)$ and $|\cdot|_{\mathrm{HS}}(x)$, respectively.

Proposition 2.1.12. - The norms $|\cdot|_{\bar{V}, \varepsilon}(x), x \in X^{\text {an }}$ define a continuous metric on the locally free $\mathcal{O}_{X}$-module $\pi^{*}(V)$.

Proof. - Let $U$ be a Zariski open subset of $X$ and $s$ be a section of $\pi^{*}(V)$ over $U$. It suffices to prove that the function $\left(x \in U^{\mathrm{an}}\right) \mapsto|s|_{\bar{V}, \varepsilon}(x)$ is continuous on $U^{\text {an }}$.

We first treat the non-Archimedean case. By Proposition [1.2.7 for any $\alpha \in] 0,1[$, there exists an $\alpha$-orthogonal basis $\left\{e_{i}\right\}_{i=1}^{n}$ of $V$. By Proposition 1.3.13, for any $x \in$ $X^{\text {an }},\left\{e_{i}\right\}_{i=1}^{n}$ is also an $\alpha$-orthogonal basis of $\left(V \otimes_{k} \widehat{\kappa}(x),|\cdot|_{\bar{V}, \varepsilon}(x)\right)$. We can write $s$ into the form $s=f_{1} e_{1}+\cdots+f_{n} e_{n}$, where $f_{1}, \ldots, f_{n}$ are regular functions on $U$. Since $\left\{e_{i}\right\}_{i=1}^{n}$ is an $\alpha$-orthogonal basis and the norm $|\cdot|_{\bar{V}, \varepsilon}(x)$ is ultrametric for any $x \in U^{\text {an }}$, one has

$$
\forall x \in U^{\text {an }}, \quad \alpha \max _{i \in\{1, \ldots, n\}}\left|f_{i}\right|_{x} \cdot\left|e_{i}\right|_{\bar{V}, \varepsilon}(x) \leqslant|s|_{\bar{V}, \varepsilon}(x) \leqslant \max _{i \in\{1, \ldots, n\}}\left|f_{i}\right|_{x} \cdot\left|e_{i}\right|_{\bar{V}, \varepsilon}(x) .
$$

By Proposition 1.3.1, one has $\left|e_{i}\right|_{\bar{V}, \varepsilon}(x)=\left\|e_{i}\right\|_{* *}$ for any $x \in X^{\text {an }}$. Hence

$$
\forall x \in U^{\mathrm{an}}, \quad \alpha \max _{i \in\{1, \ldots, n\}}\left|f_{i}\right|_{x} \cdot\left\|e_{i}\right\|_{* *} \leqslant|s|_{\bar{V}, \varepsilon}(x) \leqslant \max _{i \in\{1, \ldots, n\}}\left|f_{i}\right|_{x} \cdot\left\|e_{i}\right\|_{* *} .
$$

Note that the function $\left(x \in U^{\text {an }}\right) \mapsto\left|f_{i}\right|_{x}$ is continuous for any $i$. Hence the function

$$
\left(x \in U^{\mathrm{an}}\right) \longmapsto \max _{i \in\{1, \ldots, n\}}\left|f_{i}\right|_{x} \cdot\left\|e_{i}\right\|_{* *}
$$

is also continuous. Since $\alpha \in] 0,1[$ is arbitrary, by Lemma 2.1.10 we obtain that the function $\left(x \in U^{\text {an }}\right) \mapsto|s|_{\bar{V}, \varepsilon}(x)$ is continuous.

We now consider the Archimedean case. Let $\left\{e_{i}\right\}_{i=1}^{n}$ be a basis of $V$. We write the section $s$ in the form $f_{1} e_{1}+\cdots+f_{n} e_{n}$, where $f_{1}, \ldots, f_{n}$ are regular functions on $U$. Note that $f_{1}^{\text {an }}, \ldots, f_{n}^{\text {an }}$ are continuous complex functions on $U^{\text {an }}$. Since the norm $\|\cdot\|_{\mathbb{C}, \varepsilon}$ is a continuous function on $V_{\mathbb{C}}$, we obtain that the map (see Remark 2.1.4)

$$
\left(x \in X^{\mathrm{an}}\right) \longmapsto|s|_{\bar{V}, \varepsilon}(x)=\left\|f_{1}^{\mathrm{an}}(x) e_{1}+\cdots+f_{n}^{\mathrm{an}}(x) e_{n}\right\|_{\mathbb{C}, \varepsilon}
$$

is a continuous function on $U^{\text {an }}$. The proposition is thus proved.

Proposition 2.1.13. - We assume that the absolute value $|\cdot|$ is Archimedean. Let $\bar{V}=(V,\|\cdot\|)$ be a finite-dimensional normed vector space over $k$. 
(1) The norms $|\cdot|_{\bar{V}, \pi}(x), x \in X^{\text {an }}$ define a continuous metric on the locally free $\mathcal{O}_{X}$-module $\pi^{*}(V)$.

(2) If the norm $\|\cdot\|$ is induced by an inner product, then the norms $|\cdot|_{\bar{V} \text {.HS }}(x), x \in$ $X^{\text {an }}$ define a continuous metric on the locally free $\mathcal{O}_{X}$-module $\pi^{*}(V)$.

Proof. - The proof is quite similar to the second part of the proof of Proposition 2.1.12, where we use the continuity of the norms $\|\cdot\|_{\mathbb{C}, \pi}$ and $\|\cdot\|_{\mathbb{C}, H S}$ (in the case where the norm $\|\cdot\|$ is induced by an inner product) on the topological space $V_{\mathbb{C}}$.

Proposition 2.1.14. - We assume that the field $k$ is Archimedean. Let $\pi: X \rightarrow$ $\operatorname{Spec}(k)$ be a $k$-scheme and $\bar{V}=(V,\|\cdot\|)$ be a finite-dimensional normed vector space over $\mathbb{R}$. For any $x \in X^{\mathrm{an}}$, let $|\cdot|_{\bar{V}}(x)$ be the norm on $V \otimes_{k} \widehat{\kappa}(x)$ induced by $\|\cdot\|$ by $\natural$-extension of scalars, where $\downarrow=\varepsilon, \pi$ or HS (in the case where $\|\cdot\|$ is induced by an inner product) and let $|\cdot|_{\bar{V}}(x)_{*}$ be the dual norm on $V^{\vee} \otimes_{k} \widehat{\kappa}(x)$ of $|\cdot|_{\bar{V}}(x)$. Then the norms $|\cdot|_{V}(x)_{*}, x \in X^{\text {an }}$ define a continuous metric on the locally free $\mathcal{O}_{X}$-module $\pi^{*}\left(V^{\vee}\right)$.

Proof. - Let $\left\{\alpha_{i}\right\}_{i=1}^{n}$ be a basis of $V^{\vee}$. Locally on a Zariski open subset $U$ of $X$, any element $s \in H^{0}\left(U, \pi^{*}\left(V^{\vee}\right)\right)$ can be written in the form $s=f_{1} \alpha_{1}+\cdots+f_{n} \alpha_{n}$, where $f_{1}, \ldots, f_{n}$ are regular functions on $U$. Let $\|\cdot\|_{\mathbb{C}, \natural, *}$ be the dual norm of $\|\cdot\|_{\mathbb{C}, \natural}$ (the norm on $V_{\mathbb{C}}$ induced by $\|\cdot\|$ by

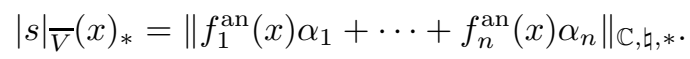

The equality follows from the definition of $|\cdot|_{V}(x)_{*}$ when $\kappa(x)=\mathbb{C}$. In the case where $\kappa(x)=\mathbb{R}$, the norm $|\cdot|_{\bar{V}}(x)_{*}$ is the dual norm of $\|\cdot\|$. Hence it coincides with the restriction of $\|\cdot\|_{\mathbb{C}, \natural, *}$ to $V^{\vee}$ (see Proposition 1.3.20 (1) [(2) and Remark 1.3.2 (2) for the cases $\downarrow=\varepsilon, \pi$ and HS, respectively). Thus the equality (2.1) also holds in this case. Since the norm $\|\cdot\|_{\mathbb{C}, \natural, *}$ is a continuous function on $V_{\mathbb{C}}^{\vee}$, we obtain that the function $\left(x \in U^{\mathrm{an}}\right) \mapsto|s|_{V}(x)_{*}$ is continuous.

Definition 2.1.15. - If the $k$-scheme $X$ is proper, then the associated Berkovich space $X^{\text {an }}$ is compact (see [8] Proposition 3.4.8). In particular, if $E$ is a locally free $\mathcal{O}_{X}$-module equipped with a continuous metric $\varphi$, for any global section $s \in H^{0}(X, E)$, the number

$$
\|s\|_{\varphi}:=\sup _{x \in X^{\text {an }}}|s|_{\varphi}(x)
$$

is finite. Thus we obtain a map $\|\cdot\|_{\varphi}: H^{0}(X, E) \rightarrow \mathbb{R}_{+}$, which is actually a seminorm on the $k$-vector space $H^{0}(X, E)$.

Let $X_{\text {red }}$ be the reduced scheme associated with $X$ and $E_{\text {red }}:=E \otimes \mathcal{O}_{X} \mathcal{O}_{X_{\text {red }}}$. Note that the natural morphism $X_{\text {red }}^{\text {an }} \rightarrow X^{\text {an }}$ is a homeomorphism (see Proposition 2.1.5), so that to give a continuous metric of $E$ on $X^{\text {an }}$ is equivalent to give a continuous metric of $E_{\text {red }}$ on $X_{\text {red }}^{\text {an }}$. The corresponding metric of $E_{\text {red }}$ is denoted by $\varphi_{\text {red. }}$. Moreover, if $X$ is proper and we denote the natural homomorphism $H^{0}(X, E) \rightarrow H^{0}\left(X_{\text {red }}, E_{\text {red }}\right)$ 
by $\gamma_{E}$, then it is easy to see that $\|s\|_{\varphi}=\left\|\gamma_{E}(s)\right\|_{\varphi_{\text {red }}}$ for any $s \in H^{0}(X, E)$. By (1) in the following proposition, the null space of $\|\cdot\|_{\varphi}$ coincides with the kernel of $\gamma_{E}$, which is denoted by $\mathcal{N}(X, E)$. The induced norm on $H^{0}(X, E) / \mathcal{N}(X, E)$ is denoted by $\|\cdot\| \tilde{\varphi}$.

Proposition 2.1.16. — (1) If $X$ is reduced, then $\|\cdot\|_{\varphi}$ is actually a norm.

(2) For any $x \in X^{\text {an }}$, the image of $\mathcal{N}(X, E) \otimes_{k} \widehat{\kappa}(x)$ by the natural homomorphism $H^{0}(X, E) \otimes_{k} \widehat{\kappa}(x) \rightarrow E \otimes_{\mathcal{O}_{X}} \widehat{\kappa}(x)$ is zero, so that one has the induced homomorphism $\left(H^{0}(X, E) / \mathcal{N}(X, E)\right) \otimes_{k} \widehat{\kappa}(x) \rightarrow E \otimes_{\mathcal{O}_{X}} \widehat{\kappa}(x)$. Moreover, if $E$ is generated by global sections, then $\left(H^{0}(X, E) / \mathcal{N}(X, E)\right) \otimes_{k} \widehat{\kappa}(x) \rightarrow E \otimes_{\mathcal{O}_{X}} \widehat{\kappa}(x)$ is surjective for all $x \in X^{\text {an }}$.

(3) If $E$ is invertible and $s \in \mathcal{N}(X, E)$, then there is a positive integer $n_{0}$ such that $s^{\otimes n}=0$ for all $n \geqslant n_{0}$.

Proof. - (1) It is sufficient to see that if $\|s\|_{\varphi}=0$, then $s=0$. Let $\eta_{1}, \ldots, \eta_{r}$ be the generic points of the irreducible components of $X$. Let $\tilde{\eta}_{i}$ be a point of $X^{\text {an }}$ such that $j\left(\tilde{\eta}_{i}\right)=\eta_{i}$. By our assumption, $|s|_{\varphi}\left(\tilde{\eta}_{i}\right)=0$, so that $s\left(\eta_{i}\right)=0$ for all $i$. Therefore, one has the assertion because $X$ is reduced.

(2) We denote the natural homeomorphism $X^{\text {an }} \rightarrow X_{\text {red }}^{\text {an }}$ by $p$. Then we have the following commutative diagram:

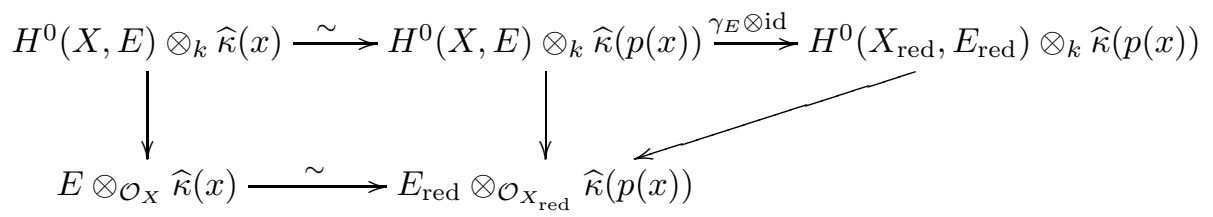

because $E_{\text {red }}=E \otimes \mathcal{O}_{X} \mathcal{O}_{X_{\text {red }}}$. Therefore one has (2).

(3) Let $X=\bigcup_{i=1}^{N} \operatorname{Spec}\left(A_{i}\right)$ be an affine open covering of $X$ such that, for each $i$, there is a local basis $\omega_{i}$ of $E$ over $\operatorname{Spec}\left(A_{i}\right)$. For any $i \in\{1, \ldots, N\}$, let $a_{i} \in A_{i}$ such that $s=a_{i} \omega_{i}$ on $\operatorname{Spec} A_{i}$. As $\left.s\right|_{X_{\text {red }}}=0, a_{i}$ is a nilpotent element of $A_{i}$, so that we can find a positive integer $n_{0}$ such that $s^{\otimes n_{0}}=0$. Therefore $s^{\otimes n}=0$ for $n \geqslant n_{0}$ because $s^{\otimes n}=s^{\otimes n_{0}} s^{\otimes n-n_{0}}$.

2.1.3. Base change. - Let $k^{\prime} / k$ be a field extension equipped with an absolute value $|\cdot|^{\prime}$ which extends $|\cdot|$ on $k$. We assume that $k^{\prime}$ is complete with respect to this absolute value.

Let $X$ be a scheme over Spec $k, X^{\prime}$ be the fibre product $X \times_{\text {Spec } k}$ Spec $k^{\prime}$ and $p: X^{\prime} \rightarrow X$ be the morphism of projection. If $\left(K,|\cdot|_{K}\right)$ is a valued extension of $k^{\prime}$ and $f:$ Spec $K \rightarrow X^{\prime}$ is a $k^{\prime}$-point of $X^{\prime}$ valued in $K$, then the composition morphism $\pi \circ f$ is a $k$-point of $X$ valued in $K$. This construction is functorial and thus determines by passing to colimit a surjective map between Berkovich spaces from $X^{\prime \text { an }}$ to $X^{\text {an }}$ which we denote by $p^{\natural}$. We emphasise that $X^{\prime \text { an }}$ is constructed from the projective 
$k^{\prime}$-scheme $X^{\prime}$. Thus $p^{\natural}$ differs from the map between Berkovich spaces associated with $p$ (considered as a $k$-morphism of schemes) as in Definition 2.1.2.

Proposition 2.1.1\%. - The map $p^{\natural}: X^{\prime a n} \rightarrow X^{\text {an }}$ defined above is continuous with respect to the Berkovich topology.

Proof. - Let $U$ be a Zariski open subset of $X$ and $g$ be a regular function on $U$. We denote by $g^{\prime}$ the pull-back of $g$ by $p$, which is a regular function on $p^{-1}(U)$. For any $y \in X^{\prime a n}$ one has $\left|g^{\prime}\right|(y)=|g|\left(p^{\natural}(y)\right)$. Hence $|g| \circ p^{\natural}$ is a continuous function on $p^{-1}(U)^{\text {an }}$. Therefore the map $p^{\natural}$ is continuous.

Definition 2.1.18. - Let $E$ be a locally free $\mathcal{O}_{X}$-module of finite rank, equipped with a metric $\varphi$ and let $E_{k^{\prime}}$ be the pull-back of $E$ by the projection morphism $p: X^{\prime} \rightarrow$ $X$. If $y$ is a point of $X^{\prime a n}$ and if $x=p^{\natural}(y)$, then the norm $|\cdot|_{\varphi}(x)$ on $E(x)=E \otimes \widehat{\kappa}(x)$ induces by $\varepsilon$-extension (resp. $\pi$-extension) of scalars a norm on $E_{k^{\prime}}(y) \cong E(x) \otimes_{\widehat{\kappa}(x)}$ $\widehat{\kappa}(y)$, denoted by $|\cdot|_{\varphi_{k^{\prime}, \varepsilon}}(y)$ (resp. $\left.|\cdot|_{\varphi_{k^{\prime}, \pi}}(y)\right)$. These norms define a metric on $E_{k^{\prime}}$, denoted by $\varphi_{k^{\prime}, \varepsilon}$ (resp. $\varphi_{k^{\prime}, \pi}$ ), called the metric induced by $\varphi$ by $\varepsilon$-extension (resp. $\pi$-extension) of scalars.

Assume that the norm $|\cdot|_{\varphi}(x)$ on $E(x)$ is induced by an inner product. For any point $y \in X^{\prime \text { an }}$ such that $x=p^{\natural}(y)$, we let $|\cdot|_{\varphi_{k^{\prime}, \mathrm{HS}}}(y)$ be the norm on $E_{k^{\prime}}(y)$ induced by $\left.\right|_{\varphi}(x)$ by orthogonal extension of scalars. These norms define a metric on $E_{k^{\prime}}$, denoted by $\varphi_{k^{\prime}, \text { HS }}$ and called the metric induced by $\varphi$ by orthogonal extension of scalars.

In the case where $E$ is an invertible $\mathcal{O}_{X}$-module, the three metrics $\varphi_{k^{\prime}, \varepsilon}, \varphi_{k^{\prime}, \pi}$ and $\varphi_{k, \mathrm{HS}}$ are the same (see Remark 1.3.2](3)], and are just denoted by $\varphi_{k^{\prime}}$.

Proposition 2.1.19. - Let $L$ be an invertible $\mathcal{O}_{X}$-module equipped with a continuous metric $\varphi$. Then one has the following:

(1) The metric $\varphi_{k^{\prime}}$ is continuous.

(2) For all $s \in H^{0}(X, L),\|s\|_{\varphi}=\left\|p^{*}(s)\right\|_{\varphi_{k^{\prime}}}$.

Proof. - Let $U$ be a Zariski open subset of $X$ and $s \in H^{0}(U, L)$. Then one has

$$
\left|p^{*}(s)\right|_{\varphi_{k^{\prime}}}=\left.|s|_{\varphi} \circ p^{\natural}\right|_{p^{-1}(U)^{\text {an }}},
$$

so that the assertion (2) follows. If we assume that $L$ is trivialised by $s$ over $U$, then $p^{*}(s)$ is a section in $H^{0}\left(p^{-1}(U), L_{k^{\prime}}\right)$ which trivialises $L_{k^{\prime}}$ on $p^{-1}(U)$. Moreover, by the above (2.2) together with Proposition 2.1.17, we obtain that $\left|p^{*}(s)\right|_{\varphi_{k^{\prime}}}$ is a continuous function on $p^{-1}(U)^{\text {an }}$. Therefore one has the assertion $(1)$.

\subsection{Metrics on invertible sheaves}

Let $X$ be a scheme over Spec $k$. In this section, we discuss constructions and properties of metrics on invertible $\mathcal{O}_{X}$-modules. 
2.2.1. Dual metric and tensor product metric. - Let $L$ be an invertible $\mathcal{O}_{X}$ module and $\varphi$ be a metric on $L$. Note that for any $x \in X^{\text {an }}$, the norm $|\cdot|_{\varphi}(x)$ is determined by its value on any non-zero element of $L \otimes \widehat{\kappa}(x)$. In particular, to verify that the metric $\varphi$ is continuous, it suffices to prove that there exists a covering $\left\{U_{i}\right\}_{i \in I}$ of $X$ by affine open subsets and for each $i \in I$ there exists a section $s_{i} \in H^{0}\left(U_{i}, L\right)$ which trivialises the invertible sheaf $L$ on $U_{i}$ such that the function $\left|s_{i}\right|_{\varphi}$ is continuous on the topological space $U_{i}^{\text {an }}$.

Definition 2.2.1. - Let $L$ be an invertible $\mathcal{O}_{X}$-module. If $\varphi$ is a metric on $L$, then the dual $\mathcal{O}_{X}$-module $L^{\vee}$ is naturally equipped with a metric $\varphi^{\vee}$ such that, for sections $\alpha$ and $s$ of $L^{\vee}$ and $L$ over a Zariski open subset $U$ of $X$ respectively, one has

$$
\forall x \in U^{\mathrm{an}}, \quad|\alpha(s)|(x)=|s|_{\varphi}(x) \cdot|\alpha|_{\varphi^{\vee}}(x) .
$$

We call $\varphi^{\vee}$ the dual metric of $\varphi$ and we also use the expression $-\varphi$ to denote the metric $\varphi^{\vee}$.

Proposition 2.2.2. - Let $X$ be a $k$-scheme, $L$ be an invertible $\mathcal{O}_{X}$-module and $\varphi$ be a metric on L. If $\varphi$ is a continuous metric, then $\varphi^{\vee}$ is also continuous.

Proof. - Let $U$ be a Zariski open subset of $X$ on which the invertible sheaves $L$ and $L^{\vee}$ are trivialised by sections $s \in \Gamma(U, L)$ and $\alpha \in \Gamma\left(U, L^{\vee}\right)$ respectively. Then $\alpha(s)$ is a regular function, and

$$
|\alpha|_{\varphi \vee}=\frac{|\alpha(s)|}{|s|_{\varphi}}
$$

on $U^{\text {an }}$. Since the functions $|\alpha(s)|$ and $|s|_{\varphi}$ are all continuous, also is $|\alpha|_{\varphi} \vee$. Since $U$ is arbitrary, we obtain that $\varphi^{\vee}$ is a continuous metric.

Definition 2.2.3. - Let $L$ be an invertible $\mathcal{O}_{X}$-module and $n$ be a positive integer. Suppose given a metric $\varphi$ on $L^{\otimes n}$. Then the maps

$$
\left(s \in H^{0}(U, L)\right) \longmapsto\left|s^{n}\right|_{\varphi}^{1 / n},
$$

with $U$ running over the set of all Zariski open subsets of $X$, define a metric on $L$, denoted by $\frac{1}{n} \varphi$. If the metric $\varphi$ is continuous, then also is $\frac{1}{n} \varphi$.

Definition 2.2.4. - Suppose given two invertible $\mathcal{O}_{X}$-modules $L_{1}$ and $L_{2}$, equipped with metrics $\varphi_{1}$ and $\varphi_{2}$ respectively. We denote by $\varphi_{1}+\varphi_{2}$ the metric on $L_{1} \otimes L_{2}$ such that, for any Zariski open subset $U$ of $X$ and all sections $s_{1} \in H^{0}\left(U, L_{1}\right)$, $s_{2} \in H^{0}\left(U, L_{2}\right)$, one has

$$
\forall x \in U^{\text {an }}, \quad\left|s_{1} \cdot s_{2}\right|_{\varphi_{1}+\varphi_{2}}(x)=\left|s_{1}\right|_{\varphi_{1}}(x) \cdot\left|s_{2}\right|_{\varphi_{2}}(x) .
$$

The metric $\varphi_{1}+\varphi_{2}$ is called tensor product of $\varphi_{1}$ and $\varphi_{2}$. Note that, if the metrics $\varphi_{1}$ and $\varphi_{2}$ are continuous, then also is $\varphi_{1}+\varphi_{2}$. We also use the expression $\varphi_{1}-\varphi_{2}$ to denote the metric $\varphi_{1}+\varphi_{2}^{\vee}$ on $L_{1} \otimes L_{2}^{\vee}$. If $L$ is an invertible $\mathcal{O}_{X}$-module equipped 
with a metric $\varphi$, for any integer $n \in \mathbb{N}_{\geqslant 1}$, we use the expression $n \varphi$ to denote the metric $\varphi+\cdots+\varphi(n$ copies $)$ on $L^{\otimes n}$.

Proposition 2.2.5. - Let $X$ be a scheme over Spec $k, L_{1}$ and $L_{2}$ be invertible $\mathcal{O}_{X}$-modules, and $\varphi_{1}$ and $\varphi_{2}$ be continuous metrics on $L_{1}$ and $L_{2}$, respectively. Then the canonical $k$-linear homomorphism $H^{0}\left(X, L_{1}\right) \otimes_{k} H^{0}\left(X, L_{2}\right) \rightarrow H^{0}\left(X, L_{1} \otimes L_{2}\right)$, sending $s_{1} \otimes s_{2} \in H^{0}\left(X, L_{1}\right) \otimes_{k} H^{0}\left(X, L_{2}\right)$ to $s_{1} \cdot s_{2}$, has operator norm $\leqslant 1$, where we consider the $\pi$-tensor product of $\|\cdot\|_{\varphi_{1}}$ and $\|\cdot\|_{\varphi_{2}}$ on the tensor product space, and the norm $\|\cdot\|_{\varphi_{1}+\varphi_{2}}$ on $H^{0}\left(X, L_{1} \otimes L_{2}\right)$. In particular, if $s_{1}$ and $s_{2}$ are elements in $H^{0}\left(X, L_{1}\right)$ and $H^{0}\left(X, L_{2}\right)$, respectively, then the following inequality holds

$$
\left\|s_{1} \cdot s_{2}\right\|_{\varphi_{1}+\varphi_{2}} \leqslant\left\|s_{1}\right\|_{\varphi_{1}} \cdot\left\|s_{2}\right\|_{\varphi_{2}} .
$$

Proof. - Let $\eta$ be an element of $H^{0}\left(X, L_{1}\right) \otimes_{k} H^{0}\left(X, L_{2}\right)$, which is written as

$$
\eta=\sum_{i=1}^{N} s_{1}^{(i)} \otimes s_{2}^{(i)},
$$

where $s_{1}^{(1)}, \ldots, s_{1}^{(N)}$ are elements in $H^{0}\left(X, L_{1}\right), s_{2}^{(1)}, \ldots, s_{2}^{(N)}$ are elements in $H^{0}\left(X, L_{2}\right)$. Let $s$ be the element

$$
\sum_{i=1}^{N} s_{1}^{(i)} \cdot s_{2}^{(i)}
$$

in $H^{0}\left(X, L_{1} \otimes L_{2}\right)$, which is the image of $\eta$ by the canonical homomorphism

$$
H^{0}\left(X, L_{1}\right) \otimes_{k} H^{0}\left(X, L_{2}\right) \longrightarrow H^{0}\left(X, L_{1} \otimes L_{2}\right) .
$$

For any $x \in X^{\text {an }}$ one has

$$
\begin{aligned}
& |s|_{\varphi_{1}+\varphi_{2}}(x)=\left|\sum_{i=1}^{N} s_{1}^{(i)} \cdot s_{2}^{(i)}\right|_{\varphi_{1}+\varphi_{2}}(x) \leqslant \sum_{i=1}^{N}\left|s_{1}^{(i)} \cdot s_{2}^{(i)}\right|_{\varphi_{1}+\varphi_{2}}(x) \\
= & \sum_{i=1}^{N}\left|s_{1}^{(i)}\right|_{\varphi_{1}}(x) \cdot\left|s_{2}^{(i)}\right|_{\varphi_{2}}(x) \leqslant \sum_{i=1}^{N}\left\|s_{1}^{(i)}\right\|_{\varphi_{1}} \cdot\left\|s_{2}^{(i)}\right\|_{\varphi_{2}} .
\end{aligned}
$$

Since $x \in X^{\text {an }}$ is arbitrary, we obtain

$$
\|s\|_{\varphi_{1}+\varphi_{2}} \leqslant \sum_{i=1}^{N}\left\|s_{1}^{(i)}\right\|_{\varphi_{1}} \cdot\left\|s_{2}^{(i)}\right\|_{\varphi_{2}} .
$$

Therefore $\|s\|_{\varphi_{1}+\varphi_{2}} \leqslant\|\eta\|_{\pi}$, where $\|\cdot\|_{\pi}$ denotes the $\pi$-tensor product of $\|\cdot\|_{\varphi_{1}}$ and $\|\cdot\|_{\varphi_{2}}$. The first assertion is thus proved.

If $s_{1}$ and $s_{2}$ are elements in $H^{0}\left(X, L_{1}\right)$ and $H^{0}\left(X, L_{2}\right)$ respectively, then one has $\left\|s_{1} \cdot s_{2}\right\|_{\varphi_{1}+\varphi_{2}}=\sup _{x \in X^{\mathrm{an}}}\left|s_{1} \cdot s_{2}\right|_{\varphi_{1}+\varphi_{2}}(x)=\sup _{x \in X^{\mathrm{an}}}\left|s_{1}\right|_{\varphi_{1}}(x) \cdot\left|s_{2}\right|_{\varphi_{2}}(x) \leqslant\left\|s_{1}\right\|_{\varphi_{1}} \cdot\left\|s_{2}\right\|_{\varphi_{2}}$, as required. 
Remark 2.2.6. - Assume that the absolute value $|\cdot|$ is non-Archimedean. The statement of Proposition 2.2.5 remains true if we consider the $\varepsilon$-tensor product $\|\cdot\|_{\varepsilon}$ of $\|\cdot\|_{\varphi_{1}}$ and $\|\cdot\|_{\varphi_{2}}$ on the tensor product space $H^{0}\left(X, L_{1}\right) \otimes_{k} H^{0}\left(X, L_{2}\right)$. In fact, by Proposition 1.2.19 if $\left\{e_{i}\right\}_{i=1}^{n}$ and $\left\{f_{j}\right\}_{j=1}^{m}$ are $\alpha$-orthogonal basis of $\left(H^{0}\left(X, L_{1}\right),\|\cdot\|_{\varphi_{1}}\right)$ and $\left(H^{0}\left(X, L_{2}\right),\|\cdot\|_{\varphi_{2}}\right)$ respectively, then

$$
\left\{e_{i} \otimes f_{j}\right\}_{(i, j) \in\{1, \ldots, n\} \times\{1, \ldots, m\}}
$$

is an $\alpha^{2}$-orthogonal basis with respect to $\|\cdot\|_{\varepsilon}$, where $\left.\alpha \in\right] 0,1[$. For any

$$
\eta=\sum_{i=1}^{n} \sum_{j=1}^{m} a_{i j} e_{i} \otimes e_{j} \in H^{0}\left(X, L_{1}\right) \otimes_{k} H^{0}\left(X, L_{2}\right)
$$

one has

$$
\|\eta\|_{\varepsilon} \geqslant \alpha^{2} \max _{(i, j) \in\{1, \ldots, n\} \times\{1, \ldots, m\}}\left|a_{i j}\right| \cdot\left\|e_{i}\right\| \cdot\left\|f_{j}\right\|,
$$

which is bounded from below by $\alpha^{2}$ times the norm of the canonical image of $\eta$ in $H^{0}\left(X, L_{1} \otimes L_{2}\right)$ since the norm $\|\cdot\|_{\varphi_{1}+\varphi_{2}}$ is ultrametric.

2.2.2. Distance between metrics. - Let $\varphi$ be a metric on $\mathcal{O}_{X}$. Then $-\ln |\mathbf{1}|_{\varphi}$ is a function on $X^{\text {an }}$, where $\mathbf{1}$ denotes the section of unity of $\mathcal{O}_{X}$. If $\varphi$ is a continuous metric, then $-\ln |\mathbf{1}|_{\varphi}$ is a continuous function. Conversely, any real-valued function $g$ on $X^{\text {an }}$ determines a metric on $\mathcal{O}_{X}$ such that the norm at $x \in X^{\text {an }}$ of the section of unity of $\mathcal{O}_{X}$ is $\mathrm{e}^{-g(x)}$. The metric is continuous if and only if the function $g$ is continuous. Therefore the set of all metrics on $\mathcal{O}_{X}$ is canonically in bijection with the set of all real-valued function on $X^{\text {an }}$. This correspondance also maps bijectively the set of all continuous metrics on $\mathcal{O}_{X}$ to the set $C^{0}\left(X^{\text {an }}\right)$ of all continuous real-valued functions on $X^{\text {an }}$.

Definition 2.2.7. - Let $L$ be an invertible $\mathcal{O}_{X}$-module. If $\varphi$ and $\varphi^{\prime}$ are two metrics on $L$, then $\varphi^{\prime}-\varphi$ is a metric on $L \otimes L^{\vee} \cong \mathcal{O}_{X}$, hence corresponds to a real valued function on $X^{\text {an }}$. By abuse of notation, we use the expression $\varphi^{\prime}-\varphi$ to denote this function. We say that the metric $\varphi^{\prime}$ is larger than $\varphi$ if $\varphi^{\prime}-\varphi$ is a non-negative function and we use the expressions $\varphi^{\prime} \geqslant \varphi$ or $\varphi \leqslant \varphi^{\prime}$ to denote the relation " $\varphi^{\prime}$ is larger than $\varphi^{\prime \prime}$. If $\varphi$ and $\varphi^{\prime}$ are metrics on $L$, we denote by $d\left(\varphi, \varphi^{\prime}\right)$ the element

$$
\sup _{x \in X^{\text {an }}}\left|\varphi^{\prime}-\varphi\right|(x) \in \mathbb{R}_{\geqslant 0} \cup\{+\infty\},
$$

called the distance between $\varphi$ and $\varphi^{\prime}$. Note that one has

$$
d\left(\varphi, \varphi^{\prime}\right)=\left.\sup _{x \in X^{\mathrm{an}}}|\ln | \cdot\right|_{\varphi}(x)-\ln \left|\cdot \varphi_{\varphi^{\prime}}(x)\right| .
$$

Proposition 2.2.8. - If the $k$-scheme $X$ is proper (so that the sup seminorms are defined), then

$$
d\left(\|\cdot\|_{\varphi}^{\tilde{\varphi}},\|\cdot\|_{\varphi^{\prime}}^{\tilde{w}}\right) \leqslant d\left(\varphi, \varphi^{\prime}\right)
$$


(see 1.1 .9 for the notion of distance between two norms and \$1.1 for the notion of norm associated with a seminorm).

Proof. - Fix $s \in H^{0}(X, L) \backslash \mathcal{N}(X, L)$. For $\epsilon>0$, one can choose $x \in X^{\text {an }}$ such that $\mathrm{e}^{-\epsilon}\|s\|_{\varphi_{1}} \leqslant|s|_{\varphi_{1}}(x)$. Then

$$
\ln \|s\|_{\varphi_{1}}-\ln \|s\|_{\varphi_{2}} \leqslant \ln |s|_{\varphi_{1}}(x)-\ln |s|_{\varphi_{2}}(x)+\epsilon \leqslant d\left(\varphi_{1}, \varphi_{2}\right)+\epsilon,
$$

so that one has $\ln \|s\|_{\varphi_{1}}-\ln \|s\|_{\varphi_{2}} \leqslant d\left(\varphi_{1}, \varphi_{2}\right)$ because $\epsilon$ is an arbitrary positive number. In the same way, $\ln \|s\|_{\varphi_{2}}-\ln \|s\|_{\varphi_{1}} \leqslant d\left(\varphi_{1}, \varphi_{2}\right)$, and hence one obtains

$$
\left|\ln \|s\|_{\varphi_{1}}-\ln \|s\|_{\varphi_{2}}\right| \leqslant d\left(\varphi_{1}, \varphi_{2}\right),
$$

which implies the assertion of the proposition.

For any integer $n \in \mathbb{Z}$ one has $n \varphi^{\prime}-n \varphi=n\left(\varphi^{\prime}-\varphi\right)$ and hence

$$
d\left(n \varphi^{\prime}, n \varphi\right)=|n| d\left(\varphi^{\prime}, \varphi\right) .
$$

The distance function verifies the triangle inequality: if $\varphi_{1}, \varphi_{2}$ and $\varphi_{3}$ are three continuous metrics on $L$, then one has

$$
d\left(\varphi_{1}, \varphi_{3}\right) \leqslant d\left(\varphi_{1}, \varphi_{2}\right)+d\left(\varphi_{2}, \varphi_{3}\right)
$$

because

$$
\left.|\ln | \cdot\right|_{\varphi_{1}}(x)-\ln |\cdot|_{\varphi_{3}}(x)|\leqslant| \ln |\cdot|_{\varphi_{1}}(x)-\ln |\cdot|_{\varphi_{2}}(x)|+| \ln |\cdot|_{\varphi_{2}}(x)-\ln |\cdot|_{\varphi_{3}}(x) \mid
$$

for any $x \in X^{\text {an }}$

Definition 2.2.9. - Let $Y$ and $X$ be two schemes over $\operatorname{Spec} k$, and $f: Y \rightarrow X$ be a $k$-morphism. Suppose given an invertible $\mathcal{O}_{X}$-module $L$, equipped with a metric $\varphi$. Then the metric $\varphi$ induces by pull-back a metric $f^{*}(\varphi)$ on $Y$ such that, for any $y \in Y^{\text {an }}$, the norm $|\cdot|_{f^{*}(\varphi)}(y)$ is induced by $|\cdot|_{\varphi}(f(y))$ by extension of scalars. The metric $f^{*}(\varphi)$ is called the pull-back of $\varphi$ by $f$. For any section $s$ of $L$ on a Zariski open subset $U$ of $X$, one has

$$
\left|f^{*}(s)\right|_{f^{*}(\varphi)}=\left.|s|_{\varphi} \circ f^{\mathrm{an}}\right|_{f^{-1}(U)^{\mathrm{an}}} .
$$

In particular, if the metric $\varphi$ is continuous, then also is $f^{*}(\varphi)$.

Proposition 2.2.10. - Let $Y$ and $X$ be two schemes over $\operatorname{Spec} k, f: Y \rightarrow X$ be a $k$-morphism, $L$ be an invertible $\mathcal{O}_{X}$-module, and $\varphi$ and $\varphi^{\prime}$ be two metrics on $L$. Then one has

$$
d\left(f^{*}(\varphi), f^{*}\left(\varphi^{\prime}\right)\right) \leqslant d\left(\varphi, \varphi^{\prime}\right) .
$$

Moreover, the equality holds if $f: Y \rightarrow X$ is surjective.

Proof. - By (2.8), one has $f^{*}(\varphi)-f^{*}\left(\varphi^{\prime}\right)=\left(\varphi-\varphi^{\prime}\right) \circ f^{\text {an }}$. Hence

$$
d\left(f^{*}(\varphi), f^{*}\left(\varphi^{\prime}\right)\right)=\sup _{y \in Y^{\text {an }}}\left|f^{*}(\varphi)-f^{*}\left(\varphi^{\prime}\right)\right|(y) \leqslant \sup _{y \in Y^{\text {an }}}\left|\varphi-\varphi^{\prime}\right|(y)=d\left(\varphi, \varphi^{\prime}\right) .
$$


If $f: Y \rightarrow X$ is surjective, then $f^{\text {an }}: Y^{\text {an }} \rightarrow X^{\text {an }}$ is also surjective, so that the last assertion follows.

2.2.3. Fubini-Study metric. — Let $V$ be a finite-dimensional vector space over $k$. We denote by $\pi: \mathbb{P}(V) \rightarrow \operatorname{Spec}(k)$ the projective space of $V$. Note that the functor $F_{\mathbb{P}(V)}$ from the category $\mathbf{A}_{k}$ of $k$-algebras to the category of sets corresponding to $\mathbb{P}(V)$ (see 2.1 sends any $k$-algebra $A$ to the set of all projective quotient $A$-modules of $V \otimes_{k} A$ which are of rank 1. By gluing morphisms of schemes, we obtain that, for any $k$-scheme $f: X \rightarrow \operatorname{Spec}(k)$, the set of all $k$-morphisms from $X$ to $\mathbb{P}(V)$ is in functorial bijection with the set of all invertible quotient $\mathcal{O}_{X}$-module of $f^{*}(V)$. In the case where $X$ is the projective space $\mathbb{P}(V)$, the invertible quotient $\mathcal{O}_{X}$-module of $\pi^{*}(V)$ corresponding to the identity map $\mathbb{P}(V) \rightarrow \mathbb{P}(V)$ is called the universal invertible sheaf, denoted by $\mathcal{O}_{V}(1)$. It verifies the following universal property: for any $k$-scheme $f: X \rightarrow$ Spec $k$, a $k$-morphism $g: X \rightarrow \mathbb{P}(V)$ corresponds to the invertible quotient

$$
g^{*}(p): g^{*}\left(\pi^{*}(V)\right) \cong f^{*}(V) \longrightarrow g^{*}\left(\mathcal{O}_{V}(1)\right),
$$

where $p: \pi^{*}(V) \rightarrow \mathcal{O}_{V}(1)$ is the quotient homomorphism defining the universal invertible sheaf.

Let $\bar{V}=(V,\|\cdot\|)$ be a normed vector space of finite rank over $k$. For any point $x$ in the Berkovich space $\mathbb{P}(V)^{\text {an }}$, if the absolute value $|\cdot|$ is non-Archimedean, we denote by $|\cdot|_{\bar{V}}(x)$ the norm on $V \otimes_{k} \widehat{\kappa}(x)$ induced by $\|\cdot\|$ by $\varepsilon$-extension of scalars; if the absolute value $|\cdot|$ is Archimedean, we denote by $|\cdot|_{\bar{V}}(x)$ the norm on $V \otimes_{k} \widehat{\kappa}(x)$ induced by $\|\cdot\|$ by $\pi$-extension of scalars. We emphasise that, in the case where $\widehat{\kappa}(x)=k$ (namely $x$ corresponds to a rational point of $\mathbb{P}(V))$, the vector space $V \otimes_{k} \widehat{\kappa}(x)$ is canonically isomorphic to $V$ and the norm $|\cdot|_{\bar{V}}(x)$ identifies with the double dual norm of $\|\cdot\|$. We denote by $|\cdot|_{\bar{V} \text {,FS }}(x)$ the quotient norm on $\mathcal{O}_{V}(1)(x)=\mathcal{O}_{V}(1) \otimes_{\mathcal{O}_{\mathbb{P}}(V)} \widehat{\kappa}(x)$ induced by the norm $|\cdot|_{V}(x)$ on $V \otimes_{k} \widehat{\kappa}(x)$, called the Fubini-Study norm on $\mathcal{O}_{V}(1)(x)$ induced by $\|\cdot\|$. For simplicity, the norm $|\cdot|_{\bar{V}, \mathrm{FS}}(x)$ is often denoted by $|\cdot|_{\mathrm{FS}}(x)$.

Remark 2.2.11. - It is a natural question to determine if the Fubini-Study metric can be defined in a uniform way (for non-Archimedean and Archimedean cases). Let $\bar{V}=(V,\|\cdot\|)$ be a finite-dimensional vector space over $k$. For any point $x \in$ $X^{\text {an }}$, we let $|\cdot|_{\bar{V}, \varepsilon}(x)$ and $|\cdot|_{\bar{V}, \pi}(x)$ be the norms on $V \otimes_{k} \widehat{\kappa}(x)$ induced by $\|\cdot\|$ by $\varepsilon$-extension and $\pi$-extension of scalars respectively. If the absolute value $|\cdot|$ is nonArchimedean, then both norms $|\cdot|_{\bar{V}, \varepsilon}(x)$ and $|\cdot|_{\bar{V}, \pi}(x)$ induce the same quotient norm on $\mathcal{O}_{V}(1)(x)$. In fact, by Proposition 1.3.20 (1), (2) the dual norms of both $|\cdot|_{\bar{V}, \varepsilon}$ and $|\cdot|_{\bar{V}, \pi}$ identify with $\|\cdot\|_{*, \widehat{\kappa}(x), \varepsilon}$, and hence induce the same restricted norm on $\mathcal{O}_{V}(1)(x)^{\vee}$. By Proposition 1.1.20, the dual norms of the quotient norms on $\mathcal{O}_{V}(1)(x)$ of $|\cdot|_{\bar{V}, \varepsilon}$ and $|\cdot|_{\bar{V}, \pi}$ are the same. Since $\mathcal{O}_{V}(1)(x)$ is a vector space of rank 1 on $\widehat{\kappa}(x)$, we obtain that these quotient norms are the same. In other words, in both the Archimedean and non-Archimedean cases, we may use the $\pi$-extension of scalars to 
define the Fubini-Study metric. However, for the reason of applications in the study of adelic vector bundles, it is more convenient to consider the $\varepsilon$-extension of scalars for the non-Archimedean case. We emphasis however that, in the Archimedean case, if we apply the $\varepsilon$-extension of scalars instead of the $\pi$-extension of scalars, in general we obtain a different metric from the Fubini-Study metric.

Proposition 2.2.12. - Let $\bar{V}=(V,\|\cdot\|)$ be a finite-dimensional normed vector space over $k$. Then the norms $|\cdot|_{\bar{V}, \mathrm{FS}}(x), x \in \mathbb{P}(V)^{\text {an }}$ described above define a continuous metric on the universal invertible sheaf $\mathcal{O}_{V}(1)$.

Proof. - By Proposition 1.2.14 (see also Remark 1.3.2), for any $x \in \mathbb{P}(V)^{\text {an }}$, the norms $\|\cdot\|$ and $\|\cdot\|_{* *}$ induce the same Fubini-Study norm on $\mathcal{O}_{V}(1)(x)$. Hence we may assume without loss of generality that the norm $\|\cdot\|$ is ultrametric when $(k,|\cdot|)$ is non-Archimedean.

For any $x \in \mathbb{P}(V)^{\text {an }}$, let $|\cdot|_{V}(x)$ be the norm on $V \otimes_{k} \widehat{\kappa}(x)$ induced by $\|\cdot\|$ by $\pi$ extension of scalars, and let $|\cdot|_{V}(x)_{*}$ be the dual norm of $|\cdot| \bar{V}(x)$. The norms $|\cdot|_{\bar{V}}(x)_{*}$ define a metric $\varphi$ on $\pi^{*}\left(V^{\vee}\right)$. By Proposition 1.3.20 (1), (2), the norm $|\cdot|_{V}(x)_{*}$ coincides with the norm induced by $\|\cdot\|_{*}$ by $\varepsilon$-extension of scalars. Therefore, by Proposition 2.1.12 we obtain that the metric $\varphi$ is continuous.

The dual norm of the Fubini-Study norm $|\cdot|_{\bar{V}, \mathrm{FS}}(x)$ then coincides with the restriction of $|\cdot|_{V}(x)_{*}$ to $\mathcal{O}_{V}(1)^{\vee} \otimes \widehat{\kappa}(x)$ by Proposition 1.1.20. Hence these dual norms (for $\left.x \in \mathbb{P}(V)^{\text {an }}\right)$ form a continuous metric on $\mathcal{O}_{V}(1)^{\vee}$. Therefore the Fubini-Study norms $|\cdot|_{\bar{V}, \mathrm{FS}}(x), x \in \mathbb{P}(V)^{\text {an }}$ define a continuous metric on $\mathcal{O}_{V}(1)$ (see Proposition 2.2 .2 .

Definition 2.2.13. — Let $\bar{V}=(V,\|\cdot\|)$ be a finite-dimensional normed vector space over $k$. The continuous metric on $\mathcal{O}_{V}(1)$ formed by the Fubini-Study norms $|\cdot|_{\bar{V}, \text { FS }}(x)$ with $x \in \mathbb{P}(V)^{\text {an }}$ is called the Fubini-Study metric on $\mathcal{O}_{V}(1)$ associated with the norm $\|\cdot\|$ on $V$.

Remark 2.2.14. - Let $\bar{V}=(V,\|\cdot\|)$ be a normed vector space over $k$ and $s$ be an element of $V$. For any $x \in \mathbb{P}(V)^{\text {an }}$ such that $s(x) \neq 0$, by definition one has

$$
|s|_{\bar{V}, \mathrm{FS}}(x)=\inf _{\substack{t \in V \otimes_{k} \widehat{\kappa}(x) \\ t(x)=s(x)}}\|t\|_{\widehat{\kappa}(x), \downarrow}
$$

with $\downarrow=\varepsilon$ if $|\cdot|$ is non-Archimedean, and $\natural=\pi$ if $|\cdot|$ is Archimedean. In particular, one has (see Proposition 1.3.1)

$$
|s|_{\bar{V}, \mathrm{FS}}(x) \leqslant\|s\|_{\widehat{\kappa}(x), \natural}=\|s\|_{* *} .
$$

Moreover, any rational point $y \in \mathbb{P}(V)(k)$ corresponds to a non-zero element $\beta_{y}$ : $V \rightarrow k$ in the dual vector space $V^{\vee}$. The dual norm of $\beta_{y}$ identifies with the inverse 
of the quotient norm of $1 \in k$. Therefore one has

$$
|s|_{\overline{V, F S}}(y)=\frac{\left|\beta_{y}(s)\right|}{\left\|\beta_{y}\right\|_{*}}
$$

and hence

$$
\sup _{y \in \mathbb{P}(V)(k)}|s(y)|_{\bar{V}, \mathrm{FS}}(y)=\sup _{y \in \mathbb{P}(V)(k)} \frac{\left|\beta_{y}(s)\right|}{\left\|\beta_{y}\right\|_{*}}=\|s\|_{* *} .
$$

Combing with (2.9), we obtain $\|\cdot\|_{\bar{V}, \mathrm{FS}}=\|\cdot\|_{* *}$.

Let $\left(k^{\prime},|\cdot|\right)$ be a valued extension of $(k,|\cdot|)$. Note that the fibre product $\mathbb{P}(V) \times$ Spec $k$ Spec $k^{\prime}$ identifies with the projective space of $V^{\prime}:=V \otimes_{k} k^{\prime}$. The Fubini-Study metric on $\mathcal{O}_{V}(1)$ induces by base change a continuous metric on $\mathcal{O}_{V^{\prime}}(1)$ which we denote by

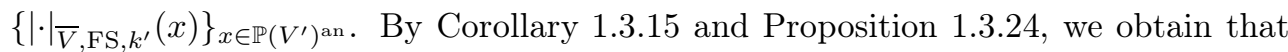
this metric coincides with the Fubini-Study metric associated with the norm $\|\cdot\|_{k^{\prime}, \natural}$ on $V^{\prime}$, where $\downarrow=\varepsilon$ if $|\cdot|$ is non-Archimedean and $\downarrow=\pi$ if $|\cdot|$ is Archimedean. In particular, one has

$$
\|\cdot\|_{\bar{V}, \mathrm{FS}, k^{\prime}}=\|\cdot\|_{\bar{V}^{\prime}, \mathrm{FS}} \cdot
$$

Definition 2.2.15. — Let $f: X \rightarrow$ Spec $k$ be a $k$-scheme and $L$ be an invertible $\mathcal{O}_{X}$-module. Suppose given a finite-dimensional vector space $V$ over $k$ and a surjective $\mathcal{O}_{X}$-homomorphism $\beta: f^{*}(V) \rightarrow L$. Then the homomorphism $\beta$ corresponds to a $k$ morphism of schemes $g: X \rightarrow \mathbb{P}(V)$ such that $g^{*}\left(\mathcal{O}_{V}(1)\right)$ is canonically isomorphic to $L$. If $V$ is equipped with a norm $\|\cdot\|$, then the Fubini-Study metric on $\mathcal{O}_{V}(1)$ induces by pull-back a continuous metric on $L$, called the quotient metric induced by the normed vector space $(V,\|\cdot\|)$ and the surjective homomorphism $\beta$.

Definition 2.2.16. - Let $\ell$ be a section of $L$ over a Zariski open set $U$, which trivialises the invertible sheaf $L$. The section $\ell$ yields the isomorphism $\iota:\left.\mathcal{O}_{U} \rightarrow L\right|_{U}$ given by $a \mapsto a \ell$. We define $\vec{\ell}:\left.f^{*}(V)\right|_{U} \rightarrow \mathcal{O}_{U}$ by $\iota^{-1} \circ \beta_{U}$, that is, the following diagram is commutative:

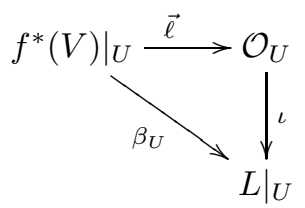

If $\left\{e_{i}\right\}_{i=1}^{r}$ is a basis of $V,\left\{e_{i}^{\vee}\right\}_{i=1}^{r}$ is the dual basis of $\left\{e_{i}\right\}_{i=1}^{r}$ and $\beta_{U}\left(e_{i}\right)=a_{i} \ell$ for $i \in\{1, \ldots, n\}$, then $\vec{\ell}$ is given by

$$
\vec{\ell}=a_{1} e_{1}^{\vee}+\cdots+a_{r} e_{r}^{\vee} .
$$

For each $x \in U^{\text {an }}$, the evaluation of $\vec{\ell}$ at $x$ is denoted by $\vec{\ell}_{x}$, that is,

$$
\vec{\ell}_{x}=a_{1}(x) e_{1}^{\vee}+\cdots+a_{r}(x) e_{r}^{\vee} \in \operatorname{Hom}_{\widehat{\kappa}(x)}\left(V_{\widehat{\kappa}(x)}, \widehat{\kappa}(x)\right) .
$$


Proposition 2.2.17. - Let $f: X \rightarrow$ Spec $k$ be a $k$-scheme and $L$ be an invertible $\mathcal{O}_{X}$-module. Suppose given a finite-dimensional normed vector space $(V,\|\cdot\|)$ over $k$ and a surjective $\mathcal{O}_{X}$-homomorphism $\beta: f^{*}(V) \rightarrow$ L. Let $\varphi$ be the quotient metric induced by $(V,\|\cdot\|)$ and $\beta$. For any section $\ell$ of $L$ on a Zariski open subset $U$ of $X$ which trivialises $L$ on $U$, one has

$$
\forall x \in U^{\text {an }}, \quad|\ell|_{\varphi}(x)=\left\|\vec{\ell}_{x}\right\|_{\widehat{\kappa}(x), \natural, *}^{-1}=\left\|\vec{\ell}_{x}\right\|_{*, \widehat{\kappa}(x), \varepsilon}^{-1},
$$

where $\emptyset=\varepsilon$ if $|\cdot|$ is non-Archimedean and $\emptyset=\pi$ if $|\cdot|$ is Archimedean.

Proof. - For each $x \in U^{\text {an }}$, one has the following commutative diagram:

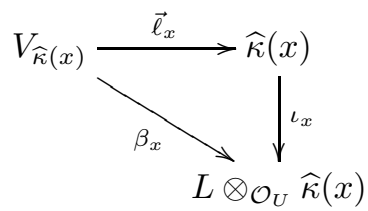

By (1) in Lemma 1.1.15, the operator norm of $\beta_{x}$ is 1. Moreover, the operator norm of $\vec{\ell}_{x}$ is $\left\|\vec{\ell}_{x}\right\|_{\widehat{\kappa}(x), \natural, *}$ and that of $\iota_{x}$ is $|\ell|_{\varphi}(x)$. As the operator norm of $\beta_{x}$ is the product of the operator norms of $\vec{\ell}_{x}$ and $\iota_{x}$ by (2) in Proposition 1.1.15, we obtain $|\ell|_{\varphi}(x)=$ $\left\|\vec{\ell}_{x}\right\|_{\widehat{\kappa}(x), \natural, *}$. The equality $\left\|\vec{\ell}_{x}\right\|_{\widehat{\kappa}(x), \downarrow, *}^{-1}=\left\|\vec{\ell}_{x}\right\|_{*, \widehat{\kappa}(x), \varepsilon}$ follows from Proposition 1.3 .20 (1), (2) for the non-Archimedean and Archimedean cases, respectively.

Remark 2.2.18. - Let $X$ be a quasi-projective $k$-scheme. The above construction shows that any ample invertible $\mathcal{O}_{X}$-module admits a continuous metric. By Proposition 2.2.2, we deduce that, more generally, any invertible $\mathcal{O}_{X}$-module admits a continuous metric.

Remark 2.2.19. - Let $f: X \rightarrow$ Spec $k$ be a $k$-scheme, $L$ be an invertible $\mathcal{O}_{X^{-}}$ module, $V$ be a finite-dimensional vector space over $k$, and $\beta: f^{*}(V) \rightarrow L$ be a surjective $\mathcal{O}_{X}$-homomorphism. Let $n$ be an integer, $n \geqslant 1$. Then $\beta$ induces a surjective $\mathcal{O}_{X}$-homomorphism $\beta^{\otimes n}: f^{*}\left(V^{\otimes n}\right) \rightarrow L^{\otimes n}$. Let $\|\cdot\|$ be a norm on $V$ and $\varphi$ be the quotient metric induced by $(V,\|\cdot\|)$ and $\beta$. We claim that $n \varphi$ is the quotient metric induced by $\left(V^{\otimes n},\|\cdot\|_{\natural}\right)$ and $\beta^{\otimes n}$, where $\|\cdot\|_{\natural}$ denotes the $\lfloor$-tensor power of the norm $\|\cdot\|, \downarrow=\varepsilon$ if $|\cdot|$ is non-Archimedean and $\downarrow=\pi$ if $|\cdot|$ is Archimedean. In fact, if $x$ is an point in $X^{\text {an }}$ and $\ell$ is a non-zero element of $L \otimes \widehat{\kappa}(x)$, then $\ell^{\otimes n}$ is a non-zero element of $L^{\otimes n} \otimes \widehat{\kappa}(x)$. By Propositions 1.3.21 and 1.3.5 the norm $\|\cdot\|_{\natural, \widehat{\kappa}(x), \natural}$ on $V^{\otimes n} \otimes \widehat{\kappa}(x)$ coincides with the $\sharp$-tensor power of $\|\cdot\|_{\widehat{\kappa}(x), \natural}$. Consider the dual homomorphism

$$
\beta_{x}^{\vee \otimes n}:\left(L^{\vee} \otimes \widehat{\kappa}(x)\right)^{\otimes n} \longrightarrow\left(V^{\vee} \otimes \widehat{\kappa}(x)\right)^{\otimes n} .
$$

By Proposition 1.1.57 and Corollary 1.2.20, the dual norm $\|\cdot\|_{\natural, \widehat{\kappa}(x), \natural, *}$ coincides with the $\varepsilon$-tensor power of $\|\cdot\|_{\widehat{\kappa}(x), \natural, *}=\|\cdot\|_{*, \widehat{\kappa}(x), \varepsilon}$ (see Proposition 1.3.20](1)[(2)]. In both cases one has

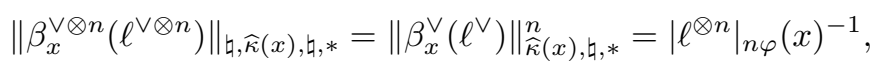


where the first equality comes from Remark 1.1.56, and the second comes from Proposition 1.1.20,

Proposition 2.2.20. - Let $f: X \rightarrow \operatorname{Spec} k$ be a scheme over $\operatorname{Spec} k$ and $L$ be an invertible $\mathcal{O}_{X}$-module. Let $V$ be a finite-dimensional vector space and $\beta: f^{*}(V) \rightarrow L$ be a surjective homomorphism. If $\|\cdot\|$ and $\|\cdot\|^{\prime}$ are two norms on $V$ and if $\varphi$ and $\varphi^{\prime}$ are quotient metrics on $L$ induced by $\bar{V}=(V,\|\cdot\|)$ and $\bar{V}^{\prime}=\left(V,\|\cdot\|^{\prime}\right)$ (and the surjective homomorphism $\beta$ ) respectively, then one has $d\left(\varphi, \varphi^{\prime}\right) \leqslant d\left(\|\cdot\|,\|\cdot\|^{\prime}\right)$.

Proof. - Let $x$ be a point of $X^{\text {an }},|\cdot|_{\bar{V}}(x)$ and $|\cdot|_{\bar{V}^{\prime}}(x)$ be the norms on $V \otimes \widehat{\kappa}(x)$ induced by $\|\cdot\|$ and $\|\cdot\|^{\prime}$ by extension of scalars. Proposition 1.3.7 leads to

$$
d\left(|\cdot|_{\bar{V}}(x),|\cdot|_{\bar{V}^{\prime}}(x)\right) \leqslant d\left(\|\cdot\|,\|\cdot\|^{\prime}\right) .
$$

Since $|\cdot|_{\varphi}(x)$ and $|\cdot|_{\varphi^{\prime}}(x)$ are respectively the quotient norms of $|\cdot|_{V}(x)$ and $|\cdot|_{V^{\prime}}(x)$, by Proposition 1.1 .42 one has $d\left(|\cdot|_{\varphi}(x),|\cdot|_{\varphi^{\prime}}(x)\right) \leqslant d\left(|\cdot|_{V}(x),|\cdot|_{V^{\prime}}(x)\right)$. Therefore

$$
d\left(\varphi, \varphi^{\prime}\right)=\sup _{x \in X^{\text {an }}} d\left(|\cdot|_{\varphi}(x),|\cdot|_{\varphi^{\prime}}(x)\right) \leqslant \sup _{x \in X^{\text {an }}} d\left(|\cdot|_{\bar{V}}(x),|\cdot|_{\bar{V}^{\prime}}(x)\right) \leqslant d\left(\|\cdot\|,\|\cdot\|^{\prime}\right),
$$

as required.

Definition 2.2.21. - Let $\pi: X \rightarrow \operatorname{Spec}(k)$ be a projective $k$-scheme, $L$ be an invertible $\mathcal{O}_{X}$-module, which is generated by global sections, and $\varphi$ be a continuous metric on $L$. By Proposition 2.1.16, for each $x \in X^{\text {an }}$, the homomorphism $\left(H^{0}(X, L) / \mathcal{N}(X, L)\right) \otimes \widehat{\kappa}(x) \rightarrow L \otimes \widehat{\kappa}(x)$ induced by $H^{0}(X, L) \otimes \widehat{\kappa}(x) \rightarrow L \otimes \widehat{\kappa}(x)$ is surjective, so that one has a quotient norm $|\cdot|^{\prime}(x)$ on $L \otimes \widehat{\kappa}(x)$ induced by $\|\cdot\|_{\varphi}$. The family $\left\{|\cdot|^{\prime}(x)\right\}_{x \in X^{\text {an }}}$ of metrics is denoted by $\varphi_{\mathrm{FS}}$, that is, $|\cdot|_{\varphi_{\mathrm{FS}}}(x):=|\cdot|^{\prime}(x)$ for $x \in X^{\text {an }}$, called the Fubini-Study metric associated with $\varphi$. Let $X_{\text {red }}$ be the reduced scheme associated with $X$ and $L_{\text {red }}:=L \otimes_{\mathcal{O}_{X}} \mathcal{O}_{X_{\text {red }}}$. Let $V$ be the image of $H^{0}(X, L) \rightarrow H^{0}\left(X_{\text {red }}, L_{\text {red }}\right)$. Then $\|\cdot\|_{\varphi}$ is a norm of $V$ and $\varphi_{\mathrm{FS}}$ is the quotient metric induced by the surjection $V \otimes \mathcal{O}_{X_{\mathrm{red}}} \rightarrow L_{\mathrm{red}}$ and $\|\cdot\|_{\varphi}$. In particular, $\varphi_{\mathrm{FS}}$ is continuous. For an integer $n \geqslant 1$, we set $\varphi_{n}=(n \varphi)_{\mathrm{FS}}$.

By Propositions 1.3.26 and 1.3.25, for any point $x \in X^{\text {an }}$ and any non-zero element $\ell \in L \otimes_{\mathcal{O}_{X}} \widehat{\kappa}(x)$, one has

$$
|\ell|_{\varphi_{\mathrm{FS}}}(x)=\inf _{\substack{s \in H^{0}(X, L), \lambda \in \widehat{\kappa}(x)^{\times} \\ s(x)=\lambda \ell}}|\lambda|_{x}^{-1} \cdot\|s\|_{\varphi} .
$$

This equality is fundamental in the study of quotient metrics.

Proposition 2.2.22. - Let $\pi: X \rightarrow$ Spec $k$ be a projective $k$-scheme and $L$ be an invertible $\mathcal{O}_{X}$-module generated by global sections, equipped with a continuous metric $\varphi$. Then the following assertions hold.

(1) For any integer $n \in \mathbb{N}_{\geqslant 1}$, one has $\varphi_{n} \geqslant n \varphi$, where $\varphi_{n}$ denotes the Fubini-Study metric associated with $n \varphi$. 
(2) The sup seminorm $\|\cdot\|_{\varphi_{n}}$ on $V_{n}:=H^{0}\left(X, L^{\otimes n}\right)$ induced by $\varphi_{n}$ coincides with $\|\cdot\|_{n \varphi}$.

(3) Let $M$ be another invertible $\mathcal{O}_{X}$-module generated by global sections, equipped with a continuous metric $\psi$. Then $(\varphi+\psi)_{\mathrm{FS}} \leqslant \varphi_{\mathrm{FS}}+\psi_{\mathrm{FS}}$. In particular, for any pair $(m, n)$ of positive integers one has $\varphi_{n+m} \leqslant \varphi_{n}+\varphi_{m}$.

(4) For any integer $n \geqslant 1$, one has $d\left(\varphi_{n}, n \varphi\right) \leqslant n d\left(\varphi_{1}, \varphi\right)$. In particular, if $\varphi_{1}=\varphi$ then $\varphi_{n}=n \varphi$ for any $n \in \mathbb{N}_{\geqslant 1}$.

(5) Let $\varphi^{\prime}$ be another continuous metric on $L$. Then one has $d\left(\varphi_{n}, \varphi_{n}^{\prime}\right) \leqslant n d\left(\varphi, \varphi^{\prime}\right)$ for any $n \in \mathbb{N}_{\geqslant 1}$.

Proof. - For any $n \in \mathbb{N}_{\geqslant 1}$, we denote by $V_{n}$ the vector space $H^{0}\left(X, L^{\otimes n}\right)$ over $k$.

(1) Let $x$ be a point of $X^{\text {an }}$ and $\ell$ be an element of $L^{\otimes n} \otimes \widehat{\kappa}(x)$. Note that, for any $s \in V_{n}$, one has $|s|_{n \varphi}(x) \leqslant\|s\|_{n \varphi}$, so that, by (2.12), one obtains

$$
|\ell|_{\varphi_{n}}(x)=\inf _{\substack{s \in V_{n}, \lambda \in \widehat{\kappa}(x)^{\times} \\ s(x)=\lambda \ell}}|\lambda|_{x}^{-1} \cdot\|s\|_{n \varphi} \geqslant \inf _{\substack{s \in V_{n}, \lambda \in \widehat{\kappa}(x)^{\times} \\ s(x)=\lambda \ell}}|\lambda|_{x}^{-1} \cdot|s|_{n \varphi}(x)=|\ell|_{n \varphi}(x),
$$

as desired.

(2) By (1), one has $\|\cdot\|_{\varphi_{n}} \geqslant\|\cdot\|_{n \varphi}$. In the following, we prove the converse inequality. If $s$ is a global section of $L^{\otimes n}$, for any $x \in X^{\text {an }}$, by (2.12), one has

$$
|s|_{\varphi_{n}}(x)=\inf _{\substack{t \in V_{n}, \lambda \in \widehat{\kappa}(x)^{\times} \\ t(x)=\lambda s(x)}}|\lambda|_{x}^{-1} \cdot\|t\|_{n \varphi} \leqslant\|s\|_{n \varphi} .
$$

Hence $\|s\|_{\varphi_{n}}=\sup _{x \in X \text { an }}|s|_{\varphi_{n}}(x) \leqslant\|s\|_{n \varphi}$.

(3) Let $x$ be a point of $X^{\text {an }}, \ell$ and $\ell^{\prime}$ be elements of $L \otimes \widehat{\kappa}(x)$ and $M \otimes \widehat{\kappa}(x)$ respectively. By (2.12) together with (2.3) one has

$$
\begin{aligned}
& \left|\ell \cdot \ell^{\prime}\right|_{(\varphi+\psi)_{\mathrm{FS}}}(x)=\inf _{\substack{s \in H^{0}(X, L \otimes M), \lambda \in \widehat{\kappa}(x)^{\times} \\
s(x)=\lambda \ell \cdot \ell^{\prime}}}|\lambda|_{x}^{-1} \cdot\|s\|_{\varphi+\psi} \\
& \leqslant \inf _{\left(t, t^{\prime}\right) \in H^{0}(X, L) \times H^{0}(X, M)}|\mu \eta|_{x}^{-1} \cdot\left\|t \cdot t^{\prime}\right\|_{\varphi+\psi} \\
& (\mu, \eta) \in\left(\widehat{\kappa}(x)^{\times}\right)^{2} \\
& t(x)=\mu \ell, t^{\prime}(x)=\eta \ell^{\prime} \\
& \leqslant \inf _{\left(t, t^{\prime}\right) \in H^{0}(X, L) \times H^{0}(X, M)}\left(|\mu|_{x}^{-1} \cdot\|t\|_{\varphi}\right)\left(|\eta|_{x}^{-1} \cdot\left\|t^{\prime}\right\|_{\psi}\right) \\
& \begin{array}{c}
(\mu, \eta) \in\left(\widehat{\kappa}(x)^{\times}\right)^{2} \\
t(x)=\mu \ell, t^{\prime}(x)=\eta \ell^{\prime}
\end{array} \\
& =|\ell|_{\varphi_{\mathrm{FS}}}(x) \cdot\left|\ell^{\prime}\right|_{\psi_{\mathrm{FS}}}(x) \text {, }
\end{aligned}
$$

For the last assertion, note that

$$
\varphi_{n+m}=(n \varphi+m \varphi)_{\mathrm{FS}} \leqslant(n \varphi)_{\mathrm{FS}}+(m \varphi)_{\mathrm{FS}}=\varphi_{n}+\varphi_{m}
$$


(4) By (3), one has $\varphi_{n} \leqslant n \varphi_{1}$. Moreover, by (1), one has $\varphi_{n} \geqslant n \varphi$ and $\varphi_{1} \geqslant \varphi$. Hence $0 \leqslant \varphi_{n}-n \varphi \leqslant n \varphi_{1}-n \varphi=n\left(\varphi_{1}-\varphi\right)$, which implies

$$
d\left(\varphi_{n}, n \varphi\right)=\sup _{x \in X^{\mathrm{an}}}\left(\varphi_{n}-n \varphi\right)(x) \leqslant n \sup _{x \in X^{\mathrm{an}}}\left(\varphi_{1}-\varphi\right)(x)=n d\left(\varphi_{1}, \varphi\right) .
$$

(5) By Proposition 2.2.20 together with (2.5), one has

$$
d\left(\varphi_{n}, \varphi_{n}^{\prime}\right) \leqslant d\left(\|\cdot\|_{n \varphi},\|\cdot\|_{n \varphi^{\prime}}\right) \leqslant d\left(n \varphi, n \varphi^{\prime}\right)=n d\left(\varphi, \varphi^{\prime}\right),
$$

where the equality comes from (2.6).

Proposition 2.2.23. - Let $\pi: X \rightarrow$ Spec $k$ be a projective $k$-scheme and $L$ be an invertible $\mathcal{O}_{X}$-module. Suppose given a normed vector space $(V,\|\cdot\|)$ and a surjective homomorphisms $\beta: \pi^{*}(V) \rightarrow L$. Let $\varphi$ be the quotient metric on $L$ induced by $(V,\|\cdot\|)$ and $\beta$. Then, one has the following:

(1) Let $f: V \rightarrow H^{0}(X, L)$ be the adjoint homomorphism of $\beta: \pi^{*}(V) \rightarrow L$. Then, $\|f(v)\|_{\varphi} \leqslant\|v\|$ for any $v \in V$.

(2) For any integer $n \geqslant 1, \varphi_{n}=n \varphi$.

Proof. - (1) By Propositions 1.3.26 and 1.3.25, for $x \in X^{\text {an }}$,

$$
|f(v)|_{\varphi}(x)=\inf _{\substack{t \in V, \lambda \in \widetilde{\kappa}(x)^{\times} \\ f(t)(x)=\lambda f(v)(x)}}|\lambda|_{x}^{-1} \cdot\|t\| \leqslant\|v\|,
$$

so that one has (1)

(2) By Proposition 2.2.22 (4), it suffices to verify that $\varphi_{1}=\varphi$. Note that $\varphi_{1} \geqslant \varphi$ (by Proposition 2.2.22 (1) . In the following, we prove the converse inequality. Let $x$ be a point of $X^{\text {an }}$ and $\ell$ be an element of $L \otimes \widehat{\kappa}(x)$. By (1) and (2.12) together with Propositions 1.3.26 and 1.3.25, one has

$$
\begin{aligned}
|\ell|_{\varphi_{1}}(x) & =\inf _{\substack{s \in H^{0}(X, L) \\
\lambda \in \widehat{\kappa}(x) \\
s(x)=\lambda \ell}}|\lambda|_{x}^{-1} \cdot\|s\|_{\varphi} \leqslant \inf _{\substack{s^{\prime} \in V, \lambda \in \widehat{\kappa}(x)^{\times} \\
f\left(s^{\prime}\right)(x)=\lambda \ell}}|\lambda|_{x}^{-1} \cdot\left\|f\left(s^{\prime}\right)\right\|_{\varphi} \\
& \leqslant \inf _{\substack{s^{\prime} \in V, \lambda \in \widehat{\kappa}(x)^{\times} \\
f\left(s^{\prime}\right)(x)=\lambda \ell}}|\lambda|_{x}^{-1} \cdot\left\|s^{\prime}\right\|=|\ell|_{\varphi}(x) .
\end{aligned}
$$

Therefore one has $\varphi_{1}=\varphi$.

\subsection{Semi-positive metrics}

Let $(k,|\cdot|)$ be a complete valued field and $\pi: X \rightarrow \operatorname{Spec} k$ be a projective $k$ scheme. In this section, we discuss positivity conditions of continuous metrics on invertible $\mathcal{O}_{X}$-modules. 
2.3.1. Definition and basic properties. - Let $L$ be an invertible $\mathcal{O}_{X}$-module equipped with a continuous metric $\varphi$. We assume that $L$ is generated by global sections. We have constructed in Definition 2.2.21 a sequence of quotient metrics $\left\{\varphi_{n}\right\}_{n \in \mathbb{N} \geqslant 1}$. By Proposition 2.2.22 (1) and (4), we obtain that $\left\{d\left(\varphi_{n}, n \varphi\right)\right\}_{n \in \mathbb{N} \geqslant 1}$ is a sub-additive non-negative sequence and the normalised sequence $\left\{d\left(\varphi_{n}, n \varphi\right) / n\right\}_{n \in \mathbb{N} \geqslant 1}$ is bounded from above. Hence the sequence $\left\{d\left(\varphi_{n}, n \varphi\right) / n\right\}_{n \in \mathbb{N} \geqslant 1}$ converges in $\mathbb{R}_{+}$. We denote by $\operatorname{dp}(\varphi)$ the limit

$$
\operatorname{dp}(\varphi):=\lim _{n \rightarrow+\infty} \frac{1}{n} d\left(\varphi_{n}, n \varphi\right),
$$

called the default of positivity of the metric $\varphi$. By definition, for any integer $m \in \mathbb{N} \geqslant 1$, one has

$$
\operatorname{dp}(m \varphi)=m \operatorname{dp}(\varphi) .
$$

We say that the metric $\varphi$ is semipositive if one has $\operatorname{dp}(\varphi)=0$. Clearly, if the metric $\varphi$ is semipositive, then for any integer $m \geqslant 1$, the metric $m \varphi$ on $L^{\otimes m}$ is also semipositive. Conversely, if there is an integer $m \geqslant 1$ such that $m \varphi$ is semipositive, then the metric $\varphi$ is also semipositive.

More generally, we assume that $L$ is semiample (namely a positive tensor power of $L$ is generated by global sections). Let $n$ be a positive integer such that $L^{\otimes n}$ is generated by global sections. The quantity $\operatorname{dp}(n \varphi) / n$ does not depend on the choice of $n$ by (2.14), so that we define $\operatorname{dp}(\varphi)$ to be $\operatorname{dp}(n \varphi) / n$. It is easy to see that (2.14) still holds under the assumption that $L$ is semiample. We say that $\varphi$ is semipositive if $\operatorname{dp}(\varphi)=0$.

Remark 2.3.1. - Let $(V,\|\cdot\|)$ be a normed vector space of finite rank over $k$ and $\beta: \pi^{*}(V) \rightarrow L$ be a surjective homomorphism. Let $\varphi$ be the quotient metric on $L$ induced by $(V,\|\cdot\|)$ and $\beta$. Then, by Proposition 2.2.23, $\varphi$ is semipositive.

Proposition 2.3.2. - Let $L$ be a semiample invertible $\mathcal{O}_{X}$-module, equipped with a continuous metric $\varphi$. If $\varphi$ is semipositive, then $n \varphi$ is semipositive for any $n \in \mathbb{N}_{\geqslant 1}$. Conversely, if there exists an integer $n \in \mathbb{N}_{\geqslant 1}$ such that $n \varphi$ is semipositive, then the metric $\varphi$ is also semipositive.

The following proposition shows that semipositive metrics form a closed subset in the topological space of continuous metrics.

Proposition 2.3.3. - Let $L$ be a semiample invertible $\mathcal{O}_{X}$-module, equipped with a continuous metric $\varphi$. Suppose that there is a sequence of semipositive metrics $\left\{\varphi^{(m)}\right\}_{n \in \mathbb{N}}$ on L such that

$$
\lim _{m \rightarrow+\infty} d\left(\varphi^{(m)}, \varphi\right)=0 .
$$

Then the metric $\varphi$ is also semipositive. 
Proof. - For any integer $p \geqslant 1$, one has

$$
d\left(p \varphi^{(m)}, p \varphi\right)=p d\left(\varphi^{(m)}, \varphi\right) .
$$

Therefore, by replacing $L$ by a certain tensor power $L^{\otimes p}$ and $\varphi$ by $p \varphi$, we may assume without loss of generality that $L$ is generated by global sections. Thus the metrics $\varphi_{n}^{(m)}$ and $\varphi_{n}$ are well defined for any $m \in \mathbb{N}$ and any $n \in \mathbb{N}_{\geqslant 1}$. Moreover, by Proposition 2.2 .22 (5) we obtain that

$$
d\left(\varphi_{n}^{(m)}, \varphi_{n}\right) \leqslant n d\left(\varphi^{(m)}, \varphi\right) .
$$

Note that for $m \in \mathbb{N}$ and $n \in \mathbb{N}_{\geqslant 1}$ one has

$$
\begin{aligned}
d\left(\varphi_{n}, n \varphi\right) & \leqslant d\left(\varphi_{n}, \varphi_{n}^{(m)}\right)+d\left(\varphi_{n}^{(m)}, n \varphi^{(m)}\right)+d\left(n \varphi^{(m)}, n \varphi\right) \\
& \leqslant d\left(\varphi_{n}^{(m)}, n \varphi^{(m)}\right)+2 d\left(\varphi^{(m)}, \varphi\right) .
\end{aligned}
$$

By taking the limit when $n$ tends to the infinity, we obtain

$$
\operatorname{dp}(\varphi) \leqslant 2 d\left(\varphi^{(m)}, \varphi\right)+\operatorname{dp}\left(\varphi^{(m)}\right)=2 d\left(\varphi^{(m)}, \varphi\right),
$$

where the equality comes from the hypothesis that the metrics $\varphi^{(m)}$ are semipositive. By taking the limit when $m$ tends to the infinity, we obtain the semipositivity of the metric $\varphi$.

Remark 2.3.4. - Proposition 2.2.23 shows that quotient metrics on an invertible $\mathcal{O}_{X}$-module are semipositive. Let $L$ be a semiample invertible $\mathcal{O}_{X}$-module and $\varphi$ be a continuous metric on $L$. For any $n \in \mathbb{N}_{\geqslant 1}$ such that $L^{\otimes n}$ is generated by global sections, let $\varphi^{(n)}$ be a continuous metric on $L$ such that $n \varphi^{(n)}$ is a quotient metric. If $\lim _{n \rightarrow+\infty} d\left(\varphi^{(n)}, \varphi\right)=0$, then the metric $\varphi$ is semipositive. This is a consequence of Propositions 2.3.2 and 2.3.3

Proposition 2.3.5. - Let $X$ be a projective $k$-scheme, $L$ and $L^{\prime}$ be semiample invertible $\mathcal{O}_{X}$-modules, equipped with continuous metrics $\varphi$ and $\varphi^{\prime}$, respectively. One has $\operatorname{dp}\left(\varphi+\varphi^{\prime}\right) \leqslant \operatorname{dp}(\varphi)+\operatorname{dp}\left(\varphi^{\prime}\right)$. In particular, if both metrics $\varphi$ and $\varphi^{\prime}$ are semipositive, then the metric $\varphi+\varphi^{\prime}$ on the tensor product $L \otimes L^{\prime}$ is also semipositive.

Proof. - By (2.14), we may assume that $L$ and $L^{\prime}$ are generated by global sections. For any integer $n \geqslant 1$, one has a natural $k$-linear homomorphism

$$
H^{0}\left(X, L^{\otimes n}\right) \otimes H^{0}\left(X, L^{\prime \otimes n}\right) \longrightarrow H^{0}\left(X,\left(L \otimes L^{\prime}\right)^{\otimes n}\right)
$$

given by the tensor product. Moreover, by Proposition 2.2.5. for $s \in H^{0}\left(X, L^{\otimes n}\right)$ and $s^{\prime} \in H^{0}\left(X, L^{\prime \otimes n}\right)$ one has

$$
\left\|s s^{\prime}\right\|_{n\left(\varphi+\varphi^{\prime}\right)} \leqslant\|s\|_{n \varphi} \cdot\left\|s^{\prime}\right\|_{n \varphi^{\prime}} .
$$

By Proposition 2.2 .22 (3), we obtain $\left(\varphi+\varphi^{\prime}\right)_{n} \leqslant \varphi_{n}+\varphi_{n}^{\prime}$ and hence

$$
d\left(\left(\varphi+\varphi^{\prime}\right)_{n}, n\left(\varphi+\varphi^{\prime}\right)\right) \leqslant d\left(\varphi_{n}, n \varphi\right)+d\left(\varphi_{n}^{\prime}, n \varphi^{\prime}\right) .
$$


Dividing the two sides of the inequality by $n$, by passing to limit when $n$ tends to the infinity we obtain $\operatorname{dp}\left(\varphi+\varphi^{\prime}\right) \leqslant \operatorname{dp}(\varphi)+\operatorname{dp}\left(\varphi^{\prime}\right)$.

Proposition 2.3.6. — Let $L$ be a semiample invertible $\mathcal{O}_{X}$-module, equipped with a continuous metric $\varphi$. Then the following are equivalent:

(1) The metric $\varphi$ is semipositive.

(2) For any $\epsilon>0$, there is a positive integer $n$ such that, for all $x \in X^{\text {an }}$, we can find $s \in H^{0}\left(X, L^{\otimes n}\right)_{\widehat{\kappa}(x)} \backslash\{0\}$ with $\|s\|_{n \varphi, \widehat{\kappa}(x)} \leqslant \mathrm{e}^{n \epsilon}|s|_{n \varphi}(x)$.

Proof. - (1) $\Longrightarrow(2)$ : By our assumption, there is a positive integer $n$ such that

$$
|\cdot|_{n \varphi}(x) \leqslant|\cdot|_{\varphi_{n}}(x) \leqslant \mathrm{e}^{n \epsilon / 2}|\cdot|_{n \varphi}(x)
$$

for all $x \in X^{\text {an }}$. Moreover, there is an $s \in H^{0}\left(X, L^{\otimes n}\right)_{\widehat{\kappa}(x)} \backslash\{0\}$ such that $\|s\|_{n \varphi, \widehat{\kappa}(x)} \leqslant$ $\mathrm{e}^{n \epsilon / 2}|s|_{\varphi_{n}}(x)$. Therefore,

$$
\|s\|_{n \varphi, \widehat{\kappa}(x)} \leqslant \mathrm{e}^{n \epsilon / 2}|s|_{\varphi_{n}}(x) \leqslant \mathrm{e}^{n \epsilon}|s|_{n \varphi}(x) .
$$

$(2) \Longrightarrow(1)$ : For a positive integer $m$, there is a positive integer $a_{m}$ such that, for any $x \in X^{\text {an }}$, we can find $s \in H^{0}\left(X, L^{\otimes a_{m}}\right)_{\widehat{\kappa}(x)} \backslash\{0\}$ with $\|s\|_{a_{m} \varphi, \widehat{\kappa}(x)} \leqslant \mathrm{e}^{a_{m} / m}|s|_{a_{m} \varphi}(x)$. Note that

$$
|s|_{a_{m} \varphi}(x) \leqslant|s|_{\varphi_{a_{m}}}(x) \leqslant \mathrm{e}^{a_{m} / m}|s|_{a_{m} \varphi}(x)
$$

which implies that

$$
0 \leqslant \frac{1}{a_{m}}\left(\ln |\cdot|_{\varphi_{a_{m}}}(x)-\ln |\cdot|_{a_{m} \varphi}(x)\right) \leqslant \frac{1}{m}
$$

for all $x \in X^{\text {an }}$, so that $\operatorname{dp}(\varphi)=0$.

Theorem 2.3.7. - Let $X$ be an irreducible and reduced projective scheme over Spec $\mathbb{C}, L$ be a semiample invertible $\mathcal{O}_{X}$-module and $\varphi$ be a continuous metric of $L$. Then the following are equivalent:

(1) The first Chern current $c_{1}(L, \varphi)$ is positive.

(2) For any positive number $\epsilon>0$, there is a positive integer $n$ such that, for all $x \in X$, we can find $s \in H^{0}\left(X, L^{\otimes n}\right) \backslash\{0\}$ with $\|s\|_{n \varphi} \leqslant \mathrm{e}^{n \epsilon}|s|_{n \varphi}(x)$.

(3) The metric $\varphi$ is semipositive.

Proof. - The proof of ' $[(1) \Longrightarrow[(2)]$ is very technical. For the proof, we refer to the papers [148 and [107, Theorem 0.2]. ' $\sqrt{(2)]} \Longrightarrow[(3)]$ ' is nothing more than Proposition 2.3.6. Here let us consider the following claim:

Claim 2.3.8. - Let $M$ be an invertible $\mathcal{O}_{X}$-module, $\bar{V}=(V,\|\cdot\|)$ be a finitedimensional normed vector space over $\mathbb{C}$ and $V \otimes_{\mathbb{C}} \mathcal{O}_{X} \rightarrow M$ be a surjective homomorphism. We assume that there is a basis $\left\{e_{i}\right\}_{i=1}^{r}$ of $V$ such that

$$
\forall\left(a_{1}, \ldots, a_{r}\right) \in \mathbb{C}^{r}, \quad\left\|a_{1} e_{1}+\cdots+a_{r} e_{r}\right\|=\max \left\{\left|a_{1}\right|, \ldots,\left|a_{r}\right|\right\} .
$$


Let $\psi$ be the quotient metric of $M$ induced by $\bar{V}$ and $V \otimes_{\mathbb{C}} \mathcal{O}_{X} \rightarrow M$. Then the first Chern current $c_{1}(M, \psi)$ is semipositive.

Proof. - Let $\left\{e_{i}^{\vee}\right\}_{i=1}^{r}$ be the dual basis of $V$. Then it is easy to see that the dual norm $\|\cdot\|_{*}$ of $\|\cdot\|$ is given by

$$
\forall a_{1}, \ldots, a_{r} \in \mathbb{C}, \quad\left\|a_{1} e_{1}^{\vee}+\cdots+a_{r} e_{r}^{\vee}\right\|_{*}=\left|a_{1}\right|+\cdots+\left|a_{r}\right| .
$$

For $v \in V$, the induced global section of $M$ over $X$ is denoted by $\tilde{v}$. Let $s$ be a local basis of $M$ over a Zariski open set $U$. We set $\tilde{e}_{i}=a_{i} s$ for some holomorphic function $a_{i}$ on $U$. Then, by Proposition 2.2.17, the function

$$
x \longmapsto-\ln |s|_{\psi}(x)=\ln \left(\left|a_{1}\right|(x)+\cdots+\left|a_{r}\right|(x)\right)
$$

is plurisubharmonic on $U^{\text {an }}$ because $\ln \left(\left|a_{1}\right|(\cdot)+\cdots+\left|a_{r}\right|(\cdot)\right)$ is plurisubharmonic on $U^{\text {an }}$.

Let us see that $\Longrightarrow(3)$ Clearly we may assume that $L$ is generated by global sections. For each $n \geqslant 1$, let $r_{n}:=\operatorname{dim}_{\mathbb{C}} H^{0}\left(X, L^{\otimes n}\right)$ and $\left\{e_{n, i}\right\}_{i=1}^{r_{n}}$ be an orthonormal basis of $H^{0}\left(X, L^{\otimes n}\right)$ with respect to $\|\cdot\|_{n \varphi}$. If we set

$$
\left\|a_{1} e_{n, 1}+\cdots+a_{r_{n}} e_{n, r}\right\|_{n}^{\prime}:=\max \left\{\left|a_{1}\right|, \ldots,\left|a_{r_{n}}\right|\right\}
$$

for $a_{1}, \ldots, a_{r_{n}} \in \mathbb{C}$, then $\|\cdot\|_{n}^{\prime} \leqslant\|\cdot\|_{n \varphi} \leqslant r_{n}\|\cdot\|_{n}^{\prime}$. Let $\psi_{n}$ be the quotient metric of $L^{\otimes n}$ by $\|\cdot\|_{n}^{\prime}$. Then $d\left(\varphi_{n}, \psi_{n}\right) \leqslant \ln \left(r_{n}\right)$ because $\psi_{n} \leqslant \varphi_{n} \leqslant r_{n} \psi_{n}$. Therefore, as

$$
d\left(\frac{1}{n} \psi_{n}, \varphi\right) \leqslant d\left(\frac{1}{n} \psi_{n}, \frac{1}{n} \varphi_{n}\right)+d\left(\frac{1}{n} \varphi_{n}, \varphi\right) \leqslant \frac{1}{n} \ln \left(r_{n}\right)+d\left(\frac{1}{n} \varphi_{n}, \varphi\right),
$$

one has $\lim _{n \rightarrow \infty} d\left(\frac{1}{n} \psi_{n}, \varphi\right)=0$ by our assumption. This means that, for a local basis $s$ of $L$ over an open set $U$, the sequence $\left\{-\frac{1}{n} \ln \left|s^{\otimes n}\right|_{\psi_{n}}\right\}_{n=1}^{\infty}$ converges to $-\ln |s|_{\varphi}$ uniformly on any compact set in $U$. As $-\frac{1}{n} \ln \left|s^{\otimes n}\right|_{\psi_{n}}$ is plurisubharmonic by the above claim, $-\ln |s|_{\varphi}$ is also plurisubharmonic, as required.

Corollary 2.3.9. - Let $T$ be a reduced complex analytic space and $\|\cdot\|$ be a norm of $\mathbb{C}^{n}$. If $f_{1}, \ldots, f_{n}$ are holomorphic functions on $T$, then $\log \left\|\left(f_{1}, \ldots, f_{n}\right)\right\|$ is plurisubharmonic on $T$.

Proof. - First of all, recall the following fact (cf. [95, Corollary 2.9.5]):

If $u$ is a plurisubharmonic function on $\mathbb{C}^{n}$ and $f_{1}, \ldots, f_{n}$ are holomorphic functions on $T$, then $u\left(f_{1}, \ldots, f_{n}\right)$ is plurisubharmonic on $T$.

Thus it is sufficient to see that $f\left(z_{1}, \ldots, z_{n}\right):=\log \left\|\left(z_{1}, \ldots, z_{n}\right)\right\|$ is plurisubharmonic on $\mathbb{C}^{n} \backslash\{(0, \ldots, 0)\}$. Let $\mathbb{C}^{n} \otimes_{\mathbb{C}} \mathcal{O}_{\mathbb{P}_{\mathbb{C}}^{n-1}} \rightarrow \mathcal{O}_{\mathbb{P}_{\mathbb{C}}^{n-1}}(1)$ be the surjective homomorphism given by $e_{i} \mapsto X_{i}$, where $\left\{e_{i}\right\}_{i=1}^{n}$ is the standard basis of $\mathbb{C}^{n}$ and $\left(X_{1}: \cdots: X_{n}\right)$ is a homogeneous coordinate of $\mathbb{P}_{\mathbb{C}}^{n-1}$. Let us consider the dual norm $\|\cdot\|_{*}$ of $\|\cdot\|$ on $\mathbb{C}^{n}$, that is, we identify the dual space $\left(\mathbb{C}^{n}\right)^{\vee}$ with $\mathbb{C}^{n}$ in the natural way. Let $\varphi$ be the quotient metric of $\mathcal{O}_{\mathbb{P}_{\mathbb{C}}^{n-1}}(1)$ induced by $\|\cdot\|_{*}$ and $\mathbb{C}^{n} \otimes_{\mathbb{C}} \mathcal{O}_{\mathbb{P}_{\mathbb{C}}^{n-1}} \rightarrow \mathcal{O}_{\mathbb{P}_{\mathbb{C}}^{n-1}}(1)$. Note that $X_{i}$ gives a local basis of $\mathcal{O}_{\mathbb{P}_{\mathbb{C}}^{n-1}}(1)$ over $\left\{X_{i} \neq 0\right\}$ and $\vec{X}_{i}=\sum_{j=1}^{n}\left(X_{j} / X_{i}\right) e_{j}$ (see 
Definition 2.2.16), so that by Proposition 2.2.17 together with the fact $\|\cdot\|_{* *}=\|\cdot\|$, one has $-\log \left|X_{i}\right|_{\varphi}=\log \left\|\vec{X}_{i}\right\|$ on $\left\{X_{i} \neq 0\right\}$. Therefore, by Theorem 2.3.7 together with the previous fact, the function

$$
\left(z_{1}, \ldots, z_{n}\right) \longmapsto \log \left\|\left(\frac{z_{1}}{z_{i}}, \ldots, \frac{z_{i-1}}{z_{i}}, 1, \frac{z_{i+1}}{z_{i}}, \ldots, \frac{z_{n}}{z_{i}}\right)\right\|
$$

is plurisubharmonic on $\mathbb{C}^{n} \backslash\left\{z_{i}=0\right\}$. Note that

$$
f\left(z_{1}, \ldots, z_{n}\right)=\log \left|z_{i}\right|+\log \left\|\left(\frac{z_{1}}{z_{i}}, \ldots, \frac{z_{i-1}}{z_{i}}, 1, \frac{z_{i+1}}{z_{i}}, \ldots, \frac{z_{n}}{z_{i}}\right)\right\|,
$$

so that $f$ is plurisubharmonic on $\mathbb{C}^{n} \backslash\left\{z_{i}=0\right\}$ for all $i$, and hence $f$ is plurisubharmonic on $\mathbb{C}^{n} \backslash\{(0, \ldots, 0)\}$.

2.3.2. Model metrics. - In this subsection, we assume that the absolute value $|\cdot|$ is non-Archimedean and non-trivial. We denote by $\mathfrak{o}_{k}$ the valuation ring of $(k,|\cdot|)$. Let $X \rightarrow \operatorname{Spec}(k)$ be a projective $k$-scheme and $L$ be an invertible $\mathcal{O}_{X}$-module. By model of $(X, L)$, we refer to a projective and flat $\mathfrak{o}_{k}$-scheme $\mathscr{X}$ equipped with an invertible $\mathcal{O}_{\mathscr{X}}$-module $\mathscr{L}$ such that the generic fibre of $\mathscr{X}$ coincides with $X$ and that the restriction of $\mathscr{L}$ to $X$ coincides with $L$. As in Definition 2.1.1, we denote by $j: X^{\text {an }} \rightarrow X$ the specification map.

Let $x$ be a point in $X^{\text {an }}$ and let $p_{x}: \operatorname{Spec} \widehat{\kappa}(x) \rightarrow X$ be the $k$-morphism of schemes defined by $x$, where $\widehat{\kappa}(x)$ is the completion of the residue field $\kappa(x)$ of $j(x)$ with respect to the absolute value $|\cdot|_{x}$. The composition of $p_{x}$ with the inclusion morphism $X \rightarrow \mathscr{X}$ then defines a $\mathfrak{o}_{k}$-morphism from $\operatorname{Spec} \widehat{\kappa}(x)$ to $\mathscr{X}$. By definition $L \otimes \widehat{\kappa}(x)$ is the pull-back sheaf $p_{x}^{*}(L)$. By the valuative criterion of properness (see [71 Chapter II, Theorem 7.3.8), there exists a unique $\mathfrak{o}_{k}$-morphism $\mathscr{P}_{x}$ from $\operatorname{Spec}\left(\mathfrak{o}_{x}\right)$ to $\mathscr{X}$ which identifies with $p_{x}$ on the generic fibre, where $\mathfrak{o}_{x}$ is the valuation ring of $\widehat{\kappa}(x)$. The image of the maximal ideal of $\mathfrak{o}_{x}$ by $\mathscr{P}_{x}$, denoted by $r_{\mathscr{X}}(x)$, is called the reduction point of $x$. Note that $r_{\mathscr{X}}(x)$ belongs to the special fibre of $\mathscr{X} \rightarrow \operatorname{Spec}\left(\mathfrak{o}_{k}\right)$. Furthermore $\mathscr{P}_{x}^{*}(\mathscr{L})$ is a lattice in $L \otimes \widehat{\kappa}(x)$ (see $\left.\$ 1.1 .7\right)$. We denote by $|\cdot| \mathscr{L}(x)$ the norm on $L \otimes \widehat{\kappa}(x)$ defined by this lattice, namely

$$
\forall \ell \in L \otimes \widehat{\kappa}(x), \quad|\ell|_{\mathscr{L}}(x):=\inf \left\{|a|_{x}: a \in \widehat{\kappa}(x)^{\times}, a^{-1} \ell \in \mathscr{P}_{x}^{*}(\mathscr{L})\right\} .
$$

The family of norms $\{|\cdot| \mathscr{L}(x)\}_{x \in X}$ an forms a metric on $L$ which we denote by $\varphi \mathscr{L}$, called the metric induced by the model $(\mathscr{X}, \mathscr{L})$.

Remark 2.3.10. - Let $(\mathscr{X}, \mathscr{L})$ be a model of $(X, L)$. Then $H^{0}(\mathscr{X}, \mathscr{L})$ is a lattice of $H^{0}(X, L)$. Indeed, since $X$ is a proper $k$-scheme, $H^{0}(\mathscr{X}, \mathscr{L})$ is an $\mathfrak{o}_{k}$-module of finite type such that (see [70, Chapter I, Proposition 9.3.2)

$$
H^{0}(X, L)=H^{0}(\mathscr{X}, \mathscr{L}) \otimes_{\mathfrak{o}_{k}} k .
$$

Moreover, since $\mathscr{X}$ is a flat $\mathfrak{o}_{k}$-scheme, the $\mathfrak{o}_{k}$-module $H^{0}(\mathscr{X}, \mathscr{L})$ is torsion free. Therefore the canonical map $H^{0}(\mathscr{X}, \mathscr{L}) \rightarrow H^{0}(X, L)$ is injective and hence it is a lattice in $H^{0}(X, L)$ (see Definition 1.1.23). 
Remark 2.3.11. - Let $\mathscr{X}$ and $\mathscr{Y}$ be projective $\mathfrak{o}_{k}$-schemes and $f: \mathscr{Y} \rightarrow \mathscr{X}$ an $\mathfrak{o}_{k}$-morphisme. Let $X$ and $Y$ be the generic fibres of $\mathscr{X}$ and $\mathscr{Y}$ respectively, and $f_{k}: X \rightarrow Y$ be the morphism induced by $f$. Let $\mathscr{L}$ be an invertible sheaf on $\mathscr{X}$ and $L$ be the restriction of $\mathscr{L}$ to the generic fibre $X$. Then the model $(\mathscr{X}, \mathscr{L})$ induces a metric $|\cdot|_{\mathscr{L}}$ on the invertible sheaf $L$. The couple $\left(\mathscr{Y}, f^{*}(\mathscr{L})\right)$ forms a model of $\left(Y, f_{k}^{*}(L)\right)$. Note that the model metric $|\cdot|_{f^{*}(\mathscr{L})}$ on $f_{k}^{*}(L)$ coincides with the pull-back of the metric $|\cdot|_{\mathscr{L}}$ by $f_{k}$ (see Definition [2.2.9).

Proposition 2.3.12. - Let $L$ be an invertible $\mathcal{O}_{X}$-module which is generated by global sections. Let $\varphi_{\mathscr{L}}$ be the metric induced by a model $(\mathscr{X}, \mathscr{L})$ of $(X, L)$. Let $\varphi$ be a continuous metric of $L$ and

$$
\mathscr{H}=\left\{s \in H^{0}(\mathscr{X}, \mathscr{L}):\|s\|_{\varphi} \leqslant 1\right\} .
$$

Then one has the following:

(1) If $\mathscr{H} \otimes_{\mathfrak{o}_{k}} \mathcal{O}_{\mathscr{X}} \rightarrow \mathscr{L}$ is surjective, then $\varphi \leqslant \varphi \mathscr{L}$.

(2) If $\varphi$ is the quotient metric on $L$ induced by $\|\cdot\|_{H^{0}(\mathscr{X}, \mathscr{L})}$ (see Definition 1.1.27 for the norm induced by a lattice), then $\varphi \geqslant \varphi_{\mathscr{L}}$.

(3) If $\varphi$ is the quotient metric on $L$ induced by $\|\cdot\|_{H^{0}(\mathscr{X}, \mathscr{L})}$, and the natural homomorphism $H^{0}(\mathscr{X}, \mathscr{L}) \otimes_{\mathfrak{o}_{k}} \mathcal{O}_{\mathscr{X}} \rightarrow \mathscr{L}$ is surjective, then $\varphi=\varphi \mathscr{L}$.

Proof. - For $x \in X^{\text {an }}$, let $p_{x}: \operatorname{Spec} \widehat{\kappa}(x) \rightarrow X$ be the $k$-morphism of schemes defined by $x$, and $\mathscr{P}_{x}: \operatorname{Spec} \mathfrak{o}_{x} \rightarrow \mathscr{X}$ be the $\mathfrak{o}_{k}$-morphism extending $p_{x}$. Moreover, let $\pi_{x}: H^{0}(X, L) \otimes_{k} \widehat{\kappa}(x) \rightarrow p_{x}^{*}(L)$ be the natural homomorphism.

(1) By our assumption, $\mathscr{H} \otimes_{\mathfrak{o}_{k}} \mathfrak{o}_{x} \rightarrow \mathscr{P}_{x}^{*}(\mathscr{L})$ is surjective, so that, if $\ell$ lies in $\mathscr{P}_{x}^{*}(\mathscr{L})$, then there exist $s_{1}, \ldots, s_{n}$ in $\mathscr{H}$ and $a_{1}, \ldots, a_{n}$ in $\mathfrak{o}_{x}$ such that $\pi_{x}\left(a_{1} s_{1}+\cdots+\right.$ $\left.a_{n} s_{n}\right)=\ell$. For any $i \in\{1, \ldots, n\}$, let $\ell_{i}=\pi_{x}\left(s_{i}\right)$. Then one has $\ell=a_{1} \ell_{1}+\cdots+a_{n} \ell_{n}$. As $s_{i} \in \mathscr{H}$, one has $\left|\ell_{i}\right|_{\varphi}(x) \leqslant 1$ for any $i$, which leads to $|\ell|_{\varphi}(x) \leqslant 1$. By Proposition 1.1.24, one obtains $|\cdot|_{\varphi}(x) \leqslant|\cdot|_{\varphi \mathscr{L}}(x)$. The assertion (1) is thus proved.

(2) Note that $p_{x}^{*}(L)$ is a quotient vector space of rank 1 of $H^{0}(X, L) \otimes_{k} \widehat{\kappa}(x)$ and $|\cdot|_{\varphi}(x)$ is the quotient norm on $p_{x}^{*}(L)$ induced by $\|\cdot\|_{H^{0}(\mathscr{X}, \mathscr{L}), \widehat{\kappa}(x)}$. By Proposition 1.3.26, for $\ell \in p_{x}^{*}(L) \backslash\{0\}$ one has

$$
|\ell|_{\varphi}(x)=\inf _{\substack{s \in H^{0}(X, L), \lambda \in \widehat{K}(x)^{\times} \\ \pi_{x}(s)=\lambda \ell}}|\lambda|^{-1}\|s\|_{H^{0}(\mathscr{X}, \mathscr{L})} .
$$

Let $s \in H^{0}(X, L)$ and $\lambda \in \widehat{\kappa}(x)^{\times}$such that $\pi_{x}(s)=\lambda \ell$. By definition one has

$$
\|s\|_{H^{0}(\mathscr{X}, \mathscr{L})}=\inf \left\{|a|: a \in k^{\times}, a^{-1} s \in H^{0}(\mathscr{X}, \mathscr{L})\right\} .
$$

If $a$ is an element in $k^{\times}$such that $a^{-1} s \in H^{0}(\mathscr{X}, \mathscr{L})$, then $a^{-1} \lambda \ell \in \mathscr{P}_{x}^{*}(\mathscr{L})$ because $\mathscr{P}_{x}^{*}(\mathscr{L})$ contains the image of $H^{0}(\mathscr{X}, \mathscr{L}) \otimes_{\mathfrak{o}_{k}} \mathfrak{o}_{x}$ in $p_{x}^{*}(L)$ by $\pi_{x}$. Hence

$$
\left|a^{-1} \lambda \ell\right|_{\varphi_{\mathscr{L}}}(x)=|a|^{-1}|\lambda| \cdot|\ell|_{\varphi_{\mathscr{L}}}(x) \leqslant 1,
$$


which implies that $|\ell|_{\varphi_{\mathscr{L}}}(x) \leqslant|\lambda|^{-1}|a|$. Since $a$ is arbitrary with $a^{-1} s \in H^{0}(\mathscr{X}, \mathscr{L})$, one obtains $|\ell|_{\varphi_{\mathscr{L}}}(x) \leqslant|\lambda|^{-1}\|s\|_{H^{0}(\mathscr{X}, \mathscr{L})}$, which leads to $|\ell|_{\varphi_{\mathscr{L}}}(x) \leqslant|\ell|_{\varphi}(x)$.

(3) By (2), it is sufficient to see $\varphi \leqslant \varphi \mathscr{L}$. Note that for $s \in H^{0}(\mathscr{X}, \mathscr{L})$, one has $\|s\|_{\varphi} \leqslant 1$, so that $\mathscr{H}=H^{0}(\mathscr{X}, \mathscr{L})$. Thus, by (1), one obtains $\varphi \leqslant \varphi \mathscr{L}$.

Corollary 2.3.13. - Let $E$ be a finite-dimensional vector space over $k, \mathcal{E}$ be a lattice in $E$ and $\|\cdot\|_{\mathcal{E}}$ be the norm on $E$ induced by the lattice $\mathcal{E}$ (see Definition 1.1.27). Then the Fubini-Study metric (see Definition 2.2.13) on the invertible $\mathcal{O}_{\mathbb{P}(E) \text {-module }}$ $\mathcal{O}_{E}(1)$ induced by $\|\cdot\|_{\mathcal{E}}$ coincides with the metric induced by the model $\left(\mathbb{P}(\mathcal{E}), \mathcal{O}_{\mathcal{E}}(1)\right)$ of $\left(\mathbb{P}(E), \mathcal{O}_{E}(1)\right)$.

Remark 2.3.14. - (1) Recall that a valuation ring is a Prüfer domain, which is a generalisation of Dedekind domain (non-necessarily Noetherian). In particular, an $\mathfrak{o}_{k}$-module is flat if and only if it is torsion free (see [26] Chapter VII, §2, Exercices 12 and 14).

(2) Let $\mathscr{A}$ be a flat $\mathfrak{o}_{k}$-algebra, and $A=S^{-1} \mathscr{A}$, where $S=\mathfrak{o}_{k} \backslash\{0\}$. Note that the canonical homomorphism $\mathscr{A} \rightarrow A$ is injective. If $B$ is a quotient ring of $A$ with respect to the ideal $I$ and if $\mathscr{I}=I \cap \mathscr{A}$, then the quotient ring $\mathscr{B}=\mathscr{A} / \mathscr{I}$ is a torsion-free $\mathfrak{o}_{k}$-module, and hence is flat. The $\operatorname{scheme} \operatorname{Spec}(\mathscr{B})$ is called the Zariski closure of $\operatorname{Spec}(B)$ in $\operatorname{Spec}(\mathscr{A})$.

(3) Let $X$ be a projective scheme over Spec $k$, equipped with an invertible $\mathcal{O}_{X^{-}}$ module $L$. Let $(\mathscr{X}, \mathscr{L})$ be a model of $(X, L)$. Then the global section space $H^{0}(\mathscr{X}, \mathscr{L})$ is a torsion-free $\mathfrak{o}_{k}$-module. Moreover, if $Y$ is a closed subscheme of $X$ and if $\mathscr{Y}$ is the Zariski closure of $Y$ in $\mathscr{Y}$, then $\left(\mathscr{Y},\left.\mathscr{L}\right|_{\mathscr{Y}}\right)$ is a model of $\left(Y,\left.L\right|_{Y}\right)$. Moreover, the metric on $\left.L\right|_{Y}$ induced by $\left.\mathscr{L}\right|_{\mathscr{Y}}$ coincides with the restriction of the metric $\varphi_{\mathscr{L}}$ to $\left.L\right|_{Y}$.

(4) Let $\pi: X \rightarrow \operatorname{Spec} k$ be a projective scheme over Spec $k, E$ be a finite-dimensional vector space over $k$ and $\beta: \pi^{*}(E) \rightarrow L$ be a surjective homomorphism, which defines a $k$-morphism $f: X \rightarrow \mathbb{P}(E)$. Suppose that $f$ is a closed immersion. Let $\mathcal{E}$ be a lattice in $E, \mathscr{X}$ be the Zariski closure of $X$ in $\mathbb{P}(\mathcal{E})$ and $\mathscr{L}$ be the restriction of $\mathcal{O}_{\mathcal{E}}(1)$ to $\mathscr{X}$. Then Proposition 2.3 .12 shows that the quotient metric on $L$ induced by the norm $\|\cdot\|_{\mathcal{E}}$ coincides with the metric induced by the model $(\mathscr{X}, \mathscr{L})$.

Proposition 2.3.15. - Let $L$ and $M$ are two invertible $\mathcal{O}_{X}$-modules. Suppose that $\mathscr{X}$ is a proper and flat $\mathfrak{o}_{k}$-scheme such that $\mathscr{X}_{k}=X$. If $\mathscr{L}$ and $\mathscr{M}$ are invertible $\mathcal{O}_{\mathscr{X}}$ modules such that $(\mathscr{X}, \mathscr{L})$ and $(\mathscr{X}, \mathscr{M})$ are models of $(X, L)$ and $(X, M)$ respectively, then one has $\varphi_{\mathscr{L} \otimes \mathscr{M}}=\varphi_{\mathscr{L}}+\varphi_{\mathscr{M}}$.

Proof. - Let $x$ be a point of $X^{\text {an }}, s$ and $t$ are element in $\mathscr{L}_{r_{\mathscr{C}}(x)}$ and $\mathscr{M}_{r_{\mathscr{X}}(x)}$ which trivialise the invertible sheaves $\mathscr{L}$ and $\mathscr{M}$ around $r_{\mathscr{X}}(x)$ respectively, and $\ell=\mathscr{P}_{x}^{*}(s)$, 
$m=\mathscr{P}_{x}^{*}(t)$, where $\mathscr{P}_{x}: \operatorname{Spec}\left(\mathfrak{o}_{x}\right) \rightarrow \mathscr{X}$ is the unique $\mathfrak{o}_{k}$-morphism extending the $k$ morphism $\operatorname{Spec} \widehat{\kappa}(x) \rightarrow X$ corresponding to the point $x$. Since $\ell$ and $m$ are generators of the free $\mathfrak{o}_{x}$-modules (of rank 1) $\mathscr{P}_{x}^{*}(\mathscr{L})$ and $\mathscr{P}_{x}^{*}(\mathscr{M})$, one has $|\ell|_{\mathscr{L}}(x)=|m|_{\mathscr{M}}(x)=$ 1. Moreover, $\mathscr{P}_{x}^{*}(s \otimes t)$ is a generator of the free $\mathfrak{o}_{x}$-module $\mathscr{P}_{x}^{*}(\mathscr{L} \otimes \mathscr{M})$, we obtain that $|\ell \otimes m|_{\mathscr{L} \otimes \mathscr{M}}(x)=1$. Hence one has $\varphi_{\mathscr{L} \otimes \mathscr{M}}=\varphi_{\mathscr{L}}+\varphi_{\mathscr{M}}$.

Proposition 2.3.16. - Let $X$ be a proper $k$-scheme and $L$ be an invertible $\mathcal{O}_{X}$ module. Let $(\mathscr{X}, \mathscr{L})$ be a model of $(X, L)$.

(1) The metric $\varphi_{\mathscr{L}}$ on $L$ is continuous.

(2) The $\mathfrak{o}_{k}$-module $H^{0}(\mathscr{X}, \mathscr{L})$ is a lattice in $H^{0}(X, L)$, which is contained in the unit ball of $H^{0}(X, L)$ with respect to the seminorm $\|\cdot\|_{\varphi_{\mathscr{L}}}$.

(3) If the valuation $|\cdot|$ is discrete, $X$ is reduced and the central fibre of $\mathscr{X} \rightarrow$ $\operatorname{Spec}\left(\mathfrak{o}_{k}\right)$ is reduced, then

$$
\left\{s \in H^{0}(X, L):\|s\|_{\varphi_{\mathscr{L}}} \leqslant 1\right\}=H^{0}(\mathscr{X}, \mathscr{L})
$$

and $\|s\|_{\varphi_{\mathscr{L}}}=\|\cdot\|_{H^{0}(\mathscr{X}, \mathscr{L})}$.

Proof. - (1) We choose an ample invertible $\mathcal{O}_{\mathscr{X}}$-module $\mathscr{A}$ such that $\mathscr{L} \otimes \mathscr{A}$ and $\mathscr{A}$ are generated by global sections. Let $\varphi_{1}$ and $\varphi_{2}$ be the quotient metrics of $L \otimes A$ and $A$ induced by $\|\cdot\|_{H^{0}(\mathscr{X}, \mathscr{L} \otimes \mathscr{A})}$ and $\|\cdot\|_{H^{0}(\mathscr{X}, \mathscr{A})}$, respectively, where $A=\left.\mathscr{A}\right|_{X}$. Then $\varphi_{1}$ and $\varphi_{2}$ are continuous by Prposition 2.2.12 and Definition 2.2.15. Moreover, by Proposition 2.3.12, $\varphi_{\mathscr{L} \otimes \mathscr{A}}=\varphi_{1}$ and $\varphi_{\mathscr{A}}=\varphi_{2}$. Therefore one has the assertion because $\varphi_{\mathscr{L}}=\varphi_{\mathscr{L} \otimes \mathscr{A}}-\varphi_{\mathscr{A}}=\varphi_{1}-\varphi_{2}$ by Proposition 2.3.15.

$(2)$ Let $s$ be a section in $H^{0}(\mathscr{X}, \mathscr{L})$, viewed as an element in $H^{0}(X, L)$, by definition one has

$$
\forall x \in X^{\text {an }}, \quad\|s\|_{\mathscr{L}}(x) \leqslant 1 .
$$

Hence $H^{0}(\mathscr{X}, \mathscr{L})$ is contained in the closed unit ball of $\left(H^{0}(X, L),\|\cdot\|_{\varphi_{\mathscr{L}}}\right)$.

(3) Let $\mathscr{E}$ be the unit ball of $H^{0}(X, L)$ with respect to $\|\cdot\|_{\varphi_{\mathscr{L}}}$. First let us see that

$$
H^{0}(\mathscr{X}, \mathscr{L})=\mathscr{E} .
$$

By $(2) H^{0}(\mathscr{X}, \mathscr{L}) \subseteq \mathscr{E}$. Let $\mathscr{X}=\bigcup_{i=1}^{N} \operatorname{Spec}\left(\mathscr{A}_{i}\right)$ be an affine open covering of $\mathscr{X}$ such that $\mathscr{A}_{i}$ is of finite type over $\mathfrak{o}_{k}$ and $\mathscr{L}$ has a local basis $\ell_{i}$ over $\operatorname{Spec}\left(\mathscr{A}_{i}\right)$. For $s \in \mathscr{E}$, we set $s=f_{i} \ell_{i}$ and $f_{i} \in A_{i}:=S^{-1} \mathscr{A}_{i}$, where $S=\mathfrak{o}_{k} \backslash\{0\}$. Then, for $x \in\left(\operatorname{Spec} A_{i}\right)_{\mathscr{A}_{i}}^{\text {an }}$ (cf. Remark 2.1.6),$|s|_{\varphi_{\mathscr{L}}}(x)=\left|f_{i}\right|_{x} \leqslant 1$. Therefore, as the central fibre $\mathscr{X}_{\circ}$ of $\mathscr{X} \rightarrow \operatorname{Spec}\left(\mathfrak{o}_{k}\right)$ is reduced, by the last assertion of Proposition 2.1.7, one has $f_{i} \in \mathscr{A}_{i}$, and hence $s \in H^{0}(\mathscr{X}, \mathscr{L})$.

Next we need to see that

$$
\|\cdot\|_{\varphi \mathscr{L}}=\|\cdot\|_{\mathscr{E}} .
$$

Let $\varpi$ be a uniformising parameter of $\mathfrak{o}_{k}, \mathscr{X}_{\circ}$ be the fibre of $\mathscr{X}$ over the maximal ideal of $\mathfrak{o}_{k}$ and $\mathscr{L}_{\circ}$ be the restriction of $\mathscr{L}$ to $\mathscr{X}_{\circ}$, that is, $\mathscr{L}_{\circ}=\mathscr{L} / \varpi \mathscr{L}$. The short 
exact sequence

$$
0 \longrightarrow \mathscr{L} \stackrel{\varpi \cdot}{\longrightarrow} \mathscr{L} \longrightarrow \mathscr{L}_{\circ} \longrightarrow 0
$$

gives rise to an exact sequence:

$$
0 \longrightarrow H^{0}(\mathscr{X}, \mathscr{L}) \stackrel{\varpi \cdot}{\longrightarrow} H^{0}(\mathscr{X}, \mathscr{L}) \longrightarrow H^{0}\left(\mathscr{X}_{\circ}, \mathscr{L}_{\circ}\right),
$$

that is, the natural homomorphism

$$
H^{0}(\mathscr{X}, \mathscr{L}) / \varpi H^{0}(\mathscr{X}, \mathscr{L}) \longrightarrow H^{0}\left(\mathscr{X}_{\circ}, \mathscr{L}_{\circ}\right)
$$

is injective. Moreover, by Proposition 1.1.30, one has

$$
\|\cdot\|_{\varphi_{\mathscr{L}}} \leqslant\|\cdot\|_{\mathscr{E}} .
$$

Here we claim that if $\|s\|_{\mathscr{E}}=1$ for $s \in H^{0}(X, L)$, then $\|s\|_{\varphi_{\mathscr{L}}}=1$. Obviously $\|s\|_{\varphi_{\mathscr{L}}} \leqslant 1$ by (2.19). As $H^{0}(\mathscr{X}, \mathscr{L})=\mathscr{E}$ by (2.16), one has $s \in H^{0}(\mathscr{X}, \mathscr{L})$ and $s$ is not zero in $H^{0}(\mathscr{X}, \mathscr{L}) / \varpi H^{0}(\mathscr{X}, \mathscr{L})$, so that by the injectivity of (2.18), $s$ is not zero in $H^{0}\left(\mathscr{X}_{\circ}, \mathscr{L}_{\circ}\right)$. Let $\xi$ be a closed point $\mathscr{X}_{\circ}$ with $s(\xi) \neq 0$. Let $\ell_{\xi}$ be a local basis of $\mathscr{L}$ around $\xi$. Then $s=f \ell_{\xi}$ and $f \in \mathcal{O}_{\mathscr{X}, \xi}^{\times}$. On the other hand, since the reduction map $r: X^{\text {an }} \rightarrow \mathscr{X}_{\mathrm{o}}$ is surjective, one can find $x \in X^{\text {an }}$ with $r(x)=\xi$. Then $|s|_{\varphi_{\mathscr{L}}}(x)=|f|_{x}=1$, so that $\|s\|_{\varphi_{\mathscr{L}}}=1$, as desired.

In general, for $s \in H^{0}(X, L) \backslash\{0\}$, there is an integer $e$ such that $\left\|\varpi^{e} s\right\|_{\mathscr{E}}=1$, so that $\left\|\varpi^{e} s\right\|_{\varphi_{\mathscr{L}}}=1$, and hence $\|s\|_{\mathscr{E}}=\|s\|_{\varphi_{\mathscr{L}}}=|\varpi|^{-e}$.

Proposition 2.3.17. - Let $X$ be a projective $k$-scheme and $L$ be an ample invertible $\mathcal{O}_{X}$-module. If $(\mathscr{X}, \mathscr{L})$ is a model of $(X, L)$ such that $L$ is ample and $\mathscr{L}$ is nef, then the metric $\varphi_{\mathscr{L}}$ is semipositive.

Proof. - Let $\pi: \mathscr{X} \rightarrow$ Spec $\mathfrak{o}_{k}$ be the structural morphism. First we assume that $\mathscr{L}$ is ample. We choose a positive integer $n$ such that $\mathscr{L}^{\otimes n}$ is very ample. Then we have a closed embedding $\iota: \mathscr{X} \rightarrow \mathbb{P}\left(\mathcal{E}_{n}\right)$ with $\mathcal{E}_{n}:=H^{0}\left(\mathscr{X}, \mathscr{L}^{\otimes n}\right)$, which is induced by the canonical (surjective) homomorphism $\pi^{*}\left(\pi_{*}\left(\mathscr{L}^{\otimes n}\right)\right)=\pi^{*}\left(\mathcal{E}_{n}\right) \rightarrow \mathscr{L}^{\otimes n}$. Note that one has $\mathscr{L}^{\otimes n}=\iota^{*}\left(\mathcal{O}_{\mathcal{E}_{n}}(1)\right)$. Moreover, $\mathcal{E}_{n}$ is a lattice in $E_{n}:=H^{0}\left(X, L^{\otimes n}\right)$. By Proposition 2.3.12, the metric $\varphi_{\mathscr{L} \otimes n}=n \varphi_{\mathscr{L}}$ (see Proposition 2.3.15 for this equality) coincides with the quotient metric on $L^{\otimes n}$ induced by the norm $\|\cdot\|_{\mathcal{E}_{n}}$, hence is semipositive (see Proposition 2.2.23). By Proposition 2.3.2, we obtain that the metric $\varphi_{\mathscr{L}}$ is also semipositive.

In the following, we treat the general nef case. Let $\mathscr{M}$ be an ample invertible sheaf on $\mathscr{X}$ and $M$ be the restriction of $\mathscr{M}$ to $X$. Since $\mathscr{L}$ is nef, we obtain that $\mathscr{L}^{\otimes n} \otimes \mathscr{M}$ is ample for any $n \in \mathbb{N}_{n \geqslant 1}$. Moreover, since $L$ is ample, for a sufficiently positive integer $n_{0} \geqslant 1$, the invertible $\mathcal{O}_{X}$-module $L^{\otimes n_{0}} \otimes M^{\vee}$ is generated by global sections. Thus for any integer $n>n_{0}$ one has (by Proposition 2.3.5)

$$
n \operatorname{dp}\left(\varphi_{\mathscr{L}}\right)=\operatorname{dp}\left(\varphi_{\mathscr{L} \otimes n}\right) \leqslant \operatorname{dp}\left(\varphi_{\mathscr{L} \otimes n_{0} \otimes \mathscr{M}^{\vee}}\right)+\operatorname{dp}\left(\varphi_{\mathscr{L} \otimes\left(n-n_{0}\right) \otimes \mathscr{M}}\right)=\operatorname{dp}\left(\varphi_{\mathscr{L} \otimes n_{0} \otimes \mathscr{M}^{\vee}}\right),
$$


where the first equality comes from (2.14), and the second equality comes from the semi-positivity of the metric $\varphi_{\mathscr{L} \otimes\left(n-n_{0}\right) \otimes \mathscr{M}}$. Since $n \geqslant n_{0}$ is arbitrary, we obtain that $\operatorname{dp}\left(\varphi_{\mathscr{L}}\right)=0$, namely $\varphi_{\mathscr{L}}$ is a semipositive metric.

Proposition 2.3.18. - Let $\pi: X \rightarrow$ Spec $k$ be a projective scheme over Spec $k$ and $L$ be an ample invertible $\mathcal{O}_{X}$-module, equipped with a continuous metric $\varphi$ which is semipositive. Then, for sufficiently positive integer $n$, there exists a sequence $\left\{\left(\mathscr{X}_{n}, \mathscr{L}_{n}\right)\right\}_{n \in \mathbb{N}, n \geqslant 1}$ of models of $\left(X, L^{\otimes n}\right)$ such that each $\mathscr{L}_{n}$ is ample, and that

$$
\lim _{n \rightarrow+\infty} \frac{1}{n} d\left(\varphi \mathscr{L}_{n}, n \varphi\right)=0
$$

Proof. - Let $\lambda \in] 0,1[$ be a number such that

$$
\lambda<\sup \left\{|a|: a \in k^{\times},|a|<1\right\} .
$$

For any $n \in \mathbb{N}_{\geqslant 1}$, let $V_{n}=H^{0}\left(X, L^{\otimes n}\right)$. Since $L$ is an ample, for sufficiently positive integer $n$, the canonical homomorphism $\pi^{*}\left(V_{n}\right) \rightarrow L^{\otimes n}$ is surjective, and the corresponding $k$-morphism $X \rightarrow \mathbb{P}\left(V_{n}\right)$ is a closed embedding. By Proposition 1.2.22 there exists a lattice of finite type $\mathcal{V}_{n}$ of $V_{n}$ such that

$$
d\left(\|\cdot\|_{\mathcal{V}_{n}},\|\cdot\|_{n \varphi}\right) \leqslant \ln \left(\lambda^{-1}\right) .
$$

Let $\mathscr{X}_{n}$ be the Zariski closure of $X$ in $\mathbb{P}\left(\mathcal{V}_{n}\right)$ and $\mathscr{L}_{n}$ be the restriction of $\mathcal{O}_{\mathbb{P}\left(\mathcal{V}_{n}\right)}(1)$ to $\mathscr{X}$, then $\left(\mathscr{X}, \mathscr{L}_{n}\right)$ is a model of $(X, L)$ with $\mathscr{L}_{n}$ being ample. Moreover, the metric on $L^{\otimes n}$ induced by $\mathscr{L}_{n}$ coincides with the quotient metric on $L^{\otimes n}$ induced by $\left(V_{n},\|\cdot\|_{\mathcal{V}_{n}}\right)$ and the canonical quotient homomorphism $\pi^{*}\left(V_{n}\right) \rightarrow L^{\otimes n}$. Therefore by Propositon 2.2 .20 one has

$$
d\left(\varphi_{\mathscr{L}_{n}}, n \varphi\right) \leqslant d\left(\|\cdot\|_{\mathcal{V}_{n}},\|\cdot\|_{n \varphi}\right) \leqslant \ln \left(\lambda^{-1}\right)
$$

as required.

2.3.3. Purity. - Let $X \rightarrow$ Spec $k$ be a projective $k$-scheme, $L$ be an invertible $\mathcal{O}_{X}$-module and $\varphi$ be a continuous metric on $X$. If the norm $\|\cdot\|_{\varphi}$ on $H^{0}(X, L)$ is pure, we say that the metric $\varphi$ is pure. If $n \varphi$ is pure for all $n \in \mathbb{N}_{\geqslant 1}$, we say that $\varphi$ is stably pure. Note that, if the absolute value $|\cdot|$ is not discrete, then any continuous metric on $L$ is stably pure (cf. Proposition 1.1.32).

Proposition 2.3.19. - We assume that the absolute value $|\cdot|$ is discrete. Let $X$ be a projective $k$-scheme and $L$ be an invertible $\mathcal{O}_{X}$-module. If $(\mathscr{X}, \mathscr{L})$ is a model of $(X, L)$ such that the central fibre of $\mathscr{X} \rightarrow \mathrm{Spec}_{\mathfrak{o}_{k}}$ is reduced, then the metric $\varphi_{\mathscr{L}}$ is stably pure.

Proof. - Note that for any $n \in \mathbb{N}_{\geqslant 1}$ one has $n \varphi_{\mathscr{L}}=\varphi_{\mathscr{L} \otimes n}$ and $\left(\mathscr{X}, \mathscr{L}^{\otimes n}\right)$ is a model of $\left(X, L^{\otimes n}\right)$. Therefore it suffices to show that $\varphi \mathscr{L}$ is a pure metric. By Proposition 2.3.16, the norm $\|\cdot\|_{\varphi_{\mathscr{L}}}$ is induced by the lattice $H^{0}(\mathscr{X}, \mathscr{L})$, hence it is pure. 
2.3.4. Extension property. - In this subsection, we introduce the extension property of an ample invertible module with a semipositive continuous metric, that is, an extension of a section with a control on the norm.

Throughout this subsection, let $\pi: X \rightarrow \operatorname{Spec}(k)$ be a projective $k$-scheme and $L$ be an invertible $\mathcal{O}_{X}$-module, equipped with a continuous metric $\varphi$. Let us begin with the following lemma.

Lemma 2.3.20. - Let $Y$ be a closed subscheme of $X$. For $n \in \mathbb{N}, n \geqslant 1$, let $\gamma_{n}: H^{0}\left(X, L^{\otimes n}\right) \rightarrow H^{0}\left(Y,\left.L^{\otimes n}\right|_{Y}\right)$ be the restriction map. For any element $\ell$ of $H^{0}\left(Y,\left.L\right|_{Y}\right) \backslash \mathcal{N}\left(Y,\left.L\right|_{Y}\right)$, we define $a_{\varphi, n}(\ell) \in[0, \infty]$ to be

$$
a_{\varphi, n}(\ell):= \begin{cases}\infty & \text { if } \gamma_{n}^{-1}\left(\left\{\ell^{\otimes n}\right\}\right)=\varnothing, \\ \inf _{\substack{\left.s \in H^{0}\left(X, L^{\otimes n}\right) \\ s\right|_{Y}=\ell^{\otimes n}}}\left(\ln \|s\|_{n \varphi}-\ln \|\ell\|_{\left.\varphi\right|_{Y}}^{n}\right) & \text { otherwise, }\end{cases}
$$

where $\left.\varphi\right|_{Y}$ denotes the restriction of $\varphi$ to $\left.L\right|_{Y}$, defined as the pull-back of $\varphi$ by the inclusion morphisme $Y \rightarrow X$ (see Definition [2.2.9). Then we have the following:

(1) The sequence $\left\{a_{\varphi, n}(\ell)\right\}_{n \in \mathbb{N}}$ is subadditive, that is, $a_{\varphi, n+n^{\prime}}(\ell) \leqslant a_{\varphi, n}(\ell)+a_{\varphi, n^{\prime}}(\ell)$ for $n, n^{\prime} \in \mathbb{N}$.

(2) Let $\varphi^{\prime}$ be another continuous metric of $L$. If $\gamma_{n}^{-1}\left(\left\{\ell^{\otimes n}\right\}\right) \neq \varnothing$, then

$$
\left|a_{\varphi, n}(\ell)-a_{\varphi^{\prime}, n}(\ell)\right| \leqslant 2 n d\left(\varphi, \varphi^{\prime}\right) .
$$

Proof. - (1) Clearly we may assume that $\gamma_{n}^{-1}\left(\left\{\ell^{\otimes n}\right\}\right) \neq \varnothing$ and $\gamma_{n^{\prime}}^{-1}\left(\left\{\ell^{\otimes n^{\prime}}\right\}\right) \neq \varnothing$. Then $\gamma_{n+n^{\prime}}^{-1}\left(\left\{\ell^{\otimes n+n^{\prime}}\right\}\right) \neq \varnothing$, so that

$$
\begin{aligned}
a_{\varphi, n+n^{\prime}}(\ell) & =\inf _{\substack{\left.s^{\prime \prime} \in H^{0}\left(L^{\otimes n+n^{\prime}}\right) \\
s^{\prime \prime}\right|_{Y}=\ell^{\otimes n+n^{\prime}}}}\left(\ln \left\|s^{\prime \prime}\right\|_{\left(n+n^{\prime}\right) \varphi}-\ln \|\ell\|_{\left.\varphi\right|_{Y}}^{n+n^{\prime}}\right) \\
& \leqslant \inf _{\substack{\left(s, s^{\prime}\right) \in H^{0}\left(L^{\otimes n}\right) \times\left. H^{0}\left(L^{\otimes n^{\prime}}\right) \\
s\right|_{Y}=\ell^{\otimes n},\left.s^{\prime}\right|_{Y}=\ell^{\otimes n^{\prime}}}}\left(\ln \left\|s \otimes s^{\prime}\right\|_{\left(n+n^{\prime}\right) \varphi}-\ln \|\ell\|_{\left.\varphi\right|_{Y}}^{n+n^{\prime}}\right) \\
\leqslant & \inf _{\substack{\left(s, s^{\prime}\right) \in H^{0}\left(L^{\otimes n}\right) \times\left. H^{0}\left(L^{\otimes n^{\prime}}\right) \\
s\right|_{Y}=\ell^{\otimes n},\left.s^{\prime}\right|_{Y}=\ell^{\otimes n^{\prime}}}}\left(\ln \|s\|_{n \varphi}+\ln \left\|s^{\prime}\right\|_{n^{\prime} \varphi}-\ln \|\ell\|_{\left.\varphi\right|_{Y}}^{n+n^{\prime}}\right) \\
& =a_{\varphi, n}(\ell)+a_{\varphi, n^{\prime}}(\ell),
\end{aligned}
$$

as required.

(2) Clearly we may assume that $a_{\varphi, n}(\ell) \geqslant a_{\varphi^{\prime}, n}(\ell)$. For any $\epsilon>0$, choose $s \in$ $H^{0}\left(X, L^{\otimes n}\right)$ such that $\left.s\right|_{Y}=\ell^{\otimes n}$ and

$$
\ln \|s\|_{n \varphi^{\prime}}-\ln \|\ell\|_{\varphi^{\prime} \mid Y}^{n} \leqslant a_{\varphi^{\prime}, n}(\ell)+\epsilon .
$$


Then, by using (2.5) and (2.6),

$$
\begin{aligned}
a_{\varphi, n}(\ell)-a_{\varphi^{\prime}, n}(\ell) & \leqslant\left(\ln \|s\|_{n \varphi}-\ln \|\ell\|_{\left.\varphi\right|_{Y}}^{n}\right)-\left(\ln \|s\|_{n \varphi^{\prime}}-\ln \|\ell\|_{\left.\varphi^{\prime}\right|_{Y}}^{n}\right)+\epsilon \\
& \leqslant\left|\ln \|s\|_{n \varphi}-\ln \|s\|_{n \varphi^{\prime}}\right|+\left|\ln \|\ell\|_{\left.\varphi\right|_{Y}}^{n}-\ln \|\ell\|_{\left.\varphi^{\prime}\right|_{Y}}^{n}\right|+\epsilon \\
& \leqslant d\left(\|\cdot\| n \varphi,\|\cdot\|_{n \varphi^{\prime}}\right)+n d\left(\|\cdot\|_{\left.\varphi\right|_{Y}},\|\cdot\|_{\left.\varphi^{\prime}\right|_{Y}}\right)+\epsilon \\
& \leqslant d\left(n \varphi, n \varphi^{\prime}\right)+n d\left(\left.\varphi\right|_{Y},\left.\varphi^{\prime}\right|_{Y}\right)+\epsilon \leqslant 2 n d\left(\varphi, \varphi^{\prime}\right)+\epsilon,
\end{aligned}
$$

so that the assertion follows because $\epsilon$ is an arbitrary positive number.

Definition 2.3.21. - Let $Y$ be a closed subscheme of $X$. For $\ell \in H^{0}\left(Y,\left.L\right|_{Y}\right)$, we say that $\ell$ has the extension property for the metric $\varphi$ if, for any $\epsilon>0$, there exists $n_{0} \in \mathbb{N}, n_{0} \geqslant 1$, such that for any integer $n, n \geqslant n_{0}$, there exists a section $s \in H^{0}\left(X, L^{\otimes n}\right)$ satisfying

$$
\left.s\right|_{Y}=\ell^{\otimes n} \quad \text { and } \quad\|s\|_{n \varphi} \leqslant \mathrm{e}^{\epsilon n}\|\ell\|_{\left.\varphi\right|_{Y}}^{n} .
$$

If $\ell \in \mathcal{N}\left(Y,\left.L\right|_{Y}\right)$, then $\ell$ has the extension property for the metric $\varphi$. Indeed, by Proposition 2.1.16 (3), there is a positive integer $n_{0}$ such that $\ell^{\otimes n}=0$ for all integer $n \geqslant n_{0}$, so that if we choose $s=0 \in H^{0}\left(X, L^{\otimes n}\right)$, then the above properties (2.21) hold. In this sense, in order to check the extension property, we may assume that $\ell \notin \mathcal{N}\left(Y,\left.L\right|_{Y}\right)$.

For any non-zero element $\ell$ of $H^{0}\left(Y,\left.L\right|_{Y}\right) \backslash \mathcal{N}\left(Y,\left.L\right|_{Y}\right)$, we let

$$
\lambda_{\varphi}(\ell)=\limsup _{n \rightarrow+\infty} \frac{a_{\varphi, n}(\ell)}{n} \in[0,+\infty] .
$$

We call $\lambda_{\varphi}(\ell)$ the extension obstruction index of $\ell$.

Definition 2.3.22. - We assume that $H^{0}\left(X, L^{\otimes n}\right) \rightarrow H^{0}\left(Y,\left.L\right|_{Y} ^{\otimes n}\right)$ is surjective for all $n \geqslant 1$. Let $\|\cdot\|_{n \varphi, \text { quot }}$ be the quotient seminorm of $H^{0}\left(Y,\left.L\right|_{Y} ^{\otimes n}\right)$ induced by $\|\cdot\|_{n \varphi}$ and the surjective homomorphism $H^{0}\left(X, L^{\otimes n}\right) \rightarrow H^{0}\left(Y,\left.L\right|_{Y} ^{\otimes n}\right)$. For $\ell \in H^{0}\left(Y,\left.L\right|_{Y} ^{\otimes n}\right)$, we define $\|\ell\|_{\varphi, \text { quot }}^{(n)}$ to be

$$
\|\ell\|_{\varphi, \text { quot }}^{(n)}:=\left(\left\|\ell^{\otimes n}\right\|_{n \varphi, \text { quot }}\right)^{1 / n}
$$

It is easy to see that

$$
\|\ell\|_{\varphi, \text { quot }}^{(\infty)}:=\lim _{n \rightarrow \infty}\|\ell\|_{\varphi, \text { quot }}^{(n)}=\inf _{n>0}\|\ell\|_{\varphi, \text { quot }}^{(n)} \in \mathbb{R}_{\geqslant 0}
$$

and

$$
\|\ell\|_{\left.\varphi\right|_{Y}} \leqslant\|\ell\|_{\varphi, q u o t}^{(\infty)}
$$

because

$$
\left\|\ell_{n} \otimes \ell_{n^{\prime}}\right\|_{\left(n+n^{\prime}\right) \varphi, \text { quot }} \leqslant\left\|\ell_{n}\right\|_{n \varphi, \text { quot }}\left\|\ell_{n^{\prime}}\right\|_{n^{\prime} \varphi, \text { quot }} \text { and }\left\|\ell_{n}\right\|_{\left.n \varphi\right|_{Y}} \leqslant\left\|\ell_{n}\right\|_{n \varphi, \text { quot }}
$$

for all $\ell_{n} \in H^{0}\left(Y,\left.L\right|_{Y} ^{\otimes n}\right)$ and $\ell_{n^{\prime}} \in H^{0}\left(Y,\left.L\right|_{Y} ^{\otimes n^{\prime}}\right)$. 
Proposition 2.3.23. - We assume that $\ell \notin \mathcal{N}\left(Y,\left.L\right|_{Y}\right)$ and there exists a positive integer $n_{1}$ such that, for all $n \geqslant n_{1}, \ell^{\otimes n}$ lies in the image of the restriction map $H^{0}\left(X, L^{\otimes n}\right) \rightarrow H^{0}\left(Y,\left.L\right|_{Y} ^{\otimes n}\right)$. Then one has the following:

(1) $\lambda_{\varphi}(\ell)=\lim _{n \rightarrow+\infty} \frac{a_{\varphi, n}(\ell)}{n}=\inf _{n \geqslant 1} \frac{a_{\varphi, n}(\ell)}{n}$.

(2) The following are equivalent:

(2.a) $\ell$ has the extension property for $\varphi$.

(2.b) For any $\epsilon>0$, there are a positive integer $n$ and a section $s \in H^{0}\left(X, L^{\otimes n}\right)$ such that $\left.s\right|_{Y}=\ell^{\otimes n}$ and $\|s\|_{n \varphi} \leqslant \mathrm{e}^{\epsilon n}\|\ell\|_{\left.\varphi\right|_{Y}}^{n}$.

(2.c) $\lambda_{\varphi}(\ell)=0$

(3) We assume that $H^{0}\left(X, L^{\otimes n}\right) \rightarrow H^{0}\left(Y,\left.L\right|_{Y} ^{\otimes n}\right)$ is surjective for all $n \geqslant 1$. Then, the above equivalent properties are also equivalent to $\|\ell\|_{\left.\varphi\right|_{Y}}=\|\ell\|_{\varphi, \text { quot }}^{(\infty)}$.

Proof. - (1) is a consequence of Fekete's lemma because the sequence $\left\{a_{\varphi, n}\right\}_{n \in \mathbb{N}}$ is subadditive by Lemma 2.3 .20

'(2.a) $\Longrightarrow(2 . \mathrm{b})$ ' is obvious.

'(2.b) $\Longrightarrow(2 . \mathrm{c})$ ': For any $\epsilon>0$, there is a positive integer $n$ such that $a_{\varphi, n}(\ell) \leqslant n \epsilon$, so that, by (1).

$$
0 \leqslant \lambda_{\varphi}(\ell)=\inf _{n \geqslant 1} \frac{a_{\varphi, n}(\ell)}{n} \leqslant \epsilon
$$

and hence one has (2.c)

$$
\text { '2(2.c) } \Longrightarrow(2 . \mathrm{a}) \text { ': Since } \lambda_{\varphi}(\ell)=\lim _{n \rightarrow+\infty} \frac{a_{\varphi, n}(\ell)}{n} \text { by (1), we can see (2.a) }
$$

(3) Note that $a_{\varphi, n}(\ell) / n=\ln \|\ell\|_{\varphi, \text { qout }}^{(n)}-\ln \|\ell\|_{\left.\varphi\right|_{Y}}$, so that $\lambda_{\varphi}(\ell)=\ln \|\ell\|_{\varphi, \text { qout }}^{(\infty)}-$ $\ln \|\ell\|_{\left.\varphi\right|_{Y}}$. Thus the assertion follows.

Remark 2.3.24. - (1) Let $\varphi^{\prime}$ be another metric on L. By Lemma 2.3.20 one has

$$
\left|a_{\varphi, n}(\ell)-a_{\varphi^{\prime}, n}(\ell)\right| \leqslant 2 n d\left(\varphi, \varphi^{\prime}\right),
$$

provided that $a_{\varphi, n}(\ell)$ or $a_{\varphi^{\prime}, n}(\ell)$ is finite. We deduce from this inequality that, $\lambda_{\varphi}(\ell)$ is finite if and only if $\lambda_{\varphi^{\prime}}(\ell)$ is finite. Moreover, when these numbers are finite, one has

$$
\left|\lambda_{\varphi}(\ell)-\lambda_{\varphi^{\prime}}(\ell)\right| \leqslant 2 d\left(\varphi, \varphi^{\prime}\right) .
$$

(2) We assume that $\lambda_{\varphi}(\ell)<\infty$. Then one has

$$
\lambda_{n \varphi}\left(\ell^{\otimes n}\right)=n \lambda_{\varphi}(\ell)
$$

for all $n>0$. Indeed,

$$
\lambda_{n \varphi}\left(\ell^{\otimes n}\right)=\lim _{m \rightarrow \infty} \frac{a_{n \varphi, m}\left(\ell^{\otimes n}\right)}{m}=\lim _{m \rightarrow \infty} \frac{a_{\varphi, n m}(\ell)}{m}=n \lim _{m \rightarrow \infty} \frac{a_{\varphi, n m}(\ell)}{n m}=n \lambda_{\varphi}(\ell) .
$$


(3) Let $X^{\prime}$ be a closed subscheme of $X$ such that $Y \subseteq X^{\prime}$. We assume that there is a positive integer $n_{0}$ such that, for all $n \geqslant n_{0}, H^{0}\left(X, L^{\otimes n}\right) \rightarrow H^{0}\left(X^{\prime},\left.L^{\otimes n}\right|_{X^{\prime}}\right)$ is surjective. Then

$$
\lambda_{\left.\varphi\right|_{X^{\prime}}}(\ell) \leqslant \lambda_{\varphi}(\ell)
$$

Indeed, as $\left\|\left.s\right|_{X^{\prime}}\right\|_{\left.\varphi\right|_{X^{\prime}}} \leqslant\|s\|_{\varphi}$ for all $s \in H^{0}\left(X, L^{\otimes n}\right)$ and $H^{0}\left(X, L^{\otimes n}\right) \rightarrow$ $H^{0}\left(X^{\prime},\left.L^{\otimes n}\right|_{X^{\prime}}\right)$ is surjective for all $n \geqslant n_{0}$, one has $a_{\left.\varphi\right|_{X^{\prime}}, n}(\ell) \leqslant a_{\varphi, n}(\ell)$ for all $n \geqslant n_{0}$, so that the assertion follows.

2.3.4.1. A generalisation of a result in 148 and $[\mathbf{1 0 6}$. - Let $X$ be a $d$-dimensional integral smooth scheme over $\mathbb{C}$. Let $Y$ be a closed and reduced subscheme of $X$ defined by an ideal sheaf $I$ on $X$, that is, $I=\sqrt{I}$ and $Y=\operatorname{Spec}\left(\mathcal{O}_{X} / I\right)$. Let $\mu_{I}: X_{I} \rightarrow X$ be the blowing-up along $I$, that is, $X_{I}=\operatorname{Proj}\left(\bigoplus_{m=0}^{\infty} I^{m}\right)$. Let $\tilde{\mu}: \widetilde{X}_{I} \rightarrow X_{I}$ be the normalisation of $X_{I}$. Furthermore, let $\mu^{\prime}: X^{\prime} \rightarrow \widetilde{X}_{I}$ be a desingularisation of $\widetilde{X}_{I}$ such that $\mu^{\prime}$ yields an isomorphism

$$
X^{\prime} \backslash \mu^{\prime-1}\left(\operatorname{Sing}\left(\widetilde{X}_{I}\right)\right) \stackrel{\sim}{\longrightarrow} \widetilde{X}_{I} \backslash \operatorname{Sing}\left(\widetilde{X}_{I}\right) .
$$

We denote the compositions

$$
X^{\prime} \stackrel{\mu^{\prime}}{\longrightarrow} \tilde{X}_{I} \stackrel{\tilde{\mu}}{\longrightarrow} X_{I} \stackrel{\mu_{I}}{\longrightarrow} X .
$$

by $\mu$, that is, $\mu:=\mu_{I} \circ \tilde{\mu} \circ \mu^{\prime}$. Note that $X^{\prime} \backslash \mu^{-1}(Y) \stackrel{\sim}{\longrightarrow} X \backslash Y$ via $\mu$.

Lemma 2.3.25. - There are positive integers $m_{0}$ and $c$ such that $\mu_{*}\left(I^{m} \mathcal{O}_{X^{\prime}}\right) \subseteq$ $I^{m-c}$ for all $m \geqslant m_{0}+c$.

Proof. - Let us consider the following claim:

Claim 2.3.26. - (a) $\mu_{*}^{\prime}\left(I^{m} \mathcal{O}_{X^{\prime}}\right)=I^{m} \mathcal{O}_{\widetilde{X}_{I}}$ for all integer $m \geqslant 0$.

(b) There is a positive integer $c$ such that $\tilde{\mu}_{*}\left(I^{m} \mathcal{O}_{\widetilde{X}_{I}}\right) \cap \mathcal{O}_{X_{I}} \subseteq I^{m-c} \mathcal{O}_{X_{I}}$ for all integer $m \geqslant c$.

(c) There is a positive integer $m_{0}$ such that $\mu_{I, *}\left(I^{m} \mathcal{O}_{X_{I}}\right)=I^{m}$ for all integer $m \geqslant m_{0}$.

Proof. - (a) Note that $I^{m} \mathcal{O}_{\widetilde{X}_{I}}$ is invertible and $I^{m} \mathcal{O}_{X^{\prime}}=\mu^{\prime *}\left(I^{m} \mathcal{O}_{\widetilde{X}_{I}}\right)$. Moreover as $\widetilde{X}_{I}$ is normal, $\mu_{*}^{\prime}\left(\mathcal{O}_{X^{\prime}}\right)=\mathcal{O}_{\widetilde{X}_{I}}$, so that the assertion follows from the projection formula.

(b) We choose an affine open covering $X_{I}=\bigcup_{i=1}^{N} \operatorname{Spec}\left(A_{i}\right)$. Let $\widetilde{A}_{i}$ be the normalisation of $A_{i}$. Then $\widetilde{X}_{I}=\bigcup_{i=1}^{N} \operatorname{Spec}\left(\widetilde{A}_{i}\right)$ is an affine open covering. Note that $\widetilde{A}_{i}$ is a finitely generated $A_{i}$-module, so that, by Artin-Lees lemma (cf. [6, Corollary 10.10]), there is a positive constant $c_{i}$ such that $I^{m} \widetilde{A}_{i} \cap A_{i}=I^{m-c_{i}}\left(I^{c} \widetilde{A}_{i} \cap A_{i}\right)$ for all $m \geqslant c_{i}$, which implies $I^{m} \widetilde{A}_{i} \cap A_{i} \subseteq I^{m-c_{i}} A_{i}$. Therefore, if we set $c=\max \left\{c_{1}, \ldots, c_{N}\right\}$, then one has the assertion. 
(c) This is essentially proved in [82, Chapter II, Theorem 5.19]. Indeed, at the final line in the proof of the above reference, it says that " $S_{n}^{\prime}=S_{n}$ for all sufficiently large $n$ ", which is nothing more than the assertion of (c) because $\mathcal{O}_{X_{I}}(m)=I^{m} \mathcal{O}_{X_{I}}$.

Let us go back to the proof of the lemma. This is a local question, so that we may assume that $X=\operatorname{Spec}(A)$ for some finitely generated regular $\mathbb{C}$-algebra $A$. Note that $\mu_{*}\left(I^{m} \mathcal{O}_{X^{\prime}}\right) \subseteq \mathcal{O}_{X}$. Therefore, it is sufficient to see that, if $f \in I^{m} \mathcal{O}_{X^{\prime}}$ for $f \in A$, then $f \in I^{m-c}$. First of all, by (a) $f \in I^{m} \mathcal{O}_{\tilde{X}_{I}}$, so that $f \in \tilde{\mu}_{*}\left(I^{m} \mathcal{O}_{\tilde{X}_{I}}\right) \cap \mathcal{O}_{X_{I}}$, and hence, by (b) $f \in I^{m-c} \mathcal{O}_{X_{I}}$. Note that $m-c \geqslant m_{0}$. Therefore, one has $f \in I^{m-c}$, as required.

We assume that $X$ is projective. Let $L$ be an ample invertible $\mathcal{O}_{X}$-module and $\varphi$ be a $C^{\infty}$-metric of $L$ such that $c_{1}(L, \varphi)$ is positive. Let $U$ be an open set (in the sense of the classical topology) of $X$ such that $Y \subseteq U$. The proof of [106, Theorem 7.6] works well even if we change the exponent $d$ of $\rho$ by a positive number $\delta$ except (3) in Claim 2, which should be

"If $\delta \geqslant d$, then $\rho^{-\delta}$ is not integrable on any neighborhood of $Y$ ".

At page 231, line 6 from the bottom, one constructs a $C^{\infty}$-section $l^{\prime}$ of $L^{\otimes n}$ over $X$, which is holomorphic on $U^{\prime}$ and satisfies the integrability condition

$$
\int_{X}\left|l^{\prime}\right|^{2} \rho^{-\delta} \Phi<\infty
$$

Let $E_{1}, \ldots, E_{r}$ be irreducible components of $\mu^{-1}(Y)$. We set

$$
I \mathcal{O}_{X^{\prime}}=-\left(a_{1} E_{1}+\cdots+a_{r} E_{r}\right) \quad \text { and } \quad K_{X^{\prime}}=\mu^{*}\left(K_{X}\right)+b_{1} E_{1}+\cdots+b_{r} E_{r} .
$$

Note that $a_{i}>0$ and $b_{i}>0$ for all $i$.

Lemma 2.3.2\%. - If $e_{i}$ is the multiplicity of $l^{\prime}$ along $E_{i}$, then $e_{i}>a_{i} \delta-b_{i}-1$ for $i=1, \ldots, r$.

Proof. - Let $\eta$ be a closed point of $E_{i} \backslash \operatorname{Sing}\left(E_{1}+\cdots+E_{r}\right)$ and $\xi=\mu(\eta)$. Let $y_{1}, \ldots, y_{d}$ be a local coordinate of $X^{\prime}$ on an open neighborhood $W$ of $\eta$ such that $E_{i}$ is defined by $y_{1}=0$. Let $x_{1}, \ldots, x_{d}$ be a local coordinate of $X^{\prime}$ on an open neighborhood $V$ of $\xi$. In the following, if it is necessary, we will shrink $V$ and $W$ freely. First of all, we may assume that

$$
\mu(W) \subseteq V
$$

Moreover, we can find a positive constant $C$ such that

$$
\Phi \geqslant C(\sqrt{-1})^{d}\left(d x_{1} \wedge d \bar{x}_{1}\right) \wedge \cdots \wedge\left(d x_{d} \wedge d \bar{x}_{d}\right)
$$

on $V$. Let $\omega$ be a local basis of $L$ on $V$. Note that $\rho$ can be writen by

$$
\rho=\left(\left|f_{1}\right|^{2}+\cdots+\left|f_{N}\right|^{2}\right)\left|\omega^{m}\right|
$$


on $V$, where $f_{1}, \ldots, f_{N}$ are generators of $I$ on $V$, so that there is a positive constant $C^{\prime}$ such that

$$
\mu^{*}(\rho) \leqslant C^{\prime}\left|y_{1}\right|^{2 a_{i}}
$$

on $W$. Further one has

$$
\begin{aligned}
& \mu^{*}\left((\sqrt{-1})^{d}\left(d z_{1} \wedge d \bar{z}_{1}\right) \wedge \cdots \wedge\right.\left.\left(d z_{d} \wedge d \bar{z}_{d}\right)\right) \\
& \quad=\left|y_{1}\right|^{2 b_{i}}|u|^{2}(\sqrt{-1})^{d}\left(d y_{1} \wedge d \bar{y}_{1}\right) \wedge \cdots \wedge\left(d y_{d} \wedge d \bar{y}_{d}\right)
\end{aligned}
$$

on $W$, where $u$ is a nowhere vanishing holomorphic function $W$, so that

$$
\begin{aligned}
\mu^{*}\left((\sqrt{-1})^{d}\left(d z_{1} \wedge d \bar{z}_{1}\right) \wedge\right. & \left.\cdots \wedge\left(d z_{d} \wedge d \bar{z}_{d}\right)\right) \\
& \geqslant C^{\prime \prime}\left|y_{1}\right|^{2 b_{i}}(\sqrt{-1})^{d}\left(d y_{1} \wedge d \bar{y}_{1}\right) \wedge \cdots \wedge\left(d y_{d} \wedge d \bar{y}_{d}\right)
\end{aligned}
$$

holds on $W$ for some positive constant $C^{\prime \prime}$. If we set $l^{\prime}=f^{\prime} \omega^{n}$, then $f^{\prime}=y_{1}^{e_{i}} g$ on $W$ such that $g$ is not identically zero on $\left.E_{i}\right|_{W}$, so that one can find $\left.\left(0, \alpha_{2}, \ldots, \alpha_{n}\right) \in E_{i}\right|_{W}$ and a positive number $r$ such that $g \neq 0$ on

$$
W_{r}=\left\{\left(y_{1}, \ldots, y_{r}\right) \in W:\left|y_{j}-\alpha_{j}\right| \leqslant r \text { for all } j=1, \ldots, d\right\},
$$

where $\alpha_{1}=0$. Therefore one can find a positive constant $C^{\prime \prime \prime}$ such that

$$
\mu^{*}\left(\left|l^{\prime}\right|^{2}\right) \geqslant C^{\prime \prime \prime}\left|y_{1}\right|^{2 e_{i}}
$$

on $W_{r}$. Thus, if we set $y_{j}-\alpha_{j}=r_{j} \exp \left(\sqrt{-1} \theta_{j}\right)$ for $j=1, \ldots, d$, then, by (2.28), (2.29), (2.30), (2.31), (2.32) and (2.33),

$$
\begin{aligned}
\infty & >\int_{X}\left|l^{\prime}\right|^{2} \rho^{-\delta} \Phi \geqslant \int_{V}\left|l^{\prime}\right|^{2} \rho^{-\delta} \Phi \\
& \geqslant \int_{V} C\left|l^{\prime}\right|^{2} \rho^{-\delta}(\sqrt{-1})^{d}\left(d x_{1} \wedge d \bar{x}_{1}\right) \wedge \cdots \wedge\left(d x_{d} \wedge d \bar{x}_{d}\right) \\
& \geqslant C \int_{W_{r}} \mu^{*}\left(\left|l^{\prime}\right|^{2} \rho^{-\delta}(\sqrt{-1})^{d}\left(d x_{1} \wedge d \bar{x}_{1}\right) \wedge \cdots \wedge\left(d x_{d} \wedge d \bar{x}_{d}\right)\right) \\
& \geqslant C C^{\prime-\delta} C^{\prime \prime} C^{\prime \prime \prime} \int_{W_{r}}\left|y_{1}\right|^{2 e_{i}+2 b_{i}-2 a_{i} \delta}(\sqrt{-1})^{d}\left(d y_{1} \wedge d \bar{y}_{1}\right) \wedge \cdots \wedge\left(d y_{d} \wedge d \bar{y}_{d}\right) \\
& \geqslant C C^{\prime-\delta} C^{\prime \prime} C^{\prime \prime \prime} \int_{[0, r]^{d} \times[0,2 \pi]^{d}} r_{1}^{2 e_{i}+2 b_{i}-2 a_{i} \delta+1} r_{2} \cdots r_{d} d r_{1} \cdots d r_{d} d \theta_{1} \cdots d \theta_{d} \\
& \geqslant C C^{\prime-\delta} C^{\prime \prime} C^{\prime \prime \prime} 2 \pi^{d} r^{2(d-1)} \int_{[0, r]} r_{1}^{2 e_{i}+2 b_{i}-2 a_{i} \delta+1} d r_{1},
\end{aligned}
$$

so that $2 e_{i}+2 b_{i}-2 a_{i} \delta+1>-1$, as required.

By virtue of Lemma 2.3.27 together with Lemma 2.3.25, one has the following generalisation of [148, Lemma 2.6] and [106, Theorem 7.6].

Theorem 2.3.28. - Let $Y^{\prime}$ be a closed subscheme of $X$ defined by an ideal sheaf $J$ on $X$, that is, $Y^{\prime}=\operatorname{Spec}\left(\mathcal{O}_{X} / J\right)$. Let $U$ be a Zariski open subset of $X$ containing $Y^{\prime}$. Then there are a positive integer $n_{0}$ and a positive constant $C$ such that, for any 
integer $n \geqslant n_{0}$ and $l_{U} \in H^{0}\left(U, L^{\otimes n}\right)$ with $\left\|l_{U}\right\|_{n \varphi_{U}}<\infty$, there is $l \in H^{0}\left(X, L^{\otimes n}\right)$ such that $\left.l\right|_{Y^{\prime}}=\left.l_{U}\right|_{Y^{\prime}}$ and $\|l\|_{n \varphi} \leqslant C n^{2 d}\left\|l_{U}\right\|_{n \varphi_{U}}$, where $\left\|l_{U}\right\|_{n \varphi_{U}}:=\sup \left\{\left|l_{U}\right|_{n \varphi}(x)\right.$ : $\left.x \in U^{\mathrm{an}}\right\}$.

Proof. - Let $I:=\sqrt{J}$ and $Y:=\operatorname{Spec}\left(\mathcal{O}_{X} / I\right)$. One can find $a \in \mathbb{Z}_{\geqslant 1}$ such that $I^{a} \subseteq J$. We fix a positive number $\delta$ with

$$
\delta \geqslant \max _{i=1, \ldots, r}\left\{\frac{b_{i}+1}{a_{i}}\right\}+c+m_{0}+a,
$$

where $c$ and $m_{0}$ are the positive integers in Lemma 2.3.25, The proof of 106 , Theorem 7.6] is carried out by using the exponent $\delta$ instead of $d$. The point is to show that $\left.l\right|_{Y^{\prime}}=\left.l_{U}\right|_{Y^{\prime}}$. By Lemma 2.3.27,

$$
e_{i}>a_{i} \delta-b_{i}-1 \geqslant a_{i}\left(c+m_{1}+a\right),
$$

so that, there is a Zariski closed set $Z$ of $\mu^{-1}(Y) \operatorname{such}$ that $\operatorname{dim} Z \leqslant d-2$ and

$$
\left.\mu^{*}\left(l^{\prime}\right) \in I^{c+m_{1}+a} \mu^{*}\left(L^{\otimes n}\right)\right|_{\mu^{-1}\left(U^{\prime}\right) \backslash Z} .
$$

As $I^{c+m_{1}+a} \mu^{*}\left(L^{\otimes n}\right)$ is invertible, one can see that $\left.\mu^{*}\left(l^{\prime}\right) \in I^{c+m_{1}+a} \mu^{*}\left(L^{\otimes n}\right)\right|_{\mu^{-1}\left(U^{\prime}\right)}$, and hence, by Lemma 2.3.25, $l^{\prime} \in I^{m_{1}+a} L^{\otimes n} \subseteq I^{a} L^{\otimes n}$. Therefore the class of $l^{\prime}$ in $L^{\otimes n} / J L^{\otimes n}$ is zero over $Y^{\prime}$, and hence $\left.l\right|_{Y^{\prime}}=\left.l_{U}\right|_{Y^{\prime}}$. The remaining estimates are same as the proof of [106. Theorem 7.6].

Corollary 2.3.29. - Let $X, L$ and $Y^{\prime}$ be the same as in Theorem 2.3.28. Let $\varphi$ a continuous metric of $L$ such that the first Chern current $c_{1}(L, \varphi)$ is positive. Then, for $\ell \in H^{0}\left(Y^{\prime},\left.L\right|_{Y^{\prime}}\right)$, $\ell$ has the extension property for $\varphi$.

Proof. - Clearly we may assume that $\ell \notin \mathcal{N}\left(Y^{\prime},\left.L\right|_{Y^{\prime}}\right)$. As $L$ is ample, there is a $C^{\infty}$-metric $\psi$ on $L$ such that $c_{1}(L, \psi)$ is a positive form.

Claim 2.3.30. - If the corollary holds for any $C^{\infty}$-metric of $L$ with the semipositive Chern form, then the corollary holds in general.

Proof. - Let $\phi$ be a continuous function such that $\psi-\varphi=\phi$. It is well known that there is a sequence $\left\{\phi_{n}\right\}_{n=1}^{\infty}$ of $C^{\infty}$-functions on $X^{\text {an }}$ such that $\varphi_{n}:=\psi-\phi_{n}$ is a $C^{\infty}$ metric of $L$ with the semipositive Chern form and $\left\{\phi_{n}\right\}_{n=1}^{\infty}$ converges uniformly to $\phi$ (for example, see [9, Theorem 1] or [105, Lemma 4.2]). Thus $\lim _{n \rightarrow \infty} d\left(\varphi, \varphi_{n}\right)=0$. By our assumption, $\lambda_{\varphi_{n}}(\ell)=0$, so that $\lambda_{\varphi}(\ell) \leqslant 2 d\left(\varphi, \varphi_{n}\right)$, and hence the assertion follows.

We fix a positive number $\epsilon$. By the above claim, we may assume that $\varphi$ is $C^{\infty}$, so that if we set $f=\psi-\varphi$, then $f$ is a $C^{\infty}$ function. Note that for $\left.\lambda \in\right] 0,1[, \varphi+\lambda f$ gives rise to a positive Chern form because $\varphi+\lambda f=(1-\lambda) \varphi+\lambda \psi$. We choose $\left.\lambda_{0} \in\right] 0,1[$ such that

$$
\lambda_{0} \sup \left\{|f(x)|: x \in X^{\text {an }}\right\} \leqslant \epsilon .
$$


We set $\varphi^{\prime}=\varphi+\lambda_{0} f$. Then

$$
\mathrm{e}^{-\epsilon}|\cdot|_{\varphi}(x) \leqslant|\cdot|_{\varphi^{\prime}}(x) \leqslant \mathrm{e}^{\epsilon}|\cdot|_{\varphi}(x)
$$

for all $x \in X^{\text {an }}$. We choose a positive integer $a$ such that

$$
H^{0}\left(X, L^{\otimes a}\right) \rightarrow H^{0}\left(Y^{\prime},\left.L^{\otimes a}\right|_{Y^{\prime}}\right)
$$

is surjective, so that one can find $t$ such that $t \in H^{0}\left(X, L^{\otimes a}\right)$ and $\left.t\right|_{Y^{\prime}}=\ell^{\otimes a}$. We also choose an open set $U$ of $X$ such that $Y^{\prime} \subseteq U$ and

$$
\|t\|_{a \varphi_{U}^{\prime}} \leqslant \mathrm{e}^{a \epsilon}\left\|l^{\otimes a}\right\|_{\left.a \varphi^{\prime}\right|_{Y^{\prime}}} .
$$

By the above theorem, there are a positive integer $n_{1}$ and a positive constant $C$ such that, for any $n \geqslant n_{1}$, one can find $s \in H^{0}\left(X, L^{\otimes a n}\right)$ such that $\left.s\right|_{Y^{\prime}}=\ell^{\otimes a n}$ and

$$
\|s\|_{n a \varphi^{\prime}} \leqslant C n^{2 d}\left\|t^{\otimes n}\right\|_{n a \varphi_{U}^{\prime}} .
$$

Let $n_{2}$ be a positive integer such that $n_{2} \geqslant n_{1}$ and

$$
C n^{2 d} \leqslant \mathrm{e}^{\epsilon a n}
$$

for $n \geqslant n_{2}$. Therefore, using (2.34), (2.35), (2.36) and (2.37), one has

$$
\begin{aligned}
\|s\|_{n a \varphi} & \leqslant \mathrm{e}^{n a \epsilon}\|s\|_{n a \varphi^{\prime}} \leqslant \mathrm{e}^{n a \epsilon}\left(C n^{2 d}\|t\|_{a \varphi_{U}^{\prime}}^{n}\right) \\
& \leqslant \mathrm{e}^{2 n a \epsilon}\|t\|_{a \varphi_{U}^{\prime}}^{n} \leqslant \mathrm{e}^{3 n a \epsilon}\|l\|_{\left.\varphi^{\prime}\right|_{Y^{\prime}}}^{a n} \leqslant \mathrm{e}^{4 n a \epsilon}\|l\|_{\left.\varphi\right|_{Y^{\prime}}}^{a n},
\end{aligned}
$$

which means that $\lambda_{a \varphi}\left(l^{\otimes a}\right) \leqslant 4 a \epsilon$, so that $\lambda_{\varphi}(l) \leqslant 4 \epsilon$. Therefore one has $\lambda_{\varphi}(l)=0$ because $\epsilon$ is an arbitrary positive number.

2.3.4.2. Extension property over an Archimedean field. - We assume that $k$ is either $\mathbb{R}$ or $\mathbb{C}$ and the absolute value of $k$ is the standard absolute value.

Theorem 2.3.31. - Let $X$ be a projective scheme over $k, L$ be an ample invertible $\mathcal{O}_{X}$-module and $\varphi$ be a semipositive continuous metric metric of L. For any closed subscheme $Y$ of $X$ and any $\ell \in H^{0}\left(Y,\left.L\right|_{Y}\right)$, $\ell$ has the extension property for $\varphi$.

Proof. - Clearly we may assume that $\ell \notin \mathcal{N}\left(Y,\left.L\right|_{Y}\right)$. Let us see the following claim:

Claim 2.3.32. - (1) We assume that $k=\mathbb{C}, X=\mathbb{P}^{n}, L=\mathcal{O}(1)$ and $\varphi$ is the Fubini-Study metric arising from a norm $\|\cdot\|$ on $H^{0}\left(\mathbb{P}^{n}, \mathcal{O}(1)\right)$. Then the assertion of the theorem holds.

(2) We assume that $k=\mathbb{R}, X=\mathbb{P}^{n}, L=\mathcal{O}(1)$ and $\varphi$ is the Fubini-Study metric arising from a norm $\|\cdot\|$ on $H^{0}\left(\mathbb{P}^{n}, \mathcal{O}(1)\right)$. Then the assertion of the theorem holds.

Proof. - (1) By Theorem 2.3.7 the first Chern current $c_{1}(L, \varphi)$ is positive, so that (1) is a consequence of Corollary 2.3.29.

(2) We consider $X_{\mathbb{C}}, L_{\mathbb{C}}$ and $\varphi_{\mathbb{C}}$. Then $\varphi_{\mathbb{C}}$ is the Fubini-Study metric induced by the norm $\|\cdot\|_{\mathbb{C}}$ on $H^{0}(X, L) \otimes_{\mathbb{R}} \mathbb{C}$ by Proposition 1.3.24. Thus, by using the 
case (1), for any $\epsilon>0$, there is a positive integer $n_{0}$ such that, for any $n \geqslant n_{0}$, we can find $s \in H^{0}\left(X_{\mathbb{C}}, L_{\mathbb{C}}^{\otimes n}\right)$ with $\left.s\right|_{Y_{\mathbb{C}}}=\ell^{\otimes n}$ and $\|s\|_{n \varphi_{\mathbb{C}}} \leqslant \mathrm{e}^{n \epsilon}\|\ell\|_{\left.\varphi_{\mathbb{C}}\right|_{Y}}^{n}$. First of all, note that $\|\ell\|_{\left.\varphi_{\mathbb{C}}\right|_{Y}}=\|\ell\|_{\left.\varphi\right|_{Y}}$. If we set $s=\sigma+i \tau\left(\sigma, \tau \in H^{0}\left(X, L^{\otimes n}\right)\right)$, then $\left(\left.\sigma\right|_{Y}\right)+i\left(\left.\tau\right|_{Y}\right)=\ell^{\otimes n}$, so that $\left.\tau\right|_{Y}=0$, and hence $\left.\sigma\right|_{Y}=\ell^{\otimes n}$. Moreover, for any $x \in X_{\mathbb{C}}^{\text {an }}$ one has $\|\sigma+i \tau\|_{n \varphi_{\mathbb{C}}}(x)=\|\sigma-i \tau\|_{n \varphi_{\mathbb{C}}}(\bar{x})$, so that $\|\sigma+i \tau\|_{n \varphi_{\mathbb{C}}}=\|\sigma-i \tau\|_{n \varphi_{\mathbb{C}}}$. We then deduce that

$$
2\|\sigma\|_{n \varphi}=2\left\|\sigma_{n \varphi_{\mathbb{C}}}\right\| \leqslant\|\sigma+i \tau\|_{n \varphi_{\mathbb{C}}}+\|\sigma-i \tau\|_{n \varphi_{\mathbb{C}}}=2\|s\|_{n \varphi_{\mathbb{C}}} .
$$

Thus one has the assertion in this case.

We choose $n_{1}$ such that, for all $n \geqslant n_{1}, L^{\otimes n}$ is very ample. Then $\varphi_{n}$ is the restriction of the Fubini-Study metric $\varphi_{\|\cdot\|_{n \varphi}}$ of $\mathcal{O}(1)$ to $\mathbb{P}\left(H^{0}\left(X, L^{\otimes n}\right)\right)$ induced by the norm $\|\cdot\|_{n \varphi}$. Thus, by the above claim together with (2.27),

$$
0 \leqslant \lambda_{\varphi_{n}}\left(\ell^{\otimes n}\right) \leqslant \lambda_{\varphi_{\|\cdot\|_{n} \varphi}}\left(\ell^{\otimes n}\right)=0
$$

and hence $\lambda_{n \varphi}\left(\ell^{\otimes n}\right) \leqslant 2 d\left(n \varphi, \varphi_{n}\right)$ by (2.25). Since $\lambda_{n \varphi}\left(\ell^{\otimes n}\right)=n \lambda_{\varphi}(\ell)$ by (2.26) , one has

$$
0 \leqslant \lambda_{\varphi}(\ell) \leqslant 2 d\left(\varphi, \frac{1}{n} \varphi_{n}\right)
$$

Therefore, the assertion follows.

2.3.4.3. Extension property over a non-Archimedean field. - In this subsection, we fix a field $k$ equipped with a non-Archimedean absolute value $|\cdot|$, under which the field $k$ is complete.

Proposition 2.3.33. - We assume that $|\cdot|$ is non-trivial. Let $X$ be a projective $k$-scheme, $L$ be an invertible $\mathcal{O}_{X}$-module and $(\mathscr{X}, \mathscr{L})$ be a model of $(X, L)$. Let $s$ be a global section of $L$ such that $\|s\|_{\varphi_{\mathscr{L}}} \leqslant 1$. Then there exists an element $a \in \mathfrak{o}_{k} \backslash\{0\}$ such that as ${ }^{n}$ belongs to $H^{0}\left(\mathscr{X}, \mathscr{L}^{\otimes n}\right)$ for all integers $n \geqslant 1$.

Proof. - Let $\left(\mathscr{U}_{i}\right)_{i=1}^{N}$ be a covering of $\mathscr{X}$ by affine open subsets, such that $\mathscr{U}_{i}=$ $\operatorname{Spec}\left(\mathscr{A}_{i}\right)$ and the invertible sheaf $\mathscr{L}$ trivialises on each $\mathscr{U}_{i}$, that is, $\left.\mathscr{L}\right|_{\mathscr{U}_{i}}=\mathscr{A}_{i} s_{i}$ for some $s_{i} \in \mathscr{L}\left(\mathscr{U}_{i}\right)$. Then the restriction of $s$ to $U_{i}:=\mathscr{U}_{i} \cap X$ can be written in the form $\lambda_{i} s_{i}$, where $\lambda_{i} \in A_{i}=S^{-1} \mathscr{A}_{i}, S=\mathfrak{o}_{k} \backslash\{0\}$. Note that for $x \in\left(U_{i}\right)_{\mathscr{A}_{i}}^{\text {an }}$, the reduction point of $x$ is in $\mathscr{U}_{i}$ (cf. Remark 2.1.6), so that since $\|s\|_{\varphi_{\mathscr{L}}} \leqslant 1$, we obtain that

$$
\left|\lambda_{i}\right|_{x}=\left|\lambda_{i}\right|_{x} \cdot\left|s_{i}\right|_{\varphi_{\mathscr{L}}}(x)=|s|_{\varphi_{\mathscr{L}}}(x) \leqslant\|s\|_{\varphi_{\mathscr{L}}} \leqslant 1
$$

for any $x \in\left(U_{i}\right)_{\mathscr{A}_{i}}^{\text {an }}$. By Proposition 2.1.7, $\lambda_{i}$ is integral over the ring $\mathscr{A}_{i}$, namely $\mathscr{A}_{i}\left[\lambda_{i}\right]$ is an $\mathscr{A}_{i}$-module of finite type. In particular, there exists an integer $d_{i} \geqslant 1$ such that, for any integer $n \geqslant 1$,

$$
\lambda_{i}^{n} \in \mathscr{A}_{i}+\mathscr{A}_{i} \lambda_{i}+\cdots+\mathscr{A}_{i} \lambda_{i}^{d_{i}} .
$$

Moreover, there exists $a_{i} \in \mathfrak{o}_{k} \backslash\{0\}$ such that

$$
\left\{a_{i} \lambda_{i}, \ldots, a_{i} \lambda_{i}^{d_{i}}\right\} \subset \mathscr{A}_{i} .
$$


We then obtain that $a_{i} \lambda_{i}^{n} \in \mathscr{A}_{i}$ for any integer $n \geqslant 1$. Finally, let $a=\prod_{i=1}^{N} a_{i} \in$ $\mathfrak{o}_{k} \backslash\{0\}$. For any integer $n \geqslant 1$ and any $i \in\{1, \ldots, N\}$, one has

$$
\left.\left(a s^{n}\right)\right|_{U_{i}}=\left(a \lambda_{i}^{n}\right) s_{i}^{n} \in H^{0}\left(\mathscr{U}_{i}, \mathscr{L}^{\otimes n}\right) .
$$

Since $\mathscr{X}$ is flat over $\operatorname{Spec}\left(\mathfrak{o}_{k}\right)$, these sections glue together to be a global section of $\mathscr{L}^{\otimes n}$. The proposition is thus proved.

Proposition 2.3.34. - We assume that $|\cdot|$ is non-trivial. Let $X$ be a projective $k$-scheme, $L$ be an ample invertible $\mathcal{O}_{X}$-module and $Y$ be a closed subscheme of $X$. Let $u \geqslant 1$ be an integer and $(\mathscr{X}, \mathscr{L})$ be a model of $\left(X, L^{\otimes u}\right)$ such that $\mathscr{L}$ is ample. Assume that $\varphi=\frac{1}{u} \varphi_{\mathscr{L}}$. Let $\varphi_{Y}$ be the restriction of the metric $\varphi$ to $\left.L\right|_{Y}$. For any positive number $\epsilon$ and any $\ell \in H^{0}\left(Y,\left.L\right|_{Y}\right)$, there exists an integer $n \geqslant 1$ and a section $s \in H^{0}\left(X, L^{\otimes n}\right)$ such that $\left.s\right|_{Y}=\ell^{n}$ and

$$
\|s\|_{n \varphi} \leqslant \mathrm{e}^{n \epsilon}\|\ell\|_{\varphi_{Y}}^{n} .
$$

In other words, one has $\lambda_{\varphi}(\ell)=0$ if $\ell \notin N\left(Y,\left.L\right|_{Y}\right)$.

Proof. - We choose a positive integer $m$ such that

$$
\mathrm{e}^{-m \epsilon / 2}<\sup \left\{|a|: a \in k^{\times},|a|<1\right\} .
$$

By Proposition 1.1.4 on $H^{0}\left(Y,\left.L\right|_{Y} ^{\otimes m}\right) / \mathcal{N}\left(Y,\left.L\right|_{Y} ^{\otimes m}\right)$, there is $\alpha \in k^{\times}$such that

$$
\mathrm{e}^{-m \epsilon / 2} \leqslant\left\|\alpha \ell^{m}\right\|_{m \varphi_{Y}} \leqslant 1 .
$$

Let $\mathscr{Y}$ be the Zariski closure of $Y$ in $\mathscr{X}$. By Proposition 2.3.33, there exists an element $\beta \in \mathfrak{o}_{k} \backslash\{0\}$ such that

$$
\beta\left(\alpha \ell^{m}\right)^{p u} \in H^{0}\left(\mathscr{Y}, \mathscr{L}^{\otimes m p} \mid \mathscr{Y}\right)
$$

for any integer $p \geqslant 1$. Moreover, since the invertible sheaf $\mathscr{L}$ is ample, for sufficiently positive integer $p$, the restriction map

$$
H^{0}\left(\mathscr{X}, \mathscr{L}^{\otimes m p}\right) \longrightarrow H^{0}\left(\mathscr{Y}, \mathscr{L}^{\otimes m p} \mid \mathscr{Y}\right)
$$

is surjective. Hence we can choose $p \in \mathbb{N}_{\geqslant 1}$ such that

$$
|\beta|^{-1} \leqslant \mathrm{e}^{m p u \epsilon / 2}
$$

and that there exists $t \in H^{0}\left(\mathscr{X}, \mathscr{L}^{\otimes m p}\right)$ verifying $\left.t\right|_{\mathscr{Y}}=\beta\left(\alpha \ell^{m}\right)^{p u}$. We then take $n=m p u$ and $s=\beta^{-1} \alpha^{-p u} t \in H^{0}\left(X, L^{\otimes n}\right)$. One has $\left.s\right|_{\mathscr{Y}}=\ell^{n}$ and

$$
\begin{aligned}
\|s\|_{n \varphi} & =|\beta|^{-1} \cdot|\alpha|^{-p u} \cdot\|t\|_{n \varphi} \leqslant|\beta|^{-1} \cdot|\alpha|^{-p u} \\
& \leqslant \mathrm{e}^{m p u \epsilon / 2} \cdot\left(\mathrm{e}^{m \epsilon / 2}\left\|\ell^{m}\right\|_{m \varphi_{Y}}\right)^{p u}=\mathrm{e}^{n \varepsilon} \cdot\left\|\ell^{m}\right\|_{m \varphi_{Y}}^{p u} \leqslant \mathrm{e}^{n \epsilon}\|\ell\|_{\varphi_{Y}}^{n},
\end{aligned}
$$

where the first inequality comes from Proposition 2.3.16(2), the second one from (2.39) and (2.38), and the last one from (2.3). The first part of the proposition is thus proved, so that the last assertion follows from Proposition 2.3.23. 
Theorem 2.3.35. - Let $X$ be a projective $k$-scheme and $L$ be an ample invertible $\mathcal{O}_{X}$-module, equipped with a semipositive continuous metric $\varphi$. Let $Y$ be a closed subscheme of $X$ and $\ell$ be an element in $H^{0}\left(Y,\left.L\right|_{Y}\right)$. Then $\ell$ has the extension property for $\varphi$.

Proof. - Clearly we may assume that $\ell \notin \mathcal{N}\left(Y,\left.L\right|_{Y}\right)$. First we assume that the absolute value $|\cdot|$ on $k$ is non-trivial. By Proposition 2.3.18, for sufficiently positive integer $n$, there exists a sequence $\left(\mathscr{X}_{n}, \mathscr{L}_{n}\right)$ of models of $\left(X, L^{\otimes n}\right)$ such that each $\mathscr{L}_{n}$ is ample, and that

$$
\lim _{n \rightarrow+\infty} \frac{1}{n} d\left(\varphi \mathscr{L}_{n}, n \varphi\right)=0
$$

For any $n \in \mathbb{N}_{\geqslant 1}$, let $\varphi^{(n)}=\frac{1}{n} \varphi_{\mathscr{L}_{n}}$. By Proposition 2.3.34 (see also Remark 2.3.24), one has $\lambda_{\varphi^{(n)}}(\ell)=0$. Therefore, by the relations (2.25) and (2.40), we obtain that $\lambda_{\varphi}(\ell)=0$.

In the following, we treat the trivial valuation case. The main idea is to introduce the field of formal Laurent series over $k$ in order to reduce the problem to the nontrivial valuation case. We assume that the absolute value $|\cdot|$ is trivial. We denote by $k^{\prime}$ the field $k((T))$ of formal Laurent series over $k$, namely $k^{\prime}$ is the fraction field of the ring $k \llbracket T \rrbracket$ of formal series over $k$. Note that $k \llbracket T \rrbracket$ is a discrete valuation ring.

Claim 2.3.36. — The field extension $k \subseteq k^{\prime}$ is separable.

Proof. - We may assume that the characteristic $p$ of $k$ is positive. First let us see the following claim:

SubClaim 2.3.37. - Let $E$ be a finite extension of $k$ and $\left\{\omega_{i}\right\}_{i=1}^{e}$ be a basis of $E$ over $k$. Then we have the following:

(i) Let $\left(g_{1}, \ldots, g_{e}\right) \in\left(k^{\prime}\right)^{e}$. If $\omega_{1} g_{1}+\cdots+\omega_{e} g_{e}=0$ in $E((T))$, then $g_{1}=\cdots=g_{e}=$ 0 .

(ii) Let $\left\{f_{i}\right\}_{i=1}^{s}$ be a family of elements in $k^{\prime}$ which is linearly independent over $k$ and $\left(c_{1}^{\prime}, \ldots, c_{s}^{\prime}\right)$ be an element of $E^{s}$. If $c_{1}^{\prime} f_{1}+\cdots+c_{s}^{\prime} f_{s}=0$ in $E((T))$, then $c_{1}^{\prime}=\cdots=c_{s}^{\prime}=0$.

Proof. - (i) is trivial if we consider the coefficients of $g_{1}, \ldots, g_{e}$.

(ii) We set $c_{i}^{\prime}=\sum_{j=1}^{e} c_{i j} \omega_{j}$ for some $c_{i j} \in k$. Then

$$
\sum_{i=1}^{s} c_{i}^{\prime} f_{i}=\sum_{j=1}^{e}\left(\sum_{i=1}^{s} c_{i j} f_{i}\right) \omega_{j}=0,
$$

so that, by (i), $\sum_{i=1}^{s} c_{i j} f_{i}=0$ for all $j$. Therefore $c_{i j}=0$ for all $i, j$, as desired. 
By [29, Théorème 2 in Chapter $\left.\mathrm{V}, \S 25, \mathrm{n}^{\circ} 4\right]$, it is sufficient to see that if $f_{1}, \ldots, f_{s} \in$ $k((T))$ are linearly independent over $k$, then $f_{1}^{p}, \ldots, f_{s}^{p}$ are linearly independent over $k$. We assume that $c_{1} f_{1}^{p}+\cdots+c_{s} f_{s}^{p}=0$ for some $c_{1}, \ldots, c_{s} \in k$. Let $E$ be a finite extension field of $k$ such that we can find $c_{i}^{\prime} \in E$ with $c_{i}=\left(c_{i}^{\prime}\right)^{p}$ for all $i$. Then $\sum_{i=1}^{s} c_{i}^{\prime} f_{i}=0$ because

$$
0=\sum_{i=1}^{s} c_{i} f_{i}^{p}=\left(\sum_{i=1}^{s} c_{i}^{\prime} f_{i}\right)^{p}
$$

Thus, by (ii), one has $c_{i}^{\prime}=0$, as requested.

Let us consider a subset $\Sigma$ of $\mathbb{R}$ given by

$$
\Sigma=\bigcup_{n=0}^{\infty} \bigcup_{\left(v, v^{\prime}\right) \in\left(H^{0}\left(X, L^{\otimes n}\right) \backslash \mathcal{N}\left(X, L^{\otimes n}\right)\right)^{2}} \mathbb{Q}\left(\ln \|v\|_{n \varphi}-\ln \left\|v^{\prime}\right\|_{n \varphi}\right) .
$$

Since

$$
\left\{\|v\|_{n \varphi}: v \in H^{0}\left(X, L^{\otimes n}\right)\right\}
$$

is a finite set by Corollary 1.1.6, one has $\#(\Sigma) \leqslant \aleph_{0}$, so that one can choose $\alpha \in$ $\mathbb{R}_{>0} \backslash \Sigma$. We denote by $v_{T}(\cdot)$ the corresponding valuation on $k^{\prime}$, and by $|\cdot|^{\prime}$ the absolute value on $k^{\prime}$ defined as

$$
\forall a \in k^{\prime},|a|^{\prime}=\mathrm{e}^{-\alpha v_{T}(a)} .
$$

Note that this absolute value extends the trivial absolute value on $k$. We denote by $X_{k^{\prime}}$ and $Y_{k^{\prime}}$ the fibre products $X \times_{\text {Spec } k} \operatorname{Spec} k^{\prime}$ and $Y \times_{\text {Spec } k}$ Spec $k^{\prime}$, respectively, and by $p: X_{k^{\prime}} \rightarrow X$ and $p_{Y}: Y_{k^{\prime}} \rightarrow Y$ the morphism of projections.

As explained in $\$ 2.1 .3$, the morphism $p$ corresponds to a map $p^{\natural}: X_{k^{\prime}}^{\text {an }} \rightarrow X^{\text {an }}$. This map is actually surjective. In fact, if $K$ is a field extension of $k$, equipped with an absolute value extending the trivial absolute value on $k$, then we can equip the field $K(T)$ of rational functions of one variable with the absolute value such that

$$
\forall F=a_{0}+a_{1} T+\cdots+a_{n} T^{n} \in K[T], \quad|F|=\max _{i \in\{0, \ldots, n\}}\left|a_{i}\right| \cdot \mathrm{e}^{-\alpha i} .
$$

This absolue value extends the restriction of $|\cdot|^{\prime}$ to $k(T)$. Hence the completion $\widehat{K(T)}$ of $K(T)$ with this absoute value is a valued extension of $k^{\prime}$. If $f: \operatorname{Spec} K \rightarrow X$ is a $k$-morphism defining a point $x$ in $X^{\text {an }}$, then it gives rise to a $k^{\prime}$-morphism from $\operatorname{Spec} \widehat{K(T)}$ to $X_{k^{\prime}}$, which defines a point $y$ in $X^{\prime \text { an }}$ such that $p^{\natural}(y)=x$.

The surjectivity of $p^{\natural}$ implies that the restriction of the seminorm $\|\cdot\|_{n \varphi_{k^{\prime}}}$ to $H^{0}(X, L)$ coincides with $\|\cdot\|_{n \varphi}$. In fact, if $s$ is a section in $H^{0}\left(X, L^{\otimes n}\right)$, then one has $\left\|p^{*}(s)\right\|_{n \varphi_{k^{\prime}}}=\|s\|_{n \varphi} \circ p^{\natural}$. Therefore

$$
\left\|p^{*}(s)\right\|_{n \varphi_{k^{\prime}}}=\sup _{y \in X^{\prime a n}}\left\|p^{*}(s)\right\|_{n \varphi_{k^{\prime}}}(y)=\sup _{y \in X^{\prime a n}}\|s\|_{n \varphi}\left(p^{\natural}(y)\right)=\|s\|_{n \varphi},
$$


where the last equality comes from the surjectivity of the map $p^{\natural}$. For $\left(v, v^{\prime}\right) \in$ $\left(H^{0}\left(X, L^{\otimes n}\right) \backslash \mathcal{N}\left(X, L^{\otimes n}\right)\right)^{2}$, if $\|v\|_{n \varphi} /\left\|v^{\prime}\right\|_{n \varphi} \in\left|k^{\prime \times}\right|$, then

$$
\ln \|v\|_{n \varphi}-\ln \left\|v^{\prime}\right\|_{n \varphi}=-\alpha v_{T}(a(T))
$$

for some $a(T) \in k^{\prime \times}$. As

$$
\alpha \notin \bigcup_{\left(v, v^{\prime}\right) \in\left(H^{0}\left(X, L^{\otimes n}\right) \backslash \mathcal{N}\left(X, L^{\otimes n}\right)\right)^{2}} \mathbb{Q}\left(\ln \|v\|_{n \varphi}-\ln \left\|v^{\prime}\right\|_{n \varphi}\right),
$$

we obtain $v_{T}(a(T))=0$, so that $\|v\|_{n \varphi}=\left\|v^{\prime}\right\|_{n \varphi}$. By Proposition 1.3.22, the seminorm $\|\cdot\|_{n \varphi_{k^{\prime}}}$ identifies with $\|\cdot\|_{n \varphi, k^{\prime}, \varepsilon}$, the $\varepsilon$-extension of scalars of $\|\cdot\|_{n \varphi}$.

Let $X_{\text {red }}$ and $Y_{\text {red }}$ be the reduced schemes associated with $X$ and $Y$, respectively. By Claim 2.3.36, $X_{\text {red, } k^{\prime}}:=X_{\text {red }} \times_{\text {Spec } k}$ Spec $k^{\prime}$ and $Y_{\text {red }, k^{\prime}}:=Y_{\text {red }} \times_{\text {Spec } k}$ Spec $k^{\prime}$ are reduced (see [72, Proposition IV.(4.6.1)]), so that

$$
\mathcal{N}\left(X_{k^{\prime}}, L_{k^{\prime}}^{\otimes n}\right)=\mathcal{N}\left(X, L^{\otimes n}\right) \otimes_{k} k^{\prime} \quad \text { and } \quad \mathcal{N}\left(Y_{k^{\prime}},\left.L_{k^{\prime}}\right|_{k^{\prime}} ^{\otimes n}\right)=\mathcal{N}\left(Y,\left.L\right|_{Y}\right) \otimes_{k} k^{\prime},
$$

where $L_{k^{\prime}}=L \otimes_{k} k^{\prime}$.

By (2.25), without loss of generality, we may assume that $L$ is very ample and that $\varphi$ is the quotient metric on $L$ induced by a ultrametric norm $\|\cdot\|$ on $V=$ $H^{0}(X, L) / \mathcal{N}(X, L)$ and the natural surjection $\beta:\left.V \otimes \mathcal{O}_{X_{\text {red }}} \rightarrow L\right|_{X_{\text {red }}}$. We may also assume that the restriction map $H^{0}\left(X, L^{\otimes n}\right) \rightarrow H^{0}\left(Y,\left.L\right|_{Y} ^{\otimes n}\right)$ is surjective for all $n \geqslant 1$.

For $n \geqslant 1$, let

$$
V_{n}:=H^{0}\left(X, L^{\otimes n}\right) / \mathcal{N}\left(X, L^{\otimes n}\right) \quad \text { and } \quad V_{Y, n}=H^{0}\left(Y,\left.L\right|_{Y} ^{\otimes n}\right) / \mathcal{N}\left(Y,\left.L\right|_{Y} ^{\otimes n}\right) .
$$

Note that $V_{1}=V$, and $V_{n}$ and $V_{Y, n}$ are isomorphic to the images of

$$
H^{0}\left(X, L^{\otimes n}\right) \rightarrow H^{0}\left(X_{\text {red }},\left.L\right|_{X_{\text {red }}} ^{\otimes n}\right) \quad \text { and } \quad H^{0}\left(Y,\left.L\right|_{Y} ^{\otimes n}\right) \rightarrow H^{0}\left(Y_{\text {red }},\left.L\right|_{Y_{\text {red }}} ^{\otimes n}\right),
$$

respectively. Let $V_{k^{\prime}}$ be the vector space $V \otimes_{k} k^{\prime}$ and let $\|\cdot\|_{k^{\prime}, \varepsilon}$ be the norm on $V_{k^{\prime}}$ induced by $V$ by $\varepsilon$-extension of scalars. Then the surjective homomorphism $\beta:\left.V \otimes \mathcal{O}_{X_{\text {red }}} \rightarrow L\right|_{X_{\text {red }}}$ induces a surjective homomorphism $\beta_{k^{\prime}}: V_{k^{\prime}} \otimes \mathcal{O}_{X_{\text {red }, k^{\prime}}} \rightarrow$ $\left.L_{k^{\prime}}\right|_{X_{\mathrm{red}, k^{\prime}}}$. By Proposition 1.3.24, the metric $\varphi_{k^{\prime}}$ of $L_{k^{\prime}}$ obtained by $\varphi$ by extension of scalars coincides with the quotient metric on $L_{k^{\prime}}$ induced by $\left(V_{k^{\prime}},\|\cdot\|_{k^{\prime}, \varepsilon}\right)$ and $\beta_{k^{\prime}}$. Therefore, by Theorem 2.3.35 for any $\epsilon>0$, there exist an integer $n \geqslant 1$ and a section $s^{\prime} \in H^{0}\left(X_{k^{\prime}}, L_{k^{\prime}}^{\otimes n}\right)$ such that $\left.s^{\prime}\right|_{{k^{\prime}}^{\prime}}=p_{Y}^{*}(\ell)^{n}$ and

$$
\left\|s^{\prime}\right\|_{n \varphi_{k^{\prime}}} \leqslant \mathrm{e}^{n \epsilon}\left\|p_{Y}^{*}(\ell)\right\|_{\varphi_{Y, k^{\prime}}}^{n}
$$

where $\varphi_{Y, k^{\prime}}$ is the metric on $\left.\left(\left.L\right|_{Y}\right) \otimes_{k} k^{\prime} \cong L_{k^{\prime}}\right|_{k_{k^{\prime}}}$ induced by $\varphi_{Y}$ by extension of scalars, which equals the restriction of $\varphi_{k^{\prime}}$ to $Y_{k^{\prime}}$.

Let $\left\{e_{1}, \ldots, e_{\alpha_{n}}, f_{1}, \ldots, f_{\beta_{n}}\right\}$ be an orthogonal basis of $H^{0}\left(X, L^{\otimes n}\right)$ with respect to $\|\cdot\|_{n \varphi}$ such that $\left\{f_{1}, \ldots, f_{\beta_{n}}\right\}$ form a basis of the kernel of the restriction map 
$H^{0}\left(X, L^{\otimes n}\right) \rightarrow H^{0}\left(Y,\left.L\right|_{Y} ^{\otimes n}\right)$ (see Proposition 1.2.30 for the existence of such an orthogonal basis). We set

$$
s^{\prime}=\sum_{i=1}^{\alpha_{n}} a_{i}(T) e_{i}+\sum_{j=1}^{\beta_{n}} b_{j}(T) f_{j},
$$

where $\left(a_{1}(T), \ldots, a_{\alpha_{n}}(T), b_{1}(T), \ldots, b_{\beta_{n}}(T)\right) \in\left(k^{\prime}\right)^{\alpha_{n}+\beta_{n}}$. Note that

$$
\left.s^{\prime}\right|_{Y_{k^{\prime}}}=\left.a_{1}(T) e_{1}\right|_{Y}+\cdots+\left.a_{\alpha_{n}}(T) e_{\alpha_{n}}\right|_{Y}
$$

and $\left\{\left.e_{1}\right|_{Y}, \ldots,\left.e_{\alpha_{n}}\right|_{Y}\right\}$ forms a basis of $H^{0}\left(Y,\left.L\right|_{Y} ^{\otimes n}\right)$. Since the restriction of $s^{\prime}$ to $Y_{k^{\prime}}$ can be written as the pull-back of an element in $H^{0}\left(Y,\left.L\right|_{Y} ^{\otimes n}\right)$, one can see that $a_{1}(T), \ldots, a_{\alpha_{n}}(T) \in k$, so that $a_{1}(T), \ldots, a_{\alpha_{n}}(T)$ are denoted by $a_{1}, \ldots, a_{\alpha_{n}}$. Therefore if we set $s=a_{1} e_{1}+\cdots+a_{\alpha_{n}} e_{\alpha_{n}}$, then $s \in H^{0}\left(X, L^{\otimes n}\right),\left.s\right|_{Y}=\ell^{\otimes n}$, and

$$
\begin{aligned}
\|s\|_{n \varphi} & \leqslant \max _{i \in\left\{1, \ldots, \alpha_{n}\right\}}\left\{\left|a_{i}\right| \cdot\left\|e_{i}\right\|_{n \varphi}\right\} \\
& \leqslant \max \left\{\max _{i \in\left\{1, \ldots, \alpha_{n}\right\}}\left|a_{i}\right| \cdot\left\|e_{i}\right\|_{n \varphi}, \max _{j \in\left\{1, \ldots, \beta_{n}\right\}}\left|b_{j}(T)\right|^{\prime} \cdot\left\|f_{j}\right\|_{n \varphi}\right\} \\
& =\left\|s^{\prime}\right\|_{n \varphi_{k^{\prime}}} \leqslant \mathrm{e}^{\epsilon n}\left\|p_{Y}^{*}(\ell)\right\|_{\varphi_{Y, k^{\prime}}}^{n}=\mathrm{e}^{\epsilon n}\|\ell\|_{\varphi_{Y}}^{n},
\end{aligned}
$$

where the first equality comes from the fact that $\left\{e_{1}, \ldots, e_{\alpha_{n}}, f_{1}, \ldots, f_{\beta_{n}}\right\}$ forms an orthogonal basis of $H^{0}\left(X_{k^{\prime}}, L_{k^{\prime}}^{\otimes n}\right)$ with respect to $\|\cdot\|_{n \varphi_{k^{\prime}}}$ (see Proposition 1.3.13). The theorem is thus proved.

Remark 2.3.38. - Let $X$ be a scheme of finite type over $k$ and $\mathcal{F}$ be a coherent $\mathcal{O}_{X}$-module. Let $X_{\text {red }}$ be the reduced structure of $X$. The reduced $i$-th cohomology group of $\mathcal{F}$, denoted by $H_{\text {red }}^{i}(X, \mathcal{F})$, is defined to be the image of the homomorphism

$$
H^{i}(X, \mathcal{F}) \rightarrow H^{i}\left(X_{\text {red }},\left.F\right|_{X_{\text {red }}}\right) .
$$

Using the reduced cohomology group, one has the following variant of Theorem 2.3.35

We assume that $X$ is projective. Let $L$ be an ample invertible $\mathcal{O}_{X}$ module, equipped with a semipositive continuous metric $\varphi$. Let $Y$ be a closed subscheme of $X$ and $\ell$ be an element of $H_{\mathrm{red}}^{0}\left(Y,\left.L\right|_{Y}\right)$. Then, for any $\epsilon>0$, there exist a positive integer $n$ and $s \in H_{\mathrm{red}}^{0}\left(X, L^{\otimes n}\right)$ such that $\left.s\right|_{Y}=l^{\otimes n}$ and $\|s\|_{n \varphi}^{\prime} \leqslant \mathrm{e}^{n \epsilon}\left(\|l\|_{\varphi_{Y}}^{\prime}\right)^{n}$, where $\left.s\right|_{Y}$ is the image of $s$ by the homomorphism $H_{\mathrm{red}}^{0}\left(X, L^{\otimes n}\right) \rightarrow H_{\mathrm{red}}^{0}\left(Y,\left.L\right|_{Y} ^{\otimes n}\right)$.

Since $H_{\text {red }}^{0}\left(X, L^{\otimes n}\right)$ and $H_{\text {red }}^{0}\left(Y,\left.L\right|_{Y} ^{\otimes n}\right)$ are subgroups of $H^{0}\left(X_{\text {red }},\left.L\right|_{X_{\text {red }}} ^{\otimes n}\right)$ and $H^{0}\left(Y_{\text {red }},\left.L\right|_{Y_{\text {red }}} ^{\otimes n}\right)$, the proof of the above result can be done in the similar way as Theorem 2.3.35 


\subsection{Cartier divisors}

In this section, we recall Cartier divisors and linear systems. Further we introduce $\mathbb{Q}$-Cartier and $\mathbb{R}$-Cartier divisors on an integral scheme and study their basic properties.

2.4.1. Reminder on Cartier divisors. - Let $X$ be a scheme. For any open subset $U$ of $X$, we denote by $S_{X}(U)$ the set of all elements $a \in \mathcal{O}_{X}(U)$ such that the homomorphisme of $\mathcal{O}_{U}$-modules $\mathcal{O}_{U} \rightarrow \mathcal{O}_{U}$ defined as the homothety by $a$ is injective. This is a multiplicative subset of $\mathcal{O}_{X}(U)$. Moreover, $S_{X}$ is a subsheaf of sets of $\mathcal{O}_{X}$. We denote by $\mathscr{M}_{X}$ the sheaf of rings associated with the presheaf

$$
U \longmapsto \mathcal{O}_{X}(U)\left[S_{X}(U)^{-1}\right]
$$

called the sheaf of meromorphic functions ${ }^{(3)}$ on $X$. The canonical homomorphisms $\mathcal{O}_{X}(U) \rightarrow \mathcal{O}_{X}(U)\left[S_{X}(U)^{-1}\right]$ of rings induce a homomorphism of sheaves of rings $\mathcal{O}_{X} \rightarrow \mathscr{M}_{X}$. The sections of $\mathscr{M}_{X}$ on an open subset $U$ of $X$ are called meromorphic fonctions on $U$.

Remark 2.4.1. - Let $U$ be an open subset of $X$. Any element $a$ in $S_{X}(U)$ is a regular element (namely the homothety $\mathcal{O}_{X}(U) \rightarrow \mathcal{O}_{X}(U)$ defined by $a$ is injective). of $\mathcal{O}_{X}(U)$. It is not true in general that $S_{X}(U)$ contains all regular elements of $\mathcal{O}_{X}(U)$. However, if $U$ is an affine open subset of $X, S_{X}(U)$ identifies with the set of all regular elements in $\mathcal{O}_{X}(U)$. In fact, an element $a \in \mathcal{O}_{X}(U)$ is in $S_{X}(U)$ if and only if its image in the local ring $\mathcal{O}_{X, x}$ is a regular element for any $x \in U$. The announced property thus results from the faithful flatness of

$$
\mathcal{O}_{X}(U) \longrightarrow \bigoplus_{x \in U} \mathcal{O}_{X, x}
$$

provided that $U$ is an affine open subset. In particular, if $X$ is an integral scheme, then $\mathscr{M}_{X}$ is the constant sheaf associated to the local ring of the generic point of $X$ (which is a field). We use the notation $R(X)$ to denote the field of all meromorphic functions on $X$.

Denote by $\mathcal{O}_{X}^{\times}$the sheaf of abelian groupes described as follows. For any open subset $U$ of $X, \mathcal{O}_{X}^{\times}(U)$ is the set of elements $a \in \mathcal{O}_{X}(U)$ such that the homothety $\mathcal{O}_{U} \rightarrow \mathcal{O}_{U}$ defined by $a$ is an isomorphism. Similarly, denote by $\mathscr{M}_{X}^{\times}$the sheaf of abelian groupes on $X$ whose section space over any open subset $U \subseteq X$ is the set of all meromorphic functions $\varphi$ on $U$ such that the homothety $\mathscr{M}_{U} \rightarrow \mathscr{M}_{U}$ defined by $\varphi$ is an isomorphism. This is a subsheaf of multiplicative monoids of $\mathscr{M}_{X}$.

Definition 2.4.2. - We call Cartier divisor on $X$ any global section of the quotient sheaf $\mathscr{M}_{X}^{\times} / \mathcal{O}_{X}^{\times}$, that is, a data of a Zariski open covering $X=\bigcup_{\alpha} U_{\alpha}$ of $X$ and a

3. The definition in [74, IV.20] is not adequate, see [93 for details. 
section $s_{\alpha} \in \mathscr{M}_{X}^{\times}\left(U_{\alpha}\right)$ over $U_{\alpha}$ for each $\alpha$, which is called a local equation over $U_{\alpha}$, such that $s_{\alpha} s_{\beta}^{-1} \in \mathcal{O}_{X}^{\times}\left(U_{\alpha} \cap U_{\beta}\right)$ for all $\alpha$ and $\beta$. The group of all Cartier divisors on $X$ is denoted by $\operatorname{Div}(X)$, where the group law is written additively. We say that a Cartier divisor $D$ is effective if it is a section of $\left(\mathscr{M}_{X}^{\times} \cap \mathcal{O}_{X}\right) / \mathcal{O}_{X}^{\times}$. We use the expression $D \geqslant 0$ to denote the condition " $D$ is effective". Moreover, for $D_{1}, D_{2} \in \operatorname{Div}(X)$, an expression $D_{1} \geqslant D_{2}$ is defined by $D_{1}-D_{2} \geqslant 0$.

The Cartier divisors are closely related to invertible sheaves. Let $D$ be a Cartier divisor on $X$. Denote by $\mathcal{O}_{X}(D)$ the sub- $\mathcal{O}_{X}$-module of $\mathscr{M}_{X}$ generated by $-D$. Namely, if $X=\bigcup_{\alpha} U_{\alpha}$ is an open covering of $X$ and $s_{\alpha}$ is a local equation of $D$ over $U_{\alpha}$, then $\left.\mathcal{O}_{X}(D)\right|_{U_{\alpha}}=\mathcal{O}_{U_{\alpha}} s_{\alpha}^{-1}$. Note that $\mathcal{O}_{X}(D)$ is an invertible $\mathcal{O}_{X}$-module since it is locally generated by a regular element. We say that the Cartier divisor $D$ is ample (resp. very ample) if the invertible sheaf $\mathcal{O}_{X}(D)$ is ample (resp. very ample). By definition, one has $\mathcal{O}_{X}(-D) \cong \mathcal{O}_{X}(D)^{\vee}$. Moreover, if $D_{1}$ and $D_{2}$ are two Cartier divisors, then $\mathcal{O}_{X}\left(D_{1}+D_{2}\right) \cong \mathcal{O}_{X}\left(D_{1}\right) \otimes \mathcal{O}_{X}\left(D_{2}\right)$. Thus the map sending a divisor $D$ to the isomorphism class of $\mathcal{O}_{X}(D)$ defines a homomorphism from $\operatorname{Div}(X)$ to the Picard group $\operatorname{Pic}(X)$ (namely the group of isomorphism classes of invertible $\mathcal{O}_{X^{-}}$ modules). This homomorphism is surjective notably when $X$ is a reduced scheme with locally finite irreducible components, or a quasi-projective scheme over a Noetherian ring (cf. [74, IV.21.3.4-5]). We recall a simple proof of this result for the particular case where $X$ is an integral scheme.

Proposition 2.4.3. — Let $X$ be an integral scheme. Then the homomorphism $\operatorname{Div}(X) \rightarrow \operatorname{Pic}(X)$ constructed above is surjective.

Proof. - Let $\eta$ be the generic point of $X$ and $R(X)$ be the field of all meromorphic functions on $X$. Let $L$ be an invertible sheaf and $s$ be a non-zero element in $L_{\eta}$. Then the maps $H^{0}(U, L) \rightarrow R(X), t \mapsto t_{\eta} / s$ (where $U$ denotes an open subset of $X$ ) define a $\mathcal{O}_{X}$-linear homomorphisme from $L$ to $\mathscr{M}_{X}$. The images of local trivialisations of $L$ by this homomorphism define a global section of $\mathscr{M}_{X}^{\times} / \mathcal{O}_{X}^{\times}$, whose opposite $D$ is a Cartier divisor such that $\mathcal{O}_{X}(D) \cong L$.

Remark 2.4.4. - Let $X$ be an integral scheme and $L$ be an invertible $\mathcal{O}_{X}$-module. Let $\eta$ be the generic point of $X$. We call rational section of $L$ any element in $L_{\eta}$. Note that for any non-empty open subset $U$ of $X$, the restriction map $H^{0}(U, L) \rightarrow L_{\eta}$ is injective. By abuse of language, we also call a section of $L$ on a non-empty open subset of $X$ a rational section of $L$. The proof of the above proposition shows that any non-zero rational section $s$ of $L$ defines a Cartier divisor of $X$, which we denote by $\operatorname{div}(s)$. One can verify that, if $L$ and $L^{\prime}$ are two invertible $\mathcal{O}_{X}$-modules and if $s$ and $s^{\prime}$ are respectively non-zero rational sections of $L$ and $L^{\prime}$, then one has $\operatorname{div}\left(s s^{\prime}\right)=\operatorname{div}(s)+\operatorname{div}\left(s^{\prime}\right)$. 
The exact sequence of abelian sheaves

$$
1 \longrightarrow \mathcal{O}_{X}^{\times} \longrightarrow \mathscr{M}_{X}^{\times} \longrightarrow \mathscr{M}_{X}^{\times} / \mathcal{O}_{X}^{\times} \longrightarrow 0
$$

induces a long exact sequence of cohomology groups

$$
1 \longrightarrow H^{0}\left(X, \mathcal{O}_{X}^{\times}\right) \longrightarrow H^{0}\left(X, \mathscr{M}_{X}^{\times}\right) \stackrel{\operatorname{div}}{\longrightarrow} \operatorname{Div}(X) \stackrel{\theta}{\longrightarrow} H^{1}\left(X, \mathcal{O}_{X}^{\times}\right) .
$$

Note that the cohomology group $H^{1}\left(X, \mathcal{O}_{X}^{\times}\right)$identifies with the Picard $\operatorname{group} \operatorname{Pic}(X)$ of $X$ (cf. [70, 0.5.6.3]), and $\theta$ is just the group homomorphism sending any Cartier divisor $D$ to the isomorphism class of the invertible $\mathcal{O}_{X}$-module $\mathcal{O}_{X}(D)$. The image of the group homomorphism $\operatorname{div}(\cdot)$ is denoted by $\operatorname{PDiv}(X)$. The divisors in $\operatorname{PDiv}(X)$ are called principal divisors. The quotient group $\operatorname{Div}(X) / \operatorname{PDiv}(X)$ is called the divisor class group of $X$, denoted by $\mathrm{Cl}(X)$. The exactness of the sequence (2.41) shows that the homomorphism from $\mathrm{Cl}(X)$ to $\mathrm{Pic}(X)$, sending the equivalent class of a Cartier divisor $D$ to the isomorphism class of the invertible sheaf $\mathcal{O}_{X}(D)$ is injective. It is an isomorphism once $X$ is a reduced scheme with locally finite irreducible components, or a quasi-projective scheme over a Noetherian ring. We write this result as a corollary of Proposition 2.4.3 in the particular case where $X$ is an integral scheme.

Corollary 2.4.5. - Let $X$ be an integral scheme. The homomorphism from $\mathrm{Cl}(X)$ to $\operatorname{Pic}(X)$ sending the equivalence class of a divisor class $D$ to the isomorphism class of $\mathcal{O}_{X}(D)$ is an isomorphism.

If two Cartier divisors $D$ and $D^{\prime}$ of $X$ differ by a principal divisor, namely lie in the same class in $\mathrm{Cl}(X)$, we say that they are linearly equivalent.

Proposition 2.4.6. - We assume that $X$ is locally Noetherian and normal. Let D be a Cartier divisor on $X$ and $D=\sum_{\Gamma \in X^{(1)}} a_{\Gamma} \Gamma$ be the expansion as a Weil divisor, where $X^{(1)}$ is the set of all codimension one points of $X$. Then $D \geqslant 0$ if and only if $a_{\Gamma} \geqslant 0$ for all $\Gamma \in X^{(1)}$.

Proof. - It is suuffuent to show that if $a_{\Gamma} \geqslant 0$ for all $\Gamma \in X^{(1)}$, then $D \geqslant 0$. Let $f_{x}$ be a local equation of $D$ at $x \in X$. By our assumption, $f_{x} \in \mathcal{O}_{X, \Gamma}$ for all $\Gamma \in X^{(1)}$ and $x \in \overline{\{\Gamma\}}$, so that, by virtue of [100, THEOREM 38],

$$
f_{x} \in \bigcap_{x \in \overline{\{\Gamma\}}, \Gamma \in X^{(1)}} \mathcal{O}_{X, \Gamma}=\mathcal{O}_{X, x},
$$

and hence the assertion follows.

2.4.2. Linear system of a divisor. - In this subsection, we fix an integral scheme $X$, and denote by $R(X)$ the field of all rational functions on $X$.

Definition 2.4.7. - Let $D$ be a Cartier divisor of $X$. We define

$$
H^{0}(D):=\left\{f \in R(X)^{\times}: \operatorname{div}(f)+D \geqslant 0\right\} \cup\{0\},
$$


called the complete linear system of the divisor $D$. It forms a subgroup of $R(X)$ with respect to the additive composition law and is invariant by the multiplication by a scalar in $K$. Hence it is a $K$-vector subspace of $R(X)$.

We obtain from the definition that, if $D$ and $D^{\prime}$ are two Cartier divisors which are linearly equivalent, and $g$ is a non-zero rational function such that $D^{\prime}=D+\operatorname{div}(g)$. Then the map $f \mapsto f g$ defines a bijection from $H^{0}(D)$ to $H^{0}\left(D^{\prime}\right)$.

Let $D$ be a Cartier divisor of $X$. Being a sub- $\mathcal{O}_{X}$-module of $\mathscr{M}_{X}$, the invertible sheaf $\mathcal{O}_{X}(D)$ shares the same generic fibre with $\mathscr{M}_{X}$, which is also canonically isomorphic to the field $R(X)$. Therefore the unit element in $R(X)$ defines a rational section of $D$ which we denote by $s_{D}$. One can verify that $\operatorname{div}\left(s_{D}\right)=D$ and $s_{D+D^{\prime}}=s_{D} s_{D^{\prime}}$ for any couple $\left(D, D^{\prime}\right)$ of Cartier divisors of $X$.

Lemma 2.4.8. - Let $D$ be a Cartier divisor of $X$ and $s_{D}$ be the meromorphic section of $\mathcal{O}_{X}(D)$ constructed above. Then $D$ is an effective divisor if and only if $s_{D}$ extends to a global section of $\mathcal{O}_{X}(D)$.

Proof. - Assume that $D$ is an effective divisor. Then the invertible $\mathcal{O}_{X}$-module $\mathcal{O}_{X}(-D)$ is actually an invertible ideal sheaf of $\mathcal{O}_{X}$ since it is generated by $D$. Let $s: \mathcal{O}_{X} \rightarrow \mathcal{O}_{X}(D)$ be the homomorphism of $\mathcal{O}_{X}$-modules which is dual to the inclusion map $\mathcal{O}_{X}(-D) \rightarrow \mathcal{O}_{X}$. It defines a global section of $\mathcal{O}_{X}(D)$ whose value at the generic point coincides with $s_{D}$.

Conversely, if $L$ is an invertible sheaf on $X$ and if $s$ is a non-zero global section of $L$, then $\operatorname{div}(s)$ is an effective Cartier divisor. In particular, if $s_{D}$ extends to a global section of $\mathcal{O}_{X}(D)$, then $D=\operatorname{div}\left(s_{D}\right)$ is an effective divisor.

Proposition 2.4.9. - Let $D$ be a Cartier divisor of $X$. A rational function $f$ lies in $H^{0}(D)$ if and only if $f s_{D}$ extends to a global section of $\mathcal{O}_{X}(D)$.

Proof. - By definition, for any non-zero meromorphic function $f \in K$, the relation $f s_{D}=s_{\operatorname{div}(f)+D}$ holds. The Lemma 2.4.8 shows that $f s_{D}$ extends to a global section of $\mathcal{O}_{X} \otimes \mathcal{O}_{X}(D) \cong \mathcal{O}_{X}(D)$ if and only if $\operatorname{div}(f)+D$ is an effective divisor. The proposition is thus proved.

Remark 2.4.10. - (1) Let $L$ be an invertible $\mathcal{O}_{X}$-module. The Proposition 2.4.9 shows that, if $s$ is a non-zero meromorphic section of $L$, then the relation $t \mapsto$ $t / s$ defines an isomorphism between the groups $H^{0}(X, L)$ and $H^{0}(\operatorname{div}(s))$. In particular, if $D$ is a Cartier divisor, then $H^{0}(D)$ is canonically isomorphic to $H^{0}\left(X, \mathcal{O}_{X}(D)\right)$.

(2) Assume that the scheme $X$ is defined over a ground field $k$, then the field of rational functions $R(X)$ is an extension of $k$. Moreover, for any Cartier divisor $D$ of $X, H^{0}(D)$ is a $k$-vector subspace of $R(X)$. 
2.4.3. $\mathbb{Q}$-Cartier and $\mathbb{R}$-Cartier divisors. - As in the previous subsection, $X$ denotes an integral scheme. Let $\mathbb{K}$ be either $\mathbb{Z}, \mathbb{Q}$ or $\mathbb{R}$. An element of $\operatorname{Div}_{\mathbb{K}}(X):=$ $\operatorname{Div}(X) \otimes_{\mathbb{Z}} \mathbb{K}$ is called a $\mathbb{K}$-Cartier divisor on $X$. Note that a $\mathbb{Z}$-Cartier divisor is a usual Cartier divisor. A $\mathbb{K}$-Cartier divisor can be regarded as an element of

$$
H^{0}\left(X,\left(\mathscr{M}_{X}^{\times} / \mathcal{O}_{X}^{\times}\right) \otimes_{\mathbb{Z}} \mathbb{K}\right)=H^{0}\left(X,\left(\mathscr{M}_{X}^{\times} \otimes_{\mathbb{Z}} \mathbb{K}\right) /\left(\mathcal{O}_{X}^{\times} \otimes_{\mathbb{Z}} \mathbb{K}\right)\right),
$$

so that, for any point $x \in X$, there are an open neighborhood $U$ of $x$ and $f \in$ $\left.\left(\mathscr{M}_{X}^{\times} \otimes_{\mathbb{Z}} \mathbb{K}\right)\right|_{U}$ such that $D$ is defined by $f$ over $U$. Note that if $\left.f^{\prime} \in\left(\mathscr{M}_{X}^{\times} \otimes_{\mathbb{Z}} \mathbb{K}\right)\right|_{U}$ also defines $D$ over $U$, then $f /\left.f^{\prime} \in\left(\mathcal{O}_{X}^{\times} \otimes_{\mathbb{Z}} \mathbb{K}\right)\right|_{U}$. The element $f$ is called a local equation of $D$. Moreover, the morphism of groups $K(X)^{\times} \rightarrow \operatorname{Div}(X)$ induces by extension of scalars a $\mathbb{K}$-linear map $K(X)^{\times} \otimes_{\mathbb{Z}} \mathbb{K} \rightarrow \operatorname{Div}_{\mathbb{K}}(X)$ which we denote by $\operatorname{div}_{\mathbb{K}}(\cdot)$.

Let $D$ be a $\mathbb{K}$-Cartier divisor on $X$. Let $f_{x}$ be a local equation of $D$ around $x$. Note that the condition $f_{x} \in \mathcal{O}_{X}^{\times} \otimes_{\mathbb{Z}} \mathbb{K}$ does not depend on the choice of the local equation of $D$ around $x$, so that we define $\operatorname{Supp}_{\mathbb{K}}(D)$ to be

$$
\operatorname{Supp}_{\mathbb{K}}(D)=\left\{x \in X: f_{x} \notin \mathcal{O}_{X}^{\times} \otimes_{\mathbb{Z}} \mathbb{K}\right\} .
$$

Proposition 2.4.11. - (1) $\operatorname{Supp}_{\mathbb{K}}(D)$ is a closed subset of $X$.

(2) If $D$ is a Cartier divisor, then $\operatorname{Supp}_{\mathbb{Q}}(D)=\bigcap_{n=1}^{\infty} \operatorname{Supp}_{\mathbb{Z}}(n D)$. In particular, $\operatorname{Supp}_{\mathbb{Q}}(D) \subseteq \operatorname{Supp}_{\mathbb{Z}}(D)$. Moreover, if $X$ is normal, then $\operatorname{Supp}_{\mathbb{Q}}(D)=$ $\operatorname{Supp}_{\mathbb{Z}}(D)$.

(3) If $D$ is a $\mathbb{Q}$-Cartier divisor, then $\operatorname{Supp}_{\mathbb{Q}}(D)=\operatorname{Supp}_{\mathbb{R}}(D)$.

Proof. - The proof can be found in [108, Section 1.2].

Definition 2.4.12. - Let $D$ be a $\mathbb{K}$-Cartier divisor on $X$. We say that $D$ is $\mathbb{K}$-effective, denoted by $D \geqslant_{\mathbb{K}} 0$, if, for every $x \in X$, a local equation of $D$ can be expressed by $f_{1}^{a_{1}} \cdots f_{r}^{a_{r}}$, where $f_{1}, \ldots, f_{r} \in \mathcal{O}_{X, x} \backslash\{0\}$ and $a_{1}, \ldots, a_{r} \in \mathbb{R}_{>0}$. Similarly as Definition 2.4.7 we define $H_{\mathbb{K}}^{0}(D)$ to be

$$
H_{\mathbb{K}}^{0}(X, D):=\left\{\varphi \in R(X)^{\times}: \operatorname{div}(\varphi)+D \geqslant_{\mathbb{K}} 0\right\} \cup\{0\} .
$$

Note that in the case where $\mathbb{K}=\mathbb{Z}, H_{\mathbb{Z}}^{0}(X, D)$ coincides with $H^{0}(D)$ in Definition 2.4.7.

Proposition 2.4.13. - Let $D$ be a $\mathbb{K}$-Cartier divisor on $X$. Then we have the following:

(1) We assume that $\mathbb{K}=\mathbb{Q}$. Then $D \geqslant \mathbb{Q} 0$ if and only if $D \geqslant \mathbb{R} 0$.

(2) We assume that $\mathbb{K}=\mathbb{Q}$. Then the natural map $H_{\mathbb{Q}}^{0}(X, D) \rightarrow H_{\mathbb{R}}^{0}(X, D)$ is bijective.

(3) We assume that $\mathbb{K}=\mathbb{Z}$ and $X$ is locally Noetherian and normal. Then $D \geqslant_{\mathbb{Z}} 0$ if and only if $D \geqslant \mathbb{Q} 0$.

(4) We assume that $\mathbb{K}=\mathbb{Z}$ and $X$ is locally Noetherian and normal. Then the natural map $H_{\mathbb{Z}}^{0}(X, D) \rightarrow H_{\mathbb{Q}}^{0}(X, D)$ is bijective. 
(5) If $a \in H^{0}\left(X, \mathcal{O}_{X}\right)$ and $\varphi \in H_{\mathbb{K}}^{0}(X, D)$, then $a \varphi \in H_{\mathbb{K}}^{0}(X, D)$.

(6) If $X$ is locally Noetherian and normal, then $H_{\mathbb{K}}^{0}(X, D)$ forms a $H^{0}\left(X, \mathcal{O}_{X}\right)$ submodule of $R(X)$.

Proof. - (1) Obviously $D \geqslant_{\mathbb{Q}} 0$ implies $D \geqslant_{\mathbb{R}} 0$. Conversely we assume that $D \geqslant_{\mathbb{R}} 0$. Let $f_{x}$ be a local equation of $D$ at $x \in X$. Then there are $f_{1}, \ldots, f_{r} \in \mathcal{O}_{X, x} \backslash\{0\}$ and $a_{1}, \ldots, a_{r} \in \mathbb{R}_{>0}$ such that $f_{x}=f_{1}^{a_{1}} \cdots f_{r}^{a_{r}}$. Note that $f_{x} \in R(X)^{\times} \otimes_{\mathbb{Z}} \mathbb{Q}$, so that, by Lemma 2.4.14 as below, there are $a_{1}^{\prime}, \ldots, a_{r}^{\prime} \in \mathbb{Q}_{>0}$ such that $f_{x}=f_{1}^{a_{1}^{\prime}} \cdots f_{r}^{a_{r}^{\prime}}$. Therefore, $D$ is $\mathbb{Q}$-effective.

$(2)$ Let $\varphi \in H_{\mathbb{R}}^{0}(X, D) \backslash\{0\}$. Then $D+\operatorname{div}(\varphi) \geqslant_{\mathbb{R}} 0$. Note that $D+\operatorname{div}(\varphi)$ is a $\mathbb{Q}$-Cartier divisor, so that, by $(1), D+\operatorname{div}(\varphi) \geqslant \mathbb{Q} 0$, which means $\varphi \in H_{\mathbb{Q}}^{0}(X, D) \backslash\{0\}$.

(3) We assume that $D \geqslant \geqslant_{\mathbb{Q}} 0$. Let $D=\sum_{\Gamma} a_{\Gamma} \Gamma$ be the expansion as a Weil divisor. Then $a_{\Gamma} \geqslant 0$ for all $\Gamma$. Thus $D \geqslant 0$ by Proposition 2.4.6.

(4) is a consequence of (3)

(5) is obvious.

(6) By (5) it is sufficient to show that if $\varphi, \psi \in H_{\mathbb{K}}^{0}(X, D)$, then $\varphi+\psi \in H_{\mathbb{K}}^{0}(X, D)$. If we set $D=\sum_{\Gamma} \alpha_{\Gamma} \Gamma\left(\alpha_{\Gamma} \in \mathbb{K}\right)$ and $\operatorname{div}(\varphi)=\sum_{\Gamma} \operatorname{ord}_{\Gamma}(\varphi) \Gamma$ as a Weil divisor for $\varphi \in R(X)^{\times}$, then

$$
\operatorname{div}(\varphi)+D \geqslant_{\mathbb{K}} 0 \quad \Longleftrightarrow \quad \forall \Gamma, \operatorname{ord}_{\Gamma}(\varphi)+\alpha_{\Gamma} \geqslant 0
$$

by [108, Lemma 1.2.4] together with (1), Moreover, for $\varphi, \psi \in R(X)$,

$$
\operatorname{ord}_{\Gamma}(\varphi+\psi) \geqslant \min \left\{\operatorname{ord}_{\Gamma}(\varphi), \operatorname{ord}_{\Gamma}(\psi)\right\} .
$$

Therefore (6) follows.

Lemma 2.4.14. - Let $V$ be a vector space over $\mathbb{Q}$. Then we have the following:

(1) $W_{\mathbb{R}} \cap V=W$ for any vector subspace $W$ of $V$.

(2) Let $x, x_{1}, \ldots, x_{r} \in V$ such that $x=a_{1} x_{1}+\cdots+a_{r} x_{r}$ for some $a_{1}, \ldots, a_{r} \in \mathbb{R}$. Then, for any $\epsilon>0$, there are $a_{1}^{\prime}, \ldots, a_{r}^{\prime} \in \mathbb{Q}$ such that $x=a_{1}^{\prime} x_{1}+\cdots+a_{r}^{\prime} x_{r}$ and $\left|a_{i}^{\prime}-a_{i}\right| \leqslant \epsilon$ for all $i$.

Proof. - (1) is obvious because $V / W \rightarrow(V / W)_{\mathbb{R}}$ is injective and $(V / W)_{\mathbb{R}}=V_{\mathbb{R}} / W_{\mathbb{R}}$.

(2) We consider the homomorphism $\psi: \mathbb{Q}^{r} \rightarrow V$ sending $\left(t_{1}, \ldots, t_{r}\right) \in \mathbb{Q}^{r}$ to $t_{1} x_{1}+\cdots+t_{r} x_{r}$. Denote by $W$ the image of $\psi$. By (1), the point $x$ belongs to $W$. We pick an element $b$ in $\psi^{-1}(\{x\})$. Let $\psi_{\mathbb{R}}: \mathbb{R}^{r} \rightarrow V_{\mathbb{R}}$ be the scalar extension of $\psi$, that is, $\psi_{\mathbb{R}}\left(\alpha_{1}, \ldots, \alpha_{r}\right)=\alpha_{1} x_{1}+\cdots+\alpha_{r} x_{r}$ for any $\left(\alpha_{1}, \ldots, \alpha_{r}\right) \in \mathbb{R}^{r}$, whose image is $W_{\mathbb{R}}$. As $\operatorname{Ker}\left(\psi_{\mathbb{R}}\right)=\operatorname{Ker}(\psi)_{\mathbb{R}}, \operatorname{Ker}(\psi)$ is dense in $\operatorname{Ker}\left(\psi_{\mathbb{R}}\right)$. Therefore, $\psi^{-1}(\{x\})=b+\operatorname{Ker}(\psi)$ is dense in $\psi_{\mathbb{R}}^{-1}(\{x\})=b+\operatorname{Ker}\left(\psi_{\mathbb{R}}\right)$, which implies the assertion of (2) 
Example 2.4.15. - The study of effective $\mathbb{Q}$-Cartier or $\mathbb{R}$-Cartier divisors on nonnormal schemes is more subtle than that in the normal case. This phenomenon can be shown by the following examples, which have been discussed in 45. Let $X:=\operatorname{Proj}\left(k\left[T_{0}, T_{1}, T_{2}\right] /\left(T_{0} T_{2}^{2}-T_{1}^{3}\right)\right.$ over a field $k, U_{i}:=\left\{T_{i} \neq 0\right\} \cap X(i=0,1,2)$ and $x:=T_{1} / T_{0}, y:=T_{2} / T_{0}$ on $U_{0}$. Then $U_{0}=X \backslash\{(0: 0: 1)\}$ and $U_{2}=X \backslash\{(1: 0: 0)\}$, so that $X=U_{0} \cup U_{2}$. Note that $y / x$ is not regular at $(1: 0: 0)$ and $y / x \in \mathcal{O}_{X, \zeta}^{\times}$for all $\zeta \in U_{0} \cap U_{2}$. Let $D$ be a Cartier divisor on $X$ given by

$$
D= \begin{cases}(y / x) & \text { on } U_{0}, \\ (1) & \text { on } U_{2} .\end{cases}
$$

(1) As $y / x$ is not regular at $(1: 0: 0), D$ is not effective as a Cartier divisor. On the other hand, since

$$
2 D= \begin{cases}(x) & \text { on } U_{0}, \\ (1) & \text { on } U_{2},\end{cases}
$$

$D$ is effective as a $\mathbb{Q}$-Cartier divisor. As a consequence, $1 \notin H^{0}(X, D)$ and $1 \in H_{\mathbb{Q}}^{0}(X, D)$, that is, $H^{0}(X, D) \rightarrow H_{\mathbb{Q}}^{0}(X, D)$ is not surjective.

(2) We assume that $\operatorname{char}(k)=0$. We set $\varphi:=x / y$. As $\varphi=T_{1} / T_{2}$ is regular on $U_{2}$, $\varphi \in H^{0}(X, D)$. Here let us see $1+\varphi \notin H_{\mathbb{Q}}^{0}(X, D)$. We assume the contrary, that is, $1+\varphi \in H_{\mathbb{Q}}^{0}(X, D)$. Then

$$
(1+\varphi)(y / x)=1+y / x
$$

is $\mathbb{Q}$-effective on $U_{0}$, so that there is a positive integer $N$ such that $(1+y / x)^{N}$ is regular on $U_{0}$. Here we claim that $(y / x)^{i}$ is regular over $U_{0}$ for an integer $i \geqslant 2$. Indeed, we set $i=2 j+\epsilon$, where $j \geqslant 1$ and $\epsilon \in\{0,1\}$. Then as

$$
(y / x)^{i}=(y / x)^{2 j+\epsilon}=\left(y^{2}\right)^{j} y^{\epsilon} x^{-2 j-\epsilon}=x^{j-\epsilon} y^{\epsilon},
$$

the assertion follows. Note that

$$
y / x=(1 / N)\left((1+y / x)^{N}-1-\sum_{i=2}^{N}\left(\begin{array}{c}
N \\
i
\end{array}\right)(y / x)^{i}\right),
$$

so that $y / x$ is regular on $U_{0}$. This is a contradiction because $y / x$ is not regular on $U_{0}$.

(3) Next we assume that $\operatorname{char}(k)=2$. We set $U_{0}^{\prime}:=U_{0} \backslash\{(1: 1: 1)\}$. Note that $X=U_{0}^{\prime} \cup U_{2}$ and $1+y / x \in \mathcal{O}_{X, \zeta}^{\times}$for all $\zeta \in U_{0}^{\prime} \cap U_{2}$, so that we set

$$
D^{\prime}:= \begin{cases}(1+y / x) & \text { on } U_{0}^{\prime}, \\ (1) & \text { on } U_{2} .\end{cases}
$$

Since $y / x$ is not regular at $(1: 0: 0)$, we have $D^{\prime} \neq 0$. Moreover, as $(1+y / x)^{2}=$ $1+x$, we have

$$
2 D^{\prime}= \begin{cases}(1+x) & \text { on } U_{0}^{\prime}, \\ (1) & \text { on } U_{2},\end{cases}
$$


and hence $2 D^{\prime}=0$ because $1+x \in \mathcal{O}_{X, \zeta}^{\times}$for all $\zeta \in U_{0}^{\prime}$. Therefore, the natural homomorphism $\operatorname{Div}(X) \rightarrow \operatorname{Div}_{\mathbb{K}}(X)$ is not injective. Furthermore $\operatorname{Supp}_{\mathbb{K}}\left(D^{\prime}\right)=$ $\varnothing$, but $\operatorname{Supp}_{\mathbb{Z}}\left(D^{\prime}\right)=\{(1: 0: 0)\}$.

Proposition 2.4.16. - We assume that $X$ is locally Noetherian and normal. Let $D$ be an $\mathbb{R}$-effective $\mathbb{R}$-Cartier divisor on $X$. Then there are effective Cartier divisors $D_{1}, \ldots, D_{n}$ and positive real numbers $a_{1}, \ldots, a_{n}$ such that $D=a_{1} D_{1}+\cdots+a_{n} D_{n}$.

Proof. - If $D=0$, then the assertion is obvious, so that we may assume that $D \neq 0$. We choose prime divisors $\Gamma_{1}, \ldots, \Gamma_{n}$ and $a_{1}, \ldots, a_{n} \in \mathbb{R}_{>0}$ such that $D=a_{1} \Gamma_{1}+\cdots+$ $a_{n} \Gamma_{n}$ as a Weil divisor. We set

$$
\left\{\begin{array}{l}
V=\left\{E=c_{1} \Gamma_{1}+\cdots+c_{n} \Gamma_{n}:\left(c_{1}, \ldots, c_{n}\right) \in \mathbb{Q}^{n} \text { and } E \text { is a } \mathbb{Q} \text {-Cartier divisor }\right\}, \\
V_{\mathbb{R}}:=V \otimes_{\mathbb{Q}} \mathbb{R}, \quad P=V_{\mathbb{R}} \cap\left(\mathbb{R}_{>0} \Gamma_{1}+\cdots+\mathbb{R}_{>0} \Gamma_{n}\right) .
\end{array}\right.
$$

Then $P$ is an open cone in $V_{\mathbb{R}}$ and $D \in P$. Thus the assertion follows.

We assume that $X$ is projective over a field $k$. An $\mathbb{K}$-Cartier divisor $D$ on $X$ is said to be ample if there are ample Cartier divisors $D_{1}, \ldots, D_{n}$ and $\left(a_{1}, \ldots, a_{n}\right) \in \mathbb{K}_{>0}^{n}$ such that $D=a_{1} D_{1}+\cdots+a_{n} D_{n}$.

Proposition 2.4.17. - Let $A$ be an ample $\mathbb{R}$-Cartier divisor on $X$ and $D_{1}, \ldots, D_{m}$ be Cartier divisors on $X$. Then there is a positive number $\delta$ such that $A+\sum_{j=1}^{m} \delta_{j} D_{j}$ is ample for all $\delta_{1}, \ldots, \delta_{m} \in \mathbb{R}$ with $\left|\delta_{1}\right|+\cdots+\left|\delta_{m}\right|<\delta$. In particular, the ampleness of $\mathbb{R}$-Cartier divisors is an open condition.

Proof. - We choose ample Cartier divisors $A_{1}, \ldots, A_{n}$ and $\left(a_{1}, \ldots, a_{n}\right) \in \mathbb{R}_{>0}^{n}$ such that $A=a_{1} A_{1}+\cdots+a_{n} A_{n}$. Let $l$ be a positive rational number such that $l A_{1} \pm D_{j}$ is ample for all $j=1, \ldots, m$. Note that

$$
A+\sum_{j=1}^{m} \delta_{j} D_{j}=\sum_{j=1}^{m}\left|\delta_{j}\right|\left(l A_{1}+\operatorname{sign}\left(\delta_{j}\right) D_{j}\right)+\left(a_{1}-l\left(\left|\delta_{1}\right|+\cdots+\left|\delta_{m}\right|\right)\right) A_{1}+\sum_{i=2}^{m} a_{i} A_{i}
$$

where

$$
\operatorname{sign}(a)= \begin{cases}1 & \text { if } a \geqslant 0 \\ -1 & \text { if } a<0\end{cases}
$$

Therefore, if we choose $\delta=a_{1} / l$, then $A+\sum_{j=1}^{m} \delta_{j} D_{j}$ is ample.

Proposition 2.4.18. - We assume that $X$ is locally Noetherian and normal. Let $D$ be an $\mathbb{R}$-effective $\mathbb{R}$-Cartier divisor on $X$. Let $s_{1}, \ldots, s_{n} \in \operatorname{Rat}(X)^{\times} \otimes_{\mathbb{Z}} \mathbb{Q}$ and $\left(a_{1}, \ldots, a_{n}\right) \in \mathbb{R}^{n}$ such that $a_{1}, \ldots, a_{n}$ are linearly independent over $\mathbb{Q}$ and $D+$ $\left(s_{1}^{a_{1}} \cdots s_{n}^{a_{n}}\right)$ is $\mathbb{R}$-effective. Then, for any $\epsilon>0$, there is a positive number $\delta$ such that if $\left|a_{1}^{\prime}-a_{1}\right|+\cdots+\left|a_{n}^{\prime}-a_{n}\right| \leqslant \delta$, then $(1+\epsilon) D+\left(s_{1}^{a_{1}^{\prime}} \cdots s_{n}^{a_{n}^{\prime}}\right)$ is $\mathbb{R}$-effective. 
Proof. - We set $\phi=s_{1}^{a_{1}} \cdots s_{n}^{a_{n}}$. Let us see that $\operatorname{Supp}\left(\left(s_{i}\right)\right) \subseteq \operatorname{Supp}((\phi))$ for all $i$. Otherwise there is a prime divisor $\Gamma$ such that $\operatorname{ord}_{\Gamma}\left(s_{i}\right) \neq 0$ and $\operatorname{ord}_{\Gamma}(\phi)=0$, so that $\sum_{j=1}^{n} a_{j} \operatorname{ord}_{\Gamma}\left(s_{j}\right)=0$, which contradicts to the linear independency of $a_{1}, \ldots, a_{n}$ over $\mathbb{Q}$. If $\operatorname{Supp}((\phi))=\emptyset$, then $\operatorname{Supp}\left(\left(s_{i}\right)\right)=\emptyset$ for all $i$, and hence the assertion is obvious, so that we may assume that $\operatorname{Supp}((\phi)) \neq \emptyset$. Let $\Gamma_{1}, \ldots, \Gamma_{m}$ be distinct prime divisors such that $\operatorname{Supp}((\phi))=\Gamma_{1} \cup \cdots \cup \Gamma_{m}$. Then we can set $\left(s_{i}\right)=\sum_{l=1}^{m} h_{i l} \Gamma_{l}$ for some $h_{i l} \in$ $\mathbb{Q}$. If we set $\gamma_{l}=\sum_{i=1}^{n} a_{i} h_{i l}$, then $((\phi))=\sum_{l=1}^{m} \gamma_{l} \Gamma_{l}$. As $\operatorname{Supp}((\phi))=\Gamma_{1} \cup \cdots \cup \Gamma_{m}$, one has $\gamma_{l} \neq 0$ for all $l$. We set

$$
L_{+}=\left\{l \in\{1, \ldots, m\} \mid \gamma_{l}>0\right\} \quad \text { and } \quad L_{-}=\left\{l \in\{1, \ldots, m\} \mid \gamma_{l}<0\right\} .
$$

As $\operatorname{ord}_{\Gamma_{l}}(D)+\gamma_{l} \geqslant 0$, one has $\operatorname{ord}_{\Gamma_{l}}(D)>0$ for all $l \in L_{-}$. We set $C=\max _{i, l}\left\{\left|h_{i l}\right|\right\}$ and choose $\delta>0$ such that

$$
C \delta<\min \left\{\left|\gamma_{1}\right|, \ldots,\left|\gamma_{m}\right|\right\} \quad \text { and } \quad C \delta \leqslant \epsilon \min _{l \in L_{-}}\left\{\operatorname{ord}_{\Gamma_{l}}(D)\right\} .
$$

Let $a_{1}^{\prime}, \ldots, a_{n}^{\prime} \in \mathbb{R}$ such that $\left|a_{1}^{\prime}-a_{1}\right|+\cdots+\left|a_{n}^{\prime}-a_{n}\right| \leqslant \delta$. If we set $\gamma_{l}^{\prime}=\sum_{i=1}^{n} a_{i}^{\prime} h_{i l}$, then $\left|\gamma_{l}^{\prime}-\gamma_{l}\right| \leqslant C \delta$, so that $\left\{l \mid \gamma_{l}^{\prime}>0\right\}=L_{+}$and $\left\{l \mid \gamma_{l}^{\prime}<0\right\}=L_{-}$. Further, for $l \in L_{-}$, we have

$(1+\epsilon) \operatorname{ord}_{\Gamma_{l}}(D)+\gamma_{l}^{\prime}=\left(\operatorname{ord}_{\Gamma_{l}}(D)+\gamma_{l}\right)+\left(\epsilon \operatorname{ord}_{\Gamma_{l}}(D)+\left(\gamma_{l}^{\prime}-\gamma_{l}\right)\right) \geqslant \epsilon \operatorname{ord}_{\Gamma_{l}}(D)-C \delta \geqslant 0$.

Therefore $(1+\epsilon) D+\left(s_{1}^{a_{1}^{\prime}} \cdots s_{n}^{a_{n}^{\prime}}\right)$ is $\mathbb{R}$-effective because

$$
\operatorname{ord}_{\Gamma_{l}}\left((1+\epsilon) D+\left(s_{1}^{a_{1}^{\prime}} \cdots s_{n}^{a_{n}^{\prime}}\right)\right)=(1+\epsilon) \operatorname{ord}_{\Gamma_{l}}(D)+\gamma_{l}^{\prime}
$$

for $l=1, \ldots, m$.

\subsection{Green functions}

Let $k$ be a field equipped with an absolute value $|\cdot|$, which is complete. If $|\cdot|$ is Archimedean, we assume that it is the usual absolute value on $\mathbb{R}$ or $\mathbb{C}$. Let $X$ be an integral projective scheme over $\operatorname{Spec} k$.

2.5.1. Green functions of Cartier divisors. - Let $X^{\text {an }}$ be the Berkovich topological space associated with $X$. We denote by $C_{\text {gen }}^{0}\left(X^{\text {an }}\right)$ the set of all continuous functions on a non-empty Zariski open subset of $X^{\text {an }}$, modulo the following equivalence relation

$$
f \sim g \stackrel{\text { def }}{\Longleftrightarrow} f \text { and } g \text { coincide on a non-empty Zariski open subset. }
$$

Note that the addition and the multiplication of functions induce a structure of $\mathbb{R}$ algebra on the set $C_{\text {gen }}^{0}\left(X^{\text {an }}\right)$. Moreover, for any non-empty Zariski open subset $U$ of $X$, we have a natural $\mathbb{R}$-algebra homomorphism from $C^{0}\left(U^{\text {an }}\right)$ to $C_{\mathrm{gen}}^{0}\left(X^{\text {an }}\right)$. Since $U^{\text {an }}$ is dense in $X^{\text {an }}$ (see [8] Corollary 3.4.5), we obtain that this homomorphism is injective. Moreover, the $\mathbb{R}$-algebra $C_{\text {gen }}^{0}\left(X^{\text {an }}\right)$ is actually the colimit of the system $C^{0}\left(U^{\text {an }}\right)$ in the category of $\mathbb{R}$-algebras, where $U$ runs over the set of all non-empty 
Zariski open subsets of $X$. We say that an element of $C_{\mathrm{gen}}^{0}\left(X^{\mathrm{an}}\right)$ extends to a continuous function on $U^{\text {an }}$ if it belongs to the image of the canonical homomorphism $C^{0}\left(U^{\text {an }}\right) \rightarrow C_{\text {gen }}^{0}\left(X^{\text {an }}\right)$.

Remark 2.5.1. - Let $f$ be an element of $C_{\mathrm{gen}}^{0}\left(X^{\mathrm{an}}\right)$. If $U$ is a non-empty Zariski open subset of $X$ such that $f$ extends to a continuous function on $U^{\text {an }}$, then, by the injectivity of the canonical homomorphism $C^{0}\left(U^{\mathrm{an}}\right) \rightarrow C_{\mathrm{gen}}^{0}\left(X^{\mathrm{an}}\right)$ there exists a unique continuous function on $U^{\text {an }}$ whose canonical image in $C_{\text {gen }}^{0}\left(X^{\text {an }}\right)$ is $f$. Therefore, by gluing of continuous functions we obtain the existence of a largest Zariski open subset $U_{f}$ of $X$ such that $f$ extends to a continuous function on $U_{f}^{a n}$. The set $U^{\text {an }}$ is called the domain of definition of the element $f$. By abuse of notation, we still use the expression $f$ to denote the continuous function on $U_{f}^{\text {an }}$ corresponding to the element $f \in C_{\text {gen }}^{0}\left(X^{\text {an }}\right)$.

If $f$ is a non-zero rational function on $X$, then it is an invertible regular function on a non-empty Zariski open subset $U$ of $X$. Therefore $\ln |f|$ is a continuous function on $U^{\text {an }}$, which determines an element of $C_{\text {gen }}^{0}\left(X^{\text {an }}\right)$. Note that this element does not depend on the choice of the non-empty Zariski open subset $U$. We still denote by $\ln |f|$ this element by abuse of notation.

Definition 2.5.2. — Let $D$ be a Cartier divisor on $X$. We call Green function of $D$ any element $g$ of $C_{\text {gen }}^{0}\left(X^{\text {an }}\right)$ such that, for any local equation $f$ of $D$ over a non-empty Zariski open subset $U$, the element $g+\ln |f|$ of $C_{\text {gen }}^{0}\left(X^{\text {an }}\right)$ extends to a continuous function on $U^{\text {an }}$.

Example 2.5.3. - Let $f$ be a non-zero rational function on $X$. Then $\operatorname{div}(f)$ is a Cartier divisor. By definition, $-\ln |f|$ is a Green function of $\operatorname{div}(f)$. More generally, let $L$ be an invertible $\mathcal{O}_{X}$-module, equipped with a continuous metric $\varphi$. Let $s$ a nonzero rational section of $L$. Then the function $-\ln |s|_{\varphi}$, which is well defined outside of the zero points and poles of the section $s$ and is continuous, determines an element of $C_{\text {gen }}^{0}\left(X^{\text {an }}\right)$. It is actually a Green function of the $\operatorname{divisor} \operatorname{div}(s)$. In particular, we deduce from Remark 2.2.18 that, for any Cartier divisor $D$ on $X$, there exists a Green function of $D$.

Remark 2.5.4. - One can also construct a metrized invertible sheaf from a Cartier divisor equipped with a Green function. Let $D$ be a Cartier divisor on $X$ and $g$ be a Green function of $D$. If $f$ is a rational function of $X$ which defines the divisor $D$ on a non-empty Zariski open subset, then the element $f^{-1} s_{D}$ is a section of the invertible sheaf $\mathcal{O}_{X}(D)$ which trivialises the latter on $U$. Note that the element $-(g+\ln |f|)$ of $C_{\text {gen }}^{0}\left(X^{\text {an }}\right)$ extends to a continuous function on $U^{\text {an }}$. We denote by $\left|f^{-1} s_{D}\right|_{g}$ the exponential of this function, which defines a continuous metric on the restriction of $L$ to $U$. By gluing we obtain a continuous metric on $L$ which we denote by $\varphi_{g}$. By definition one has $g=-\ln \left|s_{D}\right|_{g}$ in $C_{\text {gen }}^{0}\left(X^{\text {an }}\right)$. 
Proposition 2.5.5. - (1) An element $g$ in $C_{\text {gen }}^{0}\left(X^{\text {an }}\right)$ is a Green function of the trivial Cartier divisor if and only if it extends to a continuous function on $X^{\text {an }}$.

(2) Let $D$ and $D^{\prime}$ be Cartier divisors on $X$ and $g, g^{\prime}$ be Green functions of $D$ and $D^{\prime}$, respectively. Then, for $\left(a, a^{\prime}\right) \in \mathbb{Z}^{2}, a g+a^{\prime} g^{\prime}$ is a Green function of $a D+a^{\prime} D^{\prime}$.

Proof. - (1) is obvious.

(2) Let $f$ and $f^{\prime}$ be local equations of $D$ and $D^{\prime}$, respectively. Then $f^{a} f^{\prime a^{\prime}}$ is a local equation of $a D+a^{\prime} D^{\prime}$. As $g+\ln |f|$ and $g^{\prime}+\ln \left|f^{\prime}\right|$ extend to continuous functions locally,

$$
a(g+\ln |f|)+a^{\prime}\left(g^{\prime}+\ln \left|f^{\prime}\right|\right)=a g+a^{\prime} g^{\prime}+\ln \left|f^{a} \cdot f^{\prime a^{\prime}}\right|
$$

is locally continuous, as required.

We denote by $\widehat{\operatorname{Div}}(X)$ the set of all pairs of the form $(D, g)$, where $D$ is a Cartier divisor on $X$ and $g$ is a Green function of $D$. The above proposition shows that $\widehat{\operatorname{Div}}(X)$ forms a commutative group with the composition law

$$
\left(\left(D_{1}, g_{1}\right),\left(D_{2}, g_{2}\right)\right) \longmapsto\left(D_{1}+D_{2}, g_{1}+g_{2}\right) .
$$

One has a natural homomorphism of groups $\widehat{\operatorname{Div}}(X) \rightarrow \operatorname{Div}(X)$ sending $(D, g)$ to $D$. The kernel of this homomorphism is $C^{0}\left(X^{\text {an }}\right)$.

2.5.2. Green functions for $\mathbb{Q}$-Cartier and $\mathbb{R}$-Cartier divisors. - Let $\mathbb{K}$ be either $\mathbb{Q}$ or $\mathbb{R}$. Let $f$ be an element of $R(X)^{\times} \otimes_{\mathbb{Z}} \mathbb{K}$, that is,

$$
f=f_{1}^{a_{1}} \cdots f_{r}^{a_{r}}, \quad\left(f_{1}, \ldots, f_{r}\right) \in\left(R(X)^{\times}\right)^{r} \text { and }\left(a_{1}, \ldots, a_{r}\right) \in \mathbb{K}^{r} .
$$

Then one can consider an element of $C_{\text {gen }}^{0}\left(X^{\text {an }}\right)$ given by $a_{1} \ln \left|f_{1}\right|+\cdots+a_{r} \ln \left|f_{r}\right|$, which dose not depend on the choice of the expression $f=f_{1}^{a_{1}} \cdots f_{r}^{a_{r}}$. Indeed, let $f=g_{1}^{b_{1}} \cdots g_{l}^{b_{l}}$ be another expression of $f$. Let us choose an affine open set $U=\operatorname{Spec}(A)$ such that $f_{1}, \ldots, f_{r}, g_{1}, \ldots, g_{l}$ belong to $A^{\times}$. For $x \in U^{\text {an }}$, as the seminorm $|\cdot|_{x}$ is multiplicative, $|\cdot|_{x}$ naturally extends to a map $|\cdot|_{x}: A^{\times} \otimes_{\mathbb{Z}} \mathbb{K} \rightarrow \mathbb{R}$, so that $\left|f_{1}\right|_{x}^{a_{1}} \cdots\left|f_{r}\right|_{x}^{a_{r}}=\left|g_{1}\right|_{x}^{b_{1}} \cdots\left|g_{l}\right|_{x}^{b_{l}}$. Therefore,

$$
a_{1} \ln \left|f_{1}\right|+\cdots+a_{r} \ln \left|f_{r}\right|=b_{1} \ln \left|g_{1}\right|+\cdots+b_{l} \ln \left|g_{l}\right|
$$

on $U^{\text {an }}$, which shows the assertion. We denote the above function by $\ln |f|$.

Definition 2.5.6. - Let $D$ be a $\mathbb{K}$-Cartier divisor on $X$. We say an element $g \in C_{\mathrm{gen}}^{0}\left(X^{\mathrm{an}}\right)$ is a $D$-Green function or Green function of $D$ if, for any point $x \in X^{\text {an }}$ and any local equation $f$ of $D$ on a Zariski neighbourhood of $j(x), g+\log |f|$ extends to a continuous function around $x$. We denote by $\widehat{\operatorname{Div}}_{\mathbb{K}}(X)$ the set of all pairs of the form $(D, g)$, where $D$ is a $\mathbb{K}$-Cartier divisor on $X$ and $g$ is a Green function of $D$. Note that $\widehat{\operatorname{Div}}_{\mathbb{K}}(X)$ is actually a vector space over $\mathbb{K}$, which is the quotient of $\widehat{\operatorname{Div}}(X) \otimes_{\mathbb{Z}} \mathbb{K}$ by the vector subspace generated by elements of the form $\lambda(D, g)-(\lambda D, \lambda g)$, where $(D, g) \in \widehat{\operatorname{Div}}(X)$ and $\lambda \in \mathbb{K}$. 
Proposition 2.5.7. - (1) Let $g$ be a Green function of the trivial $\mathbb{K}$-Cartier divisor. Then $g$ extends to a continuous function on $X^{\text {an }}$.

(2) Let $D$ and $D^{\prime}$ be $\mathbb{K}$-Cartier divisors on $X$ and $g, g^{\prime}$ be Green functions of $D$ and $D^{\prime}$, respectively. Then, for $a, a^{\prime} \in \mathbb{K}, a g+a^{\prime} g^{\prime}$ is a Green function of $a D+a^{\prime} D^{\prime}$.

Proof. - It can be proven in the same way as Proposition 2.5.5.

Proposition 2.5.8. - Let $\mathbb{K}$ be either $\mathbb{Z}$ or $\mathbb{Q}$ or $\mathbb{R}$. Let $D$ be an effective $\mathbb{K}$ Cartier divisor on $X$ and $g$ be a Green function of D. Then the element $\mathrm{e}^{-g}$ of $C_{\text {gen }}^{0}(X)$ extends to a non-negative continuous function on $X^{\text {an }}$.

Proof. - Locally on a Zariski open subset $U=\operatorname{Spec}(A)$ of $X$, the divisor $D$ is defined by $f_{1}^{a_{1}} \ldots f_{r}^{a_{r}}$ (where $f_{1}, \ldots, f_{r}$ are elements of $A \backslash\{0\}$ and $a_{1}, \ldots, a_{r}$ are elements of $\left.\mathbb{K}_{>0}\right)$ and the element $g+\ln |f|$ of $C_{\text {gen }}^{0}\left(X^{\text {an }}\right)$ extends to a continuous function on $U^{\text {an }}$. Hence $\mathrm{e}^{-g}=|f| \cdot \mathrm{e}^{-(g+\ln |f|)}$ extends to a continuous function on $X^{\text {an }}$, which is non-negative.

Definition 2.5.9. - Let $\mathbb{K}$ be either $\mathbb{Z}$ or $\mathbb{Q}$ or $\mathbb{R}$.

(1) For $f \in H_{\mathbb{K}}^{0}(D),|f| \exp (-g)$ extends to a continuous function. Indeed, as $D+(f)$ is effective and $g-\ln |f|$ is a Green function of $D+(f)$, by the above proposition, $|f| \exp (-g)=\exp (-(g-\ln |f|))$ is a continuous function. We denote the function $|f| \exp (-g)$ by $|f|_{g}$. Moreover, $\sup \left\{|f|_{g}(x): x \in X^{\text {an }}\right\}$ is denoted by $\|f\|_{g}$.

(2) Let $D$ be an effective $\mathbb{K}$-Cartier divisor on $X$ and $g$ be a Green function of $X$. By abuse of notation, we use the expression $g$ to denote the map $-\ln \left(\mathrm{e}^{-g}\right)$ : $X^{\text {an }} \rightarrow \mathbb{R} \cup\{+\infty\}$, where $\mathrm{e}^{-g}$ is the non-negative continuous function on $X^{\text {an }}$ described in Proposition 2.5.8. We say that an element $(D, g)$ of $\widehat{\operatorname{Div}}(X)$ or $\widehat{\operatorname{Div}}_{\mathbb{R}}(X)$ is effective if $D$ is effective and the map $g$ takes non-negative values.

(3) Let $\bar{D}=(D, g)$ be an element of $\widehat{\operatorname{Div}}_{\mathbb{K}}(X)$. We define $\widehat{H}_{\mathbb{K}}^{0}(\bar{D})$ to be

$$
\widehat{H}_{\mathbb{K}}^{0}(\bar{D}):=\left\{f \in R(X)^{\times}: \bar{D}+\widehat{(f)} \text { is effective }\right\} \cup\{0\} .
$$

Note that $\widehat{H}_{\mathbb{K}}^{0}(\bar{D})=\left\{f \in H_{\mathbb{K}}^{0}(D):\|f\|_{g} \leqslant 1\right\}$.

Remark 2.5.10. - Let $(D, g)$ be an element of $\widehat{\operatorname{Div}}_{\mathbb{K}}(X)$ and $s$ be an element of $R(X)^{\times}$. Let $\left(D^{\prime}, g^{\prime}\right)=(D+\operatorname{div}(s), g-\ln |s|)$. Then the map $H_{\mathbb{K}}^{0}\left(D^{\prime}\right) \rightarrow H_{\mathbb{K}}^{0}(D)$ sending $f \in H_{\mathbb{K}}^{0}\left(D^{\prime}\right)$ to $f s$ is a bijection. Moreover, for any $f \in H_{\mathbb{K}}^{0}\left(D^{\prime}\right)$ one has $\|f\|_{g^{\prime}}=\|f s\|_{g}$. 



\section{CHAPTER 3}

\section{ADELIC CURVES}

The theory of adèles in the study of global fields was firstly introduced by Chevalley [46. Chapitre III] in the function field setting and by Weil [140] in the number field setting. This theory allows to consider all places of a global field in a unified way. It also leads to a uniform approach in the geometry of numbers in global fields, either via the adelic version of Minkowski's theorems and Siegel's lemma developed by McFeat [101, Bombieri-Vaaler [10, Thunder [135, Roy-Thunder [121], or via the study of adelic vector bundles developed by Gaudron [60, generalising the slope theory introduced by Stuhler [131], Grayson [68] and Bost [15, 17. The adelic point of view is also closely related to the Arakelov geometry approach to the height theory in arithmetic geometry. Recall that the Arakelov height theory has been developed by Arakelov [4, 3, Szpiro [133, Faltings [58, Bost-Gillet-Soulé [22], (compare to the approach of Philippon [15], see also [129 for the comparison of these approaches). We refer the readers to [149] for an application of the Arakelov height theory in the adelic setting to the Bogomolov problem. The Arakelov height theory has been generalized by Moriwaki [102] to setting of finitely generated field over a number field (see also 104 for a panoramic view of this theory).

The purpose of this chapter is to develop a formal setting of adelic curves, which permits to include the above examples of global fields and finitely generated extensions of global fields, as well as less standard examples such as the trivial absolute value, polarised projective varieties and arithmetic varieties, and the combination of different adelic structures. More concretely, we consider a field equipped with a family of absolute values on the field, indexed by a measure space, which verifies a "product formula" (see Section 3.1 below). This construction is similar to that of $M$-field introduced by Gubler [76] (see [33] for the height theory of toric varieties in this setting, and the work of Ben Yaakov and Hrushovski in the model theory framework). Moreover, Gaudron and Rémond [63 have studied Siegel's lemma for fields of algebraic numbers with a similar point of view. However, our main concern is to 
establish a general setting with which we can develop not only the height theory but also the geometry of adelic vector bundles and birational Arakelov theory. Therefore our choice is different from the previous works. In particular, we require that the absolute values are well defined for all places (same as the setting of globally valued field of Ben Yaakov and Hrushovski, compare to [76, §2]) and we pay a particular attention to the algebraic coverings of adelic curves and the measurability properties (see Sections 3.3 3.4). We prove that, for any adelic curve $S$ with underlying field $K$ and any algebraic extension $L$ of $K$, there exists a natural structure of adelic curve on $L$ whose measure space is fibred over that of $S$ with a disintegration kernel (compare to 63 ). Curiously, even in the simplest case of finite separable extensions, this result is far from simple (see for example Theorem 3.3.4). The main subtleties appear in the proof of the measurability of the fibre integral, which is neither classic in the theory of disintegration of measures nor in the extension of absolute values in algebraic number theory. We combine the monotone class theorem (in a functional form) in measure theory with divers technics in algebra and number theory such as symmetric polynomials and Vandermonde matrix to resolve this problem.

The chapter is organized as follows. In Section 3.1 we give the definition of adelic curves and discuss several basic measurability properties concerning Archimedean absolute values. Various examples of adelic curves are presented in Section 1.2. In the subsequent two sections, we discuss algebraic extensions of adelic curves. The finite separable extension case is treated in Section 1.3, where we establish the measurability of fibre integrals (Theorem 3.3.4) and the construction of the extended adelic curve (Theorem 3.3.7). Section 3.4 is devoted to the generalisation of these results to arbitrary algebraic extensions case, where the compatibility of the construction in the situation of successive extensions (Theorem 3.4.12) is proved. These results will serve as the fundament for the geometry of adelic vector bundles and birational Arakelov geometry over adelic curves developed in further chapters.

\subsection{Definition of Adelic curves}

Let $K$ be a commutative field and $M_{K}$ be the set of all absolute values on $K$. We call adelic structure on $K$ a measure space $(\Omega, \mathcal{A}, \nu)$ equipped with a map $\phi: \omega \mapsto|\cdot|_{\omega}$ from $\Omega$ to $M_{K}$ satisfying the following properties:

(i) $\mathcal{A}$ is a $\sigma$-algebra on $\Omega$ and $\nu$ is a measure on $(\Omega, \mathcal{A})$;

(ii) for any $a \in K^{\times}:=K \backslash\{0\}$, the function $\omega \mapsto \ln |a|_{\omega}$ is $\mathcal{A}$-measurable, integrable with respect to the measure $\nu$.

The data $(K,(\Omega, \mathcal{A}, \nu), \phi)$ is called an adelic curve. Moreover, the space $\Omega$ and the map $\phi: \Omega \rightarrow M_{K}$ are called a parameter space of $M_{K}$ and a parameter map, respectively. We do not require neither the injectivity nor the surjectivity of $\phi$. Further, if the 
equality

$$
\int_{\Omega} \ln |a|_{\omega} \nu(\mathrm{d} \omega)=0
$$

holds for each $a \in K^{\times}$, then the adelic curve $(K,(\Omega, \mathcal{A}, \nu), \phi)$ is said to be proper. The equation (3.1) is called a product formula.

The set of all $\omega \in \Omega$ such that $|\cdot|_{\omega}$ is Archimedean (resp. non-Archimedean) is written as $\Omega_{\infty}$ (resp. $\Omega_{\text {fin }}$ ). For any element $\omega \in \Omega$, let $K_{\omega}$ be the completion of $K$ with respect to the absolute value $|\cdot|_{\omega}$. Note that $|\cdot|_{\omega}$ extends by continuity to an absolute value on $K_{\omega}$ which we still denote by $|\cdot|_{\omega}$.

Proposition 3.1.1. - Let $(K,(\Omega, \mathcal{A}, \nu), \phi)$ be an adelic curve. The set $\Omega_{\infty}$ of all $\omega \in \Omega$ such that the absolute value $|\cdot|_{\omega}$ is Archimedean belongs to $\mathcal{A}$.

Proof. - The result of the proposition is trivial if the characteristic of $K$ is positive since in this case the set $\Omega_{\infty}$ is empty. In the following, we assume that the characteristic of $K$ is zero. Let $f$ be the function on $\Omega$ defined as $f(\omega)=\ln |2|_{\omega}$. Then $\Omega_{\infty}=\{\omega \in \Omega: f(\omega)>0\}$. Hence $\Omega_{\infty}$ is a measurable set.

In the case where $|\cdot|_{\omega}$ is Archimedean, the field $K_{\omega}$ is equal to $\mathbb{R}$ or $\mathbb{C}$. However, the absolute value $|\cdot|_{\omega}$ does not necessarily identify with the usual absolute value on $K_{\omega}$. By Ostrowski's theorem (see 110 Chapter II, Theorem 4.2), there exists a number $\kappa(\omega) \in] 0,1]$ such that $|\cdot|_{\omega}$ equals $|\cdot| \kappa(\omega)$ on $\mathbb{Q}$, where $|\cdot|$ denotes the usual absolute value on $\mathbb{C}$. Therefore one has $|\cdot|_{\omega}=|\cdot|^{\kappa(\omega)}$ on $K_{\omega}=\mathbb{R}$ or $\mathbb{C}$.

Proposition 3.1.2. - If we extend the domain of definition of the function $\kappa$ to $\Omega$ by taking the value 0 on $\Omega \backslash \Omega_{\infty}$, then the function $\kappa$ is $\mathcal{A}$-measurable and integrable with respect to $\nu$. In particular, if the function $\kappa$ is bounded from below on $\Omega_{\infty}$ by a positive number, then one has $\nu\left(\Omega_{\infty}\right)<+\infty$.

Proof. - The result of the proposition is trivial if $\Omega_{\infty}$ is empty. In the following, we assume that $\Omega_{\infty}$ is non-empty. In this case the field $K$ is of characteristic zero. One has

$$
\forall \omega \in \Omega_{\infty}, \quad \ln |2|_{\omega}=\kappa(\omega) \ln (2),
$$

so that

$$
\forall \omega \in \Omega, \quad \kappa(\omega)=\frac{\max \left\{0, \ln |2|_{\omega}\right\}}{\ln (2)} .
$$

Therefore the $\mathcal{A}$-measurability and $\nu$-integrability of the function $\omega \mapsto \ln |2|_{\omega}$ imply the results of the proposition.

Proposition 3.1.3. - Let $S=(K,(\Omega, \mathcal{A}, \nu), \phi)$ be an adelic curve. We assume that the field $K$ is countable. Let $\Omega_{0}$ be the set of points $\omega \in \Omega$ such that the absolute value $|\cdot|_{\omega}$ on $K$ is trivial. Then $\Omega_{0}$ belongs to $\mathcal{A}$. 
Proof. - By definition,

$$
\Omega_{0}=\bigcap_{a \in K^{\times}}\left\{\omega \in \Omega:|a|_{\omega}=1\right\} .
$$

Since the function $(\omega \in \Omega) \mapsto|a|_{\omega}$ is $\mathcal{A}$-measurable, the set $\left\{\omega \in \Omega:|a|_{\omega}=1\right\}$ belongs to $\mathcal{A}$. Since $K^{\times}$is a countable set, we obtain that $\Omega_{0}$ also belongs to $\mathcal{A}$.

\subsection{Examples}

We introduce several fundamental examples of proper adelic curves. Some of them are very classic objects in algebraic geometry or in arithmetic geometry.

3.2.1. Function fields. - Let $k$ be a field, $C$ be a regular projective curve over Spec $k$ and $K$ be the field of all rational functions on $C$. We denote by $\Omega$ the set of all closed points of the curve $C$, equipped with the discrete $\sigma$-algebra $\mathcal{A}$ (namely $\mathcal{A}$ is the $\sigma$-algebra of all subsets of $\Omega$ ). For any closed point $x$ of $C$, the local ring $\mathcal{O}_{C, x}$ is a discrete valuation ring whose fraction field is $K$. We let $\operatorname{ord}_{x}(\cdot): K \rightarrow \mathbb{Z} \cup\{+\infty\}$ be the discrete valuation on $K$ of valuation ring $\mathcal{O}_{C, x}$ and $|\cdot|_{x}$ be the absolute value on $K$ defined as

$$
\forall a \in K^{\times}, \quad|a|_{x}=\mathrm{e}^{-\operatorname{ord}_{x}(a)} .
$$

Let $n_{x}:=[k(x): k]$ be the degree of the residue field of $x$. Thus we obtain a map $\phi: \Omega \rightarrow M_{K}$ sending $x \in \Omega$ to $|\cdot|_{x}$. We equip the measurable space $(\Omega, \mathcal{A})$ with the measure $\nu$ such that $\nu(\{x\})=n_{x}$. The relation

$$
\forall a \in K^{\times}, \quad \sum_{x \in \Omega} n_{x} \operatorname{ord}_{x}(a)=0
$$

shows that the equality

$$
\int_{\Omega} \ln |a|_{x} \nu(\mathrm{d} x)=0
$$

holds for any $a \in K^{\times}$. Therefore $(K,(\Omega, \mathcal{A}, \nu), \phi)$ is a proper adelic curve.

3.2.2. Number fields. - Let $K$ be a number field. Denote by $\Omega$ the set of all places of $K$, equipped with the discrete $\sigma$-algebra. For any $\omega \in \Omega$, let $|\cdot|_{\omega}$ be the absolute value on $K$ in the equivalence class $\omega$, which extends either the usual Archimedean absolute value on $\mathbb{Q}$ or one of the $p$-adic absolute values (such that the absolute value of $p$ is $1 / p)$. Thus we obtain a map $\phi: \Omega \rightarrow M_{K}$ sending $\omega \in \Omega$ to $|\cdot|_{\omega}$. For each $\omega \in \Omega$, let $n_{\omega}$ be the local degree $\left[K_{\omega}: \mathbb{Q}_{\omega}\right]$, where $K_{\omega}$ and $\mathbb{Q}_{\omega}$ denote respectively the completion of $K$ and $\mathbb{Q}$ with respect to the absolute value $|\cdot|_{\omega}$. Let $\nu$ be the measure on $(\Omega, \mathcal{A})$ such that $\nu(\{\omega\})=n_{\omega}$ for any $\omega \in \Omega$. Note that the usual product formula (cf. [110] Chapter III, Proposition 1.3) asserts that

$$
\forall a \in K^{\times}, \quad \prod_{\omega \in \Omega}|a|_{\omega}^{\left[K_{\omega}: \mathbb{Q}_{\omega}\right]}=1,
$$


which can also be written in the form

$$
\forall a \in K^{\times}, \quad \int_{\Omega} \ln |a|_{\omega} \nu(\mathrm{d} \omega)=0 .
$$

Therefore $(K,(\Omega, \mathcal{A}, \nu), \phi)$ is a proper adelic curve.

3.2.3. Copies of the trivial absolute value. - Let $K$ be any field and $(\Omega, \mathcal{A}, \nu)$ be an arbitrary measure space. For each $\omega \in \Omega$, let $|\cdot|_{\omega}$ be the trivial absolute value on $K$, namely one has $|a|_{\omega}=1$ for any $a \in K^{\times}$. We denote by $\phi: \Omega \rightarrow M_{K}$ the map sending all elements of $\Omega$ to the trivial absolute value on $K$. Then the equality

$$
\forall a \in K^{\times}, \quad \int_{\Omega} \ln |a|_{\omega} \nu(\mathrm{d} \omega)=0
$$

is trivially satisfied. Therefore the data $(K,(\Omega, \mathcal{A}, \nu), \phi)$ form a proper adelic curve.

3.2.4. Polarised varieties. - Let $k$ be a field and $X$ be an integral and normal projective scheme of dimension $d \geqslant 1$ over Spec $k$. Let $K=k(X)$ be the field of rational functions on $X$ and $\Omega=X^{(1)}$ be the set of all prime divisors in $X$, equipped with the discrete $\sigma$-algebra $\mathcal{A}$. We also fix a family $\left\{D_{i}\right\}_{i=1}^{d-1}$ of ample divisors on $X$. Let $\nu$ be the measure on $(\Omega, \mathcal{A})$ such that

$$
\forall Y \in \Omega=X^{(1)}, \quad \nu(\{Y\})=\operatorname{deg}\left(D_{1} \cdots D_{d-1} \cap[Y]\right) .
$$

Thus we obtain a measure space $(\Omega, \mathcal{A}, \nu)$.

For each $Y \in \Omega$, let $\mathcal{O}_{X, Y}$ be the local ring of $X$ on the generic point of $Y$. It is a discrete valuation ring since it is a Noetherian normal domain of Krull dimension 1. Moreover, its fraction field is $K$. We denote by $\operatorname{ord}_{Y}(\cdot)$ the corresponding valuation on $K$ and by $|\cdot|_{Y}$ the absolute value on $K$ with $|\cdot|_{Y}:=\mathrm{e}^{-\operatorname{ord}_{Y}(\cdot)}$. Thus we obtain a map $\phi$ from $\Omega$ to the set of all absolute values on $K$, sending $Y \in \Omega$ to $|\cdot|_{Y}$.

For any rational function $f \in K^{\times}$, let $(f)$ be the principal divisor associated with $f$, which is

$$
(f):=\sum_{Y \in \Omega} \operatorname{ord}_{Y}(f) \cdot Y .
$$

Therefore, the relation $\operatorname{deg}\left(D_{1} \cdots D_{d-1} \cdot(f)\right)=0$ can be written as

$$
\int_{\Omega} \ln |f|_{Y} \nu(\mathrm{d} Y)=0
$$

Hence $(K,(\Omega, \mathcal{A}, \nu), \phi)$ is a proper adelic curve.

3.2.5. Function field over $\mathbb{Q}$. - Let $K=\mathbb{Q}(T)$ be the field of rational functions of one variable $T$ and with coefficients in $\mathbb{Q}$. We consider $K$ as the field of all rational functions on $\mathbb{P}_{\mathbb{Q}}^{1}$. Any closed point $x \in \mathbb{P}_{\mathbb{Q}}^{1}$ defines a discrete valuation on $K$ which we denote by $\operatorname{ord}_{x}(\cdot)$. Let $\infty$ be the rational point of $\mathbb{P}_{\mathbb{Q}}^{1}$ such that

$$
\operatorname{ord}_{\infty}(f / g)=\operatorname{deg}(g)-\operatorname{deg}(f)
$$


for polynomials $f$ and $g$ in $\mathbb{Q}[T]$ such that $g \neq 0$. Then the open subscheme $\mathbb{P}_{\mathbb{Q}}^{1} \backslash\{\infty\}$ is isomorphic to $\mathbb{A}_{\mathbb{Q}}^{1}$. Therefore any closed point $x$ of $\mathbb{P}_{\mathbb{Q}}^{1}$ different from $\infty$ corresponds to an irreducible polynomial $F_{x}$ in $\mathbb{Q}[T]$ (up to dilation by a scalar in $\mathbb{Q}^{\times}$). By convention we assume that $F_{x} \in \mathbb{Z}[T]$ and that the coefficients of $F_{x}$ are coprime. Let $H(x)$ be the Mahler measure of the polynomial $F_{x}$, defined as

$$
H(x):=\exp \left(\int_{0}^{1} \ln \left|F_{x}\left(\mathrm{e}^{2 \pi i t}\right)\right| \mathrm{d} t\right) .
$$

Note that if the polynomial $F_{x}$ is written in the form

$$
F_{x}(T)=a_{d} T^{d}+\cdots+a_{1} T+a_{0}=a_{d}\left(T-\alpha_{1}\right) \cdots\left(T-\alpha_{d}\right),
$$

then one has (by Jensen's formula, see [87])

$$
H(x)=\left|a_{d}\right| \prod_{j=1}^{d} \max \left\{1,\left|\alpha_{j}\right|\right\} \geqslant 1 .
$$

Let $|\cdot|_{x}$ be the absolute value on $\mathbb{Q}(T)$ such that

$$
\forall \varphi \in \mathbb{Q}(T), \quad|\varphi|_{x}=H(x)^{-\operatorname{ord}_{x}(\varphi)} .
$$

For any prime number $p$, let $|\cdot|_{p}$ be the natural extension to $\mathbb{Q}(T)$ of the $p$-adic absolute value on $\mathbb{Q}$ constructed as follows. For any

$$
f=a_{d} T^{d}+\cdots+a_{1} T+a_{0} \in \mathbb{Q}[T]
$$

let

$$
|f|_{p}:=\max _{j \in\{0, \ldots, d\}}\left|a_{j}\right|_{p} .
$$

Note that one has $|f g|_{p}=|f|_{p} \cdot|g|_{p}$ for $f$ and $g$ in $\mathbb{Q}[T]$ (see [12] Lemma 1.6.3 for example) and thus the function $|\cdot|_{p}$ on $\mathbb{Q}[T]$ extends in a unique way to a multiplicative function on $\mathbb{Q}(T)$. Moreover, the extended function satisfies the strong triangle inequality and therefore defines a non-Archimedean absolute value on $\mathbb{Q}(T)$.

Denote by $[0,1]_{*}$ the set of $t \in[0,1]$ such that $\mathrm{e}^{2 \pi i t}$ is transcendental. For any $t \in[0,1]_{*}$, let $|\cdot|_{t}$ be the absolute value on $\mathbb{Q}(T)$ such that

$$
\forall \varphi \in \mathbb{Q}(T), \quad|\varphi|_{t}:=\left|\varphi\left(\mathrm{e}^{2 \pi i t}\right)\right|,
$$

where $|\cdot|$ denotes the usual absolute value of $\mathbb{C}$. The absolute value $|\cdot|_{t}$ is Archimedean.

Denote by $\Omega$ the disjoint union $\Omega_{h} \amalg \mathcal{P} \amalg[0,1]_{*}$, where $\Omega_{h}$ is the set of all closed points of $\mathbb{P}_{\mathbb{Q}}^{1} \backslash\{\infty\}$, and $\mathcal{P}$ denotes the set of all prime numbers. Let $\phi: \Omega \rightarrow M_{K}$ be the map sending $\omega \in \Omega$ to $|\cdot|_{\omega}$. We equip $\Omega_{h}$ and $\mathcal{P}$ with the discrete $\sigma$-algebras, and $[0,1]_{*}$ with the restriction of the Borel $\sigma$-algebra on $[0,1]$. Let $\mathcal{A}$ be the $\sigma$-algebra on $\Omega$ generated by the above $\sigma$-algebras on $\Omega_{h}, \mathcal{P}$ and $[0,1]_{*}$ respectively. Let $\nu$ be the measure on $\Omega$ such that $\nu(\{x\})=1$ for $x \in \Omega_{h}$, that $\nu(\{p\})=1$ for any prime number 
$p$ and that the restriction of $\nu$ on $[0,1]_{*}$ coincides with the Lebesgue measure. Then for any $f \in K[T] \backslash\{0\}$ one has

$$
\int_{\Omega} \ln |f|_{\omega} \nu(\mathrm{d} \omega)=\sum_{x \in \Omega_{h}} \ln |f|_{x}+\sum_{p \in \mathcal{P}} \ln |f|_{p}+\int_{[0,1]_{*}} \ln \left|f\left(\mathrm{e}^{2 \pi i t}\right)\right| \mathrm{d} t .
$$

Since $[0,1] \backslash[0,1]_{*}$ is negligible with respect to the Lebesgue measure, we obtain that

$$
\int_{[0,1]_{*}} \ln \left|f\left(\mathrm{e}^{2 \pi i t}\right)\right| \mathrm{d} t=\int_{0}^{1} \ln \left|f\left(\mathrm{e}^{2 \pi i t}\right)\right| \mathrm{d} t
$$

is equal to the logarithm of the Mahler measure of the polynomial $f$. In particular, if we write the polynomial $f$ in the form

$$
f=a F_{x_{1}}^{r_{1}} \cdots F_{x_{n}}^{r_{n}}
$$

where $x_{1}, \ldots, x_{n}$ are distinct closed points of $\mathbb{P}_{\mathbb{Q}}^{1} \backslash\{\infty\}$, and $a \in \mathbb{Q}^{\times}$. Then one has

$$
\int_{[0,1]_{*}} \ln \left|f\left(\mathrm{e}^{2 \pi i t}\right)\right| \mathrm{d} t=\ln |a|+\sum_{j=1}^{n} r_{j} \ln H\left(x_{j}\right) .
$$

Therefore one has

$$
\int_{\Omega} \ln |f|_{\omega} \nu(\mathrm{d} \omega)=\sum_{j=1}^{n}\left(-r_{j}\right) \ln H\left(x_{j}\right)+\sum_{p \in \mathcal{P}} \ln |a|_{p}+\int_{[0,1]_{*}} \ln \left|f\left(\mathrm{e}^{2 \pi i t}\right)\right| \mathrm{d} t=0 .
$$

Hence $(K,(\Omega, \mathcal{A}, \nu), \phi)$ is a proper adelic curve.

This example of proper adelic curve is much less classic and may looks artificial. However, it is actually very natural from the Arakelov geometry point of view. In fact, one can consider $\mathbb{Q}(T)$ as the field of the rational functions on the arithmetic variety $\mathbb{P}_{\mathbb{Z}}^{1}:=\operatorname{Proj}(\mathbb{Z}[X, Y])$ with $T=X / Y$. Then the relation

$$
\forall \varphi \in K^{\times}, \quad \int_{\Omega} \ln |\varphi|_{\omega} \nu(\mathrm{d} \omega)=0
$$

can be interpreted as

$$
\widehat{\operatorname{deg}}\left(\widehat{c}_{1}\left(\mathcal{O}_{\mathbb{P}_{\mathbb{Z}}^{1}}(1),\|\cdot\|\right) \cdot \widehat{(\varphi)}\right)=0,
$$

where $\|\cdot\|$ is the continuous Hermitian metric of $\mathcal{O}_{\mathbb{P}_{\mathbb{Z}}^{\frac{1}{Z}}}(1)$ given by

$$
\|a X+b Y\|\left(\xi_{1}: \xi_{2}\right)=\frac{\left|a \xi_{1}+b \xi_{2}\right|}{\max \left\{\left|\xi_{1}\right|,\left|\xi_{2}\right|\right\}}
$$

and $\widehat{(\varphi)}$ is the arithmetic divisor associated to the rational function $\varphi$. The integrals of $\ln |\varphi|_{\omega}$ on $\Omega_{h}, \mathcal{P}$ and $I_{*}$ correspond to the horizontal, vertical and Archimedean contributions in the arithmetic intersection product, respectively. 
3.2.6. Polarised arithmetic variety. - The previous example treated in Subsection 3.2.5 can be considered as a very particular case of adelic structures arising from polarised arithmetic varieties. Let $K$ be a finitely generated field over $\mathbb{Q}$ and $d$ be its transcendental degree over $\mathbb{Q}$. Let $k$ be the set of all algebraic elements of $K$ over $\mathbb{Q}$. Note that $k$ is a finite extension over $\mathbb{Q}$. A normal model of $K$ over $\mathbb{Q}$ means an integral and normal projective scheme $X$ over $\mathbb{Q}$ such that the rational function field of $X$ is $K$.

For simplicity, the set of all $\mathbb{C}$-valued points of $\operatorname{Spec}(K)$ is denoted by $K(\mathbb{C})$, that is, $K(\mathbb{C})$ is the set of all embeddings of $K$ into $\mathbb{C}$. Let $X$ be a normal projective model of $K$ over $\mathbb{Q}$, namely $X$ is an integral normal projective $\mathbb{Q}$-scheme, whose field of rational functions identifies with $K$. Let $\operatorname{Spec}(K) \rightarrow X$ be the canonical morphism. Considering the composition

$$
\operatorname{Spec}(\mathbb{C}) \longrightarrow \operatorname{Spec}(K) \longrightarrow X,
$$

we may treat $K(\mathbb{C})$ as a subset of $X(\mathbb{C})$. Note that

$$
K(\mathbb{C})=X(\mathbb{C}) \backslash \bigcup_{Y \subsetneq X} Y(\mathbb{C}),
$$

where $Y$ runs over all prime divisors on $X$. Indeed, " $\subseteq$ " is obvious. Conversely, let $x \in X(\mathbb{C}) \backslash \bigcup_{Y \subsetneq X} Y(\mathbb{C})$. Then, for any $f \in K^{\times}, f$ has no zero and pole at $x$ as a rational function on $X(\mathbb{C})$, so that we have a homomorphism $K \rightarrow \mathbb{C}$ given by $f \mapsto f(x)$, as required. Note that the restriction to $K(\mathbb{C})$ of the Zariski topology on $X(\mathbb{C})$ does not depend on the choice of $X$. In fact, for any non-empty Zariski open set $U$ of $X, K(\mathbb{C})$ is a subset of $U(\mathbb{C})$, so that if $X^{\prime}$ is another normal model of $K$ over $\mathbb{Q}$ and $U$ is a common open set of $X$ and $X^{\prime}$, then $K(\mathbb{C})$ is a subset of $U(\mathbb{C})$. For $x \in K(\mathbb{C})$, we set $|\cdot|_{x}:=\left|\sigma_{x}(\cdot)\right|$, where $\sigma_{x}$ is the corresponding embedding $K \hookrightarrow \mathbb{C}$.

Let $O_{k}$ be the ring of integers in $k$. For any maximal ideal $\mathfrak{p}$ of $O_{k}$, let $v_{\mathfrak{p}}$ be the absolute value of $k$ given by

$$
v_{\mathfrak{p}}(\cdot)=\#\left(O_{k} / \mathfrak{p}\right)^{-\operatorname{ord}_{\mathfrak{p}}(\cdot)} .
$$

Let $k_{\mathfrak{p}}$ be the completion of $k$ with respect to $v_{\mathfrak{p}}$. By abuse of notation, the natural extension of $v_{\mathfrak{p}}$ to $k_{\mathfrak{p}}$ is also denoted by $v_{\mathfrak{p}}$. Let $X$ be a normal projective model of $K, X_{\mathfrak{p}}:=X \times_{\operatorname{Spec}(k)} \operatorname{Spec}\left(k_{\mathfrak{p}}\right)$ and $h: X_{\mathfrak{p}} \rightarrow X$ the natural projection. Let $X_{\mathfrak{p}}^{\text {an }}$ be the analytification of $X_{\mathfrak{p}}$ in the sense of Berkovich [8]. For $x \in X_{\mathfrak{p}}^{\text {an }}$, the associated scheme point of $X_{\mathfrak{p}}$ is denoted by $p_{x}$. We say that $x$ is a generic point of $X_{\mathfrak{p}}^{\text {an }}$ if $h\left(p_{x}\right)$ is the generic point of $X$. The set of all generic points of $X_{\mathfrak{p}}^{\text {an }}$ is denoted by $K_{\mathfrak{p}}^{\text {an }}$. If $U$ is a non-empty Zariski open set of $X$, then $K_{\mathfrak{p}}^{\text {an }} \subseteq U_{\mathfrak{p}}^{\text {an }}$, so that $K_{\mathfrak{p}}^{\text {an }}$ and the Berkovich topology of $K_{\mathfrak{p}}^{\mathrm{an}}$ do not depend on the choice of the model $X$. Moreover, as before, we can see that

$$
K_{\mathfrak{p}}^{\mathrm{an}}=X_{\mathfrak{p}}^{\mathrm{an}} \backslash \bigcup_{Y \subsetneq X} Y_{\mathfrak{p}}^{\mathrm{an}}
$$


where $Y$ runs over all prime divisors on $X$. For $x \in K_{\mathfrak{p}}^{\text {an }}$, the corresponding seminorm $|\cdot|_{x}$ induces an absolute value of $K$ because $K$ is contained in the residue field of $X_{\mathfrak{p}}$ at $p_{x}$. By abuse of notation, it is also denoted by $|\cdot|_{x}$.

The Zariski-Riemann space $\mathrm{ZR}(K / k)$ of $K$ over $k$ is defined by the set of all discrete valuation rings $\mathcal{O}$ such that $k \subseteq \mathcal{O} \subseteq K$ and the fraction field of $\mathcal{O}$ is $K$. For $\mathcal{O} \in \mathrm{ZR}(K / k)$, the associated valuation of $K$ is denoted by $\operatorname{ord}_{\mathcal{O}}$. We set $|\cdot|_{\mathcal{O}}:=$ $\exp \left(-\operatorname{ord}_{\mathcal{O}}(\cdot)\right)$.

Let $\Omega_{\text {geom }}^{\text {fin }}:=\mathrm{ZR}(K / k), \Omega_{\mathfrak{p}}^{\text {fin }}:=K_{\mathfrak{p}}^{\text {an }}, \Omega^{\infty}:=K(\mathbb{C})$ and

$$
\Omega:=\Omega_{\text {geom }}^{\text {fin }} \amalg \coprod_{\mathfrak{p} \in \operatorname{Max}\left(O_{k}\right)} \Omega_{\mathfrak{p}}^{\text {fin }} \amalg \Omega^{\infty},
$$

where $\operatorname{Max}\left(O_{k}\right)$ is the set of all maximal ideals of $O_{k}$. Let $\phi: \Omega \rightarrow M_{K}$ be the map $\omega \mapsto|\cdot|_{\omega}$. Here we consider the $\sigma$-algebra $\mathcal{A}$ on $\Omega$ generated by the discrete $\sigma$-algebra on $\Omega^{\text {fin }}$, the Borel $\sigma$-algebra on $\Omega_{\mathfrak{p}}^{\text {fin }}$ with respect to the topology of $K_{\mathfrak{p}}^{\text {an }}$ for each $\mathfrak{p} \in \operatorname{Max}\left(O_{k}\right)$, and the Borel $\sigma$-algebra on $\Omega^{\infty}$ with respect to the topology of $K(\mathbb{C})$. In order to introduce a measure on $(\Omega, \mathcal{A})$, let us fix a normal model $X$ of $K$ and nef adelic arithmetic $\mathbb{R}$-Cartier divisors

$$
\bar{D}_{1}=\left(D_{1}, g_{1}\right), \ldots, \bar{D}_{d}=\left(D_{d}, g_{d}\right)
$$

of $C^{0}$-type on $X$ (for details of adelic arithmetic $\mathbb{R}$-Cartier divisors, see [108). The collection $\left(X ; \bar{D}_{1}, \ldots, \bar{D}_{d}\right)$ is called a polarisation of $K$. Let $X^{(1)}$ be the set of all prime divisors on $X$. The Radon measure on $X_{\mathfrak{p}}^{\text {an }}$ given by

$$
\varphi \longmapsto \widehat{\operatorname{deg}}_{\mathfrak{p}}\left(\left(D_{1}, g_{1, \mathfrak{p}}\right) \cdots\left(D_{d}, g_{d, \mathfrak{p}}\right) ; \varphi\right)
$$

is denoted by $\mu_{\left(D_{1}, g_{1, \mathfrak{p}}\right), \ldots,\left(D_{d}, g_{d, \mathfrak{p}}\right)}$. A measure $\nu$ on $\Omega$ is defined as follows: $\nu$ on $\Omega_{\text {geom }}^{\text {fin }}$ is a discrete measure given by

$$
\nu(\{\mathcal{O}\})= \begin{cases}\widehat{\operatorname{deg}}\left(\bar{D}_{1} \cdots \bar{D}_{d} \cdot(\Gamma, 0)\right) & \text { if } \mathcal{O}=\mathcal{O}_{X, \Gamma} \text { for some } \Gamma \in X^{(1)}, \\ 0 & \text { otherwise }\end{cases}
$$

$\nu$ on $\Omega_{\mathfrak{p}}^{\text {fin }}$ is the restriction of $2 \mu_{\left(D_{1}, g_{1, \mathfrak{p}}\right), \ldots,\left(D_{d}, g_{d, \mathfrak{p}}\right)}$ to $K_{\mathfrak{p}}^{\text {an }}$, and $\nu$ on $\Omega^{\infty}$ is given by $2 c_{1}\left(D_{1}, g_{1, \infty}\right) \wedge \ldots \wedge c_{1}\left(D_{d}, g_{d, \infty}\right)$. Then $(K,(\Omega, \mathcal{A}, \nu))$ yields a proper adelic structure of $K$. Indeed, for each $f \in K^{\times}$, the product formula can be checked as follows:

$$
\begin{aligned}
\int_{\Omega} \ln |f|_{\omega} \nu(d \omega)=\sum_{\Gamma \in X^{(1)}} & -\operatorname{ord}_{\Gamma}(f) \widehat{\operatorname{deg}}\left(\bar{D}_{1} \ldots \bar{D}_{d} \cdot(\Gamma, 0)\right) \\
& +\sum_{\mathfrak{p} \in \operatorname{Max}\left(O_{k}\right)} \int_{K_{\mathfrak{p}}^{\text {an }}} \ln |f|^{2} d \mu_{\left(D_{1}, g_{1, \mathfrak{p}}\right), \ldots,\left(D_{d}, g_{d, \mathfrak{p}}\right)} \\
& \quad+\int_{K(\mathbb{C})} \ln |f|^{2} c_{1}\left(D_{1}, g_{1, \infty}\right) \wedge \ldots \wedge c_{1}\left(D_{d}, g_{d, \infty}\right) .
\end{aligned}
$$

For a proper subvariety of $Y$ of $X, Y_{\mathfrak{p}}^{\text {an }}$ and $Y(\mathbb{C})$ are null sets with respect to the measures $\mu_{\left(D_{1}, g_{1, \mathfrak{p}}\right), \ldots,\left(D_{d}, g_{d, \mathfrak{p}}\right)}$ and $c_{1}\left(D_{1}, g_{1, \infty}\right) \wedge \ldots \wedge c_{1}\left(D_{d}, g_{d, \infty}\right)$, respectively. In 
addition, we have only countably many prime divisors on $X$. Therefore, the above equation implies

$$
\begin{aligned}
\int_{\Omega} \ln |f|_{\omega} \nu(d \omega)=-\widehat{\operatorname{deg}} & \left(\bar{D}_{1} \ldots \bar{D}_{d} \cdot((f), 0)\right) \\
& +\sum_{\mathfrak{p} \in \operatorname{Max}\left(O_{k}\right)} \int_{X_{\mathfrak{p}}^{\text {an }}} \ln |f|^{2} d \mu_{\left(D_{1}, g_{1, \mathfrak{p}}\right), \ldots,\left(D_{d}, g_{d, \mathfrak{p}}\right)} \\
& \quad+\int_{X(\mathbb{C})} \ln |f|^{2} c_{1}\left(D_{1}, g_{1, \infty}\right) \wedge \ldots \wedge c_{1}\left(D_{d}, g_{d, \infty}\right) .
\end{aligned}
$$

On the other hand,

$$
\begin{aligned}
& 0= \widehat{\operatorname{deg}}\left(\bar{D}_{1} \cdots \bar{D}_{d} \cdot \widehat{(f)}\right) \\
&=\widehat{\operatorname{deg}}\left(\bar{D}_{1} \cdots \bar{D}_{d} \cdot\left((f), \sum_{\mathfrak{p} \in \operatorname{Max}\left(O_{k}\right)}-\ln |f|^{2}[\mathfrak{p}]-\ln |f|^{2}[\infty]\right)\right) \\
&=\widehat{\operatorname{deg}}\left(\bar{D}_{1} \cdots \bar{D}_{d} \cdot((f), 0)\right) \\
& \quad-\sum_{\mathfrak{p} \in \operatorname{Max}\left(O_{k}\right)} \int_{X_{\mathfrak{p}}^{\operatorname{an}}} \ln |f|^{2} d \mu_{\left(D_{1}, g_{1, \mathfrak{p}}\right), \ldots,\left(D_{d}, g_{d, \mathfrak{p}}\right)} \\
& \quad-\int_{X(\mathbb{C})} \ln |f|^{2} c_{1}\left(D_{1}, g_{1, \infty}\right) \wedge \ldots \wedge c_{1}\left(D_{d}, g_{d, \infty}\right),
\end{aligned}
$$

as desired.

This proper adelic structure is denoted by $S\left(X ; \bar{D}_{1}, \ldots, \bar{D}_{d}\right)$.

3.2.7. Amalgamation of adelic structures. - Let $K$ be a field,

$$
\left((\Omega, \mathcal{A}, \nu), \phi: \Omega \rightarrow M_{K}\right) \text { and }\left(\left(\Omega^{\prime}, \mathcal{A}^{\prime}, \nu^{\prime}\right), \phi^{\prime}: \Omega^{\prime} \rightarrow M_{K}\right)
$$

be two adelic structures on $K$. Then the disjoint union of measure spaces

$$
(\Omega, \mathcal{A}, \nu) \amalg\left(\Omega^{\prime}, \mathcal{A}^{\prime}, \nu^{\prime}\right)
$$

together with the map $\Phi: \Omega \amalg \Omega^{\prime} \rightarrow M_{K}$ extending both $\phi$ and $\phi^{\prime}$ form also an adelic structure on $K$. If $S$ and $S^{\prime}$ denote the adelic curves $(K,(\Omega, \mathcal{A}, \nu), \phi)$ and $\left(K,\left(\Omega^{\prime}, \mathcal{A}^{\prime}, \nu^{\prime}\right), \phi^{\prime}\right)$ respectively, then we use the expression $S \amalg S^{\prime}$ to denote the adelic curve

$$
\left(K,(\Omega, \mathcal{A}, \nu) \amalg\left(\Omega^{\prime}, \mathcal{A}^{\prime}, \nu^{\prime}\right), \Phi\right),
$$

called the amalgamation of the adelic curves $S$ and $S^{\prime}$. Similarly, one can define the amalgamation for any finite family of adelic arithmetic structures on the field $K$. Note that if $S$ and $S^{\prime}$ are proper, then $S \amalg S^{\prime}$ is also proper. In fact, for any $a \in K^{\times}$ one has

$$
\int_{\Omega} \ln |a|_{\omega} \nu(\mathrm{d} \omega)+\int_{\Omega^{\prime}} \ln |a|_{\omega} \nu^{\prime}(\mathrm{d} \omega)=0 .
$$


3.2.8. Restriction of adelic structure to a subfield. - Let $S=(K,(\Omega, \mathcal{A}, \nu), \phi)$ be an adelic curve and let $K_{0}$ be a subfield of $K$. Let $\phi_{0}: \Omega \rightarrow M_{K_{0}}$ be the map sending $\omega \in \Omega$ to the restriction of $|\cdot|_{\omega}$ to $K_{0}$. Then $\phi_{0}$ defines an adelic structure on $K_{0}$, called the restriction of the adelic structure of $S$ to $K_{0}$. If $S$ is proper, then its restriction to $K_{0}$ is also proper.

3.2.9. Restriction of adelic structure to a measurable subset. - Let $S=$ $(K,(\Omega, \mathcal{A}, \nu), \phi)$ be an adelic curve and $\Omega_{0}$ be an element of $\mathcal{A}$. Let $\mathcal{A}_{0}$ be the restriction of the $\sigma$-algebra $\mathcal{A}$ to $\Omega_{0}$ and $\nu_{0}$ be the restriction of the measure to $\Omega_{0}$. Then $\left(K,\left(\Omega_{0}, \mathcal{A}_{0}, \nu_{0}\right),\left.\phi\right|_{\Omega_{0}}\right)$ is an adelic curve, called the restriction of $S$ to $\Omega_{0}$. Note that this adelic curve is not necessarily proper, even if the adelic curve $S$ is proper.

\subsection{Finite separable extensions}

Let $S=\left(K,\left(\Omega_{K}, \mathcal{A}_{K}, \nu_{K}\right), \phi_{K}\right)$ be an adelic curve. Let $K^{\prime} / K$ be a finite and separable extension. For each $\omega \in \Omega_{K}$, let $M_{K^{\prime}, \omega}$ be the set of all absolute values on $K^{\prime}$ which extend the absolute value $|\cdot|_{\omega}$ on $K$. Let $\Omega_{K^{\prime}}$ be the disjoint union

$$
\coprod_{\omega \in \Omega_{K}} M_{K^{\prime}, \omega}
$$

One has a natural projection $\pi_{K^{\prime} / K}: \Omega_{K^{\prime}} \rightarrow \Omega_{K}$ which sends the elements of $M_{K^{\prime}, \omega}$ to $\omega$. Let $\phi_{K^{\prime}}: \Omega_{K^{\prime}} \rightarrow M_{K^{\prime}}$ be the map induced by the inclusion maps $M_{K^{\prime}, \omega} \rightarrow M_{K^{\prime}}$. If $x$ is an element of $\Omega_{K^{\prime}}$, we also use the expression $|\cdot|_{x}$ to denote the corresponding absolute value. Note that the following diagram is commutative

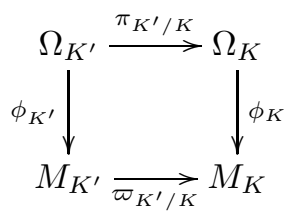

and identifies $\Omega_{K^{\prime}}$ with the fibre product of $M_{K^{\prime}}$ and $\Omega_{K}$ over $M_{K}$ in the category of sets, where $\varpi_{K^{\prime} / K}$ sends any absolute value on $K^{\prime}$ to its restriction to $K$. We equip the set $\Omega_{K^{\prime}}$ with the $\sigma$-algebra $\mathcal{A}_{K^{\prime}}$ generated by $\pi_{K^{\prime} / K}$ and all real-valued functions of the form $\left(x \in \Omega_{K^{\prime}}\right) \mapsto|a|_{x}$, where a runs over $K^{\prime}$. Namely it is the smallest $\sigma$-algebra on $\Omega_{K^{\prime}}$ which makes these maps measurable ${ }^{(1)}$, where we consider the $\sigma$-algebra $\mathcal{A}_{K}$ on $\Omega_{K}$ and the Borel $\sigma$-algebra on $\mathbb{R}$.

We aim to construct a measure $\nu_{K^{\prime}}$ on the measurable space $\left(\Omega_{K^{\prime}}, \mathcal{A}_{K^{\prime}}\right)$ such that the direct image of $\nu_{K^{\prime}}$ by $\pi_{K^{\prime} / K}$ coincides with $\nu_{K}$. Note that on each fibre $M_{K^{\prime}, \omega}$

1. A map $f: X^{\prime} \rightarrow X$ of measurable spaces $\left(X^{\prime}, \mathcal{A}^{\prime}\right)$ and $(X, \mathcal{A})$ is said to be measurable if $f^{-1}(B) \in \mathcal{A}^{\prime}$ for all $B \in \mathcal{A}$. 
of $\pi_{K^{\prime} / K}$ there is a natural probability measure $\mathbb{P}_{K^{\prime}, \omega}$ such that

$$
\forall x \in M_{K^{\prime}, \omega}, \quad \mathbb{P}_{K^{\prime}, \omega}(\{x\})=\frac{\left[K_{x}^{\prime}: K_{\omega}\right]}{\left[K^{\prime}: K\right]} .
$$

We refer to [110] Chapter II, Corollary 8.4 for a proof of the equality

$$
\sum_{x \in M_{K^{\prime}, \omega}} \frac{\left[K_{x}^{\prime}: K_{w}\right]}{\left[K^{\prime}: K\right]}=1
$$

Intuitively the family of probability measures $\left\{\mathbb{P}_{K^{\prime}, \omega}\right\}_{\omega \in \Omega_{K}}$ should form the disintegration of the measure $\nu_{K^{\prime}}$ with respect to $\nu_{K}$. However, as we will show below, the construction of the measure $\nu_{K^{\prime}}$ relies actually on a subtil application of the monotone class theorem and the properties of extensions of absolute values.

3.3.1. Integration along fibres. - If $f$ is a function on $\Omega_{K^{\prime}}$ valued in $\mathbb{R}$, we define $I_{K^{\prime} / K}(f)$ to be the function on $\Omega_{K}$ which sends $\omega \in \Omega_{K}$ to

$$
\sum_{x \in M_{K^{\prime}, \omega}} \frac{\left[K_{x}^{\prime}: K_{\omega}\right]}{\left[K^{\prime}: K\right]} f(x)
$$

This is an $\mathbb{R}$-linear operator from the vector space of all real-valued functions on $\Omega_{K^{\prime}}$ to that of all real-valued functions on $\Omega_{K}$. The equality (3.3) shows that $I_{K^{\prime} / K}$ sends the constant function 1 on $\Omega_{K^{\prime}}$ to that on $\Omega_{K}$. The following properties of the linear operator $I_{K^{\prime} / K}$ are straightforward.

Proposition 3.3.1. - Let $f$ be a real-valued function on $\Omega_{K^{\prime}}$ and $\varphi$ be a real-valued function on $\Omega_{K}$. Let $\widetilde{\varphi}=\varphi \circ \pi_{K^{\prime} / K}$. Then

$$
I_{K^{\prime} / K}(\widetilde{\varphi} f)=\varphi I_{K^{\prime} / K}(f) .
$$

Proof. - By definition, for any $\omega \in \Omega_{K}$ one has

$$
\left(I_{K^{\prime} / K}(\widetilde{\varphi} f)\right)(\omega)=\sum_{x \in M_{K^{\prime}, \omega}} \frac{\left[K_{x}^{\prime}: K_{\omega}\right]}{\left[K^{\prime}: K\right]} \widetilde{\varphi}(x) f(x)=\sum_{x \in M_{K^{\prime}, \omega}} \frac{\left[K_{x}^{\prime}: K_{\omega}\right]}{\left[K^{\prime}: K\right]} \varphi(\omega) f(x) .
$$

Proposition 3.3.2. - Let $K^{\prime \prime} / K^{\prime} / K$ be successive finite separable extensions of fields. Let $f$ be a real-valued function on $\Omega_{K^{\prime}}$ and $\widetilde{f}=f \circ \pi_{K^{\prime \prime} / K^{\prime}}$, where $\pi_{K^{\prime \prime} / K^{\prime}}$ : $\Omega_{K^{\prime \prime}} \rightarrow \Omega_{K^{\prime}}$ sends any absolute value in $\Omega_{K^{\prime \prime}, \omega}$ to its restriction to $K^{\prime}$, viewed as an element in $\Omega_{K^{\prime}, \omega}\left(\omega \in \Omega_{K}\right)$. Then one has

$$
I_{K^{\prime \prime} / K}(\widetilde{f})=I_{K^{\prime} / K}(f) .
$$


Proof. - For any $\omega \in \Omega_{K}$, one has

$$
\begin{aligned}
& \left(I_{K^{\prime \prime} / K}(\widetilde{f})\right)(\omega)=\sum_{y \in M_{K^{\prime \prime}, \omega}} \frac{\left[K_{y}^{\prime \prime}: K_{\omega}\right]}{\left[K^{\prime \prime}: K\right]} \widetilde{f}(y) \\
= & \sum_{x \in M_{K^{\prime}, \omega}} \sum_{\substack{\left.y \in M_{K^{\prime \prime}, \omega} \\
y\right|_{K^{\prime}}=x}} \frac{\left[K_{y}^{\prime \prime}: K_{x}^{\prime}\right]}{\left[K^{\prime \prime}: K^{\prime}\right]} \cdot \frac{\left[K_{x}^{\prime}: K_{\omega}\right]}{\left[K^{\prime}: K\right]} f(x),
\end{aligned}
$$

which can also be written as

$$
\sum_{x \in M_{K^{\prime}, \omega}} \frac{\left[K_{x}^{\prime}: K_{\omega}\right]}{\left[K^{\prime}: K\right]} f(x) \sum_{\substack{y \in M_{K^{\prime \prime}},\left.\omega \\ y\right|_{K^{\prime}}=x}} \frac{\left[K_{y}^{\prime \prime}: K_{x}^{\prime}\right]}{\left[K^{\prime \prime}: K^{\prime}\right]} .
$$

Therefore the desired equality follows from the relation

$$
\sum_{\substack{y \in M_{K^{\prime \prime}},\left.\omega \\ y\right|_{K^{\prime}}=x}} \frac{\left[K_{y}^{\prime \prime}: K_{x}^{\prime}\right]}{\left[K^{\prime \prime}: K^{\prime}\right]}=1 .
$$

Corollary 3.3.3. - Let $\varphi$ be an $\mathcal{A}_{K}$-measurable function on $\Omega_{K}$. One has

$$
I_{K^{\prime} / K}\left(\varphi \circ \pi_{K^{\prime} / K}\right)=\varphi .
$$

Proof. - It suffices to apply the previous proposition to the successive extensions $K^{\prime} / K / K$ and then use the fact that $I_{K / K}$ is the identity map to obtain the result.

3.3.2. Measurability of fibre integrals. - The following theorem shows that the operator $I_{K^{\prime} / K}$ sends an $\mathcal{A}_{K^{\prime}}$-measurable function to an $\mathcal{A}_{K^{-}}$-measurable function. This result is fundamental in the construction of a suitable measure on the measurable space $\left(\Omega_{K^{\prime}}, \mathcal{A}_{K^{\prime}}\right)$.

Theorem 3.3.4. - For any real-valued $\mathcal{A}_{K^{\prime}}$-measurable function $f$, the function $I_{K^{\prime} / K}(f)$ is $\mathcal{A}_{K}$-measurable.

Proof. - Step 1: We first prove that, if $a$ is a primitive element of the finite separable extension $K^{\prime} / K$ (namely $K^{\prime}=K(a)$ ) and if $f_{a}$ is the function on $\Omega_{K^{\prime}}$ sending $x \in \Omega_{K^{\prime}}$ to $|a|_{x}$, then the function $I_{K^{\prime} / K}\left(f_{a}\right)$ is $\mathcal{A}_{K^{-}}$-measurable.

Let $K^{\text {ac }}$ be an algebraic closure of $K$ containing $K^{\prime}$. For each $\omega \in \Omega_{K}$, we extend the absolute value $|\cdot|_{\omega}$ to $K^{\text {ac }}$ via an embedding of $K^{\text {ac }}$ into an algebraic closure $K_{\omega}^{\mathrm{ac}}$ of $K_{\omega}$. We still denote by $|\cdot|_{\omega}$ the extended absolute value on $K^{\text {ac }}$ by abuse of notation. 
Lemma 3.3.5. - Let $d \in \mathbb{N}_{\geqslant 1}$ and $\left\{\alpha_{1}, \ldots, \alpha_{d}\right\}$ be a finite family of distinct elements in $K^{\mathrm{ac}}$. For any $\omega \in \Omega_{K}$, one has

$$
\max _{j \in\{1, \ldots, d\}}\left|\alpha_{j}\right|_{\omega}=\limsup _{N \rightarrow+\infty}\left|\sum_{i=1}^{d} \alpha_{i}^{N}\right|_{\omega}^{\frac{1}{N}} .
$$

Moreover, for any $c \in K^{\mathrm{ac}}$, the function

$$
\left(\omega \in \Omega_{K}\right) \longmapsto \max _{\tau \in \operatorname{Aut}_{K}\left(K^{\mathrm{ac}}\right)}|\tau(c)|_{\omega}
$$

is $\mathcal{A}_{K}$-measurable.

Proof. - First of all, by the triangle inequality one has

$$
\left|\sum_{i=1}^{d} \alpha_{i}^{N}\right|_{\omega}^{\frac{1}{N}} \leqslant d^{1 / N} \max \left\{\left|\alpha_{1}\right|_{\omega}, \ldots,\left|\alpha_{d}\right|_{\omega}\right\}
$$

Therefore

$$
\max \left\{\left|\alpha_{1}\right|_{\omega}, \ldots,\left|\alpha_{d}\right|_{\omega}\right\} \geqslant \limsup _{N \rightarrow+\infty}\left|\sum_{i=1}^{d} \alpha_{i}^{N}\right|_{\omega}^{\frac{1}{N}} .
$$

Without loss of generality, we can assume that

$$
\left|\alpha_{1}\right|_{\omega}=\ldots=\left|\alpha_{\ell}\right|_{\omega}>\left|\alpha_{\ell+1}\right|_{\omega} \geqslant \ldots \geqslant\left|\alpha_{d}\right|_{\omega},
$$

where $\ell \in\{1, \ldots, d\}$. For $i \in\{1, \ldots, \ell\}$, let $\beta_{i}=\alpha_{i} / \alpha_{1}$. One has $\beta_{1}=1$ and

$$
\left|\beta_{1}\right|_{\omega}=\ldots=\left|\beta_{\ell}\right|_{\omega}=1 \text {. }
$$

For any integer $N \geqslant 1$, one has

$$
\left(\begin{array}{c}
\beta_{1}^{N}+\cdots+\beta_{\ell}^{N} \\
\vdots \\
\beta_{1}^{N+\ell-1}+\cdots+\beta_{\ell}^{N+\ell-1}
\end{array}\right)=\left(\begin{array}{ccc}
1 & \cdots & 1 \\
\beta_{1}^{1} & \ldots & \beta_{\ell}^{1} \\
\vdots & \ddots & \vdots \\
\beta_{1}^{\ell-1} & \ldots & \beta_{\ell}^{\ell-1}
\end{array}\right)\left(\begin{array}{c}
\beta_{1}^{N} \\
\vdots \\
\beta_{\ell}^{N}
\end{array}\right)
$$

Let $\widehat{K^{\mathrm{ac}}}$ be the completion of $K^{\text {ac }}$ with respect to $|\cdot|_{\omega}$. We equip the vector space $\left(\widehat{K^{\mathrm{ac}}}\right)^{\ell}$ with the following norm

$$
\forall\left(z_{1}, \ldots, z_{\ell}\right) \in\left(\widehat{K^{\mathrm{ac}}}\right)^{\ell}, \quad\left\|\left(z_{1}, \ldots, z_{\ell}\right)\right\|:=\max _{i \in\{1, \ldots, \ell\}}\left|z_{i}\right|_{\omega} .
$$

Then the vector $\left(\beta_{1}^{N}, \ldots, \beta_{\ell}^{N}\right)$ has norm 1 with respect to $\|\cdot\|$. Moreover, the Vandermonde matrix above is invertible since $\beta_{1}, \ldots, \beta_{\ell}$ are distinct. Therefore the norm of the vector

$$
\left(\sum_{i=1}^{\ell} \beta_{i}^{N}, \ldots, \sum_{i=1}^{\ell} \beta_{i}^{N+\ell-1}\right)
$$


is bounded from below by a positive constant which does not depend on $N$, which shows that the sequence

$$
\left|\sum_{i=1}^{d}\left(\frac{\alpha_{i}}{\alpha_{1}}\right)^{N}\right|_{\omega}, \quad N \in \mathbb{N}, N \geqslant 1
$$

does not converge to zero when $N \rightarrow+\infty$. This implies that

$$
\limsup _{N \rightarrow+\infty}\left|\sum_{i=1}^{d} \alpha_{i}^{N}\right|_{\omega}^{\frac{1}{N}} \geqslant\left|\alpha_{1}\right|_{\omega}=\max \left\{\left|\alpha_{1}\right|_{\omega}, \ldots,\left|\alpha_{d}\right|_{\omega}\right\} .
$$

The equality (3.5) is thus proved.

We now proceed with the proof of the second statement. Let

$$
T^{d}-\lambda_{1} T^{d-1}+\cdots+(-1)^{d} \lambda_{d} \in K[T]
$$

be the minimal polynomial of $c$ over $K$, and $\alpha_{1}, \ldots, \alpha_{d}$ be its roots in $K^{\text {ac }}$. Since the extension $K^{\prime} / K$ is separable, these roots are distinct. By definition, for $k \in\{1, \ldots, d\}$ one has

$$
\lambda_{k}=\sum_{\substack{\left(i_{1}, \ldots, i_{k}\right) \in\{1, \ldots, d\}^{k} \\ i_{1}<\ldots<i_{k}}} \alpha_{i_{1}} \cdots \alpha_{i_{k}} .
$$

By the fundamental theorem on symmetric polynomials (see for example $\mathbf{5 3}, \S 10$ $11]$ ), if $F$ is a polynomial in $K\left[X_{1}, \ldots, X_{d}\right]$ which is invariant by the action of the symmetric group $\mathfrak{S}_{d}$ by permuting the variables, then there exists a polynomial $G \in$ $K\left[T_{1}, \ldots, T_{d}\right]$ such that

$$
F\left(\alpha_{1}, \ldots, \alpha_{d}\right)=G\left(\lambda_{1}, \ldots, \lambda_{d}\right) .
$$

In particular, one has $F\left(\alpha_{1}, \ldots, \alpha_{d}\right) \in K$ and hence the function

$$
\left(\omega \in \Omega_{K}\right) \longmapsto\left|F\left(\alpha_{1}, \ldots, \alpha_{d}\right)\right|_{\omega}
$$

is $\mathcal{A}_{K}$-measurable. For any $N \in \mathbb{N}, N \geqslant 1$, the sum $\sum_{i=1}^{d} \alpha_{i}^{N}$ can be written as a symmetric polynomial evaluated at $\left(\alpha_{1}, \ldots, \alpha_{d}\right)$, thus the function

$$
\left(\omega \in \Omega_{K}\right) \longmapsto\left|\sum_{i=1}^{d} \alpha_{i}^{N}\right|_{\omega}
$$

is $\mathcal{A}_{K}$-measurable. Combining this observation with the equality (3.5), we obtain that the function

$$
\left(\omega \in \Omega_{K}\right) \longmapsto \max _{\tau \in \operatorname{Aut}_{K}\left(K^{\mathrm{ac}}\right)}|\tau(c)|_{\omega}=\max _{i \in\{1, \ldots, d\}}\left|\alpha_{i}\right|_{\omega}
$$

is $\mathcal{A}_{K}$-measurable.

We now continue with the proof of the statement that the function $I_{K^{\prime} / K}$ is $\mathcal{A}_{K^{-}}$ measurable. Let $\left\{\gamma_{1}, \ldots, \gamma_{n}\right\}$ be the orbit of $a$ under the action of $\operatorname{Aut}_{K}\left(K^{\mathrm{ac}}\right)$. For any 
$\omega \in \Omega_{K}$, let $\left(s_{1}(\omega), \ldots, s_{n}(\omega)\right)$ be the array $\left(\left|\gamma_{1}\right|_{\omega}, \ldots,\left|\gamma_{n}\right|_{\omega}\right)$ sorted in the decreasing order. Let $k$ be an arbitrary element of $\{1, \ldots, n\}$. For any $\omega \in \Omega_{K}$, one has

$$
s_{1}(\omega) \cdots s_{k}(\omega)=\max _{\substack{\left(i_{1}, \ldots, i_{k}\right) \in\{1, \ldots, n\}^{k} \\ i_{1}<\ldots<i_{k}}} \max _{\tau \in \operatorname{Aut}_{K}\left(K^{\mathrm{ac}}\right)}\left|\tau\left(\gamma_{i_{1}} \cdots \gamma_{i_{k}}\right)\right|_{\omega} .
$$

By Lemma 3.3.5, we obtain that the function $s_{1} \cdots s_{k}$ is $\mathcal{A}_{K}$-measurable. Therefore all the functions $s_{1}, \ldots, s_{n}$ on $\Omega_{K}$ are $\mathcal{A}_{K}$-measurable. In particular, if $f_{a}: \Omega_{K^{\prime}} \rightarrow \mathbb{R}$ is the function sending $x \in \Omega_{K^{\prime}}$ to $|a|_{x}$, where $a$ is the primitive element of the finite separable extension $K^{\prime} / K$ fixed in the beginning of the step, then for any $\omega \in \Omega_{K}$ one has (we refer the readers to [110, page 163] for the second equality)

$$
\left(I_{K^{\prime} / K}\left(f_{a}\right)\right)(\omega)=\sum_{x \in M_{K^{\prime}, \omega}} \frac{\left[K_{x}^{\prime}: K_{\omega}\right]}{\left[K^{\prime}: K\right]} f_{a}(x)=\frac{1}{n} \sum_{i=1}^{n}\left|\gamma_{i}\right|_{\omega}=\frac{1}{n} \sum_{i=1}^{n} s_{i}(\omega),
$$

which implies that $I_{K^{\prime} / K}\left(f_{a}\right)$ is $\mathcal{A}_{K^{-}}$-measurable.

Step 2: We then prove that, for any element $b \in K^{\prime}$, the function $I_{K^{\prime} / K}\left(f_{b}\right)$ on $\Omega_{K}$ is $\mathcal{A}_{K}$-measurable, where $f_{b}$ denotes the function on $\Omega_{K^{\prime}}$ sending $x \in \Omega_{K^{\prime}}$ to $|b|_{x}$.

We consider the sub-extension $K(b) / K$ of $K^{\prime} / K$. It is a finite and separable extension of $K$ and $b$ is a primitive element. Let $g$ be the function on $\Omega_{K(b)}$ sending $y \in \Omega_{K(b)}$ to $|b|_{y}$. One has

$$
f_{b}=g \circ \pi_{K^{\prime} / K(b)},
$$

where the map $\pi_{K^{\prime} / K(b)}: \Omega_{K^{\prime}} \rightarrow \Omega_{K(b)}$ is defined as in Proposition 3.3.2. By (3.4), one obtains

$$
I_{K^{\prime} / K}\left(f_{b}\right)=I_{K(b) / K}(g) .
$$

By the result obtained in Step 1, the function $I_{K(b) / K}(g)$ on $\Omega_{K}$ is $\mathcal{A}_{K}$-measurable. This proves the measurability of the function $I_{K^{\prime} / K}\left(f_{b}\right)$.

Step 3: We are now able to apply the monotone class theorem to prove the announced measurability property.

Let $\mathcal{H}$ be the set of all non-negative and bounded functions $f$ on $\Omega_{K^{\prime}}$ such that the function $I_{K^{\prime} / K}(f)$ on $\Omega_{K}$ is $\mathcal{A}_{K}$-measurable. Note that the constant function 1 on $\Omega_{K^{\prime}}$ belongs to $\mathcal{H}$ since $I_{K^{\prime} / K}(1)$ coincides with the constant function 1 on $\Omega_{K}$. If $f$ and $g$ are two functions in $\mathcal{H}$ such that $f \geqslant g$, then $f-g \in \mathcal{H}$ since $I_{K^{\prime} / K}(f-g)=I_{K^{\prime} / K}(f)-I_{K^{\prime} / K}(g)$ is $\mathcal{A}_{K^{-}}$-measurable. Moreover, Proposition 3.3.1 shows that, if $f$ and $g$ are two functions in $\mathcal{H}$, and $\varphi$ and $\psi$ are two non-negative and bounded $\mathcal{A}_{K}$-measurable functions on $\Omega_{K}, \widetilde{\varphi}=\varphi \circ \pi_{K^{\prime} / K}$ and $\widetilde{\psi}=\psi \circ \pi_{K^{\prime} / K}$, then the function $\widetilde{\varphi} f+\widetilde{\psi} g$ belongs to $\mathcal{H}$ since

$$
I_{K^{\prime} / K}(\widetilde{\varphi} f+\widetilde{\psi} g)=\varphi I_{K^{\prime} / K}(f)+\psi I_{K^{\prime} / K}(g)
$$

is $\mathcal{A}_{K}$-measurable. Finally, the operator $I_{K^{\prime} / K}$ preserves pointwise limit. Therefore, if $\left\{f_{n}\right\}_{n \in \mathbb{N}}$ is a uniformly bounded sequence of functions in $\mathcal{H}$ which converges pointwisely to a function $f$, then one has $f \in \mathcal{H}$. These properties show that $\mathcal{H}$ is a $\lambda$-family (see Definition A.1.1) on $\Omega_{K^{\prime}}$. 
Let $\mathcal{C}$ be the set of all non-negative and bounded functions on $\Omega_{K^{\prime}}$ which can be written in the form $f_{b} \widetilde{\varphi}$, where $b$ is an element of $K^{\prime}, \varphi$ is a non-negative and bounded $\mathcal{A}_{K}$-measurable function on $\Omega_{K}$, and $\widetilde{\varphi}=\varphi \circ \pi_{K^{\prime} / K}$. Note that if $b_{1}$ and $b_{2}$ are two elements of $K^{\prime}$ then one has

$$
f_{b_{1} b_{2}}=f_{b_{1}} f_{b_{2}} \text {. }
$$

Therefore the family $\mathcal{C}$ is stable by multiplication. By the result obtained in Step 2 and Proposition 3.3.1 we obtain that $\mathcal{C} \subseteq \mathcal{H}$. The monotone class theorem (Theorem A.1.3 then implies that the family $\mathcal{H}$ contains all non-negative and bounded $\sigma(\mathcal{C})$ measurable functions. Finally, any non-negative $\sigma(\mathcal{C})$-measurable function on $\Omega_{K^{\prime}}$ can be written as the limit of an increasing sequence of non-negative and bounded $\sigma(\mathcal{C})$ measurable functions, and any real-valued $\sigma(\mathcal{C})$-measurable function is the difference of two non-negative $\sigma(\mathcal{C})$-measurable functions. Therefore, for any real-valued $\sigma(\mathcal{C})$ measurable function $f$, the function $I_{K^{\prime} / K}(f)$ is $\mathcal{A}_{K}$-measurable.

Step 4: It remains to prove that the $\sigma$-algebras $\sigma(\mathcal{C})$ and $\mathcal{A}_{K^{\prime}}$ are the same. Clearly one has $\sigma(\mathcal{C}) \subseteq \mathcal{A}_{K^{\prime}}$ since any function in $\mathcal{C}$ is $\mathcal{A}_{K^{\prime}}$-measurable. To prove the equality it suffices to show that any function of the form $f_{b}$ with $b \in K^{\prime}$ is $\sigma(\mathcal{C})$-measurable. Let

$$
T^{m}+\mu_{1} T^{m-1}+\cdots+\mu_{m} \in K[T]
$$

be the minimal polynomial of $b$ over $K$. Then for any $\omega \in \Omega_{K}$ and any $x \in M_{K^{\prime}, \omega}$, one has

$$
|b|_{x} \leqslant m \cdot \max \left\{1,\left|\mu_{1}\right|_{\omega}, \ldots,\left|\mu_{m}\right|_{\omega}\right\}
$$

since otherwise one should have

$$
1>\frac{\left|\mu_{1}\right|_{\omega}}{|b|_{x}}+\cdots+\frac{\left|\mu_{m}\right|_{\omega}}{|b|_{x}} \geqslant \sum_{i=1}^{m} \frac{\left|\mu_{i}\right|_{\omega}}{|b|_{x}^{i}},
$$

which contradicts the equality

$$
b^{m}=-\mu_{1} b^{m-1}-\cdots-\mu_{m} .
$$

For any $N \in \mathbb{N}$, let $A_{N}$ be the set

$$
\left\{\omega \in \Omega_{K}: \max \left\{\left|\mu_{1}\right|_{\omega}, \ldots,\left|\mu_{m}\right|_{\omega}\right\} \leqslant N\right\} \in \mathcal{A}_{K} .
$$

The relation (3.6) shows that the function $f_{b} \cdot\left(\mathbb{1}_{A_{N}} \circ \pi_{K^{\prime} / K}\right)$ is non-negative and bounded. Hence it belongs to $\mathcal{C}$. Finally, since

$$
f_{b}=\lim _{N \rightarrow+\infty} f_{b} \cdot\left(\mathbb{1}_{A_{N}} \circ \pi_{K^{\prime} / K}\right)
$$

we obtain that the function $f_{b}$ is $\sigma(\mathcal{C})$-integrable. The theorem is thus proved. 
3.3.3. Construction of the measure. - In this subsection, we describe the construction of a suitable measure on the measurable space $\left(\Omega_{K^{\prime}}, \mathcal{A}_{K^{\prime}}\right)$ to form an adelic structure on $K^{\prime}$ and prove some compatibility results.

Definition 3.3.6. - We denote by $\nu_{K^{\prime}}: \mathcal{A}_{K^{\prime}} \rightarrow \mathbb{R}_{+} \cup\{+\infty\}$ the map defined as follows:

$$
\forall A \in \mathcal{A}_{K^{\prime}}, \quad \nu_{K^{\prime}}(A):=\int_{\Omega_{K}} I_{K^{\prime} / K}\left(\mathbb{1}_{A}\right) \mathrm{d} \nu_{K} .
$$

By Theorem 3.3.4, this map is well defined.

Theorem 3.3.7. - (1) The map $\nu_{K^{\prime}}$ is a measure on the measurable space $\left(\Omega_{K^{\prime}}, \mathcal{A}_{K^{\prime}}\right)$ such that, for any non-negative $\mathcal{A}_{K^{\prime}}$-measurable function $f$ on $\Omega_{K^{\prime}}$ one has

$$
\int_{\Omega_{K^{\prime}}} f \mathrm{~d} \nu_{K^{\prime}}=\int_{\Omega_{K}} I_{K^{\prime} / K}(f) \mathrm{d} \nu_{K}
$$

(2) A real-valued $\mathcal{A}_{K^{\prime}}$-measurable function $f$ on $\Omega_{K^{\prime}}$ is integrable with respect to $\nu_{K^{\prime}}$ if and only if $I_{K^{\prime} / K}(|f|)$ is integrable with respect to $\nu_{K}$. Moreover, the equality (3.7) also holds for all real-valued $\mathcal{A}_{K}^{\prime}$-measurable functions on $\Omega_{K^{\prime}}$ which are integrable with respect to $\nu_{K^{\prime}}$.

(3) The direct image of the measure $\nu_{K^{\prime}}$ by the measurable map $\pi_{K^{\prime} / K}$ coincides with $\nu_{K}$, namely for any real-valued $\mathcal{A}_{K}$-measurable function $\varphi$ on $\Omega_{K}$ which is non-negative (resp. integrable with respect to $\nu_{K}$ ), the function $\varphi \circ \pi_{K^{\prime} / K}$ is non-negative (resp. integrable with respect to $\nu_{K^{\prime}}$ ), and one has

$$
\int_{\Omega_{K^{\prime}}}\left(\varphi \circ \pi_{K^{\prime} / K}\right) \mathrm{d} \nu_{K^{\prime}}=\int_{\Omega_{K}} \varphi \mathrm{d} \nu_{K}
$$

(4) $S^{\prime}=\left(K^{\prime},\left(\Omega_{K^{\prime}}, \mathcal{A}_{K^{\prime}}, \nu_{K^{\prime}}\right), \phi_{K^{\prime}}\right)$ is an adelic curve.

(5) For $b \in K^{\prime} \backslash\{0\}$, one has

$$
\left[K^{\prime}: K\right] \int_{\Omega_{K^{\prime}}} \ln |b|_{x} \mathrm{~d} \nu_{K^{\prime}}=\int_{\Omega_{K}} \ln \left|N_{K^{\prime} / K}(b)\right|_{\omega} \mathrm{d} \nu_{K},
$$

where $N_{K^{\prime} / K}(b)$ is the norm of $b$ with respect to the extension $K^{\prime} / K$. In particular, if $S$ is proper, then $S^{\prime}$ is also proper.

Proof. - (1) The operator $I_{K^{\prime} / K}$ preserve pointwise limits. Therefore, if $\left\{A_{n}\right\}_{n \in \mathbb{N}}$ is a countable family of disjoint sets in $\mathcal{A}_{K^{\prime}}$ and if $A=\bigcup_{n \in \mathbb{N}} A_{n}$, one has

$$
\nu_{K^{\prime}}(A)=\int_{\Omega_{K}} I_{K^{\prime} / K}\left(\mathbb{1}_{A}\right) \mathrm{d} \nu_{K}=\int_{\Omega_{K}} \sum_{n \in \mathbb{N}} I_{K^{\prime} / K}\left(\mathbb{1}_{A_{n}}\right) \mathrm{d} \nu_{K}=\sum_{n \in \mathbb{N}} \nu_{K^{\prime}}\left(A_{n}\right),
$$

where the last equality comes from the monotone convergence theorem.

The set of all non-negative and bounded $\mathcal{A}_{K^{\prime}}$-measurable functions $f$ which verify the equality (3.7) forms a $\lambda$-family. Moreover, this $\lambda$-family contains the set of all functions of the form $\mathbb{1}_{A}\left(A \in \mathcal{A}_{K^{\prime}}\right)$, which is stable by multiplication. By Theorem A.1.3, we obtain that the equality (3.7) actually holds for all non-negative and 
bounded $\mathcal{A}_{K^{\prime}}$-measurable functions, and hence holds for general non-negative $\mathcal{A}_{K^{\prime}}$ measurable functions by the monotone convergence theorem again.

(2) The equality (3.7) clearly implies that a real-valued $\mathcal{A}_{K^{\prime}}$-measurable function $f$ on $\Omega_{K^{\prime}}$ is integrable with respect to $\nu_{K^{\prime}}$ if and only if $I_{K^{\prime} / K}(|f|)$ is integrable with respect to $\nu_{K}$. Moreover, if $f$ is a real-valued $\mathcal{A}_{K^{\prime}}$-measurable function on $\Omega_{K^{\prime}}$ which is integrable with respect to $\nu_{K^{\prime}}$, then the equality (3.7) applied to $\max (f, 0)$ and $-\min (f, 0)$ shows that

$$
\int_{\Omega_{K^{\prime}}} \max (f, 0) \mathrm{d} \nu_{K^{\prime}}=\int_{\Omega_{K}} I_{K^{\prime} / K}(\max (f, 0)) \mathrm{d} \nu_{K}
$$

and

$$
\int_{\Omega_{K^{\prime}}}(-\min (f, 0)) \mathrm{d} \nu_{K^{\prime}}=\int_{\Omega_{K}} I_{K^{\prime} / K}(-\min (f, 0)) \mathrm{d} \nu_{K} .
$$

Since these numbers are finite, the difference of the above two equalities leads to

$$
\int_{\Omega_{K^{\prime}}} f \mathrm{~d} \nu_{K^{\prime}}=\int_{\Omega_{K}} I_{K^{\prime} / K}(f) \mathrm{d} \nu_{K} .
$$

(3) By the two assertions proved above, one has

$$
\int_{\Omega_{K^{\prime}}}\left(\varphi \circ \pi_{K^{\prime} / K}\right) \mathrm{d} \nu_{K^{\prime}}=\int_{\Omega_{K}} I_{K^{\prime} / K}\left(\varphi \circ \pi_{K^{\prime} / K}\right) \mathrm{d} \nu_{K} .
$$

By Corollary 1.2.33, one has

$$
I_{K^{\prime} / K}\left(\varphi \circ \pi_{K^{\prime} / K}\right)=\varphi .
$$

Thus we obtain (3.8).

(4) Let $b$ be an element in $K^{\prime} \backslash\{0\}$ and $f_{b}$ be the function on $\Omega_{K^{\prime}}$ sending $x \in \Omega_{K^{\prime}}$ to $|b|_{x}$. Let $\lambda=N_{K^{\prime} / K}(b)$ be the norm of $b$ with respect to the extension $K^{\prime} / K$. For any $\omega \in \Omega_{K}$ one has (see [110 Chapter II, Corollary 8.4 and page 161)

$$
\prod_{x \in M_{K^{\prime}, \omega}}|b|_{x}^{\left[K_{x}^{\prime}: K_{\omega}\right]}=|\lambda|_{\omega}
$$

which implies that

$$
I_{K / K^{\prime}}\left(\ln f_{b}\right)=\frac{1}{\left[K^{\prime}: K\right]} \ln f_{\lambda},
$$

where $f_{\lambda}$ is the function on $\Omega_{K}$ which sends $\omega \in \Omega_{K}$ to $|\lambda|_{\omega}$.

Let $\Omega_{K, \infty}$ be the set of all $\omega \in \Omega_{K}$ such that $|\cdot|_{\omega}$ is an Archimedean absolute value. By Proposition 3.1.1 this is an element of $\mathcal{A}_{K}$. Similarly, let $\Omega_{K^{\prime}, \infty}$ be the set of all $x \in \Omega_{K^{\prime}}$ such that the absolute value $|\cdot|_{x}$ is Archimedean. One has $\Omega_{K^{\prime}, \infty}=\pi_{K^{\prime} / K}^{-1}\left(\Omega_{K, \infty}\right)$. We will prove the integrability of $\ln f_{b}$ on $\Omega_{K^{\prime}} \backslash \Omega_{K^{\prime}, \infty}$ and on $\Omega_{K^{\prime}, \infty}$, respectively. For this purpose we use a refinement of the method in the Step 4 of the proof of Theorem 3.3.4.

Let

$$
T^{m}+\mu_{1} T^{m-1}+\cdots+\mu_{m} \in K[T]
$$


be the minimal polynomial of $b$ over $K$. Then for any $\omega \in \Omega_{K} \backslash \Omega_{K, \infty}$ and any $x \in M_{K^{\prime}, \omega}$, one has

$$
|b|_{x} \leqslant \max \left\{1,\left|\mu_{1}\right|_{\omega}, \ldots,\left|\mu_{m}\right|_{\omega}\right\} .
$$

Otherwise one should have

$$
1>\max _{i \in\{1, \ldots, m\}} \frac{\left|\mu_{i}\right|_{\omega}}{|b|_{x}} \geqslant \max _{i \in\{1, \ldots, m\}} \frac{\left|\mu_{i}\right|_{\omega}}{|b|_{x}^{i}} .
$$

However, the equality

$$
b^{m}=-\mu_{1} b^{m-1}-\cdots-\mu_{m} .
$$

implies that

$$
|b|_{x}^{m} \leqslant \max _{i \in\{1, \ldots, m\}}\left|\mu_{i}\right|_{\omega} \cdot|b|_{x}^{m-i},
$$

which leads to a contradiction. Therefore, if we denote by $g$ the function

$$
\omega \longmapsto \max \left\{0, \ln \left|\mu_{1}\right|_{\omega}, \ldots \ln \left|\mu_{m}\right|_{\omega}\right\}
$$

on $\Omega_{K}$, then $\ln f_{b}$ is bounded from above by $g \circ \pi_{K^{\prime} / K}$ on $\Omega_{K} \backslash \Omega_{K, \infty}$. Moreover, by the definition of adelic curves, the functions $\omega \mapsto \ln \left|\mu_{i}\right|_{\omega}$ is integrable for any $i \in\{1, \ldots, m\}$, hence also is the function $g$. The function

$$
\left(g \circ \pi_{K^{\prime} / K}-\ln f_{b}\right) \mathbb{1}_{\Omega_{K^{\prime}} \backslash \Omega_{K^{\prime}, \infty}}
$$

is non-negative, and

$$
\begin{aligned}
& I_{K^{\prime} / K}\left(\left(g \circ \pi_{K^{\prime} / K}-\ln f_{b}\right) \mathbb{1}_{\Omega_{K^{\prime}} \backslash \Omega_{K^{\prime}, \infty}}\right) \\
= & \left(I_{K^{\prime} / K}\left(g \circ \pi_{K^{\prime} / K}\right)-I_{K^{\prime} / K}\left(\ln f_{b}\right)\right) \mathbb{1}_{\Omega_{K} \backslash \Omega_{K, \infty}} \\
= & \left(g-\frac{1}{\left[K^{\prime}: K\right]} \ln f_{\lambda}\right) \mathbb{1}_{\Omega_{K} \backslash \Omega_{K, \infty}}
\end{aligned}
$$

is an integrable function with respect to $\nu_{K}$, where the first equality comes from Proposition 3.3.1 and the fact that $I_{K^{\prime} / K}$ is a linear operator, and the second equality comes from Corollary 1.2 .33 and (3.10). By the second assertion of the theorem, the function $\left(g \circ \pi_{K^{\prime} / K}-\ln f_{b}\right) \mathbb{1}_{\Omega_{K^{\prime}} \backslash \Omega_{K^{\prime}, \infty}}$ is integrable, and hence also is $\left(\ln f_{b}\right) \mathbb{1}_{\Omega_{K^{\prime}} \backslash \Omega_{K^{\prime}, \infty}}$.

We now consider the Archimedean case. We assume that $\Omega_{\infty}$ is non-empty. Then the characteristic of the field $K$ is zero. In particular, it contains $\mathbb{Q}$ as its prime field. Moreover, for any $x \in M_{K^{\prime}, \omega}$, one has

$$
|b|_{x} \leqslant m^{\kappa(\omega)} \cdot \max \left\{1,\left|\mu_{1}\right|_{\omega}, \ldots,\left|\mu_{m}\right|_{\omega}\right\},
$$

where $\kappa(\omega)$ is the exponent of $|\cdot|_{\omega}$ as a power of the usual absolute value on $K_{\omega}=\mathbb{R}$ or $\mathbb{C}$. Otherwise one should have

$$
1>\frac{\left|\mu_{1}\right|_{\omega}^{1 / \kappa(\omega)}}{|b|_{x}^{1 / \kappa(\omega)}}+\cdots+\frac{\left|\mu_{m}\right|_{\omega}^{1 / \kappa(\omega)}}{|b|_{x}^{1 / \kappa(\omega)}} \geqslant \sum_{i=1}^{m} \frac{\left|\mu_{i}\right|_{\omega}^{1 / \kappa(\omega)}}{|b|_{x}^{i / \kappa(\omega)}} .
$$

However, the equality

$$
b^{m}=-\mu_{1} b^{m-1}-\cdots-\mu_{m} .
$$


implies that

$$
|b|_{x}^{m / \kappa(\omega)} \leqslant\left|\mu_{1}\right|_{\omega}^{1 / \kappa(\omega)}|b|_{x}^{(m-1) / \kappa(\omega)}+\cdots+\left|\mu_{m}\right|_{\omega}^{1 / \kappa(\omega)}
$$

since $|\cdot|_{\omega}^{1 / \kappa(\omega)}$ and $|\cdot|_{x}^{1 / \kappa(\omega)}$ are absolute values on $K^{\prime}$ and $K$ respectively (which extend the usual absolute value on $\mathbb{Q}$ ). Therefore, the function $\ln f_{b}$ is bounded from above by $(\ln (m) \kappa+g) \circ \pi_{K^{\prime} / K}$ on $\Omega_{K, \infty}$, where $g$ is the function defined in (3.11), and we have extended the function $\kappa$ on $\Omega_{K}$ by taking the value 0 on $\Omega_{K} \backslash \Omega_{K, \infty}$. Since the function $\ln (m) \kappa+g$ is integrable, by the same argument as in the non-Archimedean case, we obtain the integrability of the function $\ln f_{b}$ on $\Omega_{K^{\prime}, \infty}$.

(5) follows from (3.7) and (3.10).

\subsection{General algebraic extensions}

Let $S=\left(K,\left(\Omega_{K}, \mathcal{A}_{K}, \nu_{K}\right), \phi_{K}\right)$ be an adelic curve. In this section, we consider the construction of adelic curves from $S$ whose underlying field are general algebraic extensions of $K$.

3.4.1. Finite extension. - Let $K^{\prime \prime}$ be a finite extension of $K$ and $K^{\prime}$ be the separable closure of $K$ in the field $K^{\prime \prime}$. By the result of the previous section, one can construct an adelic structure on the field $K^{\prime}$ which we denote by $\left(\left(\Omega_{K^{\prime}}, \mathcal{A}_{K^{\prime}}, \nu_{K^{\prime}}\right), \phi_{K^{\prime}}\right)$.

Note that $K^{\prime \prime}$ is a purely inseparable extension of $K^{\prime}$ (see [29] Chapter V, §7, no.7, Proposition 13.a). If $q$ is the degree of the extension $K^{\prime \prime} / K^{\prime}$, then for any $\alpha \in K^{\prime \prime}$ one has $\alpha^{q} \in K^{\prime}$ (see [29] Chapter V, §5, no.1, Proposition 1). In particular, any absolute value $|\cdot|$ on $K^{\prime}$ extends in a unique way to $K^{\prime \prime}$ and one has

$$
\forall \alpha \in K^{\prime \prime}, \quad|\alpha|=\left|\alpha^{q}\right|^{1 / q},
$$

where $|\alpha|$ denotes the extended absolute value on $K^{\prime \prime}$ evaluated on $\alpha$, and $\left|\alpha^{q}\right|$ denotes the initial absolute value on $K^{\prime}$ evaluated on $\alpha^{q}$. In other words, the sets $M_{K^{\prime}}$ and $M_{K^{\prime \prime}}$ are in canonical bijection. This observation permits to construct, for any $\alpha \in K^{\prime \prime} \backslash\{0\}$ the function

$$
\Omega_{K^{\prime}} \rightarrow \mathbb{R}, \quad\left(x \in \Omega_{K^{\prime}}\right) \mapsto \ln |\alpha|_{x}
$$

This function is clearly $\mathcal{A}_{K^{\prime}}$-measurable since one has

$$
\forall x \in \Omega_{K^{\prime}}, \quad \ln |\alpha|_{x}=\frac{1}{q} \ln \left|\alpha^{q}\right|_{x} .
$$

Moreover, it is also integrable with respect to $\nu_{K^{\prime}}$ and one has

$$
\int_{\Omega_{K^{\prime}}} \ln |\alpha|_{x} \nu_{K^{\prime}}(\mathrm{d} x)=\frac{1}{q} \int_{\Omega_{K^{\prime}}} \ln \left|\alpha^{q}\right|_{x} \nu_{K^{\prime}}(d x) .
$$

This fact shows that

$$
\left(K^{\prime \prime},\left(\Omega_{K^{\prime}}, \mathcal{A}_{K^{\prime}}, \nu_{K^{\prime}}\right), \phi_{K^{\prime}}\right)
$$


is actually an adelic curve, where we identify $M_{K^{\prime}}$ with $M_{K^{\prime \prime}}$. Note that the relation (3.13) shows that $\mathcal{A}_{K^{\prime}}$ is also the smallest $\sigma$-algebra on $\Omega_{K^{\prime}}$ which makes the canonical projection map $\Omega_{K^{\prime}} \rightarrow \Omega_{K}$ and the functions $\left(x \in \Omega_{K^{\prime}}\right) \mapsto|\alpha|_{x}$ measurable, where $\alpha \in K^{\prime \prime}$.

Definition 3.4.1. - We denote by $S \otimes_{K} K^{\prime \prime}$ the adelic curve

$$
\left(K^{\prime \prime},\left(\Omega_{K^{\prime}}, \mathcal{A}_{K^{\prime}}, \nu_{K^{\prime}}\right), \phi_{K^{\prime}}\right)
$$

constructed as above, called the finite extension of $S$ induced by the extension of fields $K^{\prime \prime} / K$. We also use the expression $\pi_{K^{\prime \prime} / K}$ to denote the projection map $\pi_{K^{\prime} / K}$ : $\Omega_{K^{\prime}} \rightarrow \Omega_{K}$ described in the previous section. Similarly, we also use the expression $I_{K^{\prime \prime} / K}$ to denote the operator $I_{K^{\prime} / K}$. Note that $\Omega_{K}^{\prime}$ identifies also with the fibre product of $\Omega_{K}$ and $M_{K^{\prime \prime}}$ over $M_{K}$ in the category of sets since $M_{K^{\prime}}$ and $M_{K^{\prime \prime}}$ are the same. Similarly, $\phi_{K^{\prime}}$ identifies with the projection map from $\Omega_{K^{\prime}}=\Omega_{K} \times_{M_{K}} M_{K^{\prime \prime}}$ to $M_{K^{\prime \prime}}=M_{K^{\prime}}$. Note that if $S$ is proper, then $S \otimes_{K} K^{\prime \prime}$ is also proper (cf. (3.14) and Theorem 3.3.7 (5).

In the following, we will prove that the above construction of adelic curves is compatible with successive finite extensions. The lemma below is important for the proof.

Lemma 3.4.2. - Let $L / K$ be a finite extension of fields, $|\cdot|_{v}$ be an absolute value on $K$, and $|\cdot|_{w}$ be an absolute value on $L$ extending $|\cdot|_{v}$. Let $K^{\mathrm{sc}}$ be the separable closure of $K$ in $L$. Then the completion $K_{w}^{\mathrm{sc}}$ of $K^{\mathrm{sc}}$ with respect to the absolute value $|\cdot|_{w}$ identifies with the separable closure of $K_{v}$ in $L_{w}$.

Proof. - The case where $|\cdot|_{v}$ is Archimedean is trivial since the characteristic of the field $K$ is then zero and hence $K^{\mathrm{sc}}=L$. In the following, we assume that $|\cdot|_{v}$ is nonArchimedean. We first prove that the extension $K_{w}^{\text {sc }} / K_{v}$ is separable. Let $\alpha \in K^{\text {sc }}$ be a primitive element (see 29 Chapter $\mathrm{V}, \S 7$, no.4 Theorem 1 for its existence) of the separable extension $K^{\text {sc }} / K$ and let $F$ be its minimal polynomial. Assume that $F$ is decomposed in $K_{v}^{\mathrm{sc}}[T]$ into the product of distinct irreducible polynomials as $F=F_{1} \cdots F_{m}$. Since $F$ is a separable polynomial, the same are the polynomials $F_{1}, \ldots, F_{m}$. For any extension $|\cdot|_{w}$ of the absolute value $|\cdot|_{v}$ to $K^{\text {sc }}$, there exists an index $i \in\{1, \ldots, m\}$ such that $K_{w}^{\mathrm{sc}} \cong K_{v}[T] /\left(F_{i}\right)$ (see [110 Chapter II, Propositions 8.2 and 8.3). Therefore the extension $K_{w}^{\mathrm{sc}} / K_{v}$ is separable.

In the following, we prove that the extension $L_{w} / K_{w}^{\text {sc }}$ is purely inseparable. Note that $L_{w}=K_{v}(L)=K_{w}^{\mathrm{sc}}(L)$. Since the extension $L / K^{\mathrm{sc}}$ is purely inseparable, we obtain that the extension $L_{w} / K_{w}^{\mathrm{sc}}$ is also purely inseparable since it is generated by purely inseparable elements (see 29] Chapter V, $\S 7$, no.2, the corollary of Proposition 2). By [29] Chapter V, $\S 7$, no.7, Proposition 13.c, we obtain that $K_{w}^{\mathrm{sc}}$ is the separable closure of $K_{v}$ in $L_{w}$ since $K_{w}^{\mathrm{sc}} / K_{v}$ is separable and $L_{w} / K_{w}^{\mathrm{sc}}$ is purely inseparable. 
Remark 3.4.3. - Let $K^{\prime \prime} / K$ be a finite extension of fields and denote by $\left(K^{\prime \prime},\left(\Omega^{\prime \prime}, \mathcal{A}^{\prime \prime}, \nu^{\prime \prime}\right), \phi^{\prime \prime}\right)$ the adelic curve $S \otimes_{K} K^{\prime \prime}$. The above lemma allows to write the operator $I_{K^{\prime \prime} / K}$ in the following form: for any real-valued $\mathcal{A}^{\prime \prime}$-measurable function $f$ on $\Omega^{\prime \prime}$

$$
\forall \omega \in \Omega_{K}, \quad\left(I_{K^{\prime \prime} / K}(f)\right)(\omega)=\sum_{x \in M_{K^{\prime \prime}, \omega}} \frac{\left[K_{x}^{\prime \prime}: K_{\omega}\right]_{s}}{\left[K^{\prime \prime}: K\right]_{s}} f(x),
$$

where for any finite extension $L_{2} / L_{1}$ of fields, the expression $\left[L_{2}: L_{1}\right]_{s}$ denotes the separable degree of the extension $L_{2} / L_{1}$.

Proposition 3.4.4. - Let $K_{2} / K_{1} / K$ be successive finite extensions of fields. If $\left(\left(\Omega_{K}, \mathcal{A}_{K}, \nu_{K}\right), \phi_{K}\right)$ is an adelic structure on $K$ and $S$ is the corresponding adelic curve, then one has

$$
\left(S \otimes_{K} K_{1}\right) \otimes_{K_{1}} K_{2}=S \otimes_{K} K_{2}
$$

Moreover, one has

$$
\pi_{K_{1} / K} \circ \pi_{K_{2} / K_{1}}=\pi_{K_{2} / K}, \quad I_{K_{1} / K} \circ I_{K_{2} / K_{1}}=I_{K_{2} / K},
$$

where we have used the conventions of notation described in Definition 3.4.1.

Proof. - We denote by $\left(K_{1},\left(\Omega_{1}, \mathcal{A}_{1}, \nu_{1}\right), \phi_{1}\right)$ and $\left(K_{2},\left(\Omega_{2}, \mathcal{A}_{2}, \nu_{2}\right), \phi_{2}\right)$ the adelic curves $S \otimes_{K} K_{1}$ and $S \otimes_{K} K_{2}$ respectively. First of all, set-theoretically one has

$$
\Omega_{2}=\coprod_{\omega \in \Omega_{K}} M_{K_{2}, \omega}=\coprod_{\omega \in \Omega_{K}} \coprod_{x \in M_{K_{1}, \omega}} M_{K_{2}, x}=\coprod_{x \in \Omega_{1}} M_{K_{2}, x}
$$

and hence

$$
\pi_{K_{2} / K}=\pi_{K_{1} / K} \circ \pi_{K_{2} / K_{1}}
$$

Moreover, if $f$ is a real-valued function on $\Omega_{2}$, by Lemma 3.4.2 and Remark 3.4.3, for any $\omega \in \Omega_{K}$ one has

$$
\begin{aligned}
& \left(I_{K_{2} / K}(f)\right)(\omega)=\sum_{y \in M_{K_{2}, \omega}} \frac{\left[K_{2, y}: K_{\omega}\right]_{s}}{\left[K_{2}: K\right]_{s}} f(y) \\
= & \sum_{x \in M_{K_{1}, \omega}} \frac{\left[K_{1, x}: K_{\omega}\right]_{s}}{\left[K_{1}: K\right]_{s}} \sum_{y \in M_{K_{2}, x}} \frac{\left[K_{2, y}: K_{1, x}\right]_{s}}{\left[K_{2}: K_{1}\right]_{s}} f(y)=I_{K_{1} / K}\left(I_{K_{2} / K_{1}}(f)\right)(\omega),
\end{aligned}
$$

where in the second equality we have used the multiplicativity of the separable degree (see 29] Chapter V, §6, no.5).

We then show that the map $\pi_{K_{2} / K_{1}}$ is $\mathcal{A}_{2}$-measurable. Since the $\sigma$-algebra $\mathcal{A}_{1}$ is generated by $\pi_{K_{1} / K}$ and functions of the form $f_{a}:\left(x \in \Omega_{1}\right) \mapsto|a|_{x}$ with $a \in K_{1}$, it suffices to prove that the maps $\pi_{K_{1} / K} \circ \pi_{K_{2} / K_{1}}$ and $f_{a} \circ \pi_{K_{2} / K_{1}}$ are $\mathcal{A}_{2}$-measurable. We have shown that $\pi_{K_{1} / K} \circ \pi_{K_{2} / K_{1}}=\pi_{K_{2} / K}$, which is clearly $\mathcal{A}_{2}$-measurable by the definition of the adelic curve $S \otimes_{K} K_{2}$. Moreover, if $a$ is an element in $K_{1}$, then the map $f_{a} \circ \pi_{K_{2} / K_{1}}$ sends $y \in \Omega_{2}$ to $|a|_{y}$. Hence it is also $\mathcal{A}_{2}$-measurable. In particular, the $\sigma$-algebra $\mathcal{A}_{2}$ contains the $\sigma$-algebra $\mathcal{A}_{2}^{\prime}$ in the adelic structure of 
$\left(S \otimes_{K} K_{1}\right) \otimes_{K_{1}} K_{2}$, namely the smallest $\sigma$-algebra which makes the map $\pi_{K_{2} / K_{1}}$ and all functions of the form $\left(y \in \Omega_{K}\right) \mapsto|\alpha|_{y}$ measurable, where $\alpha$ runs over $K_{2}$.

From the equality $\pi_{K_{1} / K} \circ \pi_{K_{2} / K_{1}}=\pi_{K_{2} / K}$ we also obtain that $\pi_{K_{2} / K}^{-1}\left(\mathcal{A}_{K}\right)$ is contained in $\pi_{K_{2} / K_{1}}^{-1}\left(\mathcal{A}_{1}\right)$, and thus is contained in $\mathcal{A}_{2}^{\prime}$ since $\pi_{K_{2} / K_{1}}$ is $\mathcal{A}_{2}^{\prime}$-measurable. Since $\mathcal{A}_{2}$ is the smallest $\sigma$-algebra on $\Omega_{2}$ which makes the map $\pi_{K_{2} / K}$ and all functions of the form $\left(y \in \Omega_{K}\right) \mapsto|\alpha|_{y}$ measurable, we obtain that $\mathcal{A}_{2} \subseteq \mathcal{A}_{2}^{\prime}$. Combining with the result obtained above, we obtain that the $\sigma$-algebras $\mathcal{A}_{2}$ and $\mathcal{A}_{2}^{\prime}$ coincide.

Finally, the relation $I_{K_{1} / K} \circ I_{K_{2} / K_{1}}=I_{K_{2} / K}$ shows that the measure $\nu_{2}$ coincides with the measure in the adelic structure of the adelic curve $\left(S \otimes_{K} K_{1}\right) \otimes_{K_{1}} K_{2}$. The proposition is thus proved.

3.4.2. General algebraic extensions. - We now consider an algebraic extension $L$ of $K$ which is not necessarily finite. Let $\mathscr{E}_{L / K}$ be the set of all finite extensions of $K$ which are contained in $L$. This set is ordered by the relation of inclusion. Moreover, it is also filtered in the sense that, if $K_{1}$ and $K_{2}$ are two finite extensions of $K$ which are contained in $L$, then there exists a finite extension $K_{3} \in \mathscr{E}_{L / K}$ such that $K_{3} \supseteq K_{1} \cup K_{2}$.

By the result obtained in the previous subsection, for each element $K^{\prime \prime}$ in $\mathscr{E}_{L / K}$, we can equipped $K^{\prime \prime}$ with a natural adelic structure induced from the adelic structure of $S$, as described in Definition 3.4.1 We denote by $\left(\left(\Omega_{K^{\prime \prime}}, \mathcal{A}_{K^{\prime \prime}}, \nu_{K^{\prime \prime}}\right), \phi_{K^{\prime \prime}}\right)$ this adelic structure. Moreover, Proposition 3.4 .4 shows that, for successive finite extensions $K_{2} / K_{1} / K$ of the field $K$ which are contained in $L$, there exist a natural projection

$$
\pi_{K_{2} / K_{1}}:\left(\Omega_{K_{2}}, \mathcal{A}_{K_{2}}\right) \longrightarrow\left(\Omega_{K_{1}}, \mathcal{A}_{K_{1}}\right)
$$

together with a disintegration operator $I_{K_{2} / K_{1}}$ from the vector space of all real-valued $\mathcal{A}_{K_{2}}$-measurable functions on $\Omega_{K_{2}}$ to that of all real-valued $\mathcal{A}_{K_{1}}$-measurable functions on $\Omega_{K_{1}}$, which sends $\nu_{K_{2}}$-integrable functions to $\nu_{K_{1}}$-integrable functions. These data actually define a functor from a filtered ordered set to the category of measure spaces. Intuitively one can define an adelic structure on $L$ whose measure space part is the projective limit of this functor. However, the projective limite in the category of measure spaces does not exist in general (the product of infinitely many measures need not make sense). Therefore more careful treatment is needed for our setting of projective system of finite extensions of adelic curves. Our strategy is to construct the fibres as projective limits of probability spaces, which always exist.

For any $\omega \in \Omega_{K}$, let $M_{L, \omega}$ be the set of all absolute values on $L$ which extend $|\cdot|_{\omega}$. Let $\Omega_{L}$ be the disjoint union of all $M_{L, \omega}$ with $\omega$ runs through $\Omega_{K}$. In other words, $\Omega_{L}$ is the fibre product of $\Omega_{K}$ and $M_{L}$ over $M_{K}$. The inclusion maps $M_{L, \omega} \rightarrow M_{L}$ define a map from $\Omega_{L}$ to $M_{L}$ which we denote by $\phi_{L}$. Moreover, for any extension $K^{\prime \prime} \in \mathscr{E}_{L / K}$ and any $\omega \in \Omega_{K}$, one has a natural map from $M_{L, \omega}$ to $M_{K^{\prime \prime}, \omega}$ defined by restriction of absolute values. These maps induce a map from $\Omega_{L}$ to $\Omega_{K^{\prime \prime}}$ which we denote by $\pi_{L / K^{\prime \prime}}$. If $x$ is an element of $\Omega_{K^{\prime \prime}}$, we denote by $M_{L, x}$ the set of absolute 
values on $L$ which extends $|\cdot|_{x}$. It identifies with the inverse image of $\{x\}$ by $\pi_{L / K^{\prime \prime}}$. If $K_{1} \subseteq K_{2}$ are extensions in $\mathscr{E}_{L / K}$, then one has

$$
\pi_{L / K_{1}}=\pi_{K_{2} / K_{1}} \circ \pi_{L / K_{2}}
$$

Proposition 3.4.5. - For any $K^{\prime \prime} \in \mathscr{E}_{L / K}$, the map $\pi_{L / K^{\prime \prime}}: \Omega_{L} \rightarrow \Omega_{K^{\prime \prime}}$ is surjective.

Proof. - Let $x$ be an element in $\Omega_{K^{\prime \prime}}$. The absolute value $|\cdot|_{\omega}$ extends in a unique way to the algebraic closure $\left(K_{x}^{\prime \prime}\right)^{\text {ac }}$ of $K_{x}^{\prime \prime}$ (see [110] Chapter II, Theorem 4.8). Therefore, if we choose an embedding of $L$ into $\left(K_{x}^{\prime \prime}\right)^{\text {ac }}$, then we obtain an absolute value on $L$ which extends $|\cdot|_{x}$.

Similarly to [63. Lemma 2.1], the set $\Omega_{L}$ described above gives an explicit construction of the projective limit of the projective system $\left\{\Omega_{K^{\prime \prime}}\right\}_{K^{\prime \prime} \in \mathscr{E}_{L / K}}$ in the category of sets, where $\left\{\pi_{L / K^{\prime \prime}}\right\}_{K^{\prime \prime} \in \mathscr{E}_{L / K}}$ are universal maps. In fact, any absolute value on $L$ is uniquely determined by its restrictions on the subfields in $\mathscr{E}_{L / K}$. We equip $\Omega_{L}$ with the $\sigma$-algebra $\mathcal{A}_{L}$ generated by the maps $\pi_{L / K^{\prime \prime}}$ (namely the smallest $\sigma$-algebra which makes all maps $\pi_{L / K^{\prime \prime}}$ measurable) where $K^{\prime \prime}$ runs over $\mathscr{E}_{L / K}$. Thus $\left(\Omega_{L}, \mathcal{A}_{L}\right)$ identifies with the projective limit of the projective system $\left\{\left(\Omega_{K^{\prime \prime}}, \mathcal{A}_{K^{\prime \prime}}\right)\right\}_{K^{\prime \prime} \in \mathscr{E}_{L / K}}$ in the category of mesurable spaces.

Let $\omega$ be an element in $\Omega_{K}$. We equip $M_{L, \omega}$ with the smallest $\sigma$-algebra $\mathcal{A}_{L, \omega}$ such that the restriction of $\pi_{L / K^{\prime \prime}}$ on $M_{L, \omega}$ is measurable for any $K^{\prime \prime} \in \mathscr{E}_{L / K}$, where we consider the discrete $\sigma$-algebra on $M_{K^{\prime \prime}, \omega}=\pi_{K^{\prime \prime} / K}^{-1}(\{\omega\})$. Let $V_{L, \omega}$ be the set of all real-valued functions on $\Omega_{L}$ which can be written in the form $f \circ\left(\left.\pi_{L / K^{\prime \prime}}\right|_{M_{L, \omega}}\right)$, where $K^{\prime \prime}$ is an element of $\mathscr{E}_{L / K}$, and $f$ is a function on $M_{K^{\prime \prime}, \omega}$. Let

$$
I_{L / K, \omega}: V_{L, \omega} \longrightarrow \mathbb{R}
$$

be the map which sends any function of the form $f \circ\left(\left.\pi_{L / K^{\prime \prime}}\right|_{M_{L, \omega}}\right)$ to the integral

$$
\int_{M_{K^{\prime \prime}, \omega}} f \mathrm{~d} \mathbb{P}_{K^{\prime}, \omega}
$$

where $K^{\prime}$ is the separable closure of $K$ in $K^{\prime \prime}$, and $\mathbb{P}_{K^{\prime}, \omega}$ is the probability measure on $M_{K^{\prime}, \omega}$ defined in (3.2). Similarly to (3.15), the fibre integral is compatible with successive finite extensions of the field $K$ and the map $I_{L / K, \omega}$ is well defined since the value of the integral (3.17) does not depend on the choice of the field $K^{\prime \prime}$ upon which we write the function in $V_{L, \omega}$ as the composition of a function on $M_{K^{\prime \prime}, \omega}$ with $\left.\pi_{L / K^{\prime \prime}}\right|_{M_{L, \omega}}$.

Proposition 3.4.6. - The set $V_{L, \omega}$ forms an algebra over $\mathbb{R}$ with respect to the composition laws of addition and multiplication of functions, and the map $I_{L / K, \omega}$ : $V_{L, \omega} \rightarrow \mathbb{R}$ is an $\mathbb{R}$-linear operator. Moreover, it induces a probability measure on the measurable space $\left(M_{L, \omega}, \mathcal{A}_{L, \omega}\right)$. 
Proof. - The first assertion comes from the fact that the set $\mathscr{E}_{L / K}$ is filtered, which implies that any finite collection of functions in $V_{L, \omega}$ descend on the same space $M_{K^{\prime \prime}, \omega}$, where $K^{\prime \prime} \in \mathscr{E}_{L / K}$. In particular, the family $\mathcal{D}$ of subsets $A \subseteq M_{L, \omega}$ such that $\mathbb{1}_{A} \in V_{L, \omega}$ is an algebra (of sets), which generates $\mathcal{A}_{L, \omega}$ as a $\sigma$-algebra. Moreover, the map $\mathbb{P}_{L, \omega}: \mathcal{D} \rightarrow \mathbb{R}_{+}$which sends $A \in \mathcal{D}$ to $I_{L / K, \omega}\left(\mathbb{1}_{A}\right)$ is an additive functional. Clearly it sends $M_{L, \omega}$ to 1 .

The $\sigma$-algebra $\mathcal{A}_{L, \omega}$ is actually the Borel algebra of the projective limit topology on $M_{L, \omega}$ (namely the most coarse topology on $M_{L, \omega}$ which makes all maps $\pi_{L / K^{\prime \prime}}$ continuous, where $K^{\prime \prime} \in \mathscr{E}_{L / K}$ ). This topology also identifies with the induced topology on $M_{L, \omega}$ viewed as a subset of $\prod_{F \in \mathscr{E}_{L / K}} M_{F, \omega}$ (equipped with the product topology), where on each set $M_{F, \omega}$ we consider the discrete topology. Note that $M_{L, \omega}$ is actually a closed subset of this product space since it is the intersection of closed subsets of the form

$$
W_{K^{\prime \prime}}:=\left\{\left(x_{F}\right)_{F \in \mathscr{E}_{L / K}} \in \prod_{F \in \mathscr{E}_{L / K}} M_{F, \omega}: \pi_{K^{\prime \prime} / F}\left(x_{K^{\prime \prime}}\right)=x_{F} \text { for } F \subseteq K^{\prime \prime}\right\} .
$$

Therefore, by Tychonoff's theorem, we obtain that $M_{L, \omega}$ is actually a compact topological space. Moreover, any set in $\mathcal{D}$ is open and closed since it is the inverse image of a discrete set by a continuous map. Therefore, the sets in $\mathcal{D}$ are open and compact. As a consequence, if $\left\{A_{n}\right\}_{n \in \mathbb{N}}$ is a sequence of disjoint sets in $\mathcal{D}$ whose union also lies in $\mathcal{D}$, then for sufficiently large $n$ one has $A_{n}=\varnothing$. Hence the function $\mathbb{P}_{L, \omega}: \mathcal{D} \rightarrow \mathbb{R}_{+}$is actually $\sigma$-additive. By Carathéodory's extension theorem, the function $\mathbb{P}_{L, \omega}$ extends to a Borel probability measure on $\left(M_{L, \omega}, \mathcal{A}_{L, \omega}\right)$ such that

$$
I_{L / K, \omega}(f)=\int_{M_{L, \omega}} f \mathrm{~d} \mathbb{P}_{L, \omega} .
$$

The proposition is thus proved.

Remark 3.4.7. - Let $V_{L}$ be the vector space of all real-valued functions $f$ on $\Omega_{L}$ which can be written as $g \circ \pi_{L / K^{\prime \prime}}$, where $K^{\prime \prime} / K$ is a finite extension which is contained in $L$. Then the above construction leads to a linear operator $I_{L / K}$ from $V_{L}$ to the vector space of all real-valued functions on $\Omega$, sending $f \in V_{L}$ to the function

$$
(\omega \in \Omega) \longmapsto I_{L / K, \omega}\left(\left.f\right|_{M_{L, \omega}}\right) .
$$

Clearly, if $g$ is real-valued function on $\Omega$, then $I_{L / K}\left(g \circ \pi_{L / K}\right)=g$.

The above proposition allows to define the fibre integration for non-negative $\mathcal{A}_{L^{-}}$ measurable functions on $\Omega_{L}$.

Proposition 3.4.8. - Let $f$ be a non-negative $\mathcal{A}_{L}$-measurable function on $\Omega_{L}$. For any $\omega \in \Omega_{K}$, the restriction of $f$ to $M_{L, \omega}$ is $\mathcal{A}_{L, \omega}$-measurable. Moreover, the map $I_{L / K}(f)$ from $\Omega_{K}$ to $[0,+\infty]$ which sends $\omega \in \Omega_{K}$ to $\int_{M_{L, \omega}} f(x) \mathbb{P}_{L, \omega}(\mathrm{d} x)$ is $\mathcal{A}_{K^{-}}$ measurable. 
Proof. - Let $\mathcal{H}$ be the set of all bounded non-negative functions $f$ on $\Omega_{K}$ such that $\left.f\right|_{M_{L, \omega}}$ is $\mathcal{A}_{L, \omega}$-measurable for any $\omega \in \Omega_{K}$ and that the map

$$
\left(\omega \in \Omega_{K}\right) \longmapsto \int_{M_{L, \omega}} f(x) \mathbb{P}_{L, \omega}(\mathrm{d} x)
$$

is $\mathcal{A}_{K}$-measurable. Then $\mathcal{H}$ is a $\lambda$-family of non-negative functions on $\Omega_{L}$ (see Definition A.1.1). Moreover, the set $\mathcal{H}$ contains the subset $\mathcal{C}$ of all bounded non-negative functions of the form $g \circ \pi_{L / K^{\prime \prime}}$, where $K^{\prime \prime}$ is an element in $\mathscr{E}_{L / K}$ and $g$ is an $\mathcal{A}_{K^{\prime \prime}-}$ measurable function on $\Omega_{K^{\prime \prime}}$. In fact, for any $\omega \in \Omega_{K}$, one has

$$
\int_{M_{L, \omega}} g\left(\pi_{L / K^{\prime \prime}}(x)\right) \mathbb{P}_{L, \omega}(\mathrm{d} x)=I_{K^{\prime \prime} / K}(g)(\omega) .
$$

Since $\mathcal{C}$ is stable under multiplication, by the monotone class theorem A.1.3 we obtain that the family $\mathcal{H}$ actually contains all non-negative, bounded and $\sigma(\mathcal{C})$-measurable functions. By definition, $\mathcal{A}_{L}$ is the $\sigma$-algebra generated by the maps $\pi_{L / K^{\prime \prime}}$ with $K^{\prime \prime} \in \mathscr{E}_{L / K}$. Therefore one has $\mathcal{A}_{L}=\sigma(\mathcal{C})$. Thus we obtain the result of the proposition for bounded non-negative $\mathcal{A}_{L}$-measurable functions. For general nonnegative $\mathcal{A}_{L}$-measurable function $f$, we can apply the assertion of the proposition to the functions $\{\min (f, n)\}_{n \in \mathbb{N}}$ which form an increasing sequence converging to $f$. Passing to limit when $n$ goes to the infinity, we obtain the result for $f$.

The above proposition allows to construct a measure $\nu_{L}$ on the measurable space $\left(\Omega_{L}, \mathcal{A}_{L}\right)$ such that, for any subset $A$ of $\mathcal{A}_{L}$, one has

$$
\nu_{L}(A)=\int_{\Omega_{K}}\left(\int_{M_{L, \omega}} \mathbb{1}_{A}(x) \mathbb{P}_{L, \omega}(\mathrm{d} x)\right) \nu_{K}(\mathrm{~d} \omega) .
$$

For any non-negative $\mathcal{A}_{L}$-measurable function $f$ on $\Omega_{L}$, one has

$$
\int_{\Omega_{L}} f(x) \nu_{L}(\mathrm{~d} x)=\int_{\Omega_{K}}\left(\int_{M_{L, \omega}} f(x) \mathbb{P}_{L, \omega}(\mathrm{d} x)\right) \nu_{K}(\mathrm{~d} \omega) .
$$

We denote by $I_{L / K}(f)$ the map from $\Omega_{K}$ to $[0,+\infty]$ which sends $\omega \in \Omega_{K}$ to $\int_{M_{L, \omega}} f(x) \mathbb{P}_{L, \omega}(\mathrm{d} x)$. More generally, for any $\mathcal{A}_{L}$-measurable function $f$ such that $I_{L / K}(|f|)$ is a real-valued function, we define $I_{L / K}(f)$ as the real-valued function

$$
I_{L / K}(\max (f, 0))-I_{L / K}(-\min (f, 0)) .
$$

Note that, if $f$ is of the form $g \circ \pi_{L / K^{\prime \prime}}$ where $K^{\prime \prime} \in \mathscr{E}_{L / K}$ and $g$ is an $\mathcal{A}_{K^{\prime \prime}}$-measurable function, then $I_{L / K}(f)$ is always well defined, and one has

$$
I_{L / K}\left(g \circ \pi_{L / K^{\prime \prime}}\right)=I_{K^{\prime \prime} / K}(g) .
$$

With this notation, the equality (3.18) can also be written as

$$
\int_{\Omega_{L}} f \mathrm{~d} \nu_{L}=\int_{\Omega_{K}} I_{L / K}(f) \mathrm{d} \nu_{K}
$$

Thus we obtain the following result. 
Proposition 3.4.9. $-A n \mathcal{A}_{L}$-measurable function $f$ is $\nu_{L}$-integrable if and only if $I_{L / K}(|f|)$ is $\nu_{K}$-integrable. Moreover, $I_{L / K}$ defines a continuous linear operator from $\mathscr{L}^{1}\left(\Omega_{L}, \mathcal{A}_{L}, \nu_{L}\right)$ to $\mathscr{L}^{1}\left(\Omega_{K}, \mathcal{A}_{K}, \nu_{K}\right)$, and the equality (3.20) also holds for $\nu_{L}$ integrable functions.

Note that the relations (3.19) and (3.20) also imply that, if $g$ is a function in $\mathscr{L}^{1}\left(\Omega_{K}, \mathcal{A}_{K}, \nu_{K}\right)$, then $g \circ \pi_{L / K}$ belongs to $\mathscr{L}^{1}\left(\Omega_{L}, \mathcal{A}_{L}, \nu_{L}\right)$, and one has

$$
\int_{\Omega_{L}}\left(g \circ \pi_{L / K}\right) \mathrm{d} \nu_{L}=\int_{\Omega_{K}} g \mathrm{~d} \nu_{K} .
$$

The following proposition shows that $\left(L,\left(\Omega_{L}, \mathcal{A}_{L}, \nu_{L}\right), \phi_{L}\right)$ forms an adelic curve.

Proposition 3.4.10. - For any non-zero element $a \in L$, the function

$$
\left(z \in \Omega_{L}\right) \longmapsto \ln |a|_{z}
$$

is $\mathcal{A}_{L}$-measurable. Moreover, if $S=\left(K,\left(\Omega_{K}, \mathcal{A}_{K}, \nu_{K}\right), \phi_{K}\right)$ is proper, then $\left(L,\left(\Omega_{L}, \mathcal{A}_{L}, \nu_{L}\right), \phi_{L}\right)$ is also proper.

Proof. - Denote by $g$ the function on $\Omega_{L}$ such that $g(z)=\ln |a|_{z}$. We choose a finite extension $K^{\prime \prime} \in \mathscr{E}_{L / K}$ which contains $a$. Let $f: \Omega_{K^{\prime \prime}} \rightarrow \mathbb{R}$ be the function which sends $x \in \Omega_{K^{\prime \prime}}$ to $\ln |a|_{x}$. Then $f$ is an $\mathcal{A}_{K^{\prime \prime}}$-measurable function on $\Omega_{K^{\prime \prime}}$. Since the function $g$ identifies with the composition $f \circ \pi_{L / K^{\prime \prime}}$, we obtain that $g$ is $\mathcal{A}_{L}$-measurable.

We assume that $S$ is proper. For any $\omega \in \Omega_{K}$, one has

$$
I_{L / K}(g)(\omega)=I_{L / K, \omega}(g)=I_{K^{\prime \prime} / K}(f)(\omega) .
$$

Therefore we obtain

$$
\int_{\Omega_{L}} g \mathrm{~d} \nu_{L}=\int_{\Omega_{K}} I_{K^{\prime \prime} / K}(f) \mathrm{d} \nu_{K}=0,
$$

where the second equality comes from Theorem 3.3.7 (1) and (5)

Definition 3.4.11. - Let $S=\left(K,\left(\Omega_{K}, \mathcal{A}_{K}, \nu_{K}\right), \phi_{K}\right)$ be an adelic curve and $L / K$ be an algebraic extension. The adelic curve $\left(L,\left(\Omega_{L}, \mathcal{A}_{L}, \nu_{L}\right), \phi_{L}\right)$ is called an algebraic extension of $S$, denoted by $S \otimes_{K} L$.

The following result, which is similar to Proposition 3.4.4 shows the compatibility property of the algebraic extensions of adelic curves.

Theorem 3.4.12. - Let $S=\left(K,\left(\Omega_{K}, \mathcal{A}_{K}, \nu_{K}\right), \phi_{K}\right)$ be an adelic curve and $L_{2} / L_{1} / K$ be successive algebraic extensions of fields. Then one has

$$
\left(S \otimes_{K} L_{1}\right) \otimes_{L_{1}} L_{2}=S \otimes_{K} L_{2} .
$$

Moreover, the following relations hold

$$
\pi_{L_{1} / K} \circ \pi_{L_{2} / L_{1}}=\pi_{L_{2} / K}, \quad I_{L_{1} / K} \circ I_{L_{2} / L_{1}}=I_{L_{2} / K} .
$$


Proof. - Let $\left(L_{1},\left(\Omega_{1}, \mathcal{A}_{1}, \nu_{1}\right), \phi_{1}\right)$ and $\left(L_{2},\left(\Omega_{2}, \mathcal{A}_{2}, \nu_{2}\right), \phi_{2}\right)$ be the adelic curves $S \otimes_{K}$ $L_{1}$ and $S \otimes_{K} L_{2}$ respectively. First of all, set-theoretically one has

$$
\Omega_{2}=\coprod_{\omega \in \Omega_{K}} M_{L_{2}, \omega}=\coprod_{\omega \in \Omega_{K}} \coprod_{x \in M_{L_{1}, \omega}} M_{L_{2}, x}=\coprod_{x \in \Omega_{1}} M_{L_{2}, x},
$$

and hence

$$
\pi_{L_{2} / K}=\pi_{L_{1} / K} \circ \pi_{L_{2} / L_{1}}
$$

Moreover, for any extension $K_{1} \in \mathscr{E}_{L_{1} / K}$ the map $\pi_{L_{2} / K_{1}}$ is $\mathcal{A}_{2}$-measurable. Therefore $\pi_{L_{2} / L_{1}}: \Omega_{2} \rightarrow \Omega_{1}$ is an $\mathcal{A}_{2}$-measurable map since the $\sigma$-algebra $\mathcal{A}_{1}$ is generated by the maps $\pi_{L_{1} / K_{1}}$ with $K_{1} \in \mathscr{E}_{L_{1} / K}$.

We now proceed with the proof of the equalities (3.22) and (3.23) with the supplementary assumption that the extension $L_{2} / L_{1}$ is finite. We first show that the $\sigma$-algebra $\mathcal{A}_{2}$ coincides with that in the adelic structure of $\left(S \otimes_{K} L_{1}\right) \otimes L_{2}$, namely the smallest $\sigma$-algebra $\mathcal{A}_{2}^{\prime}$ on $\Omega_{2}$ such that $\pi_{L_{2} / L_{1}}$ and all functions of the form $\left(y \in \Omega_{2}\right) \mapsto|a|_{y}$ are $\mathcal{A}_{2}^{\prime}$-measurable, where $a \in L_{2}$. We have already shown that the map $\pi_{L_{2} / L_{1}}$ is measurable. Hence by proposition 3.4.10, we obtain that $\mathcal{A}_{2}^{\prime} \subseteq \mathcal{A}_{2}$. Conversely, for any extension $K_{1} \in \mathscr{E}_{L_{1} / K}$ one has

$$
\pi_{L_{2} / K_{1}}=\pi_{L_{1} / K_{1}} \circ \pi_{L_{2} / L_{1}},
$$

and hence

$$
\pi_{L_{2} / K_{1}}^{-1}\left(\mathcal{A}_{K_{1}}\right)=\pi_{L_{2} / L_{1}}^{-1}\left(\pi_{L_{1} / K_{1}}^{-1}\left(\mathcal{A}_{K_{1}}\right)\right) \subseteq \pi_{L_{2} / L_{1}}^{-1}\left(\mathcal{A}_{1}\right) \subseteq \mathcal{A}_{2}^{\prime}
$$

If $K_{2}$ is an extension in $\mathscr{E}_{L_{2} / K}$, then $K_{1}:=K_{2} \cap L_{1} \in \mathscr{E}_{L_{1} / K}$. Moreover, the $\sigma$-algebra $\mathcal{A}_{K_{2}}$ is generated by $\pi_{K_{2} / K_{1}}$ and the functions of the form $x \mapsto|a|_{x}$ on $\Omega_{K_{2}}$, where $a \in K_{2}$. Note that $\pi_{L_{2} / K_{1}}=\pi_{L_{1} / K_{1}} \circ \pi_{L_{2} / L_{1}}$ is $\mathcal{A}_{2}^{\prime}$-measurable, and for any $a \in K_{2}$, the composition of the function $x \mapsto|a|_{x}$ on $\Omega_{K_{2}}$ with $\pi_{L_{2} / K_{2}}$, which identifies with the function $y \mapsto|a|_{y}$ on $\Omega_{L_{2}}$, is also $\mathcal{A}_{2}^{\prime}$-measurable, we obtain that the map $\pi_{L_{2} / K_{2}}$ is actually $\mathcal{A}_{2}^{\prime}$-measurable. Since $\mathcal{A}_{2}$ is the smallest $\sigma$-algebra which makes all $\pi_{L_{2} / K_{2}}$ measurable, where $K_{2} \in \mathscr{E}_{L_{2} / K}$, we obtain $\mathcal{A}_{2} \subseteq \mathcal{A}_{2}^{\prime}$. Therefore one has $\mathcal{A}_{2}=\mathcal{A}_{2}^{\prime}$.

It remains to establish the relation $I_{L_{1} / K}\left(I_{L_{2} / L_{1}}(f)\right)=I_{L_{2} / K}(f)$ for any nonnegative $\mathcal{A}_{2}$-measurable function on $\Omega_{2}$. By induction it suffices to treat the case where $\left[L_{2}: L_{1}\right]$ is a prime number. Moreover, similarly to the proof of Proposition 3.4 .8 , by using the monotone class theorem, we only need to verify the equality $I_{L_{1} / K}\left(I_{L_{2} / L_{1}}(f)\right)=I_{L_{2} / K}(f)$ for functions $f$ of the form $g \circ \pi_{L_{2} / K_{2}}$, where $K_{2} \in \mathscr{E}_{L_{2} / K}$ and $g$ is a non-negative $\mathcal{A}_{K_{2}}$-measurable function on $\Omega_{K_{2}}$. If $K_{2}$ belongs to $\mathscr{E}_{L_{1} / K}$, one has $I_{L_{2} / L_{1}}(f)=g \circ \pi_{L_{1} / K_{2}}$, and therefore

$$
I_{L_{1} / K}\left(I_{L_{2} / L_{1}}(f)\right)=I_{K_{2} / K}(g)=I_{L_{2} / K}(f),
$$

where the second equality comes from (3.19). Otherwise one has $\left[K_{2}: K_{1}\right]=\left[L_{2}: L_{1}\right]$ since $\left[K_{2}: K_{1}\right]$ divides $\left[L_{2}: L_{1}\right]$ which is a prime number, where $K_{1}$ denotes the intersection of $K_{2}$ with $L_{1}$. Moreover, there exists an element $a \in K_{2}$ such that $K_{2}=K_{1}(a)$ and $L_{2}=L_{1}(a)$. If $a$ is totally inseparable over $K_{1}$, then it is also 
totally inseparable over $L_{1}$. In this case $I_{L_{2} / L_{1}}(f)=f=g \circ \pi_{L_{1} / K_{1}}$ and therefore the equality $I_{L_{1} / K}\left(I_{L_{2} / L_{1}}(f)\right)=I_{L_{2} / K}(f)$ also holds in this case.

In the following, we assume that the element $a$ is separable over $K_{1}$. Let

$$
P(T)=T^{p}+b_{1} T^{p-1}+\cdots+b_{p} \in K_{1}[T]
$$

be the minimal polynomial of $a$ over $K_{1}$. Since $p$ is a prime number, and $a \notin L_{1}$, we obtain that it is also the minimal polynomial of $a$ over $L_{1}$. In particular, the element $a$ is also separable over $L_{1}$. Let $y$ be an element in $\Omega_{1}$ and $x=\pi_{L_{1} / K_{1}}(y)$. Assume that

$$
P=P_{1} \cdots P_{r}
$$

is the splitting of the polynomial $P$ in the ring $K_{1, x}[T]$ into the product of distinct irreducible polynomials. Then the polynomials $P_{1}, \ldots, P_{r}$ correspond to points $x_{1}, \ldots, x_{r}$ which form the set $\pi_{K_{2} / K_{1}}^{-1}(\{x\})$. Moreover, one has (see (3.2) for the definition of $\left.\mathbb{P}_{K_{2}, x}\right)$

$$
\mathbb{P}_{K_{2}, x}\left(\left\{x_{i}\right\}\right)=\frac{\operatorname{deg}\left(P_{i}\right)}{p} .
$$

Assume that each $P_{i}$ splits in $L_{1, y}$ into the product of distinct irreducible polynomials as

$$
P_{i}=Q_{i, 1} \cdots Q_{i, n_{i}}
$$

Then each factor $Q_{i, j}$ corresponds to a point $y_{i, j}$ in $\pi_{L_{2} / L_{1}}^{-1}(\{y\})$ and one has

$$
\mathbb{P}_{L_{2}, y}\left(x_{i, j}\right)=\frac{\operatorname{deg}\left(Q_{i, j}\right)}{p} .
$$

Therefore, if a non-negative function $f$ on $\Omega_{2}$ is of the form $g \circ \pi_{L_{2} / K_{2}}$, where $g$ is a $\mathcal{A}_{K_{2}}$-measurable function on $\Omega_{K_{2}}$, then one has

$$
\left(I_{L_{2} / L_{1}}(f)\right)(y)=\sum_{i=1}^{r} \sum_{j=1}^{n_{i}} \frac{\operatorname{deg}\left(Q_{i, j}\right)}{p} g(x)=\frac{\operatorname{deg}\left(P_{i}\right)}{p} g(x)=\left(I_{K_{2} / K_{1}}(g)\right)(x),
$$

which shows that

Therefore one has

$$
I_{L_{2} / L_{1}}(f)=I_{K_{2} / K_{1}}(g) \circ \pi_{L_{1} / K_{1}}
$$

$$
\begin{aligned}
& I_{L_{1} / K}\left(I_{L_{2} / L_{1}}(f)\right)=I_{L_{1} / K}\left(I_{K_{2} / K_{1}}(g) \circ \pi_{L_{1} / K_{1}}\right) \\
= & I_{K_{1} / K}\left(I_{K_{2} / K_{1}}(g)\right)=I_{K_{2} / K}(g)=I_{L_{2} / K}(f),
\end{aligned}
$$

where the second and the last equalities come from (3.19). Thus we have established the second equality in (3.23), which implies that the measure in the adelic structure of $\left(S \otimes_{K} L_{1}\right) \otimes_{L_{1}} L_{2}$ coincides with the measure $\nu_{2}$ in the adelic structure of $S \otimes_{K} L_{2}$. The theorem is then established in the particular case where $\left[L_{2}: L_{1}\right]$ is finite.

In the following, we will prove the general case of the theorem. Note that the previously proved case actually implies that, for any finite extension $L^{\prime \prime}$ of $L_{1}$, the map $\pi_{L_{2} / L^{\prime \prime}}$ is $\mathcal{A}_{2}$-measurable since the $\sigma$-algebra $\mathcal{A}_{L^{\prime \prime}}$ in the adelic structure of $\left(S \otimes_{K} L_{1}\right) \otimes L^{\prime \prime}$ coincides with that in the adelic structure of $S \otimes_{K} L^{\prime \prime}$, which is 
generated by the maps $\pi_{L^{\prime \prime} / K^{\prime \prime}}$ with $K^{\prime \prime} \in \mathscr{E}_{L^{\prime \prime} / K}$. In particular, if we denote by $\mathcal{A}_{2}^{\prime}$ the $\sigma$-algebra in the adelic structure of $\left(S \otimes_{K} L_{1}\right) \otimes_{L_{1}} L_{2}$, then one has $\mathcal{A}_{2}^{\prime} \subseteq \mathcal{A}_{2}$. Conversely, for any $K_{2} \in \mathscr{E}_{L_{2} / K}$, one has $\pi_{L_{2} / K_{2}}=\pi_{L^{\prime \prime} / K_{2}} \circ \pi_{L_{2} / L^{\prime \prime}}$, where $L^{\prime \prime}=L_{1} K_{2}$ is an element in $\mathscr{E}_{L_{2} / L_{1}}$. Hence $\pi_{L_{2} / K_{2}}$ is $\mathcal{A}_{2}^{\prime}$-measurable. Since $K_{2} \in \mathscr{E}_{L_{2} / L}$ is arbitrary, we obtain that $\mathcal{A}_{2} \subseteq \mathcal{A}_{2}^{\prime}$ and hence $\mathcal{A}_{2}=\mathcal{A}_{2}^{\prime}$.

Again it remains to establish the equality $I_{L_{1} / K}\left(I_{L_{2} / L_{1}}(f)\right)=I_{L_{2} / K}(f)$ for any non-negative $\mathcal{A}_{2}$-measurable function $f$ on $\Omega_{2}$, which can be written in the form $g \circ \pi_{L_{2} / K_{2}}$, where $K_{2} \in \mathscr{E}_{L_{2} / K}$. Let $L^{\prime \prime}=K_{2} L_{1}$. One has $L^{\prime \prime} \in \mathscr{E}_{L_{2} / L_{1}}$ and $g \circ \pi_{L_{2} / K_{2}}=$ $g \circ \pi_{L^{\prime \prime} / K_{2}} \circ \pi_{L_{2} / L^{\prime \prime}}$. Therefore (3.19) implies

$$
I_{L_{2} / K}\left(g \circ \pi_{L_{2} / K_{2}}\right)=I_{L^{\prime \prime} / K}\left(g \circ \pi_{L^{\prime \prime} / K_{2}}\right)=I_{K_{2} / K}(g) .
$$

Moreover, also by (3.19) one obtains

$$
\begin{aligned}
& \left.I_{L_{1} / K}\left(I_{L_{2} / L_{1}}\left(g \circ \pi_{L_{2} / K_{2}}\right)\right)=I_{L_{1} / K}\left(I_{L_{2} / L_{1}}\left(g \circ \pi_{L^{\prime \prime} / K_{2}} \circ \pi_{L_{2} / L^{\prime \prime}}\right)\right)\right) \\
= & I_{L_{1} / K}\left(I_{L^{\prime \prime} / L_{1}}\left(g \circ \pi_{L^{\prime \prime} / K_{2}}\right)\right)=I_{L^{\prime \prime} / K}\left(g \circ \pi_{L^{\prime \prime} / K_{2}}\right)=I_{K_{2} / K}(g),
\end{aligned}
$$

where the third equality comes from the proved case of finite extensions. Thus we establish the relation $I_{L_{1} / K} \circ I_{L_{2} / L_{1}}=I_{L_{2} / K}$ which implies that the measure $\nu_{2}$ identifies with that in the adelic structure of $\left(S \otimes_{K} L_{1}\right) \otimes_{L_{1}} L_{2}$. The theorem is thus proved.

Proposition 3.4.13. - Let $S=\left(K,\left(\Omega_{K}, \mathcal{A}_{K}, \nu_{K}\right), \phi_{K}\right)$ be an adelic curve and $L / K$ be an algebraic extension. Let $\left(L,\left(\Omega_{L}, \mathcal{A}_{L}, \nu_{L}\right), \phi_{L}\right)$ be the adelic curve $S \otimes_{K} L$. Then $\mathcal{A}_{L}$ is the smallest $\sigma$-algebra making the canonical projection map $\pi_{L / K}: \Omega_{L} \rightarrow \Omega_{K}$ and the functions $\left(x \in \Omega_{L}\right) \mapsto|a|_{x}$ measurable for all $a \in L$.

Proof. - By definition the projection map $\pi_{L / K}$ is $\mathcal{A}_{L}$-measurable. Moreover, by Proposition 3.4.10, for any $a \in L$, the function $\left(x \in \Omega_{L}\right) \mapsto|a|_{x}$ is $\mathcal{A}_{L}$-measurable.

Suppose that $F$ is a map from a measurable space $(E, \mathcal{E})$ to $\Omega_{L}$ such that the composed map $\pi_{L / K} \circ F$ and the functions $(y \in E) \mapsto|a|_{F(y)}$ are measurable, where $a \in L$. We will show that $F$ is measurable if we consider the $\sigma$-algebra $\mathcal{A}_{L}$ on $\Omega_{L}$. This implies that $\mathcal{A}_{L}$ is contained in the smallest $\sigma$-algebra making the canonical projection $\Omega_{L} \rightarrow \Omega_{K}$ and the functions $\left(x \in \Omega_{L}\right) \mapsto|a|_{x}$ measurable, where $a \in L$.

Recall that $\mathcal{A}_{L}$ is the smallest $\sigma$-algebra making the projection maps $\pi_{L / K^{\prime \prime}}: \Omega_{L} \rightarrow$ $\Omega_{K^{\prime \prime}}$ measurable, where $K^{\prime \prime} / K$ runs over the set of finite extensions contained in $L$. To show the measurability of $F$ it suffices to verify the measurability of $\pi_{L / K^{\prime \prime}} \circ F$ for any finite extension $K^{\prime \prime} / K$ contained in $L$. Moreover, since $\mathcal{A}_{K^{\prime \prime}}$ is the smallest $\sigma$-algebra making the projection map $\pi_{K^{\prime \prime} / K}: \Omega_{K^{\prime \prime}} \rightarrow \Omega_{K}$ and the functions $(x \in$ $\left.\Omega_{K^{\prime \prime}}\right) \mapsto|a|_{x}$ measurable, where $a \in K^{\prime \prime}$, we are reduced to verify the measurability of $\pi_{K^{\prime \prime} / K} \circ \pi_{L / K^{\prime \prime}} \circ F=\pi_{L / K} \circ F$ and

$$
(y \in E) \longmapsto|a|_{\pi_{L / K^{\prime \prime}}(F(y))}, \quad \text { where } a \in K^{\prime \prime} .
$$


By the assumption on $F$, the map $\pi_{L / K} \circ F$ is measurable. Moreover, since $a \in K^{\prime \prime}$, one has

$$
|a|_{\pi_{L / K^{\prime \prime}}(F(y))}=|a|_{F(y)} .
$$

Hence the function in (3.24) is also measurable. The proposition is thus proved.

\subsection{Height function and Northcott property}

Let $S=(K,(\Omega, \mathcal{A}, \nu), \phi)$ be a proper adelic curve and $K^{\text {ac }}$ be an algebraic closure of $K$. Let $S \otimes_{K} K^{\mathrm{ac}}=\left(K^{\mathrm{ac}},\left(\Omega_{K^{\mathrm{ac}}}, \mathcal{A}_{K^{\mathrm{ac}}}, \nu_{K^{\mathrm{ac}}}\right), \phi_{K^{\mathrm{ac}}}\right)$ be the algebraic extension of $S$ by $K^{\mathrm{ac}}$.

Definition 3.5.1. - For $\left(a_{0}, a_{1}, \ldots, a_{n}\right) \in\left(K^{\text {ac }}\right)^{n+1} \backslash\{(0, \ldots, 0)\}$, we define $h_{S}\left(a_{0}, \ldots, a_{n}\right)$ to be

$$
h_{S}\left(a_{0}, \ldots, a_{n}\right):=\int_{\Omega_{K^{\mathrm{ac}}}} \ln \left(\max \left\{\left|a_{0}\right|_{\chi}, \ldots,\left|a_{n}\right|_{\chi}\right\}\right) \nu_{K^{\mathrm{ac}}}(d \chi) .
$$

By the product formula, $h_{S}\left(\lambda a_{0}, \ldots, \lambda a_{n}\right)=h_{S}\left(a_{0}, \ldots, a_{n}\right)$ for all $\lambda \in K^{\text {ac }} \backslash\{0\}$, so that there is a map $\mathbb{P}^{n}\left(K^{\mathrm{ac}}\right) \rightarrow \mathbb{R}$ such that the following diagram is commutative:

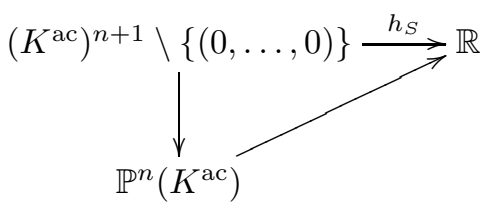

By abuse of notation, the map $\mathbb{P}^{n}\left(K^{\mathrm{ac}}\right) \rightarrow \mathbb{R}$ is also denoted by $h_{S}$. For $x \in \mathbb{P}^{n}\left(K^{\mathrm{ac}}\right)$, the value $h_{S}(x)$ is called the height of $x$ with respect to the adelic curve $S$.

Definition 3.5.2. - We say that $S$ has the Northcott property if the set $\left\{a \in K: h_{S}(1: a) \leqslant C\right\}$ is finite for any $C \geqslant 0$. In the cases of Example 3.2.2 Example 3.2.5 and Example 3.2.6, the Northcott property holds (for details, see [102 and [104]).

The purpose of this section is to prove the following theorem:

Theorem 3.5.3 (Northcott's theorem). - If $S$ has the Northcott property, then the set $\left\{x \in \mathbb{P}^{n}\left(K^{\mathrm{ac}}\right): h_{S}(x) \leqslant C,[K(x): K] \leqslant \delta\right\}$ is finite for any $C$ and $\delta$.

Before starting the proof of Theorem 3.5 .3 , we need to prepare two lemmas.

Lemma 3.5.4. - Let $K^{\prime}$ be a finite normal extension of $K$. Then $h_{S}(1: \sigma(\alpha))=$ $h_{S}(1: \alpha)$ for all $\alpha \in K^{\prime}$ and $\sigma \in \operatorname{Aut}_{K}\left(K^{\prime}\right)$.

Proof. - Let $K^{\prime \prime}$ be the separable closure of $K$ in $K^{\prime}$ and $q=\left[K^{\prime}: K^{\prime \prime}\right]$. Then $\left.\sigma\right|_{K^{\prime \prime}} \in \operatorname{Aut}_{K}\left(K^{\prime \prime}\right)$ and $a^{q} \in K^{\prime \prime}$. If the assertion holds for the extension $K^{\prime \prime} / K$, then

$$
q h_{S}(1: \sigma(\alpha))=h_{S}\left(1: \sigma\left(\alpha^{q}\right)\right)=h_{S}\left(1: \alpha^{q}\right)=q h_{S}(1: \alpha),
$$


so that we may assume that the extension $K^{\prime} / K$ is separable.

For $\chi \in \pi_{K^{\prime} / K}^{-1}(\{\omega\})$ and $\tau \in \operatorname{Gal}\left(K^{\prime} / K\right)$, a map $\left(\beta \in K^{\prime}\right) \mapsto|\tau(\beta)|_{\chi}$ gives rise to an element of $\pi_{K^{\prime} / K}^{-1}(\{\omega\})$, which is denoted by $\chi^{\tau}$. In this way, one has an action $\operatorname{Gal}\left(K^{\prime} / K\right) \times \pi_{K^{\prime} / K}^{-1}(\{\omega\}) \rightarrow \pi_{K^{\prime} / K}^{-1}(\{\omega\})$ given by $(\tau, \chi) \mapsto \chi^{\tau}$. Note that the action is transitive (cf. [110, Chapter II, Proposition 9.1]) and $\operatorname{Gal}\left(K_{\chi}^{\prime} / K_{\omega}\right)=$ $\operatorname{Stab}_{\operatorname{Gal}\left(K^{\prime} / K\right)}(\chi)$ (cf. 110, Chapter II, Proposition 9.6]). In particular, $\left[K_{\chi}^{\prime}: K_{\omega}\right]=$ $\left[K_{\chi^{\prime}}^{\prime}: K_{\omega}\right]$ for all $\chi, \chi^{\prime} \in \pi_{K^{\prime} / K}^{-1}(\{\omega\})$. Therefore,

$$
\begin{aligned}
I_{K^{\prime} / K}(\ln (\max \{1,|\sigma(\alpha)|\}))(\omega) & =\sum_{\chi \in \pi_{K^{\prime} / K}^{-1}(\{\omega\})} \frac{\left[K_{\chi}^{\prime}: K_{\omega}\right]}{\left[K^{\prime}: K\right]} \ln \left(\max \left\{1,|\sigma(\alpha)|_{\chi}\right\}\right) \\
& =\sum_{\chi \in \pi_{K^{\prime} / K}^{-1}(\{\omega\})} \frac{\left[K_{\chi^{\sigma}}^{\prime}: K_{\omega}\right]}{\left[K^{\prime}: K\right]} \ln \left(\max \left\{1,|\alpha|_{\chi^{\sigma}}\right\}\right) \\
& =I_{K^{\prime} / K}(\ln (\max \{1,|\alpha|\}))(\omega),
\end{aligned}
$$

and hence the assertion follows.

For a polynomial $F=a_{n} X^{n}+\cdots+a_{1} X+a_{0} \in K^{\text {ac }}[X] \backslash\{0\}$, we define $h_{S}(F)$ to be $h_{S}(F):=h_{S}\left(a_{n}: \cdots: a_{1}: a_{0}\right)$. If we set $\|F\|_{\chi}:=\max \left\{\left|a_{n}\right|_{\chi}, \ldots,\left|a_{0}\right|_{\chi}\right\}$ for $\chi \in \Omega_{K^{\text {ac }}}$ as in Subsection 1.1.15, then

$$
h_{S}(F)=\int_{\Omega_{K^{\mathrm{ac}}}} \ln \left(\|F\|_{\chi}\right) \nu_{K^{\mathrm{ac}}}(d \chi) .
$$

Lemma 3.5.5. - For $F, G \in K^{\mathrm{ac}}[X] \backslash\{0\}$, one has

$$
h_{S}(F G) \leqslant h_{S}(F)+h_{S}(G)+\ln \min \{\operatorname{deg}(F)+1, \operatorname{deg}(G)+1\} \int_{\Omega_{\infty}} \nu(d \omega) .
$$

Proof. - By Proposition 1.1.72.

$$
\begin{aligned}
h_{S}(F G)= & \int_{\Omega_{K^{\mathrm{ac}}}} \ln \left(\|F G\|_{\chi}\right) \nu_{K^{\mathrm{ac}}}(d \chi) \\
\leqslant & \int_{\Omega_{K^{\mathrm{ac}}}}\left(\ln \left(\|F\|_{\chi}\right)+\ln \left(\|G\|_{\chi}\right)\right) \nu_{K^{\mathrm{ac}}}(d \chi) \\
& \quad+\int_{\Omega_{\infty}} \ln (\min \{\operatorname{deg}(F)+1, \operatorname{deg}(G)+1\}) \nu(d \omega),
\end{aligned}
$$

so that the assertion follows.

Proof of Theorem 3.5.3. - Clearly we may assume that $C \geqslant 0$ and $\delta \geqslant 1$. Let us begin with the following special case:

Claim 3.5.6. - The set $\left\{\alpha \in K^{\mathrm{ac}}: h_{S}(1: \alpha) \leqslant C,[K(\alpha): K] \leqslant \delta\right\}$ is finite. 
Proof. - Let $F$ be the minimal monic polynomial of $\alpha$ over $K$. We set $F=X^{n}+$ $a_{n-1} X^{n-1}+\cdots+a_{1} X+a_{0}=\left(X-\alpha_{1}\right) \cdots\left(X-\alpha_{n}\right)$ and $K^{\prime}=K\left(\alpha_{1}, \ldots, \alpha_{n}\right)$, where $\alpha_{1}=\alpha$. Then, by Lemma 3.5.4 and Lemma 3.5.5

$$
\begin{aligned}
h_{S}(F) & \leqslant \sum_{i=1}^{n} h_{S}\left(X-\alpha_{i}\right)+(n-1) \ln (2) \int_{\Omega_{\infty}} \nu(d \omega) \\
& =\sum_{i=1}^{n} h_{S}\left(1: \alpha_{i}\right)+(n-1) \ln (2) \int_{\Omega_{\infty}} \nu(d \omega) \\
& =n h_{S}(1: \alpha)+(n-1) \ln (2) \int_{\Omega_{\infty}} \nu(d \omega) \leqslant \delta C+(\delta-1) \ln (2) \int_{\Omega_{\infty}} \nu(d \omega) .
\end{aligned}
$$

Note that $h_{S}\left(1: a_{i}\right) \leqslant h_{S}(F)$ and $a_{i} \in K$ for all $i=0, \ldots, n-1$, so that one can see that there are finitely many possibilities of $F$ because $S$ has the Northcott property. Therefore the assertion of the claim follows.

Let us go back to the proof of Theorem 3.5.3. For $i=0, \ldots, n$, let

$$
\Upsilon_{i}:=\left\{x=\left(x_{0}: \cdots: x_{n}\right) \in \mathbb{P}^{n}\left(K^{a c}\right): h_{S}(x) \leqslant C,[K(x): K] \leqslant \delta, x_{i} \neq 0\right\} .
$$

It is sufficient to show that $\#\left(\Upsilon_{i}\right)<\infty$ for all $i$. Without of loss of generality, we may assume that $i=0$. Then

$$
\Upsilon_{0}=\left\{\left(a_{1}, \ldots, a_{n}\right) \in\left(K^{a c}\right)^{n}: h_{S}\left(1, a_{1}, \ldots, a_{n}\right) \leqslant C,\left[K\left(a_{1}, \ldots, a_{n}\right): K\right] \leqslant \delta\right\} .
$$

Note that $\left[K\left(a_{i}\right): K\right] \leqslant\left[K\left(a_{1}, \ldots, a_{n}\right): K\right]$ and $h_{S}\left(1: a_{i}\right) \leqslant h_{S}\left(1, a_{1}, \ldots, a_{n}\right)$ for all $i=1, \ldots, n$. Thus the assertion is a consequence of the above special case.

Corollary 3.5.7. - We assume that $S$ has the Northcott property. Let $K^{\prime}$ be a finite extension of $K$. Then $S \otimes_{K} K^{\prime}$ has also the Northcott property.

Remark 3.5.8. - Theorem 3.5.3 can be generalized to the case of an adelic vector bundle. For details, see Proposition 6.2.3.

\subsection{Measurability of automorphism actions}

Let $S=(K,(\Omega, \mathcal{A}, \nu), \phi)$ be an adelic curve and $L / K$ be an algebraic extension. We denote by $\operatorname{Aut}_{K}(L)$ the group of field automorphisms of $L$ which are $K$-linear. The group $\operatorname{Aut}_{K}(L)$ acts on $M_{L}$ as follows: for any $\tau \in \operatorname{Aut}_{K}(L)$ and any $x \in M_{L}$, one has

$$
\forall a \in L, \quad|a|_{\tau(x)}=|\tau(a)|_{x} .
$$

Moreover, by definition the restrictions of the absolute values $|\cdot|_{x}$ and $|\cdot|_{\tau(x)}$ on $K$ are the same. Therefore we obtain an action of the $K$-linear automorphism group $\operatorname{Aut}_{K}(L)$ on the set $\Omega_{L}=\Omega \times_{M_{K}} M_{L}$ (where we consider trivial actions of $\operatorname{Aut}_{K}(L)$ on $\Omega$ and on $M_{K}$ ). 
Proposition 3.6.1. - Let $S=\left(K,\left(\Omega_{K}, \mathcal{A}_{K}, \nu_{K}\right), \phi_{K}\right)$ be an adelic curve and $L / K$ be an algebraic extension. For any $\tau \in \operatorname{Aut}_{K}(L)$, the action of $\tau$ on $\Omega_{L}$ is measurable, where on $\Omega_{L}$ we consider the $\sigma$-algebra $\mathcal{A}_{L}$ in the adelic structure of $S \otimes_{K} L$.

Proof. - By Proposition 3.4.13 the $\sigma$-algebra $\mathcal{A}_{L}$ is the smallest $\sigma$-algebra which makes measurable the canonical projection map $\pi_{L / K}: \Omega_{L} \rightarrow \Omega_{K}$ and the functions $\left(x \in \Omega_{L}\right) \mapsto|a|_{x}$, where $a \in L$. Let $\tau \in \operatorname{Aut}_{K}(L)$. To show the measurability of the action of $\tau$ on $\Omega_{L}$, it suffices to verify the measurability of the map $\pi_{L / K} \circ \tau$ and the functions $\left(x \in \Omega_{L}\right) \mapsto|a|_{\tau(x)}$ with $a \in L$. Note that by definition $\pi_{L / K} \circ \tau=\pi_{L / K}$ and $|\alpha|_{\tau(x)}=|\tau(\alpha)|_{x}$. The proposition is thus proved.

Proposition 3.6.2. - Let $S=\left(K,\left(\Omega_{K}, \mathcal{A}_{K}, \nu_{K}\right), \phi_{K}\right)$ be an adelic curve and $L / K$ be a finite extension. Let $F: \Omega_{L} \rightarrow \mathbb{R}$ be an $\mathcal{A}_{L}$-measurable function. For any $\omega \in \Omega$, let

$$
f(\omega)=\max _{x \in \pi_{L / K}^{-1}(\{\omega\})} F(x) .
$$

Then the function $f: \Omega_{K} \rightarrow \mathbb{R}$ is $\mathcal{A}_{K}$-measurable.

Proof. - We first assume that the extension $L / K$ is normal. By [26], Chapitre VI, $\S 8, \mathrm{n}^{\circ} 6$, Proposition 7 , for any $\omega \in \Omega_{K}$, the action of the $K$-linear automorphism group $\operatorname{Aut}_{K}(L)$ on $M_{L, \omega}$ is transitive. As a consequence, if we denote by $\widetilde{F}$ the function

$$
\max _{\tau \in \operatorname{Aut}_{K}(L)} F \circ \tau,
$$

then for each $\omega \in \Omega_{K}$, the restriction of $\widetilde{F}$ on $\pi_{L / K}^{-1}(\{\omega\})$ is constante, the value of which is equal to $f(\omega)$. By Proposition 3.6.1 for any $\tau \in \operatorname{Aut}_{K}(L)$, the action of $\tau$ on $\Omega_{L}$ is measurable and hence the function $F \circ \tau$ is $\mathcal{A}_{L}$-measurable. Since $\operatorname{Aut}_{K}(L)$ is a finite set, we deduce that the function $\widetilde{F}$ is also $\mathcal{A}_{L}$-measurable. By Proposition 3.4.8, the function $f=I_{L / K}(\widetilde{F})$ is $\mathcal{A}_{K}$-measurable.

In the general case, we pick a finite normal extension $L_{1} / K$ which contains $L$. By applying the proved result to the function $F \circ \pi_{L_{1} / L}$, we still obtain the measurability of the function $f$. The proposition is thus proved.

\subsection{Morphisms of adelic curves}

In this section, we consider morphism of adelic curves.

Definition 3.7.1. - Let $S=(K,(\Omega, \mathcal{A}, \nu), \phi)$ and $S^{\prime}=\left(K^{\prime},\left(\Omega^{\prime}, \mathcal{A}^{\prime}, \nu^{\prime}\right), \phi^{\prime}\right)$ be two adelic curves, we call morphism from $S^{\prime}$ to $S$ any triplet $\alpha=\left(\alpha^{\#}, \alpha_{\#}, I_{\alpha}\right)$, where

(a) $\alpha^{\#}: K \rightarrow K^{\prime}$ is a field homomorphism, 
(b) $\alpha_{\#}:\left(\Omega^{\prime}, \mathcal{A}^{\prime}\right) \rightarrow(\Omega, \mathcal{A})$ is a measurable map such that the following diagram is commutative

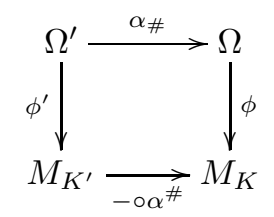

and that the direct image of $\nu^{\prime}$ by $\alpha_{\#}$ coincides with $\nu$, namely, for any function $f \in \mathscr{L}^{1}(\Omega, \mathcal{A}, \nu)$, one has

$$
\int_{\Omega^{\prime}} f \circ \alpha_{\#} \mathrm{~d} \nu^{\prime}=\int_{\Omega} f \mathrm{~d} \nu .
$$

(c) $I_{\alpha}: L^{1}\left(\Omega^{\prime}, \mathcal{A}^{\prime}, \nu^{\prime}\right) \rightarrow L^{1}(\Omega, \mathcal{A}, \nu)$ is a disintegration kernel of $\alpha_{\#}$, namely $I_{\alpha}$ is a linear map such that, for any element $g \in L^{1}\left(\Omega^{\prime}, \mathcal{A}^{\prime}, \nu^{\prime}\right)$, one has

$$
\int_{\Omega} I_{\alpha}(g) \mathrm{d} \nu=\int_{\Omega^{\prime}} g \mathrm{~d} \nu^{\prime},
$$

and for any function $f \in \mathscr{L}^{1}(\Omega, \mathcal{A}, \nu)$ one has $I_{\alpha}$ sends the equivalence class of $f \circ \alpha_{\#}$ to that of $f$.

Naturally, if $S, S^{\prime}$ and $S^{\prime \prime}$ are adelic curves and if $\alpha=\left(\alpha^{\#}, \alpha_{\#}, I_{\alpha}\right): S^{\prime} \rightarrow S$ and $\beta=\left(\beta^{\#}, \beta_{\#}, I_{\beta}\right): S^{\prime \prime} \rightarrow S$ are morphisms of adelic curves, then $\alpha \circ \beta:=$ $\left(\beta^{\#} \circ \alpha^{\#}, \alpha_{\#} \circ \beta_{\#}, I_{\alpha} \circ I_{\beta}\right)$ forms a morphism of adelic curves from $S^{\prime \prime}$ to $S$. Thus the adelic curves and their morphisms form a category.

Example 3.7.2. — Let $S=(K,(\Omega, \mathcal{A}, \nu), \phi)$ be an adelic curve.

(1) If $S \otimes_{K} K^{\prime}=\left(K^{\prime},\left(\Omega_{K^{\prime}}, \mathcal{A}_{K^{\prime}}, \nu_{K^{\prime}}\right), \phi_{K^{\prime}}\right)$ is an algebraic extension of $S$, then the triplet $\left(K \hookrightarrow K^{\prime}, \pi_{K^{\prime} / K}, \bar{I}_{K^{\prime} / K}\right)$ is a morphism of adelic curves from $S \otimes_{K} K^{\prime}$ to $S$, where $\bar{I}_{K^{\prime} / K}: L^{1}\left(\Omega_{K^{\prime}}, \mathcal{A}_{K^{\prime}}, \nu_{K^{\prime}}\right) \rightarrow L^{1}(\Omega, \mathcal{A}, \nu)$ is the linear map induced by $I_{K^{\prime} / K}: \mathscr{L}^{1}\left(\Omega_{K^{\prime}}, \mathcal{A}_{K^{\prime}}, \nu_{K^{\prime}}\right) \rightarrow \mathscr{L}^{1}(\Omega, \mathcal{A}, \nu)$.

(2) Assume that $K_{0}$ is a subfield of $K$. Let $S_{0}$ be the field $K_{0}$ equipped with the restriction to $K_{0}$ of the adelic structure of $S$ (see Subsection 3.2.8). Then the triplet $\left(K_{0} \hookrightarrow K, \operatorname{Id}_{\Omega}, \operatorname{Id}_{L^{1}(\Omega, \mathcal{A}, \nu)}\right)$ forms a morphism of adelic curves from $S$ to $S_{0}$.

(3) Let $K=\mathbb{Q}(T)$ be the field of rational functions of one variable $T$ with coefficients in $\mathbb{Q}$. Let $S=(K,(\Omega, \mathcal{A}, \nu), \phi)$ constructed in Subsection 3.2.5. Recall that $(\Omega, \mathcal{A}, \nu)$ is written as a disjoint union $\Omega_{h} \coprod \mathcal{P} \coprod[0,1]_{*}$, where $\Omega_{h}$ is the set of closed points of $\mathbb{P}_{\mathbb{Q}}^{1}, \mathcal{P}$ is the set of prime numbers, and $[0,1]_{*}$ is the subset of $[0,1]$ of $t$ such that $\mathrm{e}^{2 \pi i t}$ is transcendental. Let $S_{\mathbb{Q}}=\left(\mathbb{Q},\left(\Omega_{\mathbb{Q}}, \mathcal{A}_{\mathbb{Q}}, \nu_{\mathbb{Q}}\right), \phi_{\mathbb{Q}}\right)$ be the adelic curve defined in Subsection 3.2 .2 and $\widetilde{S}_{\mathbb{Q}}=\left(\mathbb{Q},\left(\widetilde{\Omega}_{\mathbb{Q}}, \widetilde{\mathcal{A}}_{\mathbb{Q}}, \widetilde{\nu}_{\mathbb{Q}}\right), \widetilde{\phi}_{\mathbb{Q}}\right)$ be the adelic curve consisting of the filed $\mathbb{Q}$ equipped with the amalgamation of the adelic structure of $S_{\mathbb{Q}}$ and a family of copies of the trivial absolute value on $\mathbb{Q}$ indexed by $\Omega_{h}$. We can also write $\widetilde{\Omega}$ as the disjoint union of three subsets 
$\Omega_{h} \amalg \mathcal{P} \amalg\{\infty\}$, where 0 denotes the trivial absolute value on $\mathbb{Q}$ and $\infty$ denotes the infinite place of $\mathbb{Q}$. Let $\alpha^{\#}: \mathbb{Q} \rightarrow \mathbb{Q}(T)$ be the inclusion map. Let $\alpha_{\#}: \Omega \rightarrow$ $\widetilde{\Omega}_{\mathbb{Q}}$ be the map which sends any element of $\Omega_{h} \amalg \mathcal{P}$ to itself and send any element of $[0,1]_{*}$ identically to $\infty$. Finally, let $I_{\alpha}: L^{1}(\Omega, \mathcal{A}, \nu) \rightarrow L^{1}\left(\widetilde{\Omega}_{\mathbb{Q}}, \widetilde{\mathcal{A}}_{\mathbb{Q}}, \widetilde{\nu}_{\mathbb{Q}}\right)$ be the linear map sending the equivalence class of any function $f \in \mathscr{L}^{1}(\Omega, \mathcal{A}, \nu)$ to that of the function $I_{\alpha}(f)$ sending $\omega \in \Omega_{h} \amalg \mathcal{P}$ to $f(\omega)$ and $\infty$ to $\int_{[0,1]_{*}} f(t) \mathrm{d} t$. Then the triplet $\left(\alpha^{\#}, \alpha_{\#}, I_{\alpha}\right)$ forms a morphism of adelic curves from $S$ to $\widetilde{S}_{\mathbb{Q}}$. 



\section{CHAPTER 4}

\section{VECTOR BUNDLES ON ADELIC CURVES: GLOBAL THEORY}

The purpose of this chapter is to study the geometry of adelic curves, notably the divisors and vector bundles.

\subsection{Norm families}

Let $S=(K,(\Omega, \mathcal{A}, \nu), \phi)$ be an adelic curve (see $\$ 3.1)$. Recall that for any $\omega \in \Omega$, we denote by $|\cdot|_{\omega}$ the absolute value of $K$ indexed by $\omega$. Note that in the case where $|\cdot|_{\omega}$ is Archimedean, there exists a constant $\kappa(\omega), 0<\kappa(\omega) \leqslant 1$, such that $|\cdot|_{\omega}=|\cdot|^{\kappa(\omega)}$, where $|\cdot|$ denotes the usual absolute value on $\mathbb{R}$ or $\mathbb{C}$. For simplicity, we assume that $\kappa(\omega)=1$ for any $\omega \in \Omega_{\infty}$, namely $|\cdot|_{\omega}$ identifies with the usual absolute value on $\mathbb{R}$ or $\mathbb{C}$. Note that this assumption is harmless for the generality of the theory since in general case we can replace the absolute values $\left\{|\cdot|_{\omega}\right\}_{\omega \in \Omega_{\infty}}$ by the usual ones and consider the measure $\mathrm{d} \widetilde{\nu}=\left(\mathbb{1}_{\Omega \backslash \Omega_{\infty}}+\kappa \mathbb{1}_{\Omega_{\infty}}\right) \mathrm{d} \nu$ instead.

4.1.1. Definition and algebraic constructions. - Let $E$ be a vector space of finite rank over $K$. We denote by $\mathcal{N}_{E}$ the set of norm families $\left\{\|\cdot\|_{\omega}\right\}_{\omega \in \Omega}$, where each $\|\cdot\|_{\omega}$ is a norm on $E_{K_{\omega}}:=E \otimes_{K} K_{\omega}$. We say that a norm family $\xi=\left\{\|\cdot\|_{\omega}\right\}_{\omega \in \Omega}$ is ultrametric on $\Omega \backslash \Omega_{\infty}$ if the norm $\|\cdot\|_{\omega}$ is ultrametric for any $\omega \in \Omega \backslash \Omega_{\infty}$. We say that a norm family $\left\{\|\cdot\|_{\omega}\right\}_{\omega \in \Omega}$ in $\mathcal{N}_{E}$ is Hermitian if the following conditions are satisfied:

(a) for any $\omega \in \Omega \backslash \Omega_{\infty}$, the norm $\|\cdot\|_{\omega}$ is ultrametric (namely the norm family is ultrametric on $\Omega \backslash \Omega_{\infty}$ );

(b) for any $\omega \in \Omega_{\infty}$, the norm $\|\cdot\|_{\omega}$ is induced by an inner product (see \$1.2.1), namely there exists an inner product $\langle,\rangle_{\omega}$ on $E_{K_{\omega}}$ such that $\|\ell\|_{\omega}=\langle\ell, \ell\rangle_{\omega}^{1 / 2}$ for any $\ell \in E_{K_{\omega}}$.

We denote by $\mathcal{H}_{E}$ the subset of $\mathcal{N}_{E}$ consisting of all Hermitian norm families. In the following, we describe some algebraic constructions of norm families. 
4.1.1.1. Multiplication by a numerical function. - Let $E$ be a finite-dimensional vector space over $K, \xi=\left\{\|\cdot\|_{\omega}\right\}_{\omega \in \Omega} \in \mathcal{N}_{E}$ and $\left.f: \Omega \rightarrow\right] 0,+\infty$ [ be a positive function on $\Omega$. We denote by $f \xi$ the norm family $\left\{f(\omega)\|\cdot\|_{\omega}\right\}_{\omega \in \Omega}$ in $\mathcal{N}_{E}$.

4.1.1.2. Restrict and quotient norm families. - Let $E$ be a finite-dimensional vector space over $K$ and $\xi=\left\{\|\cdot\|_{E, \omega}\right\}_{\omega \in \Omega}$ be a norm family in $\mathcal{N}_{E}$. Let $F$ be a vector subspace of $E$. For any $\omega \in \Omega$, let $\|\cdot\|_{F, \omega}$ be the restriction of the norm $\|\cdot\|_{E, \omega}$ to $F_{K_{\omega}}$ (see Definition 1.1.2). Then $\left\{\|\cdot\|_{F, \omega}\right\}_{\omega \in \Omega}$ forms a norm family in $\mathcal{N}_{F}$, called the restriction of $\xi$ to $F$. Similarly, if $G$ is a quotient vector space of $E$, then each norm $\|\cdot\|_{E, \omega}$ induces by quotient a norm $\|\cdot\|_{G, \omega}$ on $G_{K_{\omega}}$ (see 1.1.3). Thus we obtain a norm family $\left\{\|\cdot\|_{G, \omega}\right\}_{\omega \in \Omega}$ in $\mathcal{N}_{G}$, called the quotient of $\xi$ on $G$. Note that, if the norm family $\xi$ is ultrametric on $\Omega \backslash \Omega_{\infty}$ (resp. Hermitian), then all its restrictions and quotients are also ultrametric on $\Omega \backslash \Omega_{\infty}$ (resp. Hermitian).

4.1.1.3. Direct sums. - Let $\mathscr{S}$ be the set of all convex and continuous functions $f:[0,1] \rightarrow[0,1]$ such that $\max \{t, 1-t\} \leqslant f(t)$ for any $t \in[0,1]$. If $E$ and $F$ are finitedimensional vector spaces over $K$ and if $\xi_{E}=\left\{\|\cdot\|_{E, \omega}\right\}_{\omega \in \Omega}$ and $\xi_{F}=\left\{\|\cdot\|_{F, \omega}\right\}_{\omega \in \Omega}$ are respectively norm families in $\mathcal{N}_{E}$ and $\mathcal{N}_{F}$, for any family $\psi=\left\{\psi_{\omega}\right\}_{\omega \in \Omega}$ of elements in $\mathscr{S}$ we define a norm family $\xi_{E} \oplus_{\psi} \xi_{F}=\left\{\|\cdot\|_{\omega}\right\}_{\omega \in \Omega}$ in $\mathcal{N}_{E \oplus F}$ (see Subsection 1.1.10) such that, for any $(x, y) \in E_{K_{\omega}} \oplus F_{K_{\omega}}$,

$$
\|(x, y)\|_{\omega}:=\left(\|x\|_{E, \omega}+\|y\|_{F, \omega}\right) \psi_{\omega}\left(\frac{\|x\|_{E, \omega}}{\|x\|_{E, \omega}+\|y\|_{F, \omega}}\right) .
$$

We call $\xi_{E} \oplus_{\psi} \xi_{F}$ the $\psi$-direct sum of $\xi_{E}$ and $\xi_{F}$. If both norm families $\xi_{E}$ and $\xi_{F}$ are Hermitian, and if

$$
\psi_{\omega}(a, b)= \begin{cases}\max \{a, b\}, & \omega \in \Omega \backslash \Omega_{\infty}, \\ \left(a^{2}+b^{2}\right)^{1 / 2}, & \omega \in \Omega_{\infty},\end{cases}
$$

then the direct sum $\xi_{E} \oplus_{\psi} \xi_{F}$ belongs to $\mathcal{H}_{E \oplus F}$. We call it the orthogonal direct sum of $\xi_{E}$ and $\xi_{F}$.

4.1.1.4. Dual norm family. - Let $E$ be a vector space of finite rank over $K$ and $\xi=\left\{\|\cdot\|_{\omega}\right\}_{\omega \in \Omega}$ be a norm family in $\mathcal{N}_{E}$. The dual norms (see 1.1 .5$)\left\{\|\cdot\|_{\omega, *}\right\}_{\omega \in \Omega}$ form a norm family in $\mathcal{N}_{E^{\vee}}$, called the dual of $\xi$, denoted by $\xi^{\vee}$. Note that $\xi^{\vee}$ is always ultrametric on $\Omega \backslash \Omega_{\infty}$, and it is Hermitian if $\xi$ is. Moreover, if for any $\omega \in \Omega \backslash \Omega_{\infty}$ the norm $\|\cdot\|_{\omega}$ is ultrametric, then one has $\left(\xi^{\vee}\right)^{\vee}=\xi$, where we identify $E$ with its double dual space $E^{\vee \vee}$ (see Proposition 1.1.18 and Corollary 1.2.12). In particular, if $E$ is a $K$-vector space of dimension 1 , then one has $\left(\xi^{\vee}\right)^{\vee}=\xi$.

4.1.1.5. Tensor products. - Let $\left\{E_{i}\right\}_{i=1}^{n}$ be a family of finite-dimensional vector spaces over $K$. For any $i \in\{1, \ldots, n\}$, let $\xi_{i}=\left\{\|\cdot\|_{i, \omega}\right\} \omega \in \Omega$ be an element in $\mathcal{N}_{E_{i}}$. We denote by $\xi_{1} \otimes_{\pi} \cdots \otimes_{\pi} \xi_{n}$ the norm family $\left\{\|\cdot\|_{\omega, \pi}\right\}_{\omega \in \Omega}$ in $\mathcal{N}_{E_{1} \otimes \cdots \otimes E_{n}}$, where $\|\cdot\|_{\omega, \pi}$ is the $\pi$-tensor product of the norms $\|\cdot\|_{i, \omega}, i \in\{1, \ldots, n\}$ (see 1.1 .11 ). The norm family $\xi_{1} \otimes_{\pi} \cdots \otimes_{\pi} \xi_{n}$ is called the $\pi$-tensor product of $\xi_{1}, \ldots, \xi_{n}$. Similarly, we denote 
by $\xi_{1} \otimes_{\varepsilon} \cdots \otimes_{\varepsilon} \xi_{n}$ the norm family in $\mathcal{N}_{E_{1} \otimes \cdots \otimes E_{n}}$ consisting of $\varepsilon$-tensor products (see 1.1.11) of the norms $\|\cdot\|_{1, \omega}, \ldots,\|\cdot\|_{n, \omega}$. We call $\xi_{1} \otimes_{\varepsilon} \cdots \otimes_{\varepsilon} \xi_{n}$ the $\varepsilon$-tensor product of $\xi_{1}, \ldots, \xi_{n}$. We also introduce the following mixed version of $\varepsilon$-tensor product and $\pi$-tensor product. We denote by $\xi_{1} \otimes_{\varepsilon, \pi} \cdots \otimes_{\varepsilon, \pi} \xi_{n}$ consisting of the norms $\|\cdot\|_{\omega, \varepsilon}$ if $\omega \in \Omega \backslash \Omega_{\infty}$ and $\|\cdot\|_{\omega, \pi}$ if $\omega \in \Omega_{\infty}$. This norm family is called the $\varepsilon, \pi$-tensor product of $\xi_{1}, \ldots, \xi_{n}$. Note that the $\varepsilon$-tensor product and the $\varepsilon, \pi$-tensor product are both ultrametric on $\Omega \backslash \Omega_{\infty}$.

If all norm families $\xi_{1}, \ldots, \xi_{n}$ are Hermitian, we denote by $\xi_{1} \otimes \cdots \otimes \xi_{n}$ the norm family $\left\{\|\cdot\|_{\omega}\right\}_{\omega \in \Omega}$ in $\mathcal{H}_{E_{1} \otimes \cdots \otimes E_{n}}$, where for each $\omega \in \Omega \backslash \Omega_{\infty}$, the norm $\|\cdot\|_{\omega}$ is the $\varepsilon$-tensor product of $\left\{\|\cdot\|_{i, \omega}\right\}_{i \in\{1, \ldots, n\}}$, and for each $\omega \in \Omega_{\infty}$, the norm $\|\cdot\|_{\omega}$ is the orthogonal tensor product (see $\left.\S_{1.2 .9}\right)$ of $\left\{\|\cdot\|_{i, \omega}\right\}_{i \in\{1, \ldots, n\}}$. We call $\xi_{1} \otimes \cdots \otimes \xi_{n}$ the orthogonal tensor product of $\xi_{1}, \ldots, \xi_{n}$.

4.1.1.6. Exterior powers. — Let $E$ be a finite-dimensional vector space over $K$ and $\xi=\left\{\|\cdot\|_{E, \omega}\right\}_{\omega \in \Omega}$ be a norm family in $\mathcal{N}_{E}$. Let $i$ be a non-negative integer. We equip $E^{\otimes i}$ with the $\varepsilon, \pi$-tensor power of the norm family $\xi$, which induces by quotient a norm family on the exterior power $\Lambda^{i}(E)$ which we denote by $\Lambda^{i} \xi$.

4.1.1.7. Determinant. — Let $E$ be a finite-dimensional vector space over $K$ and $\xi=\left\{\|\cdot\|_{E, \omega}\right\}_{\omega \in \Omega}$ be a norm family in $\mathcal{N}_{E}$. Each norm $\|\cdot\|_{E, \omega}$ induces a determinant norm $\|\cdot\|_{\operatorname{det}(E), \omega}$ on $\operatorname{det}(E) \otimes_{K} K_{\omega} \cong \operatorname{det}\left(E_{K_{\omega}}\right)$ (see 1.1 .13 ). The norm family $\left\{\|\cdot\|_{\operatorname{det}(E), \omega}\right\}_{\omega \in \Omega}$ is called the determinant of $\xi$, denoted by $\operatorname{det}(\xi)$. By Proposition 1.2.15 we obtain that $\operatorname{det}(\xi)$ coincides with $\Lambda^{r} \xi$, where $r$ is the rank of $E$ over $K$.

4.1.1.8. Extension of scalars. - Let $E$ be a vector space of finite rank over $K$ and $\xi=\left\{\|\cdot\|_{\omega}\right\}_{\omega \in \Omega}$ be a norm family in $\mathcal{N}_{E}$. Let $L / K$ be an algebraic extension of the field $K$ and $\left(\left(\Omega_{L}, \mathcal{A}_{L}, \nu_{L}\right), \phi_{L}\right)$ be the adelic structure of the adelic curve $S \otimes_{K} L$. We construct a norm family $\xi_{L}=\left\{\|\cdot\|_{x}\right\}_{x \in \Omega_{L}} \in \mathcal{N}_{E \otimes_{K} L}$ as follows: for any $x \in \Omega_{L}$ whose canonical image in $\Omega$ is $\omega$, if $|\cdot|_{\omega}$ is non-Archimedean, $\|\cdot\|_{x}$ is the norm $\|\cdot\|_{\omega, L_{x}, \varepsilon}$ on $\left(E_{K_{\omega}}\right) \otimes_{K_{\omega}} L_{x}$ induced by $\|\cdot\|_{\omega}$ by $\varepsilon$-extension of scalars; otherwise $\|\cdot\|_{x}$ is the norm $\|\cdot\|_{\omega, L_{x}, \pi}$ on $\left(E_{K_{\omega}}\right) \otimes_{K_{\omega}} L_{x}$ induced by $\|\cdot\|_{\omega}$ by $\pi$-extension of scalars (see $\S 1.3)$. By Proposition 1.3.20 (1), if the rank of $E$ over $K$ is 1 , then the norm family $\left(\xi^{\vee}\right)_{L}$ identifies with the dual norm family of $\xi_{L}$. Moreover, by Corollary 1.3.15, if $L_{2} / L_{1} / K$ are successive algebraic extensions, then one has $\left(\xi_{L_{1}}\right)_{L_{2}}=\xi_{L_{2}}$, where we identify $E \otimes_{K} L_{2}$ with $\left(E \otimes_{K} L_{1}\right) \otimes_{L_{1}} L_{2}$.

Let $\xi=\left\{\|\cdot\|_{\omega}\right\}_{\omega \in \Omega} \in \mathcal{H}_{E}$ be a Hermitian norm family, where for $\omega \in \Omega$, the norm $\|\cdot\|_{\omega}$ is induced by an inner product $\langle,\rangle_{\omega}$. We denote by $\xi_{L}^{H}=\left\{\|\cdot\|_{x}\right\}_{x \in \Omega_{L}} \in \mathcal{H}_{E \otimes_{K} L}$ the Hermitian norm family defined as follows. For any $x \in \Omega_{L} \backslash \Omega_{L, \infty}$ over $\omega \in \Omega \backslash \Omega_{\infty}$, one has $\|\cdot\|_{x}=\|\cdot\|_{\omega, L_{x}, \varepsilon} ;$ for any $x \in \Omega_{L, \infty}$ over $\omega \in \Omega_{\infty},\|\cdot\|_{x}$ is the norm $\|\cdot\|_{\omega, L_{x}, \mathrm{HS}}$ induced by the inner product $\langle,\rangle_{\omega, L_{x}}$ on $E_{L_{x}}$ which extends $\langle,\rangle_{\omega}$ on $E_{K_{\omega}}$ (namely $\|\cdot\|_{x}$ is the orthogonal tensor product norm of $\|\cdot\|_{\omega}$ and $|\cdot|_{x}$ if we identify $E_{L_{x}}$ with 
$E_{K_{\omega}} \otimes_{K_{\omega}} L_{x}$, see Remark 1.3.2). One has $\left(\xi^{\vee}\right)_{L}^{H}=\left(\xi_{L}^{H}\right)^{\vee}$ (see Proposition 1.3.20 (1) for the ultrametric part and Remark 1.3.2 for the inner product part).

4.1.1.9. Comparison of norm families. — Let $E$ be a finite-dimensional vector space over $K$, and $\xi=\left\{\|\cdot\|_{\omega}\right\}_{\omega \in \Omega}$ and $\xi^{\prime}=\left\{\|\cdot\|_{\omega}^{\prime}\right\}_{\omega \in \Omega}$ be two elements of $\mathcal{N}_{E}$. We say that $\xi$ is smaller than $\xi^{\prime}$ if for any $\omega \in \Omega$ one has $\|\cdot\|_{\omega} \leqslant\|\cdot\|_{\omega}^{\prime}$. The condition " $\xi$ is smaller than $\xi^{\prime \prime}$ is denoted by $\xi \leqslant \xi^{\prime}$ or $\xi^{\prime} \geqslant \xi$.

4.1.1.10. Local distance. - Let $E$ be a finite-dimensional vector space over $K$, and $\xi=\left\{\|\cdot\|_{\omega}\right\}_{\omega \in \Omega}$ and $\xi^{\prime}=\left\{\|\cdot\|_{\omega}^{\prime}\right\}_{\omega \in \Omega}$ be two norm families in $\mathcal{N}_{E}$. For any $\omega \in \Omega$, let

$$
d_{\omega}\left(\xi, \xi^{\prime}\right):=\sup _{s \in E_{K_{\omega} \backslash\{0\}}}\left|\ln \|s\|_{\omega}-\ln \|s\|_{\omega}^{\prime}\right| .
$$

We call $d_{\omega}\left(\xi, \xi^{\prime}\right)$ the local distance on $\omega$ of the norm families $\xi$ and $\xi^{\prime}$. By Proposition 1.1.43, one has

$$
d_{\omega}\left(\xi^{\vee},\left(\xi^{\prime}\right)^{\vee}\right) \leqslant d_{\omega}\left(\xi, \xi^{\prime}\right)
$$

and the equality holds if $\omega \in \Omega_{\infty}$ or if $\|\cdot\|_{\omega}$ and $\|\cdot\|_{\omega}^{\prime}$ are both ultrametric.

4.1.2. Dominated norm families. - Let $E$ be a finite-dimensional vector space over $K$ and $\xi=\left\{\|\cdot\|_{\omega}\right\}_{\omega \in \Omega}$ be a norm family in $\mathcal{N}_{E}$.

Definition 4.1.1. - We say that the norm family $\xi$ is upper dominated if, for any non-zero element $s \in E$, there exists a $\nu$-integrable function $A(\cdot)$ on $\Omega$ such that $\ln \|s\|_{\omega} \leqslant A(\omega) \nu$-almost everywhere. Note that the upper dominancy is equivalent to

$$
\forall s \in E \backslash\{0\}, \quad \bar{\int}_{\Omega} \ln \|s\|_{\omega} \nu(d \omega)<+\infty
$$

with the notation of Definition A.4.1. Similarly, we say that the norm family $\xi$ is lower dominated if, for any non-zero element $s \in E$, there exists a $\nu$-integrable function $B(\cdot)$ on $\Omega$ such that $B(\omega) \leqslant \ln \|s\|_{\omega} \nu$-almost everywhere. Note that the lower dominancy is equivalent to

$$
\forall s \in E \backslash\{0\}, \quad \underline{\int}_{\Omega} \ln \|s\|_{\omega} \nu(d \omega)>-\infty .
$$

Definition 4.1.2. - We say that $\xi$ is dominated if $\xi$ and $\xi^{\vee}$ are both upper dominated. Note that the upper dominancy of $\xi$ and $\xi^{\vee}$ implies the lower dominancy of $\xi^{\vee}$ and $\xi$, respectively, because (see Proposition A.4.7) $\ln \|\alpha\|_{\omega, *}+\ln \|s\|_{\omega} \geqslant 0$ for all $s \in E$ and $\alpha \in E^{\vee}$ with $\alpha(s)=1$, so that if $\xi$ is dominated, then $\xi$ and $\xi^{\vee}$ are upper and lower dominated.

Remark 4.1.3. - If $\xi$ is a dominated norm family, then also is $\xi^{\vee}$. In fact, for any $\omega \in \Omega$ one has $\|\cdot\|_{\omega, * *} \leqslant\|\cdot\|_{\omega}$ (see (1.5)). Therefore the upper dominancy of $\xi$ implies that of $\xi^{\vee \vee}$. The converse is true when $\|\cdot\|_{\omega}$ is ultrametric for $\omega \in \Omega \backslash \Omega_{\infty}$ since in 
this case one has $\|\cdot\|_{\omega, * *}=\|\cdot\|_{\omega}$ for any $\omega \in \Omega$ (see Proposition 1.1.18 and Corollary $1.2 .12)$.

Remark 4.1.4. - It is not true that if $\xi$ is upper and lower dominated then it is dominated. Consider the following example. Let $K$ be an infinite field. We equip $K$ with the discrete $\sigma$-algebra $\mathcal{A}$ and let $\nu$ be the atomic measure on $K$ such that $\nu(\{a\})=1$ for any $a \in K$. For any $a \in K$, let $|\cdot|_{a}$ be the trivial absolute value on $K$. Then $S=\left(K,(K, \mathcal{A}, \nu),\left\{|\cdot|_{a}\right\}_{a \in K}\right)$ forms an adelic curve. Consider now the vector space $E=K^{2}$ over $K$. For any $a \in K$ let $\|\cdot\|_{a}$ be the norm on $K^{2}$ such that

$$
\|(x, y)\|_{a}= \begin{cases}0, & \text { if } x=y=0 \\ 1 / 2, & \text { if } y=a x, x \neq 0 \\ 1, & \text { else. }\end{cases}
$$

Then for any vector $s \in K^{2}, s \neq 0$, one has $\|s\|_{a}=1$ for all except at most one $a \in K$. Therefore the function $(a \in K) \mapsto \ln \|s\|_{a}$ on $K$ is integrable, and in particular dominated. Therefore the norm family $\xi=\left\{\|\cdot\|_{a}\right\}_{a \in K}$ is upper dominated and lower dominated. However, for any $a \in K$, the dual norm $\|\cdot\|_{a, *}$ on $K^{2}$ (we identify $K^{2}$ with the dual vector space of itself in the canonical way) satisfies

$$
\|(x, y)\|_{a, *}= \begin{cases}0, & \text { if } x=y=0 \\ 1, & \text { if } x=-a y, y \neq 0 \\ 2, & \text { else. }\end{cases}
$$

Therefore, for any non-zero element $s \in K^{2}$, one has $\ln \|s\|_{a, *}=\ln (2)$ for all except at most one element $a \in K$. The dual norm family $\xi^{\vee}$ is thus not upper dominated.

Example 4.1.5. - A fundamental example of dominated norm family is that arising from a basis. Let $E$ be a vector space of finite rank $r$ over $K$, and $\boldsymbol{e}=\left\{e_{1}, \ldots, e_{r}\right\}$ be a basis of $E$ over $K$. For any algebraic extension $L / K$ and any $x \in \Omega_{L}$, let $\|\cdot\|_{e, x}$ be the norm on $E \otimes_{K} L_{x}$ such that, for any $\left(\lambda_{1}, \ldots, \lambda_{r}\right) \in L_{x}^{r}$,

$$
\left\|\lambda_{1} e_{1}+\cdots+\lambda_{r} e_{r}\right\|_{\boldsymbol{e}, x}:= \begin{cases}\max _{i \in\{1, \ldots, r\}}\left|\lambda_{i}\right|_{x}, & \text { if } x \in \Omega_{L} \backslash \Omega_{L, \infty} \\ \left|\lambda_{1}\right|_{x}+\cdots+\left|\lambda_{r}\right|_{x}, & \text { if } x \in \Omega_{L, \infty},\end{cases}
$$

where $\Omega_{L, \infty}$ denotes the set of all $x \in \Omega_{L}$ such that the absolute value $|\cdot|_{x}$ is Archimedean. Let $\xi_{\boldsymbol{e}}$ be the norm family $\left\{\|\cdot\|_{\boldsymbol{e}, \omega}\right\}_{\omega \in \Omega}$. Note that one has $\xi_{\boldsymbol{e}, L}=$ $\left\{\|\cdot\|_{e, x}\right\}_{x \in \Omega_{L}}$ for any algebraic extension $L / K$. Moreover, for any non-zero vector $s=a_{1} e_{1}+\cdots+a_{r} e_{r} \in E \otimes_{K} L$, with $\left(a_{1}, \ldots, a_{r}\right) \in L^{r}$, one has

$$
\forall x \in \Omega_{L}, \quad \ln \|s\|_{e, x} \leqslant \max _{\substack{i \in\{1, \ldots, r\} \\ a_{i} \neq 0}} \ln \left|a_{i}\right|_{x}+\ln (r) \mathbb{1}_{\Omega_{L}, \infty}(x) .
$$

Since the functions $x \mapsto \ln |a|_{x}$ are $\nu_{L}$-integrable for all $a \in L \backslash\{0\}$ and since $\nu_{L}\left(\Omega_{L, \infty}\right)<+\infty$ (see Proposition 3.1.2), we obtain that the function $\left(x \in \Omega_{L}\right) \mapsto$ 
$\ln \|s\|_{\boldsymbol{e}, x}$ is $\nu_{L}$-integrable. If we denote by $\left\{e_{1}^{\vee}, \ldots, e_{r}^{\vee}\right\}$ the dual basis of $\boldsymbol{e}$, then for any $\alpha=a_{1} e_{1}^{\vee}+\cdots+a_{r} e_{r}^{\vee} \in E^{\vee} \otimes_{K} L$ with $\left(a_{1}, \ldots, a_{r}\right) \in L^{r}$ and any $x \in \Omega_{L}$ one has

$$
\|\alpha\|_{e, x, *}=\max \left\{\left|a_{1}\right|_{x}, \ldots,\left|a_{r}\right|_{x}\right\} \text {. }
$$

Therefore the function $\left(x \in \Omega_{L}\right) \mapsto\|\alpha\|_{\boldsymbol{e}, x, *}$ is $\mathcal{A}$-measurable. If $\alpha \neq 0$, then the function $\left(x \in \Omega_{L}\right) \mapsto \ln \|\alpha\|_{\mathbf{e}, x, *}$ is $\nu_{L}$-integrable. Hence the norm family $\xi_{\boldsymbol{e}, L}$ is dominated. Note that (see page 216 for the definition of the local distance function)

$$
\forall x \in \Omega_{L}, \quad d_{x}\left(\left(\xi_{\boldsymbol{e}, L}\right)^{\vee}, \xi_{\boldsymbol{e}^{\vee}, L}\right) \leqslant \ln (r) \mathbb{1}_{\Omega_{L, \infty}}(x),
$$

where $\boldsymbol{e}^{\vee}$ denotes the dual basis of $\boldsymbol{e}$.

Proposition 4.1.6. - Let $E$ be a finite-dimensional vector space over $K$, $\xi_{1}$ and $\xi_{2}$ be norm families in $\mathcal{N}_{E}$. We assume that $\xi_{1}$ is dominated. If the local distance function $(\omega \in \Omega) \mapsto d_{\omega}\left(\xi_{1}, \xi_{2}\right)$ is $\nu$-dominated (see Definition A.4.9), then the norm family $\xi_{2}$ is dominated. In particular, if there exists a basis $\boldsymbol{e}$ of $E$ over $K$ such that the function $(\omega \in \Omega) \mapsto d_{\omega}\left(\xi_{\boldsymbol{e}}, \xi_{2}\right)$ is $\nu$-dominated, then the norm family $\xi_{2}$ is dominated.

Proof. - Assume that $\xi_{i}$ is of the form $\left\{\|\cdot\|_{i, \omega}\right\}_{\omega \in \Omega}, i \in\{1,2\}$. Let $s$ be a non-zero element in $E$. For any $\omega \in \Omega$, one has,

$$
\ln \|s\|_{2, \omega}-\ln \|s\|_{1, \omega} \leqslant d_{\omega}\left(\xi_{1}, \xi_{2}\right) \quad \nu \text {-almost everywhere. }
$$

Moreover, since the norm family $\xi_{1}$ is dominated, one has $\bar{\int}_{\Omega} \ln \|s\|_{1, \omega} \nu(d \omega)<+\infty$; since the local distance function $d\left(\xi_{1}, \xi_{2}\right)$ is dominated, one has $\bar{\int}_{\Omega} d_{\omega}\left(\xi_{1}, \xi_{2}\right) \nu(d \omega)<$ $+\infty$. Therefore by Proposition A.4.4 one has

$$
\bar{\int}_{\Omega} \ln \|s\|_{2, \omega} \nu(d \omega) \leqslant \overline{\int_{\Omega}} \ln \|s\|_{1, \omega} \nu(d \omega)+\bar{\int}_{\Omega} d_{\omega}\left(\xi_{1}, \xi_{2}\right) \nu(d \omega)<+\infty .
$$

By (4.1), one has $d_{\omega}\left(\xi_{1}^{\vee}, \xi_{2}^{\vee}\right) \leqslant d_{\omega}\left(\xi_{1}, \xi_{2}\right)$ for any $\omega$. Hence the same argument as above applied to the dual norm families shows that

$$
\forall \alpha \in E^{\vee} \backslash\{0\}, \quad \bar{\int}_{\Omega} \ln \|\alpha\|_{2, \omega, *} \nu(d \omega)<+\infty .
$$

Therefore, the norm family $\xi_{2}$ is dominated. To establish the last assertion, it suffices to apply the obtained result to the case where $\xi_{1}=\xi_{\boldsymbol{e}}$ (see Example 4.1.5 for the fact that the norm family $\xi_{\boldsymbol{e}}$ is dominated).

Proposition 4.1.7. - Let $E$ be a vector space of finite rank over $K$ and $\xi=\left\{\|\cdot\|_{\omega}\right\}_{\omega \in \Omega}$ be an element of $\mathcal{N}_{E}$ which is dominated. Then for any basis $\boldsymbol{e}=\left\{e_{1}, \ldots, e_{r}\right\}$ of $E$, there exists a $\nu$-integrable function $A_{\boldsymbol{e}}$ on $\Omega$ such that, for any algebraic extension $L / K$ and any $x \in \Omega_{L}$ one has (note that $\xi_{K}=\xi^{\vee \vee}$ in the case where $L=K$ )

$$
d_{x}\left(\xi_{L}, \xi_{\boldsymbol{e}, L}\right) \leqslant A_{\boldsymbol{e}}\left(\pi_{L / K}(x)\right)
$$

In particular, the local distance function $\left(x \in \Omega_{L}\right) \mapsto d_{x}\left(\xi_{L}, \xi_{\boldsymbol{e}, L}\right)$ is $\nu_{L}$-dominated. 
Proof. - Let $x$ be an element in $\Omega_{L}$. Assume that $s$ is a non-zero vector of $E_{L_{x}}$ which is written as $s=a_{1} e_{1}+\cdots+a_{r} e_{r}$ with $\left(a_{1}, \ldots, a_{r}\right) \in L_{x}^{r}$. Then one has

$$
\|s\|_{x} \leqslant \begin{cases}\max _{i \in\{1, \ldots, r\}}\left(\left|a_{i}\right|_{x} \cdot\left\|e_{i}\right\|_{x}\right), & \text { if } x \in \Omega_{L} \backslash \Omega_{L, \infty}, \\ \sum_{i=1}^{r}\left|a_{i}\right|_{x} \cdot\left\|e_{i}\right\|_{x}, & \text { if } x \in \Omega_{L, \infty} .\end{cases}
$$

Thus $\ln \|s\|_{x} \leqslant \ln \|s\|_{e, x}+\max _{i \in\{1, \ldots, r\}} \ln \left\|e_{i}\right\|_{x}$. Moreover, one can also interpret

$$
\sup _{0 \neq s \in E_{L_{x}}} \frac{\|s\|_{\boldsymbol{e}, x}}{\|s\|_{x}}
$$

as the operator norm of the $L_{x}$-linear map

$$
\operatorname{Id}_{E_{L_{x}}}:\left(E_{L_{x}},\|\cdot\|_{x}\right) \longrightarrow\left(E_{L_{x}},\|\cdot\|_{e, x}\right)
$$

which is equal to the operator norm of the dual $L_{x}$-linear map

$$
\operatorname{Id}_{E_{L_{x}}^{\vee}}:\left(E_{L_{x}}^{\vee},\|\cdot\|_{e, x, *}\right) \longrightarrow\left(E_{L_{x}}^{\vee},\|\cdot\|_{x, *}\right)
$$

since the norm $\|\cdot\|_{e, x}$ is reflexive (see Proposition 1.1.22). Let $\left\{e_{i}^{\vee}\right\}_{i=1}^{r}$ be the dual basis of $\boldsymbol{e}$. For any $\alpha=b_{1} e_{1}^{\vee}+\cdots+b_{r} e_{r}^{\vee} \in E_{L_{x}}^{\vee}$, one has

$$
\|\alpha\|_{x, *} \leqslant \begin{cases}\max _{i \in\{1, \ldots, r\}}\left(\left|b_{i}\right|_{x} \cdot\left\|e_{i}^{\vee}\right\|_{x, *}\right), & \text { if } x \in \Omega_{L} \backslash \Omega_{L, \infty}, \\ \sum_{i=1}^{r}\left|b_{i}\right|_{x} \cdot\left\|e_{i}^{\vee}\right\|_{x, *}, & \text { if } x \in \Omega_{L, \infty}\end{cases}
$$

Thus we obtain

$$
\ln \|\alpha\|_{x, *}-\ln \|\alpha\|_{e, x, *} \leqslant \max _{i \in\{1, \ldots, r\}} \ln \left\|e_{i}^{\vee}\right\|_{x, *}+\ln (r) \mathbb{1}_{\Omega_{L, \infty}}(x) .
$$

Therefore, for any $s \in E_{L}$, one has

$$
-\max _{i \in\{1, \ldots, r\}} \ln \left\|e_{i}\right\|_{x} \leqslant \ln \|s\|_{e, x}-\ln \|s\|_{x} \leqslant \max _{i \in\{1, \ldots, r\}} \ln \left\|e_{i}^{\vee}\right\|_{x, *}+\ln (r) \mathbb{1}_{\Omega_{L, \infty}}(x) .
$$

Note that, if $\omega=\pi_{L / K}(x)$, then one has (see Proposition 1.3.1)

$$
\forall i \in\{1, \ldots, r\}, \quad\left\|e_{i}\right\|_{x}=\left\|e_{i}\right\|_{\omega, * *} .
$$

Moreover, if $\omega=\pi_{L / K}(x)$ belongs to $\Omega \backslash \Omega_{\infty}$, then

$$
\left\|e_{i}^{\vee}\right\|_{x, *}=\left\|e_{i}^{\vee}\right\|_{\omega, L_{x}, \varepsilon, *}=\left\|e_{i}^{\vee}\right\|_{\omega, *, L_{x}, \varepsilon}=\left\|e_{i}^{\vee}\right\|_{\omega, *},
$$

where the second equality comes from Proposition 1.3.20 (1) and the last one comes from Proposition 1.3.1. If $\omega=\pi_{L / K}(x) \in \Omega_{\infty}$, then

$$
\left\|e_{i}^{\vee}\right\|_{x, *}=\left\|e_{i}^{\vee}\right\|_{\omega, L_{x}, \pi, *}=\left\|e_{i}^{\vee}\right\|_{\omega, *, L_{x}, \varepsilon}=\left\|e_{i}^{\vee}\right\|_{\omega, *},
$$


where the second equality comes from Proposition $1.3 .20(2)$, and the last one comes from Proposition 1.3.1. Since the norm family $\xi$ is dominated, there exists a $\nu$ integrable function $A$ on $\Omega$ such that (see Remark 4.1.3)

$$
\max _{i \in\{1, \ldots, r\}} \max \left\{\ln \left\|e_{i}\right\|_{\omega, * *}, \ln \left\|e_{i}^{\vee}\right\|_{\omega, *}\right\} \leqslant A(\omega) \quad \nu \text {-almost everywhere. }
$$

Therefore, by (4.4), we obtain

$$
d_{x}\left(\xi_{L}, \xi_{\boldsymbol{e}, L}\right) \leqslant A_{\boldsymbol{e}}\left(\pi_{L / K}(x)\right) \quad \nu \text {-almost everywhere, }
$$

with

$$
\forall \omega \in \Omega, \quad A_{\boldsymbol{e}}(\omega):=A(\omega)+\ln (r) \mathbb{1}_{\Omega_{\infty}}(\omega) .
$$

Note that the function $A_{\boldsymbol{e}}$ is $\nu$-integrable on $(\Omega, \mathcal{A})$. The proposition is thus proved.

Corollary 4.1.8. - Let $E$ be a vector space of finite rank over $K, \xi_{1}$ and $\xi_{2}$ be norm families in $\mathcal{N}_{E}$ which are dominated and ultrametric on $\Omega \backslash \Omega_{\infty}$. Then the local distance function $(\omega \in \Omega) \mapsto d_{\omega}\left(\xi_{1}, \xi_{2}\right)$ is $\nu$-dominated.

Proof. - Let $\boldsymbol{e}$ be a basis of $E$ over $K$. By Proposition 4.1.7, the local distance functions $(\omega \in \Omega) \mapsto d_{\omega}\left(\xi_{1}, \xi_{\boldsymbol{e}}\right)$ and $(\omega \in \Omega) \mapsto d_{\omega}\left(\xi_{2}, \xi_{\boldsymbol{e}}\right)$ are both $\nu$-dominated. Since for any $\omega \in \Omega$ one has

$$
d_{\omega}\left(\xi_{1}, \xi_{2}\right) \leqslant d_{\omega}\left(\xi_{1}, \xi_{\boldsymbol{e}}\right)+d_{\omega}\left(\xi_{2}, \xi_{\boldsymbol{e}}\right)
$$

by Propositions A.4.2 and A.4.4 the function $(\omega \in \Omega) \mapsto d_{\omega}\left(\xi_{1}, \xi_{2}\right)$ is $\nu$-dominated.

Remark 4.1.9. - The assertion of Corollary 4.1.8 does not necessarily hold without the condition that the norm families are ultrametric on $\Omega \backslash \Omega_{\infty}$. Consider the following counter-example. Let $K$ be an infinite field, $\mathcal{A}$ be the discrete $\sigma$-algebra on $K$ and $\nu$ be the atomic measure on $K$ such that $\nu(\{a\})=1$ for any $a \in K$. For any $a \in K$, let $|\cdot|_{a}$ be the trivial absolute value on $K$. We consider the adelic curve $S=\left(K,(K, \mathcal{A}, \nu),\left\{|\cdot|_{a}\right\}_{a \in K}\right)$. For any $a \in K$, let $\|\cdot\|_{a}$ be the norm on $K^{2}$ such that

$$
\|(x, y)\|_{a}= \begin{cases}0, & \text { if } x=y=0 \\ 2, & \text { if } y=a x, x \neq 0 \\ 1, & \text { else. }\end{cases}
$$

Note that for any $s \in K^{2} \backslash\{(0,0)\}$, one has $\|s\|_{a}=1$ for all except at most one $a \in K$. Therefore the norm family $\xi=\left\{\|\cdot\|_{a}\right\}_{a \in K}$ is upper dominated. If we identify $K^{2}$ with the dual vector space of itself in the canonical way, then for any $a \in K$ one has

$$
\forall(x, y) \in K^{2}, \quad\|(x, y)\|_{a, *}= \begin{cases}0, & \text { if } x=y=0, \\ 1, & \text { else. }\end{cases}
$$


Hence the dual norm family $\xi^{\vee}$ is also upper dominated. Now let $\boldsymbol{e}=\{(1,0),(0,1)\}$ be the canonical basis of $K^{2}$. For any $a \in K$ one has

$$
\forall(x, y) \in K^{2}, \quad\|(x, y)\|_{e, a}= \begin{cases}0, & \text { if } x=y=0, \\ 1, & \text { else. }\end{cases}
$$

Therefore one has $d_{a}\left(\xi, \xi_{\boldsymbol{e}}\right)=\ln (2)$ for any $a \in K$. Since $K$ is an infinite set, the local distance function $(a \in K) \mapsto d_{a}\left(\xi, \xi_{\boldsymbol{e}}\right)$ is clearly not upper dominated.

Corollary 4.1.10. - Let $E$ be a finite-dimensional vector space over $K$ and $\xi=$ $\left\{\|\cdot\|_{\omega}\right\}_{\omega \in \Omega}$ be a norm family in $\mathcal{N}_{E}$. The following assertions are equivalent:

(1) the norm family $\xi$ is dominated and the local distance function $(\omega \in \Omega) \mapsto$ $d_{\omega}\left(\xi, \xi^{\vee \vee}\right)$ is $\nu$-dominated;

(2) for any basis $\boldsymbol{e}$ of $E$, the local distance function $(\omega \in \Omega) \mapsto d_{\omega}\left(\xi, \xi_{\boldsymbol{e}}\right)$ is $\nu$ dominated;

(3) there exists a basis $\boldsymbol{e}$ of $E$ such that the local distance function $(\omega \in \Omega) \mapsto$ $d_{\omega}\left(\xi, \xi_{\boldsymbol{e}}\right)$ is $\nu$-dominated.

Proof. - $\sqrt{(1)} \Longrightarrow \sqrt{(2)]}$ : Note that the norm family $\xi^{\vee \vee}$ is ultrametric on $\Omega \backslash \Omega_{\infty}$. Moreover it is dominated since $\xi$ is dominated (see Remark 4.1.3). By Proposition 4.1.7, we obtain that, for any basis $\boldsymbol{e}$ of $E$, the local distance function $(\omega \in \Omega) \mapsto d_{\omega}\left(\xi^{\vee \vee}, \xi_{\boldsymbol{e}}\right)$ is $\nu$-dominated. By the assumption that the function $(\omega \in \Omega) \mapsto d_{\omega}\left(\xi, \xi^{\vee \vee}\right)$ is $\nu$ dominated, we deduce that the function $(\omega \in \Omega) \mapsto d_{\omega}\left(\xi, \xi_{\boldsymbol{e}}\right)$ is also $\nu$-dominated.

' $\Rightarrow(2) \Longrightarrow$ ' is trivial.

' $\Rightarrow(3) \Longrightarrow$ ': By Proposition 4.1.6, the norm family $\xi$ is $\nu$-dominated. By Proposition 4.1.7, the function $(\omega \in \Omega) \mapsto d_{\omega}\left(\xi^{\vee \vee}, \xi_{\boldsymbol{e}}\right)$ is $\nu$-dominated. Since $d_{\omega}\left(\xi, \xi^{\vee \vee}\right) \leqslant$ $d_{\omega}\left(\xi, \xi_{\boldsymbol{e}}\right)+d_{\omega}\left(\xi^{\vee \vee}, \xi_{\boldsymbol{e}}\right)$, we obtain that the function $(\omega \in \Omega) \mapsto d_{\omega}\left(\xi, \xi^{\vee \vee}\right)$ is $\nu$ dominated.

Definition 4.1.11. - Let $E$ be a finite-dimensional vector space over $K$ and $\xi$ be a norm family on $E$. We say that $\xi$ is strongly dominated if it is dominated and if the function $(\omega \in \Omega) \mapsto d_{\omega}\left(\xi, \xi^{\vee \vee}\right)$ is $\nu$-dominated (or equivalently, $(E, \xi)$ satisfies any of the assertions in Corollary 4.1.10).

Remark 4.1.12. - Let $E$ be a finite-dimensional vector space over $K$ and $\xi$ be a norm family on $E$. If the norm family $\xi$ is ultrametric on $\Omega \backslash \Omega_{\infty}$, then the function $(\omega \in \Omega) \mapsto d_{\omega}\left(\xi, \xi^{\vee \vee}\right)$ is identically zero (and hence is $\nu$-dominated). Therefore, in this case $\xi$ is dominated if and only if it is strongly dominated. In particular, in the case where $E$ is of dimension 1 over $K$, the norm family $\xi$ is dominated if and only if it is strongly dominated. Moreover, if $\xi$ is a dominated norm family on a finitedimensional vector space, then the dual norm family $\xi$ is strongly dominated since it is dominated (see Remark 4.1.3) and ultrametric on $\Omega \backslash \Omega_{\infty}$. 
Corollary 4.1.13. - Let $E$ be a vector space of finite rank over $K$ and $\xi=$ $\left\{\|\cdot\|_{\omega}\right\}_{\omega \in \Omega}$ be a norm family in $\mathcal{N}_{E}$. If $\xi$ is dominated, then for any algebraic extension $L / K$ the norm family $\xi_{L}$ is strongly dominated. Conversely, if there exists an algebraic extension $L / K$ such that the norm family $\xi_{L}$ is dominated and if the function $(\omega \in \Omega) \mapsto d_{\omega}\left(\xi, \xi^{\vee \vee}\right)$ is $\nu$-dominated, then the norm family $\xi$ is also dominated.

Proof. - Assume that the norm family $\xi$ is dominated. By Proposition 4.1.7, for any basis $\boldsymbol{e}$ of $E$, the local distance function $\left(x \in \Omega_{L}\right) \mapsto d_{x}\left(\xi_{L}, \xi_{\boldsymbol{e}, L}\right)$ is $\nu_{L}$-dominated. Therefore, by Proposition 4.1.6 we obtain that the norm family $\xi_{L}$ is strongly dominated.

Conversely, we assume that $L / K$ is an algebraic extension and $\xi_{L}$ is dominated. Let $\boldsymbol{e}$ be a basis of $E$ over $K$. Since $\xi_{L}$ is dominated and since the norms in the family $\xi_{L}$ corresponding to non-Archimedean absolute values are ultrametric, the function $f:\left(x \in \Omega_{L}\right) \mapsto d_{x}\left(\xi_{L}, \xi_{e, L}\right)$ is $\nu_{L}$-dominated. Moreover, by Proposition 1.3.7, one has $f=g \circ \pi_{L / K}$, where $g$ sends $\omega \in \Omega$ to $d_{\omega}\left(\xi^{\vee \vee}, \xi_{\boldsymbol{e}}\right)$ (one has $\xi_{\boldsymbol{e}}=\xi_{\boldsymbol{e}}^{\vee \vee}$ since it is ultrametric on $\left.\Omega \backslash \Omega_{\infty}\right)$. Since the function $f$ is $\nu_{L}$-dominated, there exists an $\nu_{L}$-integrable function $A$ on $\Omega_{L}$ such that $|f| \leqslant A$ almost everywhere. By Proposition 3.4.9, the function $I_{L / K}(A)$ is $\nu$-integrable. Moreover, one has $I_{L / K}(|f|)=|g|$ since $|f|=|g| \circ \pi_{L / K}$ (see Remark 3.4.7). Therefore, the function $g$ is $\nu$-dominated by the $\nu$ integrable function $I_{L / K}(A)$, which implies that the norm family $\xi^{\vee \vee}$ is dominated. Finally, by Proposition 4.1.6 and the assumption that the function $(\omega \in \Omega) \mapsto d_{\omega}\left(\xi, \xi^{\vee \vee}\right)$ is $\nu$-dominated, we obtain that the norm family $\xi$ is dominated.

Remark 4.1.14. - Let $E$ be a finite-dimensional vector space over $K$. Corollary 4.1 .10 implies that there exist Hermitian norm families on $E$ which are dominated. In fact, let $e=\left\{e_{i}\right\}_{i=1}^{r}$ be a basis of $E$ over $K$. Consider the following norm family $\xi=\left\{\|\cdot\|_{\omega}\right\}_{\omega \in \Omega}$ with

$$
\forall\left(a_{1}, \ldots, a_{r}\right) \in K_{\omega}^{r}, \quad\left\|a_{1} e_{1}+\cdots+a_{r} e_{r}\right\|_{\omega}= \begin{cases}\max _{i \in\{1, \ldots, r\}}\left|a_{i}\right|_{\omega}, & \text { if } \omega \in \Omega \backslash \Omega_{\infty}, \\ \left(\sum_{i=1}^{r}\left|a_{i}\right|_{\omega}^{2}\right)^{1 / 2}, & \text { if } \omega \in \Omega_{\infty} .\end{cases}
$$

It is a Hermitian norm family on $E$. Note that one has $d_{\omega}\left(\xi, \xi_{e}\right) \leqslant \frac{1}{2} \ln (r) \mathbb{1}_{\Omega_{\infty}}(\omega)$. Therefore $(\omega \in \Omega) \mapsto d_{\omega}\left(\xi, \xi_{\boldsymbol{e}}\right)$ is a $\nu$-dominated function on $\Omega$. By Corollary 4.1.10, we obtain that $\xi$ is a dominated norm family.

Let $\xi$ be a Hermitian norm family on $E$. If $\xi$ is dominated, then for any algebraic extension $L / K$, the norm family $\xi_{L}^{H}$ is dominated. In fact, by Corollary 4.1.10, the norm family $\xi_{L}$ is dominated. By Proposition 1.3.1 (3), the local distance function $\left(x \in \Omega_{L}\right) \mapsto d_{x}\left(\xi_{L}, \xi_{L}^{H}\right)$ is bounded from above by $\frac{1}{2} \ln (2) \mathbb{1}_{\Omega_{L, \infty}}$. By Proposition 4.1.6. we obtain that the norm family $\xi_{L}^{H}$ is dominated.

Proposition 4.1.15. - Let $E$ be a finite-dimensional vector space over $K$ and $L / K$ be an algebraic extension of fields. For any $\omega \in \Omega$, we fix an extension $|\cdot|_{L, \omega}$ on $L$ of the absolute value $|\cdot|_{\omega}$ and denote by $L_{\omega}$ the completion of $L$ with respect to the 
extended absolute value. Let $\boldsymbol{e}=\left\{e_{i}\right\}_{i=1}^{r}$ be a basis of $E \otimes_{K} L$. For any $\omega \in \Omega$, let $\|\cdot\|_{\omega}^{\prime}$ be the norm on $E \otimes_{K} L_{\omega}$ defined as

$$
\forall\left(\lambda_{1}, \ldots, \lambda_{r}\right) \in L_{\omega}^{r}, \quad\left\|\lambda_{1} e_{1}+\cdots+\lambda_{r} e_{r}\right\|_{\omega}^{\prime}=\max _{i \in\{1, \ldots, r\}}\left|\lambda_{i}\right|_{L, \omega}
$$

and let $\|\cdot\|_{\omega}$ be the restriction of $\|\cdot\|_{\omega}^{\prime}$ to $E \otimes_{K} K_{\omega}$. Then the norm family $\xi=$ $\left\{\|\cdot\|_{\omega}\right\}_{\omega \in \Omega}$ in $\mathcal{N}_{E}$ is strongly dominated.

Proof. - We first prove that, for any element $b \in L$, the function

$$
(\omega \in \Omega) \longmapsto \ln |b|_{L, \omega}
$$

is bounded from above by a $\nu$-integrable function. Let

$$
F(X)=X^{n}+a_{n-1} X^{n-1}+\cdots+a_{0} \in K[X]
$$

be the minimal polynomial of $b$. By the same argument as in the proof of Theorem 3.3.7 (4) we obtain that

$$
\ln |b|_{\omega} \leqslant \mathbb{1}_{\Omega_{\infty}}(\omega) \ln (n)+\max \left\{0, \ln \left|a_{0}\right|_{\omega}, \ldots, \ln \left|a_{n-1}\right|_{\omega}\right\} .
$$

By Proposition 3.1.2 the function $\mathbb{1}_{\Omega_{\infty}}$ is $\nu$-integrable. Moreover, by the definition of adelic curves, for any $i \in\{0, \ldots, n-1\}$ such that $a_{i} \neq 0$, the function $(\omega \in \Omega) \mapsto$ $\ln \left|a_{i}\right|_{\omega}$ is also $\nu$-integrable, we thus obtain the assertion.

Let $\boldsymbol{f}=\left\{f_{i}\right\}_{i=1}^{r}$ be a basis of $E$ over $K$ and $A=\left(a_{i j}\right)_{(i, j) \in\{1, \ldots, r\}^{2}} \in M_{r \times r}(L)$ be the transition matrix between $\boldsymbol{e}$ and $\boldsymbol{f}$, namely

$$
\forall i \in\{1, \ldots, r\}, \quad f_{i}=\sum_{j=1}^{r} a_{i j} e_{j} .
$$

Let $\left(b_{i j}\right)_{(i, j) \in\{1, \ldots, r\}^{2}} \in M_{r \times r}(L)$ be the inverse matrix of $A$. Then one has

$$
\forall i \in\{1, \ldots, r\}, \quad e_{i}=\sum_{j=1}^{r} b_{i j} f_{j} .
$$

By the above assertion, there exists a $\nu$-integrable function $g$ on $\Omega$ such that

$$
\forall \omega \in \Omega, \max _{(i, j) \in\{1, \ldots, r\}^{2}} \max \left\{\ln \left|a_{i j}\right|_{\omega}, \ln \left|b_{i j}\right|_{\omega}\right\} \leqslant g(\omega) .
$$

We will prove that the local distance function $d\left(\xi, \xi_{f}\right)$ is $\nu$-dominated. Let $\omega \in \Omega$ and $x=\lambda_{1} f_{1}+\cdots+\lambda_{r} f_{r}$ be an element of $E \otimes_{K} K_{\omega}$. One has

$$
x=\sum_{i=1}^{r} \lambda_{i} \sum_{j=1}^{r} a_{i j} e_{j}=\sum_{j=1}^{r}\left(\sum_{i=1}^{r} a_{i j} \lambda_{i}\right) e_{j} .
$$

Therefore

$$
\begin{aligned}
\ln \|x\|_{\omega} & =\max _{j \in\{1, \ldots, r\}} \ln \left|\sum_{i=1}^{r} a_{i j} \lambda_{i}\right|_{L, \omega} \leqslant \max _{i \in\{1, \ldots, r\}} \ln \left|\lambda_{i}\right|_{\omega}+g(\omega)+\ln (r) \mathbb{1}_{\Omega_{\infty}}(\omega) \\
& \leqslant \ln \|x\|_{\boldsymbol{f}, \omega}+g(\omega)+\ln (r) \mathbb{1}_{\Omega_{\infty}}(\omega) .
\end{aligned}
$$


Similarly, if we write $x$ as $x=\mu_{1} e_{1}+\cdots+\mu_{r} e_{r}$, with $\left(\mu_{1}, \ldots, \mu_{r}\right) \in L_{\omega}^{r}$, one has

$$
x=\sum_{i=1}^{r} \mu_{i} \sum_{j=1}^{r} b_{i j} f_{j}=\sum_{j=1}^{r}\left(\sum_{i=1}^{r} b_{i j} \mu_{i}\right) f_{j} .
$$

Namely $\lambda_{j}=\sum_{i=1}^{r} b_{i j} \mu_{i}$ for any $j \in\{1, \ldots, r\}$. If $\omega \in \Omega \backslash \Omega_{\infty}$ then

$$
\begin{aligned}
\ln \|x\|_{\boldsymbol{f}, \omega} & =\ln \left(\max _{j \in\{1, \ldots, r\}}\left|\lambda_{j}\right|_{\omega}\right)=\ln \left(\max _{j \in\{1, \ldots, r\}}\left|\sum_{i=1}^{r} b_{i j} \mu_{i}\right|_{\omega}\right) \\
& \leqslant \ln \left(\max _{i \in\{1, \ldots, r\}}\left|\mu_{i}\right|_{L, \omega}\right)+g(\omega)=\ln \|x\|_{\omega}+g(\omega) .
\end{aligned}
$$

If $\omega \in \Omega_{\infty}$, then

$$
\begin{aligned}
\ln \|x\|_{\boldsymbol{f}, \omega} & =\ln \left(\sum_{j=1}^{r}\left|\lambda_{j}\right|_{\omega}\right)=\ln \left(\sum_{j=1}^{r}\left|\sum_{i=1}^{r} b_{i j} \mu_{i}\right|_{\omega}\right) \\
& \leqslant \ln \left(\max _{i \in\{1, \ldots, r\}}\left|\mu_{i}\right|_{L, \omega}\right)+g(\omega)+\ln \left(r^{2}\right)=\ln \|x\|_{\omega}+g(\omega)+\ln \left(r^{2}\right) .
\end{aligned}
$$

Therefore, one has

$$
\forall \omega \in \Omega, \quad d_{\omega}\left(\xi, \xi_{\boldsymbol{f}}\right) \leqslant g(\omega)+2 \ln (r) \mathbb{1}_{\Omega_{\infty}}(\omega),
$$

which implies that the local distance function $d\left(\xi, \xi_{\boldsymbol{f}}\right)$ is $\nu$-dominated. By Corollary 4.1.10, we obtain that the norm family $\xi$ is dominated.

The following proposition is a criterion of the dominance property in the case where the vector space is of dimension 1.

Proposition 4.1.16. - Let $E$ be a vector space of rank 1 over $K$ and $\xi=\left\{\|\cdot\|_{\omega}\right\}_{\omega \in \Omega}$ be a norm family on $E$. Then the following conditions are equivalent:

(1) the norm family $\xi$ is dominated;

(2) for any non-zero element $s \in E$, the function $(\omega \in \Omega) \mapsto \ln \|s\|_{\omega}$ is $\nu$-dominated;

(3) there exists a non-zero element $s \in E$ such that the function $(\omega \in \Omega) \mapsto \ln \|s\|_{\omega}$ is $\nu$-dominated.

Proof. - ' $[(1) \Longrightarrow(2) \Longrightarrow(3)]$ ' are trivial. In the following, we prove ' $[(3) \Longrightarrow \sqrt{(1)}]$ '. If $s^{\prime}$ is a non-zero element in $E$, then we can write it in the form $s^{\prime}=a s$, where $a$ is a non-zero element in $K$. Then one has

$$
\forall \omega \in \Omega, \quad \ln \left\|s^{\prime}\right\|_{\omega}=\ln |a|_{\omega}+\ln \|s\|_{\omega}=\ln |a|_{\omega}+\ln \|s\|_{\omega} .
$$

Since the function $(\omega \in \Omega) \mapsto \ln \|s\|_{\omega}$ is $\nu$-dominated and the function $(\omega \in \Omega) \mapsto$ $\ln |a|_{\omega}$ is $\nu$-integrable, we obtain that the function $(\omega \in \Omega) \mapsto \ln \left\|s^{\prime}\right\|_{\omega}$ is $\nu$-dominated. Moreover, if we denote by $s^{\vee}$ the dual element of $s$ in $E^{\vee}$, then one has

$$
\ln \left\|s^{\vee}\right\|_{\omega, *}=-\ln \|s\|_{\omega}
$$


for any $\omega \in \Omega$. By the same argument as above we obtain that, for any non-zero element $\alpha \in E^{\vee}$, the function $(\omega \in \Omega) \mapsto \ln \|\alpha\|_{\omega, *}$ is $\nu$-dominated. Therefore the norm family $\xi$ is dominated.

Let $K^{\prime}$ be a finite extension field of $K$ and let

$$
S_{K^{\prime}}=S \otimes_{K} K^{\prime}=\left(K^{\prime},\left(\Omega_{K^{\prime}}, \mathcal{A}_{K^{\prime}}, \nu_{K^{\prime}}\right), \phi_{K^{\prime}}\right)
$$

be the algebraic extension of $S$ by $K^{\prime}$. Let $E$ be a finite-dimensional vector space over $K$ and $E_{K^{\prime}}=E \otimes_{K} K^{\prime}$. Note that, for any $\omega \in \Omega$ and any $\omega^{\prime} \in \Omega_{K^{\prime}}$ such that $\pi_{K^{\prime} / K}\left(\omega^{\prime}\right)=\omega$, the vector space $E \otimes_{K} K_{\omega}$ can be naturally considered as a $K_{\omega}$-vector subspace of $E_{K^{\prime}} \otimes_{K^{\prime}} K_{\omega^{\prime}}^{\prime}$

Proposition 4.1.17. - Let $\xi=\left\{\|\cdot\|_{\omega}\right\}_{\omega \in \Omega}$ and $\xi^{\prime}=\left\{\|\cdot\|_{\omega^{\prime}}^{\prime}\right\}_{\omega^{\prime} \in \Omega_{K^{\prime}}}$ be norm familes of $E$ and $E_{K^{\prime}}$, respectively, such that

$$
\forall \omega \in \Omega, \forall \omega^{\prime} \in \pi_{K^{\prime} / K}^{-1}(\{\omega\}), \forall s \in E \otimes_{K} K_{\omega}, \quad\|s\|_{\omega}=\|s\|_{\omega^{\prime}}^{\prime} .
$$

If $\xi^{\prime}$ is dominated (resp. strongly dominated), then $\xi$ is also dominated (resp. strongly dominated).

Proof. - Assume that $\xi$ is dominated. Let $s$ be a non-zero element in $E$. By the assumption (4.6) one has

$$
\overline{\int_{\Omega}} \ln \|s\|_{\omega} \nu(\mathrm{d} \omega)=\bar{\int}_{\Omega_{K^{\prime}}} \ln \|s\|_{\omega^{\prime}}^{\prime} \nu_{K^{\prime}}\left(\mathrm{d} \omega^{\prime}\right)<+\infty .
$$

Hence the norm family $\xi$ is upper dominated. Let $\alpha$ be a non-zero element in $E^{\vee}$. For any $\omega \in \Omega$ and any $\omega^{\prime} \in \pi_{K^{\prime} / K}^{-1}(\{\omega\})$, one has

$$
\|\alpha\|_{\omega, *}=\sup _{s \in\left(E \otimes_{K} K_{\omega}\right) \backslash\{0\}} \frac{|\alpha(s)|_{\omega}}{\|s\|_{\omega}}=\sup _{s \in\left(E \otimes_{K} K_{\omega}\right) \backslash\{0\}} \frac{|\alpha(s)|_{\omega}}{\|s\|_{\omega^{\prime}}^{\prime}} \leqslant\|\alpha\|_{\omega^{\prime}, *}^{\prime} .
$$

Since $\left(\xi^{\prime}\right)^{\vee}$ is upper dominated, we deduce that $\xi^{\vee}$ is also upper dominated.

Assume that $\xi^{\prime}$ is strongly dominated. Let $\boldsymbol{e}=\left\{e_{i}\right\}_{i=1}^{n}$ be a basis of $E$. Then, for $\omega \in \Omega$ and $\omega^{\prime} \in \pi_{K^{\prime} / K}^{-1}(\omega)$,

$$
\begin{aligned}
d_{\omega^{\prime}}\left(\xi^{\prime}, \xi_{\boldsymbol{e}, K^{\prime}}\right) & =\sup _{s^{\prime} \in\left(E_{K^{\prime}} \otimes_{K^{\prime}} K_{\omega^{\prime}}^{\prime}\right) \backslash\{0\}}\left|\ln \left\|s^{\prime}\right\|_{\omega^{\prime}}^{\prime}-\ln \left\|s^{\prime}\right\|_{\boldsymbol{e}, K^{\prime}, \omega^{\prime}}\right| \\
& \geqslant \sup _{s \in\left(E \otimes_{K} K_{\omega}\right) \backslash\{0\}}\left|\ln \|s\|_{\omega^{\prime}}^{\prime}-\ln \|s\|_{\boldsymbol{e}, K^{\prime}, \omega^{\prime}}\right| \\
& =\sup _{s \in\left(E \otimes_{K} K_{\omega}\right) \backslash\{0\}}\left|\ln \|s\|_{\omega}-\ln \|s\|_{\boldsymbol{e}, \omega}\right|=d_{\omega}\left(\xi, \xi_{\boldsymbol{e}}\right) .
\end{aligned}
$$

By our assumption together with Corollary 4.1.10, the function $\left(\omega^{\prime} \in \Omega_{K^{\prime}}\right) \mapsto$ $d_{\omega^{\prime}}\left(\xi^{\prime}, \xi_{\boldsymbol{e}, K^{\prime}}\right)$ is $\nu_{K^{\prime}}$-dominated, that is, there is an integrable function $A^{\prime}$ on $\Omega_{K^{\prime}}$ 
such that $d_{\omega^{\prime}}\left(\xi^{\prime}, \xi_{\boldsymbol{e}, K^{\prime}}\right) \leqslant A^{\prime}\left(\omega^{\prime}\right)$ for all $\omega^{\prime} \in \Omega_{K^{\prime}}$, so that the above estimate implies that $d_{\omega}\left(\xi, \xi_{\boldsymbol{e}}\right) \leqslant I_{K^{\prime} / K}\left(A^{\prime}\right)(\omega)$ for all $\omega \in \Omega$. By Proposition 3.4.9, one has

$$
\int_{\Omega_{K^{\prime}}} A^{\prime}\left(\omega^{\prime}\right) \nu_{K^{\prime}}\left(\mathrm{d} \omega^{\prime}\right)=\int_{\Omega} I_{K^{\prime} / K}\left(A^{\prime}\right)(\omega) \nu(\mathrm{d} \omega)
$$

and hence $\xi$ is strongly dominated by Corollary 4.1.10 again.

Corollary 4.1.18. - Let $f: X \rightarrow$ Spec $K$ be a geometrically reduced projective $K$-scheme and $L$ be an invertible $\mathcal{O}_{X}$-module. Let $X_{K^{\prime}}:=X \times_{\operatorname{Spec} K} \operatorname{Spec} K^{\prime}$ and $L_{K^{\prime}}:=L \otimes_{K} K^{\prime}$. For each $\omega \in \Omega$ and $\omega^{\prime} \in \Omega_{K^{\prime}}, X_{\omega}, L_{\omega}, X_{K^{\prime}, \omega^{\prime}}$ and $L_{K^{\prime}, \omega^{\prime}}$ are defined by

$$
\begin{cases}X_{\omega}:=X \times_{\operatorname{Spec}(K)} \operatorname{Spec}\left(K_{\omega}\right), & L_{\omega}:=L \otimes_{K} K_{\omega}, \\ X_{K^{\prime}, \omega^{\prime}}:=X_{K^{\prime}} \times_{\operatorname{Spec}\left(K^{\prime}\right)} \operatorname{Spec}\left(K_{\omega^{\prime}}^{\prime}\right), & L_{K^{\prime}, \omega^{\prime}}:=L_{K^{\prime}} \otimes_{K^{\prime}} K_{\omega^{\prime}}^{\prime}\end{cases}
$$

Moreover, for each $\omega \in \Omega$ and $\omega^{\prime} \in \pi_{K^{\prime} / K}^{-1}(\omega)$, let $\varphi_{\omega}$ be a metric of $L_{\omega}$ on $X_{\omega}$, and $\varphi_{K^{\prime}, \omega^{\prime}}$ be the metric of $L_{K^{\prime}}$ obtained by $\varphi_{\omega}$ by the extension of scalars (cf. Definition 2.1.18). Let $\|\cdot\|_{\varphi_{\omega}}$ and $\|\cdot\|_{\varphi_{K^{\prime}, \omega^{\prime}}}$ be the sup norms on $H^{0}\left(X_{\omega}, L_{\omega}\right)$ and $H^{0}\left(X_{K^{\prime}, \omega^{\prime}}, L_{K^{\prime}, \omega^{\prime}}\right)$ obtained the metrics $\varphi_{\omega}$ and $\varphi_{K^{\prime}, \omega^{\prime}}$, respectively. If $\xi_{K^{\prime}}=\left\{\|\cdot\|_{\varphi_{K^{\prime}, \omega^{\prime}}}\right\}_{\omega^{\prime} \in \Omega_{K^{\prime}}}$ on $H^{0}\left(X_{K^{\prime}}, L_{K^{\prime}}\right)$ is dominated, then $\xi=\left\{\|\cdot\|_{\varphi_{\omega}}\right\}_{\omega \in \Omega}$ on $H^{0}(X, L)$ is also dominated.

Proof. - For $\omega \in \Omega, \omega^{\prime} \in \pi_{K^{\prime} / K}^{-1}(\omega)$ and $s \in H\left(X_{\omega}, L_{\omega}\right)$, one has $\|s\|_{\varphi_{K^{\prime}, \omega^{\prime}}}=\|s\|_{\varphi_{\omega}}$ (see Proposition 2.1.19), so that the assertion follows from Proposition 4.1.17.

The following proposition shows that the dominance property is actually preserved by most of the algebraic constructions on norm families.

Proposition 4.1.19. - (1) Let E be a finite-dimensional vector space over $K$ and $\xi$ be a dominated (resp. strongly dominated) norm family on $E$. The the restriction of $\xi$ to any vector subspace of $E$ is a dominated (resp. strongly dominated) norm family.

(2) Let $E$ be a finite-dimensional vector space over $K$ and $\xi$ be a dominated (resp. strongly dominated) norm family on $E$. Then the quotient norm family of $\xi$ on any quotient vector space of $E$ is a dominated (resp. strongly dominated) norm family.

(3) Let $E$ be a finite-dimensional vector space over $K$ and $\xi$ be an element in $\mathcal{N}_{E}$. If $\xi$ is dominated, then the norm family $\xi^{\vee}$ is strongly dominated.

(4) Let $E$ and $F$ be finite-dimensional vector spaces over $K$, and $\xi_{E}$ and $\xi_{F}$ be elements in $\mathcal{N}_{E}$ and $\mathcal{N}_{F}$, respectively. Let $\psi: \Omega \rightarrow \mathscr{S}$ be a map such that $\psi=\psi_{0}$ outside of a measurable subset $\Omega^{\prime}$ of $\Omega$ with $\nu\left(\Omega^{\prime}\right)<+\infty$, where $\psi_{0}$ denotes the function in $\mathscr{S}$ sending $t \in[0,1]$ to $\max \{t, 1-t\}$. If both norm families $\xi_{E}$ and $\xi_{F}$ are dominated (resp. strongly dominated), then the $\psi$-direct sum $\xi_{E} \oplus_{\psi} \xi_{F}$ is also dominated (resp. strongly dominated). 
(5) Let $E$ and $F$ be finite-dimensional vector spaces over $K$, and $\xi_{E}$ and $\xi_{F}$ be elements in $\mathcal{N}_{E}$ and $\mathcal{N}_{F}$, respectively. Assume that both norm families $\xi_{E}$ and $\xi_{F}$ are dominated. Then the $\varepsilon$-tensor product $\xi_{E} \otimes_{\varepsilon} \xi_{F}$ and the $\varepsilon, \pi$-tensor product $\xi_{E} \otimes_{\varepsilon, \pi} \xi_{F}$ are strongly dominated. If in addition both norm families $\xi_{E}$ and $\xi_{F}$ are Hermitian, then the orthogonal tensor product $\xi_{E} \otimes \xi_{F}$ is strongly dominated.

(6) Let $E$ be a finite-dimensional vector space over $K$ and $\xi$ be an element in $\mathcal{N}_{E}$. Assume that $\xi$ is dominated. Then, for any $i \in \mathbb{N}$, the exterior power norm family $\Lambda^{i} \xi$ is strongly dominated. In particular, the determinant norm family $\operatorname{det}(\xi)$ is strongly dominated.

Proof. - (1) and (2) in the dominated case: We first show the following claim: if $\xi=\left\{\|\cdot\|_{\omega}\right\}_{\omega \in \Omega}$ is an upper dominated norm family, then all its restrictions and quotients are also upper dominated. Let $F$ be a vector space of $E$ and $\xi_{F}=\left\{\|\cdot\|_{\omega}\right\}_{\omega \in \Omega}$ be the restriction of $\xi$ to $F$. For any $s \in F \backslash\{0\}$ and any $\omega \in \Omega$ one has $\|s\|_{F, \omega}=\|s\|_{\omega}$. Since the norm family $\xi$ is upper dominated, the function $(\omega \in \Omega) \mapsto \ln \|s\|_{F, \omega}$ is upper dominated. Let $G$ be a quotient vector space of $E$ and $\xi_{G}=\left\{\|\cdot\|_{G, \omega}\right\}_{\omega \in \Omega}$ be the quotient of $\xi$ on $G$. For any $t \in G \backslash\{0\}$ and any $s \in E$ which represents the class $t$ in $G$, one has $\|t\|_{G, \omega} \leqslant\|s\|_{\omega}$ for any $\omega \in \Omega$. Therefore the function $(\omega \in \Omega) \mapsto \ln \|t\|_{G, \omega}$ is upper dominated.

Let $\xi=\left\{\|\cdot\|_{\omega}\right\}_{\omega \in \Omega}$ be a norm family on $E$ such that the dual norm family $\xi^{\vee}$ is upper dominated. Let $G$ be a quotient vector space of $E$. We identify $G^{\vee}$ with a vector subspace of $E^{\vee}$. By Proposition 1.1.20, if $\xi_{G}$ denotes the quotient norm family of $\xi$ on $G$, then $\xi_{G}^{\vee}$ identifies with the restriction of $\xi^{\vee}$ to $G^{\vee}$. By the claim proved above, we obtain that $\xi_{G}^{\vee}$ is upper dominated. Similarly, if $F$ is a vector subspace of $E$ and if $\xi_{F}=\left\{\|\cdot\|_{F, \omega}\right\}_{\omega \in \Omega}$ is the restriction of $\xi$ to $F$, then, for any $\omega \in \Omega,\|\cdot\|_{F, \omega, *}$ is bounded from above by the quotient of the norm $\|\cdot\|_{\omega, *}$ on $F^{\vee}$ (viewed as a quotient vector space of $E^{\vee}$ ). Therefore, by the claim proved above, we obtain that the norm family $\xi_{F}^{\vee}$ is upper dominated.

$(1)$ in the strongly dominated case: Let $F$ be a vector subspace of $E$. Let $\boldsymbol{f}$ be a basis of $F$. We complete it into a basis $e$ of $E$. For any $\omega \in \Omega$ one has $d_{\omega}\left(\xi_{F}, \xi_{\boldsymbol{f}}\right) \leqslant d_{\omega}\left(\xi, \xi_{\boldsymbol{e}}\right)$. Since the function $(\omega \in \Omega) \mapsto d_{\omega}\left(\xi, \xi_{\boldsymbol{e}}\right)$ is $\nu$-dominated, also is the function $(\omega \in \Omega) \mapsto d_{\omega}\left(\xi_{F}, \xi_{f}\right)$. By Corollary 4.1.10, we obtain that the norm family $\xi_{F}$ is strongly dominated.

$(2)$ in the strongly dominated case: Let $\boldsymbol{g}=\left\{g_{i}\right\}_{i=1}^{m}$ be a basis of $G$. For any $i \in\{1, \ldots, m\}$, we choose a vector $e_{i}$ in $E$ such that the canonical image of $e_{i}$ in $G$ is $g_{i}$. We complete the family $\left\{e_{i}\right\}_{i=1}^{m}$ into a basis $\boldsymbol{e}$ of $E$. Then for any $\omega \in \Omega$ one has $d_{\omega}\left(\xi_{G}, \xi_{\boldsymbol{g}}\right) \leqslant d_{\omega}\left(\xi, \xi_{\boldsymbol{e}}\right)$. Therefore, the function $(\omega \in \Omega) \mapsto d_{\omega}\left(\xi_{G}, \xi_{\boldsymbol{g}}\right)$ is $\nu$-dominated, which implies that $\xi_{G}$ is strongly dominated.

(3) has already been shown in Remark 4.1.3, see also Remark 4.1.12 for the strong dominancy. 
(4) in the dominated case: We first show the following claim: if both norm families $\xi_{E}$ and $\xi_{F}$ are upper dominated, then also is the direct sum $\xi_{E} \oplus_{\psi} \xi_{F}$. In fact, if $(s, t)$ is an element in $E \oplus F$, for $\omega \in \Omega \backslash \Omega^{\prime}$ one has

$$
\|(s, t)\|_{\omega}=\max \left\{\|s\|_{E, \omega},\|t\|_{F, \omega}\right\},
$$

and for $\omega \in \Omega^{\prime}$, one has

$$
\|(s, t)\|_{\omega} \leqslant\|s\|_{E, \omega}+\|t\|_{F, \omega} \leqslant 2 \max \left\{\|s\|_{E, \omega},\|t\|_{F, \omega}\right\},
$$

where $\|\cdot\|_{\omega}$ denotes the norm indexed by $\omega$ in $\xi_{E} \oplus_{\psi} \xi_{F}$. Therefore the function $(\omega \in \Omega) \mapsto\|(s, t)\|_{\omega}$ is upper dominated.

Let $\psi^{\prime}$ be the map from $\Omega$ to $\mathscr{S}$ sending any $\omega \in \Omega \backslash \Omega_{\infty}$ to $\psi_{0}$ and any $\omega \in$ $\Omega_{\infty}$ to $\psi(\omega)_{*}$ (see Definition 1.1.48). By Proposition 1.1.49 we obtain that $\left(\xi_{E} \oplus_{\psi}\right.$ $\left.\xi_{F}\right)^{\vee}$ identifies with $\xi_{E}^{\vee} \oplus_{\psi^{\prime}} \xi_{F}^{\vee}$. By the claim proved above, if $\xi_{E}^{\vee}$ and $\xi_{F}^{\vee}$ are upper dominated, then also is $\left(\xi_{E} \oplus_{\psi} \xi_{F}\right)^{\vee}$.

(4) in the strongly dominated case: Let $e^{\prime}$ and $\boldsymbol{e}^{\prime \prime}$ be bases of $E$ and $F$ respectively, and $\boldsymbol{e}$ be the disjoint union of $\boldsymbol{e}^{\prime}$ and $\boldsymbol{e}^{\prime \prime}$, viewed as a basis of $E \oplus F$. Since $\xi_{E}$ and $\xi_{F}$ are both dominated, by Corollary 4.1.10 there exist $\nu$-integrable functions $A^{\prime}$ and $A^{\prime \prime}$ such that

$$
d_{\omega}\left(\xi_{E}, \xi_{\boldsymbol{e}^{\prime}}\right) \leqslant A^{\prime}(\omega), \quad d_{\omega}\left(\xi_{F}, \xi_{\boldsymbol{e}^{\prime \prime}}\right) \leqslant A^{\prime \prime}(\omega) \quad \nu \text {-almost everywhere. }
$$

Moreover, if $(s, t)$ is an element in $E \oplus F$, for $\omega \in \Omega \backslash \Omega^{\prime}$ one has

$$
\|(s, t)\|_{\omega}=\max \left\{\|s\|_{E, \omega},\|t\|_{F, \omega}\right\},
$$

and for $\omega \in \Omega^{\prime}$, one has

$$
\max \left\{\|s\|_{E, \omega},\|t\|_{F, \omega}\right\} \leqslant\|(s, t)\|_{\omega} \leqslant\|s\|_{E, \omega}+\|t\|_{F, \omega} \leqslant 2 \max \left\{\|s\|_{E, \omega},\|t\|_{F, \omega}\right\},
$$

where $\|\cdot\|_{\omega}$ denotes the norm indexed by $\omega$ in $\xi_{E} \oplus_{\psi} \xi_{F}$. Therefore

$$
d_{\omega}\left(\xi_{E} \oplus_{\psi} \xi_{F}, \xi_{\boldsymbol{e}}\right) \leqslant \max \left\{A^{\prime}(\omega), A^{\prime \prime}(\omega)\right\}+\ln (2) \mathbb{1}_{\Omega^{\prime}}(\omega) \quad \nu \text {-almost everywhere. }
$$

Note that the function $(\omega \in \Omega) \mapsto \max \left\{A^{\prime}(\omega), A^{\prime \prime}(\omega)\right\}+\ln (2) \mathbb{1}_{\Omega^{\prime}}(\omega)$ is $\nu$-integrable. Hence the norm family $\xi_{E} \oplus_{\psi} \xi_{F}$ is strongly dominated (by Corollary 4.1.10).

(5) By (3), the norm families $\xi_{E}^{\vee \vee}$ and $\xi_{F}^{\vee \vee}$ are both dominated. Therefore, without loss of generality, we may assume that $\|\cdot\|_{E, \omega}$ and $\|\cdot\|_{F, \omega}$ are ultrametric norms for $\omega \in \Omega \backslash \Omega_{\infty}$ (see Definition 1.1.52, see also Proposition 1.2.14). Let $\boldsymbol{e}=\left\{e_{i}\right\}_{i=1}^{n}$ and $\boldsymbol{f}=\left\{f_{j}\right\}_{j=1}^{m}$ be bases of $E$ and $F$ over $K$, and let $\boldsymbol{e} \otimes \boldsymbol{f}=\left\{e_{i} \otimes f_{j}\right\}_{(i, j) \in\{1, \ldots, n\} \times\{1, \ldots, m\}}$. Note that $\boldsymbol{e} \otimes \boldsymbol{f}$ is a basis of $E \otimes F$. Moreover, for any $\omega \in \Omega$, the norm $\|\cdot\|_{\boldsymbol{e} \otimes \boldsymbol{f}, \omega}$ identifies with the $\varepsilon$-tensor product of the norms $\|\cdot\|_{E, \omega}$ and $\|\cdot\|_{F, \omega}$. Since the norm families $\xi_{E}$ and $\xi_{F}$ are dominated, there exist $\nu$-integrable functions $A_{E}$ and $A_{F}$ on $\Omega$ such that

$$
d_{\omega}\left(\xi_{E}, \xi_{\boldsymbol{e}}\right)=\sup _{0 \neq s \in E}\left|\ln \|s\|_{E, \omega}-\ln \|s\|_{\boldsymbol{e}, \omega}\right| \leqslant A_{E}(\omega) \quad \nu \text {-almost everywhere, }
$$


and

$$
d_{\omega}\left(\xi_{F}, \xi_{\boldsymbol{f}}\right)=\sup _{0 \neq t \in F}\left|\ln \|t\|_{F, \omega}-\ln \|t\|_{\boldsymbol{f}, \omega}\right| \leqslant A_{F}(\omega) \quad \nu \text {-almost everywhere. }
$$

By (4.1), we obtain

$$
d_{\omega}\left(\xi_{E}^{\vee}, \xi_{\boldsymbol{e}}^{\vee}\right)=\sup _{0 \neq \alpha \in E^{\vee}}\left|\ln \|\alpha\|_{E, \omega, *}-\ln \|\alpha\|_{\boldsymbol{e}, \omega, *}\right| \leqslant A_{E}(\omega) \quad \nu \text {-almost everywhere, }
$$

which implies (see (4.2)

$$
d_{\omega}\left(\xi_{E}^{\vee}, \xi_{e^{\vee}}\right) \leqslant A_{E}(\omega)+\ln (n) \mathbb{1}_{\Omega_{\infty}}(\omega) \quad \nu \text {-almost everywhere. }
$$

Therefore, for any $\omega \in \Omega$ and any non-zero tensor $\varphi \in \operatorname{Hom}_{K}\left(E^{\vee}, F\right) \cong E \otimes_{K} F$, one has (see Remark 1.1.53)

$$
\begin{aligned}
& \left|\ln \|\varphi\|_{\varepsilon, \omega}-\ln \|\varphi\|_{\boldsymbol{e} \otimes \boldsymbol{f}, \omega}\right|=\left|\sup _{0 \neq \alpha \in E^{\vee}} \ln \frac{\|\varphi(\alpha)\|_{F, \omega}}{\|\alpha\|_{E, \omega, *}}-\sup _{0 \neq \alpha \in E^{\vee}} \ln \frac{\|\varphi(\alpha)\|_{\boldsymbol{f}, \omega}}{\|\alpha\|_{\boldsymbol{e}^{\vee}, \omega}}\right| \\
& \leqslant A_{E}(\omega)+A_{F}(\omega)+\ln (n) \mathbb{1}_{\Omega_{\infty}}(\omega)
\end{aligned}
$$

$\nu$-almost everywhere, where $\|\cdot\|_{\varepsilon, \omega}$ denotes the $\varepsilon$-tensor product of $\|\cdot\|_{E, \omega}$ and $\|\cdot\|_{F, \omega}$. Therefore the $\varepsilon$-tensor product norm family $\xi_{E} \otimes_{\varepsilon} \xi_{F}$ is dominated (and hence is strongly dominated since it is ultrametric on $\Omega \backslash \Omega_{\infty}$, see Remark 4.1.12). By using the fact that $\xi_{E} \otimes_{\varepsilon, \pi} \xi_{F}=\left(\xi_{E}^{\vee} \otimes_{\varepsilon} \xi_{F}^{\vee}\right)^{\vee}$ (see Corollary 1.2.20 and Proposition 1.1.57 for the non-Archimedean and the Archimedean cases respectively), we deduce the dominance property of $\xi_{E} \otimes_{\varepsilon, \pi} \xi_{F}$ from the above result and the assertion (3) of the Proposition. Finally, by Propositions 1.2.59 and 4.1.6, we deduce that the orthogonal tensor product norm family $\xi_{E} \otimes \xi_{F}$ is also strongly dominated, provided that $\xi_{E}$ and $\xi_{F}$ are both Hermitian.

(6) is a direct consequence of (2) and $(5)$ since $\Lambda^{i} \xi$ is a quotient norm family of the $i$-th $\varepsilon, \pi$-tensor power of $\xi$.

Remark 4.1.20. — Let $E$ be a finite-dimensional vector space over $K$. We denote by $\mathcal{D}_{E}$ the subset of $\mathcal{N}_{E}$ of all strongly dominated norm families $\xi=\left\{\|\cdot\|_{\omega}\right\}_{\omega \in \Omega}$ on $E$. By Corollary 4.1.8, we obtain that, for any pair $\left(\xi, \xi^{\prime}\right)$ of norm families in $\mathcal{D}_{E}$, the local distance function $(\omega \in \Omega) \mapsto d_{\omega}\left(\xi, \xi^{\prime}\right)$ is $\nu$-dominated. This observation allows us to construct a function $\operatorname{dist}(\cdot, \cdot)$ on $\mathcal{D}_{E} \times \mathcal{D}_{E}$, defined as (see $\$$ A.4 for the definition of the upper integral $\left.\bar{\int}_{\Omega} h(\omega) \nu(d \omega)\right)$

$$
\operatorname{dist}\left(\xi, \xi^{\prime}\right):=\bar{\int}_{\Omega} d_{\omega}\left(\xi, \xi^{\prime}\right) \nu(\mathrm{d} \omega) .
$$

Clearly this function is symmetric with respect to its two variables, and verifies the triangle inequality, where the latter assertion follows from the triangle inequality of the local distance function and Proposition A.4.4. Therefore, dist $(\cdot, \cdot)$ is actually a pseudometric on $\mathcal{D}_{E}$. Moreover, for any pair $\left(\xi, \xi^{\prime}\right)$ of elements of $\mathcal{D}_{E}$, $\operatorname{dist}\left(\xi, \xi^{\prime}\right)=0$ if and only if $\xi_{\omega}=\xi_{\omega}^{\prime} \nu$-almost everywhere (see Proposition A.4.10). Therefore, 
the pseudometric dist $(\cdot, \cdot)$ induces a metric on the quotient space of $\mathcal{D}_{E}$ modulo the equivalence relation

$$
\xi \sim \xi^{\prime} \Longleftrightarrow \xi_{\omega}=\xi_{\omega}^{\prime} \quad \nu \text {-almost everywhere. }
$$

This quotient metric space is actually complete. In fact, assume that $\left\{\xi_{n}\right\}_{n \in \mathbb{N}}$ is a Cauchy sequence in $\mathcal{D}_{E}$. Then we can pick a subsequence $\left\{\xi_{n_{k}}\right\}_{k \in \mathbb{N}}$ such that

$$
\forall k \in \mathbb{N}, \quad \bar{\int}_{\Omega}\left(\mathbb{1}_{\left\{d\left(\xi_{n_{k}}, \xi_{n_{k+1}}\right) \geqslant 2^{-k}\right\}}\right) \nu(d \omega) \leqslant 2^{-k} .
$$

The set of $\omega \in \Omega$ such that $\left\{\xi_{n_{k}, \omega}\right\}_{k \in \mathbb{N}}$ is not a Cauchy sequence (with respect the the metric defined in $\$ 1.1 .9$ is a $\nu$-negligible set. Let $\xi$ be a norm family such that $\left\{\xi_{n_{k}, \omega}\right\}_{k \in \mathbb{N}}$ converges to $\xi_{\omega} \nu$-almost everywhere (see Remark 1.1.41 for the local completeness). Then, by the same argument as in the proof of Proposition A.4.14 we obtain that $\operatorname{dist}\left(\xi_{n}, \xi\right)$ converges to 0 when $n$ goes to the infinity.

4.1.3. Measurability of norm families. - Let $E$ be a vector space of finite rank over $K$ and $\xi=\left\{\|\cdot\|_{\omega}\right\}_{\omega \in \Omega}$ be a norm family in $\mathcal{N}_{E}$. We say that the norm family $\xi$ is $\mathcal{A}$-measurable (or simply measurable when there is no ambiguity on the $\sigma$-algebra $\mathcal{A})$ if for any $s \in E$ the function $(\omega \in \Omega) \mapsto\|s\|_{\omega}$ is $\mathcal{A}$-measurable. By definition, if the norm family $\xi$ is $\mathcal{A}$-measurable on $\Omega$, then also is its restriction to a vector subspace of $E$. The following proposition shows that measurable direct sums preserve the measurability of norm families.

Proposition 4.1.21. - Let $E$ and $F$ be finite-dimensional vector spaces over $K$ and $\xi_{E}=\left\{\|\cdot\|_{E, \omega}\right\}_{\omega \in \Omega}, \xi_{F}=\left\{\|\cdot\|_{F, \omega}\right\}_{\omega \in \Omega}$ be respectively norm families in $\mathcal{N}_{E}$ and $\mathcal{N}_{F}$, which are both $\mathcal{A}$-measurable. For any map $\psi: \Omega \rightarrow \mathscr{S}$ which is $\mathcal{A}$-measurable, where we consider the Borel $\sigma$-algebra on $\mathscr{S}$ induced by the topology of uniform convergence, the direct sum $\xi_{E} \oplus_{\psi} \xi_{F}=\left\{\|\cdot\|_{\psi, \omega}\right\}_{\omega \in \Omega}$ is $\mathcal{A}$-measurable.

Proof. - Consider the map $g: \mathscr{S} \times\left[0,+\infty\left[^{2} \rightarrow \mathbb{R}\right.\right.$

$$
g(\eta, a, b) \longrightarrow \begin{cases}0, & a+b=0 \\ (a+b) \eta(a /(a+b)), & a+b \neq 0\end{cases}
$$

We claim that the map $g$ is continuous. Let $\left\{\left(\eta_{n}, a_{n}, b_{n}\right)\right\}_{n \in \mathbb{N}}$ be a sequence in $\mathscr{S} \times$ $\left[0,+\infty\left[^{2}\right.\right.$ which converges to $(\eta, a, b) \in \mathscr{S} \times\left[0,+\infty\left[{ }^{2}\right.\right.$. If $a+b \neq 0$, then $a_{n} /\left(a_{n}+b_{n}\right)$ converges to $a /(a+b)$, and therefore

$$
\begin{aligned}
& \left|\eta_{n}\left(a_{n} /\left(a_{n}+b_{n}\right)\right)-\eta(a /(a+b))\right| \\
\leqslant & \left|\eta_{n}\left(a_{n} /\left(a_{n}+b_{n}\right)\right)-\eta\left(a_{n} /\left(a_{n}+b_{n}\right)\right)\right|+\left|\eta\left(a_{n} /\left(a_{n}+b_{n}\right)\right)-\eta(a /(a+b))\right| \\
\leqslant & \left\|\eta_{n}-\eta\right\|_{\text {sup }}+\left|\eta\left(a_{n} /\left(a_{n}+b_{n}\right)\right)-\eta(a /(a+b))\right|
\end{aligned}
$$

converges to 0 when $n$ tends to the infinity. We then deduce that

$$
\lim _{n \rightarrow+\infty} g\left(\eta_{n}, a_{n}, b_{n}\right)=g(\eta, a, b) .
$$


If $a+b=0$, then

$$
\lim _{n \rightarrow+\infty} g\left(\eta_{n}, a_{n}, b_{n}\right)=0=g(\eta, a, b)
$$

since the sequence of functions $\left\{\eta_{n}\right\}_{n \in \mathbb{N}}$ is uniformly bounded and $a_{n}+b_{n}$ converges to 0 when $n$ tends to the infinity.

Note that $\mathscr{S}$ is a closed subset of $C^{0}([0,1])$, the space of all continuous real functions on $[0,1]$. Since $C^{0}([0,1])$ admits a countable topological basis (see [27] Chapter $\mathrm{X}, \S 3.3$, Theorem 1), also is $\mathscr{S}$. Being a metric space, the topological space $\mathscr{S}$ is thus separable. Therefore, the Borel $\sigma$-algebra of the product topological space $\mathscr{S} \times\left[0,+\infty\left[^{2}\right.\right.$ coincides with the product $\sigma$-algebra of the Borel $\sigma$-algebras of $\mathscr{S}$ and $[0,+\infty]^{2}$ (see 90 Lemma 1.2). In particular, the function $F$ is measurable with respect to the product $\sigma$-algebra. If $(s, t)$ is an element in $E \oplus F$, then one has

$$
\|(s, t)\|_{\psi, \omega}=g\left(\psi(\omega),\|s\|_{E, \omega},\|t\|_{F, \omega}\right),
$$

which is an $\mathcal{A}$-measurable function since the maps $\psi, \omega \mapsto\|s\|_{E, \omega}$ and $\omega \mapsto\|t\|_{F, \omega}$ are all $\mathcal{A}$-measurable.

Proposition 4.1.22. - (1) Let E be a vector space of dimension 1 over $K$ and $\xi$ be a norm family in $\mathcal{N}_{E}$. Then $\xi$ is $\mathcal{A}$-measurable if and only if there exists an element $s \in E \backslash\{0\}$ such that the function $(\omega \in \Omega) \mapsto\|s\|_{\omega}$ is $\mathcal{A}$-measurable.

(2) Let $E$ be a vector space of dimension 1 over $K$ and $\xi$ be a norm family in $\mathcal{N}_{E}$ which is $\mathcal{A}$-measurable. Then the dual norm family $\xi^{\vee}$ is also $\mathcal{A}$-measurable.

(3) Let $E_{1}$ and $E_{2}$ be vector spaces of dimension 1 over $K$, and $\xi_{1}$ and $\xi_{2}$ be norm families in $\mathcal{N}_{E_{1}}$ and $\mathcal{N}_{E_{2}}$ respectively. We assume that both norm families $\xi_{1}$ and $\xi_{2}$ are $\mathcal{A}$-measurable. Then the tensor product $\xi_{1} \otimes \xi_{2}$ (which is also equal to $\xi_{1} \otimes_{\varepsilon} \xi_{2}$ and $\left.\xi_{1} \otimes_{\pi} \xi_{2}\right)$ is also $\mathcal{A}$-measurable.

(4) Let $E$ be a vector space of dimension 1 over $K$ and $\xi$ be a norm family in $\mathcal{N}_{E}$. Assume that there exists an integer $n \geqslant 1$ such that $\xi^{\otimes n}$ is $\mathcal{A}$-measurable, then the norm family $\xi$ is also measurable.

Proof. - (1) The necessity follows from the definition. For the sufficiency, we assume that there exists $s \in E \backslash\{0\}$ such that the function $(\omega \in \Omega) \mapsto\|s\|_{\omega}$ is $\mathcal{A}$-measurable. If $s^{\prime}$ is a general element in $E$, there exists $a \in K$ such that $s^{\prime}=a s$. Note that for any $\omega \in \Omega$ one has

$$
\left\|s^{\prime}\right\|_{\omega}=|a|_{\omega} \cdot\|s\|_{\omega} .
$$

Since the function $(\omega \in \Omega) \mapsto|a|_{\omega}$ is $\mathcal{A}$-measurable, we obtain that the function $(\omega \in \Omega) \rightarrow\left\|s^{\prime}\right\|_{\omega}$ is $\mathcal{A}$-measurable.

Let $s$ be a non-zero element in $E$ and $\alpha$ be the element in $E^{\vee}$ such that $\alpha(s)=1$. For any $\omega \in \Omega$ one has $\|\alpha\|_{\omega}=\|s\|_{\omega}^{-1}$. Since the function $(\omega \in \Omega) \mapsto\|s\|_{\omega}$ is $\mathcal{A}$-measurable, we obtain that the function $(\omega \in \Omega) \mapsto\|\alpha\|_{\omega}$ is also $\mathcal{A}$-measurable. Therefore, by (1) we obtain that the norm family $\xi^{\vee}$ is $\mathcal{A}$-measurable on $\Omega$. 
(3) Let $s_{1}$ and $s_{2}$ be non-zero elements of $E_{1}$ and $E_{2}$ respectively. Then $s_{1} \otimes s_{2}$ is a non-zero element of $E_{1} \otimes E_{2}$. Moreover, for any $\omega \in \Omega$ one has $\left\|s_{1} \otimes s_{2}\right\|_{\omega}=$ $\left\|s_{1}\right\|_{\omega} \cdot\left\|s_{2}\right\|_{\omega}$. Since the functions $(\omega \in \Omega) \mapsto\left\|s_{1}\right\|_{\omega}$ and $(\omega \in \Omega) \mapsto\left\|s_{2}\right\|_{\omega}$ are $\mathcal{A}$ measurable, we obtain that the function $(\omega \in \Omega) \mapsto\left\|s_{1} \otimes s_{2}\right\|_{\omega}$ is $\mathcal{A}$-measurable. By $(1)$ the norm family $\xi_{1} \otimes \xi_{2}$ is $\mathcal{A}$-measurable.

(4) Let $s$ be a non-zero element of $E$. For any $\omega \in \Omega$, one has $\left\|s^{\otimes n}\right\|_{\omega}=\|s\|_{\omega}^{n}$. Hence $\|s\|_{\omega}=\left\|s^{\otimes n}\right\|_{\omega}^{1 / n}$. Since $\xi^{\otimes n}$ is $\mathcal{A}$-measurable, the function $(\omega \in \Omega) \mapsto\left\|s^{\otimes n}\right\|_{\omega}$ is $\mathcal{A}$ measurable. As a consequence, the function $(\omega \in \Omega) \mapsto\|s\|_{\omega}$ is also $\mathcal{A}$-measurable. By (1), we obtain that the norm family $\xi$ is $\mathcal{A}$-measurable.

Remark 4.1.23. - It is not clear that other algebraic constructions of norm families preserve the $\mathcal{A}$-measurability. We consider the following counter-example. Let $K=$ $\mathbb{R}$ and $(\Omega, \mathcal{A}, \nu)$ be the set $\mathbb{R}$ equipped with the Borel $\sigma$-algebra and the Lebesgue measure. Let $\phi: \Omega \rightarrow M_{\mathbb{R}}$ be the constant map which sends any point of $\Omega$ to the trivial absolute value. Then $(K,(\Omega, \mathcal{A}, \nu), \phi)$ is an adelic curve. Let $f: \mathbb{R} \rightarrow] 0,1]$ be a map which is not Borel measurable. Let $E$ be a vector space of dimension 2 over $\mathbb{R}$ and $\left\{e_{1}, e_{2}\right\}$ be a basis of $E$. For each $t \in \Omega=\mathbb{R}$, let $\|\cdot\|_{t}$ be the norm on $E$ such that $\left\|\lambda\left(e_{1}+t e_{2}\right)\right\|_{t}=f(t)$ for $\lambda \in \mathbb{R} \backslash\{0\}$, and $\|s\|_{t}=1$ if $s \in E \backslash \mathbb{R}\left(e_{1}+t e_{2}\right)$. Then $\xi=\left\{\|\cdot\|_{t}\right\}_{t \in \mathbb{R}}$ is an element in $\mathcal{N}_{E}$. Note that, for any $s \in E$ the function $t \mapsto\|s\|_{t}$ is Borel measurable on $\mathbb{R}$ since it is constant except at most one point of $\mathbb{R}$. However, if we denote by $G$ the quotient space $E / \mathbb{R} e_{2}$ and by $\xi_{G}=\left\{\|\cdot\|_{G, t}\right\}_{t \in \mathbb{R}}$ the quotient norm family of $\xi$. The one has

$$
\forall t \in \mathbb{R},\left\|\left[e_{1}\right]\right\|_{G, t}=f(t) .
$$

Therefore the quotient norm family $\xi_{G}$ is not $\mathcal{A}$-measurable on $\Omega$.

The following results show that, at least in the particular case where $K$ is a countable set, the algebraic constructions of norm families defined in the previous subsection preserve the $\mathcal{A}$-measurability of norm families.

Proposition 4.1.24. - We assume that the field $K$ admits a countable subfield $K_{0}$ which is dense in $K_{\omega}$ for any $\omega \in \Omega$.

(1) Let $E$ be a vector space of finite rank over $K$ and $\xi=\left\{\|\cdot\|_{\omega}\right\}_{\omega \in \Omega}$ be a norm family in $\mathcal{N}_{E}$ which is $\mathcal{A}$-measurable. Then

(1.a) for any quotient space $G$ of $E$, the quotient norm family $\xi_{G}=\left\{\|\cdot\|_{G, \omega}\right\}_{\omega \in \Omega}$ on $G$ of $\xi$ is $\mathcal{A}$-measurable;

(1.b) the dual norm family $\xi^{\vee}=\left\{\|\cdot\|_{\omega, *}\right\}_{\omega \in \Omega}$ is $\mathcal{A}$-measurable;

(1.c) for any algebraic extension $L / K$, the norm family $\xi_{L}=\left\{\|\cdot\|_{x}\right\}_{x \in \Omega_{L}}$ on $E_{L}:=E \otimes_{K} L$ is $\mathcal{A}_{L}$-measurable. If in addition $\xi$ is Hermitian, then the norm family $\xi_{L}^{H}$ is $\mathcal{A}_{L}$-mesurable. 
(2) Let $E$ and $F$ be finite-dimensional vector spaces over $K, \xi_{E}=\left\{\|\cdot\|_{E, \omega}\right\}_{\omega \in \Omega}$ and $\xi_{F}=\left\{\|\cdot\|_{F, \omega}\right\}_{\omega \in \Omega}$ be respectively norm families in $\mathcal{N}_{E}$ and $\mathcal{N}_{F}$. We assume that $\xi_{E}$ and $\xi_{F}$ are both $\mathcal{A}$-measurable. Then

(2.a) the $\pi$-tensor product $\xi_{E} \otimes_{\pi} \xi_{F}$, the $\varepsilon$-tensor product $\xi_{E} \otimes_{\varepsilon} \xi_{F}$ and the $\varepsilon, \pi$-tensor product $\xi_{E} \otimes_{\varepsilon, \pi} \xi_{F}$ are all $\mathcal{A}$-measurable;

(2.b) if in addition the norm families $\xi_{E}$ and $\xi_{F}$ are Hermitian, the orthogonal tensor product $\xi_{E} \otimes \xi_{F}=\left\{\|\cdot\|_{E \otimes F, \omega}\right\}_{\omega \in \Omega}$ is $\mathcal{A}$-measurable.

(3) Let $E$ be a finite-dimensional vector space over $K$ and $\xi_{E}=\left\{\|\cdot\|_{E, \omega}\right\}_{\omega \in \Omega}$ be a norm family in $\mathcal{N}_{E}$ which is $\mathcal{A}$-measurable. Then the exterior norm family $\Lambda^{i} \xi$ is $\mathcal{A}$-measurable for any $i \in \mathbb{N}$. In particular, the determinant norm family $\operatorname{det}(\xi)$ is $\mathcal{A}$-measurable.

(4) Let $E$ be a finite-dimensional vector space over $K$, and $\xi=\left\{\|\cdot\|_{\omega}\right\}_{\omega \in \Omega}$ and $\xi^{\prime}=\left\{\|\cdot\|_{\omega}^{\prime}\right\}_{\omega \in \Omega}$ be two $\mathcal{A}$-measurable norm families in $\mathcal{N}_{E}$. Then the local distance function $\omega \mapsto d_{\omega}\left(\xi, \xi^{\prime}\right)$ is $\mathcal{A}$-measurable.

Proof. - (1.a) Let $p: E \rightarrow G$ be the projection map and $F$ be its kernel. Let $F_{0}$ be a finite-dimensional $K_{0}$-vector subspace of $F$ which generates $F$ as a vector space over $K$. Note that for any $\omega \in \Omega$ the set $F_{0}$ is dense in $F_{K_{\omega}}$. For any $\ell \in G$ and any $\omega \in \Omega$, one has

$$
\|\ell\|_{G, \omega}=\inf _{s \in E, p(s)=\ell}\|s\|_{\omega}=\inf _{t \in F_{0}}\left\|s_{0}+t\right\|_{\omega},
$$

where $s_{0}$ is an element in $E$ such that $p\left(s_{0}\right)=\ell$. As the norm family $\xi_{E}$ is $\mathcal{A}$ measurable on $\Omega$, the function $(\omega \in \Omega) \mapsto\|s\|_{\omega}$ is $\mathcal{A}$-measurable for any $s \in E$. Hence the function $\omega \mapsto\|\ell\|_{G, \omega}$ is also $\mathcal{A}$-measurable since it is the infimum of a countable family of $\mathcal{A}$-measurable functions.

(1.b) Let $E_{0}$ be a finite-dimensional $K_{0}$-vector subspace of $E$ which generates $E$ as a vector space over $K$. For any $\alpha \in E^{\vee}$ and any $\omega \in \Omega$, one has

$$
\|\alpha\|_{\omega, *}=\sup _{s \in E \backslash\{0\}} \frac{|\alpha(s)|_{\omega}}{\|s\|_{\omega}}=\sup _{s \in E_{0} \backslash\{0\}} \frac{|\alpha(s)|_{\omega}}{\|s\|_{\omega}}
$$

since $E_{0} \backslash\{0\}$ is dense in $E_{K_{\omega}} \backslash\{0\}$. As the norm family $\xi_{E}$ is $\mathcal{A}$-measurable on $\Omega$, the function $(\omega \in \Omega) \mapsto\|s\|_{\omega}$ is $\mathcal{A}$-measurable for any $s \in E$. Moreover, $\alpha(s)$ belongs to $K$, and thus the function $\omega \mapsto|\alpha(s)|_{\omega}$ is $\mathcal{A}$-measurable. Hence the function $(\omega \in \Omega) \mapsto\|\alpha\|_{\omega, *}$ is $\mathcal{A}$-measurable since it is the supremum of a countable family of $\mathcal{A}$-measurable functions.

(1.c) Let $H_{0}$ be a finite-dimensional $K_{0}$-vector subspace of $E^{\vee}$ which generates $E^{\vee}$ as a vector space over $K$. Then $H_{0} \backslash\{0\}$ is dense in $E_{K_{\omega}}^{\vee} \backslash\{0\}$ for any $\omega \in \Omega$. Let $s$ be an element in $E_{L}$. For any $x \in \Omega_{L}$, let

$$
\|s\|_{\omega, L_{x}, \varepsilon}=\sup _{\varphi \in E_{K_{\omega}}^{\vee} \backslash\{0\}} \frac{|\varphi(s)|_{x}}{\|\varphi\|_{\omega, *}}=\sup _{\varphi \in H_{0} \backslash\{0\}} \frac{|\varphi(s)|_{x}}{\|\varphi\|_{\omega, *}},
$$


where $\omega=\pi_{L / K}(x)$, and $\|\cdot\|_{\omega, *}$ denotes the dual norm of $\|\cdot\|_{\omega}$. We have seen in (1.b) that the dual norm family $\xi^{\vee}=\left\{\|\cdot\|_{\omega, *}\right\}_{\omega \in \Omega}$ is $\mathcal{A}$-mesurable on $\Omega$. Therefore the function

$$
\left(x \in \Omega_{L}\right) \longmapsto\|\varphi\|_{\pi_{L / K}(x), *},
$$

which is the composition of the $\mathcal{A}$-measurable function $\omega \mapsto\|\varphi\|_{\omega, *}$ with $\pi_{L / K}$, is $\mathcal{A}_{L^{-}}$ measurable. Moreover, the function $x \mapsto|\varphi(s)|_{x}$ on $\Omega_{L}$ is $\mathcal{A}_{L}$-mesurable. Therefore, the function $x \mapsto\|s\|_{\omega, L_{x}, \varepsilon}$ on $\Omega_{L}$, which is the supremum of a countable family of measurable functions, is also measurable. Therefore the norm family $\left\{\|\cdot\|_{x}^{\prime}\right\}_{x \in \Omega_{L}}$ is measurable. This result applied to $\xi^{\vee}$ shows that the norm family $\left\{\|\cdot\|_{\omega, *, L_{x}, \varepsilon}\right\}_{x \in \Omega_{L}}$ is measurable. By Proposition 1.3.20, (1), (2), the norm family $\xi_{L}$ identifies with the dual of $\left\{\|\cdot\|_{\omega, *, L_{x}, \varepsilon}\right\}_{x \in \Omega_{L}}$, and hence is measurable.

Assume that the norm family $\xi$ is Hermitian. Let $s$ be an element of $E_{L}$, which is written as $s_{1} \otimes \lambda_{1}+\cdots+s_{n} \otimes \lambda_{n}$, where $\left(s_{1}, \ldots, s_{n}\right) \in E^{n}$ and $\left(\lambda_{1}, \ldots, \lambda_{n}\right) \in L^{n}$. For any $x \in \Omega_{L}$ one has

$$
\|s\|_{x}^{2}=\sum_{i=1}^{n} \sum_{j=1}^{n}\left\langle s_{i}, s_{j}\right\rangle_{\pi_{L / K}(x)}\left\langle\lambda_{i}, \lambda_{j}\right\rangle_{x}
$$

Note that the function

$$
\left(\omega \in \Omega_{\infty}\right) \mapsto\left\langle s_{i}, s_{j}\right\rangle_{\omega}=\frac{1}{2}\left(\left\|s_{i}+s_{j}\right\|_{\omega}^{2}-\left\|s_{i}\right\|_{\omega}^{2}-\left\|s_{j}\right\|_{\omega}^{2}\right)
$$

is $\left.\mathcal{A}\right|_{\Omega_{\infty}}$-measurable (hence its composition with $\left.\pi_{L / K}\right|_{\Omega_{L, \infty}}$ is $\left.\mathcal{A}_{L}\right|_{\Omega_{L, \infty}}$-measurable) and the function

$$
\left(x \in \Omega_{L, \infty}\right) \longmapsto\left\langle\lambda_{i}, \lambda_{j}\right\rangle_{x}=\frac{1}{2}\left(\left|\lambda_{i}+\lambda_{j}\right|_{x}^{2}-\left|\lambda_{i}\right|_{x}^{2}-\left|\lambda_{j}\right|_{x}^{2}\right)
$$

is $\left.\mathcal{A}_{L}\right|_{\Omega_{L, \infty}}$-measurable. Therefore the function $\left(x \in \Omega_{L}\right) \mapsto\|s\|_{x}^{2}$ is $\mathcal{A}_{L}$-measurable on $\Omega_{L, \infty}$. Moreover, by the measurability of $\xi_{L}$ proved above, this function is also $\mathcal{A}_{L}$-measurable on $\Omega_{L} \backslash \Omega_{L, \infty}$. Hence it is $\mathcal{A}_{L}$-measurable.

(2.a) Let $s$ be an element in $E \otimes F, \varphi$ be the $K$-linear map from $E^{\vee}$ to $F$ which corresponds to $s$, and $r$ be the rank of $\varphi$. Let $\left\{\varphi_{i}\right\}_{i=1}^{n}$ be a basis of $E^{\vee}$ such that $\varphi_{r+1}, \ldots, \varphi_{n}$ belong to the kernel of $f$ and let $\left\{e_{i}\right\}_{i=1}^{n}$ be the dual basis of $\left\{\varphi_{i}\right\}_{i=1}^{n}$. For $i \in\{1, \ldots, r\}$, let $f_{i}$ be the image of $\alpha_{i}$ by $\varphi$. We complet the family $\left\{f_{i}\right\}_{i=1}^{r}$ to a basis $\left\{f_{j}\right\}_{j=1}^{m}$ of $F$. One has

$$
s=e_{1} \otimes f_{1}+\cdots+e_{r} \otimes f_{r} .
$$

Let $E_{0}$ and $F_{0}$ be $K_{0}$-vector subspaces of $E$ and $F$ generated by $\left\{e_{i}\right\}_{i=1}^{n}$ and $\left\{f_{j}\right\}_{j=1}^{m}$ respectively.

By definition, for $\omega \in \Omega$ one has

$$
\|s\|_{\pi, \omega}=\inf \left\{\sum_{i=1}^{N}\left\|x_{i}\right\|_{E, \omega} \cdot\left\|y_{i}\right\|_{F, \omega}: \begin{array}{l}
s=x_{1} \otimes y_{1}+\cdots+x_{N} \otimes y_{N} \text { for some } N \in \mathbb{N}, \\
\left(x_{1}, \ldots, x_{N}\right) \in E_{K_{\omega}}^{N} \text { and }\left(y_{1}, \ldots, y_{N}\right) \in F_{K_{\omega}}^{N}
\end{array}\right\} .
$$


We claim that $\|s\|_{\pi, \omega}$ is eqal to

(4.8)

$$
\|s\|_{\pi, \omega}^{\prime}:=\inf \left\{\sum_{i=1}^{N}\left\|x_{i}\right\|_{E, \omega} \cdot\left\|y_{i}\right\|_{F, \omega}: \begin{array}{l}
s=x_{1} \otimes y_{1}+\cdots+x_{N} \otimes y_{N} \text { for some } N \in \mathbb{N} \\
\left(x_{1}, \ldots, x_{N}\right) \in E_{0}^{N}, \text { and }\left(y_{1}, \ldots, y_{N}\right) \in F_{0}^{N}
\end{array}\right\} .
$$

Clearly $\|s\|_{\pi, \omega}$ is bounded from above by $\|s\|_{\pi, \omega}^{\prime}$. We will show that $\|s\|_{\pi, \omega} \geqslant\|s\|_{\pi, \omega}^{\prime}$. By Proposition 1.1.11, there exists $\alpha \in] 0,1]$ such that the bases $\left\{e_{i}\right\}_{i=1}^{n}$ and $\left\{f_{j}\right\}_{j=1}^{m}$ of $E_{K_{\omega}}$ and $F_{K_{\omega}}$ are both $\alpha$-orthogonal (see Definition 1.2.4). Assume that $s$ is written in the form

$$
s=x_{1} \otimes y_{1}+\cdots+x_{N} \otimes y_{N},
$$

where $\left(x_{1}, \ldots, x_{N}\right) \in E_{K_{\omega}}^{N}$ and $\left(y_{1}, \ldots, y_{N}\right) \in F_{K_{\omega}}^{N}$. For any $\epsilon>0$, there exists $\left(x_{1}^{\prime}, \ldots, x_{N}^{\prime}\right) \in E_{0}^{N}$ and $\left(y_{1}^{\prime}, \ldots, y_{N}^{\prime}\right) \in F_{0}^{N}$ such that

$$
\sup _{\ell \in\{1, \ldots, N\}} \max \left\{\left\|x_{\ell}-x_{\ell}^{\prime}\right\|_{E, \omega},\left\|y_{\ell}-y_{\ell}^{\prime}\right\|_{F, \omega}\right\} \leqslant \epsilon .
$$

We write $x_{\ell}-x_{\ell}^{\prime}$ into the form

$$
x_{\ell}-x_{\ell}^{\prime}=\sum_{i=1}^{n} a_{\ell, i} e_{i}, \quad \text { where }\left(a_{\ell, 1}, \ldots, a_{\ell, n}\right) \in K_{\omega}^{n} .
$$

Since the basis $\left\{e_{i}\right\}_{i=1}^{n}$ is $\alpha$-orthogonal, one has

$$
\epsilon \geqslant\left\|x_{\ell}-x_{\ell}^{\prime}\right\|_{E, \omega} \geqslant \alpha \max _{i \in\{1, \ldots, n\}}\left|a_{\ell, i}\right|_{\omega} \cdot \mid e_{i} \|_{E, \omega} .
$$

Similarly, if we write $y_{\ell}-y_{\ell}^{\prime}$ as

$$
\sum_{j=1}^{m} b_{\ell, j} f_{j}, \quad\left(a_{\ell, 1}, \ldots, a_{\ell, m}\right) \in K_{\omega}^{m}
$$

one has

$$
\epsilon \geqslant\left\|y_{\ell}-y_{\ell}^{\prime}\right\|_{F, \omega} \geqslant \alpha \max _{j \in\{1, \ldots, m\}}\left|b_{\ell, j}\right|_{\omega} \cdot\left\|f_{j}\right\|_{F, \omega}
$$

Let

$$
M=\sup _{\ell \in\{1, \ldots, N\}} \max \left\{\left\|x_{\ell}^{\prime}\right\|_{E, \omega},\left\|y_{\ell}\right\|_{F, \omega}\right\} .
$$

If we write $x_{\ell}^{\prime}$ and $y_{\ell}$ into linear combination of $\left\{e_{i}\right\}_{i=1}^{n}$ and $\left\{f_{j}\right\}_{j=1}^{m}$ respectively:

$$
x_{\ell}^{\prime}=\sum_{i=1}^{n} c_{\ell, i} e_{i}, \quad y_{\ell}=\sum_{j=1}^{m} d_{\ell, j} f_{j},
$$

one has

$$
M \geqslant \alpha \max _{i \in\{1, \ldots, n\}}\left|c_{\ell, i}\right|_{\omega} \cdot\left\|e_{i}\right\|_{E, \omega} \text { and } M \geqslant \alpha \max _{j \in\{1, \ldots, m\}}\left|d_{\ell, j}\right|_{\omega} \cdot\left\|f_{j}\right\|_{F, \omega} .
$$


Note that

$$
\begin{aligned}
& s-x_{1}^{\prime} \otimes y_{1}^{\prime}-\cdots-x_{N}^{\prime} \otimes y_{N}^{\prime}=\sum_{\ell=1}^{N}\left(x_{\ell} \otimes y_{\ell}-x_{\ell}^{\prime} \otimes y_{\ell}^{\prime}\right) \\
= & \sum_{\ell=1}^{N}\left(\left(x_{\ell}-x_{\ell}^{\prime}\right) \otimes y_{\ell}+x_{\ell}^{\prime} \otimes\left(y_{\ell}-y_{\ell}^{\prime}\right)\right) \\
= & \sum_{i=1}^{n} \sum_{j=1}^{m}\left(\sum_{\ell=1}^{N} a_{\ell, i} d_{\ell, j}+c_{\ell, i} b_{\ell, j}\right) e_{i} \otimes f_{j} .
\end{aligned}
$$

For $(i, j) \in\{1, \ldots, n\} \times\{1, \ldots, m\}$, let

$$
A_{i, j}=\sum_{\ell=1}^{N} a_{\ell, i} d_{\ell, j}+c_{\ell, i} b_{\ell, j} .
$$

Since $\left\{e_{i} \otimes f_{j}\right\}_{(i, j) \in\{1, \ldots, n\} \times\{1, \ldots, m\}}$ is a basis of $E_{0} \otimes K_{0} F_{0}$ over $K_{0}$ and a basis of $E_{K_{\omega}} \otimes_{K_{\omega}} F_{K_{\omega}}$ over $K_{\omega}$, one obtains that $A_{i, j} \in K_{0}$ for any $(i, j) \in\{1, \ldots, n\} \times$ $\{1, \ldots, m\}$. Therefore one has

$$
\begin{aligned}
\|s\|_{\pi, \omega}^{\prime} & \leqslant \sum_{\ell=1}^{N}\left\|x_{\ell}^{\prime}\right\|_{E, \omega} \cdot\left\|y_{\ell}^{\prime}\right\|_{F, \omega}+\sum_{i=1}^{n} \sum_{j=1}^{m}\left|A_{i, j}\right|_{\omega} \cdot\left\|e_{i}\right\|_{E, \omega} \cdot\left\|f_{i}\right\|_{F, \omega} \\
& \leqslant \sum_{\ell=1}^{N}\left(\left\|x_{\ell}\right\|_{E, \omega}+\epsilon\right)\left(\left\|y_{\ell}\right\|_{F, \omega}+\epsilon\right)+\sum_{i=1}^{n} \sum_{j=1}^{m}\left|A_{i, j}\right|_{\omega} \cdot\left\|e_{i}\right\|_{E, \omega} \cdot\left\|f_{i}\right\|_{F, \omega} \\
& \leqslant \sum_{\ell=1}^{N}\left(\left\|x_{\ell}\right\|_{E, \omega}+\epsilon\right)\left(\left\|y_{\ell}\right\|_{F, \omega}+\epsilon\right)+\alpha^{-2} \epsilon m n M N
\end{aligned}
$$

where the first inequality comes from the definition (4.8) of $\|\cdot\|_{\pi, \omega}^{\prime}$, the second inequality results from (4.9), and the third inequality comes from (4.10), (4.11) and (4.12). Since $\epsilon$ is arbitrary, we obtain $\|s\|_{\pi, \omega}^{\prime} \leqslant \sum_{\ell=1}^{N}\left\|x_{\ell}\right\| \cdot\left\|y_{\ell}\right\|$, which leads to $\|s\|_{\pi, \omega}^{\prime} \leqslant\|s\|_{\pi, \omega}$ since the writing $s=x_{1} \otimes y_{1}+\cdots+x_{N} \otimes y_{N}$ is arbitrary.

As the set

$$
\bigcup_{N \in \mathbb{N}}\left\{\left(x_{1}, \ldots, x_{N}, y_{1}, \ldots, y_{N}\right) \in E_{0}^{N} \times F_{0}^{N}: s=x_{1} \otimes y_{1}+\cdots+x_{N} \otimes y_{N}\right\}
$$

is countable, we obtain that the function $(\omega \in \Omega) \mapsto\|s\|_{\pi, \omega}=\|s\|_{\pi^{\prime}, \omega}$ is $\mathcal{A}$-measurable. Therefore the norm family $\xi_{E} \otimes_{\pi} \xi_{F}$ is $\mathcal{A}$-measurable.

By Proposition 1.1.57 one has $\xi_{E} \otimes_{\varepsilon} \xi_{F}=\left(\xi_{E}^{\vee} \otimes_{\pi} \xi_{F}^{\vee}\right)^{\vee}$. Hence by the (1.b) of the proposition established above, we obtain that the norm family $\xi_{E} \otimes_{\epsilon} \xi_{F}$ is also $\mathcal{A}$ measurable. Finally, by Corollary 1.2.20 and Proposition 1.1.57 one has $\xi_{E} \otimes_{\varepsilon, \pi} \xi_{F}=$ $\left(\xi_{E}^{\vee} \otimes_{\varepsilon} \xi_{F}^{\vee}\right)^{\vee}$. Therefore the norm family $\xi_{E} \otimes_{\varepsilon, \pi} \xi_{F}$ is also $\mathcal{A}$-measurable.

(2.b) We proceed with the measurability of the orthogonal tensor product norm family in assuming that both norm families $\xi_{E}$ and $\xi_{F}$ are Hermitian. In the first step, we treat a particular case where $E=F^{\vee}$ and $\xi_{E}=\xi_{F}^{\vee}$. In this case the tensor product 
space $E \otimes_{K} F$ is isomorphic to the space $\operatorname{End}_{K}(F)$ of $K$-linear endomorphisms of $F$, and the $\varepsilon$-tensor product norm family $\xi_{F}^{\vee} \otimes_{\varepsilon} \xi_{F}$ identifies with the family of operator norms. Let $\left\{x_{i}\right\}_{i=1}^{n}$ be a basis of $F$ over $K$ and $F_{0}$ be the $K_{0}$-vector subspace of $F$ generated by $\left\{x_{i}\right\}_{i=1}^{n}$. By using the basis $\left\{x_{i}\right\}_{i=1}^{n}$ one can identify $\operatorname{End}_{K_{0}}\left(F_{0}\right)$ with $M_{n \times n}\left(K_{0}\right)$, the space of all matrices of size $n \times n$ with coefficients in $K_{0}$. Similarly, for any $\omega \in \Omega$, one can identify $\operatorname{End}_{K_{\omega}}\left(F_{K_{\omega}}\right)$ with the space $M_{n \times n}\left(K_{\omega}\right)$ of all matrices of size $n \times n$ with coefficients in $K_{\omega}$. In particular, $\operatorname{End}_{K_{0}}\left(F_{0}\right)$ is dense in $\operatorname{End}_{K_{\omega}}\left(F_{K_{\omega}}\right)$.

For any $f \in \operatorname{End}_{K}(F)$ and any $\omega \in \Omega_{\infty}$, let $\|f\|_{\text {HS, } \omega}$ be the Hilbert-Schmidt norm of $f$. By Proposition 1.2.61, one has

$$
\|f\|_{\mathrm{HS}, \omega}=\left(\sum_{i=1}^{r} \inf _{\substack{g \in \operatorname{End}_{K_{\omega}\left(F_{K}\right)} \\ \operatorname{rk}(g) \leqslant i-1}}\|f-g\|_{\omega}^{2}\right)^{1 / 2},
$$

where $\|\cdot\|_{\omega}$ denotes the operator norm on $\operatorname{End}_{K_{\omega}}\left(F_{K_{\omega}}\right)$. Note that the set

$$
\left\{g \in \operatorname{End}_{K_{0}}\left(F_{0}\right): \operatorname{rk}(g) \leqslant i-1\right\}
$$

is dense in

$$
\left\{g \in \operatorname{End}_{K_{\omega}}\left(F_{K_{\omega}}\right): \operatorname{rk}(g) \leqslant i-1\right\}
$$

Hence

$$
\|f\|_{\mathrm{HS}, \omega}=\left(\sum_{i=1}^{r} \inf _{\substack{g \in \operatorname{End}_{K_{0}}\left(F_{0}\right) \\ \operatorname{rk}(g) \leqslant i-1}}\|f-g\|_{\omega}^{2}\right)^{1 / 2} .
$$

By the result of (2.a) on the measurability of $\varepsilon$-tensor product, the function

$$
\left(\omega \in \Omega_{\infty}\right) \mapsto\|f-g\|_{\omega}
$$

is $\left.\mathcal{A}\right|_{\Omega_{\infty}}$-measurable. Hence we deduce the $\left.\mathcal{A}\right|_{\Omega_{\infty}}$-measurability of the function $(\omega \in$ $\left.\Omega_{\infty}\right) \mapsto\|f\|_{\mathrm{HS}, \omega}$. Finally, since the norm $\|\cdot\|_{E \otimes F, \omega}$ identifies with the $\varepsilon$-tensor product $\|\cdot\|_{\varepsilon, \omega}$ for $\omega \in \Omega \backslash \Omega_{\infty}$, by the result of (2.a), the function $\left(\omega \in \Omega \backslash \Omega_{\infty}\right) \mapsto\|f\|_{E \otimes F, \omega}$ is $\left.\mathcal{A}\right|_{\Omega \backslash \Omega_{\infty}}$-measurable. Thus we obtain the $\mathcal{A}$-measurability of the norm family $\xi_{E} \otimes \xi_{F}$.

We now consider the general case. Let $T$ be a tensor vector in $E \otimes_{K} F$, viewed as a linear map from $E^{\vee}$ to $F$. Let $G$ be the direct sum $E^{\vee} \oplus F$ and $\xi_{G}$ be the orthogonal direct sum of $\xi_{E}^{\vee}$ and $\xi_{F}$. By Proposition 4.1.21 and the result obtained in (1.b), the norm family $\xi_{G}$ is $\mathcal{A}$-measurable. Moreover, the linear map $T: E^{\vee} \rightarrow F$ induces a $K$ linear endomorphism $f=\left(\begin{array}{cc}0 & 0 \\ T & 0\end{array}\right)$ of $E^{\vee} \oplus F$. For any $\omega \in \Omega_{\infty}$, the Hilbert-Schmidt norm of $T$ with respect to $\|\cdot\|_{E, \omega}$ and $\|\cdot\|_{F, \omega}$ identifies with the Hilbert-Schmidt norm of $f$ with respect to the orthogonal direct sum norm $\|\cdot\|_{E^{\vee} \oplus F, \omega}$. By the particular case proved above, we obtain the measurability of the function $\left(\omega \in \Omega_{\infty}\right) \mapsto\|T\|_{E \otimes F, \omega}$. Combined with the measurability of $\xi_{E} \otimes_{\varepsilon} \xi_{F}$ proved in (2.a) which implies the measurability of the function $\left(\omega \in \Omega \backslash \Omega_{\infty}\right) \mapsto\|T\|_{E \otimes F, \omega}$, we obtain that the function $(\omega \in \Omega) \mapsto\|T\|_{E \otimes F, \omega}$ is $\mathcal{A}$-measurable. The assertion is thus proved. 
(3) We equip $E^{\otimes i}$ with the $i$-th $\varepsilon, \pi$-tensor power of $\xi$. By (2.a) this norm family is $\mathcal{A}$-measurable. The exterior power norm family is its quotient. By (1.a) we obtain that the norm family $\Lambda^{i} \xi$ is $\mathcal{A}$-measurable.

(4) Let $E_{0}$ be a finite-dimensional $K_{0}$-vector subspace of $E$, which generates $E$ as a $K$-vector space. For any $\omega \in \Omega, E_{0}$ is dense in $E_{K_{\omega}}$. Therefore one has

$$
d_{\omega}\left(\xi, \xi^{\prime}\right)=\sup _{0 \neq s \in E_{0}}\left|\ln \|s\|_{\omega}-\ln \|s\|_{\omega}^{\prime}\right|
$$

Since $E_{0}$ is a countable set and since the functions $(\omega \in \Omega) \mapsto\|s\|_{\omega}$ and $(\omega \in \Omega) \mapsto$ $\|s\|_{\omega}^{\prime}$ are both measurable, we obtain that the function $(\omega \in \Omega) \mapsto d_{\omega}\left(\xi, \xi^{\prime}\right)$ is also measurable.

Remark 4.1.25. — We assume that $K$ is a number field. Let $S=(K,(\Omega, \mathcal{A}, \nu), \phi)$ be the standard adelic curve as in Subsection 3.2.2 Let $E$ be a finite-dimensional vector space over $K$ and $\xi=\left\{\|\cdot\|_{\omega}\right\}_{\omega \in \Omega}$ be a norm family of $E$. Since $\mathcal{A}$ is the disctere $\sigma$-algebra, every function on $\Omega$ is measurable, so that $(E, \xi)$ is measurable.

Theorem 4.1.26. - We assume that $K$ admits a countable subfield $\widetilde{K}$ which is dense in $K_{\omega}$ for any $\omega \in \Omega_{\infty}$. Let $E$ be a finite-dimensional vector space over $K$ and $\xi=\left\{\|\cdot\|_{\omega}\right\}_{\omega \in \Omega}$ be a measurable norm family on $E$ which is ultrametric on $\Omega \backslash \Omega_{\infty}$. For any $\epsilon>0$, there exists a measurable Hermitian norm family $\xi^{H}=\left\{\|\cdot\|_{\omega}^{H}\right\}_{\omega \in \Omega}$ on E such that $\|\cdot\|_{\omega}^{H}=\|\cdot\|_{\omega}$ for any $\omega \in \Omega \backslash \Omega_{\infty}$ and that

$$
\|\cdot\|_{\omega} \leqslant\|\cdot\|_{\omega}^{H} \leqslant(r+\epsilon)^{1 / 2}\|\cdot\|_{\omega}
$$

for any $\omega \in \Omega_{\infty}$, where $r$ denotes the rank of $E$ over $K$.

Proof. - The assertion is trivial when $\Omega_{\infty}$ is empty. In what follows, we assume that $\Omega_{\infty}$ is non-empty. In this case the field $K$ is of characteristic 0 . We divide the proof of the theorem into two steps.

Step 1: In this step, we show that there is a family $\left\{\varphi_{\omega}\right\}_{\omega \in \Omega_{\infty}}$ of embeddings $\varphi_{\omega}: K \rightarrow \mathbb{C}$ such that $|\cdot|_{\omega}=\left|\varphi_{\omega}(\cdot)\right|$ for all $\omega \in \Omega_{\infty}$ and, for any $a \in \widetilde{K}$, the function $\left(\omega \in \Omega_{\infty}\right) \mapsto\left(\varphi_{\omega}(a) \in \mathbb{C}\right)$ is $\left.\mathcal{A}\right|_{\Omega_{\infty}}$-measurable, where $|\cdot|$ denotes the usual absolute value on $\mathbb{C}$.

For each $\omega \in \Omega_{\infty}$, we fix an embedding $\widetilde{\varphi}_{\omega}: K \rightarrow \mathbb{C}$ such that $|\cdot|_{\omega}=\left|\widetilde{\varphi}_{\omega}(\cdot)\right|$. We denote by $f_{\omega}(a)$ and $g_{\omega}(a)$ the real part and the imaginary part of $\widetilde{\varphi}_{\omega}(a)$, respectively, that is, $\widetilde{\varphi}_{\omega}(a)=f_{\omega}(a)+\sqrt{-1} g_{\omega}(a)$ for $a \in K$. We claim that, for any $a \in K$, the function $\left(\omega \in \Omega_{\infty}\right) \mapsto f_{\omega}(a)$ is $\left.\mathcal{A}\right|_{\Omega_{\infty}}$-measurable. In fact, we can write $f_{\omega}(a)$ as $\left|a+\frac{1}{2}\right|_{\omega}^{2}-|a|_{\omega}^{2}-\frac{1}{4}$. Therefore the claim follows from the definition of adelic curve. Moreover, for any $a \in K$, the function $\left(\omega \in \Omega_{\infty}\right) \mapsto g_{\omega}(a)^{2}$ is measurable since we can write $g_{\omega}(a)^{2}$ as $|a|_{\omega}^{2}-f_{\omega}(a)^{2}$. In particular, the function $\left(\omega \in \Omega_{\infty}\right) \mapsto\left|g_{\omega}(a)\right|$ is measurable. As a consequence, for any couple of elements $(a, b)$ in $K$, the function 
$\left(\omega \in \Omega_{\infty}\right) \mapsto g_{\omega}(a) g_{\omega}(b)$ is measurable because

$$
g_{\omega}(a) g_{\omega}(b)=\frac{1}{2}\left(g_{\omega}(a+b)^{2}-g_{\omega}(a)^{2}-g_{\omega}(b)^{2}\right) .
$$

Claim 4.1.2\%. - Let a be an element of $K$. Assume that $s: \Omega_{\infty} \rightarrow\{1,-1\}$ is a map such that the function $\left(\omega \in \Omega_{\infty}\right) \mapsto s(\omega) g_{\omega}(a)$ is $\left.\mathcal{A}\right|_{\Omega_{\infty}}$-measurable. Then for any function $\eta: \Omega_{\infty} \rightarrow \mathbb{R}$ such that the function $\left(\omega \in \Omega_{\infty}\right) \mapsto g_{\omega}(a) \eta(\omega)$ is $\left.\mathcal{A}\right|_{\Omega_{\infty}}$-measurable, the function $\left(\omega \in \Omega_{\infty}\right) \mapsto s(\omega) \eta(\omega) \mathbb{1}_{g_{\omega}(a) \neq 0}$ is $\left.\mathcal{A}\right|_{\Omega_{\infty}}$-measurable. In particular, for any $b \in K$, the function $\left(\omega \in \Omega_{\infty}\right) \mapsto s(\omega) g_{\omega}(b) \mathbb{1}_{g_{\omega}(a) \neq 0}$ is $\left.\mathcal{A}\right|_{\Omega_{\infty}}$ measurable.

Proof. - Let $h_{a, s}: \Omega_{\infty} \rightarrow \mathbb{R}$ be the function defined by

$$
h_{a, s}(\omega):= \begin{cases}s(\omega) g_{\omega}(a)^{-1}=\left(s(\omega) g_{\omega}(a)\right)^{-1}, & \text { if } g_{\omega}(a) \neq 0, \\ 0, & \text { if } g_{\omega}(a)=0 .\end{cases}
$$

As the function $\left(\omega \in \Omega_{\infty}\right) \mapsto s(\omega) g_{\omega}(a)$ is $\left.\mathcal{A}\right|_{\Omega_{\infty}}$-measurable, also is $h_{a, s}$. Since the function $\left(\omega \in \Omega_{\infty}\right) \mapsto g_{\omega}(a) \eta(\omega)$ is measurable, we deduce that the function

$$
\left(\omega \in \Omega_{\infty}\right) \longmapsto h_{a, s}(\omega) g_{\omega}(a) \eta(\omega)=s(\omega) \eta(\omega) \mathbb{1}_{g_{\omega}(a) \neq 0}
$$

is $\left.\mathcal{A}\right|_{\Omega_{\infty}}$-measurable, which proves the first assertion. The second assertion follows from the first one and the fact that the function $\left(\omega \in \Omega_{\infty}\right) \mapsto g_{\omega}(a) g_{\omega}(b)$ is $\left.\mathcal{A}\right|_{\Omega_{\infty}}$ measurable.

In the following, we show that there exists a map $s: \Omega_{\infty} \rightarrow\{1,-1\}$ such that, for any $a \in \widetilde{K}$, the function $\left(\omega \in \Omega_{\infty}\right) \mapsto s(\omega) g_{\omega}(a)$ is $\left.\mathcal{A}\right|_{\Omega_{\infty}}$-measurable. Since $\widetilde{K}$ is countable, we can write its elements in a sequence $\left\{a_{n}\right\}_{n \in \mathbb{N}}$. We will construct by induction a decreasing sequence of functions $s_{n}: \Omega_{\infty} \rightarrow\{1,-1\}(n \in \mathbb{N})$ which satisfy the following conditions:

(1) for any $n \in \mathbb{N}$ and any $i \in\{0, \ldots, n\}$, the function $\left(\omega \in \Omega_{\infty}\right) \mapsto s_{n}(\omega) g_{\omega}\left(a_{i}\right)$ is $\left.\mathcal{A}\right|_{\Omega_{\infty}}$-measurable,

(2) for any $n \in \mathbb{N}$, one has

$$
\left\{\omega \in \Omega_{\infty}: s_{n}(\omega)=1\right\} \supseteq\left\{\omega \in \Omega_{\infty}: g_{\omega}\left(a_{0}\right)=\cdots=g_{\omega}\left(a_{n}\right)=0\right\} .
$$

In the case where $n=0$, we just choose

$$
s_{0}(\omega)= \begin{cases}1 & \text { if } g_{\omega}\left(a_{0}\right) \geqslant 0 \\ -1 & \text { if } g_{\omega}\left(a_{0}\right)<0\end{cases}
$$

Then one has $s_{0}(\omega) g_{\omega}\left(a_{0}\right)=\left|g_{\omega}\left(a_{0}\right)\right|$. Therefore the function

$$
\left(\omega \in \Omega_{\infty}\right) \mapsto s_{0}(\omega) g_{\omega}\left(a_{i}\right)
$$

is $\left.\mathcal{A}\right|_{\Omega_{\infty}}$-measurable. Moreover, by definition one has

$$
\left\{\omega \in \Omega_{\infty}: s_{0}(\omega)=1\right\} \supseteq\left\{\omega \in \Omega_{\infty}: g_{\omega}\left(a_{0}\right)=0\right\} .
$$


Assume that the functions $s_{0}, \ldots, s_{n}$ have been constructed, which satisfy the conditions above. We choose

$$
s_{n+1}(\omega)= \begin{cases}-s_{n}(\omega), & \text { if } g_{\omega}\left(a_{0}\right)=\cdots=g_{\omega}\left(a_{n}\right)=0 \text { and } g_{\omega}\left(a_{n+1}\right)<0 \\ s_{n}(\omega), & \text { otherwise. }\end{cases}
$$

Clearly, if $g_{\omega}\left(a_{0}\right)=\cdots=g_{\omega}\left(a_{n}\right)=g_{\omega}\left(a_{n+1}\right)=0$, then $s_{n+1}(\omega)=s_{n}(\omega)=1$, so that the above condition (2) for $s_{n+1}$ is satisfied. Moreover, if $g_{\omega}\left(a_{0}\right)=\cdots=g_{\omega}\left(a_{n}\right)=0$ and $g_{\omega}\left(a_{n+1}\right)<0$, then $s_{n}(\omega)=1$ and $s_{n+1}(\omega)=-s_{n}(\omega)=-1$. Hence we always have $s_{n+1}(\omega) \leqslant s_{n}(\omega)$ for any $\omega \in \Omega_{\infty}$. For any $i \in\{0, \ldots, n\}$ and any $\omega \in \Omega_{\infty}$ one has

$$
s_{n+1}(\omega) g_{\omega}\left(a_{i}\right)=s_{n}(\omega) g_{\omega}\left(a_{i}\right)
$$

since $s_{n+1}(\omega)=s_{n}(\omega)$ once $g_{\omega}\left(a_{i}\right) \neq 0$. Hence by the induction hypothesis the function $\left(\omega \in \Omega_{\infty}\right) \mapsto s_{n+1}(\omega) g_{\omega}\left(a_{i}\right)$ is $\left.\mathcal{A}\right|_{\Omega_{\infty}}$-measurable.

In what follows, we show that the function $\left(\omega \in \Omega_{\infty}\right) \mapsto s_{n+1}(\omega) g_{\omega}\left(a_{n+1}\right)$ is also $\left.\mathcal{A}\right|_{\Omega_{\infty}}$-measurable. For any $i \in\{0, \ldots, n\}$, the set

$$
\left\{\omega \in \Omega_{\infty}: g_{\omega}\left(a_{i}\right)=0\right\}=\left\{\omega \in \Omega_{\infty}: g_{\omega}\left(a_{i}\right)^{2}=0\right\}
$$

belongs to $\mathcal{A}$ since the function $\left(\omega \in \Omega_{\infty}\right) \mapsto g_{\omega}\left(a_{i}\right)^{2}$ is $\left.\mathcal{A}\right|_{\Omega_{\infty}}$-measurable. By Claim 4.1.27, the function

$$
\left(\omega \in \Omega_{\infty}\right) \longmapsto s_{n+1}(\omega) g_{\omega}\left(a_{n+1}\right) \mathbb{1}_{g_{\omega}\left(a_{0}\right) \neq 0}
$$

is $\left.\mathcal{A}\right|_{\Omega_{\infty}}$-measurable. Similarly, for any $i \in\{1, \ldots, n\}$ we deduce from the $\left.\mathcal{A}\right|_{\Omega_{\infty}}{ }^{-}$ measurability of the function

$$
(\omega \in \Omega) \longmapsto g_{\omega}\left(a_{i}\right) g_{\omega}\left(a_{n+1}\right) \mathbb{1}_{g_{\omega}\left(a_{0}\right)=0} \cdots \mathbb{1}_{g_{\omega}\left(a_{i-1}\right)=0}
$$

that the function

$$
(\omega \in \Omega) \longmapsto s_{n+1}(\omega) g_{\omega}\left(a_{n+1}\right) \mathbb{1}_{g_{\omega}\left(a_{0}\right)=0} \cdots \mathbb{1}_{g_{\omega}\left(a_{i-1}\right)=0} \mathbb{1}_{g_{\omega}\left(a_{i}\right) \neq 0}
$$

is $\left.\mathcal{A}\right|_{\Omega_{\infty}}$-measurable. Moreover, the function

$$
\left(\omega \in \Omega_{\infty}\right) \longmapsto s_{n+1}(\omega) g_{\omega}\left(a_{n+1}\right) \mathbb{1}_{g_{\omega}\left(a_{0}\right)=\cdots=g_{\omega}\left(a_{n}\right)=0}
$$

is $\left.\mathcal{A}\right|_{\Omega_{\infty}}$-measurable since $s_{n+1}(\omega) g_{\omega}\left(a_{n+1}\right)=\left|g_{\omega}\left(a_{n+1}\right)\right|$ once the condition $g_{\omega}\left(a_{0}\right)=$ $\cdots=g_{\omega}\left(a_{n}\right)=0$ holds and since the set

$$
\left\{\omega \in \Omega_{\infty}: g_{\omega}\left(a_{0}\right)=\cdots=g_{\omega}\left(a_{n}\right)=0\right\}=\left\{\omega \in \Omega_{\infty}: g_{\omega}\left(a_{0}\right)^{2}=\cdots=g_{\omega}\left(a_{n}\right)^{2}=0\right\}
$$

belongs to $\mathcal{A}$. We then obtain the $\left.\mathcal{A}\right|_{\Omega_{\infty}}$-measurability of the function $\left(\omega \in \Omega_{\infty}\right) \mapsto$ $s_{n+1}(\omega) g_{\omega}\left(a_{n+1}\right)$ since we can write the function as the sum of (4.13), (4.15) and (4.14) for $i \in\{1, \ldots, n\}$. Let $s$ be the limit of the decreasing sequence of functions $\left\{s_{n}\right\}_{n \in \mathbb{N}}$. For any $n \in \mathbb{N}$, the function $\left(\omega \in \Omega_{\infty}\right) \mapsto s_{m}(\omega) g_{\omega}\left(a_{n}\right)$ is $\left.\mathcal{A}\right|_{\Omega_{\infty}}$-measurable for any integer $m \geqslant n$. By passing to limit when $m \rightarrow+\infty$, we obtain that the function $\left(\omega \in \Omega_{\infty}\right) \mapsto s(\omega) g_{\omega}\left(a_{n}\right)$ is $\left.\mathcal{A}\right|_{\Omega_{\infty}}$-measurable. 
Here we define $\varphi_{\omega}: K \rightarrow \mathbb{C}$ to be

$$
\varphi_{\omega}:= \begin{cases}\widetilde{\varphi}_{\omega} & \text { if } s(\omega)=1, \\ \text { the complex conjugation of } \widetilde{\varphi}_{\omega} & \text { if } s(\omega)=-1 .\end{cases}
$$

By the measurability result proved above, we obtain that, for any $a \in \widetilde{K}$, the function $\left(\omega \in \Omega_{\infty}\right) \mapsto \varphi_{\omega}(a)$ is an $\left.\mathcal{A}\right|_{\Omega_{\infty}}$-measurable.

Step 2: Let $\Omega_{\infty, c}$ be the set of $\omega \in \Omega_{\infty}$ such that $K_{\omega}=\mathbb{C}$. Then one has

$$
\Omega_{\infty} \backslash \Omega_{\infty, c}=\bigcap_{a \in \widetilde{K}}\left\{\omega \in \Omega_{\infty}: \varphi_{\omega}(a) \in \mathbb{R}\right\} .
$$

Therefore, the sets $\Omega_{\infty, c}$ and $\Omega_{\infty} \backslash \Omega_{\infty, c}$ belong to $\mathcal{A}$ (by Proposition 3.1 .1 , the set $\Omega_{\infty}$ belongs to $\left.\mathcal{A}\right)$.

Let $\left\{e_{i}\right\}_{i=1}^{r}$ be a basis of $E$ over $K$. We consider $\mathbb{C}^{r \times r}$ as the space of complex matrices of size $r \times r$ and equip it with the Euclidean topology and Borel $\sigma$-algebra.

Let $r^{\prime}=r+\varepsilon$. For any $\omega \in \Omega_{\infty, c}$, let $G(\omega)$ be the set of all matrices $A \in \mathbb{C}^{r \times r}$ such that, for any $\left(\lambda_{1}, \ldots, \lambda_{r}\right) \in \widetilde{K}^{r}$, if we note $\left(x_{1}, \ldots, x_{r}\right)=\left(\varphi_{\omega}\left(\lambda_{1}\right), \ldots, \varphi_{\omega}\left(\lambda_{r}\right)\right)$, then one has

$$
\left\|x_{1} e_{1}+\cdots+x_{r} e_{r}\right\|_{\omega}^{2} \leqslant\left(\bar{x}_{1}, \ldots, \bar{x}_{r}\right) A^{*} A\left(\begin{array}{c}
x_{1} \\
\vdots \\
x_{r}
\end{array}\right) \leqslant r^{\prime}\left\|x_{1} e_{1}+\cdots+x_{r} e_{r}\right\|_{\omega}^{2},
$$

where $A^{*}={ }^{t} \bar{A}$. For any $\omega \in \Omega_{\infty} \backslash \Omega_{\infty, c}$, let $G(\omega)$ be the set of all matrices $A \in \mathbb{C}^{r \times r}$ such that, for any $\left(\lambda_{1}, \ldots, \lambda_{r}\right) \in \widetilde{K}^{r}$ and any $\left(\lambda_{1}^{\prime}, \ldots, \lambda_{r}^{\prime}\right) \in \widetilde{K}^{r}$, if we note

$$
\left(z_{1}, \ldots, z_{r}\right):=\left(\varphi_{\omega}\left(\lambda_{1}\right)+\sqrt{-1} \varphi_{\omega}\left(\lambda_{1}^{\prime}\right), \ldots, \varphi_{\omega}\left(\lambda_{r}\right)+\sqrt{-1} \varphi_{\omega}\left(\lambda_{r}^{\prime}\right)\right),
$$

then

$$
\left\|z_{1} e_{1}+\cdots+z_{r} e_{r}\right\|_{\omega, \mathbb{C}}^{2} \leqslant\left(\bar{z}_{1}, \ldots, \bar{z}_{r}\right) A^{*} A\left(\begin{array}{c}
z_{1} \\
\vdots \\
z_{r}
\end{array}\right) \leqslant r^{\prime}\left\|z_{1} e_{1}+\cdots+z_{r} e_{r}\right\|_{\omega, \mathbb{C}}^{2}
$$

where the norm $\|\cdot\|_{\omega, \mathbb{C}}$ is defined as follows

$$
\forall(x, y) \in E_{K_{\omega}}^{2}, \quad\|x+\sqrt{-1} y\|_{\omega, \mathbb{C}}:=\left(\|x\|_{\omega}^{2}+\|y\|_{\omega}^{2}\right)^{-1 / 2} .
$$

For any $A \in \mathbb{C}^{r \times r}$, let $E(A)$ be the subset of $\mathbb{C}^{r}$ defined as

$$
\left\{x \in \mathbb{C}^{r}:{ }^{t} \bar{x} A^{*} A x \leqslant 1\right\} .
$$

For any $\omega \in \Omega_{\infty}$, let

$$
B_{\omega}:=\left\{\left(x_{1}, \ldots, x_{r}\right) \in \mathbb{C}^{r}:\left\|x_{1} e_{1}+\cdots+x_{r} e_{r}\right\|_{\omega, \mathbb{C}} \leqslant 1\right\} .
$$

Then a matrix $A$ belongs to $G(\omega)$ if and only if the following conditions hold

$$
\left(r^{\prime}\right)^{-1 / 2} B_{\omega} \subseteq E(A) \subseteq B_{\omega} .
$$


In fact, this relation is equivalent to, for any $\left(z_{1}, \ldots, z_{r}\right) \in \mathbb{C}^{r}$,

$$
\left\|z_{1} e_{1}+\cdots+z_{r} e_{r}\right\|_{\omega, \mathbb{C}}^{2} \leqslant\left(\bar{z}_{1}, \ldots, \bar{z}_{r}\right) A^{*} A\left(\begin{array}{c}
z_{1} \\
\vdots \\
z_{r}
\end{array}\right) \leqslant r^{\prime}\left\|z_{1} e_{1}+\cdots+z_{r} e_{r}\right\|_{\omega, \mathbb{C}}^{2} .
$$

Hence it implies that $A \in G(\omega)$. The converse implication is also true since $\varphi_{\omega}(\widetilde{K})+$ $\sqrt{-1} \varphi_{\omega}(\widetilde{K})$ is dense in $\mathbb{C}$ if $K_{\omega}=\mathbb{R}$, and $\varphi_{\omega}(\widetilde{K})$ is dense in $\mathbb{C}$ if $K_{\omega}=\mathbb{C}$.

Let $\delta>0$ such that $(1+\delta) \sqrt{r}<\sqrt{r^{\prime}}$. By Theorem 1.2.54 (see also Remark1.2.55), there exists a Hermitian matrix $A_{0}$ such that

$$
(1+\delta)^{-1} r^{-1 / 2} B_{\omega} \subseteq E\left(A_{0}\right) \subseteq(1+\delta)^{-1} B_{\omega} .
$$

This shows that $A_{0}$ belongs to the interior of $G(\omega)$ and hence the interior $G(\omega)^{\circ}$ of $G(\omega)$ is not empty. We denote by $F(\omega)$ the closure of $G(\omega)^{\circ}$. If $U$ is an open subset of $\mathbb{C}^{r \times r}$, one has

$$
\begin{gathered}
\left\{\omega \in \Omega_{\infty}: U \cap F(\omega) \neq \varnothing\right\}=\left\{\omega \in \Omega_{\infty}: U \cap G(\omega)^{\circ} \neq \varnothing\right\} \\
=\bigcup_{A \in \overline{\mathbb{Q}}^{r \times r} \cap U}\left\{\omega \in \Omega_{\infty}: A \in G(\omega)^{\circ}\right\} .
\end{gathered}
$$

Note that for any matrix $A \in \mathbb{C}^{r \times r}$

$$
\left\{\omega \in \Omega_{\infty, c}: A \in G(\omega)^{\circ}\right\}=\bigcup_{\mu \in \mathbb{Q} \cap(0,1)} \bigcap_{\lambda=\left(\lambda_{1}, \ldots, \lambda_{r}\right) \in \widetilde{K}^{r}} \Omega_{\infty, c}(\mu, \lambda),
$$

where $\Omega_{\infty, c}\left(\mu, \lambda_{1}, \ldots, \lambda_{r}\right)$ is the set of $\omega \in \Omega_{\infty, c}$ such that

$$
\begin{array}{r}
(1+\mu)\left\|\lambda_{1} e_{1}+\cdots+\lambda_{r} e_{r}\right\|_{\omega}^{2} \leqslant\left(\overline{\varphi_{\omega}\left(\lambda_{1}\right)}, \ldots, \overline{\varphi_{\omega}\left(\lambda_{r}\right)}\right) A^{*} A\left(\begin{array}{c}
\varphi_{\omega}\left(\lambda_{1}\right) \\
\vdots \\
\varphi_{\omega}\left(\lambda_{r}\right)
\end{array}\right) \\
\leqslant(1-\mu) r^{\prime}\left\|\lambda_{1} e_{1}+\cdots+\lambda_{r} e_{r}\right\|_{\omega}^{2} .
\end{array}
$$

Note that $\Omega_{\infty}\left(\mu, \lambda_{1}, \ldots, \lambda_{r}\right)$ belongs to $\mathcal{A}$ since the functions

$$
\left(\omega \in \Omega_{\infty}\right) \longmapsto\left\|\lambda_{1} e_{1}+\cdots+\lambda_{r} e_{r}\right\|_{\omega}^{2}
$$

and

$$
\left(\omega \in \Omega_{\omega}\right) \longmapsto \varphi_{\omega}\left(\lambda_{i}\right) \quad(i \in\{1, \ldots, r\})
$$

are $\left.\mathcal{A}\right|_{\Omega_{\infty}}$-measurable. We then deduce that $\left\{\omega \in \Omega_{\infty, c}: A \in G(\omega)^{\circ}\right\}$ belongs to $\mathcal{A}$. Similarly,

$$
\left\{\omega \in \Omega_{\infty} \backslash \Omega_{\infty, c}: A \in G(\omega)^{\circ}\right\}=\bigcup_{\mu \in \mathbb{Q}(0,1)} \bigcap_{\begin{array}{c}
\lambda=\left(\lambda_{1}, \ldots, \lambda_{r}\right) \in \widetilde{K}^{r} \\
\lambda^{\prime}=\left(\lambda_{1}^{\prime}, \ldots, \lambda_{r}^{\prime}\right) \in \widetilde{K}^{r}
\end{array}}\left(\Omega_{\infty} \backslash \Omega_{\infty, c}\right)\left(\mu, \lambda, \lambda^{\prime}\right),
$$


where $\left(\Omega_{\infty} \backslash \Omega_{\infty, c}\right)\left(\mu, \lambda, \lambda^{\prime}\right)$ is the set of $\omega \in \Omega_{\infty, c}$ such that

$$
\begin{aligned}
& (1+\mu)\left(\left\|\lambda_{1} e_{1}+\cdots+\lambda_{r} e_{r}\right\|_{\omega}^{2}+\left\|\lambda_{1} e_{1}+\cdots+\lambda_{r} e_{r}\right\|_{\omega}^{2}\right) \\
& \leqslant\left(\bar{z}_{1}(\omega), \ldots, \bar{z}_{r}(\omega)\right) A^{*} A\left(\begin{array}{c}
z_{1}(\omega) \\
\vdots \\
z_{n}(\omega)
\end{array}\right) \\
& \leqslant(1-\mu) r^{\prime}\left(\left\|\lambda_{1} e_{1}+\cdots+\lambda_{r} e_{r}\right\|_{\omega}^{2}+\left\|\lambda_{1}^{\prime} e_{1}+\cdots+\lambda_{r}^{\prime} e_{r}\right\|_{\omega}^{2}\right) .
\end{aligned}
$$

Hence $\left\{\omega \in \Omega_{\infty} \backslash \Omega_{\infty, c}: A \in G(\omega)^{\circ}\right\}$ belongs to $\mathcal{A}$.

Gathering the results we obtained, one can conclude that

$$
\left\{\omega \in \Omega_{\infty}: U \cap F(\omega) \neq \varnothing\right\}
$$

belongs to $\mathcal{A}$, so that by the measurable selection theorem of Kuratowski and RyllNardzweski (see A.2.1), we obtain that there exists an $\left.\mathcal{A}\right|_{\Omega_{\infty}}$-measurable map $\alpha$ : $\Omega_{\infty} \rightarrow M$ such that $\alpha(\omega)$ belongs to $F(\omega)$ for any $(\omega \in \Omega)$. Finally, for any $\omega \in \Omega_{\infty}$ and any $\left(\lambda_{1}, \ldots, \lambda_{r}\right) \in K_{\omega}^{r}$ and we let (where we extend $\varphi_{\omega}$ by continuity to $K_{\omega} \rightarrow \mathbb{C}$ )

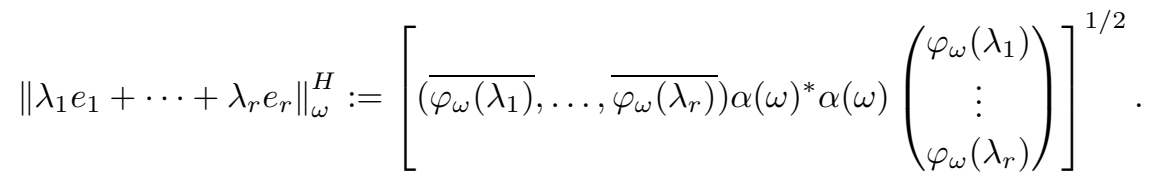

Then $\|\cdot\|_{\omega}^{H}$ is a Hermitian norm which satisfies

$$
\|\cdot\|_{\omega} \leqslant\|\cdot\|_{\omega}^{H} \leqslant(r+\varepsilon)^{1 / 2}\|\cdot\|_{\omega} .
$$

For $\omega \in \Omega \backslash \Omega_{\infty}$, let $\|\cdot\|_{\omega}^{H}:=\|\cdot\|_{\omega}$. Then by the measurability of the map $\alpha(\cdot)$ we obtain that the norm family $\xi^{H}:=\left\{\|\cdot\|_{\omega}^{H}\right\}_{\omega \in \Omega}$ is measurable. The theorem is thus proved.

4.1.4. Adelic vector bundles. - In this section, we introduce the notion of adelic vector bundles on an adelic curve $S=(K,(\Omega, \mathcal{A}, \nu), \phi)$. An adelic vector bundle is a finite-dimensional vector space $E$ over $K$ equipped with a family of norms indexed by $\Omega$, which satisfies some measurability and dominance conditions so that the height of non-zero vectors is well defined (see Definition 4.1.28). In the classic setting of global fields, the notion of adelic vector bundles was defined differently in the literature (see for example [60]): one requires that almost all norms come from a common integral model of $E$. However, in our setting it is not relevant to consider integral models. The readers will discover the link between our definition and the classic one via the dominance property described in Proposition 4.1.7

Definition 4.1.28. - Let $E$ be a finite-dimensional vector space over $K$, and $\xi$ be a norm family in $\mathcal{N}_{E}$. If both norm families $\xi$ and $\xi^{\vee}$ are $\mathcal{A}$-measurable on $\Omega$ and if 
$\xi$ is dominated (resp. strongly dominated), we say that the couple $(E, \xi)$ is an adelic vector bundle (resp. a strongly adelic vector bundle) on $S$.

If the norm family $\xi$ is Hermitian (in this case $(E, \xi)$ is necessarily a strongly adelic vector bundle), we say that $(E, \xi)$ is a Hermitian adelic vector bundle on $S$. If the rank of $E$ is 1 (in this case $\xi$ is necessarily Hermitian), we say that $(E, \xi)$ is an adelic line bundle on $S$.

Proposition 4.1.29. - Let $E$ be a vector space of rank 1 over $K$ and $\xi$ be a norm family in $\mathcal{N}_{E}$. If $\xi$ is $\mathcal{A}$-measurable and dominated, then $(E, \xi)$ is an adelic line bundle on $S$.

Proof. - Since $E$ is of rank 1 over $K$, any dominated norm family is strongly dominated (see Remark 4.1.12). Moreover, by Proposition 4.1.22, if $\xi$ is $\mathcal{A}$-measurable, then also is $\xi^{\vee}$.

Proposition 4.1.30. - Let $(E, \xi)$ be an adelic line bundle on $S$. Then $\left(E^{\vee}, \xi^{\vee}\right)$ is an adelic line bundle on $S$.

Proof. - By definition, the norm family $\xi^{\vee}$ is $\mathcal{A}$-measurable on $\Omega$. Moreover, by Proposition 4.1.19 (3), the norm family $\xi^{\vee}$ is dominated. By Proposition 4.1.29, we obtain that $\left(E^{\vee}, \xi^{\vee}\right)$ is an adelic line bundle on $S$.

The following proposition is fundamental in the height theory of rational points in a projective space over an adelic curve.

Proposition 4.1.31. - Let $(E, \xi)$ be an adelic vector bundle on $S$.

(1) Any vector subspace of rank 1 of $E$ equipped with the restriction of the norm family $\xi$ forms an adelic line bundle on $S$.

(2) Any quotient vector space of rank 1 of E equipped with the quotient norm family of $\xi$ forms an adelic line bundle on $S$.

Proof. - (1) Let $F$ be a vector subspace of rank 1 of $E$ and $\xi_{F}$ be the restriction of $\xi$ to $F$. Clearly $\xi_{F}$ is $\mathcal{A}$-measurable. Moreover, by Proposition 4.1.19 (1), the norm family $\xi_{F}$ is dominated. By Proposition 4.1.29, $\left(F, \xi_{F}\right)$ is an adelic line bundle on $S$.

(2) Let $G$ be a quotient space of rank 1 of $E$ and $\xi_{G}$ be the quotient of the norm family $\xi$ on $G$. Then $G^{\vee}$ identifies with a vector subspace of rank 1 of $E^{\vee}$ and $\xi_{G}^{\vee}$ identifies with the restriction of $\xi^{\vee}$ to $G^{\vee}$ (see Proposition 1.1.20). Therefore $\left(G^{\vee}, \xi_{G}^{\vee}\right)$ is an adelic line bundle on $S$. Finally, by Proposition 4.1.30 and the fact that $\xi_{G}=\xi_{G}^{\vee \vee}$ (where we identify $G$ with $G^{\vee \vee}$ ), we obtain that $\left(G, \xi_{G}\right)$ is an adelic line bundle on $S$.

Proposition 4.1.32. - (1) Let $(E, \xi)$ be an adelic vector bundle (resp. a strongly adelic vector bundle) on $S, F$ be a vector subspace of $E$ and $\xi_{F}$ be the restriction of $\xi$ to $F$. If the norm family $\xi_{F}^{\vee}$ is $\mathcal{A}$-measurable, then $\left(F, \xi_{F}\right)$ is an adelic vector bundle (resp. a strongly adelic vector bundle) on $S$. 
(2) Let $(E, \xi)$ be an adelic vector bundle (resp. a strongly adelic vector bundle) on $S, G$ be a quotient vector space of $E$ and $\xi_{G}$ be the quotient norm family of $\xi$. If the norm family $\xi_{G}$ is $\mathcal{A}$-measurable, then $\left(G, \xi_{G}\right)$ is an adelic vector bundle (resp. a strongly adelic vector bundle) on $S$.

(3) Let $(E, \xi)$ be an adelic vector bundle on $S$. Assume that the norm family $\xi^{\vee \vee}$ is measurable. Then $\left(E^{\vee}, \xi^{\vee}\right)$ is a strongly adelic vector bundle on $S$.

(4) Let $\left(E, \xi_{E}\right)$ and $\left(F, \xi_{F}\right)$ be adelic vector bundles on $S$. If the norm families $\xi_{E} \otimes_{\varepsilon}$ $\xi_{F}$ and $\left(\xi_{E} \otimes_{\varepsilon} \xi_{F}\right)^{\vee}$ are $\mathcal{A}$-measurable, then $\left(E \otimes F, \xi_{E} \otimes_{\varepsilon} \xi_{F}\right)$ is a strongly adelic vector bundle on $S$. Similarly, $\left(E \otimes F, \xi_{E} \otimes_{\varepsilon, \pi} \xi_{F}\right)$ is a strongly adelic vector bundle on $S$ provided that the both norm families $\xi_{E} \otimes_{\varepsilon, \pi} \xi_{F}$ and $\left(\xi_{E} \otimes_{\varepsilon, \pi} \xi_{F}\right)^{\vee}$ are measurable. If in addition $\xi_{E}$ and $\xi_{F}$ are both Hermitian, and if both norm families $\xi_{E} \otimes \xi_{F}$ and $\left(\xi_{E} \otimes \xi_{F}\right)^{\vee}$ are $\mathcal{A}$-measurable, then the orthogonal tensor product $\left(E \otimes F, \xi_{E} \otimes \xi_{F}\right)$ is a Hermitian adelic vector bundle on $S$.

(5) Let $(E, \xi)$ be an adelic vector bundle on $S$. If $\operatorname{det}(\xi)$ is $\mathcal{A}$-measurable then $(\operatorname{det}(E), \operatorname{det}(\xi))$ is an adelic line bundle on $S$.

Proof. - These assertions are direct consequences of Proposition 4.1.19, We just mention below some particular points. For the assertion (1), since $\xi$ is $\mathcal{A}$-measurable, by definition $\xi_{F}$ is also measurable. For the assertion (2) since $\xi^{\vee}$ is $\mathcal{A}$-measurable, and $\xi_{G}^{\vee}$ identifies with the restriction of $\xi^{\vee}$ to $G^{\vee}$, it is also $\mathcal{A}$-measurable. For the last assertion, since $\operatorname{det}(E)$ is of $\operatorname{rank} 1$ on $K$, the $\mathcal{A}$-measurability of $\operatorname{det}(\xi)$ implies that of its dual.

Corollary 4.1.33. - Let $\left(E_{1}, \xi_{1}\right)$ and $\left(E_{2}, \xi_{2}\right)$ be adelic line bundles on $S$. Then the tensor product $\left(E_{1} \otimes E_{2}, \xi_{1} \otimes \xi_{2}\right)$ is also an adelic line bundle on $S$.

Proof. - By Proposition 4.1.22 (3), the tensor product norm family $\xi_{1} \otimes \xi_{2}$ is $\mathcal{A}$ measurable. By Proposition 4.1.32 (4) we obtain that $\left(E_{1} \otimes E_{2}, \xi_{1} \otimes \xi_{2}\right)$ is an adelic line bundle on $S$.

Remark 4.1.34. - By using the measurability results obtained in the previous subsection (notably Proposition 4.1.24), we obtain that the assertions of Proposition 4.1 .32 remain true without measurability assumptions, if the $\sigma$-algebra $\mathcal{A}$ is discrete, or if the field $K$ admits a countable subfield which is dense in all completions $K_{\omega}$, $\omega \in \Omega$.

In the case of direct sums, the measurability result in Proposition 4.1.21 leads to the following criterion (without any condition on $K$ ).

Proposition 4.1.35. - Let $\left(E, \xi_{E}\right)$ and $\left(F, \xi_{F}\right)$ be adelic vector bundles (resp. a strongly adelic vector bundle) on $S$, and $\psi:(\omega \in \Omega) \mapsto \psi_{\omega} \in \mathscr{S}$ be an $\mathcal{A}$-measurable map. We assume that there exists a measurable subset $\Omega^{\prime}$ of $\Omega$ such that $\nu\left(\Omega^{\prime}\right)<+\infty$ and that $\psi_{\omega}=\psi_{0}$ on $\Omega \backslash \Omega^{\prime}$, where $\psi_{0}$ denotes the function in $\mathscr{S}$ sending $t \in[0,1]$ 
to $\max \{t, 1-t\}$. Then $\left(E \oplus F, \xi_{E} \oplus_{\psi} \xi_{F}\right)$ is an adelic vector bundle (resp. a strongly adelic vector bundle) on $S$.

Proof. - Since $\left(E, \xi_{E}\right)$ and $\left(F, \xi_{F}\right)$ are adelic vector bundles (resp. strongly adelic vector bundles) on $S$, the norm families $\xi_{E}, \xi_{F}, \xi_{E}^{\vee}$ and $\xi_{F}^{\vee}$ are all $\mathcal{A}$-measurable, and the norm families $\xi_{E}$ and $\xi_{F}$ are dominated (resp. strongly dominated).

By Proposition 4.1.21, the $\psi$-direct sum $\xi_{E} \oplus_{\psi} \xi_{F}$ is also $\mathcal{A}$-measurable. Let $\psi^{\prime}=$ $\left\{\psi_{\omega}^{\prime}\right\}_{\omega \in \Omega}$ be the family in $\mathscr{S}$ such that $\psi_{\omega}=\psi_{0}$ on $\Omega \backslash \Omega_{\infty}$ and $\psi_{\omega}^{\prime}=\psi_{\omega, *}$ (see Definition 1.1.48) on $\Omega_{\infty}$, then one has

$$
\left(\xi_{E} \oplus_{\psi} \xi_{F}\right)^{\vee}=\xi_{E}^{\vee} \oplus_{\psi^{\prime}} \xi_{F}^{\vee}
$$

Note that the map from $\mathscr{S}$ to itself sending $\varphi \in \mathscr{S}$ to $\varphi_{*}$ is continuous. This is a consequence of (1.11) and Proposition 1.1.43. Therefore, the map $\psi^{\prime}$ is also $\mathcal{A}$ measurable. Still by Proposition 4.1.21, we obtain that the norm family $\left(\xi_{E} \oplus \xi_{F}\right)^{\vee}$ is $\mathcal{A}$-measurable.

By Proposition 4.1.19 (4), the norm family $\xi_{E} \oplus_{\psi} \xi_{F}$ is dominated (resp. strongly dominated). Therefore $\left(E \oplus F, \xi_{E} \oplus_{\psi} \xi_{F}\right)$ is an adelic vector bundle (resp. strongly adelic vector bundle) on $S$.

4.1.5. Examples. - In this subsection, we present several examples of adelic vector bundles, which include most classical constructions.

Torsion free coherent sheaves. - Let $k$ be a field and $X$ be a normal projective scheme of dimension $d \geqslant 1$ over Spec $k$, equipped with a family $\left\{D_{i}\right\}_{i=1}^{d-1}$ of ample divisors on $X$. Let $K=k(X)$ be the field of rational functions on $X$ and $\Omega=X^{(1)}$, equipped with the discrete $\sigma$-algebra. We have seen in $\$ 3.2 .4$ that $S=(K,(\Omega, \mathcal{A}, \nu), \phi)$ is an adelic curve, where the measure $\nu$ is defined as

$$
\forall Y \in \Omega=X^{(1)}, \quad \nu(\{Y\})=\operatorname{deg}\left(D_{1} \cdots D_{d-1} \cap[Y]\right)
$$

and the map $\phi: \Omega \rightarrow M_{K}$ sends $Y \in \Omega$ to $|\cdot|_{Y}=\mathrm{e}^{-\operatorname{ord}_{Y}(\cdot)}$.

Let $\mathcal{E}$ be a torsion-free (namely the canonical homomorphism $\mathcal{E} \rightarrow \mathcal{E}^{\vee \vee}$ is injective) coherent sheaf on $X$ and $E:=\mathcal{E} \otimes_{\mathcal{O}_{X}} K$. The latter is a finite-dimensional vector space over $K$. Moreover, for any $Y \in \Omega, \mathcal{E} \otimes \mathcal{O}_{X} \mathcal{O}_{X, Y}$ is a torsion-free module of finite type over the discrete valuation ring $\mathcal{O}_{X, Y}$ (the local ring of $X$ at the generic point of $Y)$, hence is a free $\mathcal{O}_{X, Y}$-module of finite rank. We define a norm $\|\cdot\|_{Y}$ on $E \otimes_{K} K_{Y}$ as follows

$$
\forall s \in E \otimes_{K} K_{Y}, \quad\|s\|_{Y}:=\inf \left\{|a|_{Y}: a \in K_{Y}^{\times}, a^{-1} s \in \mathcal{E} \otimes_{\mathcal{O}_{X}} \widehat{\mathcal{O}}_{X, Y}\right\},
$$

where $\widehat{\mathcal{O}}_{X, Y}$ is the completion of $\mathcal{O}_{X, Y}$, which identifies with the valuation ring of $K_{Y}$. This norm is clearly ultrametric. Thus we obtain a Hermitian norm family in $\mathcal{N}_{E}$, which we denote by $\xi_{\mathcal{E}}$. Note that the dual norm family $\xi_{\mathcal{E}}^{\vee}$ identifies with $\xi_{\mathcal{E}} \vee$ where $\mathcal{E}^{\vee}$ denotes the dual $\mathcal{O}_{X}$-module of $\mathcal{E}$. 
Since torsion-free coherent sheaves are locally free on codimension 1 , we obtain that, for any basis $\boldsymbol{e}$ of $E$, the norms $\|\cdot\|_{Y}$ and $\|\cdot\|_{e, Y}$ are identical for all but a finite number of $Y \in \Omega$. Therefore, the couple $\left(E, \xi_{\mathcal{E}}\right)$ is a strongly adelic vector bundle on $S$.

Hermitian vector bundles on an arithmetic curve. - Let $K$ be a number field and $\mathcal{O}_{K}$ be the ring of algebraic integers in $K$. Recall that a Hermitian vector bundle on $\operatorname{Spec} \mathcal{O}_{K}$ is by definition a couple $\left(\mathcal{E},\left\{\|\cdot\|_{\sigma}\right\}_{\sigma: K \rightarrow \mathbb{C}}\right)$, where $\mathcal{E}$ is a projective $\mathcal{O}_{K^{-}}$ module of finite rank, and for any embedding $\sigma: K \rightarrow \mathbb{C},\|\cdot\|_{\sigma}$ is a Hermitian norm on $\mathcal{E} \otimes_{\mathcal{O}_{K}, \sigma} \mathbb{C}$. We also require that the norms $\left\{\|\cdot\|_{\sigma}\right\}_{\sigma: K \rightarrow \mathbb{C}}$ are invariant under the complex conjugation, namely for $s_{1}, \ldots, s_{n}$ in $\mathcal{E}, \lambda_{1}, \ldots, \lambda_{n}$ in $\mathbb{C}$, and $\sigma: K \rightarrow \mathbb{C}$, one has

$$
\left\|\lambda_{1} \otimes s_{1}+\cdots+\lambda_{n} \otimes s_{n}\right\|_{\sigma}=\left\|\bar{\lambda}_{1} \otimes s_{1}+\cdots+\bar{\lambda}_{n} \otimes s_{n}\right\|_{\bar{\sigma}}
$$

We let $E:=\mathcal{E} \otimes \mathcal{O}_{K} K$.

Let $S=(K,(\Omega, \mathcal{A}, \nu), \phi)$ be the adelic curve associated with the number field $K$, as described in 3.2 .2 Recall that $\Omega$ is the set of all places of $K, \mathcal{A}$ is the discrete $\sigma$-algebra on $\Omega$ and $\nu(\{\omega\})=\left[K_{\omega}: \mathbb{Q}_{\omega}\right]$.

Recall that any finite place of $K$ is determined by a maximal ideal $\mathfrak{p}$ of $\mathcal{O}_{K}$. Let $\widehat{\mathcal{O}}_{K, \mathfrak{p}}$ be the completion of the local ring $\mathcal{O}_{K, \mathfrak{p}}$, which is also the valuation ring of $K_{\mathfrak{p}}$. We construct a norm $\|\cdot\|_{\mathfrak{p}}$ as follows

$$
\forall s \in E \otimes_{K} K_{\mathfrak{p}}, \quad\|s\|_{\mathfrak{p}}:=\inf \left\{|a|_{\mathfrak{p}}: a \in K_{\mathfrak{p}}^{\times}, a^{-1} s \in \mathcal{E} \otimes_{\mathcal{O}_{K}} \widehat{\mathcal{O}}_{K, \mathfrak{p}}\right\}
$$

Let $v$ be an Archimedean place of $K$. Then $v$ corresponds to an embedding $\sigma$ of $K$ into $\mathbb{C}$, we let $\|\cdot\|_{v}$ be the restriction of $\|\cdot\|_{\sigma}$ to $E \otimes_{K} K_{v}$. Note that the condition that the norms $\left\{\|\cdot\|_{\sigma}\right\}_{\sigma: K \rightarrow \mathbb{C}}$ are invariant under the complex conjugation ensures that the norm $\|\cdot\|_{v}$ does not depend on the choice of the embedding $\sigma: K \rightarrow \mathbb{C}$ corresponding to $v$. Thus we obtain a norm family $\xi=\left\{\|\cdot\|_{v}\right\}_{v \in \Omega}$ in $\mathcal{N}_{E}$. Since $\mathcal{E}$ is a locally free sheaf, we obtain that, for any basis $\boldsymbol{e}$ of $E$ over $K$, one has $\|\cdot\|_{v}=\|\cdot\|_{\boldsymbol{e}, v}$ for all but a finite number of $v$.

Ultrametrically normed vector space over a trivially valued field. - Let $K$ be an arbitrary field and $\Omega$ be the one point set $\{\omega\}$. Let $|\cdot|_{\omega}$ be the trivial absolute value on $K$. We then obtain an adelic curve $S$ by taking the discrete $\sigma$-algebra $\mathcal{A}$ on $\Omega$ and the measure $\nu$ on $(\Omega, \mathcal{A})$ such that $\nu(\{\omega\})=1$. Then any ultrametrically normed finite-dimensional vector space over $K$ is a strongly adelic vector bundle on $S$.

Remark 4.1.36. - Let $K$ be a number field and $E$ be a finite-dimensional vector space over $K$. In [60, §3], a structure of adelic vector bundle on $E$ has been defined as a norm family $\xi \in \mathcal{N}_{E}$ such that, for all but finitely many $\omega \in \Omega$ (where $\Omega$ denotes the set of all places of $K$ ), the norm $\xi_{\omega}$ is induced by a projective $\mathcal{O}_{K}$-module of finite type $\mathcal{E}$. Clearly such a structure of adelic vector bundle is a dominated norm family. We denote by $\mathcal{D}_{E}^{\circ}$ the subset of $\mathcal{D}_{E}$ consisting of all structures of adelic vector 
bundles in the sense of [60. We claim that $\mathcal{D}_{E}^{\circ}$ is dense in $\mathcal{D}_{E}$ (with respect to the metric $\operatorname{dist}(\cdot, \cdot)$ defined in Remark 4.1.20). In other words, given a dominated norm family $\xi$ on $E$, there exists a sequence $\left\{\xi_{n}\right\}_{n \in \mathbb{N}}$ in $\mathcal{D}_{E}^{\circ}$ which converges to $\xi$. In fact, we can choose an arbitrary element $\xi_{0}$ in $\mathcal{D}_{E}^{\circ}$. The main point is that, if we modify finitely many norms in the family $\xi_{0}$, we still obtain a norm family in $\mathcal{D}_{E}^{\circ}$. Since the local distance function $d\left(\xi, \xi_{0}\right)$ is $\nu$-dominated, we can construct a sequence $\left\{\Omega_{n}\right\}_{n \geqslant 1}$ of subsets of $\Omega$, such that $\Omega \backslash \Omega_{n}$ is a finite set and that

$$
\lim _{n \rightarrow+\infty} \bar{\int}_{\Omega} \mathbb{1}_{\Omega_{n}}(\omega) d_{\omega}\left(\xi, \xi_{0}\right) \nu(\mathrm{d} \omega)=0 .
$$

We then let $\xi_{n}$ be the norm family such that

$$
\xi_{n, \omega}= \begin{cases}\xi_{0, \omega}, & \omega \in \Omega_{n} \\ \xi_{\omega}, & \omega \in \Omega \backslash \Omega_{n} .\end{cases}
$$

Then the sequence $\left\{\xi_{n}\right\}_{n \in \mathbb{N}}$ is contained in $\mathcal{D}_{E}^{\circ}$ and converges to $\xi$ (see (4.17)). Combined with the completeness of the space $\mathcal{D}_{E}$ explained in Remark 4.1.20, we obtain that $\mathcal{D}_{E}$ is actually the completion of the metric space $\mathcal{D}_{E}^{\circ}$.

\subsection{Adelic divisors}

Let $S=(K,(\Omega, \mathcal{A}, \nu), \phi)$ be an adelic curve. We call adelic divisor on $S$ any element $\zeta$ in the vector space $L^{1}(\Omega, \mathcal{A}, \nu)$ (see Section A.5). For the reason of customs of arithmetic geometry, we use the notation $\widehat{\operatorname{Div}}_{\mathbb{R}}(S)$ to denote the vector space $L^{1}(\Omega, \mathcal{A}, \nu)$.

If $\zeta$ is an adelic divisor on $S$, we define its Arakelov degree as

$$
\operatorname{deg}(\zeta):=\int_{\Omega} \zeta(\omega) \nu(d \omega) \in \mathbb{R} .
$$

The function deg is a continuous linear form on $\widehat{\operatorname{Div}}_{\mathbb{R}}(S)$. If $a$ is an element of $K^{\times}$, we denote by $\widehat{(a)}$ the adelic divisor represented by the function which sends $\omega \in \Omega$ to $-\ln |a|_{\omega}$, called the adelic divisor associated with a. The map

$$
\widehat{(\cdot)}: K^{\times} \longrightarrow \widehat{\operatorname{Div}}_{\mathbb{R}}(S)
$$

is additive and hence extends to an $\mathbb{R}$-linear homomorphism from $K^{\times} \otimes_{\mathbb{Z}} \mathbb{R}$ to $\widehat{\operatorname{Div}}_{\mathbb{R}}(S)$, which we denote by $\widehat{(\cdot)}_{\mathbb{R}}$. The closure of the image of this map is denoted by $\widehat{\operatorname{PDiv}}_{\mathbb{R}}(S)$ and the elements of this vector space are called principal adelic divisors. We denote by $\widehat{\mathrm{Cl}}_{\mathbb{R}}(S)$ the quotient space $\widehat{\operatorname{Div}}_{\mathbb{R}}(S) / \widehat{\mathrm{PDiv}}_{\mathbb{R}}(S)$. Note that it forms actually a Banach space with respect to the quotient norm. Two adelic divisors lying in the same equivalent class in $\widehat{\mathrm{Cl}}_{\mathbb{R}}(S)$ are said to be $\mathbb{R}$-linearly equivalent.

We say that an adelic divisor $\zeta$ on $S$ is effective if $\zeta$ is $\nu$-almost everywhere nonnegative. Denote by $\widehat{\operatorname{Div}}_{\mathbb{R}}(S)_{+}$the cone of all effective adelic divisors on $S$. Clearly, if $\zeta$ is effective, then $\operatorname{deg}(\zeta) \geqslant 0$. 
Let $S^{\prime}=\left(K^{\prime},\left(\Omega^{\prime}, \mathcal{A}^{\prime}, \nu^{\prime}\right), \phi^{\prime}\right)$ be another adelic curve and $\alpha=\left(\alpha^{\#}, \alpha_{\#}, I_{\alpha}\right): S^{\prime} \rightarrow$ $S$ be a morphism of adelic curves (see Section 3.7). If $\zeta$ is an adelic divisor on $S$, which is represented by an element $f \in \mathscr{L}^{1}(\Omega, \mathcal{A}, \nu)$, we denote by $\alpha^{*}(\zeta)$ the adelic divisor on $S^{\prime}$ represented by the function $f \circ \alpha_{\#}$ (the equivalence class of $f \circ \alpha_{\#}$ does not depend on the choice of the representative $f$ since $\nu$ identifies with the direct image of $\nu^{\prime}$ by $\left.\alpha_{\#}\right)$. If $\zeta^{\prime}$ is an adelic divisor on $S^{\prime}$, we denote by $\alpha_{*}\left(\zeta^{\prime}\right)$ the adelic divisor $I_{\alpha}\left(\zeta^{\prime}\right)$ on $S$. Since $I_{\alpha}$ is a disintegration kernel of $\nu^{\prime}$ over $\nu$, one has $\alpha_{*}\left(\alpha^{*}(\zeta)\right)=\zeta$ for any adelic divisor $\zeta$ on $S$.

From now on we assume that $S$ is proper. Then one has $\operatorname{deg}(\zeta)=0$ if $\zeta$ is a principal adelic divisor. This is a direct consequence of the product formula and the fact that $\operatorname{deg}(\cdot)$ is a continuous linear operator. Therefore the $\mathbb{R}$-linear map $\operatorname{deg}(\cdot)$ induces by passing to quotient a continuous $\mathbb{R}$-linear map from $\widehat{\mathrm{Cl}}_{\mathbb{R}}(S)$ to $\mathbb{R}$ which sends any class $[\zeta]$ to $\operatorname{deg}(\zeta)$. We still denote this linear map by $\operatorname{deg}(\cdot)$ by abuse of notation.

\subsection{Arakelov degree and slopes}

The purpose of this section is to generalise the theory of Arakelov degree and slopes to the setting of adelic vector bundles over adelic curves. Throughout the section, let $S=(K,(\Omega, \mathcal{A}, \nu), \phi)$ be a proper adelic curve. For all subsections except the first one, we assume in addition that, either the $\sigma$-algebra $\mathcal{A}$ is discrete, or there exists a countable subfield $K_{0}$ of $K$ which is dense in all $K_{\omega}, \omega \in \Omega$.

4.3.1. Arakelov degree of adelic line bundles. - In this subsection, we fix an adelic vector bundle $\bar{E}=(E, \xi)$ on $S$.

Definition 4.3.1. - If $s$ is a non-zero vector in $E$, by Proposition4.1.31, the vector space $K s$ equipped with the induced norm family forms an adelic line bundle on $S$. In particular, the function $\ln \|s\|:(\omega \in \Omega) \mapsto \ln \|s\|_{\omega}$ is $\nu$-dominated and $\mathcal{A}$-measurable, and hence is $\nu$-integrable. The adelic divisor given by $(\omega \in \Omega) \mapsto-\ln \|s\|_{\omega}$ is denoted by $\widehat{\operatorname{div}}_{\xi}(s)$, which is called the adelic divisor of $s$ with respect to $\xi$. We define the Arakelov degree of $s$ with respect to $\xi$ as the Arakelov degree of $\widehat{\operatorname{div}}_{\xi}(s)$, that is,

$$
\widehat{\operatorname{deg}}_{\xi}(s):=\operatorname{deg}\left(\widehat{\operatorname{div}}_{\xi}(s)\right)=-\int_{\Omega} \ln \|s\|_{\omega} \nu(\mathrm{d} \omega) .
$$

Moreover, by the product formula (3.1) we obtain that, for any $a \in K^{\times}$, one has

$$
\widehat{\operatorname{deg}}_{\xi}(a s)=\widehat{\operatorname{deg}}_{\xi}(s) .
$$

Remark 4.3.2. - We assume $E=K^{n}$ and $\xi=\left\{\|\cdot\|_{\omega}\right\}_{\omega \in \Omega}$ is given by

$$
\left\|\left(a_{1}, \ldots, a_{n}\right)\right\|_{\omega}=\max \left\{\left|a_{1}\right|_{\omega}, \ldots,\left|a_{n}\right|_{\omega}\right\}
$$

for each $\omega \in \Omega$. Then $h_{S}(s)=-\widehat{\operatorname{deg}}_{\xi}(s)$ for all $s \in E \backslash\{0\}$ (for the definition of $h_{S}$, see Defintion 3.5.1). 
Definition 4.3.3. - Let $(E, \xi)$ be an adelic line bundle on $S$. We call Arakelov degree of $(E, \xi)$ the number $\widehat{\operatorname{deg}}_{\xi}(s)$, where $s$ is a non-zero element of $E$. Note that the relation (4.19) shows that the definition does not depend on the choice of the non-zero element $s$ in $E$. We denote the Arakelov degrees of $(E, \xi)$ by $\widehat{\operatorname{deg}}(E, \xi)$.

Proposition 4.3.4. - Let $(E, \xi)$ be an adelic line bundle on $S$. Then $\left(E^{\vee}, \xi^{\vee}\right)$ is also an adelic line bundle on $S$. Moreover, one has

$$
\widehat{\operatorname{deg}}\left(E^{\vee}, \xi^{\vee}\right)=-\widehat{\operatorname{deg}}(E, \xi) .
$$

Proof. - By Proposition 4.1.30, the couple $\left(E^{\vee}, \xi^{\vee}\right)$ is also an adelic line bundle, so that the Arakelov degree $\widehat{\operatorname{deg}}\left(E^{\vee}, \xi^{\vee}\right)$ is well defined. If $\alpha$ is a non-zero element of $E^{\vee}$ and $s$ is a non-zero element of $E$ then one has

$$
\forall \omega \in \Omega, \quad|\alpha(s)|_{\omega}=\|\alpha\|_{\omega, *} \cdot\|s\|_{\omega} .
$$

By the product formula

$$
\int_{\Omega} \ln |\alpha(s)|_{\omega} \nu(\mathrm{d} \omega)=0
$$

we obtain the equality (4.20).

Proposition 4.3.5. - Let $\left(E_{1}, \xi_{1}\right)$ and $\left(E_{2}, \xi_{2}\right)$ be adelic line bundles on $S$. Let $E=E_{1} \otimes_{K} E_{2}$ and $\xi=\xi_{1} \otimes_{\varepsilon} \xi_{2}$ (which is also equal to $\xi_{1} \otimes \xi_{2}$ and $\xi_{1} \otimes_{\pi} \xi_{2}$ ). Then one has

$$
\widehat{\operatorname{deg}}\left(E_{1} \otimes E_{2}, \xi_{1} \otimes \xi_{2}\right)=\widehat{\operatorname{deg}}\left(E_{1}, \xi_{1}\right)+\widehat{\operatorname{deg}}\left(E_{2}, \xi_{2}\right) .
$$

Proof. - Let $s_{1}$ and $s_{2}$ be non-zero elements of $E_{1}$ and $E_{2}$, respectively. For any $\omega \in \Omega$, one has

$$
\ln \left\|s_{1} \otimes s_{2}\right\|_{\omega}=\ln \left\|s_{1}\right\|_{\omega}+\ln \left\|s_{2}\right\|_{\omega} .
$$

By taking the integral with respect to $\nu$, we obtain the equality (4.21).

4.3.2. Arakelov degree of adelic vector bundles. - From now on and until the end of the section, we assume that, either the $\sigma$-algebra $\mathcal{A}$ is discrete, or there exists a countable subfield $K_{0}$ of $K$ which is dense in each $K_{\omega}$, where $\omega \in \Omega$.

Definition 4.3.6. - Let $(E, \xi)$ be an adelic vector bundle on $S$. By Proposition 4.1.32, $(\operatorname{det}(E), \operatorname{det}(\xi))$ is an adelic line bundle on $S$. We define the Arakelov degree of $(E, \xi)$ as

$$
\widehat{\operatorname{deg}}(E, \xi):=\widehat{\operatorname{deg}}(\operatorname{det}(E), \operatorname{det}(\xi)) .
$$

Note that, the Arakelov degree of the zero adelic vector bundle is 0. By Proposition 1.2.15, one has $\operatorname{det}(\xi)=\operatorname{det}\left(\xi^{\vee \vee}\right)$. Therefore

$$
\widehat{\operatorname{deg}}(E, \xi)=\widehat{\operatorname{deg}}\left(E, \xi^{\vee \vee}\right) .
$$


Proposition 4.3.7. - Let $(E, \xi)$ be a Hermitian adelic vector bundle on $S$. One has

$$
\widehat{\operatorname{deg}}(E, \xi)=-\widehat{\operatorname{deg}}\left(E^{\vee}, \xi^{\vee}\right) .
$$

Proof. - The determinant of $E^{\vee}$ is canonically isomorphic to the dual vector space of $\operatorname{det}(E)$, and the norm family $\operatorname{det}\left(\xi^{\vee}\right)$ identifies with $\operatorname{det}(\xi)^{\vee}$ under this isomorphism (see Proposition 1.2.47), provided that $\xi$ is Hermitian. Therefore, by Proposition 4.3.4 we obtain the equalities.

Definition 4.3.8. - Let $(E, \xi)$ be an adelic vector bundle on $S$. We denote by $\delta(\xi)$ the function on $\Omega$ sending $\omega \in \Omega$ to $\delta_{\omega}(\xi):=\delta\left(E_{K_{\omega}},\|\cdot\|_{\omega}\right.$ ) (see \$1.2.7). Recall that the function $\delta(\xi)$ is identically 1 on $\Omega \backslash \Omega_{\infty}$ (see Proposition 1.2.47), and takes value in $\left[1, r^{r / 2}\right]$ on $\Omega_{\infty}$ (see Proposition 1.2.46 and the inequalities (1.44) and (1.47)), where $r$ is the rank of $E$ over $K$. In particular, the function $\ln \delta(\xi)$ is $\nu$-dominated since it is bounded and vanishes outside a set of finite measure.

Similarly, we denote by $\Delta(\xi)$ the function on $\Omega$ sending $\omega \in \Omega$ to $\Delta_{\omega}(\xi):=$ $\Delta\left(E_{K_{\omega}},\|\cdot\|_{\omega}\right)$ (see Definition 1.2.41). This function is bounded from below by the constant function 1. Moreover, it identifies with the constant function 1 if $\xi$ is Hermitian.

Proposition 4.3.9. - Let $\left(E, \xi=\left\{\|\cdot\|_{\omega}\right\}_{\omega \in \Omega}\right)$ be an adelic vector bundle on $S$. Then the function $(\omega \in \Omega) \mapsto \delta_{\omega}(\xi)$ is $\mathcal{A}$-measurable and its logarithm is integrable with respect to $\nu$. It is a constant function of value 1 when $\xi$ is Hermitian. Moreover, the following relations hold

$$
\begin{aligned}
0 \leqslant \widehat{\operatorname{deg}}(E, \xi)+\widehat{\operatorname{deg}}\left(E^{\vee}, \xi^{\vee}\right) \\
\quad=\int_{\Omega} \ln \left(\delta_{\omega}(\xi)\right) \nu(d \omega) \leqslant \frac{1}{2} \operatorname{rk}(E) \ln (\operatorname{rk}(E)) \nu\left(\Omega_{\infty}\right) .
\end{aligned}
$$

In particular, one has $\widehat{\operatorname{deg}}(E, \xi)+\widehat{\operatorname{deg}}\left(E^{\vee}, \xi^{\vee}\right)=0$ if for any $\omega \in \Omega_{\infty}$ the norm $\|\cdot\|_{\omega}$ is induced by an inner product.

Proof. - By Proposition 4.1.32, we obtain that both couples $(\operatorname{det}(E), \operatorname{det}(\xi))$ and $\left(\operatorname{det}\left(E^{\vee}\right), \operatorname{det}\left(\xi^{\vee}\right)\right)$ are adelic line bundles on $S$. Let $\eta$ be a non-zero element in $\operatorname{det}(E)$ and $\eta^{\vee}$ be its dual element in $\operatorname{det}(\xi)$. By (1.38), one has

$$
\left(-\ln \|\eta\|_{\omega, \text { det }}\right)+\left(-\ln \left\|\eta^{\vee}\right\|_{\omega, *, \text { det }}\right)=\ln \delta_{\omega}(\xi) .
$$

Therefore the function $(\omega \in \Omega) \mapsto \delta_{\omega}(\xi)$ is $\mathcal{A}$-measurable. Moreover, by Proposition 1.2 .47 we obtain that $\delta_{\omega}(\xi)=1$ if $\omega \in \Omega \backslash \Omega_{\infty}$ or if $\omega \in \Omega_{\infty}$ and the norm $\|\cdot\|_{\omega}$ is induced by an inner product. Therefore (4.25) follows from the inequalities

$$
0 \leqslant \ln \delta(\xi) \leqslant \frac{1}{2} \operatorname{rk}(E) \ln (\operatorname{rk}(E)) \mathbb{1}_{\Omega_{\infty}},
$$

which also implies the $\nu$-integrability of $\ln \delta(\xi)$. 
Proposition 4.3.10. - Let $(E, \xi)$ be a strongly adelic vector bundle on $S$. The function $\ln \Delta(\xi)$ is $\nu$-dominated.

Proof. - Let $\boldsymbol{e}=\left\{e_{i}\right\}_{i=1}^{r}$ be a basis of $E$ over $K$. By Corollary 4.1.10, the local distance function $(\omega \in \Omega) \mapsto d_{\omega}\left(\xi, \xi_{\boldsymbol{e}}\right)$ is $\nu$-dominated. We write the norm families $\xi$ and $\xi_{\boldsymbol{e}}$ in the form of $\xi=\left\{\|\cdot\|_{\omega}\right\}_{\omega \in \Omega}$ and $\xi_{\boldsymbol{e}}=\left\{\|\cdot\|_{\boldsymbol{e}, \omega}\right\}_{\omega \in \Omega}$, respectively. Let $\omega \in \Omega$. If $\|\cdot\|_{h, \omega}$ is a norm on $E_{K_{\omega}}$ bounded from below by $\|\cdot\|_{\boldsymbol{e}, \omega}$, which is either ultrametric or induced by an inner product, then the norm $\mathrm{e}^{d_{\omega}\left(\xi, \xi_{e}\right)}\|\cdot\|_{h, \omega}$ is bounded from below by $\|\cdot\|_{\omega}$. This norm is also ultrametric or induced by an inner product. Therefore we obtain that

$$
\ln \Delta_{\omega}(\xi) \leqslant \ln \Delta_{\omega}\left(\xi_{\boldsymbol{e}}\right)+d_{\omega}\left(\xi, \xi_{\boldsymbol{e}}\right) \leqslant \frac{1}{2} r \ln (r) \mathbb{1}_{\Omega_{\infty}}(\omega)+d_{\omega}\left(\xi, \xi_{\boldsymbol{e}}\right),
$$

where the second inequality comes from (1.44). Since $\nu\left(\Omega_{\infty}\right)<+\infty$ (see Proposition 3.1.2), we obtain that the function $\ln \Delta(\xi)$ is $\nu$-dominated.

Definition 4.3.11. - Let $\bar{E}=(E, \xi)$ be an adelic vector bundle on $S$. We denote by $\delta(\bar{E})$ the integral $\int_{\Omega} \ln \left(\delta_{\omega}(\xi)\right) \nu(\mathrm{d} \omega)$, which is also equal to $\widehat{\operatorname{deg}}(E, \xi)+\widehat{\operatorname{deg}}\left(E^{\vee}, \xi^{\vee}\right)$ (see Proposition 4.3.9). We denote by $\Delta(\bar{E})$ the lower integral $\int_{\Omega} \ln \left(\Delta_{\omega}(\xi)\right) \nu(\mathrm{d} \omega)$, which takes value in $[0,+\infty]$. It is finite once the function $(\omega \in \Omega) \rightarrow d_{\omega}\left(\xi, \xi^{\vee \vee}\right)$ is $\nu$-dominated (see Proposition 4.3.10), namely $\bar{E}$ is a strongly adelic vector bundle.

Let $E, F$ and $G$ be vector spaces of finite rank over $K$, and $\xi_{E}, \xi_{F}$ and $\xi_{G}$ be norm families in $\mathcal{N}_{E}, \mathcal{N}_{F}$ and $\mathcal{N}_{G}$ respectively. We say that a diagram

$$
0 \longrightarrow\left(F, \xi_{F}\right) \stackrel{f}{\longrightarrow}\left(E, \xi_{E}\right) \stackrel{g}{\longrightarrow}\left(G, \xi_{G}\right) \longrightarrow 0
$$

is an exact sequence if

$$
0 \longrightarrow \text { P } \stackrel{f}{\longrightarrow} E \stackrel{g}{\longrightarrow} G \longrightarrow 0
$$

is an exact sequence of vector spaces over $K$ and if the norm family $\xi_{F}$ is the restriction of $\xi_{E}$ to $F$, and the norm family $\xi_{G}$ is the quotient of the norm family of $\xi_{E}$ on $G$ (see 4.1.1. page 214). Note that if $\xi_{E}$ is Hermitian, then both norm families $\xi_{F}$ and $\xi_{G}$ are Hermitian.

Proposition 4.3.12. - Let $(E, \xi)$ be an adelic vector bundle over $S$ and

$$
0=E_{0} \subseteq E_{1} \subseteq \ldots \subseteq E_{n}=E
$$

be a flag of vector subspaces of $E$. For any $i \in\{1, \ldots, n\}$, let $\xi_{i}$ be the restriction of $\xi$ to $E_{i}$ and let $\eta_{i}$ be the quotient norm family of $\xi_{i}$ on $E_{i} / E_{i-1}$. Then one has

$$
\sum_{i=1}^{n} \widehat{\operatorname{deg}}\left(E_{i} / E_{i-1}, \eta_{i}\right) \leqslant \widehat{\operatorname{deg}}(E, \xi)
$$


and

$$
\widehat{\operatorname{deg}}(E, \xi)-\Delta(E, \xi) \leqslant \sum_{i=1}^{n}\left(\widehat{\operatorname{deg}}\left(E_{i} / E_{i-1}, \eta_{i}\right)-\Delta\left(E_{i} / E_{i-1}, \eta_{i}\right)\right) .
$$

If in addition $\xi$ is ultrametric on $\Omega \backslash \Omega_{\infty}$, then

$$
\widehat{\operatorname{deg}}(E, \xi)-\delta(E, \xi) \leqslant \sum_{i=1}^{n}\left(\widehat{\operatorname{deg}}\left(E_{i} / E_{i-1}, \eta_{i}\right)-\delta\left(E_{i} / E_{i-1}, \eta_{i}\right)\right) .
$$

In particular, if $\xi$ is Hermitian, then one has

$$
\widehat{\operatorname{deg}}(E, \xi)=\sum_{i=1}^{n} \widehat{\operatorname{deg}}\left(E_{i} / E_{i-1}, \eta_{i}\right) .
$$

Proof. - For any $i \in\{1, \ldots, n\}$, we have an exact sequence

$$
0 \longrightarrow\left(E_{i-1}, \xi_{i-1}\right) \longrightarrow\left(E_{i}, \xi_{i}\right) \longrightarrow\left(E_{i} / E_{i-1}, \eta_{i}\right) \longrightarrow 0
$$

In particular, one has a canonical isomorphism (see [28] Chapter III, §7, no.7, Proposition 10)

$$
\operatorname{det}\left(E_{i}\right) \cong \operatorname{det}\left(E_{i-1}\right) \otimes \operatorname{det}\left(E_{i} / E_{i-1}\right) .
$$

For any $i \in\{1, \ldots, n\}$, we pick a non-zero element $\alpha_{i} \in \operatorname{det}\left(E_{i} / E_{i-1}\right)$ and let $\beta_{i}=$ $\alpha_{1} \otimes \cdots \otimes \alpha_{i}$, viewed as an element in $\operatorname{det}\left(E_{i}\right)$. By convention, let $\beta_{0}$ be the element $1 \in \operatorname{det}\left(E_{0}\right) \cong K$. By Corollary 1.1.68 and Proposition 1.2.43, one has

$$
\ln \left\|\alpha_{i}\right\|_{\omega}+\ln \left\|\beta_{i-1}\right\|_{\omega}+\ln \frac{\Delta_{\omega}\left(\xi_{i-1}\right) \Delta_{\omega}\left(\eta_{i}\right)}{\Delta_{\omega}\left(\xi_{i}\right)} \leqslant \ln \left\|\beta_{i}\right\|_{\omega} \leqslant \ln \left\|\alpha_{i}\right\|_{\omega}+\ln \left\|\beta_{i-1}\right\|_{\omega} .
$$

If $\xi$ is ultrametric on $\Omega \backslash \Omega_{\infty}$, then by Proposition [1.2.51, one has

$$
\ln \left\|\alpha_{i}\right\|_{\omega}+\ln \left\|\beta_{i-1}\right\|_{\omega}+\ln \frac{\delta_{\omega}\left(\xi_{i-1}\right) \delta_{\omega}\left(\eta_{i}\right)}{\delta_{\omega}\left(\xi_{i}\right)} \leqslant \ln \left\|\beta_{i}\right\|_{\omega}
$$

Taking the sum with respect to $i$, we obtain

$$
\sum_{i=1}^{n}-\ln \left\|\alpha_{i}\right\|_{\omega} \leqslant-\ln \left\|\beta_{n}\right\|_{\omega} \leqslant\left(\sum_{i=1}^{n}-\ln \left\|\alpha_{i}\right\|_{\omega}\right)+\ln \Delta_{\omega}(\xi)-\left(\sum_{i=1}^{n} \ln \Delta_{\omega}\left(\eta_{i}\right)\right)
$$

and, in the case where $\xi$ is ultrametric on $\Omega \backslash \Omega_{\infty}$,

$$
-\ln \left\|\beta_{n}\right\|_{\omega} \leqslant\left(\sum_{i=1}^{n}-\ln \left\|\alpha_{i}\right\|_{\omega}\right)+\ln \delta_{\omega}(\xi)-\left(\sum_{i=1}^{n} \ln \delta_{\omega}\left(\eta_{i}\right)\right) .
$$

By taking the integrals with respect to $\nu$, we obtain the inequalities (4.26) and (4.28). Moreover, (4.30) leads to

$$
-\ln \left\|\beta_{n}\right\|_{\omega}+\sum_{i=1}^{n} \ln \Delta_{\omega}\left(\eta_{i}\right) \leqslant\left(\sum_{i=1}^{n}-\ln \left\|\alpha_{i}\right\|_{\omega}\right)+\ln \Delta_{\omega}(\xi) .
$$

Taking the lower integrals, by Proposition A.4.5 and the inequality (A.4) we obtain (4.27). 
If $\xi$ is a Hermitian norm family, then each $\eta_{i}$ is a Hermitian norm family, $i \in$ $\{1, \ldots, n\}$. By Proposition 4.3.9, all functions $\ln \delta(\xi)$ and $\ln \delta\left(\eta_{i}\right)$ vanish. Therefore the equality (4.29) holds.

Proposition 4.3.13. - Let $(E, \xi)$ be an adelic vector bundle on $S$. If $L / K$ is an algebraic extension of fields, then one has $\operatorname{det}\left(\xi_{L}\right)=\operatorname{det}(\xi)_{L} . \quad$ In particular, $\widehat{\operatorname{deg}}(E, \xi)=\widehat{\operatorname{deg}}\left(E_{L}, \xi_{L}\right)$.

Proof. - The relation $\operatorname{det}\left(\xi_{L}\right)=(\operatorname{det} \xi)_{L}$ comes from (1) (for the non-Archimedean case) and (2) (for the Archimedean case) in Proposition 1.3.19.

Let $\alpha$ be a non-zero element of $\operatorname{det}(E)$. For any $x \in \Omega_{L}$ and $\omega=\pi_{L / K}(x)$, one has $\ln \|\alpha\|_{x}=\ln \|\alpha\|_{\omega}$. By taking the integral with respect to $\nu_{L}$, we obtain $\widehat{\operatorname{deg}}(E, \xi)=\widehat{\operatorname{deg}}\left(E_{L}, \xi_{L}\right)$.

Definition 4.3.14. - Let $\left(E, \xi_{E}\right)$ and $\left(F, \xi_{F}\right)$ be adelic vector bundles on $S$. Let $f: E \rightarrow F$ be a $K$-linear map. If $f$ is non-zero, we define local height function of $f$ as the real-valued function on $\Omega$ sending $\omega \in \Omega$ to $\ln \left\|f_{K_{\omega}}\right\|$, where $f_{K_{\omega}}$ is the $K_{\omega}$-linear map $E_{K_{\omega}} \rightarrow F_{K_{\omega}}$ induced by $f$, and $\left\|f_{K_{\omega}}\right\|$ is its operator norm.

Proposition 4.3.15. - Let $\left(E, \xi_{E}\right)$ and $\left(F, \xi_{F}\right)$ be adelic vector bundles, and $f$ : $E \rightarrow F$ be a $K$-linear map. The local height function of $f$ is $\mathcal{A}$-measurable. If $\left(E, \xi_{E}\right)$ and $\left(F, \xi_{F}\right)$ are strongly adelic vector bundles, then the local height function of $f$ is $\nu$-dominated.

Proof. - We first prove the measurability of the local height function. The statement is trivial when the $\sigma$-algebra $\mathcal{A}$ is discrete. In the following, we prove the measurability under the hypothesis that there exists a countable subfield $K_{0}$ of $K$ which is dense in each $K_{\omega}$. In this case there exists a countable sub- $K_{0}$-module $E_{0}$ of $E$ which generates $E$ as a vector space over $K$. For any $\omega \in \Omega$, viewed as a subset of $E_{K_{\omega}}, E_{0}$ is dense. Therefore one has

$$
\left\|f_{K_{\omega}}\right\|=\inf _{x \in E_{0} \backslash\{0\}} \frac{\|f(x)\|_{F, \omega}}{\|x\|_{E, \omega}}
$$

Since $\left(E, \xi_{E}\right)$ and $\left(F, \xi_{F}\right)$ are adelic vector bundles, for any $x \in E_{0}$, the functions $(\omega \in \Omega) \mapsto\|x\|_{E, \omega}$ and $(\omega \in \Omega) \mapsto\|f(x)\|_{F, \omega}$ are $\mathcal{A}$-measurable. Therefore the function $(\omega \in \Omega) \mapsto \ln \left\|f_{K_{\omega}}\right\|$ is $\mathcal{A}$-measurable.

We now proceed with the proof of the dominancy of the function. We consider $f$ as an element of $E^{\vee} \otimes F$ and equip this vector space with the norm family $\xi_{E}^{\vee} \otimes_{\varepsilon} \xi_{F}$ denoted by $\left\{\|\cdot\|_{\omega, \varepsilon}\right\}_{\omega \in \Omega}$. By Remark 1.1.53, the norm of

$$
f_{K_{\omega}}:\left(E_{K_{\omega}},\|\cdot\|_{E, \omega, * *}\right) \rightarrow\left(F_{K_{\omega}},\|\cdot\|_{F, \omega, * *}\right)
$$

identifies with $\|f\|_{\omega, \varepsilon}$. By Proposition 4.1.19 (3) and (5), the norm family $\xi_{E}^{\vee} \otimes_{\varepsilon} \xi_{F}$ is dominated. Hence the function $(\omega \in \Omega) \mapsto \ln \|f\|_{\omega, \varepsilon}$ is $\nu$-dominated. Note that one has

$$
\left|\ln \left\|f_{K_{\omega}}\right\|-\ln \|f\|_{\omega, \varepsilon}\right| \leqslant d_{\omega}\left(\xi_{E}, \xi_{E}^{\vee \vee}\right)+d_{\omega}\left(\xi_{F}, \xi_{F}^{\vee \vee}\right)
$$


where $\left\|f_{K_{\omega}}\right\|$ denotes the operator norm of $f_{K_{\omega}}:\left(E_{K_{\omega}},\|\cdot\|_{E, \omega}\right) \rightarrow\left(F_{K_{\omega}},\|\cdot\|_{F, \omega}\right)$. As the local distance functions $(\omega \in \Omega) \mapsto d_{\omega}\left(\xi_{E}, \xi_{E}^{\vee \vee}\right)$ and $(\omega \in \Omega) \mapsto d_{\omega}\left(\xi_{F}, \xi_{F}^{\vee \vee}\right)$ are $\nu$-dominated, we obtain that the function $(\omega \in \Omega) \mapsto \ln \left\|f_{K_{\omega}}\right\|$ is $\nu$-dominated. The proposition is thus proved.

Definition 4.3.16. - Let $\left(E, \xi_{E}\right)$ and $\left(F, \xi_{F}\right)$ be adelic vector bundles, and $f$ : $E \rightarrow F$ be a $K$-linear map. We define the height $h(f)$ of $f$ as the lower integral

$$
\int_{\Omega} \ln \left\|f_{K_{\omega}}\right\| \nu(\mathrm{d} \omega) .
$$

By Remark 1.1.53 in the case where $\xi_{E}$ and $\xi_{F}$ are ultrametric on $\Omega \backslash \Omega_{\infty}$, one has

$$
h(f)=-\widehat{\operatorname{deg}}_{\xi_{E}^{\vee} \otimes_{\varepsilon} \xi_{F}}(f) .
$$

Proposition 4.3.17. - Let $\left(E_{1}, \xi_{1}\right)$ and $\left(E_{2}, \xi_{2}\right)$ be adelic vector bundles on $S$ and $f: E_{1} \rightarrow E_{2}$ be a K-linear isomorphism. One has

$$
\widehat{\operatorname{deg}}\left(E_{1}, \xi_{1}\right)-\widehat{\operatorname{deg}}\left(E_{2}, \xi_{2}\right)=h(\operatorname{det}(f)) .
$$

In particular,

$$
\widehat{\operatorname{deg}}\left(E_{1}, \xi_{1}\right) \leqslant \widehat{\operatorname{deg}}\left(E_{2}, \xi_{2}\right)+r h(f) .
$$

Proof. - By definition one has

$$
\begin{aligned}
h(\operatorname{det}(f)) & =-\widehat{\operatorname{deg}}\left(\operatorname{det}\left(E_{1}\right)^{\vee} \otimes \operatorname{det}\left(E_{2}\right), \operatorname{det}\left(\xi_{1}\right)^{\vee} \otimes \operatorname{det}\left(\xi_{2}\right)\right) \\
& =-\widehat{\operatorname{deg}}\left(E_{1}, \xi_{1}\right)+\widehat{\operatorname{deg}}\left(E_{2}, \xi_{2}\right),
\end{aligned}
$$

where the second equality comes from Propositions 4.3 .4 and 4.3.5. Finally the inequality (4.32) is a consequence of (4.31) and Proposition 1.1.64

4.3.3. Arakelov degree of tensor adelic vector bundles. - Let $\bar{E}=\left(E, \xi_{E}\right)$ and $\bar{F}=\left(F, \xi_{F}\right)$ be adelic vector bundles on $S$. We denote by $\bar{E} \otimes_{\varepsilon, \pi} \bar{F}$ the couple $\left(E \otimes_{K} F, \xi_{E} \otimes_{\varepsilon, \pi} \xi_{F}\right)$, called the $\varepsilon, \pi$-tensor product of $\bar{E}$ and $\bar{F}$. By Proposition 4.1.32 (see also Remark 4.1.34), $\bar{E} \otimes_{\varepsilon, \pi} \bar{F}$ is an adelic vector bundle on $S$. If both $\bar{E}$ and $\bar{F}$ are Hermitian adelic vector bundles, we denote by $\bar{E} \otimes \bar{F}$ the couple $\left(E \otimes_{K} F, \xi_{E} \otimes \xi_{F}\right)$, called the orthogonal tensor product of $\bar{E}$ and $\bar{F}$. By Proposition 4.1.32, $\bar{E} \otimes \bar{F}$ is a Hermitian adelic vector bundle on $S$.

Proposition 4.3.18. - Let $\bar{E}=\left(E, \xi_{E}\right)$ and $\bar{F}=\left(F, \xi_{F}\right)$ be adelic vector bundles on $S$. One has

$$
\widehat{\operatorname{deg}}\left(\bar{E} \otimes_{\varepsilon, \pi} \bar{F}\right)=\operatorname{rk}(F) \widehat{\operatorname{deg}}(\bar{E})+\operatorname{rk}(E) \widehat{\operatorname{deg}}(\bar{F}) .
$$

If $\bar{E}$ and $\bar{F}$ are Hermitian adelic vector bundles, then one has

$$
\widehat{\operatorname{deg}}(\bar{E} \otimes \bar{F})=\operatorname{rk}(F) \widehat{\operatorname{deg}}(\bar{E})+\operatorname{rk}(E) \widehat{\operatorname{deg}}(\bar{F}) .
$$


Proof. - Let $n$ and $m$ be the ranks of $E$ and $F$ over $K$ respectively. By Propositions 1.1.69 and 1.2.39, under the canonical isomorphism

$$
\operatorname{det}(E)^{\otimes m} \otimes \operatorname{det}(F)^{\otimes n} \cong \operatorname{det}\left(E \otimes_{K} F\right),
$$

the norm family $\operatorname{det}\left(\xi_{E}\right)^{\otimes m} \otimes \operatorname{det}\left(\xi_{F}\right)^{\otimes n}$ identifies with $\operatorname{det}\left(\xi_{E} \otimes_{\varepsilon, \pi} \xi_{F}\right)$. Therefore the equality (4.34) results from Proposition 4.3.5.

The equality (4.35) can be proved in a similar way by using Propositions 1.2.63 and 1.2 .39 .

4.3.4. Positive degree. — Let $(E, \xi)$ be an adelic vector bundle on $S$. We define the positive degree of $(E, \xi)$ as

$$
\widehat{\operatorname{deg}}_{+}(E, \xi):=\sup _{F \subseteq E} \widehat{\operatorname{deg}}\left(F, \xi_{F}\right),
$$

where $F$ runs over the set of all vector subspaces of $E$, and $\xi_{F}$ denotes the restriction of $\xi$ to $F$. Clearly one has $\widehat{\operatorname{deg}}(E, \xi) \geqslant 0$.

Proposition 4.3.19. - Let $\left(E, \xi_{E}\right)$ be an adelic vector bundle on $S, F$ be a vector subspace of $E$ and $G$ be the quotient space of $E$ by $F$. Let $\xi_{F}$ be the restriction of $\xi_{E}$ to $F$ and $\xi_{G}$ be the quotient of $\xi_{E}$ on $G$. Then one has

$$
\widehat{\operatorname{deg}}_{+}\left(F, \xi_{F}\right) \leqslant \widehat{\operatorname{deg}}_{+}\left(E, \xi_{E}\right) \leqslant \widehat{\operatorname{deg}}_{+}\left(F, \xi_{F}\right)+\widehat{\operatorname{deg}}_{+}\left(G, \xi_{G}\right)+\Delta\left(E, \xi_{E}\right) .
$$

If in addition $\left(E, \xi_{E}\right)$ is ultrametric on $\Omega \backslash \Omega_{\infty}$, then

$$
\widehat{\operatorname{deg}}_{+}\left(F, \xi_{F}\right) \leqslant \widehat{\operatorname{deg}}_{+}\left(E, \xi_{E}\right) \leqslant \widehat{\operatorname{deg}}_{+}\left(F, \xi_{F}\right)+\widehat{\operatorname{deg}}_{+}\left(G, \xi_{G}\right)+\delta\left(E, \xi_{E}\right) .
$$

Proof. - The first inequality of (4.36) follows directly from the definition of positive degree. In the following, we prove the second inequality of (4.36).

Let $E_{1}$ be a vector subspace of $E, F_{1}=F \cap E_{1}$ and $G_{1}$ be the canonical image of $E_{1}$ in $G$. Then we obtain the following short exact sequence of adelic vector bundles:

$$
0 \longrightarrow\left(F_{1}, \xi_{F_{1}}\right) \longrightarrow\left(E_{1}, \xi_{E_{1}}\right) \longrightarrow\left(G_{1}, \xi_{G_{1}}\right) \longrightarrow 0
$$

where $\xi_{E_{1}}$ is the restriction of the norm family $\xi_{E}, \xi_{F_{1}}$ is the restriction of $\xi_{E_{1}}$ to $F_{1}$ and $\xi_{G_{1}}$ is the quotient norm family of $\xi_{E_{1}}$ on $G_{1}$. Note that the norm family $\xi_{F_{1}}$ coincides with the restricted norm family of $\xi_{F}$ induced by the inclusion map $F_{1} \rightarrow F$. Moreover, if we denote by $\xi_{G_{1}}^{\prime}$ the restricted norm family induced by the inclusion map $G_{1} \rightarrow G$, then the identity map $\left(G_{1}, \xi_{G_{1}}\right) \rightarrow\left(G_{1}, \xi_{G_{1}}^{\prime}\right)$ has norm $\leqslant 1$ (see Proposition 1.1.14 (2.b) on any $\omega \in \Omega$. In particular, by Proposition 4.3.17 one has

$$
\widehat{\operatorname{deg}}\left(G_{1}, \xi_{G_{1}}\right) \leqslant \widehat{\operatorname{deg}}\left(G_{1}, \xi_{G_{1}}^{\prime}\right) \leqslant \widehat{\operatorname{deg}}_{+}\left(G, \xi_{G}\right)
$$


Therefore, by Proposition 4.3.12, one has

$$
\begin{aligned}
\widehat{\operatorname{deg}}\left(E_{1}, \xi_{E_{1}}\right) & \leqslant \widehat{\operatorname{deg}}\left(F_{1}, \xi_{F_{1}}\right)+\widehat{\operatorname{deg}}\left(G_{1}, \xi_{G_{1}}\right)+\Delta\left(E_{1}, \xi_{E_{1}}\right) \\
& \leqslant \widehat{\operatorname{deg}_{+}}\left(F, \xi_{F}\right)+\widehat{\operatorname{deg}}_{+}\left(G, \xi_{G}\right)+\Delta\left(E_{1}, \xi_{E_{1}}\right) \\
& \leqslant \widehat{\operatorname{deg}}_{+}\left(F, \xi_{F}\right)+\widehat{\operatorname{deg}}_{+}\left(G, \xi_{G}\right)+\Delta\left(E, \xi_{E}\right),
\end{aligned}
$$

where the last inequality comes from Corollary 1.2.44. Similarly, by Propositions 4.3 .12 and 1.2.51, in the case where $\xi_{E}$ is ultrametric on $\Omega \backslash \Omega_{\infty}$ we have

$$
\widehat{\operatorname{deg}}\left(E_{1}, \xi_{E_{1}}\right) \leqslant \widehat{\operatorname{deg}}_{+}\left(F, \xi_{F}\right)+\widehat{\operatorname{deg}}_{+}\left(G, \xi_{G}\right)+\delta\left(E, \xi_{E}\right) .
$$

Since $E_{1} \subseteq E$ is arbitrary, we obtain (4.37).

Proposition 4.3.20. - Let $\left(E, \xi=\left\{\|\cdot\|_{\omega}\right\}_{\omega \in \Omega}\right)$ be an adelic vector bundle on $S$. Then we have the following:

(1) Let $(F, \eta)$ be an adelic vector bundle on $S$ such that $F$ is a vector subspace of $E$ and $\eta \geqslant \xi_{F}$ on $F$. Then $\widehat{\operatorname{deg}}_{+}(F, \eta) \leqslant \widehat{\operatorname{deg}}_{+}\left(F, \xi_{F}\right) \leqslant \widehat{\operatorname{deg}}_{+}(E, \xi)$.

(2) Let $\varphi$ be an integrable function on $\Omega$. Then

$$
\left\{\begin{array}{l}
\widehat{\operatorname{deg}}_{+}(E, \exp (-\varphi) \xi) \leqslant \widehat{\operatorname{deg}}_{+}(E, \xi)+\operatorname{dim}_{K}(E)\left|\int_{S} \varphi(\omega) \nu(\mathrm{d} \omega)\right| \\
\widehat{\operatorname{deg}}_{+}(E, \exp (\varphi) \xi) \geqslant \widehat{\operatorname{deg}}_{+}(E, \xi)-\operatorname{dim}_{K}(E)\left|\int_{S} \varphi(\omega) \nu(\mathrm{d} \omega)\right| .
\end{array}\right.
$$

Moreover, if $\int_{S} \varphi \nu(\mathrm{d} \omega) \geqslant 0$, then

$\widehat{\operatorname{deg}}_{+}(E, \xi) \leqslant \widehat{\operatorname{deg}}_{+}(E, \exp (-\varphi) \xi) \quad$ and $\quad \widehat{\operatorname{deg}}_{+}(E, \exp (\varphi) \xi) \leqslant \widehat{\operatorname{deg}}_{+}(E, \xi)$.

Proof. - (1) The inequality $\widehat{\operatorname{deg}}_{+}\left(F, \xi_{F}\right) \leqslant \widehat{\operatorname{deg}}_{+}(E, \xi)$ has been proved in Proposition 4.3.19. For $\epsilon>0$, there is a vector subspace $W$ of $F$ such that

$$
\widehat{\operatorname{deg}}_{+}(F, \eta)-\epsilon \leqslant \widehat{\operatorname{deg}}\left(W, \eta_{W}\right),
$$

so that

$$
\widehat{\operatorname{deg}}_{+}(F, \eta)-\varepsilon \leqslant \widehat{\operatorname{deg}}\left(W, \eta_{W}\right) \leqslant \widehat{\operatorname{deg}}\left(W, \xi_{W}\right) \leqslant \widehat{\operatorname{deg}}_{+}\left(F, \xi_{F}\right),
$$

as required.

(2) Let $F$ be a vector subspace of $E$ over $K$. Then

$$
\widehat{\operatorname{deg}}\left(F, \exp (-\varphi) \xi_{F}\right)=\widehat{\operatorname{deg}}\left(F, \xi_{F}\right)+\operatorname{dim}_{K}(F) \int_{\Omega} \varphi(\omega) \nu(\mathrm{d} \omega),
$$

so that if $\int_{S} \varphi \nu(d \omega) \geqslant 0$, then $\widehat{\operatorname{deg}}\left(F, \exp (-\varphi) \xi_{F}\right) \geqslant \widehat{\operatorname{deg}}\left(F, \xi_{F}\right)$, which leads to the third inequality. Moreover, by (4.38),

$$
\widehat{\operatorname{deg}}\left(F, \exp (-\varphi) \xi_{F}\right) \leqslant \widehat{\operatorname{deg}}_{+}(E, \exp (-\varphi) \xi)+\operatorname{dim}_{K}(E)\left|\int_{\Omega} \varphi(\omega) \nu(\mathrm{d} \omega)\right|
$$

and hence the first inequality follows. 
If we set $\xi^{\prime}=\exp (\varphi) \xi$, then the first and third inequalities imply the second and fourth inequalities, respectively.

4.3.5. Riemann-Roch formula. - Here we consider a Riemann-Roch formula of an adelic vector bundle on $S$.

Proposition 4.3.21. - Let $\bar{V}=(V, \xi)$ be an adelic vector bundle on $S$. Then one has

$$
0 \leqslant \widehat{\operatorname{deg}}(\bar{V})-\left(\widehat{\operatorname{deg}}_{+}\left(\bar{V}^{\vee \vee}\right)-\widehat{\operatorname{deg}}_{+}\left(\bar{V}^{\vee}\right)\right) \leqslant \delta(\bar{V})
$$

If $\xi$ is ultrametric on $\Omega \backslash \Omega_{\infty}$, then one has

$$
0 \leqslant \widehat{\operatorname{deg}}(\bar{V})-\left(\widehat{\operatorname{deg}}_{+}(\bar{V})-\widehat{\operatorname{deg}}_{+}\left(\bar{V}^{\vee}\right)\right) \leqslant \delta(\bar{V}) .
$$

Proof. - Let $\epsilon>0$. We choose a vector subspace $W$ of $V$ such that $\widehat{\operatorname{deg}}\left(W, \xi_{W}\right) \geqslant$ $\widehat{\operatorname{deg}}_{+}(\bar{V})-\epsilon$, where $\xi_{W}$ is the restriction of $\xi$ to $W$. Let $\xi^{\vee}$ be the dual norm family of $\xi$ on $V^{\vee}, \xi_{V / W}$ be the quotient norm family of $\xi$ on $V / W$, and $\xi_{V / W}^{\vee}$ be the dual norm family of $\xi_{V / W}$ on $(V / W)^{\vee}$. If we consider $(V / W)^{\vee}$ as a vector subspace of $V^{\vee}$, then $\xi_{V / W}^{\vee}$ identifies with the restriction of $\xi^{\vee}$ to $(V / W)^{\vee}$ by Proposition 1.1.20, so that

$$
\widehat{\operatorname{deg}}\left((V / W)^{\vee}, \xi_{V / W}^{\vee}\right) \leqslant \widehat{\operatorname{deg}}_{+}\left(V^{\vee}, \xi^{\vee}\right)
$$

On the other hand, one has

$$
\widehat{\operatorname{deg}}\left((V / W)^{\vee}, \xi_{V / W}^{\vee}\right)+\widehat{\operatorname{deg}}\left(V / W, \xi_{V / W}\right) \geqslant 0
$$

by Proposition 4.3.9 and

$$
\widehat{\operatorname{deg}}\left(W, \xi_{W}\right)+\widehat{\operatorname{deg}}\left(V / W, \xi_{V / W}\right) \leqslant \widehat{\operatorname{deg}}(V, \xi)
$$

by Proposition 4.3.12, Therefore,

$$
\begin{aligned}
\widehat{\operatorname{deg}}_{+}\left(V^{\vee}, \xi^{\vee}\right) & \geqslant \widehat{\operatorname{deg}}\left((V / W)^{\vee}, \xi_{V / W}^{\vee}\right) \geqslant-\widehat{\operatorname{deg}}\left(V / W, \xi_{V / W}\right) \\
& \geqslant \widehat{\operatorname{deg}}\left(W, \xi_{W}\right)-\widehat{\operatorname{deg}}(V, \xi) \geqslant \widehat{\operatorname{deg}_{+}}(V, \xi)-\widehat{\operatorname{deg}}(V, \xi)-\epsilon
\end{aligned}
$$

and hence

$$
\widehat{\operatorname{deg}}_{+}(\bar{V})-\widehat{\operatorname{deg}}_{+}\left(\bar{V}^{\vee}\right) \leqslant \widehat{\operatorname{deg}}(\bar{V}) .
$$

Replacing $\bar{V}$ by $\bar{V}^{\vee}$ in (4.41), we obtain

$$
\widehat{\operatorname{deg}}_{+}\left(\bar{V}^{\vee}\right)-\widehat{\operatorname{deg}}_{+}\left(\bar{V}^{\vee \vee}\right) \leqslant \widehat{\operatorname{deg}}\left(\bar{V}^{\vee}\right),
$$

which, by Proposition 4.3.9, implies the second inequality of (4.39). Replacing $\bar{V}$ by $\bar{V}^{\vee \vee}$ in (4.41), by the fact that $\|\cdot\|_{\omega, * *, *}=\|\cdot\|_{\omega, *}$ for any $\omega \in \Omega$ (see Proposition 1.2.14 (1) and the equality (4.23) we obtain

$$
\widehat{\operatorname{deg}}_{+}\left(\bar{V}^{\vee \vee}\right)-\widehat{\operatorname{deg}}_{+}\left(\bar{V}^{\vee}\right) \leqslant \widehat{\operatorname{deg}}(\bar{V}),
$$

which leads to the first inequality of (4.39). 
In the case where $\xi$ is ultrametric on $\Omega \backslash \Omega_{\infty}$, one has $\|\cdot\|_{\omega}=\|\cdot\|_{\omega, * *}$ for all $\omega \in \Omega$ by Proposition 1.1 .18 (Archimedean case) and Corollary 1.2.12 Thus (4.40) follows from (4.39).

4.3.6. Comparison of $\widehat{\operatorname{deg}}_{+}$and $\widehat{h}^{0}$ in the classic setting. - In this subsection, we compare $\widehat{\operatorname{deg}}_{+}$with the invariant $\widehat{h}^{0}$ in the classic settings of vector bundles on a regular projective curve and Hermitian vector bundles on an arithmetic curve.

4.3.6.1. Function field case. - Let $k$ be a field, $C$ be a regular projective curve over Spec $k$ and $K=k(C)$ be the field of rational functions on $C$. Let $\Omega$ be the set of all closed points of the curve $C$, equipped with the discrete $\sigma$-algebra $\mathcal{A}$ and the measure $\nu$ such that $\nu(\{x\})=[k(x): k]$ for any $x \in \Omega$. Let $\phi: \Omega \rightarrow M_{K}$ be the map sending $x$ to $|\cdot|_{x}=\mathrm{e}^{-\operatorname{ord}_{x}(\cdot)}$. We have seen in 3.2 .1 that $S=(K,(\Omega, \mathcal{A}, \nu), \phi)$ is an adelic curve.

Let $\mathcal{E}$ be a locally free $\mathcal{O}_{C}$-module of finite type and $E=\mathcal{E}_{K}:=\mathcal{E} \otimes_{\mathcal{O}_{C}} K$ be its generic fibre. For any $x \in \Omega$, let $\|\cdot\|_{x}$ be the norm on $E \otimes_{K} K_{\omega}$ defined as

$$
\forall s \in E \otimes_{K} K_{\omega}, \quad\|s\|_{x}=\inf \left\{|a|_{x}: a \in K_{\omega}^{\times}, a^{-1} s \in \widehat{\mathcal{O}}_{C, x},\right\}
$$

where $\widehat{\mathcal{O}}_{C, x}$ is the completion of $\mathcal{O}_{C, \omega}$, which identifies with the valuation ring of $K_{\omega}$. Then $\xi_{\mathcal{E}}=\left\{\|\cdot\|_{x}\right\}_{x \in \Omega}$ forms a Hermitian norm family on $E$ and $\left(E, \xi_{\mathcal{E}}\right)$ is an adelic vector bundle on $\Omega$. Note that the Arakelov degree of $\left(E, \xi_{\mathcal{E}}\right)$ identifies with the degree of the locally free $\mathcal{O}_{C}$-module $\mathcal{E}$, namely

$$
\widehat{\operatorname{deg}}\left(E, \xi_{\mathcal{E}}\right)=\operatorname{deg}\left(c_{1}(\mathcal{E}) \cap[C]\right) .
$$

The Harder-Narasimhan flag of $\left(E, \xi_{\mathcal{E}}\right)$ is also related to the classic construction of Harder-Narasimhan filtration of $\mathcal{E}$. In fact, there exists a unique flag of locally free $\mathcal{O}_{C}$-modules

$$
0=\mathcal{E}_{0} \subsetneq \mathcal{E}_{1} \subsetneq \ldots \subsetneq \mathcal{E}_{n}=\mathcal{E}
$$

such that each sub-quotient $\mathcal{E}_{i} / \mathcal{E}_{i-1}$ is a locally free $\mathcal{O}_{C}$-module which is semistable and that

$$
\mu\left(\mathcal{E}_{1} / \mathcal{E}_{0}\right)>\mu\left(\mathcal{E}_{2} / \mathcal{E}_{1}\right)>\ldots>\mu\left(\mathcal{E}_{n} / \mathcal{E}_{n-1}\right) .
$$

Then the Harder-Narasimhan flag of the Hermitian adelic vector bundle $\left(E, \xi_{\mathcal{E}}\right)$ is given by

$$
0=\mathcal{E}_{0, K} \subsetneq \mathcal{E}_{1, K} \subsetneq \ldots \subsetneq \mathcal{E}_{n, K}=E .
$$

The notion of positive degree for locally free $\mathcal{O}_{C}$-modules of finite rank has been proposed in [40] and compared with the rank (over $k$ ) of the vector space of global sections, by using the Riemann-Roch formula on curves. For any locally free $\mathcal{O}_{C^{-}}$ module of finite rank $\mathcal{E}$, we denote by $h^{0}(\mathcal{E})$ the rank of $H^{0}(C, \mathcal{E})$ over $k$.

Theorem 4.3.22. - Let $g(C)$ be the genus of $C$ relatively to the field $k$ (namely $g(C)=h^{0}\left(\omega_{C / k}\right), \omega_{C / k}$ being the relative dualising sheaf of $C$ over $\left.\operatorname{Spec} k\right)$. For any 
locally free $\mathcal{O}_{C}$-module of finite rank $\mathcal{E}$, one has

$$
\left|h^{0}(\mathcal{E})-\widehat{\operatorname{deg}}_{+}\left(E, \xi_{\mathcal{E}}\right)\right| \leqslant \operatorname{rk}_{K}(E) \max (g(C)-1,1) .
$$

We refer the readers to [40, §2] for a proof.

4.3.6.2. Number field case. — Let $K$ be a number field and $\Omega$ be the set of all places of $K$, equipped with the discrete $\sigma$-algebra $\mathcal{A}$. For each $\omega \in \Omega$, we denote by $|\cdot|_{\omega}$ the absolute value on $K$ extending either the usual absolute value on $\mathbb{Q}$ or one of the $p$-adic absolute values (with $|p|_{\omega}=p^{-1}$ in the latter case). We let $K_{\omega}$ (resp. $\mathbb{Q}_{\omega}$ ) be the completion of $K$ (resp. $\mathbb{Q})$ with respect to the absolute value $|\cdot|_{\omega}$. Let $\nu$ be the measure on the measurable space $(\Omega, \mathcal{A})$ such that $\nu(\{\omega\})=\left[K_{\omega}: \mathbb{Q}_{\omega}\right]$. Then $S=\left(K,(\Omega, \mathcal{A}, \nu), \phi: \omega \mapsto|\cdot|_{\omega}\right)$ forms an adelic curve.

Let $\mathcal{O}_{K}$ be the ring of algebraic integers in $K$. Recall that a Hermitian vector bundle on $\operatorname{Spec} \mathcal{O}_{K}$ is by definition the data $\overline{\mathcal{E}}=\left(\mathcal{E},\left\{\|\cdot\|_{\sigma}\right\}_{\sigma \in \Omega_{\infty}}\right)$ of a projective $\mathcal{O}_{K}$-module of finite type $\mathcal{E}$ together with a family of norms, where $\|\cdot\|_{\sigma}$ is a norm on the vector space $\mathcal{E} \otimes \mathcal{O}_{K} K_{\omega}$ which is induced by an inner product. Similarly to the function field case, the $\mathcal{O}_{K}$-module structure of $\mathcal{E}$ induces, for each non-Archimedean place $\mathfrak{p} \in \Omega \backslash \Omega_{\infty}$, a ultrametric norm $\|\cdot\|_{\mathfrak{p}}$ on $\mathcal{E} \otimes_{\mathcal{O}_{K}} K_{\mathfrak{p}}$ as follows :

$$
\forall s \in \mathcal{E} \otimes \mathcal{O}_{K} K_{\mathfrak{p}}, \quad\|s\|_{\mathfrak{p}}=\inf \left\{|a|_{\mathfrak{p}}: a \in K_{\mathfrak{p}}^{\times}, a^{-1} s \in \mathcal{O}_{\mathfrak{p}}\right\}
$$

where $\mathcal{O}_{\mathfrak{p}}$ is the valuation ring of $K_{\mathfrak{p}}$. Let $E$ be $\mathcal{E} \otimes_{\mathcal{O}_{K}} K$ and let $\xi_{\overline{\mathcal{E}}}$ be the norm family $\left\{\|\cdot\|_{\omega}\right\}_{\omega \in \Omega}$. Then the couple $\left(E, \xi_{\overline{\mathcal{E}}}\right)$ form an adelic vector bundle on $S$, which is said to be induced by $\overline{\mathcal{E}}$.

Recall that the space $\widehat{H}^{0}(\overline{\mathcal{E}})$ of "global sections" of $\overline{\mathcal{E}}$ is defined as

$$
\widehat{H}^{0}(\overline{\mathcal{E}}):=\left\{s \in \mathcal{E}: \sup _{\sigma \in \Omega_{\infty}}\|s\|_{\sigma} \leqslant 1\right\}=\left\{s \in E: \sup _{\omega \in \Omega}\|s\|_{\omega} \leqslant 1\right\} .
$$

This is a finite set. However it does not possess a natural vector space structure over a base field. We define (compare to the case of function field of a regular projective curve over a finite field) $\widehat{h}^{0}(\overline{\mathcal{E}})$ to be $\ln \operatorname{card}\left(\widehat{H}^{0}(\overline{\mathcal{E}})\right)$. The invariants $\widehat{h}^{0}(\overline{\mathcal{E}})$ and $\widehat{\operatorname{deg}}_{+}\left(E, \xi_{\overline{\mathcal{E}}}\right)$ have been compared in [40, $\S 6]$ (see also [35]). We denote by

- $B_{n}$ the unit ball in $\mathbb{R}^{n}$, where $n \in \mathbb{N}$,

$-\operatorname{vol}\left(B_{n}\right)$ the Lebesgue measure of $B_{n}$, which is equal to $\pi^{n / 2} / \Gamma(n / 2+1)$,

- $r_{1}(K)$ the number of real places of $K$,

$-r_{2}(K)$ the number of complex places of $K$.

Theorem 4.3.23. — Let $\overline{\mathcal{E}}$ be a Hermitian vector bundle on $\operatorname{Spec} \mathcal{O}_{K}$, and $\left(E, \xi_{\overline{\mathcal{C}}}\right)$ be the adelic vector bundle on $S$ induced by $\overline{\mathcal{E}}$. then

$$
\left|\widehat{h}^{0}(\overline{\mathcal{E}})-\widehat{\operatorname{deg}}_{+}\left(E, \xi_{\overline{\mathcal{E}}}\right)\right| \leqslant \operatorname{rk}_{K}(E) \ln \left|\mathfrak{D}_{K}\right|+C\left(K, \mathrm{rk}_{K}(E)\right),
$$

where $\mathfrak{D}_{K}$ is the discriminant of $K$ over $\mathbb{Q}$, and for any $n \in \mathbb{N}$,

$$
\begin{aligned}
C(K, n):=n[K: \mathbb{Q}] & \ln (3)+n\left(r_{1}(K)+r_{2}(K)\right) \ln (2)+\frac{n}{2} \ln \left|\mathfrak{D}_{K}\right|-r_{1} \ln \left(\operatorname{vol}\left(B_{n}\right) n !\right) \\
& -r_{2} \ln \left(V\left(B_{2 n}\right)(2 n) !\right)+\ln (([K: \mathbb{Q}] n) !)
\end{aligned}
$$


4.3.7. Slopes and slope inequalities. - Let $(E, \xi)$ be an adelic vector bundle on $S$ such that $E \neq\{0\}$. We define the slope of $(E, \xi)$ as

$$
\widehat{\mu}(E, \xi):=\frac{\widehat{\operatorname{deg}}(E, \xi)}{\operatorname{rk}(E)} .
$$

We define the maximal slope of $(E, \xi)$ as

$$
\widehat{\mu}_{\max }(E, \xi):=\sup _{0 \neq F \subseteq E} \widehat{\mu}\left(F, \xi_{F}\right),
$$

where $F$ runs over the set of all non-zero vector subspaces of $E$ and $\xi_{F}$ denotes the restriction of $\xi$ to $F$. Similarly, we define the minimal slope of $(E, \xi)$ as

$$
\widehat{\mu}_{\min }(E, \xi)=\inf _{E \rightarrow G \neq\{0\}} \widehat{\mu}_{\max }\left(G, \xi_{G}\right),
$$

where $G$ runs over the set of all non-zero quotient spaces of $E$, and $\xi_{G}$ denotes the quotient norm family of $\xi$. By definition one has $\widehat{\mu}_{\min }(\bar{E}) \leqslant \widehat{\mu}_{\max }(\bar{E})$ and $\widehat{\mu}(\bar{E}) \leqslant$ $\widehat{\mu}_{\max }(\bar{E})$ (note that here the vector space $E$ has been assumed to be non-zero). If $\bar{E}=\overline{\mathbf{0}}$ is the zero adelic vector bundle, we define by convention

$$
\widehat{\mu}_{\max }(\overline{\mathbf{0}}):=-\infty, \quad \widehat{\mu}(\overline{\mathbf{0}}):=0, \quad \widehat{\mu}_{\min }(\overline{\mathbf{0}}):=+\infty .
$$

Proposition 4.3.24. - Let $\bar{E}=\left(E, \xi_{E}\right)$ be a non-zero adelic vector bundle on $S$. One has

$$
\widehat{\mu}_{\min }(\bar{E})+\widehat{\mu}_{\max }\left(\bar{E}^{\vee}\right) \geqslant 0,
$$

provided that $\widehat{\mu}_{\max }\left(\bar{E}^{\vee}\right)<+\infty$ (we will show in Proposition 4.3 .29 that this condition is always satisfied, and, as a consequence of the current proposition, that one has $\left.\widehat{\mu}_{\min }(\bar{E})>-\infty\right)$.

Proof. - Let $G$ be a non-zero quotient vector space of $E$ and $\xi_{G}$ be the quotient norm family of $\xi_{E}$. Note that $G^{\vee}$ identifies with a vector subspace of $E^{\vee}$ and by Proposition 1.1.20, the dual norm family $\xi_{G}^{\vee}$ identifies with the restriction of $\xi_{E}^{\vee}$ to $G^{\vee}$. By Proposition 4.3.9, one has

$$
0 \leqslant \widehat{\mu}\left(G, \xi_{G}\right)+\widehat{\mu}\left(G^{\vee}, \xi_{G}^{\vee}\right) \leqslant \widehat{\mu}_{\max }\left(G, \xi_{G}\right)+\widehat{\mu}_{\max }\left(E^{\vee}, \xi_{E}^{\vee}\right) .
$$

Since $G$ is arbitrary, we obtain the inequality (4.46).

Classically in the setting of vector bundles over a regular projective curve or in that of Hermitian vector bundle over an arithmetic curve, the minimal slope is rather defined as the minimal value of slopes of quotient bundles. A direct analogue would replace $\widehat{\mu}_{\max }$ by $\widehat{\mu}$ in (4.45) for the definition of the minimal slope. However, it can be shown that the two definitions are actually equivalent.

Proposition 4.3.25. - Let $\bar{E}$ be a non-zero adelic vector bundle on $S$. One has

$$
\widehat{\mu}_{\min }(\bar{E})=\inf _{E \rightarrow G \neq\{0\}} \widehat{\mu}(\bar{G}),
$$


where $G$ runs over the set of non-zero quotient vector space of $E$, and in $\bar{G}$ we consider the quotient norm family of that in $\bar{E}$.

Proof. - For any non-zero quotient vector space $G$ of $E$, one has $\widehat{\mu}(\bar{G}) \leqslant \widehat{\mu}_{\text {max }}(\bar{G})$. Therefore the case where $\widehat{\mu}_{\min }(\bar{E})=-\infty$ is trivial. In the following, we assume that $\widehat{\mu}_{\min }(\bar{E})>-\infty$.

Let $\epsilon>0$. Among the non-zero quotient vector spaces of $E$ of maximal slope bounded from above by $\widehat{\mu}_{\min }(\bar{E})+\epsilon$, we choose a $G$ having the least rank. We claim that the maximal slope of $\bar{G}$ does not exceed $\widehat{\mu}(\bar{G})+\epsilon$ and we will prove this assertion by contradiction. Assume the contrary, namely $\widehat{\mu}_{\max }(\bar{G})>\widehat{\mu}(\bar{G})+\epsilon$. Let $G^{\prime}$ be a non-zero vector subspace of $G$ such that $\widehat{\mu}\left(\overline{G^{\prime}}\right)>\widehat{\mu}(\bar{G})$. We suppose in addition that $G^{\prime}$ is of maximal rank among the non-zero vector subspaces verifying this condition. Note that by definition $G^{\prime} \subsetneq G$. Let $H=G / G^{\prime}$. Since $G^{\prime}$ is not equal to $G, H$ is a non-zero quotient vector space of $E$. Let $H^{\prime \prime}$ be a non-zero vector subspace of $H$ and $G^{\prime \prime}$ be the inverse image of $H^{\prime \prime}$ by the quotient map $G \rightarrow H=G / G^{\prime}$. We have a short exact sequence

$$
0 \longrightarrow G^{\prime} \longrightarrow G^{\prime \prime} \longrightarrow H^{\prime \prime} \longrightarrow 0
$$

which leads to (by Proposition 4.3.12)

$$
\widehat{\operatorname{deg}}\left(\overline{G^{\prime}}\right)+\widehat{\operatorname{deg}}\left(\overline{H^{\prime \prime}}\right) \leqslant \widehat{\operatorname{deg}}\left(\overline{G^{\prime \prime}}\right) .
$$

Since $H^{\prime \prime}$ is non-zero, the rank of $G^{\prime \prime}$ is greater than that of $G^{\prime}$, and hence

$$
\widehat{\mu}\left(\overline{G^{\prime \prime}}\right) \leqslant \widehat{\mu}(\bar{G})<\widehat{\mu}\left(\overline{G^{\prime}}\right)
$$

by the maximality assumption of $\operatorname{rk}_{K}\left(G^{\prime}\right)$. Therefore (4.47) leads to $\widehat{\mu}\left(\overline{H^{\prime \prime}}\right) \leqslant \widehat{\mu}\left(\overline{G^{\prime}}\right)$. Since $H^{\prime \prime}$ is arbitrary, we obtain

$$
\widehat{\mu}_{\max }(\bar{H}) \leqslant \widehat{\mu}\left(\overline{G^{\prime}}\right) \leqslant \widehat{\mu}_{\max }(\bar{G}) \leqslant \widehat{\mu}_{\min }(\bar{E})+\epsilon .
$$

This contradicts the minimality assumption of $\operatorname{rk}_{K}(G)$, which proves the claim. Since $\epsilon$ is arbitrary, the proposition is proved.

Corollary 4.3.26. — Let $\bar{E}=\left(E, \xi_{E}\right)$ be a non-zero adelic vector bundle on $S$. One has

$$
\widehat{\mu}_{\min }\left(\bar{E}^{\vee}\right)+\widehat{\mu}_{\max }(\bar{E}) \leqslant \frac{1}{2} \ln \left(\operatorname{rk}_{K}(E)\right) \nu\left(\Omega_{\infty}\right) .
$$

Moreover, one has $\widehat{\mu}_{\min }\left(\bar{E}^{\vee}\right)+\widehat{\mu}_{\max }(\bar{E})=0$ if $\bar{E}$ is Hermitian.

Proof. - Let $F$ be a non-zero vector subspace of $E$ and $\xi_{F}=\left\{\|\cdot\|_{F, \omega}\right\}_{\omega \in \Omega}$ be the restriction of $\xi_{E}=\left\{\|\cdot\|_{E, \omega}\right\}_{\omega \in \Omega}$ to $F$. For any $\omega \in \Omega,\|\cdot\|_{F, \omega, *}$ is bounded from above by the quotient norm of $\|\cdot\|_{E, \omega, *}$ by the canonical surjective map $E_{K_{\omega}}^{\vee} \rightarrow F_{K_{\omega}}^{\vee}$. Hence 
by Proposition 4.3.25, one has $\widehat{\mu}\left(\bar{F}^{\vee}\right) \geqslant \widehat{\mu}_{\min }\left(\bar{E}^{\vee}\right)$. Therefore,

$$
\begin{aligned}
\widehat{\mu}(\bar{F})+\widehat{\mu}_{\min }\left(\bar{E}^{\vee}\right) & \leqslant \widehat{\mu}(\bar{F})+\widehat{\mu}\left(\bar{F}^{\vee}\right)=\frac{1}{\operatorname{rk}_{K}(F)} \int_{\Omega} \ln \left(\delta_{\omega}\left(\xi_{F}\right)\right) \nu(d \omega) \\
& \leqslant \frac{1}{2} \ln \left(\operatorname{rk}_{K}(F)\right) \nu\left(\Omega_{\infty}\right),
\end{aligned}
$$

where the equality follows from Proposition 4.3 .9 and the last inequality comes from Remark 1.2.55. Since $F$ is arbitrary, we obtain (4.48).

If $\bar{E}$ is Hermitian, then for any non-zero vector subspace $F$ of $E$ one has

$$
\widehat{\mu}(\bar{F})+\widehat{\mu}_{\min }\left(\bar{E}^{\vee}\right) \leqslant \widehat{\mu}(\bar{F})+\widehat{\mu}\left(\bar{F}^{\vee}\right)=0,
$$

which leads to $\widehat{\mu}_{\min }\left(\bar{E}^{\vee}\right)+\widehat{\mu}_{\max }(\bar{E}) \leqslant 0$. As we have seen that $\widehat{\mu}_{\min }\left(\bar{E}^{\vee}\right)+\widehat{\mu}_{\max }(\bar{E}) \geqslant$ 0 in Proposition 4.3 .24 (note that $\bar{E}^{\vee \vee}=\bar{E}$ when $\bar{E}$ is Hermitian), the equality $\widehat{\mu}_{\min }\left(\bar{E}^{\vee}\right)+\widehat{\mu}_{\max }(\bar{E})=0$ holds.

4.3.8. Finiteness of slopes. - Let $\bar{E}=(E, \xi)$ be an adelic vector bundle on $S$ such that $\xi$ is Hermitian. We assume that the vector space $E$ is non-zero and we denote by $\Theta(E)$ the set of all $K$-vector subspaces of $E$. For any $F \in \Theta(E)$, the vector subspace $F$ equipped with the restricted norm family forms a Hermitian adelic vector bundle on $S$ (see Proposition 4.1.32). We denote by $\bar{F}$ this Hermitian adelic vector bundle. Note that the rank and the Arakelov degree defines two functions on $\Theta(E)$, which satisfy the following relations: for any pair $\left(E_{1}, E_{2}\right)$ of elements in $\Theta(E)$, one has

$$
\begin{gathered}
\operatorname{rk}_{K}\left(E_{1} \cap E_{2}\right)+\operatorname{rk}_{K}\left(E_{1}+E_{2}\right)=\operatorname{rk}_{K}\left(E_{1}\right)+\operatorname{rk}_{K}\left(E_{2}\right), \\
\widehat{\operatorname{deg}}\left(\overline{E_{1} \cap E_{2}}\right)+\widehat{\operatorname{deg}}\left(\overline{E_{1}+E_{2}}\right) \geqslant \widehat{\operatorname{deg}}\left(\bar{E}_{1}\right)+\widehat{\operatorname{deg}}\left(\bar{E}_{2}\right),
\end{gathered}
$$

where the inequality (4.50) comes from Corollary 1.2.52.

Proposition 4.3.27. - Let E be a non-zero vector space of finite rank over $K$ and $\Theta(E)$ be the set of all vector subspaces of $E$. Assume given two functions $r: \Theta(E) \rightarrow$ $\mathbb{R}_{+}$and $d: \Theta(E) \rightarrow \mathbb{R}$ which verify the following conditions:

(1) the function $r(\cdot)$ takes value 0 on the zero vector subspace of $E$ and takes positive values on non-zero vector subspaces of $E$;

(2) for any couple $\left(E_{1}, E_{2}\right)$ of elements in $\Theta(E)$ one has

$$
r\left(E_{1} \cap E_{2}\right)+r\left(E_{1}+E_{2}\right)=r\left(E_{1}\right)+r\left(E_{2}\right)
$$

and

$$
d\left(E_{1} \cap E_{2}\right)+d\left(E_{1}+E_{2}\right) \geqslant d\left(E_{1}\right)+d\left(E_{2}\right)
$$

(3) $d(\{0\}) \leqslant 0$. 
Then the function $\mu=d / r$ attains its maximal value $\mu_{\max }$ on the set $\Theta^{*}(E)$ of all non-zero vector subspaces of $E$. Moreover, there exists a non-zero vector subspace $E_{\mathrm{des}}$ of $E$ such that $\mu\left(E_{\mathrm{des}}\right)=\mu_{\max }$ and which contains all non-zero vector subspaces $F$ of $E$ such that $\mu(F)=\mu_{\max }$.

Proof. - The first relation in the condition (2) implies that, if $L_{1}, \ldots, L_{n}$ are vector subspaces of rank 1 of $E$, which are linearly independent, then one has

$$
r\left(L_{1}+\cdots+L_{n}\right)=r\left(L_{1}\right)+\cdots+r\left(L_{n}\right) .
$$

In particular, if $L$ and $L^{\prime}$ are different vector subspaces of rank 1 in $E$ then one has $r(L)=r\left(L^{\prime}\right)$. In fact, let $s$ and $s^{\prime}$ be non-zero vectors of $L$ and $L^{\prime}$ respectively, and let $L^{\prime \prime}$ be the vector subspace of $E$ generated by $s+s^{\prime}$ (which is of rank 1 ). Then one has

$$
r(L)+r\left(L^{\prime \prime}\right)=r\left(L+L^{\prime}\right)=r\left(L^{\prime}\right)+r\left(L^{\prime \prime}\right) .
$$

Therefore the function $r(\cdot)$ is proportional to the rank function. Without loss of generality, we may assume that the function $r(\cdot)$ identifies with the rank function.

We prove the proposition by induction on the rank of the vector space $E$. The case where the $r(E)=1$ is trivial. In the following, we assume that $r(E) \geqslant 2$ and that the proposition has been proved for vector spaces of rank $<r(E)$. If for any non-zero vector subspace $F$ of $E$ one has $\mu(F) \leqslant \mu(E)$, then there is nothing to prove since $\mu(E)=\mu_{\max }$ and $E=E_{\text {des }}$. Otherwise there exists a non-zero vector subspace $E^{\prime}$ of $E$ such that $\mu\left(E^{\prime}\right)>\mu(E)$. Moreover, we can choose $E^{\prime}$ such that $r\left(E^{\prime}\right)$ is maximal (among the non-zero vector subspaces of $E$ having this property). Clearly one has $r\left(E^{\prime}\right)<r(E)$. Hence by the induction hypothesis the restriction of the function $\mu(\cdot)$ to $\Theta^{*}\left(E^{\prime}\right)$ attains its maximum, and among the non-zero vector subspaces of $E^{\prime}$ on which the restriction of the function $\mu(\cdot)$ to $\Theta\left(E^{\prime}\right)$ attains the maximal value there exists a greatest one $E_{\text {des }}^{\prime}$ with respect to the relation of inclusion. Let $E_{\text {des }}:=E_{\text {des }}^{\prime}$ be this vector space. We claim that $E_{\text {des }}$ verifies the properties announced in the proposition.

Let $F$ be a non-zero vector subspace of $E$. If $F \subseteq E^{\prime}$, then clearly one has $\mu(F) \leqslant \mu\left(E_{\mathrm{des}}\right)$. Otherwise the rank of $F \cap E^{\prime}$ is smaller than $r(F)$ and the rank of $F+E^{\prime}$ is greater than $r\left(E^{\prime}\right)$. Moreover, since $F \cap E^{\prime} \subseteq E^{\prime}$, one has (here we use the condition that $d(\{0\}) \leqslant 0$ to treat the case where $\left.F \cap E^{\prime}=\{0\}\right)$

$$
d\left(F \cap E^{\prime}\right) \leqslant \mu\left(E_{\mathrm{des}}\right) r\left(F \cap E^{\prime}\right) ;
$$

since $F+E^{\prime} \supsetneq E^{\prime}$, one has $\mu\left(F+E^{\prime}\right) \leqslant \mu(E)<\mu\left(E^{\prime}\right)$. Therefore

$$
\begin{aligned}
d\left(F \cap E^{\prime}\right)+d\left(F+E^{\prime}\right) & =\mu\left(F \cap E^{\prime}\right) r\left(F \cap E^{\prime}\right)+\mu\left(F+E^{\prime}\right) r\left(F+E^{\prime}\right) \\
& <\mu\left(E_{\text {des }}\right) r\left(F \cap E^{\prime}\right)+\mu\left(E^{\prime}\right) r\left(F+E^{\prime}\right) .
\end{aligned}
$$

Combining this relation with the inequality in the condition (2) of the proposition, we obtain

$$
\mu\left(E_{\mathrm{des}}\right) r\left(F \cap E^{\prime}\right)+\mu\left(E^{\prime}\right) r\left(F+E^{\prime}\right)>\mu\left(E^{\prime}\right) r\left(E^{\prime}\right)+\mu(F) r(F) .
$$


By the equality in the condition (2), we deduce

$$
\mu(F) r(F)<\mu\left(E_{\mathrm{des}}\right) r\left(F \cap E^{\prime}\right)+\mu\left(E^{\prime}\right)\left(r(F)-r\left(F \cap E^{\prime}\right)\right) \leqslant \mu\left(E_{\mathrm{des}}\right) r(F) .
$$

Therefore, the function $\mu(\cdot)$ attains its maximal value $\mu_{\max }$ at $E_{\mathrm{des}}$. Moreover, if $F$ is a non-zero vector subspace of $E$ such that $\mu(F)=\mu\left(E_{\mathrm{des}}\right)$, then one should have $F \subseteq E^{\prime}$, and hence $F \subseteq E_{\text {des }}$ by the induction hypothesis. The proposition is thus proved.

Definition 4.3.28. - Let $\bar{E}$ be a non-zero Hermitian adelic vector bundle on $S$. We can apply the above proposition to the functions of rank and of Arakelov degree to obtain the existence of a (unique) non-zero vector subspace $E_{\text {des }}$ of $E$ such that

$$
\widehat{\mu}\left(\bar{E}_{\mathrm{des}}\right)=\widehat{\mu}_{\max }(\bar{E})=\sup _{0 \neq F \in \Theta(E)} \widehat{\mu}(\bar{F})
$$

and containing all non-zero vector subspaces of $E$ on which the function $\widehat{\mu}$ attains the maximal slope of $\bar{E}$. The vector subspace $E_{\text {des }}$ is called the destabilising vector subspace of the Hermitian adelic vector bundle $\bar{E}$. If $\bar{E}_{\text {des }}=\bar{E}$, we say that the Hermitian adelic vector bundle $\bar{E}$ is semistable. In particular, for any non-zero Hermitian adelic vector bundle $\bar{E}$ on $S$, the Hermitian adelic vector bundle $\bar{E}_{\text {des }}$ is always semistable.

Proposition 4.3.29. - Let $(E, \xi)$ be a non-zero adelic vector bundle on $S$. Then one has $\widehat{\mu}_{\max }(E, \xi)<+\infty$ and $\widehat{\mu}_{\min }(E, \xi)>-\infty$.

Proof. - Let $r$ be the rank of $E$ over $K$. We first prove the inequality $\widehat{\mu}_{\max }(E, \xi)<$ $+\infty$ in the particular case where $\xi$ is ultrametric on $\Omega \backslash \Omega_{\infty}$. By Theorem 4.1.26 there exists a measurable Hermitian norm family $\xi^{H}$ on $E$ such that

$$
\forall \omega \in \Omega, \quad d_{\omega}\left(\xi, \xi^{H}\right) \leqslant \frac{1}{2} \ln (r+1) \mathbb{1}_{\Omega_{\infty}}(\omega) .
$$

Therefore, for any non-zero vector subspace $F$ of $E$ one has

$$
\left|\widehat{\mu}\left(F, \xi_{F}\right)-\widehat{\mu}\left(F, \xi_{F}^{H}\right)\right| \leqslant \frac{1}{2} \ln (r+1) \nu\left(\Omega_{\infty}\right) .
$$

Moreover, by Proposition 4.3.27 the maximal slope $\widehat{\mu}_{\max }\left(E, \xi^{H}\right)$ is finite. Therefore one has $\widehat{\mu}_{\max }(E, \xi)<+\infty$.

We now proceed with the proof of the relation $\widehat{\mu}_{\max }(E, \xi)<+\infty$ in the general case. We write $\xi$ in the form $\left\{\|\cdot\|_{\omega}\right\}_{\omega \in \Omega}$. Note that for any $\omega \in \Omega$ one has $\|\cdot\|_{\omega, * *} \leqslant\|\cdot\|_{\omega}$. Therefore $\widehat{\mu}_{\max }\left(E, \xi^{\vee \vee}\right) \geqslant \widehat{\mu}_{\max }(E, \xi)$. Note that the norm family $\xi^{\vee \vee}$ is ultrametric on $\Omega \backslash \Omega_{\infty}$, and $\left(E, \xi^{\vee \vee}\right)$ is an adelic vector bundle (see Proposition 4.1.32 (1)). By the particular case proved above, one has $\widehat{\mu}_{\max }\left(E, \xi^{\vee \vee}\right)<+\infty$. Thus we obtain $\widehat{\mu}_{\max }(E, \xi)<+\infty$.

Applying the above proved result to $\left(E^{\vee}, \xi^{\vee}\right)$ we obtain $\widehat{\mu}_{\max }\left(E^{\vee}, \xi^{\vee}\right)<+\infty$. Therefore, by Proposition 4.3.24 we obtain $\widehat{\mu}_{\min }(E, \xi)>-\infty$. 
4.3.9. Some slope estimates. - The following proposition is a natural generalisation of the slope inequalities to the setting of adelic curves. We refer the readers to [17, §4.1] for this theory in the classic setting of Hermitian vector bundles over an algebraic integer ring.

Proposition 4.3.30. - Let $\left(E_{1}, \xi_{1}\right)$ and $\left(E_{2}, \xi_{2}\right)$ be adelic vector bundles on $S$, and $f: E_{1} \rightarrow E_{2}$ be a K-linear map.

(1) If $f$ is injective, then one has $\widehat{\mu}_{\max }\left(E_{1}, \xi_{1}\right) \leqslant \widehat{\mu}_{\max }\left(E_{2}, \xi_{2}\right)+h(f)$.

(2) If $f$ is surjective, then one has $\widehat{\mu}_{\min }\left(E_{1}, \xi_{1}\right) \leqslant \widehat{\mu}_{\min }\left(E_{2}, \xi_{2}\right)+h(f)$.

(3) If $f$ is non-zero, then one has $\widehat{\mu}_{\min }\left(E_{1}, \xi_{1}\right) \leqslant \widehat{\mu}_{\max }\left(E_{2}, \xi_{2}\right)+h(f)$.

Proof. - (1) The assertion is trivial if $f$ is the zero map since in this case $E_{1}=\{0\}$ and $\widehat{\mu}_{\max }\left(E_{1}, \xi_{1}\right)=-\infty$ by convention. In the following, we assume that the linear map $f$ is non-zero. Let $F_{1}$ be a non-zero vector subspace of $E_{1}$ and $F_{2}$ be its image in $E_{2}$. Let $g: F_{1} \rightarrow F_{2}$ be the restriction of $f$ to $F_{1}$. It is an isomorphism of vector spaces. Moreover, if we equip $F_{1}$ and $F_{2}$ with induced norm families, by Proposition 4.3.17 one has

$$
\widehat{\mu}\left(F_{1}, \xi_{F_{1}}\right) \leqslant \widehat{\mu}\left(F_{2}, \xi_{F_{2}}\right)+h(g) \leqslant \widehat{\mu}_{\max }\left(E_{2}, \xi_{2}\right)+h(f),
$$

where $\xi_{F_{1}}$ and $\xi_{F_{2}}$ are restrictions of $\xi_{1}$ and $\xi_{2}$ to $F_{1}$ and $F_{2}$, respectively. Since $F_{1}$ is arbitrary, we obtain the inequality $\widehat{\mu}_{\max }\left(E_{1}, \xi_{1}\right) \leqslant \widehat{\mu}_{\max }\left(E_{2}, \xi_{2}\right)+h(f)$.

(2) The assertion is trivial if $f$ is the zero map since in this case $E_{2}=\{0\}$ and $\widehat{\mu}_{\min }\left(E_{2}, \xi_{2}\right)=+\infty$ by convention. In the following, we assume that the linear map $f$ is non-zero. Let $G_{2}$ be a non-zero quotient vector space of $E_{2}$ and $\widetilde{f}$ be the composition of $f$ with the quotient map $E_{2} \rightarrow G_{2}$. Let $F_{1}$ be the kernel of $\widetilde{f}, G_{1}$ be the quotient space $E_{1} / F_{1}$, and $g: G_{1} \rightarrow G_{2}$ be the $K$-linear map induced by $\tilde{f}$. It is a $K$-linear isomorphism. By (1), one has

$$
\widehat{\mu}_{\min }\left(E_{1}, \xi_{1}\right) \leqslant \widehat{\mu}_{\max }\left(G_{1}, \xi_{G_{1}}\right) \leqslant \widehat{\mu}_{\max }\left(G_{2}, \xi_{G_{2}}\right)+h(g) \leqslant \widehat{\mu}_{\max }\left(G_{2}, \xi_{G_{2}}\right)+h(f),
$$

where $\xi_{G_{1}}$ and $\xi_{G_{2}}$ are the quotient norm family of $\xi_{1}$ and $\xi_{2}$, respectively. Since $G_{2}$ is arbitrary, one obtains $\widehat{\mu}_{\min }\left(E_{1}, \xi_{1}\right) \leqslant \widehat{\mu}_{\min }\left(E_{2}, \xi_{2}\right)+h(f)$.

(3) Let $G$ be the image of $E_{1}$ by $f$, which is non-zero since $f$ is non-zero. We equip $G$ with the restriction $\xi_{G}$ of $\xi_{2}$ to $G$. As $G$ is non-zero, one has $\widehat{\mu}_{\min }\left(G, \xi_{G}\right) \leqslant$ $\widehat{\mu}_{\max }\left(G, \xi_{G}\right) \leqslant \widehat{\mu}_{\max }\left(E_{2}, \xi_{2}\right)$. By $(2)$, one has $\widehat{\mu}_{\min }\left(E_{1}, \xi_{1}\right) \leqslant \widehat{\mu}_{\min }\left(G, \xi_{G}\right)+h(f)$. Hence $\widehat{\mu}_{\min }\left(E_{1}, \xi_{1}\right) \leqslant \widehat{\mu}_{\max }\left(E_{2}, \xi_{2}\right)+h(f)$.

Proposition 4.3.31. - Let $\left(E^{\prime}, \xi^{\prime}\right)$ and $(E, \xi)$ be adelic vector bundles on $S$, and $f: E^{\prime} \rightarrow E$ be an injective $K$-linear map. Let $E^{\prime \prime}$ be the quotient vector space $E / f\left(E^{\prime}\right)$ and $\xi^{\prime \prime}$ be the quotient norm family of $\xi$ on $E^{\prime \prime}$. Then the following inequality holds

$$
\widehat{\mu}_{\min }(E, \xi) \geqslant \min \left(\widehat{\mu}_{\min }\left(E^{\prime}, \xi^{\prime}\right)-h(f), \widehat{\mu}_{\min }\left(E^{\prime \prime}, \xi^{\prime \prime}\right)\right) \text {. }
$$

If in addition $\widehat{\mu}_{\min }\left(E^{\prime}, \xi^{\prime}\right)-h(f) \geqslant \widehat{\mu}_{\min }\left(E^{\prime \prime}, \xi^{\prime \prime}\right)$, then the equality $\widehat{\mu}_{\min }(E, \xi)=$ $\widehat{\mu}_{\min }\left(E^{\prime \prime}, \xi^{\prime \prime}\right)$ holds. 
Proof. - The inequality (4.51) is trivial if $E=\{0\}$ since in this case one has $\widehat{\mu}_{\min }(E, \xi)=+\infty$ by convention. Moreover, one has $E^{\prime \prime}=\{0\}$ since $E^{\prime \prime}$ is a quotient vector space of $E$. Therefore the equality $\widehat{\mu}_{\min }(E, \xi)=\widehat{\mu}_{\min }\left(E^{\prime \prime}, \xi^{\prime \prime}\right)$ holds.

In the following, we assume that $E \neq\{0\}$. Let $Q$ be a quotient vector space of $E$ and $\xi_{Q}$ be the quotient norm family of $\xi$. Let $\pi: E \rightarrow Q$ be the quotient map. If the composed map $\pi f$ is non-zero, by Proposition 4.3.30 (3) one has

$$
\widehat{\mu}_{\min }\left(E^{\prime}, \xi^{\prime}\right) \leqslant \widehat{\mu}_{\max }\left(Q, \xi_{Q}\right)+h(\pi f) \leqslant \widehat{\mu}_{\max }\left(Q, \xi_{Q}\right)+h(f) .
$$

Otherwise the quotient map $\pi: E \rightarrow Q$ factorises through $E^{\prime \prime}$ and by Proposition 4.3.30 (2) one has

$$
\widehat{\mu}_{\min }\left(E^{\prime \prime}, \xi^{\prime \prime}\right) \leqslant \widehat{\mu}_{\min }\left(Q, \xi_{Q}\right) \leqslant \widehat{\mu}_{\max }\left(Q, \xi_{Q}\right) .
$$

Since $Q$ is arbitrary, the inequality (4.51) is true.

If $\widehat{\mu}_{\min }\left(E^{\prime}, \xi^{\prime}\right)-h(f) \geqslant \widehat{\mu}_{\min }\left(E^{\prime \prime}, \xi^{\prime \prime}\right)$, then (4.51) implies $\widehat{\mu}_{\min }(E, \xi) \geqslant \widehat{\mu}_{\min }\left(E^{\prime \prime}, \xi^{\prime \prime}\right)$. Moreover, by Proposition 4.3.30 $(2)$ one has $\widehat{\mu}_{\min }(E, \xi) \leqslant \widehat{\mu}_{\min }\left(E^{\prime \prime}, \xi^{\prime \prime}\right)$. Hence the equality $\widehat{\mu}_{\min }(E, \xi)=\widehat{\mu}_{\min }\left(E^{\prime \prime}, \xi^{\prime \prime}\right)$ holds.

Proposition 4.3.32. - Let $\left\{\left(E_{i}, \xi_{E_{i}}\right)\right\}_{i=1}^{n}$ be a family of adelic vector bundles, where $n \in \mathbb{N}, n \geqslant 2$. Assume that

$$
E_{0}:=\{0\} \stackrel{\alpha_{1}}{\longrightarrow} E_{1} \stackrel{\alpha_{2}}{\longrightarrow} E_{2} \stackrel{\alpha_{3}}{\longrightarrow} E_{3} \longrightarrow \cdots \stackrel{\alpha_{n-1}}{\longrightarrow} E_{n-1} \stackrel{\alpha_{n}}{\longrightarrow} E_{n}
$$

is a sequence of injective $K$-linear maps. For any $i \in\{1, \ldots, n\}$, let $\beta_{i}=$ $\alpha_{n} \circ \cdots \circ \alpha_{i+1}$, where by convention $\beta_{n}:=\operatorname{Id}_{E_{n}}$. For any $i \in\{1, \ldots, n\}$, let $Q_{i}$ be the quotient space $E_{i} / \alpha_{i}\left(E_{i-1}\right)$ and $\xi_{Q_{i}}$ be the quotient norm family of $\xi_{E_{i}}$. Then one has

$$
\widehat{\mu}_{\min }\left(E_{n}, \xi_{E_{n}}\right) \geqslant \min _{i \in\{1, \ldots, n\}}\left(\widehat{\mu}_{\min }\left(Q_{i}, \xi_{Q_{i}}\right)-h\left(\beta_{i}\right)\right)
$$

Proof. - The case where $n=2$ was proved in Proposition 4.3.31, In the following, we assume that $n \geqslant 3$ and that the proposition has been proved for the case of $n-1$ adelic vector bundles. For any $i \in\{2, \ldots, n\}$, let $E_{i}^{\prime}$ be the cokernel of the composed linear map

$$
\alpha_{i} \circ \cdots \circ \alpha_{2}: E_{1} \longrightarrow E_{i}
$$

and let $\xi_{E_{i}^{\prime}}$ be the quotient norm family of $\xi_{E_{i}}$ on $E_{i}^{\prime}$. Let $E_{1}^{\prime}=\{0\}$. Then the sequence (4.52) induces a sequence of $K$-linear maps

$$
E_{1}^{\prime}:=\{0\} \stackrel{\alpha_{2}^{\prime}}{\longrightarrow} E_{2}^{\prime} \stackrel{\alpha_{3}^{\prime}}{\longrightarrow} E_{3}^{\prime} \longrightarrow \cdots \stackrel{\alpha_{n-1}^{\prime}}{\longrightarrow} E_{n-1}^{\prime} \stackrel{\alpha_{n}^{\prime}}{\longrightarrow} E_{n}^{\prime} .
$$

For any $i \in\{2, \ldots, n\}$, let $\beta_{i}^{\prime}=\alpha_{n}^{\prime} \circ \cdots \circ \alpha_{i+1}^{\prime}$, where by convention $\beta_{n}^{\prime}=\operatorname{Id}_{E_{n}^{\prime}}$. For any $i \in\{2, \ldots, n\}$, let $Q_{i}^{\prime}$ be the quotient space $E_{i}^{\prime} / \alpha_{i}^{\prime}\left(E_{i-1}^{\prime}\right)$ and $\xi_{Q_{i}^{\prime}}$ be the quotient norm family of $\xi_{E_{i}^{\prime}}$. Note that $Q_{i}^{\prime}$ is canonically isomorphic to $Q_{i}$, and under the canonical isomorphism $Q_{i} \cong Q_{i}^{\prime}$, the norm family $\xi_{Q_{i}}$ identifies with $\xi_{Q_{i}^{\prime}}$ 
(see Proposition 1.1.14). Therefore one has $\widehat{\mu}_{\min }\left(Q_{i}, \xi_{Q_{i}}\right)=\widehat{\mu}_{\min }\left(Q_{i}^{\prime}, \xi_{Q_{i}^{\prime}}\right)$ for any $i \in\{2, \ldots, n\}$. Applying the induction hypothesis to (4.53) we obtain

$$
\widehat{\mu}_{\min }\left(E_{n}^{\prime}, \xi_{E_{n}^{\prime}}\right) \geqslant \min _{i \in\{2, \ldots, n\}}\left(\widehat{\mu}_{\min }\left(Q_{i}, \xi_{Q_{i}}\right)-h\left(\beta_{i}^{\prime}\right)\right) \geqslant \min _{i \in\{2, \ldots, n\}}\left(\widehat{\mu}_{\min }\left(Q_{i}, \xi_{Q_{i}}\right)-h\left(\beta_{i}\right)\right),
$$

where the second inequality comes from Proposition 1.1.14 Finally, by Proposition 4.3.31 one has

$$
\widehat{\mu}_{\min }\left(E_{n}, \xi_{E_{n}}\right) \geqslant \min \left\{\widehat{\mu}_{\min }\left(E_{n}^{\prime}, \xi_{E_{n}^{\prime}}\right), \widehat{\mu}_{\min }\left(E_{1}, \xi_{E_{1}}\right)-h\left(\beta_{1}\right)\right\} .
$$

The proposition is thus proved.

Proposition 4.3.33. - Let $\bar{E}$ and $\bar{F}$ be adelic vector bundles on $S$. One has

$$
\widehat{\mu}\left(\bar{E} \otimes_{\varepsilon, \pi} \bar{F}\right)=\widehat{\mu}(\bar{E})+\widehat{\mu}(\bar{F}) .
$$

If $\bar{E}$ and $\bar{F}$ are both Hermitian, then

$$
\widehat{\mu}(\bar{E} \otimes \bar{F})=\widehat{\mu}(\bar{E})+\widehat{\mu}(\bar{F}) .
$$

Proof. - These equalities are direct consequences of Proposition 4.3.18.

Proposition 4.3.34. - Let $\bar{E}=\left(E, \xi_{E}\right)$ and $\bar{F}=\left(F, \xi_{F}\right)$ be adelic vector bundles on $S$. One has

$$
\widehat{\mu}_{\max }\left(\bar{E} \otimes_{\varepsilon, \pi} \bar{F}\right) \geqslant \widehat{\mu}_{\max }(\bar{E})+\widehat{\mu}_{\max }(\bar{F}) .
$$

If $\bar{E}$ and $\bar{F}$ are Hermitian adelic vector bundles, then

$$
\widehat{\mu}_{\max }(\bar{E} \otimes \bar{F}) \geqslant \widehat{\mu}_{\max }(\bar{E})+\widehat{\mu}_{\max }(\bar{F}) .
$$

Proof. - Let $E_{1}$ and $F_{1}$ be vector subspaces of $E$ and $F$ respectively. Let $\xi_{E_{1}}$ and $\xi_{F_{1}}$ be the restrictions of $\xi_{E}$ and $\xi_{F}$ to $E_{1}$ and $F_{1}$ respectively. By Proposition 4.3.33, one has

$$
\widehat{\mu}\left(\bar{E}_{1} \otimes_{\varepsilon, \pi} \bar{F}_{1}\right)=\widehat{\mu}\left(\bar{E}_{1}\right)+\widehat{\mu}\left(\bar{F}_{1}\right) .
$$

If $\xi_{E}$ and $\xi_{F}$ are both Hermitian, then

$$
\widehat{\mu}\left(\bar{E}_{1} \otimes \bar{F}_{1}\right)=\widehat{\mu}\left(\bar{E}_{1}\right)+\widehat{\mu}\left(\bar{F}_{1}\right) .
$$

By Proposition 1.1.60, if we denote by $\xi$ the restriction of $\xi_{E} \otimes_{\varepsilon, \pi} \xi_{F}$ to $E_{1} \otimes F_{1}$, then the identity map from $\bar{E}_{1} \otimes_{\varepsilon, \pi} \bar{F}_{1}$ to $\left(E_{1} \otimes_{k} F_{1}, \xi\right)$ has height $\leqslant 0$ and therefore

$$
\widehat{\mu}\left(\bar{E}_{1}\right)+\widehat{\mu}\left(\bar{F}_{1}\right)=\widehat{\mu}\left(\bar{E}_{1} \otimes_{\varepsilon, \pi} \bar{F}_{1}\right) \leqslant \widehat{\mu}\left(E_{1} \otimes_{k} F_{1}, \xi\right) \leqslant \widehat{\mu}_{\max }\left(\bar{E} \otimes_{\varepsilon, \pi} \bar{F}\right) .
$$

Similarly, if both norm families $\xi_{E}$ and $\xi_{F}$ are Hermitian, then by Proposition 1.2 .58 the restriction of $\xi_{E} \otimes \xi_{F}$ to $E_{1} \otimes_{K} F_{1}$ identifies with $\xi_{E_{1}} \otimes \xi_{F_{1}}$. Hence

$$
\widehat{\mu}\left(\bar{E}_{1}\right)+\widehat{\mu}\left(\bar{F}_{1}\right)=\widehat{\mu}\left(\bar{E}_{1} \otimes \bar{F}_{1}\right) \leqslant \widehat{\mu}_{\max }(\bar{E} \otimes \bar{F}) .
$$

Since $E_{1}$ and $F_{1}$ are arbitrary, we obtain the inequalities (4.54) and (4.55).

Lemma 4.3.35. — Let $(E, \xi)$ be an adelic vector bundle over $S$. Then we have the following: 
(1) Let $\psi$ be an integrable function on $\Omega$. Then

$$
\widehat{\mu}_{\max }\left(E, \mathrm{e}^{\psi} \xi\right)=\widehat{\mu}_{\max }(E, \xi)-\int_{\Omega} \psi \nu(\mathrm{d} \omega) .
$$

(2) If $\widehat{\mu}_{\max }(E, \xi) \leqslant 0$, then $\widehat{\operatorname{deg}}_{+}(E, \xi)=0$.

Proof. - (1) Let $F$ be a non-zero vector subspace of $E$. Then, as

$$
\widehat{\mu}\left(F, \mathrm{e}^{\psi} \xi_{F}\right)=\widehat{\mu}\left(F, \xi_{F}\right)-\int_{\Omega} \psi \nu(\mathrm{d} \omega),
$$

we obtain (1).

(2) Let $F$ be a non-zero vector subspace of $E$. By our assumption, $\widehat{\mu}\left(F, \xi_{F}\right) \leqslant 0$, that is, $\widehat{\operatorname{deg}}\left(F, \xi_{F}\right) \leqslant 0$, so that the assertion follows.

4.3.10. Harder-Narasimhan filtration: Hermitian case. - It had been discovered by Stuhler [131] (generalised by Grayson [68) that the Euclidean lattices and vector bundles on projective algebraic curves share some common constructions and properties such as slopes and Harder-Narasimhan filtration etc. Later Bost has developed the slope theory of Hermitian vector bundles over spectra of algebraic integer rings, see [15. Appendice] (see also [130 and [17, §4.1] for more details, and 60, 21, 63] for further generalisations).

The Hermitian adelic vector bundles on $S$ form a category in which a theory of Hader-Narasimhan filtration can be developed in a functorial way. We refer the readers to [1, 39] for more details. In this subsection, we adopt a more direct approach as in $[21]$.

Let $\bar{E}$ be a non-zero Hermitian adelic vector bundle on $S$. We can construct in a recursive way a flag

$$
0=E_{0} \subsetneq E_{1} \subsetneq \ldots \subsetneq E_{n}=E
$$

of vector subspaces of $E$ such that $\overline{E_{i} / E_{i-1}}=\overline{\left(E / E_{i-1}\right)}$ des, called the HarderNarasimhan flag of $\bar{E}$, where $E / E_{i-1}$ is equipped with the quotient norm family, and $E_{i} / E_{i-1}$ is equipped with the subquotient norm family (namely the restriction of the norm family of $\overline{E / E_{i-1}}$ to $\left.E_{i} / E_{i-1}\right)$.

Proposition 4.3.36. — Let $\bar{E}$ be a non-zero Hermitian adelic vector bundle on $S$ and

$$
0=E_{0} \subsetneq E_{1} \subsetneq \ldots \subsetneq E_{n}=E
$$

be the Harder-Narasimhan flag of $\bar{E}$. Then each subquotient $\overline{E_{i} / E_{i-1}}$ is a semistable Hermitian adelic vector bundle. Moreover, if we let $\mu_{i}=\widehat{\mu}\left(\overline{E_{i} / E_{i-1}}\right)$ for $i \in\{1, \ldots, n\}$, then one has $\mu_{1}>\ldots>\mu_{n}$. 
Proof. - We reason by induction on the length $n$ of the Harder-Narasimhan flag. When $n=1$, the assertion is trivial. In the following, we suppose that $n \geqslant 2$. By definition

$$
0=E_{1} / E_{1} \subsetneq E_{2} / E_{1} \subsetneq \ldots \subsetneq E_{n} / E_{1}
$$

is the Harder-Narasimhan flag of $\overline{E / E_{1}}$. Therefore the induction hypothesis leads to $\mu_{2}>\ldots>\mu_{n}$. It remains to establish $\mu_{1}>\mu_{2}$. Since $E_{1}$ is the destabilising vector subspace of $E$ and $E_{2}$ contains strictly $E_{1}$, one has

$$
\mu_{1}=\widehat{\mu}\left(\bar{E}_{1}\right)>\widehat{\mu}\left(\bar{E}_{2}\right) .
$$

Moreover,

$$
0 \longrightarrow \bar{E}_{1} \longrightarrow \bar{E}_{2} \longrightarrow \overline{E_{2} / E_{1}} \longrightarrow 0
$$

forms an exact sequence of adelic vector bundles on $S$. Therefore one has

$$
\widehat{\operatorname{deg}}\left(\bar{E}_{2}\right)=\widehat{\operatorname{deg}}\left(\bar{E}_{1}\right)+\widehat{\operatorname{deg}}\left(\overline{E_{2} / E_{1}}\right)=\mu_{1} \operatorname{rk}_{K}\left(E_{1}\right)+\mu_{2} \operatorname{rk}_{K}\left(E_{2} / E_{1}\right) .
$$

By (4.56) we obtain

$$
\mu_{1} \operatorname{rk}_{K}\left(E_{1}\right)+\mu_{2} \operatorname{rk}_{K}\left(E_{2} / E_{1}\right)<\mu_{1} \operatorname{rk}\left(E_{2}\right)
$$

and hence $\mu_{1}>\mu_{2}$. The proposition is thus proved.

The Harder-Narasimhan flag and the slopes of the successive subquotients in the previous proposition permit to construct an $\mathbb{R}$-filtration $\mathcal{F}_{\text {hn }}$ on the vector space $E$, called the Harder-Narasimhan $\mathbb{R}$-filtration as follows:

$$
\forall t \in \mathbb{R}, \quad \mathcal{F}_{\mathrm{hn}}^{t}(\bar{E}):=E_{i} \text { if } \mu_{i+1}<t \leqslant \mu_{i},
$$

where by convention $\mu_{0}=+\infty$ and $\mu_{n+1}=-\infty$. If $\bar{E}$ is the zero Hermitian adelic vector bundle, by convention its Harder-Narasimhan $\mathbb{R}$-filtration is defined as the only $\mathbb{R}$-filtration of the zero vector space: for any $t \in \mathbb{R}$ one has $\mathcal{F}^{t}(\bar{E})=\{0\}$. Note that the Harder-Narasimhan $\mathbb{R}$-filtration is locally constant on the left, namely $\mathcal{F}_{\mathrm{hn}}^{t-\varepsilon}(\bar{E})=\mathcal{F}_{\mathrm{hn}}^{t}(\bar{E})$ if $\varepsilon>0$ is sufficiently small. Moreover, each subquotient

$$
\mathrm{Sq}_{\mathrm{hn}}^{t}(\bar{E}):=\mathcal{F}_{\mathrm{hn}}^{t}(\bar{E}) / \mathcal{F}_{\mathrm{hn}}^{t+}(\bar{E})
$$

with $\mathcal{F}_{\text {hn }}^{t+}(\bar{E}):=\bigcup_{\varepsilon>0} \mathcal{F}_{\text {hn }}^{t+\varepsilon}(\bar{E})$, viewed as a Hermitian adelic vector bundle in considering the subqutient norm family, namely the quotient of the restricted norm family on $\mathcal{F}_{\text {hn }}^{t}(\bar{E})$, is either zero or a semistable Hermitian adelic vector bundle of slope $t$. The following proposition shows that the Harder-Narasimhan $\mathbb{R}$-filtration is actually characterized by these properties.

Proposition 4.3.37. - Let $\bar{E}$ be a non-zero Hermitian adelic vector bundle on $S$ and $\mathcal{F}$ be a decreasing $\mathbb{R}$-filtration of $E$ which is separated, exhaustive $(1)$ and locally constant on the left. Assume that each subquotient $\mathrm{Sq}(\bar{E}):=\mathcal{F}^{t}(E) / \mathcal{F}^{t+}(E)$ equipped

1. Let $E$ be a vector space over $K$ and $\left(\mathcal{F}^{t}(E)\right)_{t \in \mathbb{R}}$ be a decreasing $\mathbb{R}$-filtration of $E$. We say that the filtration $\mathcal{F}$ is separated if $\mathcal{F}^{t}(E)=\{0\}$ for sufficiently positive $t$. We say that the filtration $\mathcal{F}$ is exhaustive if $\mathcal{F}^{t}(E)=E$ for sufficiently negative $t$. 
with the subquotient norm family, is either zero or a semistable Hermitian adelic vector bundle of slope $t$. Then the $\mathbb{R}$-filtration $\mathcal{F}$ coincides with the Harder-Narasimhan $\mathbb{R}$-filtration of $\bar{E}$.

Proof. — We will prove an alternative statement as follows. Let

$$
0=F_{0} \subsetneq F_{1} \subsetneq \ldots \subsetneq F_{m} \subsetneq E
$$

be a flag of vector subspaces of $E$. We will prove that, if each subquotient $\overline{F_{i} / F_{i-1}}$ $(i \in\{1, \ldots, m\})$ is a semistable Hermitian adelic vector bundle and if the relations

$$
\widehat{\mu}\left(\overline{F_{1} / F_{0}}\right)>\ldots>\widehat{\mu}\left(\overline{F_{m} / F_{m-1}}\right)
$$

hold, then (4.58) identifies with the Harder-Narasimhan flag of $\bar{E}$. This alternative statement is actually equivalent to the form announced in the proposition. In fact, the data of an $\mathbb{R}$-filtration of $E$ is equivalent to that of a flag (of vector subspaces figuring in the $\mathbb{R}$-filtration) and a decreasing sequenc of real numbers indicating the indices where the $\mathbb{R}$-filtration has jumps see $\$ 1.1 .8$ notably Remark 1.1.40.

We will prove the statement by induction on the rank of $E$. The case where $\operatorname{rk}_{K}(E)=1$ is trivial. In the following, we assume that $\operatorname{rk}_{K}(E) \geqslant 2$ and that the alternative assertion has been proved for any Hermitian adelic vector bundle of rank $<\operatorname{rk}_{K}(E)$. Let

$$
0=E_{0} \subsetneq E_{1} \subsetneq \ldots \subsetneq E_{n}=E
$$

be the Harder-Narasimhan flag of $\bar{E}$. We begin by showing that $F_{1}=E_{1}$. Since $E_{1}$ is the destabilizing vector subspace of $\bar{E}$, one has $\widehat{\mu}\left(\bar{F}_{1}\right) \leqslant \widehat{\mu}\left(\bar{E}_{1}\right)$. Moreover one has

$$
0=F_{0} \cap E_{1} \subseteq F_{1} \cap E_{1} \subseteq F_{2} \cap E_{1} \subseteq \ldots F_{m} \cap E_{1}=E_{1} .
$$

Note that each subquotient $\left(F_{i} \cap E_{1}\right) /\left(F_{i-1} \cap E_{1}\right)$ identifies with a vector subspace of $F_{i} / F_{i-1}$. Since $\overline{F_{i} / F_{i-1}}$ is semistable, one has

$$
\begin{aligned}
\widehat{\operatorname{deg}}\left(\overline{\left(F_{i} \cap E_{1}\right) /\left(F_{i-1} \cap E_{1}\right)}\right) & \leqslant \widehat{\mu}\left(\overline{F_{i} / F_{i-1}}\right) \operatorname{rk}_{K}\left(\left(F_{i} \cap E_{1}\right) /\left(F_{i-1} \cap E_{1}\right)\right) \\
& \leqslant \widehat{\mu}\left(\bar{F}_{1}\right) \operatorname{rk}_{K}\left(\left(F_{i} \cap E_{1}\right) /\left(F_{i-1} \cap E_{1}\right)\right),
\end{aligned}
$$

where the second inequality is strict if $i>1$ and if $\left(F_{i} \cap E_{1}\right) /\left(F_{i-1} \cap E_{1}\right)$ is non-zero. Therefore we obtain

$$
\widehat{\operatorname{deg}}\left(\bar{E}_{1}\right)=\sum_{i=1}^{m} \widehat{\operatorname{deg}}\left(\overline{\left(F_{i} \cap E_{1}\right) /\left(F_{i-1} \cap E_{1}\right)}\right) \leqslant \widehat{\mu}\left(\bar{F}_{1}\right) \operatorname{rk}\left(E_{1}\right) .
$$

Combining with the inequality $\widehat{\mu}\left(\bar{F}_{1}\right) \leqslant \widehat{\mu}\left(\bar{E}_{1}\right)=\widehat{\mu}_{\max }(\bar{E})$, we deduce that the inequality (4.59) is actually an equality, which also implies that $\left(F_{i} \cap E_{1}\right) /\left(F_{i-1} \cap E_{1}\right)=$ $\{0\}$ once $i>1$. Therefore one has $F_{1}=E_{1}$, which also leads to the alternative assertion in the particular case where $\bar{E}$ is semistable.

In the case where $\bar{E}$ is not semistable, namely $n \geqslant 2$, note that

$$
0=E_{1} / E_{1} \subsetneq E_{2} / E_{1} \subsetneq \ldots \subsetneq E_{n} / E_{1}=E / E_{1}
$$


is the Harder-Narasimhan flag of $\overline{E / E_{1}}=\overline{E / F_{1}}$. By the induction hypothesis applied to $\overline{E / F_{1}}$, we obtain that the flag

$$
0=F_{1} / F_{1} \subsetneq F_{2} / F_{1} \subsetneq \ldots \subsetneq F_{m} / F_{1}=E / F_{1}
$$

coincides with (4.60). The proposition is thus proved.

Definition 4.3.38. - Let $\bar{E}$ be a non-zero Hermitian adelic vector bundle on $S$, and

$$
0=E_{0} \subsetneq E_{1} \subsetneq \ldots \subsetneq E_{n}=E
$$

be its Harder-Narasimhan flag. For any $i \in\left\{1, \ldots, \operatorname{rk}_{K}(E)\right\}$, there exists a unique $j \in\{1, \ldots, n\}$ such that $\operatorname{rk}_{K}\left(E_{j-1}\right)<i \leqslant \operatorname{rk}_{K}\left(E_{j}\right)$. We let $\widehat{\mu}_{i}(\bar{E})$ be the slope $\widehat{\mu}\left(\overline{E_{j} / E_{j-1}}\right)$, called the $i$-th slope of $\bar{E}$. Clearly one has

$$
\widehat{\mu}_{1}(\bar{E}) \geqslant \ldots \geqslant \widehat{\mu}_{r}(\bar{E})
$$

where $r$ is the rank of $E$ over $K$. Moreover, by definition $\widehat{\mu}_{1}(\bar{E})$ coincides with the maximal slope of $\bar{E}$.

Remark 4.3.39. - As in the classic case of vector bundles on projective curves or Hermitian vector bundles over algebraic integer rings, one can naturally construction Harder-Narasimhan polygones associated with Hermitian adelic vector bundles on adelic curves. Let $\bar{E}$ be a non-zero Hermitian adelic vector bundle on the adelic curve $S$. We consider the convex hull $C_{\bar{E}}$ in $\mathbb{R}^{2}$ of the points $\left(\operatorname{rk}_{K}(F), \widehat{\operatorname{deg}}(\bar{F})\right)$, where $F$ runs over the set of all vector subspaces of $E$. The upper boundary of this convex set identifies with the graph of a concave function $P_{\bar{E}}$ on $\left[0, \mathrm{rk}_{K}(E)\right]$ which is affine on each interval $[i-1, i]$ with $i \in\left\{1, \ldots, \operatorname{rk}_{K}(E)\right\}$. This function is called HarderNarasimhan polygon of $\bar{E}$. If

$$
0=E_{0} \subsetneq E_{1} \subsetneq \ldots \subsetneq E_{n}=E
$$

is the Harder-Narasimhan flag of $\bar{E}$, then the abscissae on which the HarderNarasimhan polygon $P_{\bar{E}}$ changes slopes are exactly $\operatorname{rk}_{K}\left(E_{i}\right)$ for $i \in\{0, \ldots, n\}$. Moreover, the value of $P_{\bar{E}}$ on $\operatorname{rk}_{K}\left(E_{i}\right)$ is $\widehat{\operatorname{deg}}\left(\bar{E}_{i}\right)$.

Proposition 4.3.40. - Let $\bar{E}$ be a non-zero Hermitian adelic vector bundle on $S$ and $r$ be the rank of $E$ over $K$. One has

$$
\widehat{\mu}_{r}(\bar{E})=-\widehat{\mu}_{1}\left(\bar{E}^{\vee}\right) \text {. }
$$

In particular, $\widehat{\mu}_{r}(\bar{E})$ is equal to $\widehat{\mu}_{\min }(\bar{E})$.

Proof. — Let

$$
0=E_{0} \subsetneq E_{1} \subsetneq \ldots \subsetneq E_{n}=E
$$

be the Harder-Narasimhan flag of $\bar{E}$. Note that

$$
0=\left(E / E_{n}\right)^{\vee} \subsetneq\left(E / E_{n-1}\right)^{\vee} \subsetneq \ldots \subsetneq\left(E / E_{0}\right)^{\vee}=E^{\vee}
$$


is a flag of vector subspaces of $E^{\vee}$, and for $i \in\{1, \ldots, n\}$ one has

$$
\left(E / E_{i-1}\right)^{\vee} /\left(E / E_{i}\right)^{\vee} \cong\left(E_{i} / E_{i-1}\right)
$$

By Proposition 4.3.37 (notably the alternative form stated in the proof), we obtain that (4.62) is actually the Harder-Narasimhan flag of $\bar{E}^{\vee}$. Therefore

$$
\widehat{\mu}_{1}\left(\bar{E}^{\vee}\right)=\widehat{\mu}\left(\left(\overline{E / E_{n-1}}\right)^{\vee}\right)=-\widehat{\mu}\left(\overline{E / E_{n-1}}\right)=-\widehat{\mu}_{r}(\bar{E}),
$$

where the second equality comes from Proposition 4.3.9.

Note that $\overline{E / E_{n-1}}$ is a non-zero quotient Hermitian adelic bundle of $\bar{E}$ which is semistable (so that $\widehat{\mu}\left(\overline{E / E_{n-1}}\right)=\widehat{\mu}_{\max }\left(\overline{E / E_{n-1}}\right)$ ). Therefore one has $\widehat{\mu}_{\min }(\bar{E}) \leqslant$ $\widehat{\mu}_{r}(\bar{E})$. Conversely, if $F$ is a vector subspace of $E$ such that $F \subsetneq E$ and $G$ is the quotient space $E / F$. Then $G^{\vee}$ identifies with a non-zero vector subspace of $E^{\vee}$. Moreover, by Proposition 1.1.20 the dual of the quotient norm family of $\bar{G}$ identifies with the restriction of the dual norm family in the adelic vector bundle structure of $\bar{E}^{\vee}$. Hence one has

$$
\widehat{\mu}\left(\bar{G}^{\vee}\right) \leqslant \widehat{\mu}_{1}\left(\bar{E}^{\vee}\right)=-\widehat{\mu}_{r}(\bar{E}) .
$$

Still by Proposition 4.3.9, one obtains

$$
\widehat{\mu}_{\max }(\bar{G}) \geqslant \widehat{\mu}(\bar{G}) \geqslant \widehat{\mu}_{r}(\bar{E}) .
$$

The equality $\widehat{\mu}_{\min }(\bar{E})=\widehat{\mu}_{r}(\bar{E})$ is thus proved.

The following proposition, which results from the slope inequalities, provides the functoriality of Harder-Narasimhan $\mathbb{R}$-filtration (see 39 for the meaning of the functoriality of Harder-Narasimhan $\mathbb{R}$-filtration).

Proposition 4.3.41. - Let $\bar{E}$ and $\bar{F}$ be two Hermitian adelic vector bundles on $S$, and $f: E \rightarrow F$ be a non-zero $K$-linear map. For any $t \in \mathbb{R}$ one has

$$
f\left(\mathcal{F}_{\mathrm{hn}}^{t}(\bar{E})\right) \subseteq \mathcal{F}_{\mathrm{hn}}^{t-h(f)}(\bar{F}) .
$$

Proof. — We will actually show by contradiction that the composition of maps

$$
\mathcal{F}_{\mathrm{hn}}^{t}(\bar{E}) \stackrel{f}{\longrightarrow} F \longrightarrow F / \mathcal{F}_{\mathrm{hn}}^{t-h(f)}(\bar{F})
$$

is zero. If this map is not zero, then by Proposition 4.3.30 (3) one obtains

$$
\widehat{\mu}_{\min }\left(\mathcal{F}_{\mathrm{hn}}^{t}(\bar{E})\right) \leqslant \widehat{\mu}_{\max }\left(\bar{F} / \mathcal{F}_{\mathrm{hn}}^{t-h(f)}(\bar{F})\right)+h(f) .
$$

By (4.57) we obtain

$$
t \leqslant \widehat{\mu}_{\min }\left(\mathcal{F}_{\mathrm{hn}}^{t}(\bar{E})\right) \leqslant \widehat{\mu}_{\max }\left(\bar{F} / \mathcal{F}_{\mathrm{hn}}^{t-h(f)}(\bar{F})\right)+h(f)<t-h(f)+h(f)=t,
$$

which leads to a contradiction. 
Corollary 4.3.42. - Let $\bar{E}$ be a non-zero Hermitian adelic vector bundle on $S$. One has

$$
\mathcal{F}_{\mathrm{hn}}^{t}(\bar{E})=\sum_{\substack{0 \neq F \in \Theta(E) \\ \widehat{\mu}_{\min }(\bar{F}) \geqslant t}} F
$$

where $F$ runs over the set $\Theta(E)$ of all non-zero vector subspaces of $E$ with minimal slope $\geqslant t$. In other words, $\mathcal{F}_{\mathrm{hn}}^{t}(\bar{E})$ is the largest vector subspace of $E$ whose minimal slope is bounded from below by $t$.

Proof. - By the definition of the Harder-Narasimhan $\mathbb{R}$-filtration (see (4.57)), for any $t \in \mathbb{R}$ one has $\widehat{\mu}_{\min }\left(\mathcal{F}_{\text {hn }}^{t}(\bar{E})\right) \geqslant t$. Moreover, if $F$ is a non-zero vector subspace of $E$, then one has $\mathcal{F}_{\text {hn }}^{t}(\bar{F})=F$ provided that $t \leqslant \widehat{\mu}_{\text {min }}(\bar{F})$. Therefore the proposition 4.3 .41 applied to the inclusion map $F \rightarrow E$ leads to $F \subseteq \mathcal{F}_{\mathrm{hn}}^{t}(E)$.

Proposition 4.3.43. - Let $\bar{E}$ be a non-zero Hermitian adelic vector bundle on $S$ and $r$ be the rank of $E$ over $K$. The following equalities hold:

$$
\begin{gathered}
\widehat{\operatorname{deg}}(\bar{E})=\sum_{i=1}^{r} \widehat{\mu}_{i}(\bar{E})=-\int_{\mathbb{R}} t \operatorname{drk}\left(\mathcal{F}_{\mathrm{hn}}^{t}(\bar{E})\right), \\
\widehat{\operatorname{deg}}_{+}(\bar{E})=\sum_{i=1}^{r} \max \left\{\widehat{\mu}_{i}(\bar{E}), 0\right\}=\int_{0}^{+\infty} \operatorname{rk}\left(\mathcal{F}_{\mathrm{hn}}^{t}(\bar{E})\right) \mathrm{d} t .
\end{gathered}
$$

Proof. - By definition the sum of Dirac measures

$$
\sum_{i=1}^{r} \delta_{\widehat{\mu}_{i}(\bar{E})}
$$

identifies with the derivative $-\operatorname{drk}\left(\mathcal{F}_{\text {hn }}^{t}(\bar{E})\right)$ in the sense of distribution. Therefore, the second equality in (4.63) is true, and the second equality in (4.64) follows from the relation

$$
\sum_{i=1}^{r} \max \left\{\widehat{\mu}_{i}(\bar{E}), 0\right\}=-\int_{0}^{+\infty} t \operatorname{drk}\left(\mathcal{F}_{\text {hn }}^{t}(\bar{E})\right)
$$

and the formula of integration by part.

Let

$$
0=E_{0} \subsetneq E_{1} \subsetneq \ldots \subsetneq E_{n}=E
$$

be the Harder-Narasimhan flag of $\bar{E}$. By Proposition 4.3 .12 one has

$$
\widehat{\operatorname{deg}}(\bar{E})=\sum_{j=1}^{n} \widehat{\operatorname{deg}}\left(\overline{E_{j} / E_{j-1}}\right)=\sum_{j=1}^{n} \widehat{\mu}\left(\overline{E_{j} / E_{j-1}}\right) \operatorname{rk}_{K}\left(E_{j} / E_{j-1}\right)=\sum_{i=1}^{r} \widehat{\mu}_{i}(\bar{E}),
$$

which proves (4.63).

Let $\ell$ be the largest element in $\{1, \ldots, n\}$ such that $\widehat{\mu}\left(\bar{E}_{\ell} / \bar{E}_{\ell-1}\right) \geqslant 0$. If $\widehat{\mu}\left(\overline{E_{j} / E_{j-1}}\right)<0$ for any $j \in\{1, \ldots, n\}$, by convention we let $\ell=0$. Then by (4.63) 
one has

$$
\widehat{\operatorname{deg}}\left(\bar{E}_{\ell}\right)=\sum_{i=1}^{r} \max \left\{\widehat{\mu}_{i}(\bar{E}), 0\right\}
$$

Hence we obtain

$$
\sum_{i=1}^{r} \max \left\{\widehat{\mu}_{i}(\bar{E}), 0\right\} \leqslant \widehat{\operatorname{deg}}_{+}(\bar{E}) .
$$

Conversely, if $F$ is a non-zero vector subspace of $E$ and $m$ is its rank over $K$, by Proposition 4.3 .41 one has $\widehat{\mu}_{i}(\bar{F}) \leqslant \widehat{\mu}_{i}(\bar{E})$ for any $i \in\{1, \ldots, m\}$. Therefore by (4.63) one obtains

$$
\widehat{\operatorname{deg}}(\bar{F})=\sum_{i=1}^{m} \widehat{\mu}_{i}(\bar{F}) \leqslant \sum_{i=1}^{m} \widehat{\mu}_{i}(\bar{E}) \leqslant \sum_{i=1}^{r} \max \left\{\widehat{\mu}_{i}(\bar{E}), 0\right\} .
$$

4.3.11. Harder-Narasimhan filtration: general case. - Inspired by Corollary 4.3.42, we extend the definition of Harder-Narasimhan $\mathbb{R}$-filtration to the setting of general adelic vector bundles.

Definition 4.3.44. - Let $\bar{E}$ be a non-zero adelic vector bundle on $S$. For any $t \in \mathbb{R}$, let

$$
\mathcal{F}_{\text {hn }}^{t}(\bar{E}):=\bigcap_{\epsilon>0} \sum_{\substack{\{0\} \neq F \in \Theta(E) \\ \hat{\mu}_{\min }(\bar{F}) \geqslant t-\epsilon}} F,
$$

where $\Theta(E)$ denotes the set of vector subspaces of $E$. By the finiteness of maximal and minimal slopes proved in Proposition 4.3.29, we obtain that $\mathcal{F}_{\mathrm{hn}}^{t}(\bar{E})=E$ when $t$ is sufficiently negative, and $\mathcal{F}_{\mathrm{hn}}^{t}(\bar{E})=\{0\}$ is sufficiently positive. By convention we let $\mathcal{F}_{\mathrm{hn}}^{+\infty}(\bar{E})=\{0\}$ and $\mathcal{F}_{\mathrm{hn}}^{-\infty}=E$.

Proposition 4.3.45. - Let $\bar{E}$ be a non-zero adelic vector bundle on $S$. For any $t \in \mathbb{R}$, the vector space $\mathcal{F}_{\mathrm{hn}}^{t}(\bar{E})$ equipped with the induced norm family has a minimal slope $\geqslant t$. In particular, one has

$$
\forall t \in \mathbb{R}, \quad \mathcal{F}_{\text {hn }}^{t}(\bar{E})=\sum_{\substack{\{0\} \neq F \in \Theta(E) \\ \widehat{\mu}_{\min }(\bar{F}) \geqslant t}} F
$$

and

$$
\widehat{\mu}_{\text {min }}(\bar{E})=\max \left\{t \in \mathbb{R}: \mathcal{F}_{\text {hn }}^{t}(\bar{E})=E\right\} .
$$

Proof. - Let $t \in \mathbb{R}$. For sufficiently small $\varepsilon>0$, one has

$$
F_{\text {hn }}^{t}(\bar{E})=\sum_{\substack{\{0\} \neq F \in \Theta(E) \\ \widehat{\mu}_{\min }(\bar{F}) \geqslant t-\epsilon}} F
$$


Let $M$ be a non-zero quotient vector space of $\mathcal{F}_{\mathrm{hn}}^{t}(\bar{E})$. By definition, for any $\epsilon>0$ there exists a vector subspace $F_{\epsilon}$ of $E$ such that $\widehat{\mu}_{\min }\left(\overline{F_{\epsilon}}\right) \geqslant t-\epsilon$ and that the composed map $F_{\epsilon} \rightarrow \mathcal{F}_{\text {hn }}^{t}(\bar{E}) \rightarrow M$ is non-zero. By the slope inequality (see Proposition 4.3.30) we have $t-\epsilon \leqslant \widehat{\mu}_{\min }\left(\overline{F_{\epsilon}}\right) \leqslant \widehat{\mu}_{\max }(\bar{M})$, which leads to $\widehat{\mu}_{\max }(\bar{M}) \geqslant t$ since $\epsilon>0$ is arbitrary. As $M$ is arbitrary, we obtain the first statement.

By the first statement of the proposition, for any $t \in \mathbb{R}$ such that $\mathcal{F}_{\mathrm{hn}}^{t}(\bar{E})=E$, one has $\widehat{\mu}_{\min }(\bar{E}) \geqslant t$. Conversely, by definition, if $t$ is a real number such that $\widehat{\mu}_{\min }(\bar{E}) \geqslant t$, then $E \subseteq \mathcal{F}_{\text {hn }}^{t}(\bar{E})$ and hence $E=\mathcal{F}_{\text {hn }}^{t}(\bar{E})$. Therefore, the equality $\widehat{\mu}_{\min }(\bar{E})=\max \left\{t \in \mathbb{R}: \mathcal{F}_{\text {hn }}^{t}(\bar{E})=E\right\}$ holds.

Definition 4.3.46. - Let $\bar{E}$ be a non-zero adelic vector bundle on $S$. For any $i \in\left\{1, \ldots, \operatorname{rk}_{K}(E)\right\}$, we let

$$
\widehat{\mu}_{i}(\bar{E}):=\sup \left\{t \in \mathbb{R}: \operatorname{rk}_{K}\left(\mathcal{F}_{\text {hn }}^{t}(\bar{E})\right) \geqslant i\right\} .
$$

The number $\widehat{\mu}_{i}(\bar{E})$ is called the $i$-th slope of $\bar{E}$. Proposition 4.3 .45 shows that the last slope of $\bar{E}$ identifies with the minimal slope $\widehat{\mu}_{\min }(\bar{E})$ of $\bar{E}$.

Remark 4.3.4\%. - Let $\bar{E}$ be a non-zero adelic vector bundle. In general the first slope $\widehat{\mu}_{1}(\bar{E})$ does not coincide with $\widehat{\mu}_{\max }(\bar{E})$ and we only have an inequality $\widehat{\mu}_{1}(\bar{E}) \leqslant$ $\widehat{\mu}_{\max }(\bar{E})$. Moreover, if the norm family of $\bar{E}$ is ultrametric on $\Omega \backslash \Omega_{\infty}$, then one has $\widehat{\mu}_{\max }(\bar{E}) \leqslant \widehat{\mu}_{1}(\bar{E})+\frac{1}{2} \ln \left(\operatorname{rk}_{K}(E)\right) \nu\left(\Omega_{\infty}\right)$. This follows from (4.69) and (4.25).

With the extended definition, the statement of Proposition 4.3.41 still holds for general adelic vector bundles.

Proposition 4.3.48. - Let $\bar{E}$ and $\bar{F}$ be adelic vector bundles on $S$, and $f: E \rightarrow F$ be a non-zero K-linear map. For any $t \in \mathbb{R}$ one has

$$
f\left(\mathcal{F}_{\mathrm{hn}}^{t}(\bar{E})\right) \subseteq \mathcal{F}_{\mathrm{hn}}^{t-h(f)}(\bar{F}) .
$$

Proof. - Let $M$ be a non-zero vector subspace of $E$ such that $\widehat{\mu}_{\min }(\bar{M}) \geqslant t$. By Proposition $4.3 .30(2)$, one has

$$
\widehat{\mu}_{\min }(\bar{M}) \leqslant \widehat{\mu}_{\min }(\overline{f(M)})+h\left(\left.f\right|_{M}\right) \leqslant \widehat{\mu}_{\min }(\overline{f(M)})+h(f) .
$$

Therefore $f(M) \subseteq \mathcal{F}_{\text {hn }}^{t-h(f)}(F)$.

Proposition 4.3.49. - Let $\bar{E}$ be a non-zero adelic vector bundle on $S$. Let $r$ be the rank of $E$ over $K$. Then the following inequalities hold:

$$
\begin{gathered}
\widehat{\operatorname{deg}}(\bar{E}) \geqslant \sum_{i=1}^{r} \widehat{\mu}_{i}(\bar{E})=-\int_{\mathbb{R}} t \mathrm{~d} \operatorname{rk}\left(\mathcal{F}_{\mathrm{hn}}^{t}(\bar{E})\right), \\
\widehat{\operatorname{deg}}_{+}(\bar{E}) \geqslant \sum_{i=1}^{r} \max \left\{\widehat{\mu}_{i}(\bar{E}), 0\right\}=\int_{0}^{+\infty} \operatorname{rk}\left(\mathcal{F}_{\mathrm{hn}}^{t}(\bar{E})\right) \mathrm{d} t .
\end{gathered}
$$


Proof. - For $i \in\{1, \ldots, r\}$, let $E_{i}$ be $\mathcal{F}_{\mathrm{hn}}^{\widehat{\mu}_{i}(\bar{E})}(\bar{E})$. Let $E_{0}=\{0\}$. Then for each $i \in\{1, \ldots, r\}$, such that $E_{i} \supsetneq E_{i-1}$ one has

$$
\widehat{\mu}\left(\overline{E_{i} / E_{i-1}}\right) \geqslant \widehat{\mu}_{\min }\left(\bar{E}_{i}\right)=\widehat{\mu}_{i}(\bar{E}),
$$

where the last equality comes from Proposition 4.3.45 and the fact that the restriction of the $\mathbb{R}$-filtration $\mathcal{F}_{\text {hn }}$ to $E_{i}$ coincides with the Harder-Narasimhan $\mathbb{R}$-filtration of $\bar{E}_{i}$. Therefore, by Proposition 4.3 .12 one has

$$
\widehat{\operatorname{deg}}(\bar{E}) \geqslant \sum_{\substack{i \in\{1, \ldots, r\} \\ E_{i} \supsetneq E_{i-1}}} \widehat{\operatorname{deg}}\left(\overline{E_{i} / E_{i-1}}\right) \geqslant \sum_{\substack{i \in\{1, \ldots, r\} \\ E_{i} \supsetneq E_{i-1}}} \operatorname{rk}\left(E_{i} / E_{i-1}\right) \widehat{\mu}_{i}(\bar{E})=\sum_{i=1}^{r} \widehat{\mu}_{i}(\bar{E}),
$$

which proves (4.66). Finally, if we let $j$ be the largest index in $\{1, \ldots, r\}$ such that $\widehat{\mu}_{j}(\bar{E}) \geqslant 0$. Then by what we have proved

$$
\widehat{\operatorname{deg}}\left(\bar{E}_{j}\right) \geqslant \sum_{i=1}^{j} \widehat{\mu}_{i}(\bar{E})=\sum_{i=1}^{r} \max \left(\widehat{\mu}_{i}(\bar{E}), 0\right) .
$$

Therefore, the inequality (4.67) holds.

Proposition 4.3.50. - Let $\bar{E}=(E, \xi)$ be a non-zero adelic vector bundle on $S$. Let $r$ be the rank of $E$ over $K$. Then the following inequalities hold:

$$
\widehat{\operatorname{deg}}(\bar{E}) \leqslant \sum_{i=1}^{r} \widehat{\mu}_{i}(\bar{E})+\Delta(\bar{E}) .
$$

If in addition $\xi$ is ultrametric on $\Omega \backslash \Omega_{\infty}$, then one has

$$
\widehat{\operatorname{deg}}(\bar{E}) \leqslant \sum_{i=1}^{r} \widehat{\mu}_{i}(\bar{E})+\delta(\bar{E}) .
$$

Proof. - We reason by induction on the rank of $E$ over $K$. The case where $\operatorname{rk}_{K}(E)=$ 1 is trivial since in this case $\bar{E}$ is Hermitian. In the following, we assume that $\operatorname{rk}_{K}(E)>$ 1 and that the proposition has been proved for adelic vector bundles of $\operatorname{rank}<\operatorname{rk}_{K}(E)$.

The Harder-Narasimhan $\mathbb{R}$-filtration corresponds to an increasing flag

$$
0=E_{1} \subsetneq E_{2} \subsetneq \ldots \subsetneq E_{n}=E
$$

and a decreasing sequence of numbers $\mu_{1}>\ldots>\mu_{n}$ corresponding to the points of jump of the $\mathbb{R}$-filtration. By Proposition 4.3.45, the minimal slope of $\bar{E}$ is equal to $\mu_{n}$

Let $\epsilon$ be a positive number such that $\epsilon<\mu_{n-1}-\mu_{n}$ and $E^{\prime}$ be a vector subspace of $E$ such that $E^{\prime} \subsetneq E$ and $\widehat{\mu}_{\max }\left(\overline{E / E^{\prime}}\right) \leqslant \widehat{\mu}_{\min }(\bar{E})+\epsilon=\mu_{n}+\epsilon$. By Proposition 4.3.25, one has

$$
\widehat{\mu}\left(\overline{E / E^{\prime}}\right) \geqslant \widehat{\mu}_{\min }(\bar{E})=\mu_{n} .
$$

Therefore, one has

$$
\mu_{n} \leqslant \widehat{\mu}\left(\overline{E / E^{\prime}}\right) \leqslant \widehat{\mu}_{\max }\left(\overline{E / E^{\prime}}\right) \leqslant \mu_{n}+\epsilon .
$$


Moreover, by Proposition 4.3.45, one has

$$
\widehat{\mu}_{\min }\left(\bar{E}_{n-1}\right) \geqslant \mu_{n-1}>\mu_{n}+\epsilon .
$$

By Proposition 4.3.30, we obtain that the composed map $E_{n-1} \rightarrow E \rightarrow E / E^{\prime}$ is zero, or equivalently, $E_{n-1}$ is contained in $E^{\prime}$. Note that for any vector subspace $F$ of $E^{\prime}$ such that $F \supsetneq E_{n-1}$ one has $\widehat{\mu}_{\text {min }}(\bar{F}) \leqslant \mu_{n}$, otherwise the Harder-Narasimhan $\mathbb{R}$ filtration of $\bar{E}$ could not correspond to the flag $0=E_{1} \subsetneq E_{2} \subsetneq \ldots \subsetneq E_{n}=E$ and the decreasing sequence $\mu_{1}>\ldots>\mu_{n}$. Therefore, the Harder-Narasimhan $\mathbb{R}$-filtration of $\bar{E}^{\prime}$ corresponds to a flag of the form $(\ell \in \mathbb{N})$

$$
0=E_{1} \subsetneq \ldots \subsetneq E_{n-1} \subsetneq E_{n}^{\prime} \subsetneq \ldots \subsetneq E_{n-1+\ell}^{\prime}=E^{\prime}
$$

together with a decreasing sequence $\mu_{1}>\ldots>\mu_{n-1}>\mu_{n}^{\prime}>\ldots>\mu_{n-1+\ell}^{\prime}$, where $\mu_{n}^{\prime} \leqslant \mu_{n}$ whenever $\ell \geqslant 1$. We apply the induction hypothesis to $\bar{E}^{\prime}$ and obtain

$$
\widehat{\operatorname{deg}}\left(\bar{E}^{\prime}\right) \leqslant \sum_{i=1}^{n-1} \mu_{i} \operatorname{rk}_{K}\left(E_{i} / E_{i-1}\right)+\sum_{i=n}^{n-1+\ell} \mu_{i}^{\prime} \operatorname{rk}_{K}\left(E_{i}^{\prime} / E_{i-1^{\prime}}\right)+\Delta\left(\bar{E}^{\prime}\right),
$$

with the convention $E_{n-1}=E_{n-1}^{\prime}$. By the condition that $\mu_{n}^{\prime} \leqslant \mu_{n}$ whenever $\ell \geqslant 1$ we obtain

$$
\widehat{\operatorname{deg}}\left(\bar{E}^{\prime}\right) \leqslant \sum_{i=1}^{n-1} \mu_{i} \operatorname{rk}_{K}\left(E_{i} / E_{i-1}\right)+\mu_{n} \operatorname{rk}_{K}\left(E^{\prime} / E_{n-1}\right)+\Delta\left(\bar{E}^{\prime}\right) .
$$

Finally, by Proposition 4.3 .12 (notably the inequality (4.27)) one obtains

$$
\begin{aligned}
\widehat{\operatorname{deg}}(\bar{E}) & \leqslant \widehat{\operatorname{deg}}\left(\bar{E}^{\prime}\right)+\widehat{\operatorname{deg}}\left(\overline{E / E^{\prime}}\right)+\Delta(\bar{E})-\Delta\left(\overline{E^{\prime}}\right)-\Delta\left(\overline{E / E^{\prime}}\right) \\
& \leqslant \sum_{i=1}^{n-1} \mu_{i} \operatorname{rk}_{K}\left(E_{i} / E_{i-1}\right)+\mu_{n} \operatorname{rk}_{K}\left(E^{\prime} / E_{n-1}\right)+\left(\mu_{n}+\epsilon\right) \operatorname{rk}_{K}\left(E / E^{\prime}\right)+\Delta(\bar{E}) \\
& =\sum_{j=1}^{r} \widehat{\mu}_{j}(\bar{E})+\epsilon \operatorname{rk}\left(E / E^{\prime}\right)+\Delta(\bar{E}) \leqslant \sum_{j=1}^{r} \widehat{\mu}_{j}(\bar{E})+\epsilon \operatorname{rk}_{K}(E)+\Delta(\bar{E}) .
\end{aligned}
$$

Since $\epsilon$ is arbitrary, we obtain the inequality (4.68). In the case where $\xi$ is ultrametric on $\Omega \backslash \Omega_{\infty}$ as above allows to deduce (4.69) from (4.28). The proposition is thus proved.

Corollary 4.3.51. - Let $\bar{E}=(E, \xi)$ be a non-zero adelic vector bundle on $S$ and $r$ be the rank of $E$ over $K$. One has

$$
\widehat{\operatorname{deg}}_{+}(\bar{E}) \leqslant \sum_{i=1}^{r} \max \left\{\widehat{\mu}_{i}(\bar{E}), 0\right\}+\Delta(\bar{E}) .
$$

If in addition $\xi$ is ultrametric on $\Omega \backslash \Omega_{\infty}$, then one has

$$
\widehat{\operatorname{deg}}_{+}(\bar{E}) \leqslant \sum_{i=1}^{r} \max \left\{\widehat{\mu}_{i}(\bar{E}), 0\right\}+\delta(\bar{E}) \text {. }
$$


Proof. - Let $F$ be a non-zero vector subspace of $E$ and $m$ be the rank of $F$ over $K$. By (4.68) one has

$$
\widehat{\operatorname{deg}}(\bar{F}) \leqslant \sum_{j=1}^{m} \widehat{\mu}_{j}(\bar{F})+\Delta(\bar{F}) \leqslant \sum_{j=1}^{m} \max \left\{\widehat{\mu}_{j}(\bar{F}), 0\right\}+\Delta(\bar{F}) .
$$

Note that

$$
\sum_{j=1}^{m} \max \left\{\widehat{\mu}_{j}(\bar{F}), 0\right\}=-\int_{\mathbb{R}} \max \{t, 0\} \mathrm{d}\left(\operatorname{rk}_{K}\left(\mathcal{F}_{\mathrm{hn}}^{t}(\bar{F})\right)\right)=\int_{0}^{+\infty} \operatorname{rk}_{K}\left(\mathcal{F}_{\text {hn }}^{t}(\bar{F})\right) \mathrm{d} t .
$$

Moreover, by Proposition 4.3.48, for any $t \in \mathbb{R}$, one has

$$
\operatorname{rk}_{K}\left(\mathcal{F}_{\mathrm{hn}}^{t}(\bar{F})\right) \leqslant \operatorname{rk}_{K}\left(\mathcal{F}_{\mathrm{hn}}^{t}(\bar{E})\right) .
$$

Therefore,

$$
\widehat{\operatorname{deg}}(\bar{F}) \leqslant \int_{0}^{+\infty} \operatorname{rk}_{K}\left(\mathcal{F}_{\mathrm{hn}}^{t}(\bar{E})\right) \mathrm{d} t+\Delta(\bar{F})=\sum_{i=1}^{r} \max \left\{\widehat{\mu}_{i}(\bar{E}), 0\right\}+\Delta(\bar{F}) .
$$

Note that $\Delta(\bar{F}) \leqslant \Delta(\bar{E})$ (see Corollary 1.2.44). By taking the supremum with respect to $F$, we obtain the inequality (4.70).

The proof of the inequality (4.71) is quite similar, where we combine the above argument with the inequality (4.69).

Remark 4.3.52. - Let $(E, \xi)$ be an adelic vector bundle on $S$. Then one has the following inequality: if $\widehat{\operatorname{deg}}_{+}(E, \xi)>0$, then

$$
\widehat{\operatorname{deg}}_{+}(E, \xi) \leqslant \operatorname{rk}_{K}(E) \widehat{\mu}_{\max }(E, \xi) .
$$

As a consequence, we obtain

$$
\widehat{\operatorname{deg}}_{+}(E, \xi) \leqslant \operatorname{rk}_{K}(E) \max \left\{\widehat{\mu}_{\max }(E, \xi), 0\right\}
$$

in general. The inequality (4.73) is weaker than (4.70) and (4.71), but it holds without an error term. Moreover, the inequality (4.72) can be proved as follow: for any $\epsilon \in] 0, \widehat{\operatorname{deg}}_{+}(E, \xi)[$, one can find a non-zero vector subspace $F$ of $E$ such that $0 \leqslant$ $\widehat{\operatorname{deg}}_{+}(E, \xi)-\epsilon \leqslant \widehat{\operatorname{deg}}\left(F, \xi_{F}\right)$, so that

$$
0<\frac{\widehat{\operatorname{deg}_{+}}(E, \xi)-\epsilon}{\operatorname{rk}_{K}(E)} \leqslant \frac{\widehat{\operatorname{deg}}\left(F, \xi_{F}\right)}{\operatorname{rk}_{K}(E)} \leqslant \widehat{\mu}\left(F, \xi_{F}\right) \leqslant \widehat{\mu}_{\max }(E, \xi),
$$

which implies (4.72).

Definition 4.3.53. - Let $\bar{E}$ be an adelic vector bundle on $S$ and $r$ be the rank of $E$ over $K$. We denote by $\widetilde{\operatorname{deg}}(\bar{E})$ the sum $\widehat{\mu}_{1}(\bar{E})+\cdots+\widehat{\mu}_{r}(\bar{E})$. If $\bar{E}$ is the zero adelic vector bundle on $S$, then by convention $\widehat{\operatorname{deg}}(\bar{E})$ is defined to be 0 . If $\bar{E}$ is non-zero, we define $\widetilde{\mu}(\bar{E})$ to be the quotient $\widetilde{\operatorname{deg}}(\bar{E}) / \mathrm{rk}_{K}(E)$.

Proposition 4.3.54. - Let $\bar{E}$ and $\bar{F}$ be non-zero adelic vector bundles on $S$ and $f: E \rightarrow F$ be a $K$-linear map. 
(1) Suppose that $f$ is a bijection. Then one has

$$
\widetilde{\operatorname{deg}}(\bar{E}) \leqslant \widetilde{\operatorname{deg}}(\bar{F})+\operatorname{rk}_{K}(F) \cdot h(f) .
$$

(2) Suppose that $f$ is injective. Then $\widehat{\mu}_{1}(\bar{E}) \leqslant \widehat{\mu}_{1}(\bar{F})+h(f)$.

Proof. - (1) By Proposition 4.3.48, for any $t \in \mathbb{R}$ one has

$$
f\left(\mathcal{F}_{\mathrm{hn}}^{t}(\bar{E})\right) \subseteq \mathcal{F}_{\mathrm{hn}}^{t-h(f)}(\bar{F}) .
$$

Therefore the inequality (4.74) follows from Proposition 1.1.39.

$(2)$ Let $\lambda=\widehat{\mu}_{1}(\bar{E})$. Then $\mathcal{F}_{\text {hn }}^{\lambda}(\bar{E}) \neq\{0\}$. Since $f$ is injective, by Proposition 4.3.48, this implies that $\mathcal{F}_{\mathrm{hn}}^{\lambda-h(f)}(\bar{E}) \neq\{0\}$ and hence $\lambda-h(f) \leqslant \widehat{\mu}_{1}(\bar{F})$.

Proposition 4.3.55. - Let $\bar{E}=(E, \xi)$ be an adelic vector bundle on $S$ and

$$
0=E_{0} \subseteq E_{1} \subseteq \ldots \subseteq E_{n}
$$

be a flag of vector subspaces of E. One has

$$
\widehat{\operatorname{deg}}(\bar{E})-\Delta(\bar{E}) \leqslant \sum_{i=1}^{n} \widetilde{\operatorname{deg}}\left(\overline{E_{i} / E_{i-1}}\right) \leqslant \widehat{\operatorname{deg}}(\bar{E})
$$

If in addition $\xi$ is ultrametric on $\Omega \backslash \Omega_{\infty}$, one has

$$
\widehat{\operatorname{deg}}(\bar{E})-\delta(\bar{E}) \leqslant \sum_{i=1}^{n} \widetilde{\operatorname{deg}}\left(\overline{E_{i} / E_{i-1}}\right) \leqslant \widehat{\operatorname{deg}}(\bar{E})
$$

Proof. - By Propositions 4.3.49 and 4.3.50 (notably the inequality (4.68)), for any $i \in\{1, \ldots, n\}$, one has

$$
\widehat{\operatorname{deg}}\left(\overline{E_{i} / E_{i-1}}\right)-\Delta\left(\overline{E_{i} / E_{i-1}}\right) \leqslant \widetilde{\operatorname{deg}}\left(\overline{E_{i} / E_{i-1}}\right) \leqslant \widehat{\operatorname{deg}}\left(\overline{E_{i} / E_{i-1}}\right) .
$$

Taking the sum with respect to $i$, by Proposition 4.3.12, we obtain

$$
\widehat{\operatorname{deg}}(\bar{E})-\Delta(\bar{E}) \leqslant \sum_{i=1}^{n} \widetilde{\operatorname{deg}}\left(\overline{E_{i} / E_{i-1}}\right) \leqslant \widehat{\operatorname{deg}}(\bar{E}) .
$$

In the case where $\xi$ is ultrametric on $\Omega \backslash \Omega_{\infty}$, the above argument combined with (4.69) leads to (4.76).

Definition 4.3.56. - Let $\bar{E}$ be a non-zero adelic vector bundle on $S$. We say that $\bar{E}$ is semistable if its Harder-Narasimhan $\mathbb{R}$-filtration only have one jump point, namely one has $\widehat{\mu}_{1}(\bar{E})=\cdots=\widehat{\mu}_{r}(\bar{E})$ with $r=\operatorname{rk}_{K}(E)$. By definition, the following conditions are equivalent:

(1) $\bar{E}$ is semistable;

(2) for any non-zero vector subspace $F$ of $E$, one has $\widehat{\mu}_{\min }(\bar{F}) \leqslant \widehat{\mu}_{\min }(\bar{E})$;

(3) $\widetilde{\mu}(\bar{E})=\widehat{\mu}_{\min }(\bar{E})$. 
Theorem 4.3.5\%. - Let $\bar{E}$ be a non-zero adelic vector bundle on $S$. We assume that the Harder-Narasimhan $\mathbb{R}$-filtration corresponds to the flag

$$
0=E_{0} \subsetneq E_{1} \subsetneq \ldots \subsetneq E_{n}=E
$$

and the decreasing sequence $\mu_{1}>\ldots>\mu_{n}$ of real numbers. Then each subquotient $\overline{E_{i} / E_{i-1}}$ is semistable and $\widetilde{\mu}\left(\overline{E_{i} / E_{i-1}}\right)=\mu_{i}, i \in\{1, \ldots, n\}$. Moreover, (4.77) is the only flag of vector subspaces of $E$ such that each subquotient $\overline{E_{i} / E_{i-1}}$ is semistable and

$$
\widetilde{\mu}\left(\overline{E_{1} / E_{0}}\right)>\ldots>\widetilde{\mu}\left(\overline{E_{n} / E_{n-1}}\right) .
$$

Proof. - We begin with showing that each subquotient $\overline{E_{i} / E_{i-1}}$ is semistable and that $\widehat{\mu}\left(\overline{E_{i} / E_{i-1}}\right)=\mu_{i}$. The case where $i=1$ results from the definition of HarderNarasimhan $\mathbb{R}$-filtration. In what follows, we suppose that $i \geqslant 2$.

By Proposition 4.3.45, for any $j \in\{1, \ldots, n\}$ one has $\widehat{\mu}_{\min }\left(\bar{E}_{j}\right) \geqslant \mu_{j}$. Moreover, by definition of the Harder-Narasimhan $\mathbb{R}$-filtration, one has $\widehat{\mu}_{\min }\left(\bar{E}_{j}\right) \leqslant \mu_{j}$. Hence we obtain the equality $\widehat{\mu}_{\min }\left(\bar{E}_{j}\right)=\mu_{j}$.

We claim that any vector subspace $G^{\prime}$ of $E_{i} / E_{i-1}$ has a minimal slope $\leqslant \mu_{i}$. Let $\pi: E_{i} \rightarrow E_{i} / E_{i-1}$ be the canonical quotient map and $E_{i}^{\prime}$ be the preimage of $G^{\prime}$ by the quotient map $\pi$. Since $E_{i}^{\prime}$ contains strictly $E_{i-1}$, one has $\widehat{\mu}_{\min }\left(\overline{E_{i}^{\prime}}\right) \leqslant \mu_{i}$. For any $\epsilon>0$ there exists a quotient vector space $H^{\prime}$ of $E_{i}^{\prime}$ such that $\widehat{\mu}_{\max }\left(\bar{H}^{\prime}\right) \leqslant \mu_{i}+\epsilon$. If $\epsilon<\mu_{i-1}-\mu_{i}$, then $\widehat{\mu}_{\min }\left(\bar{E}_{i-1}\right)=\mu_{i-1}>\mu_{i}+\epsilon$. By Proposition 4.3 .30 (3), we obtain that the composed map $E_{i-1} \rightarrow E_{i}^{\prime} \rightarrow H^{\prime}$ is zero, or equivalently, $H^{\prime}$ is actually a quotient vector space of $E_{i}^{\prime} / E_{i-1}=G^{\prime}$. Hence we obtain $\widehat{\mu}_{\min }\left(\bar{G}^{\prime}\right) \leqslant \mu_{i}$. Therefore one has $\widehat{\mu}_{1}\left(\overline{E_{i} / E_{i-1}}\right) \leqslant \mu_{i} \leqslant \mu_{\min }\left(\overline{E_{i} / E_{i-1}}\right)$, which implies that $\overline{E_{i} / E_{i-1}}$ is semistable and $\widetilde{\mu}\left(\overline{E_{i} / E_{i-1}}\right)=\mu_{i}$.

We now proceed with the proof of the uniqueness by induction on the rank of $E$ over $K$. The case where $\operatorname{rk}_{K}(E)=1$ is trivial. In the following, we assume that the assertion has been proved for non-zero adelic vector bundles of $\operatorname{rank}<\operatorname{rk}_{K}(E)$. We still denote by

$$
0=E_{0} \subsetneq E_{1} \subsetneq \ldots \subsetneq E_{n}=E
$$

the Harder-Narasimhan flag of $\bar{E}$. Let

$$
0=F_{0} \subsetneq F_{1} \subsetneq \ldots \subsetneq F_{m}=E
$$

be a flag of vector subspaces of $E$ such that each subquotient $\overline{F_{j} / F_{j-1}}$ is semistable and that

$$
\widetilde{\mu}\left(\overline{F_{1} / F_{0}}\right)>\ldots>\widetilde{\mu}\left(\overline{F_{m} / F_{m-1}}\right) .
$$

Since the subquotients $\overline{F_{j} / F_{j-1}}$ are semistable, we can rewrite these inequalities as

$$
\widehat{\mu}_{\min }\left(\overline{F_{1} / F_{0}}\right)>\ldots>\widehat{\mu}_{\min }\left(\overline{F_{m} / F_{m-1}}\right) .
$$

We claim that $E_{1}$ is actually contained in $F_{1}$. Assume that $i$ is the smallest index in $\{1, \ldots, m\}$ such that $E_{1} \subset F_{i}$. We identifie $E_{1} /\left(E_{1} \cap F_{i-1}\right)$ with a vector subspace of 
$F_{i} / F_{i-1}$. Since $\overline{F_{i} / F_{i-1}}$ is semistable, one has

$$
\widehat{\mu}_{\min }\left(\overline{E_{1} /\left(E_{1} \cap F_{i-1}\right)}\right) \leqslant \widehat{\mu}_{\min }\left(\overline{F_{i} / F_{i-1}}\right) .
$$

If $i>1$, then by (4.78) one has $\widehat{\mu}_{\min }\left(\overline{F_{i} / F_{i-1}}\right)<\widehat{\mu}_{\min }\left(\overline{F_{1}}\right) \leqslant \widehat{\mu}_{\min }\left(\overline{E_{1}}\right)$, which leads to a contradiction since $\widehat{\mu}_{\min }\left(\overline{E_{1} /\left(E_{1} \cap F_{i-1}\right)}\right) \geqslant \widehat{\mu}_{\min }\left(\bar{E}_{1}\right)$. Therefore one has $E_{1} \subseteq F_{1}$. If the inclusion is strict, then by the definition of Harder-Narasimhan filtration one has $\widehat{\mu}_{\min }\left(\overline{F_{1}}\right)<\widehat{\mu}_{\min }\left(\overline{E_{1}}\right)$. This contradicts the semi-stability of $\overline{F_{1}}$. Therefore we have $E_{1}=F_{1}$. Moreover, for any vector subspace $M$ of $E$ which contains strictly $E_{1}$, one has $\widehat{\mu}_{\min }(\bar{M})<\widehat{\mu}_{\min }\left(\bar{E}_{1}\right)$. Hence, by Proposition 4.3.31 one has $\widehat{\mu}_{\min }(\bar{M})=$ $\widehat{\mu}_{\min }\left(\overline{M / E_{1}}\right)$. Therefore, if $E / E_{1}$ is non-zero, then

$$
0=E_{1} / E_{1} \subsetneq \ldots \subsetneq E_{n} / E_{1}=E / E_{1}
$$

is the Harder-Narasimhan flag of $E / E_{1}$. By the induction hypothesis one has $n=m$ and $E_{i}=F_{i}$ for any $i \in\{2, \ldots, n\}$. The uniqueness is thus proved.

Proposition 4.3.58. - Let $\bar{E}$ be a non-zero adelic vector bundle on $S$. The following assertions are equivalent:

(1) $\bar{E}$ is semistable,

(2) for any non-zero vector subspace $F$ of $E$, one has $\widetilde{\mu}(\bar{F}) \leqslant \widetilde{\mu}(\bar{E})$

(3) for any non-zero quotient vector space $G$ of $E$, one has $\widetilde{\mu}(\bar{G}) \geqslant \widetilde{\mu}(\bar{E})$.

Proof. - Let $r$ be the rank of $E$ over $K$. Assume that $\bar{E}$ is semistable. Then one has $\widehat{\mu}_{1}(\bar{E})=\cdots=\widehat{\mu}_{r}(\bar{E})=\widetilde{\mu}(\bar{E})$. If $F$ is a non-zero vector subspace of $E$, then by Proposition 4.3 .48 we obtain that, for any $t \in \mathbb{R}$, one has $\mathcal{F}_{\text {hn }}^{t}(\bar{F}) \subseteq \mathcal{F}_{\mathrm{hn}}^{t}(\bar{E})$. Therefore, for any $i \in\left\{1, \ldots, \operatorname{rk}_{K}(F)\right\}$ one has $\widehat{\mu}_{i}(\bar{F}) \leqslant \widetilde{\mu}(\bar{E})$, which implies $\widetilde{\mu}(\bar{F}) \leqslant$ $\widetilde{\mu}(\bar{E})$. Similarly, if $G$ is a non-zero quotient vector space of $E$ and $\pi: E \rightarrow G$ is the quotient map, then, by Proposition 4.3.48, one has $\pi\left(\mathcal{F}_{\mathrm{hn}}^{t}(\bar{E})\right) \subseteq \mathcal{F}_{\mathrm{hn}}^{t}(\bar{G})$ for any $t \in \mathbb{R}$. Therefor for any $i \in\left\{1, \ldots, \operatorname{rk}_{K}(G)\right\}$ one has $\widehat{\mu}_{i}(\bar{G}) \geqslant \widetilde{\mu}(\bar{E})$, which implies that $\widetilde{\mu}(\bar{G}) \geqslant \widetilde{\mu}(\bar{E})$. Hence we have proved the implications $(1) \Rightarrow(2)$ and $(1) \Rightarrow(3)$.

We will prove the converse implications by contraposition. Suppose that $\bar{E}$ is not semistable and its Harder-Narasimhan $\mathbb{R}$-filtration corresponds to the flag

$$
0=E_{0} \subsetneq E_{1} \subsetneq \ldots \subsetneq E_{n}=E,
$$

and the successive jump points $\mu_{1}<\ldots<\mu_{n}$, where $n \in \mathbb{N}, n \geqslant 1$. Then one has

$$
\widetilde{\mu}(\bar{E})=\frac{1}{\operatorname{rk}_{K}(E)} \sum_{i=1}^{n} \mu_{i} \operatorname{rk}_{K}\left(E_{i} / E_{i-1}\right) .
$$

By Theorem 4.3.57 we obtain that $\widetilde{\mu}\left(\bar{E}_{1}\right)=\mu_{1}>\widetilde{\mu}(\bar{E})$ and $\widetilde{\mu}\left(\overline{E_{n} / E_{n-1}}\right)=\mu_{n}<$ $\widetilde{\mu}(\bar{E})$. The proposition is thus proved.

Remark 4.3.59. - Consider the particular case where the adelic curve consists of exactly one copy of the trivial absolute value on $K$ (of measure 1 with respect to $\nu$ ). In this case an adelic vector bundle on $S$ is just a finite-dimensional vector space $E$ over 
$K$ equipped with a norm $\|\cdot\|$ (which is not necessary ultrametric), where we consider the trivial absolute value on $K$. We have shown in $\$ 1.1 .8$ that ultrametric norms on a finite-dimensional vector space over $K$ correspond bijectively to $\mathbb{R}$-filtrations on the same vector space. In particular, if $(E,\|\cdot\|)$ is a Hermitian adelic vector bundle on $S$, then the $\mathbb{R}$-filtration on $E$ corresponding to $\|\cdot\|$ identifies with the Harder-Narasimhan $\mathbb{R}$-filtration of $(E,\|\cdot\|)$.

Proposition 4.3.60. - We equip $K$ with the trivial absolute value. Let $(E,\|\cdot\|)$ be a finite-dimensional normed vector space over $K$, which is also considered as an adelic vector bundle as in Remark 4.3.59. The adelic vector bundle $(E,\|\cdot\|)$ is semistable if and only if the double dual norm $\|\cdot\|_{* *}$ is constant on $E \backslash\{0\}$. Moreover, in this case one has

for any $x \in E \backslash\{0\}$.

$$
-\ln \|x\|_{* *}=\widehat{\mu}(E,\|\cdot\|)=\widehat{\mu}_{\min }(E,\|\cdot\|)
$$

Proof. - First we assume that $(E,\|\cdot\|)$ is semistable. Let $\left\{e_{i}\right\}_{i=1}^{r}$ be an $\alpha$-orthogonal basis of $(E,\|\cdot\|)$, where $\alpha \in] 0,1\left[\right.$. Without loss of generality, we assume that $\left\|e_{1}\right\| \leqslant$ $\ldots \leqslant\left\|e_{r}\right\|$. Moreover, by Proposition 1.2 .23 one has

$$
\widehat{\operatorname{deg}}(E,\|\cdot\|) \leqslant-r \ln (\alpha)-\sum_{i=1}^{r} \ln \left\|e_{i}\right\| .
$$

In particular, if $\left\|e_{r}\right\| /\left\|e_{1}\right\|>\alpha^{-r}$, that is,

$$
-\frac{1}{r} \ln \left\|e_{1}\right\|>-\frac{1}{r} \ln \left\|e_{r}\right\|-\ln (\alpha),
$$

then

$$
\begin{aligned}
-\ln \left\|e_{1}\right\| & =-\frac{r-1}{r} \ln \left\|e_{1}\right\|-\frac{1}{r} \ln \left\|e_{1}\right\|>-\frac{1}{r} \sum_{i=1}^{r-1} \ln \left\|e_{i}\right\|-\frac{1}{r} \ln \left\|e_{r}\right\|-\ln (\alpha) \\
& \geqslant \widehat{\mu}(E,\|\cdot\|) \geqslant \widehat{\mu}_{\min }(E,\|\cdot\|),
\end{aligned}
$$

which shows that $(E,\|\cdot\|)$ is not semistable, so that $\left\|e_{r}\right\| /\left\|e_{1}\right\| \leqslant \alpha^{-r}$. This observation shows that, for any $\alpha$-orthogonal basis $\left\{e_{i}\right\}_{i=1}^{r}$ of $E$, one has

$$
\max _{(i, j) \in\{1, \ldots, r\}^{2}}\left|\ln \left\|e_{i}\right\|-\ln \left\|e_{j}\right\|\right| \leqslant-r \ln (\alpha) .
$$

Note that $\left\{e_{i}\right\}_{i=1}^{r}$ is also an $\alpha$-orthogonal basis of $\left(E,\|\cdot\|_{* *}\right)$ (see Proposition 1.2.11). Moreover, we deduce from (4.79) and (1.25) that

$$
\max _{(i, j) \in\{1, \ldots, r\}^{2}}\left|\ln \left\|e_{i}\right\|_{* *}-\ln \left\|e_{j}\right\|_{* *}\right| \leqslant-(r+1) \ln (\alpha) .
$$

Note that one has $\widehat{\operatorname{deg}}(E,\|\cdot\|)=\widehat{\operatorname{deg}}\left(E,\|\cdot\|_{* *}\right)$ (see Proposition 1.2.15). Moreover, by Propositions 1.1.66 and 1.2.23 one has

$$
-\sum_{i=1}^{r} \ln \left\|e_{i}\right\|_{* *} \leqslant \widehat{\operatorname{deg}}(E,\|\cdot\|) \leqslant-r \ln (\alpha)-\sum_{i=1}^{r} \ln \left\|e_{i}\right\|_{* *} .
$$


Combining this estimate with (4.80) we obtain

$$
\max _{i \in\{1, \ldots, r\}}\left|\ln \left\|e_{i}\right\|_{* *}-\widehat{\mu}(E,\|\cdot\|)\right| \leqslant-(r+2) \ln (\alpha) .
$$

In particular, for any $\left(\lambda_{1}, \ldots, \lambda_{r}\right) \in K^{r} \backslash\{(0, \ldots, 0)\}$, one has

$$
\left|\ln \left\|\lambda_{1} e_{1}+\cdots+\lambda_{r} e_{r}\right\|_{* *}-\widehat{\mu}(E,\|\cdot\|)\right| \leqslant-(r+3) \ln (\alpha)
$$

Since $(E,\|\cdot\|)$ admits an $\alpha$-orthogonal basis for any $\alpha \in] 0,1[$ (see Corollary [1.2.9), we obtain that the restriction of $\ln \|\cdot\|_{* *}$ to $E \backslash\{0\}$ is constant (which is equal to $-\widehat{\mu}(E,\|\cdot\|))$.

Assume now that the double dual norm $\|\cdot\|_{* *}$ is constant on $E \backslash\{0\}$. Since $\|\cdot\|_{* *}$ and $\|\cdot\|$ induce the same dual norm on $E^{\vee}$, we obtain that the restriction of $\ln \|\cdot\|_{*}$ to $E^{\vee} \backslash$ $\{0\}$ is constant and takes $-\widehat{\mu}\left(E^{\vee},\|\cdot\|_{*}\right)$ as its value. Note that one has $-\widehat{\mu}\left(E^{\vee},\|\cdot\|_{*}\right)=$ $\widehat{\mu}(E,\|\cdot\|)$ by Proposition 4.3.9. We will show that $(E,\|\cdot\|)$ is semistable. First we show that $\widehat{\mu}_{\min }(E,\|\cdot\|)=\widehat{\mu}(E,\|\cdot\|)$. Let $G$ be a non-zero quotient vector space of $E$ and $\|\cdot\|_{G}$ be the quotient norm of $\|\cdot\|$ on $G$. By Proposition 1.1.20, $\|\cdot\|_{G, *}$ coincides with the restriction of $\|\cdot\|_{*}$ to $G^{\vee}$. Since the function $\ln \|\cdot\|_{*}$ takes constant value $\widehat{\mu}(E,\|\cdot\|)$ on $E^{\vee} \backslash\{0\}$ we obtain that

$$
\widehat{\mu}\left(G,\|\cdot\|_{G}\right)=-\widehat{\mu}\left(G^{\vee},\|\cdot\|_{G, *}\right)=\widehat{\mu}(E,\|\cdot\|) .
$$

Therefore $\widehat{\mu}_{\min }(E,\|\cdot\|)=\widehat{\mu}(E,\|\cdot\|)$. Now for any non-zero vector subspace $F$ of $E$ one has

$$
\widehat{\mu}_{\min }\left(F,\|\cdot\|_{F}\right) \leqslant \widehat{\mu}\left(F,\|\cdot\|_{F}\right) \leqslant \widehat{\mu}\left(E,\|\cdot\|_{E}\right),
$$

where $\|\cdot\|_{F}$ denotes the restriction of $\|\cdot\|$ to $F$. In fact, $\ln \|\cdot\|_{F}$ is bounded from below by the restriction of $\ln \|\cdot\|_{* *}$ to $F$, which is constant on $F \backslash\{0\}$ of value $-\widehat{\mu}(E,\|\cdot\|)$. Therefore $(E,\|\cdot\|)$ is semistable.

Remark 4.3.61. - We keep the notation of the previous proposition. Note that the norms $\|\cdot\|$ and $\|\cdot\|_{* *}$ induce the same dual norm on $E^{\vee}$ (see Proposition 1.2.14 $(1)$, so that we obtain that the adelic vector bundle $(E,\|\cdot\|)$ is semistable if and only if the restriction of the function $\|\cdot\|_{*}$ on $E^{\vee} \backslash\{0\}$ is constant. Moreover, in this case one has (see Proposition 1.2.47)

$$
\forall \varphi \in E^{\vee} \backslash\{0\}, \quad-\ln \|\varphi\|_{*}=-\widehat{\operatorname{deg}}(E,\|\cdot\|) .
$$

Remark 4.3.62. - In the case where $\|\cdot\|$ is ultrmetric, the normed vector space $(E,\|\cdot\|)$ corresponds to a sequence

$$
0=E_{0} \subsetneq E_{1} \subsetneq \ldots \subsetneq E_{n}=E
$$

of vector subspaces of $E$ and a decreasing sequence $\mu_{1}>\ldots>\mu_{n}$ of real numbers (see Remark 1.1.40). Note that, for any $i \in\{1, \ldots, n\}$ the restriction of the subquotient norm $\|\cdot\|_{E_{i} / E_{i-1}}$ to $\left(E_{i} / E_{i-1}\right) \backslash\{0\}$ is constant and takes $\mathrm{e}^{-\mu_{i}}$ as its value. Therefore 
Proposition 4.3 .60 implies that $\left(E_{i} / E_{i-1},\|\cdot\|_{E_{i} / E_{i-1}}\right)$ is semistable and admits $\mu_{i}$ as its minimal slope. Therefore Theorem 4.3 .57 shows that

$$
0=E_{0} \subsetneq E_{1} \subsetneq \ldots \subsetneq E_{n}=E
$$

is the Harder-Narasimhan flag of the adelic vector bundle $(E,\|\cdot\|)$.

Proposition 4.3.63. - We equip $K$ with the trivial absolute value. Consider a finite-dimensional non-zero normed vector space $(E,\|\cdot\|)$ over $K$. The HarderNarasimhan flags of $(E,\|\cdot\|)$ and $\left(E,\|\cdot\|_{* *}\right)$ are the same. Moreover, for any $i \in$ $\left\{1, \ldots, \operatorname{rk}_{K}(E)\right\}$ one has $\widehat{\mu}_{i}(E,\|\cdot\|)=\widehat{\mu}_{i}\left(E,\|\cdot\|_{* *}\right)$.

Proof. - Let $n$ be the rank of $E$ over $K$. We reason by induction on $n$. First of all, if $(E,\|\cdot\|)$ is semistable, then by Proposition 4.3 .60 (see also its proof), the function - $\ln \|\cdot\|_{* *}$ is constant on $E \backslash\{0\}$ and takes $\widehat{\mu}(E,\|\cdot\|)=\widehat{\mu}_{\min }(\|\cdot\|)$ as its value. Therefore the assertion of the proposition holds in this case, and in particular the assertion is true when $n=1$. In the following we suppose that $(E,\|\cdot\|)$ is not semistable (hence $n \geqslant 2$ ) and that the proposition has been proved for normed vector spaces of dimension $\leqslant n-1$.

For any $i \in\{1, \ldots, n\}$, let $\mu_{i}=\widehat{\mu}_{i}\left(E,\|\cdot\|_{* *}\right)$ and $E_{i}$ be the ball of radius $\mathrm{e}^{-\mu_{i}}$ in $\left(E,\|\cdot\|_{* *}\right)$ centered at the origin. Let

$$
\beta=\min \left\{\mu_{i}-\mu_{i-1}: i \in\{2, \ldots, n\}, \mu_{i}>\mu_{i-1}\right\}
$$

and $\alpha$ be an element of ]0,1[ such that $\alpha>\mathrm{e}^{-\beta / n}$. Let $\left\{e_{i}\right\}_{i=1}^{n}$ be an $\alpha$-orthogonal bases of $(E,\|\cdot\|)$. By Proposition 1.2.11, it is also an $\alpha$-orthgonal basis of $\left(E,\|\cdot\|_{* *}\right)$. By Proposition 1.2.26, $\left\{e_{i}\right\}_{i=1}^{n}$ is an orthogonal basis of $\left(E,\|\cdot\|_{* *}\right)$. Without loss of generality, we may assume that

$$
\left\{e_{i}\right\}_{i=1}^{n} \cap E_{1}=\left\{e_{1}, \ldots, e_{m}\right\},
$$

where $m$ is the rank of $E_{1}$ over $K$ (see Proposition 1.2.26 (1)]. Let $\left\{e_{i}^{\vee}\right\}_{i=1}^{n}$ be the dual basis of $\left\{e_{i}\right\}_{i=1}^{n}$ and $\|\cdot\|_{1}$ be the restriction of the norm $\|\cdot\|$ to $E_{1}$. For any $i \in\{1, \ldots, m\}$, let $\varphi_{i}$ be the restriction of $e_{i}^{\vee}$ on $E_{1}$. Then $\left\{\varphi_{i}\right\}_{i=1}^{m}$ forms a basis of $E_{1}^{\vee}$, which is the dual basis of $\left\{e_{i}\right\}_{i=1}^{m}$. By lemma 1.2.10 $\left\{\varphi_{i}\right\}_{i=1}^{m}$ is an $\alpha$-orthogonal basis of $E_{1}^{\vee}$, and one has

$$
\forall i \in\{1, \ldots, m\}, \quad\left\|e_{i}\right\|^{-1} \leqslant\left\|\varphi_{i}\right\|_{1, *} \leqslant \alpha^{-1}\left\|e_{i}\right\|^{-1} .
$$

By Proposition 1.2.11, one has

$$
\forall i \in\{1, \ldots, m\}, \quad \alpha\left\|e_{i}\right\| \leqslant\left\|e_{i}\right\|_{* *}=\mathrm{e}^{-\mu_{1}} \leqslant\left\|e_{i}\right\| .
$$

Therefore one obtains

$$
\forall i \in\{1, \ldots, m\}, \quad \alpha \mathrm{e}^{\mu_{1}} \leqslant\left\|\varphi_{i}\right\|_{1, *} \leqslant \alpha^{-1} \mathrm{e}^{\mu_{1}} .
$$


As a consequence, for a general non-zero element $\varphi$ of $E_{1}^{\vee}$, which is written in the form $\lambda_{1} \varphi_{1}+\cdots+\lambda_{m} \varphi_{m}$, one has

$$
\|\varphi\|_{1, *} \leqslant \max _{\substack{i \in\{1, \ldots, m\} \\ \lambda_{i} \neq 0}}\left\|\varphi_{i}\right\|_{1, *} \leqslant \alpha^{-1} \mathrm{e}^{\mu_{1}}
$$

and

$$
\|\varphi\|_{1, *} \geqslant \alpha \max _{\substack{i \in\{1, \ldots, m\} \\ \lambda_{i} \neq 0}}\left\|\varphi_{i}\right\|_{1, *} \geqslant \alpha^{2} \mathrm{e}^{\mu_{1}} .
$$

Since $(E,\|\cdot\|)$ admits an $\alpha$-orthogonal basis for any $\alpha \in] 0,1[$, we obtain that the restriction of $\|\cdot\|_{1, *}$ on $E_{1} \backslash\{0\}$ is constant and takes $\mathrm{e}^{\mu_{1}}$ as its value. Therefore, Proposition 4.3.60 (see also Remark 4.3.61), we obtain that $\left(E_{1},\|\cdot\|\right)$ is semistable and admits $\mu_{1}$ as its minimal slope.

By Proposition 1.1.20, one has (see Definition 1.1.2 and Subsection 1.1.3 for notation)

$$
\|\cdot\|_{*,\left(E / E_{1}\right)^{\vee} \hookrightarrow E^{\vee}}=\|\cdot\|_{E \rightarrow E / E_{1}, *} \cdot
$$

Moreover, since $\|\cdot\|_{*}$ is ultrametric, by Proposition 1.2.35 one has

$$
\|\cdot\|_{* *, E \rightarrow E / E_{1}}=\|\cdot\|_{*,\left(E / E_{1}\right)^{\vee} \hookrightarrow E^{\vee}, *}=\|\cdot\|_{E \rightarrow E / E_{1}, * *} \cdot
$$

Applying the induction hypothesis to $\left(E / E_{1},\|\cdot\|_{E \rightarrow E / E_{1}}\right)$ we obtain that the Harder-Narasimhan flags and the successive slopes of $\left(E / E_{1},\|\cdot\|_{E \rightarrow E / E_{1}}\right)$ and $\left(E / E_{1},\|\cdot\|_{* *, E \rightarrow E / E_{1}}\right)$ are the same. Therefore, by Theorem 4.3.57 we obtain that the Harder-Narasimhan flag and the successive slopes of $(E,\|\cdot\|)$ coincides with those of $\left(E,\|\cdot\|_{* *}\right)$. The proposition is thus proved.

Remark 4.3.64. - In the framework of linear code, Randriambololona [18] has proposed a Harder-Narasimhan theory based on semimodular degree functions on the modular lattice of vector subspaces. Note that our approach, which relies on the Arakelov degree function of quotient vector spaces, has a very different nature from the classic method (due to the fact that the equality (4.29) and the inequality (4.50) fail in general for non-Hermitian adelic vector bundles). It is an intriguing question to compare the Harder-Narasimhan filtrations constructed in our setting and in $\mathbf{1 1 8}$.

4.3.12. Absolute positive degree and absolute maximal slope. - We have seen in Proposition 4.3.13 that the Arakelov degree is preserved by extension of scalars. In this subsection, we discuss the behaviour of the maximal slope and the positive degree under extension of scalars to the algebraic closure of $K$. We denote by $K^{\text {ac }}$ the algebraic closure of the field $K$.

Definition 4.3.65. - Let $(E, \xi)$ be an adelic vector bundle on $S$. We denote by $\widehat{\operatorname{deg}}_{+}^{\mathrm{a}}(E, \xi)$ the positive degree of $\left(E_{K^{\mathrm{ac}}}, \xi_{K^{\mathrm{ac}}}\right)$, called the absolute positive degree of $(E, \xi)$. If $E$ is non-zero, we denote by $\widehat{\mu}_{\max }^{\mathrm{a}}(E, \xi)$ the maximal slope of $\left(E_{K^{\mathrm{ac}}}, \xi_{K^{\mathrm{ac}}}\right)$, called the absolute maximal slope of $(E, \xi)$. 
Proposition 4.3.66. — Let $(E, \xi)$ be an adelic vector bundle on $S$. One has

$$
\widehat{\operatorname{deg}}_{+}(E, \xi) \leqslant \widehat{\operatorname{deg}}_{+}^{\mathrm{a}}(E, \xi) \text { and } \widehat{\mu}_{\max }(E, \xi) \leqslant \widehat{\mu}_{\max }^{\mathrm{a}}(E, \xi) \text {. }
$$

Moreover, for any algebraic extension $L$ of $K$, one has

$$
\widehat{\operatorname{deg}}_{+}^{\mathrm{a}}(E, \xi)=\widehat{\operatorname{deg}}_{+}^{\mathrm{a}}\left(E_{L}, \xi_{L}\right) \text { and } \widehat{\mu}_{\max }^{\mathrm{a}}(E, \xi)=\widehat{\mu}_{\max }^{\mathrm{a}}\left(E_{L}, \xi_{L}\right) .
$$

Proof. - Let $F$ be a vector subspace of $E$ and $\xi_{F}$ be the restriction of $\xi$ to $F$. Let $\xi_{F_{K} \text { ac }}$ be the restriction of $\xi_{K^{\text {ac }}}$ to $F_{K^{\mathrm{ac}}}$. By Proposition 1.3.17 (1), (2), the identity $\operatorname{map}\left(F_{K^{\text {ac }}}, \xi_{F, K^{\mathrm{ac}}}\right) \rightarrow\left(F_{K^{\mathrm{ac}}}, \xi_{F_{K^{\mathrm{ac}}}}\right)$ has norm $\leqslant 1$ on any $\omega \in \Omega$. By Proposition 4.3.17, one has

$\widehat{\operatorname{deg}}\left(F, \xi_{F}\right)=\widehat{\operatorname{deg}}\left(F_{K^{\mathrm{ac}}}, \xi_{F, K^{\mathrm{ac}}}\right) \leqslant \widehat{\operatorname{deg}}\left(F_{K^{\mathrm{ac}}}, \xi_{F_{K^{\mathrm{ac}}}}\right) \leqslant \widehat{\operatorname{deg}_{+}}\left(E_{K^{\mathrm{ac}}}, \xi_{K^{\mathrm{ac}}}\right)=\widehat{\operatorname{deg}}_{+}^{\mathrm{a}}(E, \xi)$,

where the first equality comes from Proposition 4.3.13. Similarly, if $F$ is non-zero, one has

$$
\widehat{\mu}\left(F, \xi_{F}\right) \leqslant \widehat{\mu}\left(F_{K^{\mathrm{ac}}}, \xi_{F, K^{\mathrm{ac}}}\right) \leqslant \widehat{\mu}\left(F_{K^{\mathrm{ac}}}, \xi_{F_{K^{\mathrm{ac}}}}\right) \leqslant \widehat{\mu}_{\max }\left(F_{K^{\mathrm{ac}}}, \xi_{F_{K^{\mathrm{ac}}}}\right)=\widehat{\mu}_{\max }^{\mathrm{a}}\left(F, \xi_{F}\right) .
$$

Since $F$ is arbitrary, we obtain

$$
\widehat{\operatorname{deg}}_{+}(E, \xi) \leqslant \widehat{\operatorname{deg}}_{+}^{\mathrm{a}}(E, \xi) \text { and } \widehat{\mu}_{\max }(E, \xi) \leqslant \widehat{\mu}_{\max }^{\mathrm{a}}(E, \xi) .
$$

By Corollary 1.3.15, if $L$ is an algebraic extension of $K$, then one has $\left(\xi_{L}\right)_{K^{\mathrm{ac}}}=$ $\xi_{K^{\mathrm{ac}}}$. Therefore $\widehat{\operatorname{deg}}_{+}^{\mathrm{a}}(E, \xi)=\widehat{\operatorname{deg}}_{+}^{\mathrm{a}}\left(E_{L}, \xi_{L}\right)$, and $\widehat{\mu}_{\max }^{\mathrm{a}}(E, \xi)=\widehat{\mu}_{\max }^{\mathrm{a}}\left(E_{L}, \xi_{L}\right)$.

Proposition 4.3.67. - Assume that the field $K$ is perfect. Let $(E, \xi)$ be a Hermitian adelic vector bundle on $S$. Then one has

$$
\widehat{\operatorname{deg}}_{+}(E, \xi)=\widehat{\operatorname{deg}}_{+}^{\mathrm{a}}(E, \xi) \quad \text { and } \quad \widehat{\mu}_{\max }(E, \xi)=\widehat{\mu}_{\max }^{\mathrm{a}}(E, \xi) \text {. }
$$

Proof. - Without loss of generality, we may assume that the vector space $E$ is nonzero. Let

$$
\{0\}=\widetilde{E}_{0} \subsetneq \widetilde{E}_{1} \subsetneq \ldots \subsetneq \widetilde{E}_{n}=E_{K^{\mathrm{a}}}
$$

be the Harder-Narasimhan flag of $\left(E_{K^{\mathrm{ac}}}, \xi_{K^{\mathrm{ac}}}\right)$. By the uniqueness of HarderNarasimhan filtration (see Proposition 4.3.37), for any $K$-automorphism $\tau$ of $K^{\text {ac }}$ and any $i \in\{1, \ldots, n\}$, the vector space $\widetilde{E}_{i}$ is stable by $\tau$. Since the filed $K$ is perfect, by Galois descent (see 29], Chapter V, $§ 10$, no.4, Corollary of Proposition 6), there exists a flag

$$
\{0\}=E_{0} \subsetneq E_{1} \subsetneq \ldots \subsetneq E_{n}=E
$$

such that $\widetilde{E}_{i}=E_{i, K^{\text {a }}}$ for any $i \in\{1, \ldots, n\}$. Moreover, by Propositions 1.3.17 (1) (2) (here we use the hypothesis that $\xi$ is Hermitian), if we denote by $\xi_{i}$ the restriction of $\xi$ to $E_{i}$, the $\xi_{i, K^{\text {ac }}}$ coincides with the restriction $\widetilde{\xi}_{i}$ of $\xi_{K^{\text {ac }}}$ to $\widetilde{E}_{i}$. Therefore by Proposition 4.3.13 one has $\widehat{\operatorname{deg}}\left(E_{i}, \xi_{i}\right)=\widehat{\operatorname{deg}}\left(\widetilde{E}_{i}, \widetilde{\xi}_{i}\right)$. We then deduce that the slopes of $E_{i} / E_{i-1}$ and $\widetilde{E}_{i} / \widetilde{E}_{i-1}$ (equipped with subquotient norm families) are the same. 
Hence by Proposition 4.3.37 we obtain that (4.81) is the Harder-Narasimhan flag of $(E, \xi)$. Therefore,

$$
\widehat{\mu}_{\max }(E, \xi)=\widehat{\mu}\left(E_{1}, \xi_{1}\right)=\widehat{\mu}\left(\widetilde{E}_{1}, \widetilde{\xi}_{1}\right)=\widehat{\mu}_{\max }^{\mathrm{a}}(E, \xi)
$$

and

$$
\widehat{\operatorname{deg}}_{+}(E, \xi)=\max _{i \in\{0, \ldots, n\}} \widehat{\operatorname{deg}}\left(E_{i}, \xi_{i}\right)=\max _{i \in\{0, \ldots, n\}} \widehat{\operatorname{deg}}\left(\widetilde{E}_{i}, \widetilde{\xi}_{i}\right)=\widehat{\operatorname{deg}}_{+}^{\mathrm{a}}(E, \xi)
$$

4.3.13. Successive minima. - The successive minima are classic invariants of Hermitian vector bundles on an arithmetic curve. In this subsection, we extend their construction (more precisely, the construction of successive minima of Roy-Thunder 121]) to the setting of adelic vector bundles on an adelic curve.

Definition 4.3.68. - Let $(E, \xi)$ be an adelic vector bundle on $S$ and $r$ be the rank of $E$ over $K$. For any $i \in\{1, \ldots, r\}$, let

$$
\nu_{i}(E, \xi):=\sup \left\{t \in \mathbb{R}: \operatorname{rk}_{K}\left(\operatorname{Vect}_{K}\left(\left\{s \in E_{K}: \widehat{\operatorname{deg}}_{\xi}(s) \geqslant t\right\}\right)\right) \geqslant i\right\}
$$

called the $i^{\text {th }}$ (logarithmic) minimum of $(E, \xi)$. In other words, $\nu_{i}(E, \xi)$ is the supremum of the set of real numbers $t$ such that there exist at list $i$ linearly independent vectors of Arakelov degree $\geqslant t$. Clearly one has

$$
\nu_{1}(E, \xi) \geqslant \ldots \geqslant \nu_{r}(E, \xi)
$$

The first minimum $\nu_{1}(E, \xi)$ is also denoted by $\nu_{\max }(E, \xi)$, and the last minimum $\nu_{r}(E, \xi)$ is also denoted by $\nu_{\min }(E, \xi)$. For any $t \in \mathbb{R}$, let

$$
\mathcal{F}_{\mathrm{m}}^{t}(E, \xi):=\bigcap_{\varepsilon>0} \operatorname{Vect}_{K}\left(\left\{s \in E \backslash\{0\}: \widehat{\operatorname{deg}}_{\xi}(s) \geqslant t-\varepsilon\right\}\right) .
$$

By definition

$$
\nu_{i}(E, \xi)=\sup \left\{t \in \mathbb{R}: \operatorname{rk}_{K}\left(\mathcal{F}_{\mathrm{m}}^{t}(\bar{E})\right) \geqslant i\right\} .
$$

If $E$ is the zero vector space, then by convention we define

$$
\nu_{\max }(E, \xi):=-\infty \quad \text { and } \quad \nu_{\min }(E, \xi):=+\infty
$$

We also define the absolute version of the successive minima as follows. For any $i \in\{1, \ldots, r\}$, let $\nu_{i}^{\mathrm{a}}(E, \xi):=\nu_{i}\left(E_{K^{\mathrm{a}}}, \xi_{K^{\mathrm{a}}}\right)$, where $K^{\mathrm{a}}$ denotes the algebraic closure of $(E, \xi)$. Similarly, we let

$$
\nu_{\max }^{\mathrm{a}}(E, \xi):=\nu_{\max }\left(E_{K^{\mathrm{a}}}, \xi_{K^{\mathrm{a}}}\right) \quad \text { and } \quad \nu_{\min }^{\mathrm{a}}(E, \xi):=\nu_{\min }\left(E_{K^{\mathrm{a}}}, \xi_{K^{\mathrm{a}}}\right) .
$$

Proposition 4.3.69. - Let $\bar{E}=(E, \xi)$ be a non-zero adelic vector bundle on $S$. For any $t \in \mathbb{R}$ one has

$$
\mathcal{F}_{\mathrm{m}}^{t}(\bar{E})=\bigcap_{\varepsilon>0} \sum_{\substack{0 \neq F \subseteq E \\ \nu_{\min }(\bar{F}) \geqslant t-\varepsilon}} F
$$


where $F$ runs over the set of all non-zero vector subspaces of $E$, and in the structure of adelic vector bundle of $\bar{F}$ we consider the restricted norm family.

Proof. - Let $\varepsilon>0$ and $F$ be a non-zero vector subspace of $E$ such that $\nu_{\min }(\bar{F})>$ $t-\varepsilon$. There exists a basis $\left\{s_{i}\right\}_{i=1}^{n}$ of $F$ over $K$ such that

$$
\min _{i \in\{1, \ldots, n\}} \widehat{\operatorname{deg}}_{\xi}\left(s_{i}\right) \geqslant t-2 \varepsilon \text {. }
$$

Therefore one has

$$
\sum_{\substack{0 \neq F \subseteq E \\ \widehat{\mu}_{\text {min }}(F) \geqslant t-\varepsilon}} F \subseteq \operatorname{Vect}_{K}\left(\left\{s \in E \backslash\{0\}: \widehat{\operatorname{deg}}_{\xi}(s) \geqslant t-2 \varepsilon\right\}\right) .
$$

Conversely, for any $t \in \mathbb{R}$ such that $\mathcal{F}_{\mathrm{m}}^{t}(\bar{E}) \neq\{0\}$ and any $\varepsilon>0$, there exist elements $u_{1}, \ldots, u_{r}$ in $E$ which generates $\mathcal{F}_{\mathrm{m}}^{t}(\bar{E})$ as vector space over $K$ and such that

$$
\forall j \in\{1, \ldots, r\}, \quad \widehat{\operatorname{deg}}_{\xi}\left(u_{j}\right) \geqslant t-\varepsilon .
$$

Therefore $\nu_{\min }\left(\mathcal{F}_{\mathrm{m}}^{t}(\bar{E})\right) \geqslant t$.

Proposition 4.3.70. - Let $(E, \xi)$ be an adelic vector bundle on $S$ and $r$ be the rank of $E$ over $K$. For any $i \in\{1, \ldots, r\}$, one has $\nu_{i}(E, \xi) \leqslant \nu_{i}^{\mathrm{a}}(E, \xi)$. Moreover, for any algebraic extension $L$ of $K$ and any $i \in\{1, \ldots, r\}$ one has $\nu_{i}^{\mathrm{a}}(E, \xi)=\nu_{i}^{\mathrm{a}}\left(E_{L}, \xi_{L}\right)$.

Proof. - Let $\left\{s_{j}\right\}_{j=1}^{i}$ be a linearly independent family in $E$. Then it is also a linearly independent family in $E_{K^{\mathrm{a}}}$. Moreover, by the same argument as in the proof of Proposition 4.3.66, for any $j \in\{1, \ldots, i\}$ one has $\widehat{\operatorname{deg}}_{\xi}\left(s_{j}\right) \leqslant \widehat{\operatorname{deg}}_{\xi_{K^{\mathrm{a}}}}\left(s_{j}\right)$. Therefore $\nu_{i}(E, \xi) \leqslant \nu_{i}\left(E_{K^{\mathrm{a}}}, \xi_{K^{\mathrm{a}}}\right)=\nu_{i}^{\mathrm{a}}(E, \xi)$. The equality $\nu_{i}^{\mathrm{a}}\left(E_{L}, \xi_{L}\right)=\nu_{i}^{\mathrm{a}}(E, \xi)$ comes from the relation $\left(\xi_{L}\right)_{K^{\mathrm{a}}}=\xi_{K^{\mathrm{a}}}$, which is a consequence of Corollary 1.3.15.

The following proposition is straightforward from the definition of the (absolute) fist minimum and the (absolute) maximal slope.

Proposition 4.3.71. - If $(E, \xi)$ is an adelic vector bundle on $S$, then one has

$$
\nu_{1}(E, \xi) \leqslant \widehat{\mu}_{\max }(E, \xi) \quad \text { and } \quad \nu_{1}^{\mathrm{a}}(E, \xi) \leqslant \widehat{\mu}_{\max }^{\mathrm{a}}(E, \xi) \text {. }
$$

\subsubsection{Minkowski property. -}

Definition 4.3.72. - Let $S=(K,(\Omega, \mathcal{A}, \nu), \phi)$ be an adelic curve. Let $C$ be a nonnegative real number. We say that the adelic curve $S$ satisfies the Minkowski property of level $\geqslant C$ if, for any adelic vector bundle $(E, \xi)$ on $S$ such that $\xi$ is ultrametric on $\Omega \backslash \Omega_{\infty}$, one has

$$
\nu_{1}(E, \xi) \geqslant \widehat{\mu}_{\max }(E, \xi)-C \ln \left(\operatorname{rk}_{K}(E)\right) .
$$

We say that $S$ satisfies the absolute Minkowski property of level $\geqslant C$ if, for any adelic vector bundle $(E, \xi)$ on $S$, one has

$$
\nu_{1}^{\mathrm{a}}(E, \xi) \geqslant \widehat{\mu}_{\max }^{\mathrm{a}}(E, \xi)-C \ln \left(\mathrm{rk}_{K}(E)\right) .
$$


Remark 4.3.73. - Let $\bar{V}$ be an Euclidean lattice. The first theorem of Minkowski can be stated as (see [23, $\S 3.2]$ for more details)

$$
\nu_{1}(\bar{V}) \geqslant \widehat{\mu}_{\max }(\bar{V})-\frac{1}{2} \ln (\operatorname{rk}(V)) .
$$

Hence the Minkowski property should be considered as an analogue in the general setting of adelic curve of the statement of the first theorem of Minkowski. For general number fields, it has been shown in [64, §5] that, for any adelic vector bundle $\bar{E}$ of rank $n$ over a number field $K$, one has

$$
\nu_{1}(\bar{E}) \geqslant \widehat{\mu}_{\max }(\bar{E})-\frac{1}{2} \ln (n)-\frac{1}{2} \ln \left|\mathfrak{D}_{K / \mathbb{Q}}\right|
$$

and

$$
\nu_{1}^{\mathrm{a}}(\bar{E}) \geqslant \widehat{\mu}_{\max }(\bar{E})-\frac{1}{2} \sum_{\ell=2}^{n} \frac{1}{\ell}=\widehat{\mu}_{\max }^{\mathrm{a}}(\bar{E})-\frac{1}{2} \sum_{\ell=2}^{n} \frac{1}{\ell}
$$

where $\mathfrak{D}_{K / \mathbb{Q}}$ is the discriminant of $K$ over $\mathbb{Q}$. Therefore the adelic curve corresponding to a number field satisfies the Minkowski property of level $\geqslant \frac{1}{2}+\frac{1}{2} \ln \left|\mathfrak{D}_{K / \mathbb{Q}}\right|$ and the absolute Minkowski property of level $\geqslant \frac{1}{2}$.

In the function field case, given a regular projective curve (over a base field), by Riemann-Roch formula there exists a constant $A>0$ which only depends on the curve, such that

$$
\nu_{1}(E) \geqslant \mu_{\max }(E)-A
$$

for any vector bundle $E$ on the curve (see 40, Remark 8.3]). Therefore the Minkowski property of level $\geqslant A / \ln (2)$ is satisfied in this case. Moreover, if the base field is of characteristic zero, then it has been shown in [21 that the absolute Minkowski property of level $\geqslant 0$ is satisfied.

The Minkowski property may fail for general adelic curves. Consider the adelic curve $S=(\mathbb{Q},(\mathbb{Q}, \mathcal{A}, \nu), \phi)$ consisting of the field of rational numbers, the measure space of $\mathbb{Q}$ equipped with the discrete $\sigma$-algebra and the atomic measure such that $\nu(\{\omega\})=1$ for any $\omega \in \mathbb{Q}$, together with the map $\phi$ sending any $\omega \in \mathbb{Q}$ to the trivial absolute value on $\mathbb{Q}$. We write the rational numbers into a sequence $\left\{q_{n}\right\}_{n \in \mathbb{N}}$. For any $n \in \mathbb{N}_{\geqslant 2}$, consider the following adelic vector bundle $\bar{E}_{n}$ on $S$. Let $E_{n}=K^{2}$. For $m \in \mathbb{N}$ such that $m<n$ let $\|\cdot\|_{q_{m}}$ be the norm on $E_{n}$ defined as

$$
\|(x, y)\|_{q_{m}}= \begin{cases}\mathrm{e}^{-1}, & \text { if there exists } a \in K^{\times} \text {such that }(x, y)=a\left(1, q_{m}\right), \\ 0, & \text { if }(x, y)=(0,0) \\ 1, & \text { else. }\end{cases}
$$

For $m \in \mathbb{N}$ such that $m \geqslant n$, let $\|\cdot\|_{q_{m}}$ be the norm on $K^{2}$ such that $\|(x, y)\|=1$ for any $(x, y) \in K^{2} \backslash\{(0,0)\}$. Then by definition one has $\widehat{\operatorname{deg}}\left(\bar{E}_{n}\right)=n$ and hence $\widehat{\mu}\left(\bar{E}_{n}\right)=n / 2$. Moreover, for any vector subspace $F$ of $E$, either there exists $m \in$ $\{0, \ldots, n-1\}$ such that $F=K\left(1, q_{m}\right)$ and thus $\widehat{\mu}(\bar{F})=1$, or one has $\widehat{\mu}(\bar{F})=0$. Since $n \geqslant 2$, we obtain that the adelic vector bundle $\bar{E}_{n}$ is semistable and of slope 
$n / 2$. Moreover the first minimum of $\bar{E}_{n}$ is 1 . Therefore it is not possible to find a constant $C$ only depending on $S$ such that $\widehat{\mu}_{\max }\left(\bar{E}_{n}\right)$ is bounded from above by $\nu_{1}\left(\bar{E}_{n}\right)+C \ln (2)$.

In the literature, the (absolute) Minkowski property is closely related to the semistability of tensor vector bundles and the estimation of the maximal slope of them. We refer the readers to [2, 21, 62 for more detailed discussions. In the following, we prove several slope estimates in assuming the Minkowski property.

Proposition 4.3.74. - Let $\bar{E}=\left(E, \xi_{E}\right)$ and $\bar{F}=\left(F, \xi_{F}\right)$ be adelic vector bundles on $S$. We assume that $\xi_{E}$ and $\xi_{F}$ are ultrametric on $\Omega \backslash \Omega_{\infty}$. One has

$$
\nu_{1}\left(\bar{E} \otimes_{\varepsilon} \bar{F}\right) \leqslant \widehat{\mu}_{\max }(\bar{E})+\widehat{\mu}_{\max }(\bar{F}) .
$$

Proof. - Let $f$ be a non-zero element of $E \otimes_{K} F$, viewed as a $K$-linear map from $E^{\vee}$ to $F$. By Proposition 4.3.30 (3), one has

$$
\widehat{\mu}_{\min }\left(\bar{E}^{\vee}\right) \leqslant \widehat{\mu}_{\max }(\bar{F})+h(f)=\widehat{\mu}_{\max }(\bar{F})-\widehat{\operatorname{deg}}_{\xi_{E} \otimes_{\varepsilon} \xi_{F}}(f) .
$$

By Proposition 4.3.24, we obtain

$$
0 \leqslant \widehat{\mu}_{\max }(\bar{E})+\widehat{\mu}_{\max }(\bar{F})-\widehat{\operatorname{deg}}_{\xi_{E} \otimes_{\varepsilon} \xi_{F}}(f) .
$$

Since $f$ is arbitrary, we obtain the inequality 4.84 .

Corollary 4.3.75. - Let $C$ be a non-negative real number. We assume that the adelic curve $S$ satisfies the Minkowski property of level $\geqslant C$. Let $\bar{E}=\left(E, \xi_{E}\right)$ and $\bar{F}=\left(F, \xi_{F}\right)$ be adelic vector bundles on $S$.

(1) Assume that $\xi_{E}$ and $\xi_{F}$ are ultrametric on $\Omega \backslash \Omega_{\infty}$. Then

$$
\widehat{\mu}_{\max }\left(\bar{E} \otimes_{\varepsilon} \bar{F}\right) \leqslant \widehat{\mu}_{\max }(\bar{E})+\widehat{\mu}_{\max }(\bar{F})+C \ln \left(\mathrm{rk}_{K}(E) \cdot \mathrm{rk}_{K}(F)\right),
$$

(2) One has

(4.86) $\widehat{\mu}_{\min }\left(\bar{E} \otimes_{\varepsilon, \pi} \bar{F}\right) \geqslant \widehat{\mu}_{\min }(\bar{E})+\widehat{\mu}_{\min }(\bar{F})-\left(C+\frac{1}{2} \nu\left(\Omega_{\infty}\right)\right) \ln \left(\operatorname{rk}_{K}(E) \cdot \operatorname{rk}_{K}(F)\right)$.

Proof. - By the assumption of Minkowski property, we have

$$
\nu_{1}\left(\bar{E} \otimes_{\varepsilon} \bar{F}\right) \geqslant \widehat{\mu}_{\max }\left(\bar{E} \otimes_{\varepsilon} \bar{F}\right)-C \ln \left(\mathrm{rk}_{K}(E) \cdot \mathrm{rk}_{K}(F)\right) .
$$

Hence (4.85) follows from (4.84).

If we apply the inequality (4.85) to $\bar{E}^{\vee}$ and $\bar{F}^{\vee}$ (note that $\xi_{E}^{\vee}$ and $\xi_{F}^{\vee}$ are always ultrametric on $\Omega \backslash \Omega_{\infty}$ ), we obtain

$$
\widehat{\mu}_{\max }\left(\bar{E}^{\vee} \otimes_{\varepsilon} \bar{F}^{\vee}\right) \leqslant \widehat{\mu}_{\max }\left(\bar{E}^{\vee}\right)+\widehat{\mu}_{\max }\left(\bar{F}^{\vee}\right)+C \ln \left(\mathrm{rk}_{K}(E) \cdot \mathrm{rk}_{K}(F)\right) .
$$

By Proposition 4.3 .24 we deduce that

$$
\widehat{\mu}_{\min }\left(\bar{E} \otimes_{\varepsilon, \pi} \bar{F}\right) \geqslant-\widehat{\mu}_{\max }\left(\bar{E}^{\vee}\right)-\widehat{\mu}_{\max }\left(\bar{F}^{\vee}\right)-C \ln \left(\mathrm{rk}_{K}(E) \cdot \mathrm{rk}_{K}(F)\right) .
$$

Finally, by Corollary 4.3 .26 we obtain (4.86). 
Proposition 4.3.76. - Let $\bar{E}$ be a non-zero adelic vector bundle on $S$. One has

$$
\nu_{\min }(\bar{E}) \leqslant \widehat{\mu}_{\min }(\bar{E}) .
$$

Proof. - Let $r$ be the rank of $E$ over $K$. Assume that $\bar{E}$ is of the form $\bar{E}=(E, \xi)$, with $\xi=\left\{\|\cdot\|_{\omega}\right\}_{\omega \in \Omega}$. Let $t$ be a real number and $\left\{s_{i}\right\}_{i=1}^{r}$ be a basis of $E$ over $K$ such that $\widehat{\operatorname{deg}}_{\xi}\left(s_{i}\right) \geqslant t$ for any $i \in\{1, \ldots, r\}$.

Let $G$ be a quotient vector space of $E$ and $\xi_{G}=\left\{\|\cdot\|_{G, \omega}\right\}_{\omega \in \Omega}$ be the quotient norm family of $\xi$ on $G$. For any $i \in\{1, \ldots, r\}$, let $\alpha_{i}$ be the canonical image of $s_{i}$ in $G$. Without loss of generality, we may assume that $\left\{\alpha_{1}, \ldots, \alpha_{n}\right\}$ form a basis of $G$ over $K$. For any $\omega \in \Omega$, one has

$$
\left\|\alpha_{1} \wedge \cdots \wedge \alpha_{n}\right\|_{G, \omega, \operatorname{det}} \leqslant \prod_{i=1}^{n}\left\|\alpha_{i}\right\|_{G, \omega} \leqslant \prod_{i=1}^{n}\left\|s_{i}\right\|_{\omega},
$$

where the first inequality comes from Proposition 1.1.63. Therefore one has

$$
\widehat{\operatorname{deg}}(\bar{G}) \geqslant \sum_{i=1}^{n} \widehat{\operatorname{deg}}_{\xi}\left(s_{i}\right) \geqslant n t,
$$

which implies $\widehat{\mu}(\bar{G}) \geqslant t$. Therefore we obtain $\nu_{\min }(\bar{E}) \leqslant \widehat{\mu}_{\min }(\bar{E})$.

Corollary 4.3.77. - Let $\bar{E}=(E, \xi)$ be a non-zero adelic vector bundle on $S$. For any $i \in\left\{1, \ldots, \operatorname{rk}_{K}(E)\right\}$ one has $\nu_{i}(\bar{E}) \leqslant \widehat{\mu}_{i}(\bar{E})$.

Proof. - By the relations (4.82) and (4.65), Proposition 4.3.76 leads to $\mathcal{F}_{\mathrm{m}}^{t}(\bar{E}) \subseteq$ $\mathcal{F}_{\text {hn }}^{t}(\bar{E})$ for any $t \in \mathbb{R}$. Therefore, by Proposition 1.1 .39 we obtain that $\nu_{i}(\bar{E}) \leqslant \widehat{\mu}_{i}(\bar{E})$ for any $i \in\left\{1, \ldots, \operatorname{rk}_{K}(E)\right\}$.

Definition 4.3.78. - Let $S=(K,(\Omega, \mathcal{A}, \nu), \phi)$ be an adelic curve. Let $C$ be a non-negative real number. We say that $S$ satisfies the strong Minkowski property of level $\geqslant C$ if for any adelic vector bundle $(E, \xi)$ on $S$ such that $\xi$ is ultrametric on $\Omega \backslash \Omega_{\infty}$ one has

$$
\nu_{\min }(E, \xi) \geqslant \widehat{\mu}_{\min }(E, \xi)-C \ln \left(\mathrm{rk}_{K}(E)\right)
$$

Proposition 4.3.79. - Assume that the adelic curve $S$ satisfies the strong Minkowski property of level $\geqslant C$. For any non-zero adelic vector bundle $\bar{E}=(E, \xi)$ on $S$ one has

$$
\widehat{\mu}_{i}(\bar{E}) \leqslant \nu_{i}(\bar{E})+C \ln \left(\mathrm{rk}_{K}(E)\right) .
$$

Proof. - Since the adelic curve $S$ satisfies the strong Minkowski property of level $\geqslant C$, by the relation (4.87) we obtain that

$$
\forall t \in \mathbb{R}, \quad \mathcal{F}_{\mathrm{hn}}^{t}(\bar{E}) \subseteq \mathcal{F}_{\mathrm{m}}^{t-C \ln \left(\mathrm{rk}_{K}(E)\right)}(\bar{E}) .
$$

Therefore, by Proposition 1.1.39 we obtain the inequality (4.88). 
Remark 4.3.80. - Proposition 4.3.79 shows that, if the adelic curve $S$ satisfies the strong Minkowski property of level $\geqslant C$, then it also satisfies Minkowski property of level $\geqslant C$. Moreover, the transference theorem of Gaudron [61, Theorem 36] shows that, for any Hermitian adelic vector bundle $\bar{E}$ of rank $n$ over a number field $K$, one has

$\nu_{\min }(\bar{E})-\widehat{\mu}_{\min }(\bar{E})=\nu_{\min }(\bar{E})+\widehat{\mu}_{\max }\left(\bar{E}^{\vee}\right) \geqslant \nu_{\min }(\bar{E})+\widehat{\nu}_{\max }\left(\bar{E}^{\vee}\right) \geqslant \ln (n)+\ln \left|\mathfrak{D}_{K / \mathbb{Q}}\right|$, where $\mathfrak{D}_{K / \mathbb{Q}}$ is the discriminant of $K$ over $\mathbb{Q}$. We then deduce that, if $\bar{E}$ is a general adelic vector bundle of rank $n$ over $K$, which is not necessarily Hermitian, one has (by Theorem 4.1.26)

$$
\nu_{\min }(\bar{E}) \geqslant \widehat{\mu}_{\min }(\bar{E})-\left(1+\frac{1}{2}[K: \mathbb{Q}]\right) \ln (n)+\ln \left|\mathfrak{D}_{K / \mathbb{Q}}\right| .
$$

Therefore the adelic curve corresponding to a number field $K$ satisfies the strong Minkowski property of level $1+\frac{1}{2}[K: \mathbb{Q}] \ln \left|\mathfrak{D}_{K / \mathbb{Q}}\right|$.

\subsection{Adelic vector bundles over number fields}

Throughout this section, we fix a number field $K$ and the standard adelic curve $S=(K,(\Omega, \mathcal{A}, \nu), \phi)$ of $K$ as in Subsection 3.2.2. Note that $S$ is proper. Denote by $\Omega_{\mathrm{fin}}$ the set $\Omega \backslash \Omega_{\infty}$ of finite places of $K$, and by $\mathfrak{o}_{K}$ the ring of algebraic integers in $K$. Note that the absolute value $|\cdot|_{\omega}$ at $\omega$ is given by

$\forall x \in K_{\omega}, \quad|x|_{\omega}= \begin{cases}\text { the standard absolute value of } x \text { in either } \mathbb{R} \text { or } \mathbb{C} & \text { if } \omega \in \Omega_{\infty}, \\ \exp \left(\frac{-\log p_{\omega} \operatorname{ord}_{\omega}(x)}{\operatorname{ord}_{\omega}\left(p_{\omega}\right)}\right) & \text { if } \omega \in \Omega_{\mathrm{fin}},\end{cases}$

where $p_{\omega}$ is the characteristic of the residue field of the valuation ring of $K_{\omega}$. Further, for $\omega \in \Omega_{\mathrm{fin}}$, let $\mathfrak{o}_{K, \omega}$ be the localisation of $\mathfrak{o}_{K}$ at $\omega$ and $\mathfrak{o}_{\omega}$ be the valuation ring of the completion $K_{\omega}$ of $K$ with respect to $\omega$, that is,

$$
\mathfrak{o}_{K, \omega}=\left\{\left.a \in K|| a\right|_{\omega} \leqslant 1\right\} \quad \text { and } \quad \mathfrak{o}_{\omega}=\left\{\left.a \in K_{\omega}|| a\right|_{\omega} \leqslant 1\right\} .
$$

Moreover, $\nu(\{\omega\})=\left[K_{\omega}: \mathbb{Q}_{\omega}\right]$ for $\omega \in \Omega$ and $\sum_{\omega \in \Omega_{\infty}} \nu(\{\omega\})=[K: \mathbb{Q}]$.

Let $E$ be a finite-dimensional vector space over $K$ and $\xi=\left\{\|\cdot\|_{\omega}\right\}_{\omega \in \Omega}$ be a norm family of $E$ over $S$. In this section, we always assume that $\|\cdot\|_{\omega}$ is ultrametric for every $\omega \in \Omega_{\text {fin }}$. For $\omega \in \Omega_{\text {fin }}$, we set

$$
E_{\omega}:=E \otimes_{K} K_{\omega} \quad \text { and } \quad \mathscr{E}_{\omega}:=\left\{x \in E_{\omega} \mid\|x\|_{\omega} \leqslant 1\right\} .
$$

By Proposition 1.1.25 and 1.1.30, $\mathscr{E}_{\omega}$ is a free $\mathfrak{o}_{\omega}$-module of rank $\operatorname{dim}_{K} E$ and $\mathscr{E}_{\omega} \otimes_{\mathfrak{o}_{\omega}}$ $K_{\omega}=E_{\omega}$.

Remark 4.4.1. - As in the next subsection, let $(E, \xi)_{\leqslant 1}^{\omega}:=\left\{x \in E \mid\|x\|_{\omega} \leqslant 1\right\}$. Then one can see the following:

(1) $(E, \xi)_{\leqslant 1}^{\omega}$ is a free $\mathfrak{o}_{K, \omega}$-module. 
(2) $(E, \xi)_{\leqslant 1}^{\omega} \otimes_{\mathfrak{o}_{K, \omega}} \mathfrak{o}_{\omega}=\mathscr{E}_{\omega}$.

(1) Fix a basis of $\left(x_{i}\right)_{i=1}^{r}$ of $E$. We consider a norm $\|\cdot\|_{\omega}^{\prime}$ on $E_{\omega}$ given by

$$
\forall \lambda_{1}, \ldots, \lambda_{r} \in K_{\omega}, \quad\left\|\lambda_{1} x_{1}+\cdots+\lambda_{r} x_{r}\right\|_{\omega}^{\prime}=\max \left\{\left|\lambda_{1}\right|_{\omega}, \ldots,\left|\lambda_{r}\right|_{\omega}\right\} .
$$

By Proposition 1.1.11, there is a positive integer $n$ such that $\left|\varpi_{\omega}\right|^{n}\|\cdot\|_{\omega}^{\prime} \leqslant\|\cdot\|_{\omega}$, where $\varpi_{\omega}$ is a uniformizing parameter of $\mathfrak{o}_{K, \omega}$. Therefore,

$$
(E, \xi)_{\leqslant 1}^{\omega} \subseteq \mathfrak{o}_{K, \omega} e_{1} \varpi_{\omega}^{-n}+\cdots+\mathfrak{o}_{K, \omega} e_{r} \varpi_{\omega}^{-n},
$$

as required.

(2) Obviously $(E, \xi)_{\leqslant 1}^{\omega} \otimes_{\mathfrak{o}_{K, \omega}} \mathfrak{o}_{\omega} \subseteq \mathscr{E}_{\omega}$. Let $\left(e_{i}\right)_{i=1}^{r}$ be a free basis of $(E, \xi)_{\leqslant 1}^{\omega}$ over $\mathfrak{o}_{K, \omega}$. For $x \in \mathscr{E}_{\omega}$, we choose $a_{1}, \ldots, a_{r} \in K_{\omega}$ such that $x=a_{1} e_{1}+\cdots+a_{r} e_{r}$. One can find $a_{1}^{\prime}, \ldots, a_{r}^{\prime} \in K$ such that

$$
\left|a_{i}-a_{i}^{\prime}\right|_{\omega} \leqslant \frac{1}{2}\left|a_{i}\right|_{\omega}(\forall i) \quad \text { and } \quad\left\|x-\left(a_{1}^{\prime} e_{1}+\cdots+a_{r}^{\prime} e_{r}\right)\right\|_{\omega} \leqslant \frac{1}{2}\|x\|_{\omega} .
$$

If we set $x^{\prime}=a_{1}^{\prime} e_{1}+\cdots+a_{r}^{\prime} e_{r}$, then $\|x\|_{\omega}=\left\|x^{\prime}\right\|_{\omega}$ and $\left|a_{i}\right|_{\omega}=\left|a_{i}^{\prime}\right|_{\omega}$ for all $i$. In particular $x^{\prime} \in(E, \xi)_{\leqslant 1}^{\omega}$, so that $a_{i}^{\prime} \in \mathfrak{o}_{K, \omega}$, and hence $\left|a_{i}\right|_{\omega}=\left|a_{i}^{\prime}\right|_{\omega} \leqslant 1$. Therefore, $x \in(E, \xi)_{\leqslant 1}^{\omega} \otimes_{\mathfrak{o}_{K, \omega}} \mathfrak{o}_{\omega}$.

4.4.1. Coherency for a norm family. - Let $E$ be a finite-dimensional vector space over $K$. Let $\xi=\left\{\|\cdot\|_{\omega}\right\}_{\omega \in \Omega}$ be a norm family of $E$ over $S$. We define $(E, \xi)_{\leqslant 1}^{\text {fin }}$ and $(E, \xi)_{\leqslant 1}^{\omega}\left(\omega \in \Omega_{\text {fin }}\right)$ to be

$$
\left\{\begin{array}{l}
(E, \xi)_{\leqslant 1}^{\mathrm{fin}}:=\left\{x \in E \mid\|x\|_{\omega} \leqslant 1 \text { for all } \omega \in \Omega_{\mathrm{fin}}\right\} \\
(E, \xi)_{\leqslant 1}^{\omega}:=\left\{x \in E \mid\|x\|_{\omega} \leqslant 1\right\} .
\end{array}\right.
$$

Note that $(E, \xi)_{\leqslant 1}^{\text {fin }}$ and $(E, \xi)_{\leqslant 1}^{\omega}$ are an $\mathfrak{o}_{K}$-module and an $\mathfrak{o}_{K, \omega}$-module, respectively. Furthermore, by Remark 4.4.1. $(E, \xi)_{\leqslant 1}^{\omega}$ is a free $\mathfrak{o}_{K, \omega}$-module and $(E, \xi)_{\leqslant 1}^{\omega} \otimes_{\mathfrak{o}_{K, \omega}} \mathfrak{o}_{\omega}=$ $\mathscr{E}_{\omega}$. Let us begin with the following proposition.

Proposition 4.4.2. - The following are equivalent:

(1) For any $v \in E,\|v\|_{\omega} \leqslant 1$ except finitely many $\omega \in \Omega_{\text {fin }}$.

(2) $(E, \xi)_{\leqslant 1}^{\mathrm{fin}} \otimes_{\mathfrak{o}_{K}} \mathfrak{o}_{K, \omega}=(E, \xi)_{\leqslant 1}^{\omega}$ for all $\omega \in \Omega_{\mathrm{fin}}$.

(3) $(E, \xi)_{\leqslant 1}^{\mathrm{fin}} \otimes_{\mathfrak{o}_{K}} \mathfrak{o}_{K, \omega}=(E, \xi)_{\leqslant 1}^{\omega}$ for some $\omega \in \Omega_{\mathrm{fin}}$.

(4) $(E, \xi)_{\leqslant 1}^{\text {fin }} \otimes_{\mathfrak{o}_{K}} K=E$.

Moreover, under the above equivalent conditions, $(E, \xi)_{\leqslant 1}^{\mathrm{fin}} \otimes_{\mathbb{Z}} \mathbb{Q}=E$.

Proof. - First of all, let us see the following claim:

Claim 4.4.3. - (a) Let $S$ be a finite subset of $\Omega_{\mathrm{fin}}$. Then there is $f \in \mathfrak{o}_{K} \backslash\{0\}$ such that

$$
\operatorname{ord}_{\omega}(f) \begin{cases}>0 & \text { if } \omega \in S, \\ =0 & \text { if } \omega \notin S .\end{cases}
$$

(b) $(E, \xi)_{\leqslant 1}^{\omega} \otimes_{\mathfrak{o}_{K, \omega}} K=E$ for all $\omega \in \Omega_{\mathrm{fin}}$. 
Proof. - (a) Let us consider the ideal given by $\mathfrak{I}=\prod_{\mathfrak{p} \in S} \mathfrak{p}$. As the class group of $K$ is finite, there are a positive integer $a$ and $f \in \mathfrak{o}_{K}$ such that $f \mathfrak{o}_{K}=\mathfrak{I}^{a}$, as required.

(b) Obviously $(E, \xi)_{\leqslant 1}^{\omega} \otimes_{\mathfrak{o}_{K, \omega}} K \subseteq E$. For $v \in E$, there is $a \in \mathfrak{o}_{K, \omega} \backslash\{0\}$ such that $a v \in(E, \xi) \underset{\leqslant}{\omega}$, which shows the converse inclusion.

$(1) \Longrightarrow(2)$ : Clearly $(E, \xi)_{\leqslant 1}^{\text {fin }} \otimes_{\mathfrak{o}_{K}} \mathfrak{o}_{K, \omega} \subseteq(E, \xi)_{\leqslant 1}^{\omega}$. Conversely, for $v \in(E, \xi)_{\leqslant 1}^{\omega}$, as $S=\left\{\omega^{\prime} \in \Omega_{\mathrm{fin}} \mid\|v\|_{\omega^{\prime}}>1\right\}$ is finite, there is $f \in \mathfrak{o}_{K} \backslash\{0\}$ such that $|f|_{\omega^{\prime}}<1$ for $\omega^{\prime} \in S$ and $|f|_{\omega^{\prime}}=1$ for $\omega^{\prime} \in \Omega_{\text {fin }} \backslash S$ by the above claim (a). Thus, there is a positive integer $n$ such that $f^{n} v \in(E, \xi)_{\leqslant 1}^{\text {fin }}$. Note that $f \in \mathfrak{o}_{K, \omega}^{\times}$. Thus the converse inclusion holds.

"(2) $\Longrightarrow(3)$ " is obvious and "(3) $\Longrightarrow(4)$ " follows from (b) in the claim. Let us see that "(4) $\Longrightarrow(1)$ ". For $v \in E$, there is $a \in \mathfrak{o}_{K} \backslash\{0\}$ such that $a v \in(E, \xi)_{\leqslant 1}^{\mathrm{fin}}$, that is, $|a|_{\omega}\|v\|_{\omega} \leq 1$ for all $\omega \in \Omega_{\mathrm{fin}}$. Note that $|a|_{\omega}=1$ except finitely many $\omega$, so that one has (1).

Note that $(E, \xi)_{\leqslant 1}^{\text {fin }} \otimes_{\mathfrak{o}_{K}} K$ and $(E, \xi)_{\leqslant 1}^{\text {fin }} \otimes_{\mathbb{Z}} \mathbb{Q}$ are the localizations of $(E,\|\cdot\|)_{\leqslant 1}^{\text {fin }}$ with respect to $\mathfrak{o}_{K} \backslash\{0\}$ and $\mathbb{Z} \backslash\{0\}$, respectively. Therefore, for the last assertion, it is sufficient to show that, for $\alpha \in \mathfrak{o}_{K} \backslash\{0\}$, there is $\alpha^{\prime} \in \mathfrak{o}_{K} \backslash\{0\}$ such that $\alpha \alpha^{\prime} \in \mathbb{Z} \backslash\{0\}$. Indeed, one can find $a_{1}, \ldots, a_{n} \in \mathbb{Z}$ such that $\alpha^{n}+a_{1} \alpha^{n-1}+\cdots+a_{n-1} \alpha+a_{n}=0$. We may assume that $a_{n} \neq 0$. Thus $\alpha\left(\alpha^{n-1}+a_{1} \alpha^{n-2}+\cdots+a_{n-1}\right)=-a_{n} \in \mathbb{Z} \backslash\{0\}$.

Definition 4.4.4. - We say that $(E, \xi)$ is coherent if the equivalent conditions of Proposition 4.4 .2 are satisfied.

Proposition 4.4.5. - If there are a non-empty open set $U$ of $\operatorname{Spec}\left(\mathfrak{o}_{K}\right)$ and a locally free $\mathfrak{o}_{U}$-module $\mathscr{E}$ such that $\mathscr{E} \otimes_{\mathfrak{o}_{U}} K=E$ and $\|\cdot\|_{\omega}=\|\cdot\|_{\mathscr{E} \otimes_{\mathfrak{o}_{U}} \mathfrak{o}_{\omega}}$ for all $\omega \in U \cap \Omega_{\mathrm{fin}}$, then $(E, \xi)$ is coherent and dominated, where $\mathfrak{o}_{U}$ is the ring of regular functions on the open set $U$.

Proof. - For $s \in E \backslash\{0\}$, we can find a non-empty open set $U^{\prime} \subseteq U$ such that $s \in \mathscr{E} \otimes_{\mathfrak{o}_{U}} \mathfrak{o}_{\omega}$ and $\mathscr{E} \otimes_{\mathfrak{o}_{U}} \mathfrak{o}_{\omega} / \mathfrak{o}_{\omega} s$ is torsion free for all $\omega \in U^{\prime} \cap \Omega_{\mathrm{fin}}$, so that

$$
\|s\|_{\omega}=\|s\|_{\mathscr{E} \otimes_{\mathfrak{o}_{U}} \mathfrak{o}_{\omega}}=1 .
$$

In particular, $(E, \xi)$ is upper-dominated and coherent. Let $\mathscr{E}^{\vee}$ be the dual of $\mathscr{E}$ over $U$. Note that $\mathscr{E}^{\vee} \otimes_{\mathfrak{o}_{U}} \mathfrak{o}_{\omega}=\left(\mathscr{E} \otimes_{\mathfrak{o}_{U}} \mathfrak{o}_{\omega}\right)^{\vee}$, so that by Propsotion 1.1.34, $\|\cdot\|_{\omega, *}=\|\cdot\|_{\mathscr{E} \vee} \otimes_{\mathbb{v}_{U} \mathfrak{o}_{\omega}}$ for all $\omega \in U \cap \Omega_{\mathrm{fin}}$. Therefore, in the same way as above, one can see that $\left(E^{\vee}, \xi^{\vee}\right)$ is upper-dominated, and hence $(E, \xi)$ is dominated.

4.4.2. Finite generation of a dominated vector bundle over $S$. - Let $E$ be a finite-dimensional vector space over $K$ and $\xi=\left\{\|\cdot\|_{\omega}\right\}_{\omega \in \Omega}$ be a norm family of $E$ over $S$. The purpose of this subsection is to prove the following theorem. 
Proposition 4.4.6. - If $\xi$ is dominated, then

$$
(E, \xi)_{\leqslant 1}^{\mathrm{fin}}:=\left\{x \in E:\|x\|_{\omega} \leqslant 1 \text { for all } \omega \in \Omega_{\text {fin }}\right\}
$$

is a finitely generated $\mathfrak{o}_{K}$-module.

Proof. - First we assume that $\operatorname{dim}_{K} E=1$. Fix $x \in E \backslash\{0\}$. For each $\omega \in \Omega_{\text {fin }}$, let $a_{\omega}$ be the smallest integer $a$ with $a \geqslant-\ln \|x\|_{\omega} / \ln \left|\varpi_{\omega}\right|_{\omega}$, where $\varpi_{\omega}$ is a local parameter of $\mathfrak{o}_{K, \omega}$.

As $\xi$ is lower dominated, there is an integrable function $A(\omega)$ on $\Omega$ such that

$$
\forall \omega \in \Omega, \quad-\ln \|x\|_{\omega} \leqslant A(\omega) .
$$

Here we assume that there are infinitely many $\omega \in \Omega_{\text {fin }}$ with $a_{\omega} \leqslant-1$. As $a_{\omega} \leqslant-1$ implies $-\ln \left|\varpi_{\omega}\right|_{\omega} \leqslant-\ln \|x\|_{\omega}$, one has

$$
\begin{aligned}
A(\omega) \nu(\{\omega\}) & \geqslant-\ln \|x\|_{\omega} \nu(\{\omega\}) \\
& \geqslant-\ln \left|\varpi_{\omega}\right|_{\omega} \nu(\{\omega\})=\ln \#\left(\mathfrak{o}_{K} / \mathfrak{p}_{\omega}\right) \geqslant \ln p_{\omega},
\end{aligned}
$$

where $\mathfrak{p}_{\omega}$ is the maximal ideal of $\mathfrak{o}_{K}$ and $p_{\omega}$ is the characteristic of the residue field $\mathfrak{o}_{K} / \mathfrak{p}_{\omega}$, which gives a contradiction to the integrability of the function $A(\cdot)$. Therefore, $a_{\omega} \geqslant 0$ except finitely many $\omega \in \Omega_{\text {fin }}$.

Note that

$$
\begin{aligned}
\|a x\|_{\omega}=|a|_{\omega}\|x\|_{\omega} \leqslant 1 & \Longleftrightarrow\left|\varpi_{\omega}\right|_{\omega}^{\operatorname{ord}}(a)+\ln \|x\|_{\omega} / \ln \left|\varpi_{\omega}\right|_{\omega} \leqslant 1 \\
& \Longleftrightarrow \operatorname{ord}_{\omega}(a) \geqslant-\ln \|x\|_{\omega} / \ln \left|\varpi_{\omega}\right|_{\omega} \\
& \Longleftrightarrow \operatorname{ord}_{\omega}(a) \geqslant a_{\omega} .
\end{aligned}
$$

Therefore

$\left\{a \in K:\|a x\|_{\omega} \leqslant 1\right.$ for all $\left.\omega \in \Omega_{\text {fin }}\right\}=\left\{a \in K: \operatorname{ord}_{\omega}(a)-a_{\omega} \geqslant 0\right.$ for all $\left.\omega \in \Omega_{\text {fin }}\right\}$

is finitely generated over $\mathfrak{o}_{K}$ by Lemma 4.4.7. Thus one has the assertion in the case where $\operatorname{dim}_{K} E=1$.

In general, we prove the theorem by induction on $\operatorname{dim}_{K} E$. By the previous observation, we may assume $\operatorname{dim}_{K} E \geqslant 2$. Fix $x \in E \backslash\{0\}$. We set $E^{\prime}=K x$ and $E^{\prime \prime}=E / E^{\prime}$. Let $\xi^{\prime}$ be the norm family on $E^{\prime}$ given by the restriction of $\xi$, and $\xi^{\prime \prime}$ be the norm family on $E^{\prime \prime}$ given by the quotient of $\xi$. Then $\xi^{\prime}$ and $\xi^{\prime \prime}$ are dominated by Proposition 4.1.19, so that, by the hypothesis of induction, $\left(E^{\prime}, \xi^{\prime}\right)_{\leqslant 1}^{\text {fin }}$ and $\left(E^{\prime \prime}, \xi^{\prime \prime}\right)_{\leqslant 1}^{\text {fin }}$ are finitely generated over $\mathfrak{o}_{K}$. Note that $\beta\left((E, \xi)_{\leqslant 1}^{\mathrm{fin}}\right) \subseteq\left(E^{\prime \prime}, \xi^{\prime \prime}\right)_{\leqslant 1}^{\mathrm{fin}}$, where $\beta$ is the canonical homomorphism $E \rightarrow E^{\prime \prime}$. In particular, $\beta\left((E, \xi)_{\leqslant 1}^{\mathrm{fin}}\right)$ is finitely generated over $\mathfrak{o}_{K}$ because $\mathfrak{o}_{K}$ is Noetherian. Therefore, one has the exact sequence

$$
0 \longrightarrow\left(E^{\prime}, \xi^{\prime}\right)_{\leqslant 1}^{\mathrm{fin}} \longrightarrow(E, \xi)_{\leqslant 1}^{\mathrm{fin}} \longrightarrow \beta\left((E, \xi)_{\leqslant 1}^{\mathrm{fin}}\right) \longrightarrow 0
$$

and hence the assertion follows. 
Lemma 4.4.7. - Let $\left\{b_{\omega}\right\}_{\omega \in \Omega_{\mathrm{fin}}}$ be a family of integers indexed by $\Omega_{\mathrm{fin}}$. Then

$$
\mathfrak{o}_{K}\left(\left\{b_{\omega}\right\}_{\omega \in \Omega_{\mathrm{fin}}}\right):=\left\{a \in K: \operatorname{ord}_{\omega}(a)+b_{\omega} \geqslant 0 \text { for all } \omega \in \Omega_{\mathrm{fin}}\right\}
$$

is finitely generated over $\mathfrak{o}_{K}$ if and only if either $b_{\omega} \leqslant 0$ except finitely many $\omega$, or $b_{\omega}<0$ for infinitely many $\omega$.

Proof. - We set $S=\left\{\omega \in \Omega_{\text {fin }}: b_{\omega} \geqslant 1\right\}$ and $T=\left\{\omega \in \Omega_{\text {fin }}: b_{\omega} \leqslant-1\right\}$

First we assume that $b_{\omega} \leqslant 0$ except finitely many $\omega$, that is, $\#(S)<\infty$. Then one can choose $f \in \mathfrak{o}_{K} \backslash\{0\}$ such that $\operatorname{ord}_{\omega}(f) \geqslant b_{\omega}$ for all $\omega \in \Omega_{\text {fin. }}$. Note that $\left\{a \in K: \operatorname{ord}_{\omega}(a) \geqslant 0\right.$ for all $\left.\omega \in \Omega_{\text {fin }}\right\}=\mathfrak{o}_{K}$, so that $\mathfrak{o}_{K}\left(\left\{b_{\omega}\right\}_{\omega \in \Omega_{\text {fin }}}\right) f \subseteq \mathfrak{o}_{K}$. Thus $\mathfrak{o}_{K}\left(\left\{b_{\omega}\right\}_{\omega \in \Omega_{\mathrm{fin}}}\right) f$ is finitely generated over $\mathfrak{o}_{K}$ because $\mathfrak{o}_{K}$ is Noetherian. Therefore $\mathfrak{o}_{K}\left(\left\{b_{\omega}\right\}_{\omega \in \Omega_{\text {fin }}}\right)$ is also finitely generated over $\mathfrak{o}_{K}$.

Next we assume that $b_{\omega}<0$ for infinitely many $\omega$, that is, $\#(T)=\infty$. In this case, $\mathfrak{o}_{K}\left(\left\{b_{\omega}\right\}_{\omega \in \Omega_{\text {fin }}}\right)=\{0\}$. Indeed, if $x \in \mathfrak{o}_{K}\left(\left\{b_{\omega}\right\}_{\omega \in \Omega_{\text {fin }}}\right) \backslash\{0\}$, then $\operatorname{ord}_{\omega}(x) \geq 1$ for all $\omega \in T$, which is a contradiction.

Finally we assume that $\#(S)=\infty$ and $\#(T)<\infty$. In this case, we need to show that $\mathfrak{o}_{K}\left(\left\{b_{\omega}\right\}_{\omega \in \Omega_{\mathrm{fin}}}\right)$ is not finitely generated over $\mathfrak{o}_{K}$. We set $S=\left\{\omega_{1}, \omega_{2}, \ldots, \omega_{n}, \ldots\right\}$. For each positive integer $N$, let us consider a family $\left\{b_{N, \omega}\right\}_{\omega \in \Omega_{\text {fin }}}$ of integers given by

$$
b_{N, \omega}= \begin{cases}0 & \text { if } \omega \in\left\{\omega_{n}: n \geqslant N+1\right\}, \\ b_{\omega} & \text { otherwise. }\end{cases}
$$

Then one has a strictly increasing sequence of finitely generated $\mathfrak{o}_{K}$-modules:

$$
\mathfrak{o}_{K}\left(\left\{b_{1, \omega}\right\}_{\omega \in \Omega_{\mathrm{fin}}}\right) \subsetneq \mathfrak{o}_{K}\left(\left\{b_{2, \omega}\right\}_{\omega \in \Omega_{\mathrm{fin}}}\right) \subsetneq \cdots \subsetneq \mathfrak{o}_{K}\left(\left\{b_{N, \omega}\right\}_{\omega \in \Omega_{\mathrm{fin}}}\right) \subsetneq \cdots
$$

such that $\bigcup_{N=1}^{\infty} \mathfrak{o}_{K}\left(\left\{b_{N, \omega}\right\}_{\omega \in \Omega_{\text {fin }}}\right)=\mathfrak{o}_{K}\left(\left\{b_{\omega}\right\}_{\omega \in \Omega_{\text {fin }}}\right)$. Therefore $\mathfrak{o}_{K}\left(\left\{b_{\omega}\right\}_{\omega \in \Omega_{\text {fin }}}\right)$ is not finitely generated over $\mathfrak{o}_{K}$.

Example 4.4.8. - Let $\left\{b_{\omega}\right\}_{\omega \in \Omega_{\text {fin }}}$ be a family of integers indexed by $\Omega_{\text {fin }}$. To each $\omega \in \Omega_{\mathrm{fin}}$, we assign a norm $\|\cdot\|_{\omega}$ of $K_{\omega}$ given by

$$
\|x\|_{\omega}=\exp \left(\frac{-b_{\omega} \log p_{\omega}}{\operatorname{ord}_{\omega}\left(p_{\omega}\right)}\right)|x|_{\omega}
$$

for $x \in K_{\omega}$. Moreover, for $\omega \in \Omega_{\infty}$ let $\|\cdot\|_{\omega}$ be the standard absolute value of either $\mathbb{R}$ or $\mathbb{C}$. Then $\xi:=\left\{\|\cdot\|_{\omega}\right\}_{\omega \in \Omega}$ yields a norm family on $K$. Note that, for $\omega \in \Omega_{\mathrm{fin}}$, $\|x\|_{\omega} \leqslant 1$ if and only if $\operatorname{ord}_{\omega}(x)+b_{\omega} \geqslant 0$ for $x \in K$, that is,

$$
(K, \xi)_{\leqslant 1}^{\text {fin }}=\mathfrak{o}_{K}\left(\left\{b_{\omega}\right\}_{\omega \in \Omega_{\text {fin }}}\right) .
$$

For example, if we set $b_{\omega}=1$ for all $\omega \in \Omega_{\mathrm{fin}}$, then $(K, \xi)_{\leqslant 1}^{\text {fin }}$ is not finitely generated over $\mathfrak{o}_{K}$ by Lemma 4.4 .7 
4.4.3. Invariants $\lambda$ and $\sigma$. - Let $\left(E, \xi=\left\{\|\cdot\|_{\omega}\right\}_{\omega \in \Omega}\right)$ be an adelic vector bundle on $S$. Let $\mathscr{E}:=(E, \xi)_{\leqslant 1}^{\text {fin }}=\left\{x \in E:\|x\|_{\omega} \leqslant 1\right.$ for all $\left.\omega \in \Omega_{\text {fin }}\right\}$. If $\xi$ is coherent and dominated, then, by Proposition 4.4.2, 4.4.6 and Remark 4.4.1. $\mathscr{E}$ is finitely generated $\mathfrak{o}_{K}$-module, $\mathscr{E} \otimes_{\mathfrak{o}_{K}} K=E$ and $\mathscr{E} \otimes_{\mathfrak{o}_{K}} \mathfrak{o}_{\omega}=\mathscr{E}_{\omega}$ for all $\omega \in \Omega_{\mathrm{fin}}$.

We define $\|\cdot\|_{\infty}$ to be

$$
\forall x \in E, \quad\|x\|_{\infty}:=\max _{\omega \in \Omega_{\infty}}\left\{\left\|\iota_{\omega}(x)\right\|_{\sigma}\right\},
$$

where $\iota_{\omega}$ is the canonical homomorphism $E \rightarrow E_{\omega}$. Under the assumption that $\xi$ is coherent and dominated, the invariant $\lambda(E, \xi)$ is defined to be

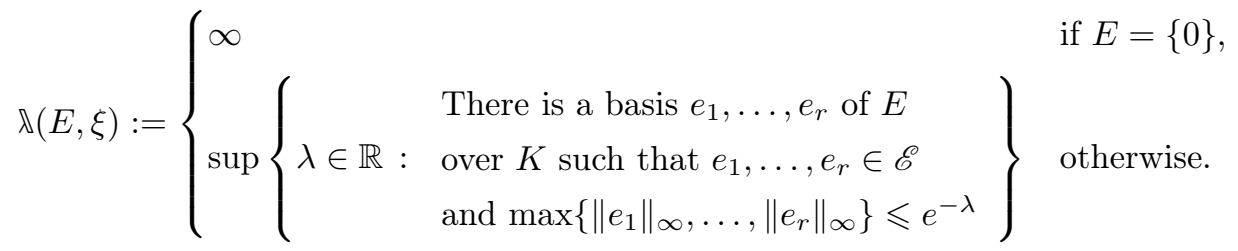

By Proposition 1.1.30,

$$
0 \leqslant \sup _{x \in E_{\omega} \backslash\{0\}} \ln \left(\frac{\|x\|_{\mathscr{E}_{\omega}}}{\|x\|_{\omega}}\right) \leqslant-\log \left|\varpi_{\omega}\right|_{\omega}
$$

for any $\omega \in \Omega_{\mathrm{fin}}$, where $\|\cdot\|_{\mathscr{E}_{\omega}}$ is the norm arising from $\mathscr{E}_{\omega}$ (cf. Subsection 1.1.7). The impurity $\sigma(E, \xi)$ of $(E, \xi)$ is defined to be

$$
\sigma(E, \xi):=\sum_{\omega \in \Omega_{\mathrm{fin}}} \sup _{x \in E_{\omega} \backslash\{0\}} \ln \left(\frac{\|x\|_{\mathscr{E}_{\omega}}}{\|x\|_{\omega}}\right) \nu(\{\omega\}) \in[0, \infty] .
$$

Note that $\sigma(E, \xi)=0$ if and only if $\|\cdot\|_{\omega}=\|\cdot\|_{\mathscr{E}_{\omega}}$ for all $\omega \in \Omega_{\mathrm{fin}}$. Moreover, if $\xi$ is coherent and dominated, then, by Proposition 4.4.5, $\xi^{\prime}=\left\{\|\cdot\|_{\mathscr{E}_{\omega}}\right\}_{\omega \in \Omega_{\text {fin }}} \cup\left\{\|\cdot\|_{\omega}\right\}_{\omega \in \Omega_{\infty}}$ is also coherent and dominated, so that $\sigma(E, \xi)<\infty$ by Corollary 4.1.10.

Proposition 4.4.9. - We assume that $\xi$ is coherent. Then the following are equivalent:

(1) $\xi$ is dominated.

(2) $\mathscr{E}$ is finitely generated over $\mathfrak{o}_{K}$ and $\sigma(E, \xi)<\infty$.

Proof. - It is sufficient to see that $(2) \Longrightarrow(1)$. If we set

$$
\xi^{\prime}=\left\{\|\cdot\|_{\mathscr{E}_{\omega}}\right\}_{\omega \in \Omega_{\text {fin }}} \cup\left\{\|\cdot\|_{\omega}\right\}_{\omega \in \Omega_{\infty}}
$$

then $\xi^{\prime}$ is dominated by Proposition 4.4.5 together with Proposition 4.4 .2 and Remark 4.4.1. Therefore the assertion follows from the assumption $\sigma(E, \xi)<\infty$.

Proposition 4.4.10. - We assume that $\xi$ is coherent and dominated. There is a constants $c_{K}$ depending only on $K$ such that

$$
[K: \mathbb{Q}] \lambda(E, \xi) \leqslant \nu_{\min }(E, \xi) \leqslant[K: \mathbb{Q}] \lambda(E, \xi)+\sigma(E, \xi)+c_{K} .
$$


Proof. - First we consider the inequality $[K: \mathbb{Q}] \lambda(E, \xi) \leqslant \nu_{\min }(E, \xi)$. We set $\lambda=$ $\lambda(E, \xi)$. For $\epsilon>0$, there is a basis $\left\{e_{1}, \ldots, e_{r}\right\}$ of $E$ over $K$ such that $e_{i} \in \mathscr{E}$ and $\left\|e_{i}\right\|_{\infty} \leqslant e^{-\lambda+\epsilon}$ for all $i$. On the other hand,

$$
\begin{aligned}
\widehat{\operatorname{deg}}_{\xi}\left(e_{i}\right) & =\sum_{\omega \in \Omega}-\ln \left\|e_{i}\right\|_{\omega} \nu(\{\omega\}) \geqslant \sum_{\omega \in \Omega_{\infty}}-\ln \left\|e_{i}\right\|_{\omega} \nu(\{\omega\}) \geqslant \sum_{\omega \in \Omega_{\infty}}(\lambda-\epsilon) \nu(\{\omega\}) \\
& =[K: \mathbb{Q}](\lambda-\epsilon),
\end{aligned}
$$

so that the assertion follows.

(2) Next let us see the second inequality

$$
\nu_{\min }(E, \xi) \leqslant[K: \mathbb{Q}] \lambda(E, \xi)+\sigma(E, \xi)+c_{K} .
$$

For $\epsilon>0$, there is a basis $\left\{e_{1}^{\prime}, \ldots, e_{r}^{\prime}\right\}$ of $E$ over $K$ such that $\widehat{\operatorname{deg}}\left(e_{i}^{\prime}\right) \geq \nu_{\min }(E, \xi)-\epsilon$ for all $i$. We set $E_{i}=K e_{i}^{\prime}$ and $\mathscr{E}_{i}=\mathscr{E} \cap E_{i}$. By Lemma 4.4.11 below, there is an $e_{i}^{\prime \prime} \in \mathscr{E}_{i}$ such that $\#\left(\mathscr{E}_{i} / \mathfrak{o}_{K} e_{i}^{\prime \prime}\right) \leqslant C_{K}^{\prime}$, where $C_{K}^{\prime}$ is a constant depending only on the number field $K$. Therefore $\nu_{\min }(E, \xi)-\epsilon$ is bounded from above by

$$
\begin{aligned}
& \widehat{\operatorname{deg}}_{\xi}\left(e_{i}^{\prime}\right)=\widehat{\operatorname{deg}}_{\xi}\left(e_{i}^{\prime \prime}\right)=\sum_{\omega \in \Omega_{\mathrm{fin}}}-\ln \left\|e_{i}^{\prime \prime}\right\|_{\omega} \nu(\{\omega\})+\sum_{\omega \in \Omega_{\infty}}-\ln \left\|e_{i}^{\prime \prime}\right\|_{\omega} \nu(\{\omega\}) \\
= & \sum_{\omega \in \Omega_{\mathrm{fin}}}-\ln \left\|e_{i}^{\prime \prime}\right\|_{\mathscr{E}_{\omega}} \nu(\{\omega\})+\sum_{\omega \in \Omega_{\mathrm{fin}}} \ln \left(\frac{\left\|e_{i}^{\prime \prime}\right\|_{\mathscr{E}_{\omega}}}{\left\|e_{i}^{\prime \prime}\right\|_{\omega}}\right) \nu(\{\omega\}) \\
& +\sum_{\omega \in \Omega_{\infty}}-\ln \left\|e_{i}^{\prime \prime}\right\|_{\omega} \nu(\{\omega\}) \\
\leqslant & \ln \#\left(\mathscr{E}_{i} / \mathfrak{o}_{K} e_{i}^{\prime \prime}\right)+\sum_{\omega \in \Omega_{\mathrm{fin}}} \ln \left(\left\|\operatorname{Id}_{E_{\omega}}\right\|_{\omega}^{\mathrm{op}}\right) \nu(\{\omega\})+\sum_{\omega \in \Omega_{\infty}}-\ln \left\|e_{i}^{\prime \prime}\right\|_{\omega} \nu(\{\omega\}) \\
\leqslant & \ln C_{K}^{\prime}+\sigma(E, \xi)+\sum_{\omega \in \Omega_{\infty}}-\ln \left\|e_{i}^{\prime \prime}\right\|_{\omega} \nu(\{\omega\}) .
\end{aligned}
$$

If we set

$$
A=\frac{1}{[K: \mathbb{Q}]} \sum_{\omega \in \Omega_{\infty}} \ln \left\|e_{i}^{\prime \prime}\right\|_{\omega} \nu(\{\omega\})
$$

then $\sum_{\omega \in \Omega_{\infty}}\left(\ln \left\|e_{i}^{\prime \prime}\right\|_{\omega}-A\right) \nu(\{\omega\})=0$. Let $\left\{u_{1}, \ldots, u_{s}\right\}$ be a free basis of $\mathfrak{o}_{K}^{\times}$modulo the torsion subgroup. Then, by Dirichlet's unit theorem, there are $a_{i 1}^{\prime}, \ldots, a_{i s}^{\prime} \in \mathbb{R}$ such that

$$
\ln \left\|e_{i}^{\prime \prime}\right\|_{\omega}-A=\sum_{j=1}^{s} a_{i j}^{\prime} \ln \left|u_{j}\right|_{\omega}
$$

for all $\omega \in \Omega_{\infty}$. Let $a_{i j}$ be the round-up of $a_{i j}^{\prime}$. Then

$$
\sum_{j=1}^{s}\left(a_{i j}^{\prime}-a_{i j}\right) \ln \left|u_{j}\right|_{\omega} \leqslant\left.\sum_{j=1}^{s}\left|a_{i j}^{\prime}-a_{i j}\right| \cdot|\ln | u_{j}\right|_{\omega}\left|\leqslant \sum_{j=1}^{s}\right| \ln \left|u_{j}\right|_{\omega} \mid \leqslant C_{K}^{\prime \prime},
$$


where $C_{K}^{\prime \prime}=\sum_{\omega \in \Omega_{\infty}} \sum_{j=1}^{s}|\ln | u_{j}|\omega|$. Therefore,

$$
\begin{aligned}
-A & =\sum_{j=1}^{s} a_{i j}^{\prime} \ln \left|u_{j}\right|_{\omega}-\ln \left\|e_{i}^{\prime \prime}\right\|_{\omega} \leqslant C_{K}^{\prime \prime}+\sum_{j=1}^{s} a_{i j} \ln \left|u_{j}\right|_{\omega}-\ln \left\|e_{i}^{\prime \prime}\right\|_{\omega} \\
& =C_{K}^{\prime \prime}-\ln \left\|v_{i} e_{i}^{\prime \prime}\right\|_{\omega},
\end{aligned}
$$

where $v_{i}=\prod_{j=1}^{s} u_{j}^{-a_{i j}}$, and hence, if we set $e_{i}=v_{i} e_{i}^{\prime \prime}$, then $e_{i} \in \mathscr{E}$ and

$$
\nu_{\min }(E, \xi)-\epsilon \leqslant \ln C_{K}^{\prime}+\sigma(E, \xi)+[K: \mathbb{Q}] C_{K}^{\prime \prime}-[K: \mathbb{Q}] \ln \left\|e_{i}\right\|_{\omega},
$$

that is, there is a constant $c_{K}$ depending only on $K$ such that

$$
\nu_{\min }(E, \xi)-\epsilon \leqslant c_{K}+\sigma(E, \xi)-[K: \mathbb{Q}] \ln \left\|e_{i}\right\|_{\omega}
$$

for all $i$ and $\omega \in \Omega_{\infty}$. We choose $i$ and $\omega$ such that $\max \left\{\left\|e_{1}\right\|_{\infty}, \ldots,\left\|e_{r}\right\|_{\infty}\right\}=\left\|e_{i}\right\|_{\omega}$. Then, as $e^{-\lambda(E, \xi)} \leqslant\left\|e_{i}\right\|_{\omega}$, that is, $-\ln \left\|e_{i}\right\|_{\omega} \leqslant \lambda(E, \xi)$,

$$
\nu_{\min }(E, \xi)-\epsilon \leqslant c_{K}+\sigma(E, \xi)+[K: \mathbb{Q}] \lambda(E, \xi)
$$

and hence the assertion follows.

Lemma 4.4.11. - There is a constant $e_{K}$ depending only on $K$ such that, for any invertible $\mathfrak{o}_{K}$-module $\mathscr{L}$, we can find $l \in \mathscr{L} \backslash\{0\}$ such that $\#\left(\mathscr{L} / \mathfrak{o}_{K} l\right) \leqslant e_{K}$.

Proof. - Since the class group is finite, there are finitely many invertible $\mathfrak{o}_{K}$-modules $\mathscr{L}_{1}, \ldots, \mathscr{L}_{h}$ such that, for any invertible $\mathfrak{o}_{K}$-module $\mathscr{L}$, there is $\mathscr{L}_{i}$ such that $\mathscr{L}_{i} \simeq \mathscr{L}$. For each $i=1, \ldots, h$, fix $l_{i} \in \mathscr{L}_{i} \backslash\{0\}$. Let $\mathscr{L}$ be an invertible $\mathfrak{o}_{K}$-module. Then there are $\mathscr{L}_{i}$ and an isomorphism $\varphi: \mathscr{L}_{i} \rightarrow \mathscr{L}$. If we set $l=\varphi\left(l_{i}\right)$, then $\mathscr{L}_{i} / \mathfrak{o}_{K} l_{i} \simeq \mathscr{L} / \mathfrak{o}_{K} l$, as required. 


\section{CHAPTER 5}

\section{SLOPES OF TENSOR PRODUCT}

The purpose of this chapter is to study the minimal slope of the tensor product of a finite of adelic vector bundles on an adelic curve. More precisely, give a family $\bar{E}_{1}, \ldots, \bar{E}_{d}$ of adelic vector bundles over an adelic curve $S$, we give a lower bound of $\widehat{\mu}_{\min }\left(\bar{E}_{1} \otimes_{\varepsilon, \pi} \cdots \otimes_{\varepsilon, \pi} \bar{E}_{d}\right)$ in terms of the sum of the minimal slopes of $\bar{E}_{i}$ minus a term which is the product of three half of the measure of the infinite places and the sum of $\ln \left(\operatorname{rk}\left(E_{i}\right)\right)$, see Corollary 5.6 .2 for details. This result, whose form is similar to the main results of [62, 21, 36], does not rely on the comparison of successive minima and the height proved in [148, which des not hold for general adelic curves. Our method inspires the work of Totaro [136] on $p$-adic Hodge theory and relies on the geometry invariant theory on Grassmannian. The chapter is organised as follows. In the first section, we regroup several fundamental property of $\mathbb{R}$-filtrations. We then recall in the second section some basic notions and results of the geometric invariant theory, in particular the Hilbert-Mumford criterion of the semistability. In the third section we give an estimate for the slope of a quotient adelic vector bundle of the tensor product adelic vector bundle, under the assumption that the underlying quotient space, viewed as a rational point of the Grassmannian (with the Plücker coordinates), is semistable in the sense of geometric invariant theory. In the fifth section, we prove a non-stability criterion which generalises [136, Proposition 1]. Finally, we prove in the sixth section the lower bound of the minimal slope of the tensor product adelic vector bundle in the general case.

\subsection{Reminder on $\mathbb{R}$-filtrations}

Let $K$ be a field. We equip $K$ with the trivial absolute value $|\cdot|$ such that $|a|=1$ for any $a \in K \backslash\{0\}$. Note that $K$ equipped with the trivial absolute value forms an adelic curve whose underlying measure space is a one point set equipped with the counting measure (which is a probability measure), see $\$ 3.2 .3$. Moreover, any finitedimensional normed vector space over $(K,|\cdot|)$ can be considered as an adelic vector 
bundle on $S$. In fact, if $V$ is a finite-dimensional vector space over $K$, any norm on $V$ can be considered as a norm family indexed by the one point set. This norm family is clearly measurable. It is also dominated since all norms on $V$ are equivalent (see Corollaries 1.1.13 and 4.1.10).

Let $V$ be a finite-dimensional vector space over $K$. Recall that the set of ultrametric norms on $V$ are canonically in bijection with the set of $\mathbb{R}$-filtrations on $V$ (see Remark1.1.40). If $\|\cdot\|$ is an ultrametric norm on $V$, then the balls centered at the origin are vector subspaces of $V$, and $\left\{(V,\|\cdot\|)_{\leqslant \mathrm{e}^{-t}}\right\}_{t \in \mathbb{R}}$ is the corresponding $\mathbb{R}$-filtration. Conversely, given an $\mathbb{R}$-filtration $\mathcal{F}$ on $V$, we define a function $\lambda_{\mathcal{F}}: V \rightarrow \mathbb{R} \cup\{+\infty\}$ as follows

$$
\forall x \in V, \quad \lambda_{\mathcal{F}}(x):=\sup \left\{t \in \mathbb{R}: x \in \mathcal{F}^{t}(V)\right\} .
$$

Then the ultrametric norm $\|\cdot\|_{\mathcal{F}}$ corresponding to the $\mathbb{R}$-filtration $\mathcal{F}$ is given by

$$
\forall x \in V, \quad\|x\|_{\mathcal{F}}=\mathrm{e}^{-\lambda_{\mathcal{F}}(x)} .
$$

Definition 5.1.1. - Let $V$ be a finite-dimensional vector space over $K$ and $\mathcal{F}$ be an $\mathbb{R}$-filtration on $V$. For any $t \in \mathbb{R}$, we denote by $\operatorname{sq}_{\mathcal{F}}^{t}(V)$ the quotient vector space

$$
\mathcal{F}^{t}(V) / \bigcup_{\varepsilon>0} \mathcal{F}^{t+\varepsilon}(V) \text {. }
$$

Clearly, if $\mathcal{F}$ corresponds to the flag

$$
0=V_{0} \subsetneq V_{1} \subsetneq \ldots \subsetneq V_{n}=V
$$

of vector subspaces of $V$ together with the sequence

$$
\mu_{1}>\ldots>\mu_{n}
$$

in $\mathbb{R}$, then

$$
\forall i \in\{1, \ldots, n\}, \quad \operatorname{sq}_{\mathcal{F}}^{\mu_{i}}(V)=V_{i} / V_{i-1},
$$

and $\operatorname{sq}_{\mathcal{F}}^{t}(V)=\{0\}$ if $t \notin\left\{\mu_{1}, \ldots, \mu_{n}\right\}$.

Proposition 5.1.2. - Let $(V,\|\cdot\|)$ be a finite-dimensional ultrametrically normed vector space over $K$. The following assertions hold.

(1) The normed vector space $(V,\|\cdot\|)$ admits an orthogonal basis.

(2) If $\boldsymbol{e}=\left\{e_{i}\right\}_{i=1}^{r}$ is an orthogonal basis of $(V,\|\cdot\|)$, then the Arakelov degree of $(V,\|\cdot\|)$ is equal to $\lambda_{\mathcal{F}}\left(e_{1}\right)+\cdots+\lambda_{\mathcal{F}}\left(e_{r}\right)$.

(3) A basis $\boldsymbol{e}=\left\{e_{i}\right\}_{i=1}^{r}$ of $V$ is orthogonal if and only if it is compatible with the $\mathbb{R}$-filtration $\mathcal{F}$, namely $\#\left(\mathcal{F}^{t}(V) \cap \boldsymbol{e}\right)=\operatorname{rk}\left(\mathcal{F}^{t}(V)\right)$ for any $t \in \mathbb{R}$.

(4) Assume that the vector space $V$ is non-zero. The adelic vector bundle $(V,\|\cdot\|)$ is semistable if and only if the function $\|\cdot\|$ is constant on $V \backslash\{0\}$.

(5) The Harder-Narasimhan $\mathbb{R}$-filtration of $(V,\|\cdot\|)$ identifies with $\mathcal{F}$.

(6) Let $\boldsymbol{e}=\left\{e_{i}\right\}_{i=1}^{r}$ be an orthogonal basis of $(V,\|\cdot\|)$. Then the sequence of successive slopes of $(V,\|\cdot\|)$ identifies with the sorted sequence of $\left\{\lambda_{\mathcal{F}}\left(e_{i}\right)\right\}_{i=1}^{r}$. 
(7) Let

$$
0=V_{0} \subsetneq V_{1} \subsetneq \ldots \subsetneq V_{r}=V
$$

be a complete flag of vector subspaces of $V$. For any $i \in\{1, \ldots, r\}$, let $\|\cdot\|_{i}$ be the subquotient norm of $\|\cdot\|$ on the vector space $V_{i} / V_{i-1}$. Then the sequence of successive slopes of $(V,\|\cdot\|)$ identifies with the sorted sequence of

$$
\left\{\widehat{\operatorname{deg}}\left(V_{i} / V_{i-1},\|\cdot\|_{i}\right)\right\}_{i=1}^{r} .
$$

Proof. - (1) Note that the valued field $(K,|\cdot|)$ is locally compact. By Proposition 1.2.30, there exists an orthogonal basis of $(V,\|\cdot\|)$.

(2) Let $\boldsymbol{e}=\left\{e_{i}\right\}_{i=1}^{r}$ be an orthogonal basis of $(V,\|\cdot\|)$. By Proposition 1.2.23, it is an Hadamard basis, namely

$$
\left\|e_{1} \wedge \cdots \wedge e_{r}\right\|=\prod_{i=1}^{r}\left\|e_{i}\right\|
$$

Therefore one has

$$
\widehat{\operatorname{deg}}(V,\|\cdot\|)=-\ln \left\|e_{1} \wedge \cdots \wedge e_{r}\right\|=-\sum_{i=1}^{r} \ln \left\|e_{i}\right\|=\sum_{i=1}^{r} \lambda_{\mathcal{F}}\left(e_{i}\right)
$$

(3) Assume that the $\mathbb{R}$-filtration $\mathcal{F}$ corresponds to the flag

$$
0=V_{0} \subsetneq V_{1} \subsetneq \ldots \subsetneq V_{n}=V
$$

together with the sequence

$$
\mu_{1}>\ldots>\mu_{n}
$$

(cf. Remark 1.1.40). Let $\boldsymbol{e}=\left\{e_{i}\right\}_{i=1}^{r}$ be a basis of $V$. Then $\boldsymbol{e}$ is compatible with the $\mathbb{R}$-filtration $\mathcal{F}$ if and only if $\#\left(\boldsymbol{e} \cap V_{j}\right)=\operatorname{rk}\left(V_{j}\right)$ for any $j \in\{1, \ldots, n\}$. By Proposition 1.2.26 (1), this condition is also equivalent to the orthogonality of the basis $\boldsymbol{e}$.

(4) follows directly from Proposition 4.3.60 since $\|\cdot\|=\|\cdot\|_{* *}$ (see Corollary 1.2.12).

(5) The $\mathbb{R}$-filtration corresponds to an increasing flag

$$
0=V_{0} \subsetneq V_{1} \subsetneq \ldots \subsetneq V_{n}=V
$$

of vector subspaces of $V$, together with a decreasing sequence of real numbers

$$
\mu_{1}>\ldots>\mu_{n} .
$$

Note that for any $i \in\{1, \ldots, n\}$ and any $x \in V_{i} \backslash V_{i-1}$ one has $\lambda_{\mathcal{F}}(x)=\mu_{i}$. In particular, the subquotient norm $\|\cdot\|_{i}$ on $V_{i} / V_{i-1}$ induced by $\|\cdot\|$ takes constant value $\mathrm{e}^{-\mu_{i}}$ on $\left(V_{i} / V_{i-1}\right) \backslash\{0\}$. Therefore, by (4) the adelic vector bundle $\left(V_{i} / V_{i-1},\|\cdot\|_{i}\right)$ is semistable of slope $\mu_{i}$. By Proposition 4.3.37, we obtain that $\mathcal{F}$ is the HarderNarasimhan $\mathbb{R}$-filtration of $(V,\|\cdot\|)$.

(6) Assume that the $\mathbb{R}$-filtration $\mathcal{F}$ corresponds to the flag

$$
0=V_{0} \subsetneq V_{1} \subsetneq \ldots \subsetneq V_{n}=V
$$


and the sequence

$$
\mu_{1}>\ldots>\mu_{n}
$$

By definition, the value $\mu_{i}$ appears exactly $\operatorname{rk}\left(V_{i} / V_{i-1}\right)$ times in the successive slopes of $(V,\|\cdot\|)$. Moreover, a basis $\boldsymbol{e}$ is orthogonal if and only if it is compatible with the flag

$$
0=V_{0} \subsetneq V_{1} \subsetneq \ldots \subsetneq V_{n}=V
$$

or equivalently, for any $i \in\{1, \ldots, n\}$, the set $e \cap\left(V_{i} \backslash V_{i-1}\right)$ contains exactly $\operatorname{rk}\left(V_{i} / V_{i-1}\right)$ elements. Since the function $\lambda_{\mathcal{F}}(\cdot)$ takes the constant value $\mu_{i}$ on $V_{i} \backslash V_{i-1}$, we obtain the assertion.

(7) By Proposition 1.2.30, there exists an orthogonal basis $\boldsymbol{e}=\left\{e_{i}\right\}_{i=1}^{r}$ which is compatible with the flag

$$
0=V_{0} \subsetneq V_{1} \subsetneq \ldots \subsetneq V_{r}=V
$$

Without loss of generality, we may assume that $e_{i} \in V_{i} \backslash V_{i-1}$ for any $i \in\{1, \ldots, r\}$ Since the basis $\boldsymbol{e}=\left\{e_{i}\right\}_{i=1}^{r}$ is orthogonal, the image of $e_{i}$ in $V_{i} / V_{i-1}$ has norm $\left\|e_{i}\right\|$. In fact, any element $x$ in $e_{i}+V_{i-1}$ can be written in the form

$$
e_{i}+\sum_{j=1}^{i-1} a_{j} e_{j}
$$

and hence $\|x\|_{i} \geqslant\left\|e_{i}\right\|$. Therefore one has

$$
\widehat{\operatorname{deg}}\left(V_{i} / V_{i-1},\|\cdot\|_{i}\right)=-\ln \left\|e_{i}\right\|=\lambda_{\mathcal{F}}\left(e_{i}\right)
$$

Proposition 5.1.3. - Let $V$ be a finite-dimensional vector space over $K$ and $\|\cdot\|$ and $\|\cdot\|^{\prime}$ be two ultrametric norms on $V$. Then there exists a basis $e$ of $V$ which is orthogonal with respect to $\|\cdot\|$ and $\|\cdot\|^{\prime}$ simultaneously.

Proof. — Let $\mathcal{F}$ be the $\mathbb{R}$-filtration on $V$ associated with the norm $\|\cdot\|$, which corresponds to a flag

$$
0=V_{0} \subsetneq V_{1} \subsetneq \ldots \subsetneq V_{n}=V
$$

together with a sequence $\mu_{1}>\ldots>\mu_{n}$. By Proposition 1.2.30, there exists an orthogonal basis $\boldsymbol{e}$ of $\left(V,\|\cdot\|^{\prime}\right)$ which is compatible with the the flag

$$
0=V_{0} \subsetneq V_{1} \subsetneq \ldots \subsetneq V_{n}=V .
$$

By Proposition 5.1.2 $(3)$, we obtain that $e$ is also orthogonal with respect to $\|\cdot\|$.

Corollary 5.1.4. - Let $V$ be a finite-dimensional vector space over $K$, and

$$
0=V_{0} \subsetneq V_{1} \subsetneq \ldots \subsetneq V_{n}=V \quad \text { and } \quad 0=W_{0} \subsetneq W_{1} \subsetneq \ldots \subsetneq W_{m}=V
$$

be two flags of vector subspaces of $V$. There exists a basis e of $V$ which is compatible with the two flags simultaneously. 
Proof. - By choosing two decreasing sequences of real numbers $\mu_{1}>\ldots>\mu_{n}$ and $\lambda_{1}>\ldots>\lambda_{m}$ we obtain two $\mathbb{R}$-filtrations on $V$, which correspond to two ultrametric norms on $V$. By Proposition 5.1.3 there exists a basis of $V$ which is orthogonal with respect to the two norms simultaneously. By Proposition [5.1.2 (3), this basis is compatible with the two flags simultaneously.

Definition 5.1.5. - Let $d \in \mathbb{N}_{\geqslant 2}$ and $\left(E_{j}\right)_{j=1}^{d}$ be a family of finite-dimensional vector spaces over $K$. For any $j \in\{1, \ldots, d\}$, let $\mathcal{F}_{j}$ be an $\mathbb{R}$-filtrations on $E_{j}$, which corresponds to an ultrametric norm $\|\cdot\|_{j}$ on $E_{j}$. Let $\|\cdot\|_{\varepsilon}$ be the $\varepsilon$-tensor product of the norms $\|\cdot\|_{1}, \ldots,\|\cdot\|_{d}$. The $\mathbb{R}$-filtration on the tensor product space

$$
E_{1} \otimes_{K} \cdots \otimes_{K} E_{d}
$$

corresponding to $\|\cdot\|_{\varepsilon}$ is called the tensor product of the $\mathbb{R}$-filtrations $\mathcal{F}_{1}, \ldots, \mathcal{F}_{d}$, which is denoted by $\mathcal{F}_{1} \otimes \cdots \otimes \mathcal{F}_{d}$.

Remark 5.1.6. - We keep the notation of Definition 5.1.5. For any $j \in\{1, \ldots, d\}$, let $\boldsymbol{e}^{(j)}=\left\{e_{i}^{(j)}\right\}_{i=1}^{n_{j}}$ be an orthogonal base of $\left(E_{j},\|\cdot\|_{j}\right)$. By Proposition 1.2.19 together with Remark 1.1.56, one has the following:

(i) $\left\{e_{i_{1}}^{(1)} \otimes \cdots \otimes e_{i_{d}}^{(d)}\right\}_{\left(i_{1}, \ldots, i_{d}\right) \in \prod_{j=1}^{d}\left\{1, \ldots, n_{j}\right\}}$ forms an orthogonal basis of the vector space $E_{1} \otimes_{K} \cdots \otimes_{K} E_{d}$ with respect to $\|\cdot\|_{\varepsilon}$.

(ii) $\left\|e_{i_{1}}^{(1)} \otimes \cdots \otimes e_{i_{d}}^{(d)}\right\|_{\varepsilon}=\prod_{j=1}^{d}\left\|e_{i_{j}}^{(j)}\right\|_{j}$ for any $\left(i_{1}, \ldots, i_{d}\right) \in \prod_{j=1}^{d}\left\{1, \ldots, n_{j}\right\}$.

Therefore, if we denote by $\mathcal{F}$ the tensor product $\mathbb{R}$-filtration $\mathcal{F}_{1} \otimes \cdots \otimes \mathcal{F}_{d}$, then the vector space $\mathcal{F}^{t}\left(E_{1} \otimes_{K} \cdots \otimes_{K} E_{d}\right)$ is generated by the vectors $e_{i_{1}}^{(1)} \otimes \cdots \otimes e_{i_{d}}^{(d)}$ such that

$$
\lambda_{\mathcal{F}_{1}}\left(e_{i_{1}}^{(1)}\right)+\cdots+\lambda_{\mathcal{F}_{d}}\left(e_{i_{d}}^{(d)}\right) \geqslant t
$$

Therefore, one has

$$
\begin{aligned}
\mathcal{F}^{t}\left(E_{1} \otimes_{K} \cdots \otimes_{K} E_{d}\right) & =\sum_{t_{1}+\cdots+t_{d} \geqslant t} \mathcal{F}_{1}^{t_{1}}\left(E_{1}\right) \otimes_{K} \cdots \otimes_{K} \mathcal{F}_{d}^{t_{d}}\left(E_{d}\right) \\
& =\sum_{t_{1}+\cdots+t_{d}=t} \mathcal{F}_{1}^{t_{1}}\left(E_{1}\right) \otimes_{K} \cdots \otimes_{K} \mathcal{F}_{d}^{t_{d}}\left(E_{d}\right) .
\end{aligned}
$$

Furthermore, by (i), (ii) and Proposition 5.1.2 (6), if $\left(E_{j},\|\cdot\|_{j}\right)$ are all semistable, where $j \in\{1, \ldots, d\}$, then $\left(E_{1} \otimes_{K} \cdots \otimes_{K} E_{d},\|\cdot\|_{\varepsilon}\right)$ is also semistable.

\subsection{Reminder on geometric invariant theory}

Let $K$ be a field. By group scheme over Spec $K$ or by $K$-group scheme, we mean a $K$-scheme $\pi: G \rightarrow$ Spec $K$ equipped with a $K$-morphism $m_{G}: G \times_{K} G \rightarrow G$ (called the group scheme structure of $G$ ) such that, for any $K$-scheme $f: S \rightarrow$ Spec $K$, the set $G(S)$ of $K$-morphisms from $S$ to $G$ equipped with the composition 
law $m_{G}(S): G(S) \times G(S) \rightarrow G(S)$ forms a group. Note that the maps of inverse $\iota_{G}(S): G(S) \rightarrow G(S)$ and the maps of unity

$$
e_{G}(S): \operatorname{Spec} K(S)=\{S \stackrel{f}{\rightarrow} \operatorname{Spec} K\} \longrightarrow G(S)
$$

actually define $K$-morphisms $\iota_{G}: G \rightarrow G$ and $e_{G}:$ Spec $K \rightarrow G$, which make the following diagrams commutative:
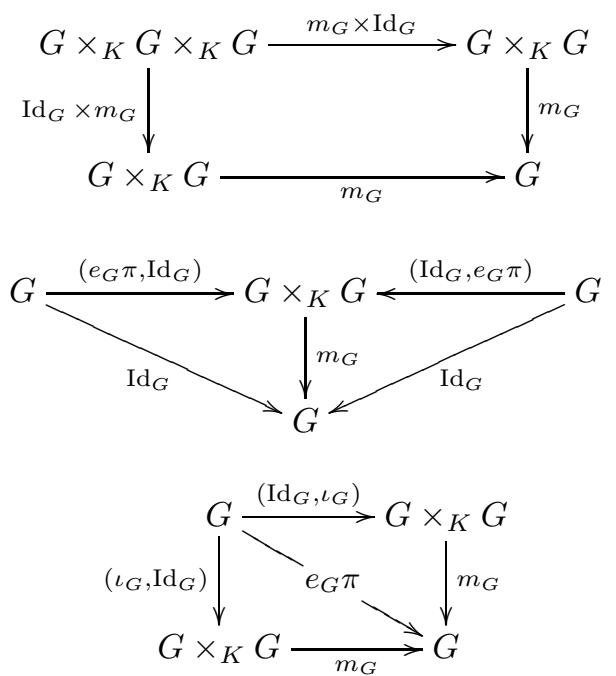

Let $G$ and $H$ be group schemes over Spec $K$. We call morphism of $K$-group schemes from $G$ to $H$ any $K$-morphism $f: G \rightarrow H$ such that, for any $K$-scheme $S$, le morphism $f$ induces a morphism of groups $f(S): G(S) \rightarrow H(S)$.

Example 5.2.1. - Let $V$ be a finite-dimensional vector space over $K$. We denote by $\mathbb{G} \mathbb{L}(V)$ the open subscheme of the affine $K$-scheme $\mathbb{A}\left(\operatorname{End}(V)^{\vee}\right)$ defined by the non-vanishing of the determinant. For any $K$-scheme $\pi: S \rightarrow \operatorname{Spec} K$, one has

$$
\mathbb{G L}(V)(S)=\operatorname{Aut}_{\mathcal{O}_{S}}\left(\pi^{*}(V)\right) .
$$

The set $\mathbb{G} \mathbb{L}(V)(S)$ is canonically equipped with a structure of group. The group structures of $\mathbb{G} \mathbb{L}(V)(S)$ where $S$ runs over the set of $K$-schemes define a $K$-morphism $\mathbb{G} \mathbb{L}(V) \times_{K} \mathbb{G L}(V) \rightarrow \mathbb{G} \mathbb{L}(V)$, which makes $\mathbb{G} L(V)$ a group scheme over $K$. The group scheme $\mathbb{G} \mathbb{L}(V)$ is called the general linear group scheme associated with $V$.

Definition 5.2.2. - Let $G$ be a group scheme over Spec $K$ and $X$ be a scheme over Spec $K$. As action of $G$ on $X$, we refer to a $K$-morphism $f: G \times_{K} X \rightarrow S$ such that, for any $K$-scheme $S$, the map

$$
f(S): G(S) \times X(S) \longrightarrow X(S)
$$

defines an action of the group $G(S)$ on $X(S)$. 
Example 5.2.3. - Let $V$ be a finite-dimensional vector space over $K$ and $X$ be the projective space $\mathbb{P}(V)$. Recall that, for any $K$-scheme $p: S \rightarrow \operatorname{Spec} K, \mathbb{P}(V)(S)$ identifies with the set of all invertible quotient modules of $p^{*}(V)$. Note that the automorphism of $p^{*}(V)$ acts naturally on the set $\mathbb{P}(V)(S)$ of invertible quotient modules of $p^{*}(V)$. Hence we obtain an action of the general linear group scheme $\mathbb{G} \mathbb{L}(V)$ on the projective space $\mathbb{P}(V)$.

More generally, let $G$ be a group scheme over Spec $K$. By (finite-dimensional) linear representation of $G$ we refer to a morphism of $K$-group schemes from $G$ to certain $\mathbb{G} \mathbb{L}(V)$, where $V$ is a finite-dimensional vector space over $K$. Note that such a morphism induces an action of $G$ on the projective space $\mathbb{P}(V)$. This action is said to be linear.

Let $G$ be a group scheme over Spec $K$ which acts on a $K$-scheme $X$. We denote by $f: G \times_{K} X \rightarrow X$ the action of $G$ on $X$ and by $\operatorname{pr}_{2}: G \times_{K} X \rightarrow X$ the projection to the second coordinate. Let $L$ be an invertible $\mathcal{O}_{X}$-module. We call $G$-linear structure on $L$ any isomorphism $\eta$ of $\mathcal{O}_{G \times_{K} X}$-modules from $f^{*}(L)$ to $\operatorname{pr}_{2}^{*}(L)$ such that the following diagram commutes

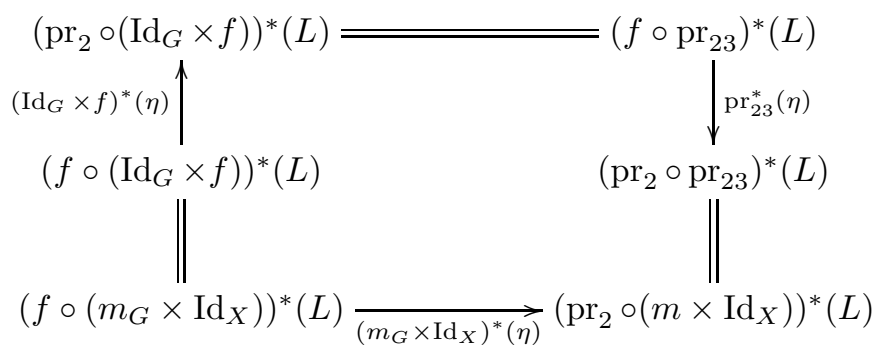

where $\mathrm{pr}_{23}: G \times_{K} G \times_{K} X \rightarrow G \times_{K} X$ is the projection to the second and the third coordinates, and $m_{G}: G \times{ }_{K} G \rightarrow G$ is the group scheme structure on $G$. The couple $(L, \eta)$ is called a $G$-linearised invertible $\mathcal{O}_{X}$-module.

Note that, if $\eta: f^{*}(L) \rightarrow \operatorname{pr}_{2}^{*}(L)$ is a $G$-linear structure on $L$, then $\eta^{\vee}: f^{*}\left(L^{\vee}\right) \rightarrow$ $\operatorname{pr}_{2}^{*}\left(L^{\vee}\right)$ is a $G$-linear structure on $L^{\vee}$. Moreover, if $\left(L_{1}, \eta_{1}\right)$ and $\left(L_{2}, \eta_{2}\right)$ are $G$ linearised invertible $\mathcal{O}_{X}$-modules, then

$\eta_{1} \otimes \eta_{2}: f^{*}\left(L_{1}\right) \otimes f^{*}\left(L_{2}\right) \cong f^{*}\left(L_{1} \otimes L_{2}\right) \longrightarrow \operatorname{pr}_{2}^{*}\left(L_{1} \otimes L_{2}\right) \cong \operatorname{pr}_{2}^{*}\left(L_{1}\right) \otimes \operatorname{pr}_{2}^{*}\left(L_{2}\right)$

is a $G$-linear structure on $L_{1} \otimes L_{2}$.

Example 5.2.4. - Let $G$ a group scheme over $K$ and $V$ be a finite-dimensional vector space over $K$. A linear action of $G$ on $\mathbb{P}(V)$ defines canonically a $G$-linear structure on the universal invertible sheaf $\mathcal{O}_{V}(1)$. Let $f: G \times_{K} \mathbb{P}(V) \rightarrow \mathbb{P}(V)$ be a linear action of $G$ on $\mathbb{P}(V)$. Let $\pi: \mathbb{P}(V) \rightarrow$ Spec $K$ be the structural morphism and $\beta: \pi^{*}(V) \rightarrow \mathcal{O}_{V}(1)$ be the tautological invertible quotient sheaf of $\pi^{*}(V)$. Note that 
the morphism

$$
G \times{ }_{K} \mathbb{P}(V) \stackrel{\left(\mathrm{pr}_{1}, f\right)}{\longrightarrow} G \times_{K} \mathbb{P}(V)
$$

is an isomorphism of $K$-schemes, the inverse of which is given by the composed $K$ morphism

$$
G \times_{K} \mathbb{P}(V) \stackrel{\left(\mathrm{pr}_{1}, \iota_{G} \mathrm{pr}_{1}, \mathrm{pr}_{2}\right)}{\longrightarrow} G \times_{K} G \times_{K} \mathbb{P}(V) \stackrel{\left(\mathrm{pr}_{1}, f \mathrm{pr}_{23}\right)}{\longrightarrow} G \times_{K} \mathbb{P}(V) .
$$

Moreover, one has $\operatorname{pr}_{2} \circ\left(\operatorname{pr}_{1}, f\right)=f$. Therefore $\left(\left(\operatorname{pr}_{1}, f\right)^{*}\right)^{-1}$ defines an isomorphism from $f^{*}\left(\mathcal{O}_{V}(1)\right)$ to $\operatorname{pr}_{2}^{*}\left(\mathcal{O}_{V}(1)\right)$ as invertible quotient modules of $f^{*}\left(\pi^{*}(V)\right) \cong$ $\operatorname{pr}_{2}^{*}\left(\pi^{*}(V)\right)$. The fact that the action of $G$ on $\mathbb{P}(V)$ is linear shows that this isomorphism actually defines a $G$-linear structure on $\mathcal{O}_{V}(1)$.

Definition 5.2.5. - We denote by $\mathbb{G}_{\mathrm{m}, K}=\operatorname{Spec} K\left[T, T^{-1}\right]$ the multiplicative group scheme over $\operatorname{Spec} K$ (recall that for any $K$-scheme $S$ one has $\mathbb{G}_{\mathrm{m}, K}(S)=$ $\left.\mathcal{O}_{S}(S)^{\times}\right)$. If $G$ is a group scheme over $\operatorname{Spec} K$, by one-parameter subgroup of $G$ any morphism of $K$-group schemes from $\mathbb{G}_{\mathrm{m}, K}$ to $G$.

Let $G$ be a group scheme over $K$, which acts on a $K$-scheme $X$. Denote by $f: G \times_{K} X \rightarrow X$ the action. If $\varphi: \mathbb{G}_{\mathrm{m}, K} \rightarrow G$ is a one-parameter subgroup of $G$, then $f$ and $\varphi$ induce an action of $\mathbb{G}_{\mathrm{m}, K}$ on $X$, denoted by $f_{\varphi}$. Note that $f_{\varphi}$ is the composed morphism

$$
\mathbb{G}_{\mathrm{m}, K} \times_{K} X \stackrel{\varphi \times \operatorname{Id}_{X}}{\longrightarrow} G \times_{K} X \stackrel{f}{\longrightarrow} X .
$$

Let $g: \mathbb{G}_{\mathrm{m}, K} \times_{K} X \rightarrow X$ be an action of the multiplicative group $\mathbb{G}_{\mathrm{m}, K}$ on a proper $K$-scheme. Suppose that $x: \operatorname{Spec} K \rightarrow X$ is a rational point of $X$. The orbit of $x$ by the action of $\mathbb{G}_{\mathrm{m}, K}$ is by definition the following composed morphism orb $\mathrm{b}_{x}$

$$
\mathbb{G}_{\mathrm{m}, K} \cong \mathbb{G}_{\mathrm{m}, K} \times_{K} \operatorname{Spec} K \stackrel{\operatorname{Id}_{\mathbb{G}_{\mathrm{m}, K}} \times x}{\longrightarrow} \mathbb{G}_{\mathrm{m}, K} \times_{K} X \stackrel{g}{\longrightarrow} X .
$$

Since $X$ is proper over $\operatorname{Spec} K$, by the valuative criterion of properness, the morphism orb $\operatorname{or}_{x}, K \rightarrow X$ extends in a unique way to a $K$-morphism $\widetilde{\operatorname{orb}}_{x}: \mathbb{A}_{K}^{1}=$ Spec $K[T] \rightarrow X$. The image by $\widetilde{\operatorname{orb}_{x}}$ of the rational point of $\mathbb{A}_{K}^{1}$ corresponding to the prime ideal $(T)$ is denoted by $\widetilde{x}_{g}$. Note that $\widetilde{x}_{g}$ is a rational point of $X$ which is invariant by the action of $\mathbb{G}_{\mathrm{m}, K}$.

Assume that $L$ is a $\mathbb{G}_{\mathrm{m}, K}$-linearised invertible $\mathcal{O}_{X}$-module. Since $\widetilde{x}_{g}$ is a fixed rational point of the action $g$, the $\mathbb{G}_{\mathrm{m}, K^{-}}$-linear structure corresponds to an action of $\mathbb{G}_{\mathrm{m}, K}$ on $\widetilde{x}_{g}^{*}(L)$, which is induced by an endomorphism of the $K$-group scheme $\mathbb{G}_{\mathrm{m}, K}$. Note that any endomorphism of the $K$-group scheme $\mathbb{G}_{\mathrm{m}, K}$ is of the form $t \mapsto t^{n}$, where the exponent $n$ is an integer. We denote by $\mu(x, L)$ the opposite of the exponent corresponding to the action of $\mathbb{G}_{\mathrm{m}, K}$ on $\widetilde{x}_{g}^{*}(L)$. Note that our choice of the constant $\mu(x, L)$ conforms with that of the book [109.

More generally, if $G$ is a $K$-group scheme, $f: G \times{ }_{K} X \rightarrow X$ is an action of $G$ on a proper $K$-scheme $X$ and if $\varphi: \mathbb{G}_{\mathrm{m}, K} \rightarrow G$ is a one-parameter subgroup of $G$, for any 
$x \in X(K)$ we denote by $\mu(x, \varphi, L)$ the exponent corresponding to the action of $\mathbb{G}_{\mathrm{m}, K}$ on $\widetilde{x}_{f_{\lambda}}^{*}(L)$.

Example 5.2.6. - Consider the one-parameter subgroups of the general linear group. Let $E$ be a finite-dimensional vector space over $K$ and $\lambda: \mathbb{G}_{m, K} \rightarrow \mathbb{G L}(E)$ be a one-parameter subgroup. By [51, II. $\left.\S 2, \mathrm{n}^{\circ} 2,2.5\right]$, we can decompose the vector space $E$ as a direct sum of $K$-vector subspaces $E_{1}, \ldots, E_{n}$ which are invariant by the action of $\mathbb{G}_{m, K}$, and integers $\left(a_{1}, \ldots, a_{n}\right)$ such that the action of $\mathbb{G}_{m, K}$ on $E_{i}$ is given by $t \mapsto t^{a_{i}} \operatorname{Id}_{E_{i}}$. Therefore the one-parameter subgroup $\varphi$ determines an $\mathbb{R}$-filtration $\mathcal{F}_{\varphi}$ on $E$ such that

$$
\mathcal{F}_{\varphi}^{t}(E)=\bigoplus_{\substack{i \in\{1, \ldots, n\} \\-a_{i} \geqslant t}} E_{i} .
$$

We now consider the canonical action of $\mathbb{G} \mathbb{L}(E)$ on the projective space $\mathbb{P}(E)$ (see Example [5.2.3). Let $x$ be a rational point of $\mathbb{P}(E)$ and $\pi_{x}: E \rightarrow K$ be the one dimensional quotient vector space of $E$ corresponding to $x$. Then the morphism $\operatorname{orb}_{x}: \mathbb{G}_{m, K}=\operatorname{Spec} K\left[T, T^{-1}\right] \rightarrow \mathbb{P}(E)$ is represented by the surjective $K\left[T, T^{-1}\right]$ linear map $p_{x}: E \otimes_{K} K\left[T, T^{-1}\right] \longrightarrow K\left[T, T^{-1}\right]$ sending $v_{i} \otimes 1$ to $\pi_{x}\left(v_{i}\right) T^{a_{i}}$ for any $v_{i} \in E_{i}$. The extended morphism $\widetilde{\operatorname{ord}}_{x}: \mathbb{A}_{K}^{1} \rightarrow \mathbb{P}(E)$ corresponds to the surjective $K[T]$-linear map

$$
\widetilde{p}_{x}: E \otimes_{K} K[T] \longrightarrow K[T] \cdot T^{-\mu\left(x, \mathcal{O}_{E}(1)\right)}
$$

given by the restriction of $p_{x}$ on $E \otimes_{K} K[T]$, where $\mathcal{O}_{E}(1)$ denotes the universal invertible sheaf. In particular, one has

$$
\mu\left(x, \varphi, \mathcal{O}_{E}(1)\right)=-\min \left\{a_{i}: i \in\{1, \ldots, n\}, \pi_{x}\left(E_{i}\right) \neq\{0\}\right\} .
$$

Therefore, we can interpret the constant $\mu\left(x, \varphi, \mathcal{O}_{E}(1)\right)$ via the $\mathbb{R}$-filtration $\mathcal{F}_{\varphi}$. In fact, the $\mathbb{R}$-filtration $\mathcal{F}_{\varphi}$ induces by the surjective map $\pi_{x}: E \rightarrow K$ an $\mathbb{R}$-filtration on $K$ (viewed as a one-dimensional vector space over $K$ ), which corresponds to the quotient norm of $\|\cdot\|_{\mathcal{F}_{\varphi}}$. Then the number $\mu\left(x, \varphi, \mathcal{O}_{E}(1)\right)$ is equal to the jump point of this quotient $\mathbb{R}$-filtration.

The following theorem of Hilbert-Mumford relates the positivity of $\mu(x, \lambda, L)$ to the non-vanishing of a global section invariant by the action of the $K$-group scheme.

Theorem 5.2.7. - We assume that the field $K$ is perfect. Let $G$ be a reductive $K$ group scheme acting on a projective $K$-scheme $X, L$ be a $G$-linearised ample invertible $\mathcal{O}_{X}$-module. For any rational point $x \in X(K)$, the following two conditions are equivalent:

(1) for any one-parameter subgroup $\lambda: \mathbb{G}_{\mathrm{m}, K} \rightarrow G$ of $G, \mu(x, \lambda, L) \geqslant 0$;

(2) there exists an integer $n \in \mathbb{N}_{\geqslant 1}$ and a section $s \in H^{0}\left(X, L^{\otimes n}\right)$ which is invariant under the action of $G(K)$ such that $x$ lies outside of the vanishing locus of $s$. 
We just explain why the condition (2) implies the positivity of $\mu(x, \lambda, L)$ for any one-parameter group. Let

$$
\widetilde{\operatorname{orb}_{x}}: \mathbb{A}_{K}^{1}=\operatorname{Spec} K[T] \longrightarrow X
$$

be the extended orbit of the rational point $x$ by the action of $\mathbb{G}_{\mathrm{m}, K}$ via $\lambda$. Then the pull-back of $L$ by $\widetilde{\operatorname{orb}}_{x}$ corresponds to a free $K[T]$-module of rank 1 , which is equipped with a linear action of $\mathbb{G}_{\mathrm{m}, K}$. This action corresponds to an invertible element of the tensorial algebra

$$
K\left[t, t^{-1}\right] \otimes_{K} K[T] \cong K\left[t, t^{-1}, T\right],
$$

where $t$ and $T$ are variables. Moreover, the compatibility condition (5.1) shows that $\eta(t, T)$ satisfies the following relation

$$
\eta(t, T) \eta(u, T)=\eta(t u, T) \text { in } K\left[t, t^{-1}, u, u^{-1}, T\right],
$$

where $t, u$, and $T$ are variables. Therefore $\eta(t, T)$ is of the form $t^{-\mu(x, \lambda, L)}$. We fix a section $m$ of $\widetilde{\operatorname{orb}}_{x}^{*}(L)$ which trivialises this invertible sheaf. Note that the pull-back of the section $s$ is an element of this free $K[T]$-module which is invariant by the action of $\mathbb{G}_{\mathrm{m}, K}(K)=K^{\times}$. We write $s$ in the form $P(T) m$, where $P \in k[T]$. Then the action of an element $a \in K^{\times}$on $s$ gives the section $P(a T) a^{-\mu(x, \lambda, L)} m$. Hence $P$ is a homogeneous polynomial and $\mu(x, \lambda, L)$ is equal to the degree of $P$, which is a nonnegative integer. We refer the readers to [109, §2.1] for a proof of the above theorem. See also [92] and [120].

Definition 5.2.8. - Under the assumption and with the notation of Theorem 5.2.7 if $x \in X(K)$ satisfies the equivalent conditions of the theorem, we say that the point $x$ is semistable with respect to the $G$-linearised invertible $\mathcal{O}_{X}$-module $L$.

Remark 5.2.9. - Let $d \in \mathbb{N}_{\geqslant 2}$ and $\left\{E_{j}\right\}_{j=1}^{d}$ be a family of finite-dimensional nonzero vector spaces over $K$. Any one-parameter subgroup

$$
\lambda: \mathbb{G}_{\mathrm{m}, K} \longrightarrow \mathbb{G} \mathbb{L}\left(E_{1}\right) \times_{K} \cdots \times_{K} \mathbb{G} \mathbb{L}\left(E_{d}\right)
$$

can be written in the form $\left(\lambda_{1}, \cdots, \lambda_{d}\right)$, where $\lambda_{j}: \mathbb{G}_{\mathrm{m}, K} \rightarrow \mathbb{G} \mathbb{L}\left(E_{j}\right)$ is a oneparameter subgroup of $\mathbb{G L}\left(E_{j}\right), j \in\{1, \ldots, d\}$. We can then decompose the vector space $E_{j}$ into the direct sum of eigenspaces of the action $\lambda_{j}$ as follows:

$$
E_{j}=E_{j, 1} \oplus \cdots \oplus E_{j, n_{j}},
$$

where each $E_{j, i}$ is stable by the action of $\lambda_{j}$, and on $E_{j, i}$ the action of $\mathbb{G}_{\mathrm{m}, K}$ is given by $t \mapsto t^{a_{j, i}} \operatorname{Id}_{E_{j, i}}, i \in\left\{1, \ldots, n_{j}\right\}$. Note that the one-parameter subgroup $\lambda$ induces an action of $\mathbb{G}_{\mathrm{m}, K}$ on the tensor product space $E_{1} \otimes_{K} \cdots \otimes_{K} E_{d}$ via the canonical morphisme of $K$-group schemes

$$
\mathbb{G} \mathbb{L}\left(E_{1}\right) \times_{K} \cdots \times_{K} \mathbb{G} \mathbb{L}\left(E_{d}\right) \longrightarrow \mathbb{G L}\left(E_{1} \otimes_{K} \cdots \otimes_{K} E_{d}\right) .
$$


For any $\left(i_{1}, \ldots, i_{d}\right) \in \prod_{j=1}^{d}\left\{1, \ldots, n_{j}\right\}$, the vector subspace $E_{1, i_{1}} \otimes_{K} \cdots \otimes_{K} E_{d, i_{d}}$ of $E_{1} \otimes_{K} \cdots \otimes_{K} E_{d}$ is invariant by the action of $\mathbb{G}_{\mathrm{m}, K}$, and on $E_{1, i_{1}} \otimes_{K} \cdots \otimes_{K} E_{d, i_{d}}$ the action of $\mathbb{G}_{\mathrm{m}, K}$ is given by

$$
t \longmapsto t^{a_{1, i_{1}}+\cdots+a_{d, i_{d}}} \operatorname{Id}_{E_{1, i_{1}} \otimes_{K} \cdots \otimes_{K} E_{d, i_{d}}} .
$$

We construct an $\mathbb{R}$-filtration $\mathcal{F}_{\lambda}$ on $E_{1} \otimes_{K} \cdots \otimes_{K} E_{d}$ as follows

$$
\mathcal{F}_{\lambda}^{t}\left(E_{1} \otimes_{K} \cdots \otimes_{K} E_{d}\right):=\sum_{\substack{\left(i_{1}, \ldots, i_{d}\right) \in \prod_{j=1}^{d}\left\{1, \ldots, n_{j}\right\} \\-a_{1, i_{1}}-\cdots-a_{d, i_{d}} \geqslant t}} E_{1, i_{1}} \otimes_{K} \cdots \otimes_{K} E_{d, i_{d}} .
$$

Moreover, if we denote by $\mathcal{F}_{\lambda_{j}}$ the $\mathbb{R}$-filtrations on $E_{j}$ defined by

$$
\mathcal{F}_{\lambda_{j}}^{t}\left(E_{j}\right)=\sum_{\substack{i \in\left\{1, \ldots, n_{j}\right\} \\-a_{i} \geqslant t}} E_{j, i}
$$

then the $\mathbb{R}$-filtration $\mathcal{F}_{\lambda}$ defined in (5.2) identifies with the tensor product of $\mathcal{F}_{\lambda_{1}}, \ldots, \mathcal{F}_{\lambda_{d}}$ (see Definition [5.1.5, see also Remark 5.1.6). Conversely, for any $\mathbb{R}$-filtration $\mathcal{F}_{j}$ with integral jump points on the vector spaces $E_{j}$, then there exists a one-parameter subgroup $\lambda_{j}: \mathbb{G}_{m, K} \rightarrow \mathbb{G} \mathbb{L}\left(E_{j}\right)$ such that $\mathcal{F}_{\lambda_{j}}=\mathcal{F}_{j}$. This comes from Proposition 1.2.30 which allows us to construct the actions of $\mathbb{G}_{m, K}$ on $E_{j}$ diagonally with respect to an orthogonal basis.

More generally, for any integer $r \geqslant 1$, any one-parameter subgroup

$$
\lambda=\left(\lambda_{1}, \ldots, \lambda_{d}\right): \mathbb{G}_{\mathrm{m}, \mathrm{K}} \rightarrow \mathbb{G} \mathbb{L}\left(E_{1}\right) \times_{K} \cdots \times_{K} \mathbb{G} \mathbb{L}\left(E_{d}\right)
$$

induces an action of $\mathbb{G}_{\mathrm{m}, K}$ on the $K$-vector space

$$
\left(E_{1} \otimes_{K} \cdots \otimes_{K} E_{d}\right)^{\otimes r}
$$

Again the $\mathbb{R}$-filtration on $\left(E_{1} \otimes_{K} \cdots \otimes_{K} E_{d}\right)^{\otimes r}$ corresponding to the eigenspace decomposition of the action of $\mathbb{G}_{\mathrm{m}, K}$ identifies with

$$
\left(\mathcal{F}_{\lambda_{1}} \otimes \cdots \otimes \mathcal{F}_{\lambda_{d}}\right)^{\otimes r}
$$

For any $j \in\{1, \ldots, d\}$, let $a_{j}$ be the rank of $E_{j}$. Consider a non-zero quotient vector space $V$ of $E_{1} \otimes_{K} \cdots \otimes_{K} E_{d}$. Let $r$ be the rank of $V$ over $K$. The canonical surjective map $E_{1} \otimes_{K} \cdots \otimes_{K} E_{d} \rightarrow V$ determines a rational point $x$ of

$$
\pi: P=\mathbb{P}\left(\left(E_{1} \otimes_{K} \cdots \otimes E_{d}\right)^{\otimes r}\right) \longrightarrow \operatorname{Spec} K,
$$

which corresponds the composed map

$$
\left(E_{1} \otimes_{K} \cdots \otimes_{K} E_{d}\right)^{\otimes r} \longrightarrow V^{\otimes r} \longrightarrow \operatorname{det}(V) .
$$

We consider the semistability of the point $x$ with respect to the $\mathbb{G} \mathbb{L}\left(E_{1}\right) \times_{K} \cdots \times_{K}$ $\mathbb{G} \mathbb{L}\left(E_{d}\right)$-invertible sheaf

$$
L:=\mathcal{O}_{P}\left(a_{1} \cdots a_{d}\right) \otimes \pi^{*}\left(\operatorname{det}\left(E_{1}^{\vee}\right)^{\otimes r b_{1}} \otimes \cdots \otimes \operatorname{det}\left(E_{d}^{\vee}\right)^{\otimes r b_{d}}\right),
$$


where for any $j \in\{1, \ldots, d\}$,

Let

$$
b_{j}:=\frac{a_{1} \cdots a_{d}}{a_{j}} .
$$

$$
\lambda=\left(\lambda_{1}, \ldots, \lambda_{d}\right): \mathbb{G}_{m, K} \longrightarrow \mathbb{G} \mathbb{L}\left(E_{1}\right) \times_{K} \cdots \times_{K} \mathbb{G} \mathbb{L}\left(E_{d}\right)
$$

be a one-parameter subgroup, which determine, for each $j \in\{1, \ldots, d\}$, an $\mathbb{R}$-filtration $\mathcal{F}_{\lambda_{j}}$ on $E_{j}$. We let $\|\cdot\|_{j}$ be the ultrametric norm on $E_{j}$ corresponding to $\mathcal{F}_{\lambda_{j}}$, where we consider the trivial absolute value on $K$. We equip $E_{1} \otimes_{K} \cdots \otimes_{K} E_{d}$ with the $\varepsilon$-tensor product of the norms $\|\cdot\|_{j}$ and equip $V$ with the quotient norm. By Example 5.2 .6 and Proposition 1.2.15, we obtain that

$$
\mu\left(x, \lambda, \mathcal{O}_{P}(1)\right)=\widehat{\operatorname{deg}}(\bar{V}) .
$$

Moreover, by definition, for any $j \in\{1, \ldots, d\}$ one has

$$
\mu\left(x, \lambda_{j}, \pi^{*}\left(\operatorname{det}\left(E_{j}^{\vee}\right)\right)\right)=-\widehat{\operatorname{deg}}\left(E_{j},\|\cdot\|_{j}\right)
$$

Therefore we obtain

$$
\mu(x, \lambda, L)=a_{1} \cdots a_{d} \widehat{\operatorname{deg}}(\bar{V})-r \sum_{j=1}^{d} b_{j} \widehat{\operatorname{deg}}\left(\bar{E}_{j}\right) .
$$

Therefore we deduce from the Hilbert-Mumford criterion the following result.

Corollary 5.2.10. - We equip $K$ with the trivial absolute value. Let $\left\{E_{j}\right\}_{j=1}^{d}$ be a finite family of finite-dimensional non-zero vector spaces over $K$, and $V$ be a nonzero quotient vector space of $E_{1} \otimes_{K} \cdots \otimes_{K} E_{d}$. Let $r$ be the rank of $V$, and, for any $j \in\{1, \ldots, d\}$, let $a_{j}$ the rank of $E_{j}$. Let

$$
P=\mathbb{P}\left(\left(E_{1} \otimes_{K} \cdots \otimes_{K} E_{d}\right)^{\otimes r}\right),
$$

$\pi: P \rightarrow \operatorname{Spec} K$ be the canonical morphism and

$$
L=\mathcal{O}_{P}\left(a_{1} \cdots a_{d}\right) \otimes \pi^{*}\left(\operatorname{det}\left(E_{1}^{\vee}\right)^{\otimes r b_{1}} \otimes \cdots \otimes \operatorname{det}\left(E_{d}^{\vee}\right)^{\otimes r b_{d}}\right),
$$

where

$$
\forall j \in\{1, \ldots, d\}, \quad b_{j}=\frac{a_{1} \cdots a_{d}}{a_{j}} .
$$

Then the composed surjective map

$$
\left(E_{1} \otimes_{K} \cdots \otimes_{K} E_{d}\right)^{\otimes r} \longrightarrow V^{\otimes r} \longrightarrow \operatorname{det}(V),
$$

viewed as a rational point $x$ of $P$, is semistable with respect to the $\mathbb{G} \mathbb{L}\left(E_{1}\right) \times_{K} \cdots \times_{K}$ $\mathbb{G L}\left(E_{d}\right)$-linearised invertible sheaf $L$ if and only if, for all ultrametric norms $\|\cdot\|_{j}$ on $E_{j}, j \in\{1, \ldots, d\}$, if we equip $V$ with the quotient norm of the $\varepsilon$-tensor product of $\|\cdot\|_{1}, \ldots,\|\cdot\|_{d}$, then one has

$$
\widehat{\mu}(\bar{V}) \geqslant \sum_{j=1}^{d} \widehat{\mu}\left(\bar{E}_{j}\right) .
$$


Proof. - Assume that the inequality (5.5) holds for any choice of norms $\|\cdot\|_{j}$. By (5.3), for any one-parameter subgroup $\lambda: \mathbb{G}_{m, K} \rightarrow \mathbb{G} \mathbb{L}\left(E_{1}\right) \times_{K} \cdots \times_{K} \mathbb{G} \mathbb{L}\left(E_{d}\right)$, one has

$$
\mu(x, \lambda, L)=a_{1} \cdots a_{d} r\left(\widehat{\mu}(\bar{V})-\sum_{j=1}^{d} \widehat{\mu}\left(\bar{E}_{j}\right)\right) \geqslant 0 .
$$

Hence the rational point $x$ of $P$ defined by (5.4) is semistable with respect to $L$.

Conversely, by Remark 5.2.9 the semi-stability of the rational points $x$ implies that the inequality (5.5) holds for any choice of ultrametric norms $\|\cdot\|_{j}$ such that $\ln \left\|E_{j} \backslash\{0\}\right\|_{j} \subseteq \mathbb{Z}$. As a consequence the inequality (5.5) holds for any choice of ultrametric norms $\|\cdot\|_{j}$ such that $\ln \left\|E_{j} \backslash\{0\}\right\|_{j} \subseteq \mathbb{Q}$. In fact, in this case there exists $n \in \mathbb{N}_{>0}$ such that the (finite) set $\ln \left\|E_{j} \backslash\{0\}\right\|_{j}$ is contained in $\frac{1}{n} \mathbb{Z}$ for any $j \in\{1, \ldots, d\}$. Note that the $n^{\text {th }}$ power of the function $\|\cdot\|_{j}$ forms a norm on $E_{j}$. If we denote by $\|\cdot\|_{V}$ the quotient norm of the $\varepsilon$-tensor product of $\|\cdot\|_{1}, \ldots,\|\cdot\|_{d}$, then the quotient norm of the $\varepsilon$-tensor product of $\|\cdot\|_{1}^{n}, \ldots,\|\cdot\|_{d}^{n}$ is $\|\cdot\|_{V}^{n}$. Note that

$$
\forall j \in\{1, \ldots, d\}, \quad \ln \left\|E_{j} \backslash\{0\}\right\|_{j}^{n}=n \ln \left\|E_{j} \backslash\{0\}\right\|_{j} \subseteq \mathbb{Z}
$$

and hence

$$
n \widehat{\mu}\left(V,\|\cdot\|_{V}\right)=\widehat{\mu}\left(V,\|\cdot\|_{V}^{n}\right) \geqslant \sum_{j=1}^{d} \widehat{\mu}\left(E_{j},\|\cdot\|_{j}^{n}\right)=n \sum_{j=1}^{d} \widehat{\mu}\left(E_{j},\|\cdot\|_{j}\right) .
$$

Finally the general case follows from a limite procedure by using Proposition 4.3.17

\subsection{Estimate for the minimal slope under semi-stability assumption}

In this section, we fix an adelic curve $S=(K,(\Omega, \mathcal{A}, \nu), \phi)$ and assume in addition that the field $K$ is perfect. We fix an integer $d \geqslant 2$ and we let $\left\{\bar{E}_{j}=\left(E_{j}, \xi_{j}\right)\right\}_{j=1}^{d}$ be a family of non-zero adelic vector bundles on $S$. Let $V$ be a quotient vector space of $E_{1} \otimes_{K} \cdots \otimes_{K} E_{d}$ and $r$ be the rank of $V$ over $K$. For any $j \in\{1, \ldots, d\}$, let $a_{j}$ be the rank of $E_{j}$. We equip $V$ with the quotient norm family of $\xi_{1} \otimes_{\varepsilon, \pi} \cdots \otimes_{\varepsilon, \pi} \xi_{d}$. Note that the quotient map $E_{1} \otimes_{K} \cdots \otimes_{K} E_{d} \rightarrow V$ induces a surjective map

$$
\Lambda^{r}\left(E_{1} \otimes_{K} \cdots \otimes_{K} E_{d}\right) \longrightarrow \Lambda^{r} V=\operatorname{det}(V) .
$$

Consider the composed map

$$
\left(E_{1} \otimes_{K} \cdots \otimes_{K} E_{d}\right)^{\otimes r} \longrightarrow \Lambda^{r}\left(E_{1} \otimes_{K} \cdots \otimes_{K} E_{d}\right) \longrightarrow \operatorname{det}(V),
$$

which permits to identify $\operatorname{det}(V)$ as a rational point of $P=\mathbb{P}\left(\left(E_{1} \otimes_{K} \cdots \otimes_{K} E_{d}\right)^{\otimes r}\right)$. Denote by $\pi: P \rightarrow$ Spec $K$ the structural morphism and by $L$ the invertible sheaf

$$
\mathcal{O}_{P}\left(a_{1} \cdots a_{d}\right) \otimes \pi^{*}\left(\operatorname{det}\left(E_{1}^{\vee}\right)^{\otimes r b_{1}} \otimes \cdots \otimes \operatorname{det}\left(E_{d}^{\vee}\right)^{r \otimes b_{d}}\right),
$$


where

$$
\forall j \in\{1, \ldots, d\}, \quad b_{j}=\frac{a_{1} \cdots a_{d}}{a_{j}} .
$$

We equip $L$ with its natural $\mathbb{G} \mathbb{L}\left(E_{1}\right) \times_{K} \cdots \times_{K} \mathbb{G} \mathbb{L}\left(E_{d}\right)$-linear structure. Note that $L$ and $\mathcal{O}_{P}\left(a_{1} \cdots a_{d}\right)$ are isomorphic as invertible $\mathcal{O}_{P}$-modules, however the natural $\mathbb{G L}\left(E_{1}\right) \times_{K} \cdots \times_{K} \mathbb{G} \mathbb{L}\left(E_{d}\right)$-linear structure on these two invertible sheaves are different.

Our purpose is to estimate $\widehat{\mu}(\bar{V})$ under the additional assumption that, as a rational point of $P=\mathbb{P}\left(\left(E_{1} \otimes_{K} \cdots \otimes_{K} E_{d}\right)^{\otimes r}\right)$, the determinant line $\operatorname{det}(V)$ is semistable with respect to the $\mathbb{G L}\left(E_{1}\right) \times_{K} \cdots \times_{K} \mathbb{G} \mathbb{L}\left(E_{d}\right)$-linearised invertible sheaf $L$.

Proposition 5.3.1. - We equip $V$ with the quotient norm family $\xi_{V}$ of $\xi_{1} \otimes_{\varepsilon, \pi}$ $\cdots \otimes_{\varepsilon, \pi} \xi_{d}$. Assume that, as a rational point of the $K$-scheme $P, \operatorname{det}(V)$ is semistable with respect to the $\mathbb{G} \mathbb{L}\left(E_{1}\right) \times_{K} \cdots \times_{K} \mathbb{G L}\left(E_{d}\right)$-linearised invertible sheaf $L$ defined in (5.6). Then the following inequality holds:

$$
\widehat{\mu}\left(V, \xi_{V}\right) \geqslant \sum_{j=1}^{d}\left(\widehat{\mu}\left(E_{j}, \xi_{j}\right)-\nu\left(\Omega_{\infty}\right) \ln \left(\operatorname{rk}\left(E_{j}\right)\right)\right) .
$$

Proof. - For any integer $m \in \mathbb{N}_{\geqslant 1}$, let $\mathfrak{S}_{m}$ be the symmetric group of $\{1, \ldots, m\}$. Let $A=a_{1} \cdots a_{d}$. By the first principal theorem of the classic invariant theory (see [141. Chapter III] and [5. Appendix 1], see also [36. Theorem 3.3]), there exist an integer $n \geqslant 1$ and an element $\left(\sigma_{1}, \ldots, \sigma_{d}\right) \in \mathfrak{S}_{n r A}^{d}$ such that the composed map

$$
\begin{gathered}
\operatorname{det}(V)^{\vee \otimes n A} \\
E_{1}^{\vee \otimes n r A} \otimes \cdots \otimes E_{d}^{\vee \otimes n r A} \\
E_{1}^{\vee \otimes n r A} \otimes \cdots \otimes E_{d}^{\vee \otimes n r A} \\
\varpi \\
\operatorname{det}\left(E_{1}^{\vee}\right)^{\otimes n r b_{1}} \otimes \cdots \otimes \operatorname{det}\left(E_{d}^{\vee}\right)^{\otimes n r b_{d}}
\end{gathered}
$$

is non-zero. Since $\xi_{V}$ is the quotient norm family of $\xi_{1} \otimes_{\varepsilon, \pi} \cdots \otimes_{\varepsilon, \pi} \xi_{d}$, the determinant norm family $\operatorname{det}\left(\xi_{V}\right)$ is the quotient norm family of the $\varepsilon, \pi$-tensor product norm family $\xi_{1}^{\otimes_{\varepsilon}, \pi} \otimes_{\varepsilon, \pi} \cdots \otimes_{\varepsilon, \pi} \xi_{d}^{\otimes_{\varepsilon}, \pi}$ by the following composed map (this is a consequence of Propositions 1.1.14 (1), 1.1.58 and 1.2.39)

$$
E_{1}^{\otimes r} \otimes_{K} \cdots \otimes_{K} E_{d}^{\otimes r} \longrightarrow \Lambda^{r}\left(E_{1} \otimes_{K} \cdots \otimes_{K} E_{d}\right) \longrightarrow \Lambda^{r}(V)=\operatorname{det}(V) .
$$

By passing to the dual vector space, we obtain that the dual of the determinant norm family $\operatorname{det}\left(\xi_{V}\right)^{\vee}$ identifies with the restrict norm family of $\xi_{1}^{\vee \otimes_{\varepsilon} r} \otimes_{\varepsilon} \cdots \otimes_{\varepsilon} \xi_{d}^{\vee \otimes_{\varepsilon} r}$. This is a consequence of Proposition 1.1.57, Corollary 1.2.20 and Proposition 1.1.20, 
By Proposition 1.2.18, the height of the $K$-linear map $\varpi$ in (5.8) is bounded from above by

$$
\sum_{j=1}^{d} n r b_{j} \ln \left(a_{j} !\right)
$$

where we consider the norm family $\xi_{1}^{\vee \otimes_{\varepsilon} n r A} \otimes_{\varepsilon} \cdots \otimes_{\varepsilon} \xi_{d}^{\vee \otimes_{\varepsilon} n r A}$ on $E_{1}^{\vee \otimes n r A} \otimes_{K} \cdots \otimes_{K}$ $E_{d}^{\vee \otimes n r A}$, and the norm family $\operatorname{det}\left(\xi_{1}\right)^{\vee \otimes n r b_{1}} \otimes \cdots \otimes \operatorname{det}\left(\xi_{d}\right)^{\vee \otimes n r b_{d}}$ on $\operatorname{det}\left(E_{1}^{\vee}\right)^{\otimes n r b_{1}} \otimes_{K}$ $\cdots \otimes_{K} \operatorname{det}\left(E_{d}^{\vee}\right)^{\otimes n r b_{d}}$.

Therefore by the slope inequality we obtain

$$
-n A \widehat{\operatorname{deg}}\left(V, \xi_{V}\right) \leqslant-\sum_{j=1}^{d}\left(n r b_{j} \widehat{\operatorname{deg}}\left(E_{j}, \xi_{j}\right)-\nu\left(\Omega_{\infty}\right) n r b_{j} \ln \left(a_{j} !\right)\right)
$$

which leads to

$$
\begin{aligned}
\widehat{\mu}\left(V, \xi_{V}\right) & \geqslant \sum_{j=1}^{d}\left(\widehat{\mu}\left(E_{j}, \xi_{j}\right)-\frac{1}{a_{j}} \ln \left(a_{j} !\right) \nu\left(\Omega_{\infty}\right)\right) \\
& \geqslant \sum_{j=1}^{d}\left(\widehat{\mu}\left(E_{j}, \xi_{j}\right)-\ln \left(a_{j}\right) \nu\left(\Omega_{\infty}\right)\right) .
\end{aligned}
$$

Remark 5.3.2. - Assume that the norm families $\xi_{1}, \ldots, \xi_{d}$ are Hermitian. If we equip $V$ with the quotient norm family $\xi_{V}^{H}$ of the orthogonal tensor product $\xi_{1} \otimes \cdots \otimes \xi_{d}$, then a similar argument as above leads to the following inequality (where we use Proposition 1.2 .62 to compute the height of $\varpi$ )

$$
\widehat{\mu}\left(V, \xi_{V}^{H}\right) \geqslant \sum_{j=1}^{d}\left(\widehat{\mu}\left(E_{j}, \xi_{j}\right)-\frac{1}{2} \nu\left(\Omega_{\infty}\right) \ln \left(\operatorname{rk}\left(E_{j}\right)\right)\right) .
$$

\subsection{An interpretation of the geometric semistability}

Let $E$ be a finite-dimensional non-zero vector space over $K$ and $r$ be the rank of $E$ over $K$. We denote by $\mathbf{F i l}(E)$ the set of $\mathbb{R}$-filtrations $\mathcal{E}$ on $E$. Let $\mathbf{F i l}_{0}(E)$ be the subset of $\mathbf{F i l}(E)$ of $\mathbb{R}$-filtrations $\mathcal{E}$ such that $\widehat{\operatorname{deg}}\left(E,\|\cdot\|_{\mathcal{E}}\right)=0$, where $\|\cdot\|_{\mathcal{E}}$ is the norm on $E$ associated with the $\mathbb{R}$-filtration $\mathcal{E}$ (here we consider the trivial absolute value on $K$ ), in other words,

$$
\forall x \in E, \quad\|x\|_{\mathcal{E}}=\exp \left(-\sup \left\{t \in \mathbb{R}: x \in \mathcal{E}^{t}(E)\right\}\right) .
$$

Let $\mathcal{E}_{1}$ and $\mathcal{E}_{2}$ be two elements in $\mathbf{F i l}(E)$. By Proposition 5.1.3, there exists a basis $\boldsymbol{e}=\left\{e_{i}\right\}_{i=1}^{r}$ of $E$ which is orthogonal with respect to the norms $\|\cdot\|_{\mathcal{E}_{1}}$ and $\|\cdot\|_{\mathcal{E}_{2}}$ 
simultaneously. We denote by $\left\langle\mathcal{E}_{1}, \mathcal{E}_{2}\right\rangle$ the number

$$
\frac{1}{r} \sum_{i=1}^{r}\left(-\ln \left\|e_{i}\right\|_{\mathcal{E}_{1}}\right)\left(-\ln \left\|e_{i}\right\|_{\mathcal{E}_{2}}\right) .
$$

As shown by the following proposition, this number actually does not depend on the choice of the basis $\boldsymbol{e}$.

Proposition 5.4.1. - Let $E$ be a finite-dimensional non-zero vector space over $K$, and $\mathcal{E}_{1}$ and $\mathcal{E}_{2}$ be $\mathbb{R}$-filtrations on $E$. If $\boldsymbol{e}=\left\{e_{i}\right\}_{i=1}^{r}$ is a basis of $E$ which is compatible with the $\mathbb{R}$-filtrations $\mathcal{E}_{1}$ and $\mathcal{E}_{2}$ simultaneously, then the following equality holds

$$
\sum_{i=1}^{r} \lambda_{\mathcal{E}_{1}}\left(e_{i}\right) \lambda_{\mathcal{E}_{2}}\left(e_{i}\right)=\sum_{t \in \mathbb{R}} t \widehat{\operatorname{deg}}\left(\mathrm{sq}_{\mathcal{E}_{1}}^{t}(E),\|\cdot\|_{\mathcal{E}_{2}, \mathrm{sq}_{\mathcal{E}_{1}}^{t}(E)}\right),
$$

where $\|\cdot\|_{\mathcal{E}_{2}, \mathrm{sq}_{\mathcal{E}_{1}}^{t}(E)}$ denotes the subquotient norm induced by $\|\cdot\|_{\mathcal{E}_{2}}$ on the vector space $\mathrm{sq}_{\mathcal{E}_{1}}^{t}(E)$.

Proof. - We assume that the $\mathbb{R}$-filtration $\mathcal{E}_{1}$ corresponds to the flag

$$
0=V_{0} \subsetneq V_{1} \subsetneq \ldots \subsetneq V_{n}=V
$$

together with the sequence

$$
\mu_{1}>\ldots>\mu_{n} .
$$

Then the right hand side of the formula can be written as

$$
\sum_{j=1}^{n} \mu_{j} \widehat{\operatorname{deg}}\left(V_{j} / V_{j-1},\|\cdot\|_{\mathcal{E}_{2}, j}\right)
$$

where $\|\cdot\|_{\mathcal{E}_{2}, j}$ is the subquotient norm on $V_{j} / V_{j-1}$ induced by $\|\cdot\|_{\mathcal{E}_{2}}$. By Proposition 5.1.2 (3) the basis $\boldsymbol{e}$ is compatible with respect to the flag

$$
0=V_{0} \subsetneq V_{1} \subsetneq \ldots \subsetneq V_{n}=V .
$$

By Proposition 1.2.6 the canonical image of $\boldsymbol{e} \cap\left(V_{j} \backslash V_{j-1}\right)$ in $V_{j} / V_{j-1}$ forms an orthogonal basis of $\left(V_{j} / V_{j-1},\|\cdot\|_{\mathcal{E}_{2}, j}\right)$. Moreover, for any $x \in \boldsymbol{e} \cap\left(V_{j} \backslash V_{j-1}\right)$ one has

$$
\|x\|_{\mathcal{E}_{2}}=\|[x]\|_{\mathcal{E}_{2}, j}
$$

and $\|x\|_{\mathcal{E}_{1}}=\mathrm{e}^{-\mu_{j}}$ Therefore,

$$
\begin{aligned}
& \sum_{i=1}^{r} \lambda_{\mathcal{E}_{1}}\left(e_{i}\right) \lambda_{\mathcal{E}_{2}}\left(e_{i}\right)=\sum_{j=1}^{n} \sum_{x \in \boldsymbol{e} \cap\left(V_{j} \backslash V_{j-1}\right)} \lambda_{\mathcal{E}_{1}}(x) \lambda_{\mathcal{E}_{2}}(x) \\
= & \sum_{j=1}^{n} \mu_{j} \sum_{x \in \boldsymbol{e} \cap\left(V_{j} \backslash V_{j-1}\right)}\left(-\ln \|[x]\|_{\mathcal{E}_{2}, j}\right)=\sum_{j=1}^{n} \mu_{j} \widehat{\operatorname{deg}}\left(V_{j} / V_{j-1},\|\cdot\|_{\mathcal{E}_{2}, j}\right),
\end{aligned}
$$

where the last equality comes from Proposition 5.1.2 (2)]. The equality (5.11) is thus proved. 
We say that an $\mathbb{R}$-filtration $\mathcal{E} \in \mathbf{F i l}(E)$ is trivial if the function $Z_{\mathcal{E}}$ is constantly zero, or equivalently, $\langle\mathcal{E}, \mathcal{E}\rangle=0$.

Lemma 5.4.2. - Let $V$ be a finite-dimensional non-zero vector space over $\mathbb{R}$, equipped with an inner product $\langle$,$\rangle . Let \left\{\ell_{i}\right\}_{i=1}^{n}$ be a finite family of linear forms on $V$, where $n \in \mathbb{N}, n \geqslant 1$. Let $\theta: V \backslash\{0\} \rightarrow \mathbb{R}$ be the function defined by

$$
\theta(x)=\max _{i \in\{1, \ldots, n\}} \frac{\ell_{i}(x)}{\|x\|},
$$

where $\|\cdot\|$ is the norm induced by the inner product $\langle$,$\rangle . Then the function \theta$ attains its minimal value on $V \backslash\{0\}$. Moreover, if $c$ is the minimal value of $\theta$ and if $x_{0}$ is a point of $V \backslash\{0\}$ minimising the function $\theta$, then for any $x \in \mathbb{R}^{n}$ one has

$$
\theta(x) \geqslant c \frac{\left\langle x, x_{0}\right\rangle}{\|x\| \cdot\left\|x_{0}\right\|} .
$$

Proof. - Note that the function $\theta$ is invariant by positive dilatations, namely for any $x \in V \backslash\{0\}$ and any $\lambda>0$ one has $\theta(\lambda x)=\theta(x)$. Moreover, the function $\theta$ is clearly continuous. Hence it attains its minimal value, which is equal to $\min _{x \in V,\|x\|=1} \theta(x)$.

To show the inequality 5.12 , we may assume without loss of generality that $\|x\|=$ $\left\|x_{0}\right\|=1$. Note that for any $t \in[0,1]$ one has

$$
\begin{aligned}
c\left\|t x+(1-t) x_{0}\right\| & \leqslant\left\|t x+(1-t) x_{0}\right\| \theta\left(t x+(1-t) x_{0}\right) \\
& =\max _{i \in\{1, \ldots, n\}} \ell_{i}\left(t x+(1-t) x_{0}\right) \leqslant t \theta(x)+(1-t) c .
\end{aligned}
$$

Note that when $t=0$ one has

$$
c\left\|t x+(1-t) x_{0}\right\|=c=t \theta(x)+(1-t) c .
$$

Therefore the right derivative at $t=0$ of the convex function

$$
t \in[0,1] \longmapsto c\left\|t x+(1-t) x_{0}\right\|
$$

is bounded from above by $\theta(x)-c$, which leads to

$$
\left.c \frac{t\|x\|^{2}-(1-t)\|x\|^{2}+(1-2 t)\left\langle x, x_{0}\right\rangle}{\left\|t x+(1-t) x_{0}\right\|}\right|_{t=0}=c\left(\left\langle x, x_{0}\right\rangle-1\right) \leqslant \theta(x)-c,
$$

namely $\theta(x) \geqslant c\left\langle x, x_{0}\right\rangle$.

Theorem 5.4.3. - Let $d \in \mathbb{N}_{\geqslant 2},\left\{E_{j}\right\}_{j=1}^{d}$ be a family of finite-dimensional nonzero vector spaces over $K$, and $V$ be a quotient vector space of $E_{1} \otimes_{K} \cdots \otimes_{K} E_{d}$. Let $r$ be the rank of $V$ over $K$. For any $j \in\{1, \ldots, d\}$, let $a_{j}$ be the rank of $E_{j}$ over $K$. Then the following conditions are equivalent.

(1) The rational point $x$ of

$$
\pi: P=\mathbb{P}\left(\left(E_{1} \otimes_{K} \cdots \otimes_{K} E_{d}\right)^{\otimes r}\right) \longrightarrow \operatorname{Spec} K
$$


corresponding to $\operatorname{det}(V)$ is not semistable with respect to the $\mathbb{G} \mathbb{L}\left(E_{1}\right) \times \cdots \times$ $\mathbb{G L}\left(E_{d}\right)$-linearised invertible sheaf

$$
L:=\mathcal{O}_{P}\left(a_{1} \cdots a_{d}\right) \otimes \pi^{*}\left(\operatorname{det}\left(E_{1}^{\vee}\right)^{\otimes r b_{1}} \otimes \cdots \operatorname{det}\left(E_{d}^{\vee}\right)^{\otimes r b_{d}}\right),
$$

where $b_{j}=a_{1} \cdots a_{d} / a_{j}$ for any $j \in\{1, \ldots, d\}$.

(2) Let $\mathbf{S}$ be the subset of $\mathbf{F i l}_{0}\left(E_{1}\right) \times \cdots \times \mathbf{F i l}_{0}\left(E_{d}\right)$ consisting of the filtrations $\left(\mathcal{F}_{1}, \ldots, \mathcal{F}_{d}\right)$ which are not simultaneously trivial. For each

$$
\mathcal{F}=\left(\mathcal{F}_{1}, \ldots, \mathcal{F}_{d}\right) \in \mathbf{F i l}\left(E_{1}\right) \times \cdots \times \mathbf{F i l}\left(E_{d}\right),
$$

let $\|\cdot\|_{\mathcal{F}, V}$ be the quotient norm on $V$ of the $\varepsilon$-tensor product of $\|\cdot\|_{\mathcal{F}_{1}}, \ldots,\|\cdot\|_{\mathcal{F}_{d}}$. Then the function $\Theta: \mathbf{S} \rightarrow \mathbb{R}$ defined as

$$
\forall \mathcal{F}=\left(\mathcal{F}_{1}, \ldots, \mathcal{F}_{d}\right) \in \mathbf{S}, \quad \Theta(\mathcal{F})=\frac{\widehat{\mu}\left(V,\|\cdot\|_{\mathcal{F}, V}\right)}{\left(\left\langle\mathcal{F}_{1}, \mathcal{F}_{1}\right\rangle+\cdots+\left\langle\mathcal{F}_{d}, \mathcal{F}_{d}\right\rangle\right)^{1 / 2}}
$$

attains its minimal value, which is negative.

Moreover, if the above conditions are satisfied and if $\mathcal{E}=\left(\mathcal{E}_{1}, \ldots, \mathcal{E}_{d}\right)$ is a minimal point of the function $\Theta$, then for any $\mathcal{F}=\left(\mathcal{F}_{1}, \ldots, \mathcal{F}_{d}\right) \in \mathbf{F i l}\left(E_{1}\right) \times \cdots \times \mathbf{F i l}\left(E_{d}\right)$ one has

$$
\widehat{\mu}\left(V,\|\cdot\|_{\mathcal{F}, V}\right) \geqslant \sum_{j=1}^{d} \widehat{\mu}\left(E_{j},\|\cdot\|_{\mathcal{F}_{j}}\right)+c \frac{\left\langle\mathcal{E}_{1}, \mathcal{F}_{1}\right\rangle+\cdots+\left\langle\mathcal{E}_{d}, \mathcal{F}_{d}\right\rangle}{\left(\left\langle\mathcal{E}_{1}, \mathcal{E}_{1}\right\rangle+\cdots+\left\langle\mathcal{E}_{d}, \mathcal{E}_{d}\right\rangle\right)^{1 / 2}},
$$

where $c$ is the minimal value of $\Theta$.

Proof. - Assume that the condition $(2)$ holds, then there exists $\mathcal{F}=\left(\mathcal{F}_{1}, \ldots, \mathcal{F}_{d}\right) \in$ $\mathbf{S}$ such that

$$
\widehat{\mu}\left(V,\|\cdot\|_{\mathcal{F}, V}\right)<0=\widehat{\mu}\left(E_{1},\|\cdot\|_{\mathcal{F}_{1}}\right)+\cdots+\widehat{\mu}\left(E_{d},\|\cdot\|_{\mathcal{F}_{d}}\right) .
$$

By Corollary 5.2.10, the point $x$ is not semistable with respect to $L$. Conversely, if the condition (1) holds, then there exist

$$
\mathcal{F}^{\prime}=\left(\mathcal{F}_{1}^{\prime}, \ldots, \mathcal{F}_{d}^{\prime}\right) \in \mathbf{F i l}\left(E_{1}\right) \times \cdots \times \mathbf{F i l}\left(E_{d}\right)
$$

such that

$$
\widehat{\mu}\left(V,\|\cdot\|_{\mathcal{F}^{\prime}, V}\right)<\sum_{j=1}^{d} \widehat{\mu}\left(E,\|\cdot\|_{\mathcal{F}_{j}^{\prime}}\right) .
$$

For $j \in\{1, \ldots, d\}$, let $\mathcal{F}_{j}$ be $\mathbb{R}$-filtrations on $E_{j}$ such that

$$
\|\cdot\|_{\mathcal{F}_{j}}=\exp \left(\widehat{\mu}\left(E,\|\cdot\|_{\mathcal{F}_{j}^{\prime}}\right)\right)\|\cdot\|_{\mathcal{F}_{j}^{\prime}} .
$$

Then one has $\mathcal{F}_{j} \in \mathbf{F i l}_{0}\left(E_{j}\right)$ for any $j \in\{1, \ldots, d\}$. Moreover, if we denote by $\mathcal{F}$ the vector $\left(\mathcal{F}_{1}, \ldots, \mathcal{F}_{d}\right)$, then one has

$$
\widehat{\mu}\left(V,\|\cdot\|_{\mathcal{F}, V}\right)=\widehat{\mu}\left(V,\|\cdot\|_{\mathcal{F}^{\prime}, V}\right)-\sum_{j=1}^{d} \widehat{\mu}\left(E,\|\cdot\|_{\mathcal{F}_{j}^{\prime}}\right)<0 .
$$


In particular, the $\mathbb{R}$-filtrations $\mathcal{F}_{j}$ are not simultaneously trivial since otherwise we should have $\widehat{\mu}\left(V,\|\cdot\|_{\mathcal{F}, V}\right)=0$. Therefore one has $\mathcal{F} \in \mathbf{S}$, which implies that the function $\Theta$ takes at least a negative value.

In the following, we show that the function $\Theta$ attains its minimal value. For any $j \in\{1, \ldots, d\}$, let $\mathscr{B}_{j}$ be the set of bases of $E_{j}$. For $n \in \mathbb{N}$, let $\Delta_{n}$ be the vector subspace of $\mathbb{R}^{n}$ of vectors $\left(z_{1}, \ldots, z_{n}\right)$ such that $z_{1}+\cdots+z_{n}=0$. For any

$$
\boldsymbol{e}=\left(\boldsymbol{e}^{(1)}, \ldots, \boldsymbol{e}^{(d)}\right) \in \mathscr{B}_{1} \times \cdots \times \mathscr{B}_{d},
$$

let

$$
\Psi_{\boldsymbol{e}}: \Delta_{a_{1}} \times \cdots \times \Delta_{a_{d}} \rightarrow \mathbf{F i l}_{0}\left(E_{1}\right) \times \cdots \times \mathbf{F i l}_{0}\left(E_{d}\right)
$$

be the map sending $\left(\boldsymbol{y}^{(1)}, \ldots, \boldsymbol{y}^{(d)}\right)$ to the vector of $\mathbb{R}$-filtrations $\left(\mathcal{F}_{1}, \ldots, \mathcal{F}_{d}\right)$ such that, for any $j \in\{1, \ldots, d\}, e^{(j)}=\left\{e_{i}^{(j)}\right\}_{i=1}^{a_{j}}$ forms an orthogonal basis of $\|\cdot\|_{\mathcal{F}_{j}}$ with

$$
\boldsymbol{y}^{(j)}=\left(\lambda_{\mathcal{F}_{j}}\left(e_{1}^{(j)}\right), \ldots, \lambda_{\mathcal{F}_{j}}\left(e_{a_{j}}^{(j)}\right)\right) .
$$

For $j \in\{1, \ldots, d\}$, let $\boldsymbol{y}^{(j)}=\left(y_{1}^{(j)}, \ldots, y_{a_{j}}^{(j)}\right)$ be an element of $\Delta_{a_{j}}$. If

$$
\mathcal{F}=\left(\mathcal{F}_{1}, \ldots, \mathcal{F}_{d}\right)=\Psi_{\boldsymbol{e}}\left(\boldsymbol{y}^{(1)}, \ldots, \boldsymbol{y}^{(d)}\right),
$$

then one has $\widehat{\mu}\left(V,\|\cdot\|_{\mathcal{F}, V}\right)$ is equal to the maximal value of

$$
\sum_{j=1}^{d} y_{i_{1}^{(j)}}^{(j)}+\cdots+y_{i_{r}^{(j)}}^{(j)}
$$

for those $\left(i_{1}^{(j)}, \ldots, i_{r}^{(j)}\right) \in\left\{1, \ldots, a_{j}\right\}^{r}$ such that the image of

$$
\left(e_{i_{1}^{(1)}}^{(1)} \otimes \cdots \otimes e_{i_{r}^{(1)}}^{(1)}\right) \otimes \cdots \otimes\left(e_{i_{1}^{(d)}}^{(d)} \otimes \cdots \otimes e_{i_{r}^{(d)}}^{(d)}\right)
$$

by the canonical composed surjective map

$$
E_{1}^{\otimes r} \otimes_{K} \cdots \otimes_{K} E_{d}^{\otimes r} \cong\left(E_{1} \otimes_{K} \cdots \otimes_{K} E_{d}\right)^{\otimes r} \longrightarrow V^{\otimes r} \longrightarrow \operatorname{det}(V)
$$

is non-zero. Moreover, for any $j \in\{1, \ldots, d\}$ one has

$$
\left\langle\mathcal{F}_{j}, \mathcal{F}_{j}\right\rangle=\sum_{i=1}^{a_{j}}\left(y_{i}^{(j)}\right)^{2} .
$$

Therefore, the composition of $\Theta$ with the restriction of $\Psi_{e}$ on

$$
\left(\Delta_{a_{1}} \times \ldots \times \Delta_{a_{d}}\right) \backslash\{(0, \ldots, 0)\}
$$

defines a continuous function on $\left(\Delta_{a_{1}} \times \ldots \times \Delta_{a_{d}}\right) \backslash\{(0, \ldots, 0)\}$ which is invariant by dilatation by elements in $\mathbb{R}_{>0}$. It hence attains its minimal value. Moreover, although $\mathscr{B}_{1} \times \cdots \times \mathscr{B}_{d}$ may contain infinitely many elements, from the expression of the value $\widehat{\mu}\left(V,\|\cdot\|_{\mathcal{E} \otimes_{\varepsilon} \mathcal{F}, V}\right)$ as above we obtain that there are only finitely many (at most $\left.2^{\left(a_{1} \cdots a_{d}\right)^{r}}\right)$ possibility for the composed function

$$
\Theta \circ\left(\left.\Psi_{e}\right|_{\Delta_{a_{1}} \times \cdots \times \Delta_{a_{d}} \backslash\{(0, \ldots, 0)\}}\right) .
$$


Therefore, the function $\Theta$ attains its minimal value, which is negative since $\Theta$ takes at least one negative value.

In the following, we prove the inequality (5.13). Let $\mathcal{E}=\left(\mathcal{E}_{1}, \ldots, \mathcal{E}_{d}\right)$ be an element of $\mathbf{F i l}_{0}\left(E_{1}\right) \times \cdots \times \mathbf{F i l}_{0}\left(E_{d}\right)$ which minimise the function $\Theta$. Let $\mathcal{F}=\left(\mathcal{F}_{1}, \ldots, \mathcal{F}_{d}\right)$ be an element of $\boldsymbol{F i l}\left(E_{1}\right) \times \cdots \times \mathbf{F i l}\left(E_{d}\right)$. Note that, if $\mathcal{F}_{j}^{\prime}$ is the $\mathbb{R}$-filtration of $E_{j}$ such that

$$
\|\cdot\|_{\mathcal{F}_{j}^{\prime}}=\exp \left(\widehat{\operatorname{deg}}\left(E_{j},\|\cdot\|_{\mathcal{F}_{j}}\right)\right)\|\cdot\|_{\mathcal{F}_{j}},
$$

then one has $\mathcal{F}_{j}^{\prime} \in \mathbf{F i l}_{0}\left(E_{j}\right)$ and $\left\langle\mathcal{E}_{j}, \mathcal{F}_{j}^{\prime}\right\rangle=\left\langle\mathcal{E}_{j}, \mathcal{F}_{j}\right\rangle$. Moreover, if we denote by $\mathcal{F}^{\prime}$ the vector $\left(\mathcal{F}_{1}^{\prime}, \ldots, \mathcal{F}_{d}^{\prime}\right)$, then

$$
\widehat{\mu}\left(V,\|\cdot\|_{\mathcal{F}^{\prime}, V}\right)=\widehat{\mu}\left(V,\|\cdot\|_{\mathcal{F}, V}\right)-\sum_{j=1}^{d} \widehat{\operatorname{deg}}\left(E_{j}, \mathcal{F}_{j}\right) .
$$

Therefore, to show the inequality (5.13), it suffices to treat the case where $\mathcal{F} \in$ $\mathbf{F i l}_{0}\left(E_{1}\right) \times \cdots \times \mathbf{F i l}_{0}\left(E_{d}\right)$.

By Proposition 5.1.3, for any $j \in\{1, \ldots, d\}$, there exists a basis $\boldsymbol{e}^{(j)}$ of $E$ which is orthogonal with respect to the norms $\|\cdot\|_{\mathcal{E}_{j}}$ and $\|\cdot\|_{\mathcal{F}_{j}}$ simultaneously. Therefore the inequality (5.13) follows from Lemma 5.4 .2

\subsection{Lifting and refinement of filtrations}

Let $V$ be a finite-dimensional vector space over $K$ and

$$
0=V_{0} \subsetneq V_{1} \subsetneq \ldots \subsetneq V_{n}=V
$$

be a flag of vector subspaces of $V$. Suppose given, for any $i \in\{1, \ldots, n\}$, an $\mathbb{R}$ filtration $\mathcal{F}_{i}$ of the sub-quotient vector space $V_{i} / V_{i-1}$. We will construct an $\mathbb{R}$-filtration on $V$ from the data of $\left\{\mathcal{F}_{i}\right\}_{i=1}^{n}$ as follows. For any $i \in\{1, \ldots, n\}$, let $\widetilde{\boldsymbol{e}}^{(i)}$ be a basis of $V_{i} / V_{i-1}$ which is orthogonal with respect to the norm $\|\cdot\|_{\mathcal{F}_{i}}$, where we consider the trivial valuation on $K$. The basis $\widetilde{\boldsymbol{e}}^{(i)}$ gives rise to a linearly independent family $\boldsymbol{e}^{(i)}$ in $V_{i}$. Let $\boldsymbol{e}=\bigcup_{i=1}^{n} \boldsymbol{e}^{(i)}$ be the (disjoint) union of $\boldsymbol{e}^{(i)}, i \in\{1, \ldots, n\}$. Note that $\boldsymbol{e}$ forms actually a basis of $V$ over $K$. We define an ultrametric norm $\|\cdot\|$ on $V$ such that $e$ is an orthogonal basis under this norm and that, for any $i \in\{1, \ldots, n\}$ and any $x \in e^{(i)}$, the norm of $x$ is $\|\widetilde{x}\|_{\mathcal{F}_{i}}$, where $\widetilde{x}$ denotes the class of $x$ in $V_{i} / V_{i-1}$.

Remark 5.5.1. - (1) For any $i \in\{1, \ldots, n\}$, the subquotient norm on $V_{i} / V_{i-1}$ induced by $\|\cdot\|$ coincides with $\|\cdot\|_{\mathcal{F}_{i}}$. In particular, one has

$$
\widehat{\operatorname{deg}}(V,\|\cdot\|)=\sum_{i=1}^{n} \widehat{\operatorname{deg}}\left(V_{i} / V_{i-1},\|\cdot\|_{\mathcal{F}_{i}}\right) .
$$

In particular, the Arakelov degree of $(V,\|\cdot\|)$ doesn't depend on the choice of $\boldsymbol{e}$. 
(2) Let $\mathcal{F}$ be the $\mathbb{R}$-filtration corresponding to the ultrametric norm $\|\cdot\|$. Assume that (5.14) is the flag of vector subspaces of $V$ defined by an $\mathbb{R}$-filtration $\mathcal{G}$ on $V$ and that $\mu_{1}>\ldots>\mu_{n}$ are jump points of the $\mathbb{R}$-filtration $\mathcal{G}$. Then we can compute $\langle\mathcal{F}, \mathcal{G}\rangle$ as follows:

$$
\langle\mathcal{F}, \mathcal{G}\rangle=\frac{1}{\operatorname{rk}(V)} \sum_{i=1}^{n} \mu_{i} \widehat{\operatorname{deg}}\left(V_{i} / V_{i-1},\|\cdot\|_{\mathcal{F}_{i}}\right) .
$$

Definition 5.5.2. — The $\mathbb{R}$-filtration on $V$ corresponding to the norm $\|\cdot\|$ constructed above is called a lifting of the family $\left\{\mathcal{F}_{i}\right\}_{i=1}^{n}$ (relatively to the basis $\boldsymbol{e}$ ). We emphasis that the lifting depends on the choice of the basis $\boldsymbol{e}$.

Definition 5.5.3. - Let $V$ be a finite-dimensional vector space over $k$ and $\mathcal{F}$ be an $\mathbb{R}$-filtration on $V$. We call refinement of $\mathcal{F}$ any flag

$$
0=V_{0} \subsetneq V_{1} \subsetneq \ldots \subsetneq V_{n}=V
$$

of vector subspaces of $V$ together with a non-increasing sequence

$$
t_{1} \geqslant \ldots \geqslant t_{n}
$$

such that, for any $i \in\{1, \ldots, n\}$ and any $x \in V_{i} \backslash V_{i-1}$, one has $\|x\|_{\mathcal{F}}=\mathrm{e}^{-t_{i}}$.

Remark 5.5.4. - Let $V$ be a finite-dimensional vector space over $K$ and $\mathcal{F}$ be an $\mathbb{R}$-filtration on $V$. Recall that the $\mathbb{R}$-filtration $\mathcal{F}$ corresponds to a flag

$$
0=W_{0} \subsetneq W_{1} \subsetneq \ldots \subsetneq W_{m}=V
$$

together with a decreasing sequence

$$
\lambda_{1}>\ldots>\lambda_{m}
$$

To choose a refinement of $\mathcal{F}$ is equivalent to specify, for any $j \in\{1, \ldots, m\}$, a flag

$$
0=V_{j}^{(0)} / W_{j-1} \subsetneq V_{j}^{(1)} / W_{j-1} \subsetneq \ldots \subsetneq V_{j}^{\left(n_{j}\right)} / W_{j-1}=W_{j} / W_{j-1}
$$

of $W_{j} / W_{j-1}$. The corresponding refinement is given by the flag

$$
0=V_{0} \subsetneq V_{1}^{(1)} \subsetneq \ldots \subsetneq V_{1}^{\left(n_{1}\right)} \subsetneq \ldots \subsetneq V_{m}^{(1)} \subsetneq \ldots \subsetneq V_{m}^{\left(n_{m}\right)}=V
$$

and the non-increasing sequence

$$
\underbrace{\lambda_{1}=\cdots=\lambda_{1}}_{n_{1} \text { copies }}>\underbrace{\lambda_{2}=\cdots=\lambda_{2}}_{n_{2} \text { copies }}>\ldots>\underbrace{\lambda_{m}=\cdots=\lambda_{m}}_{n_{m} \text { copies }} .
$$

Proposition 5.5.5. - Let $d \in \mathbb{N}_{\geqslant 2},\left\{E_{j}\right\}_{j=1}^{d}$ be a family of finite-dimensional vector spaces over $K$, and $\left(\mathcal{F}_{1}, \ldots, \mathcal{F}_{d}\right) \in \mathbf{F i l}\left(E_{1}\right) \times \cdots \mathbf{F i l}\left(E_{d}\right)$. Let $G=E_{1} \otimes_{K}$ $\cdots \otimes_{K} E_{d}$ be the tensor product space and let $\mathcal{G}$ be the tensor product $\mathbb{R}$-filtration of $\mathcal{F}_{1}, \ldots, \mathcal{F}_{d}$ (namely the $\mathbb{R}$-filtration on $G$ corresponding to the $\varepsilon$-tensor product of $\left.\|\cdot\|_{\mathcal{F}_{1}}, \ldots,\|\cdot\|_{\mathcal{F}_{d}}\right)$. Then there exists a refinement

$$
0=G_{0} \subsetneq G_{1} \subsetneq \ldots \subsetneq G_{n}=G, \quad t_{1} \geqslant \ldots \geqslant t_{n}
$$


of the $\mathbb{R}$-filtration $\mathcal{G}$, such that, for any $i \in\{1, \ldots, n\}$, the subquotient $G_{i} / G_{i-1}$ is canonically isomorphic to a tensor product of subquotients of the form

$$
\operatorname{sq}_{\mathcal{F}_{1}}^{\lambda_{i, 1}}\left(E_{1}\right) \otimes_{K} \cdots \otimes_{K} \operatorname{sq}_{\mathcal{F}_{d}}^{\lambda_{i, d}}\left(E_{d}\right)
$$

with $\lambda_{i, 1}+\cdots+\lambda_{i, d}=t_{i}$.

Proof. - Since $\mathcal{G}$ is the tensor product $\mathbb{R}$-filtration of $\mathcal{F}_{1}, \ldots, \mathcal{F}_{d}$, one has

$$
\mathcal{G}^{t}(G)=\sum_{\mu_{1}+\cdots+\mu_{d} \geqslant t} \mathcal{F}_{1}^{\mu_{1}}\left(E_{1}\right) \otimes_{K} \cdots \otimes_{K} \mathcal{F}_{d}^{\mu_{d}}\left(E_{d}\right)
$$

Therefore,

$$
\operatorname{sq}_{\mathcal{G}}^{t}(G)=\bigoplus_{\mu_{1}+\cdots+\mu_{d}=t} \operatorname{sq}_{\mathcal{F}_{1}}^{\mu_{1}}\left(E_{1}\right) \otimes_{K} \cdots \otimes_{K} \mathrm{sq}_{\mathcal{F}_{d}}^{\mu_{d}}\left(E_{d}\right)
$$

For any $t \in \mathbb{R}$ there exists clearly a flag of $\operatorname{sq}_{\mathcal{G}}^{t}(G)$ whose successive subquotient is of the form $\mathrm{sq}_{\mathcal{F}_{1}}^{\mu_{1}}\left(E_{1}\right) \otimes_{K} \cdots \otimes_{K} \mathrm{sq}_{\mathcal{F}_{d}}^{\mu_{d}}\left(E_{d}\right)$ with $\mu_{1}+\cdots+\mu_{d}=t$. Hence we can construct a refinement of the $\mathbb{R}$-filtration $\mathcal{G}$ by using the construction in Remark 5.5.4.

Remark 5.5.6. - We keep the notation of Proposition 5.5.5 and suppose that each $E_{j}$ is equipped with a norm family $\xi_{j}$ such that $\left(E_{j}, \xi_{j}\right)$ forms an adelic vector bundle, and we equip $G$ with the $\varepsilon, \pi$-tensor product norm family $\xi_{G}=\xi_{1} \otimes_{\varepsilon, \pi} \cdots \otimes_{\varepsilon, \pi} \xi_{d}$. For any $t \in \mathbb{R}$ and any $j \in\{1, \ldots, d\}$, let $\xi_{j}^{t}$ be the induced norm families of $\xi_{j}$ on $\mathcal{F}_{j}^{t}\left(E_{j}\right)$ and $\xi_{j, \text { sq }}^{t}$ be quotient norm family of $\xi_{j}^{t}$ on $\mathrm{sq}_{\mathcal{F}_{j}}^{t}\left(E_{j}\right)$. By Proposition 1.1.58 and 1.2.36. for any $\left(\mu_{1}, \ldots, \mu_{d}\right) \in \mathbb{R}^{d}$, the quotient norm family of $\xi_{1}^{\mu_{1}} \otimes_{\varepsilon, \pi} \cdots \otimes_{\varepsilon, \pi} \xi_{d}^{\mu_{d}}$ on $\mathrm{sq}_{\mathcal{F}_{1}}^{\mu_{1}}\left(E_{1}\right) \otimes_{K} \cdots \otimes_{K} \mathrm{sq}_{\mathcal{F}_{d}}^{\mu_{d}}\left(E_{d}\right)$ identifies with $\xi_{1, \mathrm{sq}}^{\mu_{1}} \otimes_{\varepsilon, \pi} \cdots \otimes_{\varepsilon, \pi} \xi_{d, \mathrm{sq}}^{\mu_{d}}$.

We consider a refinement

$$
0=G_{0} \subsetneq G_{1} \subsetneq \ldots \subsetneq G_{n}=G, \quad t_{1} \geqslant \ldots \geqslant t_{n}
$$

of the $\mathbb{R}$-filtration $\mathcal{G}$ such that each subquotient $G_{i} / G_{i-1}$ is canonically isomorphic to a tensor product of the form $\operatorname{sq}_{\mathcal{F}_{1}}^{\lambda_{i, 1}}\left(E_{1}\right) \otimes_{K} \cdots \otimes_{K} \operatorname{sq}_{\mathcal{F}_{d}}^{\lambda_{i, d}}\left(E_{i}\right)$ with $\lambda_{i, 1}+\cdots+\lambda_{i, d}=t_{i}$. Note that the canonicity of the isomorphism means that the vector space $G_{i}$ contains $\mathcal{F}^{\lambda_{i, 1}}\left(E_{1}\right) \otimes_{K} \cdots \otimes_{K} \mathcal{F}^{\lambda_{i, d}}\left(E_{d}\right)$ and the composition

$$
\mathcal{F}^{\lambda_{i, 1}}\left(E_{1}\right) \otimes_{K} \cdots \otimes_{K} \mathcal{F}^{\lambda_{i, d}}\left(E_{d}\right) \longrightarrow G_{i} \longrightarrow G_{i} / G_{i-1}
$$

of the inclusion map $\mathcal{F}^{\lambda_{i, 1}}\left(E_{1}\right) \otimes_{K} \cdots \otimes_{K} \mathcal{F}^{\lambda_{i, d}}\left(E_{d}\right) \rightarrow G_{i}$ with the quotient map $G_{i} \rightarrow G_{i} / G_{i-1}$ induces an isomorphism

$$
\varphi_{i}: \operatorname{sq}_{\mathcal{F}_{1}}^{\lambda_{i, 1}}\left(E_{1}\right) \otimes_{K} \cdots \otimes_{K} \operatorname{sq}_{\mathcal{F}_{d}}^{\lambda_{i, d}}\left(E_{d}\right) \longrightarrow G_{i} / G_{i-1}
$$

We are interested in the comparison between $\xi_{1, \mathrm{sq}}^{\lambda_{i, 1}} \otimes_{\varepsilon, \pi} \cdots \otimes_{\varepsilon, \pi} \xi_{d, \mathrm{sq}}^{\lambda_{i, d}}$ and the subquotient norm family of $\xi_{G}$ on $G_{i} / G_{i-1}$. By Propositions 1.1.60, the restriction of $\xi_{G}$ on $\mathcal{F}_{1}^{\lambda_{i, 1}}\left(E_{1}\right) \otimes_{K} \cdots \otimes_{K} \mathcal{F}_{d}^{\lambda_{i, d}}\left(E_{d}\right)$ is bounded from above by $\xi_{1}^{\lambda_{i, 1}} \otimes_{\varepsilon, \pi} \cdots \otimes_{\varepsilon, \pi} \xi_{d}^{\lambda_{i, d}}$. Therefore, for any $\omega \in \Omega$, the isomorphism $\varphi_{i, \omega}$ has an operator norm $\leqslant 1$.

Assume that the norm families $\xi_{1}, \ldots, \xi_{d}$ are Hermitian. Let $\widetilde{\xi}_{G}$ be the orthogonal tensor product of $\xi_{1}, \ldots, \xi_{d}$. If we equip $\operatorname{sq}_{\mathcal{F}_{1}}^{\lambda_{i, 1}}\left(E_{1}\right) \otimes_{K} \cdots \otimes_{K} \operatorname{sq}_{\mathcal{F}_{d}}^{\lambda_{i, d}}\left(E_{d}\right)$ with the 
orthogonal product norm family $\xi_{1, \mathrm{sq}}^{\lambda_{i, 1}} \otimes \cdots \otimes \xi_{d, \mathrm{sq}}^{\lambda_{i, d}}$ and $G_{i} / G_{i-1}$ with the subquotient norm family of $\widetilde{\xi}_{G}$ on $G_{i} / G_{i-1}$, then, for any $\omega \in \Omega$, the operator norm of $\varphi_{i, \omega}$ is bounded from above by 1 . This follows from the fact that the restriction of $\xi_{G}$ on $\mathcal{F}_{1}^{\lambda_{i, 1}}\left(E_{1}\right) \otimes_{K} \cdots \otimes_{K} \mathcal{F}_{d}^{\lambda_{i, d}}\left(E_{d}\right)$ identifies with $\xi_{1}^{\lambda_{i, 1}} \otimes \cdots \otimes \xi_{d}^{\lambda_{i, d}}$ (see Proposition 1.2.58).

\subsection{Estimation in general case}

In this section, we establish the following result.

Theorem 5.6.1. - Let $d \in \mathbb{N}_{\geqslant 2},\left\{\left(E_{j}, \xi_{j}\right)\right\}_{j=1}^{d}$ be a family of non-zero Hermitian adelic vector bundles on $S$, and $V$ be a non-zero quotient vector space of $E_{1} \otimes_{K}$ $\cdots \otimes_{K} E_{d}$. For any $j \in\{1, \ldots, d\}$ Let $\mathcal{H}_{j}$ be the Harder-Narasimhan $\mathbb{R}$-filtrations of $\left(E_{j}, \xi_{j}\right)$, and $\|\cdot\|_{V}$ be the quotient norm of the $\varepsilon$-tensor product of $\|\cdot\|_{\mathcal{H}_{1}}, \ldots,\|\cdot\|_{\mathcal{H}_{d}}$, $\xi_{V}$ be the quotient norm family of the $\varepsilon, \pi$-tensor product $\xi_{1} \otimes_{\varepsilon, \pi} \cdots \otimes_{\varepsilon, \pi} \xi_{d}$, and $\widetilde{\xi}_{V}$ be the quotient norm family of the orthogonal tensor product $\xi_{1} \otimes \cdots \otimes \xi_{d}$. Then the following inequality holds

$$
\begin{gathered}
\widehat{\mu}\left(V, \xi_{V}\right) \geqslant \widehat{\mu}\left(V,\|\cdot\|_{V}\right)-\nu\left(\Omega_{\infty}\right) \sum_{j=1}^{d} \ln \left(\operatorname{rk}\left(E_{j}\right)\right), \\
\widehat{\mu}\left(V, \widetilde{\xi}_{V}\right) \geqslant \widehat{\mu}\left(V,\|\cdot\|_{V}\right)-\frac{1}{2} \nu\left(\Omega_{\infty}\right) \sum_{j=1}^{d} \ln \left(\operatorname{rk}\left(E_{j}\right)\right) .
\end{gathered}
$$

In particular, if all adelic vector bundles $\left(E_{j}, \xi_{j}\right)$ and $\bar{F}$ are semistable, then one has

$$
\begin{aligned}
\widehat{\mu}\left(V, \widetilde{\xi}_{V}\right) & \geqslant \sum_{j=1}^{d}\left(\widehat{\mu}\left(E_{j}, \xi_{j}\right)-\nu\left(\Omega_{\infty}\right) \ln \left(\operatorname{rk}\left(E_{j}\right)\right)\right) \\
\widehat{\mu}\left(V, \xi_{V}\right) & \geqslant \sum_{j=1}^{d}\left(\widehat{\mu}\left(E_{j}, \xi_{j}\right)-\frac{1}{2} \nu\left(\Omega_{\infty}\right) \ln \left(\operatorname{rk}\left(E_{j}\right)\right)\right) .
\end{aligned}
$$

Proof. - For any $j \in\{1, \ldots, d\}$, let $a_{j}$ be the rank of $E_{j}$ over $K$. We reason by induction on $A=a_{1}+\cdots+a_{d}$. The theorem is clearly true when $A=d$ (namely $\operatorname{rk}\left(E_{j}\right)=1$ for any $\left.j\right)$. In the following, we assume that the theorem has been proved for any family of adelic vector bundles whose ranks have the sum $<A$.

Step 1: In this step, we assume that the adelic vector bundles $\left(E_{j}, \xi_{j}\right)$ are not simultaneously semistable, or equivalently, at least one of the $\mathbb{R}$-filtrations $\mathcal{H}_{j}$ has more than one jump point. Let $G$ be the tensor product space $E_{1} \otimes_{K} \cdots \otimes_{K} E_{d}$ and $\mathcal{G} \in \mathbf{F i l}(G)$ be the tensor product of the $\mathbb{R}$-filtrations $\mathcal{H}_{1}, \ldots, \mathcal{H}_{d}$, which corresponds to the $\varepsilon$-tensor product of the norms $\|\cdot\|_{\mathcal{F}_{1}}, \ldots\|\cdot\|_{\mathcal{F}_{d}}$. We choose a refinement

$$
0=G_{0} \subsetneq G_{1} \subsetneq \ldots \subsetneq G_{n}=G, \quad t_{1} \geqslant \ldots \geqslant t_{n}
$$


of the $\mathbb{R}$-filtration $\mathcal{G}$ such that, for any $i \in\{1, \ldots, n\}$, the subquotient $G_{i} / G_{i-1}$ is canonically isomorphic to a tensor product of the form

$$
\operatorname{sq}_{\mathcal{H}_{1}}^{\lambda_{i, 1}}\left(E_{1}\right) \otimes_{K} \otimes \cdots \otimes_{K} \operatorname{sq}_{\mathcal{H}_{d}}^{\lambda_{i, d}}\left(E_{d}\right)
$$

with $\lambda_{i, 1}+\cdots+\lambda_{i, d}=t_{i}$ (see Proposition 5.5.5). The assumption that at least one of the $\mathbb{R}$-filtrations $\mathcal{F}_{j}$ has more than one jump point implies that

$$
\operatorname{rk}\left(\operatorname{sq}_{\mathcal{H}_{1}}^{\lambda_{i, 1}}\left(E_{1}\right)\right)+\cdots+\operatorname{rk}\left(\operatorname{sq}_{\mathcal{H}_{d}}^{\lambda_{i, d}}\left(E_{d}\right)\right)<a_{1}+\cdots+a_{d}
$$

For any $i \in\{1, \ldots, n\}$ and any $j \in\{1, \ldots, d\}$, denoted by $\xi_{j, \mathrm{sq}}^{\lambda_{i, j}}$ the subquotient norm families of $\xi_{j}$ on $\operatorname{sq}_{\mathcal{H}_{j}}^{\lambda_{i, j}}\left(E_{j}\right)$. For any $i \in\{1, \ldots, n\}$, let $\xi_{G_{i} / G_{i-1}}$ be the subquotient norm family of $\xi_{G}$ on $G_{i} / G_{i-1}, \xi_{V_{i} / V_{i-1}}^{\prime}$ be the quotient norm family of $\xi_{G_{i} / G_{i-1}}$ on $V_{i} / V_{i-1}, \xi_{V_{i} / V_{i-1}}$ be the subquotient norm family of $\xi_{V}$ on $V_{i} / V_{i-1}$, and $\xi_{V_{i} / V_{i-1}}^{\prime \prime}$ be the quotient norm family of $\xi_{1, \mathrm{sq}}^{\lambda_{i, 1}} \otimes_{\varepsilon, \pi} \cdots \otimes_{\varepsilon, \pi} \xi_{d, \mathrm{sq}}^{\lambda_{i, d}}$ on $V_{i} / V_{i-1}$, where we identify $G_{i} / G_{i-1}$ with

$$
\operatorname{sq}_{\mathcal{H}_{1}}^{\lambda_{i, 1}}\left(E_{1}\right) \otimes_{K} \cdots \otimes_{K} \operatorname{sq}_{\mathcal{H}_{d}}^{\lambda_{i, d}}\left(E_{d}\right) .
$$

By Proposition 1.1.14 (2) one has

$$
\widehat{\operatorname{deg}}\left(V_{i} / V_{i-1}, \xi_{V_{i} / V_{i-1}}\right) \geqslant \widehat{\operatorname{deg}}\left(V_{i} / V_{i-1}, \xi_{V_{i} / V_{i-1}}^{\prime}\right) .
$$

By Remark [5.5.6, one has

$$
\widehat{\operatorname{deg}}\left(V_{i} / V_{i-1}, \xi_{V_{i} / V_{i-1}}^{\prime}\right) \geqslant \widehat{\operatorname{deg}}\left(V_{i} / V_{i-1}, \xi_{V_{i} / V_{i-1}}^{\prime \prime}\right) .
$$

Moreover, for any $(i, j) \in\{1, \ldots, n\} \times\{1, \ldots, d\}$, the Hermitian adelic vector bundle $\left(\operatorname{sq}_{\mathcal{H}_{j}}^{\lambda_{i, j}}\left(E_{j}\right), \xi_{j, \mathrm{sq}}^{\lambda_{i, j}}\right)$ is semistable of slope $\lambda_{i, j}$. Therefore, by the induction hypothesis one has

$$
\begin{aligned}
& \widehat{\operatorname{deg}}\left(V_{i} / V_{i-1}, \xi_{V_{i} / V_{i-1}}\right) \geqslant \widehat{\operatorname{deg}}\left(V_{i} / V_{i-1}, \xi_{V_{i} / V_{i-1}}^{\prime \prime}\right) \\
\geqslant & \operatorname{rk}\left(V_{i} / V_{i-1}\right) \sum_{j=1}^{d}\left(\lambda_{i, j}-\nu\left(\Omega_{\infty}\right) \ln \left(a_{j}\right)\right) .
\end{aligned}
$$

Taking the sum with respect to $i \in\{1, \ldots, n\}$, we obtain

$$
\begin{aligned}
\widehat{\operatorname{deg}}\left(V, \xi_{V}\right) & =\sum_{i=1}^{n} \widehat{\operatorname{deg}}\left(V_{i} / V_{i-1}, \xi_{V_{i} / V_{i-1}}\right) \\
& \geqslant \sum_{i=1}^{n} \operatorname{rk}\left(V_{i} / V_{i-1}\right) \sum_{j=1}^{d}\left(\lambda_{i, j}-\nu\left(\Omega_{\infty}\right) \ln \left(a_{j}\right)\right) \\
& =\sum_{i=1}^{n} \operatorname{rk}\left(V_{i} / V_{i-1}\right) t_{i}-\operatorname{rk}(V) \nu\left(\Omega_{\infty}\right) \sum_{j=1}^{d} \ln \left(a_{j}\right) \\
& =\widehat{\operatorname{deg}}\left(V,\|\cdot\|_{V}\right)-\operatorname{rk}(V) \nu\left(\Omega_{\infty}\right) \sum_{j=1}^{d} \ln \left(a_{j}\right)
\end{aligned}
$$


which leads to

$$
\widehat{\mu}\left(V, \xi_{V}\right) \geqslant \widehat{\mu}\left(V,\|\cdot\|_{V}\right)-\nu\left(\Omega_{\infty}\right) \sum_{j=1}^{d} \ln \left(a_{j}\right) .
$$

Similarly, if we denote by $\widetilde{\xi}_{V_{i} / V_{i-1}}$ the subquotient norm family of $\widetilde{\xi}_{V}$, then the induction hypothesis gives

$$
\widehat{\operatorname{deg}}\left(V_{i} / V_{i-1}, \widetilde{\xi}_{V_{i} / V_{i-1}}\right) \geqslant \operatorname{rk}\left(V_{i} / V_{i-1}\right) \sum_{j=1}^{d}\left(\lambda_{i, j}-\frac{1}{2} \nu\left(\Omega_{\infty}\right) \ln \left(a_{j}\right)\right)
$$

which leads to

$$
\widehat{\mu}\left(V, \xi_{V}\right) \geqslant \widehat{\mu}\left(V,\|\cdot\|_{V}\right)-\frac{1}{2} \nu\left(\Omega_{\infty}\right) \sum_{j=1}^{d} \ln \left(a_{j}\right) .
$$

Step 2: In this step, we assume that all adelic vector bundles $\left(E_{j}, \xi_{j}\right)$ are semistable. Note that the Harder-Narasimhan $\mathbb{R}$-filtrations of $\left(E_{j}, \xi_{j}\right)$ has then only one jump point. Therefore, it suffices to prove (5.17) and (5.18). Note that the case where $\operatorname{det}(V)$ is semistable as a rational point of

$$
P:=\mathbb{P}\left(\left(E_{1} \otimes_{K} \cdots \otimes_{K} E_{d}\right)^{\otimes \operatorname{rk}(V)}\right)
$$

has been proved in Proposition 5.3.1. In the following, we assume that $\operatorname{det}(V)$ is not semistable as a rational point of $P$.

For each

$$
\mathcal{F}=\left(\mathcal{F}_{1}, \ldots, \mathcal{F}_{d}\right) \in \mathbf{F i l}\left(E_{1}\right) \times \cdots \times \mathbf{F i l}\left(E_{d}\right),
$$

let $\|\cdot\|_{\mathcal{F}, V}$ be the quotient norm on $V$ of the $\varepsilon$-tensor product of $\|\cdot\|_{\mathcal{F}_{1}}, \ldots,\|\cdot\|_{\mathcal{F}_{d}}$. Let $\mathbf{S}$ be the subset of $\mathbf{F i l}_{0}\left(E_{1}\right) \times \cdots \times \mathbf{F i l}_{0}\left(E_{d}\right)$ consisting of vectors $\left(\mathcal{F}_{1}, \ldots, \mathcal{F}_{d}\right)$ such that the filtrations $\mathcal{F}_{1}, \ldots, \mathcal{F}_{d}$ are not simultaneously trivial. Then, by Theorem 5.4 .3 the function $\Theta: \mathbf{S} \rightarrow \mathbb{R}$

$$
\forall \mathcal{F}=\left(\mathcal{F}_{1}, \ldots, \mathcal{F}_{d}\right) \in \mathbf{S}, \quad \Theta(\mathcal{F}):=\frac{\widehat{\mu}\left(V,\|\cdot\|_{\mathcal{F}, V}\right)}{\left(\left\langle\mathcal{F}_{1}, \mathcal{F}_{1}\right\rangle+\cdots+\left\langle\mathcal{F}_{d}, \mathcal{F}_{d}\right\rangle\right)^{1 / 2}}
$$

attains its minimal value $c$, which is negative. In the following, we denote by $\mathcal{E}=$ $\left(\mathcal{E}_{1}, \ldots, \mathcal{E}_{d}\right)$ a minimal point of the function $\Theta$. Then, for any $\mathcal{F}=\left(\mathcal{F}_{1}, \ldots, \mathcal{F}_{d}\right) \in$ $\operatorname{Fil}\left(E_{1}\right) \times \cdots \times \boldsymbol{F i l}\left(E_{d}\right)$, one has

$$
\widehat{\mu}\left(V,\|\cdot\|_{\mathcal{F}, V}\right) \geqslant \sum_{j=1}^{d} \widehat{\mu}\left(E_{j},\|\cdot\|_{\mathcal{F}_{j}}\right)+c \frac{\left\langle\mathcal{E}_{1}, \mathcal{F}_{1}\right\rangle+\cdots+\left\langle\mathcal{E}_{d}, \mathcal{F}_{d}\right\rangle}{\left(\left\langle\mathcal{E}_{1}, \mathcal{E}_{1}\right\rangle+\cdots+\left\langle\mathcal{E}_{d}, \mathcal{E}_{d}\right\rangle\right)^{1 / 2}} .
$$

In the following, for each $j \in\{1, \ldots, d\}$, we denote by $\mathcal{F}_{j}$ the $\mathbb{R}$-filtration on $E$ which induces on each subquotient $\mathrm{sq}_{\mathcal{E}_{j}}^{t}\left(E_{j}\right)$ the Harder-Narasimhan filtration of this vector space equipped with the subquotient norm family $\xi_{j, \mathrm{sq}, \mathcal{E}_{j}}^{t}$ of $\xi_{j}$. By (4.63), for any $t \in \mathbb{R}$, if we denote by $\|\cdot\|_{\mathcal{F}_{j}, \mathrm{sq}, t}$ the subquotient norm of $\|\cdot\|_{\mathcal{F}_{j}}$ on $\operatorname{sq}_{\mathcal{E}_{j}}^{t}\left(E_{j}\right)$, then one has

$$
\widehat{\operatorname{deg}}\left(\operatorname{sq}_{\mathcal{E}_{j}}^{t}\left(E_{j}\right),\|\cdot\|_{\mathcal{F}_{j}, \mathrm{sq}, t}\right)=\widehat{\operatorname{deg}}\left(\operatorname{sq}_{\mathcal{E}_{j}}^{t}\left(E_{j}\right), \xi_{j, \mathrm{sq}, \mathcal{E}_{j}}^{t}\right) .
$$


Taking the sum with respect to $t$, by Proposition 4.3 .12 and the assumption that $\xi_{j}$ is Hermitian we obtain that

$$
\widehat{\operatorname{deg}}\left(E_{j},\|\cdot\|_{\mathcal{E}_{j}}\right)=\widehat{\operatorname{deg}}\left(E_{j}, \xi_{j}\right) .
$$

Moreover, by (4.63) one has

$$
\left\langle\mathcal{E}_{j}, \mathcal{F}_{j}\right\rangle=\frac{1}{a_{j}} \sum_{t \in \mathbb{R}} t \sum_{i=1}^{r_{j}(t)} \widehat{\mu}_{i}\left(\mathrm{sq}_{\mathcal{E}_{j}}^{t}\left(E_{j}\right), \xi_{j, \mathrm{sq}, \mathcal{E}_{j}}^{t}\right)=\frac{1}{a_{j}} \sum_{t \in \mathbb{R}} t \widehat{\operatorname{deg}}\left(\mathrm{sq}_{\mathcal{E}_{j}}^{t}\left(E_{j}\right), \xi_{j, \mathrm{sq}, \mathcal{E}_{j}}^{t}\right),
$$

where $r_{j}(t)=\operatorname{rk}_{K}\left(\mathrm{sq}_{\mathcal{E}_{j}}^{t}\left(E_{j}\right)\right)$, and the second equality comes from (4.63). For any $j \in\{1, \ldots, d\}$ and any $u \in \mathbb{R}$, let

$$
\Psi_{j}(u)=\sum_{t<u} \widehat{\operatorname{deg}}\left(\operatorname{sq}_{\mathcal{E}_{j}}^{t}\left(E_{j}\right), \xi_{j, \mathrm{sq}, \mathcal{E}_{j}}^{t}\right)=\widehat{\operatorname{deg}}\left(\overline{E / \mathcal{E}_{j}^{u}\left(E_{j}\right)}\right),
$$

where we consider the quotient norm family on $E / \mathcal{E}_{j}^{u}\left(E_{j}\right)$. Since $\left(E_{j}, \xi_{j}\right)$ is semistable, one has

and hence

$$
\widehat{\mu}\left(\overline{E / \mathcal{E}_{j}^{u}\left(E_{j}\right)}\right) \geqslant \widehat{\mu}_{\min }\left(E_{j}, \xi_{j}\right)=\widehat{\mu}\left(E_{j}, \xi_{j}\right)
$$

$$
\Psi_{j}(u) \geqslant \widehat{\mu}\left(E_{j}, \xi_{j}\right) \operatorname{rk}\left(E / \mathcal{E}_{j}^{u}\left(E_{j}\right)\right) .
$$

By Abel's summation formula we obtain

$$
\left\langle\mathcal{E}_{j}, \mathcal{F}_{j}\right\rangle=\frac{1}{a_{j}} \int_{\mathbb{R}} t \mathrm{~d} \Psi_{j}(t)=M_{j} \frac{\Psi_{j}\left(M_{j}\right)}{a_{j}}-\frac{1}{a_{j}} \int_{-\infty}^{M_{j}} \Psi_{j}(t) \mathrm{d} t,
$$

where $M_{j}$ is a sufficiently positive number such that $\mathcal{E}_{j}^{M_{j}}\left(E_{j}\right)=\{0\}$. Therefore one has

$$
\begin{aligned}
\left\langle\mathcal{E}_{j}, \mathcal{F}_{j}\right\rangle & \leqslant M_{j} \frac{\widehat{\operatorname{deg}}\left(E_{j}, \xi_{j}\right)}{a_{j}}-\frac{1}{a_{j}} \widehat{\mu}\left(E_{j}, \xi_{j}\right) \int_{-\infty}^{M_{j}} \operatorname{rk}\left(E_{j} / \mathcal{E}_{j}^{t}\left(E_{j}\right)\right) \mathrm{d} t \\
& =\frac{\widehat{\mu}\left(E_{j}, \xi_{j}\right)}{a_{j}} \int_{-\infty}^{M_{j}} t \operatorname{drk}\left(E_{j} / \mathcal{E}_{j}^{t}\left(E_{j}\right)\right)=\frac{\widehat{\mu}\left(E_{j}, \xi_{j}\right)}{a_{j}} \widehat{\operatorname{deg}}\left(E_{j},\|\cdot\|_{\mathcal{E}_{j}}\right)=0 .
\end{aligned}
$$

Therefore we obtain

$$
\widehat{\mu}\left(V,\|\cdot\|_{\mathcal{F}, V}\right) \geqslant \sum_{j=1}^{d} \widehat{\mu}\left(E_{j}, \xi_{j}\right)
$$

It remains to compare $\widehat{\mu}\left(V,\|\cdot\|_{\mathcal{F}, V}\right)$ with the slopes of $\left(V, \xi_{V}\right)$ and $\left(V, \widetilde{\xi}_{V}\right)$. We choose a refinement

$$
0=G_{0} \subsetneq G_{1} \subsetneq \ldots \subsetneq G_{n}=E_{1} \otimes_{K} \cdots \otimes_{K} E_{d}, \quad t_{1} \geqslant \ldots \geqslant t_{n}
$$

of the $\mathbb{R}$-filtration $\mathcal{E}_{1} \otimes \cdots \otimes \mathcal{E}_{d}$ such that, for any $i \in\{1, \ldots, n\}$, the subquotient $G_{i} / G_{i-1}$ is canonically isomorphic to a tensor product of the form

$$
\operatorname{sq}_{\mathcal{E}_{1}}^{\lambda_{i, 1}}\left(E_{1}\right) \otimes_{K} \cdots \otimes_{K} \operatorname{sq}_{\mathcal{E}_{d}}^{\lambda_{i, d}}\left(E_{d}\right)
$$

with $\lambda_{i, 1}+\cdots+\lambda_{i, d}=t_{i}$. For any $i \in\{1, \ldots, n\}$, let $\|\cdot\|_{\mathcal{F}, G_{i} / G_{i-1}}$ be the subquotient norm on $G_{i} / G_{i-1}$ of the $\varepsilon$-tensor product of $\|\cdot\|_{\mathcal{F}_{1}}, \ldots,\|\cdot\|_{\mathcal{F}_{d}}$. By 
the construction of $\mathcal{F}_{1}, \ldots, \mathcal{F}_{d}$, the subquotient norm $\|\cdot\|_{\mathcal{F}, G_{i} / G_{i-1}}$ on $G_{i} / G_{i-1}$ corresponds to the tensor product of the Harder-Narasimhan $\mathbb{R}$-filtrations of $\left(\operatorname{sq}_{\mathcal{E}_{1}}^{\lambda_{i, 1}}\left(E_{1}\right), \xi_{1, \mathrm{sq}, \mathcal{E}_{1}}^{t}\right), \ldots,\left(\mathrm{sq}_{\mathcal{E}_{d}}^{\lambda_{i, d}}\left(E_{d}\right), \xi_{d, \mathrm{sq}, \mathcal{E}_{d}}^{t}\right) . \quad$ By the induction hypothesis, and the same argument showing (5.19) and (5.20), we obtain

$$
\widehat{\operatorname{deg}}\left(V_{i} / V_{i-1}, \xi_{V_{i} / V_{i-1}}\right) \geqslant \widehat{\operatorname{deg}}\left(V_{i} / V_{i-1},\|\cdot\|_{\mathcal{F}, V_{i} / V_{i-1}}\right)-\operatorname{rk}\left(V_{i} / V_{i-1}\right) \nu\left(\Omega_{\infty}\right) \sum_{j=1}^{d} \ln \left(a_{j}\right),
$$

where $\xi_{V_{i} / V_{i-1}}$ is the subquotient norm family of $\xi_{V}$ on $V_{i} / V_{i-1},\|\cdot\|_{\mathcal{F}, V_{i} / V_{i-1}}$ is the quotient norm of $\|\cdot\|_{\mathcal{F}, G_{i} / G_{i-1}}$, which identifies with the subquotient norm of $\|\cdot\|_{F, V}$ since the flag $0=G_{0} \subsetneq G_{1} \subsetneq \ldots \subsetneq G_{n}$ is compatible with the $\mathbb{R}$-filtration $\mathcal{F}_{1} \otimes \cdots \otimes \mathcal{F}_{d}$. Taking the sum of the above formula with respect to $i \in\{1, \ldots, n\}$, we obtain

$$
\widehat{\operatorname{deg}}\left(V, \xi_{V}\right) \geqslant \widehat{\operatorname{deg}}\left(V,\|\cdot\|_{\mathcal{F}, V}\right)-\operatorname{rk}(V) \nu\left(\Omega_{\infty}\right) \sum_{j=1}^{d} \ln \left(a_{j}\right)
$$

which leads to

$$
\widehat{\mu}\left(V, \xi_{V}\right) \geqslant \widehat{\mu}\left(V,\|\cdot\|_{\mathcal{F}, V}\right)-\nu\left(\Omega_{\infty}\right) \sum_{j=1}^{d} \ln \left(a_{j}\right)
$$

Similarly, one has

$$
\widehat{\mu}\left(V, \widetilde{\xi}_{V}\right) \geqslant \widehat{\mu}\left(V,\|\cdot\|_{\mathcal{F}, V}\right)-\frac{1}{2} \nu\left(\Omega_{\infty}\right) \sum_{j=1}^{d} \ln \left(a_{j}\right) .
$$

The theorem is thus proved.

Corollary 5.6.2. - Let $d \in \mathbb{N}_{\geqslant 2},\left\{\left(E, \xi_{j}\right)\right\}_{j=1}^{d}$ be a family of adelic vector bundles on $S$ and $V$ be a non-zero quotient vector space of $E_{1} \otimes_{K} \cdots \otimes_{K} E_{d}$. Let $\xi_{V}$ be the quotient norm families of $\xi_{1} \otimes_{\varepsilon, \pi} \cdots \otimes_{\varepsilon, \pi} \xi_{d}$. Then one has

$$
\widehat{\mu}_{\min }\left(V, \xi_{V}\right) \geqslant \sum_{j=1}^{d}\left(\widehat{\mu}_{\min }\left(E_{j}, \xi_{j}\right)-\frac{3}{2} \nu\left(\Omega_{\infty}\right) \ln \left(\operatorname{rk}\left(E_{j}\right)\right)\right) .
$$

If all norm families $\xi_{1}, \ldots, \xi_{d}$ are Hermitian, then one has

$$
\begin{gathered}
\widehat{\mu}_{\min }\left(V, \xi_{V}\right) \geqslant \sum_{j=1}^{d}\left(\widehat{\mu}_{\min }\left(E_{j}, \xi_{j}\right)-\nu\left(\Omega_{\infty}\right) \ln \left(\operatorname{rk}\left(E_{j}\right)\right)\right), \\
\widehat{\mu}_{\min }\left(V, \widetilde{\xi}_{V}\right) \geqslant \sum_{j=1}^{d}\left(\widehat{\mu}_{\min }\left(E_{j}, \xi_{j}\right)-\frac{1}{2} \nu\left(\Omega_{\infty}\right) \ln \left(\operatorname{rk}\left(E_{j}\right)\right)\right),
\end{gathered}
$$

where $\widetilde{\xi}_{V}$ is the quotient norm family of the orthogonal tensor product $\xi_{1} \otimes \cdots \otimes \xi_{d}$. 
Proof. - We begin with the proof of the Hermitian case. To establish (15.22) it suffices to prove weaker inequalities

$$
\begin{gathered}
\widehat{\mu}\left(V, \xi_{V}\right) \geqslant \sum_{j=1}^{d}\left(\widehat{\mu}_{\text {min }}\left(E_{j}, \xi_{j}\right)-\nu\left(\Omega_{\infty}\right) \ln \left(\operatorname{rk}\left(E_{j}\right)\right)\right), \\
\widehat{\mu}\left(V, \widetilde{\xi}_{V}\right) \geqslant \sum_{j=1}^{d}\left(\widehat{\mu}_{\text {min }}\left(E_{j}, \xi_{j}\right)-\frac{1}{2} \nu\left(\Omega_{\infty}\right) \ln \left(\operatorname{rk}\left(E_{j}\right)\right)\right)
\end{gathered}
$$

for all non-zero quotient vector space $V$ of $E_{1} \otimes_{K} \cdots \otimes_{K} E_{d}$. For any $j \in\{1, \ldots, d\}$, let $\mathcal{H}_{j}$ be the Harder-Narasimhan $\mathbb{R}$-filtration of $\left(E_{j}, \xi_{j}\right)$. Let $\|\cdot\|_{V}$ be the quotient norm of the $\varepsilon$-tensor product of $\|\cdot\|_{\mathcal{H}_{1}}, \ldots,\|\cdot\|_{\mathcal{H}_{d}}$. By Theorem [5.6.1, one has

$$
\begin{gathered}
\widehat{\mu}\left(V, \xi_{V}\right) \geqslant \widehat{\mu}\left(V,\|\cdot\|_{V}\right)-\nu\left(\Omega_{\infty}\right) \sum_{j=1}^{d} \ln \left(\operatorname{rk}\left(E_{j}\right)\right), \\
\widehat{\mu}\left(V, \widetilde{\xi}_{V}\right) \geqslant \widehat{\mu}\left(V,\|\cdot\|_{V}\right)-\frac{1}{2} \nu\left(\Omega_{\infty}\right) \sum_{j=1}^{d} \ln \left(\operatorname{rk}\left(E_{j}\right)\right) .
\end{gathered}
$$

Moreover, since $\|\cdot\|_{V}$ is the quotient norm of the $\varepsilon$-tensor product of $\|\cdot\|_{\mathcal{H}_{1}}, \ldots,\|\cdot\|_{\mathcal{H}_{d}}$, one has (see Remark 5.1.6)

$$
\widehat{\mu}\left(V,\|\cdot\|_{V}\right) \geqslant \sum_{j=1}^{d} \widehat{\mu}_{\min }\left(E_{j}, \xi_{j}\right) .
$$

Therefore (5.24) follows from (5.26) and (5.25) follows from (5.27).

In the following, we proceed with the proof of (5.21) in the general (non-necessarily Hermitian) case. Note that one has

$$
\xi_{1}^{\vee \vee} \otimes_{\varepsilon, \pi} \cdots \otimes_{\varepsilon, \pi} \xi_{d}^{\vee \vee}=\xi_{1} \otimes_{\varepsilon, \pi} \cdots \otimes_{\varepsilon, \pi} \xi_{d} .
$$

Moreover, since $\xi_{j}^{\vee \vee} \leqslant \xi_{j}$, one has

$$
\widehat{\mu}_{\min }\left(E_{j}, \xi_{j}^{\vee \vee}\right) \geqslant \widehat{\mu}_{\min }\left(E_{j}, \xi_{j}\right)
$$

for any $j \in\{1, \ldots, d\}$. Therefore, by replacing $\xi_{j}$ by $\xi_{j}^{\vee \vee}$ we may suppose without loss of generality that all $\xi_{j}$ are non-Archimedean on $\Omega \backslash \Omega_{\infty}$.

Assume that $\xi_{j}$ is of the form $\left\{\|\cdot\|_{j, \omega}\right\}_{j=1}^{d}$. By Theorem 4.1.26, for any $\epsilon>0$ and any $j \in\{1, \ldots, d\}$ there exist measurable Hermitian norm families $\xi_{j}^{H}=\left\{\|\cdot\|_{j, \omega}^{H}\right\}_{\omega \in \Omega}$ of $E_{j}$ such that $\|\cdot\|_{j, \omega}^{H}=\|\cdot\|_{j, \omega}$ for any $\omega \in \Omega \backslash \Omega_{\infty}$ and

$$
\|\cdot\|_{j, \omega} \leqslant\|\cdot\|_{j, \omega}^{H} \leqslant\left(\operatorname{rk}\left(E_{j}\right)+\epsilon\right)^{1 / 2}\|\cdot\|_{j, \omega}
$$

for any $\omega \in \Omega_{\infty}$. By the slope inequality (see Proposition 4.3.30) one has

$$
\widehat{\mu}_{\min }\left(E_{j}, \xi_{j}^{H}\right) \geqslant \widehat{\mu}\left(E_{j}, \xi_{j}\right)-\frac{1}{2} \nu\left(\Omega_{\infty}\right) \ln \left(\operatorname{rk}\left(E_{j}\right)+\epsilon\right) .
$$


Moreover, if we denote by $\xi_{V}^{\prime}$ the quotient norm family of $\xi_{1}^{H} \otimes_{\varepsilon, \pi} \cdots \otimes_{\varepsilon, \pi} \xi_{d}^{H}$ on $V$, one has

$$
\widehat{\mu}_{\min }\left(V, \xi_{V}^{\prime}\right) \leqslant \widehat{\mu}_{\min }\left(V, \xi_{V}\right)
$$

by the slope inequality. Applying the Hermitian case of the corollary to $\left(E_{j}, \xi_{j}^{H}\right)$ $(j \in\{1, \ldots, d\})$ and $\left(V, \xi_{V}^{\prime}\right)$, we obtain

$$
\widehat{\mu}_{\min }\left(V, \xi_{V}^{\prime}\right) \geqslant \sum_{j=1}^{d}\left(\widehat{\mu}_{\min }\left(E_{j}, \xi_{j}^{H}\right)-\nu\left(\Omega_{\infty}\right) \ln \left(\operatorname{rk}\left(E_{j}\right)\right)\right) .
$$

Combining this inequality with (5.28) and (5.29), by passing to limite when $\epsilon$ tend to $0+$ we obtain (5.21). The corollary is thus proved.

Remark 5.6.3. - In the case where the adelic curve $S$ comes from an arithmetic curve. The inequality (5.23) recovers essentially the second inequality of 62, Corollary 5.4], which strengthen [36. Theorem 1]. From the methodological point of view, the arguments in this chapter rely on the geometric invariant theory without using the theorem of successive minima of Zhang, which is a key argument in [62, 21] (see [148, Theorem 5.2], see also [62, §3]). 



\section{CHAPTER 6}

\section{ADELIC LINE BUNDLES ON ARITHMETIC VARIETIES}

In this chapter, we fix a proper adelic curve $S=(K,(\Omega, \mathcal{A}, \nu), \phi)$. We assume that, either the $\sigma$-algebra $\mathcal{A}$ is discrete, or there exists a countable subfield $K_{0}$ of $K$ which is dense in the completion $K_{\omega}$ of $K$ with respect to any $\omega \in \Omega$.

\subsection{Metrised line bundles on an arithmetic variety}

Let $X$ be a projective scheme over $\operatorname{Spec} K$ and $L$ be an invertible $\mathcal{O}_{X}$-module. For any $\omega \in \Omega$, we let $X_{\omega}$ be the fibre product $X \times_{\operatorname{Spec} K} \operatorname{Spec} K_{\omega}$ (recall that $K_{\omega}$ is the completion of $K$ with respect to $\left.|\cdot|_{\omega}\right)$ and $L_{\omega}$ be the pull-back of $L$ by the canonical projection morphism $X_{\omega} \rightarrow X$. By metric family on $L$, we refer to a family of continuous metrics $\varphi=\left\{\varphi_{\omega}\right\}_{\omega \in \Omega}$, where $\varphi_{\omega}$ is a continuous metric on $L_{\omega}$. If $\varphi=\left\{\varphi_{\omega}\right\}_{\omega \in \Omega}$ and $\varphi^{\prime}=\left\{\varphi_{\omega}^{\prime}\right\}_{\omega \in \Omega}$ are two metric families on $L$, the local distance of $\varphi$ and $\varphi^{\prime}$ at $\omega \in \Omega$ is defined as (see Definition 2.2.7)

$$
d_{\omega}\left(\varphi, \varphi^{\prime}\right):=d\left(\varphi_{\omega}, \varphi_{\omega}^{\prime}\right)
$$

The global distance between $\varphi$ and $\varphi^{\prime}$ is defined as the upper integral

$$
\operatorname{dist}\left(\varphi, \varphi^{\prime}\right):=\bar{\int}_{\Omega} d_{\omega}\left(\varphi, \varphi^{\prime}\right) \nu(\mathrm{d} \omega) .
$$

If $\varphi=\left\{\varphi_{\omega}\right\}_{\omega \in \Omega}$ is a metric family on $L$, then the dual metrics $\left\{-\varphi_{\omega}\right\}_{\omega \in \Omega}$ form a metric family on $L^{\vee}$, denoted by $-\varphi$. If $L$ and $L^{\prime}$ are invertible $\mathcal{O}_{X}$-modules, and $\varphi=\left\{\varphi_{\omega}\right\}_{\omega \in \Omega}$ and $\varphi^{\prime}=\left\{\varphi_{\omega}^{\prime}\right\}_{\omega \in \Omega}$ are metric families on $L$ and $L^{\prime}$ respectively, then $\left\{\varphi_{\omega}+\varphi_{\omega}^{\prime}\right\}_{\omega \in \Omega}$ is a metric family on $L \otimes L^{\prime}$, denoted by $\varphi+\varphi^{\prime}$. The metric family $\varphi+\left(-\varphi^{\prime}\right)$ on $L \otimes L^{\prime \vee}$ is also denoted by $\varphi-\varphi^{\prime}$. Similarly, for any integer $n \geqslant 0$, $\left\{n \varphi_{\omega}\right\}_{\omega \in \Omega}$ is a metric family on $L^{\otimes n}$, denoted by $n \varphi$.

Definition 6.1.1. - Let $Y$ and $X$ be projective schemes over Spec $K$ and $f: Y \rightarrow$ $X$ be a projective $K$-morphism. Let $L$ be an invertible $\mathcal{O}_{X}$-module equipped with a metric family $\varphi=\left\{\varphi_{\omega}\right\}_{\omega \in \Omega}$. We denote by $f^{*}(\varphi)$ the metric family $\left\{f_{\omega}^{*}\left(\varphi_{\omega}\right)\right\}_{\omega \in \Omega}$ on 
$f^{*}(L)$, where for any $\omega \in \Omega, f_{\omega}: Y_{\omega} \rightarrow X_{\omega}$ is the $K_{\omega}$-morphisme induced by $f$, and $f_{\omega}^{*}\left(\varphi_{\omega}\right)$ is defined in Definition 2.2.9. The norm family $f^{*}(\varphi)$ is called the pull-back of $\varphi$ by $f$. In the particular case where $Y$ is a closed subscheme of $X$ and $f$ is the canonical immersion, the norm family $f^{*}(\varphi)$ is also denoted by $\left.\varphi\right|_{Y}$ and called the restriction of $\varphi$ on $Y$.

The following properties are straightforward from the definition.

Proposition 6.1.2. - Let $X$ be a projective scheme over $\operatorname{Spec} K$ and $f: Y \rightarrow X$ be a projective morphism of $K$-schemes.

(1) If $L_{1}$ and $L_{2}$ are two invertible $\mathcal{O}_{X}$-modules, and $\varphi_{1}$ and $\varphi_{2}$ are metric families on $L_{1}$ and $L_{2}$ respectively, then one has $f^{*}\left(\varphi_{1}+\varphi_{2}\right)=f^{*}\left(\varphi_{1}\right)+f^{*}\left(\varphi_{2}\right)$ on $f^{*}\left(L_{1} \otimes L_{2}\right) \cong f^{*}\left(L_{1}\right) \otimes f^{*}\left(L_{2}\right)$.

(2) For any invertible $\mathcal{O}_{X}$-module $L$ and any metric family $\varphi$ on $L$, one has $f^{*}(-\varphi)=-f^{*}(\varphi)$ on $f^{*}\left(L^{\vee}\right) \cong f^{*}(L)^{\vee}$.

Remark 6.1.3. - Let us consider the particular case where $X$ is the spectrum of a finite extension $K^{\prime}$ of the field $K$. For any $\omega \in \Omega$, the Berkovich space of $X_{\omega}$ identifies with the discrete set of absolute values on $K^{\prime}$ extending $|\cdot|_{\omega}$ on $K$. Moreover, any invertible $\mathcal{O}_{X}$-module $L$ could be considered as a vector space of rank 1 over $K^{\prime}$, and any metric family on $L$ is just a norm family with respect to the adelic curve $S \otimes_{K} K^{\prime}$ (cf. Definition 3.4.1) if we consider $L$ as a vector space over $K^{\prime}$.

6.1.1. Quotient metric families. - Let $E$ be a finite-dimensional vector space over $K$ and $\xi=\left\{\|\cdot\|_{\omega}\right\}_{\omega \in \Omega}$ be a norm family on $E$. Let $f: X \rightarrow \operatorname{Spec} K$ be a projective $K$-scheme and $L$ be an invertible $\mathcal{O}_{X}$-module. Suppose given a surjective homomorphism $\beta: f^{*}(E) \rightarrow L$. For any $\omega \in \Omega$, the morphism $f: X \rightarrow \operatorname{Spec} K$ induces by base change a morphism $f_{\omega}$ from $X_{\omega}:=X \times_{\operatorname{Spec} K} \operatorname{Spec} K_{\omega}$ to $\operatorname{Spec} K_{\omega}$. We denote by $L_{\omega}$ the pull-back of $L$ on $X_{\omega}$. The homomorphism $\beta$ induces a surjective homomorphism $\beta_{\omega}: f_{\omega}^{*}(E) \rightarrow L_{\omega}$. Therefore, the norm $\|\cdot\|_{\omega}$ induces a quotient metric $\varphi_{\omega}$ on $L_{\omega}$ (see Definition 2.2.15). The family $\varphi=\left\{\varphi_{\omega}\right\}_{\omega \in \Omega}$ is called the quotient metric family induced by $(E, \xi)$ and $\beta$.

Let $\boldsymbol{e}=\left\{e_{i}\right\}_{i=1}^{r}$ be a basis of $E$. For each $\omega \in \Omega$, let $\|\cdot\|_{\boldsymbol{e}, \omega}$ be the norm on $E_{\omega}:=E \otimes_{K} K_{\omega}$ given by

$\forall a_{1}, \ldots, a_{r} \in K_{\omega}, \quad\left\|a_{1} e_{1}+\cdots+a_{r} e_{r}\right\|_{e, \omega}:= \begin{cases}\max \left\{\left|a_{1}\right|_{\omega}, \ldots,\left|a_{r}\right|_{\omega}\right\} & \text { if } \omega \in \Omega \backslash \Omega_{\infty}, \\ \left|a_{1}\right|_{\omega}+\cdots+\left|a_{r}\right|_{\omega} & \text { if } \omega \in \Omega_{\infty},\end{cases}$

and let $\varphi_{\boldsymbol{e}, \omega}$ be the metric of $L_{\omega}$ induced by $\|\cdot\|_{\boldsymbol{e}, \omega}$ and the surjective homomorphism $E_{\omega} \otimes_{K_{\omega}} \mathcal{O}_{X_{\omega}} \rightarrow L_{\omega}$. Let $\xi_{\boldsymbol{e}}:=\left\{\|\cdot\|_{\boldsymbol{e}, \omega}\right\}_{\omega \in \Omega}$ and let $\varphi_{\boldsymbol{e}}:=\left\{\varphi_{\boldsymbol{e}, \omega}\right\}_{\omega \in \Omega}$. The metric family $\varphi_{\boldsymbol{e}}$ is called the quotient metric family of $L$ induced by $\beta$ and $\boldsymbol{e}$. 
Remark 6.1.4. - We keep the above notation. Given a fixed surjective homomor$\operatorname{phism} \beta: f^{*}(E) \rightarrow L$, by Proposition 1.2.14 (see also Remark 1.3.2), the norm family $\xi$ and the double dual norm family $\xi^{\vee \vee}$ induce the same quotient metric family on $L$.

Proposition 6.1.5. - Let $(E, \xi)$ and $\left(E^{\prime}, \xi^{\prime}\right)$ be finite-dimensional vector spaces equipped with dominated norm families. Let $f: X \rightarrow$ Spec $K$ be a projective scheme over $\operatorname{Spec} K, \beta: f^{*}(E) \rightarrow L$ and $\beta^{\prime}: f^{\prime *}\left(E^{\prime}\right) \rightarrow L$ be two surjective homomorphisms inducing closed immersions $i: X \rightarrow \mathbb{P}(E)$ and $i^{\prime}: X \rightarrow \mathbb{P}\left(E^{\prime}\right)$, and $\varphi$ and $\varphi^{\prime}$ be quotient metric families induced by $(E, \xi)$ and $\beta$, and by $\left(E^{\prime}, \xi^{\prime}\right)$ and $\beta^{\prime}$, respectively. Then the local distance function $(\omega \in \Omega) \mapsto d_{\omega}\left(\varphi, \varphi^{\prime}\right)$ is $\nu$-dominated.

Proof. - We begin with the particular case where $E=E^{\prime}$ and $\beta=\beta^{\prime}$. By Proposition 2.2.20 for any $\omega \in \Omega$ one has

$$
d_{\omega}\left(\varphi, \varphi^{\prime}\right) \leqslant d_{\omega}\left(\xi^{\vee \vee}, \xi^{\prime \vee \vee}\right) .
$$

Note that the norm families $\xi^{\vee \vee}$ and $\xi^{\prime \vee \vee}$ are strongly dominated (see Remark 4.1.12) By Corollary 4.1.10 and the triangle inequality of the local distance function, we obtain that the function $(\omega \in \Omega) \mapsto d_{\omega}\left(\xi^{\vee \vee}, \xi^{\prime \vee \vee}\right)$ is $\nu$-dominated and then deduce that the function $(\omega \in \Omega) \mapsto d_{\omega}\left(\varphi, \varphi^{\prime}\right)$ is $\nu$-dominated.

In the general case, since $i$ and $i^{\prime}$ are closed immersions, by Serre's vanishing therorem (cf. [82, Theorem 5.2, Chapter III]), there exists an integer $n \geqslant 1$ such that

$$
\Gamma\left(\mathbb{P}(E), \mathcal{O}_{\mathbb{P}(E)}(n)\right) \longrightarrow \Gamma\left(X, L^{\otimes n}\right) \quad \text { and } \quad \Gamma\left(\mathbb{P}\left(E^{\prime}\right), \mathcal{O}_{\mathbb{P}\left(E^{\prime}\right)}(n)\right) \longrightarrow \Gamma\left(X, L^{\otimes n}\right)
$$

are surjective, so that the natural homomorphisms

$$
E^{\otimes n} \longrightarrow \operatorname{Sym}^{n}(E)=\Gamma\left(\mathbb{P}(E), \mathcal{O}_{\mathbb{P}(E)}(n)\right) \longrightarrow \Gamma\left(X, L^{\otimes n}\right)
$$

and

$$
E^{\prime \otimes n} \longrightarrow \operatorname{Sym}^{n}\left(E^{\prime}\right)=\Gamma\left(\mathbb{P}\left(E^{\prime}\right), \mathcal{O}_{\mathbb{P}\left(E^{\prime}\right)}(n)\right) \longrightarrow \Gamma\left(X, L^{\otimes n}\right)
$$

are both surjective. Therefore both surjective homomorphisms $\beta$ and $\beta^{\prime}$ factorise through $f^{*} \Gamma\left(X, L^{\otimes n}\right)$. Moreover, by Remark 2.2.19, if we equip $E^{\otimes n}$ and $E^{\prime \otimes n}$ with the $\varepsilon, \pi$-tensor power norm families (see 4 4.1.1) of $\xi$ and $\xi^{\prime}$ respectively, then the corresponding quotient metric families are $n \varphi$ and $n \varphi^{\prime}$ respectively. Note that the $\varepsilon, \pi$-tensor powers of $\xi$ and $\xi^{\prime}$ are dominated (see Proposition 4.1.19](5)]. Therefore, by the special case proved above, we obtain that the function

$$
(\omega \in \Omega) \longmapsto d_{\omega}\left(n \varphi, n \varphi^{\prime}\right)=n d_{\omega}\left(\varphi, \varphi^{\prime}\right)
$$

is $\nu$-dominated. The proposition is thus proved.

6.1.2. Dominated metric families. - Throughout this subsection, let $f: X \rightarrow$ Spec $K$ be a projective $K$-scheme. 
Definition 6.1.6. - Let $L$ be a very ample invertible $\mathcal{O}_{X}$-module. We say that a metric family $\varphi$ on $L$ is dominated if there exist a finite-dimensional vector space $E$ over $K$, a dominated norm family $\xi$ on $E$, and a surjective homomorphism $\beta$ : $f^{*}(E) \rightarrow L$ inducing a closed immersion $X \rightarrow \mathbb{P}(E)$, such that the quotient metric family $\varphi^{\prime}$ induced by $(E, \xi)$ and $\beta$ satisfies the following condition:

the local distance function $(\omega \in \Omega) \mapsto d_{\omega}\left(\varphi, \varphi^{\prime}\right)$ is $\nu$-dominated.

Remark 6.1.7. - With the above definition, Proposition 6.1.5 implies the following assertions. Let $E$ be a finite-dimensional vector space over $K$ equipped with a dominated norm family $\xi$. Let $L$ be an invertible $\mathcal{O}_{X}$-module and $\beta: f^{*}(E) \rightarrow L$ be a surjective homomorphism inducing a closed immersion $X \rightarrow \mathbb{P}(E)$. Then the quotient metric family induced by $(E, \xi)$ and $\beta$ is dominated. Moreover, if $\varphi_{1}$ and $\varphi_{2}$ are two metric families on $L$ which are dominated, then the local distance function $(\omega \in \Omega) \mapsto d_{\omega}\left(\varphi_{1}, \varphi_{2}\right)$ is $\nu$-dominated.

Proposition 6.1.8. - Let $L_{1}$ and $L_{2}$ be very ample invertible $\mathcal{O}_{X}$-modules. Assume that $\varphi_{1}$ and $\varphi_{2}$ are dominated metric families on $L_{1}$ and $L_{2}$ respectively. Then $\varphi_{1}+\varphi_{2}$ is a dominated metric family on $L_{1} \otimes L_{2}$.

Proof. - Since the metric families $\varphi_{1}$ and $\varphi_{2}$ are dominated, there exist finitedimensional vector spaces $E_{1}$ and $E_{2}$ over $K$, dominated norm families $\xi_{1}$ and $\xi_{2}$ on $E_{1}$ and $E_{2}$ respectively, and surjective homomorphisms $\beta_{1}: f^{*}\left(E_{1}\right) \rightarrow L_{1}$ and $\beta_{2}: f^{*}\left(E_{2}\right) \rightarrow L_{2}$ inducing closed immersions $X \rightarrow \mathbb{P}\left(E_{1}\right)$ and $X \rightarrow \mathbb{P}\left(E_{2}\right)$ respectively, such that if we denote by $\widetilde{\varphi}_{1}$ and $\widetilde{\varphi}_{2}$ the quotient metric families induced by $\left(E_{1}, \xi_{1}\right)$ and $\beta_{1}$ and by $\left(E_{2}, \xi_{2}\right)$ and $\beta_{2}$ respectively, then the local distance functions

$$
(\omega \in \Omega) \longmapsto d_{\omega}\left(\varphi_{1}, \widetilde{\varphi}_{1}\right) \text { and }(\omega \in \Omega) \longmapsto d_{\omega}\left(\varphi_{2}, \widetilde{\varphi}_{2}\right)
$$

are $\nu$-dominated. Consider now the composition morphism

$$
\iota: X \stackrel{\left(\iota_{1}, \iota_{2}\right)}{\longrightarrow} \mathbb{P}\left(E_{1}\right) \times_{K} \mathbb{P}\left(E_{2}\right) \stackrel{\varsigma}{\longrightarrow} \mathbb{P}\left(E_{1} \otimes_{K} E_{2}\right),
$$

where $\iota_{1}$ and $\iota_{2}$ are closed immersions corresponding to $\beta_{1}$ and $\beta_{2}$, and $\varsigma$ is the Segre embedding. Note that $\iota$ is the closed immersion corresponding to the surjective homomorphism

$$
\beta_{1} \otimes \beta_{2}: f^{*}\left(E_{1} \otimes_{K} E_{2}\right) \cong f^{*}\left(E_{1}\right) \otimes_{\mathcal{O}_{X}} f^{*}\left(E_{2}\right) \longrightarrow L_{1} \otimes_{\mathcal{O}_{X}} L_{2} .
$$

Moreover, if we equip $E_{1} \otimes_{K} E_{2}$ with the $\varepsilon, \pi$-tensor product norm family of $\xi_{1}$ and $\xi_{2}$, then the quotient metric family on $L_{1} \otimes L_{2}$ induced by $\left(E_{1} \otimes_{K} E_{2}, \xi_{1} \otimes_{\varepsilon, \pi} \xi_{2}\right)$ and $\beta_{1} \otimes \beta_{2}$ identifies with $\widetilde{\varphi}_{1}+\widetilde{\varphi}_{2}$. This is a consequence of Proposition 1.2.36 (for the non-Archimedean case) and Proposition 1.1.58 (for the Archimedean case). Since

$$
\forall \omega \in \Omega, \quad d_{\omega}\left(\varphi_{1}+\varphi_{2}, \widetilde{\varphi}_{1}+\widetilde{\varphi}_{2}\right) \leqslant d_{\omega}\left(\varphi_{1}, \widetilde{\varphi}_{1}\right)+d_{\omega}\left(\varphi_{2}, \widetilde{\varphi}_{2}\right),
$$

we obtain that the function $(\omega \in \Omega) \mapsto d_{\omega}\left(\varphi_{1}+\varphi_{2}, \widetilde{\varphi}_{1}+\widetilde{\varphi}_{2}\right)$ is $\nu$-dominated. Therefore the metric family $\varphi_{1}+\varphi_{2}$ is dominated. 
Definition 6.1.9. - Let $L$ be an invertible $\mathcal{O}_{X}$-module and $\varphi$ be a metric family on $L$. We say that $\varphi$ is dominated if there exist two very ample invertible $\mathcal{O}_{X}$-modules $L_{1}$ and $L_{2}$ together with dominated metric families $\varphi_{1}$ and $\varphi_{2}$ on $L_{1}$ and $L_{2}$ respectively, such that $L=L_{2} \otimes L_{1}^{\vee}$ and $\varphi=\varphi_{2}-\varphi_{1}$.

Remark 6.1.10. - In the case where the invertible $\mathcal{O}_{X}$-module $L$ is very ample, the condition of dominancy in Definition 6.1.9 is actually equivalent to that in Definition 6.1.6. In order to explain this fact (in avoiding confusions), in this remark we temporary say that a metric family $\varphi$ on a very ample invertible $\mathcal{O}_{X}$-module $L$ is strictly dominated if it satisfies the condition in Definition 6.1.6. Clearly, if $\varphi$ is strictly dominated, then it is dominated (namely satisfies the condition in Definition 6.1.9) since we can write $L$ as $L^{\otimes 2} \otimes L^{\vee}$ and $\varphi$ as $2 \varphi-\varphi$. Conversely, if $\varphi$ is dominated, then there exist very ample invertible $\mathcal{O}_{X}$-modules $L_{1}$ and $L_{2}$ such that $L \cong L_{2} \otimes L_{1}^{\vee}$ and strictly dominated metric families $\varphi_{1}$ and $\varphi_{2}$ on $L_{1}$ and $L_{2}$ such that $\varphi=\varphi_{2}-\varphi_{1}$. We pick an arbitrary strictly dominated metric family $\varphi^{\prime}$ on $L$. By Proposition 6.1.8, we obtain that $\varphi^{\prime}+\varphi_{1}$ is a strictly dominated metric family on $L_{2}$. Hence the local distance function

$$
(\omega \in \Omega) \longmapsto d_{\omega}\left(\varphi_{2}, \varphi^{\prime}+\varphi_{1}\right)=d_{\omega}\left(\varphi+\varphi_{1}, \varphi^{\prime}+\varphi_{1}\right)=d_{\omega}\left(\varphi, \varphi^{\prime}\right)
$$

is $\nu$-dominated. Therefore the metric family $\varphi$ is strictly dominated.

Proposition 6.1.11. - Let $E$ be a finite-dimensional vector space over $K$ equipped with a norm family $\xi, \beta: f^{*}(E) \rightarrow L$ be a surjective homomorphism (we do not assume that $\beta$ induces a closed immersion), and $\varphi$ be the quotient metric family induced by $(E, \xi)$ and $\beta$. Suppose that $\xi$ is a dominated norm family. Then $\varphi$ is a dominated metric family.

Proof. - Since $X$ is a projective $K$-scheme, there exists a very ample invertible $\mathcal{O}_{X^{-}}$ module $L^{\prime}$. Let $E^{\prime}$ be a finite dimensional vector space over $K$ and $\beta^{\prime}: f^{*}\left(E^{\prime}\right) \rightarrow L^{\prime}$ be a surjective homomorphism, which induces a closed embedding of $X$ in $\mathbb{P}\left(E^{\prime}\right)$, which we denote by $\lambda^{\prime}$. Let $\lambda: X \rightarrow \mathbb{P}(E)$ be the $K$-morphism induced by $\beta$. Then the tensor product homomorphism

$$
\beta \otimes \beta^{\prime}: f^{*}(E) \otimes_{\mathcal{O}_{X}} f^{*}\left(E^{\prime}\right) \cong f^{*}\left(E \otimes_{K} E^{\prime}\right) \longrightarrow L \otimes_{\mathcal{O}_{X}} L^{\prime}
$$

corresponds to the composed $K$-morphisme

$$
X \stackrel{\left(\lambda, \lambda^{\prime}\right)}{\longrightarrow} \mathbb{P}(E) \times_{K} \mathbb{P}\left(E^{\prime}\right) \stackrel{\varsigma}{\longrightarrow} \mathbb{P}\left(E \otimes_{K} E^{\prime}\right),
$$

where $\varsigma$ is the Segre embedding. Since $X$ is separated over $\operatorname{Spec} K$ and $\lambda^{\prime}$ is a closed immersion, the morphism $\left(\lambda, \lambda^{\prime}\right)$ is a closed immersion. Therefore, the morphism from $X$ to $\mathbb{P}\left(E \otimes_{K} E^{\prime}\right)$ induced by $\beta \otimes \beta^{\prime}$ is a closed embedding.

Let $\xi^{\prime}$ be a dominated norm family on $E^{\prime}$ and $\varphi^{\prime}$ be the metric family on $L^{\prime}$ induced by $\left(E^{\prime}, \xi^{\prime}\right)$ and $\beta^{\prime}$. By definition the metric family $\varphi^{\prime}$ is dominated (see Proposition 4.1.19 (5) . Moreover, the metric family $\varphi+\varphi^{\prime}$ is induced by $\left(E \otimes E^{\prime}, \xi \otimes_{\varepsilon, \pi} \xi^{\prime}\right)$ and 
$\beta \otimes \beta^{\prime}$ (see the proof of Proposition 6.1.8). As $\xi$ and $\xi^{\prime}$ are dominated, we obtain that $\xi \otimes_{\varepsilon, \pi} \xi^{\prime}$ is dominated. Therefore the metric families $\varphi+\varphi^{\prime}$ is dominated. Hence the metric family $\varphi$ is also dominated.

Proposition 6.1.12. - Let $L$ and $L^{\prime}$ be invertible $\mathcal{O}_{X}$-modules, and $\varphi$ and $\varphi^{\prime}$ be metric families on $L$ and $L^{\prime}$, respectively.

(1) If $\varphi$ is dominated, then the dual metric family $-\varphi$ on $L^{\vee}$ is dominated.

(2) If $\varphi$ and $\varphi^{\prime}$ are dominated, then the tensor product metric family $\varphi+\varphi^{\prime}$ on $L \otimes L^{\prime}$ is dominated.

(3) If $L=L^{\prime}$ and $\varphi$ and $\varphi^{\prime}$ are dominated, then the local distance function $(\omega \in$ $\Omega) \mapsto d_{\omega}\left(\varphi, \varphi^{\prime}\right)$ is $\nu$-dominated.

(4) If $L=L^{\prime}, \varphi^{\prime}$ is dominated and the local distance function $(\omega \in \Omega) \mapsto d_{\omega}\left(\varphi, \varphi^{\prime}\right)$ is $\nu$-dominated, then $\varphi$ is dominated.

(5) If $r \varphi$ is dominated for some non-zero integer $r$, then $\varphi$ is dominated.

(6) Let $g: Y \rightarrow X$ be a projective morphism of $K$-schemes. If $\varphi$ is dominated, then $g^{*}(\varphi)$ is also dominated.

Proof. - (1) Let $L_{1}$ and $L_{2}$ be very ample invertible $\mathcal{O}_{X}$-modules and $\varphi_{1}$ and $\varphi_{2}$ be dominated metric families on $L_{1}$ and $L_{2}$ respectively, such that $L \cong L_{2} \otimes L_{1}^{\vee}$ and that $\varphi=\varphi_{2}-\varphi_{1}$. Then one has $L^{\vee} \cong L_{1} \otimes L_{2}^{\vee}$ and $-\varphi=\varphi_{1}-\varphi_{2}$. Hence the metric family $-\varphi$ is dominated.

(2) Let $L_{1}, L_{2}, L_{1}^{\prime}$ and $L_{2}^{\prime}$ be very ample invertible $\mathcal{O}_{X}$-modules, $\varphi_{1}, \varphi_{2}, \varphi_{1}^{\prime}$ and $\varphi_{2}^{\prime}$ be dominated metric families on $L_{1}, L_{2}, L_{1}^{\prime}$ and $L_{2}^{\prime}$ respectively, such that $L \cong L_{2} \otimes L_{1}^{\vee}$, $L^{\prime} \cong L_{2}^{\prime} \otimes L_{1}^{\prime \vee}, \varphi=\varphi_{2}-\varphi_{1}$ and $\varphi^{\prime}=\varphi_{2}^{\prime}-\varphi_{1}^{\prime}$. Note that $L \otimes L^{\prime} \cong\left(L_{2} \otimes L_{2}^{\prime}\right) \otimes\left(L_{1} \otimes L_{1}^{\prime}\right)^{\vee}$, and $\varphi+\varphi^{\prime}=\left(\varphi_{2}+\varphi_{2}^{\prime}\right)-\left(\varphi_{1}+\varphi_{1}^{\prime}\right)$. By Proposition 6.1.8, the metric families $\varphi_{2}+\varphi_{2}^{\prime}$ and $\varphi_{1}+\varphi_{1}^{\prime}$ are dominated. Hence $\varphi+\varphi^{\prime}$ is dominated.

(3) Let $L_{1}$ be a very ample invertible $\mathcal{O}_{X}$-module and $\varphi_{1}$ be dominated metric family on $L_{1}$. Let $\varphi_{2}=\varphi+\varphi_{1}$ and $\varphi_{2}^{\prime}=\varphi^{\prime}+\varphi_{1}$. By (2), the metric families $\varphi_{2}$ and $\varphi_{2}^{\prime}$ are dominated. Since the invertible $\mathcal{O}_{X}$-module $L_{2}$ is very ample, by Proposition 6.1 .5 we obtain that the local distance function $(\omega \in \Omega) \mapsto d_{\omega}\left(\varphi_{2}, \varphi_{2}^{\prime}\right)=d_{\omega}\left(\varphi, \varphi^{\prime}\right)$ is $\nu$-dominated.

(4) First we assume that $L$ is very ample. As $\varphi^{\prime}$ is dominated, there exist a finitedimensional vector space $E$ over $K$, a dominated norm family $\xi$ on $E$, and a surjective homomorphism $\beta: f^{*}(E) \rightarrow L$ inducing a closed immersion $X \rightarrow \mathbb{P}(E)$ such that, if $\psi$ is the quotient metric family induced by $(E, \xi)$ and $\beta$, then the local distance function $(\omega \in \Omega) \mapsto d_{\omega}\left(\varphi^{\prime}, \psi\right)$ is $\nu$-dominated. Note that

$$
\forall \omega \in \Omega, \quad d_{\omega}(\varphi, \psi) \leqslant d_{\omega}\left(\varphi, \varphi^{\prime}\right)+d_{\omega}\left(\varphi^{\prime}, \psi\right) .
$$

Thus $(\omega \in \Omega) \mapsto d_{\omega}(\varphi, \psi)$ is $\nu$-dominated, as required.

In general, there are very ample invertible $\mathcal{O}_{X}$-modules $L_{1}$ and $L_{2}$, and dominated metric families $\varphi_{1}^{\prime}$ and $\varphi_{2}^{\prime}$ on $L_{1}$ and $L_{2}$, respectively, such that $L=L_{1} \otimes L_{2}^{\vee}$ and 
$\varphi^{\prime}=\varphi_{1}^{\prime}-\varphi_{2}^{\prime}$. We set $\varphi_{1}=\varphi+\varphi_{2}^{\prime}$ and $\varphi_{2}=\varphi_{2}^{\prime}$. Then $\varphi=\varphi_{1}-\varphi_{2}$, and $\varphi_{1}$ and $\varphi_{2}$ are metric families of $L_{1}$ and $L_{2}$, respectively. As

$$
d_{\omega}\left(\varphi_{1}, \varphi_{1}^{\prime}\right)=d_{\omega}\left(\varphi_{1}-\varphi_{2}, \varphi_{1}^{\prime}-\varphi_{2}^{\prime}\right)=d_{\omega}\left(\varphi, \varphi^{\prime}\right),
$$

$(\omega \in \Omega) \mapsto d_{\omega}\left(\varphi_{1}, \varphi_{1}^{\prime}\right)$ is $\nu$-dominated, so that $\varphi_{1}$ is dominated by the previous observation. Therefore, $\varphi$ is also dominated.

(5) As $-r \varphi$ is dominated by (1), we may assume that $r>0$. We choose a dominated metric family $\psi$ on $L$. By (2) $r \psi$ is dominated, so that, by (3),

$$
(\omega \in \Omega) \mapsto d_{\omega}(r \varphi, r \psi)=r d_{\omega}(\varphi, \psi)
$$

is $\nu$-dominated. Therefore $(\omega \in \Omega) \mapsto d_{\omega}(\varphi, \psi)$ is also $\nu$-dominated, and hence the assertion follows from $(4)$

(6) First we assume that $L$ is very ample. Then there exist a finite-dimensional vector space $E$ over $K$, a dominated norm family $\xi$ on $E$, a surjective homomorphism $\beta: f^{*}(E) \rightarrow L$ inducing a closed immersion $X \rightarrow \mathbb{P}(E)$ such that, if $\psi$ denotes the quotient metric family induced by $(E, \xi)$ and $\beta$, then the local distance function $(\omega \in \Omega) \mapsto d_{\omega}(\varphi, \psi)$ is $\nu$-dominated. Note that $\beta: E \otimes_{K} \mathcal{O}_{X} \rightarrow L$ yields the surjective homomorphism $g^{*}(\beta): E \otimes_{K} \mathcal{O}_{Y} \rightarrow g^{*}(L)$. Moreover, $g^{*}(\psi)$ coincides with quotient metric family induced by $(E, \xi)$ and $g^{*}(\beta)$. By Proposition 6.1.11, $g^{*}(\psi)$ is a dominated metric family. Moreover, for any $\omega \in \Omega$ one has

$$
d_{\omega}\left(g^{*}(\varphi), g^{*}(\psi)\right) \leqslant d_{\omega}(\psi, \varphi) .
$$

By (4) we obtain that $g^{*}(\varphi)$ is a dominated metric family.

In general, there are very ample invertible $\mathcal{O}_{X}$-modules $L_{1}$ and $L_{2}$ such that $L=$ $L_{1} \otimes L_{2}^{\vee}$. Let $\varphi_{1}$ be dominated metric family on $L_{1}$. If we set $\varphi_{2}=\varphi_{1}-\varphi$, then $\varphi_{2}$ is dominated by (1) and (2). By the previous case, $g^{*}\left(\varphi_{1}\right)$ and $g^{*}\left(\varphi_{2}\right)$ are dominated, so that, by (1) and (2) again, $g^{*}(\varphi)=g^{*}\left(\varphi_{1}\right)-g^{*}\left(\varphi_{2}\right)$ is also dominated.

Theorem 6.1.13. - Let $f: X \rightarrow \operatorname{Spec} K$ be a geometrically reduced projective $K$ scheme and $L$ be an invertible $\mathcal{O}_{X}$-module, equipped with a dominated metric family $\varphi=\left\{\varphi_{\omega}\right\}_{\omega \in \Omega}$. For any $\omega \in \Omega$, let \|\|$_{\varphi_{\omega}}$ be the sup norm on $H^{0}(X, L) \otimes_{K} K_{\omega}$ corresponding to the metric $\varphi_{\omega}$. Then the norm family $\xi=\left\{\|\cdot\|_{\varphi_{\omega}}\right\}_{\omega \in \Omega}$ on $H^{0}(X, L)$ is strongly dominated.

Proof. - Let us begin with the following claim:

Claim 6.1.14. - If the assertion of the theorem holds under the assumption that $X$ is geometrically integral, then it holds in general.

Proof. - One can find a finite extension $K^{\prime}$ of $K$ and the irreducible decomposition $X_{1} \cup \cdots \cup X_{n}$ of $X_{K^{\prime}}$ such that $X_{1}, \ldots, X_{n}$ are geometrically integral. We use the same notation as in Corollary 4.1.18, which says that it is sufficient to see that $\xi_{K^{\prime}}$ is dominated. 
Let $\psi=\left\{\psi_{\omega}\right\}_{\omega \in \Omega}$ be another metric family of $L$. Then $d_{\omega^{\prime}}\left(\varphi_{K^{\prime}}, \psi_{K^{\prime}}\right)=d_{\omega}(\varphi, \psi)$ for all $\omega \in \Omega$ and $\omega^{\prime} \in \Omega_{K^{\prime}}$ with $\pi_{K^{\prime} / K}\left(\omega^{\prime}\right)=\omega$. Therefore, one can see that $\varphi_{K^{\prime}}=\left\{\varphi_{K^{\prime}, \omega^{\prime}}\right\}_{\omega^{\prime} \in \Omega_{K^{\prime}}}$ is dominated.

Let $\varphi_{K^{\prime}, i}$ be the restriction of $\varphi_{K^{\prime}}$ to $X_{i}$. Then, by Proposition 6.1.12, (6) $\varphi_{K^{\prime}, i}$ is also dominated for all $i$. On the other hand, one has the natural injective homomorphism

$$
H^{0}\left(X_{K^{\prime}, \omega^{\prime}}, L_{K^{\prime}, \omega^{\prime}}\right) \rightarrow H^{0}\left(X_{1, \omega^{\prime}}, L_{K^{\prime}, \omega^{\prime}}\right) \oplus \cdots \oplus H^{0}\left(X_{n, \omega^{\prime}}, L_{K^{\prime}, \omega^{\prime}}\right) .
$$

Here we give a norm $\|\cdot\|_{\omega^{\prime}}$ on $H^{0}\left(X_{1, \omega^{\prime}}, L_{K^{\prime}, \omega^{\prime}}\right) \oplus \cdots \oplus H^{0}\left(X_{n, \omega^{\prime}}, L_{K^{\prime}, \omega^{\prime}}\right)$ given by

$$
\left\|\left(x_{1}, \ldots, x_{n}\right)\right\|_{\omega^{\prime}}=\max _{i \in\{1, \ldots, n\}}\left\{\left\|x_{i}\right\|_{\varphi_{K^{\prime}, i, \omega^{\prime}}}\right\} .
$$

By our assumption, $\xi_{K^{\prime}, i}$ is dominated for all $i$, so that $\left\{\|\cdot\|_{\omega^{\prime}}\right\}_{\omega \in \Omega_{K^{\prime}}}$ is also dominated by Proposition 4.1.19, (4). Note that $\|\cdot\|_{\varphi_{K^{\prime}, \omega^{\prime}}}$ is the restriction norm of $\|\cdot\|_{\omega^{\prime}}$, and hence $\xi_{K^{\prime}}=\left\{\|\cdot\|_{\varphi_{K^{\prime}, \omega^{\prime}}}\right\}_{\omega^{\prime} \in \Omega_{K^{\prime}}}$ is dominated by Proposition 4.1.19, (1), as desired.

From now on, we assume that $X$ is geometrically integral.

Claim 6.1.15. - If $L$ is very ample, the assertion of the theorem holds.

Proof. - Let $E=H^{0}(X, L), r=\operatorname{dim}_{K}(E)$ and $\beta: f^{*}(E) \rightarrow L$ be the canonical surjective homomorphism which induces a closed immersion of $X$ in $\mathbb{P}(E)$. Note that any non-zero element of $E$ can not identically vanish on $X$. Hence there exist a finite extension $K^{\prime}$ of $K$ together with closed points $P_{1}, \ldots, P_{r}$ of $X$ such that the residue filed $\kappa\left(P_{i}\right)$ at $P_{i}$ is contained in $K^{\prime}$, and that we have a strictly decreasing sequence of $K^{\prime}$-vector spaces

$$
E_{0} \supsetneq E_{1} \supsetneq E_{2} \supsetneq \cdots \supsetneq E_{r-1} \supsetneq E_{r}=\{0\},
$$

where $E_{0}=E \otimes_{K} K^{\prime}$ and

$$
E_{i}=\left\{s \in E \otimes_{K} K^{\prime}: s\left(P_{1}\right)=\cdots=s\left(P_{i}\right)=0\right\}
$$

for $i \in\{1, \ldots, r\}$. In order to prove Claim 6.1.15, by virtue of Corollary 4.1.18, we may assume that $K^{\prime}=K$.

Let $\omega_{1}, \ldots, \omega_{r}$ be local bases of $L$ around $P_{1}, \ldots, P_{r}$, respectively. For each $i=$ $1, \ldots, r$, we define $\theta_{i} \in E^{\vee}$ to be

$$
\forall s \in E, \quad \theta_{i}(s)=f_{s}\left(P_{i}\right) \quad\left(s=f_{s} \omega_{i} \text { around } P_{i}\right) .
$$

Note that $\theta_{1}, \ldots, \theta_{r}$ are linearly independent over $K$. Let $\boldsymbol{e}=\left\{e_{i}\right\}_{i=1}^{r}$ be the dual basis of $\left\{\theta_{i}\right\}_{i=1}^{r}$ over $K$, that is, $\left(e_{1}, \ldots, e_{r}\right) \in E^{r}$ and $\theta_{i}\left(e_{j}\right)=\delta_{i j}$. Here we define a norm $\|\cdot\|_{\omega}^{\prime}$ as follows: for any element $s \in E_{\omega}$ written as $s=\lambda_{1} e_{1}+\cdots+\lambda_{r} e_{r}$ with $\left(\lambda_{1}, \ldots, \lambda_{r}\right) \in K_{\omega}^{r}$

$$
\|s\|_{\omega}^{\prime}=\max _{i \in\{1, \ldots, r\}}\left|\lambda_{i}\right|_{\omega} .
$$

Let $\xi^{\prime}$ be the norm family of $E$ given by $\left\{\|\cdot\|_{\omega}^{\prime}\right\}_{\omega \in \Omega}$. Since the volume of $\Omega_{\infty}$ is finite, $\xi^{\prime}$ is dominated by Corollary 4.1.10. 
Let $\varphi^{\prime}$ be the metric family of $L$ induced by $\left(E, \xi^{\prime}\right)$ and $\beta$. Note that $\varphi^{\prime}$ is dominated. Let us see that $\|\cdot\|_{\omega}^{\prime}=\|\cdot\|_{\varphi_{\omega}^{\prime}}$ for any $\omega \in \Omega$. First of all, by Proposition 2.2.23 $\|\cdot\|_{\varphi_{\omega}^{\prime}} \leqslant\|\cdot\|_{\omega}^{\prime}$. For any $(i, j) \in\{1, \ldots, r\}^{2}$, one has

$$
\left|e_{j}\right|_{\varphi_{\omega}^{\prime}}\left(P_{i}\right)= \begin{cases}1, & \text { if } i=j, \\ 0, & \text { if } i \neq j .\end{cases}
$$

Therefore, for any $\left(\lambda_{1}, \ldots, \lambda_{r}\right) \in K_{\omega}^{r}$,

$$
\left\|\lambda_{1} e_{1}+\cdots+\lambda_{r} e_{r}\right\|_{\varphi_{\omega}^{\prime}} \geqslant \max _{i \in\{1, \ldots, r\}}\left|\lambda_{1} e_{1}+\cdots+\lambda_{r} e_{r}\right|_{\varphi_{\omega}^{\prime}}\left(P_{i}\right)=\max _{i \in\{1, \ldots, r\}}\left|\lambda_{i}\right|_{\omega},
$$

as required.

By the inequality (2.5) in Subsection 2.2.2, one has

$$
d_{\omega}\left(\|\cdot\|_{\varphi_{\omega}},\|\cdot\|_{\omega}^{\prime}\right)=d_{\omega}\left(\|\cdot\|_{\varphi_{\omega}},\|\cdot\|_{\varphi_{\omega}^{\prime}}\right) \leqslant d_{\omega}\left(\varphi_{\omega}, \varphi_{\omega}^{\prime}\right) .
$$

Therefore, $\omega \mapsto d_{\omega}\left(\|\cdot\|_{\varphi_{\omega}},\|\cdot\|_{\omega}^{\prime}\right)$ is $\nu$-dominaited, and hence $\xi$ is dominaited by Proposition 4.1.6.

Finally let us consider the following claim:

Claim 6.1.16. - For any non-zero element $s \in H^{0}(X, L)$, the function $(\omega \in \Omega) \longmapsto$ $\ln \|s\|_{\varphi_{\omega}}$ is $\nu$-dominated.

Proof. - Fix a non-zero element $s \in H^{0}(X, L)$.

Let us construct a dominated metric family $\varphi^{\prime}$ of $L$ such that the function $(\omega \in$ $\Omega) \longmapsto \ln \|s\|_{\varphi^{\prime} \omega}$ is $\nu$-dominated. Let $L_{1}$ be a very ample invertible $\mathcal{O}_{X}$-module such that $L_{2}:=L \otimes L_{1}$ is also very ample. The multiplication by the non-zero section $s$ defines an injective $K$-linear map from $H^{0}\left(X, L_{1}\right)$ to $H^{0}\left(X, L_{2}\right)$. We choose a dominated norm family $\xi_{2}^{\prime}=\left\{\|\cdot\|_{2, \omega}^{\prime}\right\}_{\omega, \in \Omega}$ on $H^{0}\left(X, L_{2}\right)$ and let $\xi_{1}^{\prime}$ be the restriction of $\xi_{2}^{\prime}$ on $H^{0}\left(X, L_{1}\right)$ via this injective map. By Proposition 4.1.19 (1), the norm family $\xi_{1}^{\prime}$ is also dominated. Let $\varphi_{1}^{\prime}$ and $\varphi_{2}^{\prime}$ be the quotient metric family induced by $\xi_{1}^{\prime}$ and $\xi_{2}^{\prime}$ respectively, where we consider the canonical surjective homomorphisms $f^{*}\left(H^{0}\left(X, L_{1}\right)\right) \rightarrow L_{1}$ and $f^{*}\left(H^{0}\left(X, L_{2}\right)\right) \rightarrow L_{2}$. We set $\varphi^{\prime}=\varphi_{2}^{\prime}-\varphi_{1}^{\prime}$. By Propositions 1.3 .26 and 1.3.25, for all $\omega \in \Omega, x \in X_{\omega}^{\text {an }}$ such that $s(x) \neq 0$ and $\ell \in L_{1, \omega} \otimes \widehat{\kappa}(x) \backslash\{0\}$, one has

$$
\begin{aligned}
|\ell|_{\varphi_{1, \omega}^{\prime}}(x) & =\inf _{\substack{u \in H^{0}\left(X, L_{1}\right), \lambda \in \widehat{\kappa}(x)^{\times} \\
u(x)=\lambda \ell}}|\lambda|_{x}^{-1} \cdot\|s u\|_{2, \omega}^{\prime} \\
& \geqslant \inf _{\substack{v \in H^{0}\left(X, L_{2}\right), \lambda \in \widehat{\kappa}(x)^{\times} \\
v(x)=\lambda s(x) \ell}}|\lambda|_{x}^{-1} \cdot\|v\|_{2, \omega}^{\prime} \\
& =|s(x) \ell|_{\varphi_{2, \omega}^{\prime}}^{\prime}(x)=|s(x)|_{\varphi_{\omega}^{\prime}}(x) \cdot|\ell|_{\varphi_{1, \omega}^{\prime}}(x),
\end{aligned}
$$

which leads to the inequality $\|s\|_{\varphi_{\omega}^{\prime} \text {,sup }} \leqslant 1$. Moreover, by Proposition 2.2.5, for any non-zero section $u \in H^{0}\left(X, L_{1}\right)$, one has

$$
\|s u\|_{\varphi_{2, \omega}^{\prime}} \leqslant\|s\|_{\varphi_{\omega}^{\prime}} \cdot\|u\|_{\varphi_{1, \omega}^{\prime}} \cdot
$$


Therefore, the function $(\omega \in \Omega) \mapsto \ln \|s\|_{\varphi_{\omega}^{\prime}}$ is non-positive and bounded from below by a $\nu$-dominated function.

By the inequality (2.5) in Subsection 2.2.2, one has

$$
d_{\omega}\left(\|\cdot\|_{\varphi_{\omega}},\|\cdot\|_{\varphi_{\omega}^{\prime}}\right) \leqslant d_{\omega}\left(\varphi_{\omega}, \varphi_{\omega}^{\prime}\right) .
$$

On the other hand, by Proposition 6.1.12 (3), the function $(\omega \in \Omega) \longmapsto d_{\omega}\left(\varphi_{\omega}, \varphi_{\omega}^{\prime}\right)$ is $\nu$-dominated, so that the assertion follows.

We now proceed with the proof of the theorem. Let $L_{1}$ be a very ample invertible $\mathcal{O}_{X}$-module such that $L_{2}:=L \otimes L_{1}$ is also very ample. We fix a non-zero global section $t$ of $L_{1}$, which defines an injective $K$-linear map from $H^{0}(X, L)$ to $H^{0}\left(X, L_{2}\right)$. We choose a dominated metric family $\varphi_{1}=\left\{\varphi_{1, \omega}\right\}_{\omega \in \Omega}$ on $L_{1}$ such that $\|t\|_{\varphi_{1, \omega}} \leqslant 1$ for any $\omega \in \Omega$, which is possible if we take a strongly dominated norm family $\xi_{1}=\left\{\|\cdot\|_{1, \omega}\right\}_{\omega \in \Omega}$ on $H^{0}\left(X, L_{1}\right)$ such that $\|t\|_{1, \omega} \leqslant 1$ for any $\omega \in \Omega$, and choose $\varphi_{1}$ as the quotient metric family induced by $\left(H^{0}\left(X, L_{1}\right), \xi_{1}\right)$ and the canonical surjective homomorphism $f^{*}\left(H^{0}\left(X, L_{1}\right)\right) \rightarrow L_{1}$. Let $\varphi_{2}=\left\{\varphi_{2, \omega}\right\}_{\omega \in \Omega}$ be the metric family $\varphi+\varphi_{1}$ on $L_{2}$. By Proposition 6.1.12 (2) the metric family $\varphi_{2}$ is dominated. Let $\xi_{2}$ be the norm family $\left\{\|\cdot\|_{\varphi_{2, \omega}}\right\}$ on $H^{0}\left(X, L_{2}\right)$. By Claim 6.1.15, the norm family $\xi_{2}$ is strongly dominated.

Let $\left\{s_{1}, \ldots, s_{m}\right\}$ be a basis of $H^{0}(X, L)$. For any $i \in\{1, \ldots, m\}$, let $t_{i}=t s_{i} \in$ $H^{0}\left(X, L_{2}\right)$. We choose sections $t_{m+1}, \ldots, t_{n}$ in $H^{0}\left(X, L_{2}\right)$ such that $\left\{t_{1}, \ldots, t_{n}\right\}$ forms a basis of $H^{0}\left(X, L_{2}\right)$. Let $\xi_{2}^{\circ}=\left\{\|\cdot\|_{2, \omega}^{\circ}\right\}_{\omega \in \Omega}$ be the norm family on $H^{0}\left(X, L_{2}\right)$ such that, for any $\omega \in \Omega$ and any $\left(\lambda_{1}, \ldots, \lambda_{n}\right) \in K_{\omega}^{n}$,

$$
\left\|\lambda_{1} t_{1}+\cdots+\lambda_{n} t_{n}\right\|_{2, \omega}^{\circ}= \begin{cases}\max _{i \in\{1, \ldots, n\}}\left|\lambda_{i}\right|_{\omega}, & \text { if } \omega \in \Omega \backslash \Omega_{\infty}, \\ \left|\lambda_{1}\right|_{\omega}+\cdots+\left|\lambda_{n}\right|_{\omega}, & \text { if } \omega \in \Omega_{\infty} .\end{cases}
$$

For any $\omega \in \Omega$ and any $\left(\lambda_{1}, \ldots, \lambda_{\omega}\right) \in K_{\omega}^{m}$, one has

$$
\left\|\lambda_{1} s_{1}+\cdots+\lambda_{m} s_{m}\right\|_{\varphi_{\omega}} \geqslant\left\|\lambda_{1} t_{1}+\cdots+\lambda_{m} t_{m}\right\|_{\varphi_{2, \omega}}
$$

since $\|t\|_{\varphi_{1, \omega}, \text { sup }} \leqslant 1$. As the norm family $\xi_{2}$ is strongly dominated, the local distance function $(\omega \in \Omega) \mapsto d_{\omega}\left(\xi_{2}, \xi_{2}^{\circ}\right)$ is $\nu$-dominated (see Corollary 4.1.10). In particular, there exists a $\nu$-dominated function $A$ on $\Omega$ such that, for any $\omega \in \Omega$ and any $\left(\lambda_{1}, \ldots, \lambda_{m}\right) \in K_{\omega}^{m}$, one has

$$
\ln \left\|\lambda_{1} s_{1}+\cdots+\lambda_{m} s_{m}\right\|_{\varphi_{\omega}} \geqslant \ln \left\|\lambda_{1} t_{1}+\cdots+\lambda_{m} t_{m}\right\|_{2, \omega}^{\circ}-A(\omega) .
$$

Moreover,

$$
\left\|\lambda_{1} s_{1}+\cdots+\lambda_{m} s_{m}\right\|_{\varphi_{\omega}} \leqslant\left\|\lambda_{1} t_{1}+\cdots+\lambda_{m} t_{m}\right\|_{2, \omega}^{\circ} \cdot \max _{i \in\{1, \ldots, m\}}\left\|s_{i}\right\|_{\varphi_{\omega}}
$$

By Claim 6.1.16, for any $i \in\{1, \ldots, m\}$, the function $(\omega \in \Omega) \mapsto \ln \left\|s_{i}\right\|_{\varphi_{\omega}}$ is $\nu$ dominated. Therefore, there exists a $\nu$-dominated function $B$ on $\Omega$ such that, for any $\omega \in \Omega$ and any $\left(\lambda_{1}, \ldots, \lambda_{m}\right) \in K_{\omega}^{m}$, one has

$$
\ln \left\|\lambda_{1} s_{1}+\cdots+\lambda_{m} s_{m}\right\|_{\varphi_{\omega}} \leqslant \ln \left\|\lambda_{1} t_{1}+\cdots+\lambda_{m} t_{m}\right\|_{2, \omega}^{\circ}+B(\omega) .
$$


The inequalities (6.2) and (6.3) imply that the local distance function $(\omega \in \Omega) \mapsto$ $d_{\omega}\left(\xi, \xi^{\circ}\right)$ is $\nu$-dominated, where $\xi^{\circ}$ is the restricted norm family of $\xi_{2}^{\circ}$ on $H^{0}(X, L)$. The strong dominancy of $\xi$ then follows from Corollary 4.1.10. The theorem is thus proved.

Proposition 6.1.17. - Let $K^{\prime}$ be a finite extension of $K$ and $X=\operatorname{Spec} K^{\prime}$. Let $L$ be an invertible $\mathcal{O}_{X}$-module. Then a metric family $\varphi$ on $L$ is dominated if and only if the corresponding norm family $\xi_{L}$ on $L$ relatively to the adelic curve $S \otimes_{K} K^{\prime}$ (cf. Remark 6.1.3) is dominated.

Proof. - First we assume that there exists a finite-dimensional vector space $E$ over $K$, a surjective $K^{\prime}$-linear map $\beta: E \otimes_{K} K^{\prime} \rightarrow L$, and a dominated norm family $\xi_{E}$ on $E$ such that the local distance function $(\omega \in \Omega) \mapsto d_{\omega}\left(\varphi, \varphi^{\prime}\right)$ is $\nu$-dominated, where $\varphi^{\prime}$ denotes the metric family on $L$ induced by $\left(E, \xi_{E}\right)$ and $\beta$. Denote by $\xi_{L}^{\prime}$ the norm families on $L$ relatively to the adelic curve $S \otimes_{K} K^{\prime}$, which correspond to the metric families $\varphi^{\prime}$. By definition $\xi_{L}^{\prime}$ identifies with the quotient norm family of $\xi_{K^{\prime}}$ induced by $\beta$. Since $\xi$ is dominated, also is $\xi_{K^{\prime}}$ (cf. Corollary 4.1.13). Hence the norm family $\xi_{L}^{\prime}$ is also dominated (cf. Proposition 4.1.19 (2)]. By Proposition 4.1.6, we obtain that the norm family $\xi_{L}$ is dominated.

Conversely, we assume that the norm family $\xi_{L}$ is dominated. Let $e$ be a generator of $L$ as vector space over $K^{\prime}$ and let $\xi_{e}=\left\{\|\cdot\|_{e, \omega}\right\}_{\omega \in \Omega}$ be the norm family on $K e$ such that $\|\lambda e\|_{e, \omega}=|\lambda|_{\omega}$ for any $\omega \in \Omega$. Note that the norm family $\xi_{e, K^{\prime}}$ on $(K e) \otimes_{K}$ $K^{\prime} \cong L$ is dominated. By Corollary 4.1.10, the local distance function $\left(x \in \Omega_{K^{\prime}}\right) \mapsto$ $d_{x}\left(\xi_{e, K^{\prime}}, \xi_{L}\right)$ is $\nu_{K^{\prime}}$ dominated. Let $\varphi^{\prime}$ be the metric family induced by $\left(K e, \xi_{e}\right)$ and the canonical isomorphism $(K e) \otimes_{K} K^{\prime} \cong L$. For any $x \in \Omega_{K^{\prime}}$ one has $d_{x}\left(\xi_{e, K^{\prime}}, \xi_{L}\right)=$ $d_{x}\left(\varphi^{\prime}, \varphi\right)$. By Proposition 6.1.12 (4), the metric family $\varphi$ is dominated.

6.1.3. Universally dense point families. - In this subsection, we consider universally dense point families (cf. Lemma 6.1.18) and their consequences.

Lemma 6.1.18. - Let $K$ be a field, $X$ be a scheme locally of finite type over Spec $K$ and $K^{\prime} / K$ be a field extension. Let $X_{K^{\prime}}$ be the fibre product $X \times_{\operatorname{Spec} K} \operatorname{Spec} K^{\prime}$ and $\pi: X_{K^{\prime}} \rightarrow X$ be the morphism of projection. For any closed point $P$ of $X$, the set $\pi^{-1}(\{P\})$ is finite and consists of closed points of $X_{K^{\prime}}$. Moreover, if $F$ is a set consisting of closed points of $X$, which is Zariski dense in $X$, then the subset $\pi^{-1}(F)$ of $X_{K^{\prime}}$ is Zariski dense.

Proof. - Let $P^{\prime}$ be a point of $X_{K^{\prime}}$ such that $P=\pi\left(P^{\prime}\right)$ is a closed point. Since $X$ is locally of finite type over $\operatorname{Spec} K$, the residue field of $P$ is a finite extension of $K$ (this is a consequence of Zariski's lemma, see [147]). As the residue field of $P^{\prime}$ is a quotient ring of $\kappa(P) \otimes_{K} K^{\prime}$, we obtain that it is a finite extension of $K^{\prime}$. Moreover, since $\kappa(P) \otimes_{K} K^{\prime}$ is an Artinian $K^{\prime}$-algebra, the set $\pi^{-1}(\{P\})$ is finite. 
In the following, we fix an algebraic closure $K^{\prime a c}$ of the field $K^{\prime}$ and we denote by $K^{\text {ac }}$ the algebraic closure of $K$ in $K^{\prime \text { ac }}$. For any closed point $P$ of $X$, we choose an arbitrary embedding of $\kappa(P)$ in $K^{\text {ac }}$ so that we can consider the residue field $\kappa(P)$ as a subfield of $K^{\text {ac }}$. Similarly, for any $P^{\prime} \in \pi^{-1}(\{P\})$, we choose an embedding of the residue field $\kappa\left(P^{\prime}\right)$ in $K^{\prime a c}$ which extends the embedding $\kappa(P) \rightarrow K^{\text {ac }}$.

To prove the lemma it suffices to verify that, for any affine open subset $U$ of $X$, $\pi^{-1}(U \cap F)$ is Zariski dense in $U_{K^{\prime}}$. Therefore we may assume without loss of generality that $X$ is an affine scheme of finite type over $K$. We let $A$ be the coordinate ring of $X$. Thus the coordinate ring of $X_{K^{\prime}}$ is $A \otimes_{K} K^{\prime}$. Let $f$ be an element of $A \otimes_{K} K^{\prime}$ and $\tilde{f}$ be the canonical image of $f$ in $A \otimes_{K} K^{\prime a c}$. We write $\tilde{f}$ as a linear combination

$$
\tilde{f}=a_{1} g_{1}+\cdots+a_{n} g_{n}
$$

where $g_{1}, \ldots, g_{n}$ are elements in $A \otimes_{K} K^{\text {ac }}$, and $a_{1}, \ldots, a_{n}$ are elements of $K^{\prime a c}$ which are linearly independent over $K^{\mathrm{ac}}$. Let $P$ be a closed point of $X$ and $\mathfrak{m}_{P}$ be the maximal ideal of $A$ corresponding to $P$. Assume that for any $P^{\prime} \in \pi^{-1}(\{P\})$ one has $f\left(P^{\prime}\right)=0$, where $f\left(P^{\prime}\right)$ denotes the image of $f$ by the projection map $A \otimes_{K} K^{\prime} \rightarrow$ $\left(A \otimes_{K} K^{\prime}\right) / \mathfrak{m}_{P^{\prime}}$, with $\mathfrak{m}_{P^{\prime}}$ being the (maximal) ideal of $A \otimes_{K} K^{\prime}$ corresponding to $P^{\prime}$. Then the canonical image of $f$ in $\left(A / \mathfrak{m}_{P}\right) \otimes_{K} K^{\prime}$ is nilpotent, which implies that the canonical image of $\tilde{f}$ in $\left(A / \mathfrak{m}_{P}\right) \otimes_{K} K^{\prime \text { ac }}$ is nilpotent. In particular, the canonical image of $\tilde{f}$ by the composed map

$$
A \otimes_{K} K^{\prime \mathrm{ac}} \longrightarrow\left(A / \mathfrak{m}_{P}\right) \otimes_{K} K^{\prime \mathrm{ac}}=\kappa(P) \otimes_{K} K^{\prime \mathrm{ac}} \longrightarrow K^{\prime \mathrm{ac}}
$$

is zero, where the last map in the above diagram is given by $\lambda \otimes \mu \mapsto \lambda \mu$ for any $\lambda \in K(P) \subseteq K^{\mathrm{ac}}$ and $\mu \in K^{\prime \text { ac }}$. In other words, one has

$$
a_{1} g_{1}(P)+\cdots+a_{n} g_{n}(P)=0,
$$

where for each $i \in\{1, \ldots, n\}, g_{n}(P)$ denotes the image of $g_{n}$ by the composed map

$$
A \otimes_{K} K^{\mathrm{ac}} \longrightarrow\left(A / \mathfrak{m}_{P}\right) \otimes_{K} K^{\mathrm{ac}}=\kappa(P) \otimes_{K} K^{\mathrm{ac}} \longrightarrow K^{\mathrm{ac}} .
$$

Since $a_{1}, \ldots, a_{n}$ are linearly independent over $K^{\mathrm{a}}$, we obtain that $g_{1}(P)=\cdots=$ $g_{n}(P)=0$. Since this holds for any $P \in F$ and since $F$ is Zariski dense in $X$, we obtain that $g_{1}, \ldots, g_{n}$ are nilpotent elements in $A \otimes_{K} K^{\text {ac }}$. Therefore $\tilde{f}$ is a nilpotent element of $A \otimes_{K} K^{\prime a c}$. Since the extension $K^{\prime a c} / K^{\text {ac }}$ equips $K^{\prime a c}$ with a structure of $K^{\text {ac }}$-algebra which is faithfully flat, the canonical map $A \otimes_{K} K^{\text {ac }} \rightarrow A \otimes_{K} K^{\prime a c}$ is injective (see [51, Chapitre I, $\S 1, \mathrm{n}^{\circ} 2$, Lemme 2.7). Therefore, $f$ is a nilpotent element in $A \otimes_{K} K^{\prime}$. This shows that $\pi^{-1}(F)$ is Zariski dense in $X_{K^{\prime}}$.

Proposition 6.1.19. - Let $S=(K,(\Omega, \mathcal{A}, \nu), \phi)$ be an adelic curve. Let $X$ be a projective $K$-scheme, $L$ be an invertible $\mathcal{O}_{X}$-module, equipped with a metric family $\varphi$. We assume that, for any closed point $P$ in $X$, the norm family $P^{*}(\varphi)$ (cf. Remark 6.1.3) on $P^{*}(L)$ is measurable. Then, for any $s \in H^{0}(X, L)$, the function

$$
\left(\omega \in \Omega \backslash \Omega_{0}\right) \longmapsto\|s\|_{\varphi_{\omega}}
$$


is measurable, where $\Omega_{0}$ denotes the set of $\omega \in \Omega$ such that $|\cdot|_{\omega}$ is the trivial absolute value, and we consider the restriction of the $\sigma$-algebra $\mathcal{A}$ on $\Omega \backslash \Omega_{0}$.

Proof. - As $X$ is projective over $K$, considering the coefficients of defining homogeneous polynomials of $X$, we can find a subfield $K_{0}$ of $K$ which is finitely generated over the prime field of $K$ (and hence $K_{0}$ is countable) and a projective scheme $X_{0}$ over Spec $K_{0}$ such that $X \cong X_{0} \times_{\text {Spec } K_{0}}$ Spec $K$. Let $\mathscr{P}$ be the set of closed points $P$ in $X$ whose canonical image in $X_{0}$ is a closed point. By Lemma 6.1.18, $\mathscr{P}$ is a Zariski dense and countable subset of $X$.

By the assumption of the proposition, for any closed point $P$ of $X$, the function

$$
\left(x \in \Omega_{\kappa(P)}\right) \longmapsto|s|_{\varphi_{\omega}}\left(P_{x}\right), \quad\left(\omega=\pi_{\kappa(P) / K}(x)\right)
$$

is $\mathcal{A}_{\kappa(P)}$-measurable. By Proposition 3.6.2, we obtain that the function

$$
(\omega \in \Omega) \longmapsto \max _{x \in \pi_{\kappa(P) / K}^{-1}(\{\omega\})}|s|_{\varphi_{\omega}}\left(P_{x}\right)
$$

is $\mathcal{A}$-measurable. Therefore, the function

$$
(\omega \in \Omega) \longmapsto \sup _{P \in \mathscr{P}} \max _{x \in \pi_{\kappa(P) / K}^{-1}(\{\omega\})}|s|_{\varphi_{\omega}}(x)
$$

is $\mathcal{A}$-measurable since $\mathscr{P}$ is countable.

To obtain the conclusion of the proposition, it remains to show that the function coincides with $\omega \mapsto\|s\|_{\varphi_{\omega} \text {, sup }}$ on $\Omega \backslash \Omega_{0}$. For this purpose it suffices to verify that, for any $\omega \in \Omega \backslash \Omega_{0}$, the set

$$
F_{\omega}=\left\{P_{x}: P \in \mathscr{P} \text { and } x \in \pi_{\kappa(P) / K}^{-1}(\{\omega\})\right\}
$$

is dense in $X_{\omega}^{\text {an }}$, where $X_{\omega}:=X \times_{\operatorname{Spec} K} \operatorname{Spec} K_{\omega}$. Let $j_{\omega}: X_{\omega}^{\text {an }} \rightarrow X_{\omega}$ be the specification map. By Lemma 6.1.18, $j_{\omega}\left(F_{\omega}\right)$ is Zariski dense in $X_{\omega}$ and hence $F_{\omega}$ is dense in $X_{\omega}^{\text {an }}$ with respect to the Berkovich topology (see [8, Corollary 3.4.5]). The proposition is thus proved.

We assume that $K$ is equipped with the trivial absolute value $|\cdot|_{0}$. Let $F$ be a finitely generated field over $K$ such that the transcendence degree of $F$ over $K$ is one. Let $C_{F}$ be a regular projective curve over $K$ such that the function field of $C_{F}$ is $F$, that is, $C_{F}$ is the unique regular model of $F$ over $K$. It is well-known that, for any absolute value $|\cdot|$ of $F$ over $K$ (i.e. the restriction of $|\cdot|$ to $K$ is trivial), there are a closed point $\xi$ of $C_{F}$ and $q \in \mathbb{R}_{\geqslant 0}$ such that $|\varphi|=\exp \left(-q \operatorname{ord}_{\xi}(\varphi)\right)$ for all $\varphi \in F^{\times}$ (see [82, §I.6] and [110, Proposition II.(3.3)]). Note that in the case where $q=0$, the absolute value is trivial. We say that $q$ is the exponent of $|\cdot|$. The absolute value given by $\exp \left(-q \operatorname{ord}_{\xi}(\cdot)\right)$ is denoted by $|\cdot|_{(\xi, q)}$. Let $X$ be a projective scheme over $\operatorname{Spec} K$. The Berkovich space associated with $X$ is denote by $X^{\text {an }}$ (see Definition 2.1.2). Let $j: X^{\text {an }} \rightarrow X$ be the specification map. Let us consider the following subsets $X_{0}^{\text {an }}$, 
$X_{1, \mathbb{Q}}^{\text {an }}$ and $X_{\leqslant 1, \mathbb{Q}}^{\text {an }}$ in $X^{\text {an }}$ :

$\left\{\begin{array}{l}X_{0}^{\text {an }}:=\left\{x \in X^{\text {an }}: j(x) \text { is closed }\right\}, \\ X_{1, \mathbb{Q}}^{\text {an }}:=\left\{\begin{array}{l|l}x \in X^{\text {an }} & \begin{array}{l}\text { the Zariski closure of }\{j(x)\} \text { has dimension one } \\ \text { and the exponent of the corresponding absolute value } \\ \text { is rational }\end{array} \\ X_{\leqslant 1, \mathbb{Q}}^{\text {an }}:=X_{0}^{\mathrm{an}} \cup X_{1, \mathbb{Q}}^{\text {an }} .\end{array}\right.\end{array}\right.$

Lemma 6.1.20. $-X_{\leqslant 1, \mathbb{Q}}^{\mathrm{an}}$ is dense in $X^{\text {an }}$ with respect to the Berkovich topology.

Proof. - To prove the lemma, we need to show that, for any regular function $f$ over an affine open subset $U=\operatorname{Spec} A$ of $X$ and for any point $x \in U^{\text {an }}$, the value $|f|(x)$ belongs to the closure $T$ of the set $\left\{|f|(z): z \in X_{\leqslant 1, \mathbb{Q}}^{\text {an }} \cap U^{\text {an }}\right\}$ in $\mathbb{R}$. First let us see the following claim:

Claim 6.1.21. - (a) If $f$ is not a nilpotent element, then $1 \in T$.

(b) If $f$ has a zero point in $U$, then $0 \in T$.

Proof. - (a) As $f$ is not a nilpotent element, there is a closed point $z$ of $U$ such that $f(z) \neq 0$, so that $1=|f|(z) \in T$.

(b) In this case one can find a closed point $z^{\prime}$ with $f\left(z^{\prime}\right)=0$. Therefore, $0=$ $|f|\left(z^{\prime}\right) \in T$.

Let us go back to the proof of the lemma. Let $X^{\prime}=\operatorname{Spec} A^{\prime}$ be the Zariki closure of $\{j(x)\}$ in $U$, where $A^{\prime}$ is the quotient domain of $A$ by the prime ideal corresponding to $j(x)$. Let $|\cdot|_{x}$ be the absolute value on the field of fractions of $A^{\prime}$ corresponding to $x$. If $\operatorname{dim} X^{\prime}=0$, then $j(x)$ is closed, so that the assertion is obvious. Moreover, if $\left|f^{\prime}\right|_{x}$ is either 0 or 1 , then the assertion is also obvious by the above claim. In particular, if $f^{\prime}=\left.f\right|_{X^{\prime}}$ is algebraic over $K$, then $|f|(x)=\left|f^{\prime}\right|_{x}$ is either 0 or 1 , and hence the assertion is true. Therefore we may assume that $\operatorname{dim} X^{\prime} \geqslant 1, f^{\prime}$ is transcendental over $K$ and $\left|f^{\prime}\right|_{x} \in \mathbb{R}_{\geqslant 0} \backslash\{0,1\}$.

Consider the ring $A^{\prime} \otimes_{K\left[f^{\prime}\right]} K\left(f^{\prime}\right)$, where $K\left(f^{\prime}\right)$ is the fraction field of $K\left[f^{\prime}\right]$. This is a localisation of the ring $A^{\prime}$ with respect to the multiplicatively closed subset $K\left[f^{\prime}\right] \backslash\{0\}$. We pick a closed point $\zeta^{\prime} \in \operatorname{Spec}\left(A^{\prime} \otimes_{K\left[f^{\prime}\right]} K\left(f^{\prime}\right)\right)$ and let $\zeta$ be the canonical image of $\zeta^{\prime}$ in $U$. Then the point $\zeta \in U$ has dimension 1 and the canonical image $f^{\prime \prime}$ of $f^{\prime}$ in the residue field $\kappa(\zeta)$ is transcendental over $K$ because $f^{\prime}$ is an element of the constant field $K\left(f^{\prime}\right)$ of the variety $\operatorname{Spec}\left(A^{\prime} \otimes_{K\left[f^{\prime}\right]} K\left(f^{\prime}\right)\right)$. In particular, the natural homomorphism $K\left[f^{\prime}\right] \rightarrow K\left[f^{\prime \prime}\right]$ is an isomorphism, which yields an isomorphism $K\left(f^{\prime}\right) \stackrel{\sim}{\longrightarrow} K\left(f^{\prime \prime}\right)$. Let $|\cdot|_{x}^{\prime}$ be the restriction of $|\cdot|_{x}$ to $K\left(f^{\prime}\right)$, and $|\cdot|_{x}^{\prime \prime}$ be the absolute value of $K\left(f^{\prime \prime}\right)$ such that the above isomorphism gives rise to an isometry

$$
\left(K\left(f^{\prime}\right),|\cdot|_{x}^{\prime}\right) \stackrel{\sim}{\longrightarrow}\left(K\left(f^{\prime \prime}\right),|\cdot|_{x}^{\prime \prime}\right) .
$$


Then $\left|f^{\prime \prime}\right|_{x}^{\prime \prime}=\left|f^{\prime}\right|_{x}^{\prime}=|f|(x)$. Let $|\cdot|_{\zeta}$ be an extension of $|\cdot|_{x}^{\prime \prime}$ to the residue field $\kappa(\zeta)$ and $C_{\zeta}$ be a regular and projective model of $\kappa(\zeta)$ over $K$. Then there are a closed point $\xi$ of $C_{\zeta}$ and $q \in \mathbb{R}_{>0}$ such that $|\cdot|_{\zeta}=|\cdot|_{(\xi, q)}$. Thus the assertion follows if we consider a sequence $\left\{q_{n}\right\}_{n=1}^{\infty}$ of rational numbers such that $\lim _{n \rightarrow \infty} q_{n}=q$.

Remark 6.1.22. - In the case where the absolute value of $K$ is non-trivial, $X_{0}^{\text {an }}$ is dense in $X^{\text {an }}$ (cf. Lemma 6.1.18 and [8, Corollary 3.4.5]). However we need one more layer $X_{1, \mathbb{Q}}^{\text {an }}$ if the absolute value of $K$ is trivial. Moreover, if the dimension of every irreducible component of $X$ is greater than or equal to one, then $X_{1, \mathbb{Q}}^{\text {an }}$ is dense in $X^{\text {an }}$ with respect to the Berkovich topology. Indeed, it is sufficient to show that $X_{0}^{\text {an }}$ is contained in the closure of $X_{1, \mathbb{Q}}^{\text {an }}$. Let $x \in X_{0}^{\text {an }}$ and choose a subvariety $C^{\prime}$ of $X$ such that $\operatorname{dim} C^{\prime}=1$ and $j(x) \in C^{\prime}$. Let $\mu: C \rightarrow C^{\prime}$ be the normalisation of $C^{\prime}$ and $\xi \in C$ with $\mu(\xi)=j(x)$. Note that $\lim _{\substack{q \rightarrow \infty \\ q \in \mathbb{Q}>0}}|\cdot|_{(\xi, q)}$ gives rise to the trivial valuation of the residue field $\kappa(\xi)$, which means that $x$ belongs to the closure of $X_{1, \mathbb{Q}}^{\text {an }}$.

Remark 6.1.23. - If $K$ is countable, then $X_{\leqslant 1, \mathbb{Q}}^{\text {an }}$ is also countable. In fact, the set of all closed points of a projective scheme over $K$ is countable. Therefore, $X_{0}^{\mathrm{an}}$ is countable. Moreover, if we fix an increasing sequence

$$
K_{1} \subseteq K_{2} \subseteq \ldots \subseteq K_{n} \subseteq K_{n+1} \subseteq \ldots
$$

of finite extensions of the field $K(T)$ of rational functions such that $\bigcup_{n \in \mathbb{N}, n \geqslant 1} K_{n}$ is the algebraic closure of $K(T)$, then any point $z \in X_{1}^{\text {an }}$ is represented by a point of $X$ valued in certain $K_{n}$ equipped with an absolute value over $K$ of rational exponent. Suppose that $K_{n}$ identifies with the rational function field of the projective curve $C_{n}$ over $K$, there are only countably many such absolute values since $K$ is assumed to be countable. Hence $X_{1, \mathbb{Q}}^{\text {an }}$ is also countable.

Remark 6.1.24. - We assume that $K$ is uncountable and the absolute value of $K$ is trivial. In this case, we can not expect a dense countable subset of $X^{\text {an }}$. Indeed, let $S$ be a countable subset of $\mathbb{P}_{K}^{1, \text { an }}$. Let $r: \mathbb{P}_{K}^{1, \text { an }} \rightarrow \mathbb{P}_{K}^{1}$ be the reduction map. As $r(S)$ is countable and $K$ is uncountable, there is a closed point $\xi \in \mathbb{P}_{K}^{1}$ such that $\xi \notin r(S)$. We set $I:=\left\{\exp \left(-q \operatorname{ord}_{\xi}(\cdot)\right): q \in\right] 0, \infty[\}$. Then $I$ is an open set of $\mathbb{P}_{K}^{1, \text { an }}$ and $r(I)=\{\xi\}$, so that $I \cap S=\emptyset$, which shows that $S$ is not dense in $\mathbb{P}_{K}^{1 \text {,an }}$.

Let $S=(K,(\Omega, \mathcal{A}, \nu), \phi)$ be an adelic curve. Denote by $\Omega_{0}$ the set of $\omega \in \Omega$ such that the absolute value $|\cdot|_{\omega}$ on $K$ is trivial. Let $\mathcal{A}_{0}$ be the restriction of the $\sigma$-algebra on $\Omega_{0}$. Let $X$ be a projective scheme over $\operatorname{Spec} K$. We equip $K$ with the trivial absolute value and denote by $X^{\text {an }}$ be the Berkovich space associated with $X$ (see Definition 2.1.2). Suppose given an invertible $\mathcal{O}_{X}$-module $L$ equipped with a metric family $\varphi=\left\{\varphi_{\omega}\right\}_{\omega \in \Omega}$. For any point $x \in X^{\text {an }}$, the metric $\varphi$ induces, for any $\omega \in \Omega$, a norm $|\cdot|_{\omega}(x)$ on the one-dimensional vector space $L \otimes \mathcal{O}_{X} \widehat{\kappa}(x)$. 
Proposition 6.1.25. - Let $S=(K,(\Omega, \mathcal{A}, \nu), \phi)$ be an adelic curve. We assume that the field $K$ is countable. Let $X$ be a projective scheme over $\operatorname{Spec} K, L$ be an invertible $\mathcal{O}_{X}$-module and $\varphi$ be metric family on $L$. Suppose that, for any $x \in X_{\leqslant 1, \mathbb{Q}}^{\mathrm{an}}$ and any non-zero element $\ell$ in $L \otimes_{\mathcal{O}_{X}} \widehat{\kappa}(x)$, the function $\left(\omega \in \Omega_{0}\right) \mapsto|\ell|_{\varphi_{\omega}}(x)$ is $\mathcal{A}_{0}$-measurable. Then for any $s \in H^{0}(X, L)$, the function

$$
\left(\omega \in \Omega_{0}\right) \longmapsto\|s\|_{\varphi_{\omega}}
$$

is measurable on $\left(\Omega_{0}, \mathcal{A}_{0}\right)$.

Proof. - By Lemma 6.1.20, we can write $\|s\|_{\varphi_{\omega}}$ as

$$
\|s\|_{\varphi_{\omega}}=\sup _{z \in X_{\leqslant 1, \mathbb{Q}}^{\mathrm{an}}}|s|_{\varphi_{\omega}}(z) .
$$

By the assumption of the proposition, each function $\left(\omega \in \Omega_{0}\right) \mapsto|s|_{\varphi_{\omega}}(z)$ is $\mathcal{A}_{0^{-}}$ measurable. Since $X_{\leqslant 1, \mathbb{Q}}^{\text {an }}$ is a countable set (see Remark 6.1.23), we deduce that the function $\left(\omega \in \Omega_{0}\right) \mapsto\|s\|_{\varphi_{\omega}}$ is also $\mathcal{A}_{0}$-measurable. The proposition is thus proved.

\subsubsection{Measurable metric families. -}

Definition 6.1.26. - Let $S=(K,(\Omega, \mathcal{A}, \nu), \phi)$ be an adelic curve, $X$ be a projective scheme over Spec $K$ and $L$ be an invertible $\mathcal{O}_{X}$-module. We say that a metric family $\varphi=\left\{\varphi_{\omega}\right\}_{\omega \in \Omega}$ on $L$ is measurable if the following conditions are satisfied:

(a) for any closed point $P$ in $X$, the norm family $P^{*}(\varphi)$ on $P^{*}(L)$ is measurable,

(b) for any point $x \in X_{\leqslant 1, \mathbb{Q}}^{\text {an }}$ (where we consider the trivial absolute value on $K$ in the construction of the Berkovich space $\left.X^{\text {an }}\right)$ and any element $\ell$ in $L \otimes_{\mathcal{O}_{X}} \widehat{\kappa}(x)$, the function $\left(\omega \in \Omega_{0}\right) \mapsto|\ell|_{\varphi_{\omega}}(x)$ is $\mathcal{A}_{0}$-measurable, where $\Omega_{0}$ the set of $\omega \in \Omega$ such that the absolute value $|\cdot|_{\omega}$ on $K$ is trivial, and $\mathcal{A}_{0}$ is the restriction of the $\sigma$-algebra on $\Omega_{0}$.

Proposition 6.1.2\%. - Let $S=(K,(\Omega, \mathcal{A}, \nu), \phi)$ be an adelic curve and $X$ be a projective scheme over $\operatorname{Spec} K$.

(1) Let $L$ be an invertible $\mathcal{O}_{X}$-module equipped with a measurable metric family $\varphi$, then the dual metric family $-\varphi$ on $L^{\vee}$ is measurable.

(2) Let $L_{1}$ and $L_{2}$ be two invertible $\mathcal{O}_{X}$-modules. If $\varphi_{1}$ and $\varphi_{2}$ are measurable metric families on $L_{1}$ and $L_{2}$ respectively, then the metric family $\varphi_{1}+\varphi_{2}$ on $L_{1} \otimes L_{2}$ is measurable.

(3) Let $L$ be an invertible $\mathcal{O}_{X}$-module equipped with a metric family $\varphi$. Suppose that there exists an integer $n \geqslant 1$ such that $n \varphi$ is measurable, then the metric family $\varphi$ is also measurable.

(4) Let $L$ be an invertible $\mathcal{O}_{X}$-module equipped with a measurable metric family $\varphi$, and $f: Y \rightarrow X$ be a projective morphism of $K$-schemes. Then $f^{*}(\varphi)$ is measurable. 
Proof. - (11) Let $P$ be a closed point of $X$. One has $P^{*}(-\varphi)=P^{*}(\varphi)^{\vee}$. Since $\varphi$ is measurable, the norm family $P^{*}(\varphi)$ is measurable. By Proposition 4.1.22] obtain that $P^{*}(-\varphi)$ is also measurable.

Let $x$ be a point of $X_{\leqslant 1, \mathbb{Q}}^{\text {an }}, \ell$ be a non-zero element of $L \otimes_{\mathcal{O}_{X}} \widehat{\kappa}(x)$ and $\ell^{\vee}$ be the dual element of $\ell$ in $L^{\vee} \otimes_{\mathcal{O}_{X}} \widehat{\kappa}(x)$. Then for any $\omega \in \Omega_{0}$ one has

$$
|\ell|_{\varphi_{\omega}}(x) \cdot\left|\ell^{\vee}\right|_{-\varphi_{\omega}}(x)=1 \text {. }
$$

Since the function $\left(\omega \in \Omega_{0}\right) \mapsto|\ell|_{\varphi_{\omega}}(x)$ is $\mathcal{A}_{0}$-measurable, also is the function $(\omega \in$ $\left.\Omega_{0}\right) \mapsto\left|\ell^{\vee}\right|_{-\varphi_{\omega}}(x)$. Therefore the metric family $-\varphi$ is measurable.

(2) Let $P$ be a closed point of $X$. One has $P^{*}\left(\varphi_{1}+\varphi_{2}\right)=P^{*}\left(\varphi_{1}\right) \otimes P^{*}\left(\varphi_{2}\right)$. Since $\varphi_{1}$ and $\varphi_{2}$ are both measurable, the norm families $P^{*}\left(\varphi_{1}\right)$ and $P^{*}\left(\varphi_{2}\right)$ are measurable. By Proposition 4.1.22 (3) the tensor norm family $P^{*}\left(\varphi_{1}\right) \otimes P^{*}\left(\varphi_{2}\right)$ is also measurable.

Let $x$ be a point of $X_{\leqslant 1, \mathbb{Q}}^{\text {an }}, \ell_{1}$ and $\ell_{2}$ be non-zero elements of $L_{1} \otimes_{\mathcal{O}_{X}} \widehat{\kappa}(x)$ and $L_{2} \otimes_{\mathcal{O}_{X}} \widehat{\kappa}(x)$, respectively. For any $\omega \in \Omega_{0}$ one has

$$
\left|\ell_{1}\right|_{\varphi_{1, \omega}}(x) \cdot\left|\ell_{2}\right|_{\varphi_{2, \omega}}(x)=\left|\ell_{1} \otimes \ell_{2}\right|_{\left(\varphi_{1}+\varphi_{2}\right)_{\omega}}(x) .
$$

Since the metric families $\varphi_{1}$ and $\varphi_{2}$ are measurable, the functions $\left(\omega \in \Omega_{0}\right) \mapsto\left|\ell_{1}\right|_{\varphi_{1, \omega}}$ and $\left(\omega \in \Omega_{0}\right) \mapsto\left|\ell_{2}\right|_{\varphi_{2, \omega}}$ are both $\mathcal{A}_{0}$-measurable. Hence the function $\left(\omega \in \Omega_{0}\right) \mapsto$ $\left|\ell_{1} \otimes \ell_{2}\right|_{\left(\varphi_{1}+\varphi_{2}\right)_{\omega}}(x)$ is $\mathcal{A}_{0}$-measurable.

(3) Let $P$ be a closed point of $X$. One has $P^{*}(n \varphi)=P^{*}(\varphi)^{\otimes n}$. Since $n \varphi$ is measurable, the norm family $P^{*}(n \varphi)$ is measurable. By Proposition 4.1.22 (4), we obtain that the norm family $P^{*}(\varphi)$ is also measurable.

Let $x$ be a point of $X_{\leqslant 1, \mathbb{Q}}^{\text {an }}$ and $\ell$ be a non-zero element of $L \otimes_{\mathcal{O}_{X}} \widehat{\kappa}(x)$. For any $\omega \in \Omega_{0}$ one has $\left|\ell^{\otimes n}\right|_{n \varphi_{\omega}}(x)=|\ell|_{\varphi_{\omega}}(x)^{n}$ and hence $|\ell|_{\varphi_{\omega}}(x)=|\ell|_{n \varphi_{\omega}}(x)^{1 / n}$. Since the metric family $n \varphi$ is measurable, we obtain that the function $\left(\omega \in \Omega_{0}\right) \mapsto\left|\ell^{\otimes n}\right|_{n \varphi_{\omega}}(x)$ is $\mathcal{A}_{0}$-measurable. Hence the function $\left(\omega \in \Omega_{0}\right) \mapsto|\ell|_{\varphi_{\omega}}(x)$ is also $\mathcal{A}_{0}$-measurable. Therefore the metric family $\varphi$ is measurable.

(4) This is obvious by the definition of the measurability of $\varphi$.

The following proposition shows that the measurability of metric family is a property stable by pointwise limit.

Proposition 6.1.28. - Let $S=(K,(\Omega, \mathcal{A}, \nu), \phi)$ be an adelic curve, $X$ be a projective scheme over $\operatorname{Spec} K$ and $L$ be an invertible $\mathcal{O}_{X}$-module. Let $\varphi$ and $\left\{\varphi_{n}\right\}_{n \in \mathbb{N}}$ be metric families on $L$ such that, for any $\omega \in \Omega$ and any $x \in X_{\omega}^{\mathrm{an}}$ (where $X_{\omega}^{\mathrm{an}}$ is the Berkovich space associated with $X_{\omega}:=X \times_{\operatorname{Spec} K} \operatorname{Spec} K_{\omega}$ ) one has

$$
\lim _{n \rightarrow+\infty} d\left(|\cdot|_{\varphi_{n, \omega}}(x),|\cdot|_{\varphi_{\omega}}(x)\right)=0 .
$$

Assume that the metric families $\varphi_{n}, n \in \mathbb{N}$, are measurable. Then the limite metric family $\varphi$ is also measurable.

Proof. - Let $P$ be a closed point of $X$ and $s$ be a non-zero element in $P^{*}(L)$. By the assumption of the proposition, for any $\omega^{\prime} \in \Omega_{K(P)}$ over $\omega \in \Omega$, the norm $\|\cdot\|_{\omega^{\prime}}$ indexed 
by $\omega^{\prime}$ in the norm family $P^{*}(\varphi)$ is given by $|\cdot|_{\varphi_{\omega}}\left(P_{\omega^{\prime}}\right)$, where $P_{\omega^{\prime}}$ is the algebraic point of $X_{\omega}$ determined by $P$ and $|\cdot|_{\omega^{\prime}}$. As well, for any $n \in \mathbb{N}$, the norm $\|\cdot\|_{n, \omega^{\prime}}$ indexed by $\omega^{\prime}$ in $P^{*}\left(\varphi_{n}\right)$ is given by $|\cdot|_{\varphi_{n, \omega}}\left(P_{\omega^{\prime}}\right)$. Therefore, by the limit assumption of the proposition, the sequence of functions

$$
\left(\omega^{\prime} \in \Omega_{K(P)}\right) \longmapsto\|s\|_{n, \omega^{\prime}}
$$

converges pointwisely to $\left(\omega^{\prime} \in \Omega_{K(P)}\right) \mapsto\|s\|_{\omega^{\prime}}$, which implies that the latter function is $\mathcal{A}_{K(P)}$-measurable by the measurability assumption of the proposition.

Similarly, for any point $x \in X_{\leqslant 1, \mathbb{Q}}^{\text {an }}$ (where we consider the trivial absolute value on $K$ in the construction of $\left.X^{\text {an }}\right)$, and any element $\ell \in L \otimes_{\mathcal{O}_{X}} \widehat{\kappa}(x)$, the sequence of functions

$$
\left(\omega \in \Omega_{0}\right) \longmapsto|\ell|_{\varphi_{n, \omega}}(x), \quad n \in \mathbb{N}
$$

converges pointwisely to $\left(\omega \in \Omega_{0}\right) \mapsto|\ell|_{\varphi_{\omega}}(x)$. Since each function in the sequence is $\mathcal{A}_{0}$-measurable, also is the limit function. The proposition is thus proved.

Proposition 6.1.29. - Let $S=(K,(\Omega, \mathcal{A}, \nu), \phi)$ be an adelic curve, $f: X \rightarrow$ Spec $K$ be a projective $K$-scheme and $L$ be an invertible $\mathcal{O}_{X}$-module. We assume that $K$ admits a countable subfield which is dense in every $K_{\omega}, \omega \in \Omega$. Let $E$ be a finite-dimensional vector space over $K$, equipped with a measurable norm family $\xi=\left\{\|\cdot\|_{\omega}\right\}_{\omega \in \Omega}$. Suppose give a surjective homomorphism $\beta: f^{*}(E) \rightarrow L$ and let $\varphi$ be the quotient metric family on $L$ induced by $(E, \xi)$ and $\beta$. Then the metric family $\varphi$ is measurable.

Proof. - By Proposition (1.b) the double dual norm family $\xi^{\vee \vee}$ is measurable. By Remark 6.1.4, we can replace $\xi$ by $\xi^{\vee \vee}$ without changing the corresponding quotient metric family. Hence we may assume without loss of generality that the norm $\|\cdot\|_{\omega}$ is ultrametric for any $\omega \in \Omega \backslash \Omega_{\infty}$.

Let $P$ be a closed point of $X$. Then the norm family $P^{*}(\varphi)$ identifies with the quotient norm family of $\xi \otimes K(P)$ induced by the surjective homomorphism

$$
P^{*}(\beta): P^{*}\left(f^{*}(E)\right) \cong E \otimes_{K} K(P) \longrightarrow P^{*}(L) .
$$

By Proposition 4.1.24 (2.a) and (1.a), the norm family $P^{*}(\varphi)$ is measurable.

Assume that $\Omega_{0}$ is not empty. In this case the field $K$ itself is countable. Let $x$ be a point of $X_{\leqslant 1, \mathbb{Q}}^{\text {an }}$ and $\ell$ be a non-zero element of $L \otimes_{\mathcal{O}_{X}} \widehat{\kappa}(x)$. By Proposition 1.3.26. for any $\omega \in \Omega_{0}$ one has

$$
|\ell|_{\varphi_{\omega}}(x)=\inf _{\substack{s \in E, \lambda \in \widehat{\kappa}(x)^{\times} \\ s(x)=\lambda \ell}}|\lambda|_{x}^{-1} \cdot\|s\|_{\omega},
$$

where $s(x)$ denotes the image of $s$ by the quotient map

$$
\beta_{x}: E \otimes_{K} \widehat{\kappa}(x) \rightarrow L \otimes_{\mathcal{O}_{X}} \widehat{\kappa}(x) .
$$

Since the norm family $\xi$ is measurable, the function $\left(\omega \in \Omega_{0}\right) \mapsto\|s\|_{\omega}$ is $\mathcal{A}_{0^{-}}$ measurable. Moreover, since $K$ is a countable field, the vector space $E$ is a countable 
set. Hence we obtain that the function $\left(\omega \in \Omega_{0}\right) \mapsto|\ell|_{\varphi_{\omega}}(x)$ is $\mathcal{A}_{0}$-measurable. Therefore, the metric family $\varphi$ is measurable.

Definition 6.1.30. - Let $S=(K,(\Omega, \mathcal{A}, \nu), \phi)$ be an adelic curve, $\pi: X \rightarrow$ Spec $K$ be a projective $K$-scheme, $L$ be an invertible $\mathcal{O}_{X}$-module and $\varphi$ be a metric family on $L$. We denote by $\pi_{*}(\varphi)$ the norm family $\left\{\|\cdot\|_{\varphi_{\omega}}\right\}_{\omega \in \Omega}$ on $\pi_{*}(L)$.

The propositions 6.1.19 and 6.1.25 can be resumed as follows.

Theorem 6.1.31. - Let $S=(K,(\Omega, \mathcal{A}, \nu), \phi)$ be a adelic curve, $X$ be a projective $K$-scheme and $L$ be an invertible $\mathcal{O}_{X}$-module. We assume that, either $\Omega_{0}$ is empty, or the field $K$ is countable. For any measurable metric family $\varphi$ on $L$, the norm family $\pi_{*}(\varphi)$ is measurable.

\subsection{Adelic line bundle and Adelic divisors}

In this section, we fix an adelic curve $S=(K,(\Omega, \mathcal{A}, \nu), \phi)$ be an adelic curve.

Definition 6.2.1. — Let $X$ be a projective scheme over Spec $K$. We call adelic line bundle on $X$ any invertible $\mathcal{O}_{X}$-module $L$ equipped with a metric family $\varphi$ which is dominated an measurable.

6.2.1. Height function. - Let $(L, \varphi)$ be an adelic line bundle on $X$. For any closed point $P$ of $X$, the norm family $P^{*}(\varphi)$ on $P^{*}(L)$ is measurable and dominated (see Propositions 6.1.12 (6) and 6.1.17 for the dominance of $P^{*}(\varphi)$ ). Therefore $\left(P^{*}(L), P^{*}(\varphi)\right)$ is an adelic line bundle on $S$ (cf. Proposition 4.1.29). We denote by $h_{(L, \varphi)}(P)$ the Arakelov degree of this adelic line bundle, called the height of $P$ with respect to the adelic line bundle $(L, \varphi)$. By abuse of notation, we also denote by $h_{(L, \varphi)}(\cdot)$ the function on the set $X\left(K^{\mathrm{ac}}\right)$ of algebraic points of $X$ sending any $K$-morphism Spec $K^{\text {ac }} \rightarrow X$ to the height of the image of the $K$-morphism.

Proposition 6.2.2. - Let $X$ be a projective $K$-scheme, $\left(L_{1}, \varphi_{1}\right)$ and $\left(L_{2}, \varphi_{2}\right)$ be adelic line bundles on $X$.

(1) For any closed point $P$ of $X$, one has

$$
h_{\left(L_{1} \otimes L_{2}, \varphi_{1}+\varphi_{2}\right)}(P)=h_{\left(L_{1}, \varphi_{1}\right)}(P)+h_{\left(L_{2}, \varphi_{2}\right)}(P) .
$$

(2) Assume that $L_{1}$ and $L_{2}$ are the same invertible $\mathcal{O}_{X}$-module $L$. Then, for any closed point $P$ of $X$, one has

$$
\left|h_{\left(L, \varphi_{1}\right)}(P)-h_{\left(L, \varphi_{2}\right)}(P)\right| \leqslant \operatorname{dist}\left(\varphi_{1}, \varphi_{2}\right) .
$$

Proof. - (1) This follows directly from Proposition 6.1.2 and 4.3.5

(2) Let $P$ be a closed point of $X$. By definition, for any $\omega \in \Omega$ and any $x \in M_{K(P), \omega}$ one has $d_{x}\left(P^{*}\left(\varphi_{1}\right), P^{*}\left(\varphi_{2}\right)\right) \leqslant d_{\omega}\left(\varphi_{1}, \varphi_{2}\right)$. Therefore, by taking the integral with respect to $x$, we obtain the inequality (6.5). 
The following proposition shows that, if the adelic curve $S$ has the Northcott property, then the height function associated with an adelic line bundle with ample underlying invertible sheaf has a finiteness property of Northcott type.

Proposition 6.2.3. - Assume that the adelic curve $S$ has the Northcott property (cf. Definition 3.5.2). Let $X$ be a projective $K$-scheme and $(L, \varphi)$ be an adelic line bundle on $X$ such that $L$ is ample. For all positive real numbers $\delta$ and $C$, the set

$$
\left\{P \in X\left(K^{\mathrm{ac}}\right): h_{(L, \varphi)}(P) \leqslant C,[K(P): K] \leqslant \delta\right\}
$$

is finite.

Proof. - By Proposition 6.2.2, for any integer $n \geqslant 1$, one has $h_{(n L, n \varphi)}=n h_{(L, \varphi)}$ as function on $X\left(K^{\mathrm{ac}}\right)$. Therefore, without loss of generality we may assume that $L$ is very ample. Moreover, by Proposition 6.2.2 (2), to prove the proposition it suffice to check the finiteness of (6.6) for a particular choice of metric family $\varphi$. Thus we may assume without loss of generality that there exist a finite dimensional vector space $E$ over $K$ and a surjective homomorphism $\beta: E \otimes_{K} \mathcal{O}_{K} \rightarrow L$ inducing a closed immersion $X \rightarrow \mathbb{P}(E)$, together with a basis $\boldsymbol{e}=\left\{e_{i}\right\}_{i=0}^{r}$ of $E$ over $K$, such that $\varphi$ identifies with the quotient metric family induced by $\left(E, \xi_{\boldsymbol{e}}\right)$ and $\beta$ (see Example 4.1.5 for the definition of $\boldsymbol{e})$. Let $P$ be a closed point of $X$. Then $\left(P^{*}(L) \otimes_{K(P)} K^{\text {ac }}, P^{*}(\varphi)_{K^{\text {ac }}}\right)$ is a quotient adelic line bundle of $\left(E_{K^{\text {ac }}}, \xi_{e, K^{\text {ac }}}\right)$ and hence $\left(L_{K^{\text {ac }}}^{\vee}, P^{*}(\varphi)_{K^{\text {ac }}}^{\vee}\right)$ identifies with an adelic line subbundle of $\left(E_{K^{\text {ac }}}^{\vee}, \xi_{\boldsymbol{e}, K^{\text {ac }}}^{\vee}\right)$. Suppose that, as a vector subspace of rank 1 of $E_{K^{\mathrm{ac}}}^{\vee}, L_{K^{\mathrm{ac}}}^{\vee}$ is generated by the vector $a_{0} e_{0}^{\vee}+\cdots+a_{r} e_{r}^{\vee}$, where

$$
\left[a_{0}: \cdots: a_{r}\right] \in \mathbb{P}^{r}\left(K^{\mathrm{ac}}\right),
$$

then one has

$$
\begin{aligned}
& \widehat{\operatorname{deg}}\left(P^{*}(L), P^{*}(\varphi)\right)=\widehat{\operatorname{deg}}\left(L_{K^{\mathrm{ac}}}, P^{*}(\varphi)_{K^{\mathrm{ac}}}\right)=-\widehat{\operatorname{deg}}\left(L_{K^{\mathrm{ac}}}^{\vee}, P^{*}(\varphi)_{K^{\mathrm{ac}}}^{\vee}\right) \\
= & \int_{\Omega_{K^{\mathrm{ac}}}} \ln \left(\max \left\{\left|a_{0}\right|_{\chi}, \ldots,\left|a_{r}\right|_{\chi}\right\}\right) \nu_{K^{\mathrm{ac}}}(d \chi) .
\end{aligned}
$$

Therefore the finiteness of (6.6) follows from Theorem 3.5.3

6.2.2. Essential minimum. - In this subsection, we fix an integral projective scheme $X$ over Spec $K$. For any adelic line bundle $(L, \varphi)$ on $X$, we define the essential minimum of $(L, \varphi)$ as

$$
\widehat{\mu}_{\mathrm{ess}}(L, \varphi):=\sup _{Z \subsetneq X} \inf _{P \in(X \backslash Z)\left(K^{\text {ac }}\right)} h_{(L, \varphi)}(P),
$$

where $Z$ runs over the set of all strict Zariski closed subsets of $X$, and $P$ runs over the set of closed points of the open subscheme $X \backslash Z$ of $X$. By Proposition 6.2.2 (1) for any integer $n \geqslant 1$, one has $\widehat{\mu}_{\mathrm{ess}}\left(L^{\otimes n}, n \varphi\right)=n \widehat{\mu}_{\mathrm{ess}}(L, \varphi)$.

Proposition 6.2.4. - Let $\left(L_{1}, \varphi_{1}\right)$ and $\left(L_{2}, \varphi_{2}\right)$ be adelic line bundles on $X$. Then

$$
\widehat{\mu}_{\mathrm{ess}}\left(L_{1} \otimes L_{2}, \varphi_{1}+\varphi_{2}\right) \geqslant \widehat{\mu}_{\mathrm{ess}}\left(L_{1}, \varphi_{1}\right)+\widehat{\mu}_{\mathrm{ess}}\left(L_{2}, \varphi_{2}\right) \text {. }
$$


Proof. - Let $Z_{1}$ and $Z_{2}$ be strict Zariski closed subsets of $X$. Then $Z=Z_{1} \cup Z_{2}$ is also a strict Zariski closed subset of $X$. If $P$ is an element of $(X \backslash Z)\left(K^{\text {ac }}\right)$, one has

$$
\begin{aligned}
& h_{\left(L_{1} \otimes L_{2}, \varphi_{1}+\varphi_{2}\right)}(P)=h_{\left(L_{1}, \varphi_{1}\right)}(P)+h_{\left(L_{2}, \varphi_{2}\right)}(P) \\
& \geqslant \inf _{Q_{1} \in\left(X \backslash Z_{1}\right)\left(K^{\mathrm{ac}}\right)} h_{\left(L_{1}, \varphi_{1}\right)}\left(Q_{1}\right)+\inf _{Q_{2} \in\left(X \backslash Z_{2}\right)\left(K^{\mathrm{ac}}\right)} h_{\left(L_{2}, \varphi_{2}\right)}\left(Q_{2}\right) .
\end{aligned}
$$

Taking the infimum with respect to $P \in(X \backslash Z)\left(K^{\mathrm{ac}}\right)$ and then the supremum with respect to $\left(Z_{1}, Z_{2}\right)$, we obtain that

$$
\widehat{\mu}_{\mathrm{ess}}\left(L_{1} \otimes L_{2}, \varphi_{1}+\varphi_{2}\right) \geqslant \widehat{\mu}_{\mathrm{ess}}\left(L_{1}, \varphi_{1}\right)+\widehat{\mu}_{\mathrm{ess}}\left(L_{2}, \varphi_{2}\right)
$$

Proposition 6.2.5. — Let $(L, \varphi)$ be an adelic line bundle on $X$.

(1) The essential minimum of $(L, \varphi)$ identifies with the infimum of the set of real numbers $C$ such that $\left\{P \in X\left(K^{\mathrm{ac}}\right): h_{(L, \varphi)}(P) \leqslant C\right\}$ is Zariski dense in $X$.

(2) If $X^{\prime}$ is an integral projective $K$-scheme and $f: X^{\prime} \rightarrow X$ is a birational projective morphism, then one has $\widehat{\mu}_{\mathrm{ess}}(L, \varphi)=\widehat{\mu}_{\mathrm{ess}}\left(f^{*}(L), f^{*}(\varphi)\right)$.

Proof. - (1) Let $C$ be a real number such that the set

$$
M_{C}:=\left\{P \in X\left(K^{\mathrm{ac}}\right): h_{(L, \varphi)}(P) \leqslant C\right\}
$$

is Zariski dense. Then, for any Zariski closed subset $Z$ of $X$ such that $Z \subsetneq X$, the set $M_{C}$ is not contained in $Z$, namely $(X \backslash Z)\left(K^{\text {ac }}\right)$ contains at least one element of $M_{C}$. Therefore one has

$$
\inf _{P \in(X \backslash Z)\left(K^{\mathrm{ac}}\right)} h_{(L, \varphi)}(P) \leqslant C .
$$

We then obtain that $\widehat{\mu}_{\mathrm{ess}}(L, \varphi)$ is bounded from above by the infimum of the set of real numbers $C$ such that $M_{C}$ is Zariski dense in $X$.

Conversely, if $C$ is a real number such that $M_{C}$ is not Zariski dense in $X$, then one has

$$
\widehat{\mu}_{\mathrm{ess}}(L, \varphi) \geqslant \inf _{P \in\left(X \backslash \bar{M}_{C}^{\mathrm{Zar}}\right)\left(K^{\mathrm{ac}}\right)} h_{(L, \varphi)}(P) \geqslant C .
$$

Since $C$ is arbitrary, we obtain that $\widehat{\mu}_{\text {ess }}(L, \varphi)$ is bounded from below by the infimum of the set of real numbers $C$ such that $M_{C}$ is Zariski dense in $X$.

(2) Denote by $\left(L^{\prime}, \varphi^{\prime}\right)$ the adelic line bundle $\left(f^{*}(L), f^{*}(\varphi)\right)$, and by $Y$ the exceptional locus of $f$. Note that the restriction of $f$ to $Z^{\prime} \backslash f^{-1}(Y)$ is an isomorphism between $X^{\prime} \backslash f^{-1}(Y)$ and $X \backslash Y$. Let $Z$ be a Zariski closed subset of $X$ such that $Z \subsetneq X$. Let $Z^{\prime}=f^{-1}(Z)$. It is a Zariski closed subset of $X^{\prime}$ such that $Z^{\prime} \subsetneq X^{\prime}$. Therefore,

$$
\widehat{\mu}_{\text {ess }}\left(L^{\prime}, \varphi^{\prime}\right) \geqslant \inf _{P \in\left(X^{\prime} \backslash\left(Z^{\prime} \cup f^{-1}(Y)\right)\left(K^{\text {ac }}\right)\right.} h_{\left(L^{\prime}, \varphi^{\prime}\right)}(P) \geqslant \inf _{Q \in(X \backslash Y)} h_{(L, \varphi)}(Q) .
$$

Since $Y$ is arbitrary, we obtain that $\widehat{\mu}_{\mathrm{ess}}\left(L^{\prime}, \varphi^{\prime}\right) \geqslant \widehat{\mu}_{\mathrm{ess}}(L, \varphi)$.

Let $C$ be a real number such that the set $M_{C}$ of points $Q \in X\left(K^{\text {ac }}\right)$ with $h_{(L, \varphi)}(Q) \leqslant C$ is Zariski dense. Then the set $M_{C} \cap(X \backslash Y)\left(K^{\text {ac }}\right)$ is also Zariski 
dense in $X$. This implies that $f^{-1}\left(M_{C} \cap(X \backslash Y)\left(K^{\text {ac }}\right)\right)$ is Zariski dense in $X^{\prime}$. Note that for any $P \in f^{-1}\left(M_{C} \cap(X \backslash Y)\left(K^{\text {ac }}\right)\right)$ one has $h_{\left(L^{\prime}, \varphi^{\prime}\right)}(P)=h_{(L, \varphi)}(f(P))$. Therefore the set of $P \in X^{\prime}\left(K^{\text {ac }}\right)$ with $h_{\left(L^{\prime}, \varphi^{\prime}\right)}(P) \leqslant C$ is Zariski dense, which implies that $\widehat{\mu}_{\text {ess }}\left(L^{\prime}, \varphi^{\prime}\right) \leqslant C$. Since $C>\widehat{\mu}_{\text {ess }}(L, \varphi)$ is arbitrary, we obtain that $\widehat{\mu}_{\text {ess }}\left(L^{\prime}, \varphi^{\prime}\right) \geqslant \widehat{\mu}_{\text {ess }}(L, \varphi)$.

Proposition 6.2.6. - We assume that, either the $\sigma$-algebra $\mathcal{A}$ is discrete, or the field $K$ admits a countable subfield which is dense in each $K_{\omega}, \omega \in \Omega$. Let $f: X \rightarrow$ Spec $K$ be an integral projective scheme and $(L, \varphi)$ be an adelic line bundle on $X$. If $s$ is a non-zero global section of $L$, then

$$
\widehat{\mu}_{\text {ess }}(L, \varphi) \geqslant \widehat{\operatorname{deg}}_{f_{*}(\varphi)}(s) .
$$

In particular, $\widehat{\mu}_{\mathrm{ess}}(L, \varphi)>-\infty$ once $L$ admits a non-zero global section.

Proof. - For any closed point $P$ outside of the zero locus of $s$, one has

$$
\begin{aligned}
h_{(L, \varphi)}(P) & =-\int_{\chi \in \Omega_{K^{\mathrm{ac}}}} \ln |s|_{\varphi_{\pi_{K^{\mathrm{ac}} / K}}(\chi)}\left(\sigma_{\chi}(P)\right) \nu_{K^{\mathrm{ac}}}(\mathrm{d} \chi) \\
& \geqslant-\int_{\chi \in \Omega_{K^{\mathrm{ac}}}} \ln \|s\|_{\varphi_{\pi_{K^{\mathrm{ac}} / K}}(\chi)} \nu_{K^{\mathrm{ac}}}(\mathrm{d} \chi) \\
& =-\int_{\omega \in \Omega} \ln \|s\|_{\varphi_{\omega}} \nu(\mathrm{d} \omega)=\widehat{\operatorname{deg}}_{f_{*}(\varphi)}(s) .
\end{aligned}
$$

This leads to the inequality $\widehat{\mu}_{\text {ess }}(L, \varphi) \geqslant \widehat{\operatorname{deg}}_{f_{*}(\varphi)}(s)$.

Proposition 6.2.7. - Let $(L, \varphi)$ be an adelic line bundle on $X$. One has $\widehat{\mu}_{\text {ess }}(L, \varphi)<+\infty$.

Proof. - If $\varphi$ and $\varphi^{\prime}$ are metric families on $L$ such that $(L, \varphi)$ and $\left(L, \varphi^{\prime}\right)$ are adelic line bundles on $X$, then for any $P \in X\left(K^{\text {ac }}\right)$ one has

$$
\left|h_{(L, \varphi)}(P)-h_{\left(L, \varphi^{\prime}\right)}(P)\right| \leqslant \operatorname{dist}\left(\varphi, \varphi^{\prime}\right)
$$

(see (6.1) for the definition of $\operatorname{dist}\left(\varphi, \varphi^{\prime}\right)$ ). Therefore, to show the proposition, it suffice to prove the assertion for a particular choice of the metric family $\varphi$. This observation allows us to change the metric family whenever necessary in the proof.

Let $M$ be a very ample invertible $\mathcal{O}_{X}$-module such that $M \otimes L$ is also very ample. Let $\varphi_{M}$ be a metric family on $M$ such that $\left(M, \varphi_{M}\right)$ forms an adelic line bundle on $X$. By Proposition 6.2.6, one has $\widehat{\mu}_{\text {ess }}\left(M, \varphi_{M}\right)>-\infty$. Moreover, by Proposition 6.2.4 one has $\widehat{\mu}_{\text {ess }}\left(L \otimes M, \varphi+\varphi_{M}\right) \geqslant \widehat{\mu}_{\text {ess }}(L, \varphi)+\widehat{\mu}_{\text {ess }}\left(M, \varphi_{M}\right)$. Therefore, by replacing $L$ by $L \otimes M$ we may assume without loss of generality that $L$ is a very ample invertible $\mathcal{O}_{X}$-module.

By Noetherian normalisation we obtain that there existe a positive integer $n$, an integral projective $K$-scheme $X^{\prime}$, a birational projective $K$-morphism $f: X^{\prime} \rightarrow X$, together with a generically finite projective $K$-morphism $g: X^{\prime} \rightarrow \mathbb{P}_{K}^{r}$ (where $r$ is the 
Krull dimension of $X)$ such that $g^{*}(\mathcal{O}(1)) \cong f^{*}\left(L^{\otimes n}\right)$, where $\mathcal{O}(1)$ denotes the universal invertible sheaf on $\mathbb{P}_{K}^{r}$. We can for example construct first a rational morphism from $X$ to $\mathbb{P}_{K}^{r}$ corresponding to an injective finite homogeneous homomorphism from the polynomial algebra to the Cox ring of some power of $L$. This step is guaranteed by the fact that the Cox ring $\bigoplus_{m \in \mathbb{N}} H^{0}\left(X, L^{\otimes m}\right)$ is finitely generated, by using Noether normalisation, see [54, §13.1]. Then we can take $X^{\prime}$ as the blowing-up of $X$ along the locus where the rational morphism is not defined. By Proposition 6.2.5 (2), one has $n \widehat{\mu}_{\text {ess }}(L, \varphi)=n \widehat{\mu}_{\text {ess }}\left(f^{*}(L), f^{*}(\varphi)\right)=\widehat{\mu}_{\text {ess }}\left(f^{*}\left(L^{\otimes n}\right), n f^{*}(\varphi)\right)$. Therefore we can reduce the problem to the case where there exists a generically finite projective $K$-morphism $g: X \rightarrow \mathbb{P}_{K}^{r}$ such that $L \cong g^{*}(\mathcal{O}(1))$.

We identify $\mathbb{P}_{K}^{r}$ with $\mathbb{P}\left(K^{r+1}\right)$ and equip $K^{r+1}$ with the norm family $\xi$ associated with the canonical basis (see Example 4.1.5). Let $\varphi_{0}$ be the quotient metric family on $\mathcal{O}(1)$ induced by $\left(K^{r+1}, \xi\right)$ and the canonical surjective homomorphism $K^{r+1} \otimes_{K} \mathcal{O}_{\mathbb{P}_{K}^{r}} \rightarrow \mathcal{O}(1)$. As explained above, we may assume without loss of generality that $\varphi=g^{*}\left(\varphi_{0}\right)$. In particular, for any closed point $P$ of $X$, one has $h_{(L, \varphi)}(P)=h_{\left(\mathcal{O}(1), \varphi_{0}\right)}(g(P))$. Moreover, similarly as in the proof of Proposition 6.2.3, for any element $\left[a_{0}: \ldots: a_{r}\right] \in \mathbb{P}_{K}^{r}\left(K^{\text {ac }}\right)$, one has

$$
h_{\left(\mathcal{O}(1), \varphi_{0}\right)}\left(\left[a_{0}: \ldots: a_{r}\right]\right)=\int_{\Omega_{K^{\mathrm{ac}}}} \ln \left(\max \left\{\left|a_{0}\right|_{\chi}, \ldots,\left|a_{r}\right|_{\chi}\right\}\right) \nu_{K^{\mathrm{ac}}}(d \chi) .
$$

In particular, if $a_{0}, \ldots, a_{r}$ are all roots of the unity, then one has $h_{\left(\mathcal{O}(1), \varphi_{0}\right)}\left(\left[a_{0}: \ldots\right.\right.$ : $\left.\left.a_{r}\right]\right)=0$. This implies that the set of closed points in $X$ having non-positive height (with respect to $(L, \varphi)$ ) is Zariski dense. Therefore $\widehat{\mu}_{\text {ess }}(L, \varphi) \leqslant 0$. The proposition is thus proved.

6.2.3. Adelic divisors. - In this subsection we fix a geometrically integral projective scheme over $\operatorname{Spec} K$. If $D$ is a Cartier divisor on $X$, for any $\omega \in \Omega, D$ induces by base change a Cartier divisor on $X_{\omega}$, which we denote by $D_{\omega}$.

Let $D$ be a Cartier divisor on $X$. We call Green function family of $D$ any family $g=\left\{g_{\omega}\right\}_{\omega \in \Omega}$ parametrised by $\Omega$ such that each $g_{\omega}$ is a Green function of $D_{\omega}$ (cf. Subsection 2.5.1). Note that each Green function $g_{\omega}$ determines a continuous metric on the invertible sheaf $\mathcal{O}_{X_{\omega}}\left(D_{\omega}\right) \cong \mathcal{O}_{X}(D) \otimes_{\mathcal{O}_{X}} \mathcal{O}_{X_{\omega}}$, which we denote by $\varphi_{g_{\omega}}$. Thus the collection $\left\{\varphi_{g_{\omega}}\right\}_{\omega \in \Omega}$ forms a metric family on $\mathcal{O}_{X}(D)$ which we denote by $\varphi_{g}$ and called the metric family associated with $g$. We say that the Green function family $g$ is dominated (resp. measurable) if the associated metric family $\varphi_{g}$ is dominated (resp. measurable). In the case where $g$ is dominated and measurable, we say that the couple $(D, g)$ is an adelic Cartier divisor on $X$. Note that this condition is equivalent to the assertion that $\left(\mathcal{O}_{X}(D), \varphi_{g}\right)$ is an adelic line bundle on $X$. In this case we denote by $h_{(D, g)}$ the height function $h_{\left(\mathcal{O}_{X}(g), \varphi_{g}\right)}$ on $X\left(K^{\mathrm{ac}}\right)$.

Let $D$ and $D^{\prime}$ be Cartier divisors on $X, g=\left\{g_{\omega}\right\}_{\omega \in \Omega}$ and $g^{\prime}=\left\{g_{\omega}^{\prime}\right\}_{\omega \in \Omega}$ be Green function families of $D$ and $D^{\prime}$, respectively. We denote by $g+g^{\prime}$ the Green function 
family $\left\{g_{\omega}+g_{\omega}^{\prime}\right\}_{\omega \in \Omega}$ of $D+D^{\prime}$. Moreover, we denote by $-g$ the Green function family $\left\{-g_{\omega}\right\}_{\omega \in \Omega}$ of $-D$. Note that, if $(D, g)$ and $\left(D^{\prime}, g^{\prime}\right)$ are adelic Cartier divisors then $\left(D+D^{\prime}, g+g^{\prime}\right)$ and $(-D,-g)$ are also adelic Cartier divisors. This follows from Propositions 6.1.12 and 6.1.27. Therefore, the set of adelic Cartier divisors forms an abelian group, which we denote by $\widehat{\operatorname{Div}}(X)$.

Remark 6.2.8. - In the case where the Cartier divisor $D$ is trivial, a Green function family on $D$ can be considered as a family $\left\{g_{\omega}\right\}_{\omega \in \Omega}$ of continuous real-valued functions, where $g_{\omega}$ is a continuous function on $X_{\omega}^{\text {an }}$. It is dominated if and only if the function $(\omega \in \Omega) \mapsto \sup _{x \in X_{\omega}^{\text {an }}}\left|g_{\omega}\right|(x)$ is $\nu$-dominated. It is measurable if the following two conditions are satisfied (cf. Definition 6.1.26):

(a) for any closed point $P$ of $X$, the function $(\omega \in \Omega) \mapsto g_{\omega}(P)$ is $\mathcal{A}$-measurable,

(b) for any point $x \in X_{\leqslant 1, \mathbb{Q}}^{\mathrm{an}}$ (where we consider the trivial absolute value on $K$ in the construction of $\left.X^{\text {an }}\right)$, the function $\left(\omega \in \Omega_{0}\right) \mapsto g_{\omega}(x)$ is $\mathcal{A}_{0}$-measurable, where $\Omega_{0}$ is the set of $\omega \in \Omega$ such that $|\cdot|_{\omega}$ is trivial.

The set of all dominated and measurable Green function families on the trivial Cartier divisor forms actually a vector space over $\mathbb{R}$, which we denote by $\widehat{C}^{0}(X)$.

Definition 6.2.9. - Let $\mathbb{K}$ be either $\mathbb{Q}$ or $\mathbb{R}$. We denote by $\widehat{\operatorname{Div}}_{\mathbb{K}}(X)$ the $\mathbb{K}$-vector space $\widehat{\operatorname{Div}}(X) \otimes_{\mathbb{Z}} \mathbb{K}$ modulo the vector subspace generated by elements of the form

$$
\left(0, g_{1}\right) \otimes \lambda_{1}+\cdots+\left(0, g_{n}\right) \otimes \lambda_{n}-\left(0, \lambda_{1} g_{1}+\cdots+\lambda_{n} g_{n}\right),
$$

where $\left\{g_{i}\right\}_{i=1}^{n}$ is a finite family of elements in $\widehat{C}^{0}(X)$, and $\left(\lambda_{1}, \ldots, \lambda_{n}\right) \in \mathbb{K}^{n}$. In the other words, $\widehat{\operatorname{Div}}_{\mathbb{K}}(X)$ consists of pairs (see 2.5 .1 for the notation of $C_{\text {gen }}^{0}\left(X_{\omega}^{\mathrm{an}}\right)$ )

$$
\left(D,\left\{g_{\omega}\right\}_{\omega \in \Omega}\right) \in \operatorname{Div}_{\mathbb{K}}(X) \times \prod_{\omega \in \Omega} C_{\mathrm{gen}}^{0}\left(X_{\omega}^{\mathrm{an}}\right)
$$

such that $D=a_{1} D_{1}+\cdots+a_{n} D_{n}$ and $g_{\omega}=a_{1} g_{1, \omega}+\cdots+a_{n} g_{n, \omega}$ for some $\left(D_{1}, g_{1}\right), \ldots,\left(D_{n}, g_{n}\right) \in \widehat{\operatorname{Div}}(X)$ and $a_{1}, \ldots, a_{n} \in \mathbb{K}$. For $\lambda_{1}, \lambda_{2} \in \mathbb{K}$ and $\left(D_{1}, g_{1}\right),\left(D_{2}, g_{2}\right) \in \widehat{\operatorname{Div}}_{\mathbb{K}}(X), \lambda_{1}\left(D_{1}, g_{1}\right)+\lambda_{2}\left(D_{2}, g_{2}\right)$ is defined by $\left(\lambda_{1} D_{1}+\right.$ $\left.\lambda_{2} D_{2}, \lambda_{1} g_{1}+\lambda_{2} g_{2}\right)$. Note that $\lambda_{1}\left(D_{1}, g_{1}\right)+\lambda_{2}\left(D_{2}, g_{2}\right) \in \widehat{\operatorname{Div}}_{\mathbb{K}}(X)$. In this sense, $\widehat{\operatorname{Div}}_{\mathbb{K}}(X)$ forms a vector space over $\mathbb{K}$.

The elements in $\widehat{\operatorname{Div}}_{\mathbb{K}}(X)$ are called adelic $\mathbb{K}$-Cartier divisors on $X$. For any element $\bar{D}$ written in the form $\lambda_{1} \bar{D}_{1}+\cdots+\lambda_{n} \bar{D}_{n}$ with $\left(\bar{D}_{1}, \ldots, \bar{D}_{n}\right) \in \widehat{\operatorname{Div}}(X)$ and $\left(\lambda_{1}, \ldots, \lambda_{n}\right) \in \mathbb{K}^{n}$, we define a function $h_{\bar{D}}: X\left(K^{\text {ac }}\right) \rightarrow \mathbb{R}$ such that for any $P \in X\left(K^{\mathrm{ac}}\right)$,

$$
h_{\bar{D}}(P):=\sum_{i=1}^{n} \lambda_{i} h_{\bar{D}_{i}}(P) .
$$

Note that the Proposition 6.2.2 (1) shows that this map is actually well defined.

Remark 6.2.10. - Let $\bar{D}$ be an element of $\widehat{\operatorname{Div}}_{\mathbb{K}}(X)$, which is written in the form $\lambda_{1}\left(D_{1}, g_{1}\right)+\cdots+\lambda_{n}\left(D_{n}, g_{n}\right)$, where $\left(\lambda_{1}, \ldots, \lambda_{n}\right) \in \mathbb{K}^{n}$, and for any $i \in\{1, \ldots, n\}$, 
$\left(D_{i}, g_{i}\right)$ is an element of $\widehat{\operatorname{Div}}(X)$. Then, for any $\omega \in \Omega$, the element $\lambda_{1} D_{1, \omega}+\cdots+$ $\lambda_{n} D_{n, \omega}$ of $\operatorname{Div}_{\mathbb{K}}(X)$ is equal to $D_{\omega}$, where $D=\lambda_{1} D_{1}+\cdots+\lambda_{n} D_{n} \in \operatorname{Div}_{\mathbb{K}}(X)$. Moreover, assume that $g_{i}$ is written in the form $\left\{g_{i, \omega}\right\}_{\omega \in \Omega}$, where $g_{i, \omega}$ is a Green function of $D_{i, \omega}$. Then for any $\omega \in \Omega$, the element $\lambda_{1} g_{1, \omega}+\cdots+\lambda_{n} g_{n, \omega}$ is a Green function of the $\mathbb{K}$-Cartier divisor $D_{\omega}$, which does not depend on the choice of the decomposition $\bar{D}=\lambda_{1}\left(D_{1}, g_{1}\right)+\cdots+\lambda_{n}\left(D_{n}, g_{n}\right)$. Thus we can write $\bar{D}$ in the form $(D, g)$, where $D$ is a $\mathbb{K}$-Cartier divisor of $X$ and $g$ is a family of Green functions of the form $\left\{g_{\omega}\right\}_{\omega \in \Omega}$, with $g_{\omega}$ being a Green function of $D_{\omega}$. Note that the measurability of the Green function families $g_{1}, \ldots, g_{n}$ implies the following statements:

(a) for any closed point $P$ of $X$ outside of the support of $D$, the function $(\omega \in \Omega) \mapsto$ $g_{\omega}(P)$ is well defined and is $\mathcal{A}$-measurable,

(b) for any point $x \in X_{\leqslant 1, \mathbb{Q}}^{\text {an }}$ outside of the analytification of the support of $D$, the function $\left(\omega \in \Omega_{0}\right) \mapsto g_{\omega}(x)$ is well defined and is $\mathcal{A}_{0}$-measurable.

Moreover, if $D$ belongs to $\operatorname{Div}(X)$, then $g$ is a dominated Green function family of $D$. This statement results directly from the following proposition.

Example 6.2.11. - Let $s$ be a non-zero rational function on $X$. For any $\omega \in \Omega$, we consider $s$ as a non-zero rational function on $X_{\omega}$. Note that $-\ln |s|_{\omega}$ is a Green function of the principal Cartier divisor $\operatorname{div}(s)$. Note that the Green function family $\left\{-\ln |s|_{\omega}\right\}_{\omega \in \Omega}$ is measurable and dominated since the corresponding metric family on $\mathcal{O}_{X}(\operatorname{div}(f)) \cong \mathcal{O}_{X}$ is trivial. Thus

$$
\left(s \in K(X)^{\times}\right) \longmapsto \widehat{\operatorname{div}}(s):=\left(\operatorname{div}(s),\left\{-\ln |s|_{\omega}\right\}_{\omega \in \Omega}\right)
$$

defines a morphism of groups from $\left(K(X)^{\times}, \times\right)$to $\widehat{\operatorname{Div}}(X)$. The adelic Cartier divisors belonging to the image of this morphism are called principal adelic Cartier divisors. Moreover, for $\mathbb{K} \in\{\mathbb{Q}, \mathbb{R}\}$ this morphism induces a $\mathbb{K}$-linear map $\widehat{\operatorname{div}}_{\mathbb{K}}: K(X)^{\times} \otimes_{\mathbb{Z}}$ $\mathbb{K} \rightarrow \widehat{\operatorname{Div}}_{\mathbb{K}}(X)$ sending to $s_{1}^{\lambda_{1}} \cdots s_{n}^{\lambda_{n}}$ to $\lambda_{1} \widehat{\operatorname{div}}\left(s_{1}\right)+\cdots+\lambda_{n} \widehat{\operatorname{div}}\left(s_{n}\right)$. The adelic $\mathbb{K}-$ Cartier divisors belonging to the image of this $\mathbb{K}$-linear map are said to be principal.

Let $(D, g)$ be an adelic $\mathbb{K}$-Cartier divisor on $S$. For $\phi \in H_{\mathbb{K}}^{0}(X, D),|\phi|_{\omega} \exp \left(-g_{\omega}\right)$ extends to a continuous function on $X_{\omega}^{\text {an }}$ by Proposition 2.5.8. We denote by

$$
\|\phi\|_{g_{\omega}}:=\sup _{x \in X_{\omega}^{\text {an }}}\left\{\left(|\phi|_{\omega} \exp \left(-g_{\omega}\right)\right)(x)\right\} .
$$

Proposition 6.2.12. - We assume that, either the $\sigma$-algebra $\mathcal{A}$ is discrete, or the field $K$ admits a countable subfield which is dense in every $K_{\omega}, \omega \in \Omega$. Let $(D, g)$ be an adelic $\mathbb{K}$-Cartier divisor on $S$ and $\phi \in H_{\mathbb{K}}^{0}(X, D)$. The function on $\Omega$ given by

$$
(\omega \in \Omega) \mapsto \ln \|\phi\|_{g_{\omega}}=\sup _{x \in X_{\omega}^{\mathrm{an}}}\left\{\left(-g_{\omega}+\log |\phi|_{\omega}\right)(x)\right\}
$$

is $\nu$-integrable.

Proof. - Note that $D^{\prime}:=D+(\phi) \geqslant_{\mathbb{K}} 0, g_{\omega}^{\prime}:=g_{\omega}-\log |\phi|_{\omega}$ is a Green function of $D_{\omega}^{\prime}$ and $|\phi|_{g_{\omega}}=|1|_{g_{\omega}^{\prime}}$ on $X_{\omega}^{\text {an }}$, so that we may assume that $D$ is $\mathbb{K}$-effective and $\phi=1$. 
Let $X^{\prime}$ be the normalisation of $X$. Since $X$ and $X^{\prime}$ have the same function field, $X^{\prime}$ is also geometrically integral over $K$. Moreover, let $D^{\prime}$ (resp. $g_{\omega}^{\prime}$ ) be the pull-back of $D$ by $X^{\prime} \rightarrow X$ (resp. $X_{\omega}^{\prime} \rightarrow X_{\omega}$ ). Then $g^{\prime}=\left\{g_{\omega}^{\prime}\right\}_{\omega \in \Omega}$ is a family of Green functions of $D^{\prime}$ over $S$. Note that $\|1\|_{g_{\omega}}=\|1\|_{g_{\omega}^{\prime}}$, so that we may further assume that $X$ is normal.

First we consider the case $\mathbb{K}=\mathbb{Q}$. Then there is a positive integer $N$ such that $N D$ is a Cartier divisor. Then $\phi^{N} \in H^{0}(X, N D)$ and $\omega \mapsto \ln \left\|\phi^{N}\right\|_{N g_{\omega}}$ is integrable on $\Omega$ by Theorem 6.1.13 and Theorem 6.1.31. Note that $\ln \left\|\phi^{N}\right\|_{N g_{\omega}}=N \ln \|\phi\|_{g_{\omega}}$, so that $\omega \mapsto \ln \|\phi\|_{g_{\omega}}$ is also integrable on $\Omega$.

Next we consider the case $\mathbb{K}=\mathbb{R}$. By Proposition 2.4.16, there are effective Cartier divisors $D_{1}, \ldots, D_{r}$ and $a_{1}, \ldots, a_{r} \in \mathbb{R}_{\geqslant 0}$ such that $D=a_{1} D_{1}+\cdots+a_{r} D_{r}$. We choose a family of Green functions $g_{i}=\left\{g_{i, \omega}\right\}_{\omega \in \Omega}$ of $D_{i}$ over $S$ such that $\left(D_{i}, g_{i}\right)$ is an adelic Cartier divisor over $S$ for each $i$ and

$$
(D, g)=\left(a_{1} D_{1}+\cdots+a_{r} D_{r}, a_{1} g_{1}+\cdots+a_{r} g_{r}\right)
$$

If we set $\psi_{i}(\omega)=\ln \|1\|_{g_{i, \omega}}$ and $g_{i, \omega}^{\prime}:=g_{i, \omega}+\psi_{i}(\omega)$ for $i=1, \ldots, r$, then $\psi_{i}$ is integrable on $\Omega$ and

$$
\|1\|_{g_{i, \omega}^{\prime}}=\|1\|_{g_{i, \omega}} \exp \left(-\psi_{i}(\omega)\right)=1,
$$

so that $g_{i, \omega}^{\prime} \geqslant 0$ for all $i$ and $\omega$. Note that if we set $g^{\prime}=a_{1} g_{1}^{\prime}+\cdots+a_{n} g_{n}^{\prime}$, then

$$
\ln \|1\|_{g^{\prime}, \omega}=\ln \|1\|_{g, \omega}-\left(a_{1} \psi_{1}(\omega)+\cdots+a_{n} \psi_{n}(\omega)\right) .
$$

Therefore, we may assume that $g_{i, \omega} \geqslant 0$ for all $i$ and $\omega$.

For each $i$, we choose a sequence $\left\{a_{i, n}\right\}_{n=1}^{\infty}$ of non-negative rational numbers such that

for all $n$. We set

$$
0 \leqslant a_{i, n}-a_{i} \leqslant \frac{a_{i}}{n} \quad \text { and } \quad a_{i, n+1} \leqslant a_{i, n}
$$

$$
\left(D_{n}, h_{n}\right):=\left(a_{1, n} D_{1}+\cdots+a_{r, n} D_{r}, a_{1, n} g_{1}+\cdots+a_{r, n} g_{r}\right) .
$$

Then $D_{n}$ is effective and

$$
-h_{n, \omega} \leqslant-g \leqslant \frac{n}{n+1}\left(-h_{n, \omega}\right) \leqslant 0 \text { and } \quad-h_{n, \omega} \leqslant-h_{n+1, \omega}
$$

for all $n$ and $\omega$. If we set

$$
A(\omega)=\sup _{x \in X_{\omega}^{\text {an }}}\left\{-g_{\omega}(x)\right\} \text { and } A_{n}(\omega)=\sup _{x \in X_{\omega}^{\text {an }}}\left\{-h_{n, \omega}(x)\right\}
$$

then

$$
A_{n}(\omega) \leqslant A(\omega) \leqslant \frac{n}{n+1} A_{n}(\omega) \leqslant 0 \quad \text { and } \quad A_{n}(\omega) \leqslant A_{n+1}(\omega)
$$

for all $n$ and $\omega$. Thus $\lim _{n \rightarrow \infty} A_{n}(\omega)=A(\omega)$ and $A_{n}(\omega) \leqslant A(\omega) \leqslant 0$. Note that $\omega \mapsto A_{n}(\omega)$ is integrable for all $n$. Therefore, by monotone convergence theorem, $A(\omega)$ is integrable. 
Corollary 6.2.13. - We keep the hypothesis of Proposition 6.2.12. Let $(D, g)$ be an adelic $\mathbb{K}$-Cartier divisor on $X$. Let $\phi \in K(X)^{\times} \otimes_{\mathbb{Z}} \mathbb{K}$ such that $D+(\phi) \geqslant_{\mathbb{K}} 0$. Then the function

$$
(\omega \in \Omega) \longmapsto \ln \|\phi\|_{g_{\omega}}=\sup _{x \in X_{\omega}^{\text {an }}}\left\{\left(-g_{\omega}+\log |\phi|_{\omega}\right)(x)\right\}
$$

is $\nu$-integrable.

Proof. - If we set $D^{\prime}=(D)+(\phi)$ and $g_{\omega}^{\prime}=g_{\omega}-\ln |\phi|_{\omega}$, then $\left(D^{\prime}, g^{\prime}=\left\{g_{\omega}^{\prime}\right\}_{\omega \in \Omega}\right)$ is an adelic $\mathbb{K}$-Cartier divisor on $X$. Thus the assertion follows from Proposition 6.2.12.

Corollary 6.2.14. - We keep the hypothesis of Proposition 6.2.12, Let $(\mathbf{0}, g)$ be an adelic $\mathbb{K}$-Cartier divisor on $X$ whose underlying $\mathbb{K}$-Cartier divisor is trivial. Assume that $g$ is written in the form $\left\{g_{\omega}\right\}_{\omega \in \Omega}$, where $g_{\omega}$ is considered as a continuous function on $X_{\omega}^{\text {an }}$. Then the function

$$
(\omega \in \Omega) \longmapsto \sup _{x \in X_{\omega}^{\text {an }}}\left|g_{\omega}(x)\right|
$$

is $\nu$-integrable.

For any $\bar{D} \in \widehat{\operatorname{Div}}_{\mathbb{K}}(X)$, we define the essential minimum of $\bar{D}$ as

$$
\widehat{\mu}_{\text {ess }}(\bar{D}):=\sup _{Z \subsetneq X} \inf _{P \in(X \backslash Z)\left(K^{\text {ac }}\right)} h \bar{D}(P),
$$

where $Z$ runs over the set of all strict Zariski closed subsets of $X$, and $P$ runs over the set of closed points of the open subscheme $X \backslash Z$ of $X$. It turns out that the analogue of Proposition 6.2.4 and Proposition 6.2.5 (1) holds for adelic $\mathbb{K}$-Cartier divisors (with essentially the same proof). We resume these statements as follows.

Proposition 6.2.15. - Let $\bar{D}$ be an adelic $\mathbb{K}$-Cartier divisor on $X$. Then $\widehat{\mu}(\bar{D})$ identifies with the infimum of the set of real numbers $C$ such that $\left\{P \in X\left(K^{\text {ac }}\right)\right.$ : $\left.h_{\bar{D}}(P) \leqslant C\right\}$ is Zariski dense in $X$. Moreover, if $\bar{D}_{1}$ and $\bar{D}_{2}$ are adelic $\mathbb{K}$-Cartier divisors on $X$, then $\widehat{\mu}_{\mathrm{ess}}\left(\bar{D}_{1}+\bar{D}_{2}\right) \geqslant \widehat{\mu}_{\mathrm{ess}}\left(\bar{D}_{1}\right)+\widehat{\mu}_{\mathrm{ess}}\left(\bar{D}_{2}\right)$.

Similarly as in the case of adelic line bundles, the essential minimum of adelic $\mathbb{K}$-Cartier divisors never takes the value of $+\infty$.

Proposition 6.2.16. - Let $\bar{D}$ be an adelic $\mathbb{K}$-Cartier divisor on $X$. One has $\widehat{\mu}_{\text {ess }}(\bar{D})<+\infty$.

Proof. - Assume that $\bar{D}$ is written in the form $\bar{D}=\lambda_{1} \bar{D}_{1}+\cdots+\lambda_{n} \bar{D}_{n}$, where $D_{1}, \ldots, D_{n}$ are very ample Cartier divisors on $X$ and $\left(\lambda_{1}, \ldots, \lambda_{n}\right) \in \mathbb{K}^{n}$. By Proposition 6.2.6. for any $i \in\{1, \ldots, n\}$ one has $\widehat{\mu}_{\text {ess }}\left(\bar{D}_{i}\right)>-\infty$. We choose $\left(\lambda_{1}^{\prime}, \ldots, \lambda_{n}^{\prime}\right) \in$ $\left(\mathbb{K} \cap \mathbb{R}_{>0}\right)^{n}$ such that $\lambda_{i}+\mu_{i} \in \mathbb{Z}$ for any $i \in\{1, \ldots, n\}$. Let

$$
\bar{E}:=\sum_{i=1}^{n}\left(\lambda_{i}+\lambda_{i}^{\prime}\right) \bar{D}_{i}
$$


By Proposition 6.2.7, one has $\widehat{\mu}_{\text {ess }}(\bar{E})<+\infty$. Moreover, by Proposition 6.2.15, one has

$$
\widehat{\mu}_{\mathrm{ess}}(\bar{E}) \geqslant \widehat{\mu}_{\mathrm{ess}}(\bar{D})+\sum_{i=1}^{n} \lambda_{i}^{\prime} \widehat{\mu}_{\mathrm{ess}}\left(\bar{D}_{i}\right) .
$$

Since $\widehat{\mu}_{\mathrm{ess}}\left(\bar{D}_{i}\right)>-\infty$ and $\lambda_{i}^{\prime}>0$ for any $i \in\{1, \ldots, n\}$, we deduce that $\widehat{\mu}_{\mathrm{ess}}(\bar{D})<$ $+\infty$.

Definition 6.2.1\%. - We assume that $X$ is normal. Let $\bar{D}=(D, g)$ be an adelic $\mathbb{K}$-Cartier divisor, where the Green function family $g$ is written in the form $\left\{g_{\omega}\right\}_{\omega \in \Omega}$. For $\omega \in \Omega$, let $X_{\omega}^{\prime}$ be the normalization of $X_{\omega}$ and $D_{\omega}^{\prime}$ (resp. $g_{\omega}^{\prime}$ ) be the pull-back of $D$ by $X_{\omega}^{\prime} \rightarrow X_{\omega}$ (resp. the pull-back of $g_{\omega}$ by $X_{\omega}^{\prime a n} \rightarrow X_{\omega}^{\text {an }}$ ). By using the natural injective homomorphism $H_{\mathbb{K}}^{0}(X, D) \otimes_{K} K_{\omega} \rightarrow H_{\mathbb{K}}^{0}\left(X_{\omega}^{\prime}, D_{\omega}^{\prime}\right)$ and $g_{\omega}^{\prime}$, one has a norm $\|\cdot\|_{g_{\omega}}$ on $H_{\mathbb{K}}^{0}(X, D) \otimes_{K} K_{\omega}$ (cf. Definition 2.5.9). The norm family $\left\{\|\cdot\|_{g_{\omega}}\right\}_{\omega \in \Omega}$ is denoted by $\xi_{g}$.

Theorem 6.2.18. - We assume that, either the $\sigma$-algebra $\mathcal{A}$ is discrete, or the field $K$ admits a countable subfield which is dense in every $K_{\omega}, \omega \in \Omega$. Suppose that $X$ is normal. Then the couple $\left(H_{\mathbb{K}}^{0}(D), \xi_{g}\right)$ is a strictly adelic vector bundle on $S$.

Proof. - The measurability of $\xi_{g}$ is a consequence of Proposition 6.2.12 Let us consider the dominancy of $\xi_{g}$. By using [105, Lemma 5.2.3], $\bar{D}$ is written in the form

$$
\lambda_{1}\left(D_{1}, g_{1}\right)+\cdots+\lambda_{n}\left(D_{n}, g_{n}\right),
$$

where $\left(D_{i}, g_{i}\right)$ 's are elements of $\widehat{\operatorname{Div}}(X)$ such that $D_{1}, \ldots, D_{n}$ are effective, and $\left(\lambda_{1}, \ldots, \lambda_{n}\right) \in \mathbb{K}^{n}$. Let $\left(\lambda_{1}^{\prime}, \ldots, \lambda_{n}^{\prime}\right)$ be an element of $\left(\mathbb{K} \cap \mathbb{R}_{>0}\right)^{n}$ such that $\lambda_{i}+\lambda_{i}^{\prime} \in$ $\mathbb{Z}_{>0}$ for any $i \in\{1, \ldots, n\}$. Let

$$
\left(D^{\prime}, g^{\prime}\right):=\left(\lambda_{1}+\lambda_{1}^{\prime}\right)\left(D_{1}, g_{1}\right)+\cdots+\left(\lambda_{n}+\lambda_{n}^{\prime}\right)\left(D_{n}, g_{n}\right)
$$

which is viewed as an adelic Cartier divisor on $X$. Since $D_{i}$ is effective, we obtain that 1 belongs to $H^{0}\left(D_{i}\right)$. Moreover, by Proposition 6.2 .12 , the function $(\omega \in \Omega) \mapsto$ $\ln \|1\|_{g_{i, \omega}}$ is $\nu$-integrable.

Let $\boldsymbol{e}=\left\{e_{i}\right\}_{i=1}^{m}$ be a basis of $H_{\mathbb{K}}^{0}(D)$. We complete it into a basis $\boldsymbol{e}^{\prime}=\left\{e_{i}\right\}_{i=1}^{r}$ of $H_{\mathbb{K}}^{0}\left(D^{\prime}\right)$. By Theorem 6.1.13, the norm family $\xi_{g^{\prime}}:=\left\{\|\cdot\|_{g_{\omega}^{\prime}}\right\}_{\omega \in \Omega}$ is strongly dominated, so that, by Corollary 4.1.10, the local distance function $(\omega \in \Omega) \mapsto d_{\omega}\left(\xi_{g^{\prime}}, \xi_{\boldsymbol{e}^{\prime}}\right)$ is $\nu$-dominated. Further, by Proposition 6.2.12, the function $(\omega \in \Omega) \mapsto \ln \left\|e_{i}\right\|_{g_{\omega}}$ is $\nu$-integrable for each $i$.

Let $\omega \in \Omega$ and $\left(a_{1}, \ldots, a_{m}\right) \in K_{\omega}^{m}$, one has

$$
\begin{aligned}
\ln \| a_{1} e_{1}+\cdots & +a_{m} e_{m} \|_{g_{\omega}} \leqslant \max _{i \in\{1, \ldots, m\}}\left\{\ln \left|a_{i}\right|+\ln \left\|e_{i}\right\|_{g_{\omega}}\right\}+\mathbb{1}_{\Omega_{\infty}}(\omega) \ln (m) \\
& \leqslant \ln \left\|a_{1} e_{1}+\cdots+a_{m} e_{m}\right\|_{\xi_{e}}+\max _{i \in\{1, \ldots, m\}}\left\{\ln \left\|e_{i}\right\|_{g_{\omega}}\right\}+\mathbb{1}_{\Omega_{\infty}}(\omega) \ln (m) .
\end{aligned}
$$


Moreover,

$$
\begin{aligned}
\ln \left\|a_{1} e_{1}+\cdots+a_{m} e_{m}\right\|_{g_{\omega}} \geqslant \ln \left\|a_{1} e_{1}+\cdots+a_{m} e_{m}\right\|_{g_{\omega}^{\prime}}-\sum_{i=1}^{n} \lambda_{i}^{\prime} \ln \|1\|_{g_{i, \omega}} \\
\geqslant \ln \left\|a_{1} e_{1}+\cdots+a_{m} e_{m}\right\|_{\xi_{e}}-d_{\omega}\left(\xi_{g^{\prime}}, \xi_{e^{\prime}}\right)-\sum_{i=1}^{n} \lambda_{i}^{\prime} \ln \|1\|_{g_{i, \omega}},
\end{aligned}
$$

and hence one obtains

$$
\begin{aligned}
& d_{\omega}\left(\xi_{g}, \xi_{e}\right) \\
\leqslant & \max \left\{\max _{i \in\{1, \ldots, m\}}\left\{\left|\ln \left\|e_{i}\right\|_{g_{\omega}}\right|\right\}+\mathbb{1}_{\Omega_{\infty}}(\omega) \ln (m), d_{\omega}\left(\xi_{g^{\prime}}, \xi_{e^{\prime}}\right)+\sum_{i=1}^{n} \lambda_{i}^{\prime}\left|\ln \|1\|_{g_{i, \omega}}\right|\right\}
\end{aligned}
$$

Therefore the local distance function $(\omega \in \Omega) \mapsto d_{\omega}\left(\xi_{g}, \xi_{e}\right)$ is $\nu$-dominated, which implies that the norm family $\xi_{g}$ is strongly dominated (cf. Corollary 4.1.10).

\subsection{Okounkov bodies and concave transform}

6.3.1. Reminder on some facts about convex sets. - In this subsection, we recall some basic facts about convex sets in finite-dimensional vector spaces, which will be used in the subsequening subsections.

Proposition 6.3.1. - Let $V$ be a finite-dimensional vector space over $\mathbb{R}$. Suppose that $C_{1}$ and $C_{2}$ are two convex subsets of $V$ which have the same closure in $V$, then the interiors $C_{1}^{\circ}$ and $C_{2}^{\circ}$ are also the same.

Proof. - It suffices to prove that, if $C$ is a convex subset of $V$, then the interior of the closure $\bar{C}$ coincides with the interior $C^{\circ}$ of $C$. Let $x$ be an element of $V$. If $x$ does not lie in $C^{\circ}$, then (by Hahn-Banach theorem, see [123. Theorem 3.4]) there exists an affine function $q$ on $V$ such that the restriction of $q$ on $C^{\circ}$ is non-negative but $q(x) \leqslant 0$. Since the set $\{y \in V: q(y) \geqslant 0\}$ is closed, it contains $\bar{C}$. Moreover, the interior of this set is $\{y \in V: q(y)>0\}$, which contains $(\bar{C})^{\circ}$. Hence $x$ cannot lie in $(\bar{C})^{\circ}$. Therefore one has $C^{\circ} \supseteq(\bar{C})^{\circ}$, which actually implies the equality of these two sets.

Proposition 6.3.2. - Let $V$ be a finite-dimensional vector space over $\mathbb{R}$ and $\left(C_{i}\right)_{i \in I}$ be a family of convex subsets of $W$. Suppose that the family $\left(C_{i}\right)_{i \in I}$ is filtered, namely, for any couple $\left(i_{1}, i_{2}\right)$ of indices in $I$, there exists $j \in I$ such that $C_{i_{1}} \cup C_{i_{2}} \subseteq C_{j}$. Let $C$ be the union of $C_{i}, i \in I$. Then the interior of $C$ identifies with the union of $C_{i}^{\circ}$, $i \in I$.

Proof. - Since the family $\left(C_{i}\right)_{i \in I}$ is filtered, for any couple of points $(x, y)$ in $C$, there exists an index $i \in I$ such that $\{x, y\} \subseteq C_{i}$. Therefore $C$ is a convex subset of $V$. As a consequence, for any point $x$ of the interior $C^{\circ}$, there exists points $x_{1}, \ldots, x_{n}$ 
in $C$ such that the point $x$ is contained in the interior of the convex hull of $x_{1}, \ldots, x_{n}$. Still by the assumption that the family $\left(C_{i}\right)_{i \in I}$ is filtered, there exists $j \in I$ such that $\left\{x_{1}, \ldots, x_{n}\right\} \subseteq C_{j}$. Hence one has $x \in C_{j}^{\circ}$.

6.3.2. Graded semigroups. — Let $V$ be a finite-dimensional vector space over $\mathbb{R}$. By graded semigroup in $V$ we refer to a non-empty subset $\Gamma$ of $\mathbb{N}_{\geqslant 1} \times V$ which is stable by addition. If $\Gamma$ is a graded semigroup in $V$, for any $n \in \mathbb{N}_{\geqslant 1}$ we denote by $\Gamma_{n}$ the projection of $\Gamma \cap(\{n\} \times V)$ in $V$. Let $\mathbb{N}(\Gamma)$ be the set of all $n \in \mathbb{N}_{\geqslant 1}$ such that $\Gamma_{n}$ is non-empty. This is a non-empty sub-semigroup of $\mathbb{N}_{\geqslant 1}$. We denote by $\mathbb{Z}(\Gamma)$ the subgroup of $\mathbb{Z}$ generated by $\mathbb{N}(\Gamma)$.

Proposition 6.3.3. — Let $\Gamma$ be a graded semigroup in $V$. Then there exist at most finitely many positive elements of $\mathbb{Z}(\Gamma) \backslash \mathbb{N}(\Gamma)$.

Proof. - The group $\mathbb{Z}(\Gamma)$ is non-zero since $\Gamma$ is not empty. Hence there exists a positive integer $m$ such that $\mathbb{Z}(\Gamma)=m \mathbb{Z}$. Assume that $m$ is written in the form

$$
m=a_{1} n_{1}+\cdots+a_{\ell} n_{\ell},
$$

where $n_{1}, \ldots, n_{\ell}$ are elements in $\mathbb{N}(\Gamma)$ and $a_{1}, \ldots, a_{\ell}$ are integers. Since $\mathbb{N}(\Gamma) \subseteq \mathbb{Z}(\Gamma)$, there exists a positive integer $N$ such that $n_{1}+\cdots+n_{\ell}=m N$. Let

$$
b=N \cdot \max _{i \in\{1, \ldots, \ell\}}\left|a_{i}\right| \text {. }
$$

We claim that $m n \in \mathbb{N}(\Gamma)$ for any $n \geqslant N b$. In fact, we can write such $n$ in the form $n=c N+r$ where $c \in \mathbb{N}_{\geqslant b}$ and $r \in\{0, \ldots, N-1\}$. Thus

$m n=c m N+m r=c\left(n_{1}+\cdots+n_{\ell}\right)+r\left(a_{1} n_{1}+\cdots+a_{\ell} n_{\ell}\right)=\left(c+r a_{1}\right) n_{1}+\cdots+\left(c+r a_{\ell}\right) n_{\ell}$.

Since $c \geqslant b$ and $r<N$, we obtain that $c+r a_{i} \geqslant 0$ for any $i \in\{1, \ldots, \ell\}$. Hence $m n \in \mathbb{N}(\Gamma)$.

Definition 6.3.4. - Let $\Gamma$ be a graded semigroup in $V$. We denote by $\Delta(\Gamma)$ the closure of the set

$$
\bigcup_{n \in \mathbb{N}, n \geqslant 1}\left\{n^{-1} \alpha: \alpha \in \Gamma_{n}\right\} \subset V .
$$

Proposition 6.3.5. - Let $\Gamma$ be a graded semigroup in $V$. The set $\Delta(\Gamma)$ is a closed convex subset of $V$.

Proof. - It suffices to prove the convexity of the set $\Delta(\Gamma)$. Observe that, if $n$ and $m$ are two positive integers, $\alpha$ and $\beta$ are elements of $\Gamma_{n}$ and $\Gamma_{m}$, respectively. We show that, for any $\epsilon \in[0,1] \cap \mathbb{Q}$, one has $\epsilon n^{-1} \alpha+(1-\epsilon) m^{-1} \beta \in \Delta(\Gamma)$. Let $\epsilon=p / q$ be a rational number in $[0,1]$, where $q \in \mathbb{N}_{\geqslant 1}$. One has

$$
\epsilon n^{-1} \alpha+(1-\epsilon) m^{-1} \beta=\frac{p}{q n} \alpha+\frac{q-p}{q m} \beta=(q m n)^{-1}(p m \alpha+(q-p) n \beta) .
$$


Since $\alpha \in \Gamma_{n}$ and $\beta \in \Gamma_{m}$, one has $p m \alpha+(q-p) n \beta \in \Gamma_{q m n}$. Therefore

$$
\epsilon n^{-1} \alpha+(1-\epsilon) m^{-1} \beta \in \Delta(\Gamma) .
$$

Let $H$ be the set

$$
\bigcup_{n \in \mathbb{N}, n \geqslant 1}\left\{n^{-1} \alpha: \alpha \in \Gamma_{n}\right\} .
$$

Let $x$ and $y$ be two points in $\Delta(\Gamma)$, and $\epsilon \in[0,1]$. By definition, there exists two sequences $\left\{x_{n}\right\}_{n \in \mathbb{N}}$ and $\left\{y_{n}\right\}_{n \in \mathbb{N}}$ in $H$ such that

$$
\lim _{n \rightarrow+\infty} x_{n}=x, \quad \lim _{n \rightarrow+\infty} y_{n}=y .
$$

Let $\left\{\epsilon_{n}\right\}_{n \in \mathbb{N}}$ be a sequence in $[0,1] \cap \mathbb{Q}$ which converges to $\epsilon$. By what we have shown above, for any $n \in \mathbb{N}$ one has $\epsilon_{n} x_{n}+\left(1-\epsilon_{n}\right) y_{n} \in H$. Moreover, one has

$$
\lim _{n \rightarrow+\infty} \epsilon_{n} x_{n}+\left(1-\epsilon_{n}\right) y_{n}=\epsilon x+(1-\epsilon) y .
$$

Therefore $\epsilon x+(1-\epsilon) y \in \Delta(\Gamma)$.

Let $\Gamma$ be a graded semigroup in $V$. We denote by $\Gamma_{\mathbb{R}}$ the $\mathbb{R}$-vector subspace of $\mathbb{R} \times V$ generated by $\Gamma$. For $n \in \mathbb{Z}$, let $A(\Gamma)_{n}$ be the projection of $\Gamma_{\mathbb{R}} \cap(\{n\} \times V)$ to $V$. Especially, $A(\Gamma)_{1}$ is denoted by $A(\Gamma)$. Note that $A(\Gamma)_{0}$ is a vector subspace of $V$, which is a translation of the affine subspace $A(\Gamma)$. Since $A(\Gamma)_{n}$ is the image of an affine subspace of $\mathbb{R} \times V$ by a linear map, it is an affine subspace in $V$. Note that any element in $A(\Gamma)=A(\Gamma)_{1}$ can be written in the form

$$
\lambda_{1} \gamma_{1}+\cdots+\lambda_{\ell} \gamma_{\ell}
$$

where for $i \in\{1, \ldots, \ell\}, \gamma_{i} \in \Gamma_{n_{i}}, n_{i} \in \mathbb{N}, n_{i} \geqslant 1$, and $\left(\lambda_{1}, \ldots, \lambda_{\ell}\right)$ is an element in $\mathbb{R}^{\ell}$ such that $\lambda_{1} n_{1}+\cdots+\lambda_{\ell} n_{\ell}=1$. We denote by $\Gamma_{\mathbb{Z}}$ the subgroup of $\mathbb{R} \times V$ generated by $\Gamma$. For any $n \in \mathbb{Z}, n \geqslant 1$, let $\Gamma_{\mathbb{Z}, n}$ be the image of $\Gamma_{\mathbb{Z}} \cap(\{n\} \times V)$ in $V$ by the canonical projection. Note that $\Gamma_{\mathbb{Z}, n}$ is non-empty if and only if $n \in \mathbb{Z}(\Gamma)$.

Proposition 6.3.6. - Let $\Gamma$ be a graded semigroup in $V$. We assume that $\Gamma_{\mathbb{Z}}$ is a discrete subset of $\mathbb{R} \times V$.

(1) The set $\Gamma_{\mathbb{Z}, 0}$ is a lattice in $A(\Gamma)_{0}$.

(2) For any $n, n^{\prime} \in \mathbb{Z}(\Gamma)$ and any $\gamma_{0} \in \Gamma_{\mathbb{Z}, n}$, the map from $\Gamma_{\mathbb{Z}, n^{\prime}}$ to $\Gamma_{\mathbb{Z}, n+n^{\prime}}$, sending $\gamma \in \Gamma_{\mathbb{Z}, n^{\prime}}$ to $\gamma+\gamma_{0}$, is a bijection.

(3) For any convex and compact subset $K$ of $A(\Gamma)$ which is contained in the relative interior of $\Delta(\Gamma)$, one has

$$
K \cap\left\{n^{-1} \gamma: \gamma \in \Gamma_{n}\right\}=K \cap\left\{n^{-1} \gamma: \gamma \in \Gamma_{\mathbb{Z}, n}\right\}
$$

for sufficiently positive $n \in \mathbb{N}(\Gamma)$. 
Proof. - (1) Let $n$ be an element in $\mathbb{Z}(\Gamma)$ and $\gamma_{0} \in \Gamma_{\mathbb{Z}, n}$. By definition, an element $x \in V$ lies in $A(\Gamma)_{0}$ if and only if $x+n^{-1} \gamma_{0} \in A(\Gamma)$. In other words, $A(\Gamma)_{0}$ is precisely the vector subspace of $V$ of all vectors $\gamma$ which can be written in the form

$$
\gamma=\lambda_{1} \gamma_{1}+\cdots+\lambda_{\ell} \gamma_{\ell}
$$

where for any $i \in\{1, \ldots, \ell\}, \gamma_{i} \in \Gamma_{n_{i}}$ with $n_{i} \in \mathbb{N}(\Gamma)$, and $\left(\lambda_{1}, \ldots, \lambda_{\ell}\right)$ is an element in $\mathbb{R}^{\ell}$ such that $\lambda_{1} n_{1}+\cdots+\lambda_{\ell} n_{\ell}=0$. Note that the set $\Gamma_{\mathbb{Z}, 0}$ is characterized by the same condition, except that $\left(\lambda_{1}, \ldots, \lambda_{\ell}\right)$ is required to be in $\mathbb{Z}^{\ell}$ Therefore $\Gamma_{\mathbb{Z}, 0}$ is a subset (and hence a subgroup) of $A(\Gamma)_{0}$. Moreover, we can also rewrite (6.8) as

$$
\gamma=\frac{\lambda_{1}}{n}\left(n \gamma_{1}-n_{1} \gamma_{0}\right)+\cdots+\frac{\lambda_{\ell}}{n}\left(n \gamma_{\ell}-n_{\ell} \gamma_{0}\right) .
$$

Since $n \gamma_{i}-n_{i} \gamma_{0}$ belongs to $\Gamma_{\mathbb{Z}, 0}$ for $i \in\{1, \ldots, \ell\}$, we obtain that $A(\Gamma)_{0}$ is generated by $\Gamma_{\mathbb{Z}, 0}$ as a vector space over $\mathbb{R}$. Moreover, since $\Gamma_{\mathbb{Z}}$ is a discrete subspace of $\mathbb{R} \times V$, the set $\Gamma_{\mathbb{Z}, 0} \subseteq V$ is also discrete. Hence it forms a lattice in $A(\Gamma)_{0}$.

(2) This comes from the definition of $\Gamma_{\mathbb{Z}}$. In particular, the inverse map is given by $\left(\gamma^{\prime} \in \Gamma_{\mathbb{Z}, n+n^{\prime}}\right) \mapsto \gamma^{\prime}-\gamma_{0}$.

(3) Let $\Theta$ be the family of all sub-semigroups of $\Gamma$ which are finitely generated. The family of convex sets $\left\{\Delta\left(\Gamma^{\prime}\right)\right\}_{\Gamma^{\prime} \in \Theta}$ is filtered. Let $C$ be the union of all $\Delta\left(\Gamma^{\prime}\right), \Gamma^{\prime} \in \Theta$. By definition, the closure of $C$ coincides with $\Delta(\Gamma)$. Therefore (by Proposition 6.3.1), the interior of $\Delta(\Gamma)$ relatively to $A(\Gamma)$ identifies with that of $C$, which is equal to $\bigcup_{\Gamma^{\prime} \in \Theta} \Delta\left(\Gamma^{\prime}\right)^{\circ}$, where $\Delta\left(\Gamma^{\prime}\right)^{\circ}$ denotes the relative interior of $\Delta\left(\Gamma^{\prime}\right)$ in $A(\Gamma)$. Since $K$ is a compact subset of $\Delta(\Gamma)^{\circ}$ and since the family $\left(\Delta\left(\Gamma^{\prime}\right)^{\circ}\right)_{\Gamma^{\prime} \in \Theta}$ is filtered, there exists $\Gamma^{\prime} \in \Theta$ such that $K \subseteq \Delta\left(\Gamma^{\prime}\right)^{\circ}$. Moreover, since $\Gamma_{\mathbb{Z}}$ is a discrete subgroup of $\mathbb{R} \times V$, it is actually finitely generated. Hence by possibly enlarging $\Gamma^{\prime}$ we may assume that $\Gamma_{\mathbb{Z}}^{\prime}=\Gamma_{\mathbb{Z}}$. Therefore, without loss of generality, we may assume that the semigroup $\Gamma$ is finitely generated.

Let $\left\{x_{i}\right\}_{i=1}^{\ell}$ be a system of generators of $\Gamma$, where $x_{i}=\left(n_{i}, \gamma_{i}\right)$. Then $\Delta(\Gamma)$ is just the convex hull of $n_{i}^{-1} \gamma_{i}(i \in\{1, \ldots, \ell\})$. The set

$$
F=\left\{\lambda_{1} x_{1}+\cdots+\lambda_{\ell} x_{\ell} \mid\left(\lambda_{1}, \ldots, \lambda_{\ell}\right) \in[0,1]^{\ell}\right\}
$$

is a compact subset of $\mathbb{R} \times V$. Therefore the intersection of $F$ with $\Gamma_{\mathbb{Z}}$ is finite since $\Gamma_{\mathbb{Z}}$ is supposed to be discrete. In particular, there exists $x_{0}=\left(n_{0}, \gamma_{0}\right) \in \Gamma$ such that $x_{0}+y \in \Gamma$ for any $y \in F \cap \Gamma_{\mathbb{Z}}$. Let $n$ be an element of $\mathbb{Z}(\Gamma), n \geqslant 1$, and let $\gamma \in \Gamma_{\mathbb{Z}, n}$. If $n^{-1} \gamma$ belongs to $\Delta(\Gamma)$, then there exists $\left(a_{1}, \ldots, a_{\ell}\right) \in \mathbb{R}_{+}^{\ell}$ such that $a_{1} n_{1}+\cdots+a_{\ell} n_{\ell}=n$ and that $\gamma=a_{1} \gamma_{1}+\cdots+a_{\ell} \gamma_{\ell}$. Let $b_{i}=\left\lfloor a_{i}\right\rfloor$ and $\lambda_{i}=a_{i}-b_{i}$ for any $i \in\{1, \ldots, \ell\}$. We write $x=(n, \gamma)$ in the form $x=x^{\prime}+y$ with

$$
x^{\prime}=b_{1} x_{1}+\cdots+b_{\ell} x_{\ell} \in \Gamma, \quad y=\lambda_{1} x_{1}+\cdots+\lambda_{\ell} x_{\ell} \in F .
$$

Since $x \in \Gamma_{\mathbb{Z}}$, also is $y$. Hence $y \in F \cap \Gamma_{\mathbb{Z}}$. Thus $x+x_{0}=x^{\prime}+\left(y+x_{0}\right) \in \Gamma$. In particular, one has

$$
\gamma+\gamma_{0} \in \Gamma_{n+n_{0}} .
$$


Now we introduce a norm $\|\cdot\|$ on $V$. Since $K$ is a compact subset of the relative interior of $\Delta(\Gamma)$, there exists $\epsilon>0$ such that, for any $u \in K$, the ball

$$
B(u, \epsilon)=\left\{u^{\prime} \in W:\left\|u-u^{\prime}\right\| \leqslant \epsilon\right\}
$$

is contained in $\Delta(\Gamma)$. Moreover, the set $K$ is bounded. Therefore, for sufficiently positive integer $n \in \mathbb{N}(\Gamma)$, if $\beta$ is an element in $\Gamma_{\mathbb{Z}, n} \cap n K$, then one has

$$
\left(n-n_{0}\right)^{-1}\left(\beta-\gamma_{0}\right) \in \Delta(\Gamma),
$$

which implies that $\beta \in \Gamma_{n}$ by the above argument. The equality (6.7) is thus proved.

Definition 6.3.7. - Let $\Gamma$ be a graded semigroup in $V$ such that $\Gamma_{\mathbb{Z}}$ is discrete. Let $A(\Gamma)_{0}$ be the vector subspace of $V$ which is the translation of the affine subspace $A(\Gamma)$. We equip $A(\Gamma)_{0}$ with the normalised Lebesgue measure such that the mass of a fundamental domain of the lattice $\Gamma_{\mathbb{Z}, 0}$ in $A(\Gamma)_{0}$ is 1 . This measure induces by translation a Borel measure on $A(\Gamma)$. We denote by $\eta_{\Gamma}$ the restriction of this Borel measure to the closed convex set $\Delta(\Gamma)$, that is, for any function $f \in C_{c}(A(\Gamma)$ ) (namely $f$ is continuous on $A(\Gamma)$ and of compact support), one has

$$
\int_{A(\Gamma)} f(x) \eta_{\Gamma}(\mathrm{d} x)=\int_{\Delta(\Gamma)} f(\gamma) \mathrm{d} \gamma
$$

where $\mathrm{d} \gamma$ denotes the normalised Lebesgue measure.

The following theorem is the key point of the Newton-Okounkov body approach to the study of graded linear series [111, 91, 97. Here we adopte the form presented in the Bourbaki seminar lecture of Boucksom [24].

Theorem 6.3.8. - Let $\Gamma$ be a graded semigroup in $V$ such that $\Gamma_{\mathbb{Z}}$ is discrete. For any integer $n \in \mathbb{N}(\Gamma)$, we denote by $\eta_{\Gamma, n}$ the Radon measure on $A(\Gamma)$ such that, for any function $f \in C_{c}(A(\Gamma))$ one has

$$
\int_{A(\Gamma)} f(x) \eta_{\Gamma, n}(\mathrm{~d} x)=\frac{1}{n^{\kappa}} \sum_{\gamma \in \Gamma_{n}} f\left(n^{-1} \gamma\right),
$$

where $\kappa$ is the dimension of the affine space $A(\Gamma)$. Then the sequence of measures $\left\{\eta_{\Gamma, n}\right\}_{n \in \mathbb{N}(\Gamma)}$ converges vaguely (see A.3) to the Radon measure $\eta_{\Gamma}$.

Proof. - Recall that the vague convergence in the statement of the theorem signifies that the sequence $\left\{\eta_{\Gamma, n}\right\}_{n \in \mathbb{N}(\Gamma)}$, viewed as a sequence of positive linear functionals on $C_{c}(A(\Gamma))$, converges pointwisely to $\eta_{\Gamma}$. In other words, for any continuous function $f$ on $A(\Gamma)$ of compact support, one has

$$
\lim _{n \in \mathbb{N}(\Gamma), n \rightarrow+\infty} \frac{1}{n^{\kappa}} \sum_{\gamma \in \Gamma_{n}} f\left(n^{-1} \gamma\right)=\int_{\Delta(\Gamma)} f(\gamma) \mathrm{d} \gamma
$$


Note that the direct image preserves the vague convergence. Therefore, it suffices to prove that, for any non-negative continuous function $f$ on $\Delta(\Gamma)$ which is of compact support, the equality (6.9) holds.

For any $n \in \mathbb{N}(\Gamma)$ one has

$$
\frac{1}{n^{\kappa}} \sum_{\gamma \in \Gamma_{n}} f\left(n^{-1} \gamma\right) \leqslant \frac{1}{n^{\kappa}} \sum_{\gamma \in \Gamma_{\mathbb{Z}, n} \cap n \Delta(\Gamma)} f\left(n^{-1} \gamma\right) .
$$

Note that the right had side of the inequality is the $n^{\text {th }}$ Riemann sum of the function $f$ on the convex set $\Delta(\Gamma)$. Therefore one has

$$
\lim _{n \in \mathbb{N}(\Gamma), n \rightarrow+\infty} \frac{1}{n^{\kappa}} \sum_{\gamma \in \Gamma_{\mathbb{Z}, n} \cap n \Delta(\Gamma)} f\left(n^{-1} \gamma\right)=\int_{\Delta(\Gamma)} f(\gamma) \mathrm{d} \gamma,
$$

which implies

$$
\lim _{n \in \mathbb{N}(\Gamma), n \rightarrow+\infty} \frac{1}{n^{\kappa}} \sum_{\gamma \in \Gamma_{n}} f\left(n^{-1} \gamma\right) \leqslant \int_{\Delta(\Gamma)} f(\gamma) \mathrm{d} \gamma
$$

Moreover, if $g$ is a continuous function on $\Delta(\Gamma)$ whose support is contained in $\Delta(\Gamma)^{\circ}$ (the relative interior of $\Delta(\Gamma)$ in $A(\Gamma)$ ) and which is bounded from above by $f$, by Proposition 6.3.6 (3) for sufficiently positive $n$ one has

$$
\sum_{\gamma \in \Gamma_{\mathbb{Z}, n} \cap n \Delta(\Gamma)} g\left(n^{-1} \gamma\right)=\sum_{\gamma \in \Gamma_{n}} g\left(n^{-1} \gamma\right)
$$

Hence one has

$$
\liminf _{n \in \mathbb{N}(\Gamma), n \rightarrow+\infty} \frac{1}{n^{\kappa}} \sum_{\gamma \in \Gamma_{n}} f\left(n^{-1} \gamma\right) \geqslant \lim _{n \in \mathbb{N}(\Gamma), n \rightarrow+\infty} \frac{1}{n^{\kappa}} \sum_{\gamma \in \Gamma_{n}} g\left(n^{-1} \gamma\right)=\int_{\Delta(\Gamma)^{\circ}} g(\gamma) \mathrm{d} \gamma .
$$

Since the restriction of the function $f$ on $\Delta(\Gamma)^{\circ}$ can be written as the limite of an increasing sequence of continous functions with support contained in $\Delta(\Gamma)^{\circ}$, by the monotone convergence theorem of Levi, one has

$$
\liminf _{n \in \mathbb{N}(\Gamma), n \rightarrow+\infty} \frac{1}{n^{\kappa}} \sum_{\gamma \in \Gamma_{n}} f\left(n^{-1} \gamma\right) \geqslant \int_{\Delta(\Gamma)^{\circ}} f(\gamma) \mathrm{d} \gamma .
$$

Finally, since the border of $\Delta(\Gamma)$ has Lebesgue measure 0 , we obtain the desired result.

Definition 6.3.9. - Let $\Gamma$ be a graded semigroup in $V$. The dimension of the affine space $A(\Gamma)$ is called the Kodaira dimension of $\Gamma$.

Corollary 6.3.10. - We keep the notation and the hypotheses of Theorem 6.3.8. For any convex subset $C$ of $\Delta(\Gamma)$ one has

$$
\lim _{n \in \mathbb{N}(\Gamma), n \rightarrow+\infty} \frac{\#\left(\Gamma_{n} \cap n C\right)}{n^{\kappa}}=\eta_{\Gamma}(C),
$$

where $\kappa$ is the Kodaira dimension of $\Gamma$. 
Proof. - Let $C^{\circ}$ be the relative interior of $C$ in $A(\Gamma)$. If $C^{\circ}$ is empty, then one has $\eta_{\Gamma}(C)=0$. Moreover, for $n \in \mathbb{N}_{\geqslant 1}$, one has $\#\left(\Gamma_{n, \mathbb{Z}} \cap n C\right)=o\left(n^{\kappa}\right)$ since $\Gamma_{n, \mathbb{Z}}$ is a translation of lattice (see Proposition 6.3.6). Therefore, one has

$$
\lim _{n \in \mathbb{N}(\Gamma), n \rightarrow+\infty} \frac{\#\left(\Gamma_{n} \cap n C\right)}{n^{\kappa}}=0 .
$$

In the following, we assume that $C^{\circ}$ is not empty. Let $K$ be a compact convex subset of $C^{\circ}$. We can find a function $f \in C_{c}(A(\Gamma))$ with $0 \leqslant f \leqslant \mathbb{1}_{C},\left.f\right|_{K} \equiv 1$. Then one has

$$
\forall n \in \mathbb{N}(\Gamma), \frac{\#\left(\Gamma_{n} \cap n C\right)}{n^{\kappa}} \geqslant \int_{A(\Gamma)} f(x) \eta_{\Gamma, n}(\mathrm{~d} x),
$$

which leads to (by Theorem 6.3.8)

$$
\liminf _{n \in \mathbb{N}(\Gamma), n \rightarrow+\infty} \frac{\#\left(\Gamma_{n} \cap n C\right)}{n^{\kappa}} \geqslant \int_{A(\Gamma)} f(x) \eta_{\Gamma}(\mathrm{d} x) \geqslant \eta_{\Gamma}(K) .
$$

Since $K$ is arbitrary, we obtain

$$
\liminf _{n \in \mathbb{N}(\Gamma), n \rightarrow+\infty} \frac{\#\left(\Gamma_{n} \cap n C\right)}{n^{\kappa}} \geqslant \eta_{\Gamma}\left(C^{\circ}\right)=\eta_{\Gamma}(C) .
$$

In particular, if $C$ is not bounded, then

$$
\lim _{n \in \mathbb{N}(\Gamma), n \rightarrow+\infty} \frac{\#\left(\Gamma_{n} \cap n C\right)}{n^{\kappa}}=\eta_{\Gamma}(C)=+\infty .
$$

In the following, we assume in addition that the convex set $C$ is bounded. Denote by $\bar{C}$ the closure of the convex set $C$. It is a conex and compact subset of $A(\Gamma)$. Let $K$ be a compact subset of $A(\Gamma)$ such that the relative interior of $K$ contains $\bar{C}$. For any non-negative function $g \in C_{c}(A(\Gamma))$ with support contained $K$ and such that $0 \leqslant g \leqslant 1,\left.g\right|_{C} \equiv 1$, one has

$$
\forall n \in \mathbb{N}(C), \frac{\#\left(\Gamma_{n} \cap n C\right)}{n^{\kappa}} \leqslant \int_{A(\Gamma)} g(x) \eta_{\Gamma, n}(\mathrm{~d} x) .
$$

By Theorem 6.3.8, we obtain

$$
\limsup _{n \in \mathbb{N}(\Gamma), n \rightarrow+\infty} \frac{\#\left(\Gamma_{n} \cap n C\right)}{n^{\kappa}} \leqslant \int_{A(\Gamma)} g(x) \eta_{\Gamma}(\mathrm{d} x) \leqslant \eta_{\Gamma}(\mathrm{d} x) \leqslant \eta_{\Gamma}(K) .
$$

Since $K$ is arbitrary, we obtain

$$
\limsup _{n \rightarrow+\infty} \frac{\#\left(\Gamma_{n} \cap n C\right)}{n^{\kappa}} \leqslant \int_{A(\Gamma)} g(x) \eta_{\Gamma}(\mathrm{d} x) \leqslant \eta_{\Gamma}(C) .
$$


6.3.3. Concave transform. - Let $V$ be a finite-dimensional vector space over $\mathbb{R}$ and $\Gamma$ be a graded semigroup in $V$ such that $\Gamma_{\mathbb{Z}}$ is discrete. We suppose given a map $\delta: \mathbb{N}_{\geqslant 1} \rightarrow \mathbb{R}$ such that $\delta(n) / n$ tends to 0 when $n \rightarrow+\infty$.

Definition 6.3.11. - Let $g: \Gamma \rightarrow \mathbb{R}$ be a function. We say that the function $g$ is strongly $\delta$-superadditive if for any $\ell \in \mathbb{N}_{\geqslant 2}$ and for all elements $\left(n_{1}, \gamma_{1}\right), \ldots,\left(n_{\ell}, \gamma_{\ell}\right)$ in $\Gamma$, one has

$$
g\left(n_{1}+\cdots+n_{\ell}, \gamma_{1}+\cdots+\gamma_{\ell}\right) \geqslant \sum_{i=1}^{\ell}\left(g\left(n_{i}, \gamma_{i}\right)-\delta\left(n_{i}\right)\right)
$$

The purpose of this subsection is to prove the following result.

Theorem 6.3.12. - Let $\Gamma$ be a graded semigroup in $V$. We assume that $\Gamma_{\mathbb{Z}}$ is discrete and that $\Delta(\Gamma)$ is compact. Suppose given a function $g$ on $\Gamma$ which is strongly $\delta$-superadditive for certain function $\delta: \mathbb{N}_{\geqslant 1} \rightarrow \mathbb{R}$ such that

$$
\lim _{n \rightarrow+\infty} \frac{\delta(n)}{n}=0
$$

For any $n \in \mathbb{N}(\Gamma)$, let $\nu_{n}$ be the Borel probability measure on $\mathbb{R}$ given by

$$
\forall f \in C_{c}(\mathbb{R}), \quad \int_{\mathbb{R}} f(t) \nu_{n}(\mathrm{~d} t)=\frac{1}{\# \Gamma_{n}} \sum_{\gamma \in \Gamma_{n}} f\left(\frac{1}{n} g(n, \gamma)\right) .
$$

The the sequence of measures $\left\{\nu_{n}\right\}_{n \in \mathbb{N}(\Gamma)}$ converges vaguely to a Borel measure $\nu_{\Gamma}$ on $\mathbb{R}$. Moreover, $\nu_{\Gamma}$ is either the zero measure or a probability measure, and in the latter case the sequence $\left\{\nu_{n}\right\}_{n \in \mathbb{N}(\Gamma)}$ actually converges weakly to $\nu_{\Gamma}$ (see Theorem A.3.2) and there exists a concave function $G_{\Gamma}: \Delta(\Gamma)^{\circ} \rightarrow \mathbb{R}$ such that $\nu_{\Gamma}$ identifies with the direct image of

$$
\frac{1}{\eta_{\Gamma}(\Delta(\Gamma))} \eta_{\Gamma}
$$

by the map $G_{\Gamma}$.

Proof. - We introduce an auxiliary function $\widetilde{g}$ on $\Gamma$ taking values in $\mathbb{R} \cup\{+\infty\}$ as follows:

$$
\forall u \in \Gamma, \quad \widetilde{g}(u)=\limsup _{n \rightarrow+\infty} \frac{g(n u)}{n} .
$$

Note that the sequence defining $\widetilde{g}(u)$ is bounded from below and hence the sup limit does not take the value $-\infty$. The proof of the theorem is decomposed into the following steps.

Step 1: The sup limit in the formula (6.12) is actually a limit. This follows from the following generalisation of Fekete's lemma (the case where $\delta(n)=0$ for all $n$ ): let 
$\left\{a_{n}\right\}_{n \geqslant 1}$ be a sequence in $\mathbb{R}$ such that, for any $\ell \in \mathbb{N}_{\geqslant 2}$ and for all $n_{1}, \ldots, n_{\ell}$ in $\mathbb{N}_{\geqslant 1}$ one has

$$
a_{n_{1}+\cdots+n_{\ell}} \geqslant \sum_{i=1}^{\ell}\left(a_{n_{i}}-\delta\left(n_{i}\right)\right),
$$

then the sequence $\left\{a_{n} / n\right\}_{n \geqslant 1}$ converges in $\mathbb{R} \cup\{+\infty\}$. In fact, if $p$ is an integer, $p \geqslant 1$ and if $m \in \mathbb{N}, r \in\{1, \ldots, p\}$ one has

$$
a_{m p+r} \geqslant m a_{p}+a_{r}-m \delta(p)-\delta(r),
$$

and hence

Therefore

$$
\frac{a_{m p+r}}{m p+r} \geqslant \frac{m}{m p+r} a_{p}+\frac{a_{r}}{m p+r}-\frac{m \delta(p)+\delta(r)}{m p+r} .
$$

$$
\liminf _{n \rightarrow+\infty} \frac{a_{n}}{n} \geqslant \frac{a_{p}}{p}-\frac{\delta(p)}{p} .
$$

In particular, $\liminf \operatorname{in}_{n \rightarrow+\infty} a_{n} / n \geqslant a_{1}-\delta(1)>-\infty$. Moreover, this inequality also implies that

$$
\liminf _{n \rightarrow+\infty} \frac{a_{n}}{n} \geqslant \limsup _{p \rightarrow+\infty}\left(\frac{a_{p}}{p}-\frac{\delta(p)}{p}\right)=\limsup _{p \rightarrow+\infty} \frac{a_{p}}{p},
$$

which leads to the convergence of the sequence $\left\{a_{n} / n\right\}_{n \geqslant 1}$.

Step 2: Some properties of the function $\widetilde{g}$. Let $u_{1}=\left(n_{1}, \gamma_{1}\right)$ and $u_{2}=\left(n_{2}, \gamma_{2}\right)$ be two elements in $\Gamma$. For any $n \in \mathbb{N}_{\geqslant 1}$ one has

$$
g\left(n\left(u_{1}+u_{2}\right)\right) \geqslant g\left(n u_{1}\right)+g\left(n u_{2}\right)-\delta\left(n n_{1}\right)-\delta\left(n n_{2}\right)
$$

and hence

$$
\frac{g\left(n\left(u_{1}+u_{2}\right)\right)}{n} \geqslant \frac{g\left(n u_{1}\right)}{n}+\frac{g\left(n u_{2}\right)}{n}-\frac{\delta\left(n n_{1}\right)+\delta\left(n n_{2}\right)}{n} .
$$

By taking the limit when $n \rightarrow+\infty$, we obtain $\widetilde{g}\left(u_{1}+u_{2}\right) \geqslant \widetilde{g}\left(u_{1}\right)+\widetilde{g}\left(u_{2}\right)$. In other words, the function $\widetilde{g}$ is superadditive.

Let $(n, \gamma)$ be an element of $\Gamma$. Note that for any $N \in \mathbb{N}_{\geqslant 1}$ one has

$$
\frac{g(N n, N \gamma)}{N} \geqslant g(n, \gamma)-\delta(n)
$$

By taking the limit when $N \rightarrow+\infty$, we obtain

$$
\widetilde{g}(n, \gamma) \geqslant g(n, \gamma)-\delta(n) .
$$

Step 3: Construction of the function $G_{\Gamma}$. For any $t \in \mathbb{R}$, let $\Gamma^{t}$ be the set of all $(n, \gamma) \in \Gamma$ such that $\widetilde{g}(n, \gamma) \geqslant n t$. It is actually a sub-semigroup of $\Gamma$ since $\widetilde{g}$ is super-additive. Note that $\left\{\Gamma^{t}\right\}_{t \in \mathbb{R}}$ is a decreasing family of sub-semigroups of $\Gamma$ and hence $\left\{\Delta\left(\Gamma^{t}\right)\right\}_{t \in \mathbb{R}}$ is a decreasing family of closed convex subsets of $\Delta(\Gamma)$. We define the function $G_{\Gamma}: \Delta(\Gamma) \rightarrow \mathbb{R} \cup\{+\infty\}$ as follows:

$$
\forall x \in \Delta(\Gamma), \quad G_{\Gamma}(x)=\sup \left\{t \in \mathbb{R}: x \in \Delta\left(\Gamma^{t}\right)\right\} .
$$

By definition, if $t$ is a real number, then $G_{\Gamma}(x) \geqslant t$ if and only if $x \in \bigcap_{s<t} \Delta\left(\Gamma^{s}\right)$. We claim that the function $G_{\Gamma}$ is concave. In fact, since the function $\widetilde{g}$ is super-additive, 
we obtain that, if $s$ and $t$ are two real numbers and if $\epsilon \in[0,1] \cap \mathbb{Q}$, for $u \in \Gamma^{s}$ and $v \in \Gamma^{t}$ one has

$$
N(\epsilon u+(1-\epsilon) v) \in \Gamma^{\epsilon s+(1-\epsilon) t},
$$

where $N$ is an element in $\mathbb{N}_{\geqslant 1}$ such that $N \epsilon \in \mathbb{N}$. Therefore one has

$$
\epsilon \Delta\left(\Gamma^{s}\right)+(1-\epsilon) \Delta\left(\Gamma^{t}\right) \subseteq \Delta\left(\Gamma^{\epsilon s+(1-\epsilon) t}\right) .
$$

In general, if we choose a sequence $\left\{\epsilon_{n}\right\}$ of rational numbers such that $\lim _{n \rightarrow \infty} \epsilon_{n}=\epsilon$ and $\epsilon_{n} s+\left(1-\epsilon_{n}\right) t \geqslant \epsilon s+(1-\epsilon) t$ for all $n$, then

$$
\epsilon_{n} \Delta\left(\Gamma^{s}\right)+\left(1-\epsilon_{n}\right) \Delta\left(\Gamma^{t}\right) \subseteq \Delta\left(\Gamma^{\epsilon_{n} s+\left(1-\epsilon_{n}\right) t}\right) \subseteq \Delta\left(\Gamma^{\epsilon s+(1-\epsilon) t}\right),
$$

and hence $\epsilon \Delta\left(\Gamma^{s}\right)+(1-\epsilon) \Delta\left(\Gamma^{t}\right) \subseteq \Delta\left(\Gamma^{\epsilon s+(1-\epsilon) t}\right)$. Combining with the definition of the function $G_{\Gamma}$, we obtain the concavity of $G_{\Gamma}$. In particular, the restriction of the function $G_{\Gamma}$ on $\Delta(\Gamma)^{\circ}$ is either finite or identically $+\infty$, and it is a continuous function on $\Delta(\Gamma)^{\circ}$ when it is finite.

Step 4. Abundance of $\Gamma_{\mathbb{Z}}^{t}$. Let $t$ be an element of $\mathbb{R}$ such that $t<\sup _{x \in \Delta(\Gamma)} G_{\Gamma}(x)$. We will prove that $\Gamma_{\mathbb{Z}}^{t}=\Gamma_{\mathbb{Z}}$ (and hence $A\left(\Gamma^{t}\right)=A(\Gamma)$ ). Note that $\Gamma_{\mathbb{Z}}$ is finitely generated because $\Gamma_{\mathbb{Z}}$ is discrete. Let $u_{i}=\left(n_{i}, \gamma_{i}\right), i \in\{1, \ldots, \ell\}$ be a family of elements in $\Gamma$ which forms a system of generators in $\Gamma_{\mathbb{Z}}$. Since $t<\sup _{x \in \Delta(\Gamma)} G_{\Gamma}(x)$, there exists $\epsilon>0$ such that $\Gamma^{t+\epsilon}$ is not empty. Let $u_{0}=\left(n_{0}, \gamma_{0}\right)$ be an element in $\Gamma^{t+\epsilon}$. By definition, one has $\widetilde{g}\left(u_{0}\right) \geqslant n_{0}(t+\epsilon)$. Therefore, for sufficiently positive integer $p$, one has

$$
\forall i \in\{1, \ldots, \ell\}, \quad \widetilde{g}\left(p u_{0}+u_{i}\right) \geqslant p \widetilde{g}\left(u_{0}\right)+\widetilde{g}\left(u_{i}\right) \geqslant\left(p n_{0}+n_{i}\right) t,
$$

namely $p u_{0}+u_{i} \in \Gamma^{t}$ for any $i \in\{1, \ldots, \ell\}$, which leads to $\Gamma_{\mathbb{Z}}^{t}=\Gamma_{\mathbb{Z}}$.

Step 5: Lower bound of the function $g$. We fix a (closed) fundamental domain $F$ of the lattice $\Gamma_{\mathbb{Z}, 0}$ (see Proposition 6.3.6 (1)]. For $n \in \mathbb{N}(\Gamma)$, we call an $n$-cell in $A(\Gamma)_{n}$ any closed convex subset of $A(\Gamma)_{n}$ of the form $\gamma_{0}+F$, where $\gamma_{0}$ is an element in $\Gamma_{\mathbb{Z}, n}$. We say that a compact subset $K$ of $A(\Gamma)$ is $n$-tileable if it can be written as a union of $n$-cells in $A(\Gamma)_{n}$. Note that, if $K$ is $n$-tileable, then, for any integer $p \geqslant 1$, the set $p K$ is $p n$-tileable since $p F$ can be written as the union of $p^{\kappa} 0$-celles.

Let $t$ be a real number such that $t<\sup _{x \in \Delta(\Gamma)} G_{\Gamma}(x)$, and $\epsilon$ be a positive real number. Let $m \geqslant 1$ be the generator of the group $\mathbb{Z}(\Gamma)$. Suppose given a compact subset $K$ of $\Delta\left(\Gamma^{t}\right)^{\circ}$. We assume that there exists an integer $n \in \mathbb{N}(\Gamma)$ such that $n K$ is $n$-tileable.

By Proposition 6.3.6 (3) there exists an integer $n_{0} \in \mathbb{N}_{\geqslant 1}$ which verifies the following conditions (in the condition $[2]$ we also use the result of Step 4 to identify $\Gamma_{m n_{0}, \mathbb{Z}}$ with $\left.\Gamma_{m n_{0}, \mathbb{Z}}^{t}\right)$ :

(1) $m n_{0} K$ is $m n_{0}$-tileable;

(2) $m n K \cap \Gamma_{m n, \mathbb{Z}} \subseteq \Gamma_{m n}^{t}$ for any $n \in \mathbb{N}, n \geqslant n_{0}$;

(3) for any integer $q \geqslant m n_{0}, \delta(q) / q<\epsilon / 3$. 
For simplifying the notation, in the following we denote by $\Theta$ the set $m n_{0} K \cap \Gamma_{m n_{0}, \mathbb{Z}}$. Note that the condition (2) implies that $\widetilde{g}\left(m n_{0}, \gamma\right) \geqslant t$ for any $\gamma \in \Theta$. Therefore by the definition of the function $\widetilde{g}$ and the finiteness of the set $\Theta$, we obtain that there exists an integer $N_{0}$ divisible by $n_{0}$ such that

$$
\frac{1}{m N_{0}} g\left(m N_{0},\left(N_{0} / n_{0}\right) \gamma\right) \geqslant t-\frac{\epsilon}{3}
$$

for any $\gamma \in \Theta$.

Let $N$ be an integer, $N \geqslant n_{0}$. Let $\alpha$ be an element in $m N K \cap \Gamma_{m N}$ and $x=$ $\left(n_{0} / N\right) \alpha$. Since $m n_{0} K$ is $m n_{0}$-tileable, there exists an $m n_{0}$-cell $C$ such that $x$ belongs to $C$. We write $C$ as $\gamma_{0}+F$ with $\gamma_{0} \in \Gamma_{m n_{0}}$. Let $\left\{e_{1}, \ldots, e_{\kappa}\right\}$ be the basis of $\Gamma_{\mathbb{Z}}$ defining the fundamental domain $F$. Then the point $x$ can be written in a unique way as

$$
x=\gamma_{0}+\sum_{i=1}^{\kappa} \lambda_{i} e_{i}
$$

where

$$
\forall i \in\{1, \ldots, \kappa\}, \quad \lambda_{i} \in[0,1] .
$$

Moreover, since $N\left(x-\gamma_{0}\right)=n_{0} \alpha-N \gamma_{0} \in \Gamma_{0, \mathbb{Z}}$, we obtain that $N \lambda_{i} \in \mathbb{Z}$ for any $i \in\{1, \ldots, \kappa\}$. Without loss of generality, we may assume that $\lambda_{1} \geqslant \ldots \geqslant \lambda_{\kappa}$. Then we can rewrite $x$ as

$$
x=\sum_{i=0}^{\kappa}\left(\lambda_{i}-\lambda_{i+1}\right) \gamma_{i},
$$

where by convention $\lambda_{0}=1, \lambda_{\kappa+1}=0$, and for $i \in\{1, \ldots, \kappa\}, \gamma_{i}=\gamma_{0}+e_{1}+\cdots+e_{i}$. Note that $\gamma_{0}, \ldots \gamma_{\kappa}$ are vertices of the $m n_{0}$-cell $C$, hence belong to $\Theta$. For any $i \in\{0, \ldots, \kappa\}$, let $b_{i}$ be the integral part of

$$
\frac{N}{n_{0}}\left(\lambda_{i}-\lambda_{i+1}\right)
$$

One has

$$
N-(\kappa+1) n_{0}+1 \leqslant n_{0} \sum_{i=0}^{\kappa} b_{i} \leqslant N .
$$

Therefore, we can write $\alpha$ as

$$
\alpha=\sum_{i=0}^{\kappa} b_{i} \gamma_{i}+\beta^{\prime},
$$

where $\beta \in \Gamma_{m r^{\prime}, \mathbb{Z}} \cap m r^{\prime} K$, with

$$
r^{\prime}=N-n_{0} \sum_{i=0}^{\kappa} b_{i} \in\left\{0, \ldots,(\kappa+1) n_{0}-1\right\} .
$$

Note that we have assumed that $N \geqslant n_{0}$. Therefore, if $r^{\prime} \leqslant n_{0}-1$, then there exists at least an indice $b_{i}$ which is $>0$. In this case, we replace $\beta^{\prime}$ by $\beta^{\prime}+\gamma_{i}$ and $b_{i}$ by 
$b_{i}-1$. Thus we obtain the existence of a decomposition of $\alpha$ into the form

$$
\alpha=\sum_{i=0}^{\kappa} a_{i} \gamma_{i}+\beta
$$

with $a_{i} \in \mathbb{N}$ for $i \in\{0, \ldots, \kappa\}$, and $\beta \in \Gamma_{m r, \mathbb{Z}} \cap m r K$ with

$$
r \in\left\{n_{0}, \ldots,(\kappa+1) n_{0}-1\right\} .
$$

The advantage of the new decomposition is that $\beta$ actually belongs to $\Gamma_{m r}$ (see the condition (2) above). Finally, we write each $a_{i}$ in the form $a_{i}=p_{i} N_{0} / n_{0}+r_{i}$ with $p_{i} \in \mathbb{N}$ and $r_{i} \in\left\{0, \ldots, N_{0} / n_{0}-1\right\}$. Then we can decompose $\alpha$ as

$$
\alpha=\sum_{i=0}^{\kappa} p_{i}\left(N_{0} / n_{0}\right) \gamma_{i}+\omega
$$

where

$$
\omega=\beta+\sum_{i=0}^{\kappa} r_{i} \gamma_{i}
$$

The element $\omega$ belongs to certain $\Gamma_{m s} \cap m s K$ with

$$
s \in\left\{n_{0}, \ldots,(\kappa+1) N_{0}-1\right\} .
$$

Hence by (6.11) and (6.14) one obtains

$$
\begin{aligned}
& \frac{g(m N, \alpha)}{m N} \\
\geqslant & \frac{1}{m N}\left(\sum_{i=0}^{\kappa} p_{i} g\left(m N_{0},\left(N_{0} / n_{0}\right) \gamma_{i}\right)+g(m s, \omega)-\delta\left(m N_{0}\right) \sum_{i=0}^{\kappa} p_{i}-\delta(m s)\right) \\
\geqslant & \frac{N_{0} P}{N}(t-\epsilon / 3)+\frac{g(m s, \omega)}{m N}-\frac{P}{m N} \delta\left(m N_{0}\right)-\frac{\delta(m s)}{N},
\end{aligned}
$$

where

Therefore we obtain

$$
P=p_{0}+\cdots+p_{\kappa}=\frac{N-s}{N_{0}} .
$$

$$
\liminf _{N \rightarrow+\infty} \inf _{\alpha \in m N K \cap \Gamma_{m N}} \frac{g(m N, \alpha)}{m N} \geqslant t-\frac{2 \epsilon}{3},
$$

where we have used the condition (3) above to obtain

$$
\frac{P \delta\left(m N_{0}\right)}{m N}=\frac{N-s}{N} \cdot \frac{\delta\left(m N_{0}\right)}{m N_{0}} \leqslant \frac{N-s}{N} \cdot \frac{\epsilon}{3} .
$$

Therefore, there exists an integer $N^{\prime}$ depending on $t, \epsilon$ and $K$ such that $g(m N, \alpha) \geqslant$ $m N(t-\epsilon)$ for any $N \geqslant N^{\prime}$ and any $\alpha \in \Gamma_{m N} \cap m N K$.

Step 6: Convergence of measures. We now proceed with the proof of the convergence of the measures. We first consider the case where $G_{\Gamma}$ is identically $+\infty$ on the interior of $\Delta(\Gamma)$. Let $f$ be a non-negative continuous function with compact support on $\mathbb{R}$ and $t_{0} \in \mathbb{R}$ be a real number which is larger than the supremum of the support 
of the function $f$. Let $K$ be a compact subset of $\Delta(\Gamma)^{\circ}$. By the results in Step 5, we obtain that, there exists $n_{0} \in \mathbb{N}$ such that, for any $n \in \mathbb{N}(\Gamma), n \geqslant n_{0}$ and any $\alpha \in \Gamma_{n} \cap n K$, one has $g(N, \alpha) \geqslant n t_{0}$. Hence

$$
\int_{\mathbb{R}} f(t) \nu_{n}(\mathrm{~d} t) \leqslant\left(\frac{\#\left(\Gamma_{n} \backslash n K\right)}{\# \Gamma_{n}}\right) M=\left(1-\frac{\#\left(\Gamma_{n} \cap n K\right)}{\# \Gamma_{n}}\right) M,
$$

where $M=\sup _{t \in \mathbb{R}} f(t)$. By Corollary 6.3.10, one has

$$
\lim _{n \in \mathbb{N}(\Gamma), n \rightarrow+\infty} \frac{\#\left(\Gamma_{n} \cap n K\right)}{\# \Gamma_{n}}=\frac{\eta_{\Gamma}(K)}{\eta_{\Gamma}(\Delta(\Gamma))} .
$$

Since $K$ is arbitrary, we obtain

$$
\lim _{n \in \mathbb{N}(\Gamma), n \rightarrow+\infty} \int_{\mathbb{R}} f(t) \nu_{n}(\mathrm{~d} t)=0 .
$$

In the following, we assume that the function $G_{\Gamma}$ is finite. In this case, the direct image $\nu_{\Gamma}$ of $\eta_{\Gamma}(\Delta(\Gamma))^{-1} \eta_{\Gamma}$ by $G_{\Gamma}$ is a Borel provability measure on $\mathbb{R}$. We denote by $F$ its probability distribution function, namely

$$
\left.\left.\forall t \in \mathbb{R}, F(t)=\nu_{\Gamma}(]-\infty, t\right]\right)=1-\frac{\eta_{\Gamma}\left(\Delta\left(\Gamma^{t}\right)\right)}{\eta_{\Gamma}(\Delta(\Gamma))} .
$$

By Corollary 6.3 .10 , one has

$$
F(t)=1-\lim _{n \in \mathbb{N}(\Gamma), n \rightarrow+\infty} \frac{\#\left(\Gamma_{n} \cap n \Delta\left(\Gamma^{t}\right)\right)}{\# \Gamma_{n}} .
$$

The function $F$ is continuous on $\mathbb{R}$, except possibly at the point $\sup _{x \in \Delta(\Gamma)} G_{\Gamma}(x)$ (the discontinuity of the function $F$ happens precisely when the function $G_{\Gamma}$ is constant on $\left.\Delta(\Gamma)^{\circ}\right)$. For any $n \in \mathbb{N}(\Gamma)$, let $F_{n}$ be the probability distribution function of $\nu_{n}$.

If $(n, \gamma)$ is an element of $\Gamma$, then one has

$$
G\left(n^{-1} \gamma\right) \geqslant \frac{\widetilde{g}(n, \gamma)}{n} \geqslant \frac{g(n, \gamma)}{n}-\frac{\delta(n)}{n}
$$

where the second inequality comes from (6.13). Therefore we obtain

$$
\forall t \in \mathbb{R},\left\{(n, \gamma) \in \Gamma: G\left(n^{-1} \gamma\right)>t-\delta(n) / n\right\} \supseteq\{(n, \gamma) \in \Gamma: g(n, \gamma) / n>t\},
$$

which implies (by (6.16) $)$

$$
\forall \epsilon>0, \quad 1-\liminf _{n \in \mathbb{N}(\Gamma), n \rightarrow+\infty} F_{n}(t) \leqslant 1-F(t-\epsilon) .
$$

Conversely, for any $t \in \mathbb{R}$, any $\epsilon>0$ and any compact subset $K$ of $\Delta\left(\Gamma^{t+\epsilon}\right)^{\circ}$ (the relative interior of $\Delta\left(\Gamma^{t+\epsilon}\right)$ with respect to $\left.A(\Gamma)\right)$. By the result obtained in Step 5, we obtain that, there exists $N_{0} \in \mathbb{N}$ such that, for any $n \in \mathbb{N}(\Gamma), n \geqslant N_{0}$, one has

$$
\forall \gamma \in \Gamma_{n} \cap n K, \quad g(n, \gamma) \geqslant n t .
$$

Therefore, we obtain

$$
\forall \epsilon>0, \quad 1-\limsup _{n \in \mathbb{N}(\Gamma), n \rightarrow+\infty} F_{n}(t) \geqslant 1-F(t+\epsilon) .
$$


The estimates (6.17) and (6.18) leads to the convergence of $\left\{F_{n}(t)\right\}_{n \in \mathbb{N}}$ to $F(t)$ if $t \in \mathbb{R}$ is a point of continuity of the function $F$, which implies the weak convergence of the sequence $\left\{\nu_{n}\right\}_{n \in \mathbb{N}(\Gamma)}$ to $\nu_{\Gamma}$ (see [114, $\left.\S I .4\right]$ for more details about weak convergence of Borel probability measures on $\mathbb{R}$ ).

Definition 6.3.13. - Let $\Gamma$ be a graded semigroup in $V$ such that $\Gamma_{\mathbb{Z}}$ is discrete, and $g: \Gamma \rightarrow \mathbb{R}$ and $\delta: \mathbb{N}_{\geqslant 1} \rightarrow \mathbb{R}$ be functions. We say that the function $g$ is $\delta$-superadditive if for all elements $\left(n_{1}, \gamma_{1}\right)$ and $\left(n_{2}, \gamma_{2}\right)$ in $\Gamma$, one has

$$
g\left(n_{1}+n_{2}, \gamma_{1}+\gamma_{2}\right) \geqslant g\left(n_{1}, \gamma_{1}\right)+g\left(n_{2}, \gamma_{2}\right)-\delta\left(n_{1}\right)-\delta\left(n_{2}\right)
$$

Lemma 6.3.14. - Let $\delta: \mathbb{N}_{\geqslant 1} \rightarrow \mathbb{R}_{\geqslant 0}$ be an increasing function such that

$$
\sum_{a \in \mathbb{N}} \frac{\delta\left(2^{a}\right)}{2^{a}}<+\infty
$$

Then one has

$$
\lim _{n \rightarrow+\infty} \frac{\delta(n)}{n}=0
$$

and

$$
\lim _{a \rightarrow+\infty} \frac{1}{2^{a}} \sum_{i=0}^{a} \delta\left(2^{i}\right)=0 .
$$

Proof. - For $n \in \mathbb{N}_{\geqslant 1}$, let $a(n)=\left\lfloor\log _{2} n\right\rfloor$. One has $2^{a(n)} \leqslant n<2^{a(n)+1}$. Hence

By the hypothesis of the lemma, one has

$$
\frac{\delta(n)}{n} \leqslant \frac{\delta\left(2^{a(n)+1}\right)}{2^{a(n)}} .
$$

$$
\lim _{n \rightarrow+\infty} \frac{\delta\left(2^{a(n)+1}\right)}{2^{a(n)+1}}=0
$$

which implies (6.20).

For any $a \in \mathbb{N}$, let

$$
S_{a}:=\sum_{i \in \mathbb{N}, i \geqslant a} \frac{\delta\left(2^{i}\right)}{2^{i}} .
$$

By Abel's summation formula, one has

$$
\sum_{i=0}^{a} \delta\left(2^{i}\right)=\sum_{i=0}^{a}\left(S_{i}-S_{i+1}\right) 2^{i}=S_{0}-S_{a+1} 2^{a}+\sum_{i=1}^{a} S_{i} 2^{i-1} .
$$

As the sequence $\left\{S_{a}\right\}_{a \in \mathbb{N}}$ converges to 0 , one has

$$
\lim _{a \rightarrow+\infty} \frac{1}{2^{a}} \sum_{i=1}^{a} S_{i} 2^{i-1}=0
$$

which implies the relation (6.21). 
Proposition 6.3.15. - Let $\delta: \mathbb{N}_{\geqslant 1} \rightarrow \mathbb{R}_{\geqslant 0}$ be an increasing function such that

$$
\sum_{a \in \mathbb{N}_{\geqslant 1}} \frac{\delta\left(2^{a}\right)}{2^{a}}<+\infty .
$$

Let $\left\{b_{n}\right\}_{n \in \mathbb{N}}$ be a sequence of real numbers. We assume that there exists an integer $n_{0}>0$ such that, for any couple $(n, m)$ of integers which are $\geqslant n_{0}$, one has

$$
b_{n+m} \geqslant b_{n}+b_{m}-\delta(n)-\delta(m)
$$

Then the sequence $\left\{b_{n} / n\right\}_{n \in \mathbb{N} \geqslant 1}$ converges in $\mathbb{R} \cup\{+\infty\}$.

Proof. - We first treat the case where $n_{0}=1$. For any $n \in \mathbb{N}_{\geqslant 1}$ one has

$$
b_{2 n} \geqslant 2 b_{n}-2 \delta(n)
$$

and hence by induction we obtain that

$$
b_{2^{a} n} \geqslant 2^{a}\left(b_{n}-\sum_{i=0}^{a-1} \frac{\delta\left(2^{i} n\right)}{2^{i}}\right) .
$$

In particular, one has

$$
\frac{b_{2^{a}}}{2^{a}} \geqslant b_{1}-\sum_{i=0}^{a-1} \frac{\delta\left(2^{i}\right)}{2^{i}}
$$

which implies that

$$
\limsup _{n \rightarrow+\infty} \frac{b_{n}}{n}>-\infty
$$

For any $a \in \mathbb{N}$, let

$$
S_{a}=\sum_{i \in \mathbb{N}, i \geqslant a} \frac{\delta\left(2^{i}\right)}{2^{i}} .
$$

By the hypothesis (6.22), we have

$$
\lim _{a \rightarrow+\infty} S_{a}=0
$$

Let $n \in \mathbb{N}_{\geqslant 1}$ and let $a(n)$ be the unique natural number such that $2^{a(n)} \leqslant n<2^{a(n)+1}$, namely $a(n)=\left\lfloor\log _{2} n\right\rfloor$. Let $p$ be an element in $\mathbb{N}_{\geqslant 1}$, which is written in 2-adic basis as

$$
p=\sum_{i=0}^{\kappa} \epsilon_{i} 2^{i}
$$

with $\epsilon_{i} \in\{0,1\}$ for $i \in\{0, \ldots, \kappa\}$ and $\epsilon_{\kappa}=1$. For any $r \in\{0, \ldots, n-1\}$, by (6.23) one has

$$
b_{n p+r} \geqslant b_{n p}+b_{r}-\delta(n p)-\delta(r) \geqslant b_{n p}+b_{r}-2 \delta(n p) .
$$


Moreover, by induction on $\kappa$ one has

$$
\begin{aligned}
b_{n p} & \geqslant \sum_{i=0}^{\kappa} \epsilon_{i} b_{2^{i} n}-2 \sum_{i=1}^{\kappa} \epsilon_{i} \delta\left(2^{i} n\right) \\
& \geqslant \sum_{i=0}^{\kappa} \epsilon_{i} 2^{i} b_{n}-\sum_{i=1}^{\kappa} \epsilon_{i}\left(2^{i} \sum_{j=0}^{i-1} \frac{\delta\left(2^{j} n\right)}{2^{j}}+2 \delta\left(2^{i} n\right)\right) \\
& \geqslant p b_{n}-2 p \sum_{j=0}^{\kappa} \frac{\delta\left(2^{j} n\right)}{2^{j}} .
\end{aligned}
$$

Since $n \geqslant 2^{a(n)}$ we deduce that

$$
b_{n p} \geqslant p b_{n}-p 2^{\beta+1} S_{a(n)} \geqslant p b_{n}-p n S_{a(n)}
$$

Combining (6.26) and (6.27), we obtain

$$
\frac{b_{n p+r}}{n p+r} \geqslant \frac{p b_{n}+b_{r}}{n p+r}-\frac{n p}{n p+r} S_{a(n)}-2 \frac{\delta(n p)}{n p+r} .
$$

Taking the infimum limit when $p \rightarrow+\infty$, by 6.20 we obtain

$$
\liminf _{m \rightarrow+\infty} \frac{b_{m}}{m} \geqslant \frac{b_{n}}{n}-S_{a(n)},
$$

which implies, by (6.25), that

$$
\liminf _{m \rightarrow+\infty} \frac{b_{m}}{m} \geqslant \limsup _{n \rightarrow+\infty} \frac{b_{n}}{n} .
$$

Therefore the sequence $\left\{b_{n} / n\right\}_{n \in \mathbb{N} \geqslant 1}$ converges in $\mathbb{R} \cup\{+\infty\}$.

For the general case, we apply the obtained result on the sequence $\left\{b_{n_{0} k}\right\}_{k \in \mathbb{N}_{\geqslant 1}}$ and obtain the convergence of the sequence $\left\{b_{n_{0} k} / k\right\}_{k \in \mathbb{N}_{\geqslant 1}}$. Moreover, if $\ell$ is an element in $\left\{n_{0}, \ldots, 2 n_{0}-1\right\}$, then for any $k \in \mathbb{N}_{\geqslant 1}$ one has

$$
b_{n_{0}(k+2)}-b_{2 n_{0}-\ell}+\delta\left(n_{0} k+\ell\right)+\delta\left(2 n_{0}-\ell\right) \geqslant b_{n_{0} k+\ell} \geqslant b_{n_{0} k}+b_{\ell}-\delta\left(n_{0} k\right)-\delta(\ell) .
$$

Dividing this formula by $n_{0} k+\ell$ and taking the limit when $k \rightarrow+\infty$, we obtain

$$
\lim _{k \rightarrow+\infty} \frac{b_{n_{0} k+\ell}}{n_{0} k+\ell}=\lim _{k \rightarrow+\infty} \frac{b_{n_{0} k}}{n_{0} k} .
$$

Since $\ell$ is arbitrary, we obtain the statement announced in the proposition.

Theorem 6.3.16. — Let $\Gamma$ be a graded semigroup in $V$. We assume that $\Gamma_{\mathbb{Z}}$ is discrete and that $\Delta(\Gamma)$ is compact. Suppose given a function $g$ on $\Gamma$ which is $\delta$ superadditive for certain increasing function $\delta: \mathbb{N}_{\geqslant 1} \rightarrow \mathbb{R}$ such that

$$
\sum_{a \in \mathbb{N}} \frac{\delta\left(2^{a}\right)}{2^{a}}<+\infty .
$$

For any $n \in \mathbb{N}(\Gamma)$, let $\nu_{n}$ be the Borel probability measure on $\mathbb{R}$ such that

$$
\int_{\mathbb{R}} f(t) \nu_{n}(\mathrm{~d} t)=\frac{1}{\# \Gamma_{n}} \sum_{\gamma \in \Gamma_{n}} f\left(n^{-1} g(n, \gamma)\right) .
$$


The the sequence of measures $\left\{\nu_{n}\right\}_{n \in \mathbb{N}(\Gamma)}$ converges vaguely to a Borel measure $\nu_{\Gamma}$ on $\mathbb{R}$. Moreover, $\nu_{\Gamma}$ is either the zero measure or a probability measure, and in the latter case the sequence $\left\{\nu_{n}\right\}_{n \in \mathbb{N}(\Gamma)}$ actually converges weakly to $\nu_{\Gamma}$ and there exists a concave function $G: \Delta(\Gamma)^{\circ} \rightarrow \mathbb{R}$, called concave transform of $g$, such that $\nu_{\Gamma}$ identifies with the direct image of

$$
\frac{1}{\eta_{\Gamma}(\Delta(\Gamma))} \eta_{\Gamma}
$$

by the map $G_{\Gamma}$.

Proof. - The proof is very similar to that of Theorem 6.3.12, We will sketch it in emphasising the difference. Let $u=(\ell, \gamma)$ be an element in $\Gamma$, where $\ell \geqslant 1$. Since the function $g$ is $\delta$-superadditive, for any pair $(n, m) \in \mathbb{N}_{\geqslant 1}$ one has

$$
g((n+m) u) \geqslant g(n u)+g(m u)-\delta(n \ell)-\delta(m \ell) .
$$

Moreover, if we let $b$ be an integer such that $\ell \leqslant 2^{b}$, then, by the increasing property of the function $\delta$, one has

$$
\sum_{a \in \mathbb{N}} \frac{\delta\left(2^{a} \ell\right)}{2^{a}} \leqslant 2^{b} \sum_{a \in \mathbb{N}} \frac{\delta\left(2^{a+b}\right)}{2^{a+b}}<+\infty .
$$

By Proposition 6.3.15, for any $u \in \Gamma$, the sequence $\{g(n u) / n\}_{n \in \mathbb{N} \geqslant 1}$ converges in $\mathbb{R} \cup\{+\infty\}$. We denote by $\widetilde{g}(u)$ the limite of the sequence. Moreover, the convergence of the series $\sum_{a \in \mathbb{N}} \delta\left(2^{a}\right) / 2^{a}$ implies that

$$
\lim _{a \rightarrow+\infty} \frac{\delta\left(2^{a}\right)}{2^{a}}=0 .
$$

Still by the hypothesis that the function $\delta(\cdot)$ is increasing, we deduce that

$$
\lim _{n \rightarrow+\infty} \frac{\delta(n)}{n}=0
$$

Therefore, by the same argument as in the Step 2 of the proof of Theorem 6.3.12 we obtain that the function $\widetilde{g}$ is superadditive, namely, for any pair $u_{1}, u_{2}$ of elements in $\Gamma$, one has

$$
\widetilde{g}\left(u_{1}+u_{2}\right) \geqslant \widetilde{g}\left(u_{1}\right)+\widetilde{g}\left(u_{2}\right) .
$$

Moreover, for any $(n, \gamma) \in \Gamma$ and any $a \in \mathbb{N}_{\geqslant 1}$ one has

$$
g\left(2^{a} n, 2^{a} \gamma\right) \geqslant 2^{a} g(n, \gamma)-\sum_{i=0}^{a-1} 2^{a-i} \delta\left(2^{i} n\right) .
$$

Let $b(n)=\left\lfloor\log _{2} n\right\rfloor+1$. One has $2^{b(n)-1} \leqslant n<2^{b(n)}$. Let

$$
R(n)=2^{b(n)} \sum_{i=b(n)}^{+\infty} \frac{\delta\left(2^{i}\right)}{2^{i}} .
$$


Note that one has

$$
\lim _{n \rightarrow+\infty} \frac{R(n)}{n}=0
$$

by the hypothesis (6.28). By the increasing property of the function $\delta$ one has

$$
\sum_{i=0}^{a-1} 2^{a-i} \delta\left(2^{i} n\right) \leqslant 2^{a+b(n)} \sum_{i=0}^{a-1} \frac{\delta\left(2^{i+b(n)}\right)}{2^{i+b(n)}} \leqslant 2^{a} R(n) .
$$

Therefore the inequality (6.29) leads to

$$
\widetilde{g}(n, \gamma) \geqslant g(n, \gamma)-R(n)
$$

We then proceed as in the Steps 3-6 of the proof of Theorem 6.3.12 except that in the counterpart of the minoration 6.15) we need more elaborated estimate as in 6.27).

Remark 6.3.17. - We keep the notations in the proof of Theorems 6.3.12 and 6.3.16. By virtue of [25. Lemma 1.6] (see also [41]), we obtain that, for any real number $t$ such that

$$
t<\lim _{n \in \mathbb{N}(\Gamma), n \rightarrow+\infty} \max _{\gamma \in \Gamma_{n}} \frac{\widetilde{g}(n, \gamma)}{n}=\lim _{n \in \mathbb{N}(\Gamma), n \rightarrow+\infty} \frac{g(n, \gamma)}{n},
$$

the set $\left\{x \in \Delta(\Gamma)^{\circ}: G(x) \geqslant t\right\}$ has a positive measure with respect to $\eta_{\Gamma}$ (and hence is not empty). In particular, we obtain

$$
\sup _{x \in \Delta(\Gamma)^{\circ}} G(x)=\lim _{n \in \mathbb{N}(\Gamma), n \rightarrow+\infty} \frac{g(n, \gamma)}{n}
$$

6.3.4. Applications to the study of graded algebras. - Let $d \geqslant 1$ be an integer. We call monomial order on $\mathbb{Z}^{d}$ any total order $\leqslant$ on $\mathbb{Z}^{d}$ such that $0 \leqslant \alpha$ for any $\alpha \in \mathbb{N}^{d}$ and that $\alpha \leqslant \alpha^{\prime}$ implies $\alpha+\beta \leqslant \alpha^{\prime}+\beta$ for all $\alpha, \alpha^{\prime}$ and $\beta$ in $\mathbb{Z}^{d}$. For example, the lexicographic order on $\mathbb{Z}^{d}$ is a monomial order.

Given a monomial order $\leqslant$ on $\mathbb{Z}^{d}$, we construction a $\mathbb{Z}^{d}$-valuation

$$
v: k \llbracket T_{1}, \ldots, T_{d} \rrbracket \rightarrow \mathbb{Z}^{d} \cup\{\infty\}
$$

as follows. For any $\alpha=\left(a_{1}, \ldots, a_{d}\right) \in \mathbb{N}^{d}$ we denote by $T^{\alpha}$ the monomial $T_{1}^{a_{1}} \cdots T_{d}^{a_{d}}$. For any formal series $F$ written as

$$
F\left(T_{1}, \ldots, T_{d}\right)=\lambda_{\alpha} T^{\alpha}+\sum_{\alpha<\beta} \lambda_{\beta} T^{\beta}, \quad \lambda_{\alpha} \neq 0,
$$

we let $v_{\geqslant}(F):=\alpha$. If $F=0$ is the zero formal series, let $v(0)=\infty$. It is easy to check that the map $v$ satisfies the following axioms of valuation : for any $(F, G) \in$ $k \llbracket T_{1}, \ldots, T_{d} \rrbracket^{2}$, one has $v(F G)=v(F)+v(G)$ and $v(F+G) \geqslant \min (v(F), v(G))$, and the equality $v(F+G)=\min (v(F), v(G))$ holds when $v(F) \neq v(G)$. In particular, if we denote by $R$ the fraction field of $k \llbracket T_{1}, \ldots, T_{d} \rrbracket$, then the map $v: k \llbracket T_{1}, \ldots, T_{d} \rrbracket \rightarrow$ $\mathbb{Z}^{d} \cup\{\infty\}$ extends to a map $v: R \rightarrow \mathbb{Z}^{d} \cup\{\infty\}$ such that, for any $(F, G) \in k \llbracket T_{1}, \ldots, T_{d} \rrbracket$ 
with $G \neq 0$, one has $v(F / G)=v(F)-v(G)$. The valuation map $v: R \rightarrow \mathbb{Z}^{d} \cup\{\infty\}$ allows to define a $\mathbb{Z}^{d}$-filtration $\mathcal{G}$ of $R$ as follows

$$
\forall \alpha \in \mathbb{Z}^{d}, \quad \mathcal{G}_{\geqslant \alpha}(R):=\{f \in R: v(f) \geqslant \alpha\} .
$$

Note that for $(f, g) \in R^{2}$ one has $v(f g)=v(f)+v(g)$ and $v(f+g) \geqslant \min (v(f), v(g))$. Therefore, for $(\alpha, \beta) \in \mathbb{Z}^{d} \times \mathbb{Z}^{d}$ one has

$$
\mathcal{G}_{\geqslant \alpha}(R) \cdot \mathcal{G}_{\geqslant \beta}(R) \subseteq \mathcal{G}_{\geqslant \alpha+\beta}(R) .
$$

For any $\alpha \in \mathbb{Z}^{d}$, we let

$$
\mathcal{G}_{>\alpha}(R):=\{f \in R: v(f)>\alpha\} \text { and } \operatorname{gr}_{\alpha}(R):=\mathcal{G}_{\geqslant \alpha}(R) / \mathcal{G}_{>\alpha}(R)
$$

The relation (6.31) shows that the $k$-algebra structure on $R$ induces by passing to graduation a $k$-algebra structure on

$$
\operatorname{gr}(R):=\bigoplus_{\alpha \in \mathbb{Z}} \operatorname{gr}_{\alpha}(R)
$$

so that $\operatorname{gr}(R)$ is isomorphic to the group algebra $k\left[\mathbb{Z}^{d}\right]$.

Let $V_{\bullet}=\bigoplus_{n \in \mathbb{N}} V_{n}$ be a graded sub- $k$-algebra of the polynomial ring $R[T]$ (viewed as a graded $k$-algebra with the grading by the degree on $T$ ). The filtration $\mathcal{G}$ on $R$ induces an $\mathbb{Z}^{d}$-filtration on each homogeneous component $V_{n}$. The direct sum of subquotients of $V_{n}$ form an $\mathbb{N} \times \mathbb{Z}^{d}$-graded sub- $k$-algebra

$$
\operatorname{gr}\left(V_{\bullet}\right)=\bigoplus_{(n, \alpha) \in \mathbb{N} \times \mathbb{Z}^{d}} \operatorname{gr}_{(n, \alpha)}\left(V_{\bullet}\right)
$$

of $\operatorname{gr}(R)[T] \cong k\left[\mathbb{N} \times \mathbb{Z}^{d}\right]$. In particular, $\operatorname{gr}\left(V_{\bullet}\right)$ is an integral ring, and each homogeneous component $\operatorname{gr}_{(n, \alpha)}\left(V_{\bullet}\right)$ is either zero or $k$-vector space of dimension 1 . In particular, the set

$$
\Gamma\left(V_{\bullet}\right):=\left\{(n, \alpha) \in \mathbb{N}_{\geqslant 1} \times \mathbb{Z}^{d}: \operatorname{gr}_{(n, \alpha)}\left(V_{\bullet}\right) \neq\{0\}\right\}
$$

is a sub-semigroup of $\mathbb{N} \times \mathbb{Z}^{d}$, called the Newton-Okounkov semigroup of $V$. The algebra $\operatorname{gr}\left(V_{\bullet}\right)$ is canonically isomorphic to the semigroup $k$-algebra associated with $\Gamma\left(V_{\bullet}\right)$. Denote by $\Delta\left(V_{\bullet}\right)$ the closure of the subset

$$
\left\{n^{-1} \alpha:(n, \alpha) \in \Gamma\left(V_{\bullet}\right)\right\}
$$

of $\mathbb{R}^{d}$, called the Newton-Okounkov body of $V_{\bullet}$. Let $A\left(V_{\bullet}\right)$ be the affine subspace of $\mathbb{R}^{d}$ the canonical projection of $\Gamma\left(V_{\bullet}\right) \cap\left(\{1\} \times \mathbb{R}^{d}\right)$ in $\mathbb{R}^{d}$. By Proposition 6.3.5, $\Delta\left(V_{\bullet}\right)$ is a closed convex subset of $A\left(V_{\bullet}\right)$. Moreover, the relative interior of $\Delta\left(V_{\bullet}\right)$ in $A\left(V_{\bullet}\right)$ is not empty. The dimension of the affine space $A\left(V_{\bullet}\right)$ is called the Kodaira dimension of the graded linear series $V_{\bullet}$.

Proposition 6.3.18. - Let $V_{\bullet}=\bigoplus_{n \in \mathbb{N}} V_{n}$ be a graded sub-k-algebra of the polynomial ring $R[T]$. One has

$$
\lim _{n \in \mathbb{N}\left(V_{\bullet}\right), n \rightarrow+\infty} \frac{\operatorname{rk}_{k}\left(V_{n}\right)}{n^{\kappa}}=\operatorname{vol}\left(\Delta\left(V_{\bullet}\right)\right),
$$


where $\mathbb{N}\left(V_{\bullet}\right)$ is the set of $n \in \mathbb{N}$ such that $V_{n} \neq\{0\}, \kappa$ is the Kodaira dimension of $V_{\bullet}$, and $\operatorname{vol}(\cdot)$ is the Lebesgue measure which is normalised with respect to the semi-group $\Gamma\left(V_{\bullet}\right)$ as in Definition 6.3.7.

Proof. - It is a direct consequence of Corollary 6.3.10.

Definition 6.3.19. - Let $V_{\bullet}$ be a graded sub- $k$-algebra of $R[T]$ such that $V_{n}$ is of finite rank for any $n \in \mathbb{N}$.

(a) We say that $V_{\bullet}$ is of subfinite type if it is contained in a graded sub- $k$-algebra of $R[T]$ which is of finite type (over $k$ ).

(b) We call $\mathbb{R}$-filtration on $V_{\bullet}$ any collection $\mathcal{F}_{\bullet}=\left\{\mathcal{F}_{n}\right\}_{n \in \mathbb{N}}$, where $\mathcal{F}_{n}$ is an $\mathbb{R}$ filtration on $V_{n}$.

(c) Let $\delta: \mathbb{N}_{\geqslant 1} \rightarrow \mathbb{R}_{\geqslant 0}$ be a function. We say that an $\mathbb{R}$-filtration $\mathcal{F}$. on $V_{\text {. }}$ is strongly $\delta$-superadditive if for any $\ell \in \mathbb{N}_{\geqslant 1}$ and all $\left(n_{1}, \ldots, n_{\ell}\right) \in \mathbb{N}_{\geqslant 1}^{\ell}$ and $\left(t_{1}, \ldots, t_{\ell}\right) \in \mathbb{R}^{\ell}$, one has

$$
\mathcal{F}_{n_{1}}^{t_{1}}\left(V_{n_{1}}\right) \cdots \mathcal{F}_{n_{\ell}}^{t_{\ell}}\left(V_{n_{\ell}}\right) \subseteq \mathcal{F}_{n_{1}+\cdots+n_{\ell}}^{t_{1}+\cdots+t_{\ell}-\delta\left(n_{1}\right)-\cdots-\delta\left(n_{\ell}\right)} V_{n_{1}+\cdots+n_{\ell}}
$$

We say that the $\mathbb{R}$-filtration $\mathcal{F}$. is $\delta$-superadditive if the above relation holds in the particular case where $\ell=2$, namely, for any $\left(n_{1}, n_{2}\right) \in \mathbb{N}_{\geqslant 1}^{2}$ and any $\left(t_{1}, t_{2}\right) \in \mathbb{R}^{2}$

$$
\mathcal{F}_{n_{1}}^{t_{1}}\left(V_{n_{1}}\right) \mathcal{F}_{n_{2}}^{t_{2}}\left(V_{n_{2}}\right) \subseteq \mathcal{F}_{n_{1}+n_{2}}^{t_{1}+t_{2}-\delta\left(n_{1}\right)-\delta\left(n_{2}\right)} V_{n_{1}+n_{2}}
$$

In the following theorem, we fix a graded sub- $k$-algebra $V_{\bullet}$ of subfinite type of $R[T]$, which is equipped with an $\mathbb{R}$-filtration $\mathcal{F}_{\bullet}$. We suppose in addition that $\mathbb{N}\left(V_{\bullet}\right):=$ $\left\{n \in \mathbb{N}: n \geqslant 1, V_{n} \neq\{0\}\right\}$ is not empty. For each $n \in \mathbb{N}\left(V_{\bullet}\right)$, let $\mathbb{P}_{n}$ be the Borel probability measure on $\mathbb{R}$ such that, for any positive Borel function $f$ on $\mathbb{R}$, one has

$$
\int_{\mathbb{R}} f(t) \mathbb{P}_{n}(\mathrm{~d} t)=\frac{1}{\operatorname{rk}\left(V_{n}\right)} \sum_{i=1}^{\operatorname{rk}\left(V_{n}\right)} f\left(\frac{1}{n} \widehat{\mu}_{i}\left(V_{n},\|\cdot\|_{\mathcal{F}_{n}}\right)\right),
$$

where $\|\cdot\|_{\mathcal{F}_{n}}$ is the norm on $V_{n}$ associated with the $\mathbb{R}$-filtration $\mathcal{F}_{n}$.

Theorem 6.3.20. - Let $\delta: \mathbb{N}_{\geqslant 1} \rightarrow \mathbb{R}_{\geqslant 0}$ be an increasing function. We suppose that, either $\mathcal{F}_{\bullet}$ is strongly $\delta$-superadditive and $\lim _{n \rightarrow+\infty} \delta(n) / n=0$, or $\mathcal{F}$. is $\delta$ superadditive and $\sum_{a \in \mathbb{N}} \delta\left(2^{a}\right) / 2^{a}<+\infty$. Then the sequence of measures $\left\{\mathbb{P}_{n}\right\}_{n \in \mathbb{N}\left(V_{\bullet}\right)}$ converges vaguely to a limite Borel measure $\mathbb{P}_{\mathcal{F}_{\bullet}}$ on $\mathbb{R}$, which is the direct image of the uniform probability measure on $\Delta\left(V_{\bullet}\right)^{\circ}$ by a concave function $G_{\mathcal{F}_{\bullet}}: \Delta\left(V_{\bullet}\right)^{\circ} \rightarrow$ $\mathbb{R} \cup\{+\infty\}$, called the concave transform of $\mathcal{F}_{\bullet}$. Moreover, $\mathbb{P}_{\mathcal{F}}$. is either the zero measure or a probability measure, and, in the case where it is a probability measure, $\left\{\mathbb{P}_{n}\right\}_{n \in \mathbb{N}\left(V_{\bullet}\right)}$ also converges weakly to $\mathbb{P}_{\mathcal{F}_{\bullet}}$.

Proof. - Let $\Gamma\left(V_{\bullet}\right)$ be the Newton-Oknounkov semigroup of $V_{\bullet}$. Since $V_{\bullet}$ is contained in an $\mathbb{N}$-graded sub-algebra of finite type of $R[T]$, the group $\Gamma\left(V_{\bullet}\right)_{\mathbb{Z}}$ is discrete and the Newton-Okounkov body $\Delta\left(V_{\bullet}\right)$ is compact. For any $\gamma=(n, \alpha) \in \Gamma\left(V_{\bullet}\right)$, let 
$\|\cdot\|_{u}$ be the subquotient norm on $\operatorname{gr}_{\gamma}\left(V_{\bullet}\right)$ induced by $\|\cdot\|_{\mathcal{F}_{n}}$ and let $g_{\mathcal{F}_{\bullet}}(\gamma)$ be the Arakelov degree of $\left(\operatorname{gr}_{\gamma}\left(V_{\bullet}\right),\|\cdot\|_{\gamma}\right)$. Since the $\mathbb{R}$-filtration $\mathcal{F}_{\bullet}$ is strongly $\delta$-superadditive (resp. $\delta$-superadditive), the function $g_{\mathcal{F}_{\bullet}}$ on $\Gamma\left(V_{\bullet}\right)$ is strongly $\delta$-superadditive (resp. $\delta$-superadditive). Moreover, by Proposition 5.1.2(7), the sequence of successive slopes of $\left(V_{n},\|\cdot\|_{\mathcal{F}_{n}}\right)$ identifies with the sorted sequence of $\left\{g_{\mathcal{F}_{\bullet}}(n, \alpha)\right\}_{\alpha \in \Gamma\left(V_{\bullet}\right\}_{n}}$. Therefore the Borel probability measure $\mathbb{P}_{n}$ verifies

$$
\int_{\mathbb{R}} f(t) \mathbb{P}_{n}(\mathrm{~d} t)=\frac{1}{\operatorname{rk}\left(V_{n}\right)} \sum_{\alpha \in \Gamma\left(V_{\bullet}\right)_{n}} f\left(\frac{1}{n} g_{\mathcal{F}_{\bullet}}(n, \alpha)\right) .
$$

Therefore the assertion follows from Theorem 6.3.12 (resp. Theorem 6.3.16).

Remark 6.3.21. - We keep the notation and the hypothesis of Theorem 6.3.20

(1) By (6.30) we obtain that

$$
\sup _{x \in \Delta\left(V_{\bullet}\right)^{\circ}} G_{\mathcal{F}_{\bullet}}(x)=\lim _{n \in \mathbb{N}\left(V_{\bullet}\right), n \rightarrow+\infty} \frac{1}{n} \widehat{\mu}_{1}\left(V_{n},\|\cdot\|_{\mathcal{F}_{n}}\right) .
$$

(2) Let $m$ be an integer, we denote by $V_{\bullet}^{(m)}$ the graded sub- $k$-algebra of $R[T]$ such that $V_{n}^{(m)}=V_{n m}$ for any $n \in \mathbb{N}$. Then one has

$$
\Gamma\left(V_{\bullet}^{(m)}\right)=\left\{(n, \alpha) \in \mathbb{N}_{\geqslant 1} \times \mathbb{Z}^{d}: \operatorname{gr}_{(n m, \alpha)}\left(V_{\bullet}\right) \neq\{0\}\right\} .
$$

Therefore one has $\Delta\left(V_{\bullet}^{(m)}\right)=m \Delta\left(V_{\bullet}\right)$. Denote by $\mathcal{F}_{\bullet}^{(m)}$ the family of filtrations $\left\{\mathcal{F}_{m n}\right\}_{n \in \mathbb{N}}$ on $V_{\bullet}^{(m)}$, then one has

$$
\forall x \in \Delta\left(V_{\bullet}\right)^{\circ}, \quad G_{\mathcal{F}_{\bullet}^{(m)}}(m x)=m G_{\mathcal{F}_{\bullet}}(x) .
$$

In particular, $\mathbb{P}_{\mathcal{F}_{\bullet}^{(m)}}$ identifies with the direct image of $\mathbb{P}_{\mathcal{F}}$ by the dilatation $\operatorname{map}(t \in \mathbb{R}) \mapsto m t$.

Remark 6.3.22. - Let $U_{\bullet}, V_{\bullet}$ and $W_{\bullet}$ be graded sub- $k$-algebras of subfinite type of $R[T]$. Suppose that, for any $n \in \mathbb{N}$, one has

$$
U_{n}+V_{n}:=\left\{x+y: x \in U_{n}, y \in V_{n}\right\} \subseteq W_{n} .
$$

Then, for all $n \in \mathbb{N}$ and $(\alpha, \beta) \in \mathbb{N}^{d}$ such that $(n, \alpha) \in \Gamma\left(U_{\bullet}\right)$ and $(n, \beta) \in \Gamma\left(V_{\bullet}\right)$, one has $(n, \alpha+\beta) \in \Gamma\left(W_{\bullet}\right)$. Therefore, $\Delta\left(U_{\bullet}\right)+\Delta\left(V_{\bullet}\right) \subseteq \Delta\left(W_{\bullet}\right)$.

Assume that the graded sub- $k$-algebras $U_{\bullet}, V_{\bullet}$ and $W_{\bullet}$ are equipped with $\mathbb{R}$ filtrations $\mathcal{F}^{U}, \mathcal{F}^{V}$ and $\mathcal{F}^{W}$ respectively. Let $\delta: \mathbb{N}_{\geqslant 1} \rightarrow \mathbb{R}_{\geqslant 0}$. We suppose that, either $\mathcal{F}_{\bullet}^{U}, \mathcal{F}_{\bullet}^{V}$ and $\mathcal{F}_{\bullet}^{W}$ are $\delta$-superadditive and $\lim _{n \rightarrow+\infty} \delta(n) / n=0$, or $\mathcal{F}_{\bullet}^{U}, \mathcal{F}_{\bullet}^{V}$ and $\mathcal{F}_{\bullet}^{W}$ are weakly $\delta$-superadditive and $\sum_{a \in \mathbb{N}} \delta\left(2^{a}\right) / 2^{a}<+\infty$. Let $\epsilon: \mathbb{N}_{\geqslant 1} \rightarrow \mathbb{R}_{\geqslant 0}$ be a map such that $\lim _{n \rightarrow+\infty} \epsilon(n) / n=0$. Suppose that, for any $n \in \mathbb{N}_{\geqslant 1}$ and any $\left(t_{1}, t_{2}\right) \in \mathbb{R}^{2}$, one has

$$
\mathcal{F}_{n}^{U, t_{1}}\left(U_{n}\right) \cdot \mathcal{F}_{n}^{V, t_{2}}\left(V_{n}\right) \subseteq \mathcal{F}_{n}^{W, t_{1}+t_{2}-\epsilon(n)}\left(W_{n}\right) .
$$


Then, for all $(n, m) \in \mathbb{N}_{\geqslant 1}^{2}$ and $(\alpha, \beta) \in \mathbb{N}^{d}$ such that $(n, \alpha) \in \Gamma\left(U_{\bullet}\right)$ and $(n, \beta) \in$ $\Gamma\left(V_{\bullet}\right)$, one has

$$
g_{\mathcal{F}_{\bullet}}(m n, m \alpha)+g_{\mathcal{F}_{\bullet}}(m n, m \beta) \leqslant g_{\mathcal{F}_{\bullet}}(m n, m \alpha+m \beta)+\epsilon(m n) .
$$

Dividing the two sides of the inequality by $m$, by passing to limit when $m \rightarrow+\infty$, we obtain that

$$
\widetilde{g}_{\mathcal{F}_{\bullet}}(n, \alpha)+\widetilde{g}_{\mathcal{F}_{\bullet}}(n, \beta) \leqslant \widetilde{g}_{\mathcal{F}_{\bullet}}(n, \alpha+\beta) .
$$

Therefore, for $(x, y) \in \Delta\left(U_{\bullet}\right) \times \Delta\left(V_{\bullet}\right)$, one has

$$
G_{\mathcal{F}_{\bullet}}(x)+G_{\mathcal{F}_{\bullet}}(y) \leqslant G_{\mathcal{F}_{\bullet}}(x+y) .
$$

\subsubsection{Applications to the study of the volume function. -}

Definition 6.3.23. - Let $C_{0}$ be a non-negative real number. We say that the adelic curve $S$ satisfies the tensorial minimal slope property of level $\geqslant C_{0}$ if, for any couple $(\bar{E}, \bar{F})$ of adelic vector bundles on $S$, the following inequality holds

$$
\widehat{\mu}_{\min }\left(\bar{E} \otimes_{\varepsilon, \pi} \bar{F}\right) \geqslant \widehat{\mu}_{\min }(\bar{E})+\widehat{\mu}_{\min }(\bar{F})-C_{0} \ln \left(\operatorname{rk}_{K}(E) \cdot \operatorname{rk}_{K}(F)\right)
$$

Recall that we have proved in Corollary 5.6 .2 that, if the field $K$ is perfect, then the adelic curve $S$ satisfies the tensorial minimal slope property of level $\geqslant \frac{3}{2} \nu\left(\Omega_{\infty}\right)$.

We let $R=\operatorname{Frac}\left(K \llbracket T_{1}, \ldots, T_{d} \rrbracket\right)$ be the fraction field of the $K$-algebra of formal series of $d$ variables $T_{1}, \ldots, T_{d}$ and we equip $\mathbb{Z}^{d}$ with a monomial order $\leqslant$ and $R$ with the corresponding $\mathbb{Z}^{d}$-filtration as explained in Subsection 6.3.4.

Definition 6.3.24. - We call graded $K$-algebra of adelic vector bundles with respect to $R$ any family $\bar{E}_{\bullet}=\left\{\left(E_{n}, \xi_{n}\right)\right\}_{n \in \mathbb{N}}$ of adelic vector bundles on $S$ such that the following conditions are satisfied:

(a) $E_{\bullet}=\bigoplus_{n \in \mathbb{N}} E_{n} T^{n}$ forms a graded sub- $K$-algebra of subfinite type of the polynomial ring $R[T]$;

(b) for any $n \in \mathbb{N}$, the norm family $\xi_{n}$ is ultrametric on $\Omega \backslash \Omega_{\infty}$;

(c) assume that $\xi_{n}$ is of the form $\left\{\|\cdot\|_{n, \omega}\right\}_{\omega \in \Omega}$, then, for all $\omega \in \Omega,\left(n_{1}, n_{2}\right) \in \mathbb{N}_{\geqslant 1}^{2}$, and $\left(s_{1}, s_{2}\right) \in E_{n_{1}, K_{\omega}} \times E_{n_{2}, K_{\omega}}$, one has $\left\|s_{1} \cdot s_{2}\right\|_{n_{1}+n_{2}, \omega} \leqslant\left\|s_{1}\right\|_{n_{1}, \omega} \cdot\left\|s_{2}\right\|_{n_{2}, \omega}$.

Proposition 6.3.25. - Let $\bar{E}_{\bullet}=\left\{\left(E_{n}, \xi_{n}\right)\right\}_{n \in \mathbb{N}}$ be a graded K-algebra of adelic vector bundles with respect to $R$. For any $n \in \mathbb{N}$, we equip $E_{n}$ with the HarderNarasimhan $\mathbb{R}$-filtration $\mathcal{F}_{n}$. Then the collection $\mathcal{F}_{\bullet}=\left\{\mathcal{F}_{n}\right\}_{n \in \mathbb{N}}$ forms an $\mathbb{R}$-filtration on $E$. which is $\delta$-superadditive, where $\delta$ denotes the function $\mathbb{N}_{\geqslant 1} \rightarrow \mathbb{R}_{\geqslant 0}$ sending $n \in \mathbb{N}_{\geqslant 1}$ to $C \ln \left(\operatorname{rk}_{K}\left(E_{n}\right)\right)$.

Proof. - Let $n_{1}$ and $n_{2}$ be elements of $\mathbb{N}_{\geqslant 1}$. By the condition (c) in Definition 6.3.24, for any $\omega \in \Omega$ and $s_{1}^{(1)} \otimes s_{2}^{(1)}+\cdots+s_{1}^{(N)} \otimes s_{2}^{(N)} \in E_{n_{1}, K_{\omega}} \otimes_{K_{\omega}} E_{n_{2}, K_{\omega}}$, one has $\left\|s_{1}^{(1)} s_{2}^{(1)}+\cdots+s_{1}^{(N)} s_{2}^{(N)}\right\|_{n_{1}+n_{2}, \omega} \leqslant \begin{cases}\max _{i \in\{1, \ldots, N\}}\left\|s_{1}^{(i)}\right\|_{n_{1}, \omega} \cdot\left\|s_{2}^{(i)}\right\|_{n_{2}, \omega}, & \omega \in \Omega \backslash \Omega_{\infty}, \\ \sum_{i=1}^{N}\left\|s_{1}^{(i)}\right\|_{n_{1}, \omega} \cdot\left\|s_{2}^{(i)}\right\|_{n_{2}, \omega}, & \omega \in \Omega_{\infty} .\end{cases}$ 
Therefore, the canonical $K_{\omega}$-linear map $E_{n_{1}, K_{\omega}} \otimes_{K_{\omega}} E_{n_{2}, K_{\omega}} \rightarrow E_{n_{1}+n_{2}, K_{\omega}}$ is of operator norm $\leqslant 1$. Let $F_{1}$ and $F_{2}$ be non-zero vector subspace of $E_{n_{1}}$ and $E_{n_{2}}$, respectively, and let $G$ be the image of $F_{1} \otimes_{K} F_{2}$ by the canonical $K$-linear map $E_{n_{1}} \otimes_{K} E_{n_{2}} \rightarrow E_{n_{1}+n_{2}}$. By Proposition 4.3 .30 (2), one has

$$
\begin{aligned}
& \widehat{\mu}_{\min }(\bar{G}) \geqslant \widehat{\mu}_{\min }\left(\bar{F}_{1} \otimes_{\varepsilon, \pi} \bar{F}_{2}\right) \\
\geqslant & \widehat{\mu}_{\min }\left(\bar{F}_{1}\right)+\widehat{\mu}_{\min }\left(F_{2}\right)-C\left(\ln \left(\operatorname{rk}_{K}\left(F_{1}\right)\right)\right)-C\left(\ln \left(\mathrm{rk}_{K}\left(F_{2}\right)\right)\right) \\
\geqslant & \widehat{\mu}_{\min }\left(\bar{F}_{1}\right)+\widehat{\mu}_{\min }\left(F_{2}\right)-C\left(\ln \left(\operatorname{rk}_{K}\left(E_{n_{1}}\right)\right)\right)-C\left(\ln \left(\mathrm{rk}_{K}\left(E_{n_{2}}\right)\right)\right),
\end{aligned}
$$

where the second inequality comes from (7.1). By Proposition 4.3.45, we obtain that, for any $\left(t_{1}, t_{2}\right) \in \mathbb{R}^{2}$, one has

$$
\mathcal{F}_{n_{1}}^{t_{1}}\left(E_{n_{1}}\right) \cdot \mathcal{F}_{n_{2}}^{t_{2}}\left(E_{n_{2}}\right) \subseteq \mathcal{F}_{n_{1}+n_{2}}^{t_{1}+t_{2}-\delta\left(n_{1}\right)-\delta\left(n_{2}\right)}\left(E_{n_{1}+n_{2}}\right) .
$$

The proposition is thus proved.

Corollary 6.3.26. - Let $\bar{E}_{\bullet}=\left\{\left(E_{n}, \xi_{n}\right)\right\}_{n \in \mathbb{N}}$ be a graded $K$-algebra of adelic vector bundles with respect to $R$. We assume that $\mathbb{N}\left(E_{\bullet}\right)$ does not reduce to $\{0\}$ and we denote by $q \in \mathbb{N}$ a generator of the group $\mathbb{Z}\left(E_{\bullet}\right)$. Suppose in addition that

$$
\sum_{a \in \mathbb{N}, 2^{a} q \in \mathbb{N}\left(E_{\bullet}\right)} \frac{\ln \left(\mathrm{rk}_{K}\left(E_{2^{a} q}\right)\right)}{2^{a}}<+\infty .
$$

For each $n \in \mathbb{N}\left(V_{\bullet}\right)$, let $\mathbb{P}_{n}$ be the Borel probability measure on $\mathbb{R}$ such that, for any positive Borel function $f$ on $\mathbb{R}$, one has

$$
\int_{\mathbb{R}} f(t) \mathbb{P}_{n}(\mathrm{~d} t)=\frac{1}{\operatorname{rk}\left(E_{n}\right)} \sum_{\operatorname{rk}\left(E_{n}\right)} f\left(\frac{1}{n} \widehat{\mu}_{i}\left(\bar{E}_{n}\right)\right)
$$

Then the sequence of measures $\left\{\mathbb{P}_{n}\right\}_{n \in \mathbb{N}\left(E_{\bullet}\right)}$ converges vaguely to a limite Borel measure $\mathbb{P}_{\bar{E}}$, which is the direct image of the uniform distribution on $\Delta\left(E_{\bullet}\right)$ by a concave function $G_{\bar{E}_{.}}: \Delta\left(\bar{E}_{\bullet}\right) \rightarrow \mathbb{R} \cup\{+\infty\}$. Moreover, the limite measure is either zero or a Borel probability measure, and in the latter case the sequence $\left\{\mathbb{P}_{n}\right\}_{n \in \mathbb{N}\left(E_{\bullet}\right)}$ also converges weakly to $\mathbb{P}_{\bar{E}}$.

Proof. - This is a direct consequence of Proposition 6.3.25 and Theorem 6.3.20,

Remark 6.3.27. - We keep the notation and the conditions of Corollary6.3.26, We suppose that $\left(\frac{1}{n} \widehat{\mu}_{1}\left(\bar{E}_{n}\right)\right)_{n \in \mathbb{N}\left(V_{\bullet}\right), n \geqslant 1}$ is bounded from above. Then the limit measure $\mathbb{P}_{\overline{E_{\bullet}}}$ is a probability measure. The weak convergence of $\left\{\mathbb{P}_{n}\right\}_{n \in \mathbb{N}\left(E_{\bullet}\right)}$ to $\mathbb{P}_{\overline{E_{\bullet}}}$ implies that

$$
\begin{aligned}
\int_{\mathbb{R}} \max \{t, 0\} \mathbb{P}_{\bar{E}}(\mathrm{~d} t) & =\lim _{n \in \mathbb{N}\left(E_{\bullet}\right), n \rightarrow+\infty} \frac{1}{n \mathrm{rk}\left(E_{n}\right)} \sum_{i=1}^{\mathrm{rk}\left(E_{n}\right)} \max \left\{\widehat{\mu}_{i}\left(\bar{E}_{n}\right), 0\right\} \\
& =\lim _{n \in \mathbb{N}\left(E_{\bullet}\right), n \rightarrow+\infty} \frac{\widehat{\operatorname{deg}}_{+}\left(\bar{E}_{n}\right)}{n \operatorname{rk}\left(E_{n}\right)} .
\end{aligned}
$$


If in addition the sequence $\left(\frac{1}{n} \widehat{\mu}_{\min }\left(\bar{E}_{n}\right)\right)_{n \in \mathbb{N}\left(V_{\bullet}\right), n \geqslant 1}$ is bounded from below, then one has

$$
\begin{aligned}
\int_{\mathbb{R}} t \mathbb{P}_{\overline{E_{\bullet}}}(\mathrm{d} t) & =\lim _{n \in \mathbb{N}\left(E_{\bullet}\right), n \rightarrow+\infty} \frac{1}{n \operatorname{rk}\left(E_{n}\right)} \sum_{i=1}^{\operatorname{rk}\left(E_{n}\right)} \widehat{\mu}_{i}\left(\bar{E}_{n}\right) \\
& =\lim _{n \in \mathbb{N}\left(E_{\bullet}\right), n \rightarrow+\infty} \frac{\widehat{\mu}\left(\bar{E}_{n}\right)}{n} .
\end{aligned}
$$

Proposition 6.3.28. - Let

$$
\left\{\begin{array}{l}
\bar{U} \bullet=\left\{\left(U_{n},\left\{\|\cdot\|_{U_{n}, \omega}\right\}_{\omega \in \Omega}\right)\right\}_{n \in \mathbb{N}} \\
\overline{V_{\bullet}}=\left\{\left(V_{n},\left\{\|\cdot\|_{V_{n}, \omega}\right\}_{\omega \in \Omega}\right)\right\}_{n \in \mathbb{N}} \\
\bar{W} \bullet=\left\{\left(W_{n},\left\{\|\cdot\|_{W_{n}, \omega}\right\}_{\omega \in \Omega}\right)\right\}_{n \in \mathbb{N}}
\end{array}\right.
$$

be graded $K$-algebras of adelic vector bundles with respect to $R$. We assume that

$$
\sum_{a \in \mathbb{N}, 2^{a} q \in \mathbb{N}\left(W_{\bullet}\right)} \frac{\ln \left(\operatorname{rk}\left(W_{2^{a} q}\right)\right)}{2^{a}}<+\infty,
$$

where $q \in \mathbb{N}$ is a generator of the group $\mathbb{Z}\left(W_{\bullet}\right)$. Suppose that, for any $n \in \mathbb{N}$ one has $U_{n} \cdot V_{n} \subseteq W_{n}$, and

(6.38) $\forall \omega \in \Omega, \forall\left(s, s^{\prime}\right) \in U_{n, K_{\omega}} \times V_{n, K_{\omega}}, \quad\left\|s s^{\prime}\right\|_{W_{n}, \omega} \leqslant\|s\|_{U_{n}, \omega} \cdot\left\|s^{\prime}\right\|_{V_{n}, \omega}$.

Then, for any $(x, y) \in \Delta\left(U_{\bullet}\right) \times \Delta\left(V_{\bullet}\right)$, one has

$$
G_{\overline{W_{\bullet}}}(x+y) \geqslant G_{\bar{U}}(x)+G_{\bar{V}}(y) .
$$

Proof. - Denote by $\delta: \mathbb{N}_{\geqslant 1} \rightarrow \mathbb{R}_{\geqslant 0}$ the function sending $n \in \mathbb{N}_{\geqslant 1}$ to

$$
C \ln \left(\operatorname{rk}_{K}\left(U_{n}\right)\right)+C \ln \left(\operatorname{rk}_{K}\left(V_{n}\right)\right)
$$

Let $n \in \mathbb{N}, n \geqslant 1$. Suppose that $E$ is a non-zero vector subspace of $U_{n}$ and $F$ is a nonzero vector subspace of $V_{n}$. Since the adelic curve $S$ satisfies the tensorial minimal slope superadditivity of level $\geqslant C$, one has

$$
\widehat{\mu}_{\min }\left(\bar{E} \otimes_{\varepsilon, \pi} \bar{F}\right) \geqslant \widehat{\mu}_{\min }(E)+\widehat{\mu}_{\min }(F)-\delta(n) .
$$

Moreover, by (6.38) the canonical $K$-linear map $E \otimes F \rightarrow W_{n}$ has height $\leqslant 0$ if we consider the adelic vector bundles $\bar{E} \otimes_{\varepsilon, \pi} \bar{F}$ and $\left(W_{n},\left\{\|\cdot\|_{W_{n}, \omega}\right\}_{\omega \in \Omega}\right.$. Therefore, if we denote by $\mathcal{F}_{n}^{U}, \mathcal{F}_{n}^{V}$ and $\mathcal{F}_{n}^{W}$ the Harder-Narasimhan $\mathbb{R}$-filtrations of $\bar{U}_{n}, \bar{V}_{n}$ and $\bar{W}_{n}$ respectively, then, for any $\left(t, t^{\prime}\right) \in \mathbb{R}^{2}$,

$$
\mathcal{F}_{n}^{U, t}\left(U_{n}\right) \cdot \mathcal{F}_{n}^{V, t^{\prime}}\left(V_{n}\right) \subseteq \mathcal{F}_{n}^{W, t+t^{\prime}-\delta(n)} .
$$

By Remark 6.3.22, we obtain the inequality (6.39).

Remark 6.3.29. - Let $V$. be a graded $k$-algebra. We say that $V$. is of subfinite type if it is contained in a graded $k$-algebra of finite type. It is not true that any integral graded $k$-algebra of subfinite type can be identifies as a graded sub- $k$-algebra of the ring of polynomials (of one variable) with coefficients in the fraction field of the formal 
series ring (with finitely many variables) over $k$ since the latter condition implies that $V_{\bullet}$ admits a valuation of one-dimensional leaves and in particular $V_{\bullet}$ is geometrically integral. We refer to [43. Remark 5.3] for more details. Moreover, the combination of the methods in [41, §4] and [43, §5] allows to obtain a generalisation of Corollary 6.3 .26 and Proposition 6.3 .28 to the case of graded algebras of adelic vector bundles whose underlying graded $k$-algebras are integral domain and of subfinite type over $k$. Note that the $\mathbb{R}$-filtration by slopes of a graded algebra of adelic vector bundles is not necessarily superadditive and we need an argument similar to the Step 2 in the proof of Theorem 6.3.12 in order to replace the $\mathbb{R}$-filtration by slopes by a superadditive $\mathbb{R}$-filtration while keeping the asymptotic behaviour of the distribution of average on the jump points of the $\mathbb{R}$-filtrations. The approach can serve to remove the hypothesis that the scheme $X$ admits a regular rational points in Theorem 6.4.6 and Theorem 6.4 .7

Definition 6.3.30. - Let $C_{1}$ be a non-negative real number. We say that the adelic curve $S$ satisfies the strong tensorial minimal slope property of level $\geqslant C_{1}$ if, for any integer $n \in \mathbb{N}_{\geqslant 2}$ and any family $\left\{\bar{E}_{i}\right\}_{i=1}^{n}$ of $d$ non-zero adelic vector bundles on $S$, the following inequality holds

$$
\widehat{\mu}\left(\bar{E}_{1} \otimes_{\varepsilon, \pi} \cdots \otimes_{\varepsilon, \pi} \bar{E}_{n}\right) \geqslant \sum_{i=1}^{n}\left(\widehat{\mu}_{\min }\left(\bar{E}_{i}\right)-C_{1} \ln \left(\mathrm{rk}_{K}\left(E_{i}\right)\right)\right) .
$$

Note Corollary 5.6 .2 shows that the adelic curve $S$ satisfies the strong tensorial minimal slope property of level $\geqslant \frac{3}{2} \nu\left(\Omega_{\infty}\right)$, provided that the field $K$ is perfect.

Remark 6.3.31. - Let $C$ be a non-negative real number. We say that the adelic curve $S$ satisfies the strong minimal slope property of level $\geqslant C$. Let $\bar{E}$. $=\left\{\left(E_{n}, \xi_{n}\right)\right\}_{n \in \mathbb{N}}$ be a graded $K$-algebra of adelic vector bundles with respect to $R$. For any $n \in \mathbb{N}$, we equip $E_{n}$ with the Harder-Narasimhan $\mathbb{R}$-filtration $\mathcal{F}_{n}$. Then the collection $\mathcal{F}_{\bullet}=\left\{\mathcal{F}_{n}\right\}_{n \in \mathbb{N}}$ forms an $\mathbb{R}$-filtration on $E_{\text {e }}$ which is strongly $\delta$-superadditive, where $\delta$ denotes the function $\mathbb{N}_{\geqslant 1} \rightarrow \mathbb{R}_{\geqslant 0}$ sending $n \in \mathbb{N}_{\geqslant 1}$ to $C \ln \left(\operatorname{rk}_{K}\left(E_{n}\right)\right)$. Therefore, by Theorem 6.3.20, if the condition

$$
\lim _{n \rightarrow+\infty} \frac{\ln \left(\mathrm{rk}\left(E_{n}\right)\right)}{n}=0
$$

is satisfied, then the sequence of Borel probability measures $\left\{\mathbb{P}_{n}\right\}_{n \in \mathbb{N}\left(E_{\bullet}\right)}$, defined by

$$
\int_{\mathbb{R}} f(t) \mathbb{P}_{n}(\mathrm{~d} t)=\frac{1}{\operatorname{rk}\left(E_{n}\right)} \sum_{i=1}^{\operatorname{rk}\left(E_{n}\right)} \delta_{\frac{1}{n} \widehat{\mu}_{i}\left(\bar{E}_{n}\right)}
$$

converges vaguely to a Borel measure $\mathbb{P}_{\overline{E_{\bullet}}}$, which is either the zero measure or a Borel probability measure. In the latter case, the sequence $\left\{\mathbb{P}_{n}\right\}_{n \in \mathbb{N}\left(E_{\bullet}\right)}$ converges weakly to $\mathbb{P}_{\bar{E}}$. Similarly, the assertion of Proposition 6.3.28 holds under the condition

$$
\lim _{n \rightarrow+\infty} \frac{\ln \left(\operatorname{rk}\left(W_{n}\right)\right)}{n}=0 .
$$


Remark 6.3.32. - In the number field setting, Yuan [145, 146] has proposed another method to associate to each adelic line bundle a convex body which computes the arithmetic volume of the adelic line bundle. His method relies on multiplicity estimates of arithmetic global sections with respect to a flag of subvarieties of the fibre of the arithmetic variety over a finite place, which is similar to 97 . Note that in the general setting of adelic curves the set of "arithmetic global sections" is not necessarily finite and the classic formula relating the volume function and the asymptotic behaviour of "arithmetic global sections" does not hold in general.

\subsection{Asymptotic invariants of graded linear series}

In this section, we fix an integral projective $K$-scheme $X$ and denote by $\pi: X \rightarrow$ Spec $K$ the structural morphism. Let $d$ be the Krull dimension of the scheme $X$.

6.4.1. Asymptotic maximal slope. - Let $(L, \varphi)$ be an adelic line bundle on an integral projective $K$-scheme $\pi: X \rightarrow \operatorname{Spec} K$. For any $n \in \mathbb{N}_{\geqslant 1}$, the metric family $n \varphi$ on $L^{\otimes n}$ induces a norm family $\left\{\|\cdot\|_{n \varphi_{\omega}}\right\}_{\omega \in \Omega}$ on the linear series $\pi_{*}\left(L^{\otimes n}\right)=H^{0}\left(X, L^{\otimes n}\right)$ which we denote by $\pi_{*}(n \varphi)$. By Theorem 6.1.13 and 6.1.31, the pair $\left(\pi_{*}\left(L^{\otimes n}\right), \pi_{*}(n \varphi)\right)$ forms an adelic vector bundle on $S$, which we denote by $\pi_{*}\left(L^{\otimes n}, n \varphi\right)$. Note that $\pi_{*}(n \varphi)$ is ultrametric on $\Omega \backslash \Omega_{\infty}$. We can then compute divers arithmetic invariants of these adelic vector bundles. The asymptotic behaviour of these arithmetic invariants describes the positivity of the adelic line bundle $(L, \varphi)$.

Let $(L, \varphi)$ be an adelic line bundle on $X$. We define

$$
\nu_{1}^{\text {asy }}(L, \varphi):=\limsup _{n \rightarrow+\infty} \frac{\nu_{1}\left(\pi_{*}\left(L^{\otimes n}, n \varphi\right)\right)}{n},
$$

called the asymptotic first minimum of $(L, \varphi)$. Similarly, we define

$$
\widehat{\mu}_{\max }^{\text {asy }}(L, \varphi):=\limsup _{n \rightarrow+\infty} \frac{\widehat{\mu}_{\max }\left(\pi_{*}\left(L^{\otimes n}, n \varphi\right)\right)}{n},
$$

called the asymptotic maximal slope of $(L, \varphi)$. Note that all adelic vector bundles $\pi_{*}\left(L^{\otimes n}, n \varphi\right)$ are ultrametric on $\Omega \backslash \Omega_{\infty}$. Therefore, by Remark 4.3.47 and the fact that

$$
\lim _{n \rightarrow+\infty} \frac{\ln \left(\mathrm{rk}_{K}\left(H^{0}\left(X, L^{\otimes n}\right)\right)\right)}{n}=0
$$

we obtain that

$$
\widehat{\mu}_{\max }^{\operatorname{asy}}(L, \varphi)=\limsup _{n \rightarrow+\infty} \frac{\widehat{\mu}_{1}\left(\pi_{*}\left(L^{\otimes n}, n \varphi\right)\right)}{n} .
$$

Let $\mathbb{K}$ be either $\mathbb{Z}$ or $\mathbb{Q}$ or $\mathbb{R}$. From now on we assume that $\mathbb{K}=\mathbb{Z}$ or $X$ is normal. Let $(D, g)$ be an adelic $\mathbb{K}$-Cartier divisor on $X$. Note that if $X$ is not normal 
and $\mathbb{K}$ is either $\mathbb{Q}$ or $\mathbb{R}$, then $H_{\mathbb{K}}^{0}(X, D)$ is not necessarily a vector space over $K$ (cf. Example 2.4.15). Similarly we can define $\nu_{1}^{\text {asy }}(D, g)$ and $\widehat{\mu}_{\text {max }}^{\text {asy }}(D, g)$ as follows:

$$
\left\{\begin{array}{l}
\nu_{1}^{\text {asy }}(D, g):=\limsup _{n \rightarrow+\infty} \frac{\nu_{1}\left(H_{\mathbb{K}}^{0}(X, n D), \xi_{n g}\right)}{n}, \\
\widehat{\mu}_{\text {max }}^{\text {asy }}(D, g):=\limsup _{n \rightarrow+\infty} \frac{\widehat{\mu}_{\max }\left(H_{\mathbb{K}}^{0}(X, n D), \xi_{n g}\right)}{n} .
\end{array}\right.
$$

In the case where $\mathbb{K}=\mathbb{Z}$,

$$
\nu_{1}^{\text {asy }}(D, g)=\nu_{1}^{\text {asy }}\left(\mathcal{O}_{X}(D), \varphi_{g}\right) \quad \text { and } \quad \widehat{\mu}_{\max }^{\text {asy }}(D, g)=\widehat{\mu}_{\max }^{\text {asy }}\left(\mathcal{O}_{X}(D), \varphi_{g}\right),
$$

where $\varphi_{g}$ is the metric family of $L$ defined by $g$.

Proposition 6.4.1. - Let $(D, g)$ be an adelic $\mathbb{K}$-Cartier divisor on $S$. Then one has $\nu_{1}^{\text {asy }}(D, g) \leqslant \widehat{\mu}_{\max }^{\text {asy }}(D, g)$. The equality holds when $S$ satisfies the Minkowski property of certain level (see Definition 4.3.72).

Proof. - By (4.83), for any $n \in \mathbb{N}_{n \geqslant 1}$ one has

$$
\nu_{1}\left(H_{\mathbb{K}}^{0}(X, n D), \xi_{n g}\right) \leqslant \widehat{\mu}_{\max }\left(H_{\mathbb{K}}^{0}(X, n D), \xi_{n g}\right) .
$$

Therefore $\nu_{1}^{\text {asy }}(D, g) \leqslant \widehat{\mu}_{\max }^{\text {asy }}(D, g)$.

If the adelic curve $S$ satisfies the Minkowski property of level $\geqslant C$, where $C \geqslant 0$, then

$$
\nu_{1}\left(H_{\mathbb{K}}^{0}(X, n D), \xi_{n g}\right) \geqslant \widehat{\mu}_{\max }\left(H_{\mathbb{K}}^{0}(X, n D), \xi_{n g}\right)-C \ln \left(\mathrm{rk}_{K}\left(H_{\mathbb{K}}^{0}(X, n D)\right)\right) .
$$

Since $X$ is a projective scheme, one has

$$
\operatorname{rk}_{K}\left(H_{\mathbb{K}}^{0}(X, n D)\right)=O\left(n^{\operatorname{dim}(X)}\right) .
$$

Hence

$$
\lim _{n \rightarrow+\infty} \frac{\ln \left(\operatorname{rk}_{K}\left(H_{\mathbb{K}}^{0}(X, n D)\right)\right)}{n}=0 .
$$

Therefore $\nu_{1}^{\text {asy }}(D, g) \geqslant \widehat{\mu}_{\max }^{\text {asy }}(D, g)$.

Let $(D, g)$ be an adelic $\mathbb{R}$-Cartier divisor on $X$. Let $\mathbb{K}$ be either $\mathbb{Q}$ or $\mathbb{R}$. We set

$$
\left\{\begin{array}{l}
\Gamma_{\mathbb{K}}^{\times}(D)=\left\{\phi \in K(X)^{\times} \otimes_{\mathbb{Z}} \mathbb{K}: D+(\phi) \geqslant \mathbb{K} 0\right\}, \\
\nu_{1, \mathbb{K}}^{\text {asy }}(D, g)= \begin{cases}\sup \left\{\widehat{\operatorname{deg}}_{\xi_{g}}(s): s \in \Gamma_{\mathbb{K}}^{\times}(D)\right\} & \text { if } \Gamma_{\mathbb{K}}^{\times}(D) \neq \emptyset, \\
-\infty & \text { if } \Gamma_{\mathbb{K}}^{\times}(D)=\emptyset,\end{cases}
\end{array}\right.
$$

where (cf. Corollary 6.2.13)

$$
\widehat{\operatorname{deg}}_{\xi_{g}}(s)=-\int_{\Omega} \ln \|s\|_{g_{\omega}} \nu(d \omega)
$$

Note that $\nu_{1}^{\text {asy }}(D, g)=\nu_{1, \mathbb{Q}}^{\text {asy }}(D, g) \leqslant \nu_{1, \mathbb{R}}^{\text {asy }}(D, g)$.

Proposition 6.4.2. - We assume that $X$ is normal. Let $(D, g)$ and $\left(D^{\prime}, g^{\prime}\right)$ be adelic $\mathbb{R}$-Cartier divisors on $X$. Then one has the following: 
(1) $\nu_{1, \mathbb{K}}^{\text {asy }}\left((D, g)+\left(D^{\prime}, g^{\prime}\right)\right) \geqslant \nu_{1, \mathbb{K}}^{\text {asy }}(D, g)+\nu_{1, \mathbb{K}}^{\text {asy }}\left(D^{\prime}, g^{\prime}\right)$.

(2) $\nu_{1, \mathbb{K}}^{\text {asy }}(a(D, g))=a \nu_{1, \mathbb{K}}^{\text {asy }}(D, g)$ for all $a \in \mathbb{K}_{\geqslant 0}$.

Proof. - (1) Clearly we may assume that $\Gamma_{\mathbb{K}}^{\times}(D) \neq \emptyset$ and $\Gamma_{\mathbb{K}}^{\times}\left(D^{\prime}\right) \neq \emptyset$. If $\phi \in \Gamma_{\mathbb{K}}^{\times}(D)$ and $\phi^{\prime} \in \Gamma_{\mathbb{K}}^{\times}\left(D^{\prime}\right)$, then $\left\|\phi \phi^{\prime}\right\|_{g+g^{\prime}} \leqslant\|\phi\|_{g}\left\|\phi^{\prime}\right\|_{g^{\prime}}$, so that

$$
-\log \|\phi\|_{g}-\log \left\|\phi^{\prime}\right\|_{g^{\prime}} \leqslant-\log \left\|\phi \phi^{\prime}\right\|_{g+g^{\prime}} \leqslant \nu_{1, \mathbb{K}}^{\text {asy }}\left(D+D^{\prime}, g+g^{\prime}\right) .
$$

Therefore one has (1).

(2) Clearly we may assume that $a>0$ and $\Gamma_{\mathbb{K}}^{\times}(D) \neq \emptyset$. Then one has a bijective correspondence $\left(s \in \Gamma_{\mathbb{K}}^{\times}(D)\right) \mapsto\left(s^{a} \in \Gamma_{\mathbb{K}}^{\times}(a D)\right)$. Moreover, $\widehat{\operatorname{deg}}_{\xi_{a g}}\left(s^{a}\right)=a \widehat{\operatorname{deg}}_{\xi_{g}}(s)$ for $s \in \Gamma_{\mathbb{K}}^{\times}(D)$. Thus the assertion follows.

Theorem 6.4.3. - We assume that $X$ is normal. Let $(D, g)$ be an adelic $\mathbb{R}$-Cartier divisor on $X$. If $\Gamma_{\mathbb{Q}}^{\times}(D) \neq \emptyset$, then $\nu_{1, \mathbb{Q}}^{\text {asy }}(D, g)=\nu_{1, \mathbb{R}}^{\text {asy }}(D, g)$. In particular, if $D$ is big, then $\nu_{1}^{\text {asy }}(a(D, g))=a \nu_{1}^{\text {asy }}(D, g)$ for all $a \in \mathbb{R}_{\geqslant 0}$.

Proof. - By our assumption, we can find $\psi \in \Gamma_{\mathbb{Q}}^{\times}(D)$. Then the map

$$
\alpha_{\psi}: \Gamma_{\mathbb{K}}^{\times}(D) \rightarrow \Gamma_{\mathbb{K}}^{\times}(D+(\psi))
$$

given by $\phi \mapsto \phi \psi^{-1}$ is bijective and, for $\phi \in \Gamma_{\mathbb{K}}^{\times}(D),\|\phi\|_{g}=\left\|\alpha_{\psi}(\phi)\right\|_{g-\log |\psi|}$, so that

$$
\nu_{1, \mathbb{K}}^{\text {asy }}(D, g)=\nu_{1, \mathbb{K}}^{\text {asy }}(D+(\psi), g-\log |\psi|) .
$$

Therefore we may assume that $D$ is effective. Moreover, for an integrable function $\varphi$ on $\Omega$,

$$
\nu_{1, \mathbb{K}}^{\text {asy }}(D, g+\varphi)=\nu_{1, \mathbb{K}}^{\text {asy }}(D, g)+\int_{\Omega} \varphi \nu(d \omega),
$$

so that we may further assume that

$$
\int_{\Omega}-\log \|1\|_{g_{\omega}} \nu(d \omega) \geqslant 0
$$

For $\phi \in \Gamma_{\mathbb{R}}^{\times}(D)$, we choose $s_{1}, \ldots, s_{r} \in K(X)^{\times} \otimes_{\mathbb{Z}} \mathbb{Q}$ and $a_{1}, \ldots, a_{r} \in \mathbb{R}$ such that $\phi=s_{1}^{a_{1}} \cdots s_{r}^{a_{r}}$ and $a_{1}, \ldots, a_{r}$ are linearly independent over $\mathbb{Q}$. We set $\|x\|_{0}=$ $\left|x_{1}\right|+\cdots+\left|x_{r}\right|$ and $s^{x}=s_{1}^{x_{1}} \cdots s_{r}^{x_{r}}$ for $x=\left(x_{1}, \ldots, x_{r}\right) \in \mathbb{R}^{r}$, so that if we denote $\left(a_{1}, \ldots, a_{r}\right)$ by $\alpha$, then $\phi=s^{\alpha}$. By Proposition 2.4.18, for any a positive rational number $\varepsilon$, there is a positive number $\delta$ such that if $\left\|\alpha^{\prime}-\alpha\right\|_{0} \leqslant \delta$ for $\alpha^{\prime} \in \mathbb{R}^{r}$, then $(1+\varepsilon) D+\left(s^{\alpha^{\prime}}\right)$ is effective. We choose a basis $\left\{\omega_{1}, \ldots, \omega_{r}\right\}$ of $\mathbb{Q}^{r}$ such that $\left\|\omega_{j}-a\right\|_{0} \leqslant \delta$ for all $j \in\{1, \ldots, r\}$, so that $(1+\varepsilon) D+\left(s^{\omega_{j}}\right) \geqslant 0$ for all $j$. Here we set $\alpha=\lambda_{1} \omega_{1}+\cdots+\lambda_{r} \omega_{r}$. Further, if we define a norm $\|\cdot\|_{\omega}$ by $\| x_{1} \omega_{1}+\cdots+$ $x_{r} \omega_{r} \|_{\omega}=\left|x_{1}\right|+\cdots+\left|x_{r}\right|$ for $x_{1}, \ldots, x_{r} \in \mathbb{R}$, then there is a positive constant $C$ such that $C\|\cdot\|_{0} \leqslant\|\cdot\|_{\omega}$. Note that for any $t>0$, there is $\alpha^{\prime} \in \mathbb{Q}^{r}$ such that if we set $\alpha^{\prime}=\lambda_{1}^{\prime} \omega_{1}+\cdots+\lambda_{r}^{\prime} \omega_{r}$, then $\lambda_{j}^{\prime} \geqslant \lambda_{j}(\forall j)$ and $\left\|\alpha^{\prime}-\alpha\right\|_{\omega} \leqslant t$. Indeed, for each $j$, one can find $\lambda_{j}^{\prime} \in \mathbb{Q}$ such that $0 \leqslant \lambda_{j}^{\prime}-\lambda_{j} \leqslant t / r$, and hence $\left\|\alpha^{\prime}-\alpha\right\|_{\omega} \leqslant t$. Therefore, we can also choose a sequence $\left\{\alpha_{n}\right\}_{n=1}^{\infty}$ of $\mathbb{Q}^{r}$ with the following properties:

(i) $\alpha_{n} \in \alpha+\mathbb{R}_{\geqslant 0} \omega_{1}+\cdots+\mathbb{R}_{\geqslant 0} \omega_{r}$ for all $n \geqslant 1$. 
(ii) $\left\|\alpha_{n}-\alpha\right\|_{\omega} \leqslant \min \{\varepsilon /((1+\varepsilon) n), C \delta\}$ for all $n \geqslant 1$.

Since $\left\|\alpha_{n}-\alpha\right\|_{0} \leqslant \delta$ by (ii), $(1+\varepsilon) D+\left(s^{\alpha_{n}}\right) \geqslant 0$ for all $n \geqslant 1$. Moreover, if we set $\alpha_{n}-\alpha=\sum_{j=1}^{r} \lambda_{j}^{(n)} \omega_{j}$, then $\lambda_{j}^{(n)} \geqslant 0(\forall j)$ and $\sum_{j=1}^{r} \lambda_{j}^{(n)} \leqslant \varepsilon /((1+\varepsilon) n)$. Therefore, if we denote $\varepsilon-(1+\varepsilon) \sum_{j=1}^{r} \lambda_{j}^{(n)}$ by $\kappa_{n}$, then $\kappa_{n} \geqslant 0$ and, for each $\omega \in \Omega$,

$$
\left|s^{\alpha_{n}}\right|_{(1+\varepsilon) g_{\omega}}=\left|s^{\alpha}\right|_{g_{\omega}}\left|s^{\omega_{1} \lambda_{1}^{(n)}} \cdots s^{\omega_{r} \lambda_{r}^{(n)}}\right|_{\varepsilon g_{\omega}}=|\phi|_{g_{\omega}}\left|s^{\omega_{1}}\right|_{(1+\varepsilon) g_{\omega}}^{\lambda_{1}^{(n)}} \cdots\left|s^{\omega_{r}}\right|_{(1+\varepsilon) g_{\omega}}^{\lambda_{r}^{(n)}}|1|_{\kappa_{n} g_{\omega}},
$$

so that $\left\|s^{\alpha_{n}}\right\|_{(1+\varepsilon) g_{\omega}} \leqslant\|\phi\|_{g_{\omega}}\left\|s^{\omega_{1}}\right\|_{(1+\varepsilon) g_{\omega}}^{\lambda_{1}^{(n)}} \cdots\left\|s_{\omega_{r}}\right\|_{(1+\varepsilon) g_{\omega}}^{\lambda_{r}^{(n)}}\|1\|_{g_{\omega}}^{\kappa_{n}}$. Therefore, since

$$
\int_{\Omega}-\log \left\|s^{\alpha_{n}}\right\|_{(1+\varepsilon) g_{\omega}} \nu(d \omega) \leqslant \nu_{1, \mathbb{Q}}^{\text {asy }}((1+\varepsilon)(D, g))
$$

and $\kappa_{n} \int_{\Omega}-\log \|1\|_{g_{\omega}} \nu(d \omega) \geqslant 0$, one has

$$
\int_{\Omega}-\log \|\phi\|_{g_{\omega}} \nu(d \omega)+\sum_{j=1}^{r} \lambda_{j}^{(n)} \int_{\Omega}-\log \left\|s^{\omega_{j}}\right\|_{(1+\varepsilon) g_{\omega}} \nu(d \omega) \leqslant \nu_{1, \mathbb{Q}}^{\text {asy }}((1+\varepsilon)(D, g)),
$$

so that taking $n \rightarrow \infty$, we obtain

$$
\int_{\Omega}-\log \|\phi\|_{g_{\omega}} \nu(d \omega) \leqslant \nu_{1, \mathbb{Q}}^{\text {asy }}((1+\varepsilon)(D, g)),
$$

and hence, as $\varepsilon$ is a rational number, by Proposition 6.4.2, (2), one can see

$$
\nu_{1, \mathbb{R}}^{\text {asy }}(D, g) \leqslant \nu_{1, \mathbb{Q}}^{\text {asy }}((1+\varepsilon)(D, g))=(1+\varepsilon) \nu_{1, \mathbb{Q}}^{\text {asy }}(D, g),
$$

which implies $\nu_{1, \mathbb{R}}^{\text {asy }}(D, g) \leqslant \nu_{1, \mathbb{Q}}^{\text {asy }}(D, g)$, as required.

Proposition 6.4.4. - Let $(D, g)$ be an adelic $\mathbb{K}$-Cartier divisor on $X$. We assume that either $\mathbb{K}=\mathbb{Z}$ or $X$ is normal. Then one has

$$
\widehat{\mu}_{\text {ess }}(D, g) \geqslant \widehat{\mu}_{\max }\left(H_{\mathbb{K}}^{0}(X, D), \xi_{g}\right) \text {. }
$$

In particular,

$$
\widehat{\mu}_{\mathrm{ess}}(D, g) \geqslant \widehat{\mu}_{\max }^{\mathrm{asy}}(D, g) \text {. }
$$

Proof. - The second inequality is a consequence of the first inequality because $\widehat{\mu}_{\text {ess }}(n D, n g)=n \widehat{\mu}_{\text {ess }}(D, g)$.

Let $U$ be a non-empty Zariski open set of $X$ given by

$$
\left\{x \in X: X \rightarrow \operatorname{Spec} K \text { is smooth at } x \text { and } x \notin \operatorname{Supp}_{\mathbb{K}}(D)\right\} \text {. }
$$

Note that $\exp \left(-g_{\omega}\right)$ is a positive continuous function on $U_{\omega}^{\text {an }}$ for each $\omega \in \Omega$ and that, for $\phi \in H_{\mathbb{K}}^{0}(X, D)$ and $x \in U$, one has $\phi \in \mathcal{O}_{X, x}$. Let $t$ be a real number such that $\widehat{\mu}_{\text {ess }}(D, g)<t$. Then there is an infinite subset $\Lambda$ of $U\left(K^{\text {ac }}\right)$ such that $\Lambda$ is Zariski dense in $U$ and $h_{(D, g)}(P) \leqslant t$ for all $P \in \Lambda$. 
Let $F$ be a non-zero vector subspace of $H_{\mathbb{K}}^{0}(X, D)$. Then there exist $P_{1}, \ldots, P_{\operatorname{dim} F} \in$ $\Lambda$ such that the evaluation map

$$
f: F \otimes_{K} K^{\mathrm{ac}} \longrightarrow \bigoplus_{i=1}^{\operatorname{dim} F} \kappa\left(P_{i}\right)
$$

is a bijection. For $\chi \in \Omega_{K^{\text {an }}}$, let $P_{i, \chi}$ be the unique extension of $P_{i} \in X_{K^{\text {an }}}$ to $\left(X_{K^{\text {an }}}\right)_{\chi}^{\text {an }}$. Let $\|\cdot\|_{P_{i, \chi}}$ be a norm of $\kappa\left(P_{i}\right)_{\chi}$ given by $\|1\|_{P_{i, \chi}}=\exp \left(-g_{\pi(\chi)}\left(\mu\left(P_{i, \chi}\right)\right)\right)$, where $\pi$ is the canonical map $\Omega_{K^{\text {an }}} \rightarrow \Omega$ and $\mu:\left(X_{K^{\text {an }}}\right)_{\chi}^{\text {an }} \rightarrow X_{\pi(\chi)}^{\text {an }}$ is also the canonical morphism as analytic spaces. We set $\xi_{i}:=\left\{\|\cdot\|_{P_{i, \chi}}\right\}_{\chi \in \Omega_{K} \text { an }}$. We equip $\bigoplus_{i=1}^{\operatorname{dim} F} \kappa\left(P_{i}\right)$ with the $\psi_{0}$-direct sum $\xi=\left\{\|\cdot\|_{\chi}\right\}_{\chi \in \Omega_{K} \text { an }}$ of $\xi_{1}, \ldots, \xi_{\operatorname{dim} F}$, where $\psi_{0}$ denotes the function from $[0,1]$ to $[0,1]$ sending $x \in[0,1]$ to $\max (x, 1-x)$ (see Subsections 1.1.10 and 4.1.1). Note that, if we denote by $\left\{e_{i}\right\}_{i=1}^{\operatorname{dim} F}$ a basis of $\bigoplus_{i=1}^{\operatorname{dim} F} \kappa\left(P_{i}\right)$ such that $e_{i} \in \kappa\left(P_{i}\right)$, then this basis is orthogonal with respect to $\|\cdot\|_{\chi}$ for any $\chi \in \Omega_{K}$ an. By Proposition 1.2.23, this basis is also an Hadamard basis with respect to $\|\cdot\|_{\chi}$ for any $\chi \in \Omega_{K^{\text {an }}}$. In particular, one has

$$
\widehat{\operatorname{deg}}\left(\bigoplus_{i=1}^{\operatorname{dim} F}\left(\kappa\left(P_{i}\right), \xi_{i}\right)\right)=\sum_{i=1}^{\operatorname{dim} F} h_{(D, g)}\left(P_{i}\right) \leqslant(\operatorname{dim} F) t .
$$

Moreover, for any $\chi \in \Omega_{K}$ ac the operator norm of $f_{\chi}$ is $\leqslant 1$. Therefore, by Proposition 4.3.17, one has

$$
\widehat{\mu}(\bar{F})=\widehat{\mu}\left(\bar{F} \otimes K^{\mathrm{an}}\right) \leqslant \frac{1}{\operatorname{dim} F} \widehat{\operatorname{deg}}\left(\bigoplus_{i=1}^{\operatorname{dim} F}\left(\kappa\left(P_{i}\right), \xi_{i}\right)\right) \leqslant t .
$$

Since $F$ is arbitrary, we obtain $\widehat{\mu}_{\max }\left(H_{\mathbb{K}}^{0}(X, D), \xi_{g}\right) \leqslant t$. Therefore 6.42) follows because $t$ is an arbitrary real number with $t>\widehat{\mu}_{\text {ess }}(L, \varphi)$.

6.4.2. Volume function. - We assume that there exists $C \geqslant 0$ such that the adelic curve $S$ verifies the tensorial minimal slope superadditivity.

Definition 6.4.5. — Let $(L, \varphi)$ be an adelic line bundle on $X$. We define the arithmetic volume of $(L, \varphi)$ as

$$
\widehat{\operatorname{vol}}(L, \varphi):=\limsup _{n \rightarrow+\infty} \frac{\widehat{\operatorname{deg}}_{+}\left(\pi_{*}\left(L^{\otimes n}, n \varphi\right)\right)}{n^{d+1} /(d+1) !} .
$$

We say that $(L, \varphi)$ is big if $\widehat{\operatorname{vol}}(L, \varphi)>0$.

Assume that the $K$-scheme $X$ admit a regular rational point of $X$. Then the local ring $\mathcal{O}_{X, P}$ is a regular local ring. By Cohen's structure theorem of complete regular local rings [54, Proposition 10.16], the formal completion of $\mathcal{O}_{X, P}$ is isomorphic to the algebra of formal series $K \llbracket T_{1}, \ldots, T_{d} \rrbracket$, where $d$ is the Krull dimension of $X$. If $L$ is an invertible $\mathcal{O}_{X}$-module, by choosing a local generator of the $\mathcal{O}_{X, P}$-module $L_{P}$, we can identify the graded linear series $\bigoplus_{n \in \mathbb{N}} H^{0}\left(X, L^{\otimes n}\right)$ as a graded sub- $K$-algebra 
(of subfinite type) of $K \llbracket T_{1}, \ldots, T_{d} \rrbracket[T]$. We denote by $\Delta(L)$ the Newton-Okounknov body of this graded algebra (see $\$ 6.3 .4$ for the construction of $\Delta(L)$ ). For any $n \in \mathbb{N}$, let $r_{n}:=\operatorname{rk}_{K}\left(H^{0}\left(X, L^{\otimes n}\right)\right)>0$. By Proposition 6.3.18 one has

$$
\int_{\Delta(L)} 1 \mathrm{~d} x=\lim _{r_{n}>0, n \rightarrow+\infty} \frac{r_{n}}{n^{\kappa}}
$$

where $\kappa$ is the Kodaira-Iitaka dimension of the graded linear series $\bigoplus_{n \in \mathbb{N}} H^{0}\left(X, L^{\otimes n}\right)$ (which is also called the Kodaira-Iitaka dimension of $L$ ). In particular, if $L$ is a big line bundle, namely

$$
\operatorname{vol}(L):=\limsup _{n \rightarrow+\infty} \frac{r_{n}}{n^{d} / d !}>0,
$$

or equivalently, $\kappa=d$, one has

$$
\operatorname{vol}(L)=d ! \int_{\Delta(L)} 1 \mathrm{~d} x
$$

If $(L, \varphi)$ is an adelic line bundle of $X$, then the family $\left\{\left(\pi_{*}\left(L^{\otimes n}, n \varphi\right)\right)\right\}_{n \in \mathbb{N}}$ forms a graded $K$-algebra of adelic vector bundles with respect to $\operatorname{Frac}\left(K \llbracket T_{1}, \ldots, T_{d} \rrbracket\right)$ (see Definition 6.3.24). For any $n \in \mathbb{N}_{\geqslant 1}$ such that $r_{n}:=\operatorname{rk}_{K}\left(H^{0}\left(X, L^{\otimes n}\right)\right)>0$, let $\mathbb{P}_{(L, \varphi), n}$ be the Borel probability measure on $\mathbb{R}$ such that, for any positive Borel function $f$ on $\mathbb{R}$, one has

$$
\int_{\mathbb{R}} f(t) \mathbb{P}_{(L, \varphi), n}(\mathrm{~d} t)=\frac{1}{r_{n}} \sum_{i=1}^{r_{n}} f\left(\frac{1}{n} \widehat{\mu}_{i}\left(\pi_{*}\left(L^{\otimes n}, n \varphi\right)\right)\right) .
$$

Theorem 6.4.6. - Assume that the scheme $X$ admits a regular rational point. Let $(L, \varphi)$ be an adelic line bundle on $X$. For any $n \in \mathbb{N}$, let $r_{n}=\operatorname{rk}_{K}\left(H^{0}\left(X, L^{\otimes n}\right)\right)$. Assume that there exists $n \in \mathbb{N}_{\geqslant 1}$ such that $r_{n}>0$. Then the sequence of measures $\left\{\mathbb{P}_{(L, \varphi), n}\right\}_{n \in \mathbb{N}, r_{n}>0}$ converges weakly to a Borel probability measure $\mathbb{P}_{(L, \varphi)}$, which is the direct image of a concave real-valued function $G_{(L, \varphi)}$ on $\Delta(L)^{\circ}$. In particular, if $(L, \varphi)$ is big, then the invertible $\mathcal{O}_{X}$-module is big. Moreover, in the case where $L$ is big, the sequence

$$
\frac{\widehat{\operatorname{deg}}_{+}\left(\pi_{*}\left(L^{\otimes n}, n \varphi\right)\right)}{n^{d+1} /(d+1) !}, \quad n \in \mathbb{N}, r_{n}>0
$$

converges to $\widehat{\operatorname{vol}}(L, \varphi)$, which is also equal to

$$
(d+1) \operatorname{vol}(L) \int_{[0,+\infty[} t \mathbb{P}_{(L, \varphi)}(\mathrm{d} t)=(d+1) \int_{\Delta(L)^{\circ}} \max \left(G_{(L, \varphi)}(x), 0\right) \mathrm{d} x .
$$

Proof. - We deduce from Corollary 6.3.26 that the sequence $\left\{\mathbb{P}_{(L, \varphi), n}\right\}_{n \in \mathbb{N}, r_{n}>0}$ converges vaguely to a Borel measure $\mathbb{P}_{(L, \varphi)}$ on $\mathbb{R}$, which is the direct image of the uniform probability measure on $\Delta(L)^{\circ}$ by a concave function $G_{(L, \varphi)}: \Delta(L)^{\circ} \rightarrow \mathbb{R} \cup\{+\infty\}$. Moreover, by Proposition 6.4.4 and 6.2.7, we obtain that the supports of the Borel probability measures $\mathbb{P}_{(L, \varphi), n}$ are uniformly bounded from above. The function $G_{(L, \varphi)}$ 
is then bounded from above and hence limit measure $\mathbb{P}_{(L, \varphi)}$ is a Borel probability measure and the sequence $\left\{\mathbb{P}_{n}\right\}_{n \in \mathbb{N}, r_{n}>0}$ converges weakly to $\mathbb{P}_{(L, \varphi)}$. In particular, the sequence $\left\{\frac{1}{n r_{n}} \widehat{\operatorname{deg}}_{+}\left(L^{\otimes n}, n \varphi\right)\right\}_{n \in \mathbb{N}, r_{n}>0}$ converges to

$$
\int_{[0,+\infty[} t \mathbb{P}_{(L, \varphi)}(\mathrm{d} t)=\frac{1}{\operatorname{vol}(\Delta(L))} \int_{\Delta(L)^{\circ}} \max \left(G_{(L, \varphi)}(x), 0\right) \mathrm{d} x
$$

since

$$
\int_{[0,+\infty[} t \mathbb{P}_{n}(\mathrm{~d} t)=\int_{\left[0, \widehat{\mu}_{\max }^{\mathrm{asy}}(L, \varphi)[\right.} t \mathbb{P}_{n}(\mathrm{~d} t)=\frac{1}{n r_{n}} \sum_{i=1}^{r_{n}} \max \left\{\widehat{\mu}_{i}\left(\pi_{*}\left(L^{\otimes n}, n \varphi\right)\right), 0\right\},
$$

and by 4.71) and (4.67),

$$
\left|\widehat{\operatorname{deg}}_{+}\left(\pi_{*}\left(L^{\otimes n}, n \varphi\right)\right)-\sum_{i=1}^{r_{n}} \max \left\{\widehat{\mu}_{i}\left(\pi_{*}\left(L^{\otimes n}, n \varphi\right)\right), 0\right\}\right| \leqslant \frac{1}{2} \ln \left(r_{n}\right) \nu\left(\Omega_{\infty}\right) .
$$

In particular, in the case where $\widehat{\operatorname{vol}}(L, \varphi)>0$, one has

$$
\limsup _{n \rightarrow+\infty} \frac{r_{n}}{n^{d}}>0
$$

namely the invertible $\mathcal{O}_{X}$-module $L$ is big. Moreover, in the case where $L$ is big, one has

$$
\lim _{n \rightarrow+\infty} \frac{r_{n}}{n^{d} / d !}=\operatorname{vol}(L)>0 .
$$

Therefore the sequence (6.43) converges (to $\widehat{\operatorname{vol}}(L, \varphi)$ by definition), which is equal to (6.44).

Theorem 6.4.7. - Assume that the scheme $X$ admits a regular rational point. Let $\left(L_{1}, \varphi_{1}\right)$ and $\left(L_{2}, \varphi_{2}\right)$ be big adelic line bundles on $X$. Then the following inequality of Brunn-Minkowski type holds

$$
\widehat{\operatorname{vol}}\left(L_{1} \otimes L_{2}, \varphi_{1}+\varphi_{2}\right)^{1 /(d+1)} \geqslant \widehat{\operatorname{vol}}\left(L_{1}, \varphi_{1}\right)^{1 /(d+1)}+\widehat{\operatorname{vol}}\left(L_{2}, \varphi_{2}\right)^{1 /(d+1)} .
$$

Proof. - For any adelic line bundle $(L, \varphi)$ on $X$ such that $L$ is big, we denote by $\widehat{\Delta}(L, \varphi)$ the closure of the convex set $\left\{(x, t) \in \Delta(L)^{\circ} \times \mathbb{R}: 0 \leqslant t \leqslant G_{(L, \varphi)}(x)\right\}$. Then Theorem 6.4.6 implies that

$$
\widehat{\operatorname{vol}}(L, \varphi)=(d+1) \int_{\widehat{\Delta}(L, \varphi)} 1 \mathrm{~d}(x, t)
$$

By Proposition 6.3.28, one has

$$
\widehat{\Delta}\left(L_{1}, \varphi_{1}\right)+\widehat{\Delta}\left(L_{2}, \varphi_{2}\right) \leqslant \widehat{\Delta}\left(L_{1} \otimes L_{2}, \varphi_{1}+\varphi_{2}\right) .
$$

Therefore the relation (6.45) follows from the classic Brunn-Minkowski inequality. 
6.4.3. Volume of adelic $\mathbb{R}$-Cartier divisors. - We assume that $X$ is normal and geometrically integral and admits a regular rational point $P$. We identify the formal completion of $\mathcal{O}_{X, P}$ with $K \llbracket T_{1}, \ldots, T_{d} \rrbracket$, which allow us to embed the rational function field $K(X)$ into the fraction field $R=\operatorname{Frac}\left(K \llbracket T_{1}, \ldots, T_{d} \rrbracket\right)$. We also suppose that there exists $C \geqslant 0$ such that the adelic curve $S$ verifies the tensorial minimal slope superadditivity of level $\geqslant C$. In the following, the symbol $\mathbb{K}$ denotes $\mathbb{Z}, \mathbb{Q}$ or $\mathbb{R}$.

Let $D$ be a $\mathbb{K}$-Cartier divisor. We identify $\bigoplus_{n \in \mathbb{N}} H^{0}(n D)$ with a graded sub- $K$ algebra of subfinite type of $R[T]$. We denote by $\Delta(D)$ the Newton-Okounkov body of this graded algebra (see $\$ 6.3 .4$ for its construction).

Definition 6.4.8. - Let $(D, g)$ be an adelic $\mathbb{K}$-Cartier divisor. We define the arithmetic volume of $(D, g)$ as

$$
\widehat{\operatorname{vol}}(D, g):=\limsup _{n \rightarrow+\infty} \frac{\widehat{\operatorname{deg}}_{+}\left(H_{\mathbb{K}}^{0}(n D), \xi_{n g}\right)}{n^{d+1} /(d+1) !}
$$

(for the definition of the norm family $\xi_{n g}$, see Definition 6.2.17). We say that $(D, g)$ is big if $\widehat{\operatorname{vol}}(D, g)>0$. Note that for any $s \in K(X)^{\times}$one has (see Remark 2.5.10)

$$
\widehat{\operatorname{vol}}((D, g)+\widehat{\operatorname{div}}(s))=\widehat{\operatorname{vol}}(D, g) .
$$

Moreover, $(D, g)$ is said to be arithmetically $\mathbb{K}$-effective, which is denoted by $(D, g) \geqslant_{\mathbb{K}}$ $(0,0)$, if $D$ is $\mathbb{K}$-effective and $g_{\omega} \geqslant 0$ for all $\omega \in \Omega$. For adelic $\mathbb{K}$-Cartier divisors $\left(D_{1}, g_{1}\right)$ and $\left(D_{2}, g_{2}\right)$ on $X$,

$$
\left(D_{1}, g_{1}\right) \geqslant_{\mathbb{K}}\left(D_{2}, g_{2}\right) \stackrel{\text { def }}{\Longleftrightarrow}\left(D_{1}, g_{1}\right)-\left(D_{2}, g_{1}\right) \geqslant_{\mathbb{K}}(0,0) .
$$

Note that if $\left(D_{1}, g_{1}\right) \geqslant_{\mathbb{K}}\left(D_{2}, g_{2}\right)$, then $\widehat{\operatorname{deg}}_{+}\left(H_{\mathbb{K}}^{0}\left(D_{1}\right), \xi_{g_{1}}\right) \geqslant \widehat{\operatorname{deg}}_{+}\left(H_{\mathbb{K}}^{0}\left(D_{2}\right), \xi_{g_{2}}\right)$. In particular, $\widehat{\operatorname{vol}}\left(D_{1}, g_{1}\right) \geqslant \widehat{\operatorname{vol}}\left(D_{2}, g_{2}\right)$. By using Prposition 6.2.12, if $D \geqslant \mathbb{K} 0$, then there is a family of $D$-Green functions $g$ over $S$ such that $(D, g) \geqslant_{\mathbb{K}}(0,0)$.

Let $(D, g)$ be an adelic $\mathbb{K}$-Cartier divisor. The family $\left\{\left(H_{\mathbb{K}}^{0}(n D), \xi_{n g}\right)\right\}_{n \in \mathbb{N}}$ forms a graded $K$-algebra of adelic vector bundles with respect to $R=\operatorname{Frac}\left(K \llbracket T_{1}, \ldots, T_{d} \rrbracket\right)$. For any $n \in \mathbb{N}_{\geqslant 1}$ such that $r_{n}:=\operatorname{rk}_{K}\left(H^{0}(n D)\right)>0$, we let $\mathbb{P}_{(D, g), n}$ be the Borel probability measure on $\mathbb{R}$ such that, for any positive Borel function on $\mathbb{R}$, one has

$$
\int_{\mathbb{R}} f(t) \mathbb{P}_{(D, g), n}(\mathrm{~d} t)=\frac{1}{r_{n}} \sum_{i=1}^{r_{n}} f\left(\frac{1}{n} \widehat{\mu}_{i}\left(H_{\mathbb{K}}^{0}(n D), \xi_{n g}\right)\right) .
$$

Theorem 6.4.9. - Let $(D, g)$ be an adelic $\mathbb{K}$-Cartier divisor. For any $n \in \mathbb{N}$, let $r_{n}=\operatorname{rk}_{K}\left(H_{\mathbb{K}}^{0}(n D)\right)$. Assume that there exists $n \in \mathbb{N}_{n \geqslant 1}$ such that $r_{n}>0$. Then the sequence of measures $\left\{\mathbb{P}_{(D, g), n}\right\}_{n \in \mathbb{N}, r_{n}>0}$ converges weakly to a Borel probability measure $\mathbb{P}_{(D, g)}$, which is the direct image of a concave real-valued function $G_{(D, g)}$ on $\Delta(D)^{\circ}$. In particular, if $(D, g)$ is big, then the $\mathbb{K}$-Cartier divisor $D$ is big. Moreover, 
in the case where $D$ is big, the sequence

$$
\frac{\widehat{\operatorname{deg}}_{+}\left(H_{\mathbb{K}}^{0}(n D), \xi_{n g}\right)}{n^{d+1} /(d+1) !}, \quad n \in \mathbb{N}, r_{n}>0
$$

converges to $\widehat{\operatorname{vol}}(D, g)$, which is also equal to

$$
(d+1) \operatorname{vol}(L) \int_{[0,+\infty[} t \mathbb{P}_{(D, g)}(\mathrm{d} t)=(d+1) \int_{\Delta(D) \circ} \max \left(G_{(D, g)}(x), 0\right) \mathrm{d} x .
$$

Proof. - We omit the proof since it is quite similar to that of Theorem 6.4.6.

Corollary 6.4.10. — Let $(D, g)$ and $(A, h)$ be adelic $\mathbb{R}$-Cartier divisors on $X$. We assume that $D$ is big. Then

$$
\lim _{t \rightarrow \infty} \frac{\widehat{\operatorname{deg}}_{+}\left(H_{\mathbb{R}}^{0}(X, t D+A), \xi_{t g+h}\right)}{t^{d+1} /(d+1) !}=\widehat{\operatorname{vol}}(D, g)
$$

where $t$ is a positive real number.

Proof. — Let us begin with the following claim:

Claim 6.4.11. - $\widehat{\operatorname{vol}}(a D, a g)=a^{d+1} \widehat{\operatorname{vol}}(D, g)$ for a positive integer a.

Proof. — By Theorem 6.4.9,

$$
\begin{aligned}
\widehat{\operatorname{vol}}(a D, a g) & =\lim _{n \rightarrow \infty} \frac{\widehat{\operatorname{deg}}_{+}\left(H_{\mathbb{K}}^{0}(n a D), \xi_{n a g}\right)}{n^{d+1} /(d+1) !} \\
& =a^{d+1} \lim _{n \rightarrow \infty} \frac{\widehat{\operatorname{deg}}_{+}\left(H_{\mathbb{K}}^{0}(n a D), \xi_{n a g}\right)}{(n a)^{d+1} /(d+1) !}=a^{d+1} \widehat{\operatorname{vol}}(D, g),
\end{aligned}
$$

as required.

Claim 6.4.12. - If $D$ is $\mathbb{R}$-effective, then the assertion of the corollary holds.

Proof. - Choose positive integers $n_{0}$ and $n_{1}$ such that $H_{\mathbb{R}}^{0}\left(X, n_{0} D+A\right) \neq\{0\}$ and $H_{\mathbb{R}}^{0}\left(X, n_{1} D-A\right) \neq\{0\}$, so that one can take $\phi \in H_{\mathbb{R}}^{0}\left(X, n_{0} D+A\right) \backslash\{0\}$ and $\psi \in$ $H_{\mathbb{R}}^{0}\left(X, n_{1} D-A\right) \backslash\{0\}$. Let us consider the following injective homomorphisms

$$
\alpha_{t}: H_{\mathbb{R}}^{0}\left(\left(\lfloor t\rfloor-n_{0}\right) D\right) \rightarrow H_{\mathbb{R}}^{0}(t D+A) \quad \text { and } \quad \beta_{t}: H_{\mathbb{R}}^{0}(t D+A) \rightarrow H_{\mathbb{R}}^{0}\left(\left(\lceil t\rceil+n_{1}\right) D\right)
$$

given by $f \mapsto f \phi$ and $f \mapsto f \psi$, respectively. Note that $\left\|\alpha_{t}(f)\right\|_{t g_{\omega}+h_{\omega}} \leqslant\|f\|_{\left(\lfloor t\rfloor-n_{0}\right) g_{\omega}}\|\phi\|_{\left(t-\lfloor t\rfloor+n_{0}\right) g_{\omega}+h_{\omega}} \leqslant\|f\|_{\left(\lfloor t\rfloor-n_{0}\right) g_{\omega}}\|\phi\|_{n_{0} g_{\omega}+h_{\omega}}\|1\|_{g_{\omega}}^{t-\lfloor t\rfloor}$, so that, by Proposition 4.3.20, (1) and (2)

$$
\begin{aligned}
\widehat{\operatorname{deg}}_{+}( & \left.H_{\mathbb{R}}^{0}\left(\left(\lfloor t\rfloor-n_{0}\right) D\right), \xi_{\left([t]-n_{0}\right) g}\right) \leqslant \widehat{\operatorname{deg}}_{+}\left(H_{\mathbb{R}}^{0}(t D+A), \xi_{t g+h}\right) \\
& +\left(\operatorname{dim}_{K} H_{\mathbb{R}}^{0}\left(\left(\lfloor t\rfloor-n_{0}\right) D\right)\right) \int_{\Omega}\left(\left|\ln \|\phi\|_{n_{0} g_{\omega}+h_{\omega}}\right|+\left|\ln \|1\|_{g_{\omega}}\right|\right) \nu(d \omega) .
\end{aligned}
$$


In the same way, one has

$$
\begin{aligned}
\widehat{\operatorname{deg}}_{+}\left(H_{\mathbb{R}}^{0}(t D+A), \xi_{t g+h}\right) \leqslant \widehat{\operatorname{deg}}_{+}\left(H_{\mathbb{R}}^{0}\left(\left(\lceil t\rceil+n_{1}\right) D\right), \xi_{\left(\lceil t\rceil+n_{1}\right) g}\right) \\
\quad+\left(\operatorname{dim}_{K} H_{\mathbb{R}}^{0}(t D+A)\right) \int_{\Omega}\left(\left|\ln \|\psi\|_{n_{1} g_{\omega}-h_{\omega}}\right|+\left|\ln \|1\|_{g_{\omega}}\right|\right) \nu(d \omega) .
\end{aligned}
$$

Note that

$$
\lim _{t \rightarrow \infty} \frac{\widehat{\operatorname{deg}}_{+}\left(H_{\mathbb{R}}^{0}\left(\left(\lfloor t\rfloor-n_{0}\right) D\right), \xi_{\left(\lfloor t\rfloor-n_{0}\right) g}\right)}{t^{d+1} /(d+1) !}=\widehat{\operatorname{vol}}(D, g), \lim _{t \rightarrow \infty} \frac{\operatorname{dim}_{K} H_{\mathbb{R}}^{0}\left(\left(\lfloor t\rfloor-n_{0}\right) D\right)}{t^{d+1} /(d+1) !}=0,
$$

so that, by (6.48), one has

$$
\widehat{\operatorname{vol}}(D, g) \leqslant \liminf _{n \rightarrow \infty} \frac{\widehat{\operatorname{deg}}_{+}\left(H_{\mathbb{R}}^{0}(t D+A), \xi_{t g+h}\right)}{t^{d} /(d+1) !} .
$$

Similarly, by using (6.49),

$$
\limsup _{n \rightarrow \infty} \frac{\widehat{\operatorname{deg}}_{+}\left(H_{\mathbb{R}}^{0}(t D+A), \xi_{t g+h}\right)}{t^{d} /(d+1) !} \leqslant \widehat{\operatorname{vol}}(D, g) .
$$

Thus the assertion of the claim follows.

Claim 6.4.13. - If there is $\phi \in K(X)^{\times}$such that $D^{\prime}:=D+(\phi)$ is $\mathbb{R}$-effective, then the assertion of the corollary holds.

Proof. — We set $\left(D^{\prime}, g^{\prime}\right)=(D, g)+\widehat{(\phi)}$. We choose an arithmetically $\mathbb{R}$-effective adelic Cartier divisor $(B, k)$ on $X$ such that $(B, k) \pm \widehat{(\phi)}$ are arithmetically $\mathbb{R}$-effective. Then, as $(B, k) \pm(t-\lfloor t\rfloor) \widehat{(\phi)}$ are arithmetically $\mathbb{R}$-effective, one has $t\left(D^{\prime}, g^{\prime}\right)+(A, h)-(B, k)-\lfloor t\rfloor \widehat{(\phi)} \leqslant t(D, g)+(A, h) \leqslant t\left(D^{\prime}, g^{\prime}\right)+(A, h)+(B, k)+\lfloor t\rfloor \widehat{(\phi)}$.

Thus

$$
\begin{aligned}
\widehat{\operatorname{deg}}_{+}\left(H_{\mathbb{R}}^{0}\left(t D^{\prime}+A-B\right), \xi_{t g^{\prime}+h-k}\right) \leqslant \widehat{\operatorname{deg}}_{+} & \left(H_{\mathbb{R}}^{0}(t D+A), \xi_{t g+h}\right) \\
& \leqslant \widehat{\operatorname{deg}}_{+}\left(H_{\mathbb{R}}^{0}\left(t D^{\prime}+A+B\right), \xi_{t g^{\prime}+h+k}\right),
\end{aligned}
$$

so that, by using Claim 6.4.12,

$$
\begin{aligned}
& \widehat{\operatorname{vol}}\left(D^{\prime}, g^{\prime}\right)=\lim _{t \rightarrow \infty} \frac{\widehat{\operatorname{deg}}_{+}\left(H_{\mathbb{R}}^{0}\left(t D^{\prime}+A-B\right), \xi_{t g^{\prime}+h-k}\right)}{t^{d+1} /(d+1) !} \\
& \leqslant \liminf _{t \rightarrow \infty} \frac{\widehat{\operatorname{deg}_{+}}\left(H_{\mathbb{R}}^{0}(t D+A), \xi_{t g+h}\right)}{t^{d+1} /(d+1) !} \leqslant \limsup _{t \rightarrow \infty} \frac{\widehat{\operatorname{deg}}_{+}\left(H_{\mathbb{R}}^{0}(t D+A), \xi_{t g+h}\right)}{t^{d+1} /(d+1) !} \\
& \leqslant \lim _{t \rightarrow \infty} \frac{\widehat{\operatorname{deg}}_{+}\left(H_{\mathbb{R}}^{0}\left(t D^{\prime}+A+B\right), \xi_{t g^{\prime}+h+k}\right)}{t^{d+1} /(d+1) !}=\widehat{\operatorname{vol}}\left(D^{\prime}, g^{\prime}\right) .
\end{aligned}
$$

Therefore one has the claim because $\widehat{\operatorname{vol}}(D, g)=\widehat{\operatorname{vol}}\left(D^{\prime}, g^{\prime}\right)$ 
In general, there are a positive integer $a$ and $f \in K(X)^{\times}$such that $a D+(f)$ is $\mathbb{R}$-effective, so that, by using Claim 6.4.11 and Claim 6.4.13, one has

$$
\begin{aligned}
\widehat{\operatorname{vol}}(D, g) & =\frac{1}{a^{d+1}} \widehat{\operatorname{vol}}(a D, a g)=\frac{1}{a^{d+1}} \lim _{t \rightarrow \infty} \frac{\widehat{\operatorname{deg}}_{+}\left(H_{\mathbb{R}}^{0}(t a D+A), \xi_{t a g+h}\right)}{t^{d+1} /(d+1) !} \\
& =\lim _{t \rightarrow \infty} \frac{\widehat{\operatorname{deg}}_{+}\left(H_{\mathbb{R}}^{0}(t a D+A), \xi_{t a g+h}\right)}{(t a)^{d+1} /(d+1) !}=\lim _{t \rightarrow \infty} \frac{\widehat{\operatorname{deg}}_{+}\left(H_{\mathbb{R}}^{0}(t D+A), \xi_{t g+h}\right)}{t^{d+1} /(d+1) !}
\end{aligned}
$$

as required.

Corollary 6.4.14. - Let $(D, g)$ be an adelic $\mathbb{R}$-Cartier divisor on $X$. Then, for any $a \in \mathbb{R}_{\geqslant 0}, \widehat{\operatorname{vol}}(a D, a g)=a^{d+1} \widehat{\operatorname{vol}}(D, g)$.

Proof. - Clearly we may assume that $a>0$. If $D$ is not big, then $a D$ is also not big, so that $\widehat{\operatorname{vol}}(D, g)=0$ and $\widehat{\operatorname{vol}}(a D, a g)=0$. Thus the assertion follows in this case. If $D$ is big, then by Corollary 6.4.10.

$$
\begin{aligned}
\widehat{\operatorname{vol}}(a D, a g) & =\lim _{t \rightarrow \infty} \frac{\widehat{\operatorname{deg}}_{+}\left(H_{\mathbb{K}}^{0}(t a D), \xi_{\text {tag }}\right)}{t^{d+1} /(d+1) !} \\
& =a^{d+1} \lim _{t \rightarrow \infty} \frac{\widehat{\operatorname{deg}}_{+}\left(H_{\mathbb{K}}^{0}(t a D), \xi_{\text {tag }}\right)}{(t a)^{d+1} /(d+1) !}=a^{d+1} \widehat{\operatorname{vol}}(D, g),
\end{aligned}
$$

as required.

Remark 6.4.15. - Let $\left(D_{1}, g_{1}\right)$ and $\left(D_{2}, g_{2}\right)$ be adelic $\mathbb{K}$-Cartier divisors. Proposition 6.3 .28 shows that, if $(x, y) \in \Delta\left(D_{1}\right)^{\circ} \times \Delta\left(D_{2}\right)^{\circ}$, one has

$$
G_{\left(D_{1}+D_{2}, g_{1}+g_{2}\right)}(x+y) \geqslant G_{\left(D_{1}, g_{1}\right)}(x)+G_{\left(D_{2}, g_{2}\right)}(y) .
$$

Similar to Theorem 6.4.7, an analogue of Brunn-Minkowski inequality holds for adelic $\mathbb{K}$-Cartier divisors.

Theorem 6.4.16. - Let $\left(D_{1}, g_{1}\right)$ and $\left(D_{2}, g_{2}\right)$ be big adelic $\mathbb{K}$-Cartier divisors on $X$. Then the following inequality holds

$$
\widehat{\operatorname{vol}}\left(D_{1}+D_{2}, g_{1}+g_{2}\right)^{1 /(d+1)} \geqslant \widehat{\operatorname{vol}}\left(D_{1}, g_{1}\right)^{1 /(d+1)}+\widehat{\operatorname{vol}}\left(D_{2}, g_{2}\right)^{1 /(d+1)} \text {. }
$$

Proof. - The proof of (6.50) is similar to that of (6.45), which relies on the inequality

$$
\forall(x, y) \in \Delta\left(D_{1}\right) \times \Delta\left(D_{2}\right), \quad G_{\left(D_{1}+D_{2}, g_{1}+g_{2}\right)}(x+y) \geqslant G_{\left(D_{1}, g_{1}\right)}(x)+G_{\left(D_{2}, g_{2}\right)}(y) .
$$

Let us consider a criterion for the bigness of adelic $\mathbb{K}$-Cartier divisors.

Lemma 6.4.17. — Let $(D, g)$ be an adelic $\mathbb{K}$-Cartier divisor on $X$ such that $D$ is big. Then $\sup _{x \in \Delta(D)}{ }^{\circ} G_{(D, g)}(x)$ is equal to $\widehat{\mu}_{\max }^{\text {asy }}(D, g)$. 
Proof. - By (6.32), we obtain that the maximal value of $G_{(D, g)}$ is equal to $\lim _{n \rightarrow+\infty} \frac{1}{n} \widehat{\mu}_{1}\left(H_{\mathbb{K}}^{0}(n D), \xi_{n g}\right)$. Note that all norm families $\xi_{n g}$ are ultrametric on $\Omega \backslash \Omega_{\infty}$.

By Remark 4.3.47 and the relation

$$
\lim _{n \rightarrow+\infty} \frac{1}{n} \ln \operatorname{rk}_{K}\left(H_{\mathbb{K}}^{0}(n D)\right)=0,
$$

we obtain the equality $\sup _{x \in \Delta(D)^{\circ}} G_{(D, g)}(x)=\widehat{\mu}_{\max }^{\text {asy }}(D, g)$.

Proposition 6.4.18. - Let $(D, g)$ be an adelic $\mathbb{K}$-Cartier divisor on $X$. Then the following are equivalent:

(1) $(D, g)$ is big.

(2) $D$ is big and $\widehat{\mu}_{\max }^{\text {asy }}(D, g)>0$.

Proof. - First of all, note that $\sup _{x \in \Delta(D)^{\circ}} G_{(D, g)}(x)=\widehat{\mu}_{\max }^{\text {asy }}(D, g)$ by Lemma 6.4.17 Moreover, by Theorem 6.4.9

$$
\widehat{\operatorname{vol}}(D, g)=(d+1) \int_{\Delta(D)^{\circ}} \max \left(G_{(D, g)}(x), 0\right) \mathrm{d} x,
$$

(1) $\Longrightarrow(2)$ : By the above facts, one has

$$
\widehat{\operatorname{vol}}(D, g) \leqslant(d+1) \operatorname{vol}(D) \max \left(\widehat{\mu}_{\text {max }}^{\text {asy }}(D, g), 0\right) .
$$

Therefore, the assertion follows.

$(2) \Longrightarrow(1)$ : First of all, as $D$ is big, $\Delta(D)^{\circ} \neq \emptyset$. Moreover, since $\widehat{\mu}_{\max }^{\text {asy }}(D, g)>0$ and $G_{(D, g)}$ is continuous on $\Delta(D)^{\circ}$, one can find a non-empty open set $U$ of $\Delta(D)^{\circ}$ such that $G_{(D, g)}>0$ on $U$, so that the assertion follows from (6.51).

Definition 6.4.19. - An adelic $\mathbb{K}$-Cartier divisor $(D, g)$ is strongly big if $D$ is big and $\nu_{1}^{\text {asy }}(D, g)>0$, that is, $D$ is big and there are positive integer $a$ and $\phi \in H_{\mathbb{K}}^{0}(a D) \backslash\{0\}$ such that $\widehat{\operatorname{deg}}_{\xi_{a g}}(\phi)>0$. Note that strong bigness implies bigness by Proposition 6.4.1 and Proposition 6.4.18, Moreover if $S$ satisfies the Minkowski property of certain level, then strong bigness is equivalent to bigness by Proposition 6.4.1 and Proposition 6.4.18

Proposition 6.4.20. - Let $(D, g)$ be an adelic $\mathbb{K}$-Cartier divisor on $X$ such that $D$ is big. Then there is an integrable function $\varphi$ on $\Omega$ such that $(D, g+\varphi)$ is strongly big.

Proof. - Since $D$ is big, there are a positive integer $a$ and $f \in K(X)^{\times}$such that $a D+(f)$ is effective. By Proposition 6.2.12 a function given by $\omega \mapsto \ln \|f\|_{a g_{\omega}}$ is 
integrable. Thus if we set

$$
\varphi(\omega):= \begin{cases}\frac{1}{a}\left(\ln \|f\|_{a g_{\omega}}+\ln 2\right) & \text { if } \omega \in \Omega_{\infty}, \\ \frac{1}{a} \ln \|f\|_{a g_{\omega}} & \text { if } \omega \in \Omega \backslash \Omega_{\infty},\end{cases}
$$

then $(\omega \in \Omega) \mapsto \varphi(\omega)$ is integrable. Let $F_{n}$ be a vector subspace of $H_{\mathbb{K}}^{0}(n a D)$ generated by $f^{n}$. Then

$$
\begin{aligned}
\widehat{\operatorname{deg}}_{\xi_{n a(g+\varphi)}}\left(F_{n}\right) & =-\int_{\Omega} \ln \left\|f^{n}\right\|_{n a\left(g_{\omega}+\varphi(\omega)\right)} \nu(\mathrm{d} \omega)=-n \int_{\Omega}\left(\ln \|f\|_{a g_{\omega}}-a \varphi(\omega)\right) \nu(\mathrm{d} \omega) \\
& =n \int_{\Omega_{\infty}}(\ln 2) \nu(\mathrm{d} \omega),
\end{aligned}
$$

so that

$$
\nu_{1}\left(H^{0}(n a D), \xi_{n a(g+\varphi)}\right) \geqslant \widehat{\operatorname{deg}}_{\xi_{n a(g+\varphi)}}\left(F_{n}\right)=n \int_{\Omega_{\infty}}(\ln 2) \nu(\mathrm{d} \omega),
$$

which shows that $\nu_{1}^{\text {asy }}(D, g+\varphi)>0$, so that $(D, g+\varphi)$ is strongly big.

Definition 6.4.21. — Let $(D, g)$ and $\left(D^{\prime}, g^{\prime}\right)$ be adelic $\mathbb{K}$-Cartier divisors on $X$. We define $\left(D^{\prime}, g^{\prime}\right) \precsim(D, g)$ to be

$$
\left(D^{\prime}, g^{\prime}\right) \precsim(D, g) \stackrel{\text { def }}{\Longleftrightarrow} \text { either }\left(D^{\prime}, g^{\prime}\right)=(D, g) \text { or }(D, g)-\left(D^{\prime}, g^{\prime}\right) \text { is big. }
$$

Proposition 6.4.22. — (1) The relation $\precsim$ forms a partial order on the group of adelic $\mathbb{K}$-Cartier divisors on $X$.

(2) For adelic $\mathbb{K}$-Cartier divisors $(D, g),\left(D^{\prime}, g^{\prime}\right),(E, h)$ and $\left(E^{\prime}, h^{\prime}\right)$ on $X$, if $\left(D^{\prime}, g^{\prime}\right) \precsim(D, g)$ and $\left(E^{\prime}, h^{\prime}\right) \precsim(E, h)$, then $\left(D^{\prime}, g^{\prime}\right)+\left(E^{\prime}, h^{\prime}\right) \precsim(D, g)+(E, h)$ and $a\left(D^{\prime}, g^{\prime}\right) \precsim a(D, g)$ for $a \in \mathbb{K}_{\geqslant 0}$.

(3) For adelic $\mathbb{K}$-Cartier divisors $(D, g)$ and $\left(D^{\prime}, g^{\prime}\right)$ on $X$, if $\left(D^{\prime}, g^{\prime}\right) \precsim(D, g)$, then $\widehat{\operatorname{vol}}\left(D^{\prime}, g^{\prime}\right) \leqslant \widehat{\operatorname{vol}}(D, g)$.

Proof. - (1) We assume that $\left(D^{\prime}, g^{\prime}\right) \precsim(D, g)$ and $(D, g) \precsim\left(D^{\prime}, g^{\prime}\right)$. If $\left(D^{\prime}, g^{\prime}\right) \neq$ $(D, g)$, then $(D, g)-\left(D^{\prime}, g^{\prime}\right)$ and $\left(D^{\prime}, g^{\prime}\right)-(D, g)$ are big, so that

$$
(0,0)=\left((D, g)-\left(D^{\prime}, g^{\prime}\right)\right)+\left(\left(D^{\prime}, g^{\prime}\right)-(D, g)\right)
$$

is also big by Theorem 6.4.16, which is a contradiction. Next let us see that if $\left(D_{1}, g_{1}\right) \precsim\left(D_{2}, g_{2}\right)$ and $\left(D_{2}, g_{2}\right) \precsim\left(D_{3}, g_{3}\right)$, then $\left(D_{1}, g_{1}\right) \precsim\left(D_{3}, g_{3}\right)$. Indeed, this is a consequence of Theorem 6.4.16 because

$$
\left(D_{3}, g_{3}\right)-\left(D_{1}, g_{1}\right)=\left(\left(D_{3}, g_{3}\right)-\left(D_{2}, g_{2}\right)\right)+\left(\left(D_{2}, g_{2}\right)-\left(D_{1}, g_{1}\right)\right) .
$$

(2) is the consequences of Theorem 6.4.16 and Corollary 6.4.14 because

$$
\left\{\begin{array}{l}
((D, g)+(E, h))-\left(\left(D^{\prime}, g^{\prime}\right)+\left(E^{\prime}, h^{\prime}\right)\right)=\left((D, g)-\left(D^{\prime}, g^{\prime}\right)\right)+\left((E, h)-\left(E^{\prime}, h^{\prime}\right)\right), \\
a(D, g)-a\left(D^{\prime}, g^{\prime}\right)=a\left((D, g)-\left(D^{\prime}, g^{\prime}\right)\right) .
\end{array}\right.
$$


(3) We may assume that $(D, g)-\left(D^{\prime}, g^{\prime}\right)$ is big. If $\left(D^{\prime}, g^{\prime}\right)$ is big, then the assertion follows from Theorem 6.4.16 because $(D, g)=\left((D, g)-\left(D^{\prime}, g^{\prime}\right)\right)+\left(D^{\prime}, g^{\prime}\right)$. Otherwise, the assertion is obvious because $\widehat{\operatorname{vol}}\left(D^{\prime}, g^{\prime}\right)=0$.

Proposition 6.4.23. — Let $(D, g)$ be a big adelic $\mathbb{K}$-Cartier divisor on $X$ and $(A, h)$ be an adelic $\mathbb{R}$-Cartier divisor on $X$. Then there is a positive integer $n_{0}$ such that $n(D, g)+(A, h)$ is big for all $n \in \mathbb{Z}_{\geqslant n_{0}}$.

Proof. - It is sufficient to find a positive integer $n_{0}$ such that $n_{0}(D, g)+(A, h)$ is big because $n(D, g)+(A, h)=n_{0}(D, g)+(A, h)+\left(n-n_{0}\right)(D, g)$.

As $D$ is big, one can find a positive integer $m$ such that $m D+A$ is big, so that, by Prioposition 6.4.20, $(m D+A, m g+h+\phi)$ is big for some non-negative integrable function $\phi$ on $\Omega$. Let $a$ be a positive integer such that

$$
\widehat{\operatorname{vol}}(D, g)>\frac{(d+1) \operatorname{vol}(D)}{a} \int_{\Omega} \phi \nu(d \omega) \text {. }
$$

Since

$$
\widehat{\operatorname{vol}}(D, g-\phi / a) \geqslant \widehat{\operatorname{vol}}(D, g)-\frac{(d+1) \operatorname{vol}(D)}{a} \int_{\Omega} \phi \nu(d \omega)>0
$$

by using Proposition 4.3.20, (2), one obtains $(D, g-\phi / a)$ is big. Thus the assertion follows because

$$
(m+a)(D, g)+(A, h)=(m D+A, m g+h+\phi)+a(D, g-\phi / a) .
$$

Theorem 6.4.24. - Let $(D, g),\left(D_{1}, g_{1}\right), \ldots,\left(D_{n}, g_{n}\right)$ be adelic $\mathbb{R}$-Cartier divisors on $X$. Then

$$
\lim _{\varepsilon_{1} \rightarrow 0, \ldots, \varepsilon_{n} \rightarrow 0} \widehat{\operatorname{vol}}\left((D, g)+\varepsilon_{1}\left(D_{1}, g_{1}\right)+\cdots+\varepsilon_{n}\left(D_{n}, g_{n}\right)\right)=\widehat{\operatorname{vol}}(D, g) .
$$

Proof. - Let us begin with the following Claim 6.4.25, Claim 6.4.26, Claim 6.4.27 and Claim 6.4.28.

Claim 6.4.25. - Let $(E, h)$ be an adelic $\mathbb{R}$-Cartier divisor on $X$. Let $(0, f)$ be an adelic Cartier divisor on $X$. Then $\lim _{\varepsilon \rightarrow 0} \widehat{\operatorname{vol}}(E, h+\varepsilon f)=\widehat{\operatorname{vol}}(E, h)$.

Proof. - We set $\varphi_{1}(\omega)=\sup _{x \in X_{\omega}}\left\{f_{\omega}(x)\right\}$ and $\varphi_{2}(\omega)=\sup _{x \in X_{\omega}}\left\{-f_{\omega}(x)\right\}$. Then, by Proposition 6.2.12, $\varphi_{1}(\omega)$ and $\varphi_{2}(\omega)$ are integrable on $\Omega$, so that $\varphi(\omega)=\max \left\{\left|\varphi_{1}(\omega)\right|,\left|\varphi_{2}(\omega)\right|\right\}$ is also integrable on $\Omega$ and $\left|f_{\omega}(x)\right| \leqslant \varphi(\omega)$ for all $x \in X_{\omega}$ and $\omega \in \Omega$. Therefore,

$$
h_{\omega}-|\varepsilon| \varphi(\omega) \leqslant h_{\omega}+\varepsilon f_{\omega} \leqslant h_{\omega}+|\varepsilon| \varphi(\omega),
$$

so that, by Proposition 4.3.20, (1),

$$
\widehat{\operatorname{deg}}_{+}\left(H_{\mathbb{K}}^{0}(n E), \mathrm{e}^{n|\varepsilon| \varphi} \xi_{n h}\right) \leqslant \widehat{\operatorname{deg}}_{+}\left(H_{\mathbb{K}}^{0}(n E), \xi_{n(h+\varepsilon f)}\right) \leqslant \widehat{\operatorname{deg}}_{+}\left(H_{\mathbb{K}}^{0}(n E), \mathrm{e}^{-n|\varepsilon| \varphi} \xi_{n h}\right) .
$$


Moreover, by Proposition 4.3.20, (2),

$$
\begin{aligned}
\widehat{\operatorname{deg}}_{+}\left(H_{\mathbb{K}}^{0}(n E), \xi_{n h}\right) \leqslant \widehat{\operatorname{deg}}_{+}\left(H_{\mathbb{K}}^{0}(n E), \mathrm{e}^{-n|\varepsilon| \varphi} \xi_{n h}\right) \\
\quad \leqslant \widehat{\operatorname{deg}}_{+}\left(H_{\mathbb{K}}^{0}(n E), \xi_{n h}\right)+n|\varepsilon| \operatorname{dim}_{K}\left(H_{\mathbb{K}}^{0}(n E)\right) \int_{\Omega} \varphi \nu(\mathrm{d} \omega),
\end{aligned}
$$

and

$$
\begin{aligned}
\widehat{\operatorname{deg}}_{+}\left(H_{\mathbb{K}}^{0}(n E), \mathrm{e}^{n|\varepsilon| \varphi} \xi_{n h}\right) & \leqslant \widehat{\operatorname{deg}}_{+}\left(H_{\mathbb{K}}^{0}(n E), \xi_{n h}\right) \\
& \leqslant \widehat{\operatorname{deg}}_{+}\left(H_{\mathbb{K}}^{0}(n E), \mathrm{e}^{n|\varepsilon| \varphi} \xi_{n h}\right)+n|\varepsilon| \operatorname{dim}_{K}\left(H_{\mathbb{K}}^{0}(n E)\right) \int_{\Omega} \varphi \nu(\mathrm{d} \omega) .
\end{aligned}
$$

Therefore the assertion of the claim follows.

Claim 6.4.26. - Let $(B, f)$ be an adelic $\mathbb{R}$-Cartier divisor on $X$ such that $(B, f) \pm$ $\left(D_{i}, g_{i}\right)$ is big for every $i=1, \ldots, n$. Then

$$
\begin{aligned}
\widehat{\operatorname{vol}}\left((D, g)-\left(\left|\varepsilon_{1}\right|\right.\right. & \left.\left.+\cdots+\left|\varepsilon_{n}\right|\right)(B, f)\right) \\
& \leqslant \widehat{\operatorname{vol}}\left((D, g)+\varepsilon_{1}\left(D_{1}, g_{1}\right)+\cdots+\varepsilon_{n}\left(D_{n}, g_{n}\right)\right) \\
& \leqslant \widehat{\operatorname{vol}}\left((D, g)+\left(\left|\varepsilon_{1}\right|+\cdots+\left|\varepsilon_{n}\right|\right)(B, f)\right)
\end{aligned}
$$

Proof. - Since

$$
\left\{\begin{array}{l}
\left|\varepsilon_{i}\right|(B, f)-\varepsilon_{i}\left(D_{i}, g_{i}\right)=\left|\varepsilon_{i}\right|\left((B, f) \pm\left(D_{i}, g_{i}\right)\right), \\
\varepsilon_{i}\left(D_{i}, g_{i}\right)+\left|\varepsilon_{i}\right|(B, f)=\left|\varepsilon_{i}\right|\left((B, f) \pm\left(D_{i}, g_{i}\right)\right),
\end{array}\right.
$$

one has $-\left|\varepsilon_{i}\right|(B, f) \precsim \varepsilon_{i}\left(D_{i}, g_{i}\right) \precsim\left|\varepsilon_{i}\right|(B, f)$ by Proposition 6.4.22. (2), so that, by using Proposition 6.4.22, (2) again,

$$
\begin{aligned}
(D, g)-\left(\left|\varepsilon_{1}\right|+\cdots+\right. & \left.\left|\varepsilon_{n}\right|\right)(B, f) \\
& \precsim(D, g)+\varepsilon_{1}\left(D_{1}, g_{1}\right)+\cdots+\varepsilon_{n}\left(D_{n}, g_{n}\right) \\
& \precsim(D, g)+\left(\left|\varepsilon_{1}\right|+\cdots+\left|\varepsilon_{n}\right|\right)(B, f) .
\end{aligned}
$$

Therefore, by Proposition 6.4.22, (3), one obtains the claim.

Claim 6.4.27. - Let $\left(H, g_{H}\right)$ be an adelic $\mathbb{R}$-Cartier divisor on $X$. Then there is an integrable function $\psi$ on $S$ such that $\left(H, g_{H}-\psi\right)$ is not big.

Proof. - Proposition 6.2.16 and Proposition 6.4.4 one obtains $\widehat{\mu}_{\max }^{\text {asy }}\left(H, g_{H}\right)<\infty$, so that one can find an integrable function $\psi$ on $S$ such that

$$
\widehat{\mu}_{\max }^{\text {asy }}\left(H, g_{H}\right)<\int_{\Omega} \psi \nu(\mathrm{d} \omega) .
$$

We choose a positive integer $n_{0}$ such that

$$
\widehat{\mu}_{\max }\left(H_{\mathbb{R}}^{0}(X, n H), \xi_{n g_{H}}\right) \leqslant \int_{\Omega} n \psi \nu(\mathrm{d} \omega)
$$


for all $n \geqslant n_{0}$. Thus, as $\xi_{n\left(g_{H}-\psi\right)}=\exp (n \psi) \xi_{n g_{H}}$, by Lemma 4.3.35, (1),

$$
\widehat{\mu}_{\max }\left(H_{\mathbb{R}}^{0}(X, n H), \xi_{n\left(g_{H}-\psi\right)}\right)=\widehat{\mu}_{\max }\left(H_{\mathbb{R}}^{0}(X, n H), \xi_{n g_{H}}\right)-\int_{\Omega} n \psi \nu(\mathrm{d} \omega) \leqslant 0,
$$

so that the assertion follows from Lemma 4.3.35 (2).

Claim 6.4.28. - Let $\left(H, g_{H}\right)$ be an adelic $\mathbb{R}$-Cartier divisor on $X$ and $\varphi$ be an integrable function on $\Omega$. Then

$$
\widehat{\operatorname{vol}}\left(H, g_{H}+\varphi\right) \leqslant \widehat{\operatorname{vol}}\left(H, g_{H}\right)+(d+1) \operatorname{vol}(H) \int_{\Omega}|\varphi(\omega)| \nu(\mathrm{d} \omega)
$$

Proof. - As $\xi_{n\left(g_{H}+\varphi\right)}=\exp (-n \varphi) \xi_{n g_{H}}$, by using Proposition 4.3.20, (2),

$$
\begin{aligned}
\widehat{\operatorname{deg}}_{+}\left(H_{\mathbb{R}}^{0}(X, n H), \xi_{n\left(g_{H}+\varphi\right)}\right) \leqslant \widehat{\operatorname{deg}}_{+}\left(H_{\mathbb{R}}^{0}(\right. & \left.X, n H), \xi_{n g_{H}}\right) \\
& +n\left(\operatorname{dim}_{K} H_{\mathbb{R}}^{0}(n H)\right) \int_{\Omega}|\varphi(\omega)| \nu(\mathrm{d} \omega),
\end{aligned}
$$

so that the assertion follows.

First we assume that $D$ is big. By Proposition 6.4 .20 , we can choose a $D$-Green functions family $g^{\prime}$ such that $\left(D, g^{\prime}\right)$ is a big adelic $\mathbb{K}$-Cartier divisor. Then, by Proposition 6.4.23, one can choose a positive integer $a$ such that $a\left(D, g^{\prime}\right) \pm\left(D_{i}, g_{i}\right)$ is big for every $i=1, \ldots, n$. Then, by Claim 6.4.26,

$$
\begin{aligned}
\widehat{\operatorname{vol}}\left((D, g)-a\left(\left|\varepsilon_{1}\right|\right.\right. & \left.\left.+\cdots+\left|\varepsilon_{n}\right|\right)\left(D, g^{\prime}\right)\right) \\
& \leqslant \widehat{\operatorname{vol}}\left((D, g)+\varepsilon_{1}\left(D_{1}, g_{1}\right)+\cdots+\varepsilon_{n}\left(D_{n}, g_{n}\right)\right) \\
& \leqslant \widehat{\operatorname{vol}}\left((D, g)+a\left(\left|\varepsilon_{1}\right|+\cdots+\left|\varepsilon_{n}\right|\right)\left(D, g^{\prime}\right)\right)
\end{aligned}
$$

If we set $f=g^{\prime}-g$ and $\varepsilon=\left|\varepsilon_{1}\right|+\cdots+\left|\varepsilon_{n}\right|$, then

$$
\left\{\begin{array}{l}
(D, g)-a\left(\left|\varepsilon_{1}\right|+\cdots+\left|\varepsilon_{n}\right|\right)\left(D, g^{\prime}\right)=(1-a \varepsilon)\left((D, g)+\left(0, \frac{a \varepsilon}{1-a \varepsilon} f\right)\right), \\
(D, g)+a\left(\left|\varepsilon_{1}\right|+\cdots+\left|\varepsilon_{n}\right|\right)\left(D, g^{\prime}\right)=(1+a \varepsilon)\left((D, g)+\left(0, \frac{a \varepsilon}{1+a \varepsilon} f\right)\right) .
\end{array}\right.
$$

Therefore, by Claim 6.4.25

$$
\begin{aligned}
\lim _{\varepsilon_{1} \rightarrow 0, \ldots, \varepsilon_{n} \rightarrow 0} \widehat{\operatorname{vol}}\left((D, g)-a\left(\left|\varepsilon_{1}\right|+\cdots+\left|\varepsilon_{n}\right|\right)\left(D, g^{\prime}\right)\right) \\
=\lim _{\varepsilon \rightarrow 0}(1-a \varepsilon)^{d+1} \widehat{\operatorname{vol}}\left((D, g)+\left(0, \frac{a \varepsilon}{1-a \varepsilon} f\right)\right)=\widehat{\operatorname{vol}}(D, g) .
\end{aligned}
$$

In the same way,

$$
\lim _{\varepsilon_{1} \rightarrow 0, \ldots, \varepsilon_{n} \rightarrow 0} \widehat{\operatorname{vol}}\left((D, g)+a\left(\left|\varepsilon_{1}\right|+\cdots+\left|\varepsilon_{n}\right|\right)\left(D, g^{\prime}\right)\right)=\widehat{\operatorname{vol}}(D, g) .
$$

One has the theorem in the case where $D$ is big. 
Next we assume that $D$ is not big. Let $(A, h)$ be a big adelic Cartier divisor on $X$ such that $D+A$ is big and $(A, h) \pm\left(D_{i}, g_{i}\right)$ are big for every $i=1, \ldots, n$. Then, by Claim 6.4.26, if we set $\varepsilon=\left|\varepsilon_{1}\right|+\cdots+\left|\varepsilon_{n}\right|$, then

$$
0 \leqslant \widehat{\operatorname{vol}}\left((D, g)+\varepsilon_{1}\left(D_{1}, g_{1}\right)+\cdots+\varepsilon_{n}\left(D_{n}, g_{n}\right)\right) \leqslant \widehat{\operatorname{vol}}((D, g)+\varepsilon(A, h)),
$$

and hence one need to show that $\lim _{\varepsilon \downarrow 0} \widehat{\operatorname{vol}}((D, g)+\varepsilon(A, h))=0$. By Claim 6.4.27. one can choose a non-negative integrable function $\varphi$ on $\Omega$ such that $(D, g)+(A, h)-(0, \varphi)$ is not big. Then, as $(D, g)-(0, \varphi)+\varepsilon(A, h) \precsim(D, g)+(A, h)-(0, \varphi)+\varepsilon(A, h)$, one has

$$
\widehat{\operatorname{vol}}((D, g)-(0, \varphi)+\varepsilon(A, h)) \leqslant \widehat{\operatorname{vol}}((D, g)+(A, h)-(0, \varphi)+\varepsilon(A, h)) .
$$

Since $D+A$ is big, by the previous case,

$$
\lim _{\varepsilon \downarrow 0} \widehat{\operatorname{vol}}((D, g)+(A, h)-(0, \varphi)+\varepsilon(A, h))=\widehat{\operatorname{vol}}((D, g)+(A, h)-(0, \varphi))=0,
$$

and hence

$$
\lim _{\varepsilon \downarrow 0} \widehat{\operatorname{vol}}((D, g)-(0, \varphi)+\varepsilon(A, h))=0 .
$$

On the other hand, by Claim 6.4.28

$\widehat{\operatorname{vol}}((D, g)+\varepsilon(A, h)) \leqslant \widehat{\operatorname{vol}}((D, g)-(0, \varphi)+\varepsilon(A, h))+(d+1) \operatorname{vol}(D+\varepsilon A) \int_{\Omega} \varphi(\omega) \nu(d \omega)$.

As $D$ is not big, one obtains

$$
\lim _{\varepsilon \downarrow 0} \operatorname{vol}(D+\varepsilon A)=\operatorname{vol}(D)=0,
$$

and hence, by (6.53), one has $\lim _{\varepsilon \downarrow 0} \widehat{\operatorname{vol}}((D, g)+\varepsilon(A, h))=0$, as required.

Corollary 6.4.29. - Let $H$ be a finite-dimensional vector space of $\widehat{\operatorname{Div}}_{\mathbb{R}}(X)$. Then the set $\{(D, g) \in H \mid(D, g)$ is big $\}$ is an open cone in $H$.

Proof. - The openness of it is a consequence of Theorem 6.4.24 One can check that it is a cone by Theorem 6.4.16 and Corollary 6.4.14

Corollary 6.4.30. - The volume function $\widehat{\mathrm{vol}}: \widehat{\operatorname{Div}}_{\mathbb{R}}(X) \rightarrow \mathbb{R}$ factors through $\widehat{\operatorname{Div}}_{\mathbb{R}}(X)$ modulo the vector subspace over $\mathbb{R}$ generated by principal Cartier divisors, that is,

$$
\widehat{\operatorname{vol}}\left((D, g)+a_{1} \widehat{\left(f_{1}\right)}+\cdots+a_{r} \widehat{\left(f_{r}\right)}\right)=\widehat{\operatorname{vol}}(D, g)
$$

for any $r \in \mathbb{Z}_{\geqslant 1},(D, g) \in \widehat{\operatorname{Div}}_{\mathbb{R}}(X), f_{1}, \ldots, f_{r} \in K(X)^{\times}$and $a_{1}, \ldots, a_{r} \in \mathbb{R}$.

Proof. - If $a_{1}, \ldots, a_{r} \in \mathbb{Z}$, then the assertion is obvious. Next we assume that $a_{1}, \ldots, a_{r} \in \mathbb{Q}$. We choose a positive integer $N$ such that $N a_{1}, \ldots, N a_{r} \in \mathbb{Z}$. Then

$$
\begin{aligned}
N^{d+1} \widehat{\operatorname{vol}}(D, g) & =\widehat{\operatorname{vol}}(N D, N g)=\widehat{\operatorname{vol}}\left((N D, N g)+\left(N a_{1}\right) \widehat{\left(f_{1}\right)}+\cdots+\left(N a_{r}\right) \widehat{\left(f_{r}\right)}\right) \\
& =N^{d+1} \widehat{\operatorname{vol}}\left((D, g)+a_{1} \widehat{\left(f_{1}\right)}+\cdots+a_{r} \widehat{\left(f_{r}\right)}\right)
\end{aligned}
$$


as required. In general, take sequences $\left\{a_{1, n}\right\}_{n=1}^{\infty}, \ldots,\left\{a_{r, n}\right\}_{n=1}^{\infty}$ of rational numbers such that $a_{1}=\lim _{n \rightarrow \infty} a_{1, n}, \ldots, a_{r}=\lim _{n \rightarrow \infty} a_{r, n}$. Then, by Theorem 6.4 .24 .

$$
\begin{aligned}
\widehat{\operatorname{vol}}\left((D, g)+a_{1} \widehat{\left(f_{1}\right)}+\cdots+a_{r} \widehat{\left(f_{r}\right)}\right) & =\lim _{n \rightarrow \infty} \widehat{\operatorname{vol}}\left((D, g)+a_{1, n} \widehat{\left(f_{1}\right)}+\cdots+a_{r, n} \widehat{\left(f_{r}\right)}\right) \\
& =\widehat{\operatorname{vol}}(D, g),
\end{aligned}
$$

so that the assertion follows. 



\section{CHAPTER 7}

\section{NAKAI-MOISHEZON'S CRITERION}

In this chapter, we fix an adelic curve $S=(K,(\Omega, \mathcal{A}, \nu), \phi)$. We assume that, either the $\sigma$-algebra $\mathcal{A}$ is discrete, or the field $K$ admits a countable subfield which is dense in each $K_{\omega}, \omega \in \Omega$.

\subsection{Graded algebra of adelic vector bundles}

Let $C$ be a non-negative real number. In this section, we assume that the adelic curve $S$ satisfies the tensorial minimal slope property of level $\geqslant C$. Namely, for any pair $(\bar{E}, \bar{F})$ of non-zero adelic vector bundles on $S$, one has

$$
\widehat{\mu}_{\min }\left(\bar{E} \otimes_{\varepsilon, \pi} \bar{F}\right) \geqslant \widehat{\mu}_{\min }(\bar{E})+\widehat{\mu}_{\min }(\bar{F})-C\left(\ln \left(\mathrm{rk}_{K}(E) \cdot \mathrm{rk}_{K}(F)\right)\right) .
$$

Note that we have shown in Chapter 5 that, if the field $K$ is perfect, then the adelic curve $S$ satisfies the tensorial minimal slope super-additivity of level $\geqslant \frac{2}{3} \nu\left(\Omega_{\infty}\right)$.

Definition 7.1.1. - Let $R_{\bullet}=\bigoplus_{n \in \mathbb{N}} R_{n}$ be a graded $K$-algebra. We assume that, for any $n \in \mathbb{N}, R_{n}$ is of finite rank over $K$. For any $n \in \mathbb{N}$, let $\xi_{n}=\left\{\|\cdot\|_{n, \omega}\right\}_{\omega \in \Omega}$ be a norm family on $R_{n}$. We say that $\bar{R}_{\bullet}=\left\{\left(R_{n}, \xi_{n}\right)\right\}_{n \in \mathbb{N}}$ is a normed graded algebra on $S$ if, for any $\omega \in \Omega, \bar{R}_{\bullet} \omega=\left\{\left(R_{n, \omega},\|\cdot\|_{n, \omega}\right)\right\}_{n \in \mathbb{N}}$ forms a normed graded algebra over $K_{\omega}$, where $R_{n, \omega}:=R_{n} \otimes_{K} K_{\omega}$ (cf. Subsection 1.1.14). Moreover, if $\left(R_{n}, \xi_{n}\right)$ forms an adelic vector bundle on $S$ for all $n \in \mathbb{N}$, then $\bar{R}$. is called a graded algebra of adelic vector bundles on $S$. Furthermore, we say that $\bar{R}$. is of finite type if the underlying graded $K$-algebra $R_{\bullet}$ is of finite type over $K$.

Proposition 7.1.2. - Let $C_{0}$ be a non-negative real number. Assume that the adelic curve $S$ satisfies the tensorial minimal slope property of level $\geqslant C_{0}$. Let $\bar{R}_{\mathbf{0}}=$ $\left\{\left(R_{n}, \xi_{n}\right)\right\}$ be a graded algebra of adelic vector bundles on $S$ such that $\bar{R}_{0}$ is the trivial adelic line bundle, namely $R_{0}=K$ and for any $\omega \in \Omega$, one has $\|1\|_{\omega}=1$. Suppose in addition that $R_{\bullet}$ is generated as $R_{0}$-algebra by $R_{1}$. Then the sequence $\left\{\widehat{\mu}_{\min }\left(\bar{R}_{n}\right) / n\right\}_{n \in \mathbb{N}}$ converges to an element in $\mathbb{R} \cup\{+\infty\}$. 
Proof. - Let $(n, m)$ be a couple of positive integers. Since $R_{\bullet}$ is generated as $K$ algebra by $R_{1}$, the canonical $K$-linear map $f_{n, m}: R_{n} \otimes_{K} R_{m} \rightarrow R_{n+m}$ is surjective. Moreover, if we equip $R_{n} \otimes_{K} R_{m}$ with the $\varepsilon, \pi$-tensor product $\xi_{n} \otimes_{\varepsilon, \pi} \xi_{m}$, then, by the submultiplicativity condition, the homomorphism $f_{n, m}$ has height $\leqslant 1$. By Proposition 4.3.30 one has

$$
\widehat{\mu}_{\min }\left(\bar{R}_{n} \otimes_{\varepsilon, \pi} \bar{R}_{m}\right) \leqslant \widehat{\mu}_{\min }\left(\bar{R}_{n+m}\right) .
$$

Moreover, by the assumption of tensorial minimal slope property,

$$
\widehat{\mu}_{\text {min }}\left(\bar{R}_{n} \otimes_{\varepsilon, \pi} \bar{R}_{m}\right) \geqslant \widehat{\mu}_{\min }\left(\bar{R}_{n}\right)+\widehat{\mu}_{\min }\left(\bar{R}_{m}\right)-C_{0}\left(\ln \left(\mathrm{rk}_{K}\left(R_{n}\right)\right)+\ln \left(\mathrm{rk}_{K}\left(R_{m}\right)\right)\right) .
$$

Note that $R_{\bullet}$ is a quotient $K$-algebra of $K\left[R_{1}\right]$. Hence $\operatorname{rk}_{K}\left(R_{n}\right)=O\left(n^{\mathrm{rk}_{K}\left(R_{1}\right)-1}\right)$. By [38, Proposition 1.3.5] ${ }^{(1)}$, the sequence $\left\{\widehat{\mu}_{\min }\left(\bar{R}_{n}\right) / n\right\}_{n \in \mathbb{N}}$ converges to an element in $\mathbb{R} \cup\{+\infty\}$.

Definition 7.1.3. - Let $\bar{R}$. be a normed graded algebra on $S$. Let $M_{\bullet}=\bigoplus_{n \in \mathbb{Z}} M_{n}$ be a $\mathbb{Z}$-graded $K$-linear space and $h$ be a positive integer. We say that $M_{\bullet}$ is a $h$-graded $R_{\bullet}$-module if $M_{\bullet}$ is equipped with a structure of $R_{\bullet}$-module such that

$$
\forall(n, m) \in \mathbb{N} \times \mathbb{Z}, \quad \forall(a, x) \in R_{n} \times M_{m}, \quad \text { ax } \in M_{n h+m} .
$$

Let $M_{\boldsymbol{0}}$ be an $h$-graded $R_{\mathbf{0}}$-module. Assume that each homogeneous component $M_{n}$ is of finite rank over $K$ and is equipped with a norm family $\xi_{n}^{\prime}=\left\{\|\cdot\|_{n, \omega}^{\prime}\right\}_{\omega \in \Omega}$. We say that $\bar{M}_{\bullet}=\left\{\bar{M}_{n}\right\}_{n \in \mathbb{Z}}$ is a normed $h$-graded $\bar{R}_{\bullet}$-module if, for any $\omega \in \Omega$, $\bar{M}_{\bullet, \omega}=\left\{\left(M_{n, \omega},\|\cdot\|_{n, \omega}^{\prime}\right)\right\}_{n \in \mathbb{Z}}$ forms a normed $h$-graded $\bar{R}_{\bullet}, \omega$-module, where $M_{n, \omega}:=$ $M_{n} \otimes_{K} K_{\omega}$ (cf. Subsetion 1.1.14). We say that an $h$-graded $\bar{R}_{\bullet}$-module $\bar{M}_{\bullet}$. is of finite type if the underlying $h$-graded $R_{\bullet}$-module $M_{\bullet}$ is of finite type. Moreover, if $\left(M_{n}, \xi_{n}^{\prime}\right)$ forms an adelic vector bundle on $S$ for all $n \in \mathbb{Z}$, then $\bar{M}$. is called a $h$-graded $\bar{R}_{\bullet}$-module of adelic vector bundles on $S$.

Proposition 7.1.4. - Let $C_{0}$ be a non-negative constant. We assume that the adelic curve $S$ satisfies the tensorial minimal slope property of level $\geqslant C_{0}$. Let $\bar{R}$. be a graded algebra of adelic vector bundles which is of finite type, and $\bar{M}$. be an h-graded $\bar{R}$.-module of adelic vector bundles on $S$ such that $\bar{M}$. is of finite type, where $h$ is a positive integer. Then one has

$$
\liminf _{n \rightarrow+\infty} \frac{\widehat{\mu}_{\min }\left(\bar{M}_{n}\right)}{n} \geqslant \frac{1}{h} \liminf _{n \rightarrow+\infty} \frac{\widehat{\mu}_{\min }\left(\bar{R}_{n}\right)}{n}>-\infty .
$$

1. In the statement of [38. Proposition 1.3.5], we suppose given a positive sequence $\left\{b_{n}\right\}_{n \in \mathbb{N}, n \geqslant 1}$ satisfying the weak subadditivity condition $b_{n+m} \leqslant b_{n}+b_{m}+f(n)+f(m)$, where $f: \mathbb{N}_{\geqslant 1} \rightarrow \mathbb{R}_{+}$ is a non-decreasing function such that $\sum_{\alpha \geqslant 0} f\left(2^{\alpha}\right) / 2^{\alpha}<+\infty$. Then the sequence $\left\{b_{n} / n\right\}_{n \in \mathbb{N}, n \geqslant 1}$ converges in $\mathbb{R}_{+}$. However the same proof applies to a general (not necessarily positive) sequence satisfying the same weak subadditivity condition and leads to the convergence of the sequence $\left\{b_{n} / n\right\}_{n \in \mathbb{N}, n \geqslant 1}$ in $\mathbb{R} \cup\{-\infty\}$. 
Proof. - If we replace $\bar{R}_{0}$ by the trivial adelic line bundle, we obtain a new graded algebra of adelic vector bundles (denoted by $\bar{R}_{\bullet}^{\prime}$ ) and $\bar{M}$. is naturally equipped with a structure of module over this graded algebra of adelic vector bundles. Moreover, $R$. is a finite $R_{0}^{\prime}$-algebra since $R_{0}$ is supposed to be of finite rank over $K$. In particular, $M_{\bullet}$ is a module of finite type over $R_{\bullet}^{\prime}$. If $\left\{a_{i}\right\}_{i \in I}$ is a basis of $R_{0}$ over $K$ which contains $1 \in R_{0}$ and if $\left\{b_{j}\right\}_{j \in J}$ is a finite family of homogeneous elements of positive degree in $R_{\bullet}$, which generates $R_{\bullet}$ as $R_{0}$-algebra, then $R_{\bullet}^{\prime}$ is generated as $K$-algebra by $\left\{a_{i} b_{j}\right\}_{(i, j) \in I \times J}$. This shows that $R_{\bullet}^{\prime}$ is a $K$-algebra of finite type. Therefore (by replacing $\bar{R}$. by $\bar{R}_{\bullet}^{\prime}$ ) we may assume without loss of generality that $\bar{R}_{0}$ is the trivial adelic line bundle.

We first prove the proposition in the particular case where $R_{\bullet}$ is generated as $K$ algebra by $R_{1}$. Let $A$ be the infimum limit of the sequence $\left\{\widehat{\mu}_{\min }\left(\bar{R}_{n}\right) / n\right\}_{n \in \mathbb{N}, n \geqslant 1}$. By [71, Lemma 2.1.6], there exist integers $b_{1}$ and $m>0$ such that, for any integer $b$ with $b \geqslant b_{1}$ and any integer $\ell \geqslant 1$ the canonical $K$-linear map $R_{\ell m} \otimes_{K} M_{b} \rightarrow M_{b+\ell m h}$ is surjective. Hence by Proposition 4.3.30, one has

$$
\widehat{\mu}_{\min }\left(\bar{M}_{b+\ell m h}\right) \geqslant \widehat{\mu}_{\min }\left(\bar{R}_{\ell m} \otimes_{\varepsilon, \pi} \bar{M}_{b}\right)
$$

which leads to

$$
\widehat{\mu}_{\min }\left(\bar{M}_{b+\ell m h}\right) \geqslant \widehat{\mu}_{\min }\left(\bar{R}_{\ell m}\right)+\widehat{\mu}_{\min }\left(\bar{M}_{b}\right)-C_{0} \ln \left(\operatorname{rk}_{K}\left(R_{\ell m}\right) \cdot \operatorname{rk}_{K}\left(M_{b}\right)\right) .
$$

Dividing the two sides of the inequality by $\ell m h$ and then letting $\ell$ tend to the infinity, we obtain

$$
\liminf _{\ell \rightarrow+\infty} \frac{\widehat{\mu}_{\min }\left(\bar{M}_{b+\ell m h}\right)}{\ell m h} \geqslant \frac{1}{h} A
$$

where we have used the fact that

$$
\lim _{\ell \rightarrow+\infty} \frac{\ln \left(\operatorname{rk}_{K}\left(R_{\ell m}\right)\right)}{\ell}=0 .
$$

Since $b \geqslant b_{1}$ is arbitrary, we obtain

$$
\liminf _{n \rightarrow+\infty} \frac{\widehat{\mu}_{\min }\left(\bar{M}_{n}\right)}{n} \geqslant \frac{1}{h} A
$$

We now consider the general case. By [71, Lemma 2.1.6], there exists a positive integer $u$ such that $R_{\bullet}^{(u)}:=\bigoplus_{n \in \mathbb{N}} R_{u n}$ is generated as $K$-algebra by $R_{1}^{(u)}=R_{u}$. Moreover, $R_{\bullet}$ is a $u$-graded $R^{(u)}$-module of finite type and hence a finite $R_{\bullet}^{(u)}$-algebra. Therefore $M_{\bullet}$ is an $h u$-graded $R_{\bullet}^{(u)}$-algebra of finite type. Let $B$ be the infimum limit of the sequence $\left\{\widehat{\mu}_{\min }\left(\bar{R}_{n u}\right) / n\right\}_{n \in \mathbb{N}, n \geqslant 1}$. By applying the particular case of the proposition established above, we obtain

$$
\liminf _{n \rightarrow+\infty} \frac{\widehat{\mu}_{\min }\left(\bar{R}_{n}\right)}{n} \geqslant \frac{B}{u} \text { and } \liminf _{n \rightarrow+\infty} \frac{\widehat{\mu}_{\min }\left(\bar{M}_{n}\right)}{n} \geqslant \frac{B}{h u} .
$$

Note that the first inequality actually implies that

$$
\liminf _{n \rightarrow+\infty} \frac{\widehat{\mu}_{\min }\left(\bar{R}_{n}\right)}{n}=\frac{B}{u}
$$


since $\left(\widehat{\mu}_{\min }\left(\bar{R}_{n u}\right) / n\right)_{n \in \mathbb{N}, n \geqslant 1}$ converges to $B$. The inequality (7.2) is thus proved.

Finally, if the adelic curve $S$ satisfies the minimal slope property, by Proposition 7.1.2 the sequence $\left(\widehat{\mu}_{\min }\left(\bar{R}_{n u}\right) / n\right)_{n \in \mathbb{N}, n \geqslant 1}$ converges to some element $\mathbb{R} \cup\{+\infty\}$. Hence the last statement of the proposition is true.

Remark 7.1.5. - Let $\bar{R}$. be a graded algebra of adelic vector bundles, $I_{\bullet}$ be a homogeneous ideal of $R_{\bullet}$ and $R_{\bullet}^{\prime}$ be the quotient algebra $R_{\bullet} / I_{\bullet}$. If we equip $R_{n}^{\prime}$ with the quotient norm family of that of $R_{n}$, then $\bar{R}_{0}^{\prime}$. is a graded algebra of adelic vector bundles, denoted $\overline{R_{\bullet} / I_{\bullet}}$ (cf. Proposition $1.1 .71(1)$ ).

More generally, let $\bar{M}_{\bullet}$ be an $h$-graded $\bar{R}_{\bullet}$-module and $Q$. is a graded quotient $R_{\bullet}$-module of $M_{\bullet}$. If we equip each $Q_{n}$ with the quotient norm family of that of $\bar{M}_{n}$, then $\bar{Q}$. becomes an $h$-graded $\bar{R}_{\bullet}$-module (cf. Proposition 1.1.71](2)].

Let $\bar{R}$. be a graded algebra of adelic vector bundles, $\bar{M}$. be an $h$-graded module, where $h \in \mathbb{N}, h \geqslant 1$. Assume that $I_{\bullet}$ is a homogeneous ideal of $R_{\bullet}$. Assume that $M_{\bullet}$ is annihilated by $I_{\bullet}$, then $\bar{M}$. is naturally equipped with a structure of $h$-graded $\overline{R_{\bullet} / I_{\bullet}}$-module (cf. Proposition 1.1.71] (2)].

Proposition 7.1.6. - We suppose that the adelic curve $S$ satisfies the tensorial minimal slope property. Let $\bar{R}_{\bullet}$ be a graded algebra of adelic vector bundle, $I_{\bullet}, J_{\bullet}$ and $M_{\bullet}$ be homogeneous ideals of $R_{\bullet}$ such that $J_{\bullet} \subseteq M_{\bullet}$ and $I_{\bullet} \cdot M_{\bullet} \subseteq J_{\bullet}$. Let $R_{\bullet}^{\prime}=R_{\bullet} / I_{\bullet}$ and $Q_{\bullet}=M_{\bullet} / J_{\bullet}$. For each $n \in \mathbb{N}$, we equip $R_{n}^{\prime}$ and $Q_{n}$ with the quotient norm families of that of $\bar{R}_{n}$ and $\bar{M}_{n}$ respectively. Then one has

$$
\liminf _{n \rightarrow+\infty} \frac{\widehat{\mu}_{\min }\left(\bar{Q}_{n}\right)}{n} \geqslant \liminf _{n \rightarrow+\infty} \frac{\widehat{\mu}_{\min }\left(\bar{R}_{n}^{\prime}\right)}{n} .
$$

Proof. - By the above remark, $\bar{Q}$. is equipped with a structure of graded $\bar{R}_{\bullet}^{\prime}$-module. Hence the statement follows from Proposition 7.1.4.

\subsection{Fundamental estimations}

In this section, we prove some lower bound for the asymptotic minimal slope. We assume that the adelic curve $S$ satisfies the tensorial minimal slope property of level $\geqslant C_{0}$, where $C_{0} \geqslant 0$.

Let $\bar{R}_{\bullet}=\left\{\left(R_{n}, \xi_{n}\right)\right\}$ be a graded algebra of adelic vector bundles which is of finite type. We assume that $R_{\bullet}$ is an integral ring. Let $X=\operatorname{Proj}\left(R_{\bullet}\right)$ be the projective spectrum of $R_{\bullet}$. If $Y$ is an integral closed subscheme of $X$ and $P_{\bullet} \subseteq R_{\bullet}$ is the defining homogeneous prime ideal of $Y$, we denote by $R_{Y, \bullet}$ the quotient graded ring $R_{\bullet} / P_{\bullet}$. Note that each $R_{Y, n}$ is naturally equipped with the quotient norm family $\xi_{Y, n}$ of $\xi_{n}$ so that $\bar{R}_{Y, \bullet}$ becomes a graded algebra of adelic vector bundles (cf. Proposition 1.1.71, Proposition 4.1.19 and Proposition 4.1.24).

Theorem 7.2.1. - Let $\mathfrak{S}_{X}$ be the set of all integral closed subschemes of $X$. To each $Y \in \mathfrak{S}_{X}$ we assigne a real number $v_{Y}$, a positive integer $n_{Y}$ and a non-zero 
element $s_{Y}$ in $R_{Y, n_{Y}}$ such that $\widehat{\operatorname{deg}}_{\xi_{Y, n_{Y}}}\left(s_{Y}\right) \geqslant n_{Y} v_{Y}$. Then there exists a finite subset $\mathfrak{S}$ of $\mathfrak{S}_{X}$ such that

$$
\liminf _{n \rightarrow+\infty} \frac{\widehat{\mu}_{\min }\left(\bar{R}_{n}\right)}{n} \geqslant \min \{v(Y): Y \in \mathfrak{S}\}
$$

Proof. - Step 1: For any positive integer $h$, we set

$$
R_{n}^{(h)}:=R_{h n} \quad \text { and } \quad R^{(h)}=\bigoplus_{n \in \mathbb{N}} R_{n}^{(h)}
$$

If we assign $v_{Y}^{h}, h n_{Y}$ and $s_{Y}^{h}$ to each $Y \in \mathfrak{S}_{X}$, then $s_{Y}^{h} \in R_{Y, h n_{Y}} \backslash\{0\}$ and

$$
\widehat{\operatorname{deg}}_{\xi_{Y, h n_{Y}}}\left(s_{Y}^{h}\right) \geqslant h \cdot \widehat{\operatorname{deg}}_{\xi_{Y}, n_{Y}}\left(s_{Y}\right) \geqslant h v_{Y},
$$

so that the above assignment satisfies the condition of the theorem for $R^{(h)}$. Moreover, $R$ is a finitely generated $h$-graded $R^{(h)}$-module (cf. [106, Lemma 5.44]). By using Proposition [7.1.6, we can see that if the theorem holds for $R^{(h)}$, then it holds for $R$. Therefore, by [31, Chapitre III, $\S 1$, Proposition 3], we may assume that $R$ is generated by $R_{1}$ over $R_{0}$ and $n_{X}=1$. Let $\mathscr{O}_{X}(1)$ be the tautological invertible sheaf of $X$ arising from $R_{1}$.

We prove the theorem by induction on $d=\operatorname{dim} X$.

Step 2: In the case where $d=0, X=\operatorname{Spec}(F)$ for some finite extension field $F$ over $K$, so that $R_{n} \subseteq H^{0}\left(X, \mathscr{O}_{X}(n)\right) \cong F$. Therefore, $\operatorname{dim}_{K}\left(R_{n}\right) \leqslant[F: K]$ for all $n \in \mathbb{N}$. Let us consider the following sequence of homomorphisms:

$$
R_{0} \stackrel{s_{X}}{\longrightarrow} R_{1} \stackrel{s_{X}}{\longrightarrow} R_{2} \stackrel{s_{X}}{\longrightarrow} R_{3} \stackrel{s_{X}}{\longrightarrow} \cdots \stackrel{s_{X}}{\longrightarrow} R_{n-1} \stackrel{s_{X}}{\longrightarrow} R_{n} \stackrel{s_{X}}{\longrightarrow} \cdots
$$

Note that each homomorphism is injective and $\operatorname{dim}_{K}\left(R_{n}\right)$ is bounded, so that we can find a positive integer $N$ such that $R_{n} \stackrel{s_{X}}{\longrightarrow} R_{n+1}$ is an isomorphism for all $n \in \mathbb{N}_{N}$. Therefore, by Proposition 4.3.30.

$$
\widehat{\mu}_{\min }\left(\bar{R}_{n}\right) \geqslant \widehat{\mu}_{\min }\left(\bar{R}_{N}\right)+(n-N) \widehat{\operatorname{deg}}_{\xi_{1}}\left(s_{X}\right) \geqslant \widehat{\mu}_{\min }\left(\bar{R}_{N}\right)+(n-N) v_{X}
$$

which leads to

$$
\liminf _{n \rightarrow+\infty} \frac{\widehat{\mu}_{\min }\left(\bar{R}_{n}\right)}{n} \geqslant v_{X} .
$$

Step 3: We assume $d>0$. Let $I_{\bullet}$ be the homogeneous ideal generated by $s_{X}$, that is, $I_{\bullet}=R_{\bullet} s_{X}$. By using the same ideas as in [82, Chapter I, Proposition 7.4], we can find a sequence

$$
I_{\bullet}=I_{0, \bullet} \subsetneq I_{1, \bullet} \subsetneq \cdots \subsetneq I_{r, \bullet}=R \bullet
$$

of homogeneous ideals of $R_{\bullet}$ and non-zero homogeneous prime ideals $P_{1, \bullet}, \ldots, P_{r, \bullet}$ of $R_{\bullet}$ such that $P_{i, \bullet} \cdot I_{i, \bullet} \subseteq I_{i-1, \bullet}$ for $i \in\{1, \ldots, r\}$. 
Step 4: Consider the following sequence:

$$
\begin{aligned}
& R_{0} \quad \stackrel{\cdot s_{X}}{\longrightarrow} \quad I_{0,1} \quad \hookrightarrow \cdots \hookrightarrow \quad I_{i, 1} \quad \hookrightarrow \cdots \hookrightarrow \quad I_{r, 1}=R_{1} \\
& \begin{array}{cccccc}
\vdots & \vdots & \vdots & \vdots & \vdots & \vdots \\
\stackrel{s_{X}}{\longrightarrow} & I_{0, j} & \hookrightarrow \cdots \hookrightarrow & I_{i, j} & \hookrightarrow \cdots \hookrightarrow & I_{r, j}=R_{j}
\end{array} \\
& \stackrel{\cdot s_{X}}{\longrightarrow} I_{0, j+1} \quad \hookrightarrow \cdots \hookrightarrow \quad I_{i, j+1} \quad \hookrightarrow \cdots \hookrightarrow \quad I_{r, j+1}=R_{j+1} \\
& \begin{array}{cccccc}
\vdots & \vdots & \vdots & \vdots & \vdots & \vdots
\end{array} \\
& \stackrel{\cdot s_{X}}{\longrightarrow} \quad I_{0, n} \quad \hookrightarrow \cdots \hookrightarrow \quad I_{i, n} \quad \hookrightarrow \cdots \hookrightarrow \quad I_{r, n}=R_{n}
\end{aligned}
$$

By using Proposition 4.3.32, one has

$$
\widehat{\mu}_{\min }\left(\bar{R}_{n}\right) \geqslant \min \left\{\min _{\substack{i \in\{1, \ldots, r\} \\ j \in\{1, \ldots, n\}}} \widehat{\mu}_{\min }\left(\overline{I_{i, j} / I_{i-1, j}}\right)+(n-j) v_{X}, \widehat{\mu}_{\min }\left(\bar{R}_{0}\right)+n v_{X}\right\} .
$$

For any $i \in\{1, \ldots, r\}$ let $Y_{i}$ be the integral closed subscheme defined by $P_{i}$. By Proposition 7.1.6, one has

$$
\liminf _{m \rightarrow+\infty} \frac{\widehat{\mu}_{\min }\left(\overline{I_{i, m} / I_{i-1, m}}\right)}{m} \geqslant \liminf _{m \rightarrow+\infty} \frac{\widehat{\mu}_{\min }\left(\bar{R}_{Y_{i}, m}\right)}{m}
$$

Moreover, by the induction hypothesis, there are a finite subset $\mathfrak{S}_{i}$ of $\mathfrak{S}_{Y_{i}}$ such that

$$
\liminf _{m \rightarrow+\infty} \frac{\widehat{\mu}_{\min }\left(\bar{R}_{Y_{i}, m}\right)}{m} \geqslant \min \left\{v_{Z}: Z \in \mathfrak{S}_{i}\right\} .
$$

There the estimate (7.3) leads to

$$
\liminf _{n \rightarrow+\infty} \frac{\widehat{\mu}_{\min }\left(\bar{R}_{n}\right)}{n} \geqslant \min \left\{v_{Z}: Z \in\{X\} \cup \bigcup_{i=1}^{r} \mathfrak{S}_{i}\right\} .
$$

The theorem is thus proved.

Remark 7.2.2. - Consider the following variante of the above theorem. Assume that $R_{\bullet}$ is generated as $R_{0}$-algebra by $R_{1}$. By using Proposition 4.3.12, we obtain that, for integers $n$ and $m$ such that $1 \leqslant m \leqslant n$, one has

$$
\begin{aligned}
& \widehat{\operatorname{deg}}\left(\bar{R}_{n}\right) \geqslant \sum_{j=1}^{n} \sum_{i=1}^{r} \widehat{\operatorname{deg}}\left(\overline{I_{i, j} / I_{i-1, j}}\right)+\widehat{\operatorname{deg}}\left(\bar{R}_{0}\right)+v_{X} \sum_{k=0}^{n-1} \operatorname{rk}\left(R_{k}\right) \\
& \geqslant \sum_{j=1}^{m} \sum_{i=1}^{r} \widehat{\operatorname{deg}}\left(\overline{I_{i, j} / I_{i-1, j}}\right)+\min _{i \in\{1, \ldots, r\}} \inf _{\ell \in \mathbb{N} \geqslant m} \frac{\widehat{\mu}_{\min }\left(\overline{I_{i, \ell} / I_{i-1, \ell}}\right)}{\ell} \sum_{j=m+1}^{n} j \operatorname{rk}\left(R_{j} / R_{j-1}\right) \\
& \quad+\widehat{\operatorname{deg}}\left(\bar{R}_{0}\right)+v_{X} \sum_{k=0}^{n-1} \operatorname{rk}\left(R_{k}\right) .
\end{aligned}
$$

Dividing the two sides by $n \operatorname{rk}\left(R_{n}\right)$ and letting $n$ tend to the infinity, we obtain

$$
\liminf _{n \rightarrow+\infty} \frac{\widehat{\mu}\left(R_{n}\right)}{n} \geqslant \frac{d}{d+1} \min _{i \in\{1, \ldots, r\}} \inf _{\ell \in \mathbb{N} \geqslant m} \frac{\widehat{\mu}_{\min }\left(\overline{I_{i, \ell} / I_{i-1, \ell}}\right)}{\ell}+\frac{1}{d+1} v_{X},
$$


where we have used the geometric Hilbert-Samuel theorem asserting that $\operatorname{rk}\left(R_{n}\right)=$ $\operatorname{deg}(X) n^{d}+O\left(n^{d-1}\right)$, with $d$ being the Krull dimension of the scheme $X$, which leads to

$$
\lim _{n \rightarrow+\infty} \frac{1}{n \operatorname{rk}\left(R_{n}\right)} \sum_{j=0}^{n-1} \operatorname{rk}\left(R_{j}\right)=\frac{1}{d+1} .
$$

Since $m$ is arbitrary, we obtain

$$
\liminf _{n \rightarrow+\infty} \frac{\widehat{\mu}\left(R_{n}\right)}{n} \geqslant \frac{d}{d+1} \min _{i \in\{1, \ldots, r\}} \min _{Z \in \mathfrak{S}_{i}} v_{Z}+\frac{1}{d+1} v_{X} .
$$

Note that in the general case where $R_{\bullet}$ is not necessarily generated by $R_{1}$, the same argument leads to

$$
\limsup _{n \rightarrow+\infty} \frac{\widehat{\mu}\left(R_{n}\right)}{n} \geqslant \frac{d}{d+1} \min _{i \in\{1, \ldots, r\}} \min _{Z \in \mathfrak{S}_{i}} v_{Z}+\frac{1}{d+1} v_{X} .
$$

Under the strong tensorial minimal slope property (see Definition 6.3.30), Theorem 7.2 .1 admits the following analogue.

Theorem 7.2.3. - We assume that the adelic curve $S$ satisfies the strong tensorial minimal slope property of level $\geqslant C_{1}$, where $C_{1} \in \mathbb{R}_{\geqslant 0}$. Let $\mathfrak{S}_{X}$ be the set of all integral closed subschemes of $X$. Then one has

$$
\liminf _{n \rightarrow+\infty} \frac{\widehat{\mu}_{\min }\left(\bar{R}_{n}\right)}{n} \geqslant \inf _{Y \in \mathfrak{S}_{X}} \limsup _{m \rightarrow+\infty} \frac{\widehat{\mu}_{1}\left(\bar{R}_{Y, m}\right)}{m} .
$$

Proof. - We reason by induction on the dimension $d$ of the scheme $X$.

First we treat the case where $d=0$. Let $m$ be an integer, $m \geqslant 1$. Let $E$ be a vector subspace of $R_{m}$ such that $\widehat{\mu}_{\text {min }}(\bar{E})=\widehat{\mu}_{1}\left(\bar{R}_{m}\right)$. There exists any integer $N \in \mathbb{N}_{\geqslant 1}$ such that, for any $p \in \mathbb{N}_{\geqslant 1}$, the canonical $K$-linear map

$$
R_{m N} \otimes E^{\otimes p} \longrightarrow R_{m(N+p)}
$$

is surjective. Therefore, by Proposition 4.3 .30 and the strong tensorial tensor minimal slope property (by an argument similar to the Step 2 of the proof of Theorem 7.2.1), one has

$$
\begin{aligned}
\widehat{\mu}_{\text {min }}\left(\bar{R}_{m(N+p)}\right) & \geqslant \widehat{\mu}_{\min }\left(\bar{R}_{m N}\right)-C_{1} \ln \left(\operatorname{rk}\left(R_{m N}\right)\right)+p\left(\widehat{\mu}_{1}\left(\bar{R}_{m}\right)-C_{1} \ln (\operatorname{rk}(E))\right) \\
& \geqslant \widehat{\mu}_{\min }\left(\bar{R}_{m N}\right)-C_{1} \ln \left(\operatorname{rk}\left(R_{m N}\right)\right)+p\left(\widehat{\mu}_{1}\left(\bar{R}_{m}\right)-C_{1} \ln \left(\operatorname{rk}\left(R_{m}\right)\right)\right) .
\end{aligned}
$$

Dividing the two sides by $m(N+p)$ and letting $p$ tend to $+\infty$, by Proposition 7.1.6 we obtain

$$
\liminf _{n \rightarrow+\infty} \frac{\widehat{\mu}_{\min }\left(\bar{R}_{n}\right)}{n} \geqslant \frac{\widehat{\mu}_{1}\left(\bar{R}_{m}\right)}{m}-C_{1} \frac{\ln \left(\operatorname{rk}\left(R_{m}\right)\right)}{m} .
$$

Note that

$$
\lim _{m \rightarrow+\infty} \frac{\ln \left(\mathrm{rk}\left(R_{m}\right)\right)}{m}=0 .
$$


Therefore, by taking the limsup when $m \rightarrow+\infty$, we obtain

$$
\liminf _{n \rightarrow+\infty} \frac{\widehat{\mu}_{\min }\left(\bar{R}_{n}\right)}{n} \geqslant \limsup _{m \rightarrow+\infty} \frac{\widehat{\mu}_{1}\left(\bar{R}_{m}\right)}{m} .
$$

We now assume that $d \geqslant 1$. Let $m$ be an integer such that $m \geqslant 1$. Let $E$ be a vector subspace of $R_{m}$ such that $\widehat{\mu}_{\min }(\bar{E})=\widehat{\mu}_{1}\left(\bar{R}_{m}\right)$. Let $I$. be the homogeneous ideal of $R_{\bullet}^{(m)}=\bigoplus_{n \in \mathbb{N}} R_{m n}$ generated by $E$. That is, for any $n \in \mathbb{N}, I_{n}$ is the image of the canonical homomorphism

$$
R_{(n-1) m} \otimes E \longrightarrow R_{n m} .
$$

As in the the proof of Theorem 7.2.1, we let

$$
I_{\bullet}=I_{0, \bullet} \subsetneq I_{1, \bullet} \subsetneq \ldots \subsetneq I_{r, \bullet}=R_{\bullet}^{(m)}
$$

be a sequence of homogeneous ideals of $R_{\bullet}^{(m)}$ and $P_{1, \bullet}, \ldots, P_{r, \bullet}$ be non-zero homogeneous prime ideals of $R^{(m)}$ such that $P_{i, \bullet} \cdot I_{i, \bullet} \subset I_{i-1, \bullet}$ for $i \in\{1, \ldots, r\}$. Let $p$ be an integer in $\mathbb{N}_{\geqslant 1}$. We denote by $F_{p}$ the image of the canonical $K$-linear map

$$
R_{0} \otimes E^{\otimes p} \longrightarrow R_{m p}
$$

Consider the following sequence:

$$
\begin{aligned}
& F_{p}=I_{0,1} E^{p-1} \quad \hookrightarrow \cdots \hookrightarrow \quad I_{i, 1} E^{p-1} \quad \hookrightarrow \cdots \hookrightarrow \quad I_{r, 1} E^{p-1} \\
& \begin{array}{cccccc}
\vdots & \vdots & \vdots & \vdots & \vdots & \vdots
\end{array} \\
& =I_{0, j} E^{p-j} \quad \hookrightarrow \cdots \hookrightarrow \quad I_{i, j} E^{p-j} \quad \hookrightarrow \cdots \hookrightarrow \quad I_{r, j} E^{p-j} \\
& =I_{0, j+1} E^{p-j-1} \hookrightarrow \cdots \hookrightarrow I_{i, j+1} E^{p-j-1} \quad \hookrightarrow \cdots \hookrightarrow I_{r, j+1} E^{p-j-1} \\
& \begin{array}{cccccc}
\vdots & \vdots & \vdots & \vdots & \vdots & \vdots \\
= & I_{0, p} & \hookrightarrow \cdots \hookrightarrow & I_{i, p} & \hookrightarrow \cdots \hookrightarrow & I_{r, p}=R_{m p}
\end{array}
\end{aligned}
$$

By Proposition 4.3 .32 we obtain that

$$
\widehat{\mu}_{\min }\left(\bar{R}_{m p}\right) \geqslant \min \left\{\widehat{\mu}_{\min }\left(\bar{F}_{p}\right), \min _{\substack{i \in\{1, \ldots, r\} \\ j \in\{1, \ldots, p\}}} \widehat{\mu}_{\min }\left(\overline{I_{i, j} E^{p-j} / I_{i-1, j} E^{p-j}}\right)\right\} .
$$

By Proposition 4.3.30 and the strong tensorial tensor minimal slope property, one has

$$
\begin{aligned}
\widehat{\mu}_{\min }\left(\bar{F}_{p}\right) & \geqslant \widehat{\mu}_{\min }\left(\bar{R}_{0}\right)-C_{1} \ln \left(\operatorname{rk}\left(R_{0}\right)\right)+p\left(\widehat{\mu}_{\min }(\bar{E})-C_{1} \ln (\operatorname{rk}(E))\right) \\
& \geqslant \widehat{\mu}_{\min }\left(\bar{R}_{0}\right)-C_{1} \ln \left(\operatorname{rk}\left(R_{0}\right)\right)+p\left(\widehat{\mu}_{1}\left(\bar{R}_{m}\right)-C_{1} \ln \left(\operatorname{rk}\left(R_{m}\right)\right)\right) .
\end{aligned}
$$

Similarly, for any $(i, j) \in\{1, \ldots, r\} \times\{1, \ldots, n\}$, one has

$$
\begin{array}{r}
\widehat{\mu}_{\min }\left(\overline{I_{i, j} E^{p-j} / I_{i-1, j} E^{p-j}}\right) \geqslant \widehat{\mu}_{\min }\left(\overline{I_{i, j} / I_{i-1, j}}\right)-C_{1} \ln \left(\operatorname{rk}\left(I_{i, j} / I_{i-1, j}\right)\right) \\
+(p-j)\left(\widehat{\mu}_{\min }(\bar{E})-C_{1} \ln (\operatorname{rk}(E))\right) .
\end{array}
$$

For any $i \in\{1, \ldots, r\}$ let $Y_{i}$ be the integral closed subscheme defined by $P_{i}$. By Proposition 7.1.6, one has

$$
\liminf _{j \rightarrow+\infty} \frac{\widehat{\mu}_{\min }\left(\overline{I_{i, j} / I_{i-1, j}}\right)}{j} \geqslant \liminf _{j \rightarrow+\infty} \frac{\widehat{\mu}_{\min }\left(\bar{R}_{Y_{i}, j}\right)}{j} \geqslant \min _{Z \in \mathfrak{S}_{Y_{i}}} \limsup _{k \rightarrow+\infty} \frac{\widehat{\mu}_{1}\left(\bar{R}_{Z, k}\right)}{k},
$$


where the second inequality comes from the induction hypothesis. Therefore, if we denote by $v$ the value

$$
\inf _{Z \in \mathfrak{S}_{X}} \limsup _{k \rightarrow+\infty} \frac{\widehat{\mu}_{1}\left(\bar{R}_{Z, k}\right)}{k}
$$

then the inequality (7.5) leads to

$$
\liminf _{n \rightarrow+\infty} \frac{\widehat{\mu}_{\min }\left(\bar{R}_{n}\right)}{n}=\liminf _{p \rightarrow+\infty} \frac{\widehat{\mu}_{\min }\left(\bar{R}_{m p}\right)}{m p} \geqslant \min \left\{\frac{\widehat{\mu}_{1}\left(\bar{R}_{m}\right)}{m}-C_{1} \frac{\ln \left(\operatorname{rk}\left(R_{m}\right)\right)}{m}, v\right\},
$$

where the equality comes from Proposition 7.1.6. By taking the limsup when $m \rightarrow$ $+\infty$, we obtain

$$
\liminf _{n \rightarrow+\infty} \frac{\widehat{\mu}_{\min }\left(\bar{R}_{n}\right)}{n} \geqslant v
$$

as desired.

\subsection{A consequence of the extension property of semipositive metrics}

Let $S=(K,(\Omega, \mathcal{A}, \nu), \phi)$ be an adelic curve which satisfies the tensorial minimal slope property. We assume that, either (i) $\Omega_{0}$ is empty, or (ii) the field $K$ is countable, or (iii) $\Omega=\Omega_{0}$ and $\#\left(\Omega_{0}\right)=1$. The purpose of this section is to prove the following theorem as a consequence of the extension property of semipositive metrics (cf. Theorem 2.3.31 and Theorem 2.3.35).

Theorem 7.3.1. - Let $X$ be a geometrically reduced projective $K$-scheme, $L$ be a semiample invertible $\mathcal{O}_{X}$-module and $\varphi=\left\{\varphi_{\omega}\right\}_{\omega \in \Omega}$ be a metric family of L. Let $Y$ be a geometrically reduced closed subscheme of $X, \xi_{n}:=\left\{\|\cdot\|_{n \varphi_{\omega}, \text { sup }}\right\}_{\omega \in \Omega}$ and $\left.\xi_{n}\right|_{Y}:=$ $\left\{\|\cdot\|_{\left.n \varphi_{\omega}\right|_{Y_{\omega}}, \sup }\right\}_{\omega \in \Omega}$. Let $R_{Y, n}$ be the image of $H^{0}\left(X, L^{\otimes n}\right) \rightarrow H^{0}\left(Y,\left.L\right|_{Y} ^{\otimes n}\right)$ and $\xi_{Y, n}=$ $\left\{\|\cdot\|_{Y, n, \omega}\right\}_{\omega \in \Omega}$ be the quotient norm family on $R_{Y, n}$ obtained by $H^{0}\left(X, L^{\otimes n}\right) \rightarrow R_{Y, n}$ and $\xi_{n}$. If $\varphi$ is dominated and measurable and $\varphi_{\omega}$ is semipositive for all $\omega \in \Omega$, then we have the following:

(1) $\xi_{n},\left.\xi_{n}\right|_{Y}$ and $\xi_{Y, n}$ are dominated and measurable for all $n \geqslant 0$.

(2) For $s \in R_{Y, 1} \backslash\{0\}$, one has

$$
\lim _{n \rightarrow \infty} \frac{\widehat{\operatorname{deg}}_{\xi_{Y, n}}\left(s^{\otimes n}\right)}{n}=\widehat{\operatorname{deg}}_{\left.\xi_{1}\right|_{Y}}(s) .
$$

Proof. - (1) In the case (iii), the assertion is obvious, so that we assume (i) and (ii). First, by Theorem 6.1.13 and Theorem6.1.31, $\xi_{n}$ is dominated and measurable for $n \geqslant$ 0. Moreover, by Theorem 6.1.13 and Theorem 6.1.31 together with Proposition 6.1.12 and Proposition 6.1.27, $\left.\xi_{n}\right|_{Y}$ is dominated and measurable for $n \geqslant 0$. Finally, by virtue of Proposition 4.1.19 and Proposition 4.1.24, $\xi_{Y, n}$ is dominated and measurable for $n \geqslant 0$.

Before starting the proof of (2), we need to prepare several facts. Here we assume either (i) or (ii) or (iii). We set $\xi_{Y, n}=\left\{\|\cdot\|_{Y, n, \omega}\right\}_{\omega \in \Omega}$. We claim the following: 
Claim 7.3.2. - (a) For all $\omega \in \Omega, n \geqslant 0$ and $s \in R_{Y, n, \omega}$,

$$
\|s\|_{\left.n \varphi_{\omega}\right|_{Y_{\omega}}, \sup } \leqslant\|s\|_{Y, n, \omega} .
$$

(b) For all $\omega \in \Omega, n \geqslant 1$ and $s \in R_{Y, 1, \omega} \backslash\{0\}$,

$$
\ln \|s\|_{\left.\varphi_{\omega}\right|_{Y_{\omega}}, \sup } \leqslant \frac{\ln \left\|s^{\otimes n}\right\|_{Y, n, \omega}}{n} \leqslant \ln \|s\|_{Y, 1, \omega} .
$$

(c) For all $\omega \in \Omega$ and $s \in R_{Y, 1, \omega} \backslash\{0\}$,

$$
\lim _{n \rightarrow \infty} \frac{\ln \left\|s^{\otimes n}\right\|_{Y, n, \omega}}{n}=\ln \|s\|_{\left.\varphi_{\omega}\right|_{Y_{\omega}}, \text { sup }}
$$

Proof. - (a) Note that, for all $l \in H^{0}\left(X, L^{\otimes n}\right)$ with $\left.l\right|_{Y}=s$, one has $\|s\|_{\left.n \varphi_{\omega}\right|_{Y_{\omega}} \text {,sup }} \leqslant$ $\|l\|_{n \varphi_{\omega}, \text { sup }}$, so that the assertion follows.

(b) By Proposition 1.1.71 $\left\|s^{\otimes n}\right\|_{Y, n, \omega} \leqslant\left(\|s\|_{Y, 1, \omega}\right)^{n}$. Moreover, by (a),

$$
\left(\|s\|_{\left.\varphi_{\omega}\right|_{Y_{\omega}}, \text { sup }}\right)^{n}=\left\|s^{\otimes n}\right\|_{\left.n \varphi_{\omega}\right|_{Y_{\omega}} \text {,sup }} \leqslant\left\|s^{\otimes n}\right\|_{Y, n, \omega},
$$

so that one has (b).

(c) For a positive number $\epsilon$, by Theorem 2.3.31 and Theorem 2.3.35, there is a positive integer $n_{0}$ such that, for all $n \geqslant n_{0}$, we can find $l \in H^{0}\left(X_{\omega}, L_{\omega}^{\otimes n}\right)$ such that $\left.l\right|_{Y_{\omega}}=s^{\otimes n}$ and $\|l\|_{n \varphi_{\omega}, \text { sup }} \leqslant \mathrm{e}^{n \epsilon}\left(\|s\|_{\left.\varphi_{\omega}\right|_{Y_{\omega}}, \text { sup }}\right)^{n}$, and hence

$$
\ln \left\|s^{\otimes n}\right\|_{Y, n, \omega} \leqslant \ln \|l\|_{n \varphi_{\omega}, \sup } \leqslant n \epsilon+n \ln \|s\|_{\left.\varphi_{\omega}\right|_{Y_{\omega}}, \text { sup }} .
$$

Therefore, by (b),

$$
0 \leqslant \frac{\ln \left\|s^{\otimes n}\right\|_{Y, n, \omega}}{n}-\ln \|s\|_{\left.\varphi_{\omega}\right|_{Y_{\omega}}, \text { sup }} \leqslant \epsilon
$$

for all $n \geqslant n_{0}$, as required.

(2) By $(1),(\omega \in \Omega) \mapsto\left|\ln \|s\|_{\left.\varphi_{\omega}\right|_{Y_{\omega}} \text {,sup }}\right|$ and $(\omega \in \Omega) \mapsto\left|\ln \|s\|_{Y, 1, \omega}\right|$ are integrable. Moreover, by (b), one has

$$
\left|\frac{\ln \left\|s^{\otimes n}\right\|_{Y, n, \omega}}{n}\right| \leqslant \max \left\{\left|\ln \|s\|_{\left.\varphi_{\omega}\right|_{Y_{\omega}}, \sup }\right|,\left|\ln \|s\|_{Y, 1, \omega}\right|\right\},
$$

and hence, by Lebesgue's dominated convergence theorem together with (c),

$$
\lim _{n \rightarrow \infty} \frac{1}{n} \int_{\Omega} \ln \left\|s^{\otimes n}\right\|_{Y, n, \omega} \nu(d \omega)=\int_{\Omega} \ln \|s\|_{\left.\varphi_{\omega}\right|_{Y_{\omega}}, \sup } \nu(d \omega),
$$

which shows the assertion of the theorem. 


\subsection{Nakai-Moishezon's criterion in a general settings}

Let $S=(K,(\Omega, \mathcal{A}, \nu), \phi)$ be an adelic curve. We assume that, either (i) $\Omega_{0}$ is empty, or (ii) the field $K$ is countable, or (iii) $\Omega=\Omega_{0}$ and $\#\left(\Omega_{0}\right)=1$. In this section, let us consider the following Nakai-Moishezon's criterion in a general settings:

Theorem 7.4.1. - Let $X$ be a geometrically integral projective $K$-scheme, $L$ be an invertible $\mathcal{O}_{X}$-module and $\varphi=\left\{\varphi_{\omega}\right\}_{\omega \in \Omega}$ be a metric family of $L$. Let $\xi_{n}:=$ $\left\{\|\cdot\|_{n \varphi_{\omega}, \text { sup }}\right\}_{\omega \in \Omega}$ and $\left.\xi_{n}\right|_{Y}:=\left\{\|\cdot\|_{\left.n \varphi_{\omega}\right|_{Y_{\omega}} \text {,sup }}\right\}_{\omega \in \Omega}$ for a subvariety $Y$ of $X$. We assume the following:

(1) (Dominancy and measurability) The metric family $\varphi$ is dominated and measurable.

(2) (Semipositivity) $L$ is semiample and $\varphi_{\omega}$ is semipositive for all $\omega \in \Omega$.

(3) (Bigness) For every subvariety $Y$ of $X,\left.L\right|_{Y}$ is big, and there are a positive number $n_{Y}$ and $s_{Y} \in H^{0}\left(Y,\left.L^{\otimes n_{Y}}\right|_{Y}\right) \backslash\{0\}$ such that $\widehat{\operatorname{deg}}_{\left.\xi_{n_{Y}}\right|_{Y}}\left(s_{Y}\right)>0$.

Then one has

$$
\liminf _{n \rightarrow \infty} \frac{\hat{\mu}_{\min }\left(H^{0}\left(X, L^{\otimes n}\right), \xi_{n}\right)}{n}>0 .
$$

Moreover, if the adelic curve $S$ satisfies the strong Minkowski properties, then

$$
\liminf _{n \rightarrow \infty} \frac{\nu_{\min }\left(H^{0}\left(X, L^{\otimes n}\right), \xi_{n}\right)}{n}>0,
$$

so that, there are a positive integer $n$ and a basis $\left\{e_{i}\right\}_{i=1}^{N}$ of $H^{0}\left(X, L^{\otimes n}\right)$ such that $\widehat{\operatorname{deg}}_{\xi_{n}}\left(e_{i}\right)>0$ for $i=1, \ldots, N$.

Proof. - First of all, for each subvariety $Y$ of $X$, as $\left.L\right|_{Y}$ is nef and big, one has $\left(\left.L\right|_{Y} ^{\operatorname{dim} Y}\right)>0$, so that by the classical Nakai-Moishezon criterion, $L$ is ample.

For a subvariety $Y$ of $X$, we set

$$
\left\{\begin{array}{l}
R_{Y, n}:=\text { the image of the natural homomorphism } H^{0}\left(X, L^{\otimes n}\right) \rightarrow H^{0}\left(Y,\left.L\right|_{Y} ^{\otimes n}\right), \\
R_{Y, n, \omega}:=R_{Y, n} \otimes_{K} K_{\omega} \quad(\omega \in \Omega), \\
\|\cdot\|_{Y, n, \omega}:=\text { the quotient norm of }\|\cdot\|_{n \varphi_{\omega}, \text { sup }} \text { on } R_{Y, n, \omega} \quad(\omega \in \Omega), \\
\xi_{Y, n}:=\left\{\|\cdot\|_{Y, n, \omega}\right\}_{\omega \in \Omega} .
\end{array}\right.
$$

Claim 7.4.2. - There are a positive number $n_{Y}^{\prime}$ and $s_{Y}^{\prime} \in R_{Y, n_{Y}^{\prime}} \backslash\{0\}$ such that $\widehat{\operatorname{deg}}_{\xi_{Y, n_{Y}^{\prime}}}\left(s_{Y}^{\prime}\right)>0$.

Proof. - Fix a positive integer $n_{0}$ such that the natural homomorphism

$$
H^{0}\left(X, L^{\otimes n}\right) \rightarrow H^{0}\left(Y,\left.L\right|_{Y} ^{\otimes n}\right)
$$


is surjective for all $n \geqslant n_{0}$, that is, $R_{Y, n}=H^{0}\left(Y,\left.L\right|_{Y} ^{\otimes n}\right)$ for all $n \geq n_{0}$, so that $s_{Y}^{\otimes n_{0}} \in R_{Y, n_{0} n_{Y}} \backslash\{0\}$. By Theorem 7.3.1, (2), one has

$$
\lim _{n \rightarrow \infty} \frac{\widehat{\operatorname{deg}}_{\xi_{Y, n n_{0} n_{Y}}}\left(s_{Y}^{\otimes n n_{0}}\right)}{n}=\widehat{\operatorname{deg}}_{\left.\xi_{n_{0} n_{Y}}\right|_{Y}}\left(s_{Y}^{\otimes n_{0}}\right)=\left.n_{0} \widehat{\operatorname{deg}}_{\xi_{n_{Y}}}\right|_{Y}\left(s_{Y}\right)>0,
$$

so that there is a positive integer $n_{1}$ such that $\widehat{\operatorname{deg}}_{\xi_{Y, n_{1} n_{0} n_{Y}}}\left(s_{Y}^{\otimes n_{1} n_{0}}\right)>0$. Therefore, of we set $n_{Y}^{\prime}:=n_{1} n_{0} n_{Y}$ and $s_{Y}^{\prime}:=s_{Y}^{\otimes n_{1} n_{0}}$, one has the claim.

The assertion follows from (7.6) follows from the above claim together with Theorem 7.2.1. Further, if $S$ satisfies the strong Minkowski property, then there is a constant $C$ depending only on $S$ such that

$$
\nu_{\min }\left(H^{0}\left(X, L^{\otimes n}\right), \xi_{n}\right)+C \ln \left(\operatorname{dim}_{K} H^{0}\left(X, L^{\otimes n}\right)\right) \geqslant \widehat{\mu}_{\min }\left(H^{0}\left(X, L^{\otimes n}\right), \xi_{n}\right),
$$

and hence the assertion (7.7) follows.

Remark 7.4.3. - In the case (iii) (i.e. $\Omega=\Omega_{0}$ and $\#\left(\Omega_{0}\right)=1$ ), $S$ satisfies the strong Minkowski property, that is, if $E$ is a finite-dimensional vector space over $K$ and $\|\cdot\|$ is an ultrametric norm of $E$ over $(K,|\cdot|)$, then $\nu_{\min }(E,\|\cdot\|)=\widehat{\mu}_{\min }(E,\|\cdot\|)$, which can be checked as follows:

In general, one has $\nu_{\min }(E,\|\cdot\|) \leqslant \widehat{\mu}_{\min }(E,\|\cdot\|)$ by Proposition 4.3.76, so that it is sufficient to show that $\nu_{\min }(E,\|\cdot\|) \geqslant \widehat{\mu}_{\min }(E,\|\cdot\|)$. Let $\left(e_{i}\right)_{i=1}^{r}$ be an orthogonal basis of $E$ with respect to $\|\cdot\|$ (cf. Proposition 1.2.30). Clearly we may assume that $\left\|e_{r}\right\|=\max \left\{\left\|e_{1}\right\|, \ldots,\left\|e_{r}\right\|\right\}$. Let $Q:=E /\left(K e_{1}+\cdots+K e_{r-1}\right)$ and $\|\cdot\|_{Q}$ be the quotient norm of $\|\cdot\|$ on $Q$. Then $\left\|\pi\left(e_{r}\right)\right\|_{Q}=\left\|e_{r}\right\|$, where $\pi: E \rightarrow Q$ is the canonical homomorphism. Thus $-\log \left\|e_{r}\right\| \geqslant \widehat{\mu}_{\min }(E,\|\cdot\|)$, and hence $\widehat{\operatorname{deg}}\left(e_{i}\right) \geqslant \widehat{\mu}_{\min }(E,\|\cdot\|)$ for all $i$. Therefore, one has $\nu_{\min }(E,\|\cdot\|) \geqslant \widehat{\mu}_{\min }(E,\|\cdot\|)$.

Lemma 7.4.4. - We assume that the adelic curve $S$ satisfies the strong Minkowski property. Let $X$ be a geometrically integral projective $K$-scheme, $L$ be an ample invertible $\mathcal{O}_{X}$-module, $\varphi=\left\{\varphi_{\omega}\right\}_{\omega \in \Omega}$ be a dominant and measurable metric family on $L$ such that $\varphi_{\omega}$ is semipositive for any $\omega \in \Omega$. If the height function $h_{(L, \varphi)}$ on the set $X\left(K^{\mathrm{ac}}\right)$ of algebraic points of $X$ is bounded from below by a positive number, then one has

$$
\liminf _{n \rightarrow+\infty} \frac{\widehat{\mu}_{\min }\left(H^{0}\left(X, L^{\otimes n}\right), \xi_{n}\right)}{n}>0
$$

where for any $n \in \mathbb{N}, \xi_{n}=\left\{\|\cdot\|_{n \varphi_{\omega}}\right\}_{\omega \in \Omega}$.

Proof. - We reason by induction on the dimension $d$ of the scheme $X$. The case where $d=0$ comes from Theorem 7.4.1. In the following, we assume that the theorem is true for lower dimensional arithmetic varieties. Denote by

$$
\widehat{\mu}_{\min }^{\text {asy }}(L, \varphi):=\liminf _{n \rightarrow+\infty} \frac{\widehat{\mu}_{\min }\left(H^{0}\left(X, L^{\otimes n}\right), \xi_{n}\right)}{n},
$$




$$
\widehat{\mu}_{\max }^{\mathrm{asy}}(L, \varphi):=\lim _{n \rightarrow+\infty} \frac{\widehat{\mu}_{\max }\left(H^{0}\left(X, L^{\otimes n}\right), \xi_{n}\right)}{n}
$$

and

$$
\widehat{\mu}^{\text {asy }}(L, \varphi):=\liminf _{n \rightarrow+\infty} \frac{\widehat{\mu}\left(H^{0}\left(X, L^{\otimes n}\right), \xi_{n}\right)}{n} .
$$

Assume by contradiction that $\widehat{\mu}_{\text {min }}^{\text {asy }}(L, \varphi) \leqslant 0$. If $\widehat{\mu}_{\text {max }}^{\text {asy }}(L, \varphi)>0$, then there exist a positive integer $n_{X}$ and $s_{X} \in H^{0}\left(X, L^{\otimes n_{X}}\right) \backslash\{0\}$ such that $\widehat{\operatorname{deg}}_{\xi_{n_{X}}}\left(s_{X}\right)>0$. Moreover, by the induction hypothesis, for any subvariety $Y \subsetneq X$ one has

$$
\widehat{\mu}_{\min }^{\text {asy }}\left(\left.L\right|_{Y},\left.\varphi\right|_{Y}\right)>0 \text {. }
$$

As a consequence, there are a positive number $n_{Y}$ and $s_{Y} \in H^{0}\left(Y,\left.L^{\otimes n_{Y}}\right|_{Y}\right) \backslash\{0\}$ such that $\widehat{\operatorname{deg}}_{\left.\xi_{n_{Y}}\right|_{Y}}\left(s_{Y}\right)>0$. Therefore, by Theorem 7.4 .1 one has $\widehat{\mu}_{\min }^{\text {asy }}(L, \varphi)>0$, which leads to a contradiction. Therefore $\widehat{\mu}_{\max }^{\text {asy }}(L, \varphi) \leqslant 0$. This observation actually leads to

$$
\widehat{\mu}_{\max }^{\text {asy }}(L, \varphi)=\widehat{\mu}_{\min }^{\text {asy }}(L, \varphi) .
$$

In fact, if $\widehat{\mu}_{\max }^{\text {asy }}(L, \varphi)>\widehat{\mu}_{\min }^{\text {asy }}(L, \varphi)$, then we can twist $(L, \varphi)$ by the pull-back of an adelic line bundle on $S$ to obtain a metric $\varphi^{\prime}$ such that

$$
\widehat{\mu}_{\max }^{\text {asy }}\left(L, \varphi^{\prime}\right)>0 \geqslant \widehat{\mu}_{\min }^{\text {asy }}\left(L, \varphi^{\prime}\right) .
$$

Note that $h_{\left(L, \varphi^{\prime}\right)} \geqslant h_{(L, \varphi)}$. By the above argument we still obtain a contradiction.

Finally, suppose that $\widehat{\mu}_{\max }^{\text {asy }}(L, \varphi)=\widehat{\mu}_{\text {min }}^{\text {asy }}(L, \varphi) \leqslant 0$. By twisting $(L, \varphi)$ by the pull-back of an adelic line bundle on $S$, we may assume without loss of generality that $\widehat{\mu}_{\max }^{\text {asy }}(L, \varphi)=\widehat{\mu}_{\min }^{\text {asy }}(L, \varphi)=0$. In this case one has $\widehat{\mu}^{\text {asy }}(L, \varphi)=0$ since $\widehat{\mu}_{\min }^{\text {asy }}(L, \varphi) \leqslant \widehat{\mu}^{\text {asy }}(L, \varphi) \leqslant \widehat{\mu}_{\max }^{\text {asy }}(L, \varphi)$. By the induction hypothesis we obtain that, for any subvariety $Y \subsetneq X$, one has

$$
\widehat{\mu}_{\min }^{\text {asy }}\left(\left.L\right|_{Y},\left.\varphi\right|_{Y}\right)>0 .
$$

However, by Remark 7.2 .2 we obtain that $\widehat{\mu}(L, \varphi)>0$, which leads to a contradiction. The theorem is thus proved.

Definition 7.4.5. - Let $X$ be a geometrically integral scheme over $\operatorname{Spec} K, L$ be an ample invertible $\mathcal{O}_{X}$-module, $\varphi=\left\{\varphi_{\omega}\right\}_{\omega \in \Omega}$ be a dominant and measurable metric family on $L$. We denote by $\nu_{\text {abs }}(L, \varphi)$ the infimum of the height function $h_{D, g}$, called the absolute minimum of $(D, g)$.

Theorem 7.4.6. - We assume that the adelic curve $S$ satisfies the strong Minkowski property. Let $X$ be a geometrically integral projective $K$-scheme, $L$ be an ample invertible $\mathcal{O}_{X}$-module, $\varphi=\left\{\varphi_{\omega}\right\}_{\omega \in \Omega}$ be a dominant and measurable metric family on $L$ such that $\varphi_{\omega}$ is semipositive for any $\omega \in \Omega$. Then the following inequality holds.

$$
\widehat{\mu}_{\min }^{\text {asy }}(L, \varphi):=\liminf _{n \rightarrow+\infty} \frac{\widehat{\mu}_{\min }\left(H^{0}\left(X, L^{\otimes n}\right), \xi_{n}\right)}{n}=\nu_{\text {abs }}(L, \varphi),
$$

where for any $n \in \mathbb{N}, \xi_{n}=\left\{\|\cdot\|_{n \varphi_{\omega}}\right\}_{\omega \in \Omega}$, and $d$ is the Krull dimension of $X$. 
Proof. - For any $n \in \mathbb{N}$, let $E_{n}:=H^{0}\left(X, L^{\otimes n}\right)$. Since the adelic curve $S$ satisfies the strong Minkowski property, one has

$\widehat{\mu}_{\min }^{\text {asy }}(L, \varphi)=\liminf _{n \rightarrow+\infty} \frac{\nu_{\min }\left(E_{n}, \xi_{n}\right)}{n} \leqslant \liminf _{n \rightarrow+\infty} \frac{\nu_{\min }^{\mathrm{a}}\left(E_{n}, \xi_{n}\right)}{n} \leqslant \liminf _{n \rightarrow+\infty} \frac{\widehat{\mu}_{\min }\left(E_{n, K^{\mathrm{ac}}}, \xi_{n, K^{\mathrm{ac}}}\right)}{n}$,

where the second inequality comes from Proposition 4.3 .70 and the last inequality comes from Corollary 4.3.77 Let $P$ be an algebraic point of $X$. For sufficiently positive integer $n$, the invertible $\mathcal{O}_{X}$-module $L^{\otimes n}$ is very ample hence defines a closed embedding $X \rightarrow \mathbb{P}\left(E_{n}\right)$. Let $\mathcal{O}_{E_{n}}(1)$ be the universal invertible sheaf on $\mathbb{P}\left(E_{n}\right)$. Then, viewed as a quotient vector space of rank 1 of $E_{n, K^{\text {ac }}}$, the Arakelov degree of $P^{*}\left(\mathcal{O}_{E_{n}}(1)\right)$ (equipped with the quotient norm family) is bounded from above by $n h_{(L, \varphi)}(P)$ and bounded from below by $\widehat{\mu}_{\min }\left(E_{n, K^{\mathrm{ac}}}, \xi_{n, K^{\mathrm{ac}}}\right)$. Therefore we obtain $\widehat{\mu}_{\text {min }}^{\text {asy }}(L, \varphi) \leqslant h_{(L, \varphi)}(P)$. Since $P \in X\left(K^{\text {ac }}\right)$ is arbitrary, this leads to the inequality $\widehat{\mu}_{\min }^{\text {asy }}(L, \varphi) \leqslant \nu_{\text {abs }}(L, \varphi)$. Moreover, by Lemma 7.4 .4 the converse inequality also holds. Therefore the equality (7.8) is proved.

\subsection{Nakai-Moishezon's criterion over a number field}

Throughout this section, we fix a number field $K$ and the standard adelic curve $S=(K,(\Omega, \mathcal{A}, \nu), \phi)$ of $K$ as in Subsection 3.2.2. Denote by $\Omega_{\text {fin }}$ the set $\Omega \backslash \Omega_{\infty}$ of finite places of $K$, and by $\mathfrak{o}_{K}$ the ring of algebraic integers in $K$. Note that $S$ satisfies the strong Minkowski property (see [42, Theorem 1.1]). Moreover, for $\omega \in \Omega_{\text {fin }}$, the valuation ring of the completion $K_{\omega}$ of $K$ with respect to $\omega$ is denoted by $\mathfrak{o}_{\omega}$.

7.5.1. Invariants $\lambda$ and $\sigma$ for a graded algebra of adelic vector bundles. Let $\bar{R}_{\bullet}=\left\{\left(R_{n}, \xi_{n}\right)\right\}_{n \in \mathbb{Z} \geqslant 0}$ be a graded algebra of adelic vector bundles on $S$ such that $\left(R_{n}, \xi_{n}\right)$ is dominated and coherent for all $n \geqslant 0$. For the definition of the invariants $\lambda$ and $\sigma$, see Subsection 4.4 .3 .

Definition 7.5.1. - We say that $\bar{R}$. is asymptotically pure if

$$
\limsup _{n \rightarrow \infty} \frac{\sigma\left(R_{n}, \xi_{n}\right)}{n}=0
$$

As a consequence of Proposition 4.4.10, we have the following:

Proposition 7.5.2. - One has the following inequalities:

$$
\begin{aligned}
& {[K: \mathbb{Q}] \liminf _{n \rightarrow+\infty} \frac{\lambda\left(R_{n}, \xi_{n}\right)}{n} \leqslant \liminf _{n \rightarrow+\infty} \frac{\nu_{\min }\left(R_{n}, \xi_{n}\right)}{n} } \\
& \leqslant[K: \mathbb{Q}] \liminf _{n \rightarrow+\infty} \frac{\lambda\left(R_{n}, \xi_{n}\right)}{n}+\limsup _{n \rightarrow+\infty} \frac{\sigma\left(R_{n}, \xi_{n}\right)}{n} .
\end{aligned}
$$


In particular, if $\bar{R}$. is asymptotically pure, then

$$
[K: \mathbb{Q}] \liminf _{n \rightarrow+\infty} \frac{\lambda\left(R_{n}, \xi_{n}\right)}{n}=\liminf _{n \rightarrow+\infty} \frac{\nu_{\min }\left(R_{n}, \xi_{n}\right)}{n} .
$$

Let $\bar{M}_{\bullet}=\left\{\left(M_{n}, \xi_{M_{n}}\right)\right\}_{n \in \mathbb{Z}}$ be a $h$-graded $\bar{R}_{\bullet}$-module such that $\left(M_{n}, \xi_{n}\right)$ is dominated and coherent for all $n \in \mathbb{Z}$.

Proposition 7.5.3. - (1) If $R$. is generated by $R_{1}$ over $K$, then $\lim _{n \rightarrow \infty} \frac{\lambda\left(R_{n}, \xi_{n}\right)}{n}$ exists in $\mathbb{R} \cup\{\infty\}$.

(2) If $R_{\bullet}=\bigoplus_{n=0}^{\infty} R_{n}$ is of finite type over $K$ and $M_{\bullet}=\bigoplus_{n \in \mathbb{Z}} M_{n}$ is finitely generated over $R_{\bullet}$, then

$$
\frac{1}{h} \liminf _{n \rightarrow \infty} \frac{\lambda\left(R_{n}, \xi_{n}\right)}{n} \leqslant \liminf _{n \rightarrow \infty} \frac{\lambda\left(M_{n}, \xi_{M_{n}}\right)}{n} .
$$

Proof. - We set $\mathscr{R}_{n}:=\left(R_{n}, \xi_{n}\right)_{\leqslant 1}^{\text {fin }}$ for $n \geqslant 0$, and $\mathscr{M}_{n}:=\left(M_{n}, \xi_{M_{n}}\right)_{\leqslant 1}^{\text {fin }}$ for $n \in \mathbb{Z}$.

(1) For $\epsilon>0$, we choose bases $e_{1}, \ldots, e_{r}$ and $e_{1}^{\prime}, \ldots, e_{r^{\prime}}^{\prime}$ of $R_{n}$ and $R_{m}$ over $K$, respectively, such that

$$
\begin{cases}e_{1}, \ldots, e_{r} \in \mathscr{R}_{n}, & \max \left\{\left\|e_{i}\right\|_{\infty, n}\right\} \leqslant e^{-\lambda\left(R_{n}, \xi_{n}\right)+\epsilon} \\ e_{1}^{\prime}, \ldots, e_{r^{\prime}}^{\prime} \in \mathscr{R}_{m}, & \max \left\{\left\|e_{j}^{\prime}\right\|_{\infty, n}\right\} \leqslant e^{-\lambda\left(R_{m}, \xi_{m}\right)+\epsilon}\end{cases}
$$

Then $e_{i} e_{j}^{\prime} \in \mathscr{R}_{n+m}$ and $\max \left\{\left\|e_{i} e_{j}^{\prime}\right\|_{\infty, n+m}\right\} \leqslant e^{-\lambda\left(R_{n}, \xi_{n}\right)-\lambda\left(R_{n}, \xi_{n}\right)+2 \epsilon}$. Note that $\left\{e_{i} e_{j}^{\prime}\right\}$ forms generators of $R_{n+m}$ over $K$ because $R_{n} \otimes R_{m} \rightarrow R_{n+m}$ is surjective, so that $e^{-\lambda\left(R_{n+m}, \xi_{n+m}\right)} \leqslant e^{-\lambda\left(R_{n}, \xi_{n}\right)-\lambda\left(R_{n}, \xi_{n}\right)+2 \epsilon}$. Therefore, one has

$$
\lambda\left(R_{n+m}, \xi_{n+m}\right) \geqslant \lambda\left(R_{n}, \xi_{n}\right)+\lambda\left(R_{n}, \xi_{n}\right)
$$

for all $n, m$. Thus the assertion follows from Fekete's lemma.

(2) It can be proved in the similar way as in Proposition 7.1.4. First we assume that $R_{\bullet}$ is generated by $R_{1}$ over $K$. Then there exist integers $b_{1}$ and $m>0$ such that, for any integer $b$ with $b \geqslant b_{1}$ and any integer $\ell \geqslant 1$ the canonical $K$-linear map $R_{\ell m} \otimes_{K} M_{b} \rightarrow M_{b+\ell m h}$ is surjective. For $\epsilon>0$, we choose a basis $e_{1}, \ldots, e_{r}$ of $R_{\ell m}$ and a basis $m_{1}, \ldots, m_{r^{\prime}}$ of $M_{b}$ such that $e_{1}, \ldots, e_{r} \in \mathscr{R}_{\ell m}, m_{1}, \ldots, m_{r^{\prime}} \in \mathscr{M}_{b}$, $\max \left\{\left\|e_{i}\right\|_{\infty, \ell m}\right\} \leqslant e^{-\lambda\left(R_{\ell m}, \xi_{\ell m}\right)+\epsilon}$ and $\max \left\{\left\|m_{j}\right\|_{\infty, M_{b}}\right\} \leqslant e^{-\lambda\left(M_{b}, \xi_{M_{b}}\right)+\epsilon}$. Note that $e_{i} m_{j} \in \mathscr{M}_{b+\ell m h}$ and

$$
\left\|e_{i} m_{j}\right\|_{\infty, M_{b+\ell m h}} \leqslant e^{-\lambda\left(R_{\ell m}, \xi_{\ell m}\right)-\lambda\left(M_{b}, \xi_{M_{b}}\right)+2 \epsilon} .
$$

Moreover we can find a basis of $M_{b+\ell m h}$ among $\left\{e_{i} m_{j}\right\}_{1 \leqslant i \leqslant r, 1 \leqslant j \leqslant r^{\prime}}$, so that

$$
e^{-\lambda\left(M_{b+\ell m h}, \xi_{M_{b+\ell m h}}\right)} \leqslant e^{-\lambda\left(R_{\ell m}, \xi_{\ell m}\right)-\lambda\left(M_{b}, \xi_{M_{b}}\right)+2 \epsilon},
$$

and hence one has

$$
\lambda\left(M_{b+\ell m h}, \xi_{M_{b+\ell m h}}\right) \geqslant \lambda\left(R_{\ell m}, \xi_{\ell m}\right)+\lambda\left(M_{b}, \xi_{M_{b}}\right) .
$$


Therefore,

$$
\liminf _{l \rightarrow \infty} \frac{\lambda\left(M_{b+\ell m h}, \xi_{M_{b+\ell m h}}\right)}{\ell m h} \geqslant \frac{1}{h} \liminf _{l \rightarrow \infty} \frac{\lambda\left(R_{\ell m}, \xi_{\ell m}\right)}{\ell m} \geqslant \frac{1}{h} \liminf _{n \rightarrow \infty} \frac{\lambda\left(R_{n}, \xi_{n}\right)}{n},
$$

which implies

$$
\liminf _{n \rightarrow \infty} \frac{\lambda\left(M_{n}, \xi_{M_{n}}\right)}{n} \geqslant \frac{1}{h} \liminf _{n \rightarrow \infty} \frac{\lambda\left(R_{n}, \xi_{n}\right)}{n}
$$

because $b \geqslant b_{1}$ is arbitrary.

In general, we can find a positive integer $u$ such that $R_{\bullet}^{(u)}:=\bigoplus_{n=0}^{\infty} R_{u n}$ is generated by $R_{1}^{(u)}=R_{u}$ over $K$. Note that $R_{\bullet}$ is a finitely generated $R_{\bullet}^{(u)}$-module. Therefore, by the previous observation, one has

$$
\left\{\begin{array}{l}
\liminf _{n \rightarrow \infty} \frac{\lambda\left(R_{n}, \xi_{n}\right)}{n} \geqslant \frac{1}{u} \liminf _{n \rightarrow \infty} \frac{\lambda\left(R_{u n}, \xi_{u n}\right)}{n}, \\
\liminf _{n \rightarrow \infty} \frac{\lambda\left(M_{n}, \xi_{M_{n}}\right)}{n} \geqslant \frac{1}{h u} \liminf _{n \rightarrow \infty} \frac{\lambda\left(R_{u n}, \xi_{u n}\right)}{n} .
\end{array}\right.
$$

Moreover, as

one obtains

$$
\liminf _{n \rightarrow \infty} \frac{\lambda\left(R_{u n}, \xi_{u n}\right)}{u n} \geqslant \liminf _{m \rightarrow \infty} \frac{\lambda\left(R_{m}, \xi_{m}\right)}{m},
$$

$$
\liminf _{n \rightarrow \infty} \frac{\lambda\left(R_{n}, \xi_{n}\right)}{n}=\frac{1}{u} \liminf _{n \rightarrow \infty} \frac{\lambda\left(R_{u n}, \xi_{u n}\right)}{n} .
$$

Thus the assertion follows.

7.5.2. Dominancy and coherency of generically pure metric. - Let $X$ be a geometrically integral projective variety over $K$ and $L$ be an invertible sheaf on $X$. For $\omega \in \Omega$, let $X_{\omega}:=X \times_{\operatorname{Spec} K} K_{\omega}$ and $L_{\omega}:=L \otimes_{\mathcal{O}_{X}} \mathcal{O}_{X_{\omega}}$. Let $\varphi_{\omega}$ be a continuous metric of $L_{\omega}$ on $X_{\omega}^{\text {an }}$ for each $\omega \in \Omega$, and $\varphi:=\left\{\varphi_{\omega}\right\}_{\omega \in \Omega}$.

Let us begin with the definition of the generic purity of the metric family $\varphi$.

Definition 7.5.4. - We say that $\varphi$ is generically pure if there are a non-empty Zariski open set $U$ of $\operatorname{Spec}\left(\mathfrak{o}_{K}\right)$, a projective integral scheme $\mathscr{X}$ over $U$ and an invertible $\mathcal{O}_{\mathscr{X}}$-module $\mathscr{L}$ such that $\mathscr{X} \times_{U} \operatorname{Spec}(K)=X,\left.\mathscr{L}\right|_{X}=L$ and, for each $\omega \in U \cap \Omega_{\text {fin }}, \varphi_{\omega}$ coincides with the metric arising from $\mathscr{X}_{\omega}$ and $\mathscr{L}_{\omega}$, where $\mathscr{X}_{\omega}=\mathscr{X} \times_{U} \operatorname{Spec}\left(\mathfrak{o}_{\omega}\right)$ and $\mathscr{L}_{\omega}$ is the pull-back of $\mathscr{L}$ to $\mathscr{X}_{\omega}$.

Proposition 7.5.5. - (1) If $L$ is generated by global sections and $\varphi$ is generically pure, there exist a non-empty Zariski open set $U$ of $\operatorname{Spec}\left(\mathfrak{o}_{K}\right)$ and a basis $\boldsymbol{e}=$ $\left(e_{i}\right)_{i=1}^{r}$ of $H^{0}(X, L)$ such that $\varphi_{\omega}=\varphi_{\boldsymbol{e}, \omega}$ for all $\omega \in U \cap \Omega_{\mathrm{fin}}$.

(2) If $L$ is semiample and $\varphi$ is generically pure, then $\varphi$ is dominated and $\left(H^{0}\left(X, L^{\otimes n}\right),\left\{\|\cdot\|_{n \varphi_{\omega}, \text { sup }}\right\}_{\omega \in \Omega}\right)$ is coherent for all $n \geqslant 0$.

Proof. - (1): We use the notation in Definition 7.5.4. Shrinking $U$ if necessarily, we may assume that $H^{0}(\mathscr{X}, \mathscr{L})$ is a free $\mathfrak{o}_{U}$-module and $H^{0}(\mathscr{X}, \mathscr{L}) \otimes_{\mathfrak{o}_{U}} \mathcal{O}_{\mathscr{X}} \rightarrow \mathscr{L}$ is surjective. Let $\boldsymbol{e}=\left(e_{i}\right)_{i=1}^{r}$ be a free basis of $H^{0}(\mathscr{X}, \mathscr{L})$ over $\mathfrak{o}_{U}$. Then $\left(e_{i}\right)_{i=1}^{r}$ yields a 
free basis of $H^{0}\left(\mathscr{X}_{\omega}, \mathscr{L}_{\omega}\right)$ over $\mathfrak{a}_{\omega}$ for any $\omega \in U \cap \Omega_{\mathrm{fin}}$. Let $\|\cdot\|_{H^{0}\left(\mathscr{X}_{\omega}, \mathscr{L}_{\omega}\right)}$ be the norm of $H^{0}\left(X_{\omega}, L_{\omega}^{\otimes r}\right)$ arising from the lattice $H^{0}\left(\mathscr{X}_{\omega}, \mathscr{L}_{\omega}\right)$. Then, by Proposition 1.2.21, $\|\cdot\|_{H^{0}\left(\mathscr{X}_{\omega}, \mathscr{L}_{\omega}\right)}=\|\cdot\|_{\boldsymbol{e}, \omega}$ for any $\omega \in U \cap \Omega_{\mathrm{fin}}$. Moreover, $H^{0}\left(\mathscr{X}_{\omega}, \mathscr{L}_{\omega}\right) \otimes_{\mathfrak{o}_{\omega}} \mathcal{O}_{\mathscr{X}_{\omega}} \rightarrow \mathscr{L}_{\omega}$ is surjective, so that, by Proposition 2.3.12, one has $\varphi_{\omega}=\varphi_{\boldsymbol{e}, \omega}$, as required.

(2) We choose a positive integer $m$ such that $L^{\otimes m}$ is generated by global sections and $\alpha_{n}: H^{0}\left(X, L^{\otimes m}\right)^{\otimes n} \rightarrow H^{0}\left(X, L^{\otimes n m}\right)$ is surjective for all $n \geqslant 1$. Then, by (1), there are a non-empty Zariski open set $U$ of $\operatorname{Spec}\left(\mathfrak{o}_{K}\right)$ and a basis $\boldsymbol{e}=\left(e_{i}\right)_{i=1}^{r}$ of $H^{0}\left(X, L^{\otimes m}\right)$ such that $m \varphi_{\omega}=\varphi_{\boldsymbol{e}, \omega}$ for all $\omega \in U \cap \Omega_{\mathrm{fin}}$. In particular, $m \varphi$ is dominated, so that $\varphi$ is also dominated by Proposition 6.1.12.

For $\omega \in U \cap \Omega_{\mathrm{fin}}$, let $\|\cdot\|_{\boldsymbol{e}, \omega}^{\otimes n}$ be the $\varepsilon$-tensor products of $\|\cdot\|_{\boldsymbol{e}, \omega}$ on $H^{0}\left(X_{\omega}, L_{\omega}^{\otimes m}\right)^{\otimes n}$. Note that, by Proposition 1.2.19 together with (1.17) in Remark 1.1.56.

$$
\forall a_{i_{1}, \ldots, i_{N}} \in K_{\omega}, \quad\left\|\sum_{\substack{\left.i_{1}, \ldots, i_{r}\right) \in \mathbb{Z}_{\geqslant 0}^{r}, i_{1}+\cdots+i_{r}=n}} a_{i_{1}, \ldots, i_{r}} e_{1}^{\otimes i_{1}} \otimes \cdots \otimes e_{r}^{\otimes i_{r}}\right\|_{\boldsymbol{e}, \omega}^{\otimes n}=\max \left\{\left|a_{i_{1}, \ldots, i_{r}}\right|_{\omega}\right\} .
$$

Moreover, by Remark 2.2.19, $n \varphi_{\boldsymbol{e}, \omega}$ coincides with the quotient metric induced by the surjective homomorphism $H^{0}\left(X_{\omega}, L_{\omega}^{\otimes m}\right)^{\otimes n} \otimes_{K_{\omega}} \mathcal{O}_{X_{\omega}} \rightarrow L_{\omega}^{\otimes n m}$ and $\|\cdot\|_{\boldsymbol{e}, \omega}^{\otimes n}$.

Fix $s \in H^{0}\left(X, L^{\otimes n}\right)(n \geqslant 1)$. Then $s^{\otimes m} \in H^{0}\left(X, L^{\otimes m n}\right)$. As $\alpha_{n}$ is surjective, one can choose

$$
f=\sum_{\substack{\left.i_{1}, \ldots, i_{r}\right) \in \mathbb{Z}_{>}^{r}, i_{1}+\cdots+i_{r}=n}} f_{i_{1}, \ldots, i_{r}} e_{1}^{\otimes i_{1}} \otimes \cdots \otimes e_{r}^{\otimes i_{r}} \in H^{0}\left(X, L^{\otimes m}\right)^{\otimes n} \quad\left(f_{i_{1}, \ldots, i_{r}} \in K\right)
$$

such that $\alpha_{n}(f)=s^{\otimes m}$. Then, by Proposition 2.2.23,

$$
\left(\|s\|_{n \varphi_{\omega}, \text { sup }}\right)^{m}=\left\|s^{\otimes m}\right\|_{n m \varphi_{\omega}, \text { sup }}=\left\|s^{\otimes m}\right\|_{n \varphi_{\boldsymbol{e}, \omega}, \text { sup }} \leqslant\|f\|_{\boldsymbol{e}, \omega}^{\otimes n}=\max \left\{\left|f_{i_{1}, \ldots, i_{r}}\right|_{\omega}\right\},
$$

for all $\omega \in U \cap \Omega_{\text {fin }}$, so that so that $\|s\|_{n \varphi_{\omega}, \text { sup }} \leqslant 1$ for all $\omega \in \Omega$ except finitely many $\omega$ because $\Omega \backslash\left(U \cap \Omega_{\mathrm{fin}}\right)$ is finite and $\left|f_{i_{1}, \ldots, i_{r}}\right|_{\omega}=1$ for all $i_{1}, \ldots, i_{r}$ and $\omega \in \Omega_{\mathrm{fin}}$ except finitely many $\omega$.

7.5.3. Fine metric family. - Let $X$ be a geometrically integral projective variety over $K$ and $L$ be an invertible sheaf on $X$. For $\omega \in \Omega$, let $X_{\omega}:=X \times_{\operatorname{Spec} K} K_{\omega}$ and $L_{\omega}:=L \otimes_{\mathcal{O}_{X}} \mathcal{O}_{X_{\omega}}$. Let $\varphi_{\omega}$ be a continuous metric of $L_{\omega}$ on $X_{\omega}^{\text {an }}$ for each $\omega \in \Omega$, and $\varphi:=\left\{\varphi_{\omega}\right\}_{\omega \in \Omega}$.

Definition 7.5.6. - We say that $\varphi$ is very fine if $\varphi$ is dominated and there are a generically pure continuous metric family $\varphi^{\prime}=\left\{\varphi_{\omega}^{\prime}\right\}_{\omega \in \Omega}$ of $L$ and a non-empty Zariski open set $U$ of $\operatorname{Spec}\left(\mathfrak{o}_{K}\right)$ such that $|\cdot|_{\varphi_{\omega}} \leqslant|\cdot|_{\varphi_{\omega}^{\prime}}$ for all $\omega \in U \cap \Omega_{\mathrm{fin}}$. Further, $\varphi$ is said to be fine if $r \varphi$ is very fine for some positive integer $r$.

Proposition 7.5.7. - Let $L$ and $M$ be invertible $\mathcal{O}_{X}$-module, and $\varphi$ and $\psi$ be continuous metric families of $L$ and $M$, respectively.

(1) If $\varphi$ and $\psi$ are very fine, then $\varphi+\psi$ is very fine. 
(2) If $\varphi$ and $\psi$ are fine, then $\varphi+\psi$ is fine.

(3) If a $\varphi$ is fine for some positive integer a, then $\varphi$ is fine.

(4) If $\varphi$ is fine, then $\left(H^{0}(X, L),\left\{\|\cdot\|_{\varphi_{\omega}, \text { sup }}\right\}_{\omega \in \Omega}\right)$ is coherent.

Proof. - (1) is obvious.

(2) We choose positive integers $r$ and $r^{\prime}$ such that $r \varphi$ and $r^{\prime} \psi$ are very fine. Then, by (1), $r r^{\prime} \varphi$ and $r r^{\prime} \psi$ are very fine, so that $r r^{\prime}(\varphi+\psi)$ is very fine, as required.

(3) Since $a \varphi$ is fine, there is a positive integer $r$ such that $r a \varphi$ is very fine, so that $\varphi$ is fine.

(4) Let $r$ be a positive integer such that $r \varphi$ is very fine. Then there are a generically pure continuous metric family $\varphi^{\prime}=\left\{\varphi_{\omega}^{\prime}\right\}_{\omega \in \Omega}$ of $L^{\otimes r}$ and a non-empty Zariski open set $U$ of $\operatorname{Spec}\left(\mathfrak{o}_{K}\right)$ such that $|\cdot|_{r \varphi_{\omega}} \leqslant|\cdot|_{\varphi_{\omega}^{\prime}}$ for all $\omega \in U \cap \Omega_{\mathrm{fin}}$, so that, for $s \in$ $H^{0}(X, L) \backslash\{0\}$ and $\omega \in U \cap \Omega_{\text {fin }},\left\|s^{\otimes r}\right\|_{r \varphi_{\omega}, \text { sup }} \leqslant\left\|s^{\otimes r}\right\|_{\varphi_{\omega}^{\prime} \text {,sup. By Proposition 7.5.5 }}$ $\left\|s^{\otimes r}\right\|_{\varphi_{\omega}^{\prime} \text {,sup }} \leqslant 1$ expect finitely many $\omega \in \Omega$. Therefore, the same assertion holds for $\left\|s^{\otimes r}\right\|_{r \varphi_{\omega}, \text { sup }}$. Note that $\left\|s^{\otimes r}\right\|_{r \varphi_{\omega}, \text { sup }}=\|s\|_{\varphi_{\omega}, \text { sup }}^{r}$, and hence $\|s\|_{\varphi_{\omega}, \text { sup }} \leqslant 1$ expect finitely many $\omega \in \Omega$.

For $n \geqslant 0$, we set $R_{n}:=H^{0}\left(X, L^{\otimes n}\right)$ and $\xi_{n}:=\left\{\|\cdot\|_{n \varphi_{\omega}, \text { sup }}\right\}_{\omega \in \Omega}$. Note that $\bar{R}_{\mathbf{.}}=\left\{\left(R_{n}, \xi_{n}\right)\right\}_{n=0}^{\infty}$ forms a graded algebra of adelic vector bundles over $S$ (cf. Definition 7.1.11. For $n \geqslant 0$ and $\omega \in \Omega$, we denote $R_{n} \otimes_{K} K_{\omega}=H^{0}\left(X_{\omega}, L_{\omega}^{\otimes n}\right)$ by $R_{n, \omega}$. Moreover, for $n \geqslant 0$ and $\omega \in \Omega_{\text {fin }}$, we set $\mathscr{R}_{n, \omega}:=\left\{x \in R_{n, \omega} \mid\|x\|_{n, \omega} \leqslant 1\right\}$. Note that $\mathscr{R}_{n, \omega}$ is a locally free $\mathfrak{o}_{\omega}$-module and $\mathscr{R}_{n, \omega} \otimes_{\mathfrak{o}_{\omega}} K_{\omega}=R_{n, \omega}$ (cf. Proposition 1.1.25] and Proposition 1.1.30). Further we set

$$
\mathscr{R}_{n}:=\left\{x \in R_{n} \mid\|x\|_{n, \omega} \leqslant 1 \text { for all } \omega \in \Omega_{\text {fin }}\right\} .
$$

If $\left(R_{n}, \xi_{n}\right)$ is dominated and coherent, then, by Proposition 4.4 .2 and Proposition 4.4.6, $\mathscr{R}_{n}$ is finitely generated over $\mathfrak{o}_{K}, \mathscr{R}_{n} \otimes_{\mathfrak{o}_{K}} K=R_{n}, \mathscr{R}_{n} \otimes_{\mathfrak{o}_{K}} K_{\omega}=R_{n, \omega}$ and $\mathscr{R}_{n} \otimes_{\mathfrak{o}_{K}} \mathfrak{o}_{\omega}=\mathscr{R}_{n, \omega}$ for all $\omega \in \Omega_{\mathrm{fin}}$.

Proposition 7.5.8. - We assume that $L$ is ample and $\varphi$ is dominated. Then the following are equivalent:

(1) The metric family $\varphi$ is fine.

(2) $\left(R_{n}, \xi_{n}\right)$ is coherent for all $n \geqslant 0$.

Proof. - (1) $\Longrightarrow(2)$ : This is a consequence of Proposition 7.5.7.

$(2) \Longrightarrow(1)$ : First note that $\left(R_{n}, \xi_{n}\right)$ is dominated for $n \geqslant 0$ by Proposition 6.1.12 and Theorem 6.1.13. Moreover, by our assumption, $\left(R_{n}, \xi_{n}\right)$ is coherent for every $n \geqslant 0$.

Let $r$ be a positive integer such that $L^{\otimes r}$ is very ample. Let $\mathscr{X}$ be the Zariski closure of $X$ in $\mathbb{P}\left(\mathscr{R}_{r}\right)$ and $\mathscr{L}=\left.\mathcal{O}_{\mathbb{P}\left(\mathscr{R}_{r}\right)}(1)\right|_{\mathscr{X}}$. Then $\left.\mathscr{L}\right|_{X}=L^{\otimes r}$. Moreover, since $\mathscr{R}_{r} \otimes_{\mathfrak{o}_{K}} \mathcal{O}_{\mathbb{P}\left(\mathscr{R}_{r}\right)} \rightarrow \mathcal{O}_{\mathbb{P}\left(\mathscr{R}_{r}\right)}(1)$ is surjective, $\mathscr{R}_{r} \otimes_{\mathfrak{o}_{K}} \mathcal{O}_{\mathscr{X}} \rightarrow \mathscr{L}$ is also surjective. For each $\omega \in \Omega_{\text {fin }}$, let $\psi_{\omega}$ be the metric of $L_{\omega}^{\otimes r}$ arising from $\mathscr{X}_{\omega}$ and $\mathscr{L}_{\omega}$, where $\mathscr{X}_{\omega}=$ 
$\mathscr{X} \times_{\operatorname{Spec}\left(\mathfrak{o}_{K}\right)} \operatorname{Spec}\left(\mathfrak{o}_{\omega}\right)$ and $\mathscr{L}_{\omega}$ is the pull-back of $\mathscr{L}$ to $\mathscr{X}_{\omega}$. Let $\varphi^{\prime}=\left\{\varphi_{\omega}^{\prime}\right\}_{\omega \in \Omega}$ be the metric family of $L^{\otimes r}$ given by

$$
\varphi_{\omega}^{\prime}:= \begin{cases}\psi_{\omega} & \text { if } \omega \in \Omega_{\mathrm{fin}} \\ r \varphi_{\omega} & \text { otherwise }\end{cases}
$$

Here let us see

$$
\forall \omega \in \Omega, \forall x \in X_{\omega}^{\mathrm{an}}, \quad|\cdot|_{r \varphi_{\omega}}(x) \leqslant|\cdot|_{\varphi_{\omega}^{\prime}}(x) .
$$

Clearly we may assume that $\omega \in \Omega_{\mathrm{fin}}$. Note that

$$
\mathscr{R}_{r, \omega}=\left\{s \in H^{0}\left(\mathscr{X}_{\omega}, \mathscr{L}_{\omega}\right) \mid\|s\|_{r \varphi_{\omega}, \text { sup }} \leqslant 1\right\}
$$

and $\mathscr{R}_{r, \omega} \otimes \mathcal{O}_{\mathscr{X}_{\omega}} \rightarrow \mathscr{L}_{\omega}$ is surjective, so that, by Proposition 2.3.12, (7.9) follows. Therefore, $r \varphi$ is very fine, and hence $\varphi$ is fine.

Finally we consider the following theorem:

Theorem 7.5.9. - If $\varphi$ is very fine, then $\bar{R}$. is asymptotically pure.

Proof. - By our assumption, $\varphi$ is dominated and there are a generically pure continuous metric family $\varphi^{\prime}=\left\{\varphi_{\omega}^{\prime}\right\}_{\omega \in \Omega}$ of $L$ and a non-empty Zariski open set $U$ of $\operatorname{Spec}\left(\mathfrak{o}_{K}\right)$ such that $|\cdot|_{\varphi_{\omega}} \leqslant|\cdot|_{\varphi_{\omega}^{\prime}}$ for all $\omega \in U \cap \Omega_{\mathrm{fin}}$.

First note that $\left(R_{n}, \xi_{n}\right)$ is dominated for $n \geqslant 0$ by Proposition 6.1.12 and Theorem 6.1.13. Moreover, by Proposition 7.5.7 or Proposition 7.5.8, $\left(R_{n}, \xi_{n}\right)$ is coherent for every $n \geqslant 0$.

By the generic purity of $\varphi^{\prime}$, there are a non-empty Zariski open set $U^{\prime}$ of $\operatorname{Spec}\left(\mathfrak{o}_{K}\right)$, a projective integral scheme $\mathscr{X}$ over $U^{\prime}$ and an invertible $\mathcal{O}_{\mathscr{X}}$-module $\mathscr{L}$ such that $\mathscr{X} \times_{U^{\prime}} \operatorname{Spec}(K)=X,\left.\mathscr{L}\right|_{X}=L$ and, for each $\omega \in U^{\prime} \cap \Omega_{\text {fin }}, \varphi_{\omega}^{\prime}$ coincides with the metric arising from $\mathscr{X}_{\omega}$ and $\mathscr{L}_{\omega}$, where $\mathscr{X}_{\omega}=\mathscr{X}_{U} \operatorname{Spec}\left(\mathfrak{o}_{\omega}\right)$ and $\mathscr{L}_{\omega}$ is the pull-back of $\mathscr{L}$ to $\mathscr{X}_{\omega}$. Replacing $U$ and $U^{\prime}$ by $U \cap U^{\prime}$, we may assume that $U=U^{\prime}$. Moreover, as $X$ is geometrically integral over $K$, by virtue of [73, Théorème 9.7.7], shrinking $U$ if necessarily, we may also assume that, for any $\omega \in U \cap \Omega_{\mathrm{fin}}$, the fiber of $\mathscr{X} \rightarrow U$ over $\omega$ is geometrically integral over the residue field at $\omega$. Then, by Proposition 2.3.16

$$
\left\{\begin{array}{l}
\left\{x \in R_{n} \mid\|x\|_{n \varphi_{\omega}^{\prime}, \text { sup }} \leqslant 1\right\}=H^{0}\left(\mathscr{X}_{\omega}, \mathscr{L}_{\omega}^{\otimes n}\right)=H^{0}\left(\mathscr{X}, \mathscr{L}^{\otimes n}\right) \otimes_{\mathfrak{o}_{U}} \mathfrak{o}_{\omega}, \\
\|\cdot\|_{n \varphi_{\omega}^{\prime}, \text { sup }}=\|\cdot\|_{H^{0}\left(\mathscr{X}_{\omega}, \mathscr{L}_{\omega}^{\otimes n}\right)}
\end{array}\right.
$$

for all $n \geqslant 1$ and $\omega \in U \cap \Omega_{\mathrm{fin}}$.

Claim 7.5.10. $n \geqslant 1$.

(a) $|\cdot|_{n \varphi_{\omega}}(x) \leqslant|\cdot|_{n \varphi_{\omega}^{\prime}}(x)$ for all $\omega \in U \cap \Omega_{\mathrm{fin}}, x \in X_{\omega}^{\text {an }}$ and

(b) $\|\cdot\|_{n \varphi_{\omega}, \text { sup }} \leqslant\|\cdot\|_{\mathscr{R}_{n, \omega}} \leqslant \min \left\{\left|\varpi_{\omega}\right|_{\omega}^{-1}\|\cdot\|_{n \varphi_{\omega}, \text { sup }},\|\cdot\|_{n \varphi_{\omega}^{\prime}, \text { sup }}\right\}$ for all $\omega \in U \cap \Omega_{\mathrm{fin}}$ and $n \geqslant 1$, where $\varpi_{\omega}$ is a uniformizing parameter of $\mathfrak{a}_{\omega}$. 
Proof. - (a) is obvious.

(b) First of all, by Proposition 1.1.30,

$$
\|\cdot\|_{n \varphi_{\omega}, \text { sup }} \leqslant\|\cdot\|_{\mathscr{R}_{n, \omega}} \leqslant\left|\varpi_{\omega}\right|_{\omega}^{-1}\|\cdot\|_{n \varphi_{\omega}, \text { sup }} .
$$

By (a), one has $\|\cdot\|_{n \varphi_{\omega}, \text { sup }} \leqslant\|\cdot\|_{n \varphi_{\omega}^{\prime}, \text { sup }}$, so that, by (7.10), one obtains

$$
\mathscr{R}_{n, \omega} \supseteq H^{0}\left(\mathscr{X}_{\omega}, \mathscr{L}_{\omega}^{\otimes n}\right) \text {. }
$$

Therefore, by (7.10) again, (b) follows.

Claim 7.5.11. - If we set $A_{\omega}=d_{\omega}\left(\varphi_{\omega}, \varphi_{\omega}^{\prime}\right)$ for $\omega \in \Omega$, then one has the following:

(a) $\sup _{x \in R_{n} \backslash\{0\}} \ln \frac{\|s\|_{\mathscr{R}_{n, \omega}}}{\|s\|_{n \varphi_{\omega}, \text { sup }}} \leqslant A_{\omega} n$ for all $\omega \in U \cap \Omega_{\mathrm{fin}}$ and $n \geqslant 1$.

(b) $\int_{\Omega} A_{\omega} \nu(d \omega)=\sum_{\omega \in \Omega} A_{\omega} \nu(\{\omega\})<\infty$.

Proof. - (a) By using the inequality (2.5) in Subsection 2.2.2 together with Claim 7.5.10,

$$
\begin{aligned}
\sup _{x \in R_{n} \backslash\{0\}} \ln \frac{\|s\|_{\mathscr{R}_{n, \omega}}}{\|s\|_{n \varphi_{\omega}, \text { sup }}} & \leqslant \sup _{x \in R_{n} \backslash\{0\}} \ln \frac{\|s\|_{n \varphi_{\omega}^{\prime}, \text { sup }}}{\|s\|_{n \varphi_{\omega}, \text { sup }}} \\
& =d_{\omega}\left(\|\cdot\|_{n \varphi_{\omega}^{\prime}, \text { sup }},\|\cdot\|_{n \varphi_{\omega}^{\prime}, \text { sup }}\right) \\
& \leqslant d_{\omega}\left(n \varphi_{\omega}, n \varphi_{\omega}^{\prime}\right)=n A_{\omega} .
\end{aligned}
$$

(b) Note that $\varphi^{\prime}$ is dominated by Proposition 7.5.5 Moreover, $\varphi$ is dominated by our assumption, so that, by Proposition 6.1.12 the function $\omega \mapsto d_{\omega}\left(\varphi_{\omega}, \varphi_{\omega}^{\prime}\right)=A_{\omega}$ is $\nu$-dominated. Thus one obtains (b).

Fix a positive number $\epsilon$. Then, by Claim 7.5.11, there is a non-empty Zariski open set $U_{\epsilon}$ of $U$ such that

$$
\sum_{\omega \in U_{\epsilon} \cap \Omega_{\mathrm{fin}}} A_{\omega} \nu(\{\omega\}) \leqslant \epsilon .
$$

Thus, if we set $B=\sum_{\omega \in \Omega_{\mathrm{fin}} \backslash U_{\epsilon}}-\ln \left|\varpi_{\omega}\right|_{\omega} \nu(\{\omega\})$, then, by Claim 7.5.10

$$
\begin{aligned}
\sigma\left(R_{n}, \xi_{n}\right) & =\sum_{\omega \in \Omega_{\mathrm{fin}}} \sup _{x \in R_{n, \omega} \backslash\{0\}} \ln \left(\frac{\|x\|_{\mathscr{R}_{n, \omega}}}{\|x\|_{n \varphi_{\omega}, \text { sup }}}\right) \nu(\{\omega\}) \\
& \leqslant \sum_{\omega \in U_{\epsilon} \cap \Omega_{\mathrm{fin}}} \sup _{x \in R_{n, \omega} \backslash\{0\}} \ln \left(\frac{\|x\|_{\mathscr{R}_{n, \omega}}}{\|x\|_{n \varphi_{\omega}, \text { sup }}}\right) \nu(\{\omega\})+B \\
& \leqslant n \sum_{\omega \in U_{\epsilon} \cap \Omega_{\mathrm{fin}}} A_{\omega} \nu(\{\omega\})+B \leqslant n \epsilon+B
\end{aligned}
$$

for $n \geqslant 1$, and hence one has

$$
\limsup _{n \rightarrow \infty} \frac{\sigma\left(R_{n}, \xi_{n}\right)}{n} \leqslant \epsilon,
$$

so that the assertion of the theorem follows. 
7.5.4. A generalization of Nakai-Moishezon's criterion. - Let $X$ be a geometrically integral projective variety over $K$ and $L$ be an invertible sheaf on $X$. For $\omega \in \Omega$, let $X_{\omega}:=X \times_{\operatorname{Spec} K} K_{\omega}$ and $L_{\omega}:=L \otimes_{\mathcal{O}_{X}} \mathcal{O}_{X_{\omega}}$. Let $\varphi_{\omega}$ be a continuous metric of $L_{\omega}$ on $X_{\omega}^{\text {an }}$ for each $\omega \in \Omega$, and $\varphi:=\left\{\varphi_{\omega}\right\}_{\omega \in \Omega}$. For $n \geqslant 0$ and a subvariety $Y$ of $X$, let $\xi_{n}:=\left\{\|\cdot\|_{n \varphi_{\omega}, \text { sup }}\right\}_{\omega \in \Omega}$ and $\left.\xi_{n}\right|_{Y}:=\left\{\|\cdot\|_{\left.n \varphi_{\omega}\right|_{Y_{\omega}}, \text { sup }}\right\}_{\omega \in \Omega}$. In this subsection, let us consider the following Nakai-Moishezon's criterion over a number field, which gives a generalisation of Nakai-Moishezon's criterion due to Shouwu Zhang.

Theorem 7.5.12. - We assume the following:

(1) (Fineness) The metric family $\varphi$ is fine.

(2) (Semipositivity) $L$ is semiample and $\varphi_{\omega}$ is semipositive for every $\omega \in \Omega$.

(3) (Bigness) For every subvariety $Y$ of $X,\left.L\right|_{Y}$ is big, and there are a positive number $n_{Y}$ and $s_{Y} \in H^{0}\left(Y,\left.L^{\otimes n_{Y}}\right|_{Y}\right) \backslash\{0\}$ such that $\widehat{\operatorname{deg}}_{\left.\xi_{n_{Y}}\right|_{Y}}\left(s_{Y}\right)>0$.

Then one has

$$
\liminf _{n \rightarrow \infty} \frac{\lambda\left(H^{0}\left(X, L^{\otimes n}\right), \xi_{n}\right)}{n}>0 .
$$

Proof. - We set $R_{n}:=H^{0}\left(X, L^{\otimes n}\right)$ for $n \geqslant 0$. By Proposition 7.5.3 for a positive number $h$,

$$
\frac{1}{h} \liminf _{n \rightarrow \infty} \frac{\lambda\left(R_{n}, \xi_{n}\right)}{n} \leqslant \liminf _{n \rightarrow \infty} \frac{\lambda\left(R_{n}, \xi_{n}\right)}{n},
$$

so that, replacing $L, \varphi, n_{Y}$ and $s_{Y}$ by $L^{\otimes h}, h \varphi, h n_{Y}$ and $s_{Y}^{\otimes h}$ for a sufficiently large integer $h$, we may assume that $\varphi$ is very fine. Moreover, by Remark 4.1.25, we can see that $\varphi$ is measurable. Therefore, by Theorem 7.4.1, one has

$$
\liminf _{n \rightarrow \infty} \frac{\nu_{\min }\left(R_{n}, \xi_{n}\right)}{n}>0
$$

By Proposition 6.1.12, Theorem 6.1.13 and Proposition 7.5.7 $\left(R_{n}, \xi_{n}\right)$ is dominated and coherent for all $n \geqslant 0$. Therefore, by Proposition 7.5.2 and Theorem 7.5.9, one can see the assertion of the theorem.

Remark 7.5.13. - In [44, Nakai-Moishezon's criterion was proved under the following condition (7.11) instead of (Fineness) in Theroem 7.5.12

$$
\left\{\begin{array}{l}
\text { The adelic vector bundle }\left(H^{0}\left(X, L^{\otimes n}\right), \xi_{n}\right) \text { over } S \\
\text { is dominaited and coherent for every } n \geqslant 0 .
\end{array}\right.
$$





\section{APPENDIX A}

\section{REMINDERS ON MEASURE THEORY}

\section{A.1. Monotone class theorems}

We recall here a monotone class theorem in the functional form and several related results and we refer to [49, §I.2] and [144, §2.2] for reference. For convenience of readers, we include the proof here. We fix in this section a non-empty set $\Omega$. If $\mathcal{H}$ is a family of real-valued functions on $\Omega$, we denote by $\sigma(\mathcal{H})$ the $\sigma$-algebra on $\Omega$ generated by $\mathcal{H}$. It is the smallest $\sigma$-algebra on $\Omega$ with respect to which all functions in $\mathcal{H}$ are measurable.

Definition A.1.1. - Let $\mathcal{H}$ be a family of non-negative and bounded functions on $\Omega$. We say that $\mathcal{H}$ is a $\lambda$-family if it verifies the following conditions:

(i) the constant function 1 belongs to $\mathcal{H}$;

(ii) if $f$ and $g$ are two functions in $\mathcal{H}, a$ and $b$ are non-negative numbers, then $a f+b g \in \mathcal{H}$

(iii) if $f$ and $g$ are two functions in $\mathcal{H}$ such that $f \leqslant g$, then $g-f \in \mathcal{H}$;

(iv) if $\left\{f_{n}\right\}_{n \in \mathbb{N}}$ is an increasing and uniformly bounded sequence of functions in $\mathcal{H}$, then the limit of the sequence $\left\{f_{n}\right\}_{n \in \mathbb{N}}$ belongs to $\mathcal{H}$.

Lemma A.1.2. - Let $\mathcal{H}$ be a $\lambda$-family of non-negative and bounded functions on $\Omega$. If for any couple $(f, g)$ of functions in $\mathcal{H}$, one has $\min (f, g) \in \mathcal{H}$, then any nonnegative, bounded and $\sigma(\mathcal{H})$-measurable function on $\Omega$ belongs to $\mathcal{H}$. In particular, the $\sigma$-algebra $\sigma(\mathcal{H})$ is equal to the set of all $A \subseteq \Omega$ such that $\mathbb{1}_{A} \in \mathcal{H}$.

Proof. - Let $\mathcal{F}$ be the set of all $A \subseteq \Omega$ such that $\mathbb{1}_{A} \in \mathcal{H}$. Since $\mathcal{H}$ is a $\lambda$-family, we obtain that $\mathcal{F}$ is a $\lambda$-system $(1)$ and at the same time a $\pi$-system (namely for all

1. Namely $\mathcal{F}$ satisfies the following conditions: (i) $\Omega \in \mathcal{F}$; (ii) if $A \in \mathcal{F}, B \in \mathcal{F}$ and $A \subseteq B$, then $B \backslash A \in \mathcal{F}$, (iii) if $\left\{A_{n}\right\}_{n \in \mathbb{N}}$ is an increasing sequence of elements of $\mathcal{F}$, then the union $\bigcup_{n \in \mathbb{N}} A_{n}$ belongs to $\mathcal{F}$. 
$A \in \mathcal{F}$ and $B \in \mathcal{F}$ one has $A \cap B \in \mathcal{F}$ ) since the family $\mathcal{H}$ is supposed to be stable by the operator $(f, g) \mapsto \min (f, g)$. Therefore $\mathcal{F}$ is actually a $\sigma$-algebra.

If $(f, g)$ is a couple of functions in $\mathcal{H}$, one has

$$
\max (f, g)=f+g-\min (f, g) \in \mathcal{H} .
$$

In particular, if $f \in \mathcal{H}$ and $a \in \mathbb{R}_{+}$, then

$$
\max (f-a, 0)=\max (f, a)-a \in \mathcal{H} .
$$

This property actually implies that, for any $f \in \mathcal{H}$ and any integer $n \geqslant 1$, one has $f^{n} \in \mathcal{H}$. In fact, the function $x \mapsto x^{n}$ is convex on $\mathbb{R}_{+}$, which can be written as the supremum of a countable family of functions of the form

$$
x \longmapsto \max \left(n a^{n-1} x-(n-1) a^{n}, 0\right)
$$

with $a \in \mathbb{Q}_{+}:=\mathbb{Q} \cap \mathbb{R}_{+}$. Therefore by the condition (iv) in Definition A.1.1 one obtains

$$
f^{n}=\sup _{a \in \mathbb{Q}_{+}} \max \left(n a^{n-1} f-(n-1) a^{n}, 0\right) \in \mathcal{H} .
$$

If $f$ is an element of $\mathcal{H}$ and $t$ is a real number, $t>0$, one has $\min \left(t^{-1} f, 1\right) \in \mathcal{H}$. Moreover, the sequence $\left\{1-\min \left(t^{-1} f, 1\right)^{n}\right\}_{n \in \mathbb{N}, n \geqslant 1}$ is increasing and converges to $\mathbb{1}_{\{f<t\}}$, which implies that $\mathbb{1}_{\{f<t\}} \in \mathcal{H}$ and hence $\{f<t\} \in \mathcal{F}$. Therefore every function in $\mathcal{H}$ is $\mathcal{F}$-measurable, and thus $\sigma(\mathcal{H}) \subseteq \mathcal{F}$.

It remains to prove that any non-negative bounded $\mathcal{F}$-measurable function belongs to $\mathcal{H}$. Let $f$ be such a function. For any integer $n \geqslant 1$, let

$$
f_{n}=\sum_{k=0}^{n 2^{n}-1} \frac{k}{2^{n}} \mathbb{1}_{\left\{k / 2^{n} \leqslant f<(k+1) / 2^{n}\right\}}+n \mathbb{1}_{\{f \geqslant n\}} .
$$

This is a function in $\mathcal{H}$. Moreover, the sequence $\left\{f_{n}\right\}_{n \in \mathbb{N}, n \geqslant 1}$ is increasing and converges to $f$. Therefore $f \in \mathcal{H}$.

Theorem A.1.3. - Let $\mathcal{H}$ be a $\lambda$-family of non-negative and bounded functions on $\Omega$ and $\mathcal{C}$ be a subset of $\mathcal{H}$. Assume that for any couple $(f, g)$ of functions in $\mathcal{C}$, the product function $f g$ belongs to $\mathcal{C}$. Then any non-negative and bounded $\sigma(\mathcal{C})$ measurable function belongs to $\mathcal{H}$.

Proof. - By replacing $\mathcal{H}$ by the intersection of all $\lambda$-families containing $\mathcal{C}$ we may assume that $\mathcal{H}$ is the smallest $\lambda$-family which contains $\mathcal{C}$.

We first prove that $\mathcal{H}$ is stable by multiplication. Let $\mathcal{H}_{1}$ be the set of all nonnegative and bounded functions $f$ on $\Omega$ such that $f g \in \mathcal{H}$ for any $g \in \mathcal{C}$. This is a $\lambda$-family containing $\mathcal{C}$. Hence one has $\mathcal{H}_{1} \supseteq \mathcal{H}$. Let $\mathcal{H}_{2}$ be the set of all non-negative and bounded functions $f$ on $\Omega$ such that $f g \in \mathcal{H}$ for any $g \in \mathcal{H}$. This is also a $\lambda$-family. Moreover, since $\mathcal{H}_{1} \supseteq \mathcal{H}$ one obtains $\mathcal{H}_{2} \supseteq \mathcal{C}$ and hence $\mathcal{H}_{2} \supseteq \mathcal{H}$, which implies that $\mathcal{H}$ is stable by multiplication. 
Let $f$ and $g$ be two functions in $\mathcal{H}$. We will prove that $|f-g| \in \mathcal{H}$. By dilating the function $|f-g|$ by a positive constant, we may assume that $|f-g|$ is bounded from above by 1 . One has

$$
(f-g)^{2}=f^{2}+g^{2}-2 f g \in \mathcal{H} .
$$

Let $\left\{f_{n}\right\}_{n \in \mathbb{N}}$ be the sequence of functions on $\Omega$ defined by the following recursive formula

$$
f_{0}=0, \quad f_{n+1}=f_{n}+\frac{1}{2}\left((f-g)^{2}-f_{n}^{2}\right) .
$$

By induction on $n$, we can show that $f_{n} \in \mathcal{H}$ and $f_{n} \leqslant|f-g|$. In fact, these properties are trivially satisfied by $f_{0}$. If $f_{n} \in \mathcal{H}$ and $f_{n} \leqslant|f-g|$, then one has $f_{n+1} \in \mathcal{H}$. Moreover, by the relation $|f-g| \leqslant 1$ one obtains $f_{n+1} \leqslant|f-g|$ since the function $t \mapsto t-\frac{1}{2} t^{2}$ is increasing on the interval [0,1]. The properties $f_{n} \in \mathcal{H}$ and $f_{n} \leqslant|f-g|$ show that the sequence $\left\{f_{n}\right\}_{n \in \mathbb{N}}$ is increasing and converges to $|f-g|$. Hence $|f-g| \in \mathcal{H}$, which implies that

$$
\min (f, g)=\frac{1}{2}(f+g-|f-g|) \in \mathcal{H} .
$$

By Lemma A.1.2 any non-negative, bounded and $\sigma(\mathcal{H})$-measurable function belongs to $\mathcal{H}$. The theorem is thus proved.

\section{A.2. Measurable selection theorem}

In this section, we recall a measurable selection theorem due to Kuratowski and Ryll-Nardzewski [96]. See [112, Chapter 5] for more details.

Theorem A.2.1. - Let $Y$ be a complete separable metric space and $\mathscr{P}(Y)$ be the set of subsets of $Y$. Let $(\Omega, \mathcal{A})$ be a measurable space and $F: \Omega \rightarrow \mathscr{P}(Y)$ be a map. We assume that

(1) for any $\omega \in \Omega$, the set $F(\omega)$ is a non-empty closed subset of $Y$,

(2) for any open subset $U$ of $Y$, the set $\{\omega \in \Omega: F(\omega) \cap U \neq \varnothing\}$ belongs to $\mathcal{A}$.

Then there exist a measurable map $f: \Omega \rightarrow Y$ such that $f(\omega) \in F(\omega)$ for any $\omega \in \Omega$.

\section{A.3. Vague convergence and weak convergence of measures}

Let $X$ be a locally compact Hausdorff space. Recall that a Radon measure is by definition a Borel measure $\nu$ on $X$ which satisfies the following conditions:

(1) $\nu$ is tight, that is, for any Borel subset $B$ of $X, \nu(B)$ is equal to the supremum of $\nu(K)$, where $K$ runs over the set of compact subsets of $B$;

(2) $\nu$ is outer regular, that is, for any Borel subset $B$ of $X, \nu(B)$ is equal to the infimum of $\nu(U)$, where $U$ runs over the set of open subsets of $X$ containing $B$;

(3) $\nu$ is locally finite, that is, for any $x \in X$ there exists a neighbourhood $U$ of $x$ such that $\nu(U)<+\infty$. 
We denote by $\mathscr{M}(X)$ be the set of Radon measures on $X$. Let $C_{c}(X)$ be the vector space of continuous real-valued functions of compact support on $X$. We say that an $\mathbb{R}$-linear map $\varphi: C_{c}(X) \rightarrow \mathbb{R}$ is a positive functional if $\varphi(f) \geqslant 0$ for any non-negative function $f$ in $C_{c}(X)$. Recall the Riesz's representation theorem as follows. See $[\mathbf{8 0}$, $\S 56]$ for a proof.

Theorem A.3.1. — Let $X$ be a locally compact Hausdorff space. The map sending $\nu \in \mathscr{M}(X)$ to the positive functional

$$
f \in C_{c}(X) \longrightarrow \int_{X} f \mathrm{~d} \nu
$$

defines a bijection between the set $\mathscr{M}(X)$ and the set of all positive linear functionals on $C_{c}(X)$.

The vague topology on $\mathscr{M}(X)$ is an example of weak-* topology if we identify $\mathscr{M}(X)$ with a subset of the dual space of $C_{c}(X)$. More precisely, we say that a sequence $\left\{\nu_{n}\right\}_{n \in \mathbb{N}}$ of Radon measures converges vaguely if for any function $f \in C_{c}(X)$, the sequence of integrals $\left\{\int_{X} f \mathrm{~d} \nu_{n}\right\}_{n \in \mathbb{N}}$ converges in $\mathbb{R}$. Note that the limit of the above sequence defines a positive linear functional on $C_{c}(X)$ when $f$ varies, which corresponds to a Radon measure, called the vague limit of $\left\{\nu_{n}\right\}_{n \in \mathbb{N}}$.

If $\left\{\nu_{n}\right\}_{n \in \mathbb{N}}$ is a sequence of Radon probability measure which converges vaguely, the limite measure may have a total mass $<1$. In probability theory, the notion of weak convergence is also largely used. Let $\mathscr{M}_{1}(X)$ be the subset of $\mathscr{M}(X)$ of probability measures. Let $C_{b}(X)$ be the vector space of bounded continuous functions. We say that a sequence $\left\{\nu_{n}\right\}_{n \in \mathbb{N}}$ of measures in $\mathscr{M}_{1}(X)$ (they are therefore probability measures) converges weakly if for any bounded continuous function $f$ on $X$, the sequence of integrals $\left\{\int_{X} f \mathrm{~d} \nu_{n}\right\}_{n \in \mathbb{N}}$ converges in $\mathbb{R}$. Clearly, if the sequence $\left\{\nu_{n}\right\}_{n \in \mathbb{N}}$ converges weakly, then it also converges vaguely, and its vague limit is also called its weak limit. Note that in the weak convergence case the limit measure should be a probability measure. The following criterion provides a criterion of weak convergence for vaguely convergence sequence of Radon probability measures. We refer the readers to [94, Theorem 13.16] for the proof and for more details (2).

Theorem A.3.2. - Let $X$ be a locally compact metrisable space and $\left\{\nu_{n}\right\}_{n \in \mathbb{N}}$ be a sequence of Radon probability measures on $X$, which converges vaguely to a limite measure $\nu$. Assume the limite measure $\nu$ is a probability measure. Then the sequence $\left\{\nu_{n}\right\}_{n \in \mathbb{N}}$ converge weakly to $\nu$.

2. In 94, Theorem 13.16], it is assumed that the topological space is a locally compact Polish space. This condition is satisfied notably when $X$ is a locally compact Hausdorff space with countable base, see [138. However, it actually suffices that the topological space is locally compact and metrisable (see Lemma 13.10 of [94] which is used in the proof of Theorem 13.16 of loc. cit.). 


\section{A.4. Upper and lower integral}

Let $(\Omega, \mathcal{A}, \nu)$ be a measure space. We denote by $\mathscr{L}^{1}(\Omega, \mathcal{A}, \nu)$ the vector space of all real-valued $\nu$-integrable functions on $(\Omega, \mathcal{A})$. We say that a subset $A$ of $\Omega$ is $\nu$ negligible if there exists a set $B \in \mathcal{A}$ such that $\nu(B)=0$ and that $A \subseteq B$. We say that two functions $h_{1}$ and $h_{2}$ on $\Omega$ are $\nu$-indistinguishable if $\left\{h_{1} \neq h_{2}\right\}$ is a $\nu$-negligible set. Any function on $\Omega$ which is $\nu$-indistinguishable with the zero function is said to be $\nu$-negligible. In other words, a function $f$ on $\Omega$ is $\nu$-negligible if and only if $\{f \neq 0\}$ is a $\nu$-negligible set. If a formula depending on a variable $\omega \in \Omega$ is satisfied outside of a $\nu$-negligible set, we say that it holds $\nu$-almost everywhere (written in abbriviation as $\nu$-a.e.).

Definition A.4.1. - We construct two non-necessarily linear functional $\bar{I}_{v}(\cdot)$ and $\underline{I}_{\nu}(\cdot)$ as follows. For any function $h: \Omega \rightarrow \mathbb{R}$, let

$$
\begin{aligned}
\int_{\Omega} h(\omega) \nu(d \omega) & :=\inf _{\substack{f \in \mathscr{L}^{1}(\Omega, \mathcal{A}, \nu) \\
f \geqslant h \nu \text {-a.e. }}} \int_{\Omega} f(\omega) \nu(d \omega), \\
\int_{\Omega} h(\omega) \nu(d \omega) & :=\sup _{\substack{g \in \mathscr{L}^{1}(\Omega, \mathcal{A}, \nu) \\
g \leqslant h \nu \text {-a.e. }}} \int_{\Omega} g(\omega) \nu(d \omega) .
\end{aligned}
$$

If $h$ is not $\nu$-almost everywhere bounded from above by any integrable function, then $\int_{\Omega} h(\omega) \nu(d \omega)$ is defined as $+\infty$ by convention. Similarly, if $h$ is not $\nu$-almost everywhere bounded from below by any integrable function, then $\int_{\Omega} h(\omega) \nu(d \omega)$ is defined as $-\infty$ by convention. The values $\bar{\int}_{\Omega} h(\omega) \nu(d \omega)$ and $\int_{\Omega} h(\omega) \nu(d \omega)$ are called upper integral and lower integral of the function $h$, respectively. From now on, for simplicity,

$$
\bar{\int}_{\Omega} h(\omega) \nu(d \omega), \quad \int_{\Omega} h(\omega) \nu(d \omega) \text { and } \int_{\Omega} f(\omega) \nu(d \omega)
$$

are denoted by $\bar{I}_{\nu}(h), \underline{I}_{\nu}(h)$ and $I_{\nu}(f)$, respectively, for any function $h$ on $\Omega$ and any integrable function $f$ on $\Omega$.

The following properties are straightforward from the definition of upper and lower integrals.

Proposition A.4.2. - (1) For any function $h: \Omega \rightarrow \mathbb{R}$

$$
\underline{I}_{\nu}(h) \leqslant \bar{I}_{\nu}(h) .
$$

(2) If $h_{1}$ and $h_{2}$ are two real-valued functions on $\Omega$ such that $h_{1} \leqslant h_{2}$, then

$$
\underline{I}_{\nu}\left(h_{1}\right) \leqslant \underline{I}_{\nu}\left(h_{2}\right), \quad \bar{I}_{\nu}\left(h_{1}\right) \leqslant \bar{I}_{\nu}\left(h_{2}\right) .
$$

Proposition A.4.3. - Let $h$ be a real-valued function on $\Omega$. Then $h$ is $\nu$ indistinguishable with a $\nu$-integrable function if and only if $\bar{I}_{\nu}(h)=\underline{I}_{\nu}(h) \in \mathbb{R}$. 
Proof. - If $h$ is indistinguishable with a $\nu$-integrable function $\widetilde{h}$, then $\bar{I}_{\nu}(h)$ and $\underline{I}_{\nu}(h)$ are both equal to the integral of $\widetilde{h}$ with respect to the measure $\nu$, which is a real number.

Conversely, assume that $\bar{I}_{\nu}(h)=\underline{I}_{\nu}(h)$, then we can find two sequences $\left\{f_{n}\right\}_{n \in \mathbb{N}}$ and $\left\{g_{n}\right\}_{n \in \mathbb{N}}$ of functions in $\mathscr{L}^{1}(\Omega, \mathcal{A}, \nu)$ such that $g_{n} \leqslant h \leqslant f_{n} \nu$-almost everywhere. and that

$$
\lim _{n \rightarrow+\infty} I_{\nu}\left(f_{n}\right)=\bar{I}_{\nu}(h)=\underline{I}_{\nu}(h)=\lim _{n \rightarrow+\infty} I_{\nu}\left(g_{n}\right)
$$

Without loss of generality, we may assume that the sequence $\left\{f_{n}\right\}_{n \in \mathbb{N}}$ is decreasing and $\left\{g_{n}\right\}_{n \in \mathbb{N}}$ is increasing (otherwise we replace $f_{n}$ by $\widetilde{f}_{n}=\min \left\{f_{1}, \ldots, f_{n}\right\}$ and $g_{n}$ by $\left.\widetilde{g}_{n}=\max \left\{g_{1}, \ldots, g_{n}\right\}\right)$. Let $f=\inf _{n \in \mathbb{N}} f_{n}$ and $g=\sup _{n \in \mathbb{N}} g_{n}$. By Lebesgue's dominant convergence theorem, we obtain that $f$ and $g$ are both $\nu$-integrable, and

$$
I_{\nu}(f)=\bar{I}_{\nu}(h)=\underline{I}_{\nu}(h)=I_{\nu}(g) .
$$

Moreover, one has $g \leqslant h \leqslant f \nu$-almost everywhere, which implies that $f=g=h$ $\nu$-almost everywhere.

In general the operators $\bar{I}_{\nu}(\cdot)$ and $\underline{I}_{\nu}(\cdot)$ are not linear operators. However, they satisfies some convexity property.

Proposition A.4.4. - Let $h_{1}$ and $h_{2}$ be two real-valued functions on $\Omega$.

(1) Assume that $\left\{\bar{I}_{\nu}\left(h_{1}\right), \bar{I}_{\nu}\left(h_{2}\right)\right\} \neq\{+\infty,-\infty\}$. Then one has

$$
\bar{I}_{\nu}\left(h_{1}+h_{2}\right) \leqslant \bar{I}_{\nu}\left(h_{1}\right)+\bar{I}_{\nu}\left(h_{2}\right)
$$

(2) Assume that $\left\{\underline{I}_{\nu}\left(h_{1}\right), \underline{I}_{\nu}\left(h_{2}\right)\right\} \neq\{+\infty,-\infty\}$. Then one has

$$
\underline{I}_{\nu}\left(h_{1}+h_{2}\right) \geqslant \underline{I}_{\nu}\left(h_{1}\right)+\underline{I}_{\nu}\left(h_{2}\right) \text {. }
$$

Proof. - (1) We first treat the case where neither of $\bar{I}_{\nu}\left(h_{1}\right)$ and $\bar{I}_{\nu}\left(h_{2}\right)$ is $+\infty$. If $f_{1}$ and $f_{2}$ be two $\nu$-integrable functions on $\Omega$ such that $f_{1} \geqslant h_{1}$ and $f_{2} \geqslant h_{2} \nu$-almost everywhere. Then the sum $f_{1}+f_{2}$ is $\nu$-integrable, and $f_{1}+f_{2} \geqslant h_{1}+h_{2}$. Therefore $I_{\nu}\left(f_{1}\right)+I_{\nu}\left(f_{2}\right)=I_{\nu}\left(f_{1}+f_{2}\right) \geqslant \bar{I}_{\nu}\left(h_{1}+h_{2}\right)$. Since $f_{1}$ and $f_{2}$ are arbitrary, we obtain $\bar{I}_{\nu}\left(h_{1}\right)+\bar{I}_{\nu}\left(h_{2}\right) \geqslant \bar{I}_{\nu}\left(h_{1}+h_{2}\right)$.

If at least one of the upper integrals $\bar{I}_{\nu}\left(h_{1}\right)$ and $\bar{I}_{\nu}\left(h_{2}\right)$ is $+\infty$, then by the hypothesis $\left\{\bar{I}_{\nu}\left(h_{1}\right), \bar{I}_{\nu}\left(h_{2}\right)\right\} \neq\{+\infty,-\infty\}$ one has $\bar{I}_{\nu}\left(h_{1}\right)+\bar{I}_{\nu}\left(h_{2}\right)=+\infty$. Hence the inequality A.3 is trivial.

The proof of the statement (2) is very similar to that of (1). We omit the details.

Proposition A.4.5. - Let $h$ be a real-valued function on $\Omega$ and $\varphi$ be a $\nu$-integrable function. Then one has

$$
\bar{I}_{\nu}(h+\varphi)=\bar{I}_{\nu}(h)+I_{\nu}(\varphi), \quad \underline{I}_{\nu}(h+\varphi)=\underline{I}_{\nu}(h)+I_{\nu}(\varphi) .
$$

Proof. - Since $\varphi$ is $\nu$-integrable, one has

$$
\bar{I}_{\nu}(\varphi)=I_{\nu}(\varphi)=\underline{I}_{\nu}(\varphi) \in \mathbb{R} .
$$


By Proposition A.4.4, one has

$$
\bar{I}_{\nu}(h+\varphi) \leqslant \bar{I}_{\nu}(h)+I_{\nu}(\varphi) .
$$

Moreover, if we apply this inequality to $h+\varphi$ and $-\varphi$, we obtain

$$
\bar{I}_{\nu}(h) \leqslant \bar{I}_{\nu}(h+\varphi)-I_{\nu}(\varphi) .
$$

Therefore the first equality is true. The proof of the second equality is quite similar, we omit the details.

Proposition A.4.6. - Let $h$ be a real-valued function on $\Omega$. If a is a non-negative number, then one has

$$
\bar{I}_{\nu}(a h)=a \bar{I}_{\nu}(h), \quad \underline{I}_{\nu}(a h)=a \underline{I}_{\nu}(h) .
$$

Proof. - The assertions are trivial when $a=0$. In the following, we assume that $a>0$. If $f$ is a $\nu$-integrable function such that $h \leqslant f \nu$-almost everywhere, then af is a $\nu$-integrable function such that $a h \leqslant a f \nu$-almost everywhere. Therefore, we obtain that $\bar{I}_{\nu}(a h) \leqslant a \bar{I}(h)$. If we apply this inequality to $a^{-1}$ and $a h$ we get $\bar{I}_{\nu}(h) \leqslant a^{-1} \bar{I}_{\nu}(a h)$. Hence the first equality is true. The proof of the second equality is very similar, we omit the details.

Proposition A.4.7. - Let $h$ be a real-valued function on $\Omega$. One has

$$
\bar{I}_{\nu}(-h)=-\underline{I}_{\nu}(h), \quad \underline{I}_{\nu}(-h)=-\bar{I}_{\nu}(h) .
$$

Proof. - If $f$ is an $\nu$-integrable function such that $-h \leqslant f \nu$-almost everywhere, then one has $-f \leqslant h \nu$-almost everywhere. Since $f$ is arbitrary, we obtain $-\bar{I}_{\nu}(-h) \leqslant$ $\underline{I}_{\nu}(h)$. Similarly, if $g$ is an $\nu$-integrable function such that $g \leqslant-h \nu$-almost everywhere, then one has $h \leqslant-g \nu$-almost everywhere Since $g$ is arbitrary, we obtain $-\underline{I}_{\nu}(-h) \geqslant \bar{I}_{\nu}(h)$. Finally, if we apply the obtained inequality to $-h$, we obtain $-\bar{I}_{\nu}(h) \leqslant \underline{I}_{\nu}(-h)$ and $-\underline{I}_{\nu}(h) \geqslant \bar{I}_{\nu}(-h)$. Therefore the equalities hold.

Proposition A.4.8. - Let $h_{1}$ and $h_{2}$ be two real-valued functions on $\Omega$, and let $h=h_{1}+h_{2}$. Assume that $\left\{\bar{I}_{\nu}\left(h_{1}\right), \underline{I}_{\nu}\left(h_{2}\right)\right\} \neq\{+\infty,-\infty\}$. Then one has

$$
\underline{I}_{\nu}(h) \leqslant \bar{I}_{\nu}\left(h_{1}\right)+\underline{I}_{\nu}\left(h_{2}\right) \leqslant \bar{I}_{\nu}(h) .
$$

Proof. - By the equality $h=h_{1}+h_{2}$ we obtain $h_{1}=\left(-h_{2}\right)+h$. Thus Proposition A.4.4 leads to

$$
\bar{I}_{\nu}\left(h_{1}\right) \leqslant \bar{I}_{\nu}\left(-h_{2}\right)+\bar{I}_{\nu}(h)=-\underline{I}_{\nu}\left(h_{2}\right)+\bar{I}_{\nu}(h)
$$

where the equality comes from Proposition A.4.7. Hence we obtain the inequality

$$
\bar{I}_{\nu}(h) \geqslant \bar{I}_{\nu}\left(h_{1}\right)+\underline{I}_{\nu}\left(h_{2}\right) .
$$

We then apply this inequality to $-h,-h_{2}$ and $-h_{1}$ to get the other equality. 
Definition A.4.9. - Let $h: \Omega \rightarrow \mathbb{R}$ be a real valued function on $\Omega$. We say that $h$ is $\nu$-dominated if there exists a $\nu$-integrable function $f$ such that $\{\omega \in \Omega:|h(\omega)| \leqslant$ $f(\omega)\}$ is a $\nu$-negligible set (in other words, $|h| \leqslant f \nu$-almost everywhere). Note that this condition is equivalent to

$$
\bar{I}_{\nu}(h)<+\infty \text { and } \underline{I}_{\nu}(h)>-\infty .
$$

We denote by $\mathscr{D}^{1}(\Omega, \mathcal{A}, \nu)$ the vector space of $\nu$-dominated functions on $\Omega$. Clearly one has $\mathscr{D}^{1}(\Omega, \mathcal{A}, \nu) \supseteq \mathscr{L}^{1}(\Omega, \mathcal{A}, \nu)$, and $\mathscr{D}^{1}(\Omega, \mathcal{A}, \nu)$ is invariant by the operator $f \mapsto|f|$ of taking the absolute value. Moreover, if $f$ and $g$ are real valued functions on $\Omega$ such that $|f| \leqslant|g| \nu$-almost everywhere and that $g$ is $\nu$-dominated, then the function $f$ is also $\nu$-dominated.

Proposition A.4.10. - Let $\|\cdot\|_{\mathscr{D}_{\nu}^{1}}$ be the function on $\mathscr{D}^{1}(\Omega, \mathcal{A}, \nu)$ sending any $\nu$ dominated function $f$ to $\bar{I}_{\nu}(|f|)$. Then $\|\cdot\|_{\mathscr{D}_{\nu}^{1}}$ is a seminorm. Moreover, a function $f \in \mathscr{D}^{1}(\Omega, \mathcal{A}, \nu)$ satisfies $\|f\|_{\mathscr{D}_{\nu}^{1}}=0$ if and only if it is $\nu$-negligible.

Proof. - Let $f$ be a $\nu$-dominated function and $a$ be a real number. One has $|a f|=$ $|a| \cdot|f|$. By Proposition A.4.6 we obtain that

$$
\|a f\|_{\mathscr{D}_{\nu}^{1}}=\bar{I}_{\nu}(|a f|)=\bar{I}_{\nu}(|a| \cdot|f|)=|a| \cdot \bar{I}_{\nu}(|f|)=|a| \cdot\|f\|_{\mathscr{D}_{\nu}^{1}} .
$$

Moreover, if $f$ and $g$ are two $\nu$-dominated functions, then by Proposition A.4.4 one has

$$
\|f+g\|_{\mathscr{D}_{\nu}^{1}}=\bar{I}_{\nu}(|f+g|) \leqslant \bar{I}_{\nu}(|f|+|g|) \leqslant \bar{I}_{\nu}(|f|)+\bar{I}_{\nu}(|g|)=\|f\|_{\mathscr{D}_{\nu}^{1}}+\|g\|_{\mathscr{D}_{\nu}^{1}},
$$

where the first inequality comes from (A.2), and the second inequality comes from (A.4.4). Therefore $\|\cdot\|_{\mathscr{D}_{\nu}}$ is a seminorm on $\mathscr{D}_{\nu}^{1}(\Omega, \mathcal{A}, \nu)$.

Let $f$ be a $\nu$-negligible function. Then one has $|f|=0 \nu$-almost everywhere. Hence one has $\|f\|_{\mathscr{D}_{\nu}^{1}}=\bar{I}_{\nu}(|f|)=0$. Conversely, if $f$ is a $\nu$-dominated function such that $\bar{I}_{\nu}(|f|)=0$, then one has $\underline{I}_{\nu}(|f|)=\bar{I}_{\nu}(|f|)=0$. By Proposition A.4.3, $|f|$ is $\nu$ indistinguishable with a $\nu$-integrable function $g$ of integral 0 . Moreover, since $|f|$ is non-negative, we obtain that the set $\{g<0\}$ is $\nu$-negligible. Therefore $g$ vanishes $\nu$-almost everywhere. Thus $f$ is $\nu$-negligible.

Proposition A.4.11. - Let $\left\{f_{n}\right\}_{n \in \mathbb{N}}$ be an increasing sequence of non-negative functions on $\Omega$ and $f$ be the limit of $\left\{f_{n}\right\}_{n \in \mathbb{N}}$. Then one has

$$
\lim _{n \rightarrow+\infty} \bar{I}_{\nu}\left(f_{n}\right)=\bar{I}_{\nu}(f) \text {. }
$$

Proof. - Clearly one has $\bar{I}_{\nu}\left(f_{n}\right) \leqslant \bar{I}_{\nu}(f)$ for any $n \in \mathbb{N}$. Hence

$$
\lim _{n \rightarrow+\infty} \bar{I}_{\nu}\left(f_{n}\right) \leqslant \bar{I}_{\nu}(f) \text {. }
$$

If one of the functions $f_{n}$ is not dominated, then neither is $f$. Hence one has

$$
\lim _{n \rightarrow+\infty} \bar{I}_{\nu}\left(f_{n}\right)=+\infty=\bar{I}_{\nu}(f) \text {. }
$$


In the following, we assume that all the functions $f_{n}$ are dominated. Let $\epsilon>0$. For any $n \in \mathbb{N}$, let $g_{n}$ be an integrable function on $\Omega$ such that $f_{n} \leqslant g_{n}$ and that $\bar{I}_{\nu}\left(f_{n}\right) \geqslant I_{\nu}\left(g_{n}\right)-\epsilon$. Note that $\widetilde{g}_{n}:=\inf _{m \geqslant n} g_{m}$ is also an integrable function on $\Omega$ such that $f_{n} \leqslant \widetilde{g}_{n}$ and $\bar{I}_{\nu}\left(f_{n}\right) \geqslant I_{\nu}\left(\widetilde{g}_{n}\right)-\varepsilon$. Therefore, by replacing $g_{n}$ by $\widetilde{g}_{n}$, we may assume without loss of generality that the sequence $\left\{g_{n}\right\}_{n \in \mathbb{N}}$ is increasing. Let $g=\sup _{n \in \mathbb{N}} g_{n}$. By the monotone convergence theorem one has

$$
\bar{I}_{\nu}(g)=\lim _{n \rightarrow+\infty} I_{\nu}\left(g_{n}\right) \leqslant \lim _{n \rightarrow+\infty} \bar{I}_{\nu}\left(f_{n}\right)+\epsilon .
$$

Moreover, since $g \geqslant f$, one has $\bar{I}_{\nu}(f) \leqslant \bar{I}_{\nu}(g)$. Therefore the proposition is proved.

Corollary A.4.12. - Let $\left\{f_{n}\right\}_{n \in \mathbb{N}}$ be a sequence of non-negative functions on $\Omega$, and $f$ be the sum of the series $\sum_{n \in \mathbb{N}} f_{n}$. Then one has

$$
\bar{I}_{\nu}(f) \leqslant \sum_{n \in \mathbb{N}} \bar{I}_{\nu}\left(f_{n}\right)
$$

Proof. - For any $n \in \mathbb{N}$, let $g_{n}=\sum_{k=0}^{n} f_{k}$. The sequence $\left\{g_{n}\right\}_{n \in \mathbb{N}}$ is increasing, and converges to $f$. Therefore, by Proposition A.4.11, one has

$$
\bar{I}_{\nu}(f)=\lim _{n \rightarrow+\infty} \bar{I}_{\nu}\left(g_{n}\right) .
$$

Moreover, by Proposition A.4.4, for any $n \in \mathbb{N}$ one has

$$
\bar{I}_{\nu}\left(g_{n}\right) \leqslant \sum_{k=0}^{n} \bar{I}_{\nu}\left(f_{k}\right)
$$

Hence we obtain

$$
\bar{I}_{\nu}(f) \leqslant \sum_{n \in \mathbb{N}} \bar{I}_{\nu}\left(f_{n}\right)
$$

Proposition A.4.13. - Let $\left\{f_{n}\right\}_{n \in \mathbb{N}}$ be a sequence of non-negative functions on $\Omega$ and $f=\liminf _{n \rightarrow+\infty} f_{n}$. Then one has

$$
\bar{I}_{\nu}(f) \leqslant \liminf _{n \rightarrow+\infty} \bar{I}_{\nu}\left(f_{n}\right) .
$$

Proof. - For any $n \in \mathbb{N}$, let $g_{n}=\inf _{m \geqslant n} f_{m}$. Then the sequence $\left\{g_{n}\right\}_{n \in \mathbb{N}}$ is increasing and converges to $f$. By Proposition A.4.11, one has

$$
\bar{I}_{\nu}(f)=\lim _{n \rightarrow+\infty} \bar{I}_{\nu}\left(g_{n}\right) \leqslant \liminf _{n \rightarrow+\infty} \bar{I}_{\nu}\left(f_{n}\right),
$$

where the inequality comes from the fact that $g_{n} \leqslant f_{n}$ for any $n \in \mathbb{N}$. The proposition is thus proved. 
Proposition A.4.14. - Let $D^{1}(\Omega, \mathcal{A}, \nu)$ be the quotient space of $\mathscr{D}^{1}(\Omega, \mathcal{A}, \nu)$ by the vector subspace of $\nu$-negligible functions. Then the seminorm $\|\cdot\|_{\mathscr{D}_{\nu}^{1}}$ on $\mathscr{D}^{1}(\Omega, \mathcal{A}, \nu)$ induced a norm $\|\cdot\|_{D_{\nu}^{1}}$ on $D^{1}(\Omega, \mathcal{A}, \nu)$ induced by the seminorm $\|\cdot\|_{\mathscr{D}_{\nu}^{1}}$, and the vector space $D^{1}(\Omega, \mathcal{A}, \nu)$ is complete with respect to this norm.

Proof. - The first assertion is a direct consequence of Proposition A.4.10, In the following, we prove the second assertion.

Let $\left\{f_{n}\right\}_{n \in \mathbb{N}}$ be a Cauchy sequence in $\mathscr{D}^{1}(\Omega, \mathcal{A}, \nu)$. For any $\epsilon>0$ and any $m, n \in \mathbb{N}$, one has $\left|f_{n}-f_{m}\right| \geqslant \epsilon \mathbb{1}_{\left\{\left|f_{n}-f_{m}\right|>\epsilon\right\}}$, which implies that

$$
\left\|f_{n}-f_{m}\right\|_{\mathscr{D}_{\nu}^{1}}=\bar{I}_{\nu}\left(\left|f_{n}-f_{m}\right|\right) \geqslant \epsilon \bar{I}_{\nu}\left(\mathbb{1}_{\left\{\left|f_{n}-f_{m}\right|>\epsilon\right\}}\right) .
$$

Since $\left\{f_{n}\right\}_{n \in \mathbb{N}}$ is a Cauchy sequence, one has

$$
\lim _{N \rightarrow+\infty} \sup _{\substack{(n, m) \in \mathbb{N}^{2} \\ n \geqslant N, m \geqslant N}}\left\|f_{n}-f_{m}\right\|=0 .
$$

Therefore we can construct a subsequence $\left\{f_{n_{k}}\right\}_{k \geqslant 1}$ of $\left\{f_{n}\right\}_{n \in \mathbb{N}}$ such that

$$
\forall k \in \mathbb{N}_{\geqslant 1}, \quad \bar{I}_{\nu}\left(\mathbb{1}_{\left\{\left|f_{n_{k}}-f_{n_{k+1}}\right|>2^{-k}\right\}}\right)<2^{-k} .
$$

For any $m \in \mathbb{N}_{\geqslant 1}$, let $A_{m}=\bigcup_{k \geqslant m}\left\{\left|f_{n_{k}}-f_{n_{k+1}}\right|>2^{-k}\right\}$. Then the set

$$
B:=\left\{\omega \in \Omega:\left\{f_{n_{k}}(\omega)\right\}_{k \geqslant 1} \text { does not converge }\right\}
$$

is contained in $\bigcap_{m \geqslant 1} A_{m}$. Moreover, for any $m \in \mathbb{N}_{\geqslant 1}$, by Corollary A.4.12 one has

$$
\bar{I}_{\nu}\left(\mathbb{1}_{A_{m}}\right) \leqslant \sum_{k \geqslant m} \bar{I}_{\nu}\left(\mathbb{1}_{\left\{\left|f_{n_{k}}-f_{n_{k+1}}\right|>2^{-k}\right\}}\right) \leqslant 2^{-m+1} .
$$

Therefore one obtain $\bar{I}_{\nu}\left(\mathbb{1}_{B}\right)=0$, which implies that $B$ is a $\nu$-negligible set. Thus we obtain that the sequence $\left\{f_{n_{k}}\right\}_{k \geqslant 1}$ converges $\nu$-almost everywhere to some function $f$ on $\Omega$. Note that by Proposition A.4.13 one has

$$
\bar{I}_{\nu}(|f|) \leqslant \liminf _{k \rightarrow+\infty} \bar{I}_{\nu}\left(\left|f_{n_{k}}\right|\right) .
$$

Therefore $f$ is a dominated function. Finally, still by Proposition A.4.13, for any $n \in \mathbb{N}$ one has

$$
\bar{I}_{\nu}\left(\left|f_{n}-f\right|\right) \leqslant \liminf _{k \rightarrow+\infty} \bar{I}_{\nu}\left(\left|f_{n}-f_{n_{k}}\right|\right) .
$$

Hence one has

$$
\lim _{n \rightarrow+\infty} \bar{I}_{\nu}\left(\left|f_{n}-f\right|\right)=0 .
$$

The proposition is thus proved. 


\section{A.5. $L^{1}$ space}

Let $\mathscr{L}^{1}(\Omega, \mathcal{A}, \nu)$ be the vector space of all real-valued $\nu$-integrable functions on the measurable space $(\Omega, \mathcal{A})$. This vector space is equipped with the seminorm $\|\cdot\|_{\mathscr{L}_{\nu}^{1}}$ which sends a function $f \in \mathscr{L}^{1}(\Omega, \mathcal{A}, \nu)$ to

$$
\|f\|_{\mathscr{L}_{\nu}^{1}}:=\int_{\Omega}|f(\omega)| \nu(\mathrm{d} \omega) .
$$

Note that the set of all functions $f \in \mathscr{L}^{1}(\Omega, \mathcal{A}, \nu)$ such that $\|f\|_{\mathscr{L}^{1}}=0$ forms a vector subspace of $\mathscr{L}^{1}(\Omega, \mathcal{A}, \nu)$. Such functions are said to be $\nu$-negligible. The quotient space of $\mathscr{L}^{1}(\Omega, \mathcal{A}, \nu)$ by the vector subspace of $\nu$-negligible functions is denoted by $L^{1}(\Omega, \mathcal{A}, \nu)$. The seminorm $\|\cdot\|_{\mathscr{L}_{\nu}^{1}}$ induces by quotient a norm on $L^{1}(\Omega, \mathcal{A}, \nu)$, which we denote by $\|\cdot\|_{L_{\nu}^{1}}$. Note that the vector space $L^{1}(\Omega, \mathcal{A}, \nu)$ is complete with respect to this norm, and the integration with respect to $\nu$ induces a continuous linear form on $L^{1}(\Omega, \mathcal{A}, \nu)$, which we denote by

$$
\left(\zeta \in L^{1}(\Omega, \mathcal{A}, \nu)\right) \longmapsto \int_{\Omega} \zeta(\omega) \nu(\mathrm{d} \omega)
$$

by abuse of notation. 



\section{BIBLIOGRAPHY}

[1] Y. AndrÉ - "Slope filtrations", Confluentes Mathematici 1 (2009), no. 1, p. 1-85.

[2] Y. ANDRÉ - "On nef and semistable Hermitian lattices, and their behaviour under tensor product", The Tohoku Mathematical Journal. Second Series 63 (2011), no. 4, p. 629-649.

[3] S. J. ARAKELOV - "Theory of intersections on the arithmetic surface", in Proceedings of the International Congress of Mathematicians (Vancouver, B.C., 1974), Vol. 1, Canad. Math. Congress, Montreal, Que., 1975, p. 405-408.

[4] S. J. ARAKELOV - "An intersection theory for divisors on an arithmetic surface", Izvestiya Akademii Nauk SSSR. Seriya Matematicheskaya 38 (1974), p. 1179-1192.

[5] M. Aттуah, R. Botт \& V. K. Patodi - "On the heat equation and the index theorem", Inventiones Mathematicae 19 (1973), p. 279-330.

[6] M. F. AtiYAh \& I. G. Macdonald - Introduction to commutative algebra, Addison-Wesley Publishing Co., Reading, Mass.-London-Don Mills, Ont., 1969.

[7] I. Ben YaAkov \& E. Hrushovski - "Towards a model theory of global fields", Lecture notes of Ehud Hrushovski at Institut Henri Poincaré, 2016.

[8] V. G. Berkovich - Spectral theory and analytic geometry over non-Archimedean fields, Mathematical Surveys and Monographs, vol. 33, American Mathematical Society, Providence, RI, 1990.

[9] Z. BŁocki \& S. KoŁOdZIEJ - "On regularization of plurisubharmonic functions on manifolds", Proceedings of the American Mathematical Society 135 (2007), no. 7, p. 2089-2093.

[10] E. Bombieri \& J. VAaler - "On Siegel's lemma", Inventiones Mathematicae 73 (1983), no. 1, p. 11-32.

[11] E. Bombieri - "The Mordell conjecture revisited", Annali della Scuola Normale Superiore di Pisa. Classe di Scienze. Serie IV 17 (1990), no. 4, p. 615-640. 
[12] E. Bombieri \& W. Gubler - Heights in Diophantine geometry, New Mathematical Monographs, vol. 4, Cambridge University Press, Cambridge, 2006.

[13] F. F. Bonsall \& J. Duncan - Numerical ranges. II, Cambridge University Press, New York-London, 1973, London Mathematical Society Lecture Notes Series, No. 10.

[14] T. BorEK - "Successive minima and slopes of Hermitian vector bundles over number fields", Journal of Number Theory 113 (2005), no. 2, p. 380-388.

[15] J.-B. Bost - "Périodes et isogénies des variétés abéliennes sur les corps de nombres (d'après D. Masser et G. Wüstholz)", Astérisque (1996), no. 237, p. Exp. No. 795, 4, 115-161, Séminaire Bourbaki, Vol. 1994/1995.

[16] J.-B. Bost - "Hermitian vector bundle and stability", Lecture at Oberwolfach conference "Algebraische Zahlentheorie", July 24th, 1997.

[17] J.-B. Bost - "Algebraic leaves of algebraic foliations over number fields", Publications Mathématiques. Institut de Hautes Études Scientifiques (2001), no. 93, p. $161-221$.

[18] J.-B. Bost - "Germs of analytic varieties in algebraic varieties: canonical metrics and arithmetic algebraization theorems", in Geometric aspects of Dwork theory. Vol. I, II, Walter de Gruyter GmbH \& Co. KG, Berlin, 2004, p. 371-418.

[19] J.-B. Bost - "Evaluation maps, slopes, and algebraicity criteria", in Proceedings of the International Congress of Mathematicians, Vol. II (Madrid, 2006) (Switzerland), European Mathematical Society, 2007, p. 537-562.

[20] J.-B. Bost \& A. ChamberT-Loir - "Analytic curves in algebraic varieties over number fields", in Algebra, arithmetic, and geometry: in honor of Yu. I. Manin. Vol. I, Progr. Math., vol. 269, Birkhäuser Boston, Inc., Boston, MA, 2009, p. 69-124.

[21] J.-B. Bost \& H. Chen - "Concerning the semistability of tensor products in Arakelov geometry", Journal de Mathématiques Pures et Appliquées. Neuvième Série 99 (2013), no. 4, p. 436-488.

[22] J.-B. Bost, H. Gillet \& C. Soulé - "Heights of projective varieties", Journal of the American Mathematical Society 7 (1994), no. 4, p. 903-1027.

[23] J.-B. Bost \& K. KÜNnEmAnn - "Hermitian vector bundles and extension groups on arithmetic schemes. I. Geometry of numbers", Advances in Mathematics 223 (2010), no. 3, p. 987-1106.

[24] S. BoucKsom - "Corps d'Okounkov (d'après Okounkov, Lazarsfeld-Mustaţă et Kaveh-Khovanskii)", Astérisque (2014), no. 361, p. Exp. No. 1059, vii, 1-41.

[25] S. Boucksom \& H. Chen - "Okounkov bodies of filtered linear series", Compositio Mathematica 147 (2011), no. 4, p. 1205-1229. 
[26] N. Bourbaki - Éléments de mathématique. Algèbre commutative. Chapitre 5 à 6, Hermann, Paris, 1964.

[27] N. Bourbaki - Elements of Mathematics. General topology. Chapiters 5 - 10, Springer-Verlag, Berlin, 1989.

[28] N. Bourbaki - Éléments de mathématique. Algèbre. Chapitres 1 à 3, Hermann, Paris, 1970.

[29] N. Bourbaki - Éléments de mathématique. Algèbre. Chapitres 4 à \%., Masson, Paris, 1981.

[30] N. Bourbaki - Espaces vectoriels topologiques. Chapitres 1 à 5, Masson, Paris, 1981.

[31] N. BourBAKI - Éléments de mathématique. algèbre commutative. Chapitre 1 à 4, Masson, Paris, 1985.

[32] J. I. Burgos Gil, A. Moriwaki, P. Philippon \& M. Sombra - "Arithmetic positivity on toric varieties", Journal of Algebraic Geometry 25 (2016), no. 2, p. 201272.

[33] J. I. Burgos Gil, P. Philippon \& M. Sombra - "Height of varieties over finitely generated fields", Kyoto Journal of Mathematics 56 (2016), no. 1, p. 13-32.

[34] A. Chambert-Loir \& A. Ducros - "Formes différentielles réelles et courants sur les espaces de berkovich", arXiv:1204.6277.

[35] H. Chen - "Positive degree and arithmetic bigness", 2008, arXiv:0803.2583

[36] H. CHEN - "Maximal slope of tensor product of Hermitian vector bundles", Journal of algebraic geometry 18 (2009), no. 3, p. 575-603.

[37] H. CHEn - "Arithmetic Fujita approximation", Annales Scientifiques de l'École Normale Supérieure. Quatrième Série 43 (2010), no. 4, p. 555-578.

[38] H. Chen - "Convergence des polygones de Harder-Narasimhan", Mémoires de la Société Mathématique de France 120 (2010), p. 1-120.

[39] H. CHEN - "Harder-Narasimhan categories", Journal of Pure and Applied Algebra 214 (2010), no. 2, p. 187-200.

[40] H. CHEN - "Majorations explicites de fonctions de hilbert-samuel géométrique et arithmétique", Mathematische Zeitschrift 279 (2015), no. 1, p. 99-137.

[41] H. Chen - "Okounkov bodies: an approach of function field arithmetic", to appear, 2017.

[42] H. Chen - "Sur la comparaison entre les minima et les pentes", in Publications mathématiques de Besançon. Algèbre et théorie des nombres, 2015, Publ. Math. Besançon Algèbre Théorie Nr., vol. 2018, Presses Univ. Franche-Comté, Besançon, 2018, p. 5-23. 
[43] H. Chen \& H. Ikoma - "On subfiniteness of graded linear series", 2017.

[44] H. Chen \& A. Moriwaki - "Extension property of semipositive invertible sheaves over a non-archimedean field", Annali della Scuola Normale Superiore di Pisa. Classe di Scienze. Serie V. (2017), to appear.

[45] H. Chen \& A. Moriwaki - "Sufficient conditions for the dirichlet property", arXiv:1704.01410, 2017.

[46] C. Chevalley - Introduction to the Theory of Algebraic Functions of One Variable, Mathematical Surveys, No. VI, American Mathematical Society, New York, N. Y., 1951.

[47] I. S. Cohen - "On non-Archimedean normed spaces", Nederl. Akad. Wetensch., Proc. 51 (1948), p. 693-698 = Indagationes Math. 10, 244-249.

[48] P. Corvaja \& U. Zannier - "Arithmetic on infinite extensions of function fields", Boll. Un. Mat. Ital. B (7) 11 (1997), no. 4, p. 1021-1038.

[49] C. Dellacherie \& P.-A. Meyer - Probabilités et potentiel, Hermann, Paris, 1975, Chapitres I à IV, Édition entièrement refondue, Publications de l'Institut de Mathématique de l'Université de Strasbourg, No. XV, Actualités Scientifiques et Industrielles, No. 1372.

[50] M. Demazure \& P. Gabriel - Groupes algébriques. Tome I: Géométrie algébrique, généralités, groupes commutatifs, Masson \& Cie, Éditeur, Paris, 1970, Avec un appendice Corps de classes local par Michiel Hazewinkel.

[51] M. Demazure \& P. Gabriel - Groupes algébriques. Tome I: Géométrie algébrique, généralités, groupes commutatifs, Masson \& Cie, Éditeur, Paris; NorthHolland Publishing Co., Amsterdam, 1970, Avec un appendice it Corps de classes local par Michiel Hazewinkel.

[52] N. Durov - "New approach to Arakelov geometry", arxiv:0704.2030, 2007.

[53] H. M. Edwards - Galois theory, Graduate Texts in Mathematics, vol. 101, Springer-Verlag, New York, 1984.

[54] D. Eisenbud - Commutative algebra, Graduate Texts in Mathematics, vol. 150, Springer-Verlag, New York, 1995, With a view toward algebraic geometry.

[55] G. FALTINGS - "Endlichkeitssätze für abelsche Varietäten über Zahlkörpern", Inventiones Mathematicae 73 (1983), no. 3, p. 349-366.

[56] G. Faltings - "Erratum: "Finiteness theorems for abelian varieties over number fields"', Inventiones Mathematicae $\mathbf{7 5}$ (1984), no. 2, p. 381.

[57] G. Faltings - "Calculus on arithmetic surfaces", Annals of Mathematics. Second Series 119 (1984), no. 2, p. 387-424. 
[58] G. Faltings - "Diophantine approximation on abelian varieties", Annals of Mathematics. Second Series 133 (1991), no. 3, p. 549-576.

[59] G. Faltings \& G. WÜstholz - "Diophantine approximations on projective spaces", Inventiones Mathematicae 116 (1994), no. 1-3, p. 109-138.

[60] É. Gaudron - "Pentes de fibrés vectoriels adéliques sur un corps globale", Rendiconti del Seminario Matematico della Università di Padova 119 (2008), p. 21-95.

[61] É. Gaudron - "Minima and slopes of rigid adelic spaces", (2018), preprint.

[62] É. Gaudron \& G. RÉmond - "Minima, pentes et algèbre tensorielle", Israel Journal of Mathematics 195 (2013), no. 2, p. 565-591.

[63] E. Gaudron \& G. RÉmond - "Corps de Siegel", Journal für die Reine und Angewandte Mathematik 726 (2017), p. 187-247.

[64] E. Gaudron \& G. RÉmond - "Espaces adéliques quadratiques", Mathematical Proceedings of the Cambridge Philosophical Society 162 (2017), no. 2, p. 211-247.

[65] H. Gillet, D. Rössler \& C. SoulÉ - "An arithmetic Riemann-Roch theorem in higher degrees", Université de Grenoble. Annales de l'Institut Fourier 58 (2008), no. 6 , p. 2169-2189.

[66] H. Gillet \& C. SoulÉ - "Intersection sur les variétés d'Arakelov", Comptes Rendus des Séances de l'Académie des Sciences. Série I. Mathématique 299 (1984), no. 12 , p. $563-566$.

[67] H. Gillet \& C. SoulÉ - "An arithmetic Riemann-Roch theorem", Inventiones Mathematicae 110 (1992), no. 3, p. 473-543.

[68] D. Grayson - "Reduction theory using semistability", Commentarii Mathematici Helvetici 59 (1984), no. 4, p. 600-634.

[69] A. Grothendieck - "Résumé de la théorie métrique des produits tensoriels topologiques", Resenhas do Instituto de Matemática e Estatística da Universidade de São Paulo 2 (1996), no. 4, p. 401-480, Reprint of Bol. Soc. Mat. São Paulo 8 (1953), 1-79.

[70] A. Grothendieck \& J. A. Dieudonné - Eléments de géométrie algébrique. I, Grundlehren der Mathematischen Wissenschaften, vol. 166, Springer-Verlag, Berlin, 1971.

[71] A. Grothendieck \& J. Dieudonné - "Éléments de géométrie algébrique. II. Étude globale élémentaire de quelques classes de morphismes", Institut des Hautes Études Scientifiques. Publications Mathématiques (1961), no. 8, p. 222.

[72] A. Grothendieck \& J. Dieudonné - "Éléments de géométrie algébrique. IV. Étude locale des schémas et des morphismes de schémas. II", Institut des Hautes Études Scientifiques. Publications Mathématiques (1965), no. 24, p. 231. 
[73] A. Grothendieck \& J. Dieudonné - "Éléments de géométrie algébrique. IV. Étude locale des schémas et des morphismes de schémas. III", Institut des Hautes Études Scientifiques. Publications Mathématiques (1966), no. 28, p. 255.

[74] A. Grothendieck \& J. Dieudonné - "Éléments de géométrie algébrique. IV. Étude locale des schémas et des morphismes de schémas IV", Institut des Hautes Études Scientifiques. Publications Mathématiques (1967), no. 32, p. 361.

[75] A. Grothendieck - "Produits tensoriels topologiques et espaces nucléaires", Memoirs of the American Mathematical Society No. 16 (1955), p. 140.

[76] W. Gubler - "Heights of subvarieties over $M$-fields", in Arithmetic geometry (Cortona, 1994), Sympos. Math., XXXVII, Cambridge Univ. Press, Cambridge, 1997, p. 190-227.

[77] W. Gubler - "The Bogomolov conjecture for totally degenerate abelian varieties", Inventiones Mathematicae 169 (2007), no. 2, p. 377-400.

[78] W. Gubler - "A guide to tropicalizations", in Algebraic and combinatorial aspects of tropical geometry, Contemp. Math., vol. 589, Amer. Math. Soc., Providence, RI, 2013, p. 125-189.

[79] W. Gubler \& K. Künnemann - "Positivity properties of metrics and deltaforms", arXiv:1509.09079.

[80] P. R. Halmos - Measure theory, Springer-Verlag, New York, 1974, Graduate Texts in Mathematics, No. 18.

[81] G. HARDER \& M. S. NARASIMHAN - "On the cohomology groups of moduli spaces of vector bundles on curves", Mathematische Annalen 212 (1974/1975), p. $215-248$.

[82] R. Hartshorne - Algebraic geometry, Springer-Verlag, New York, 1977, Graduate Texts in Mathematics, No. 52.

[83] M. Henk - "Löwner-John ellipsoids", Documenta Mathematica (2012), no. Extra volume: Optimization stories, p. 95-106.

[84] P. HriluAC - "Heights and Arakelov's intersection theory", American Journal of Mathematics 107 (1985), no. 1, p. 23-38.

[85] E. Hrushovski - "A logic for global fields", Lecture at Séminaire d'Arithmétique et de Géométrie Algébrique, May 31st, 2016.

[86] A. W. Ingleton - "The Hahn-Banach theorem for non-Archimedean valued fields", Proc. Cambridge Philos. Soc. 48 (1952), p. 41-45.

[87] J. L. W. V. JENSEN - "Sur un nouvel et important théorème de la théorie des fonctions", Acta Mathematica 22 (1899), no. 1, p. 359-364. 
[88] F. JoHN - "Extremum problems with inequalities as subsidiary conditions", in Studies and Essays Presented to R. Courant on his 60th Birthday, January 8, 1948, Interscience Publishers, Inc., New York, N. Y., 1948, p. 187-204.

[89] S. KaKUTAni - "Some characterizations of Euclidean space", Japanese Journal of Mathematics 16 (1939), p. 93-97.

[90] O. Kallenberg - Foundations of modern probability, Probability and its Applications (New York), Springer-Verlag, New York, 1997.

[91] K. Kaveh \& A. G. Khovanskit - "Algebraic equations and convex bodies", à paraître dans Perspectives in Analysis, Topology and Geometry, Birkhäuser series "Progress in Mathematics", 2009.

[92] G. R. KEMPF - "Instability in invariant theory", Annals of Mathematics (1978), no. 108 , p. 299-316.

[93] S. L. Kleiman - "Misconceptions about $K_{x}$ ", L'Enseignement Mathématique. Revue Internationale. IIe Série 25 (1979), no. 3-4, p. 203-206 (1980).

[94] A. KLenke - Probability theory, second ed., Universitext, Springer, London, 2014, A comprehensive course.

[95] M. KLIMEK - Pluripotential theory, London Mathematical Society Monographs. New Series, vol. 6, The Clarendon Press, Oxford University Press, New York, 1991, Oxford Science Publications.

[96] K. Kuratowski \& C. Ryll-Nardzewski - "A general theorem on selectors", Bulletin de l'Académie Polonaise des Sciences. Série des Sciences Mathématiques, Astronomiques et Physiques 13 (1965), p. 397-403.

[97] R. Lazarsfeld \& M. Mustaţă - "Convex bodies associated to linear series", Annales Scientifiques de l'École Normale Supérieure. Quatrième Série 42 (2009), no. 5 , p. $783-835$.

[98] M. Maruyama - "The theorem of Grauert-Mülich-Spindler", Mathematische Annalen 255 (1981), no. 3, p. 317-333.

[99] R. C. MAson - Diophantine equations over function fields, London Mathematical Society Lecture Note Series, vol. 96, Cambridge University Press, Cambridge, 1984.

[100] H. Matsumura - Commutative algebra, second ed., Mathematics Lecture Note Series, vol. 56, Benjamin/Cummings Publishing Co., Inc., Reading, Mass., 1980.

[101] R. B. MCFEAT - "Geometry of numbers in adele spaces", Dissertationes Math. Rozprawy Mat. 88 (1971), p. 49.

[102] A. MORIWAKI - "Arithmetic height functions over finitely generated fields", Inventiones Mathematicae 140 (2000), no. 1, p. 101-142. 
[103] A. MORIWAKI - "The canonical arithmetic height of subvarieties of an abelian variety over a finitely generated field", Journal für die Reine und Angewandte Mathematik 530 (2001), p. 33-54.

[104] A. MorIWAKI - "Diophantine geometry viewed from Arakelov geometry [translation of Sūgaku 54 (2002), no. 2, 113-129]", Sugaku Expositions 17 (2004), no. 2, p. 219-234, Sugaku Expositions.

[105] A. MORIWAKI - "Zariski decompositions on arithmetic surfaces", Publications of the Research Institute for Mathematical Sciences 48 (2012), no. 4, p. 799-898.

[106] A. MORIWAKI - "Arakelov geometry", 244 (2014), p. x+285, Translated from the 2008 Japanese original.

[107] A. MORIWAKI - "Semiample invertible sheaves with semipositive continuous hermitian metrics", Algebra \& Number Theory 9 (2015), no. 2, p. 503-509.

[108] A. MorIWAKI - "Adelic divisors on arithmetic varieties", Memoirs of the American Mathematical Society 242 (2016), no. 1144, p. v+122.

[109] D. Mumford, J. Fogarty \& F. Kirwan - Geometric invariant theory, third ed., Ergebnisse der Mathematik und ihrer Grenzgebiete (2) [Results in Mathematics and Related Areas (2)], vol. 34, Springer-Verlag, Berlin, 1994.

[110] J. NEUKIRCH - Algebraic number theory, Grundlehren der Mathematischen Wissenschaften, vol. 322, Springer-Verlag, Berlin, 1999, Translated from the 1992 German original and with a note by Norbert Schappacher, With a foreword by G. Harder.

[111] A. Okounkov - "Brunn-Minkowski inequality for multiplicities", Inventiones Mathematicae 125 (1996), no. 3, p. 405-411.

[112] T. PARThASARATHY - Selection theorems and their applications, Lecture Notes in Mathematics, Vol. 263, Springer-Verlag, Berlin-New York, 1972.

[113] C. Perez-Garcia \& W. H. Schikhof - Locally convex spaces over nonArchimedean valued fields, Cambridge Studies in Advanced Mathematics, vol. 119, Cambridge University Press, Cambridge, 2010.

[114] V. V. Petrov - Sums of independent random variables, Springer-Verlag, New York-Heidelberg, 1975, Translated from the Russian by A. A. Brown, Ergebnisse der Mathematik und ihrer Grenzgebiete, Band 82.

[115] P. Philippon - "Critères pour l'indépendance algébrique", Institut des Hautes Études Scientifiques. Publications Mathématiques (1986), no. 64, p. 5-52.

[116] P. PhiLIPPON - "Sur des hauteurs alternatives. II", Université de Grenoble. Annales de l'Institut Fourier 44 (1994), no. 4, p. 1043-1065.

[117] S. Ramanan \& A. Ramanathan - "Some remarks on the instability flag", The Tohoku Mathematical Journal. Second Series 36 (1984), no. 2, p. 269-291. 
[118] H. RANDRIAmbololona - "Harder-narasimhan theory for linear codes", 2017.

[119] M. RAYNAUd - "Fibrés vectoriels instables - applications aux surfaces (d'après Bogomolov)", in Algebraic surfaces (Orsay, 1976-78), Lecture Notes in Math., vol. 868, Springer, Berlin, 1981, p. 293-314.

[120] G. Rousseau - "Immeubles sphériques et théorie des invariants", Comptes Rendus Mathématique. Académie des Sciences. Paris 286 (1978), no. 5, p. A247$\mathrm{A} 250$.

[121] D. Roy \& J. L. Thunder - "An absolute Siegel's lemma", Journal für die Reine und Angewandte Mathematik 476 (1996), p. 1-26.

[122] D. Roy \& J. L. Thunder - "Addendum and erratum to: "An absolute Siegel's lemma"", Journal für die Reine und Angewandte Mathematik 508 (1999), p. 47-51.

[123] W. Rudin - Functional analysis, McGraw-Hill Book Co., New York-DüsseldorfJohannesburg, 1973, McGraw-Hill Series in Higher Mathematics.

[124] R. A. RYAN - Introduction to tensor products of Banach spaces, Springer Monographs in Mathematics, Springer-Verlag London, Ltd., London, 2002.

[125] J. J. SACCOMAN - "On the extension of linear operators", International Journal of Mathematics and Mathematical Sciences 28 (2001), no. 10, p. 621-623.

[126] K.-S. Saito, M. Kato \& Y. Takahashi - "Absolute norms on $\mathbf{C}^{n \text { ", Journal }}$ of Mathematical Analysis and Applications 252 (2000), no. 2, p. 879-905.

[127] W. H. SchiкhоF - Ultrametric calculus, Cambridge Studies in Advanced Mathematics, vol. 4, Cambridge University Press, Cambridge, 2006, An introduction to $p$-adic analysis, Reprint of the 1984 original [MR0791759].

[128] S. S. Shatz - "The decomposition and specialization of algebraic families of vector bundles", Compositio Mathematica 35 (1977), no. 2, p. 163-187.

[129] C. Soulé - "Géométrie d'Arakelov et théorie des nombres transcendants", Astérisque (1991), no. 198-200, p. 355-371 (1992), Journées Arithmétiques, 1989 (Luminy, 1989).

[130] C. SoulÉ - "Hermitian vector bundles on arithmetic varieties", in Algebraic geometry - Santa Cruz 1995, Proc. Sympos. Pure Math., vol. 62, Amer. Math. Soc., Providence, RI, 1997, p. 383-419.

[131] U. StuhlER - "Eine Bemerkung zur Reduktionstheorie quadratischen Formen", Archiv der Mathematik 27 (1976), p. 604-610.

[132] L. Szpiro, E. Ullmo \& S. Zhang - "équirépartition des petits points", Inventiones Mathematicae 127 (1997), no. 2, p. 337-347.

[133] L. SzPIRo - "Degrés, intersections, hauteurs", Astérisque (1985), no. 127, p. 1128, Seminar on arithmetic bundles: the Mordell conjecture (Paris, 1983/84). 
[134] F. ТАкемото - "Stable vector bundles on algebraic surfaces", Nagoya Mathematical Journal 47 (1972), p. 29-48.

[135] J. L. THUNDER - "An adelic Minkowski-Hlawka theorem and an application to Siegel's lemma", Journal für die Reine und Angewandte Mathematik 475 (1996), p. $167-185$.

[136] B. Totaro - "Tensor products in p-adic Hodge theory", Duke Mathematical Journal 83 (1996), no. 1, p. 79-104.

[137] E. Ullmo - "Positivité et discrétion des points algébriques des courbes", Annals of Mathematics. Second Series 147 (1998), no. 1, p. 167-179.

[138] H. E. VAughan - "On locally compact metrisable spaces", Bulletin of the American Mathematical Society 43 (1937), no. 8, p. 532-535.

[139] P. Vouta - "Siegel's theorem in the compact case", Annals of Mathematics. Second Series 133 (1991), no. 3, p. 509-548.

[140] A. WEIL - "Sur la théorie du corps de classes", Journal of the Mathematical Society of Japan 3 (1951), p. 1-35.

[141] H. WEYL - The classical groups, Princeton Landmarks in Mathematics, Princeton University Press, Princeton, NJ, 1997, Their invariants and representations, Fifteenth printing, Princeton Paperbacks.

[142] K. YAMAKI - "Strict supports of canonical measures and applications to the geometric Bogomolov conjecture", Compositio Mathematica 152 (2016), no. 5, p. 9971040.

[143] K. YAMAKI - "Non-density of small points on divisors on abelian varieties and the Bogomolov conjecture", Journal of the American Mathematical Society 30 (2017), no. 4, p. 1133-1163.

[144] J. A. YAN - Lectures in measure theory (in chinese), Lecture Notes of Chinese Academy of Sciences, Science Press, Beijing, 2004, Second edition.

[145] X. YUAN - "On volumes of arithmetic line bundles", Compositio Mathematica 145 (2009), no. 6, p. 1447-1464.

[146] X. YUAN - "Volumes of arithmetic Okounkov bodies", Mathematische Zeitschrift 280 (2015), no. 3-4, p. 1075-1084.

[147] O. Zariski - "A new proof of Hilbert's Nullstellensatz", Bull. Amer. Math. Soc. 53 (1947), p. 362-368.

[148] S. ZHANG - "Positive line bundles on arithmetic varieties", Journal of the American Mathematical Society 8 (1995), no. 1, p. 187-221.

[149] S. ZHANG - "Small points and adelic metrics", Journal of Algebraic Geometry 4 (1995), no. 2, p. 281-300. 
[150] S. ZHANG - "Equidistribution of small points on abelian varieties", Annals of Mathematics. Second Series 147 (1998), no. 1, p. 159-165. 